This document was too large to scan as a whole document, therefore it required breaking into smaller sectlons.

Document number: $50-W / 4-T I-764$

Section_ of 2

Titlo: Yagard Analyie) Oatabase

Date: $8 / 12 / 97$ Revision:

Originator: qumi, $\lg$

Co: $h Q E S H$

Recipient:

Co:

References: $\varepsilon$ N -629254 


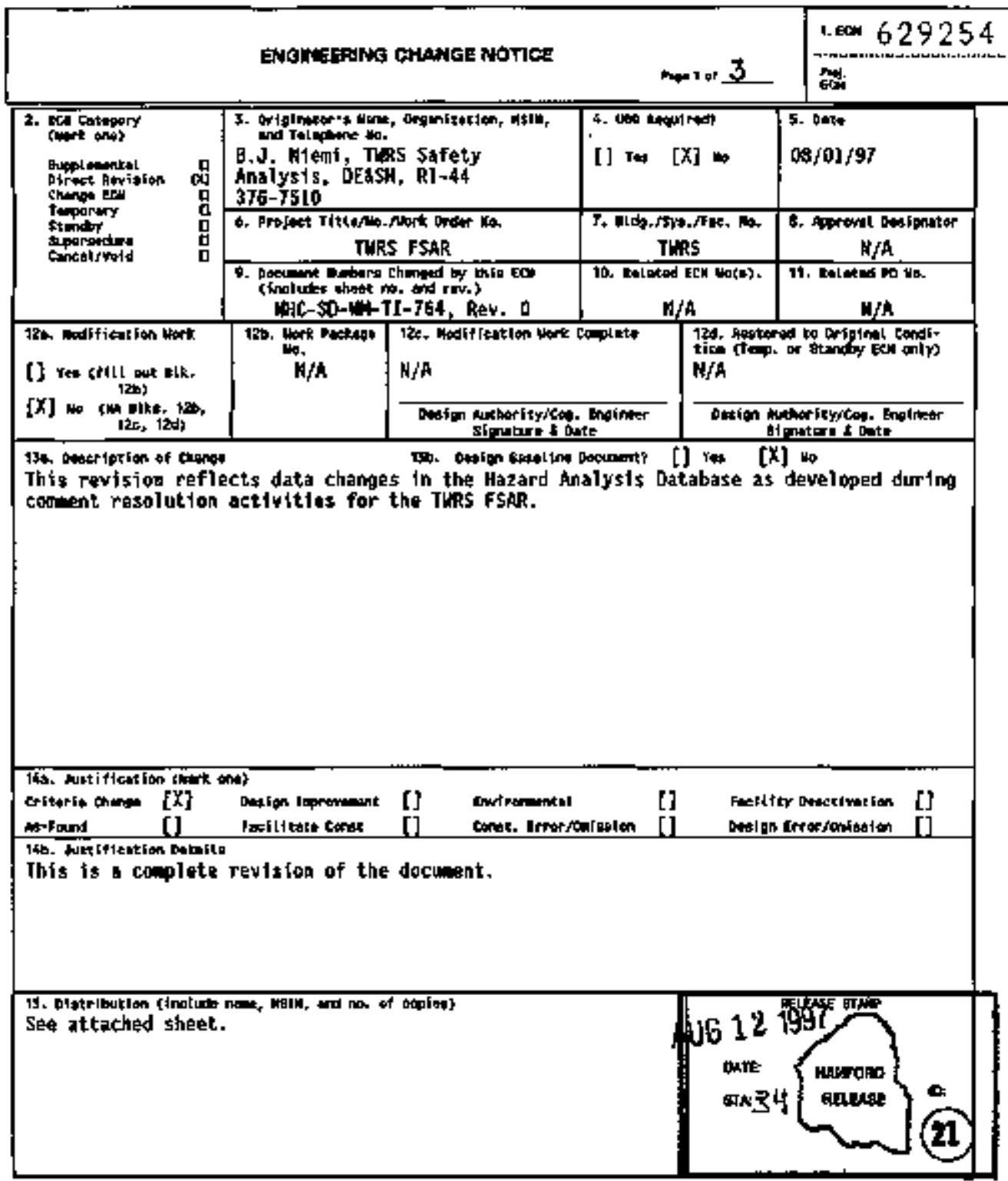




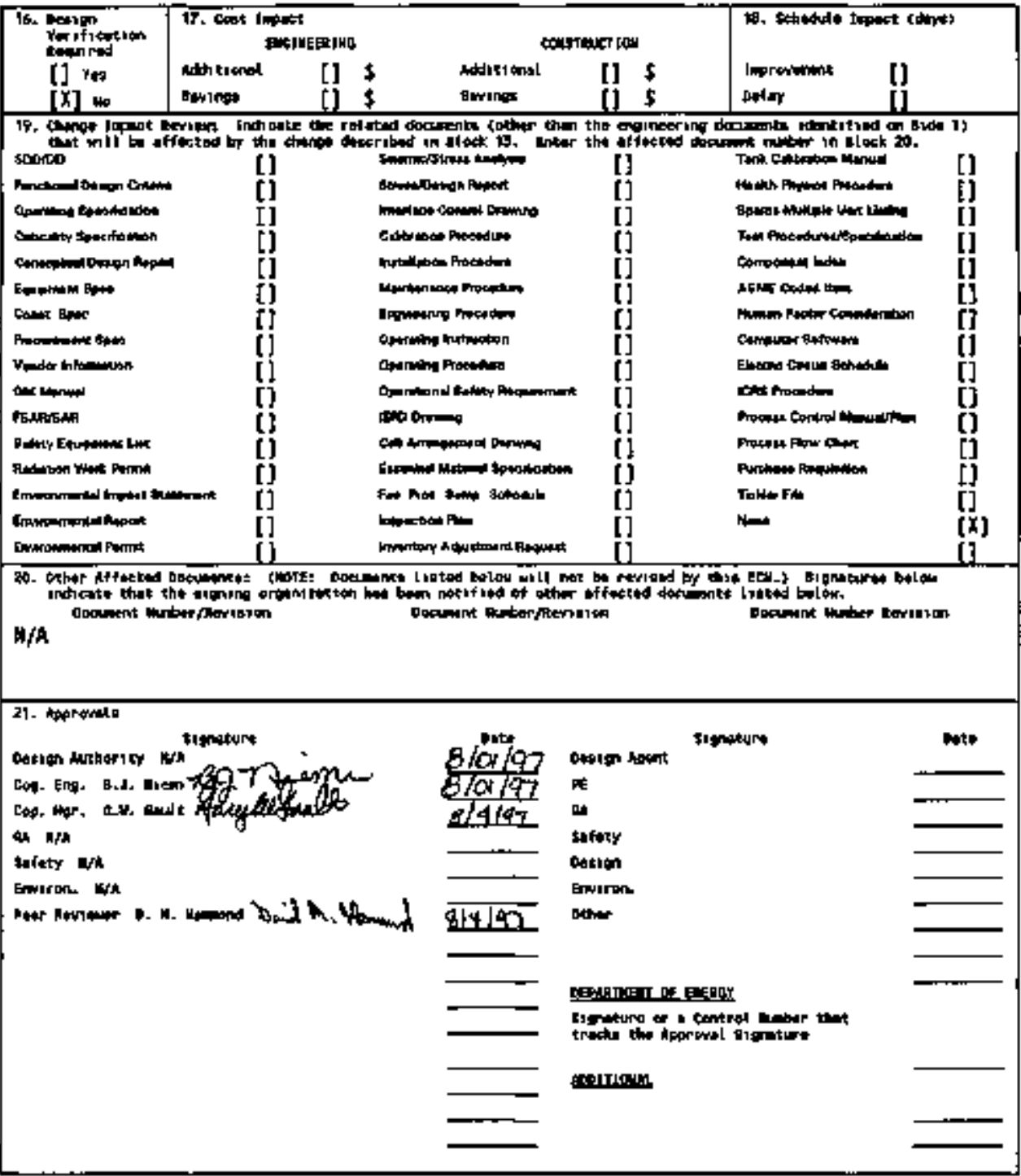


CHECRISI FOA PEER REVIEY

Oocument Rerioned: HaF-SD-lin-TI-T64, Hazard Analysis Databasa Raport

Scope of Revien: Peer Révitow

Iat Ho H

[]] [] [י] *

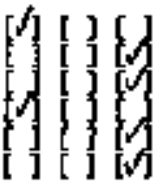

Proviaus reviaws cemplets and cowar analysis, up to scope of this review, with no gaps.

Probien completely defind.

Accldent stenarios developed in a clear and logical manar.

Hecussary assumptions explicitly steted and supperted.

Computer todes and data fiteg doclmented.

Bata used in criculations expileitiy stated in doculant.

Dato chacked for consistency with orlginal sasrce inforintion as applicable.

[ ] [ ] [ $\sqrt{ }]$

[ ] [ ] [

[ ] [ ] [V]

$\{3\}$ 约

[ ] [ ] [

$\left[\begin{array}{l}1 \\ {[}\end{array}\right][\sqrt[V]{ }]$

Mathernatical deryvations checked fncluding dimentional cons istency of results.

Hodals appropriate and uged within range of validity or use outside range of established validity Justified.

Hand colculations checked for errors. Sproadshatt results should be troated exactly the sathe as hand calculations. Software input correct and consistent with documint revlewed, Softmare output consistent with inpkt and with results reported in docunant rerimesd. Liatts/criteria/gutdelines app1ied to analygis results are appropriate and referenced. Lindts/criteria/guldel ines checked agalnst refementes.

Safoty uargins cansistent rith good eng iseering practices. Conclusions consistant wth analytical results and applicable 11utts.

[d] [ ] ]

[ ] : ] [4]

[ ] [M Results and conclusions address all points required in the problea statement.

Foralat consistent with approprlote Rage Rogulary Guide or othar standards

[ $A$ I] [ ] Docanent approved.

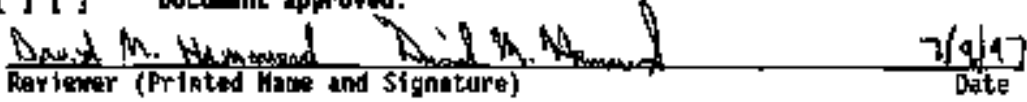

* Any calculations, coments, or notes generated as part of this rowien should be signad, dated and attached ta this thecklist. Such paterial should be labeled and racordad in such a eanner as to ba intelligible to a technically qualified third party. 


\section{Hazard Analysis Database Report}

D.J. Weat

DESS Hanford, Inc, Richl and, wh 99352

U.S. Department of Enorgy Contract DE-ACOS6-96RL]3200

EOT/ECA: 629254

Org cods: 2ni70

的负 Code: Eu3120071

Uc: 2030

Charge Code: E61380

Total Pages: 912

Kay Hords: Hazard Analysis, Safety Analysis, Accident inalysis, Hazard Evaluation, HazOp, PHA, hot-If Mnalygis, Ralational Databasa, Databose, FSAR.

Abstract: This document describes and defines the Hazard inalysis Database for the Tank kaste Remediation Syttell final Safety Analysis Report.

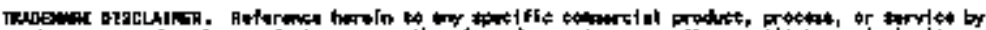

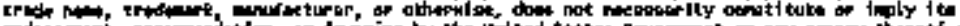

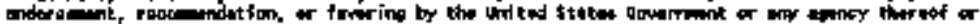

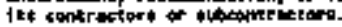

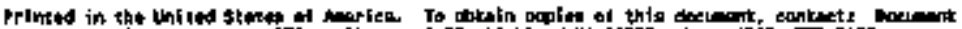

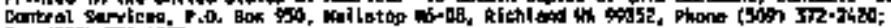

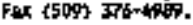
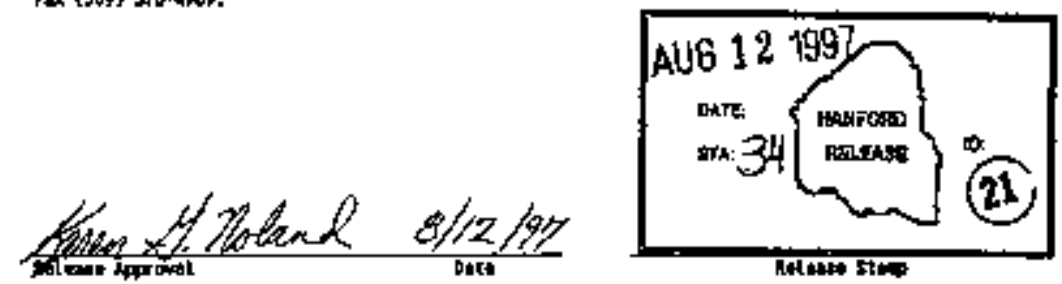

Approved for Putric Release 


\section{RECORD OF REMSOH}

(z) r|țl:

Hazard Analysis Datubase Report

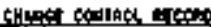

\begin{tabular}{|c|c|c|c|}
\hline \multirow{2}{*}{ (1) Aevilsian } & \multirow{2}{*}{ 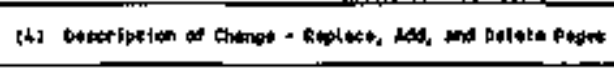 } & \multicolumn{2}{|c|}{ folborized for Prlside } \\
\hline & & (5) shellotis & 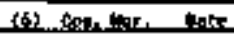 \\
\hline 0 & (7) Original 1ssure (EOT 619625) & $\begin{array}{l}\text { B.J. Migाni } \\
1] 15 / 97\end{array}$ & $\begin{array}{l}\text { 6.M. Gault } \\
\text { 11:isfor }\end{array}$ \\
\hline ] 0 & Replace eat1re docunent (ECN 629254) & B.J Jienj & AH. Gyult gid \\
\hline & & & \\
\hline & & & \\
\hline & & & \\
\hline & & & \\
\hline & & & \\
\hline & & & \\
\hline & & & \\
\hline & & & - \\
\hline & & & \\
\hline & & & \\
\hline & & & \\
\hline & & & \\
\hline & & & \\
\hline & & & \\
\hline & & & \\
\hline & & & \\
\hline & & & \\
\hline & & & \\
\hline & & & \\
\hline & & & \\
\hline & & & \\
\hline & & & \\
\hline & & & \\
\hline & & & \\
\hline & & & \\
\hline & & & \\
\hline & & & \\
\hline & & & \\
\hline & & & \\
\hline & & & \\
\hline & & & \\
\hline & & & \\
\hline
\end{tabular}


HNE-50-HA4T-704, RW, 1

HAZARD ANALYSIS DATABASE REPORT 
HWF-SD-HANTR-704, RAY, 1

Thss pege Interithanally left tank. 


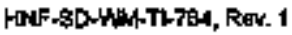

conTENTS

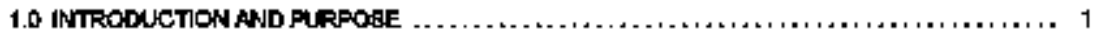

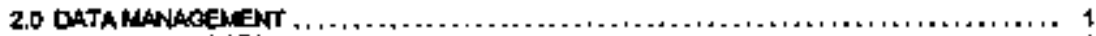

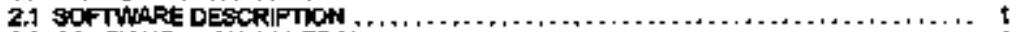

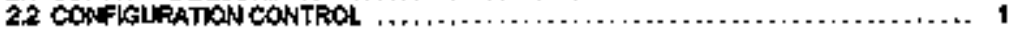

3.0 HAZARD EVALUATION DATADAEE TAELES AND FED OESCRPTKONB , . . . . . . . ..... 2

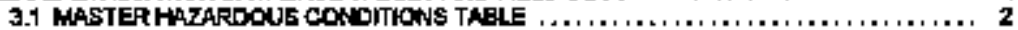

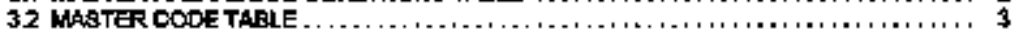

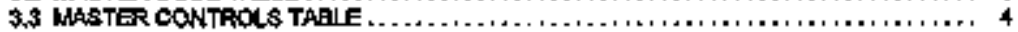

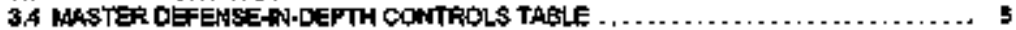

3.5 CONTRO

3.8 PROCESS HATORY TAELE $, \ldots, \ldots, \ldots, \ldots, \ldots, \ldots, \ldots, \ldots \ldots \ldots \ldots, \ldots$

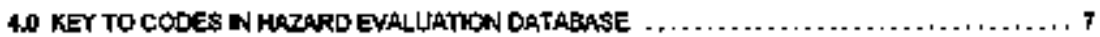

50 MAZARD TOPOGRAFHY DATABASE TAELES AND FELD DESCAPTIOWS ............ 7

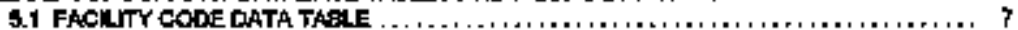

$\$ 2$ TOPOGRAFHY DATA TAE

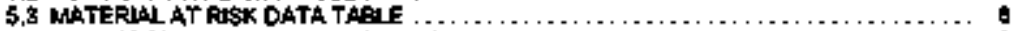

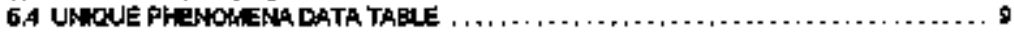

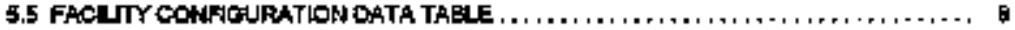

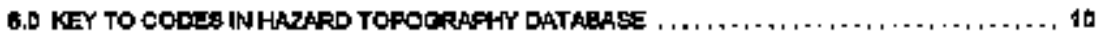

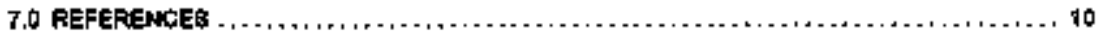

\section{APperioteds}

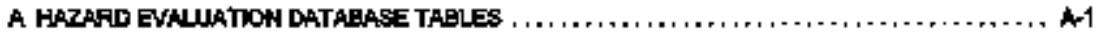

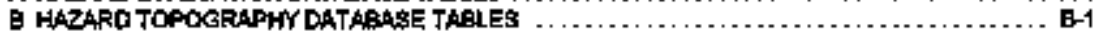

\section{LST OF THBLES}

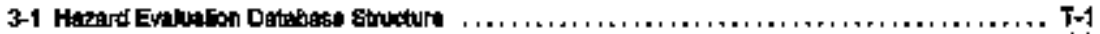

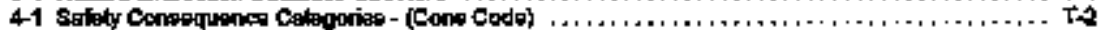

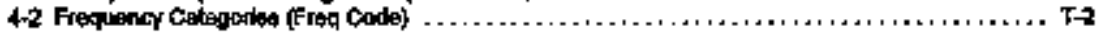

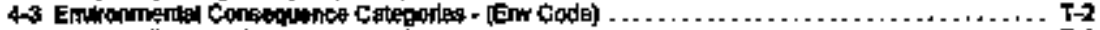

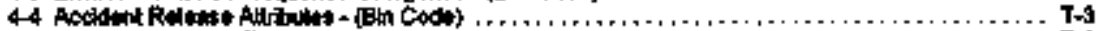

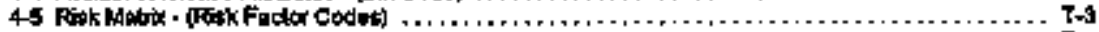

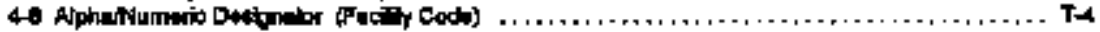

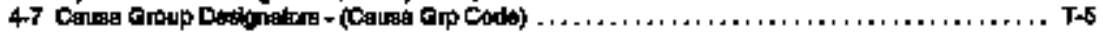

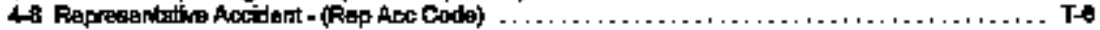

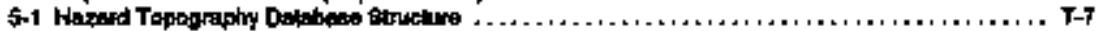

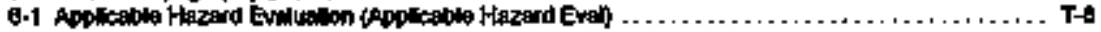




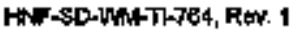

\section{UST of TERUt'}

\begin{tabular}{|c|c|}
\hline 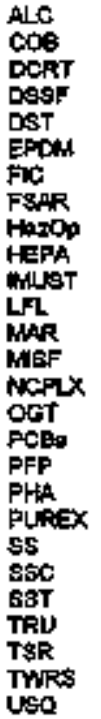 & 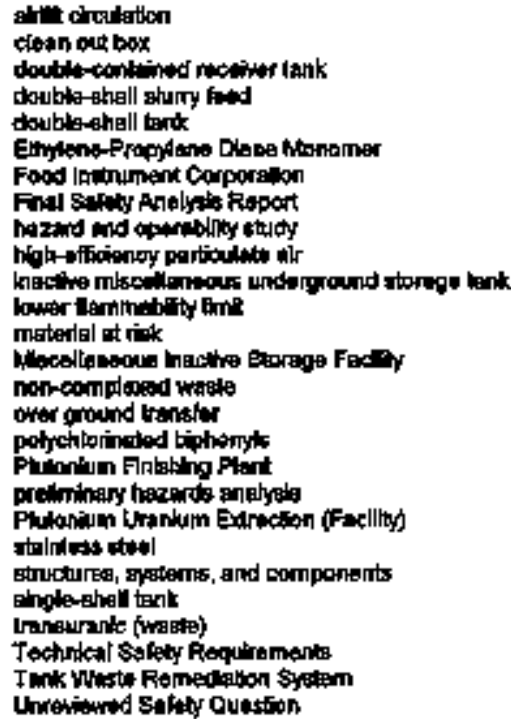 \\
\hline
\end{tabular}




\section{0 inTtRóUCTTON AND PURPOSE}

This documan descrlbeg, Ldertities, and dannes the coxtendy of the TwRS FBaR Hazard

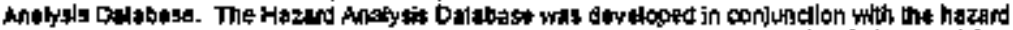

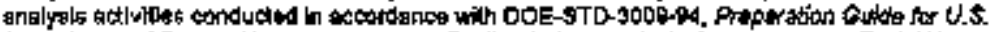

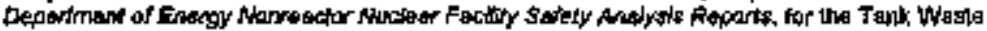

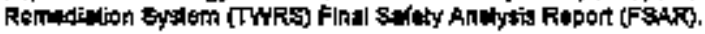

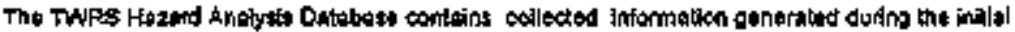

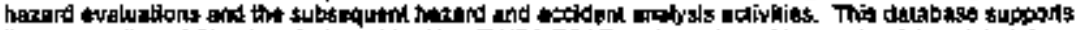

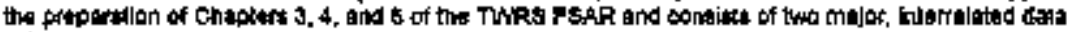
6ets:

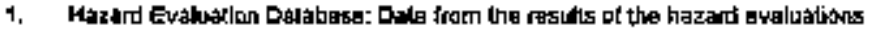

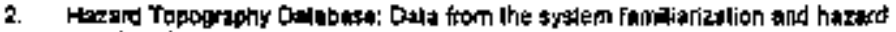
idęriliticaklon.

\subsection{DATA MRNAOERAENT}

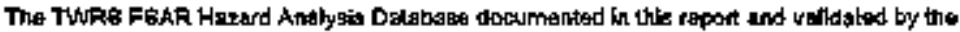

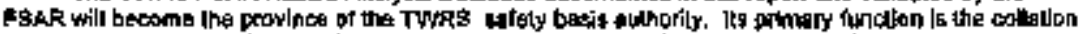

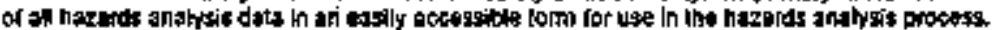

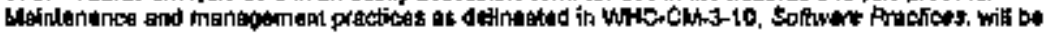

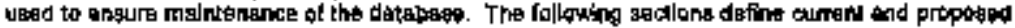
minimum confouratlon conirols ond descilbe the curserd dils set sothware package.

\section{GOFTHATE DEgCRAPTON}

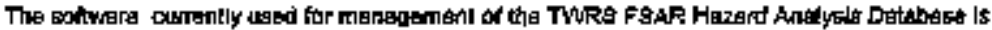

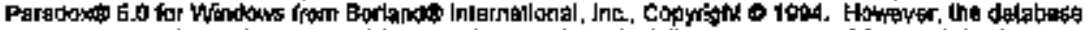

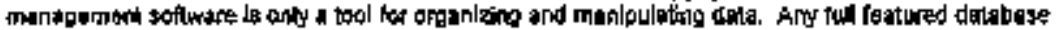

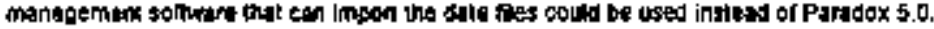

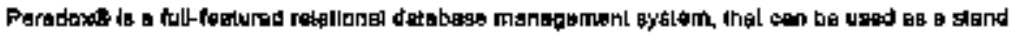
uone s) Anotyats wiviles. Parajox is belag used as a etand alone gystem).

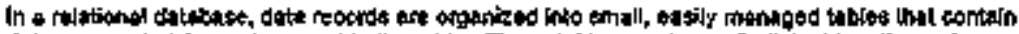

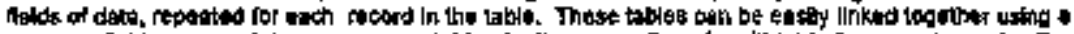

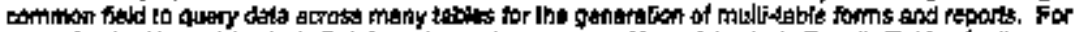

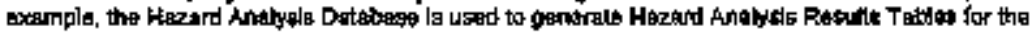
TULARS FEAR.

\subsection{CONHFURATHON CONTROL}

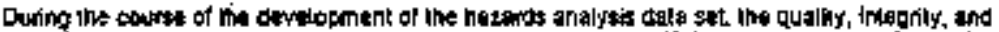

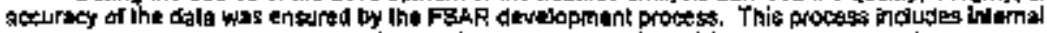

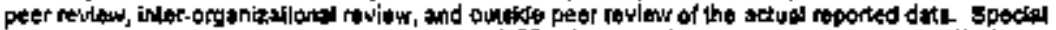

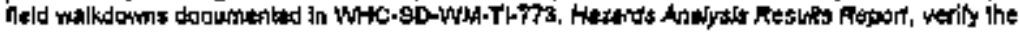




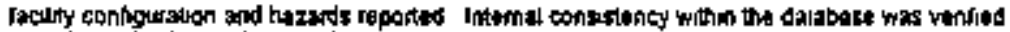
ponodictilly by the analyste pertoming the work

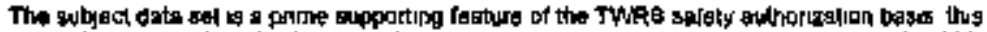

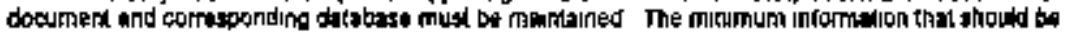

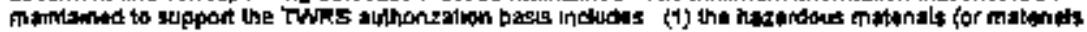

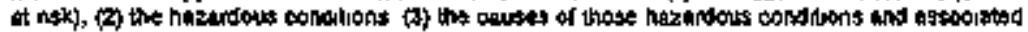

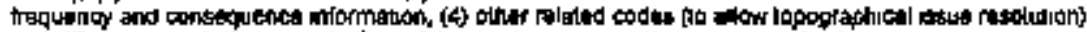

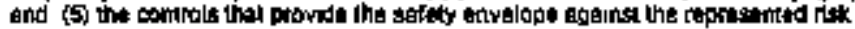

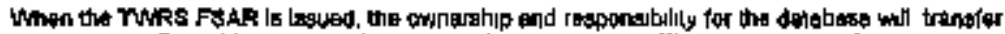

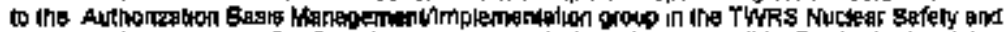

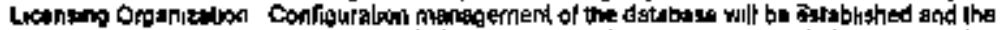

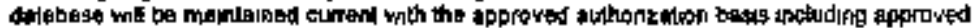

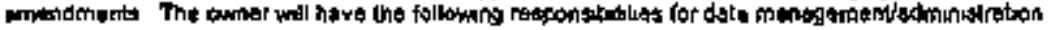

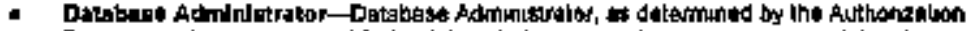

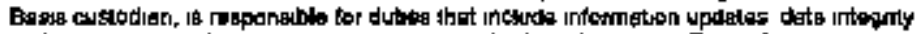

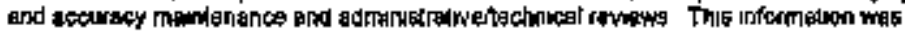

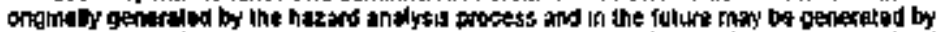

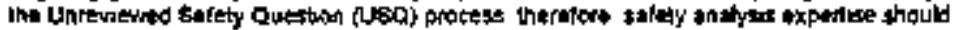

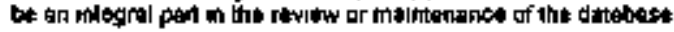

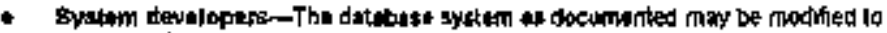
ecesmmodat tulute uph

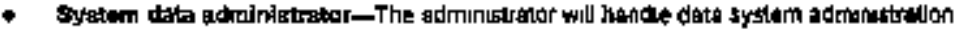

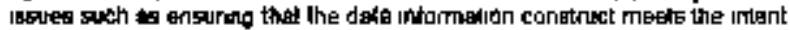

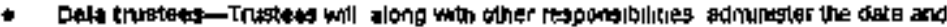
detine the data and its source

\section{IO HAZARD EVALUATION DATABASH TABLES AND FAELO DESERIPTIONO}

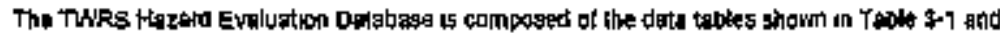

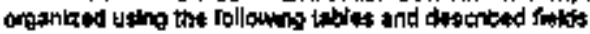

\subsection{BASTER HAZARDOUS CONOITLOAS TARU E [AppendIX A.} Tabio A-i\}

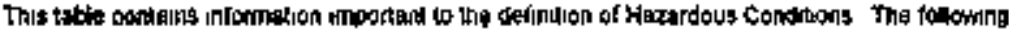

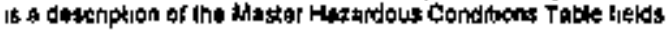

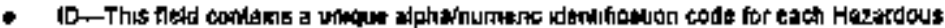

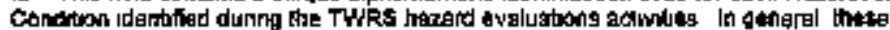

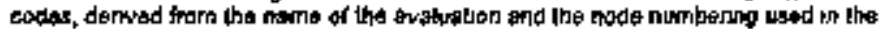

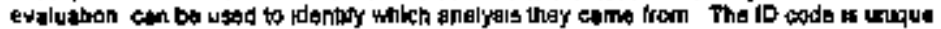

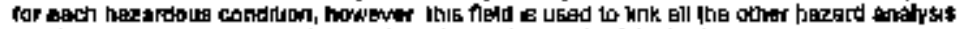
dalabage labies logether end as such se wentical in wach of lhe lables

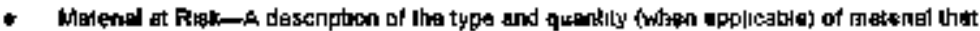
may be effeded by the ocoxisexice of a Hazardour condtion 
- Hezerdous Conditun-A desemptron of the Harerdaus Canditon sagocalad with the unique

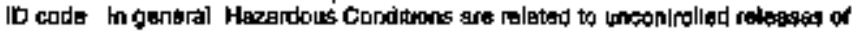

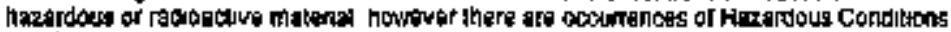

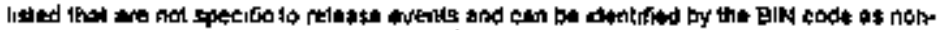

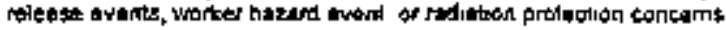

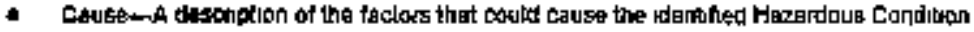

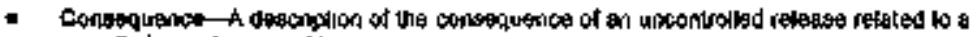
spectilo hazarions conditem

\section{MBSTLR GCOE TAELLF (APpendIX A, Tables A-z And A + J)}

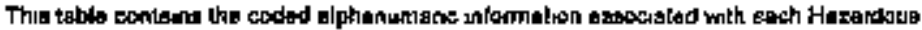

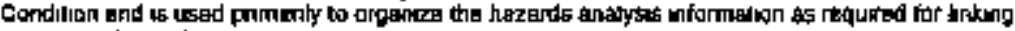
sortung and raporting

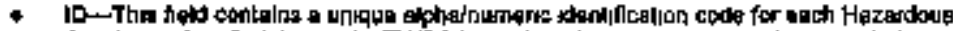

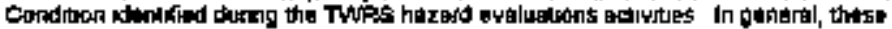

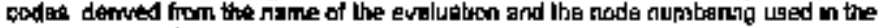

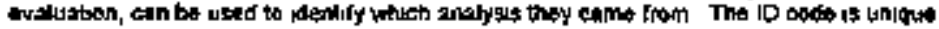

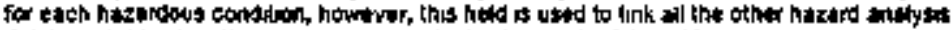

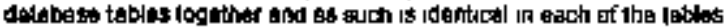

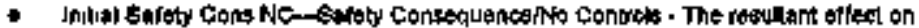

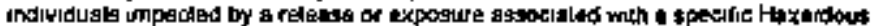

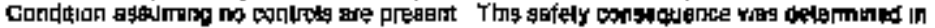

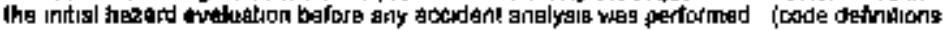
If Toble 4 1)

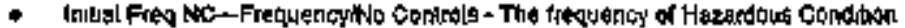

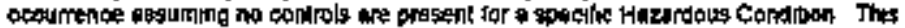

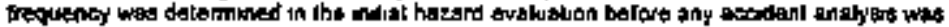

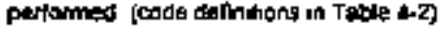

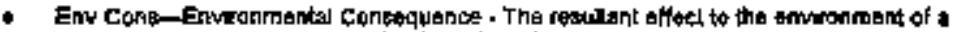

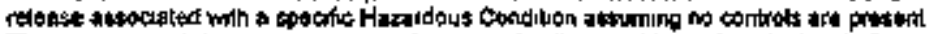

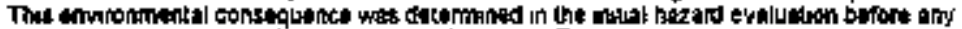
accidant anthysas wat porfomed (cotle dafinilions in Toble 4-3)

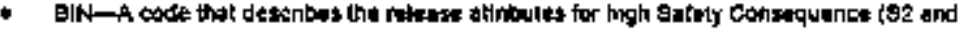

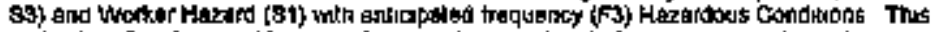

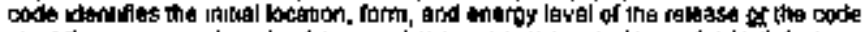

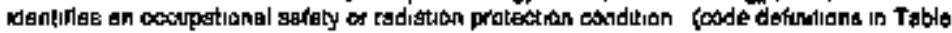
4) 4)

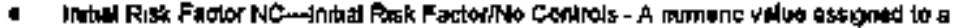

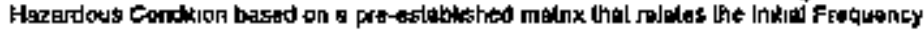

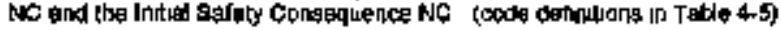

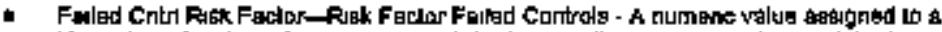

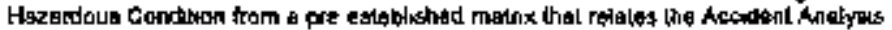

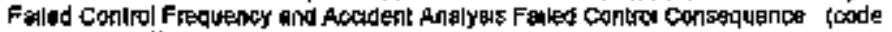
dofinilons in Topbe 4-5) 


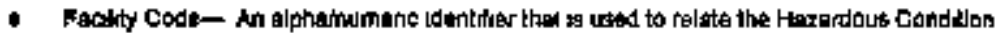

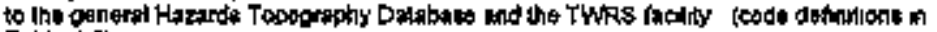
Table 45]

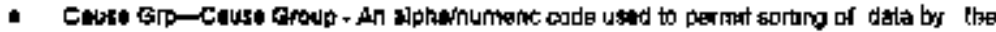

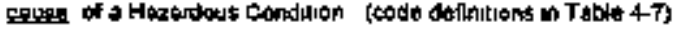

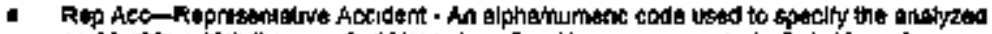

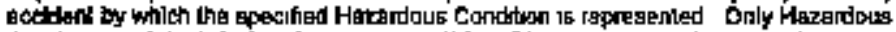

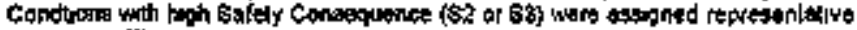

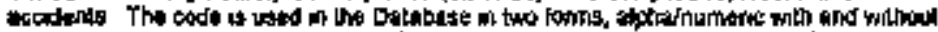

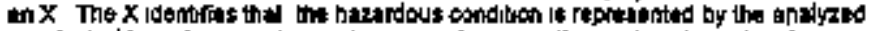

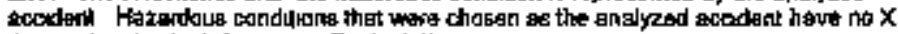

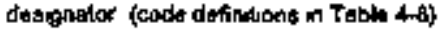

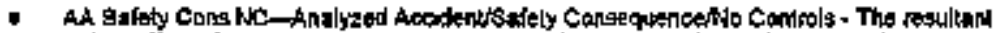

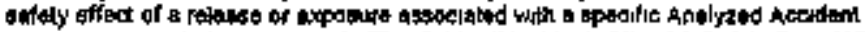

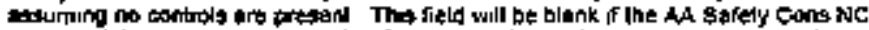

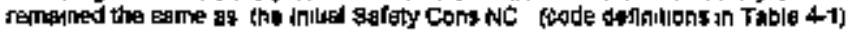

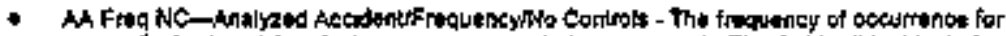

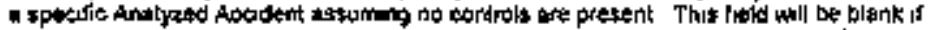

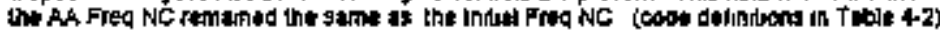

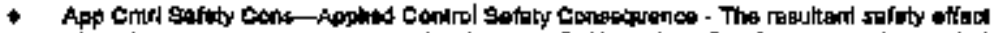

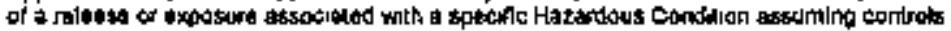

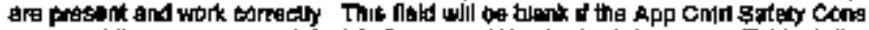

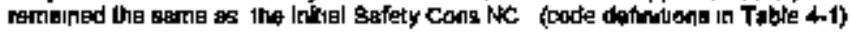

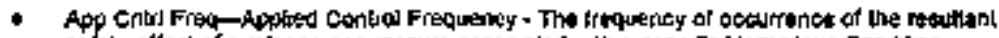

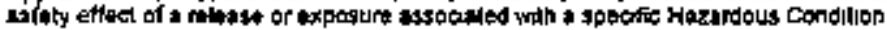

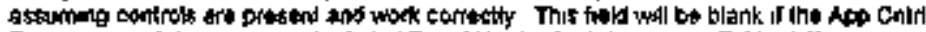

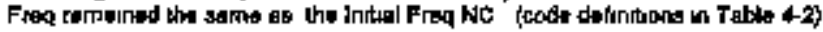

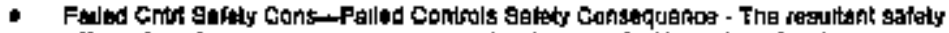

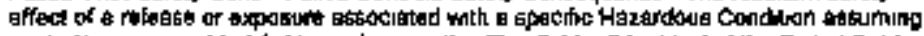

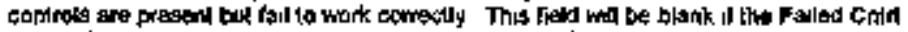

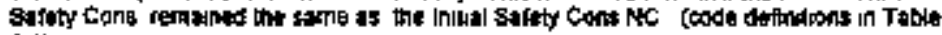
4.1)

- Faled Cntri Fraq-Falled Candrol Frequancy - The trequency of accurrence of line

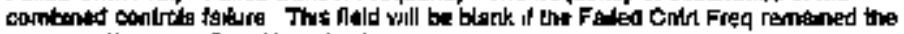

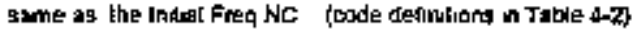

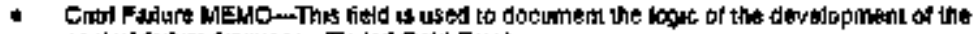
controd fallera traduency (Faled Comit Frodi

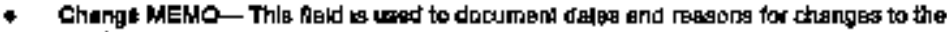
delabase

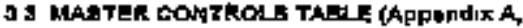 Table A-A\}}

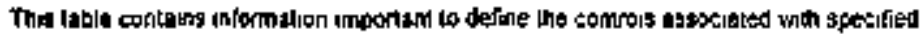

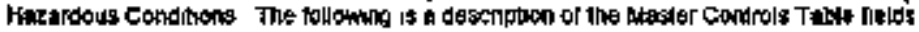




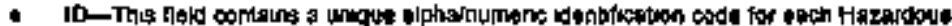

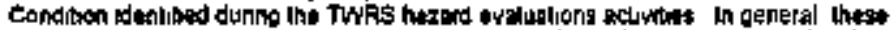

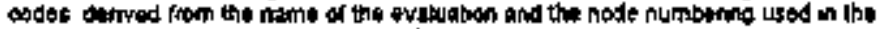

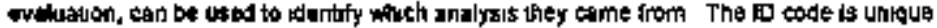

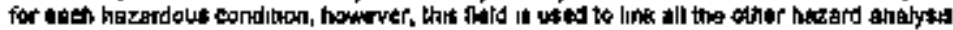

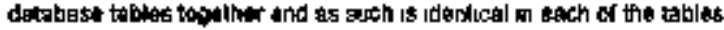

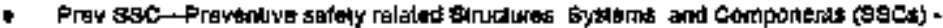

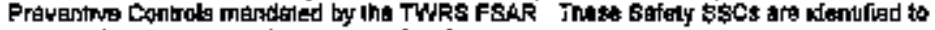

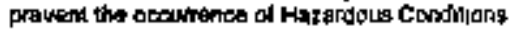

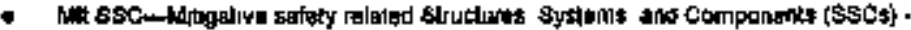

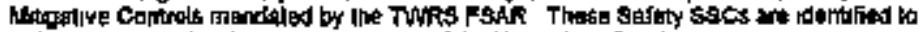
reduce the severty of the constaquence of the kazatious Condilan

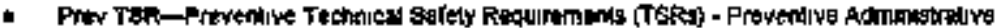

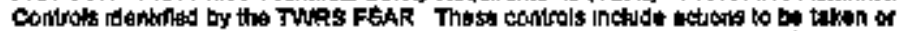

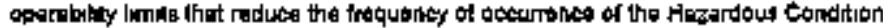

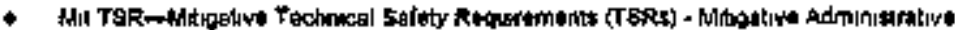

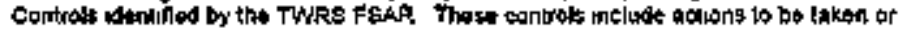

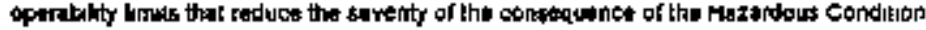

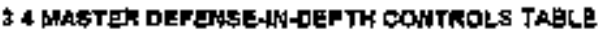

[Appandix A, Table A-S]

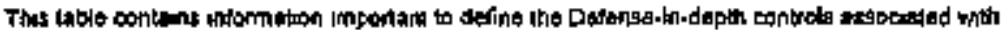
apentad Hazardoess conditions

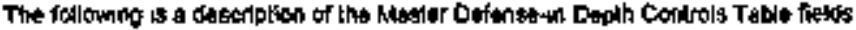

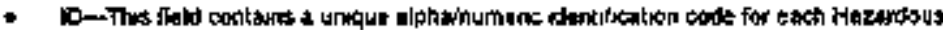

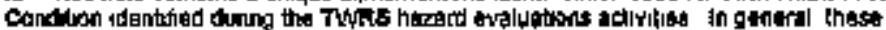
codes, denyed fiom the hams of tho evaluation and the nod numbering usad in the

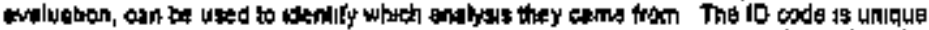

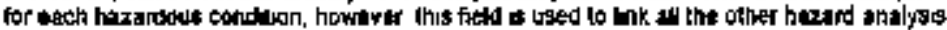

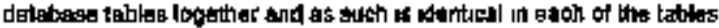

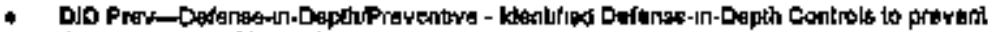

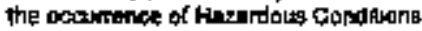

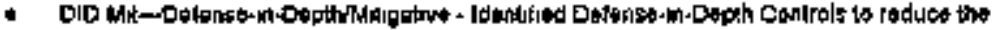
severmy of 1 he consequances of the Hazdrdous Condirons

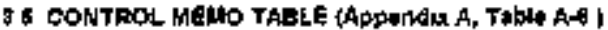

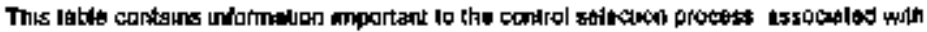
Eperailed Hazardaus Conditione

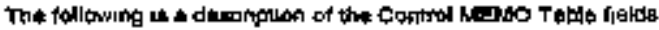

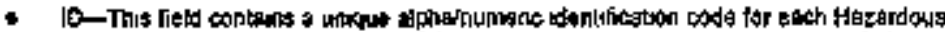

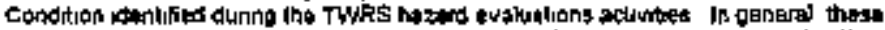

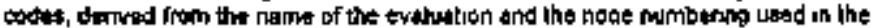

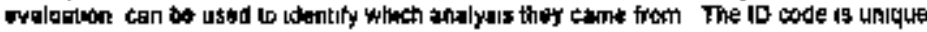




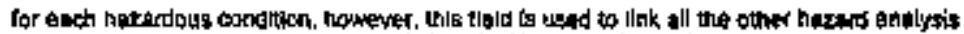

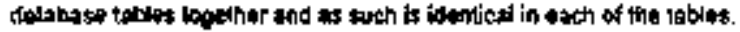

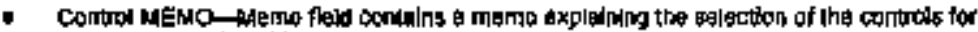
Ine Hirzardous Conditioc.

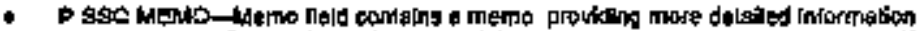

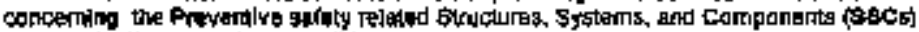
controls lor the Hagardous Contilan.

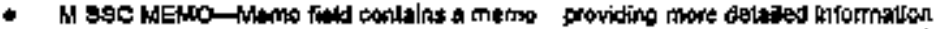

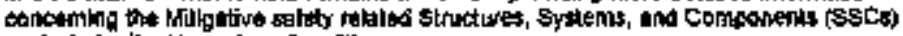
condrots for the Haztedous Condition.

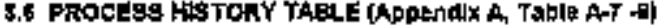

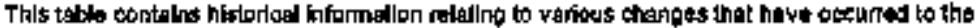

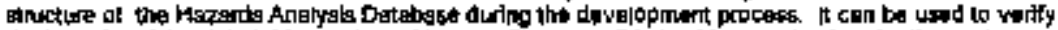

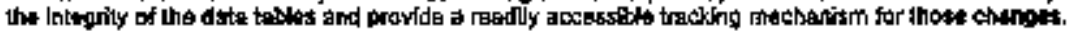

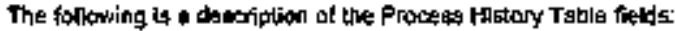

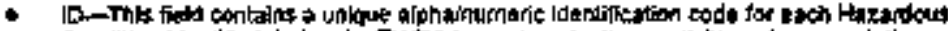

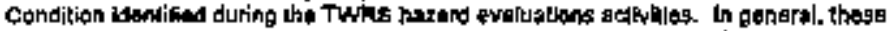

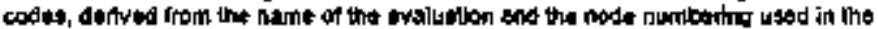

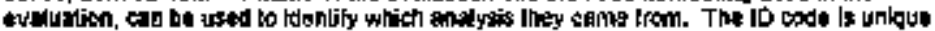

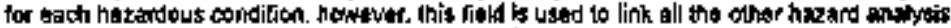

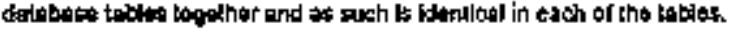

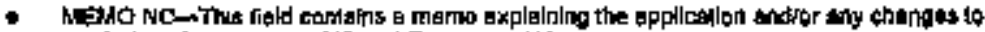
the Batety Corseguance NC and Frequarioy NG

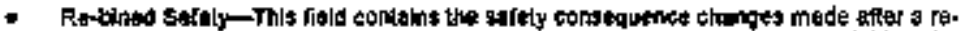

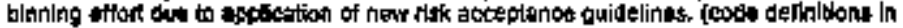
Thele 4-1)

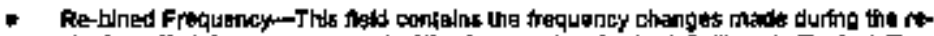

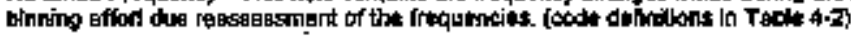

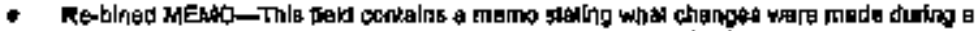

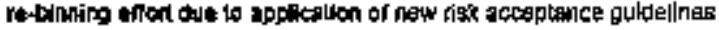

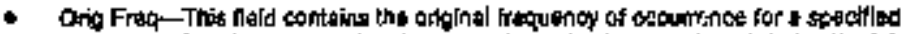

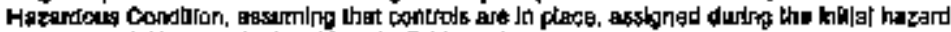

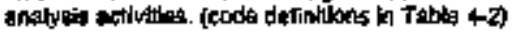

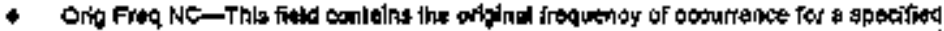

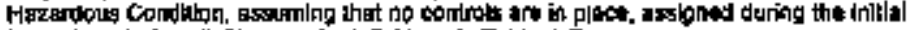
hared matysta actiniles. \{cods definildons in Table 4-2)

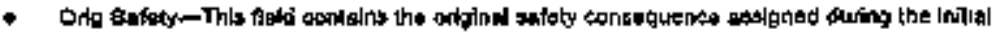

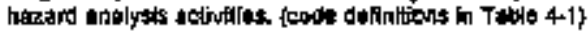

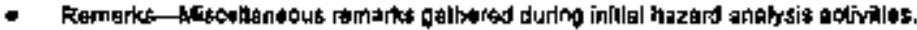




\section{KAY TO COOFS HA HAZAO EVALUATON DATABASE}

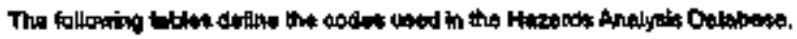

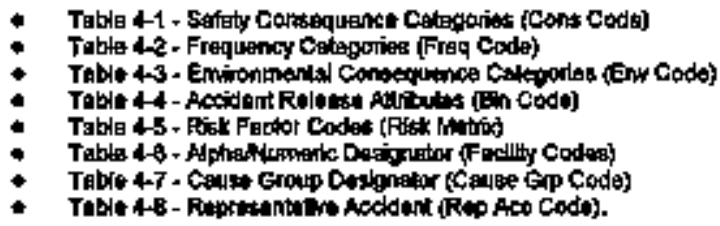

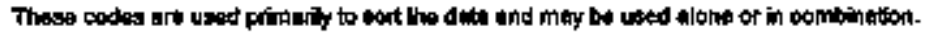

\section{HAZARO TOPDORAFEN DATARASE TABAEB AND FELD DESCRPTONG}

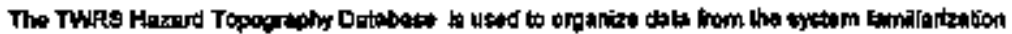

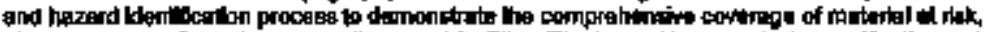

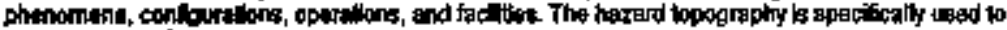

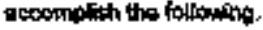

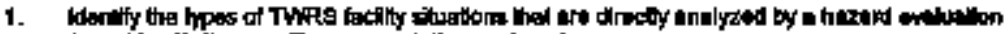

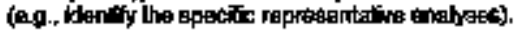

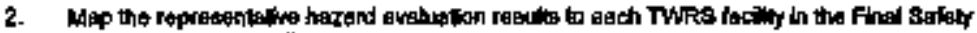
Anlytit Roporl (FSA

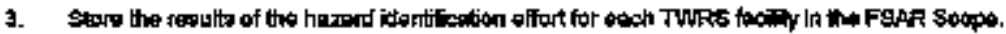

The Herant Topography Dagkbage a composed of the dats bables ahoun in Table 5-1 and

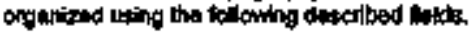

\subsection{FACHLITY CODE DATA TABLE \{Appandir B, Tahlo B-1\}}

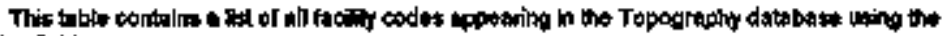

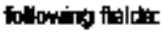

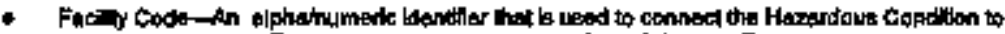

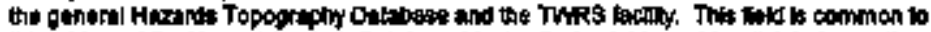

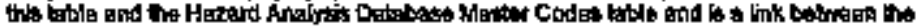

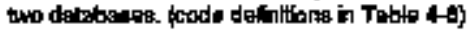

- Fard - A tark farm idenifer uegd for tormaing zeports.

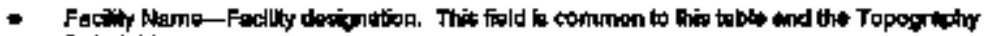
Cate table.

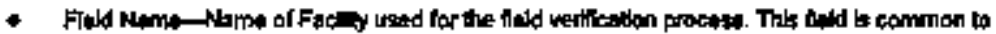

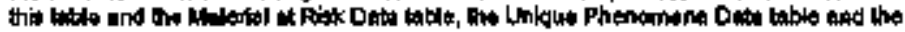
Faclity Canfoumtion babio. 


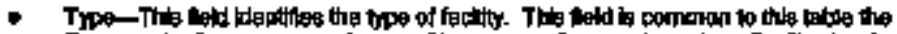

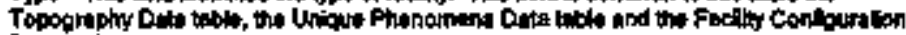
Doto mato .

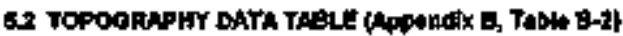

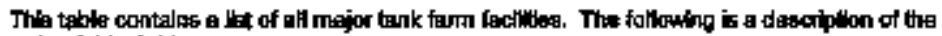
Toposraphy Table ishols:

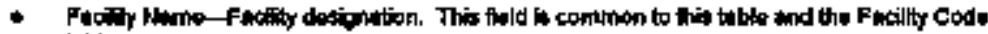
thabe.

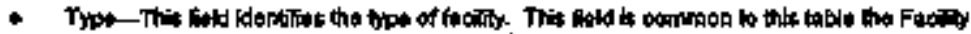

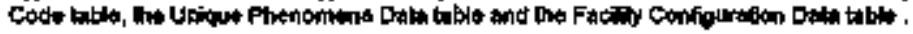

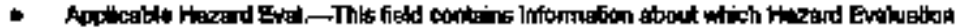

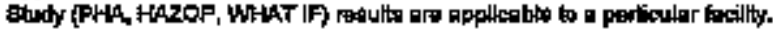

\section{S.S MATEFIALAT nisk OATA THELE}

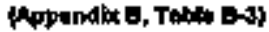

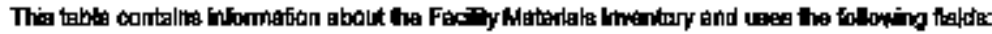

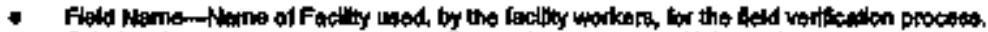

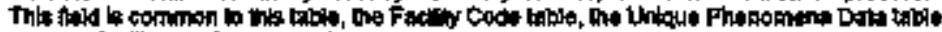

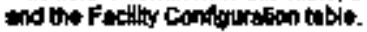

- MAR-aubject Matenfal at ilst untfoct

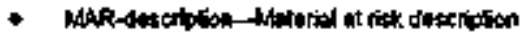

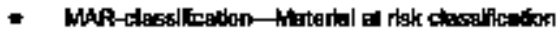

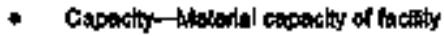

- Moterdat Jppe-Tho type of ingtarlat in the tactiry

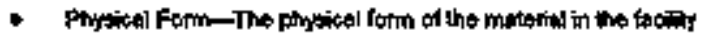

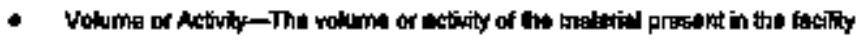

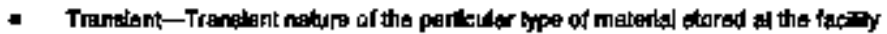

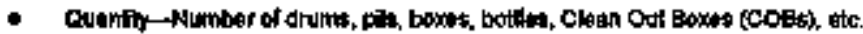

- Camment-Comments abort the matertad at itak.

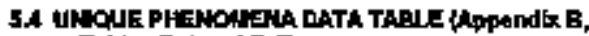 Trable B-4 and B-6]}

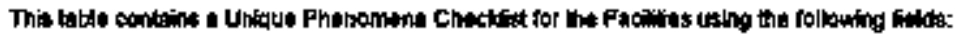




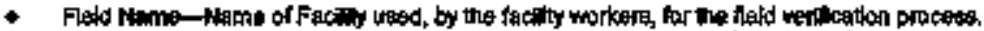

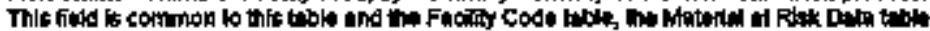
and the Factity Confiowstion tabte.

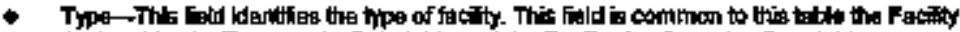

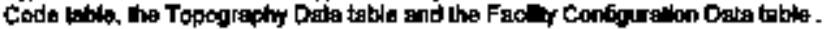

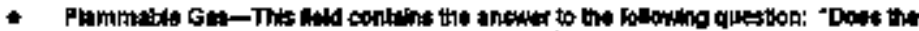

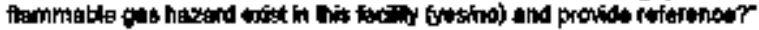

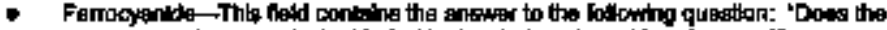

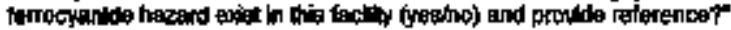

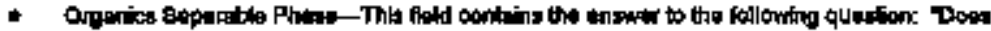

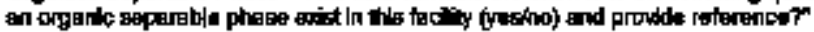

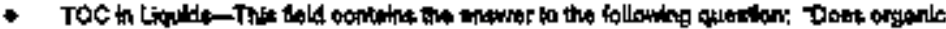

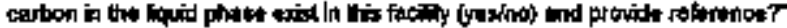

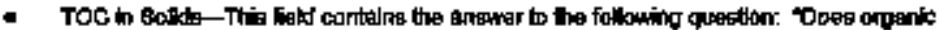

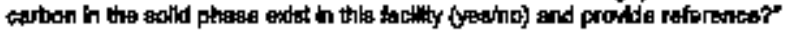

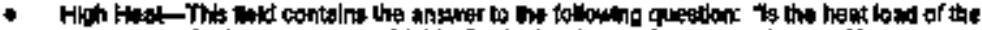

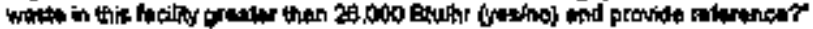

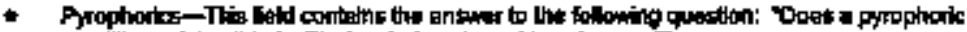
cartition wist at the facilty (ytaging) and provide referanco?

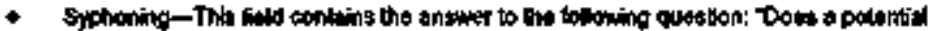

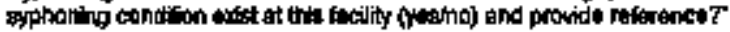

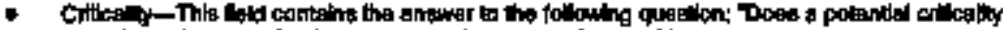

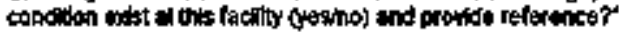

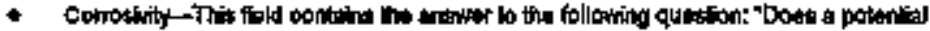

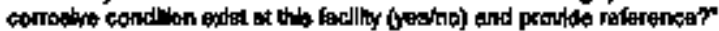

\section{S5 FACALTY CONFICURATION DATATABEE} (lopendix B, Tabo B B)

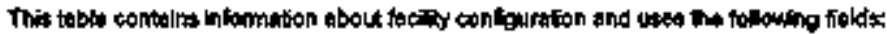

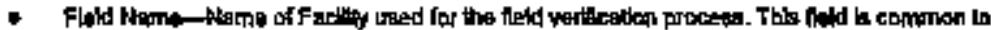

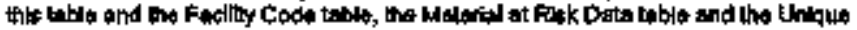

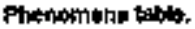

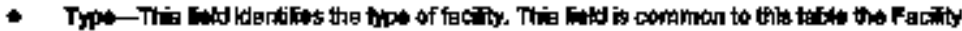

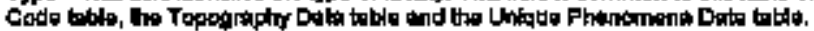

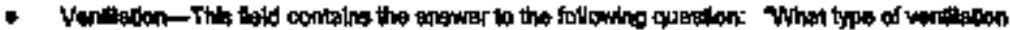

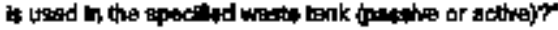

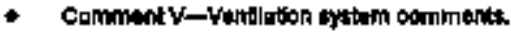




\section{HNF-SO-1HAT-764, Row. 1}

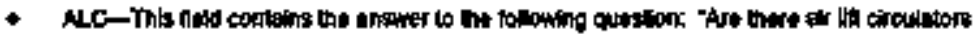
gativet in lhe wegte tantk (yeging)?

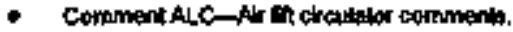

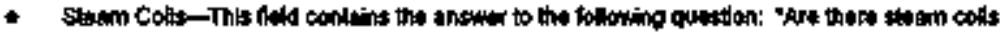

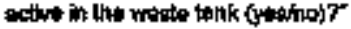

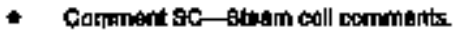

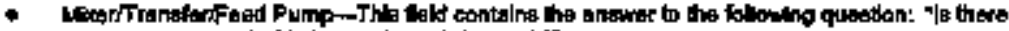

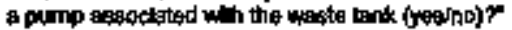

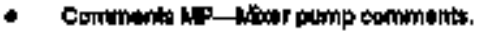

\subsection{KeY TO CODES IN HAZARD TOPQBMAPHY DATAEASE}

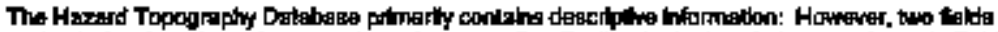

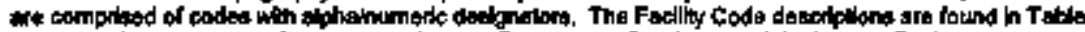

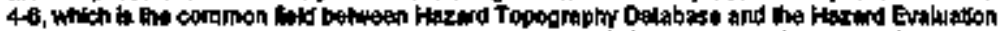

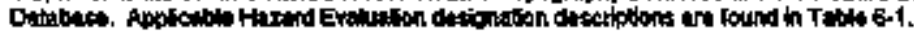

\section{7,0 REFERDEGES}

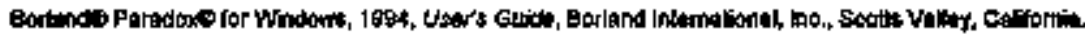

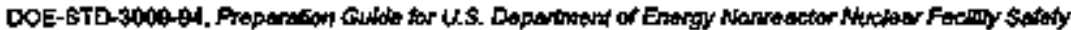
Antystis Reports, U.S. Departm ant of Enery,y, Wathington, D.C.

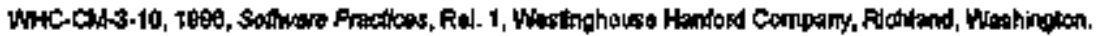

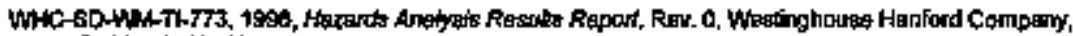
Furhiand, Whatinglon. 


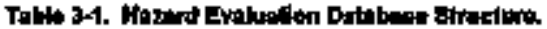

\begin{tabular}{|c|c|c|}
\hline \multicolumn{3}{|c|}{ 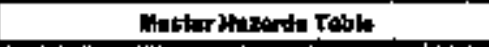 } \\
\hline$D$ & 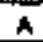 & Sol \\
\hline 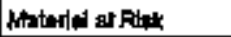 & $\mathbf{A}$ & 265 \\
\hline Hazandous Conkivinn & $\mathbf{A}$ & 255 \\
\hline Cousto & A & 255 \\
\hline Constigunot & $A$ & 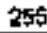 \\
\hline
\end{tabular}

\begin{tabular}{|c|c|c|}
\hline \multicolumn{3}{|c|}{ Writet Controla Table } \\
\hline$\Phi$ & A & 50 \\
\hline Prar $\$ s c$ & $A$ & 265 \\
\hline by asc & A & 25 \\
\hline Pton TSR & A. & 255 \\
\hline MR TSR & A & 255 \\
\hline
\end{tabular}

\begin{tabular}{|c|c|c|}
\hline \multicolumn{3}{|c|}{ Master Code Tuble } \\
\hline to & A & 50 \\
\hline 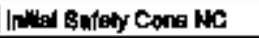 & $\mathbf{A}$ & 5 \\
\hline In:sil Freq NG & $\mathbf{A}$ & 5 \\
\hline En Cont & A & 5 \\
\hline EN & A & 10 \\
\hline 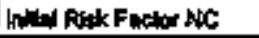 & $\mathbf{N}$ & \\
\hline 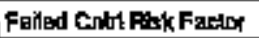 & $\mathbf{N}$ & \\
\hline Fun cods & A & 15 \\
\hline Gause Cop & A & 5 \\
\hline Rapdace & A & 5 \\
\hline NA Stoby Cons NC & $\mathbf{A}$ & 5 \\
\hline AA Fregd NG & 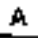 & $\$$ \\
\hline App Crutu Suftoty & A & 5 \\
\hline App Cntul Freq & A & 5 \\
\hline 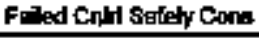 & A & 5 \\
\hline Feiled Cntw Freq & A & 5 \\
\hline Catol Fallure hENO & $\mathbf{M}$ & 240 \\
\hline Change HEMO & M & 240 \\
\hline
\end{tabular}

\begin{tabular}{|c|c|c|}
\hline \multicolumn{3}{|c|}{ Master Defenge la Depth Tahle } \\
\hline & & \\
\hline 10 & A & 50 \\
\hline MD - Proventiver & A & 250 \\
\hline DL - Nolitadie & $A$ & 2㠿 \\
\hline
\end{tabular}

\begin{tabular}{|c|c|c|}
\hline \multicolumn{3}{|c|}{ Coairal Themo Ta } \\
\hline D & A & 50 \\
\hline P ASc kano & $\mathbf{M}$ & 240 \\
\hline MSSC MENO & $\mathbf{M}$ & 240 \\
\hline Conted be & $\mathbf{M}$ & 240 \\
\hline
\end{tabular}

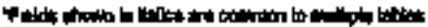

Puld tope detortors:

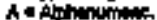

N w Nambint.

N = Vhing Fin.

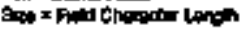

\begin{tabular}{|c|c|c|}
\hline 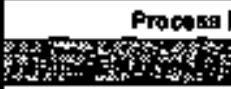 & & is: \\
\hline P & $A$ & so. \\
\hline MENO HC & M & 240 \\
\hline Fe-hined stifely & A & 5 \\
\hline Rit-bland Froquition & $\Lambda$ & 5 \\
\hline RAbiad N"FNO & A & 240 \\
\hline OipF伯 & $\boldsymbol{\lambda}$ & $\mathbf{s}$ \\
\hline Orixfraq $\mathrm{NC}$ & $A$ & 5 \\
\hline Orivstifity & 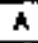 & 5 \\
\hline Remaiks & $\mathbf{A}$ & asts \\
\hline
\end{tabular}




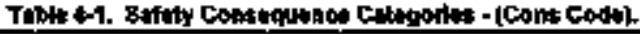

\begin{tabular}{|c|c|}
\hline coteday & Detionition \\
\hline $\mathbf{8 3}$ & 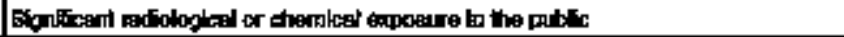 \\
\hline$\$ 2$ & 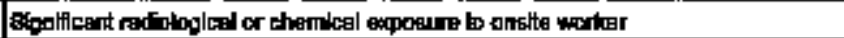 \\
\hline $\mathbf{3 1}$ & 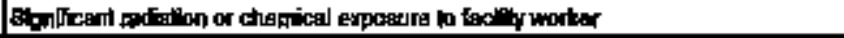 \\
\hline \$o & 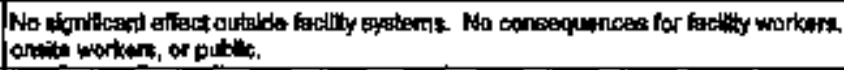 \\
\hline Prantiantad & Nar effect-Accidant Preweritod \\
\hline
\end{tabular}

Table 4-2 Frequency Cotegorleg (Frag Codaj.

\begin{tabular}{|c|c|}
\hline Cangary & Dationilon \\
\hline $\begin{array}{l}\text { P3 } \\
(0.01)\end{array}$ & 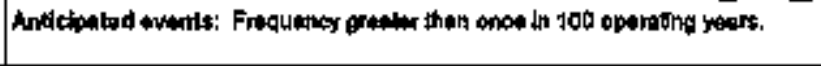 \\
\hline$\left(10^{-2}(0)+10^{-1}\right)$ & 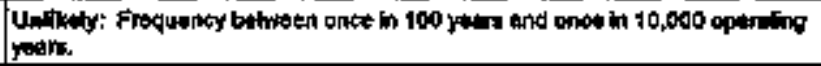 \\
\hline (10) & 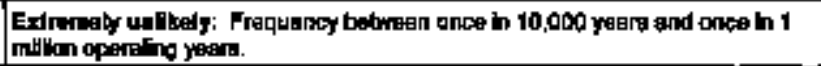 \\
\hline $\begin{array}{c}F 0 \\
{[<10 \%}\end{array}$ & 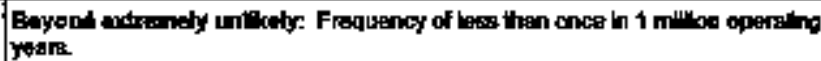 \\
\hline
\end{tabular}

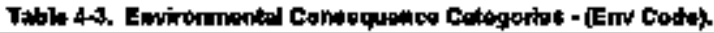

\begin{tabular}{|c|c|}
\hline Crtegary & Deflntion \\
\hline , $\mathbf{E 3}$ & 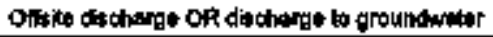 \\
\hline $\mathbf{E 2}$ & Algrificant dechajge onsolte \\
\hline E1 & 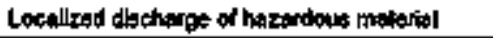 \\
\hline E0 & 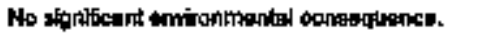 \\
\hline
\end{tabular}




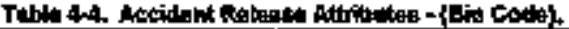

\begin{tabular}{|c|c|c|}
\hline \multicolumn{3}{|c|}{ Actsterit raloese atuibuteg } \\
\hline 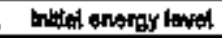 & 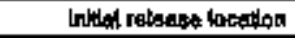 & 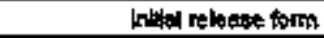 \\
\hline A. HEght & T. Nenosphere & 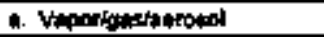 \\
\hline B. Hodorink & 2 Ground tudtaos & b. Liquldithry \\
\hline C. Low & 3. Sibanfiot & o. sonditdudot \\
\hline \multicolumn{3}{|c|}{ 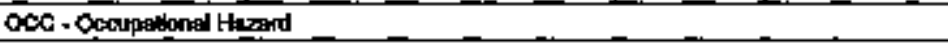 } \\
\hline \multicolumn{3}{|c|}{ RP - Radigilon Prolectikn } \\
\hline \multicolumn{3}{|c|}{ Wh. Not Appiltrable } \\
\hline NRR - Sto Raglesese & & \\
\hline
\end{tabular}

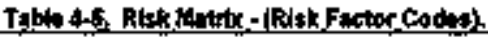

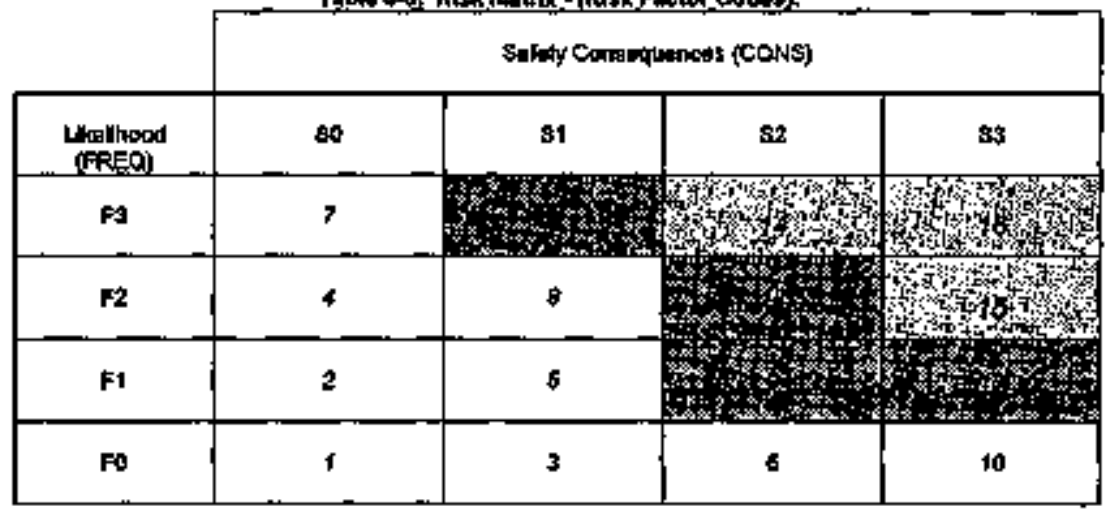

Corindored for labriticertion of Sufoty

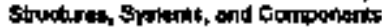

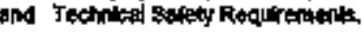

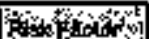
(4) Ropisos ldantiteabon of Bafoty Bthitures, Bydents, and Components and Tochiced Sality Racivienents 


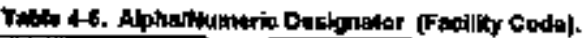

\begin{tabular}{|c|c|}
\hline \multicolumn{2}{|c|}{ IN } \\
\hline ER & Colven ftehex, pende \\
\hline \multicolumn{2}{|c|}{ 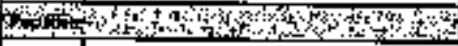 } \\
\hline Fan & 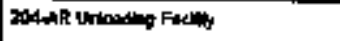 \\
\hline Frt9 & 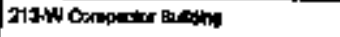 \\
\hline Fa4 & 244-Ak bual \\
\hline \multicolumn{2}{|c|}{ 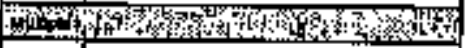 } \\
\hline$M$ & 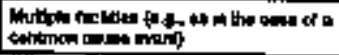 \\
\hline \multicolumn{2}{|c|}{ 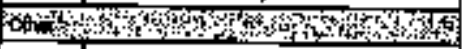 } \\
\hline A & 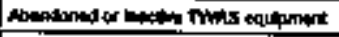 \\
\hline g & 1 \\
\hline 0 & 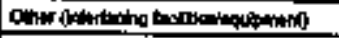 \\
\hline c & 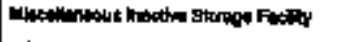 \\
\hline \multicolumn{2}{|c|}{ Pans } \\
\hline $\boldsymbol{H}$ & Mom pound plap \\
\hline PE & 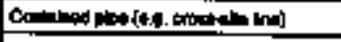 \\
\hline pos & 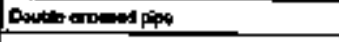 \\
\hline P8 & 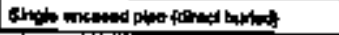 \\
\hline \multicolumn{2}{|c|}{ 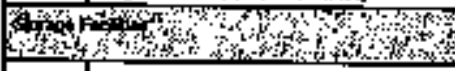 } \\
\hline$\$ 90$ & 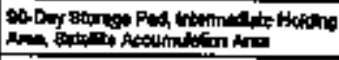 \\
\hline $\mathbf{\$ 4}$ & 272t w 3000um gtankes \\
\hline EW & Wulpod borape \\
\hline
\end{tabular}

\begin{tabular}{|c|c|}
\hline $\mathbf{T}$ & 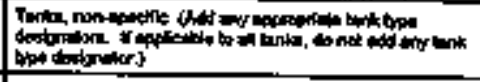 \\
\hline cb & DCRT (24+TX 24+1, $1 \leqslant 1$ \\
\hline CA & 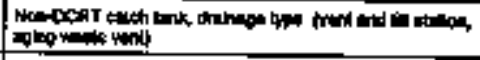 \\
\hline \multirow[t]{8}{*}{ c) } & 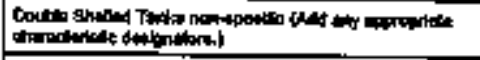 \\
\hline & 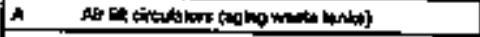 \\
\hline & 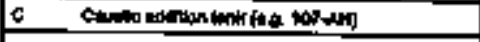 \\
\hline & Ewhower toed tork \\
\hline & 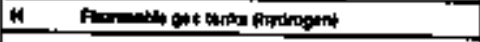 \\
\hline & Orentring \\
\hline & 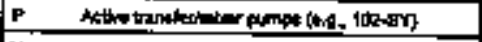 \\
\hline & 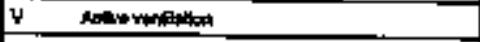 \\
\hline \multirow[t]{6}{*}{98} & 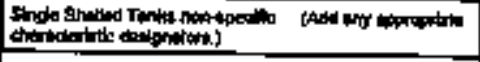 \\
\hline & Oryales lonin $\{11143 x\}$ \\
\hline & 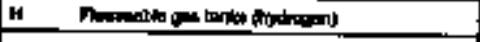 \\
\hline & 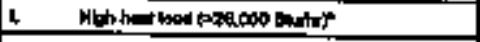 \\
\hline & Ontive the \\
\hline & 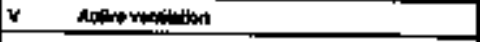 \\
\hline 80 & 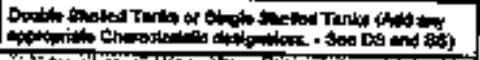 \\
\hline \multicolumn{2}{|c|}{ 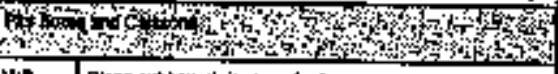 } \\
\hline W. & 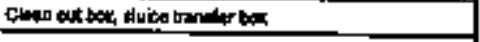 \\
\hline W & 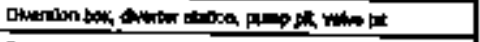 \\
\hline 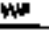 & 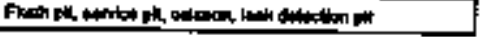 \\
\hline
\end{tabular}

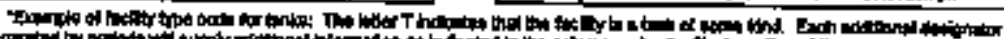

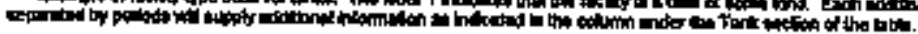

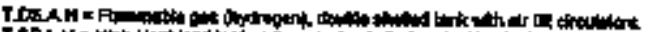

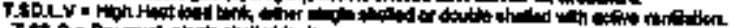

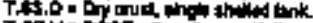

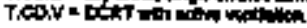

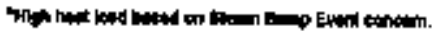


HNF-s[-Wh-Tr-7od, Rav. 1

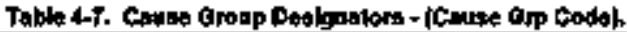

\begin{tabular}{|c|c|c|c|c|c|c|}
\hline comose descilition & Crttersy & $\begin{array}{c}\text { Fuer } \\
\text { Exploson }\end{array}$ & $\begin{array}{c}\text { Tonk } \\
\text { Dome } \\
\text { Colapese }\end{array}$ & Relange & $\begin{array}{l}\text { Hernma } \\
\text { Emor } \\
\text { Relogeg }\end{array}$ & 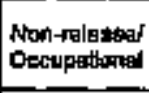 \\
\hline 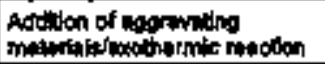 & AO1 & Bô1 & $\cos$ & DO1 & EOT & F01 \\
\hline 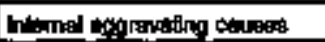 & A02 & NA & $M$ & DO2 & EOZ & Mk \\
\hline Orar conemiration & $\mathrm{AOO}$ & Ha & NA & Nh & $E 00$ & $F 03$ \\
\hline Intustum exfinty & $\mathrm{MOH}$ & $\mathbf{B O}=$ & MA & DOA & E04 & FO4 \\
\hline 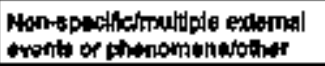 & Ads & $\mathbf{N A}$ & $\cos$ & Det5 & E05 & F05 \\
\hline 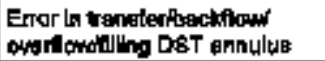 & Apes & 600 & $\cos$ & $\cos$ & EDa & $\mathrm{Fos}$ \\
\hline Vertiletbon Fintur & Ha & $\mathrm{BOP}$ & 007. & DEF & EOP & ror \\
\hline 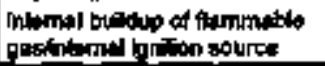 & Wh & E0B & NA & NA & $\mathbf{N a}$ & $\mathrm{Fos}$ \\
\hline 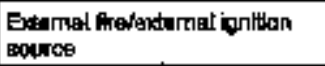 & Na & BDS & MA & $\mathbf{C O S}$ & NA & F09 \\
\hline 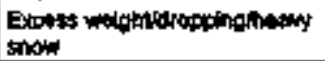 & MA & NA & C14 & D10 & E10 & F10 \\
\hline Wagnm & NAS & NA & c11 & D11 & NA & NA \\
\hline 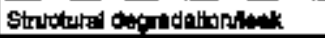 & NA & B12 & c되 & 012 & Ma & Fin \\
\hline Ower rated anghment & NA & NA & \$13 & MA & $\mathrm{E}+3$ & Wh \\
\hline 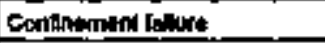 & w & NA & HA & D14 & E14 & F14 \\
\hline Expese efglittlax & NAA & NA & NA & WA & E1s & NA \\
\hline Contosith & NA & NA & NA & D19 & NA & NA \\
\hline Whter hamianter & $\mathrm{ma}$ & NA & NA & D17 & NA & MA \\
\hline 9ximic eventhoftrand actiol & NA & NA & MA & D18 & $\mathbf{N A}$ & MA \\
\hline Ploosing & NA & NA & cite & $\cos \theta$ & wa & MH \\
\hline 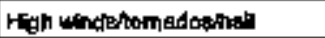 & NA & NA. & $\mathrm{NA}$ & 000 & NA & MA \\
\hline Lighluting & NA & B21 & NA & $D 21$ & MA & Mh \\
\hline 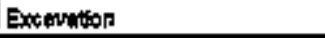 & NA & NA & Nh & $\cos 2$ & Nud & $\mathbf{F} 2$ \\
\hline Arcrall cr:ab & NA & $B 23$ & NA & 023 & MA & NA \\
\hline Low lamperalurelliwering & NA & NA & NA & 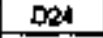 & $\mathbf{M}$ & Nh \\
\hline Vehiols aorldeits & NA & 825 & NA & 026 & E26 & F25 \\
\hline struthry demega & NA & K25 & C2: & 026 & E26 & F26 \\
\hline
\end{tabular}

DST o dowblesten bik.

AA = not appicabs. 


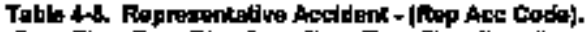

\begin{tabular}{|c|c|}
\hline$\#$ & Fupentintalive Accident Duscription \\
\hline 1 & 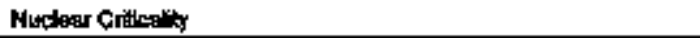 \\
\hline 2 & 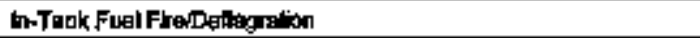 \\
\hline 3 & 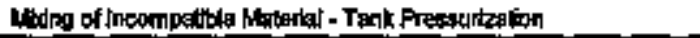 \\
\hline 4 & 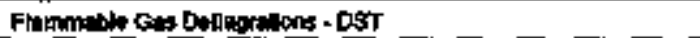 \\
\hline$\underline{5}$ & 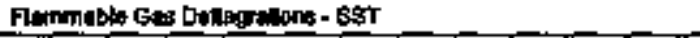 \\
\hline B & 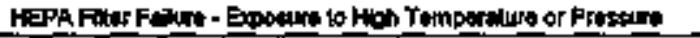 \\
\hline 7 & Firs h Contemingind Axw \\
\hline 8 & 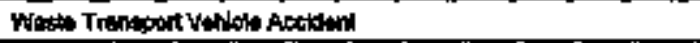 \\
\hline 9 & 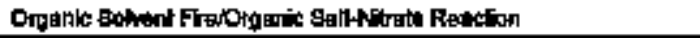 \\
\hline 10 & 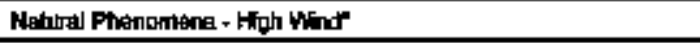 \\
\hline 11 & 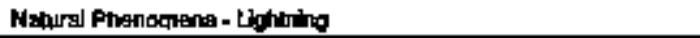 \\
\hline 12 & Tant: Falure Dure be Excbagive Loarts \\
\hline 13 & Tank Falkure Dere to Veaceum ar Degradalon \\
\hline 14 & 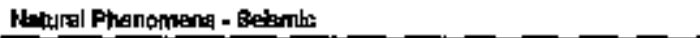 \\
\hline 15 & 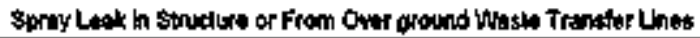 \\
\hline 18 & 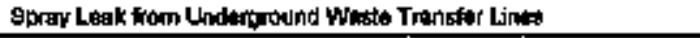 \\
\hline$\uparrow 7$ & 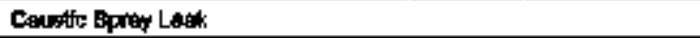 \\
\hline $18 \mathrm{~A}$ & Tank Bump \\
\hline $18 b$ & Unthered Rfolease \\
\hline 21 & Subequrtoeg Leak Rouning h Pool \\
\hline 22 & Evapontor Clamp \\
\hline 23 & 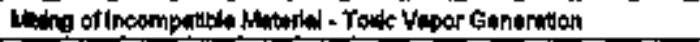 \\
\hline 25 & 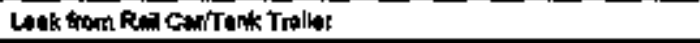 \\
\hline 23 & 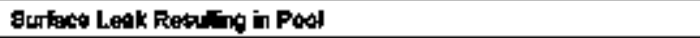 \\
\hline 28 & 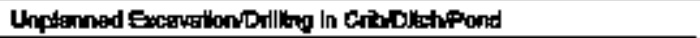 \\
\hline 20 & Suboinlace Leak Fomining Subuyrlaç \\
\hline 30 & \$odum Fut \\
\hline 31 & Abova Grould Otruchus Fallut \\
\hline 32 & 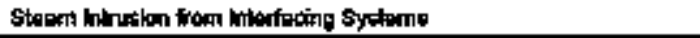 \\
\hline
\end{tabular}

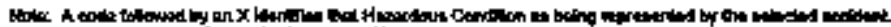

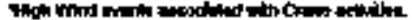

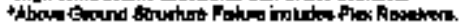


Takle 5-1. Hazard Tepograply Database 8tnuesare.

\begin{tabular}{|c|c|c|}
\hline \multicolumn{3}{|c|}{ Facity Cod Thblo } \\
\hline & & \\
\hline Fondity coda & 4 & 15 \\
\hline Facilif Nome & $\Lambda$ & 50 \\
\hline Fidef Noto & A & 90 \\
\hline The & $A$ & 50 \\
\hline
\end{tabular}

\begin{tabular}{|c|c|c|}
\hline \multicolumn{3}{|c|}{ Tapograpty Tablo } \\
\hline Fecinity Nams & 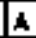 & 50 \\
\hline Type & A & 50 \\
\hline 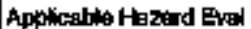 & A & 25 \\
\hline
\end{tabular}

\begin{tabular}{|c|c|c|}
\hline 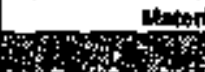 & & \\
\hline 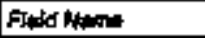 & A & 50 \\
\hline Har-subloct & A & 255 \\
\hline 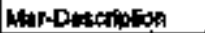 & h & 255 \\
\hline 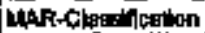 & A & 25 \\
\hline Capandy & A & 255 \\
\hline Mtglental Type & A & 15 \\
\hline Phyrated Fomp & A & 15 \\
\hline Wolume or Actithy & A & 50 \\
\hline Trantsient & A & $25 s$ \\
\hline Quantiky & A & 295 \\
\hline Connthtsts & A & 255 \\
\hline
\end{tabular}

\begin{tabular}{|c|c|c|}
\hline \multicolumn{3}{|c|}{ 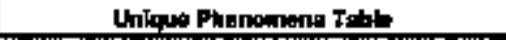 } \\
\hline Fiald Wane & A & 50 \\
\hline Type & A & 60 \\
\hline Flanmpabio $\mathrm{Gag}$ & A & 255 \\
\hline Fartocynidy & A & 25 \\
\hline Orguntes Separible Phere & h & 255 \\
\hline TOC in Linuids: & A & 255 \\
\hline TOC in Socid: & A & 256 \\
\hline Hon Hapt & A & 255 \\
\hline Pyopharice & A & 265 \\
\hline Syphoplno & M & 265 \\
\hline Griticeally & A & 265 \\
\hline Coltaghy & A & 255 \\
\hline Ollike & M & 240 \\
\hline Commonis & A & 266 \\
\hline LOC & A & 16 \\
\hline NLEM & $\mathbf{N}$ & \\
\hline
\end{tabular}

\begin{tabular}{|c|c|c|}
\hline \multicolumn{3}{|c|}{ Fatiny Confirtrion Tabto } \\
\hline Fiold Name & A & 50 \\
\hline Thp & A & 50 \\
\hline Vontion & A & 10 \\
\hline Commont $\mathbf{y}$ & A & 240 \\
\hline ALG & A & 15 \\
\hline Commo日nt ALC & A & 240 \\
\hline Stath catso & A & 15 \\
\hline Comment so & A & 240 \\
\hline Moerftransforf ood Punp & A & 15 \\
\hline Comraent MP & $A$ & 240 \\
\hline
\end{tabular}

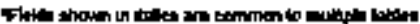

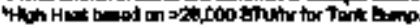

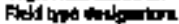

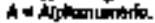

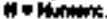

ir a Mimion Fid

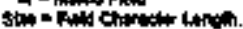




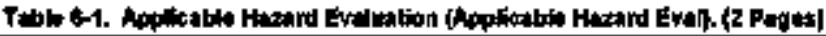

\begin{tabular}{|c|c|}
\hline Nanowitur & Dotherion \\
\hline FAP, & 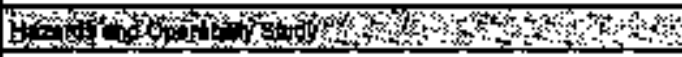 \\
\hline 204 AR & 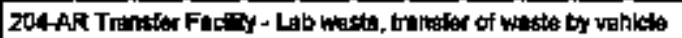 \\
\hline $2 m-8$ & 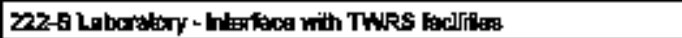 \\
\hline $212-A$ & 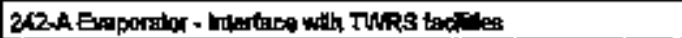 \\
\hline 212.3 & 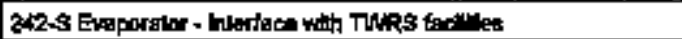 \\
\hline $242-T$ & 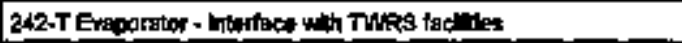 \\
\hline 24A-AR & 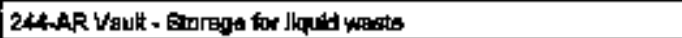 \\
\hline AN-10? & 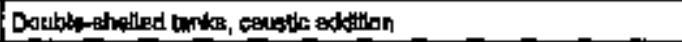 \\
\hline ANH-102 & 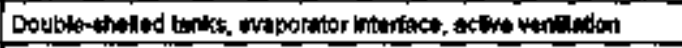 \\
\hline B-PUNT & 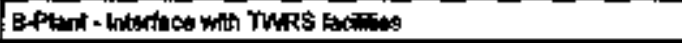 \\
\hline C.105 & 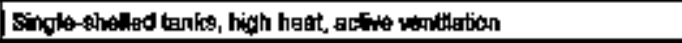 \\
\hline ogt & ONeTgTound bangier gybarth \\
\hline PFP & 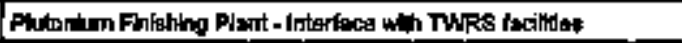 \\
\hline PUREX & PUREX - interlace with ThRs factllites \\
\hline T-PLANT & 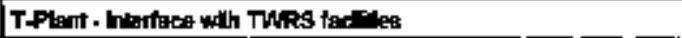 \\
\hline S-102 & 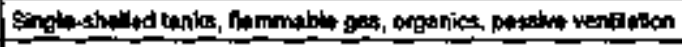 \\
\hline$\$+102$ & 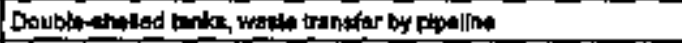 \\
\hline$\$ x-114$ & 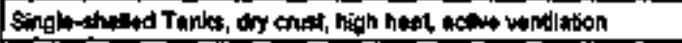 \\
\hline UPLANT & 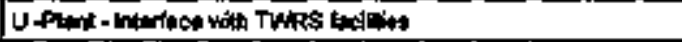 \\
\hline Hoss. & 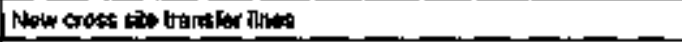 \\
\hline$x \leqslant$ & Cruen the trinefor thes \\
\hline 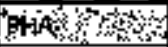 & 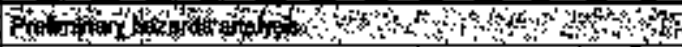 \\
\hline$\infty$ & 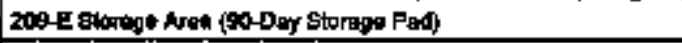 \\
\hline $213 \mathbf{W}$ & 213 Compector - Equiprient decontaminattan acth"es \\
\hline $272 \pi N$ & 2727 w Bod"um \&tprege Balliding \\
\hline $12-102$ & 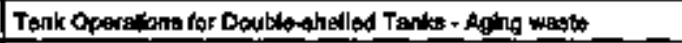 \\
\hline BX-100 & 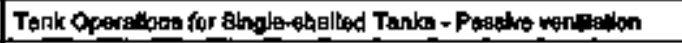 \\
\hline BND & Bondilag ectintos \\
\hline $\cos$ & 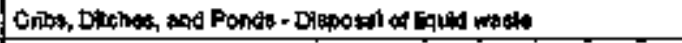 \\
\hline $\operatorname{cog}$ & 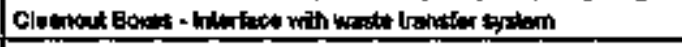 \\
\hline CFN & Crane acthilat in the thenk fams \\
\hline Gir & 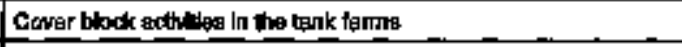 \\
\hline DCN & Coconiaminathon mGinge \\
\hline
\end{tabular}


HNWF-SD-NMATR-764, Rov. 1

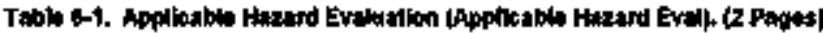

\begin{tabular}{|c|c|}
\hline detandintor & Dufnimon \\
\hline $\mathbf{F R}$ & 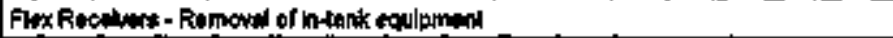 \\
\hline EF & Granhoust actititios \\
\hline ITK & 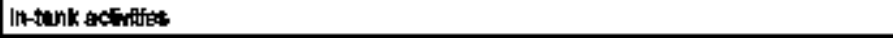 \\
\hline Mas & 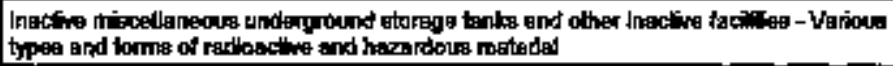 \\
\hline RAD & Rapliation potecton. \\
\hline RSR & Riper acthotites. \\
\hline 的F & 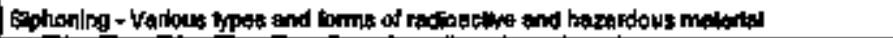 \\
\hline $5 \%-101$ & 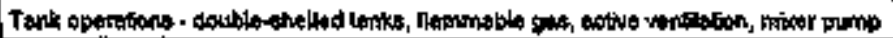 \\
\hline WH & 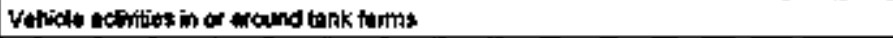 \\
\hline VSU & 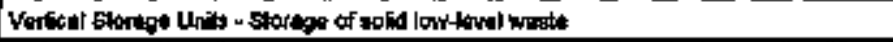 \\
\hline 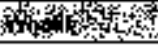 & 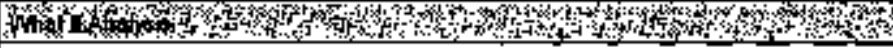 \\
\hline cor & 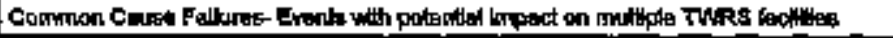 \\
\hline EE & 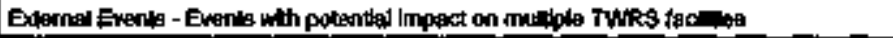 \\
\hline MP & 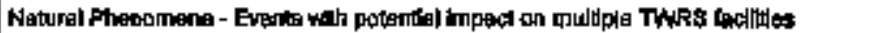 \\
\hline
\end{tabular}




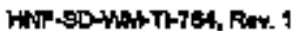

Thls pege inlentlonedly left blenk 
HWF-AD-WH-TA-764, Row. 1

APPERDX A

HAZARD gNALUATON DATARASE TAELES

A-t 
HNF-SD-WM-TI-764, Rev. 1

This pepe intentitanaly let thank. 
Hars.sonth-TR-704, Rev. 1

\section{LIST OF TAQLS}

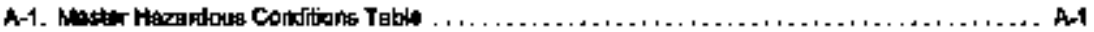

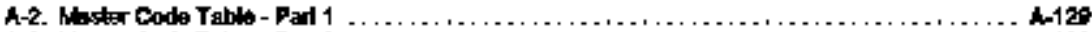

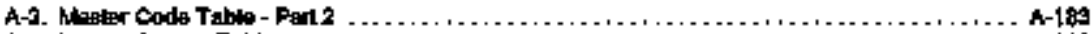

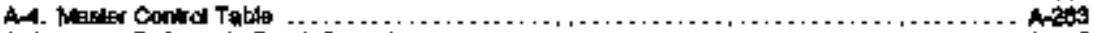

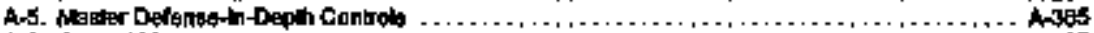

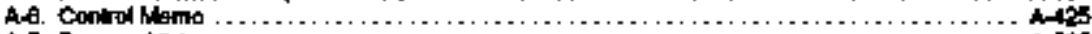

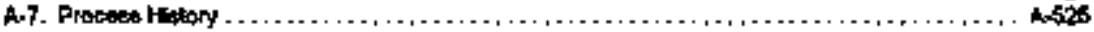


HKF-SOWWA-TL7Ed, Rov, 1

This page inkentionally let benk. 


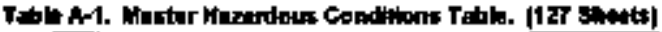

\begin{tabular}{|c|c|c|c|c|}
\hline $\mathbf{D}$ & Mandelath & Heardous Conditen & Conan & Contenquentes \\
\hline 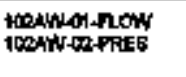 & OST tart coritedts & 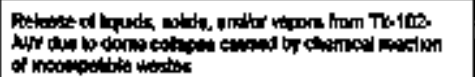 & 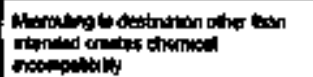 & 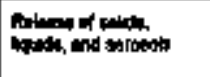 \\
\hline 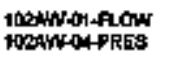 & 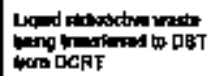 & 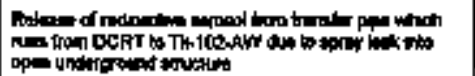 & 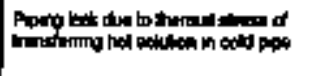 & 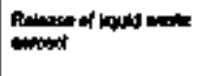 \\
\hline 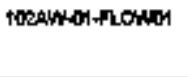 & 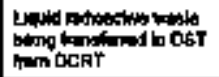 & 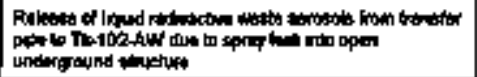 & 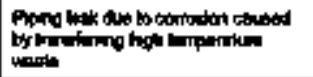 & 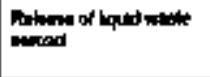 \\
\hline wantor-nowns & 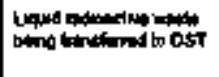 & 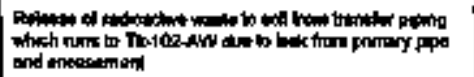 & 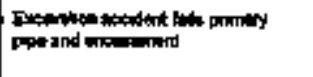 & 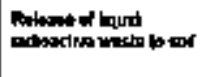 \\
\hline 1024us-01-Flones & 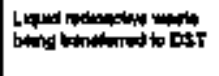 & 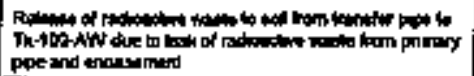 & 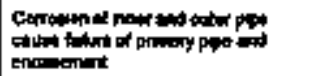 & 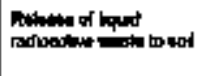 \\
\hline 1CBuW-Q1-PROAng & 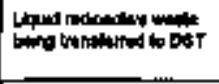 & 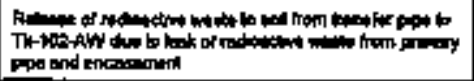 & 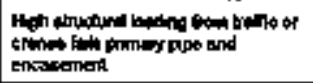 & lal \\
\hline 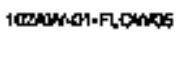 & 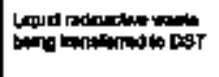 & 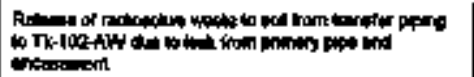 & 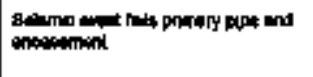 & A \\
\hline 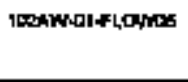 & 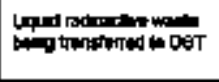 & 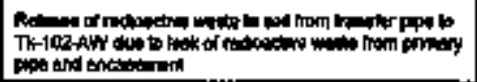 & 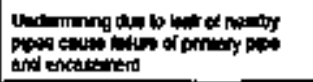 & 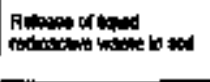 \\
\hline 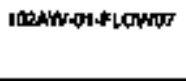 & 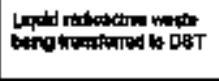 & 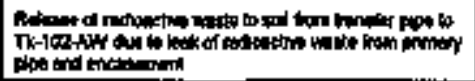 & 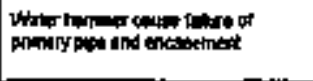 & A \\
\hline 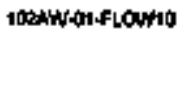 & 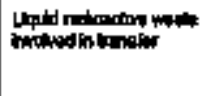 & 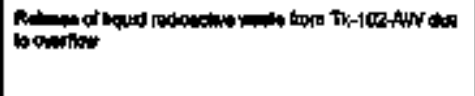 & 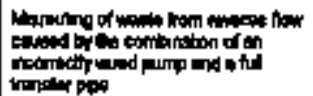 & 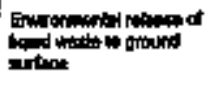 \\
\hline
\end{tabular}


Toble A-1, Hadter Herardors Condalions Talle. [t27 shoebs]

\begin{tabular}{|c|c|c|c|c|}
\hline 10 & Nownalat Ran: & 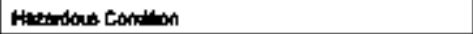 & Coun & Condepuentet \\
\hline 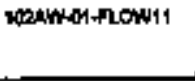 & 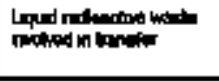 & 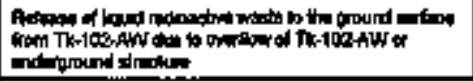 & 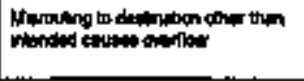 & 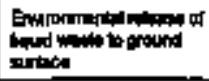 \\
\hline 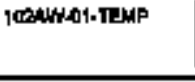 & 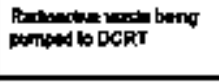 & 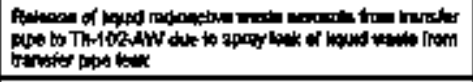 & 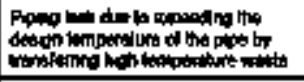 & 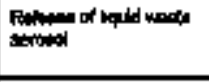 \\
\hline 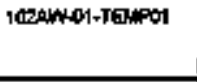 & 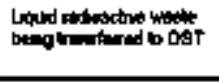 & 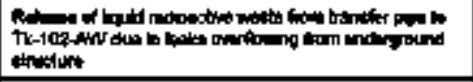 & 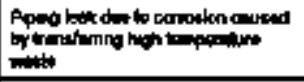 & 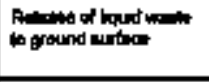 \\
\hline 1002NA-61-TEMPOAA & 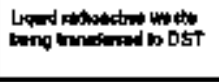 & 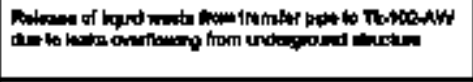 & 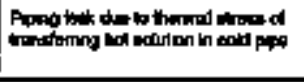 & 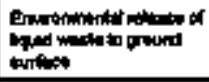 \\
\hline 10024N-DI-TENMPQ & 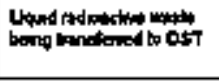 & 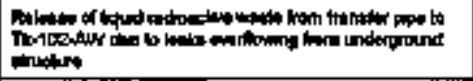 & 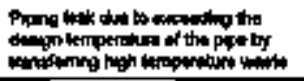 & 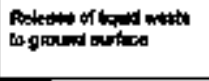 \\
\hline IOEHW-02LELE & 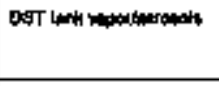 & 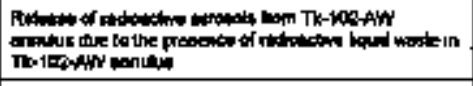 & 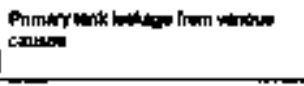 & 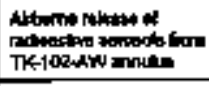 \\
\hline 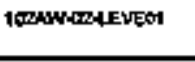 & 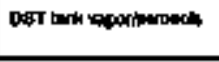 & 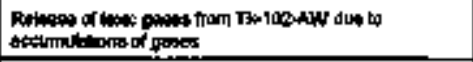 & sonry orath & 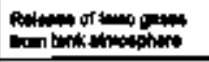 \\
\hline 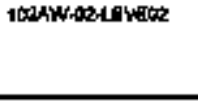 & 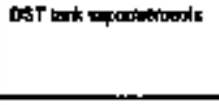 & 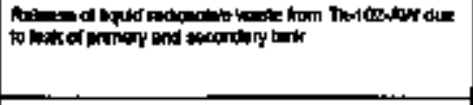 & 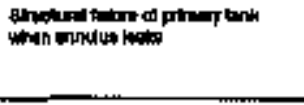 & 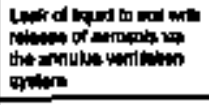 \\
\hline 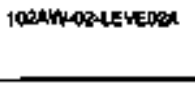 & 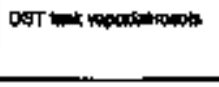 & 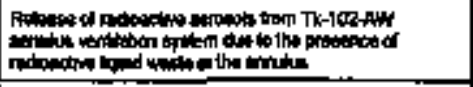 & 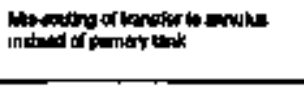 & 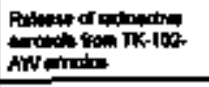 \\
\hline 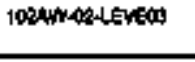 & 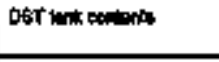 & 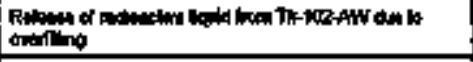 & 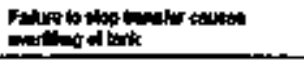 & 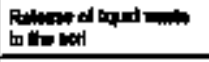 \\
\hline 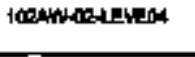 & 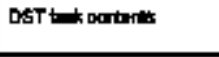 & 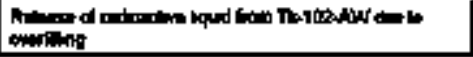 & 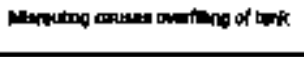 & 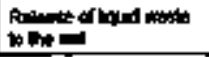 \\
\hline
\end{tabular}




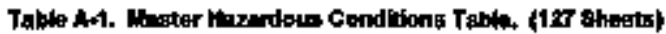

\begin{tabular}{|c|c|c|c|c|}
\hline 10 & 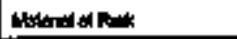 & Hearitusa Conotion & 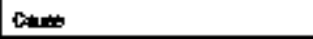 & Contictiot \\
\hline 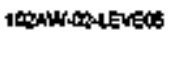 & 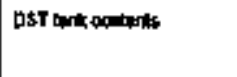 & 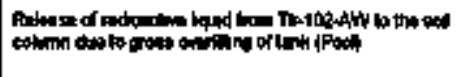 & 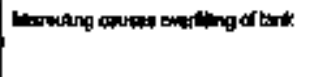 & 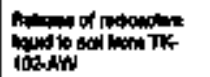 \\
\hline 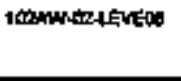 & 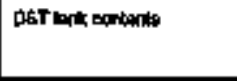 & 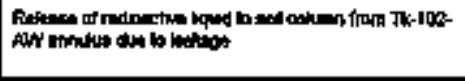 & 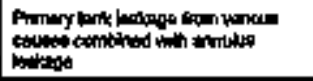 & 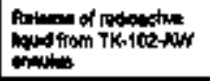 \\
\hline 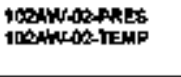 & DCRT trak noporim mant & 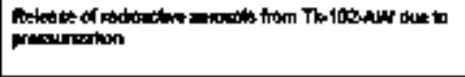 & 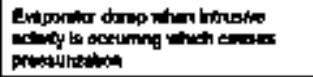 & 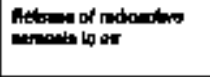 \\
\hline 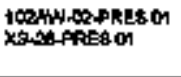 & Wh & 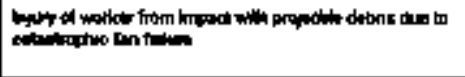 & 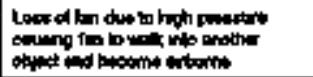 & 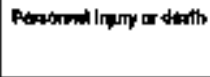 \\
\hline 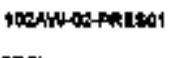 & 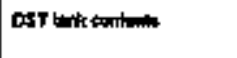 & 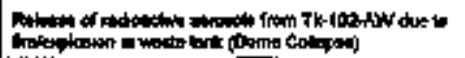 & 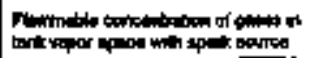 & 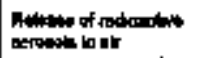 \\
\hline 10201402-Fresor & 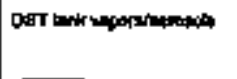 & 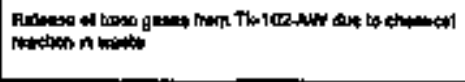 & 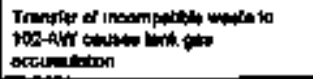 & 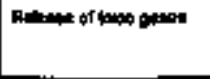 \\
\hline 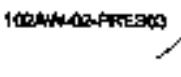 & Dost trincorinats & 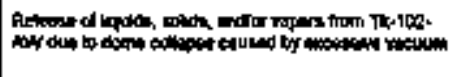 & 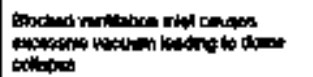 & 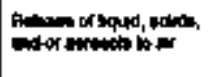 \\
\hline 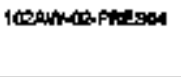 & DotT bah contmet & 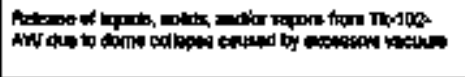 & 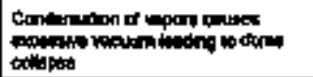 & 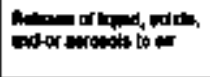 \\
\hline 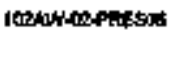 & 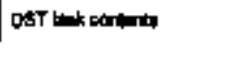 & 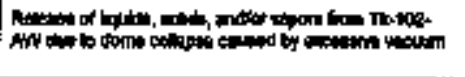 & 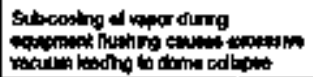 & 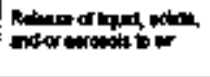 \\
\hline 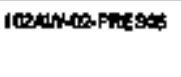 & 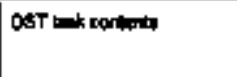 & 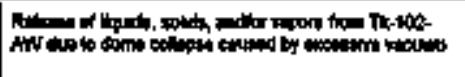 & 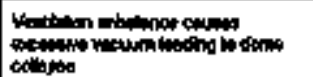 & 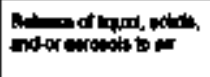 \\
\hline
\end{tabular}




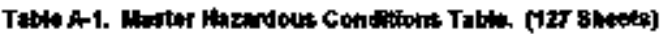

\begin{tabular}{|c|c|c|c|c|}
\hline ID & 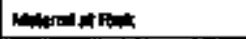 & Howrowe candbon & Crus: & Cenopipumes: \\
\hline 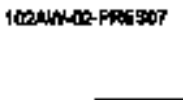 & DST tork ecemint & 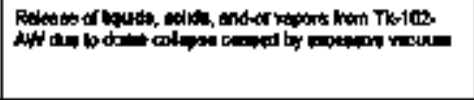 & 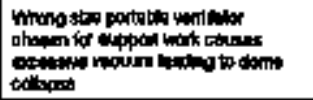 & 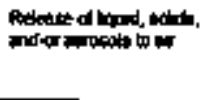 \\
\hline 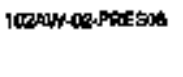 & 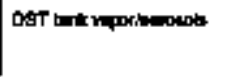 & 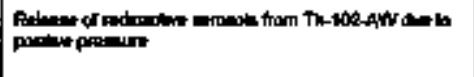 & 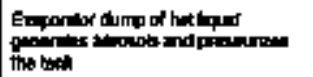 & 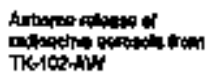 \\
\hline 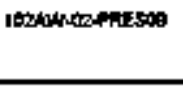 & DST tath thatiphent & 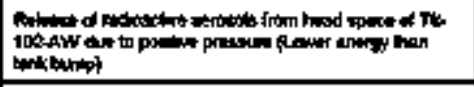 & 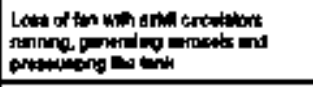 & 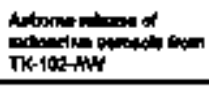 \\
\hline 1002Awatrew & 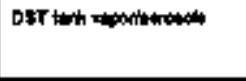 & 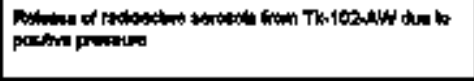 & 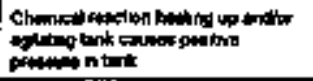 & 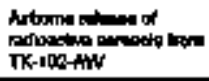 \\
\hline 102AW-02-TENOOOI & DST ENk & 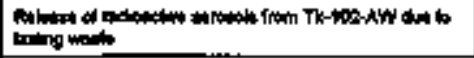 & 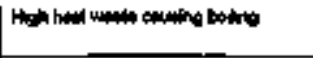 & 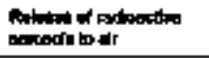 \\
\hline 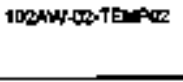 & 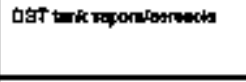 & 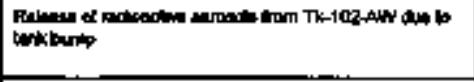 & 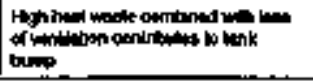 & 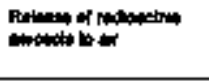 \\
\hline 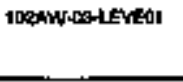 & DST tok contertes & 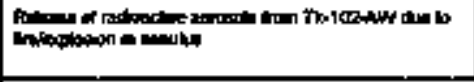 & 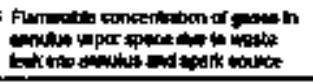 & 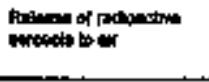 \\
\hline nozando3-Levete & 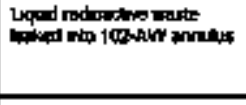 & 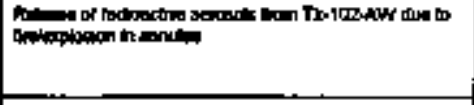 & 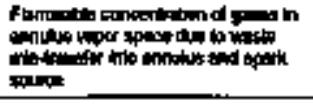 & 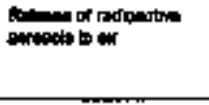 \\
\hline 102AN-0B-LENECG & 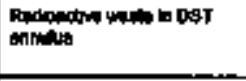 & 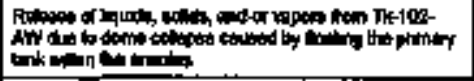 & 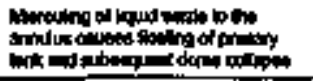 & 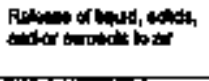 \\
\hline 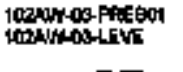 & GST unk yepundurpols & 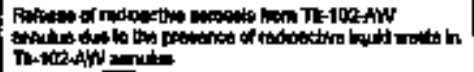 & 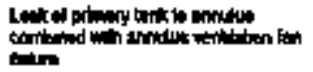 & 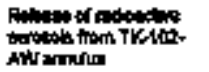 \\
\hline
\end{tabular}




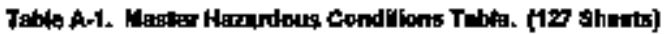

\begin{tabular}{|c|c|c|c|c|}
\hline $\mathbf{D}$ & Hutonol at Ruk & Henowe Condath & Cante & Consesqueha \\
\hline 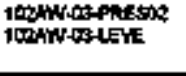 & DQT this kepathropots & 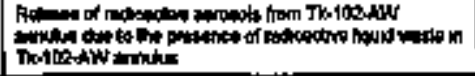 & 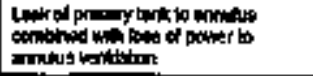 & 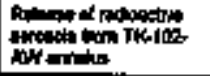 \\
\hline 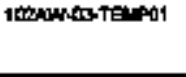 & 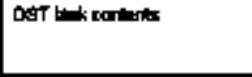 & 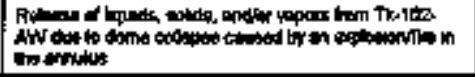 & 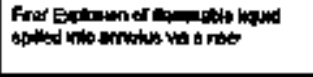 & Aninesed land wath, \\
\hline 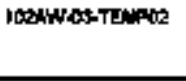 & 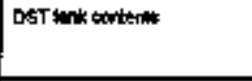 & 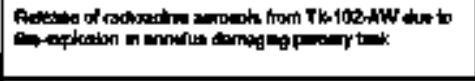 & 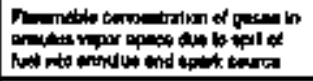 & 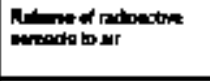 \\
\hline 102AW-OMHEYEO1 & L & 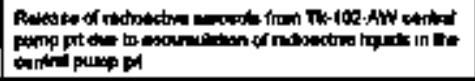 & 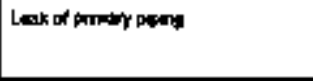 & 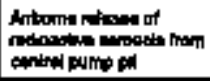 \\
\hline 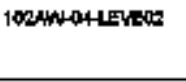 & 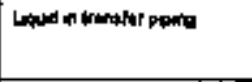 & 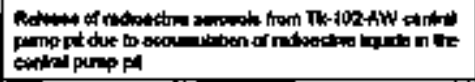 & 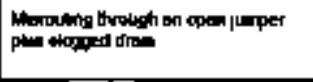 & 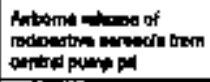 \\
\hline 10RAN-OMLEMECO & 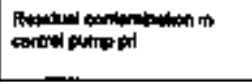 & 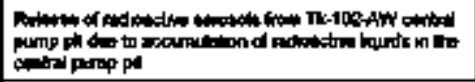 & 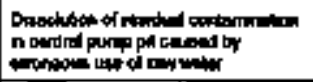 & 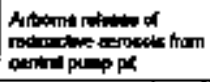 \\
\hline TRBAW-QALEVEOA & 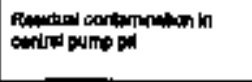 & 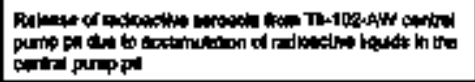 & 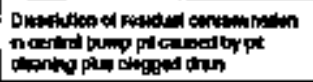 & 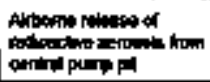 \\
\hline 1ERAMN04PFESO1 & Hah & 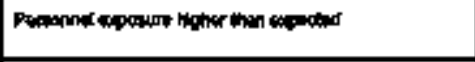 & 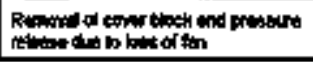 & 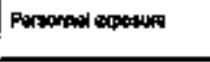 \\
\hline 10024Ho4missor & 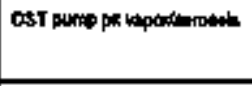 & 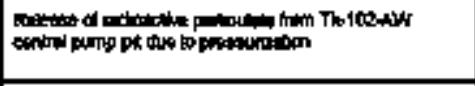 & 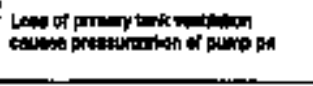 & 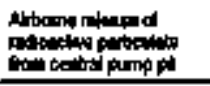 \\
\hline nodiwaspinson & 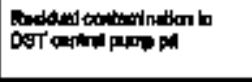 & 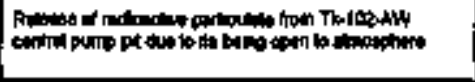 & 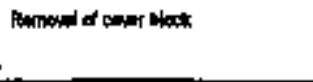 & 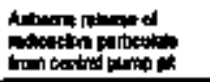 \\
\hline 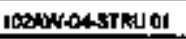 & 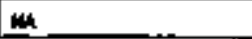 & 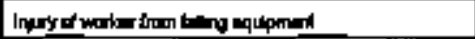 & 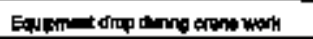 & 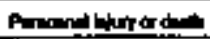 \\
\hline
\end{tabular}




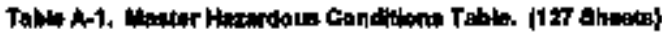

\begin{tabular}{|c|c|c|c|c|}
\hline $\boldsymbol{\sigma}$ & 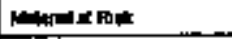 & 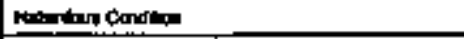 & Coust & 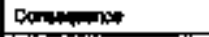 \\
\hline I0SAN-04-STRU or & 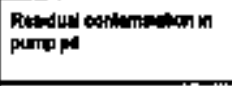 & 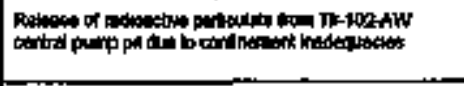 & 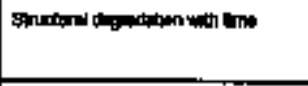 & 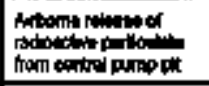 \\
\hline 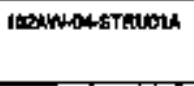 & DST tark contents & 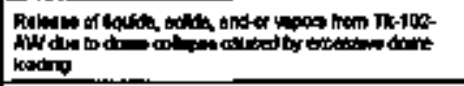 & 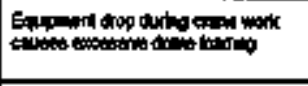 & 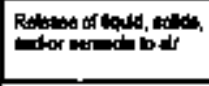 \\
\hline 100AN-01-6TRUOAB & 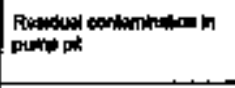 & 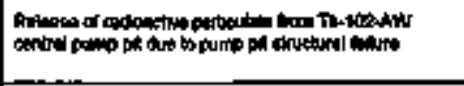 & 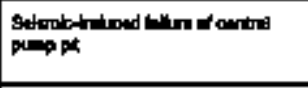 & 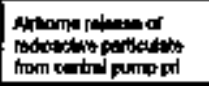 \\
\hline 102016-04-5TR4TDO & DET tentepontentas & 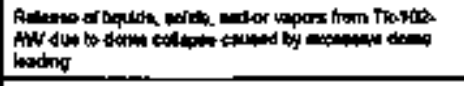 & 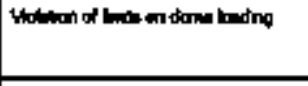 & 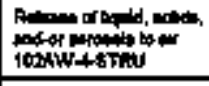 \\
\hline LONOW-OA-8TRLOOS & 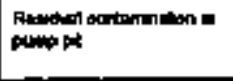 & 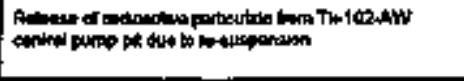 & 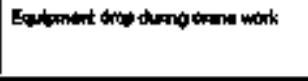 & 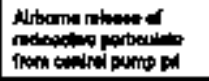 \\
\hline 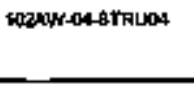 & 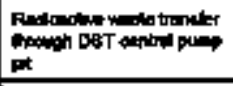 & 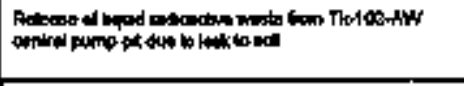 & 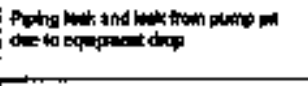 & 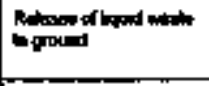 \\
\hline 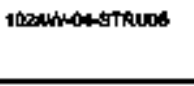 & 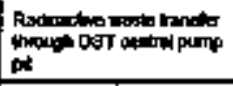 & 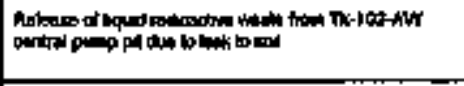 & 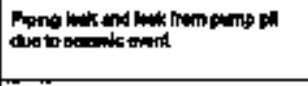 & 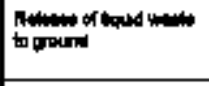 \\
\hline 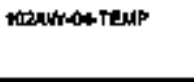 & 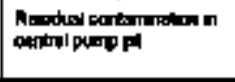 & 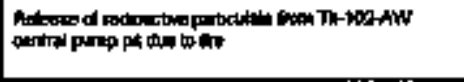 & 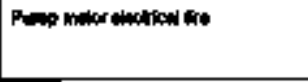 & 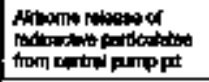 \\
\hline 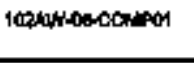 & 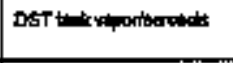 & 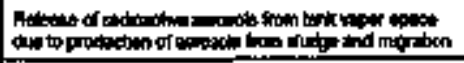 & 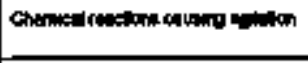 & 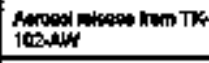 \\
\hline 10zahetosones & CST tank contente & 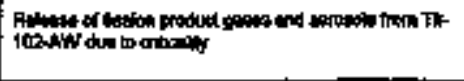 & 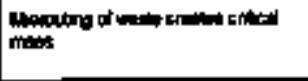 & 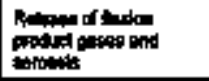 \\
\hline 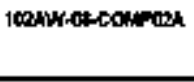 & DST tinkeonterta & 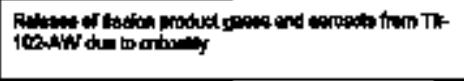 & 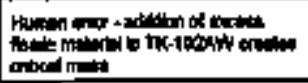 & 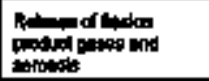 \\
\hline
\end{tabular}




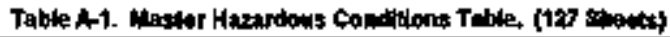

\begin{tabular}{|c|c|c|c|c|}
\hline $\mathbf{p}$ & Wherd at Rak & 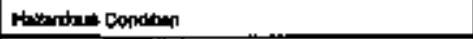 & Cawt: & COMPaqpeng: \\
\hline 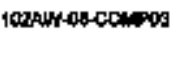 & 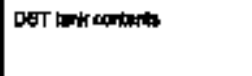 & 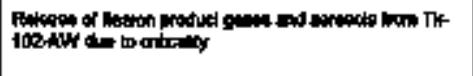 & 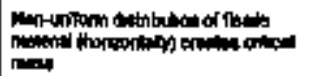 & 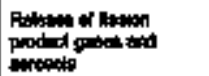 \\
\hline 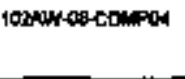 & 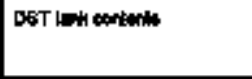 & 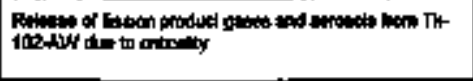 & 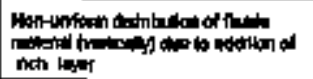 & 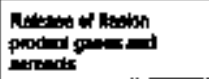 \\
\hline 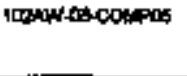 & 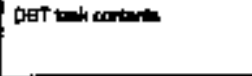 & 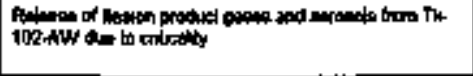 & 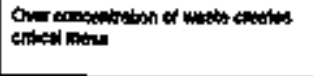 & 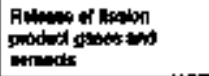 \\
\hline grawabconede & 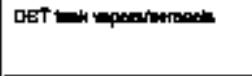 & 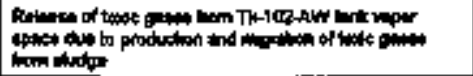 & 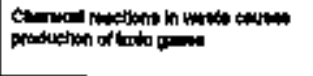 & Tape naper freman hem \\
\hline 10Bawnaconfel & QEST tak & 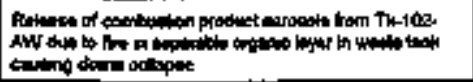 & 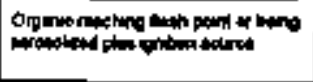 & 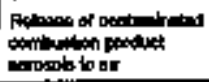 \\
\hline 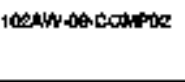 & D:89T mort morterth & 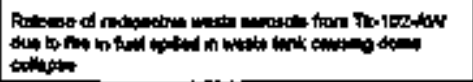 & 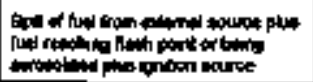 & 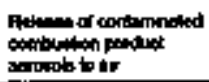 \\
\hline 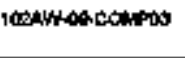 & 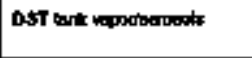 & 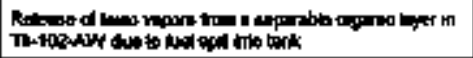 & 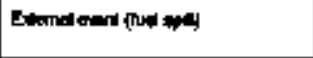 & 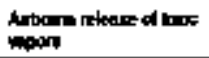 \\
\hline 102AH 00 CONPOA & 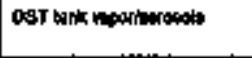 & 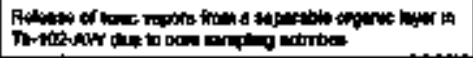 & 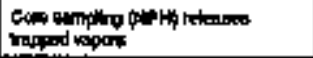 & 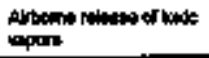 \\
\hline 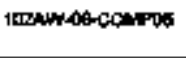 & 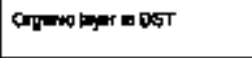 & 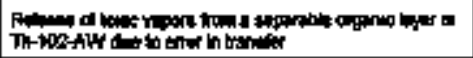 & 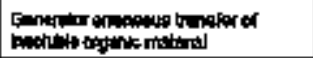 & 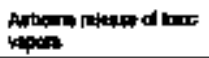 \\
\hline 10EMWOGTEP & 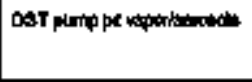 & 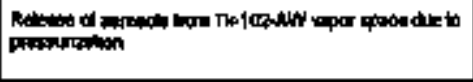 & 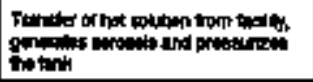 & 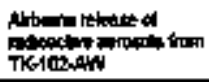 \\
\hline 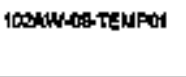 & DST & 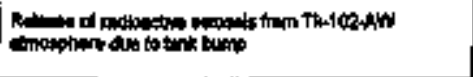 & 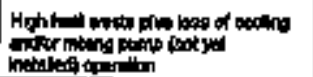 & 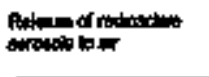 \\
\hline
\end{tabular}




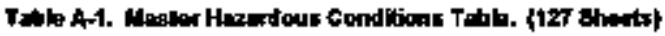

\begin{tabular}{|c|c|c|c|c|}
\hline ID & Noriend at Rak & 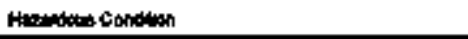 & cant & 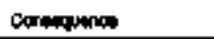 \\
\hline vedulwas TEuph & 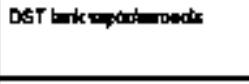 & 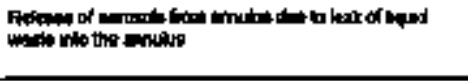 & 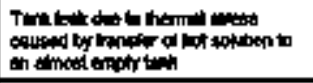 & 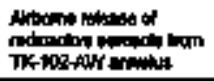 \\
\hline 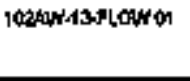 & $\mathbf{m}$ & 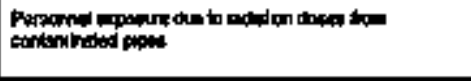 & 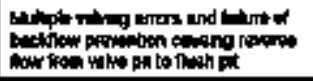 & 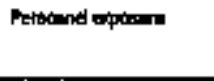 \\
\hline 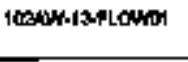 & 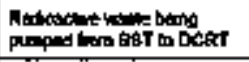 & 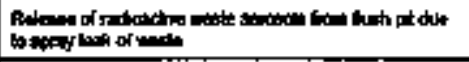 & 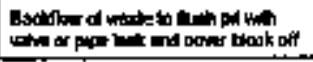 & 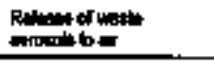 \\
\hline 10204-IFFLOWD & 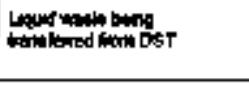 & 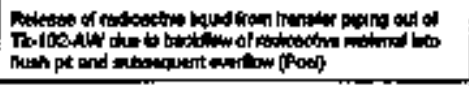 & 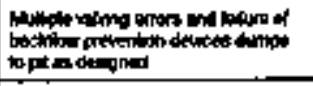 & 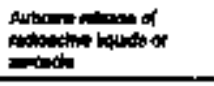 \\
\hline 1008-01 of & 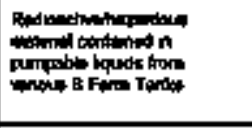 & 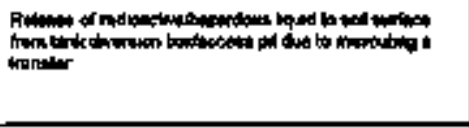 & 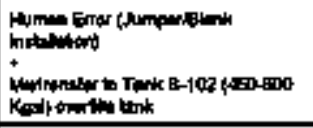 & 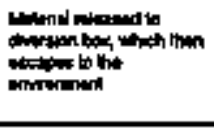 \\
\hline 100est be & 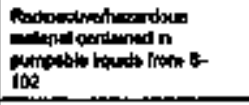 & 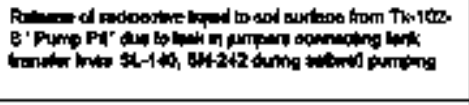 & 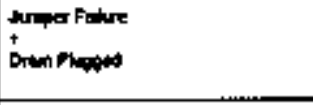 & 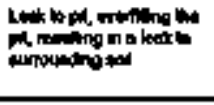 \\
\hline Fonsal bs & 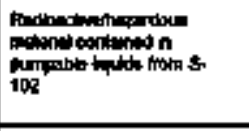 & 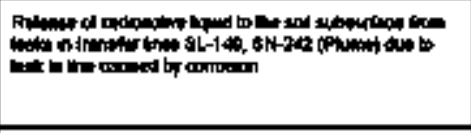 & 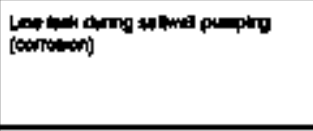 & 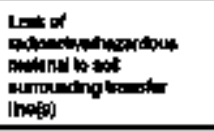 \\
\hline $100 \cos a$ & 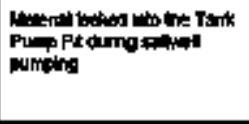 & 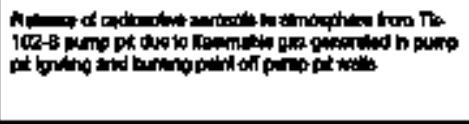 & 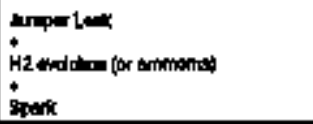 & 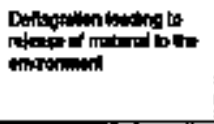 \\
\hline
\end{tabular}




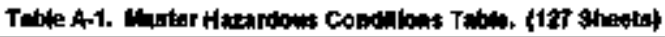

\begin{tabular}{|c|c|c|c|c|}
\hline ID & 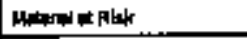 & Heringut tondinan & Cand & Contapiones \\
\hline 10090202 & 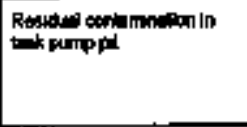 & 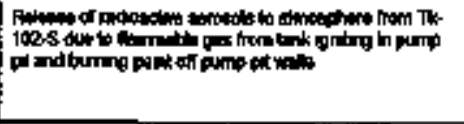 & 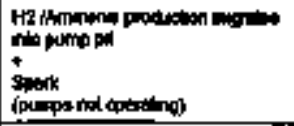 & 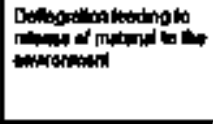 \\
\hline 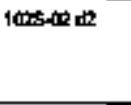 & 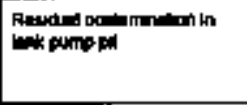 & 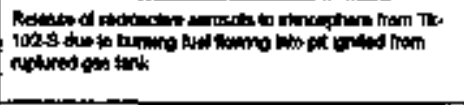 & 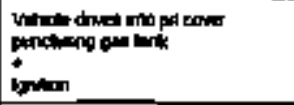 & 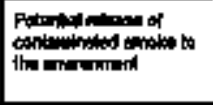 \\
\hline 140,020 & 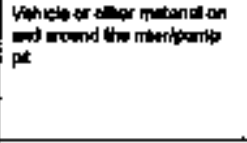 & 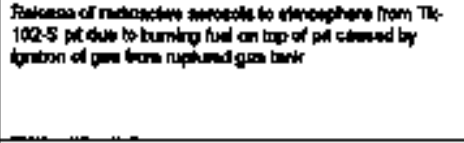 & 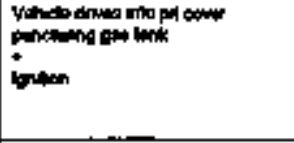 & 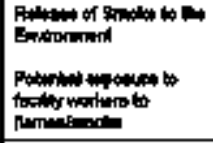 \\
\hline $1 \mathrm{trgs}-0 \mathrm{~s} \mathrm{Bg}$ & Terkenomitis & 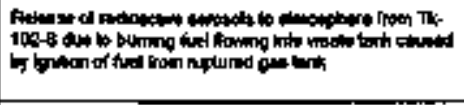 & 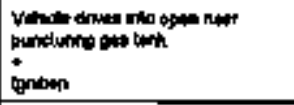 & 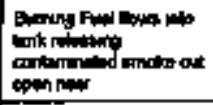 \\
\hline $1068-03$ b4 & 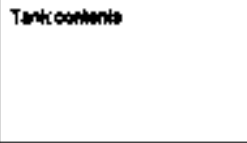 & 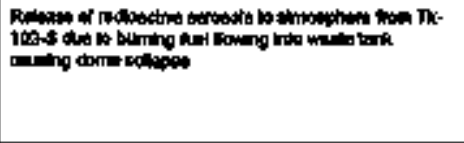 & 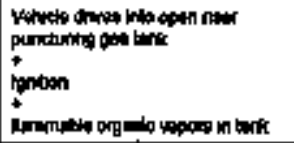 & 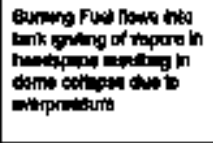 \\
\hline 100804516 & 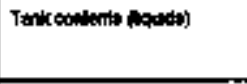 & 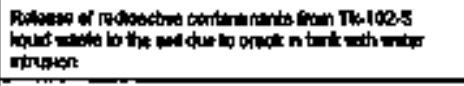 & 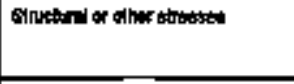 & 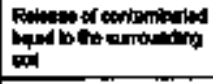 \\
\hline 1025-04ated & 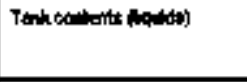 & 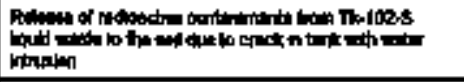 & Cominish & 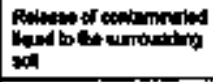 \\
\hline $102804 \%$ & 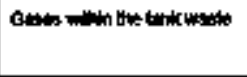 & 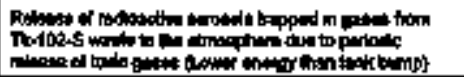 & 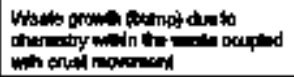 & 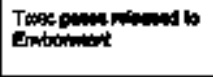 \\
\hline
\end{tabular}




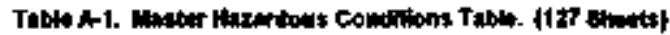

\begin{tabular}{|c|c|c|c|c|}
\hline ס & Unested a thin & Hrandoun Compingen & Emag & Comingen \\
\hline $1005-10 t$ & 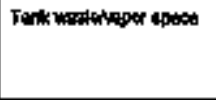 & 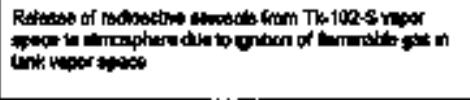 & 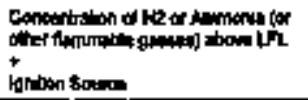 & 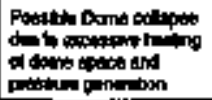 \\
\hline 1020연 머 & 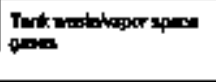 & 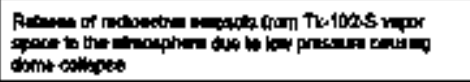 & 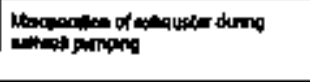 & 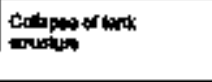 \\
\hline $1008-04$ alb & 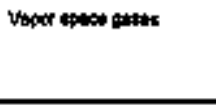 & 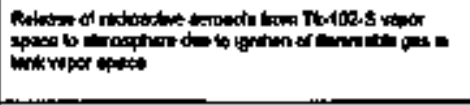 & 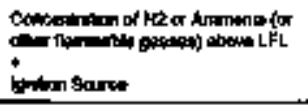 & 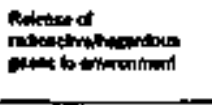 \\
\hline $1028-04-2$ & 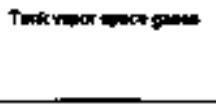 & 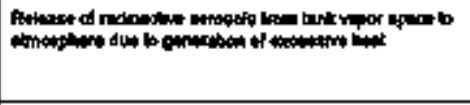 & 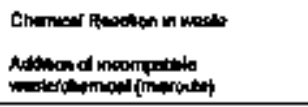 & 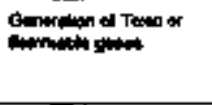 \\
\hline Inge o4 웅 & Twircorterts & 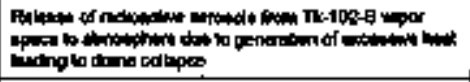 & 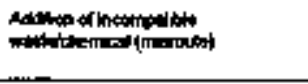 & The \\
\hline $1008-04 \times 4$ & 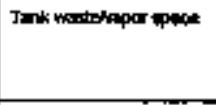 & 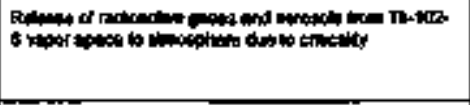 & 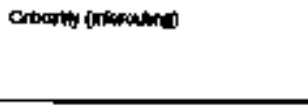 & 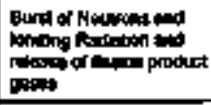 \\
\hline $10 \cos -05$ & 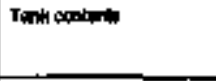 & 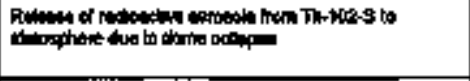 & 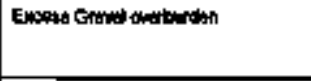 & 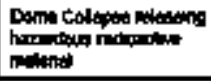 \\
\hline 10ES-05 al:A & 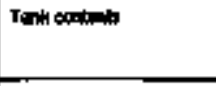 & 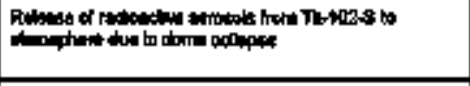 & 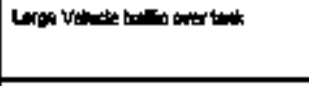 & 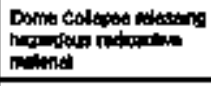 \\
\hline $1 \cos 45$ ar & Tank cortinim & 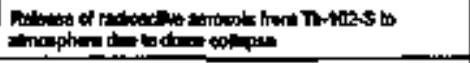 & 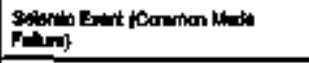 & 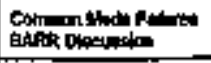 \\
\hline $1000-050$ & 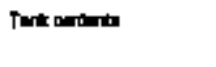 & 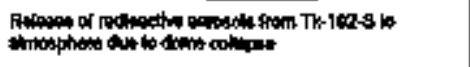 & 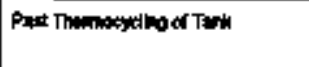 & 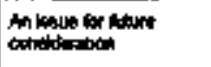 \\
\hline
\end{tabular}




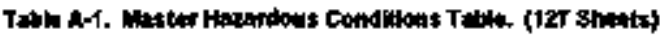

\begin{tabular}{|c|c|c|c|c|}
\hline ID & Motoriandin Rath & Nuardous Condion & Canis & Compipangy \\
\hline vosects ats & Ink contion & 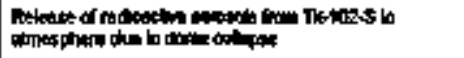 & Long Tim ing & 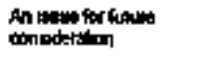 \\
\hline these-ist & 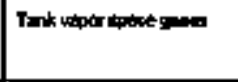 & 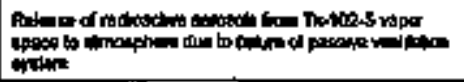 & 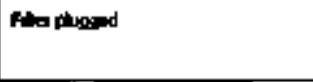 & 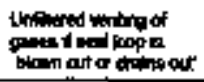 \\
\hline 10 ofolk & Tonk numiney & 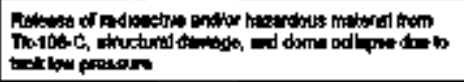 & 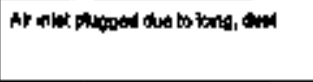 & \\
\hline $109-010$ & 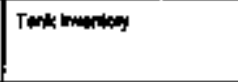 & 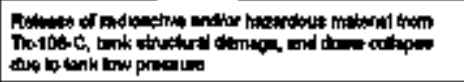 & 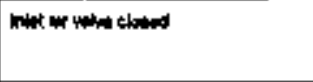 & \\
\hline 10:019-019 & Tork manisey & 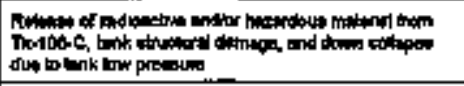 & 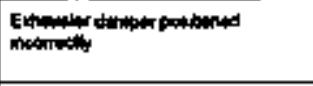 & \\
\hline IABC-010 & 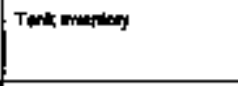 & 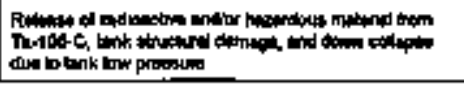 & 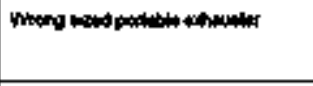 & \\
\hline 10EC-01E & 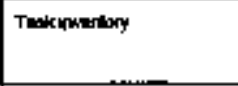 & 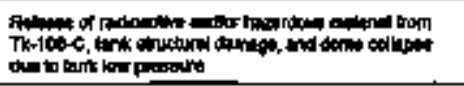 & 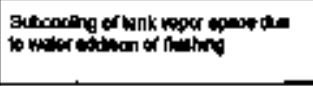 & \\
\hline 10E-G-01F & Trak; mamphere & 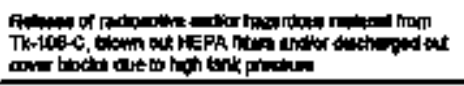 & 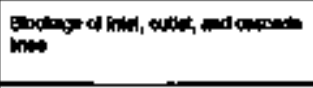 & \\
\hline toto-010 & 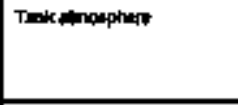 & 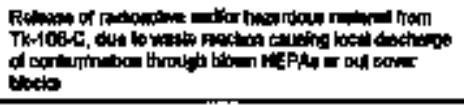 & 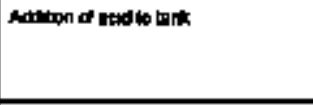 & \\
\hline NOCOOHH & 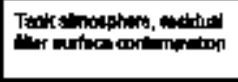 & 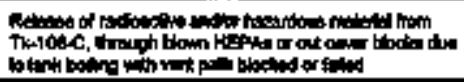 & 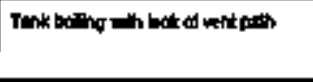 & \\
\hline
\end{tabular}




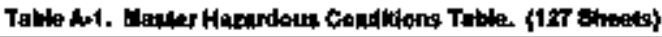

\begin{tabular}{|c|c|c|c|c|}
\hline D. & 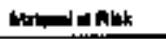 & Mentrisous Condilm & can & Conteponkt \\
\hline 1080-91 & Tutionoriory & 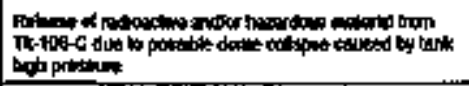 & 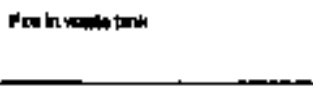 & \\
\hline $1000-01]$ & Tartimantory & 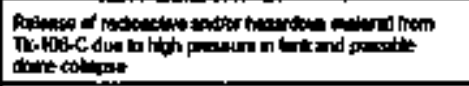 & 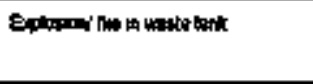 & \\
\hline 1000 & Tathompapher & 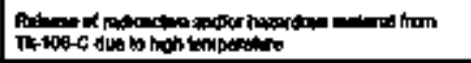 & Whiter exporition & \\
\hline notersin & Tuttmintioy & 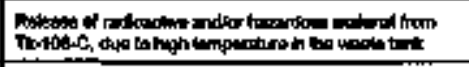 & 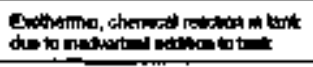 & \\
\hline 109C-OMLA & 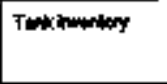 & 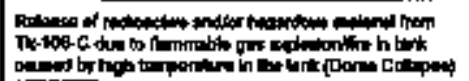 & 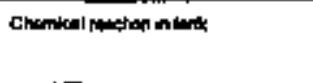 & \\
\hline $100 \mathrm{c}-0 \mathrm{gN}$ & Imk mentory & 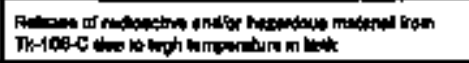 & 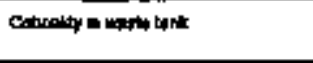 & \\
\hline Iotcourtion & 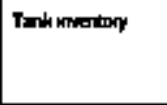 & 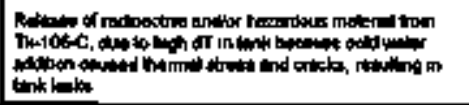 & 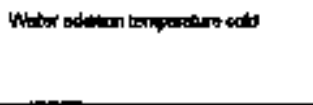 & \\
\hline 10sa-als & 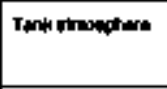 & 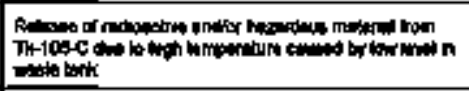 & Wher mapion & \\
\hline 145-dis & 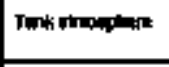 & 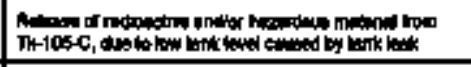 & 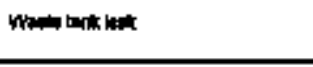 & \\
\hline 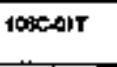 & Trik & 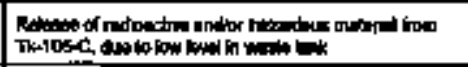 & 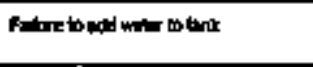 & \\
\hline 10acolu & w & 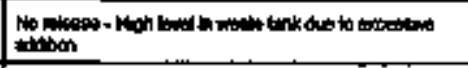 & 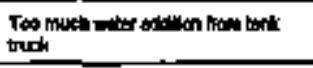 & \\
\hline $1000001 \%$ & 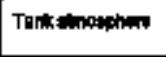 & 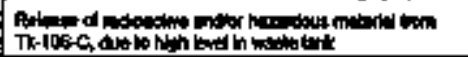 & 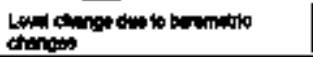 & \\
\hline
\end{tabular}




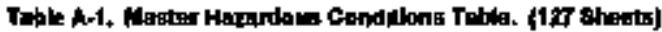

\begin{tabular}{|c|c|c|c|c|}
\hline in & Intanal st Rek & 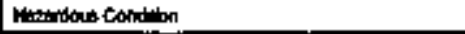 & $C \operatorname{cod}$ & Contanquention \\
\hline $10 x-01 x$ & Tank mationgy & 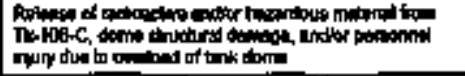 & 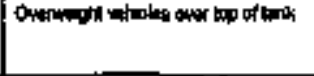 & \\
\hline $100-0 \mathrm{rr}$ & Trte mortary & 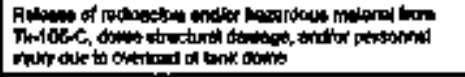 & 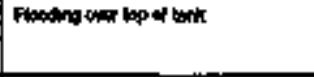 & \\
\hline $100 \mathrm{cosh}$ & Thate mentary & 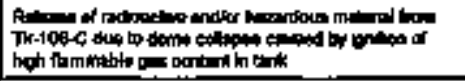 & 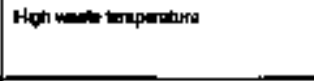 & \\
\hline $1000-02 \mathrm{~B}$ & THAtration & 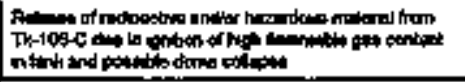 & 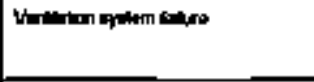 & \\
\hline 1000005 & TH: & 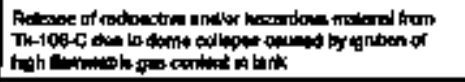 & 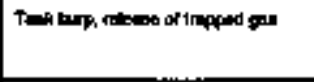 & \\
\hline 100004 & Tanh mingont & 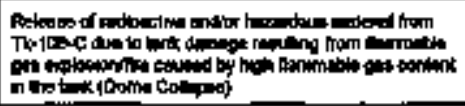 & 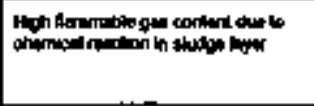 & \\
\hline IEx-0uts & Tahsimogatere & 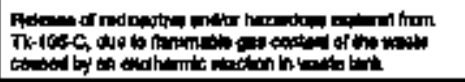 & 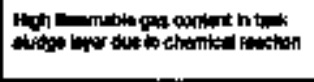 & \\
\hline 10tectous & 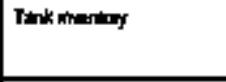 & 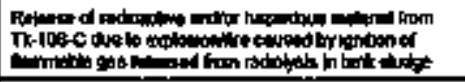 & 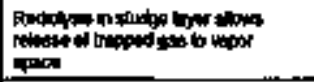 & \\
\hline 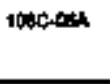 & 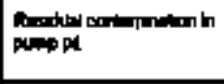 & 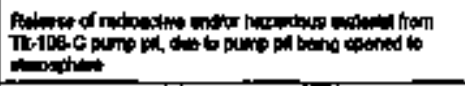 & 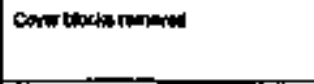 & \\
\hline $1000-008$ & Nh & 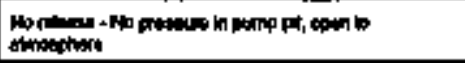 & 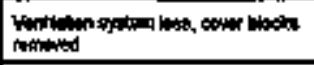 & \\
\hline $1000-000$ & 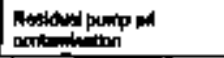 & 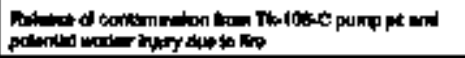 & 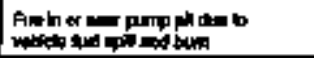 & \\
\hline
\end{tabular}




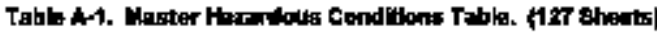

\begin{tabular}{|c|c|c|c|c|}
\hline 描 & 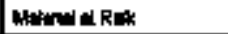 & menstion cention & Conse & Centongines \\
\hline 1000-00\% & 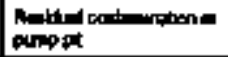 & 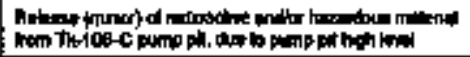 & 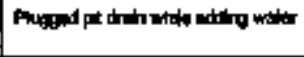 & \\
\hline $10 \cos -6$ & 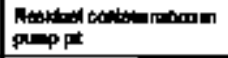 & 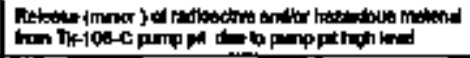 & 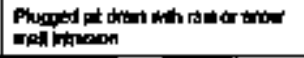 & \\
\hline Joactey & 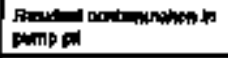 & 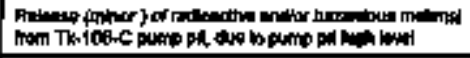 & 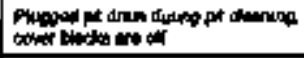 & \\
\hline $1000-005]$ & Nin & 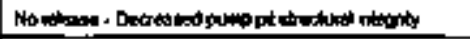 & Dome an kas & \\
\hline $\sec 0 \mathrm{ctax}$ & Geand purpept & 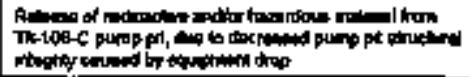 & 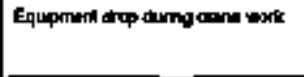 & \\
\hline 1000 ates & 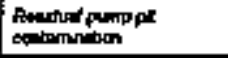 & 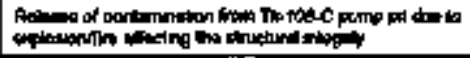 & Explocentita m parppot & \\
\hline $100=-094$ & Mh & 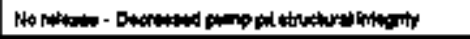 & 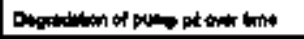 & \\
\hline $1000+165$ & Wh & 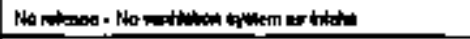 & 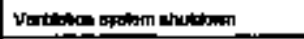 & \\
\hline $100 \mathrm{de}-10 \mathrm{c}$ & MN & 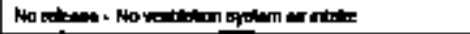 & Whet Hepen ther paps & \\
\hline $1000-100$ & wh & 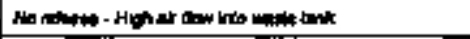 & Ind & \\
\hline \$OCE-toE & Wh & 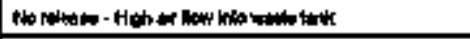 & 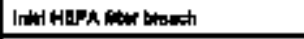 & \\
\hline Doscintir & 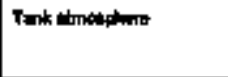 & 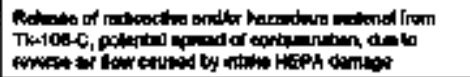 & 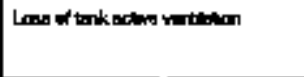 & \\
\hline roc-moH & Talk atmotphitr & 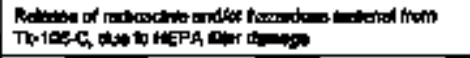 & 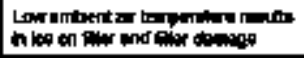 & \\
\hline $1000-10$ & 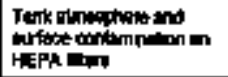 & 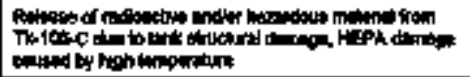 & 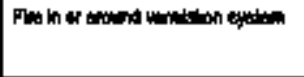 & \\
\hline $1000-10 x$ & 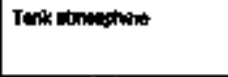 & 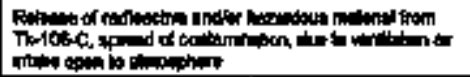 & HEPh Det weoch & \\
\hline
\end{tabular}




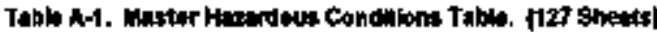

\begin{tabular}{|c|c|c|c|c|}
\hline ID & 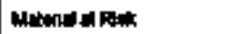 & 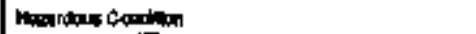 & thman & Conporumose \\
\hline thes $10 x$ & Tunk & 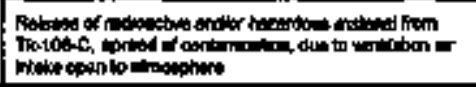 & 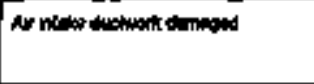 & \\
\hline $100 x-114$ & 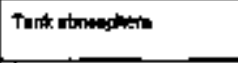 & 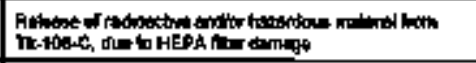 & HEPh ownts photed & \\
\hline $108 \mathrm{c}-119$ & Nak & 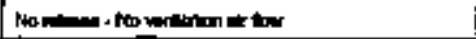 & 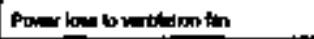 & \\
\hline $1000-11 c$ & NA & 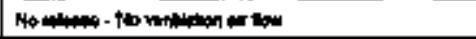 & 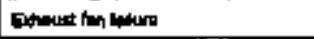 & \\
\hline $1006-110$ & wa & Not & 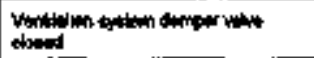 & \\
\hline 109C-11E & 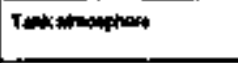 & 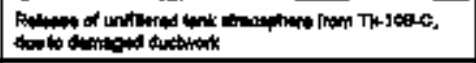 & Domare of unthonductiont & \\
\hline 1000 -1fF & $m$ & 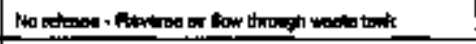 & 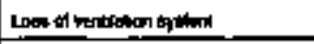 & \\
\hline 16ec-4to & Int mnophert & 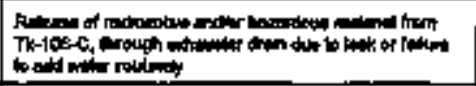 & 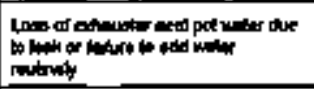 & \\
\hline $1000-11 H$ & Textanophen & 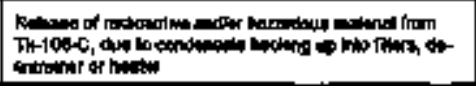 & 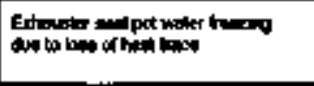 & \\
\hline$n 00=111$ & 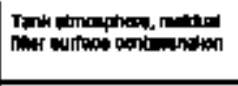 & 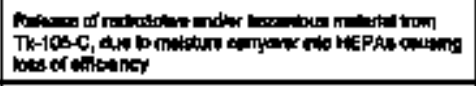 & 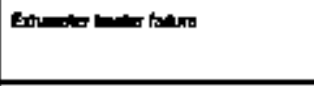 & \\
\hline $\operatorname{noc}-114$ & 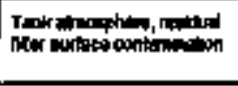 & 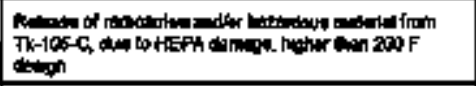 & 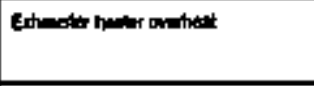 & \\
\hline lasa-11K & 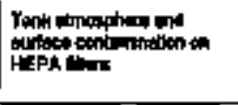 & 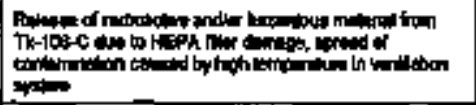 & 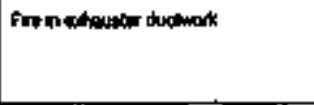 & \\
\hline IAGC-IIL & 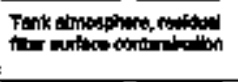 & 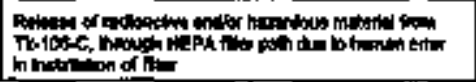 & 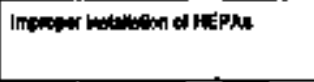 & \\
\hline
\end{tabular}




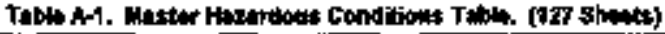

\begin{tabular}{|c|c|c|c|c|}
\hline $\mathbf{D}$ & 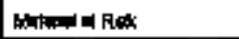 & Haxplous Conenting & $\cos$ & Contaquance \\
\hline votis & 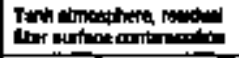 & 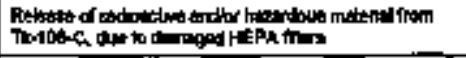 & 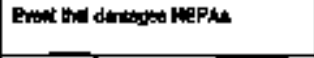 & \\
\hline $100 \mathrm{C}-11 \mathrm{~N}$ & 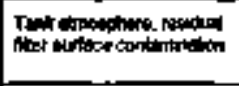 & 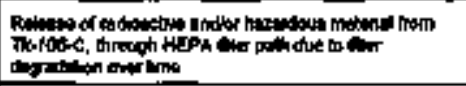 & HEPA ther wation & \\
\hline $1000-110$ & Tanil atmeaphere & 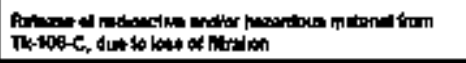 & GEPA thr wowar & \\
\hline $1620-124$ & 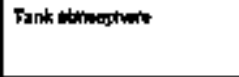 & 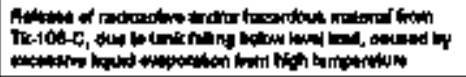 & 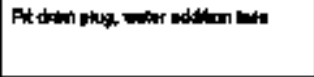 & \\
\hline laecheste & Twat theophom & 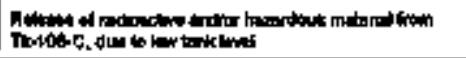 & 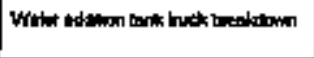 & \\
\hline $1006-12 x$ & 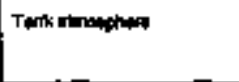 & 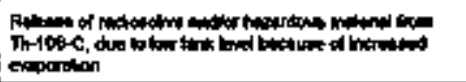 & 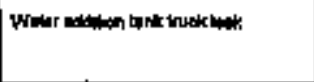 & \\
\hline $1606-120$ & 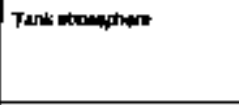 & 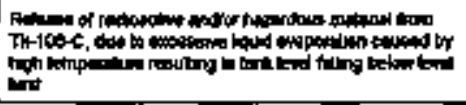 & 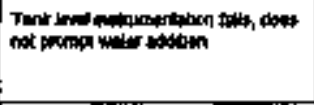 & \\
\hline $109 \mathrm{C,12F}$ & Termk inamery & 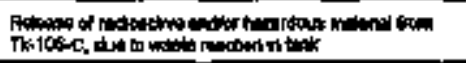 & 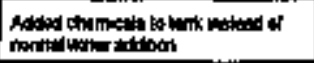 & \\
\hline voct-tas & Tutimertary & 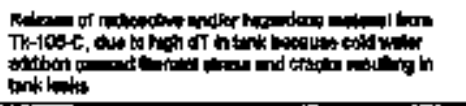 & 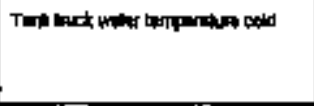 & \\
\hline 1000.139 & Tentomativing & 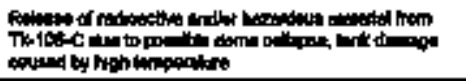 & 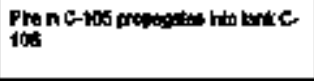 & \\
\hline $1000-13$ & $\mathbf{H i n}$ & No & 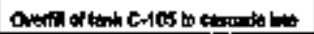 & \\
\hline
\end{tabular}




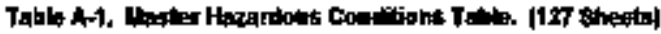

\begin{tabular}{|c|c|c|c|c|}
\hline I0 & 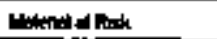 & 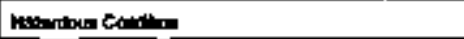 & and & 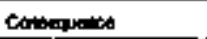 \\
\hline NOTABSOALUEM & Gurcriontan & 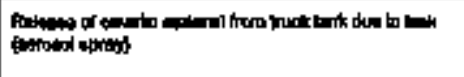 & 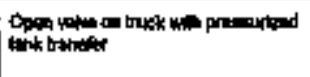 & 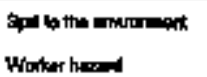 \\
\hline 10TAMal-jeving & Conube & 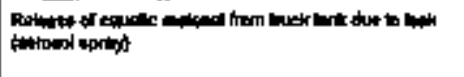 & 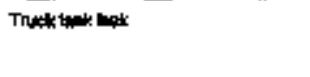 & 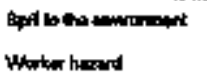 \\
\hline 10tanosditued & Cavito matinos & 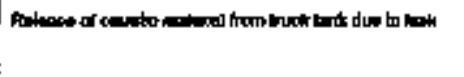 & 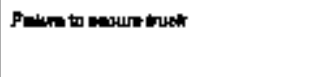 & 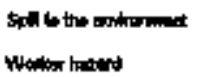 \\
\hline 107ANG1-TENOB & $\mathbf{M h}$ & 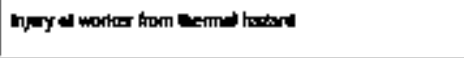 & 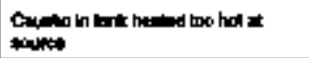 & $\begin{array}{l}\text { Thamsin bocood to } \\
\text { persorel }\end{array}$ \\
\hline 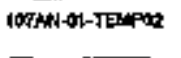 & 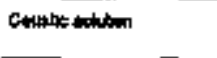 & 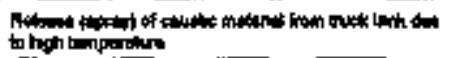 & The hathonit: & Lation hion \\
\hline IOSANACOFLOOWOH & curtents of Das & 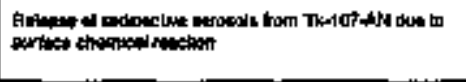 & 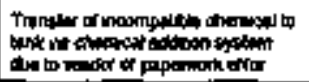 & 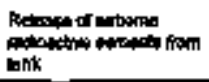 \\
\hline 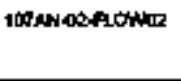 & cantent: ư Dost & 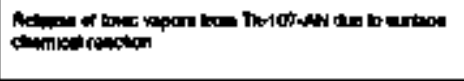 & 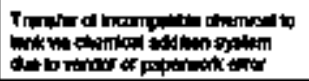 & 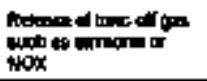 \\
\hline nFindosonos & 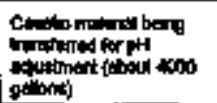 & 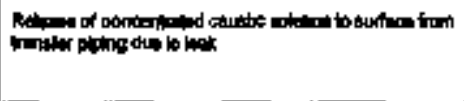 & 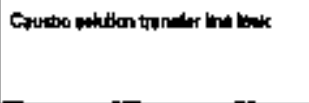 & 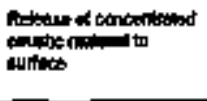 \\
\hline 1OTAHOS-PBES & 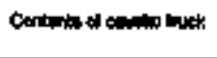 & 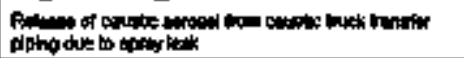 & 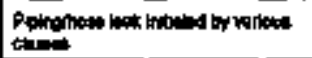 & 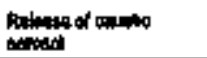 \\
\hline 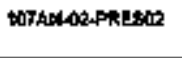 & Cenciton & 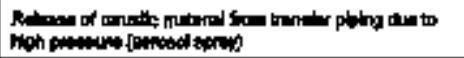 & Monedecomed & | \\
\hline 107AMO2,TEANOA & Canbut tolution & 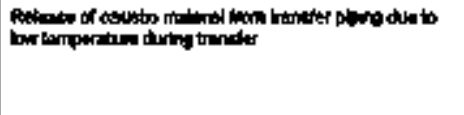 & 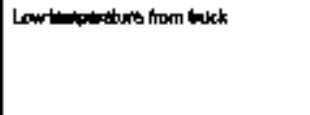 & \\
\hline
\end{tabular}




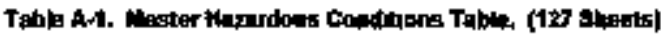

\begin{tabular}{|c|c|c|c|c|}
\hline 10 & Hets & Mazuriburt condom & Calso & 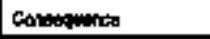 \\
\hline NTANGOFLON & 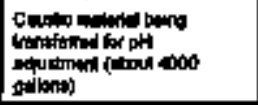 & 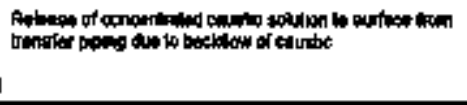 & 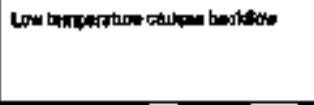 & 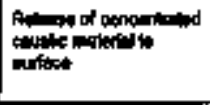 \\
\hline $1140 \times 402 b$ & 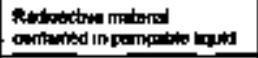 & 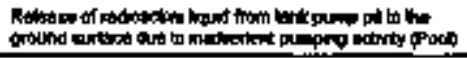 & Mamito Biver & Non \\
\hline $1148 x-04=14$ & 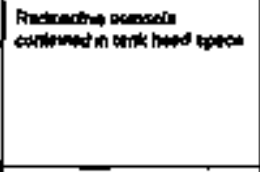 & 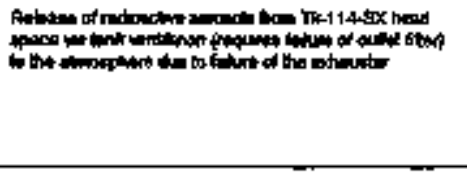 & 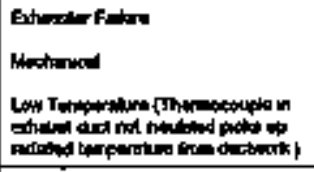 & 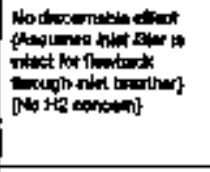 \\
\hline 114330-05 16 & 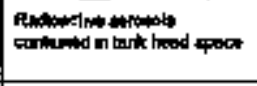 & 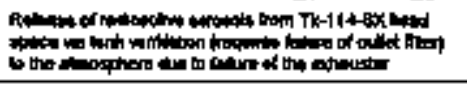 & Fen Mupar & 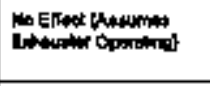 \\
\hline $110 \times-04=2$ & 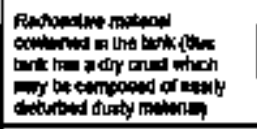 & 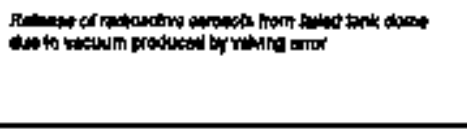 & 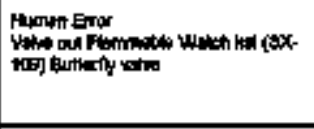 & 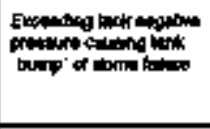 \\
\hline $1145 x-04 \backsim 3$ & 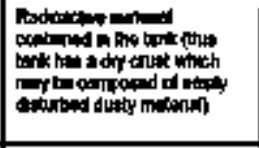 & 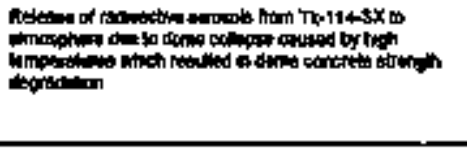 & 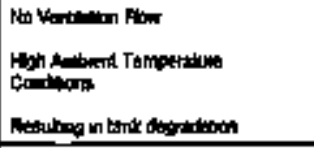 & 10 \\
\hline $1146 \times-04=4$ & 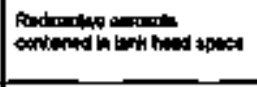 & 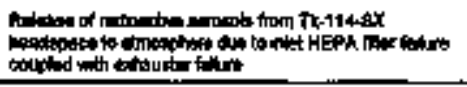 & Ftim Fand & 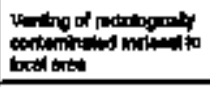 \\
\hline 11 \&x-adb 1 : 1 & 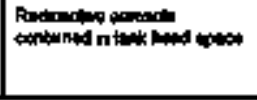 & 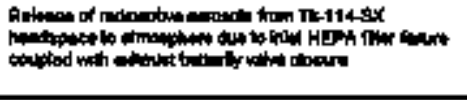 & 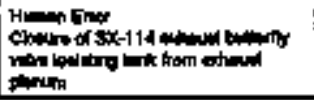 & 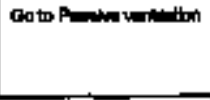 \\
\hline
\end{tabular}




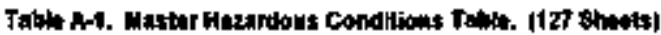

\begin{tabular}{|c|c|c|c|c|}
\hline $\mathbf{0}$ & 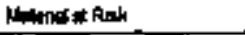 & 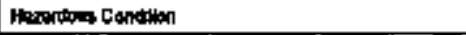 & tan & conanimanes \\
\hline $11 \leq 5 \times 04 b 1 \cdot 2$ & 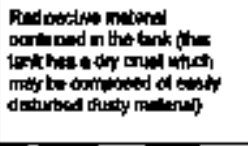 & 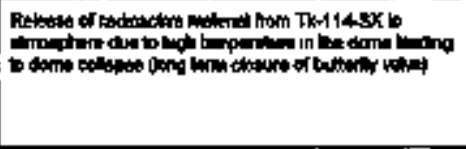 & 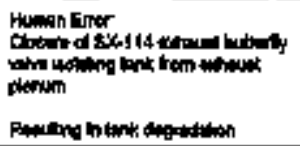 & 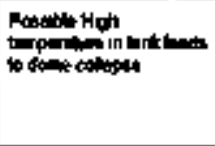 \\
\hline $1145 \times 0401+3$ & 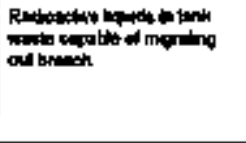 & 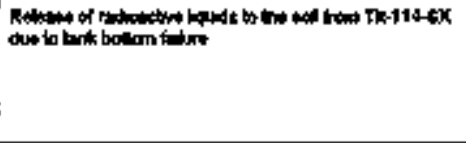 & 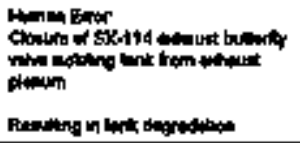 & 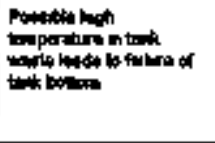 \\
\hline $1145 x-04010$ in & 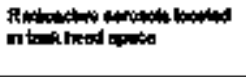 & 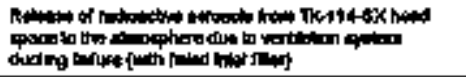 & 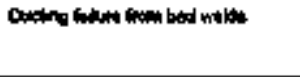 & 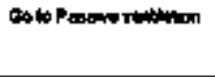 \\
\hline$A 145 x-0,1 b 101 b$ & 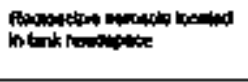 & 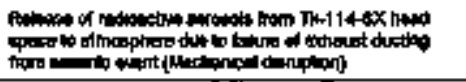 & 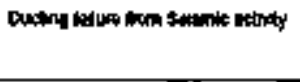 & 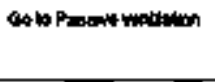 \\
\hline 1t $46 x-04$ b 1 i d 2 & 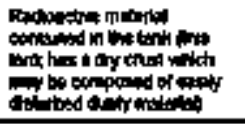 & 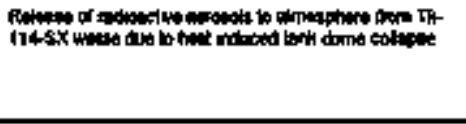 & 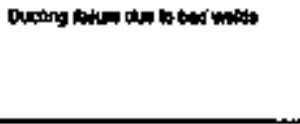 & 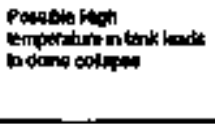 \\
\hline $1146 x-04$ b 1 b 20 & 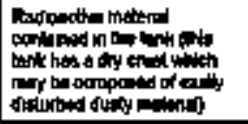 & 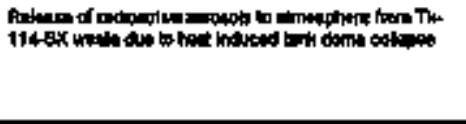 & 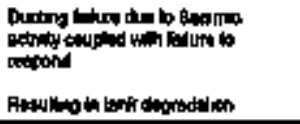 & 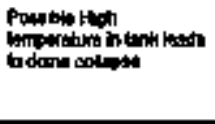 \\
\hline 1145xiat b i b ta & 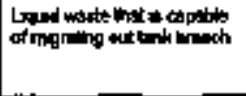 & 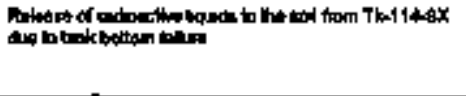 & 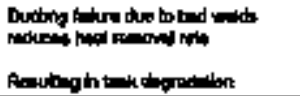 & 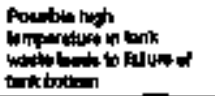 \\
\hline
\end{tabular}




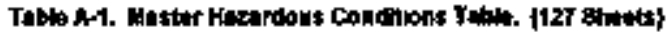

\begin{tabular}{|c|c|c|c|c|}
\hline ID & 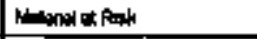 & 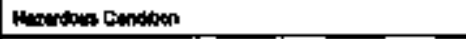 & Connen & Cenompinases \\
\hline $1145 \times-0461 b 3$ & 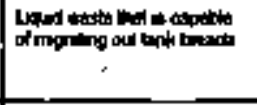 & 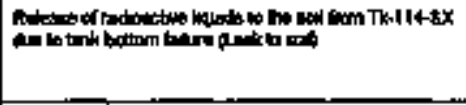 & 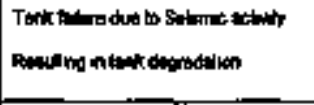 & 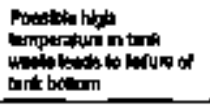 \\
\hline 1149604 b 1 व & 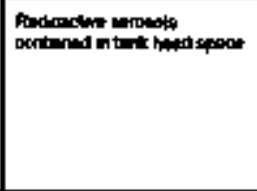 & 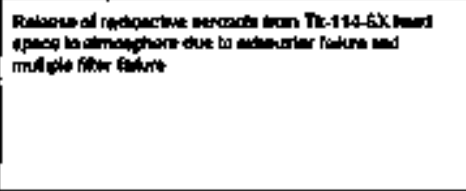 & 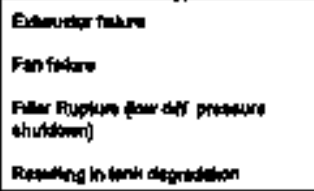 & 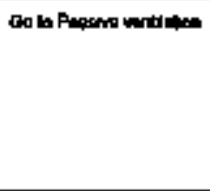 \\
\hline 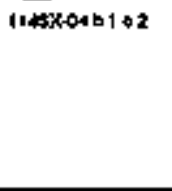 & 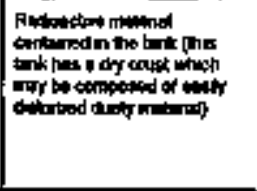 & 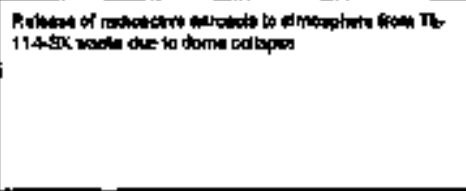 & 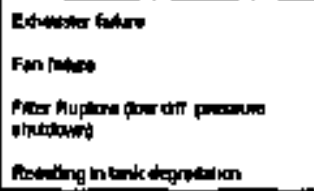 & 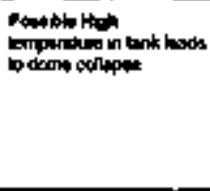 \\
\hline $1148 \times<04$ b $1=3$ & 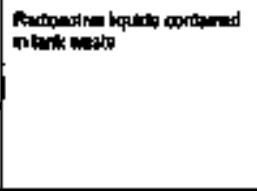 & 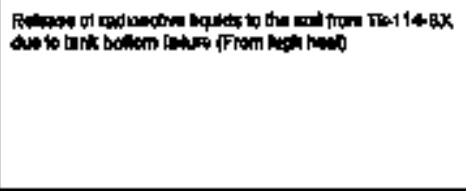 & 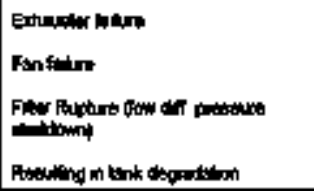 & 等 \\
\hline 1146xod of of & 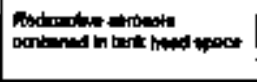 & 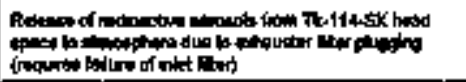 & 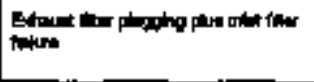 & 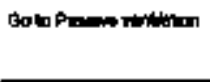 \\
\hline $1148 \times 04 b+d z$ & 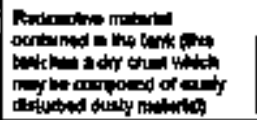 & 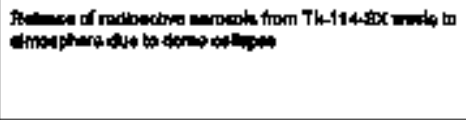 & 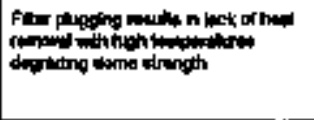 & Pandin Hoh \\
\hline
\end{tabular}




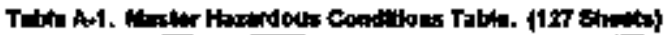

\begin{tabular}{|c|c|c|c|c|}
\hline $\mathbf{w}$ & Mncordal at Then & Heasiens condien & CNA & Conianpunans \\
\hline 114 ax<0a b 1 d 3 & 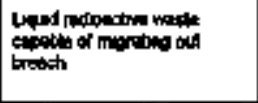 & 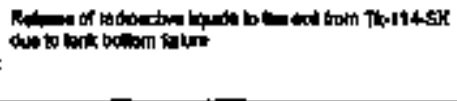 & 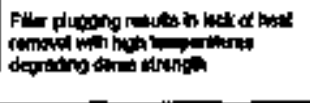 & 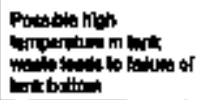 \\
\hline $1145 \times 04635$ & 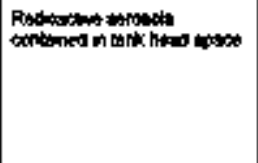 & 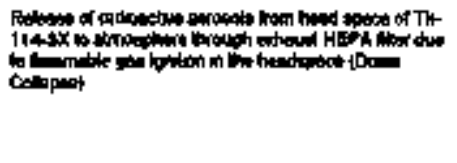 & 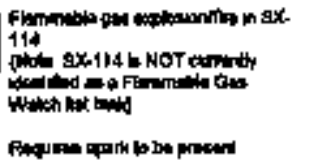 & 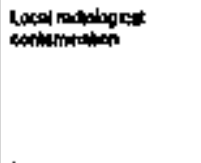 \\
\hline $11+5 \times-01 b 7$ & 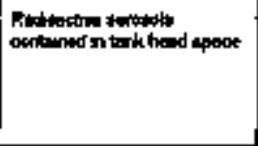 & 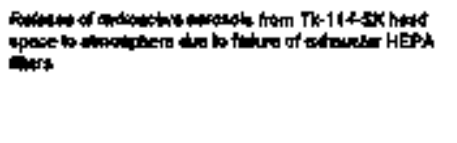 & 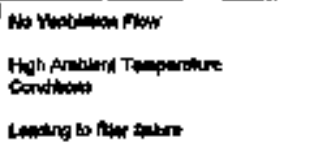 & 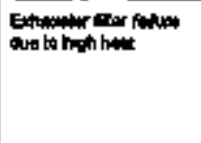 \\
\hline $1148 x-04<1$ & 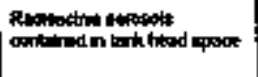 & 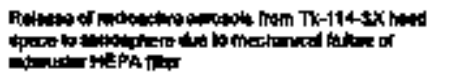 & 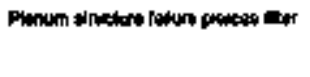 & 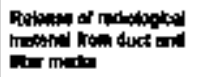 \\
\hline $1+49 x-04 c 20$ & 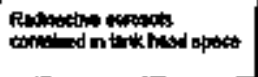 & 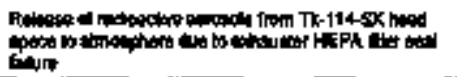 & MEPA ANer wal Mant & 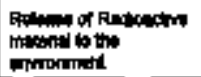 \\
\hline $1145 x-\infty \times-2$ & 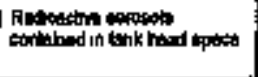 & 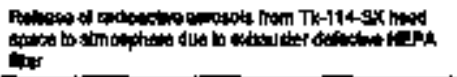 & 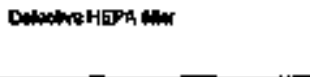 & 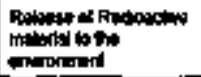 \\
\hline $1143 x-04=2 e$ & 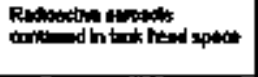 & 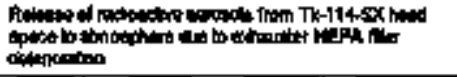 & 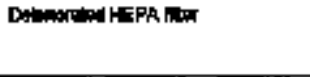 & 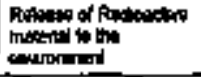 \\
\hline $1145 x=04024$ & 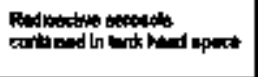 & 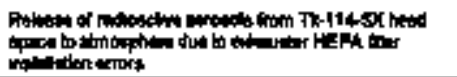 & 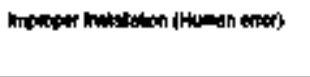 & 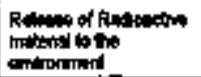 \\
\hline
\end{tabular}




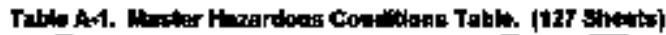

\begin{tabular}{|c|c|c|c|c|}
\hline 10 & 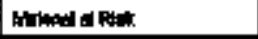 & 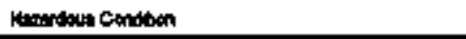 & conen & conescinumed \\
\hline $1148 \times 06$ b 1 & 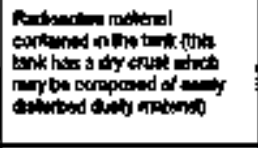 & 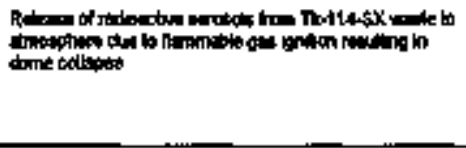 & 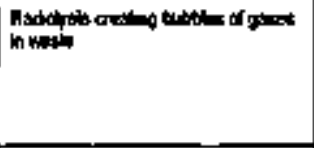 & 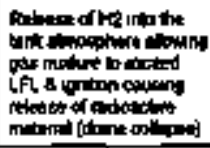 \\
\hline $1149 \times 60$ b b 2 & 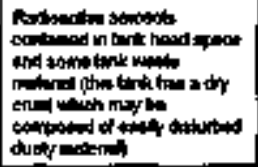 & 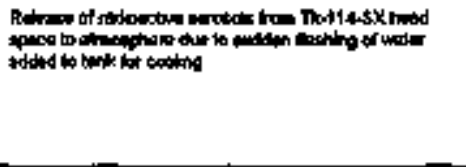 & 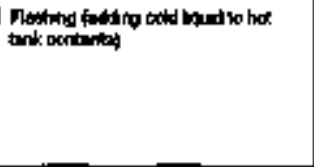 & 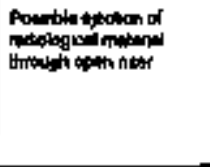 \\
\hline $1148 x-66$ b 3 & 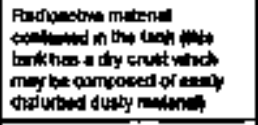 & 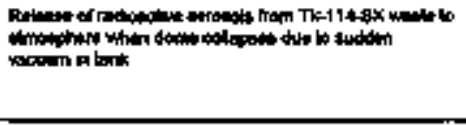 & 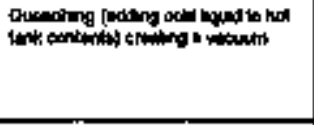 & 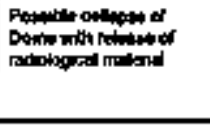 \\
\hline $11454 \omega 6=20$ & 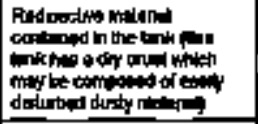 & 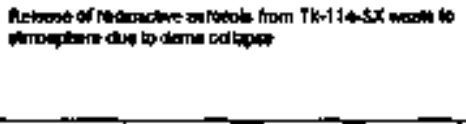 & 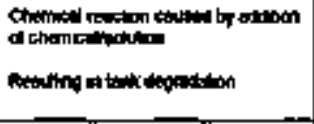 & 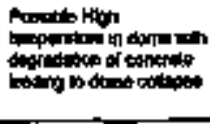 \\
\hline $1149 x-05+2 x$ & 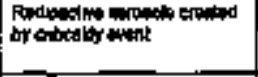 & 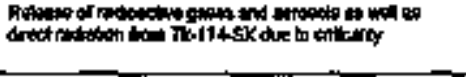 & 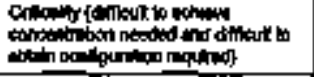 & 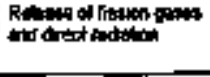 \\
\hline $1148 \times 05 d$ & 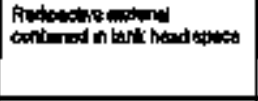 & 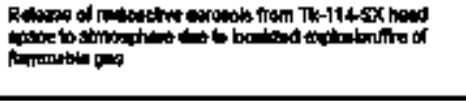 & 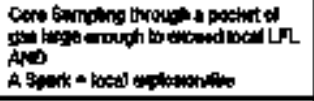 & 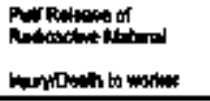 \\
\hline 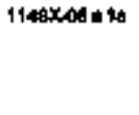 & 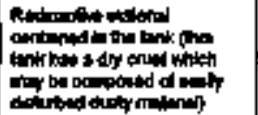 & 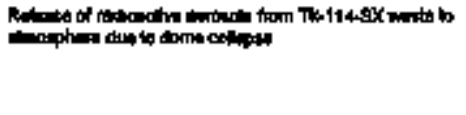 & 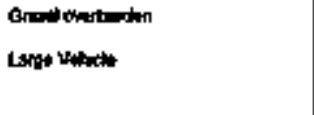 & Dan Cdhpe \\
\hline
\end{tabular}




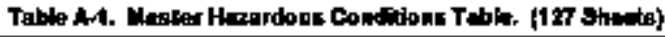

\begin{tabular}{|c|c|c|c|c|}
\hline $\mathbf{n}$ & Wrimed in fosk & Harmown Oanden & couns & 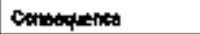 \\
\hline 1145xeace ib & 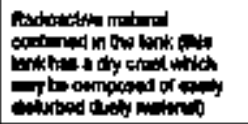 & 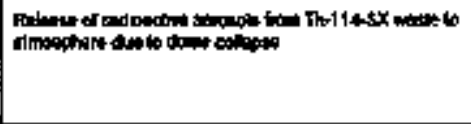 & 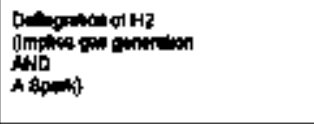 & 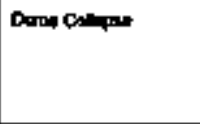 \\
\hline $1145 \times 00+2$ & 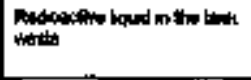 & 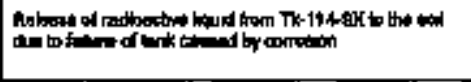 & cinipen & 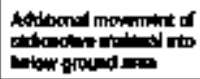 \\
\hline $1145 \times 03$ a 3 & 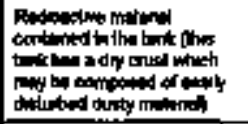 & 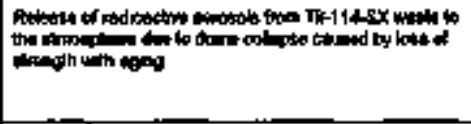 & Lenoton tong & Done Collapes \\
\hline $1948 \times-006$ & 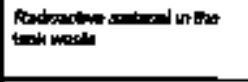 & 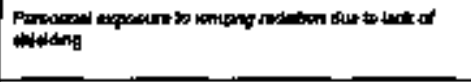 & 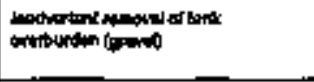 & 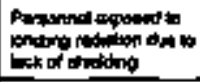 \\
\hline 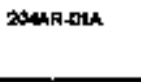 & 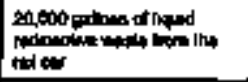 & 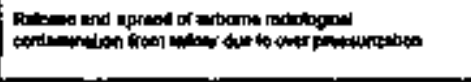 & 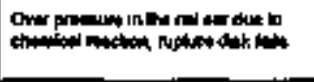 & \\
\hline 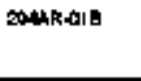 & 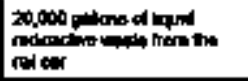 & 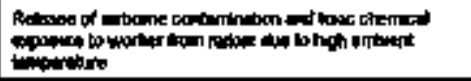 & 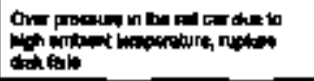 & \\
\hline 2owhorc & 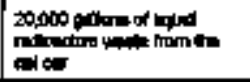 & 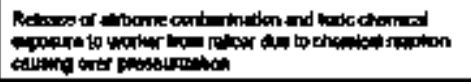 & 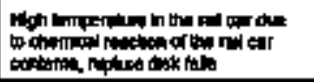 & \\
\hline 214k서엄 & 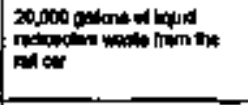 & 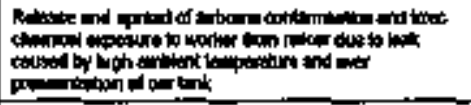 & 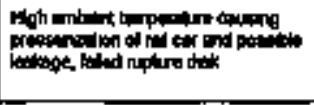 & \\
\hline SoAkR-01E & Docos ganp of lowad & 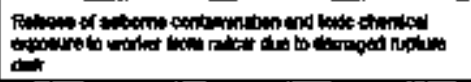 & 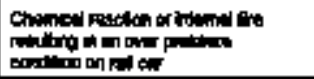 & \\
\hline
\end{tabular}




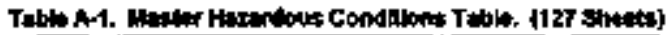

\begin{tabular}{|c|c|c|c|c|}
\hline $\boldsymbol{n}$ & Whand a Rak & Fanerdous Fondion & קחקטת & Gonsopinonge \\
\hline 20ungh & 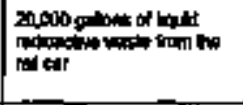 & 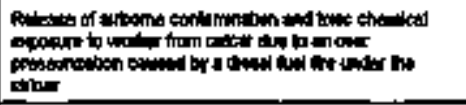 & 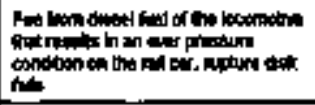 & \\
\hline 204N-019 & 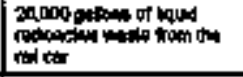 & 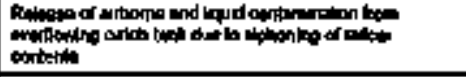 & 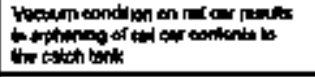 & \\
\hline DOShRuth & 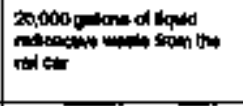 & 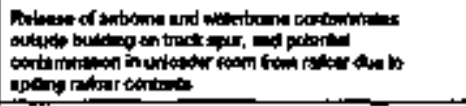 & 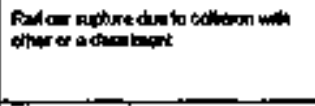 & \\
\hline 2040-0ri & 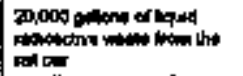 & 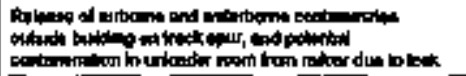 & 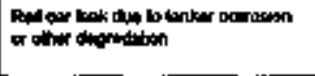 & \\
\hline 20upfatd & 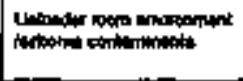 & 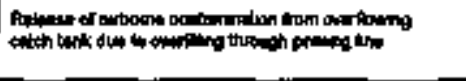 & 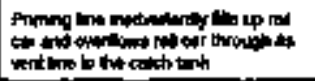 & \\
\hline DOAAR-0IK & 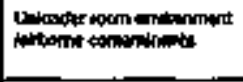 & 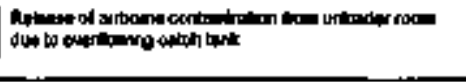 & 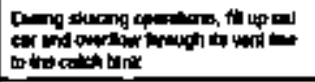 & \\
\hline $204 x \cos \alpha$ & 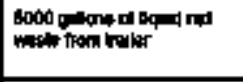 & 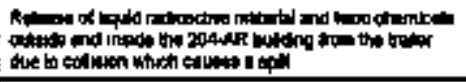 & 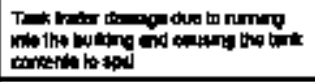 & \\
\hline adrRaca & 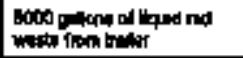 & 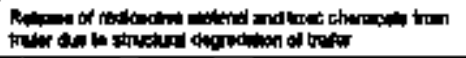 & 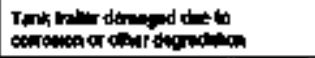 & \\
\hline 20uknect & 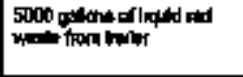 & 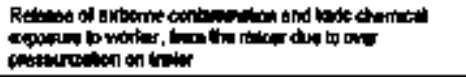 & 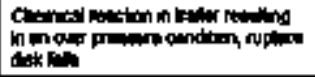 & \\
\hline 20uketest & 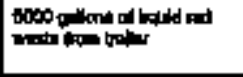 & 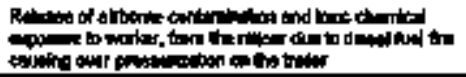 & 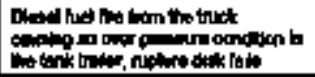 & \\
\hline
\end{tabular}




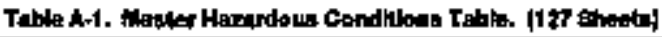

\begin{tabular}{|c|c|c|c|c|}
\hline 10 & Inth & 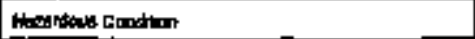 & consos & Cotrotequiner \\
\hline 204AR-02E & 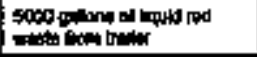 & 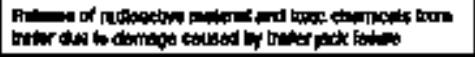 & 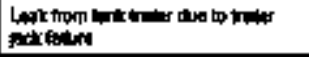 & \\
\hline $2004 \times-02^{*}$ & 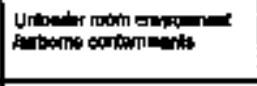 & 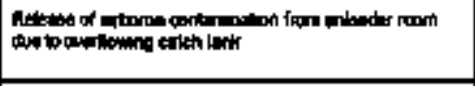 & 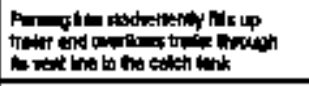 & \\
\hline 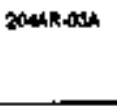 & 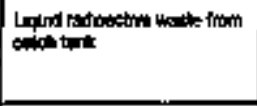 & 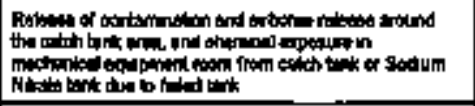 & 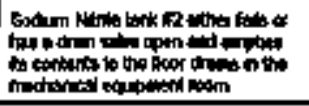 & \\
\hline 204AR-01: & 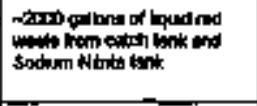 & 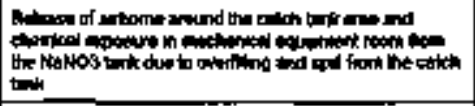 & 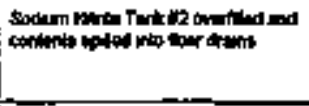 & \\
\hline 204AR-Cي & 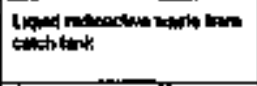 & 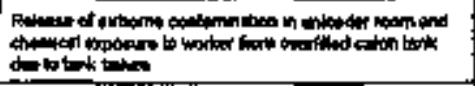 & 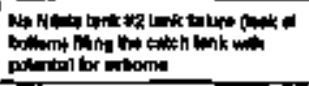 & \\
\hline SOAREAL & 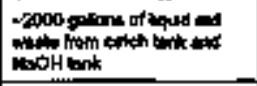 & 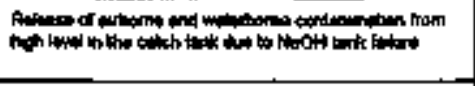 & 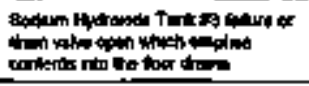 & \\
\hline Soutrates & 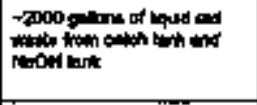 & 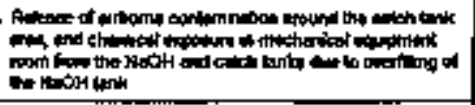 & 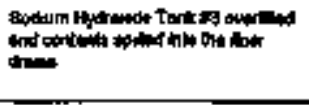 & \\
\hline 204R-04c & 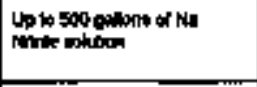 & 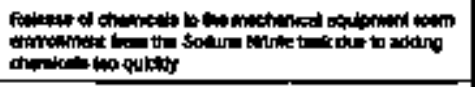 & 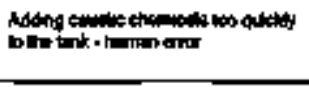 & \\
\hline 204R-040 & 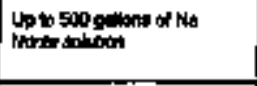 & 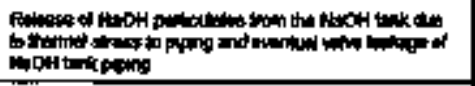 & 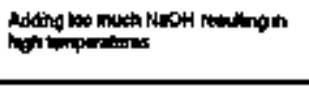 & 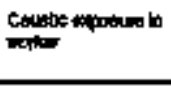 \\
\hline zouphous & 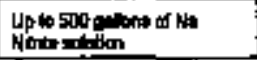 & 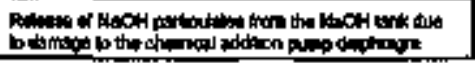 & 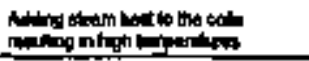 & 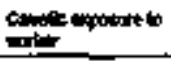 \\
\hline
\end{tabular}




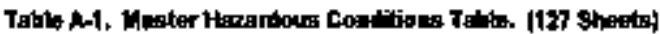

\begin{tabular}{|c|c|c|c|c|}
\hline 10 & 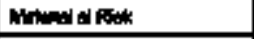 & 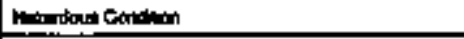 & Can & 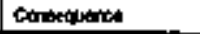 \\
\hline soleperes & 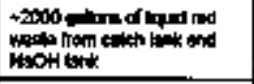 & 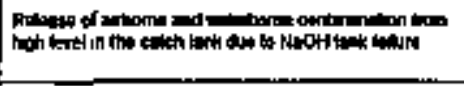 & 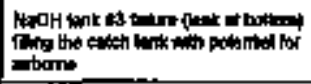 & \\
\hline PonR-5n & 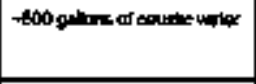 & 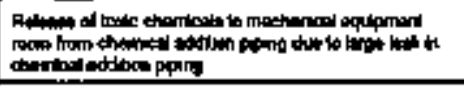 & 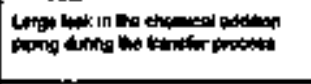 & \\
\hline TO4ARDOS & Dod plowe of com & 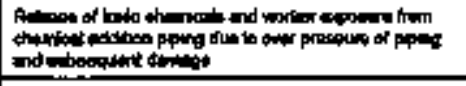 & 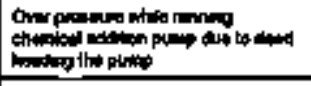 & \\
\hline DURAdect & 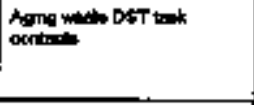 & 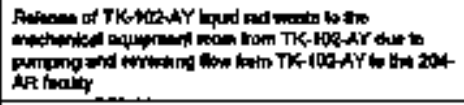 & 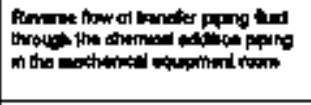 & \\
\hline aparach & 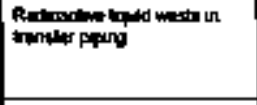 & 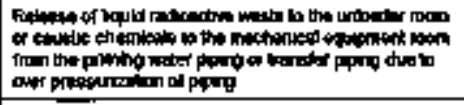 & 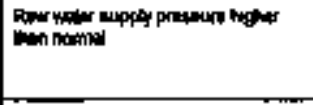 & \\
\hline 204AR-098 & 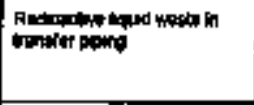 & 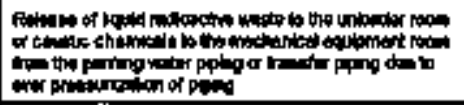 & 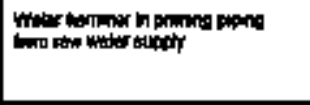 & \\
\hline xushooc & 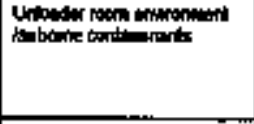 & 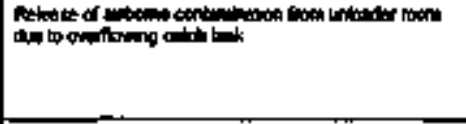 & 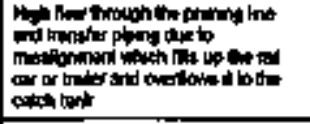 & \\
\hline 2OAR-OTA & 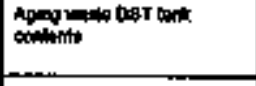 & 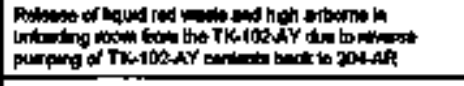 & 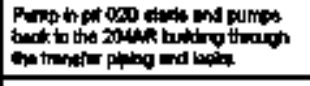 & 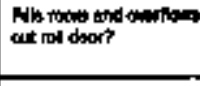 \\
\hline 204R-0T: & 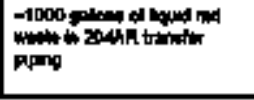 & 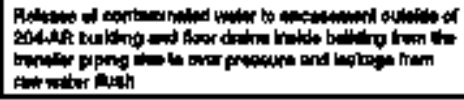 & 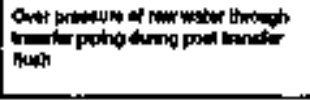 & \\
\hline
\end{tabular}




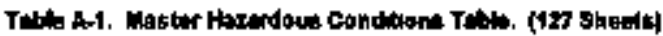

\begin{tabular}{|c|c|c|c|c|}
\hline ID & Hak & 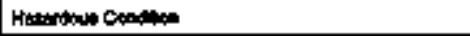 & Conte & Comanopuones \\
\hline Sounatonc & 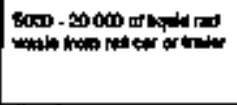 & 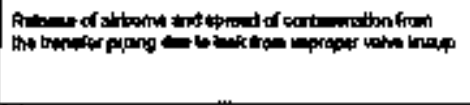 & 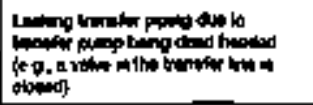 & \\
\hline Sorritoen & 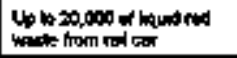 & 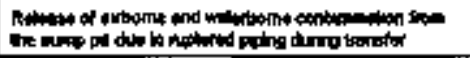 & 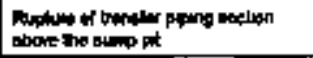 & \\
\hline zoukdost & 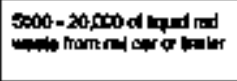 & 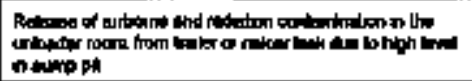 & 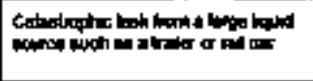 & $\lim _{\text {rith }}$ \\
\hline JUARAt & 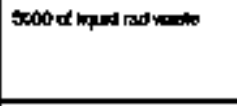 & 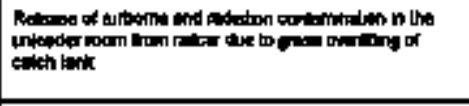 & 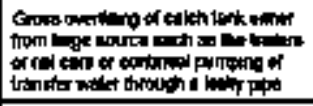 & Fils nom ind hewe at \\
\hline 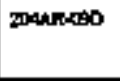 & 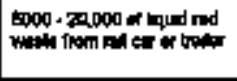 & 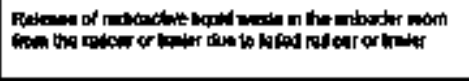 & 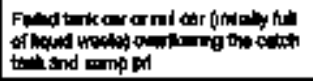 & \\
\hline Dothere & 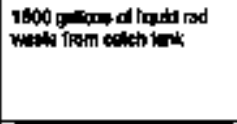 & 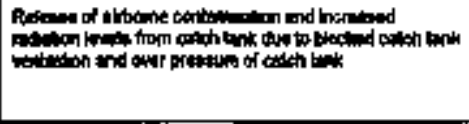 & 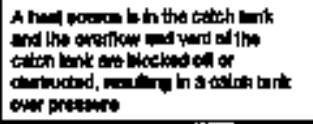 & \\
\hline 204AR.09\% & 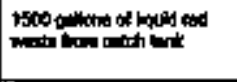 & 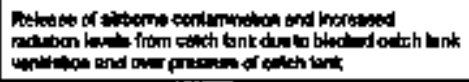 & 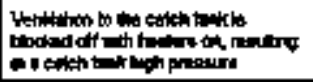 & \\
\hline Xo4hr-006 & 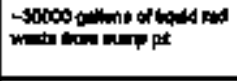 & 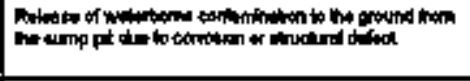 & 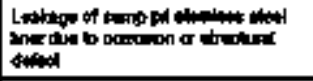 & \\
\hline somp-ow & 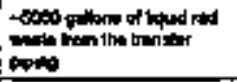 & 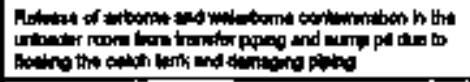 & 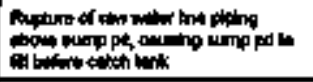 & \\
\hline 20une-11h & 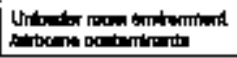 & 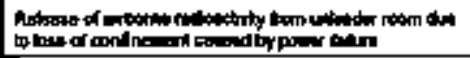 & 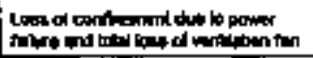 & \\
\hline
\end{tabular}




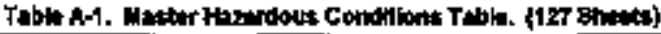

\begin{tabular}{|c|c|c|c|c|}
\hline 10 & 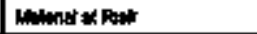 & Harious Constion & Cand & Conserpunes \\
\hline Donr-11日 & 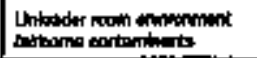 & 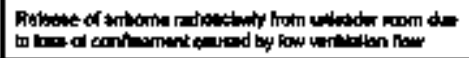 & 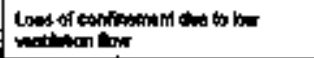 & \\
\hline 2OMR-51C & 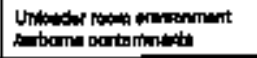 & 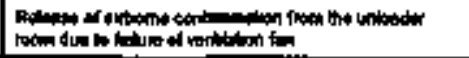 & 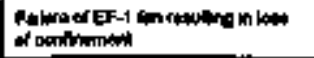 & \\
\hline 204R-110 & 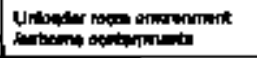 & 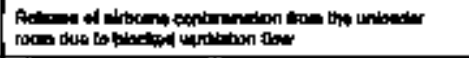 & 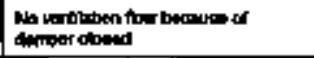 & \\
\hline $204 A R-11 E$ & 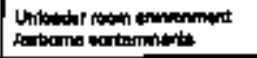 & 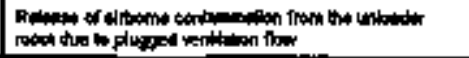 & 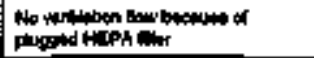 & \\
\hline 20uke-11F & 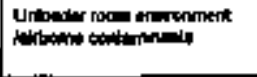 & 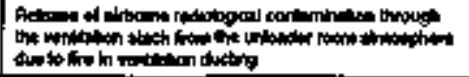 & 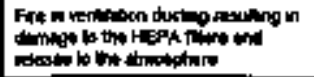 & \\
\hline 213 than & 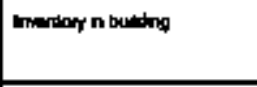 & 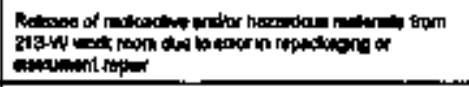 & 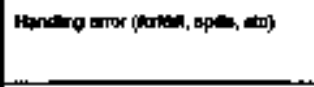 & Worker mpowion b \\
\hline $2134 \mathrm{ng}$ & 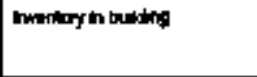 & 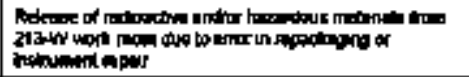 & ranet of bots & 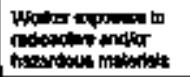 \\
\hline $213 \%-100$ & 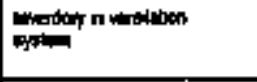 & 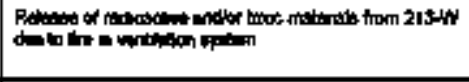 & 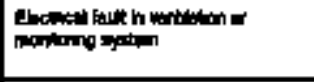 & 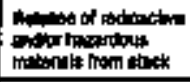 \\
\hline 2134004 & 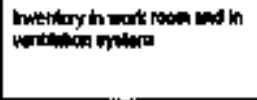 & 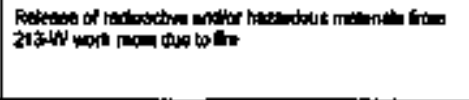 & 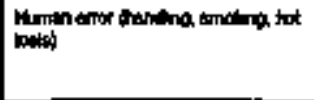 & 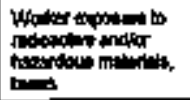 \\
\hline 213 wat & 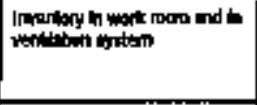 & 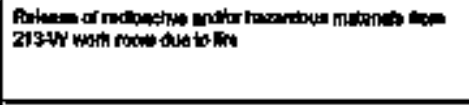 & 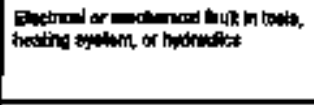 & 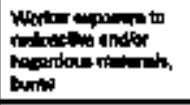 \\
\hline $213 w 09$ & 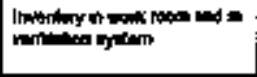 & 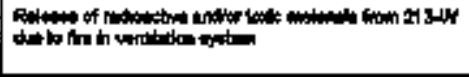 & Eghtima & 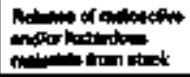 \\
\hline
\end{tabular}




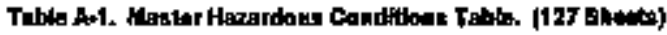

\begin{tabular}{|c|c|c|c|c|}
\hline 10 & 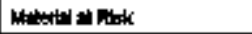 & Howandent Condton & Coups & cormagne \\
\hline 213 Whor & him & 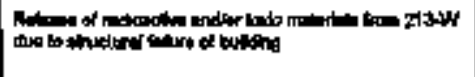 & 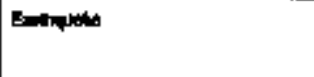 & Whier expotinets \\
\hline 293 WA & 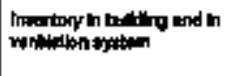 & 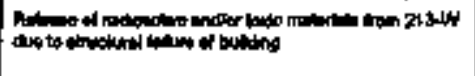 & Heph wither bonodo & 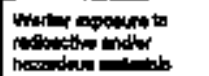 \\
\hline 212 wos & 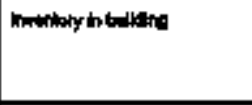 & 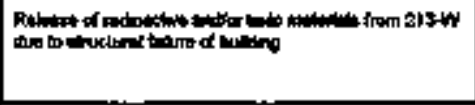 & 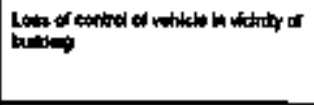 & 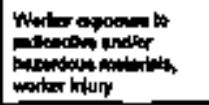 \\
\hline 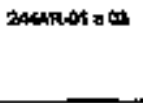 & 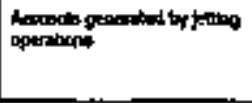 & 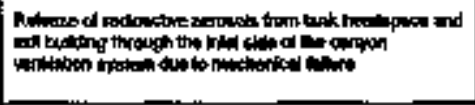 & 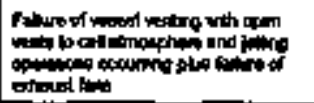 & 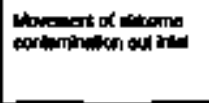 \\
\hline 24017-01 bD & 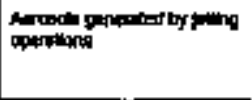 & 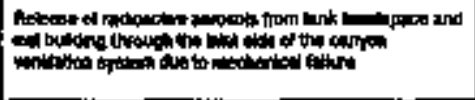 & Fan Fiture wh joth goctump & 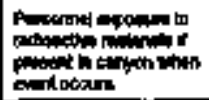 \\
\hline 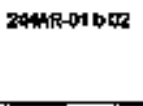 & 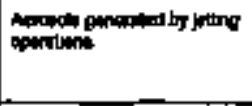 & 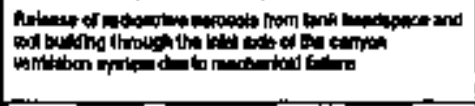 & 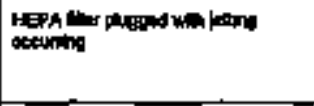 & 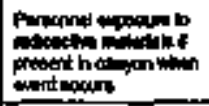 \\
\hline 244kR-01 bos & 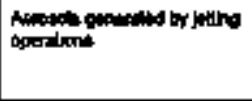 & 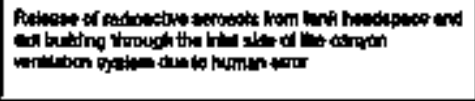 & 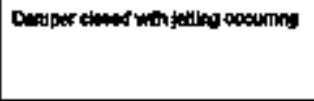 & 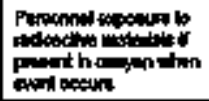 \\
\hline 24ARAS bod & 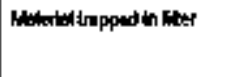 & 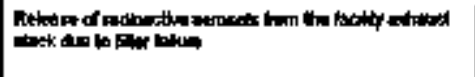 & 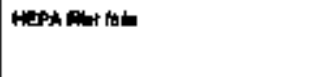 & 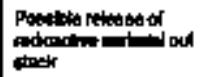 \\
\hline 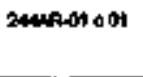 & the & 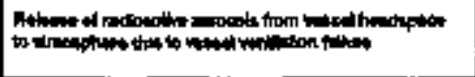 & Pagon & 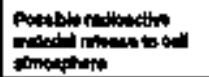 \\
\hline
\end{tabular}




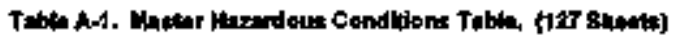

\begin{tabular}{|c|c|c|c|c|}
\hline ID & 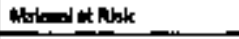 & 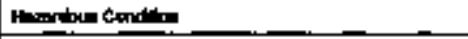 & onsen & Conawerenes \\
\hline 2AMrktil a & 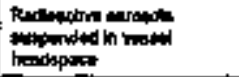 & 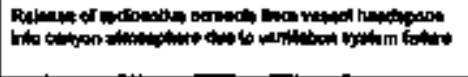 & 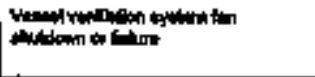 & 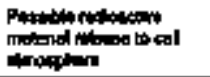 \\
\hline $244 \mathrm{APA}$ ops & 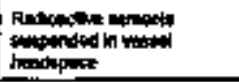 & 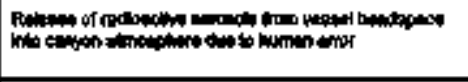 & 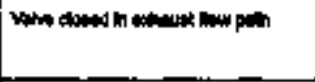 & 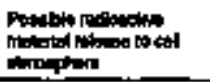 \\
\hline zwaked =o & 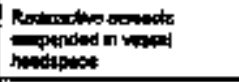 & 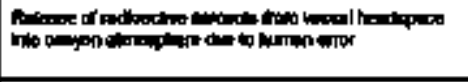 & 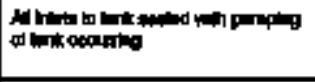 & 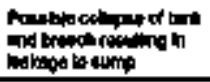 \\
\hline 24ares tot & 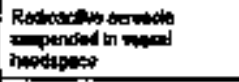 & 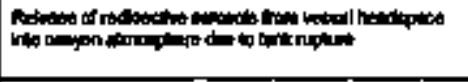 & 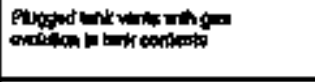 & 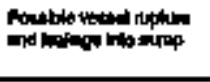 \\
\hline 24ARR-01 $=09$ & 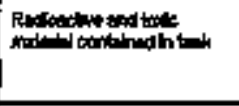 & 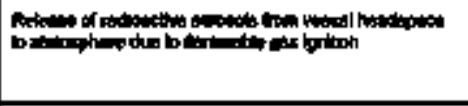 & 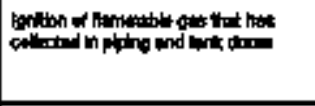 & 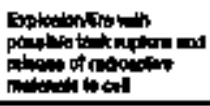 \\
\hline 244 kiol t 10 & 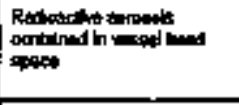 & 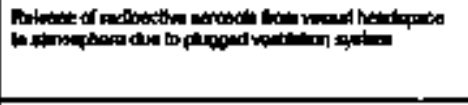 & 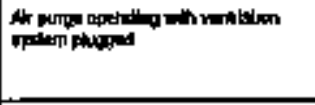 & 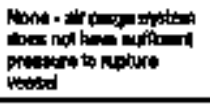 \\
\hline 24ARCA1 011 & 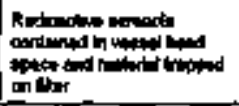 & 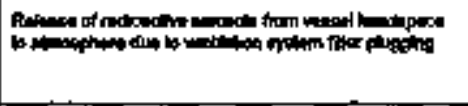 & 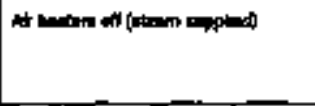 & 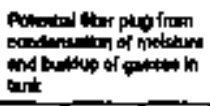 \\
\hline 24CARAD $=19$ & 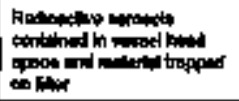 & 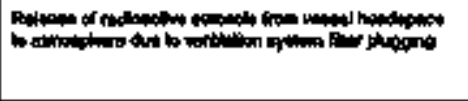 & 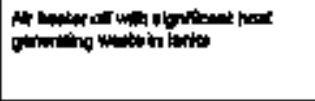 & 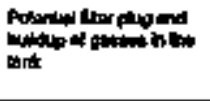 \\
\hline 2kunter as & 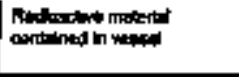 & 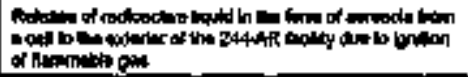 & 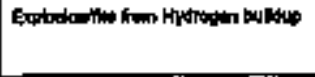 & 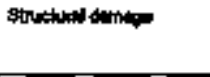 \\
\hline
\end{tabular}




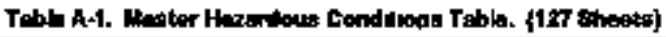

\begin{tabular}{|c|c|c|c|c|}
\hline$\omega$ & Mowerld * intin & 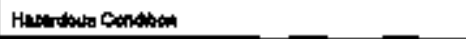 & مets & Corresparares \\
\hline 24AnRate ats & 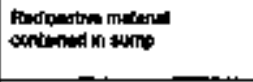 & 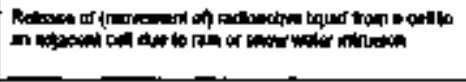 & 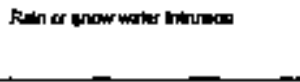 & 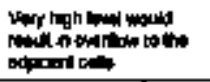 \\
\hline 2014F $00=06$ & Pantiontive ming & 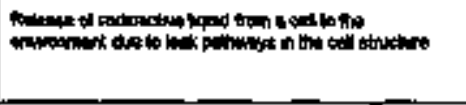 & 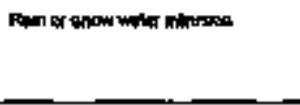 & 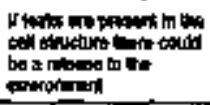 \\
\hline $244 A R-0 Z \backsim D F$ & 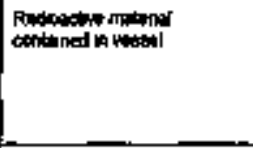 & 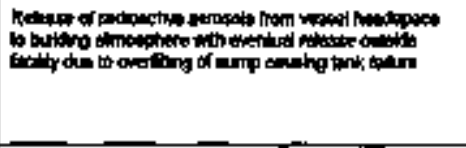 & 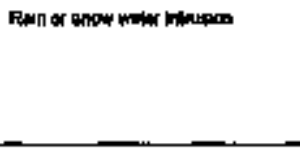 & 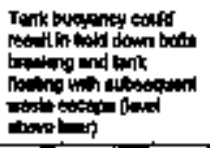 \\
\hline $2448 R-02$ 00: & 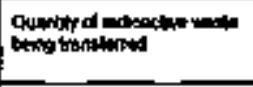 & 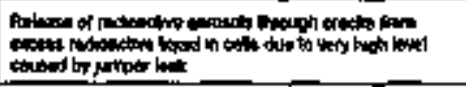 & 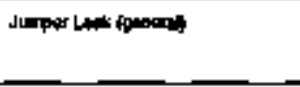 & 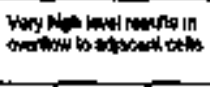 \\
\hline 24apkne pos & 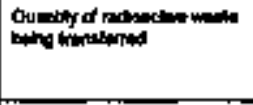 & 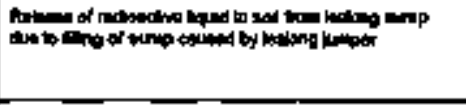 & 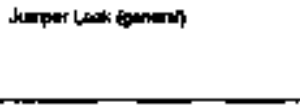 & 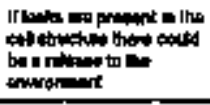 \\
\hline $240 \mathrm{R}-02+10$ & 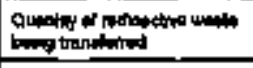 & 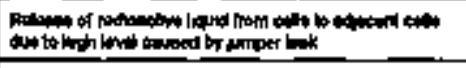 & 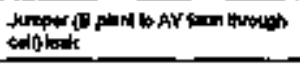 & 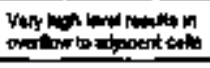 \\
\hline $2404 R-62111$ & 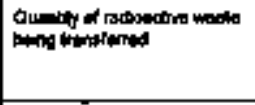 & 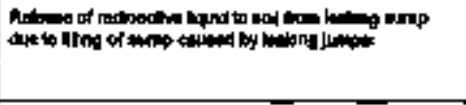 & 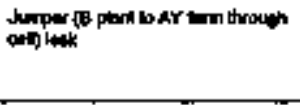 & 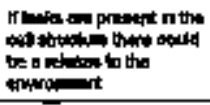 \\
\hline $244+-02+12$ & 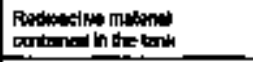 & 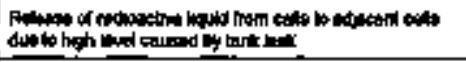 & Tank ink & Wary hion ind imila in \\
\hline $240 \mathrm{R}-0 \mathrm{C} \times 1 \mathrm{13}$ & 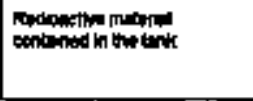 & 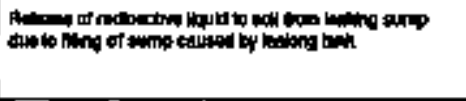 & $\operatorname{Tank} \operatorname{lank}$ & 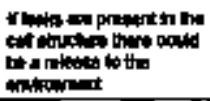 \\
\hline
\end{tabular}




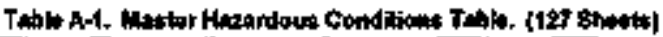

\begin{tabular}{|c|c|c|c|c|}
\hline$\pi$ & 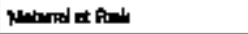 & 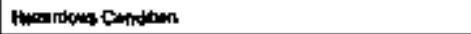 & Dence. & 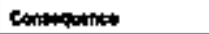 \\
\hline $244 k-08+14$ & 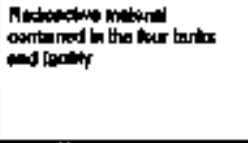 & 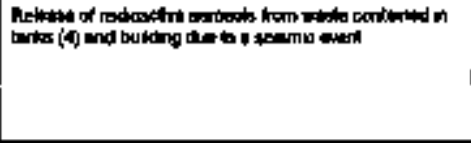 & 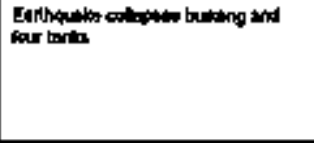 & 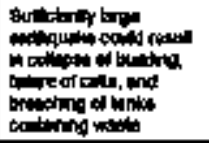 \\
\hline $244 \times 8-03 \bullet 03$ & 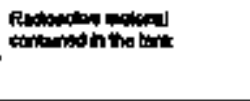 & 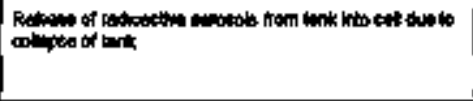 & 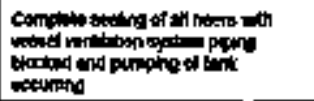 & 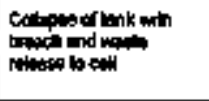 \\
\hline $244 \sin 00$ atod & 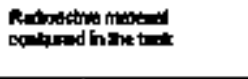 & 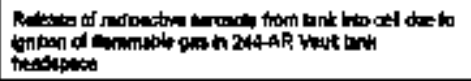 & 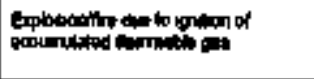 & 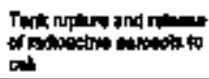 \\
\hline $244 R$ AOCS & 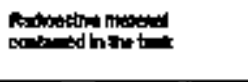 & 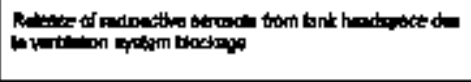 & 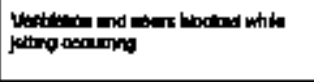 & 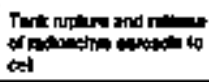 \\
\hline $244 \times 100006$ & 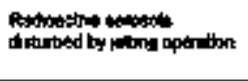 & 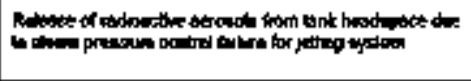 & 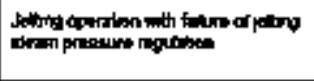 & 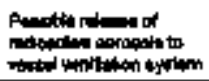 \\
\hline $2 \ln 80007$ & 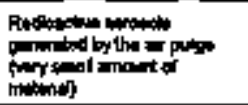 & 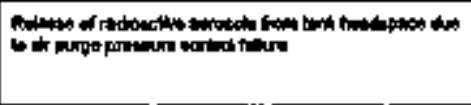 & 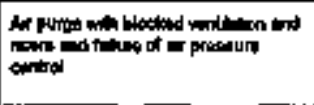 & 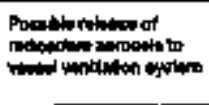 \\
\hline 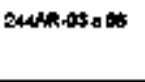 & 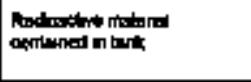 & 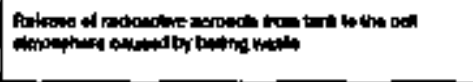 & 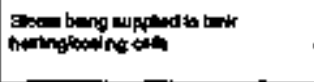 & ding of woits with \\
\hline 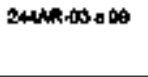 & 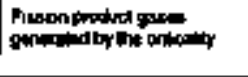 & 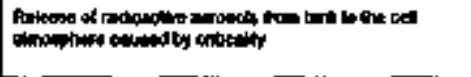 & 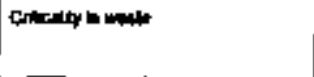 & 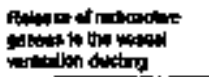 \\
\hline $244 k$ - 100 a 13 & 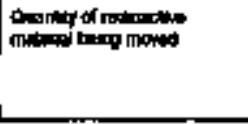 & 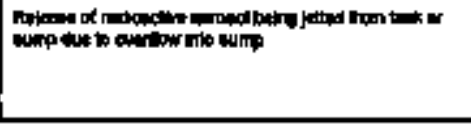 & 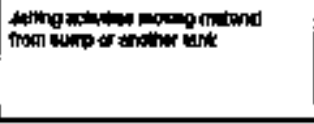 & 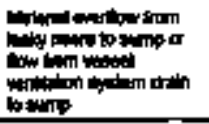 \\
\hline
\end{tabular}




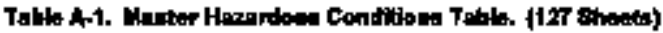

\begin{tabular}{|c|c|c|c|c|}
\hline 10 & Molond th then & Metroure tandhint & Cona & Candengerse \\
\hline 244dR:Ded = 14 & 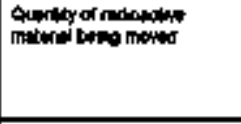 & 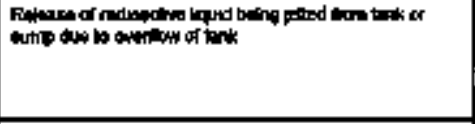 & 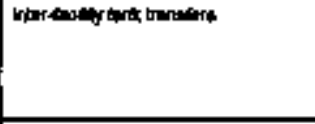 & 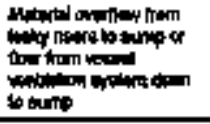 \\
\hline $2404 k 09$ a 18 & 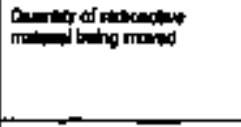 & 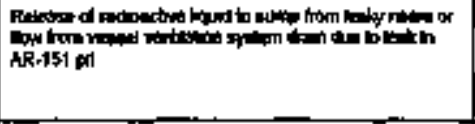 & Lat from pat.as 191 & 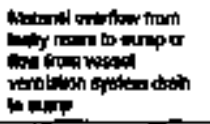 \\
\hline 244 月- 60.18 & 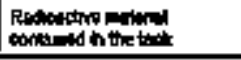 & 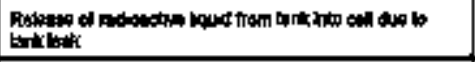 & Carpanan & Pח \\
\hline $2004 R-100$ \& 17 & 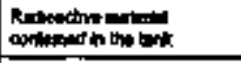 & 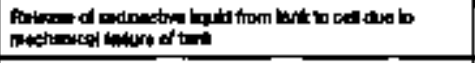 & 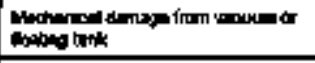 & Leirth \\
\hline $244+2-03=18$ & 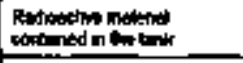 & 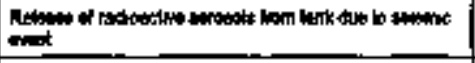 & 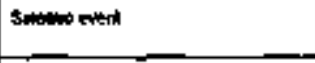 & 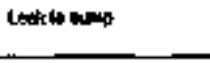 \\
\hline 2CA18.04. Di & 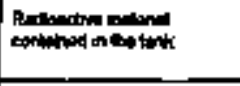 & 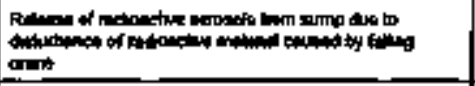 & 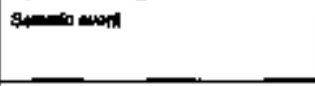 & 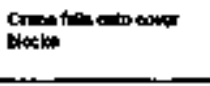 \\
\hline $244 \mathrm{k}$ as an & Wh & 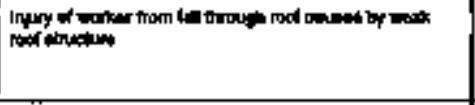 & A 190 (reol) & 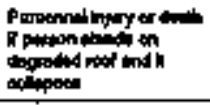 \\
\hline $244 A R-05$ 0 DR & 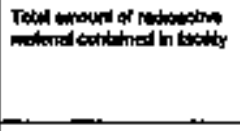 & 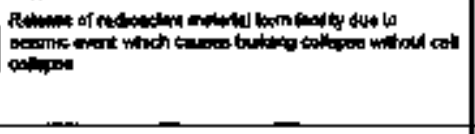 & 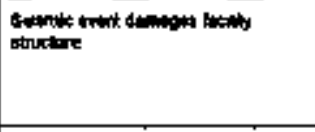 & 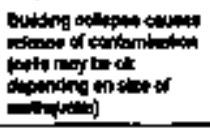 \\
\hline 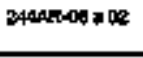 & 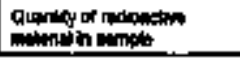 & 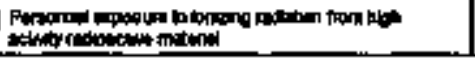 & 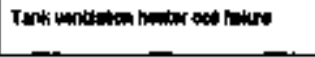 & 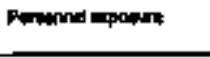 \\
\hline
\end{tabular}




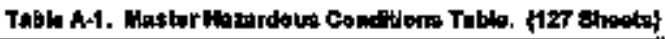

\begin{tabular}{|c|c|c|c|c|}
\hline $\boldsymbol{0}$ & Hath & 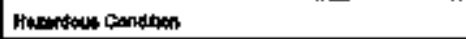 & Cans & Conanowion \\
\hline $240 \mathrm{k} \cdot \mathrm{mos}=\mathrm{O}$ or & 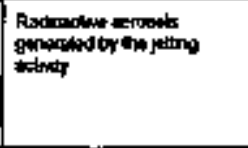 & 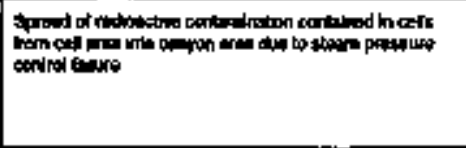 & 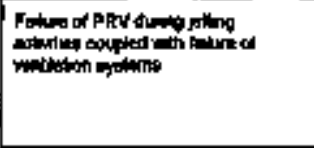 & 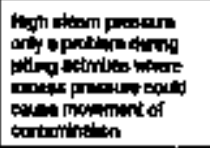 \\
\hline 244MR-10 atis & 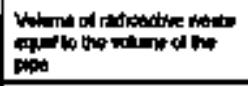 & 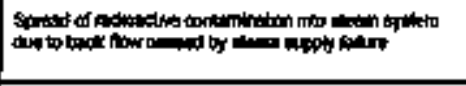 & 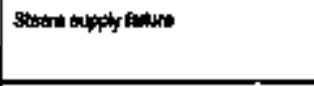 & 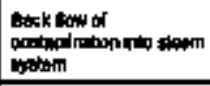 \\
\hline 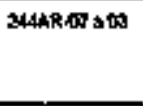 & 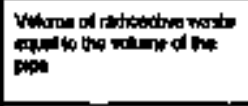 & 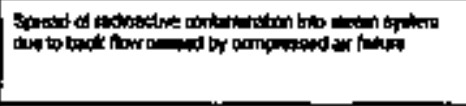 & 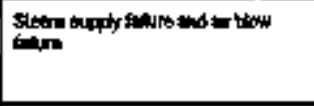 & 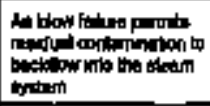 \\
\hline SAAARAT I OA & 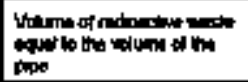 & 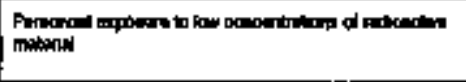 & 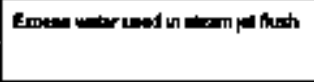 & 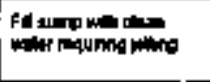 \\
\hline 244ARAmitis & inh & 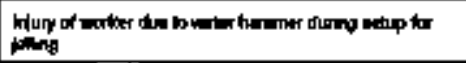 & 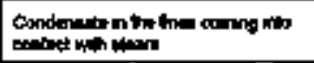 & 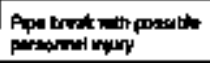 \\
\hline 2tate-pucos & un & 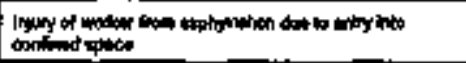 & 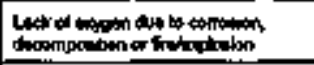 & 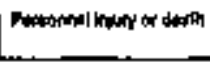 \\
\hline EALARATDS & $m$ & 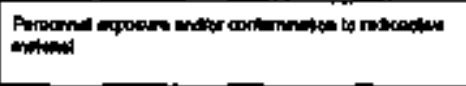 & 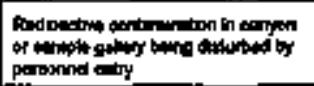 & 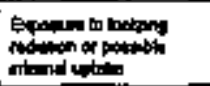 \\
\hline 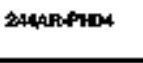 & $m$ & 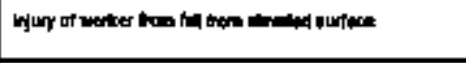 & 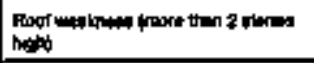 & 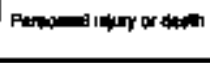 \\
\hline $244 \mathrm{R}$ PNAd & inte & 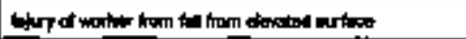 & 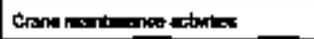 & 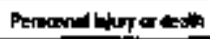 \\
\hline 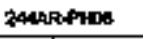 & an & 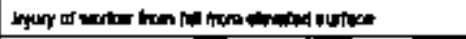 & 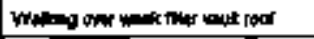 & 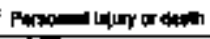 \\
\hline 2WAP-Ption & M & 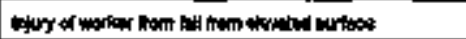 & 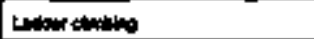 & 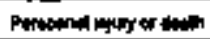 \\
\hline 24MRPHAO & $m$ & 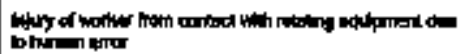 & 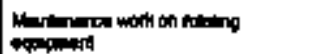 & 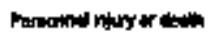 \\
\hline
\end{tabular}




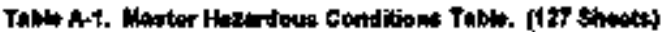

\begin{tabular}{|c|c|c|c|c|}
\hline o & 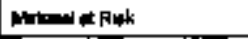 & 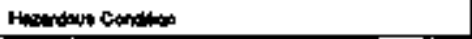 & Canes & 定 \\
\hline 24whentice & $\mathbf{m}$ & 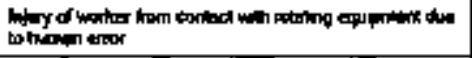 & 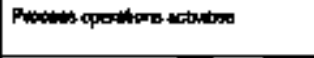 & 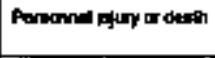 \\
\hline 2AWRPA110 & Nh & 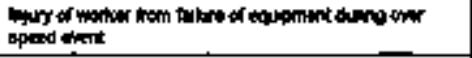 & 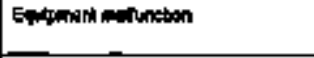 & Pabconed willy ar dealh \\
\hline 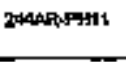 & $m$ & 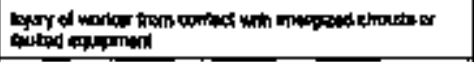 & thenthenfinte & 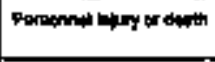 \\
\hline 24AKR+HI & $\mathbf{M R}$ & 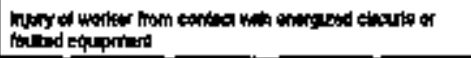 & 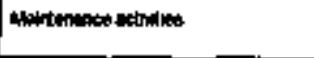 & 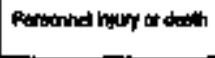 \\
\hline 2A4trarins & $\boldsymbol{m}$ & 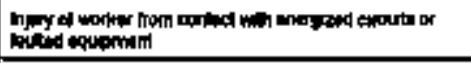 & 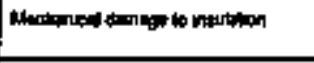 & 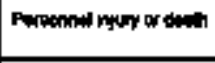 \\
\hline 244ARPAKA & m & 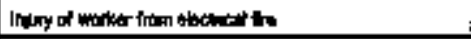 & 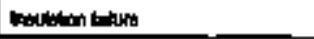 & 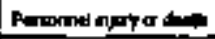 \\
\hline 204hititis & ah & 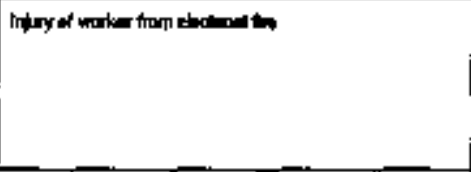 & 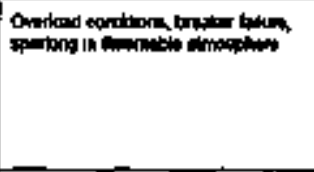 & 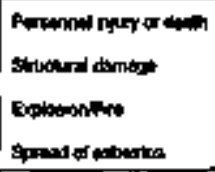 \\
\hline 261ARAPH16 & wat & 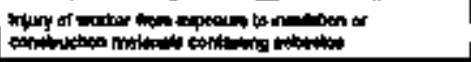 & 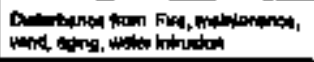 & 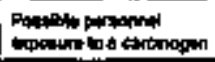 \\
\hline 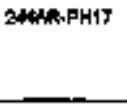 & NA & 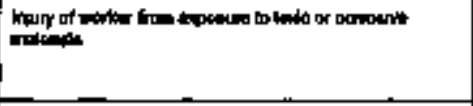 & 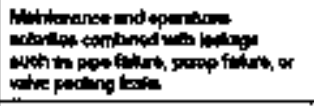 & 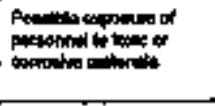 \\
\hline 24AR-PHIs & wal & 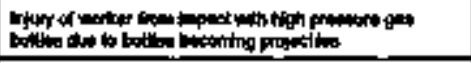 & 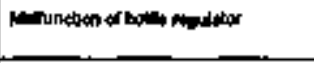 & 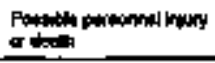 \\
\hline 24URPHID & NA & 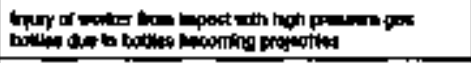 & 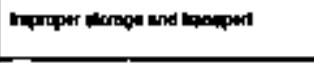 & 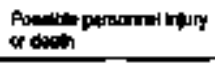 \\
\hline 27xm-91 & 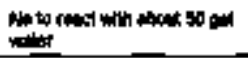 & 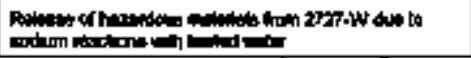 & 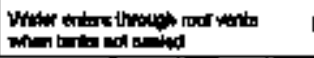 & 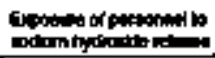 \\
\hline
\end{tabular}




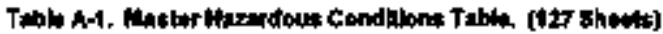

\begin{tabular}{|c|c|c|c|c|}
\hline 10 & Mat ank & 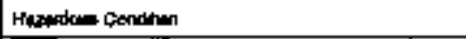 & comine & Conoseaplise \\
\hline 2tasulog & 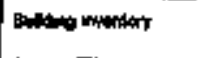 & 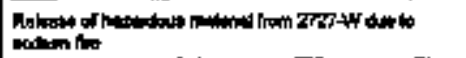 & 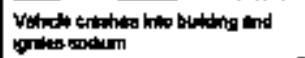 & 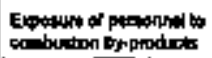 \\
\hline 272गय-03 & Bubang inveriony & 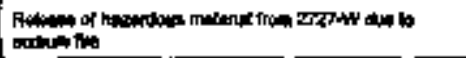 & Ltoing & 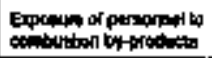 \\
\hline ZCSTW-A & Bucking weritury & 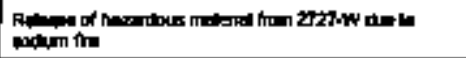 & 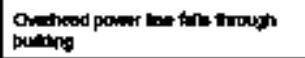 & 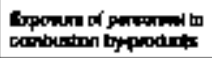 \\
\hline zrzrumas & 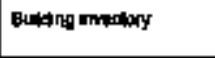 & 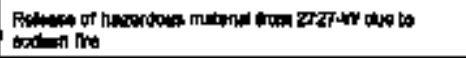 & 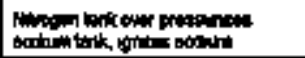 & 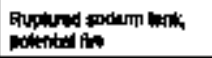 \\
\hline 2TZMHOS & Heding ioteritary & 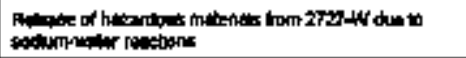 & 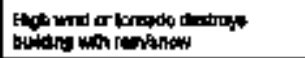 & 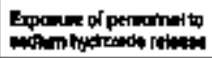 \\
\hline moos & 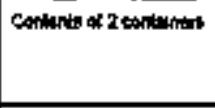 & 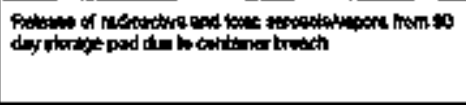 & 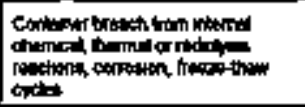 & 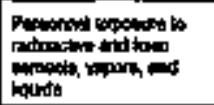 \\
\hline cooter & Content of 2 taitsers & 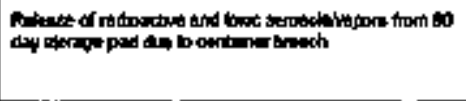 & 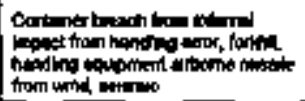 & 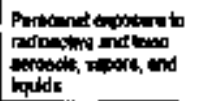 \\
\hline nows & Contents of 2 contares & 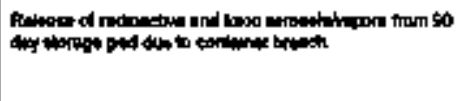 & 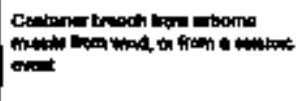 & 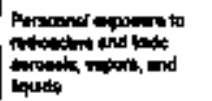 \\
\hline poo-04 & Rod muntiong & 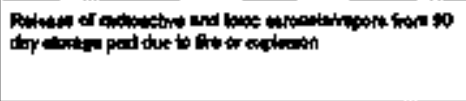 & 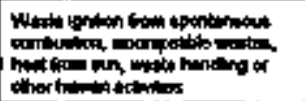 & 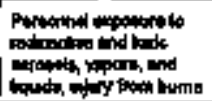 \\
\hline $\operatorname{son}$ & Pad imationy & 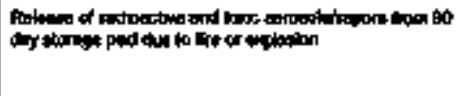 & 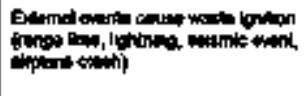 & 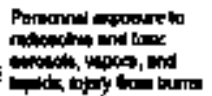 \\
\hline
\end{tabular}




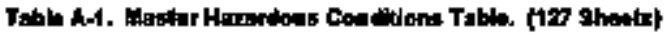

\begin{tabular}{|c|c|c|c|c|}
\hline 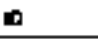 & 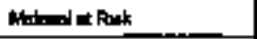 & Howerout Cindiben & Cuno & Cethisaneses \\
\hline DO06 & 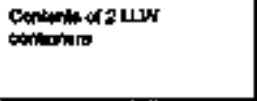 & 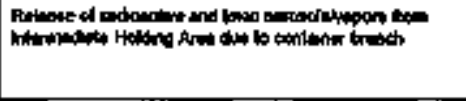 & 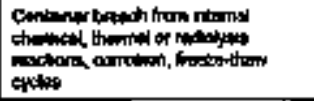 & 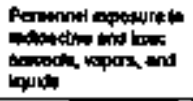 \\
\hline $000-0$ & 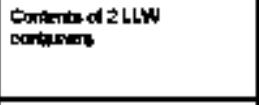 & 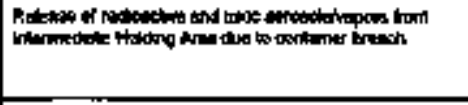 & 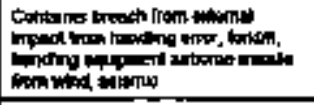 & 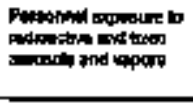 \\
\hline Boodot & 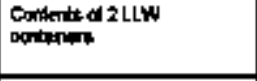 & 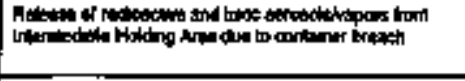 & 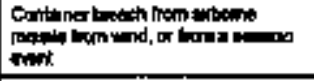 & 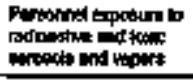 \\
\hline 9000 & 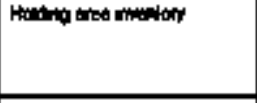 & 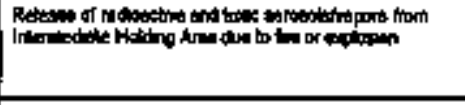 & 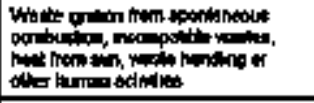 & 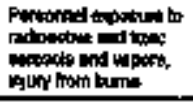 \\
\hline $\cos 10$ & 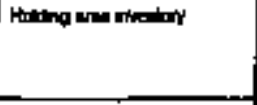 & 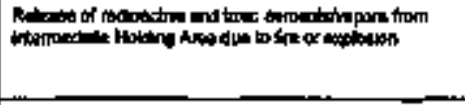 & 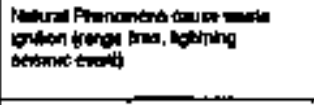 & 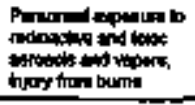 \\
\hline m-11 & 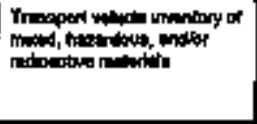 & 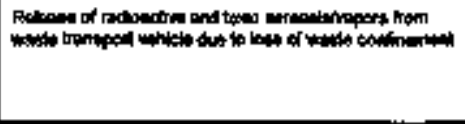 & 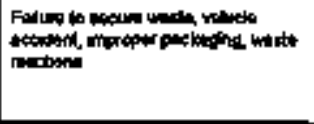 & 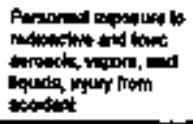 \\
\hline andant & Na & 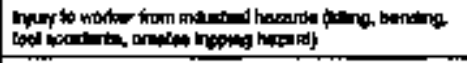 & 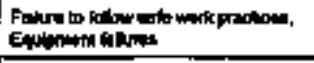 & Waber myry \\
\hline BND-GH & Nh & 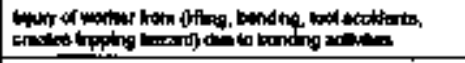 & 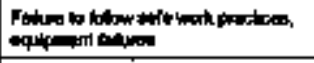 & 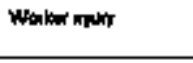 \\
\hline $\log$ & 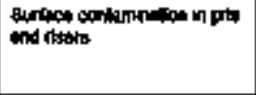 & 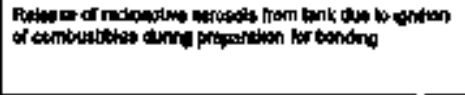 & 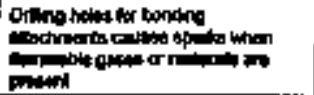 & Wootwer langy \\
\hline
\end{tabular}




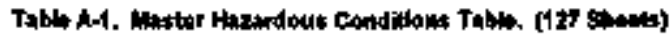

\begin{tabular}{|c|c|c|c|c|}
\hline to & Whom 며 Pict & Hepropus crondiban & Cousp & 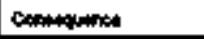 \\
\hline NDDOSH & Nh & 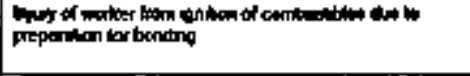 & 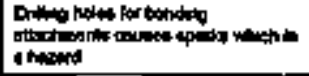 & Wonker kpipr \\
\hline Ceppor & 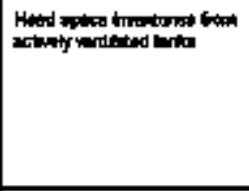 & 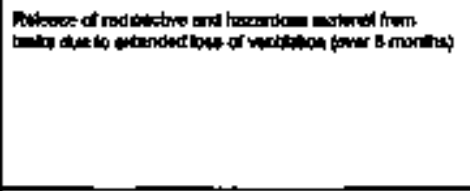 & 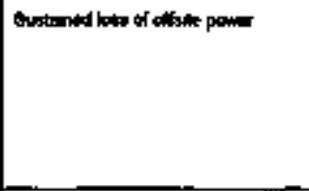 & 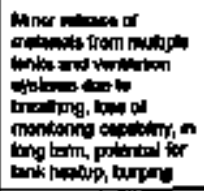 \\
\hline$C C F=.02$ & 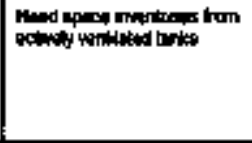 & 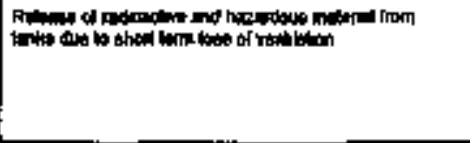 & Leop of athis power & 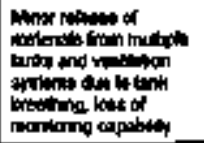 \\
\hline $\cos -00$ & wh & 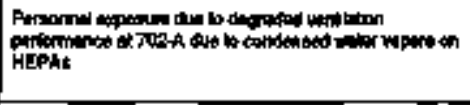 & 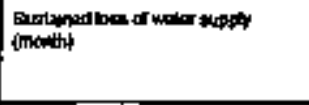 & 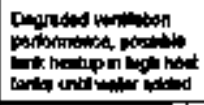 \\
\hline CCEA-OA & $\mathbf{M}$ & 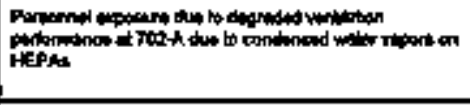 & 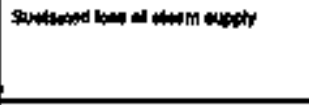 & 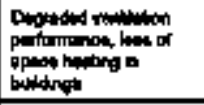 \\
\hline ecF- & H & 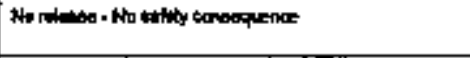 & 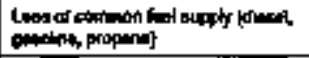 & \\
\hline CCFF $\oplus$ & NA & 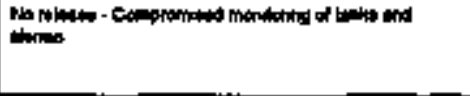 & Comed alr ansty & 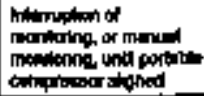 \\
\hline
\end{tabular}




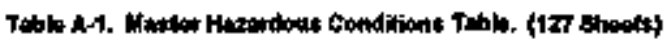

\begin{tabular}{|c|c|c|c|c|}
\hline $\mathbf{0}$ & Mtrat & 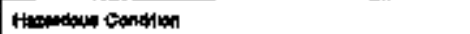 & Covin & (1) \\
\hline $\operatorname{cosen}$ & 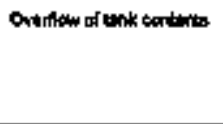 & 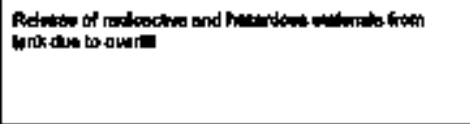 & 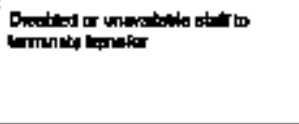 & 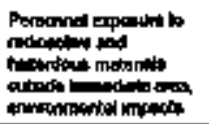 \\
\hline $\cos -0 \theta$ & $\omega$ & 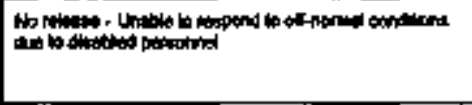 & 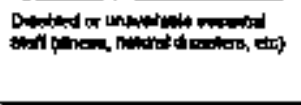 & 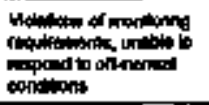 \\
\hline CCF- 00 & ath & 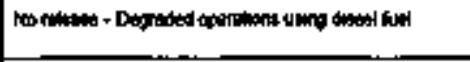 & 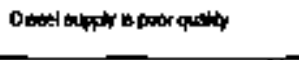 & 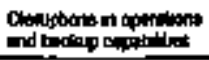 \\
\hline GCGE-10 & Me & 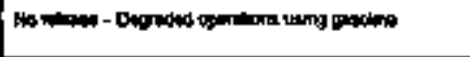 & 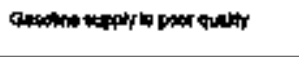 & 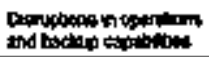 \\
\hline etrind & m & 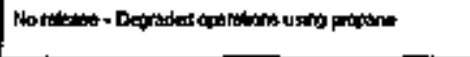 & 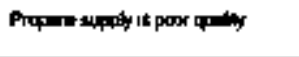 & 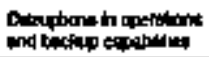 \\
\hline CefF-12 & HA & 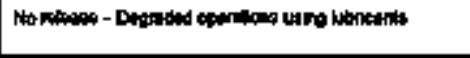 & 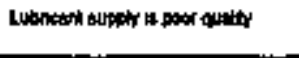 & Dampluene in cperritin: \\
\hline CEF.15 & Mat & 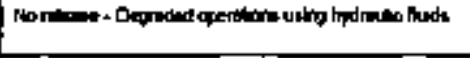 & 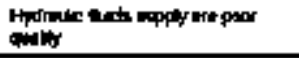 & 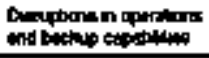 \\
\hline COF-14 & NA & 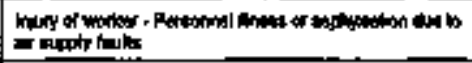 & 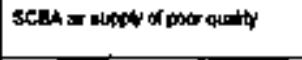 & 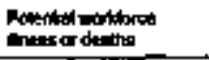 \\
\hline cef:16 & Nat & 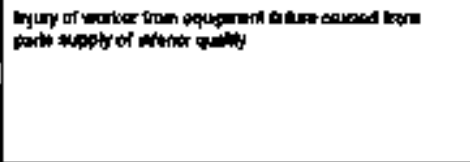 & 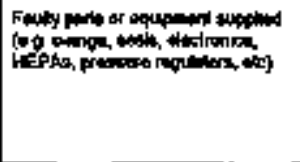 & 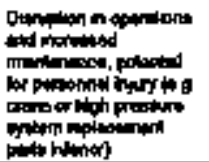 \\
\hline
\end{tabular}




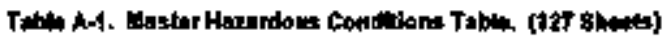

\begin{tabular}{|c|c|c|c|c|}
\hline$\rightarrow$ & 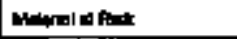 & Horadoul Condtan & Cones & connepame \\
\hline CCF -16 & 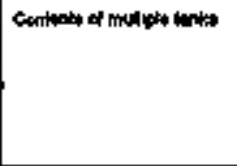 & 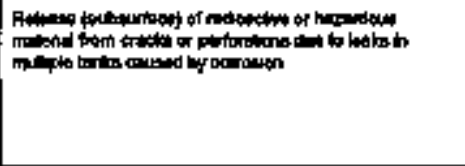 & 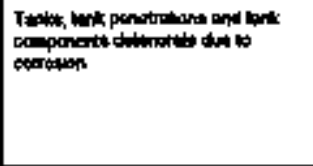 & 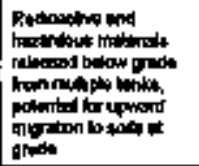 \\
\hline $\cos -17$ & Comberto of & 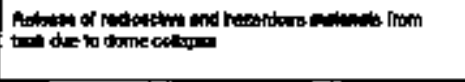 & 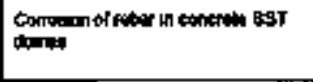 & 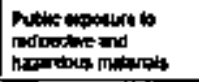 \\
\hline 065.16 & 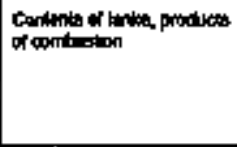 & 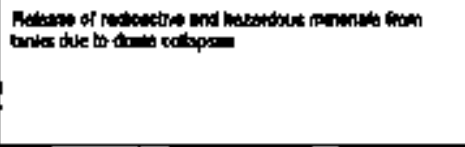 & 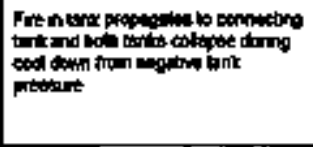 & 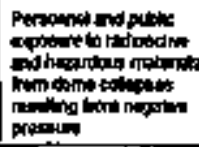 \\
\hline COF-19 & 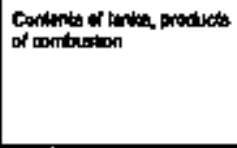 & 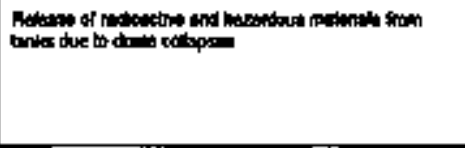 & 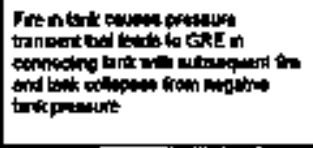 & 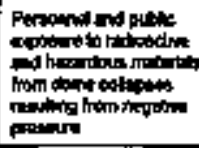 \\
\hline CEF-20 & 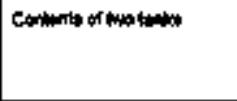 & 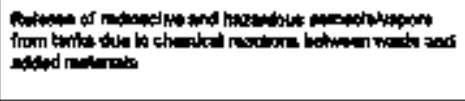 & 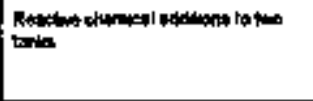 & 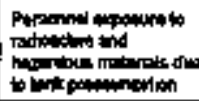 \\
\hline ODPAM & 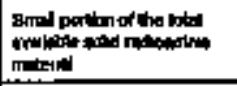 & 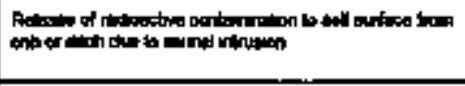 & 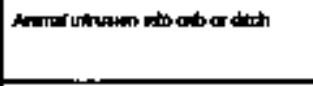 & 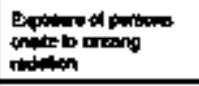 \\
\hline CDPA & 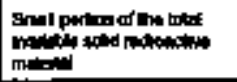 & 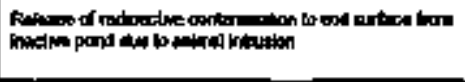 & 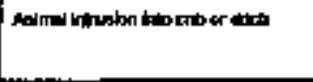 & 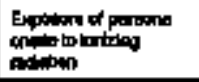 \\
\hline Dopos & 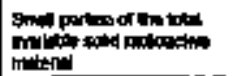 & 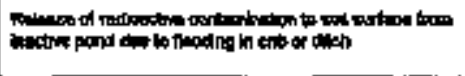 & flootng & 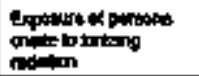 \\
\hline
\end{tabular}




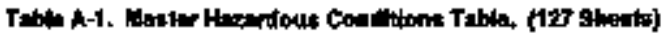

\begin{tabular}{|c|c|c|c|c|}
\hline 10 & Mtridat fot: & 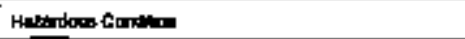 & Cons & careapon \\
\hline copen & 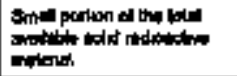 & 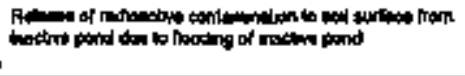 & Fonatiog & 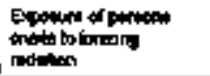 \\
\hline eop-0s & 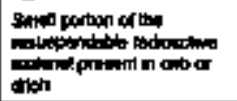 & 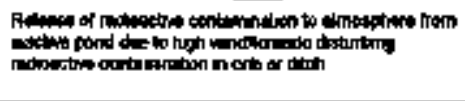 & 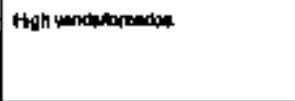 & 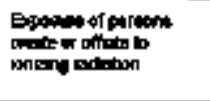 \\
\hline $\operatorname{cop}-0$ & 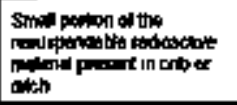 & 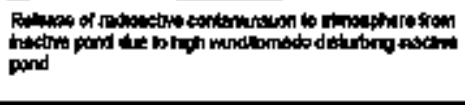 & 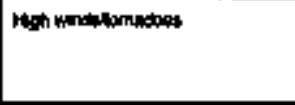 & 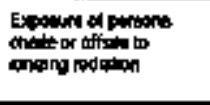 \\
\hline $\cos \theta$ & 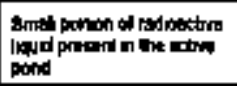 & 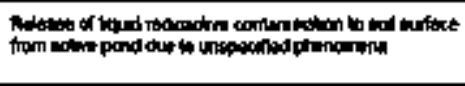 & Uhepesulas phatimim its & 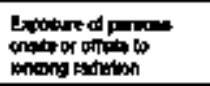 \\
\hline CDPAS & 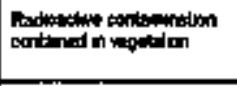 & 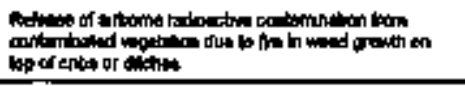 & 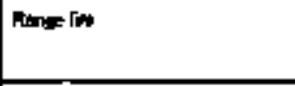 & 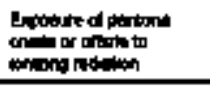 \\
\hline $\operatorname{cop} \infty$ & m & 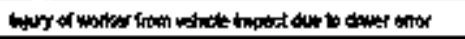 & Dhet wor & 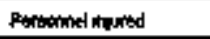 \\
\hline $\cos 40$ & 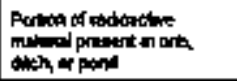 & 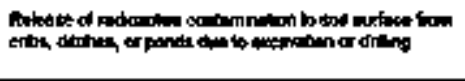 & 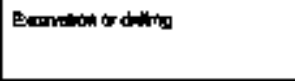 & 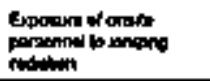 \\
\hline $\cos 14$ & Thenter motory & 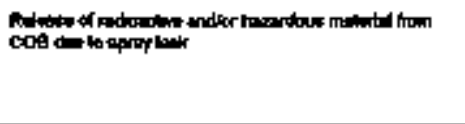 & 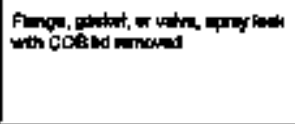 & 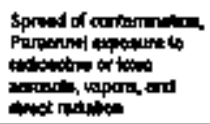 \\
\hline choos & 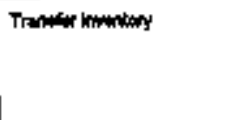 & 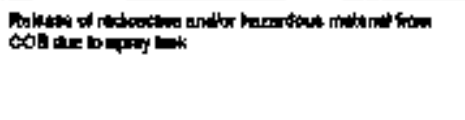 & 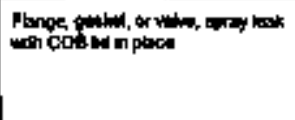 & 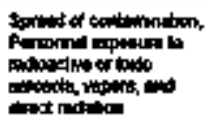 \\
\hline
\end{tabular}




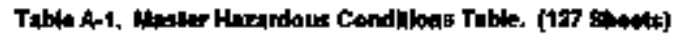

\begin{tabular}{|c|c|c|c|c|}
\hline $\boldsymbol{D}$ & 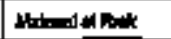 & 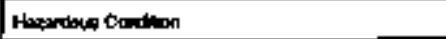 & tones & Consequanish \\
\hline copos & Thinewer mustion & 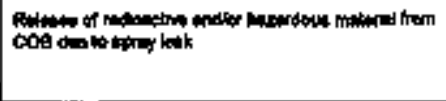 & 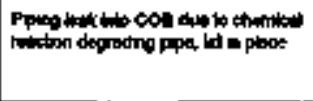 & 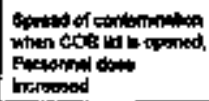 \\
\hline cosest & Trander munitior & 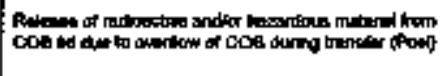 & 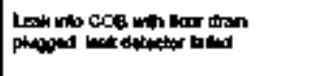 & 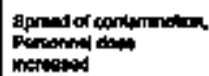 \\
\hline $\cos -6 \operatorname{ar}$ & 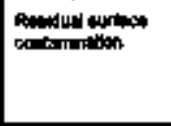 & 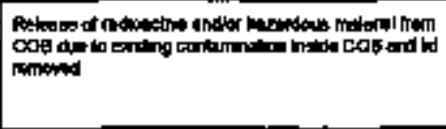 & Intopedot d COA & 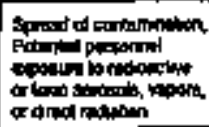 \\
\hline Copos & 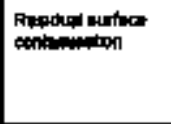 & 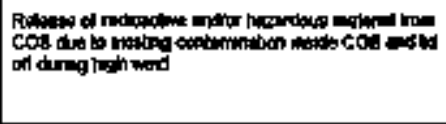 & 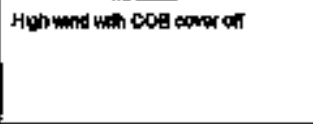 & 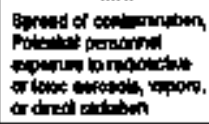 \\
\hline $\cos \cos$ & 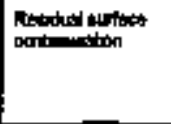 & 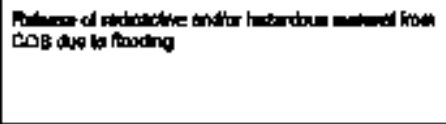 & 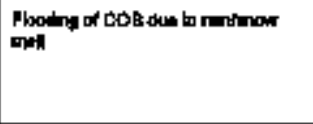 & 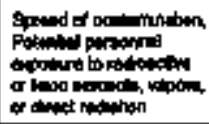 \\
\hline cosoces & 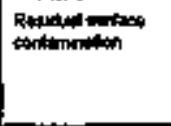 & 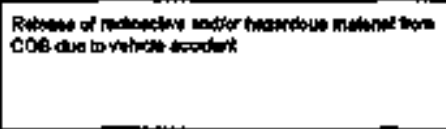 & 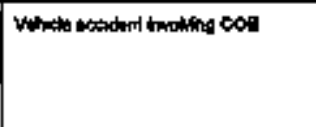 & 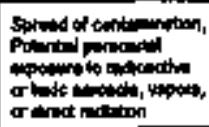 \\
\hline cobota & 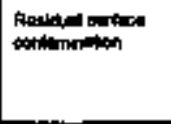 & 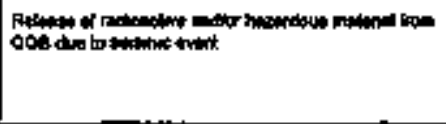 & 8umank with & 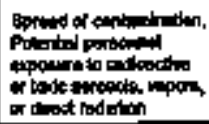 \\
\hline
\end{tabular}




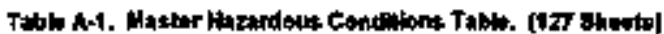

\begin{tabular}{|c|c|c|c|c|}
\hline נ] & SMmad at Ry & Havipula Conition & conve: & Cantwant \\
\hline COS-05' & Reanumintion & 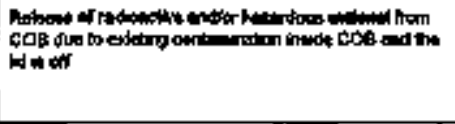 & Henten tom lowing COA w of & 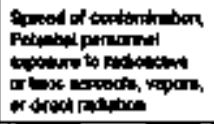 \\
\hline cososin & 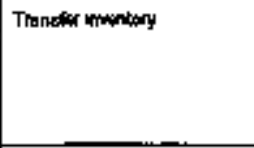 & 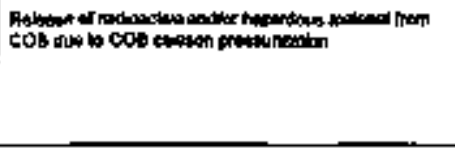 & 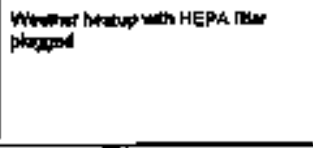 & 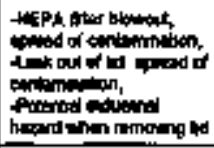 \\
\hline copBos & Tanafor mesitery & 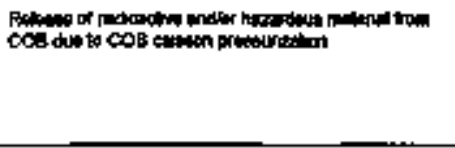 & 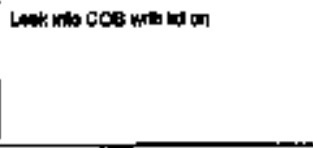 & 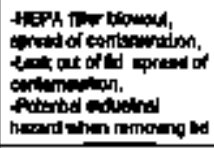 \\
\hline chater & Tanafur mestitery & 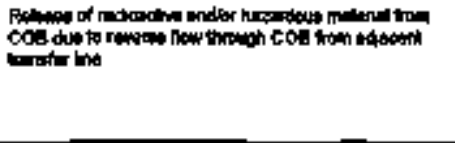 & 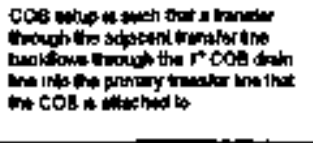 & 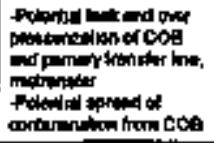 \\
\hline $\cos \theta$ & Trenectr mentory & 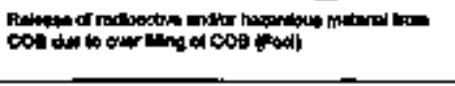 & 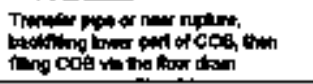 & 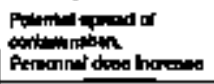 \\
\hline CRHAD & 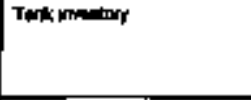 & 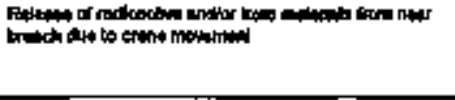 & 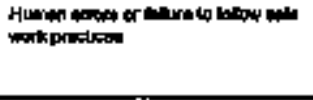 & 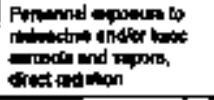 \\
\hline castont-1 & 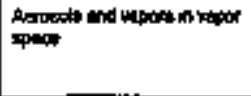 & 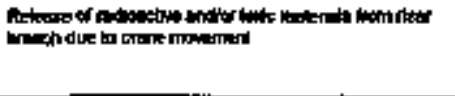 & 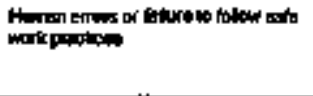 & 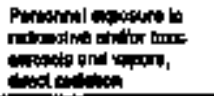 \\
\hline
\end{tabular}




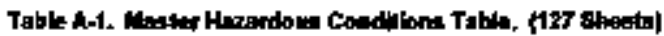

\begin{tabular}{|c|c|c|c|c|}
\hline 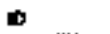 & Atak & 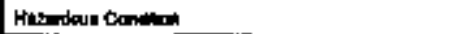 & center & Careapunse \\
\hline coneses & Twtemaritory & 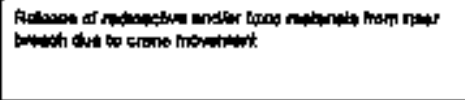 & 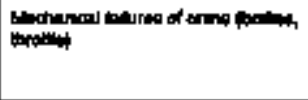 & 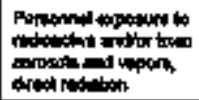 \\
\hline CRk-02.1 & 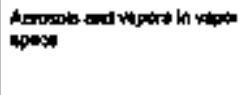 & 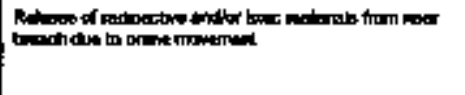 & 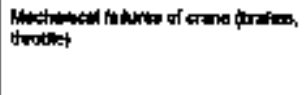 & 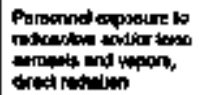 \\
\hline crunos & Tutemeritoy & 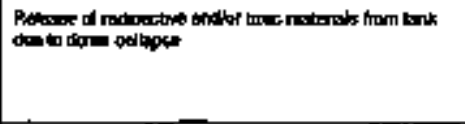 & 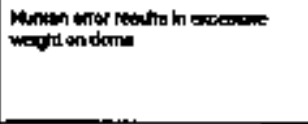 & 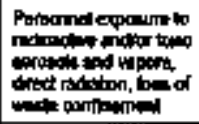 \\
\hline CRH-OSH & Corimo of leotity & 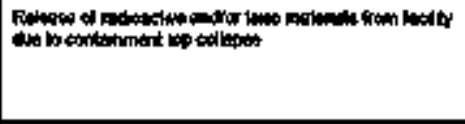 & 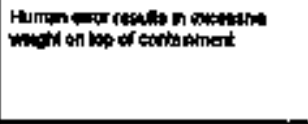 & 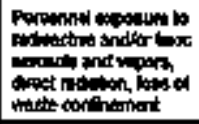 \\
\hline CRrbats.4. & Combertin of beth & 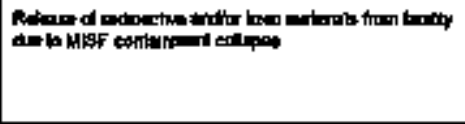 & 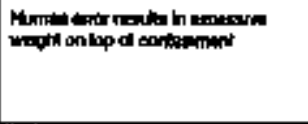 & 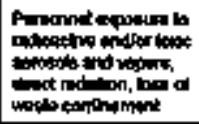 \\
\hline Conaton & Tank metrotory & 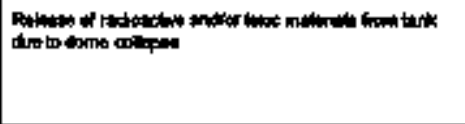 & 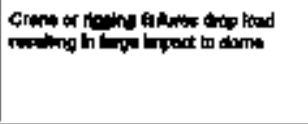 & 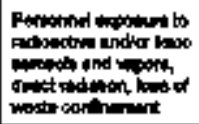 \\
\hline CRHOA-1A & Continte of 1 tons & 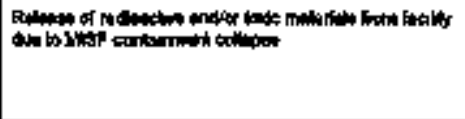 & 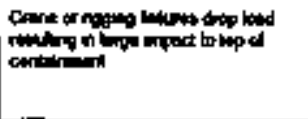 & 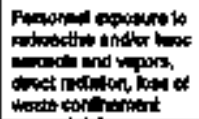 \\
\hline
\end{tabular}




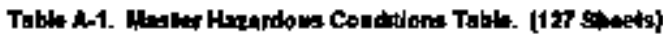

\begin{tabular}{|c|c|c|c|c|}
\hline 10 & 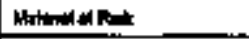 & Hagribuen Conilon & Couve & taraspenes. \\
\hline opronas & Cortention of besty & 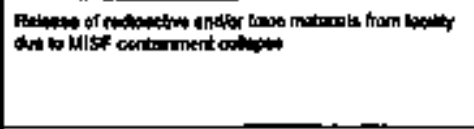 & 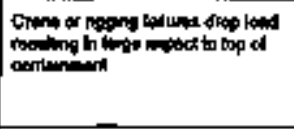 & 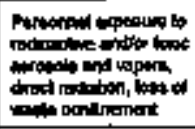 \\
\hline Cretsos & 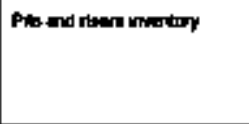 & 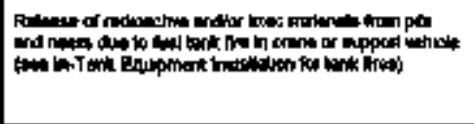 & 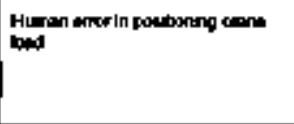 & 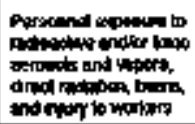 \\
\hline CRHOE-A & 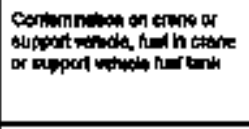 & 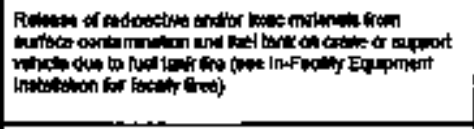 & 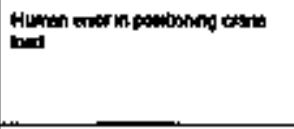 & 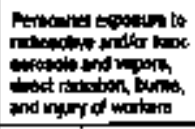 \\
\hline contod & 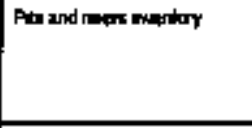 & 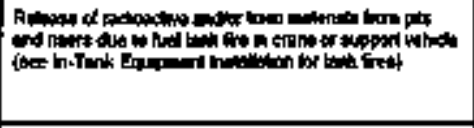 & 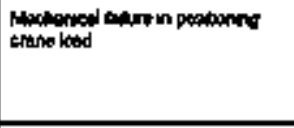 & 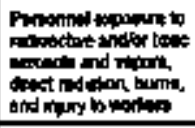 \\
\hline 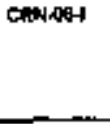 & 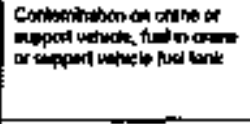 & 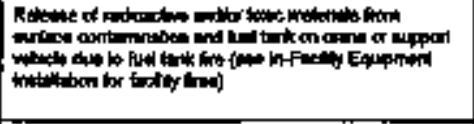 & 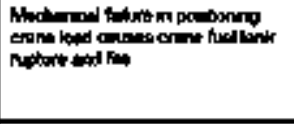 & 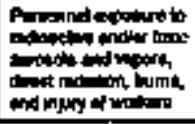 \\
\hline camor & 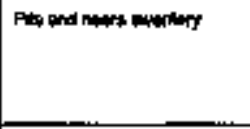 & 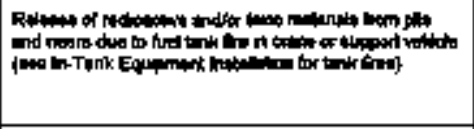 & 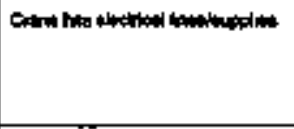 & 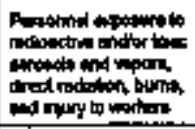 \\
\hline cribotat & 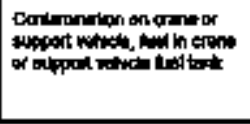 & 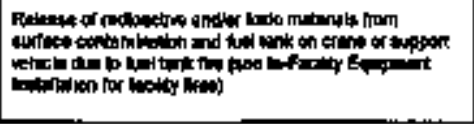 & 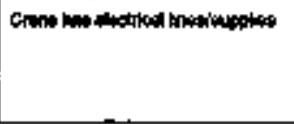 & 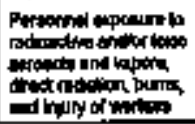 \\
\hline
\end{tabular}




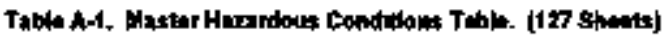

\begin{tabular}{|c|c|c|c|c|}
\hline m & Mandir a Butc & 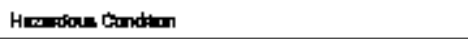 & Conum & Donochos \\
\hline CRHOA & 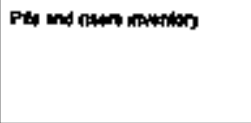 & 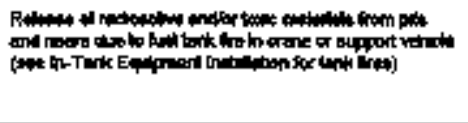 & 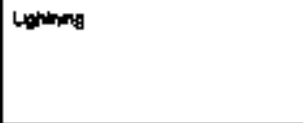 & 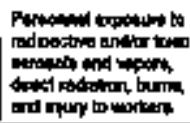 \\
\hline CRNOGH & 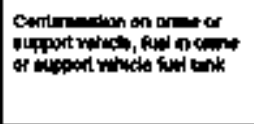 & 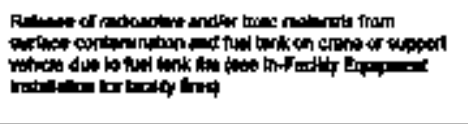 & Loning & 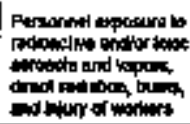 \\
\hline chatsos & 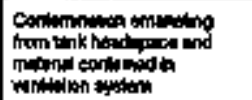 & 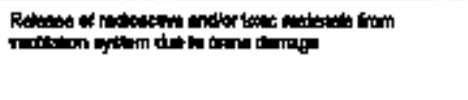 & 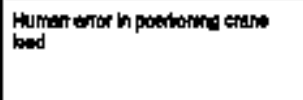 & 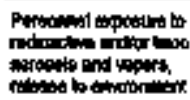 \\
\hline CNakis | & 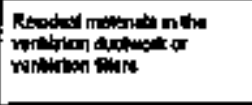 & 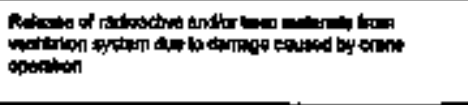 & ham & 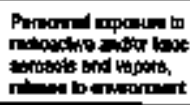 \\
\hline CAN-1D & 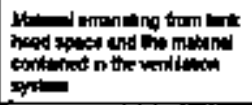 & 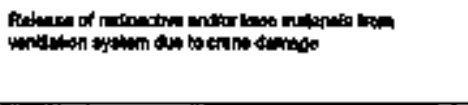 & 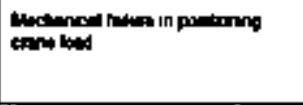 & 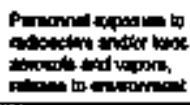 \\
\hline CRN-NOA & 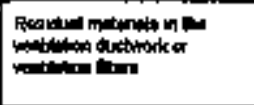 & 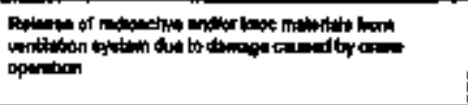 & 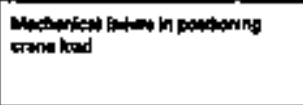 & 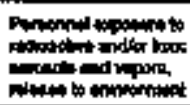 \\
\hline cow.11 & THAthomintor & 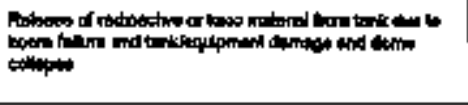 & 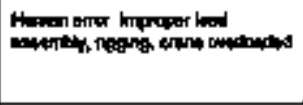 & 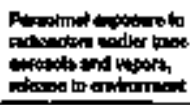 \\
\hline 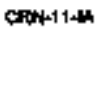 & 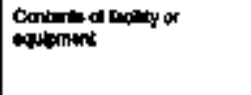 & 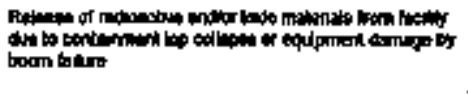 & 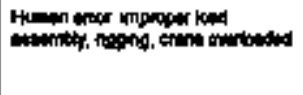 & 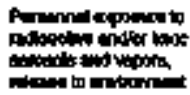 \\
\hline
\end{tabular}




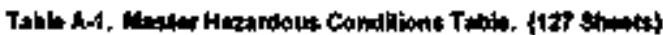

\begin{tabular}{|c|c|c|c|c|}
\hline ID & Mrotoind at Ant & 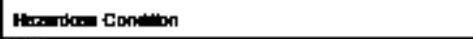 & Canas & Consequenou \\
\hline CRILAT & Contente of tallowent & 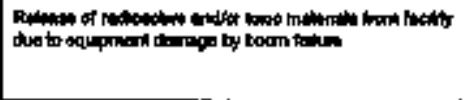 & 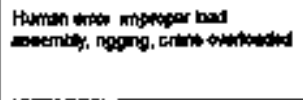 & 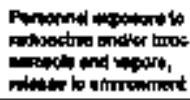 \\
\hline CAIFITE & Conimita of betsy & 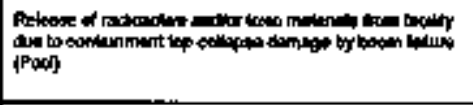 & 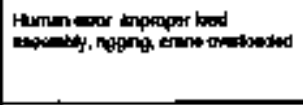 & 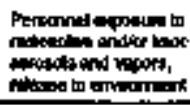 \\
\hline CRN-12 & Toratiming & 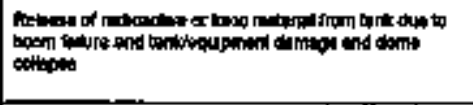 & 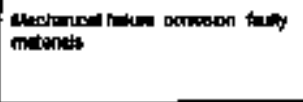 & 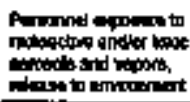 \\
\hline CRRt-12H & Contonto of & 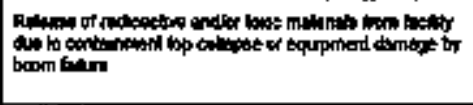 & 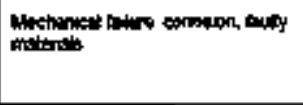 & 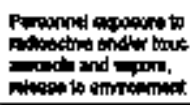 \\
\hline $\cos 12 \mathrm{As}$ & 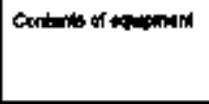 & 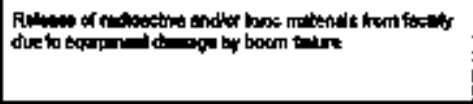 & 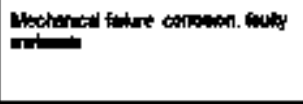 & 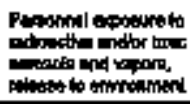 \\
\hline 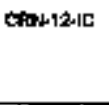 & Cartule of tacilis & 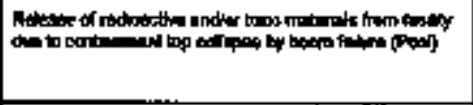 & 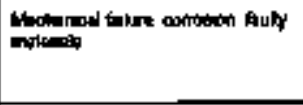 & 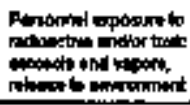 \\
\hline $\cosh -13-14$ & continele of thedisy & 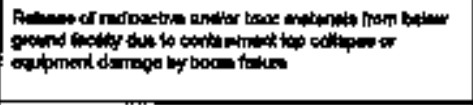 & 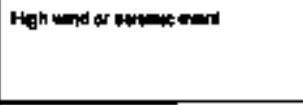 & 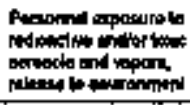 \\
\hline 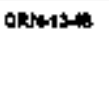 & conteds of & 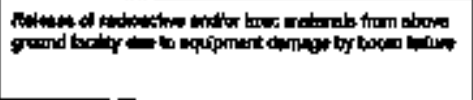 & 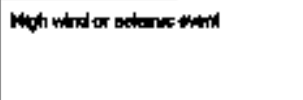 & 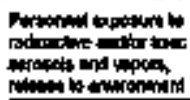 \\
\hline
\end{tabular}




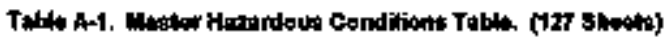

\begin{tabular}{|c|c|c|c|c|}
\hline$\omega$ & 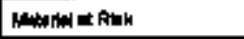 & 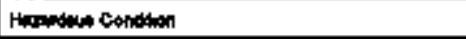 & chuna & 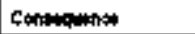 \\
\hline CRNAMU & Comberio of fats & 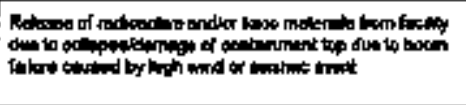 & 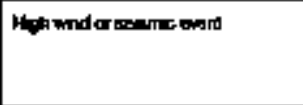 & | \\
\hline CRN-13-A & Tanki mantity & 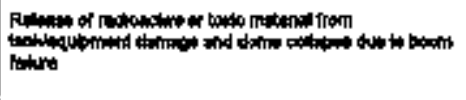 & 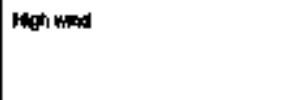 & 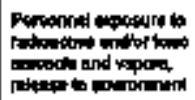 \\
\hline$c a+13-b$ & 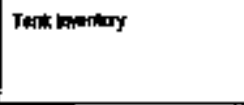 & 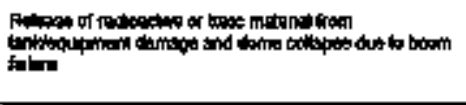 & 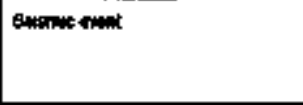 & 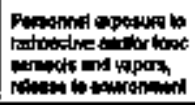 \\
\hline Crint 14 & 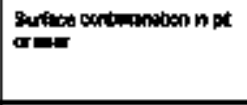 & 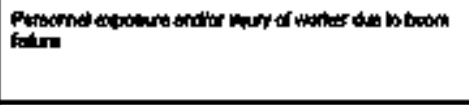 & 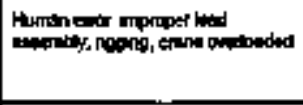 & 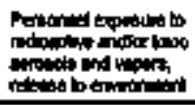 \\
\hline CRR+14-1 & Nh & 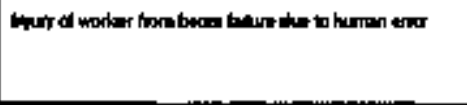 & 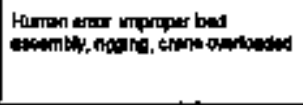 & 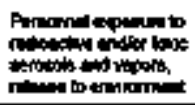 \\
\hline CRN-16 & 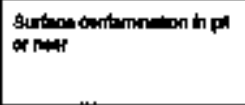 & 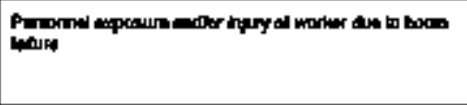 & 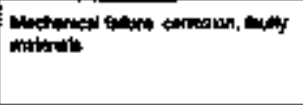 & 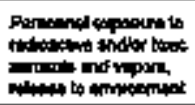 \\
\hline CON-15- & M4 & 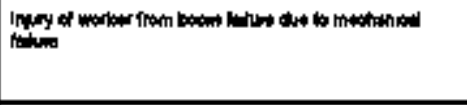 & 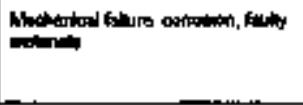 & 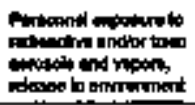 \\
\hline chtr-10 & 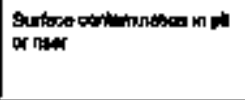 & 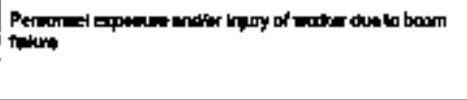 & 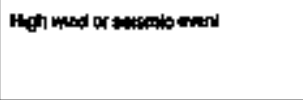 & 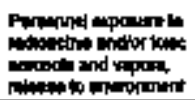 \\
\hline
\end{tabular}




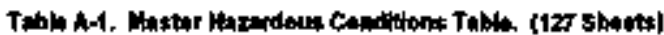

\begin{tabular}{|c|c|c|c|c|}
\hline $\boldsymbol{\omega}$ & 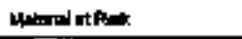 & Herantore Cendiban & Conum & Consequenes \\
\hline ORIABH & Nk & 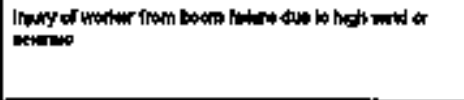 & 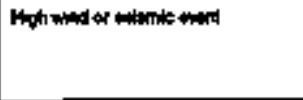 & 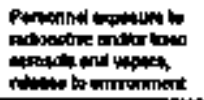 \\
\hline CARA-13 & Temt mentsiny & 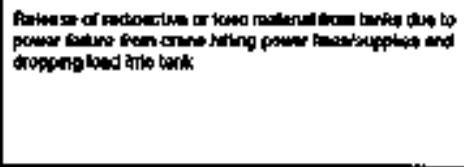 & 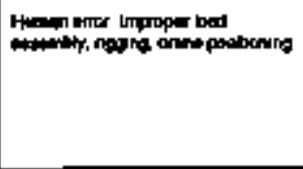 & 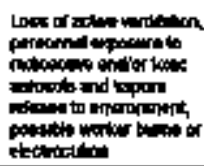 \\
\hline 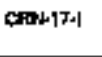 & $m$ & 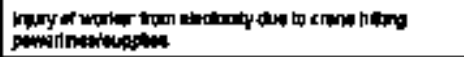 & Humangror mproper lo. & 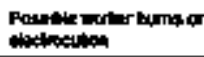 \\
\hline Cents 1t & 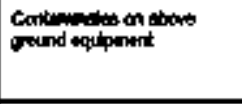 & 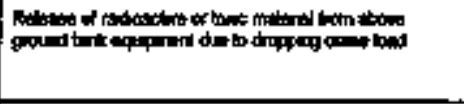 & 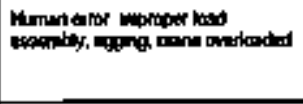 & 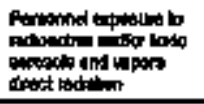 \\
\hline CAMB-1B-4 & 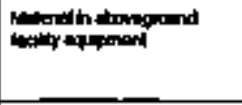 & 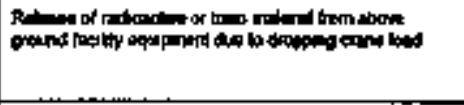 & 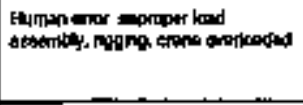 & 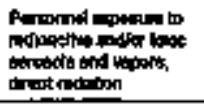 \\
\hline CRRM-1D & 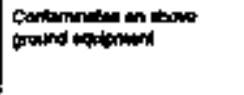 & 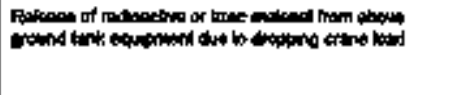 & 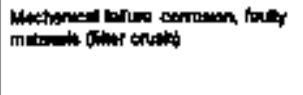 & 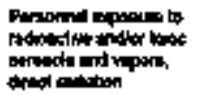 \\
\hline Exw.14 & 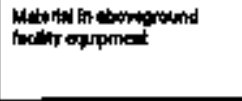 & 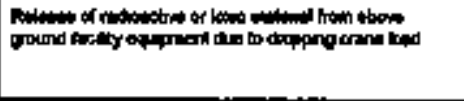 & 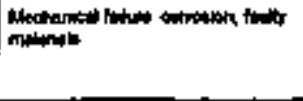 & 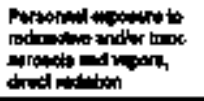 \\
\hline chango & 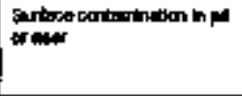 & 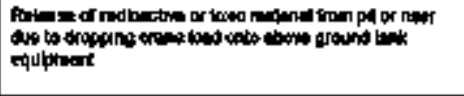 & 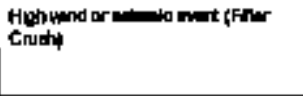 & 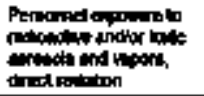 \\
\hline
\end{tabular}


Tablo A-1. Woglor Haradeus Cendlions Tob. [127 Bherts]

\begin{tabular}{|c|c|c|c|c|}
\hline $\mathbf{0}$ & 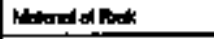 & 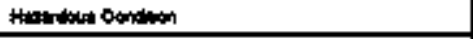 & Guve & Conociporice \\
\hline CRARDU & 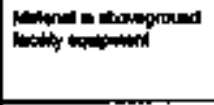 & 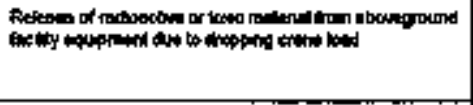 & 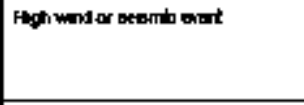 & 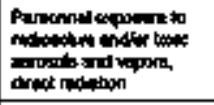 \\
\hline Centh-21 & Tahmmiton & 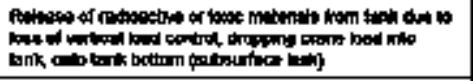 & 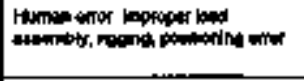 & 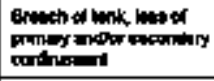 \\
\hline Cotort-21-n & 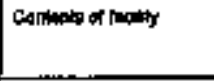 & 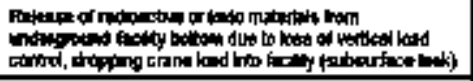 & 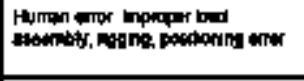 & 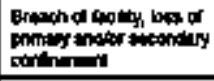 \\
\hline $\cos a+21-\theta$ & 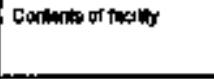 & 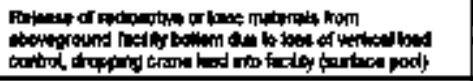 & 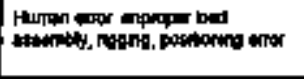 & 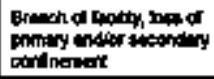 \\
\hline CAne-22 & 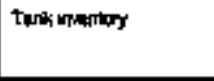 & 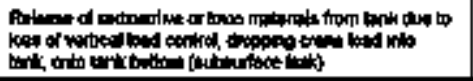 & 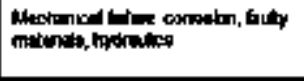 & 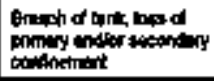 \\
\hline CAmLzar & Dartents of finctey & 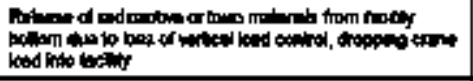 & 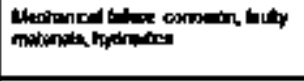 & 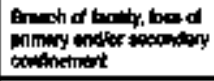 \\
\hline CAnuzas & Contents difacter & 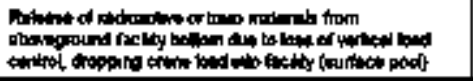 & 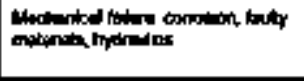 & 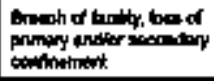 \\
\hline CARECOB & Tut mentery & 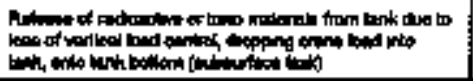 & 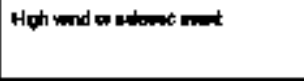 & 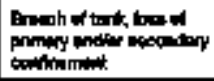 \\
\hline GRNes-sh & Cortinkat tooky & 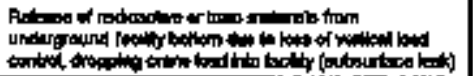 & 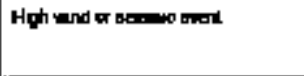 & 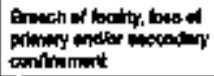 \\
\hline Gatsons & 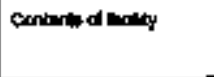 & 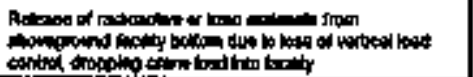 & 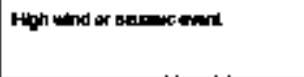 & 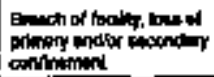 \\
\hline
\end{tabular}




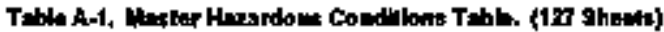

\begin{tabular}{|c|c|c|c|c|}
\hline w & 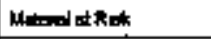 & 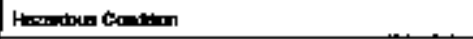 & 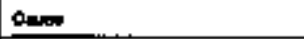 & Dansepane \\
\hline $60+24$ & Nh & 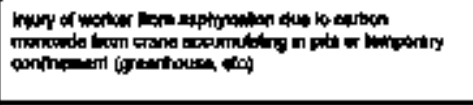 & 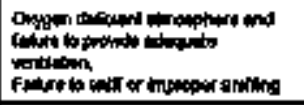 & 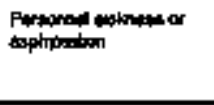 \\
\hline contrit & $\mathrm{NH}$ & 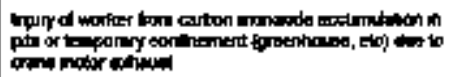 & 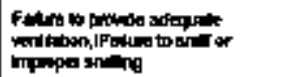 & 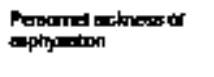 \\
\hline EPMAs & NA & 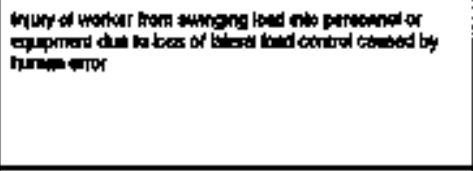 & 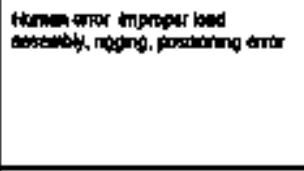 & 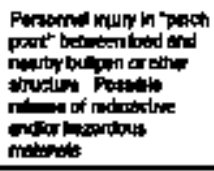 \\
\hline CRrtistih & $\mathrm{min}$ & 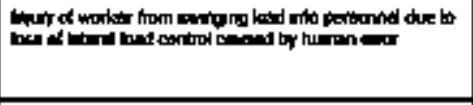 & 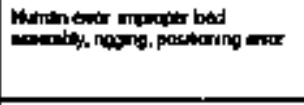 & 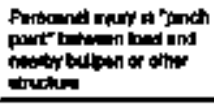 \\
\hline 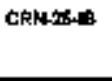 & 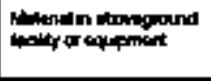 & 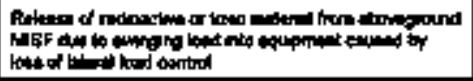 & 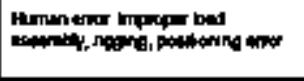 & 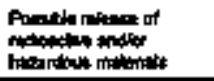 \\
\hline CRNA & $\mathbf{m}$ & 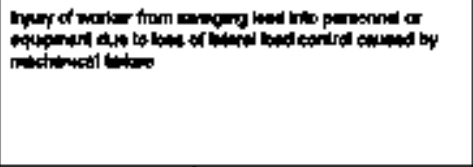 & 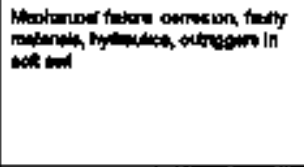 & 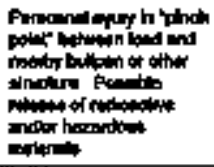 \\
\hline CPAN20HA & $\mathbf{M}$ & 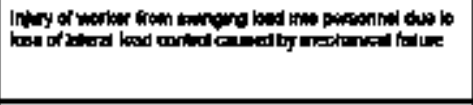 & 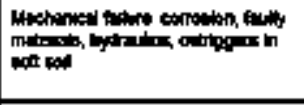 & 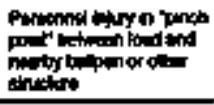 \\
\hline cratole & 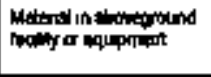 & 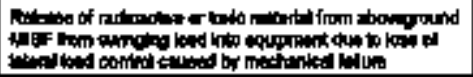 & 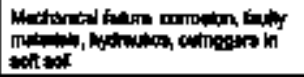 & 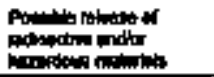 \\
\hline
\end{tabular}




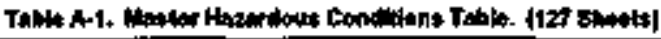

\begin{tabular}{|c|c|c|c|c|}
\hline$\omega$ & 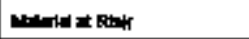 & 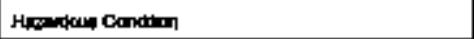 & Cman & Consercamipy \\
\hline CRN-2 & 种 & 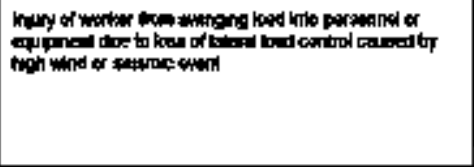 & 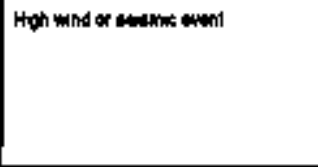 & 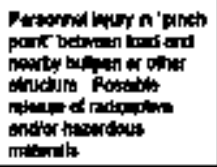 \\
\hline chatherah & Hh & 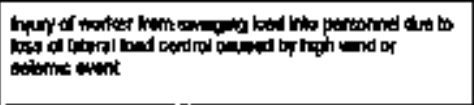 & Hoh wid or wat d atent & 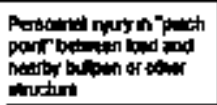 \\
\hline Croph-27al & 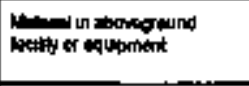 & 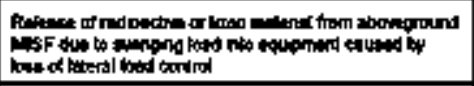 & 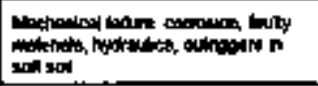 & 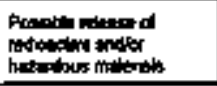 \\
\hline 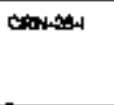 & 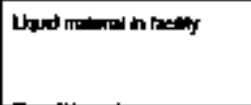 & 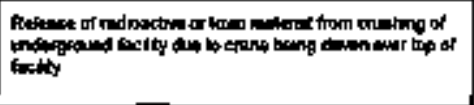 & 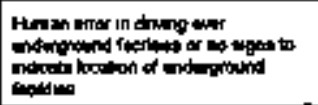 & Undeppourd muctum \\
\hline D्ञास-01 & Com in & 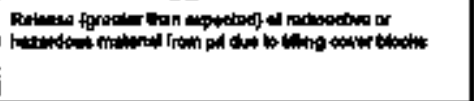 & 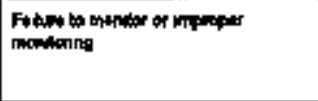 & 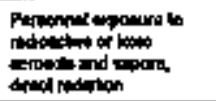 \\
\hline EيR-OI & 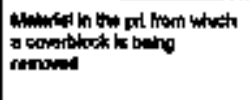 & 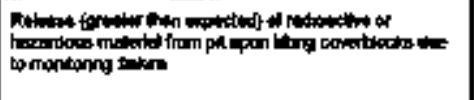 & 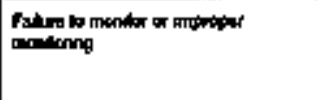 & 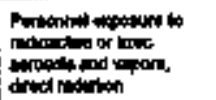 \\
\hline cuks m & 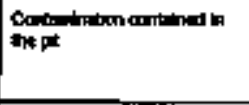 & 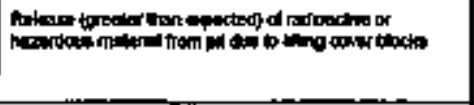 & 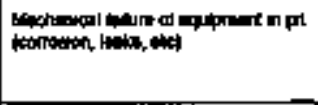 & 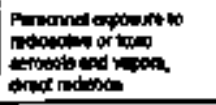 \\
\hline GuR-ä & 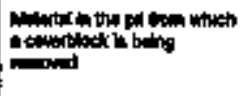 & 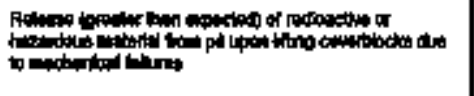 & 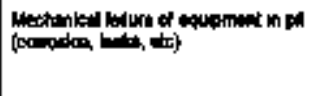 & 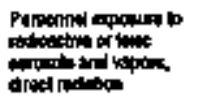 \\
\hline
\end{tabular}




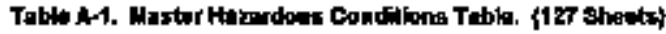

\begin{tabular}{|c|c|c|c|c|}
\hline $\mathbf{m}$ & 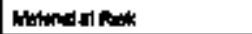 & 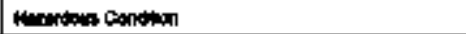 & $\cos$ & conenpuntet \\
\hline Cymas & 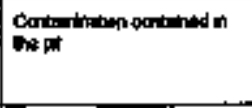 & 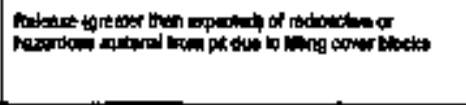 & 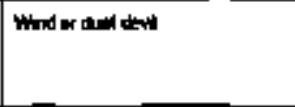 & 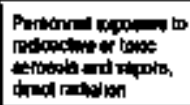 \\
\hline CथR-क्स & 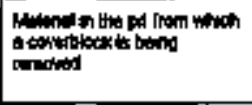 & 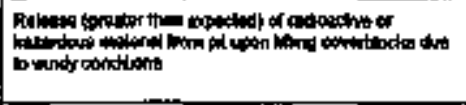 & mols or duel on & 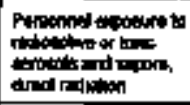 \\
\hline curpen & 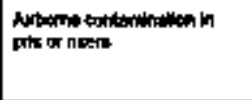 & 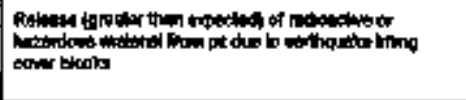 & A & 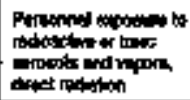 \\
\hline Chand4 & 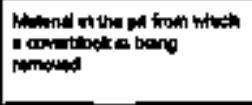 & 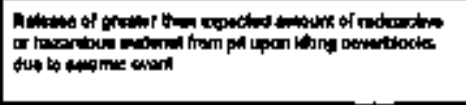 & | & 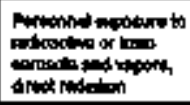 \\
\hline Evadx & 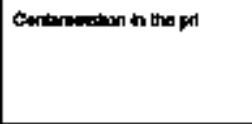 & 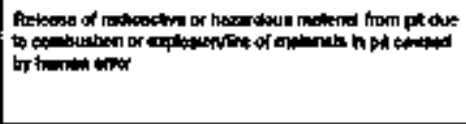 & 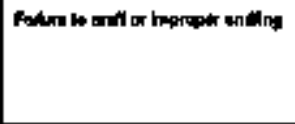 & 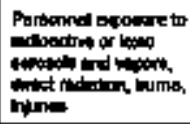 \\
\hline CYR-OS-1 & 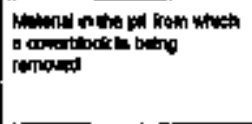 & 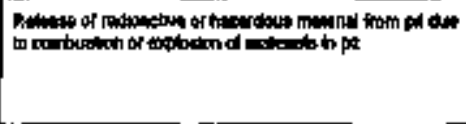 & 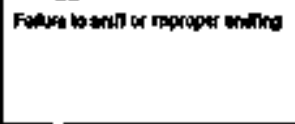 & 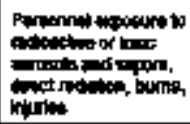 \\
\hline Cymal & Buflus conkmoniben $\mathrm{h}$ pa & 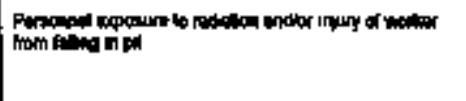 & 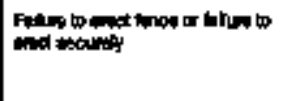 & 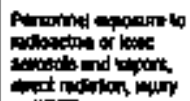 \\
\hline $\cos 0$ & 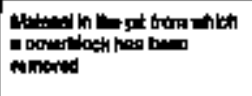 & 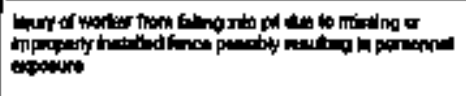 & 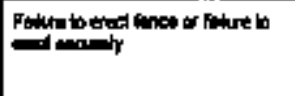 & 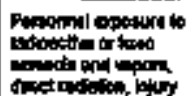 \\
\hline
\end{tabular}




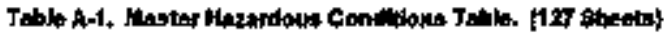

\begin{tabular}{|c|c|c|c|c|}
\hline $\mathbf{D}$ & Nemilat a then & Mesodas Conoting & CAsin & connequaran \\
\hline chipor & 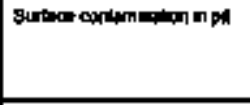 & 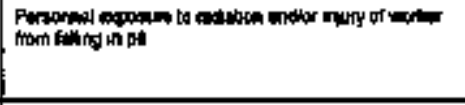 & 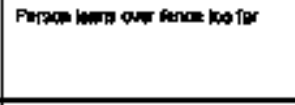 & 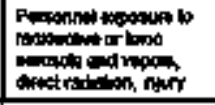 \\
\hline CWhated & 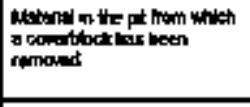 & 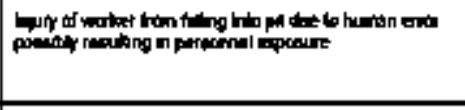 & 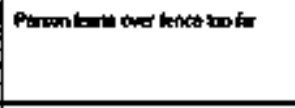 & 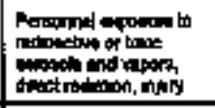 \\
\hline CUA. & 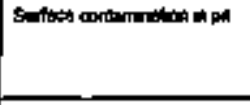 & 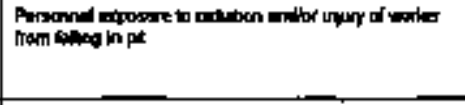 & Nand hitur & 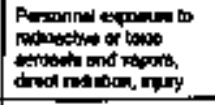 \\
\hline CWR-06- & 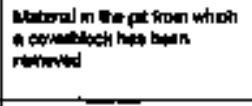 & 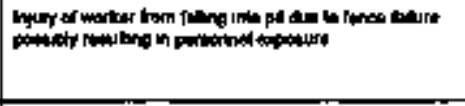 & Fangen foutso & 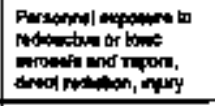 \\
\hline CWR-09 & Surtion & 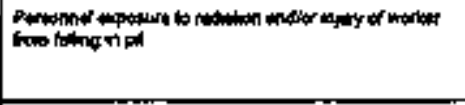 & 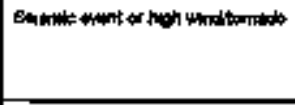 & 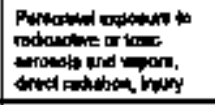 \\
\hline $6400-1$ & 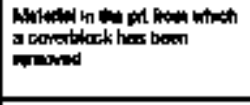 & 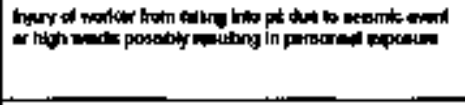 & 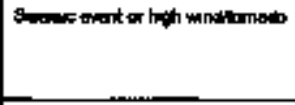 & 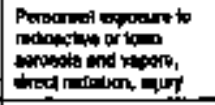 \\
\hline chint.10 & $\mathrm{m}$ & 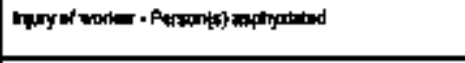 & 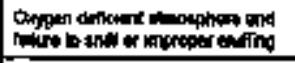 & 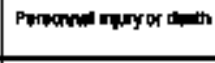 \\
\hline Con.10.1 & Mh & 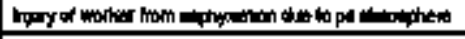 & 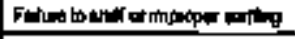 & 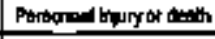 \\
\hline Civh-11 & $\mathbf{m}$ & 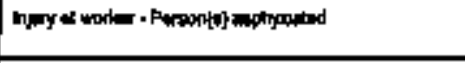 & 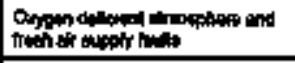 & 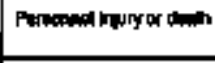 \\
\hline ERR-11A & Ha & 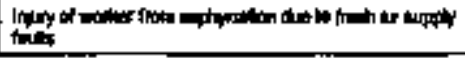 & 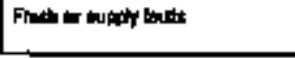 & 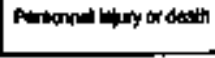 \\
\hline
\end{tabular}




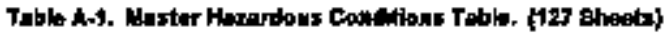

\begin{tabular}{|c|c|c|c|c|}
\hline ID & Hand at in & 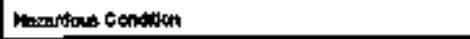 & Contbo & Constonimed \\
\hline CWR-124 & 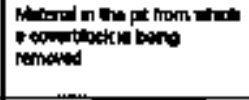 & 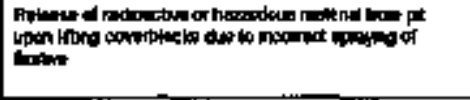 & 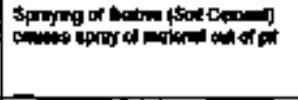 & 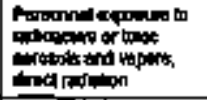 \\
\hline CYR-13 & Mrimid in opan heedtat of & 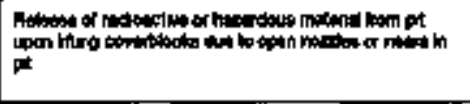 & 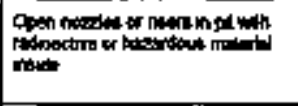 & 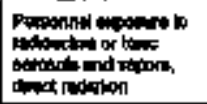 \\
\hline CYR-14 & H & 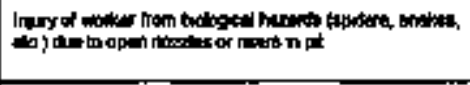 & 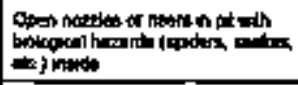 & 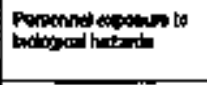 \\
\hline CUR.15H & 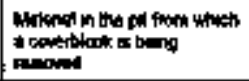 & 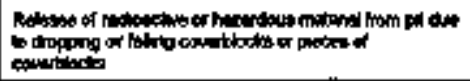 & 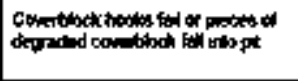 & 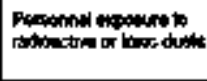 \\
\hline DCN-01 & 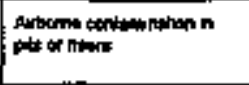 & 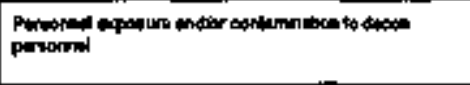 & Fatho b PPE & 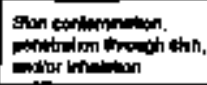 \\
\hline D다먹-1 & 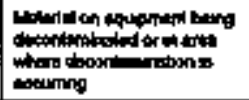 & 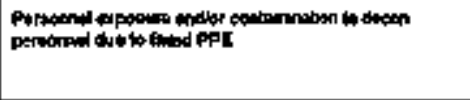 & Fullurd tod PPE & 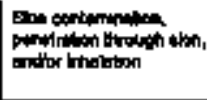 \\
\hline Dewtor & 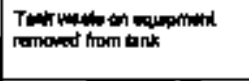 & 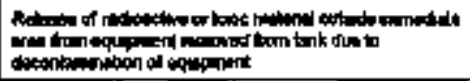 & 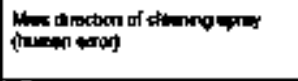 & 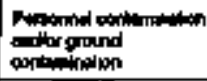 \\
\hline DGMER-A & 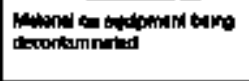 & 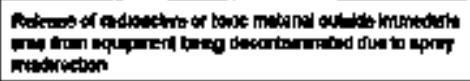 & 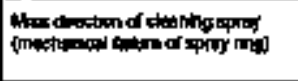 & 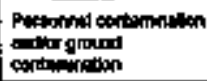 \\
\hline DCMOe.10 & 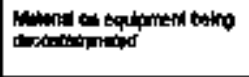 & 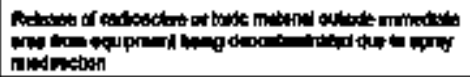 & Whection af dasing aprty & 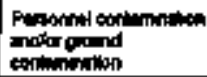 \\
\hline
\end{tabular}


Table M-1. Mnotur thrindows Condilons Table (123 aheota)

\begin{tabular}{|c|c|c|c|c|}
\hline$\rightarrow$ & 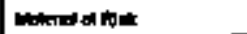 & 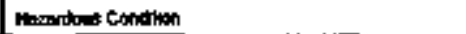 & Eauta & 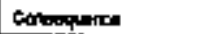 \\
\hline DCHas & 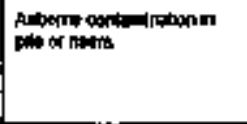 & 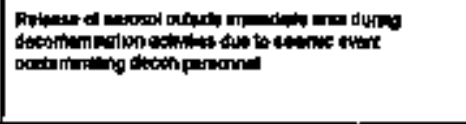 & E & 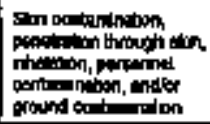 \\
\hline 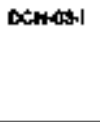 & 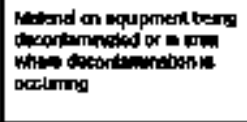 & 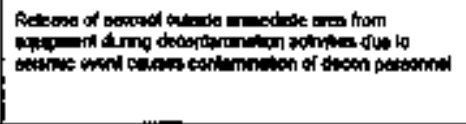 & Gadera aval & 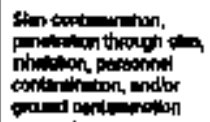 \\
\hline Doton & 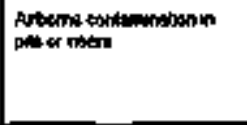 & 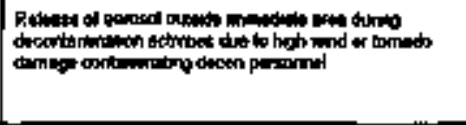 & MnNTamaso & 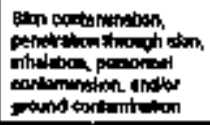 \\
\hline DCHO4| & 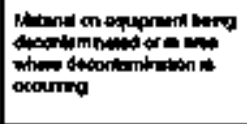 & 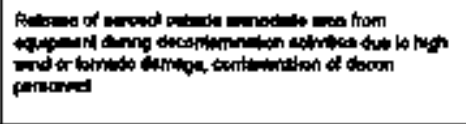 & Whitraments & 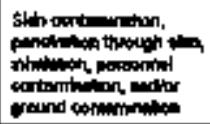 \\
\hline OCN-OS & Non & 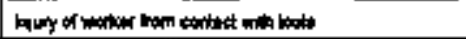 & Huthen ther & 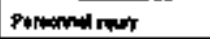 \\
\hline Octeos & $\operatorname{mos}$ & 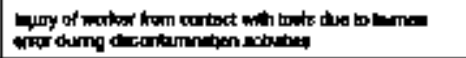 & Humeneror & Punthed apror \\
\hline DCHOA & 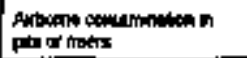 & 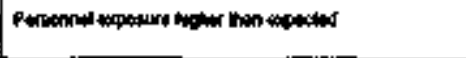 & 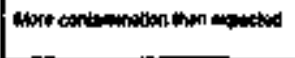 & 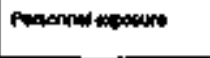 \\
\hline DCRNDOH & Nh & 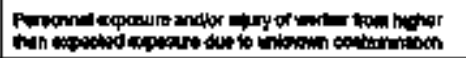 & 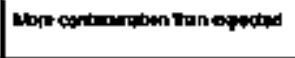 & 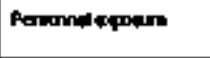 \\
\hline DEHAOS & wh & 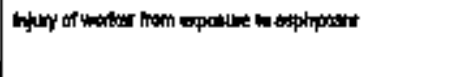 & 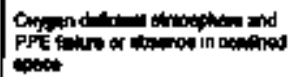 & Paconnt riply \\
\hline Dethost & Nin & 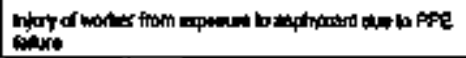 & 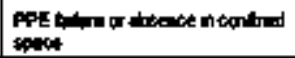 & Puawhilinpry \\
\hline
\end{tabular}




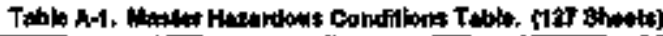

\begin{tabular}{|c|c|c|c|c|}
\hline$\omega$ & 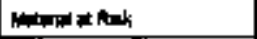 & 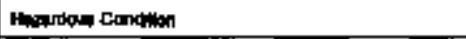 & $c=$ & Sontequence \\
\hline$E=01$ & 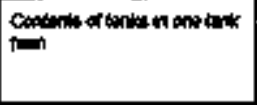 & 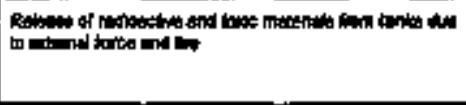 & Aromen eath & 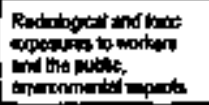 \\
\hline$\epsilon \in-R_{2}$ & 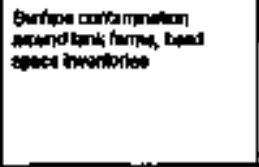 & 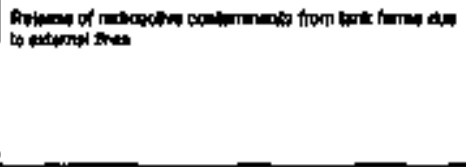 & Angen th & 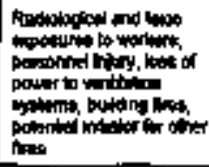 \\
\hline EE-OO & 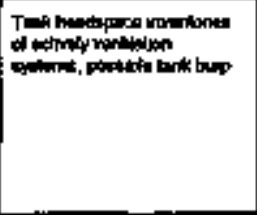 & 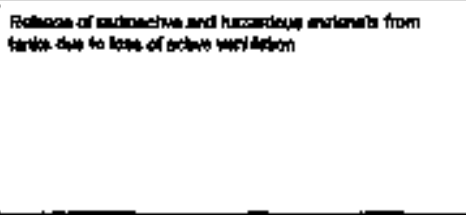 & Dem actuper & 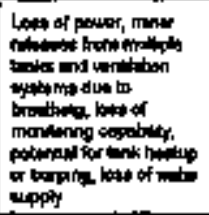 \\
\hline Eesos & क्रा thath conterti & 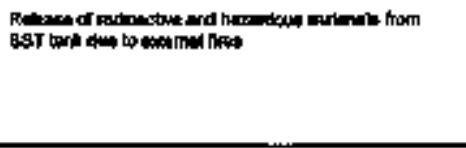 & 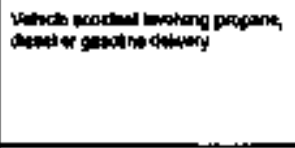 & 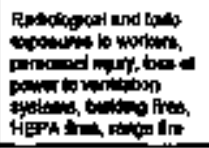 \\
\hline es.04b & 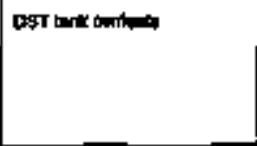 & 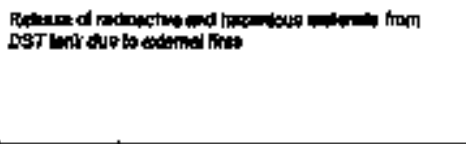 & 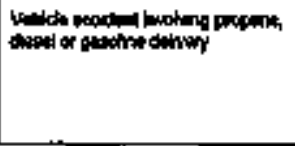 & 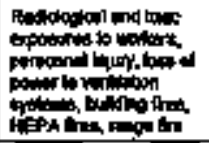 \\
\hline
\end{tabular}




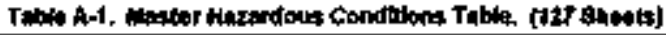

\begin{tabular}{|c|c|c|c|c|}
\hline 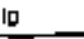 & 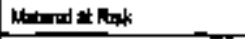 & 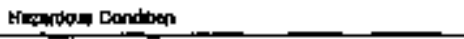 & can & Canampina \\
\hline EP.65 & Componts of BST beth hos & 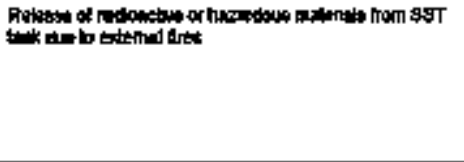 & 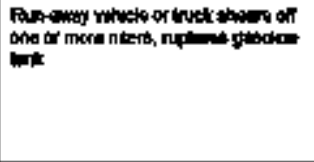 & 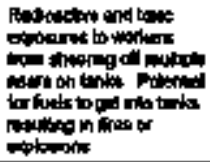 \\
\hline ex-1006 & 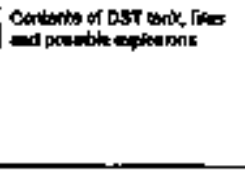 & 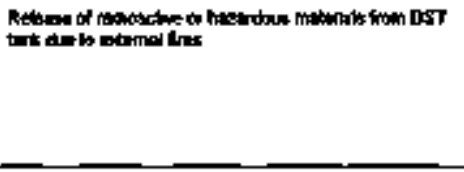 & 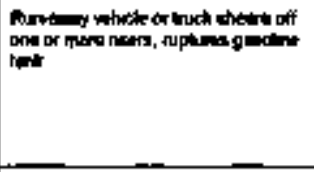 & 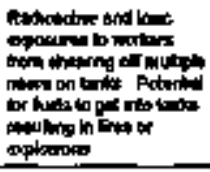 \\
\hline EE-OA & 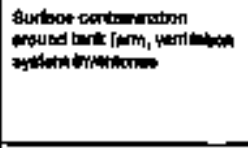 & 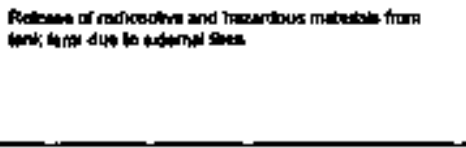 & 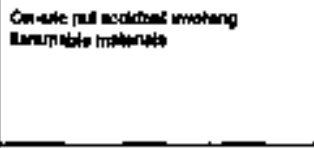 & 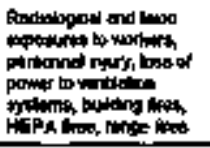 \\
\hline EE-UT & $\mathbf{m a n}$ & 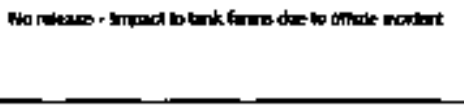 & 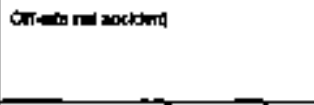 & 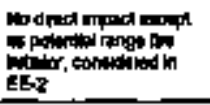 \\
\hline EE-OP & $\mathrm{Nan}$ & 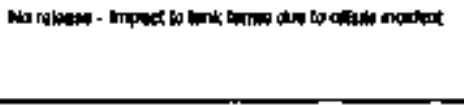 & 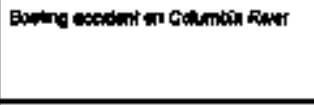 & 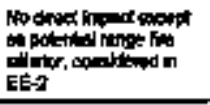 \\
\hline Ees & M & 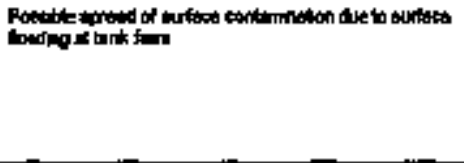 & 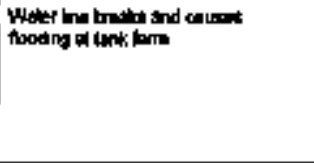 & 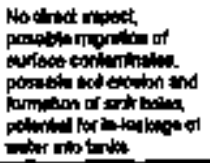 \\
\hline
\end{tabular}




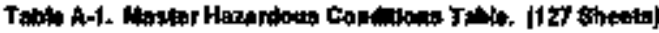

\begin{tabular}{|c|c|c|c|c|}
\hline 由 & 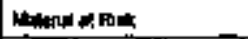 & Hethon Condthin & conat & Colited \\
\hline EE.10 & Comienta el ofrockatiming & 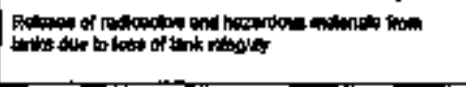 & 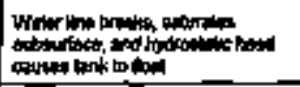 & 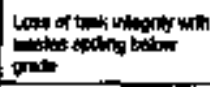 \\
\hline TEN1 & 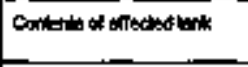 & 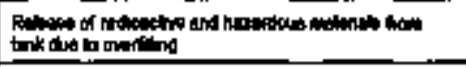 & 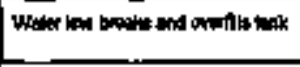 & 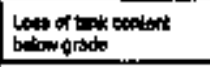 \\
\hline EE-12 & m & 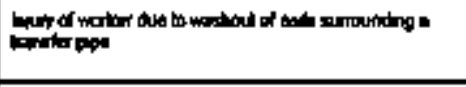 & 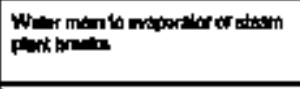 & 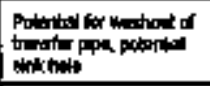 \\
\hline EE-13 & Nh & 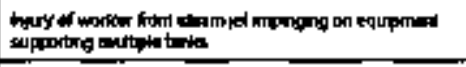 & stand ind thet & 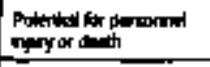 \\
\hline EE-14 & NAR & 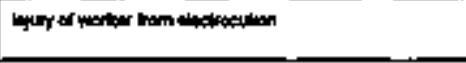 & EF & 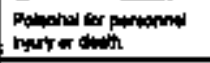 \\
\hline EE-16 & Na & 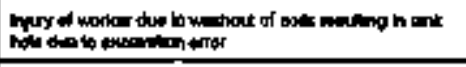 & 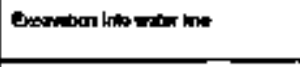 & 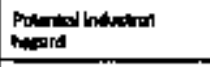 \\
\hline EI-10 & 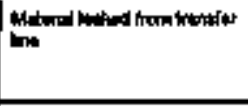 & 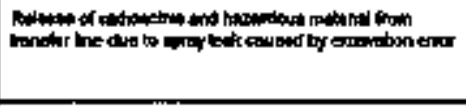 & 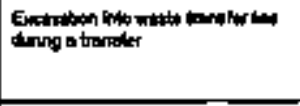 & 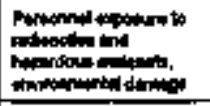 \\
\hline rE.17 & 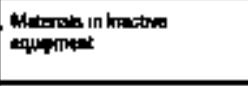 & 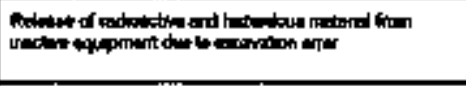 & 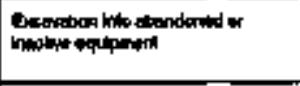 & 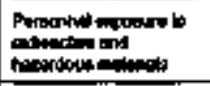 \\
\hline Grater & Nar & 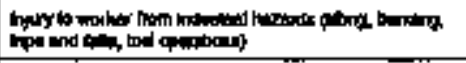 & 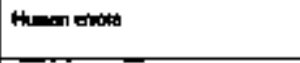 & Hotitin has \\
\hline CPanot-1 & wh & 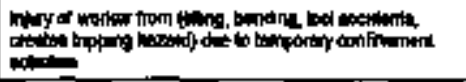 & Han who & Nextax hisny \\
\hline Gath-co & Ha & 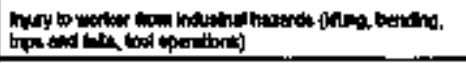 & 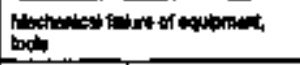 & nerier nony \\
\hline Cenotis & Wh & 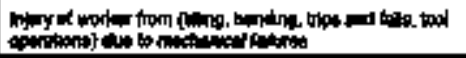 & 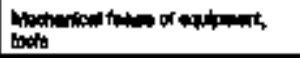 & 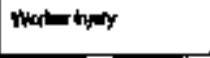 \\
\hline
\end{tabular}




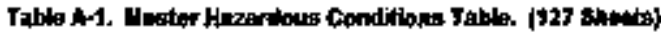

\begin{tabular}{|c|c|c|c|c|}
\hline It & (1) & Hanimut Com & Couts & contiontintes \\
\hline 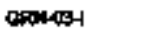 & m & 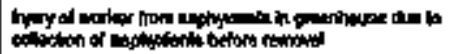 & Humb שx & Whater mpy \\
\hline Lots-1-CURA & 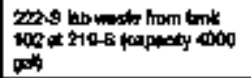 & 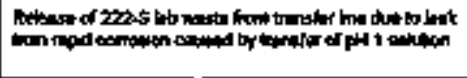 & 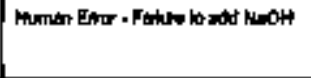 & \\
\hline 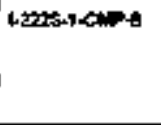 & 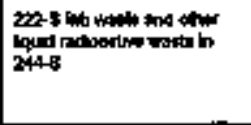 & 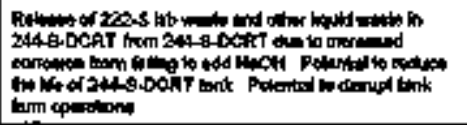 & 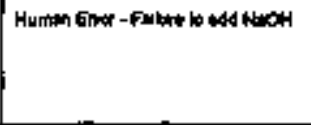 & \\
\hline 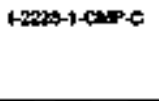 & 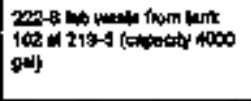 & 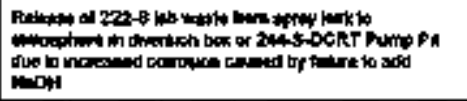 & 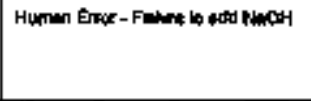 & \\
\hline WHE.1-CAN-D & 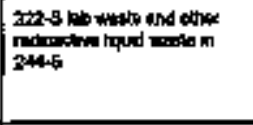 & 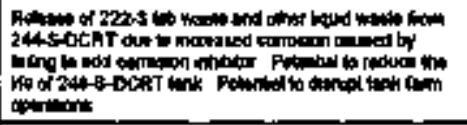 & 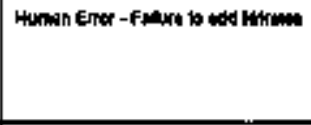 & \\
\hline MZOF-1-CMPE & 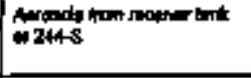 & 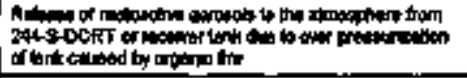 & 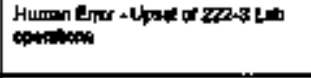 & \\
\hline $10202-1-6424$ & 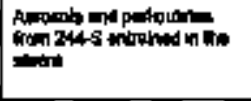 & 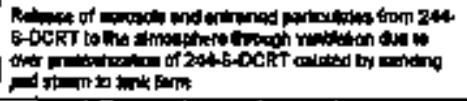 & 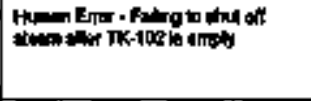 & \\
\hline |-2025-1-Round & 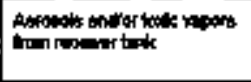 & 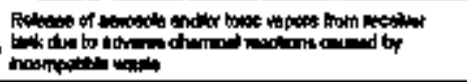 & Tranter of hesmpals wath & \\
\hline 1-20250-PLWHA & 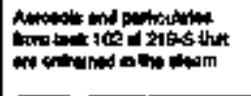 & 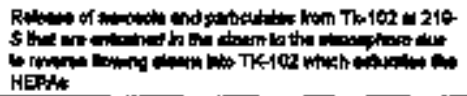 & 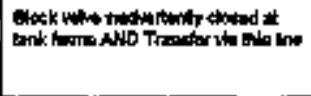 & \\
\hline
\end{tabular}




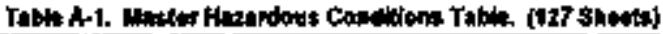

\begin{tabular}{|c|c|c|c|c|}
\hline $\boldsymbol{D}$ & 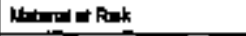 & Ht Brider Conditien & can & Carompuange \\
\hline $10205-2+2046$ & 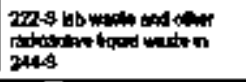 & 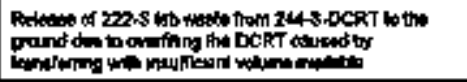 & 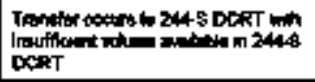 & \\
\hline 1020t-2-Nun-s & 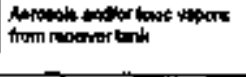 & 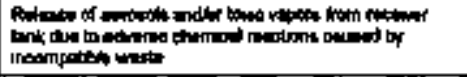 & 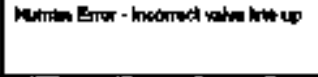 & \\
\hline $1-2025-2-7 u m-0$ & 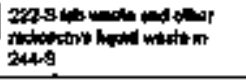 & 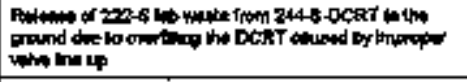 & 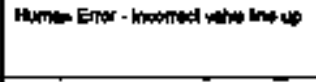 & \\
\hline $1-2025-2-6 \times 1-n$ & 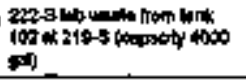 & 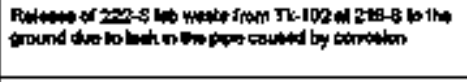 & 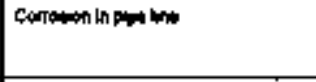 & \\
\hline $12223-310 \times 10$ & 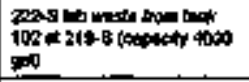 & 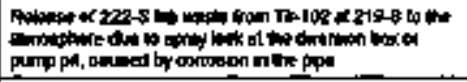 & 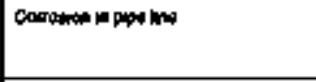 & \\
\hline $1002 \theta-2060$ & 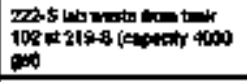 & 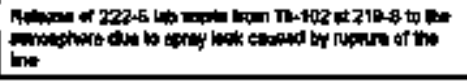 & 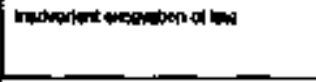 & \\
\hline 1.002024060 & 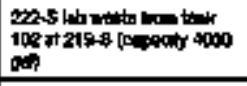 & 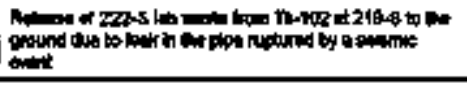 & Earthruater & \\
\hline |Hentake & 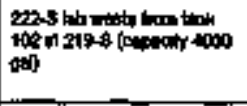 & 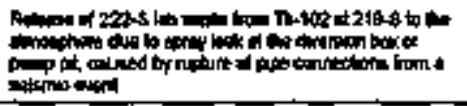 & Exithonimon & \\
\hline HOPS-2PREA & 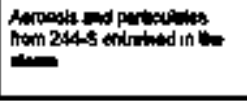 & 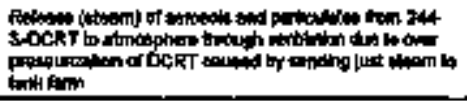 & 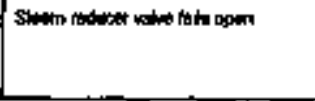 & \\
\hline
\end{tabular}




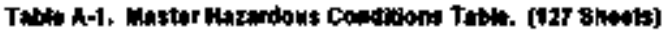

\begin{tabular}{|c|c|c|c|c|}
\hline $\mathbf{D}$ & 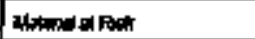 & Heardous truditen & conve & Congenpinat \\
\hline 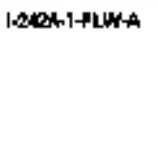 & 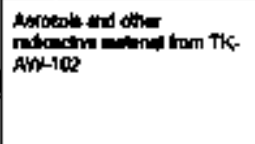 & 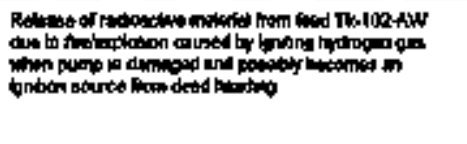 & 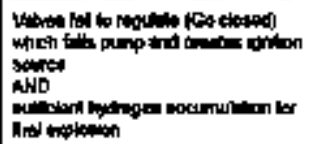 & \\
\hline 1-24at-1-PLWE & 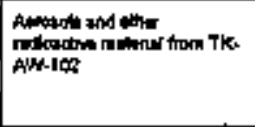 & 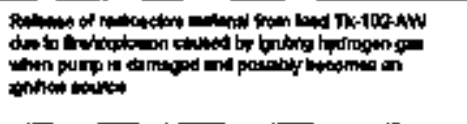 & 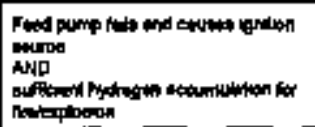 & \\
\hline 1242A-1-FUN-C & 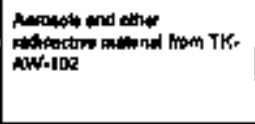 & 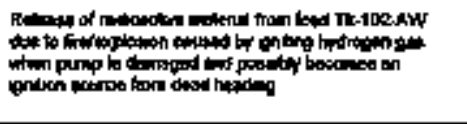 & 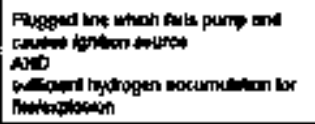 & \\
\hline $1-2 \pi n h-1-n+n d 0$ & 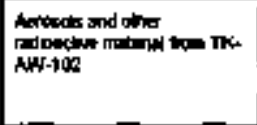 & 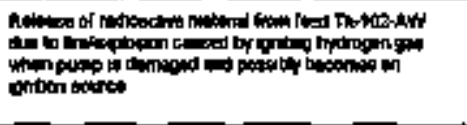 & 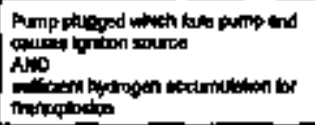 & \\
\hline HARU1-FLWE & 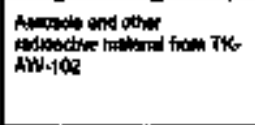 & 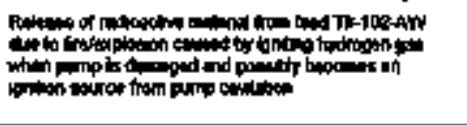 & 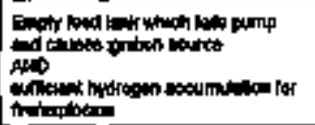 & \\
\hline 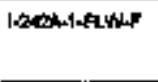 & m & 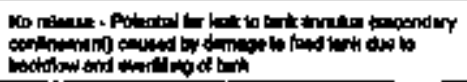 & 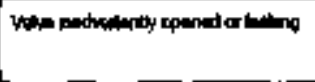 & \\
\hline LDEACLLEKA & 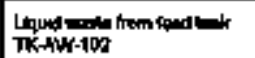 & 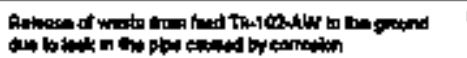 & 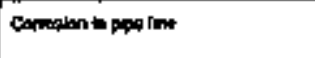 & \\
\hline 1.2WW-1 + EKKE & 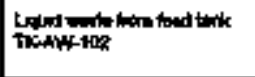 & 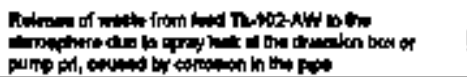 & 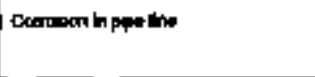 & \\
\hline
\end{tabular}




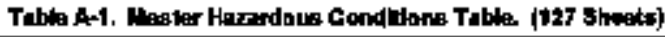

\begin{tabular}{|c|c|c|c|c|}
\hline $\mathbf{0}$ & 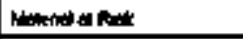 & 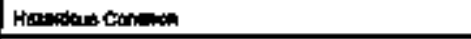 & Coun: & Contequance \\
\hline 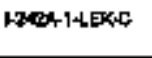 & 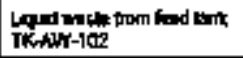 & 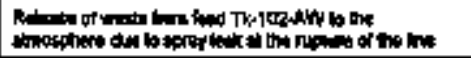 & 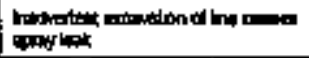 & \\
\hline 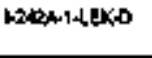 & 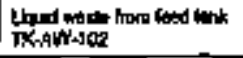 & 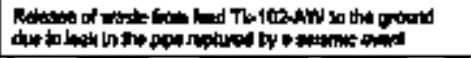 & 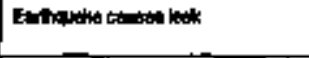 & \\
\hline 1-2WW+1-LEKE & 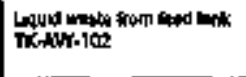 & 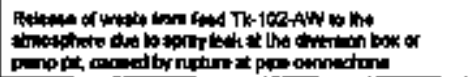 & 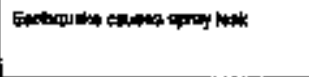 & \\
\hline 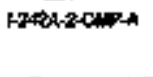 & NA & 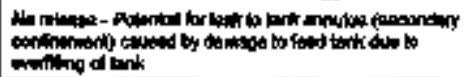 & Viste & \\
\hline $1-242 \mathrm{~A}-3 \mathrm{CA}-\mathrm{B}$ & 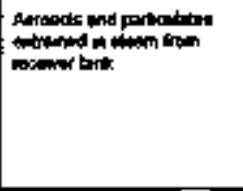 & 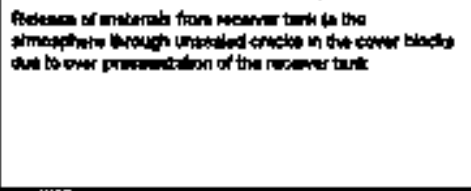 & 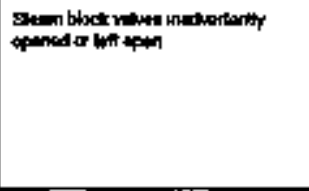 & 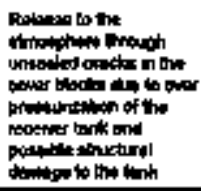 \\
\hline Hedenthon & 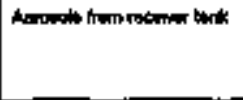 & 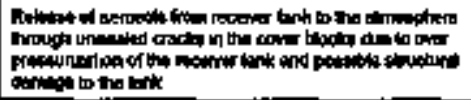 & 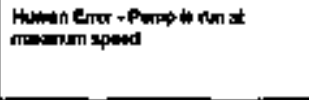 & \\
\hline 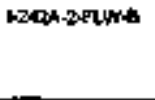 & 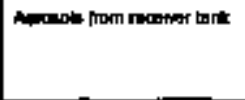 & 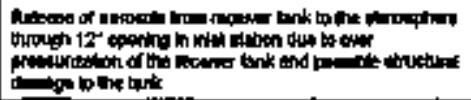 & 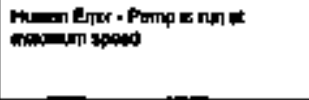 & \\
\hline 1204-2ABX-4 & 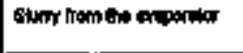 & 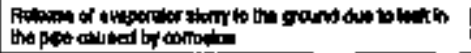 & Comearon in pope ins & \\
\hline $1264-240 \times 6$ & 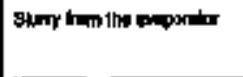 & 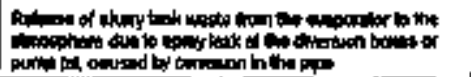 & Conchnn now & \\
\hline $124043-1, E x<$ & 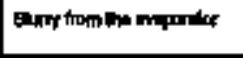 & 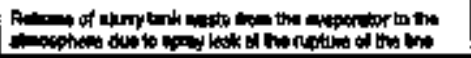 & 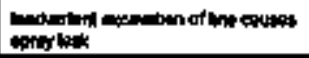 & \\
\hline
\end{tabular}




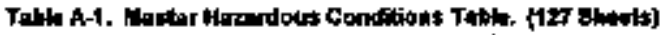

\begin{tabular}{|c|c|c|c|c|}
\hline ID & 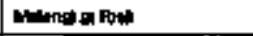 & Anordins Gandion & conat. & Connownes \\
\hline 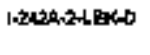 & 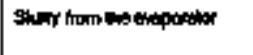 & 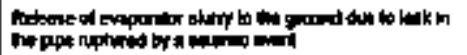 & 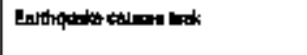 & \\
\hline HOA2A-RLEKE & 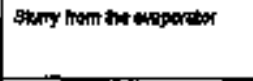 & 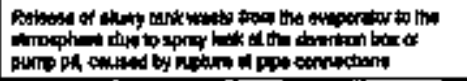 & 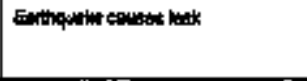 & \\
\hline 1-242A-2ATPA & $\mathrm{man}$ & 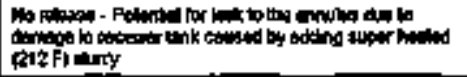 & 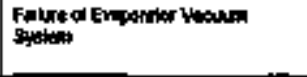 & \\
\hline $1-204-2489-A$ & 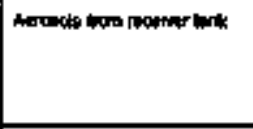 & 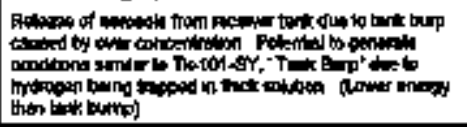 & 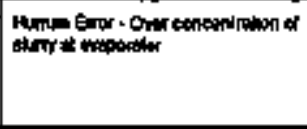 & \\
\hline 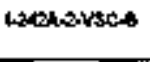 & $\mathbf{m}$ & 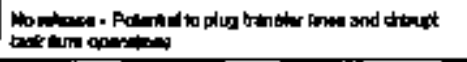 & 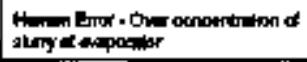 & \\
\hline 1-204-3FLWA-A & 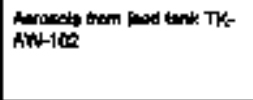 & 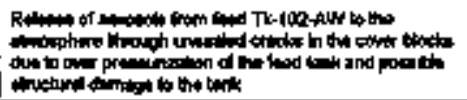 & 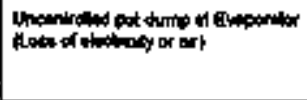 & \\
\hline Habs-3 FlW & 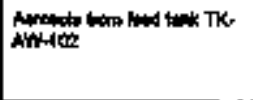 & 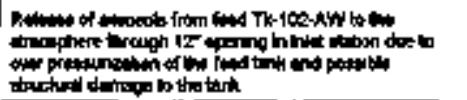 & 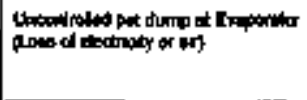 & \\
\hline nzesh+rivs & 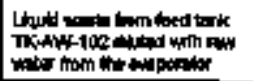 & 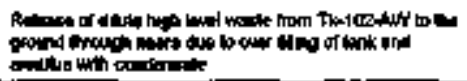 & 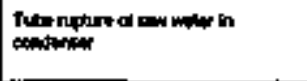 & \\
\hline 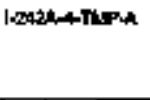 & 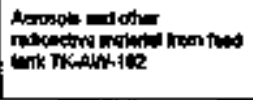 & 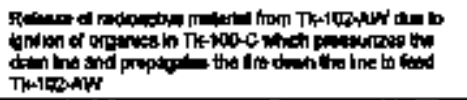 & 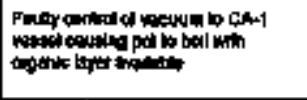 & \\
\hline
\end{tabular}




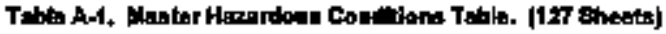

\begin{tabular}{|c|c|c|c|c|}
\hline $\mathbf{p}$ & 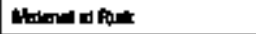 & Hera ribux Cendidon & const & 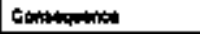 \\
\hline 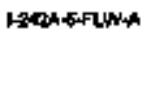 & wh & 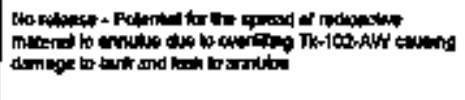 & 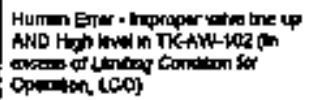 & \\
\hline $1.24246-C$ & 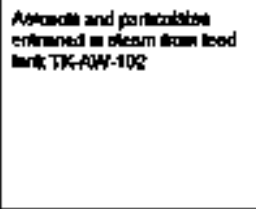 & 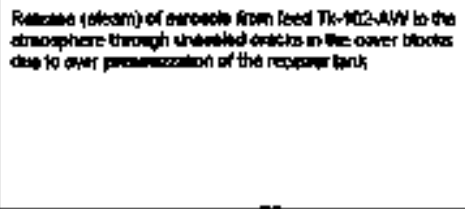 & 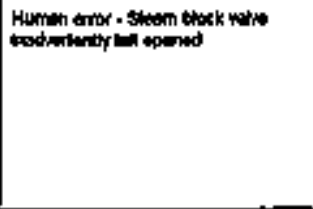 & 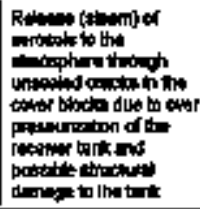 \\
\hline 1-262A-6-FLWA & 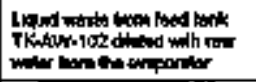 & 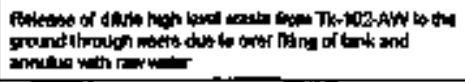 & 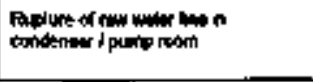 & \\
\hline $1-2424$ - & 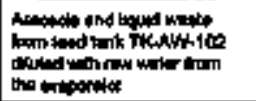 & 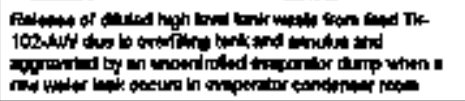 & 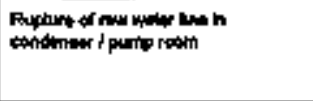 & \\
\hline 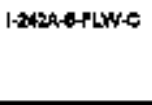 & 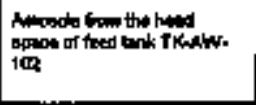 & 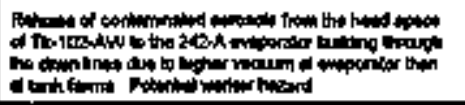 & 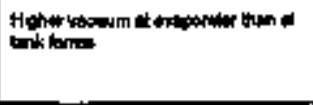 & \\
\hline hechingaph & 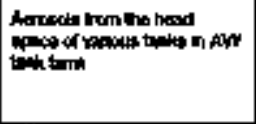 & 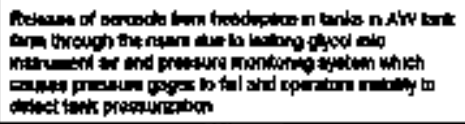 & 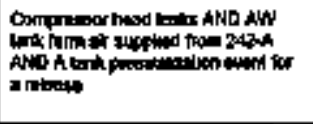 & \\
\hline 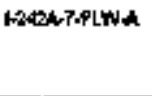 & 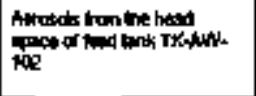 & 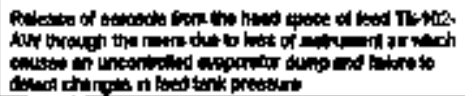 & 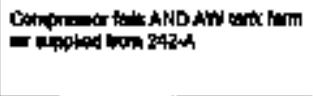 & \\
\hline
\end{tabular}




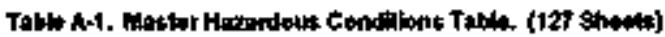

\begin{tabular}{|c|c|c|c|c|}
\hline $\mathbf{D}$ & 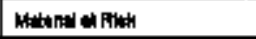 & 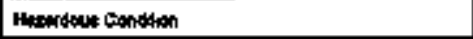 & Cana & consequance \\
\hline 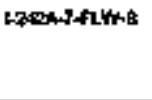 & 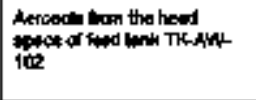 & 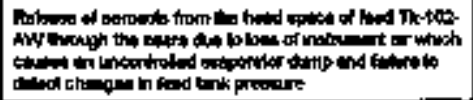 & 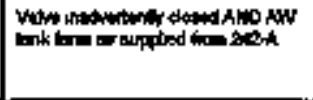 & \\
\hline 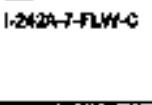 & 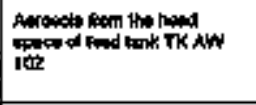 & 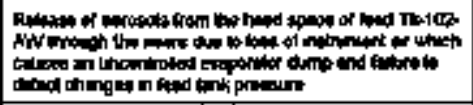 & 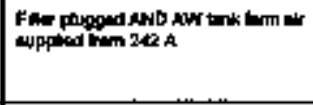 & \\
\hline $1-2624-7+2 \pi 3 x$ & Nh & 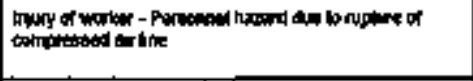 & 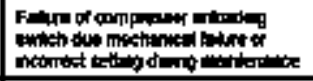 & \\
\hline $12029-1+P R B A$ & neconols hom 20-8 & 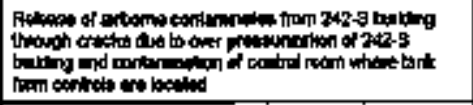 & 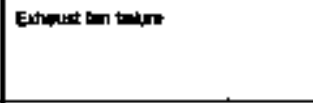 & \\
\hline 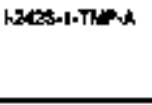 & 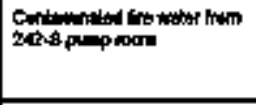 & 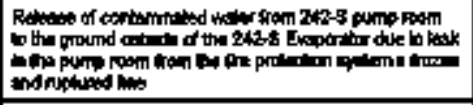 & 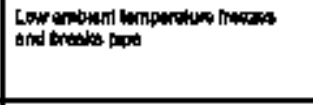 & \\
\hline 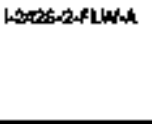 & 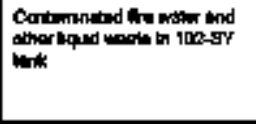 & 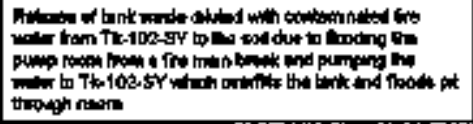 & Break in mo mant & \\
\hline+2 tos-2-PLW-A & 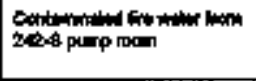 & 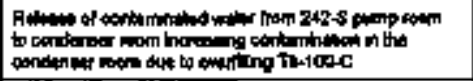 & 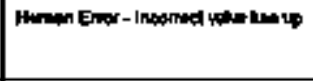 & \\
\hline WHar2.FLW & 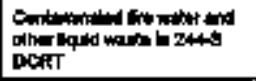 & 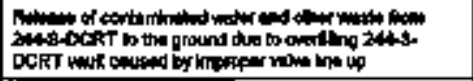 & 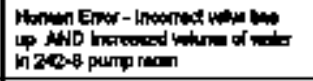 & \\
\hline Nateszintind & $\lim _{\text {ind }}$ & 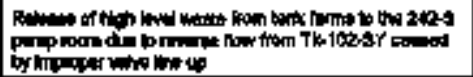 & 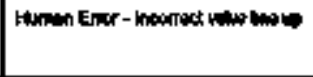 & \\
\hline
\end{tabular}


Table A-1. Marter Huxadous Conditions Thbly. (127 shoots)

\begin{tabular}{|c|c|c|c|c|}
\hline 10 & Bumbet Pent & 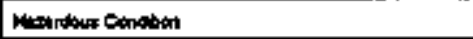 & Cwan & consmpintos \\
\hline 1-248:-2.FLWE & 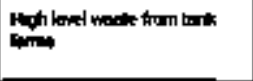 & 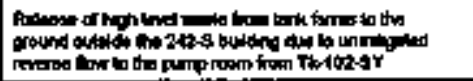 & 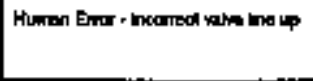 & \\
\hline 1-2438010X4 & 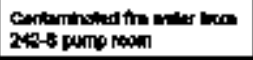 & 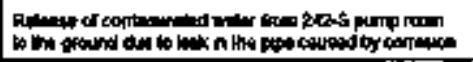 & 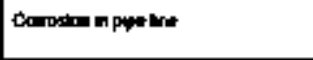 & \\
\hline $1-202-2-1 E K a$ & 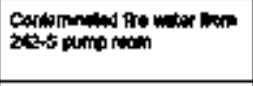 & 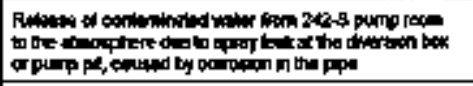 & 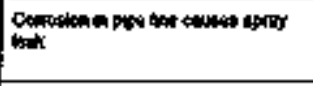 & \\
\hline M2ES-2-1EK-C & 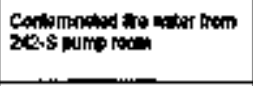 & 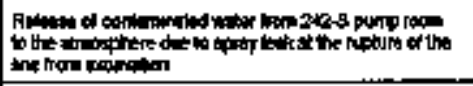 & 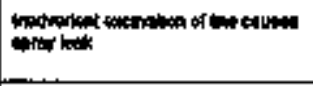 & \\
\hline 12ES-2HEK-O & 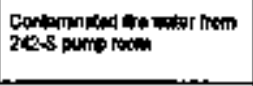 & 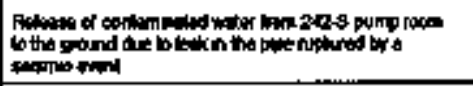 & Extrounds & \\
\hline 1-2RE-ZUEK-E & 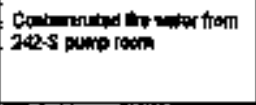 & 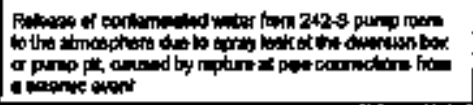 & Ematomis & \\
\hline H-2W2-4A1WA & 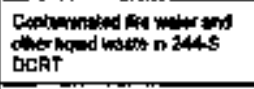 & 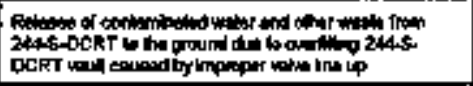 & 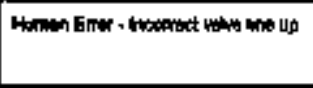 & \\
\hline $1-2625-47 W-B$ & 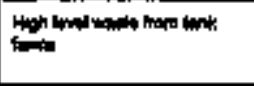 & 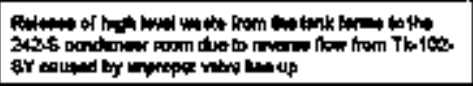 & 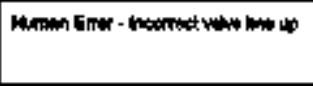 & \\
\hline $1-20 \leq-47 w 6$ & 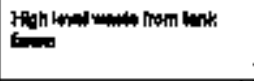 & 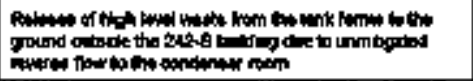 & 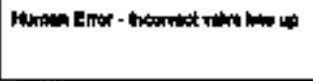 & \\
\hline$B A B+P L W-0$ & 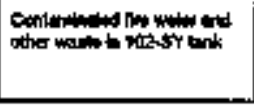 & 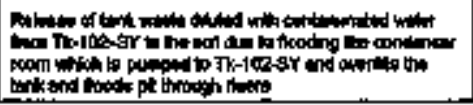 & 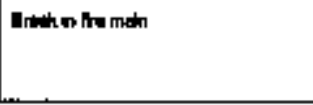 & \\
\hline
\end{tabular}




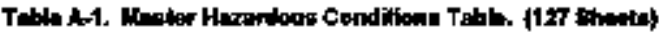

\begin{tabular}{|c|c|c|c|c|}
\hline 10 & 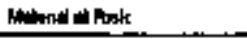 & A & Cono & 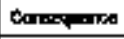 \\
\hline 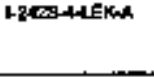 & 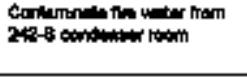 & 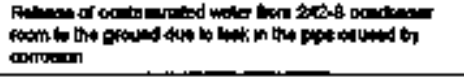 & 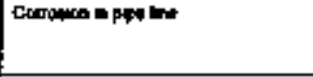 & \\
\hline 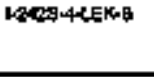 & 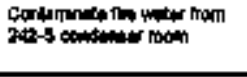 & 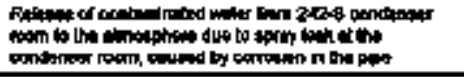 & 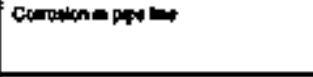 & \\
\hline 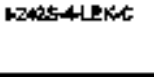 & 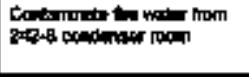 & 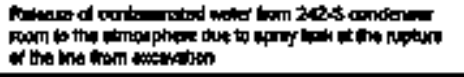 & 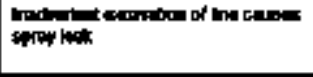 & \\
\hline L2425-4LEKO & 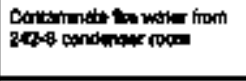 & 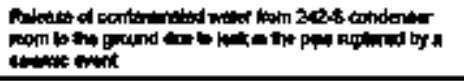 & exthquale & \\
\hline l.20estherete & 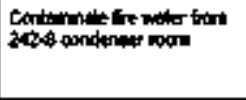 & 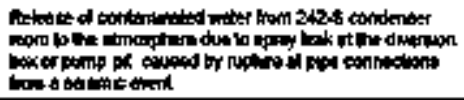 & 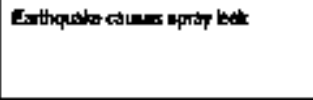 & \\
\hline lotatsonst & 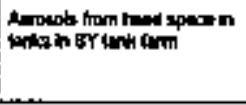 & 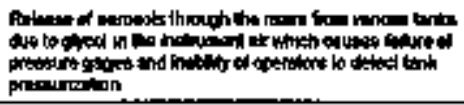 & 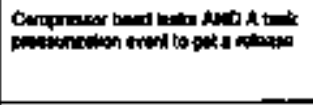 & \\
\hline $1-200-5006$ & 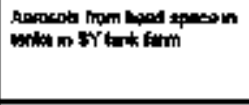 & 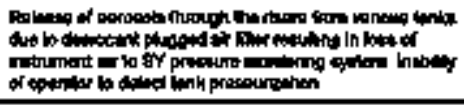 & 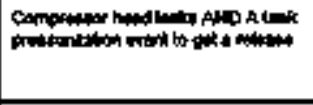 & \\
\hline 12*2s-capcc & 的 & 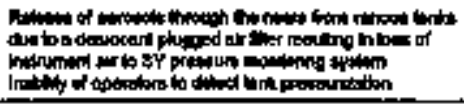 & 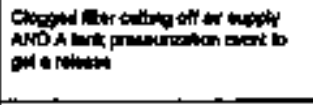 & \\
\hline 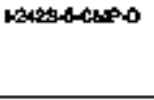 & 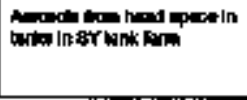 & 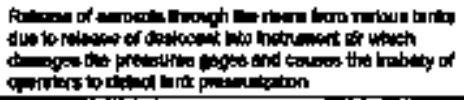 & 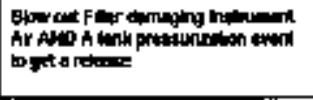 & \\
\hline
\end{tabular}




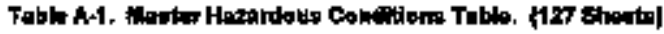

\begin{tabular}{|c|c|c|c|c|}
\hline 10 & 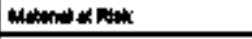 & Handens Comolum & Chat & 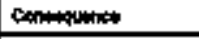 \\
\hline 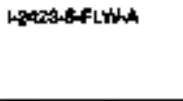 & 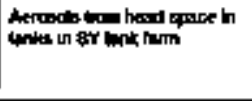 & 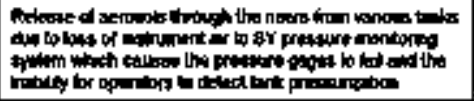 & 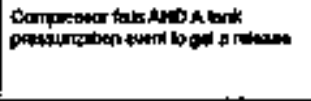 & \\
\hline $1-206-5+215-8$ & 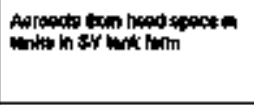 & 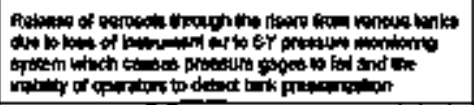 & 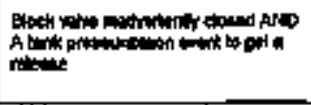 & \\
\hline L2095-71W-C & 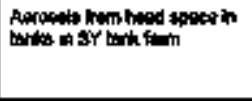 & 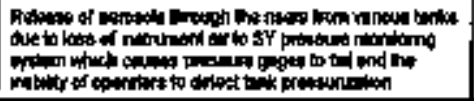 & 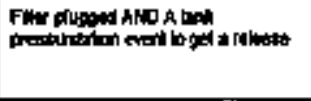 & \\
\hline 1.2425spikson & $\mathrm{me}$ & 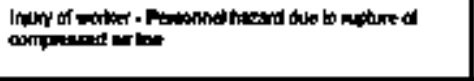 & 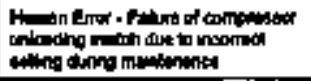 & \\
\hline 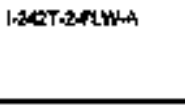 & 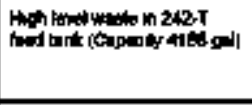 & 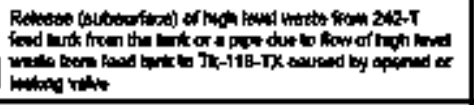 & 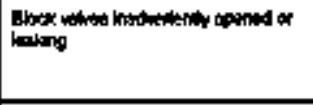 & 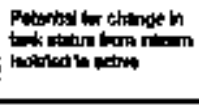 \\
\hline 1.2012T-3.FLVA & 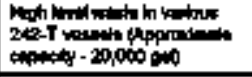 & 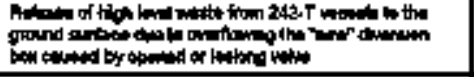 & 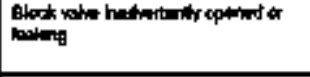 & \\
\hline latedtafikth & 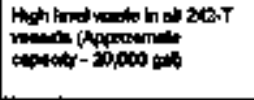 & 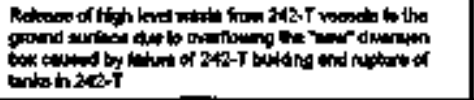 & 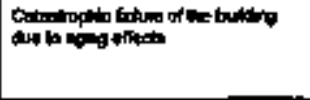 & \\
\hline IBPUANT-1-CANPA & 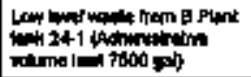 & 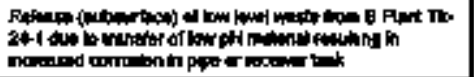 & 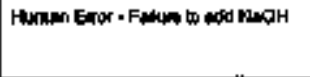 & \\
\hline 16PLAMT-1-CMPB & 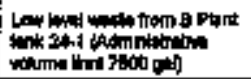 & 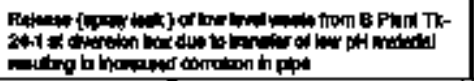 & 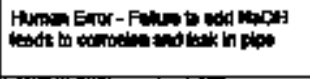 & \\
\hline
\end{tabular}




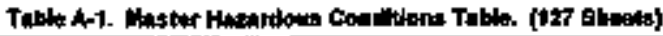

\begin{tabular}{|c|c|c|c|c|}
\hline $\mathbf{D}$ & 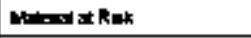 & 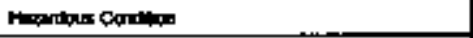 & Const & Comegrater \\
\hline 1-6PLANT-1-GM-C & 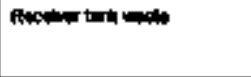 & 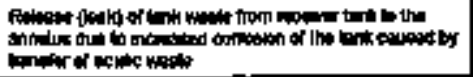 & 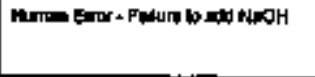 & \\
\hline IEPLNMT-1-CAMP-D & 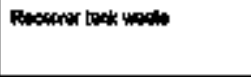 & 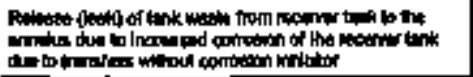 & 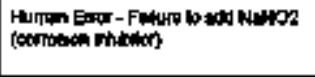 & \\
\hline HERANT-1 GUPE: & 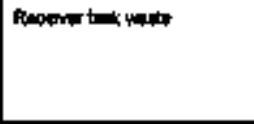 & 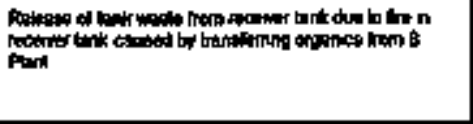 & 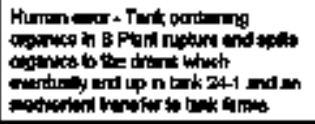 & \\
\hline LBPCANT-1-THPA & 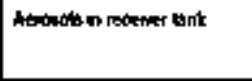 & 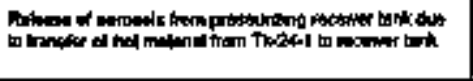 & 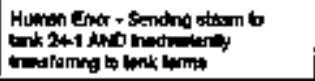 & \\
\hline CBPLANTSAWA & m & 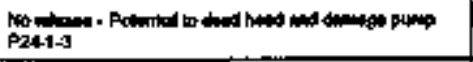 & 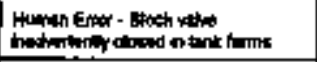 & \\
\hline 1-BPYNTI-2-FWW-B & 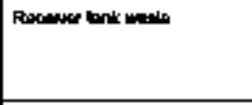 & 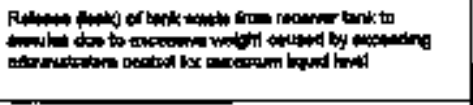 & 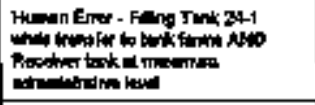 & \\
\hline 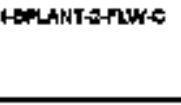 & $R=$ & 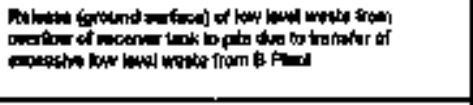 & 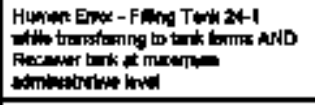 & \\
\hline 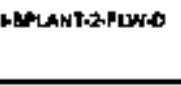 & Anomer bit wots & 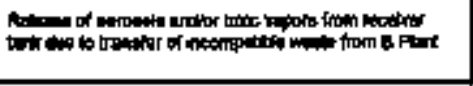 & 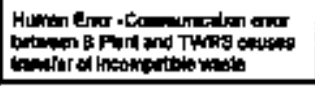 & \\
\hline LAPUAmT240KA & 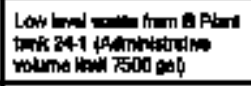 & 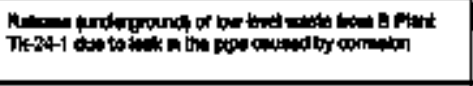 & 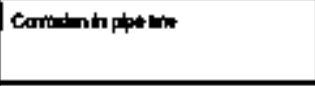 & \\
\hline LEPUATREKG: & 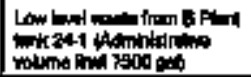 & 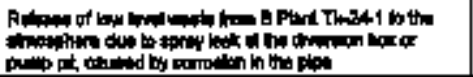 & 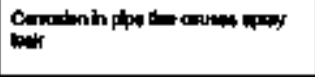 & \\
\hline
\end{tabular}




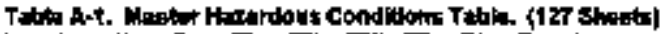

\begin{tabular}{|c|c|c|c|c|}
\hline $\boldsymbol{E}$ & 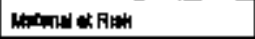 & Merrotia Conimin & $\cos$ & consequnes \\
\hline 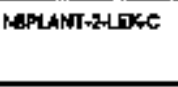 & 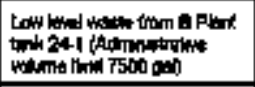 & 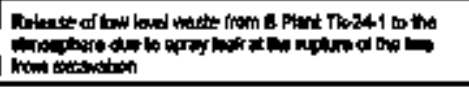 & 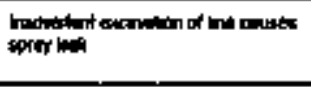 & \\
\hline LAPLANT-2LED & 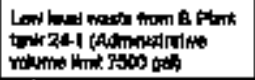 & 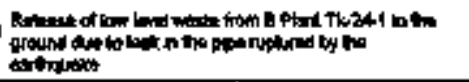 & 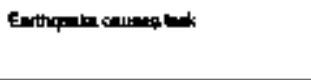 & \\
\hline IEPLWT2LLEE & 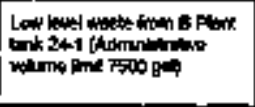 & 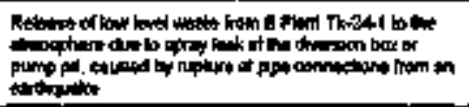 & 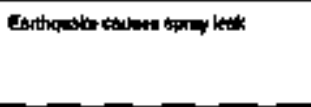 & \\
\hline IPFPI-AGTA & 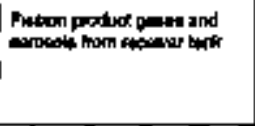 & 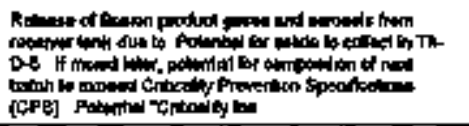 & 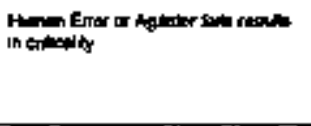 & \\
\hline HPF-A-CMP-n & 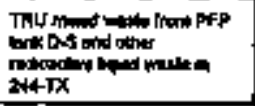 & 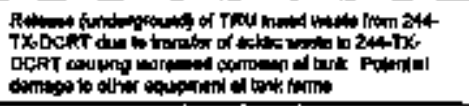 & 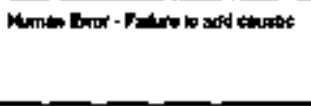 & \\
\hline 1PFP.1-CAPas & 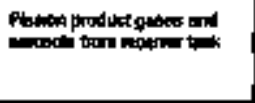 & 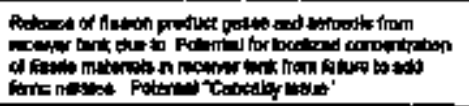 & 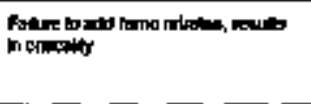 & \\
\hline IPFPS1-GAPS & 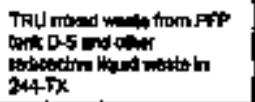 & 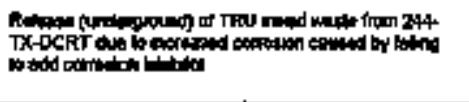 & 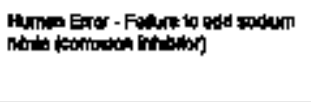 & \\
\hline 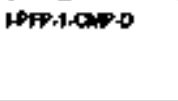 & Fation product & 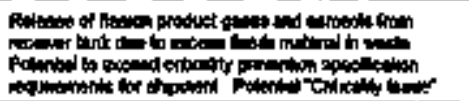 & 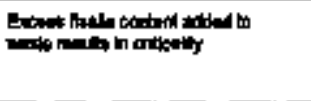 & \\
\hline IPPA ACWPE & 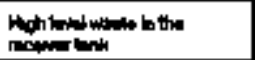 & 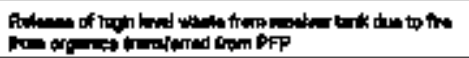 & 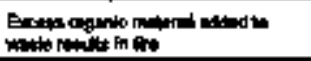 & \\
\hline
\end{tabular}




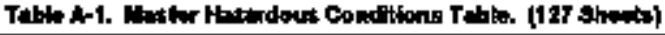

\begin{tabular}{|c|c|c|c|c|}
\hline ID & Henentit ok Plat & 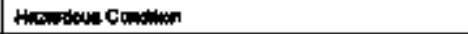 & count & Conowipart \\
\hline |APPA-1+LVLA & $\begin{array}{l}\text { TRL matid wade from FFP } \\
\text { teit D-S }\end{array}$ & 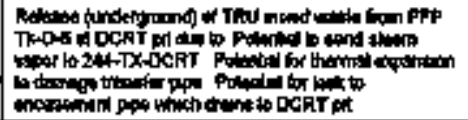 & 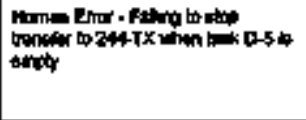 & \\
\hline LPFP. 140mkth & 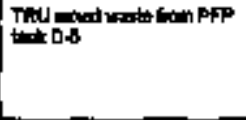 & 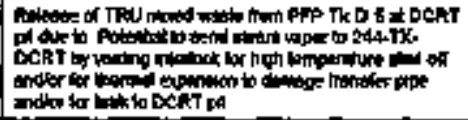 & 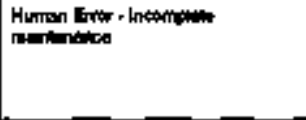 & \\
\hline 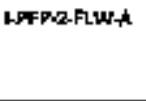 & 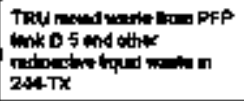 & 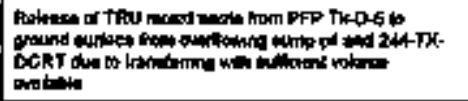 & 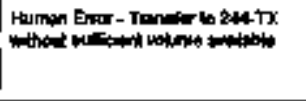 & \\
\hline LPFP-2-LEKA & 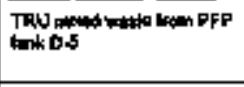 & 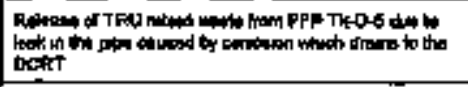 & Compon in pios ine & \\
\hline LPFAZLEKA & 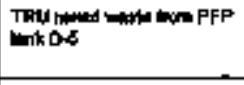 & 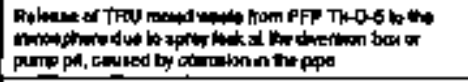 & 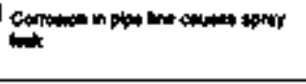 & \\
\hline IPPPQLEKCC & 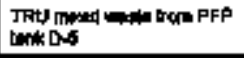 & 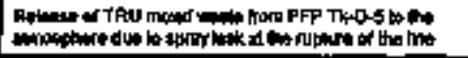 & 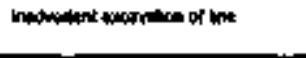 & \\
\hline 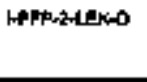 & 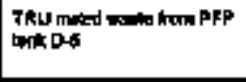 & 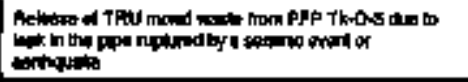 & Barthquare & \\
\hline APPA2HEKE & 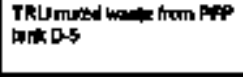 & 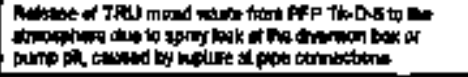 & 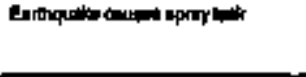 & \\
\hline APFP,2-n!Path & 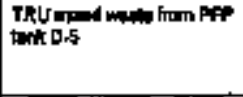 & 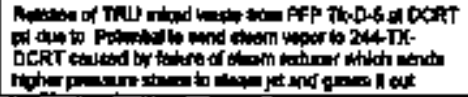 & 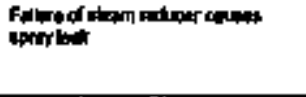 & 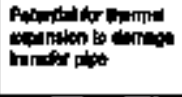 \\
\hline
\end{tabular}




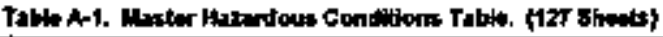

\begin{tabular}{|c|c|c|c|c|}
\hline ID & 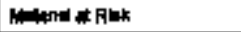 & 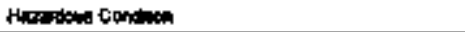 & Can & 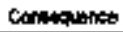 \\
\hline HPR-2TUPA & 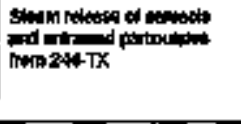 & 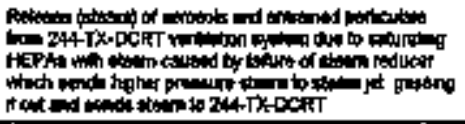 & R- & \\
\hline 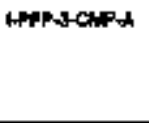 & 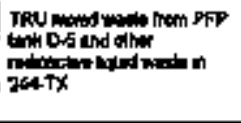 & 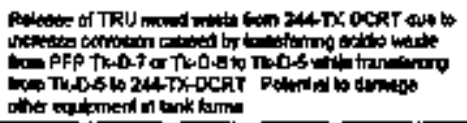 & 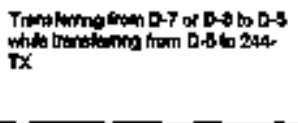 & \\
\hline MPFP-3.FWA & 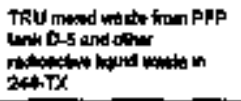 & 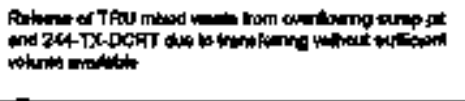 & 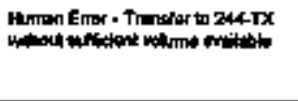 & \\
\hline IPEP-\&FLW-C & 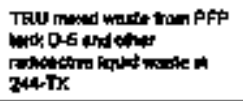 & 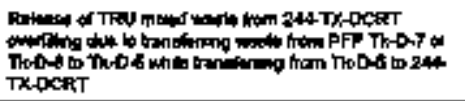 & 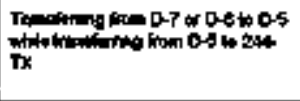 & \\
\hline IPFP-SUECh & 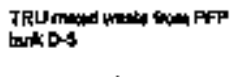 & 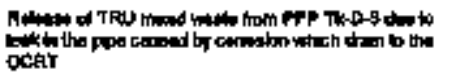 & 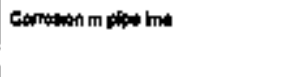 & \\
\hline HPFP-\$UEK日 & 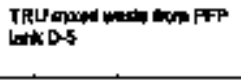 & 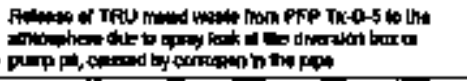 & 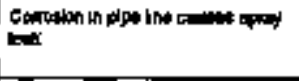 & \\
\hline LFFP-HEKGC & 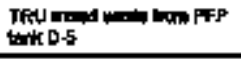 & 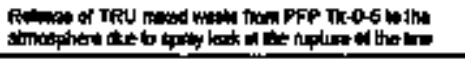 & 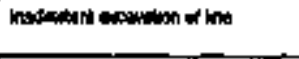 & \\
\hline APFPUJLEKD & 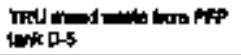 & 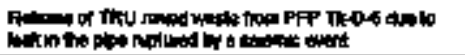 & Eatthquan & \\
\hline APPP-31EKGE & 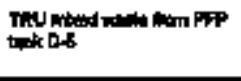 & 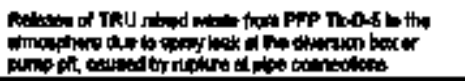 & 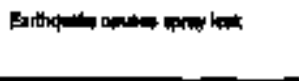 & \\
\hline
\end{tabular}


Table A-1, Matwr Handidous Condfions Tabl. (127 thents)

\begin{tabular}{|c|c|c|c|c|}
\hline 10 & Wt: & Mesartow Conditen & Gousp & Consequence \\
\hline Methex-1-NoTh & 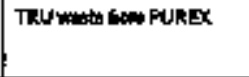 & 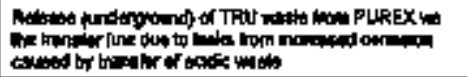 & 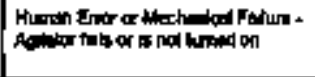 & \\
\hline |AIMEX14GT: & TRU/m furex & 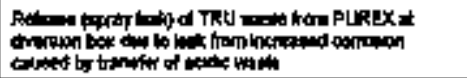 & 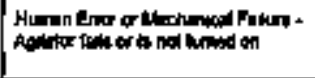 & \\
\hline IFIPEX-1AOT-S & $\mathrm{Mh}$ & 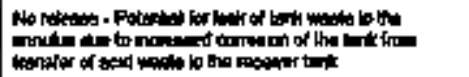 & 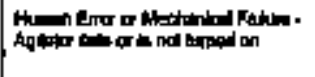 & \\
\hline 1FUREX-1-CKMP & TRU wow hom PUAdex & 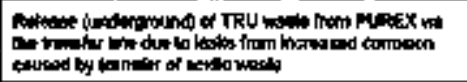 & 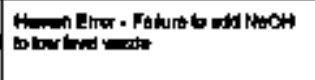 & \\
\hline 1-PUR $3 x-1-C 100$ & TRU wath hom PUPex & 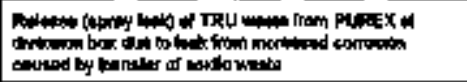 & 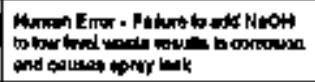 & \\
\hline HPURECA-CUP-s & $\mathrm{m}$ & 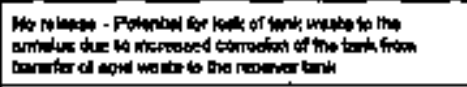 & 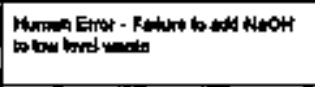 & \\
\hline LPUREK-1-DPP.D & Nh & 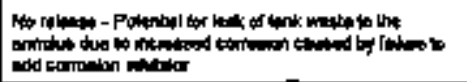 & 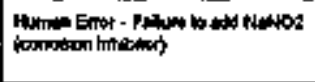 & \\
\hline 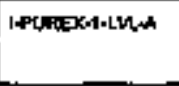 & eproseds mam bonk NOSAVr & 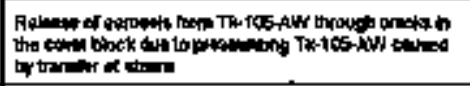 & 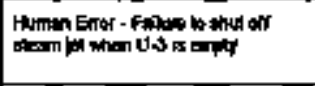 & \\
\hline HPUNEX-1-TMPA & 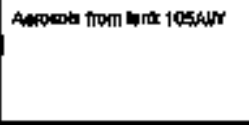 & 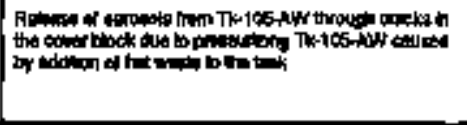 & 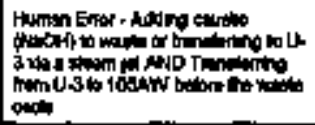 & \\
\hline 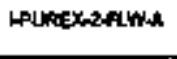 & 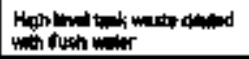 & 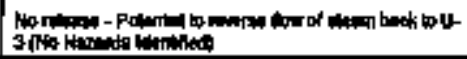 & 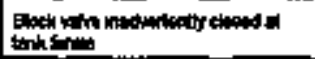 & \\
\hline
\end{tabular}




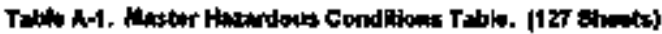

\begin{tabular}{|c|c|c|c|c|}
\hline I & Mrterid Rak & 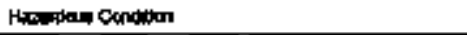 & Cown & Corntisones \\
\hline IFURESA2.1EKA & 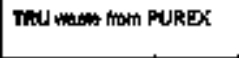 & 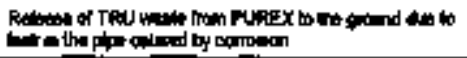 & 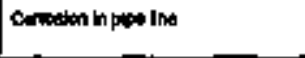 & \\
\hline 1DUFEX-2-LEK-D & TRU WRAW ITOM PUREX & 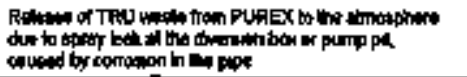 & 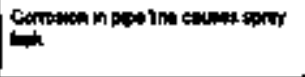 & \\
\hline 1FUREX-2AEKEC & 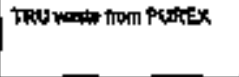 & 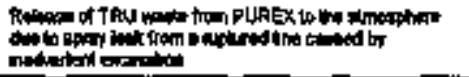 & 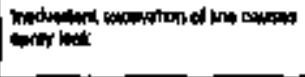 & \\
\hline 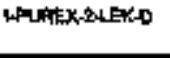 & 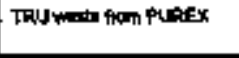 & 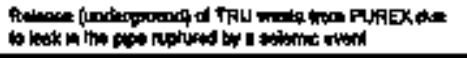 & 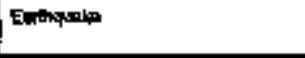 & \\
\hline 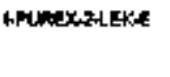 & TRU & 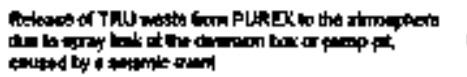 & 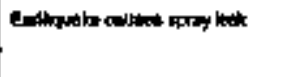 & \\
\hline 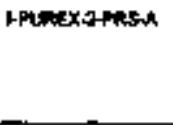 & 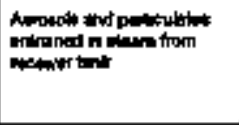 & 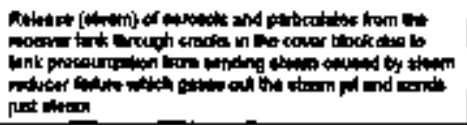 & 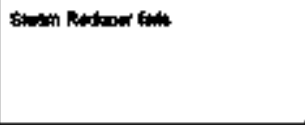 & \\
\hline LPUTEX-2-FFR-B & 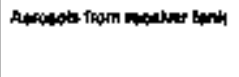 & 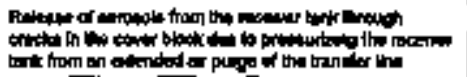 & 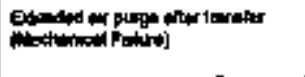 & \\
\hline HUREXAFFR-C & NAh & 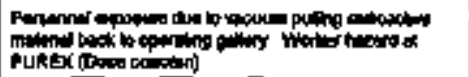 & 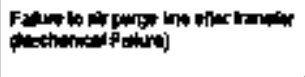 & \\
\hline IPUPEX-3ACTA & TRU Waik hon PUREX & 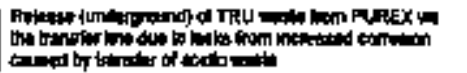 & 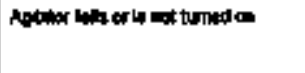 & \\
\hline 1मUAEX-3A4T-B & TRU wim hom PUREX & 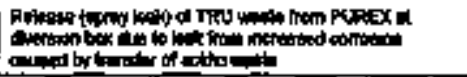 & 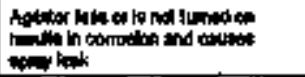 & \\
\hline
\end{tabular}




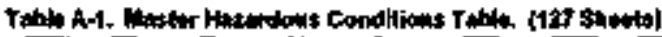

\begin{tabular}{|c|c|c|c|c|}
\hline סו & 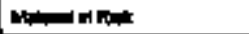 & 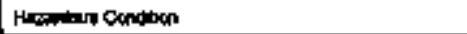 & can & Conswinest \\
\hline IPUAEESAAGTC & NA & 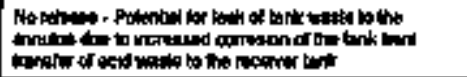 & 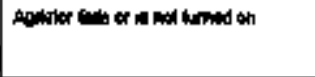 & \\
\hline IFUREX-3-Chenth & Trid wate & 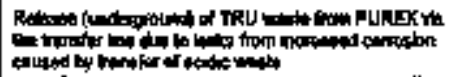 & 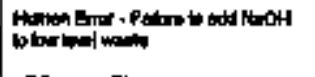 & \\
\hline HPUREX-30Am-8 & TRU masp tom PLAEX & 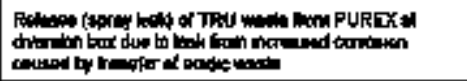 & 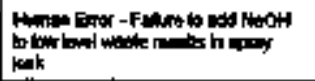 & \\
\hline HPUREX-3apec & man & 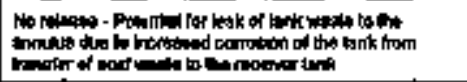 & 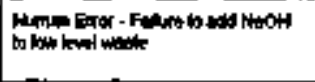 & \\
\hline HRUREX3000 & $\mathbf{m a}$ & 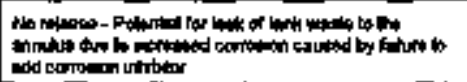 & 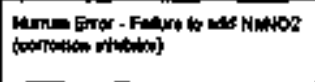 & \\
\hline LHUREX_-CNPE & Foren prodxt gaves ind & 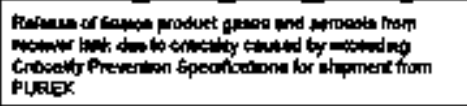 & 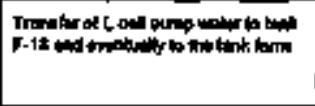 & \\
\hline 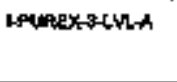 & 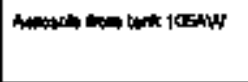 & 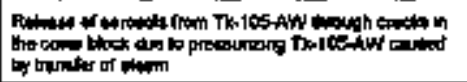 & 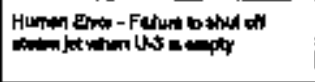 & \\
\hline 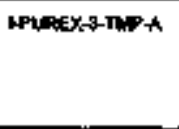 & 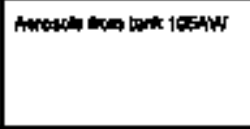 & 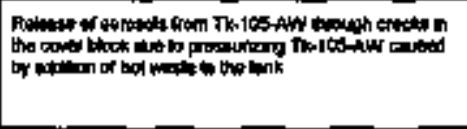 & 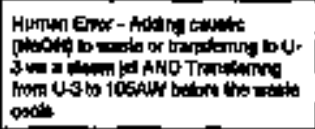 & \\
\hline |.PUREX-ARUW-B & 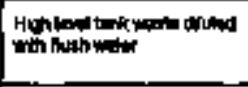 & 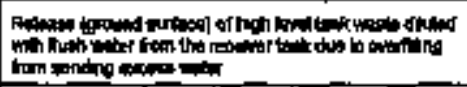 & 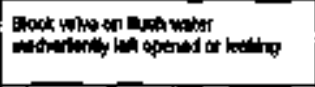 & \\
\hline LPUAEX-LLEXA & ThU wast trom PUNAEX & 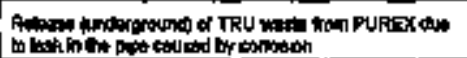 & Conotion nopa un & \\
\hline
\end{tabular}




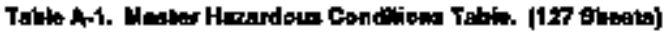

\begin{tabular}{|c|c|c|c|c|}
\hline 10 & Wrimal at Pow & 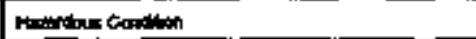 & Count & Defosquentod \\
\hline 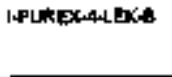 & TKL $\Rightarrow$ hom PUwax & 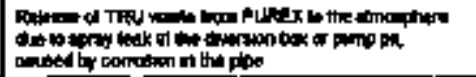 & 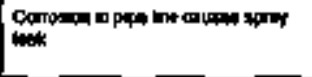 & \\
\hline 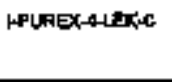 & 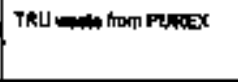 & 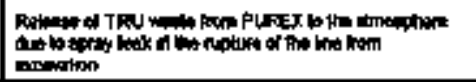 & 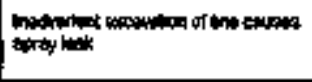 & \\
\hline HMIREKALEKO & 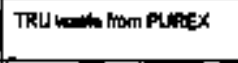 & 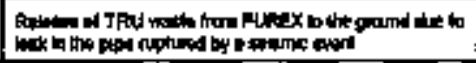 & 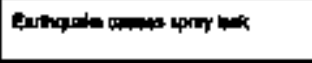 & \\
\hline 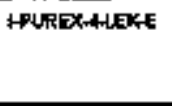 & 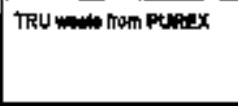 & 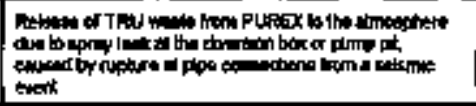 & 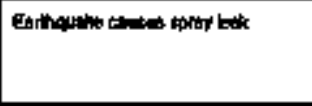 & \\
\hline IPURDCAPRSA & 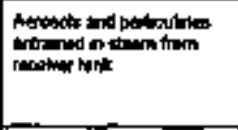 & 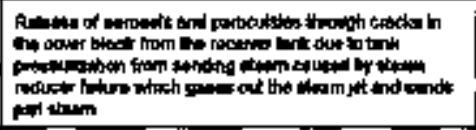 & banghenom & \\
\hline LPUEX A PRg & 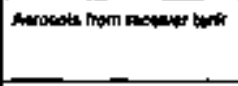 & 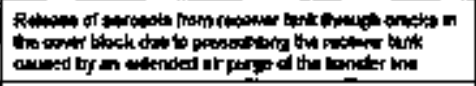 & 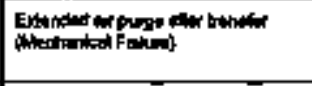 & \\
\hline IPUEEX_PRGC & ma & 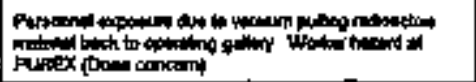 & 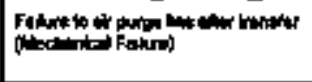 & \\
\hline 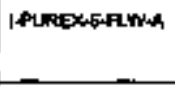 & 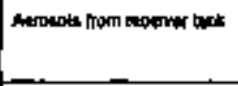 & 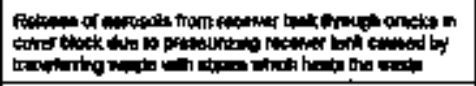 & 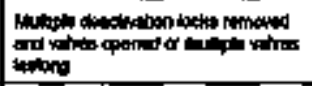 & \\
\hline HPLFEX-S-RWB & ה & 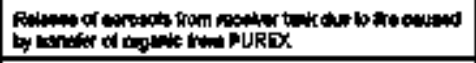 & 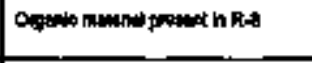 & \\
\hline 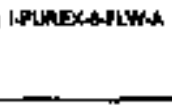 & 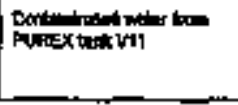 & 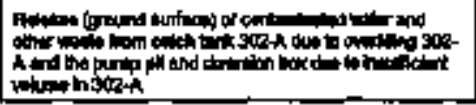 & 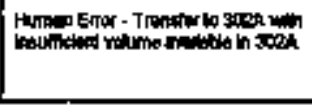 & \\
\hline
\end{tabular}




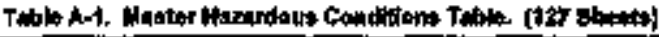

\begin{tabular}{|c|c|c|c|c|}
\hline 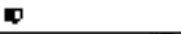 & 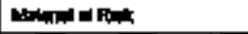 & Merantione tandwan & Gian & carequane \\
\hline HUREXEALW-8 & 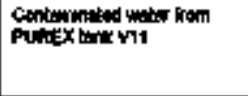 & 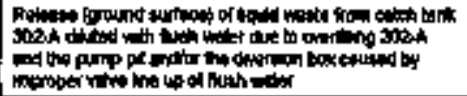 & 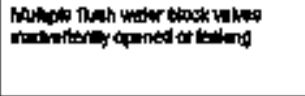 & \\
\hline LPUAEX-ANWW & 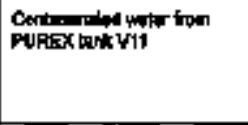 & 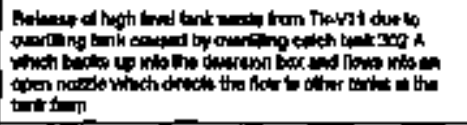 & 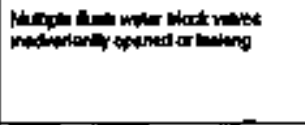 & \\
\hline 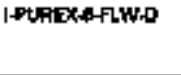 & $M 4$ & 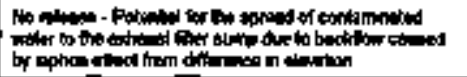 & 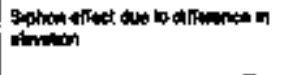 & \\
\hline LTRANTT-1-CUNA & 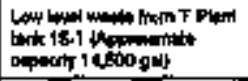 & 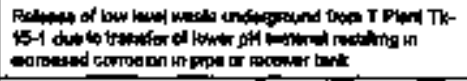 & 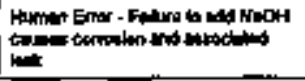 & \\
\hline I-TPLANT-1-CLPG & 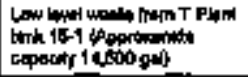 & 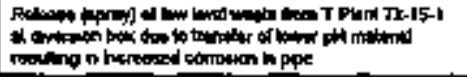 & 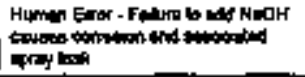 & \\
\hline 1.TPLANT-1-CTPCA & 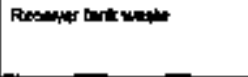 & 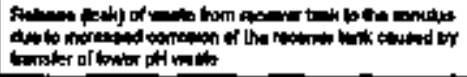 & 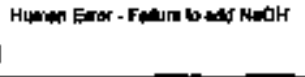 & \\
\hline 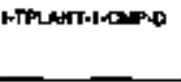 & 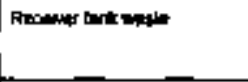 & 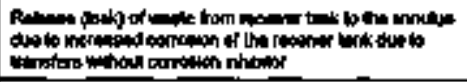 & 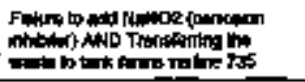 & \\
\hline ITPUNT-1-TMPA & 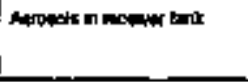 & 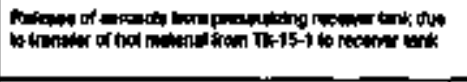 & 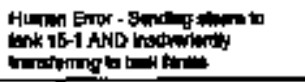 & \\
\hline ITPUAMT-20-A-A & 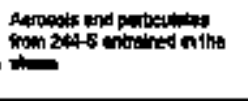 & 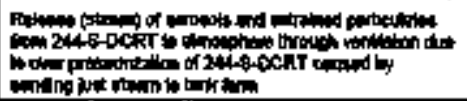 & 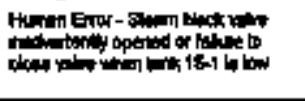 & \\
\hline
\end{tabular}




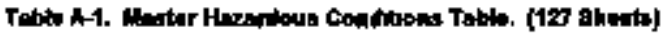

\begin{tabular}{|c|c|c|c|c|}
\hline D. & Hitum a Rt & 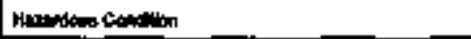 & Cuter & tangen बान \\
\hline 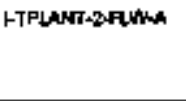 & Facan & 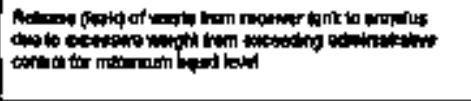 & 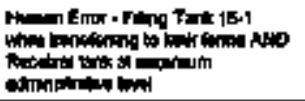 & \\
\hline LTPUAHTERLAHS & Pechar init wails & 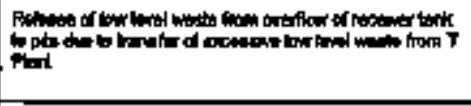 & 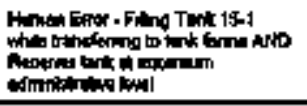 & \\
\hline ITPLANT-ZLEKA & 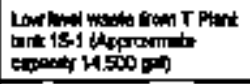 & 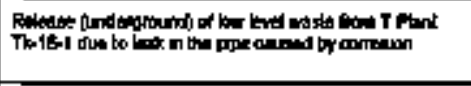 & 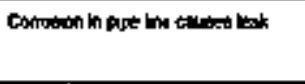 & \\
\hline ITPLANTEZLEKE & 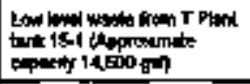 & 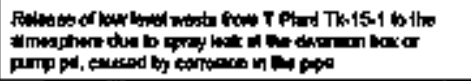 & 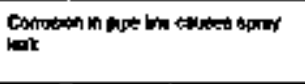 & \\
\hline RTPLAT-2LEKCC & 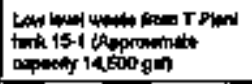 & 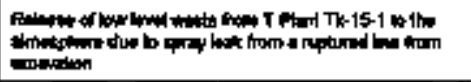 & 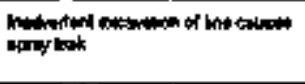 & \\
\hline LTPLAMT-2LEKO & 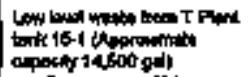 & 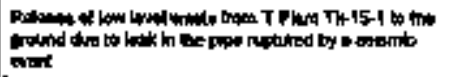 & 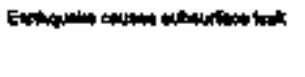 & \\
\hline HTRANT-2UEKEE & 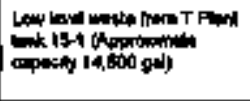 & 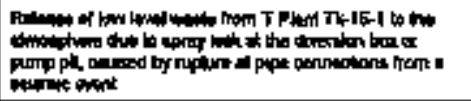 & 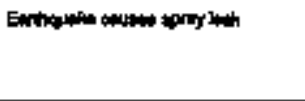 & \\
\hline HOLWWT-1.PLWA & 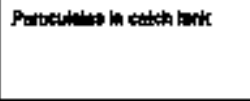 & 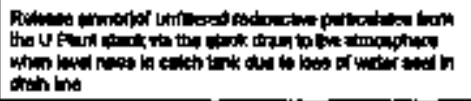 & 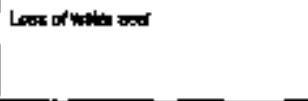 & \\
\hline LUPLAMT-14ERTh & 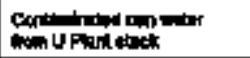 & 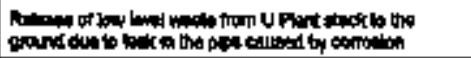 & Compationm pos he & \\
\hline HUPLAHT.1AEKE & 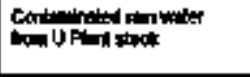 & 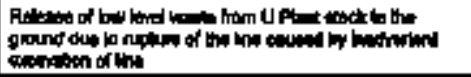 & 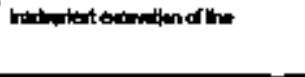 & \\
\hline
\end{tabular}




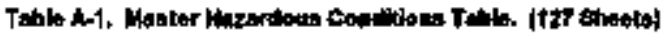

\begin{tabular}{|c|c|c|c|c|}
\hline ID & 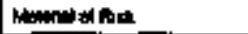 & 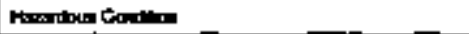 & Gabror & chrasopero: \\
\hline HMLNTSALEKCD & 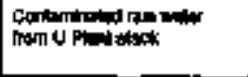 & 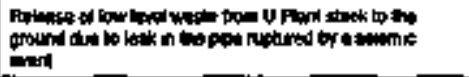 & Eirhopalos & \\
\hline 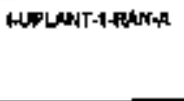 & 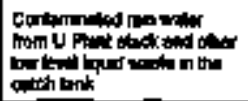 & 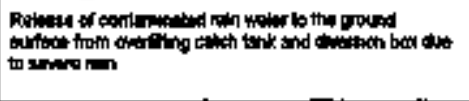 & Sanpo Ren & \\
\hline mKO1 & Tort himant & 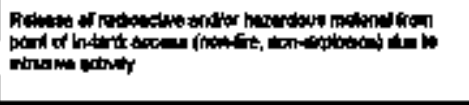 & 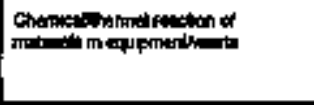 & 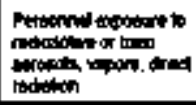 \\
\hline mKEMन & 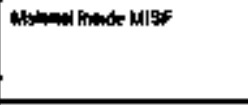 & 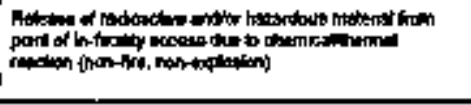 & 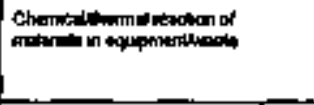 & 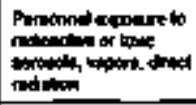 \\
\hline rTkan & 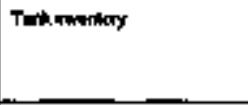 & 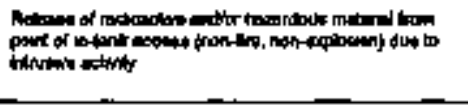 & 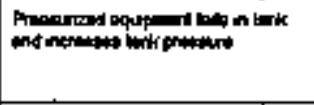 & 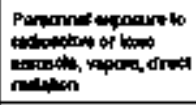 \\
\hline m<सR| & 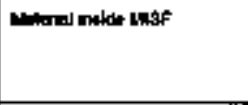 & 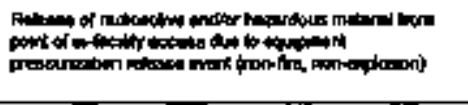 & 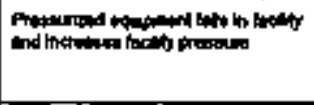 & 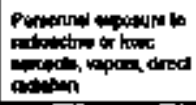 \\
\hline mk- & 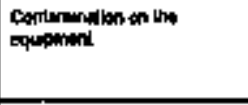 & 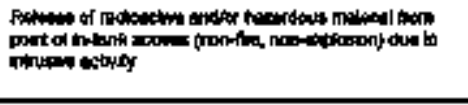 & 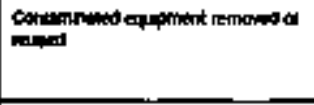 & 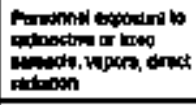 \\
\hline rthose-1 & 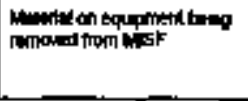 & 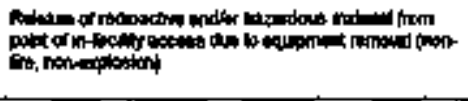 & 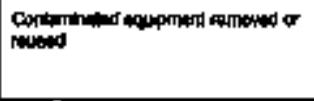 & 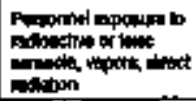 \\
\hline
\end{tabular}




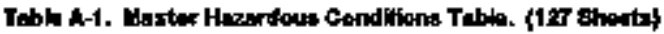

\begin{tabular}{|c|c|c|c|c|}
\hline $\mathbf{0}$ & 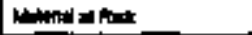 & Nenerdilu Condinon & 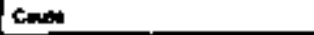 & 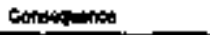 \\
\hline ImK0.04 & 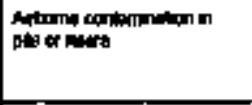 & 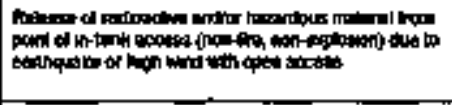 & 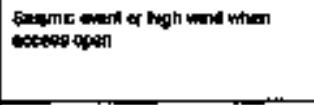 & 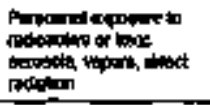 \\
\hline ITK-0.4 & 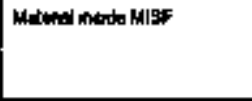 & 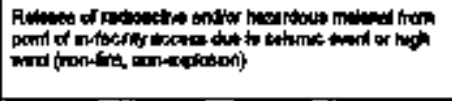 & 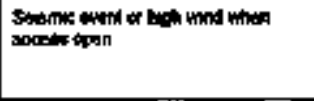 & 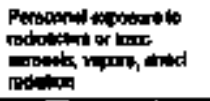 \\
\hline mKens & Trikbonteritior & 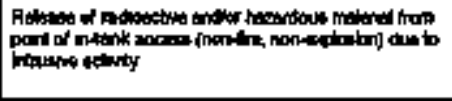 & 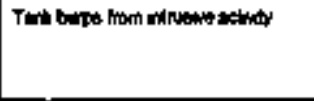 & 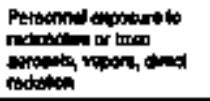 \\
\hline rTkased & 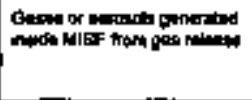 & 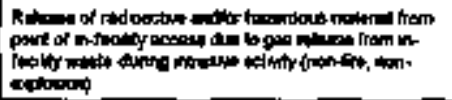 & 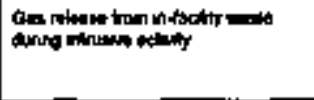 & 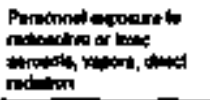 \\
\hline mK-at & 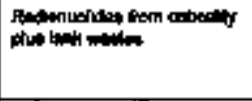 & 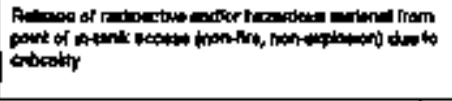 & 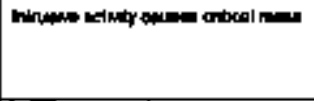 & 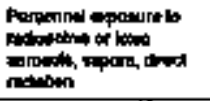 \\
\hline ITK-6⿴囗 & Huned math hegF & 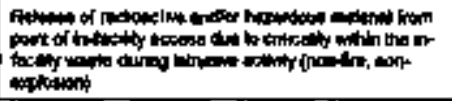 & 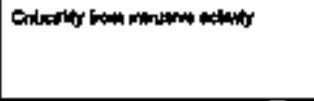 & 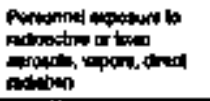 \\
\hline Ink-or & Tant Imantiony & 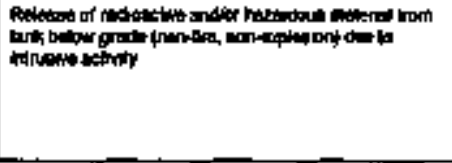 & 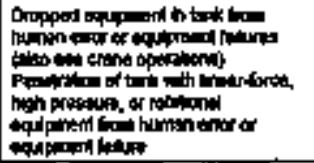 & 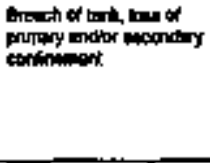 \\
\hline
\end{tabular}




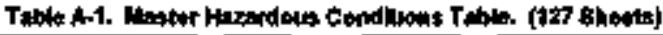

\begin{tabular}{|c|c|c|c|c|}
\hline $\mathbf{D}$ & NAm & 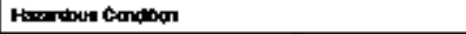 & Coust & contexputence \\
\hline InK-010 & 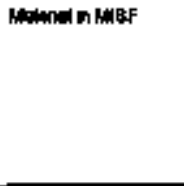 & 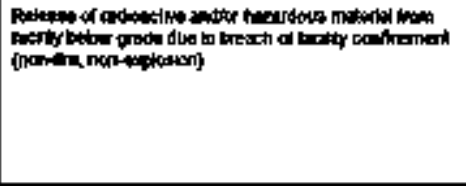 & 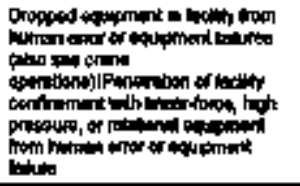 & 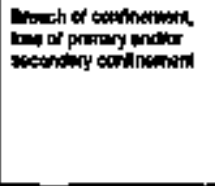 \\
\hline 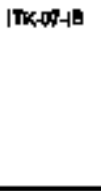 & M. & 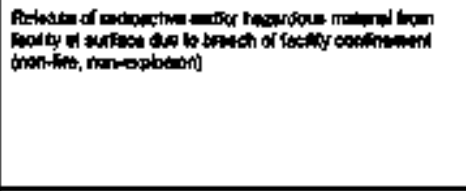 & 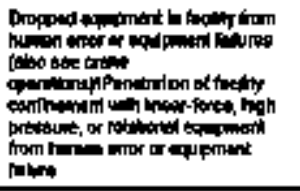 & 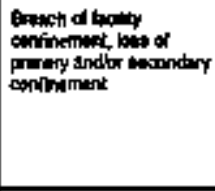 \\
\hline mkate & Tank Mmamint & 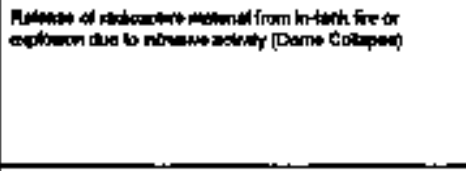 & 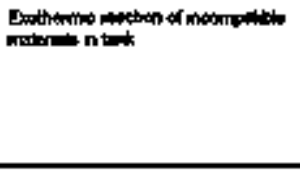 & 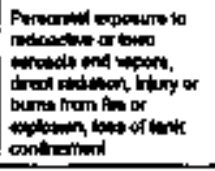 \\
\hline ITKas & 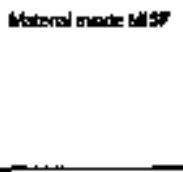 & 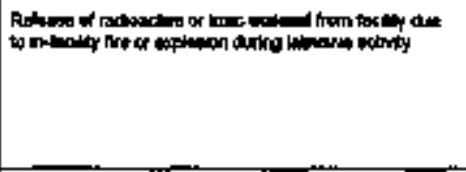 & 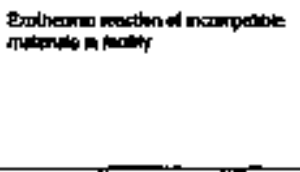 & 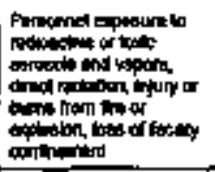 \\
\hline$\pi k-\infty$ & Tarik invionion & 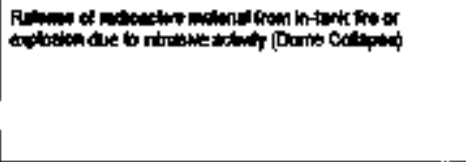 & 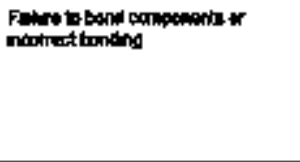 & 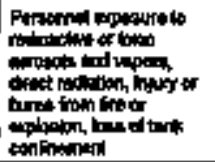 \\
\hline
\end{tabular}




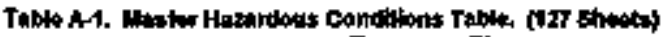

\begin{tabular}{|c|c|c|c|c|}
\hline ID & 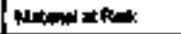 & 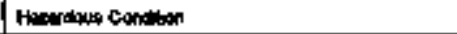 & coush & Conowipuant \\
\hline Intose- & 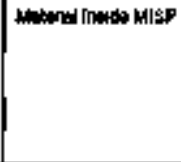 & 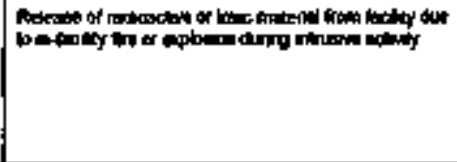 & 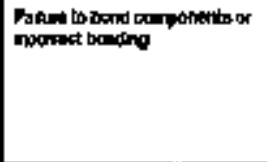 & 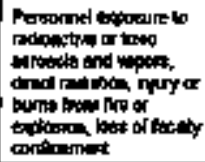 \\
\hline 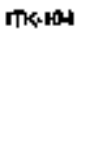 & 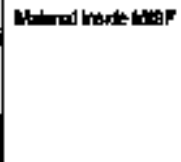 & 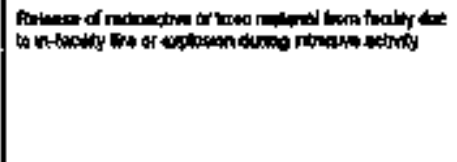 & 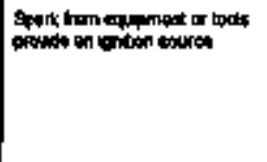 & 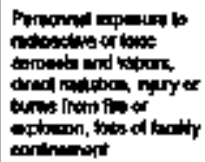 \\
\hline mk-10. & 8-9t trenk conterits & 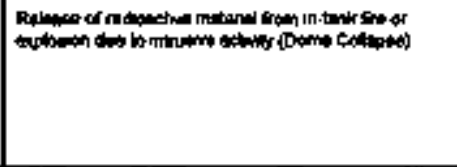 & 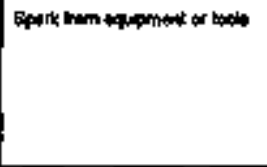 & 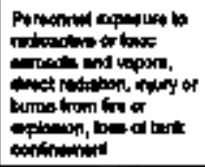 \\
\hline $\mid \pi k-10-6$ & 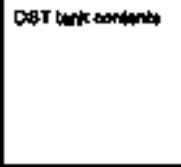 & 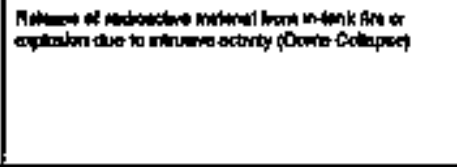 & 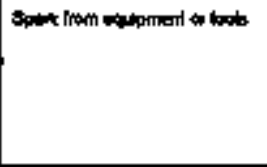 & 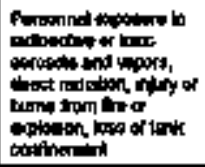 \\
\hline ITKGII & 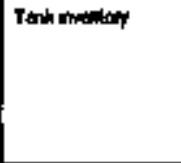 & 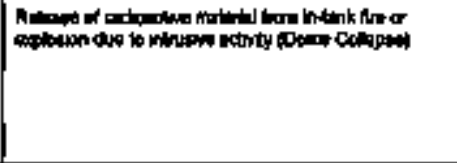 & 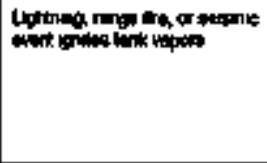 & 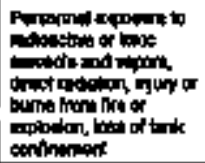 \\
\hline
\end{tabular}




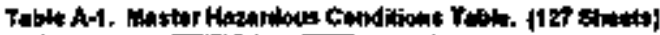

\begin{tabular}{|c|c|c|c|c|}
\hline ID & Nand and & Herartout Condtan & coning & Conquapuno \\
\hline Ink:II & 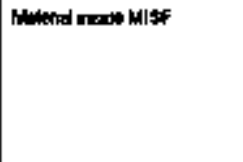 & 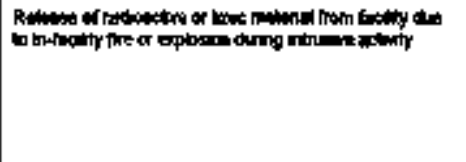 & 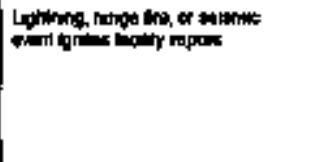 & 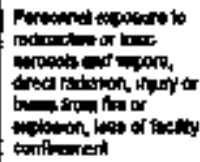 \\
\hline TKKFRAM & 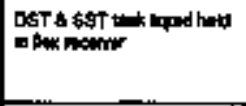 & 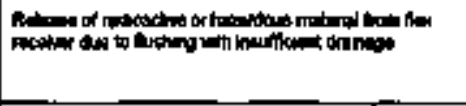 & 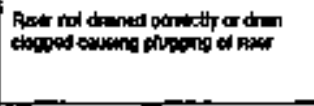 & 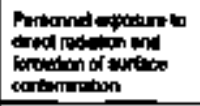 \\
\hline गास्सR-02 & 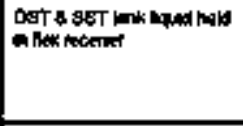 & 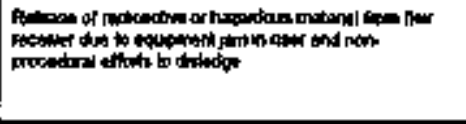 & 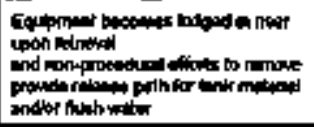 & 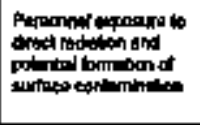 \\
\hline TTKFA- 69 & DET \& GST toli nmetony & 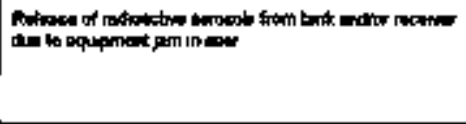 & 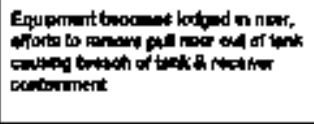 & 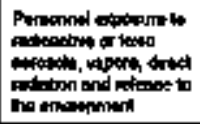 \\
\hline mK-FF-G4 & 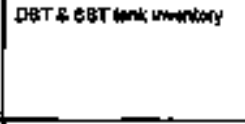 & 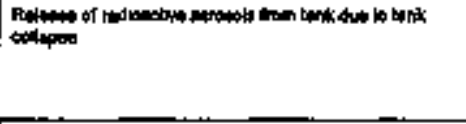 & 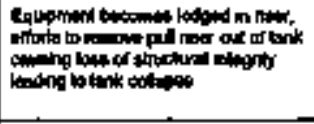 & 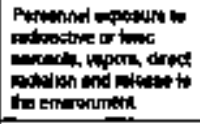 \\
\hline InKFR-CS & 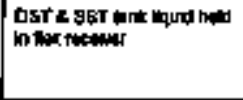 & 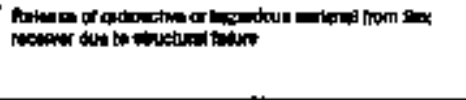 & 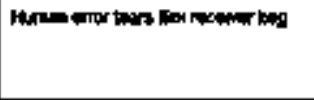 & 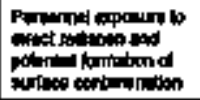 \\
\hline TIK+R-10 & 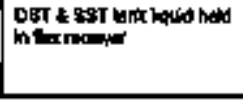 & 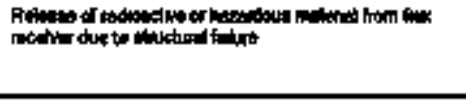 & 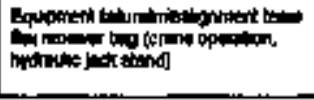 & 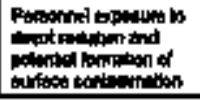 \\
\hline
\end{tabular}




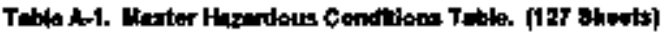

\begin{tabular}{|c|c|c|c|c|}
\hline ఐ & 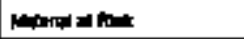 & Heanters Cindon & Cwan & Consonumes \\
\hline Irecher & 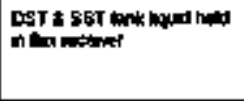 & 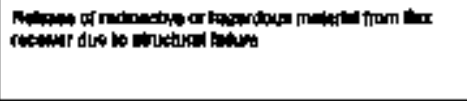 & 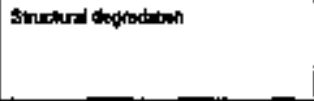 & 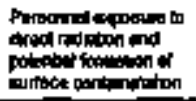 \\
\hline flkKR-09 & 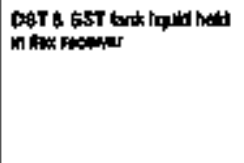 & 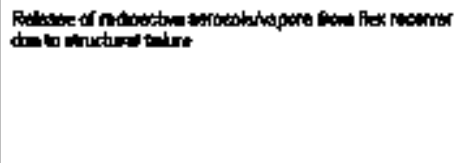 & 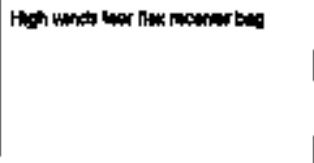 & 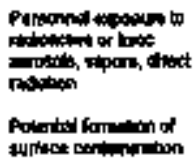 \\
\hline mk-re-0s & 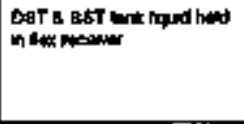 & 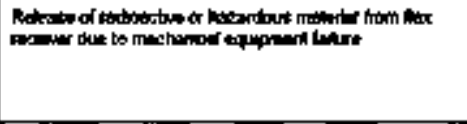 & 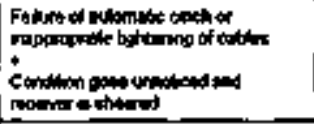 & 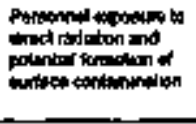 \\
\hline TKK.FRAS & 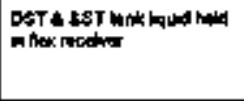 & 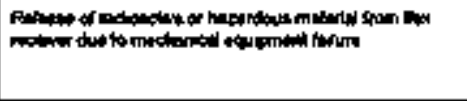 & 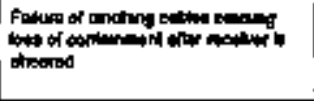 & 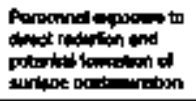 \\
\hline ITKGFR:11 & 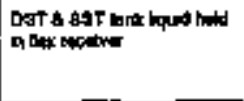 & 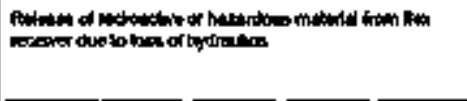 & 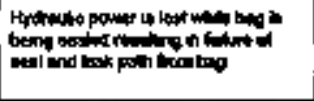 & 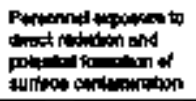 \\
\hline IEK-fR-12 & 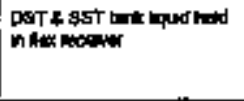 & 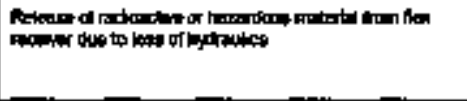 & 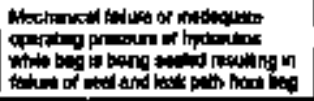 & 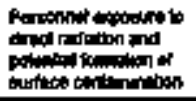 \\
\hline [TKA]R-15 & 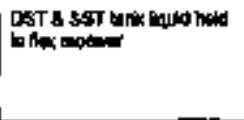 & 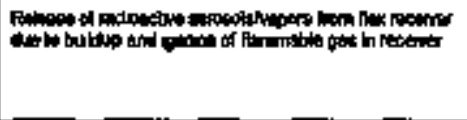 & 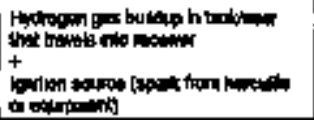 & 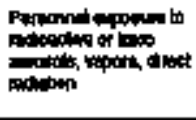 \\
\hline
\end{tabular}




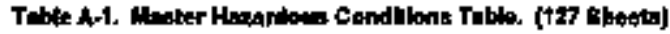

\begin{tabular}{|c|c|c|c|c|}
\hline 中 & Monnd a Aath & Nentendare tondan & oses & Cormequated \\
\hline mG-FP14 & 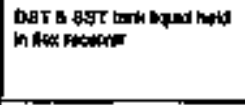 & 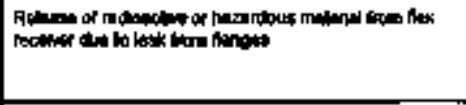 & 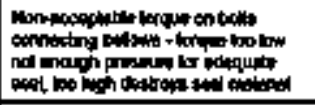 & 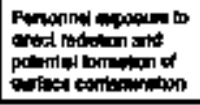 \\
\hline ITh-8R-13 & 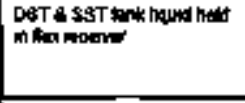 & 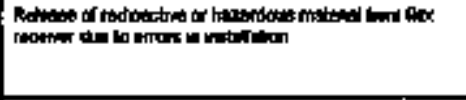 & 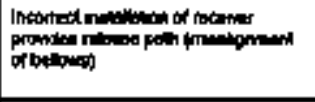 & 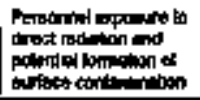 \\
\hline ITKAR-16 & 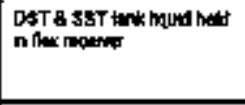 & 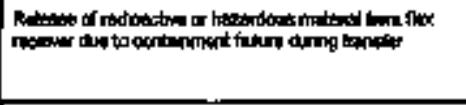 & 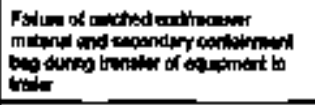 & 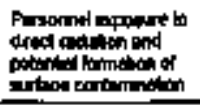 \\
\hline [nKfR-1] & 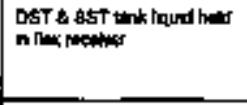 & 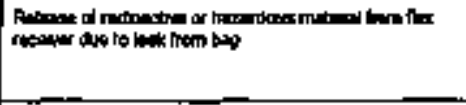 & 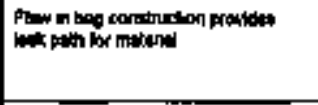 & 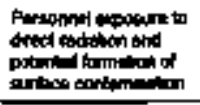 \\
\hline TKFR-18 & 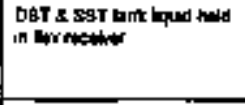 & 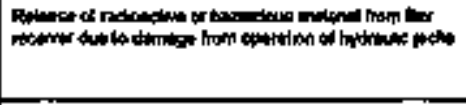 & 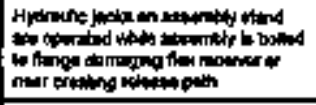 & 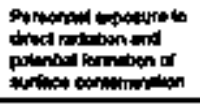 \\
\hline TKGrR-19 & 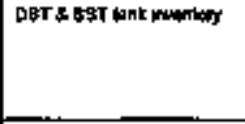 & 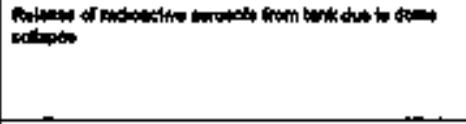 & 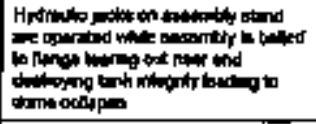 & 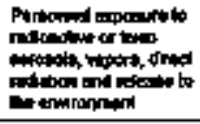 \\
\hline incFrad & 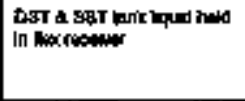 & 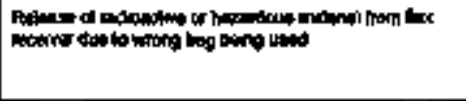 & 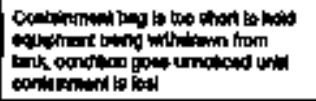 & 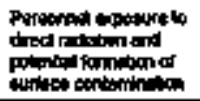 \\
\hline$|I K-f R-2|$ & 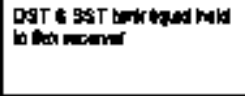 & 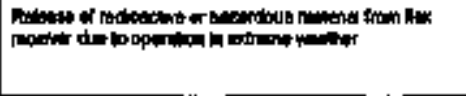 & 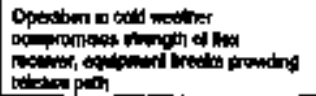 & 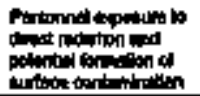 \\
\hline
\end{tabular}




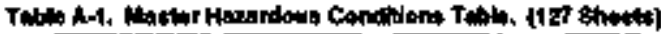

\begin{tabular}{|c|c|c|c|c|}
\hline 印 & Mript & Honedore Condion & chane & Consequeno \\
\hline TKFFA-22 & 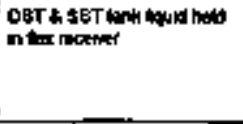 & 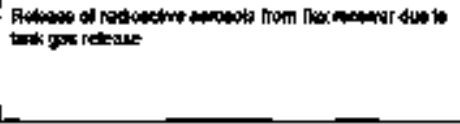 & 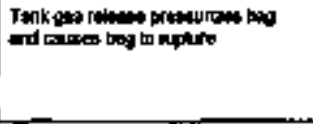 & 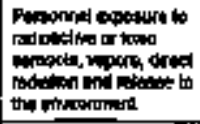 \\
\hline TRKFR-29 & 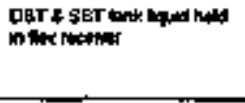 & 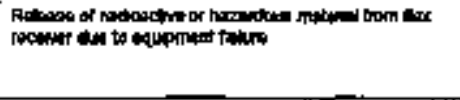 & 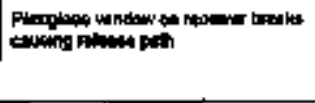 & 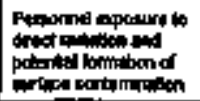 \\
\hline ITK-FR-24 & 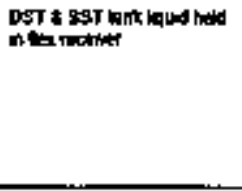 & 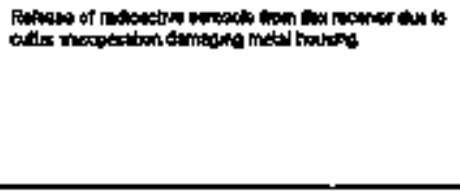 & 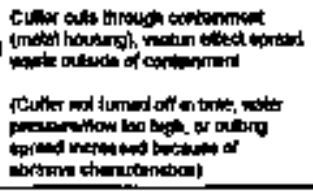 & 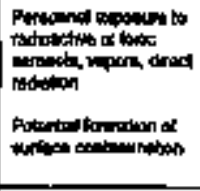 \\
\hline ITKGALS & 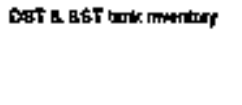 & 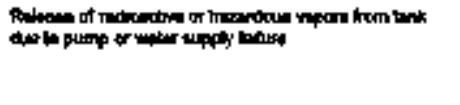 & 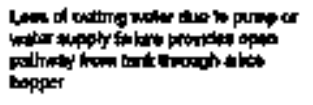 & 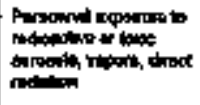 \\
\hline TKArtas & $\begin{array}{l}\text { DST \& IST boh had ind } \\
\text { n hex receng }\end{array}$ & 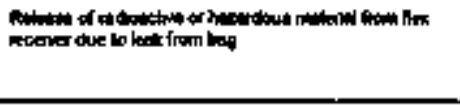 & 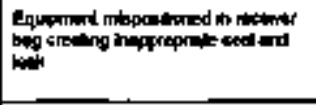 & 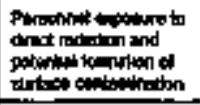 \\
\hline mkenty & 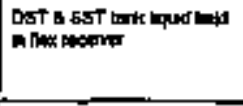 & 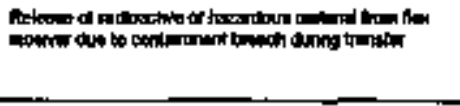 & 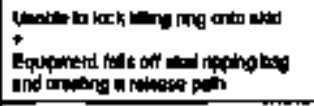 & 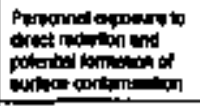 \\
\hline TKसR-29 & 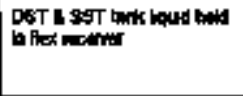 & 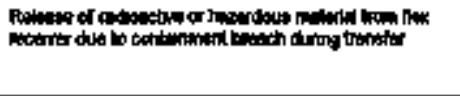 & 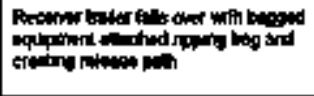 & 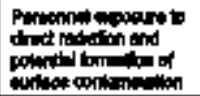 \\
\hline
\end{tabular}




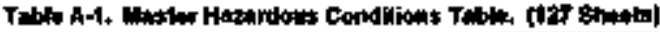

\begin{tabular}{|c|c|c|c|c|}
\hline 10 & 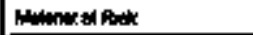 & 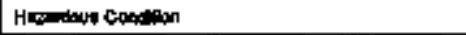 & cand & Danequare \\
\hline HPQ1 & 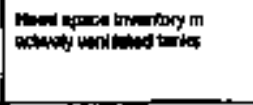 & 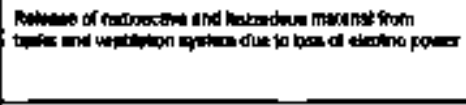 & 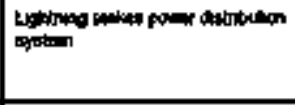 & 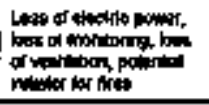 \\
\hline $\operatorname{man}$ & 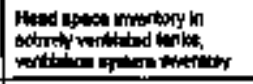 & 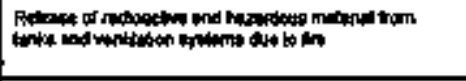 & 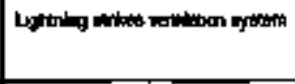 & 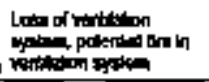 \\
\hline 100 & 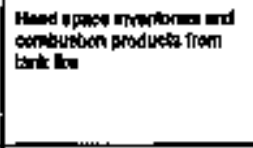 & 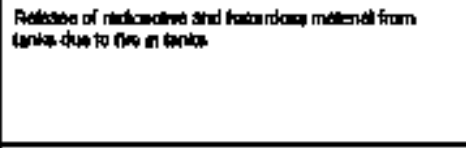 & 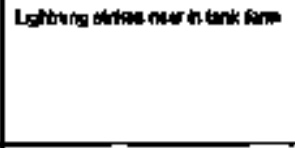 & 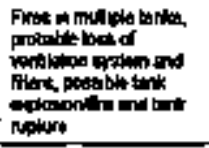 \\
\hline NPMOS & 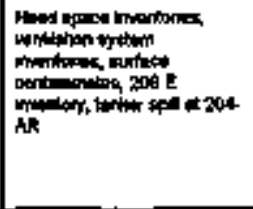 & 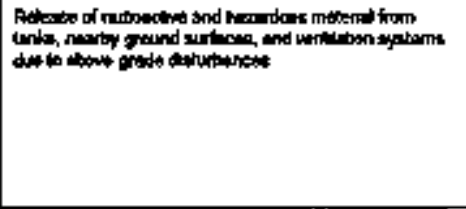 & Wah hands & 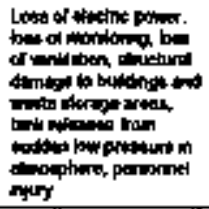 \\
\hline mping & 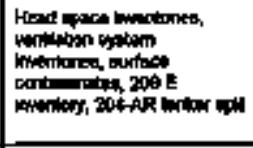 & 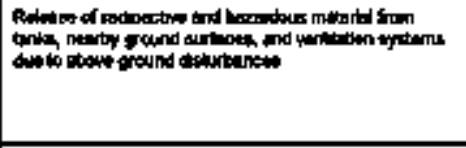 & Hath rand & 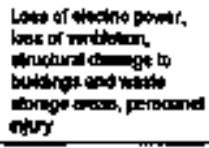 \\
\hline NPOT & 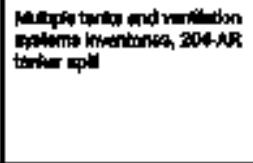 & 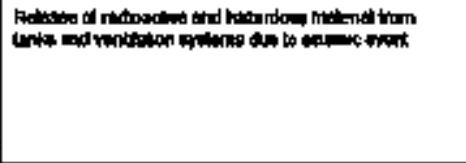 & 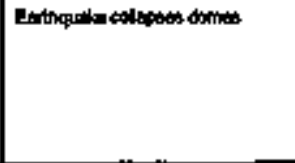 & 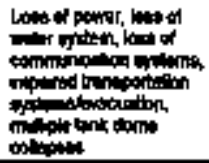 \\
\hline
\end{tabular}




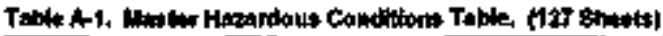

\begin{tabular}{|c|c|c|c|c|}
\hline 0 & Hala ef $P=$ & 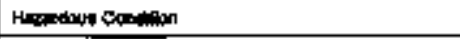 & $\cos$ & comapownes \\
\hline NPpos & 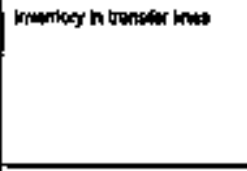 & 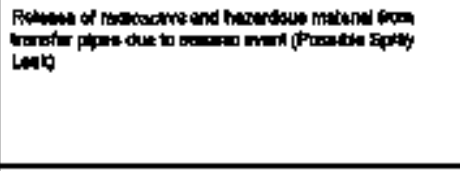 & 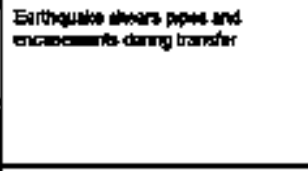 & 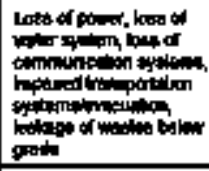 \\
\hline $\operatorname{lng} 0$ & 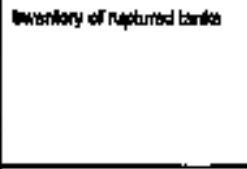 & 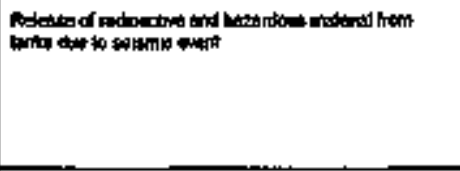 & 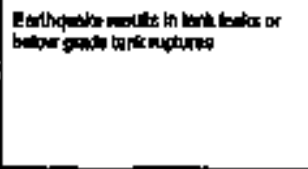 & 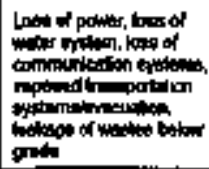 \\
\hline$N P-10$ & 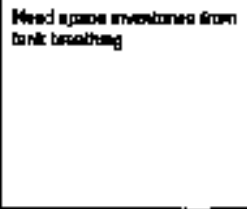 & 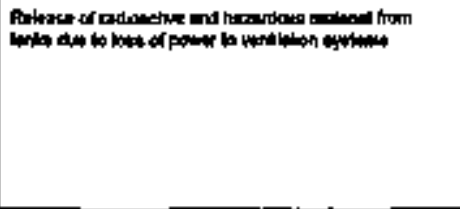 & 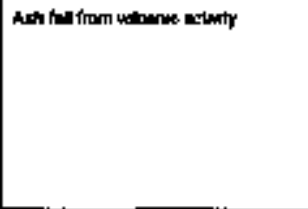 & 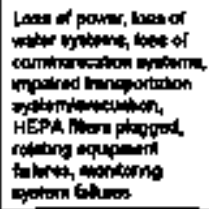 \\
\hline NP.19 & Tank mingex & 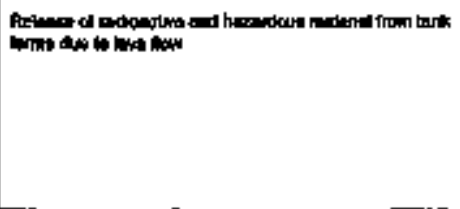 & 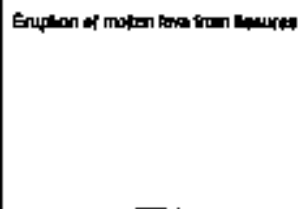 & 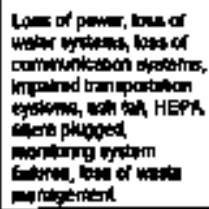 \\
\hline
\end{tabular}




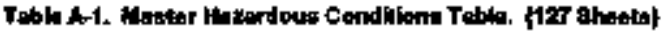

\begin{tabular}{|c|c|c|c|c|}
\hline 18 & 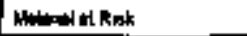 & Hatedors condition & Oane & 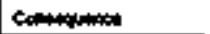 \\
\hline m.12 & 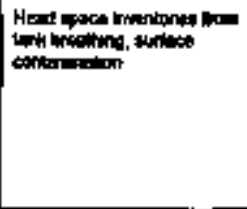 & 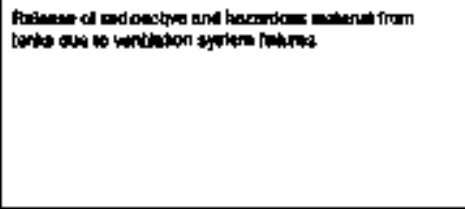 & 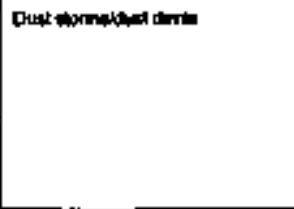 & 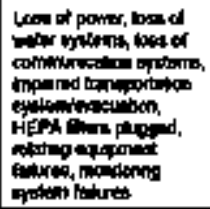 \\
\hline 15:13 & 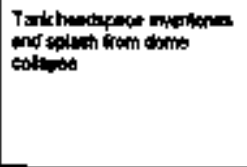 & 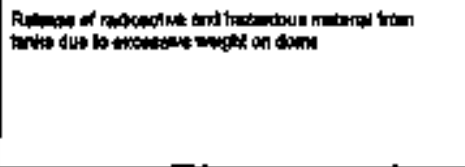 & 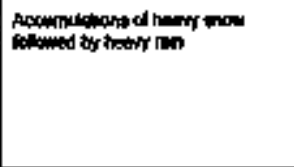 & 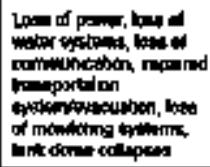 \\
\hline w.14 & Tank & 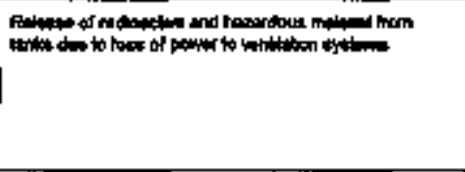 & Exwate $\mathrm{Her}$ & 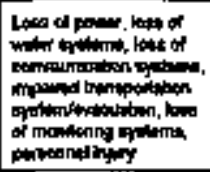 \\
\hline kP-15 & 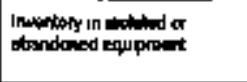 & 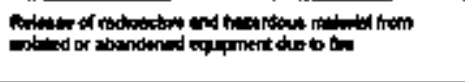 & 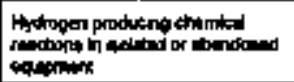 & 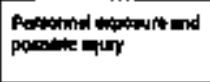 \\
\hline C01-БXT)-01R & 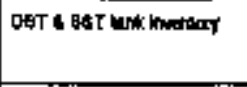 & 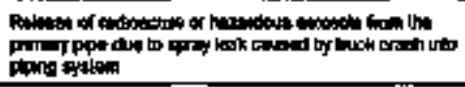 & 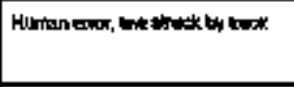 & 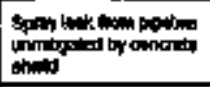 \\
\hline OOT.DKTअ OI: & 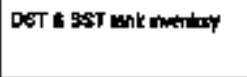 & 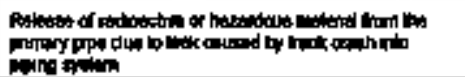 & 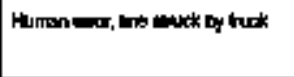 & 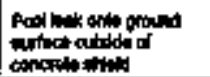 \\
\hline
\end{tabular}




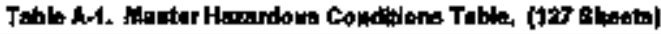

\begin{tabular}{|c|c|c|c|c|}
\hline D & 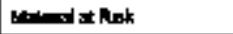 & Harndens Onithent & Canc: & Conemeres \\
\hline OETFLOWI-01 & 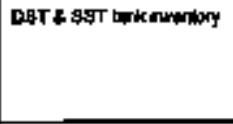 & 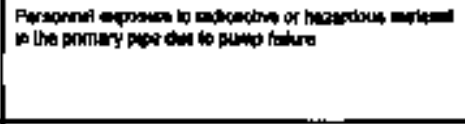 & 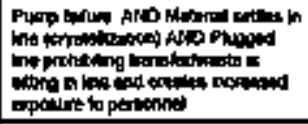 & 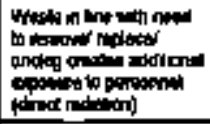 \\
\hline OGTFLWMAZ & 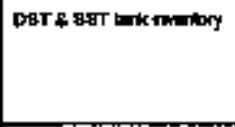 & 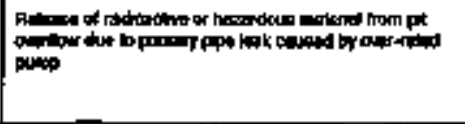 & 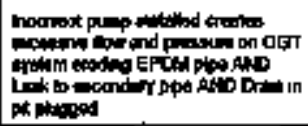 & Oneriar onte grourd \\
\hline OGTFIONAMAn & DST a S5T ind immoning & 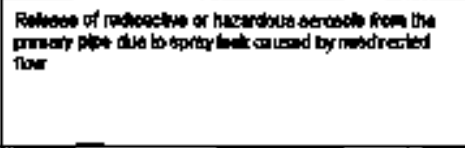 & 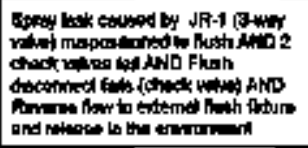 & 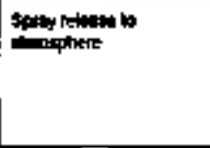 \\
\hline OOT-Rowl-es: & 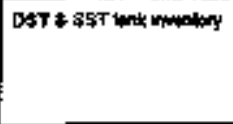 & 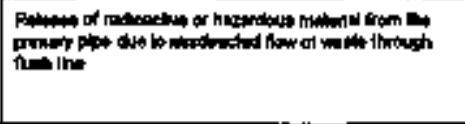 & 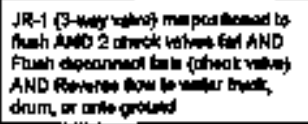 & Pind \\
\hline OATSLCOHYAS & 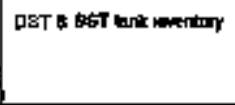 & 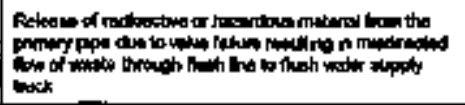 & 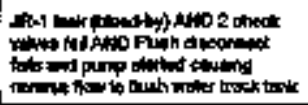 & 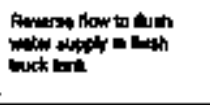 \\
\hline OATflonil-65 & 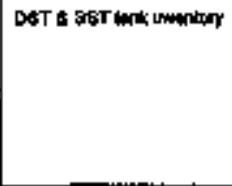 & 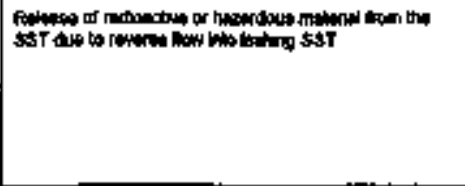 & 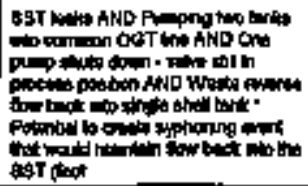 & 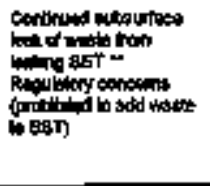 \\
\hline
\end{tabular}




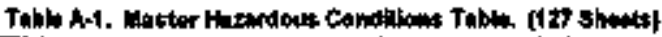

\begin{tabular}{|c|c|c|c|c|}
\hline ID & Mt & 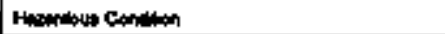 & Cons: & 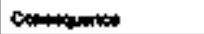 \\
\hline DOT.PLOWI- & 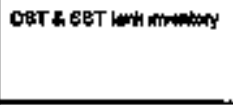 & 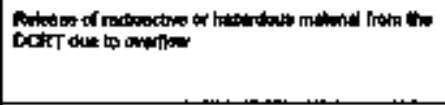 & 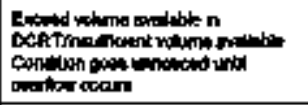 & 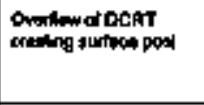 \\
\hline WIfLOMnO1A & CAsT a sst tuk unedinfy & 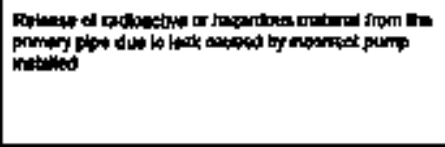 & 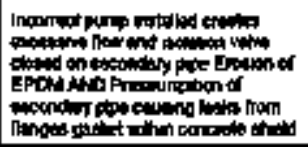 & 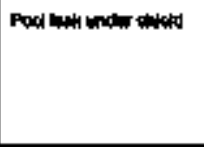 \\
\hline ọ़ा flawhM & 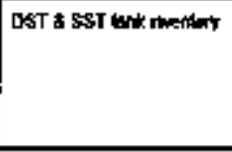 & 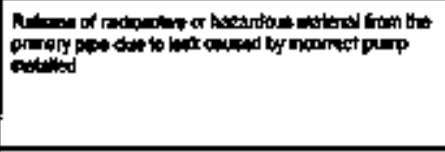 & 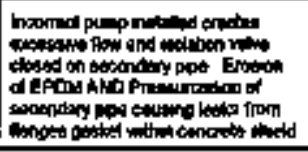 & 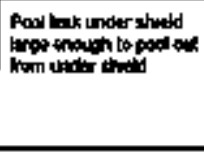 \\
\hline DOTRPLOHU202 & DST \& SST Thonthy & 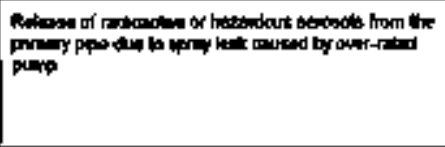 & 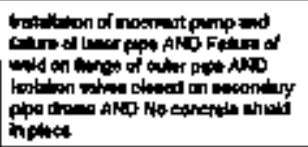 & 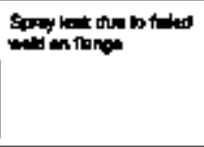 \\
\hline OOT.PLON2019 & 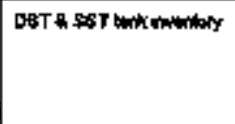 & 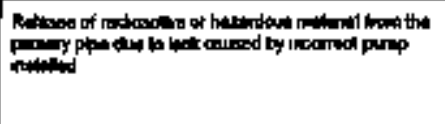 & 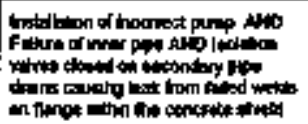 & Pad leats under shets \\
\hline OxIflonz-(es) & 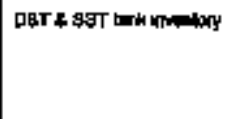 & 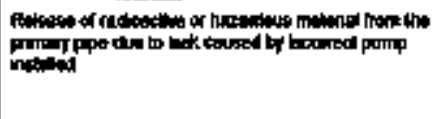 & 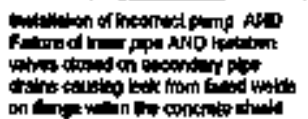 & 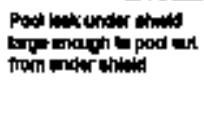 \\
\hline
\end{tabular}


Tobte A-1. Marter Heredeus Condilions Tablo. (126 ahmots]

\begin{tabular}{|c|c|c|c|c|}
\hline 由 & 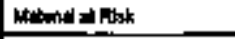 & Hetidars Condtitan & Gover & 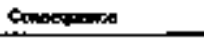 \\
\hline ostenteosat & 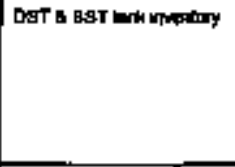 & 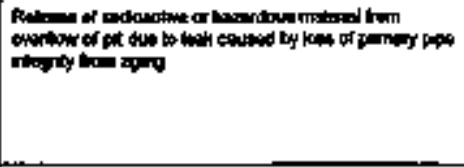 & 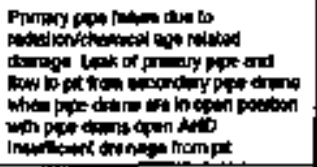 & 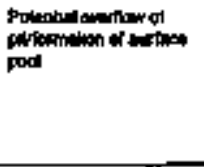 \\
\hline OGT-NFBAT & DBT \& SET Rnk Mumitry & 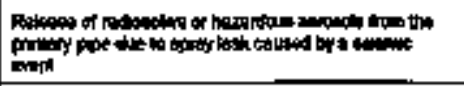 & 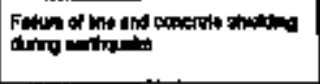 & 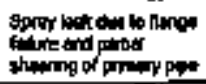 \\
\hline 00140002 & 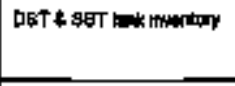 & 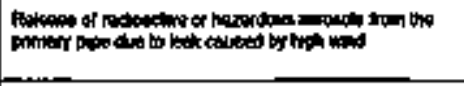 & 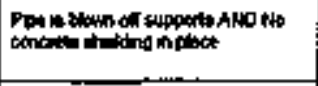 & 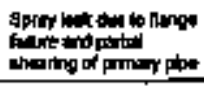 \\
\hline Dot-optial & 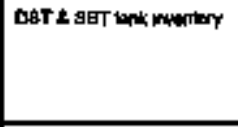 & 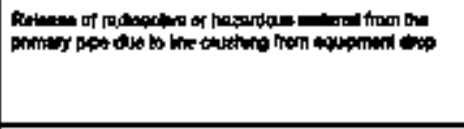 & 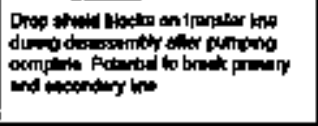 & 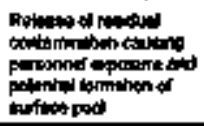 \\
\hline O\&TARTS1-OI & 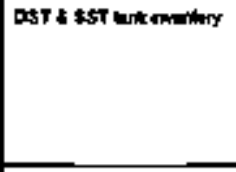 & 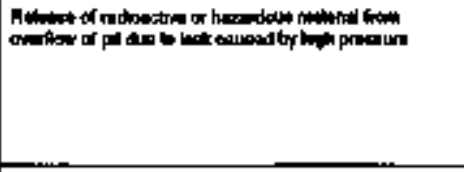 & 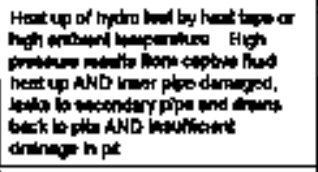 & 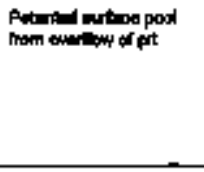 \\
\hline opatfuckicion & 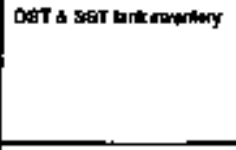 & 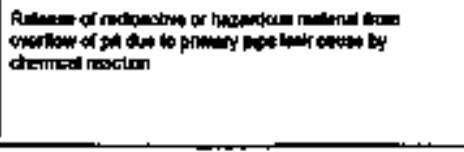 & 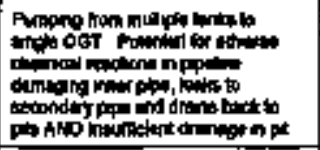 & 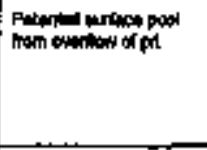 \\
\hline Dof-SPeC1.01 & प्gा स \& & 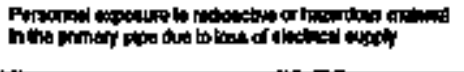 & 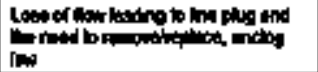 & 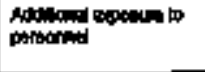 \\
\hline
\end{tabular}




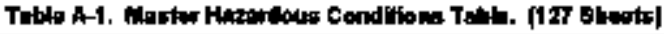

\begin{tabular}{|c|c|c|c|c|}
\hline 10 & hatord at fork & 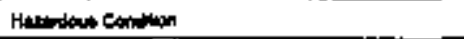 & Chat & A \\
\hline OGT-TIMPAOS & DBST \& 96T tork mentor & 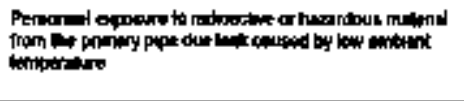 & 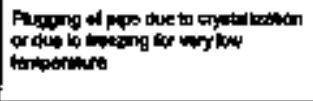 & 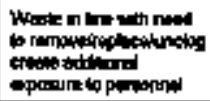 \\
\hline OOT-TENM-DR & 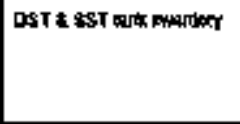 & 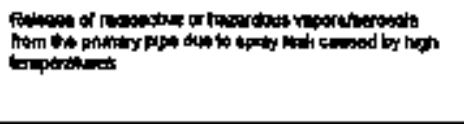 & 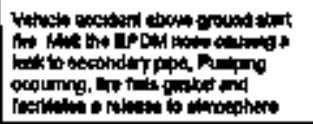 & 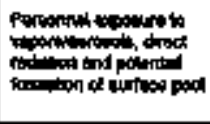 \\
\hline OGT.TENF=08 & 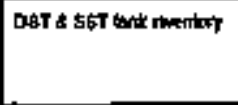 & 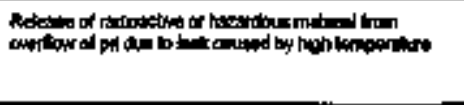 & 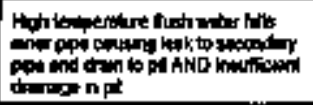 & 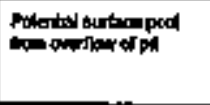 \\
\hline rear & 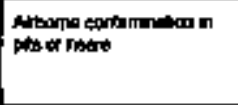 & 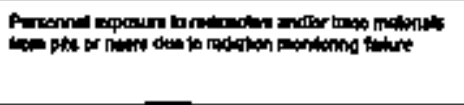 & fulu bemantor & 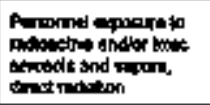 \\
\hline คAم01 & Mu & 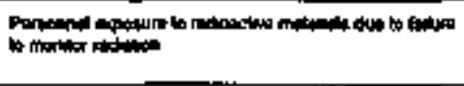 & Falun bo mentor & 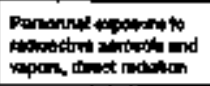 \\
\hline RADER & 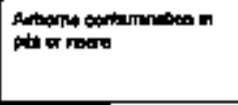 & 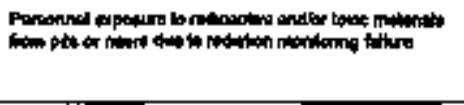 & Fulpro of & 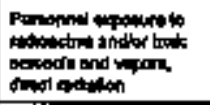 \\
\hline PADOOA & $M$ & 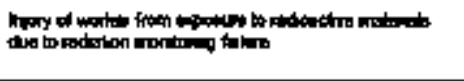 & 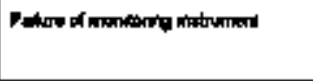 & 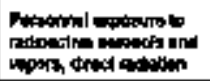 \\
\hline RADOS & 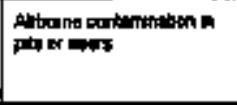 & 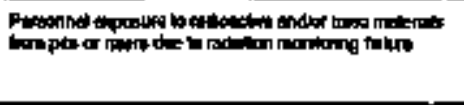 & 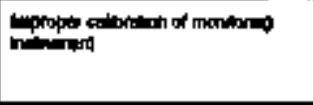 & 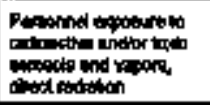 \\
\hline RAON004 & wa & 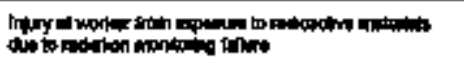 & 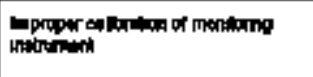 & 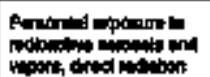 \\
\hline
\end{tabular}




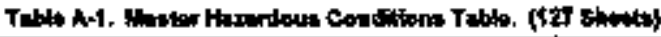

\begin{tabular}{|c|c|c|c|c|}
\hline $\boldsymbol{D}$ & 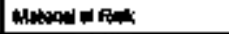 & Horaden contion & Canen & 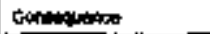 \\
\hline ingkar & 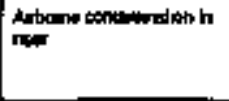 & 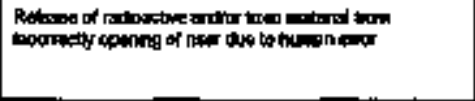 & 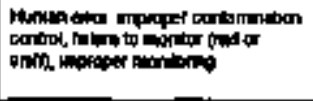 & 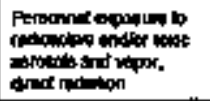 \\
\hline RSR-01-1 & 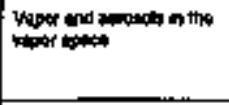 & 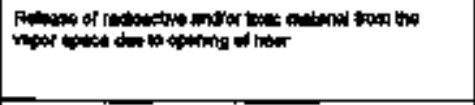 & 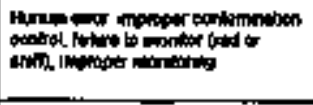 & 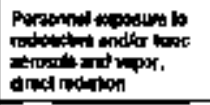 \\
\hline RPR02 & Alanden & 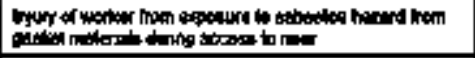 & furos bololow & 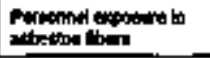 \\
\hline AERACL & nh & 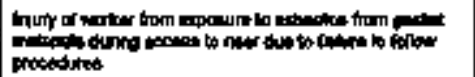 & 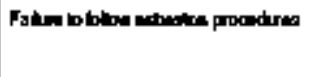 & 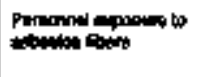 \\
\hline Ather & Antind & 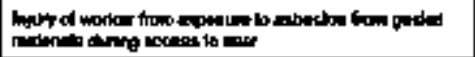 & 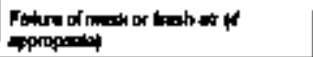 & 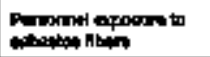 \\
\hline$R \cong R-1$ & wh & 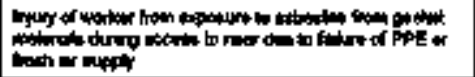 & 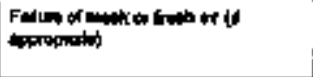 & 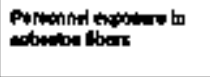 \\
\hline REROA & $M$ & 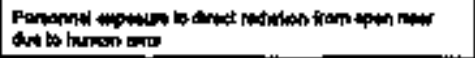 & 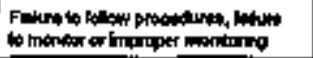 & 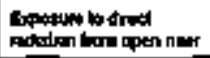 \\
\hline ค:AดAMA & Mh & 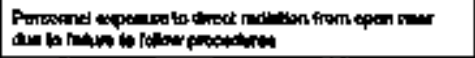 & 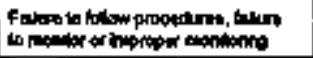 & 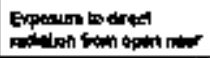 \\
\hline nom-os & 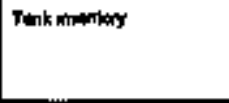 & 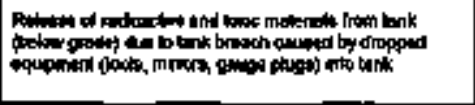 & 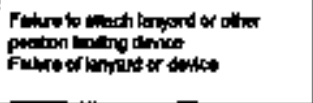 & 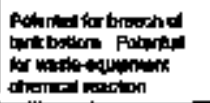 \\
\hline Mathdedn & 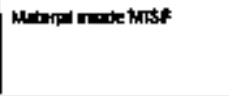 & 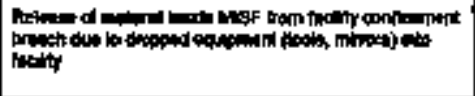 & 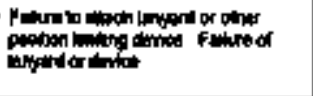 & 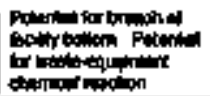 \\
\hline
\end{tabular}




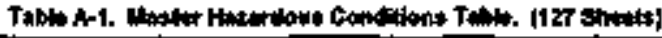

\begin{tabular}{|c|c|c|c|c|}
\hline ID & 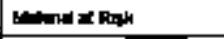 & 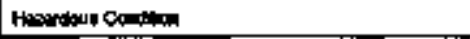 & Con & 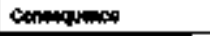 \\
\hline$R B A-05-8$ & Imiond numb MISP & 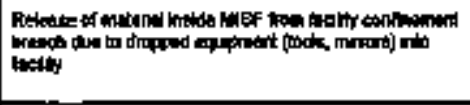 & 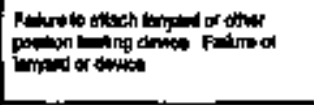 & 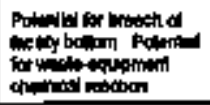 \\
\hline ABR 004 & Nore & 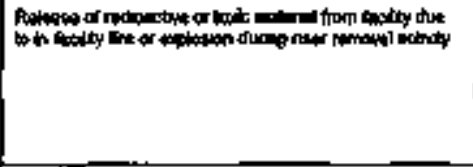 & 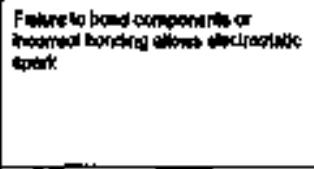 & 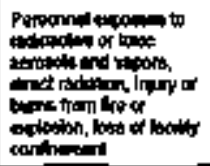 \\
\hline 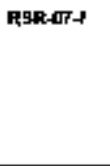 & 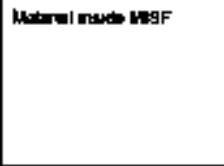 & 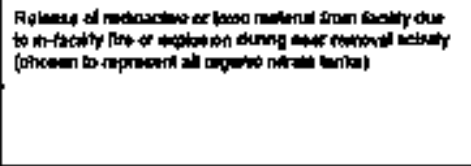 & 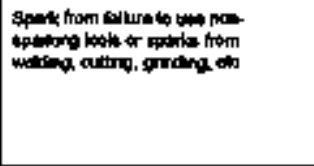 & 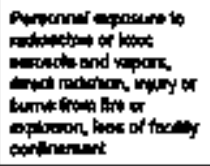 \\
\hline RER-CB-I & 蚻 & 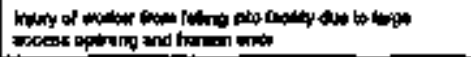 & 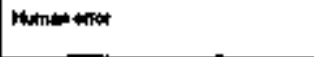 & vownen miny \\
\hline setral & 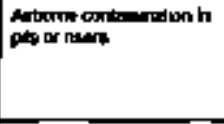 & 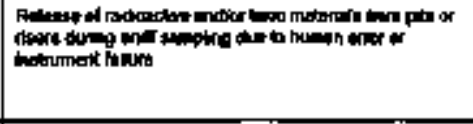 & 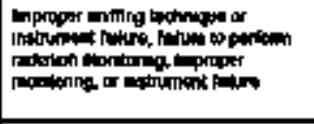 & 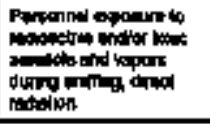 \\
\hline SxFAnd & wh & 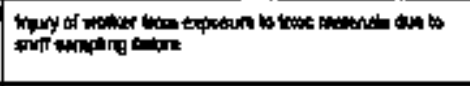 & 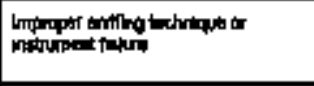 & 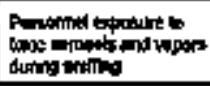 \\
\hline TFs-1 & Waw hom $1028 \mathrm{y}$ & 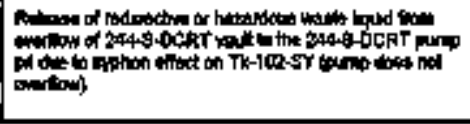 & 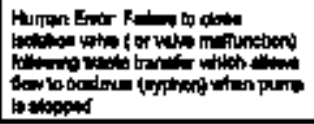 & \\
\hline
\end{tabular}




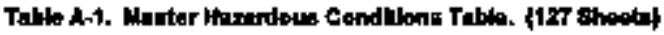

\begin{tabular}{|c|c|c|c|c|}
\hline 由 & Mternd at Rak & Heribus Sondion & Gant & Contrquenor \\
\hline พ파 & 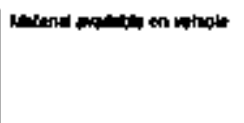 & 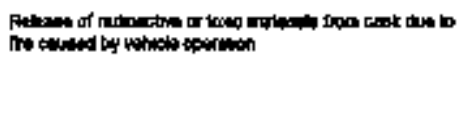 & Wehtle otrene on the & 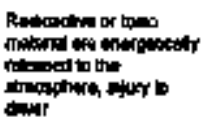 \\
\hline VEHOH 4 & 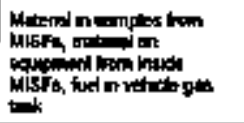 & 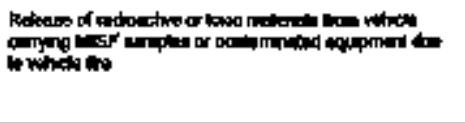 & 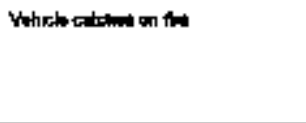 & 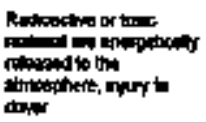 \\
\hline VEHDO & Bampla lanmod cat: & 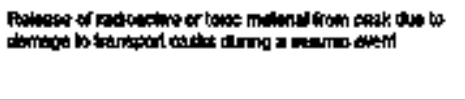 & 9oamu went & 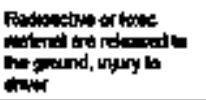 \\
\hline VEHAOI & 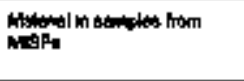 & 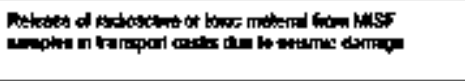 & 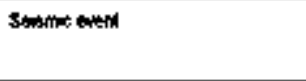 & 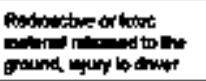 \\
\hline पed & 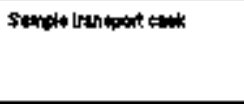 & 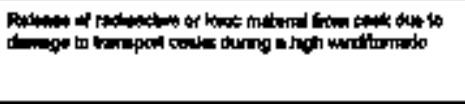 & 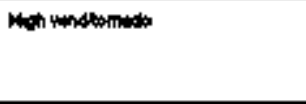 & 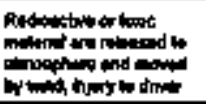 \\
\hline VEFAlat & 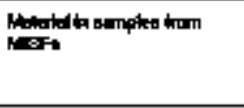 & 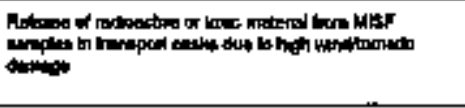 & Migh nondtomoth & 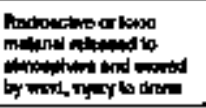 \\
\hline WEHQ4 & $M$ & 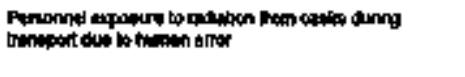 & 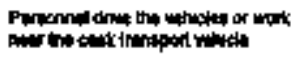 & 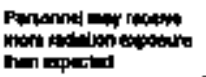 \\
\hline VEHOH & MA & 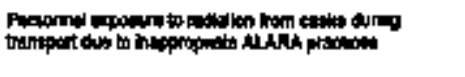 & 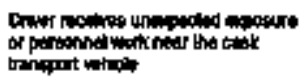 & 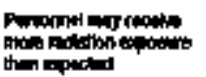 \\
\hline
\end{tabular}


Tablo h-1. Mastor Harardous Gendifiont Table (127 thets)

\begin{tabular}{|c|c|c|c|c|}
\hline+0 & 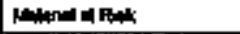 & 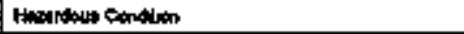 & Cwan & 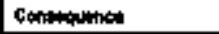 \\
\hline VELHAS & Man & 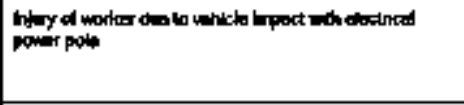 & 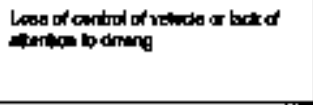 & 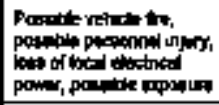 \\
\hline VEHOSA-1 & Man & In & 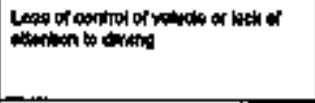 & 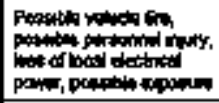 \\
\hline VEHOH & Wh & 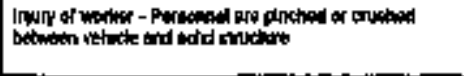 & 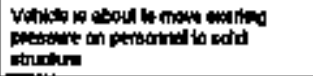 & Procmininary \\
\hline VIat06- & wid & 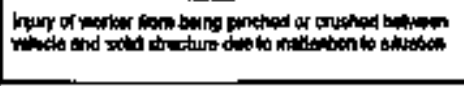 & 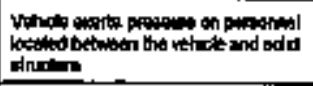 & Prowend inwy \\
\hline YaHOT & Tmat mumby & 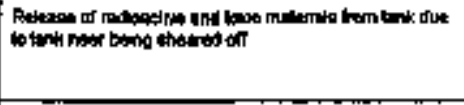 & 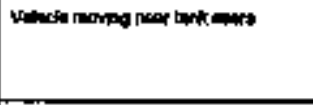 & 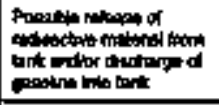 \\
\hline WEATP-1 & Naterd a NagF & 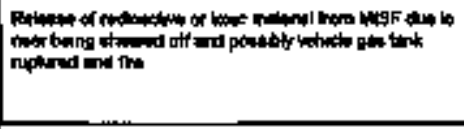 & 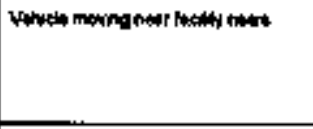 & 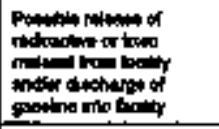 \\
\hline VEHAS-I & 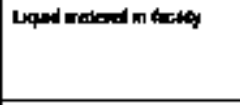 & 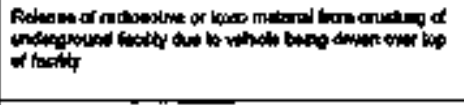 & 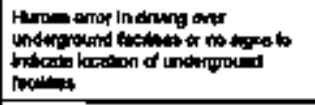 & 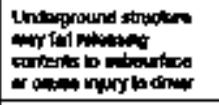 \\
\hline weu-o1 & 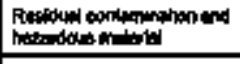 & 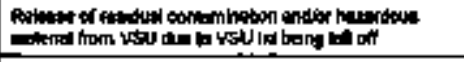 & 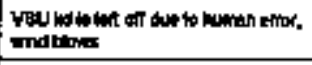 & 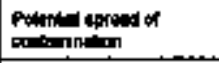 \\
\hline V\$N-02 & 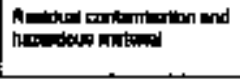 & 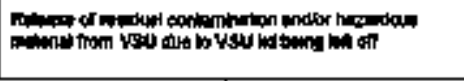 & 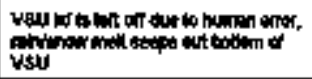 & 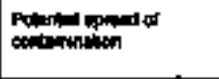 \\
\hline
\end{tabular}




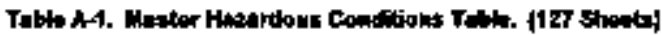

\begin{tabular}{|c|c|c|c|c|}
\hline 10 & Holmow at Rask & 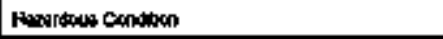 & 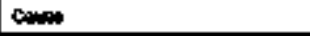 & Contrequariot \\
\hline$v \sin$ & 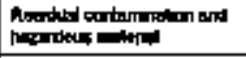 & 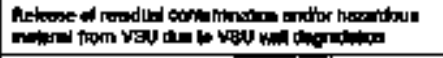 & 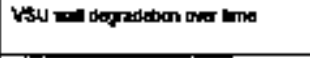 & 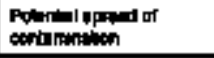 \\
\hline VESOA & 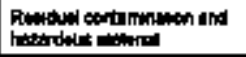 & 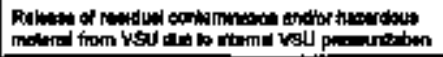 & 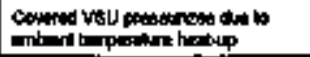 & 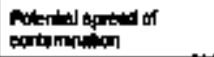 \\
\hline Ysumes & None & 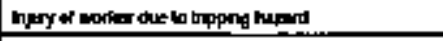 & Eponre tom gound leve & 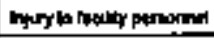 \\
\hline พक्षि05. & $\lim$ & 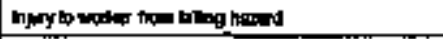 & 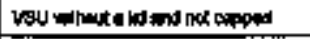 & 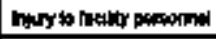 \\
\hline WOSB-FLWW01 & 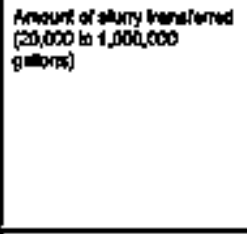 & 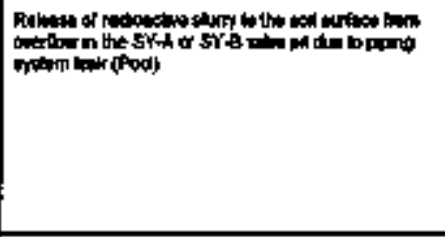 & 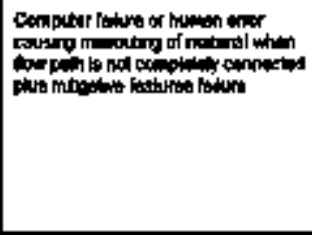 & 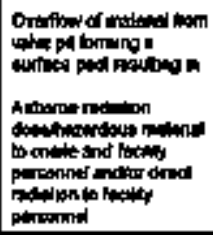 \\
\hline Wogt-PLown: & 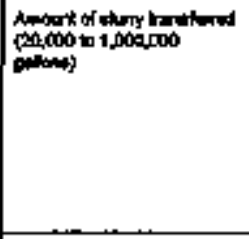 & 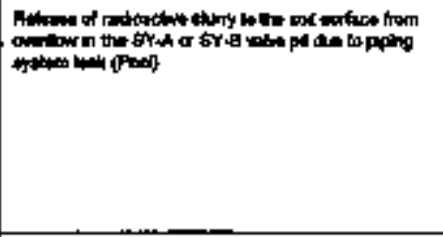 & 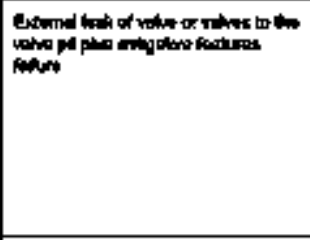 & 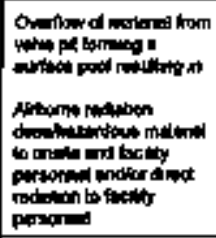 \\
\hline 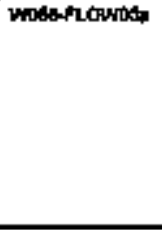 & 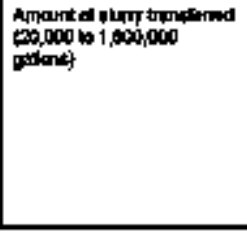 & 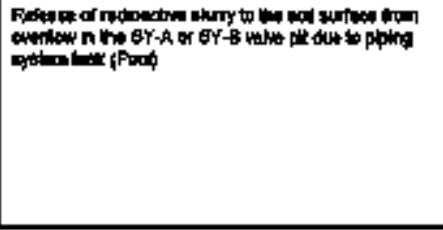 & 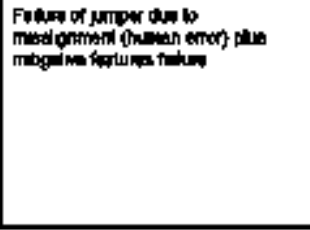 & 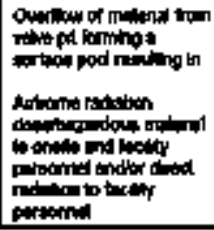 \\
\hline
\end{tabular}




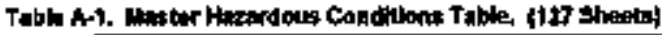

\begin{tabular}{|c|c|c|c|c|}
\hline 10 & Whates at Rak & Muswitous conition & $C$ & correqunues \\
\hline 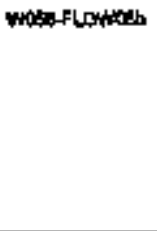 & 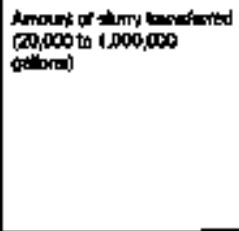 & 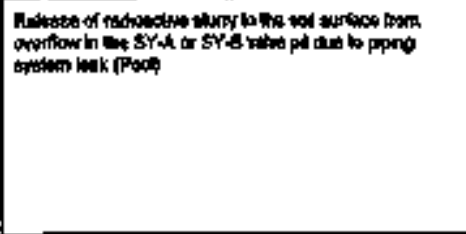 & 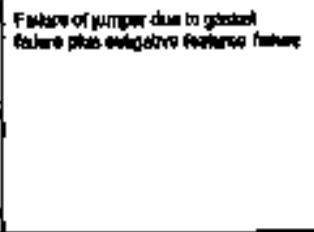 & 1 \\
\hline WOSPPLONDES & 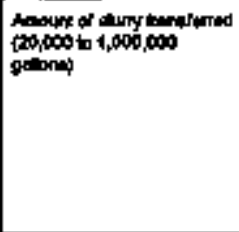 & 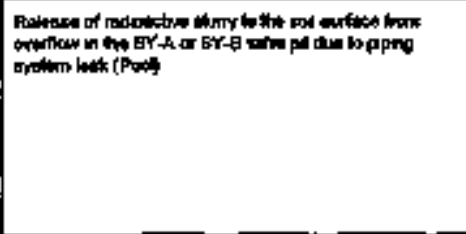 & 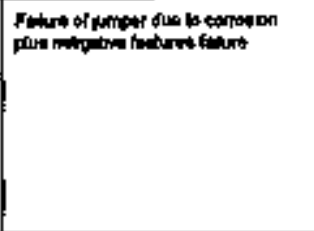 & 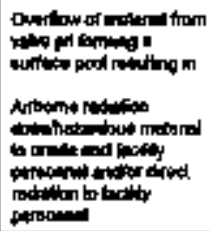 \\
\hline WOSAPLOWOT & 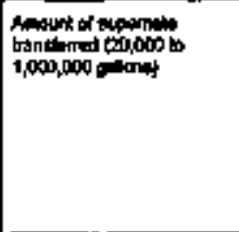 & 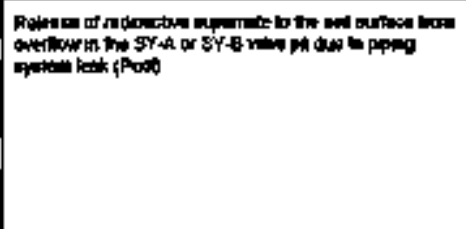 & 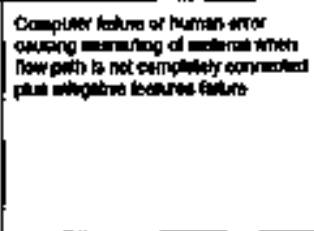 & 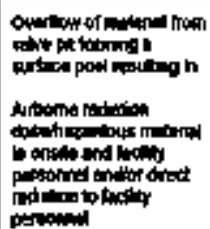 \\
\hline
\end{tabular}




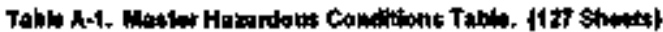

\begin{tabular}{|c|c|c|c|c|}
\hline 10 & 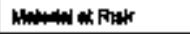 & 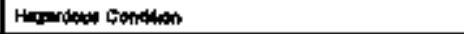 & Canaw & Goramenunine \\
\hline Noto-rowate & 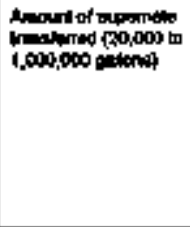 & 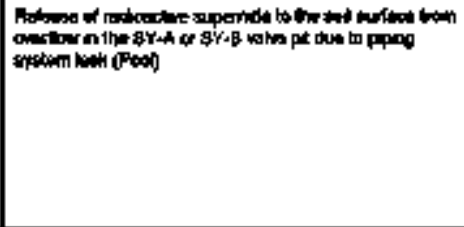 & 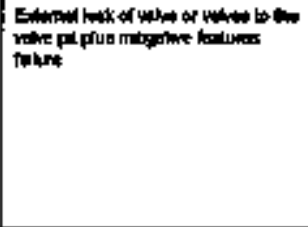 & 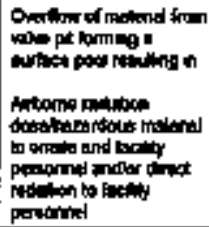 \\
\hline 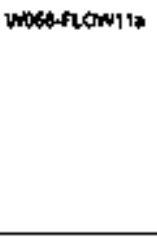 & 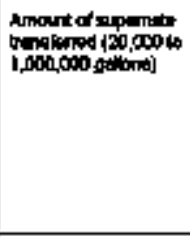 & 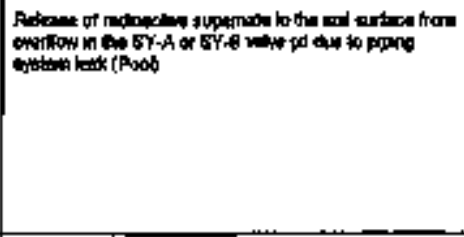 & 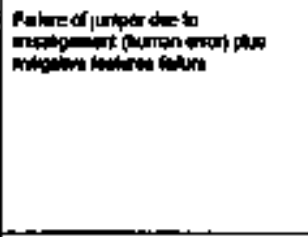 & 10 \\
\hline WOSA-FLWN11b & 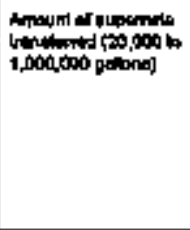 & 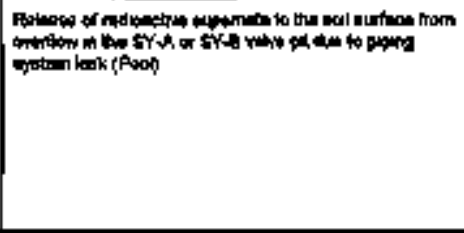 & 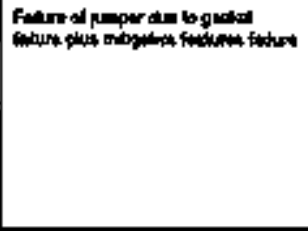 & 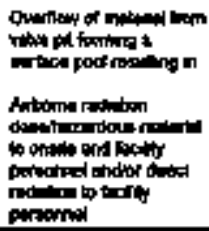 \\
\hline
\end{tabular}




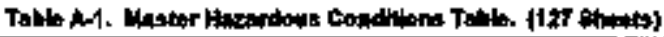

\begin{tabular}{|c|c|c|c|c|}
\hline 10 & 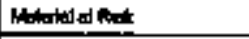 & Parantore Candon & Cona & Conampinte \\
\hline noparower is & mont of ypersin & 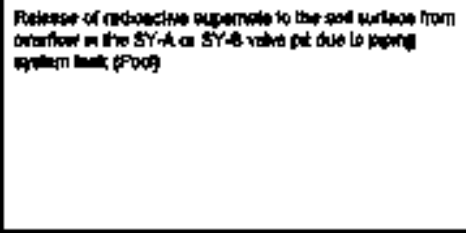 & 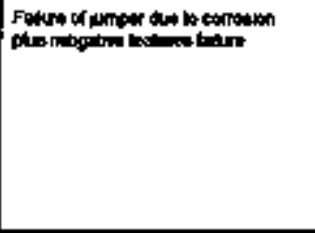 & 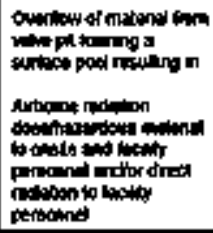 \\
\hline MOSB-FLOHI? & 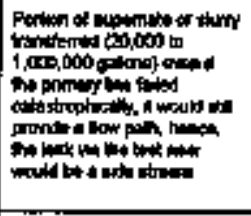 & 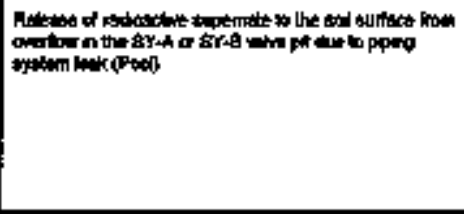 & 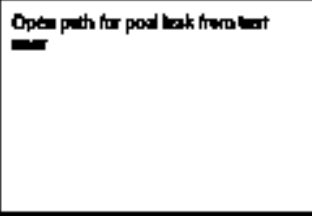 & 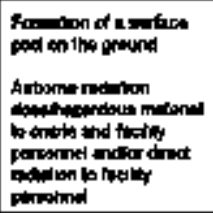 \\
\hline MOSAfLOWH & Anopind of tainy & 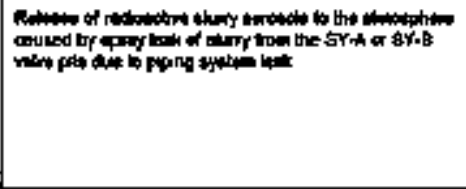 & 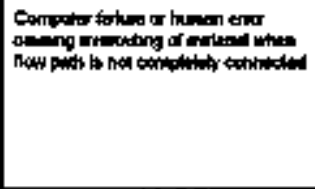 & 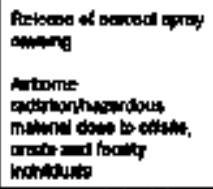 \\
\hline mogentrow16 & 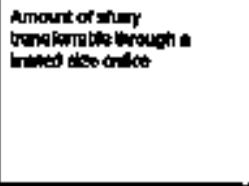 & 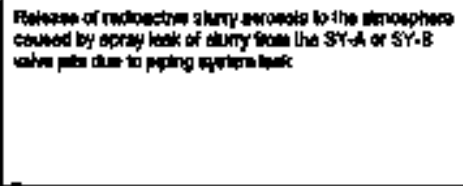 & 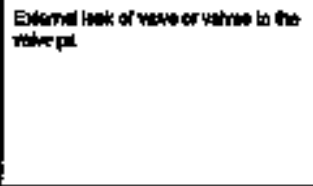 & 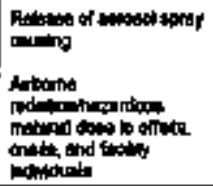 \\
\hline
\end{tabular}




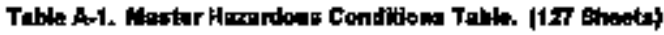

\begin{tabular}{|c|c|c|c|c|}
\hline D & thedeffent & 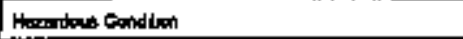 & Ctand & 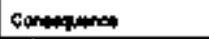 \\
\hline 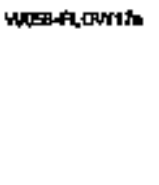 & ingortef atumy & 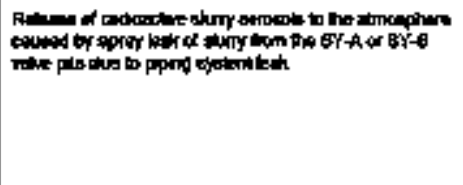 & 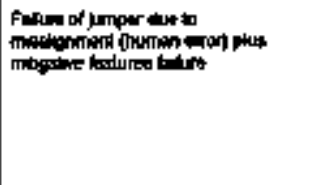 & 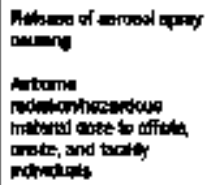 \\
\hline WOSEFLOHM & 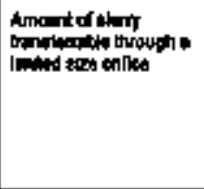 & 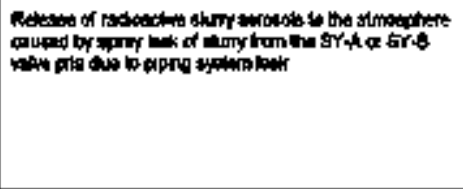 & 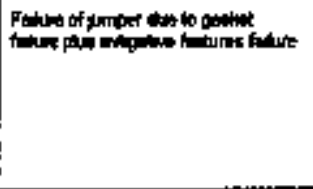 & 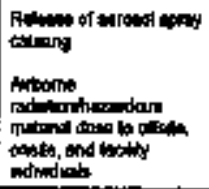 \\
\hline Mosprocowite & 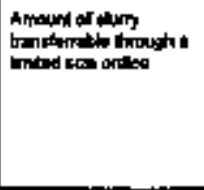 & 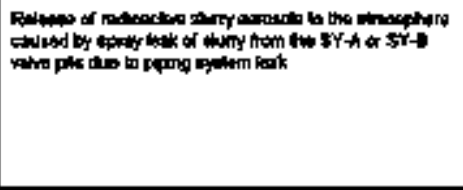 & 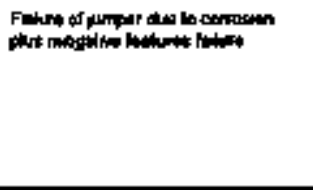 & 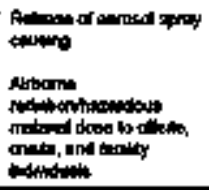 \\
\hline NHESAFLOWIS & 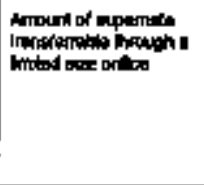 & 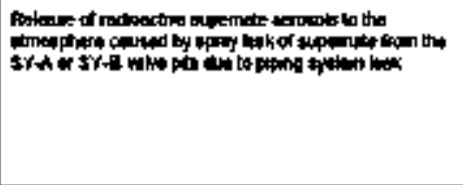 & 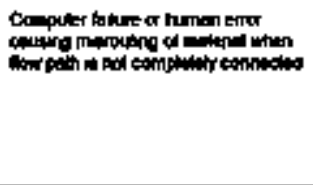 & 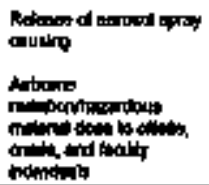 \\
\hline
\end{tabular}


Tablu A-1. Mnstar Hanrdosa Conditons Tuble, \{127 Bhesta)

\begin{tabular}{|c|c|c|c|c|}
\hline 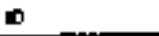 & Nond ath then & 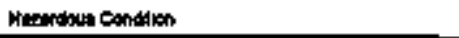 & Cand & Contapentest \\
\hline Mosincoptr & 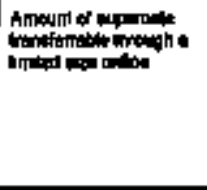 & 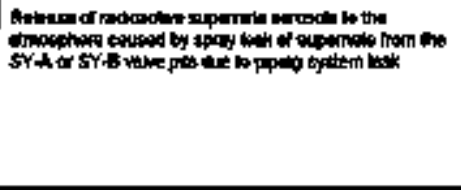 & 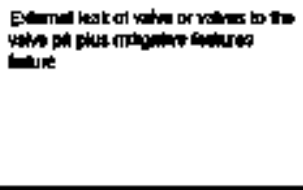 & 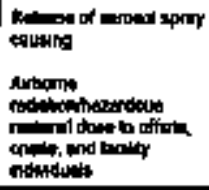 \\
\hline Moodrowasos & inourtof opentity & 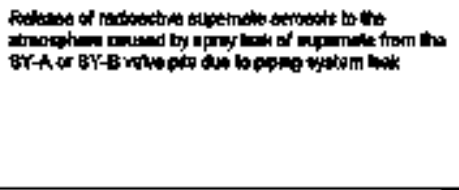 & 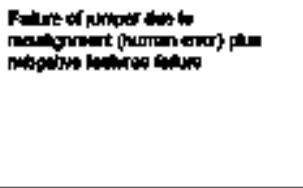 & 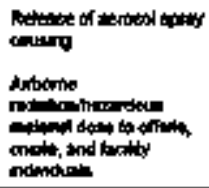 \\
\hline WOSS-FLOMatob & 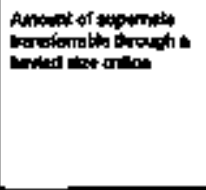 & 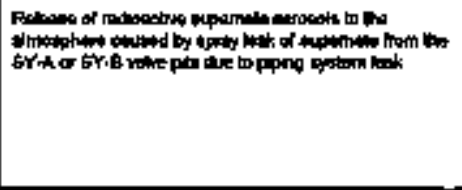 & 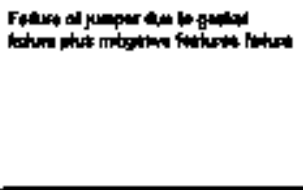 & 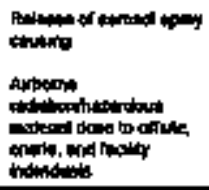 \\
\hline noserplowrsoc & 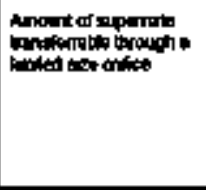 & 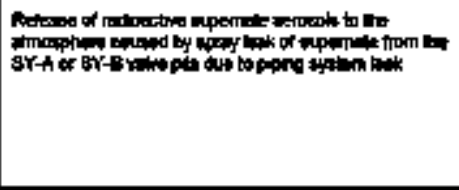 & 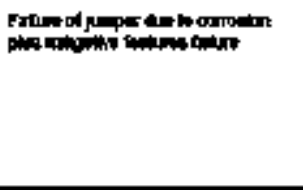 & 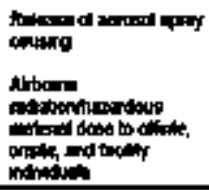 \\
\hline
\end{tabular}




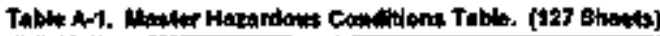

\begin{tabular}{|c|c|c|c|c|}
\hline סו & Ihters al thet & 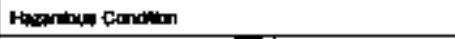 & Cosids & 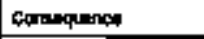 \\
\hline WO68-AMTFOOA & 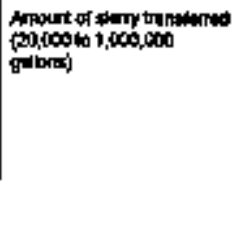 & 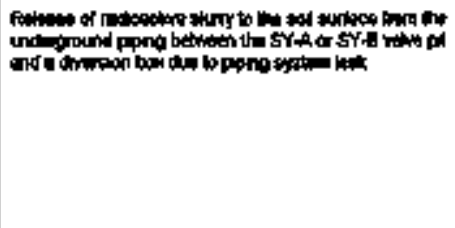 & 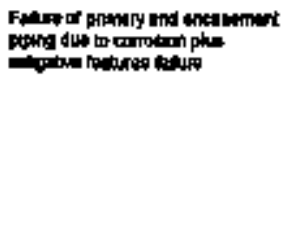 & 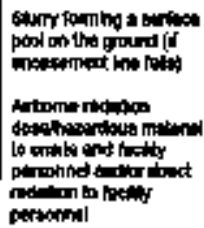 \\
\hline WOSSINTEEOHb & 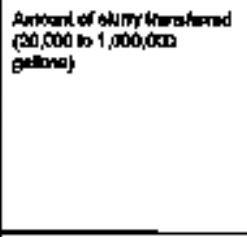 & 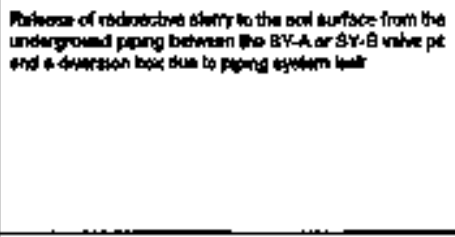 & 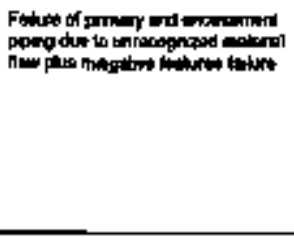 & 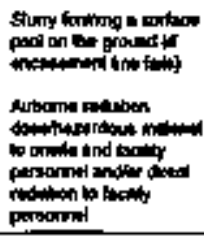 \\
\hline WOGQINTEOXIC & 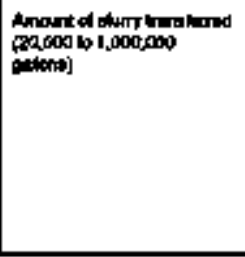 & 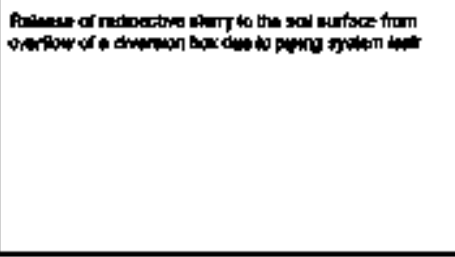 & 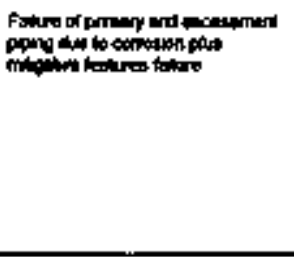 & 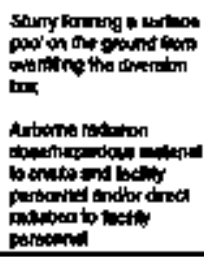 \\
\hline
\end{tabular}




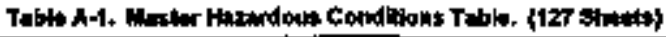

\begin{tabular}{|c|c|c|c|c|}
\hline 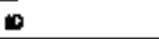 & 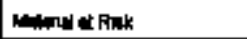 & 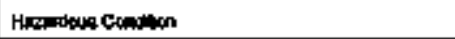 & cmant & Conangunce \\
\hline nES6-NMTECOId & 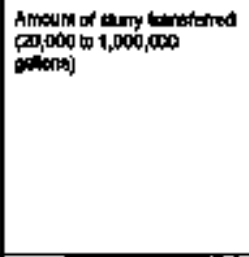 & 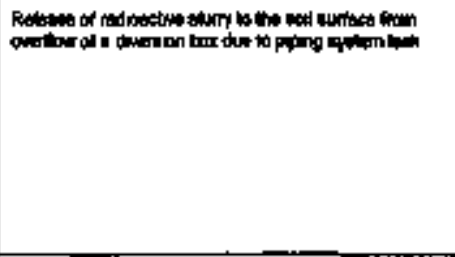 & 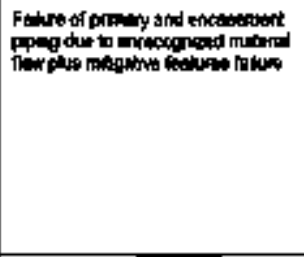 & 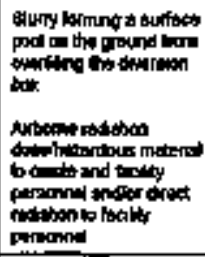 \\
\hline 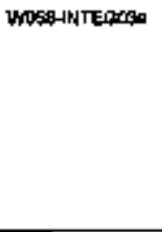 & 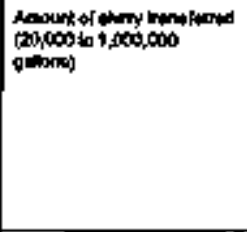 & 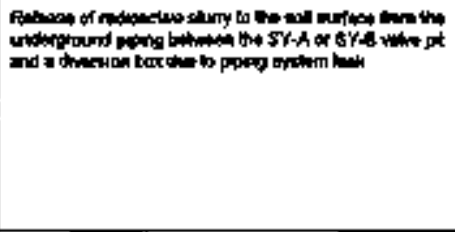 & 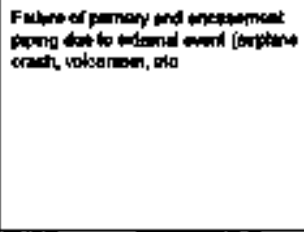 & 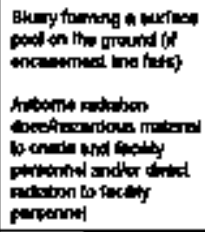 \\
\hline Nuse-NTECOOS & 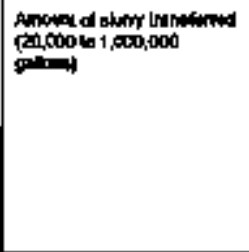 & 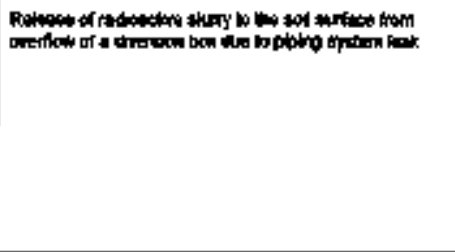 & 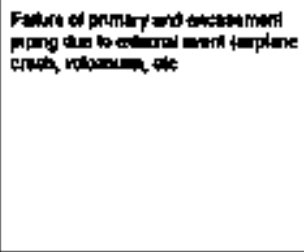 & 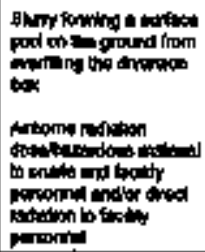 \\
\hline
\end{tabular}




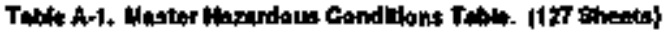

\begin{tabular}{|c|c|c|c|c|}
\hline סו & 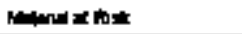 & 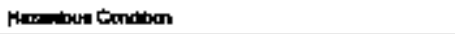 & Eorbo & Conergungan \\
\hline WNSQHKTTCOE & 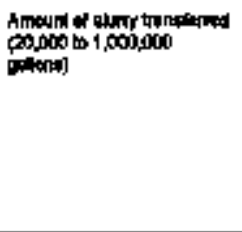 & 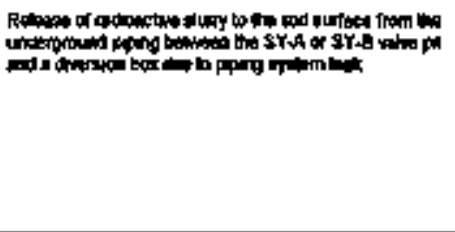 & 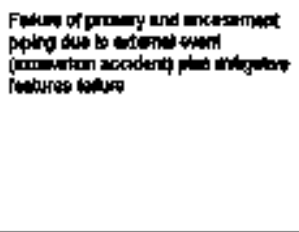 & 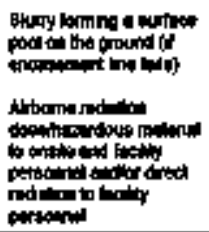 \\
\hline Nustututecold & 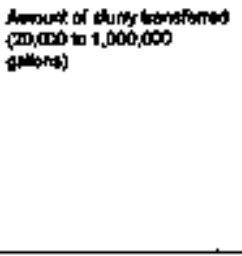 & 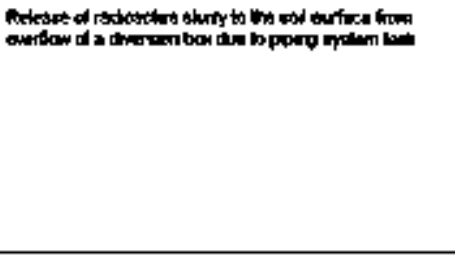 & 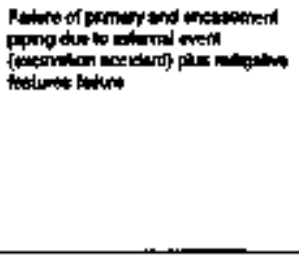 & 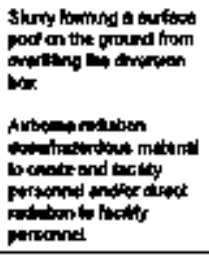 \\
\hline 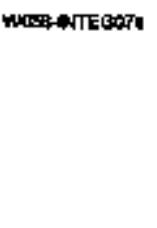 & 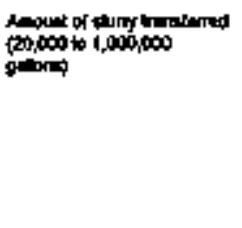 & 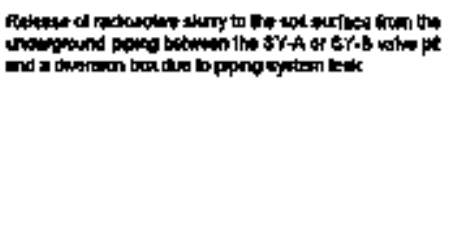 & 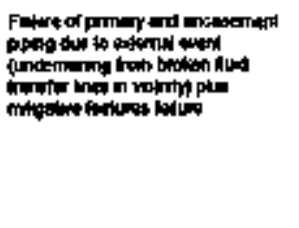 & 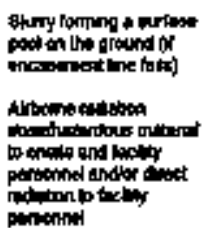 \\
\hline
\end{tabular}




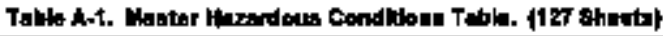

\begin{tabular}{|c|c|c|c|c|}
\hline 10 & 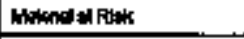 & Hrantors Cindban & canto & consequines \\
\hline WOQGINTEGOTH & 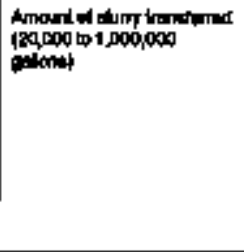 & 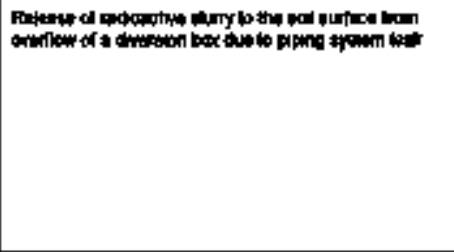 & 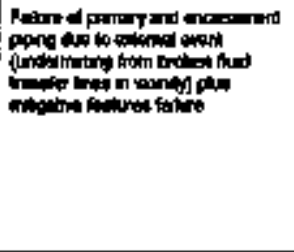 & 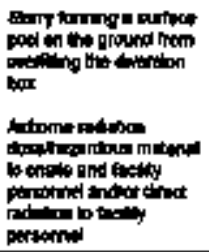 \\
\hline WDSOWTEGDG & 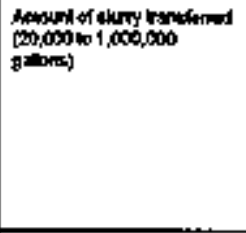 & 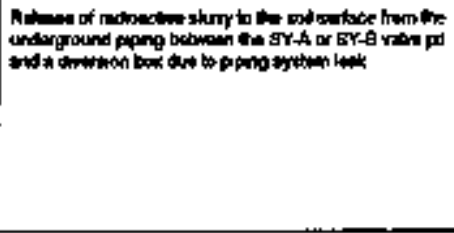 & 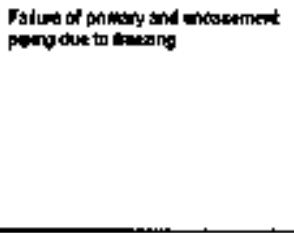 & 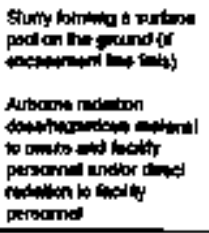 \\
\hline wots intecoth & 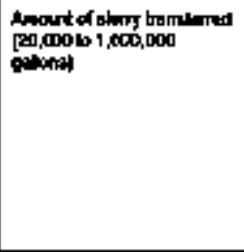 & 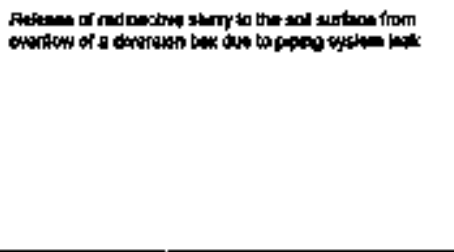 & 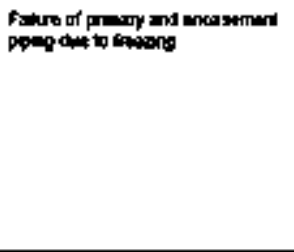 & 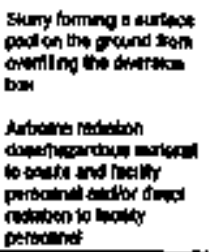 \\
\hline
\end{tabular}




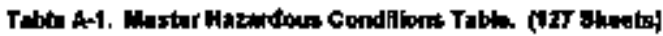

\begin{tabular}{|c|c|c|c|c|}
\hline Dו & Wwoth of flak & Morostan Conditon & conten & Gonempunest \\
\hline Whoterlmesito & 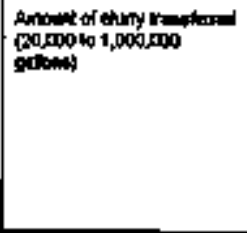 & 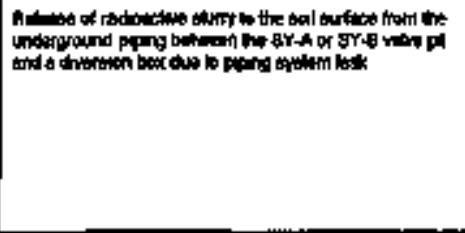 & 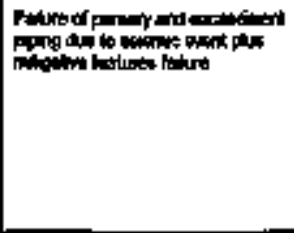 & 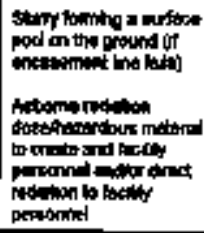 \\
\hline NUPSTIER11 & 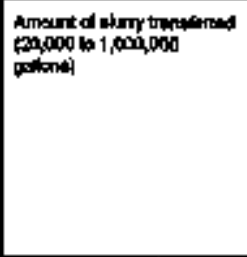 & 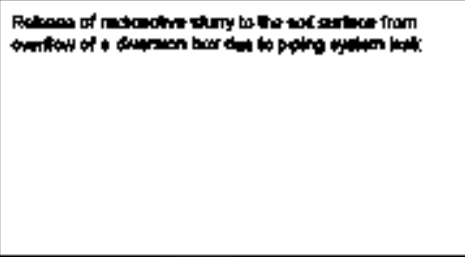 & 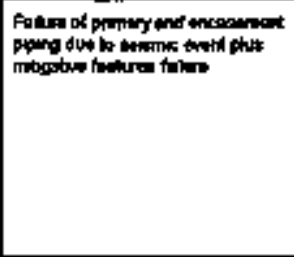 & 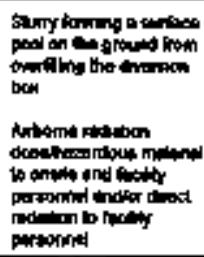 \\
\hline 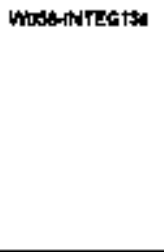 & 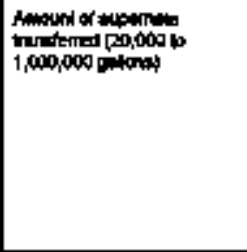 & 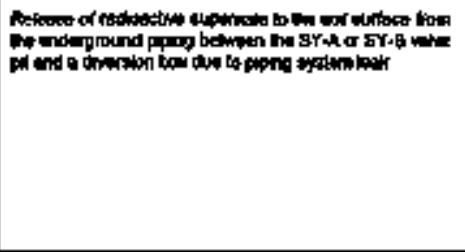 & 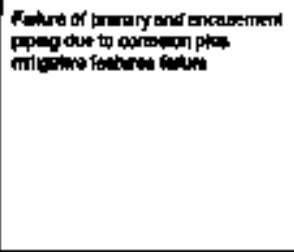 & 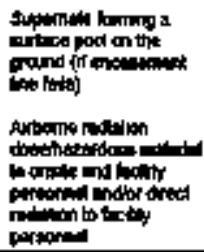 \\
\hline
\end{tabular}




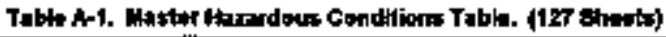

\begin{tabular}{|c|c|c|c|c|}
\hline 10 & attond at Risk & 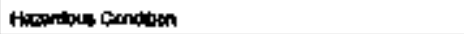 & Cann & Contecyuenist \\
\hline MpQE-WTEGA & 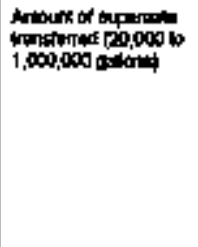 & 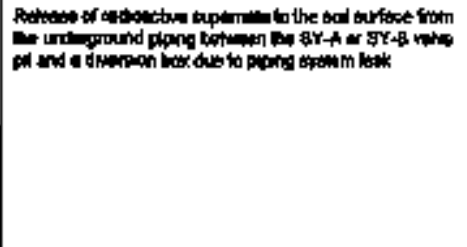 & 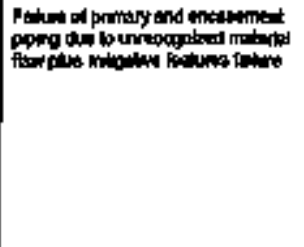 & 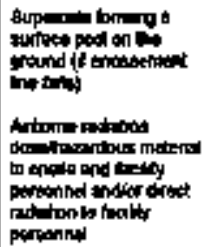 \\
\hline nKSE-4TEG12 & 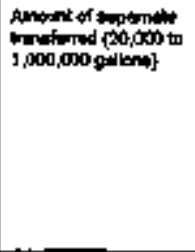 & 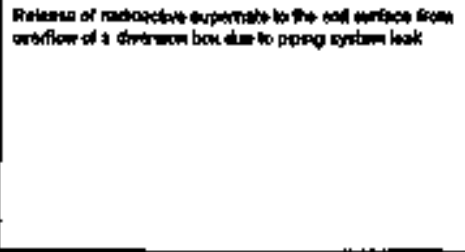 & 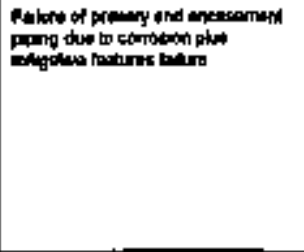 & 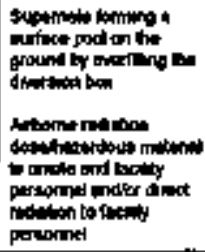 \\
\hline WOGPNNIES13d & 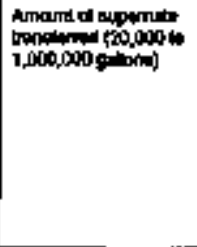 & 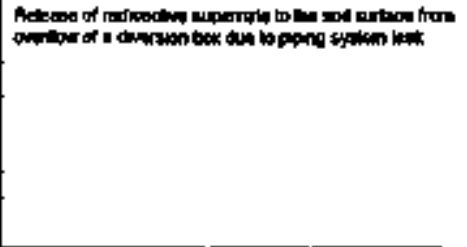 & 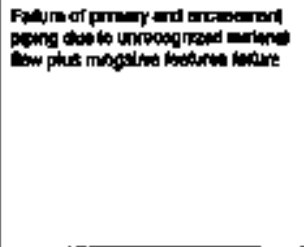 & 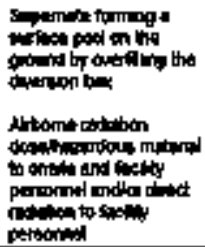 \\
\hline
\end{tabular}




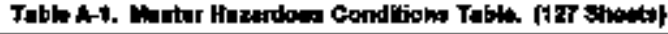

\begin{tabular}{|c|c|c|c|c|}
\hline ID & 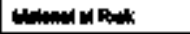 & 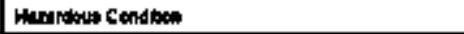 & Cenne & 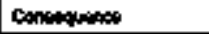 \\
\hline WhophriteGIA & 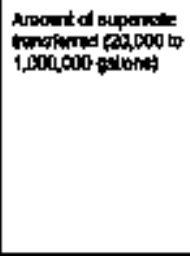 & 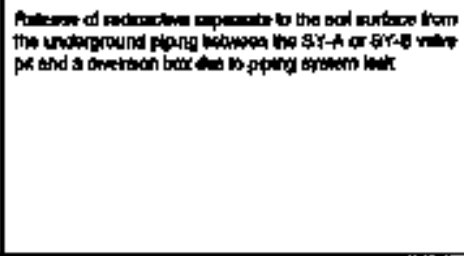 & 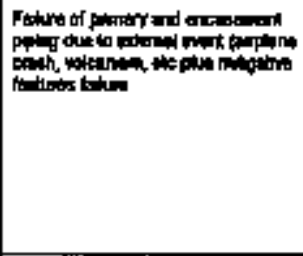 & 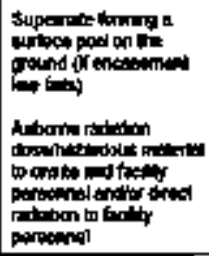 \\
\hline WOAL-INTEGAdb & 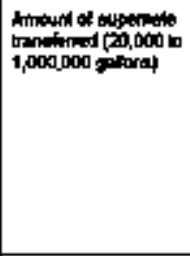 & 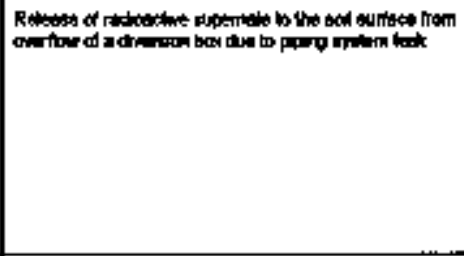 & 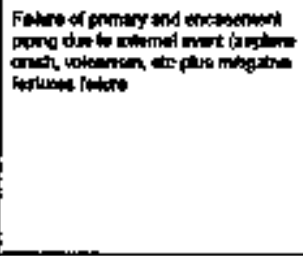 & 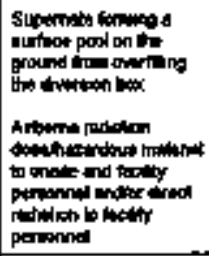 \\
\hline ИPSS-WTEBTH & 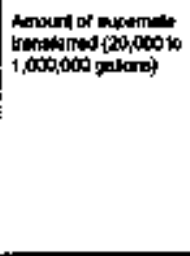 & 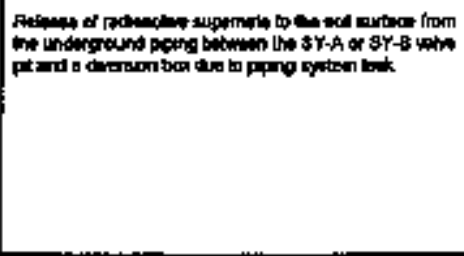 & 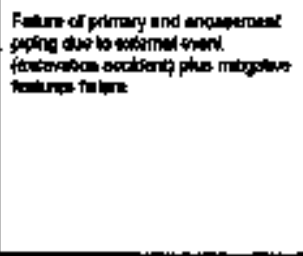 & 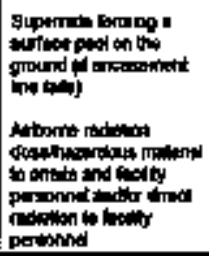 \\
\hline
\end{tabular}




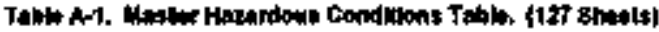

\begin{tabular}{|c|c|c|c|c|}
\hline $\mathbf{D}$ & : & 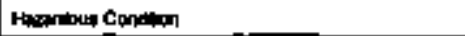 & ch & F्वाaptipt \\
\hline 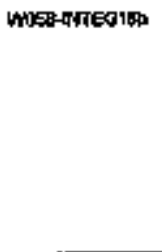 & 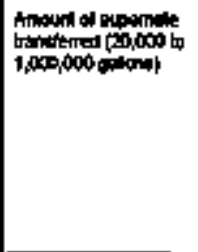 & 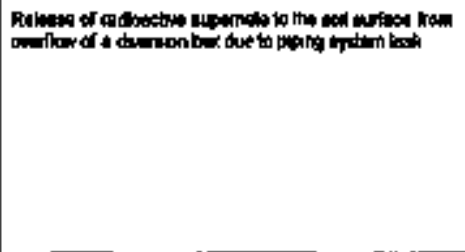 & 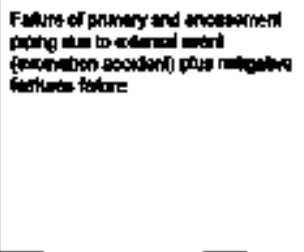 & 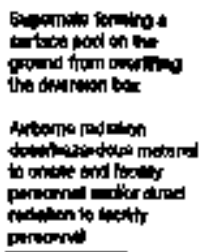 \\
\hline 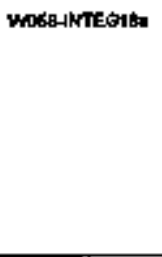 & 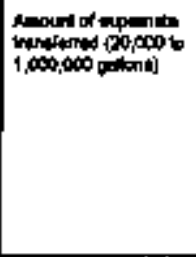 & 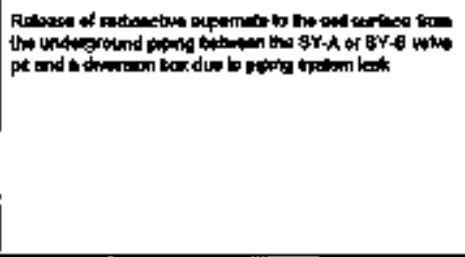 & 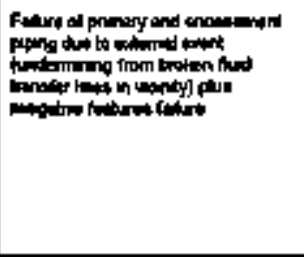 & 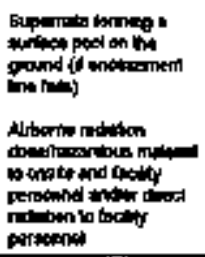 \\
\hline MUSOINTEOAR & 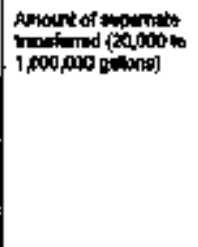 & 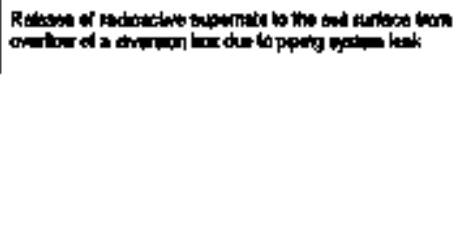 & 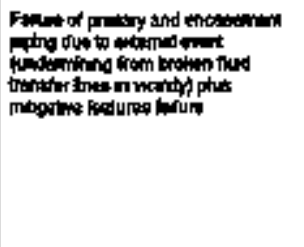 & 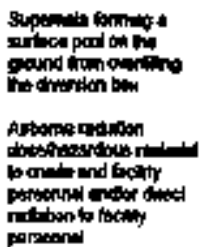 \\
\hline
\end{tabular}




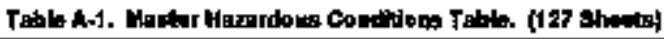

\begin{tabular}{|c|c|c|c|c|}
\hline ID & 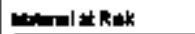 & Momplow Gondthoth & Caves & 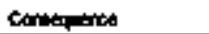 \\
\hline 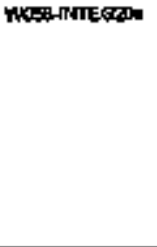 & 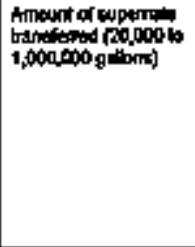 & 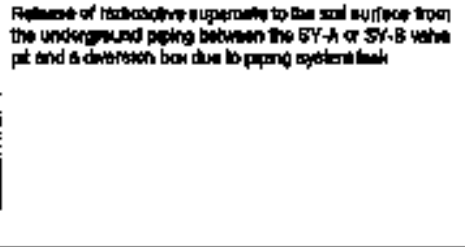 & 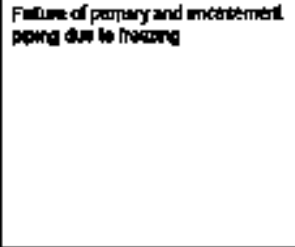 & 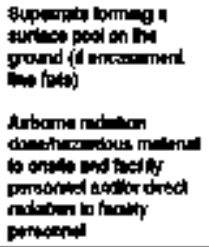 \\
\hline WW:AlNTEGSt & 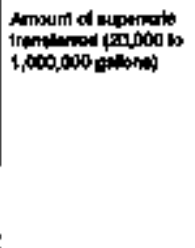 & 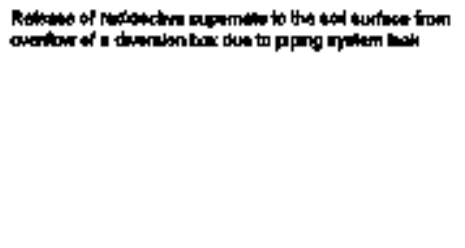 & 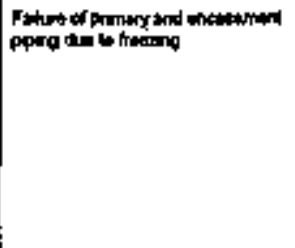 & 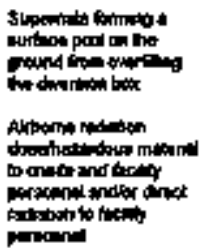 \\
\hline MOSBHTECD2 & 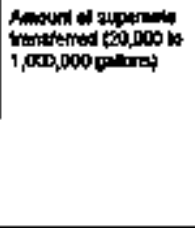 & 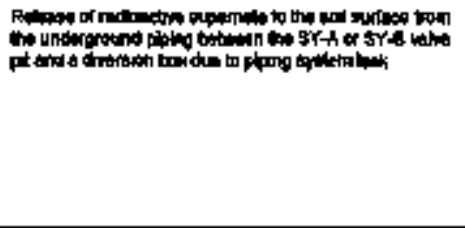 & 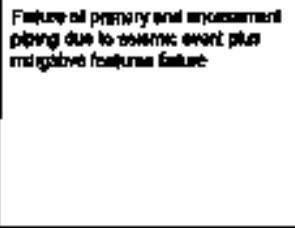 & 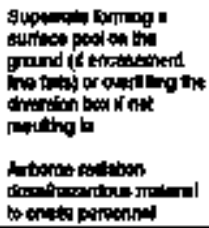 \\
\hline
\end{tabular}




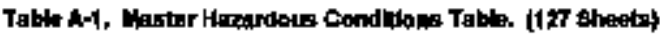

\begin{tabular}{|c|c|c|c|c|}
\hline $\boldsymbol{n}$ & 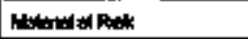 & Hessovitust Contiben & Covos & Coptraquence \\
\hline Motostect24 & 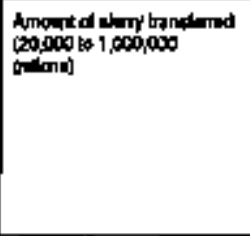 & 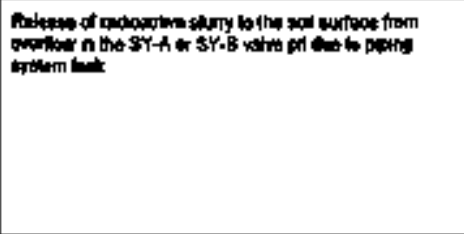 & 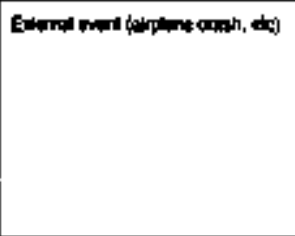 & 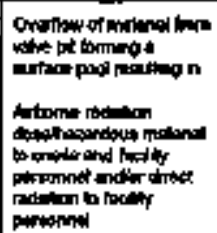 \\
\hline MUSP-WT:6as & 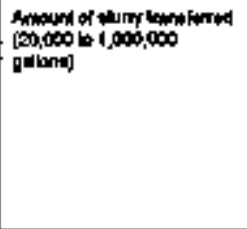 & 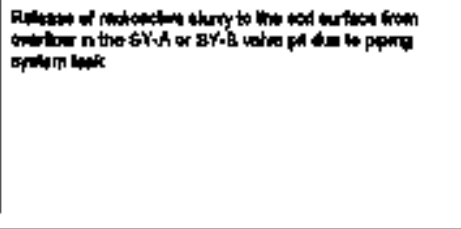 & 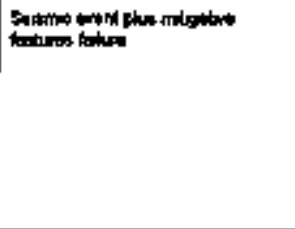 & 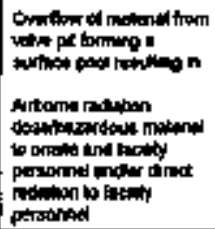 \\
\hline MASEWTEGEH & 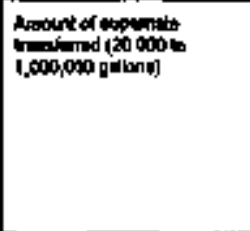 & 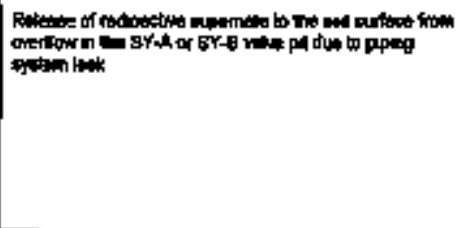 & 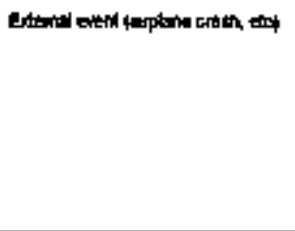 & 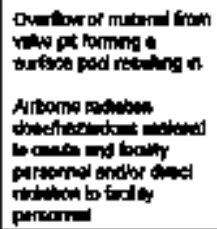 \\
\hline
\end{tabular}




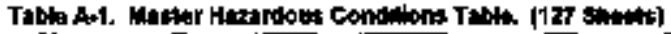

\begin{tabular}{|c|c|c|c|c|}
\hline 10 & Nonondat Risk & Heverseus condton & Conukt & Conewipunos \\
\hline HNSSWTEG: & 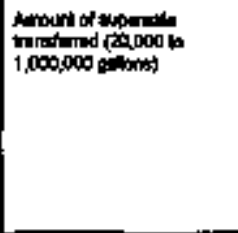 & 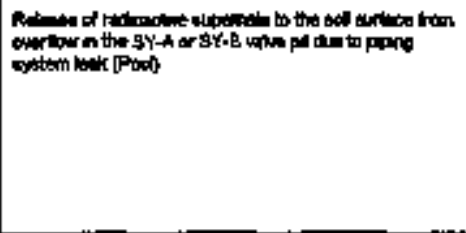 & setent mint & 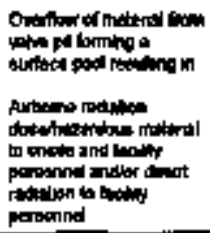 \\
\hline nutamerterato & 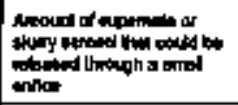 & 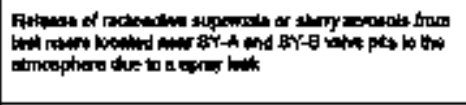 & 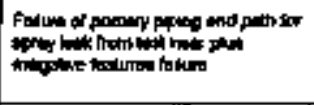 & 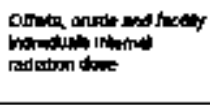 \\
\hline WMGA-WTECOß & Anover of shm & 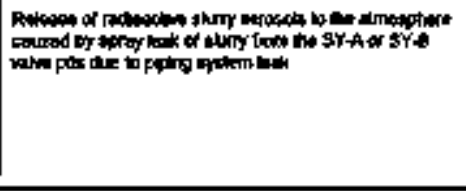 & 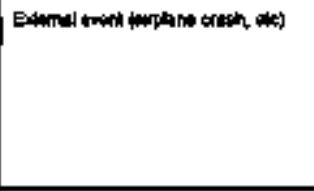 & 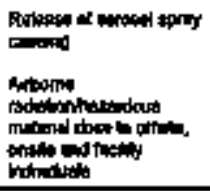 \\
\hline Wost.|NTECSS & 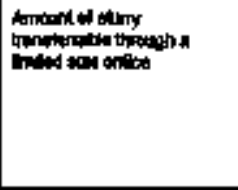 & 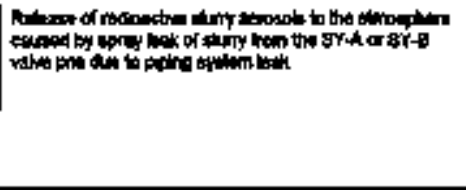 & seming anit & 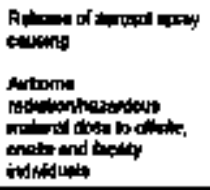 \\
\hline nosewneas & Amolit of womitis & 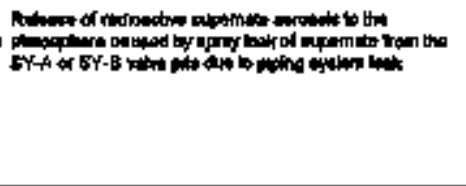 & 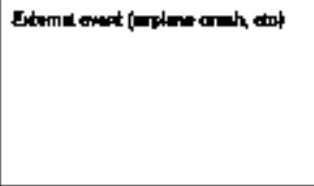 & 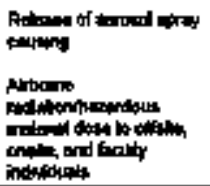 \\
\hline
\end{tabular}




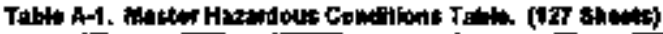

\begin{tabular}{|c|c|c|c|c|}
\hline It & 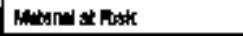 & Heradow Constion & Conas & Comonquenct \\
\hline 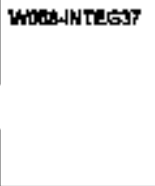 & 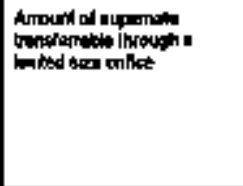 & 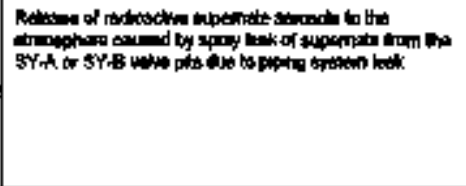 & 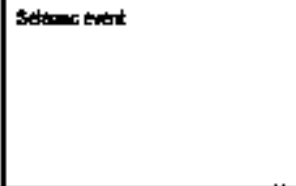 & 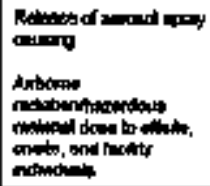 \\
\hline woak-ratest & 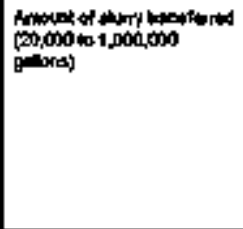 & 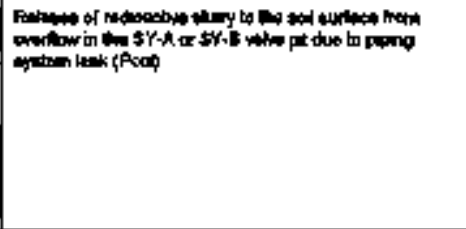 & 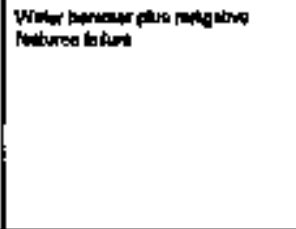 & 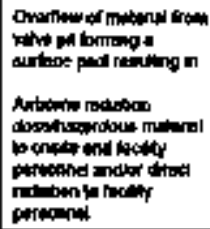 \\
\hline mosenerisgt3 & 1, & 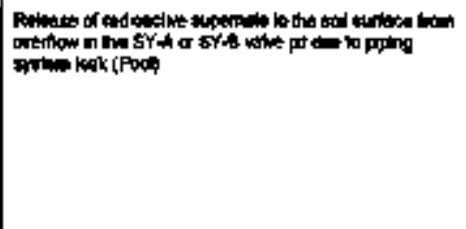 & 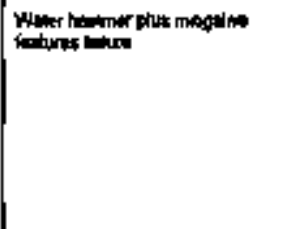 & 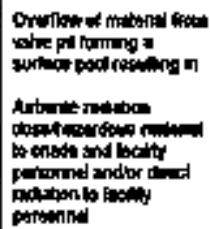 \\
\hline Xto-11-ALounts & 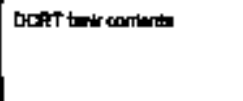 & 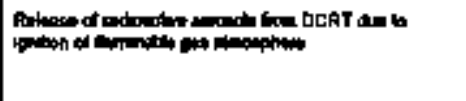 & 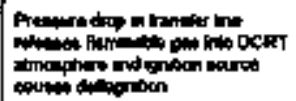 & 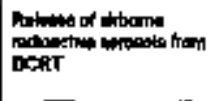 \\
\hline xS-OHFLOWOZ2 & 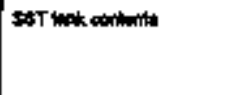 & 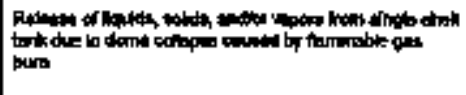 & 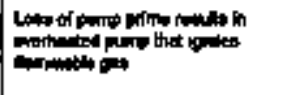 & 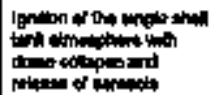 \\
\hline
\end{tabular}




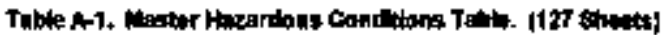

\begin{tabular}{|c|c|c|c|c|}
\hline it & 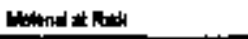 & 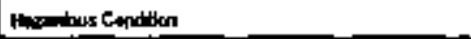 & A & Domentanes \\
\hline X5.01-rountiot & gist add bCRT mk & 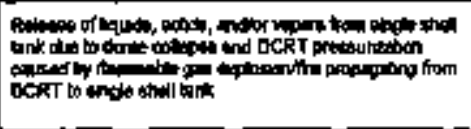 & 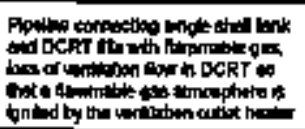 & 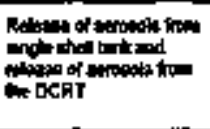 \\
\hline 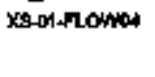 & 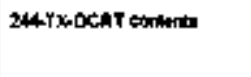 & 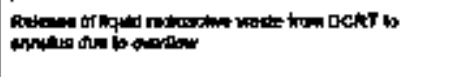 & 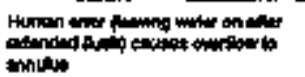 & 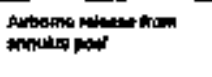 \\
\hline Xs-OMALONAS & 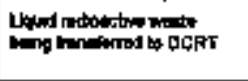 & 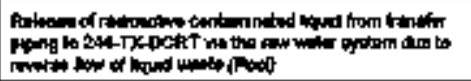 & 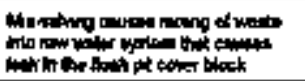 & 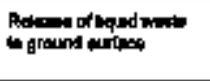 \\
\hline x:Al-nlowne & 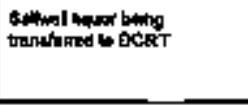 & 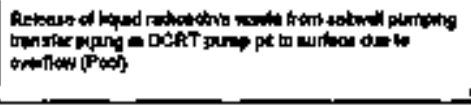 & 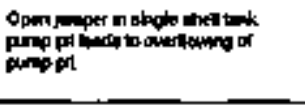 & 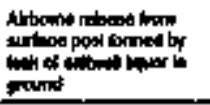 \\
\hline Xs-a1fLónth & 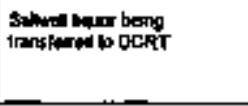 & 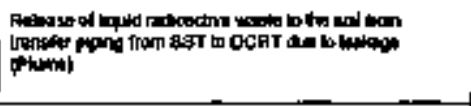 & 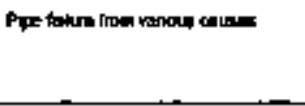 & 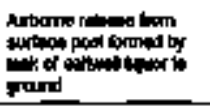 \\
\hline 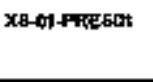 & 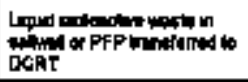 & 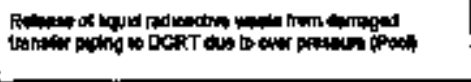 & 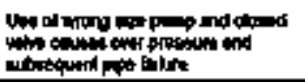 & 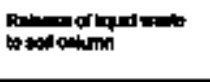 \\
\hline 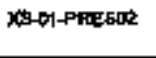 & stompurtaing & 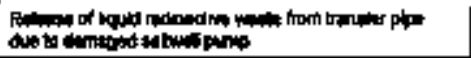 & 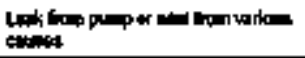 & 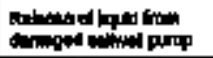 \\
\hline KS-A-TEMPOH & 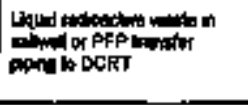 & 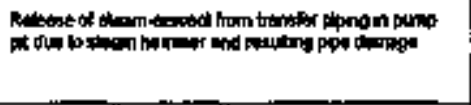 & 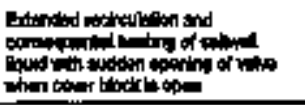 & 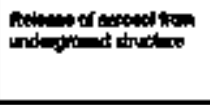 \\
\hline 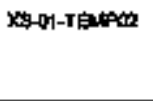 & 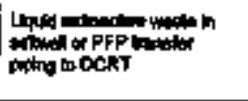 & 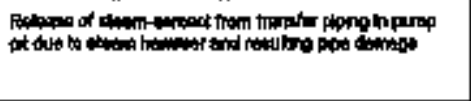 & 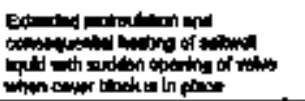 & 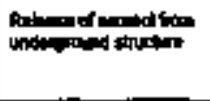 \\
\hline
\end{tabular}




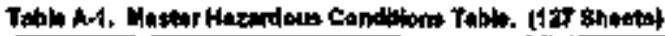

\begin{tabular}{|c|c|c|c|c|}
\hline 10 & 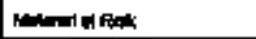 & Hsaribus condun & cenpe. & Canseremente \\
\hline Xs:-12-flophor & 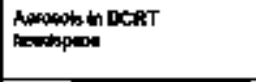 & 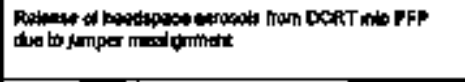 & 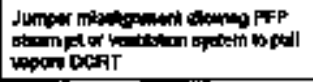 & 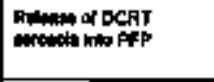 \\
\hline Xs-ib-Flowno & 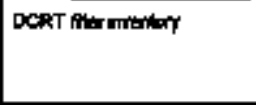 & 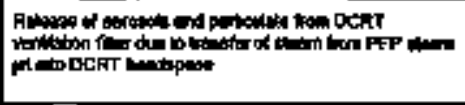 & 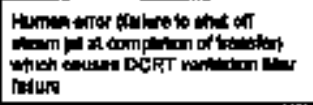 & 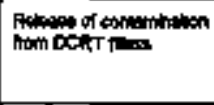 \\
\hline XS-OB.PLOWMB & 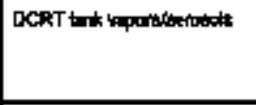 & 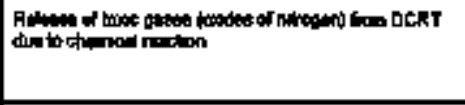 & 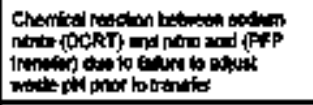 & Alwow of adeact \\
\hline xh-onstound & 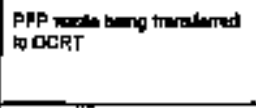 & 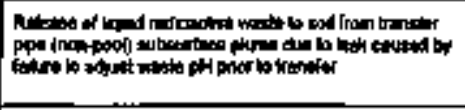 & 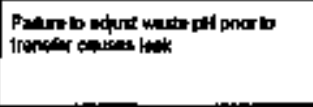 & 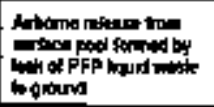 \\
\hline XE-0QRTCUAS & 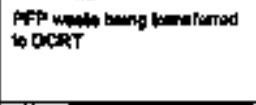 & 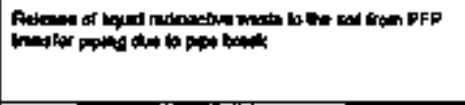 & 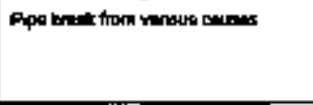 & 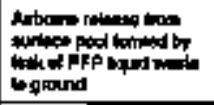 \\
\hline yo-0s-rondod & 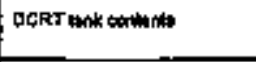 & 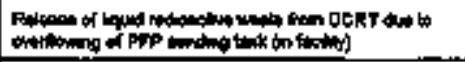 & 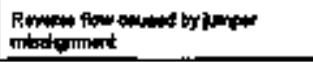 & Lapur nation in PFP \\
\hline NG-03-LEY & OtefT lank bentats & 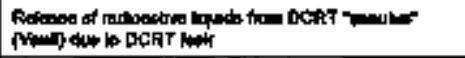 & 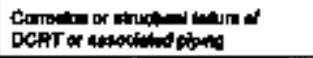 & 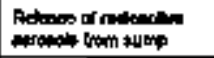 \\
\hline 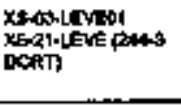 & 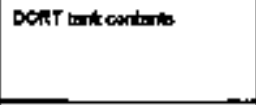 & 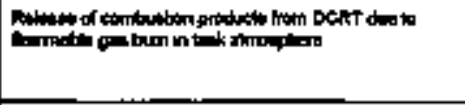 & 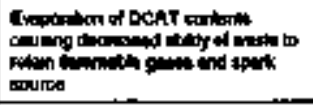 & DGRT \\
\hline 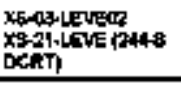 & 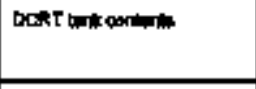 & 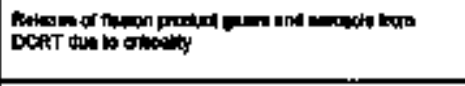 & 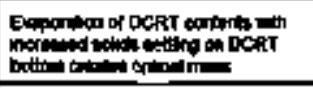 & 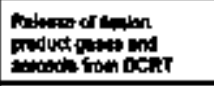 \\
\hline 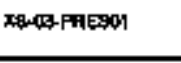 & DCHT wophuraseis & 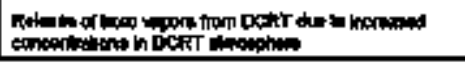 & 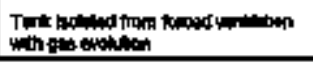 & 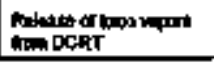 \\
\hline
\end{tabular}




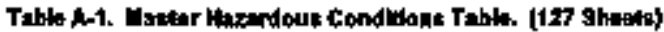

\begin{tabular}{|c|c|c|c|c|}
\hline ID & Athorimat font & Hergitors Endiban & tauss & Conechuenos \\
\hline 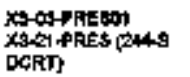 & 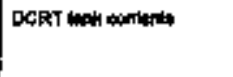 & 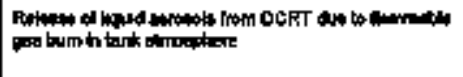 & 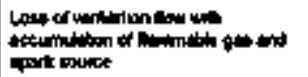 & 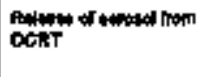 \\
\hline 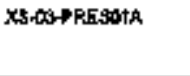 & 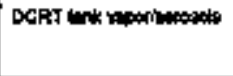 & 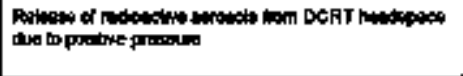 & 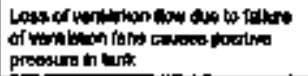 & 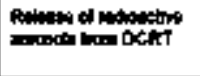 \\
\hline XS-COAPREA02 & 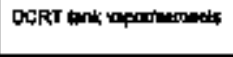 & 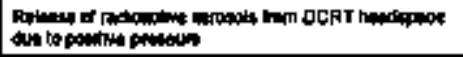 & 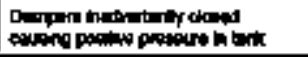 & 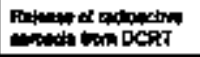 \\
\hline 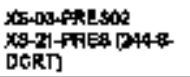 & DCRT bink ranterts & 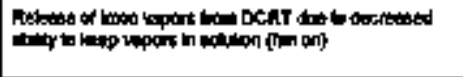 & 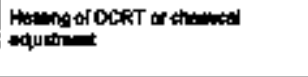 & 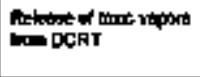 \\
\hline 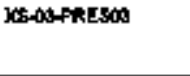 & 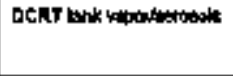 & 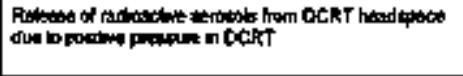 & 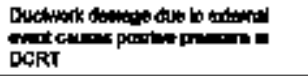 & 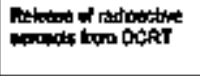 \\
\hline 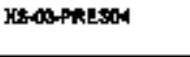 & 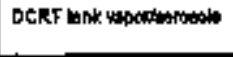 & 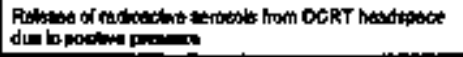 & 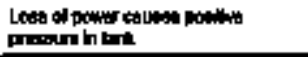 & 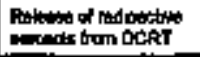 \\
\hline XE-03_FRESAR & 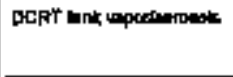 & 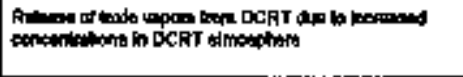 & 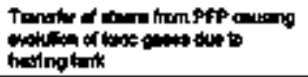 & 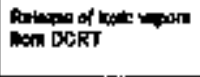 \\
\hline XE-03-TEЕศO1 & GCAT Wnk Dem & 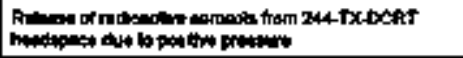 & 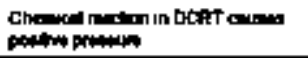 & 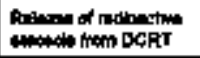 \\
\hline 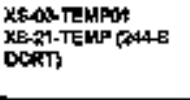 & 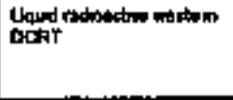 & 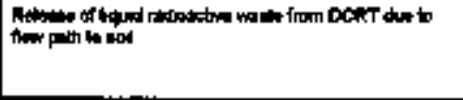 & 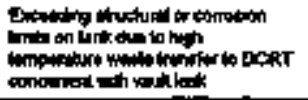 & 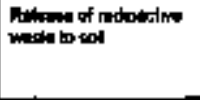 \\
\hline XE-09-TELPQD & 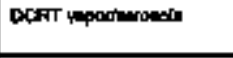 & 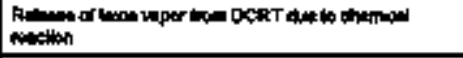 & 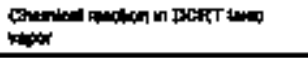 & 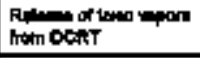 \\
\hline 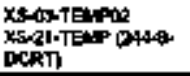 & CCRT tatemitats & 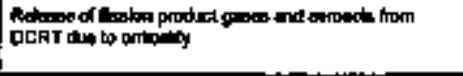 & 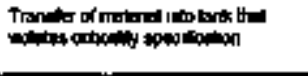 & 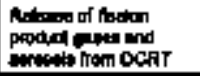 \\
\hline
\end{tabular}




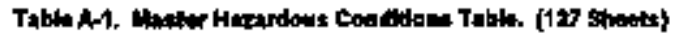

\begin{tabular}{|c|c|c|c|c|}
\hline $\mathbf{b}$ & ther at & Hontbul Conim & Corne & Canmpuman \\
\hline 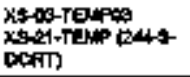 & 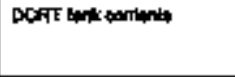 & 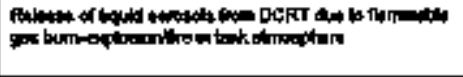 & 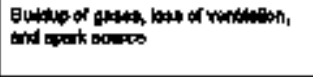 & 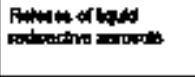 \\
\hline 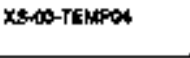 & OCRT toontentionto & 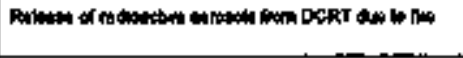 & 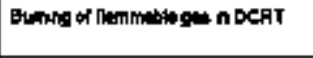 & 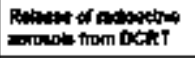 \\
\hline 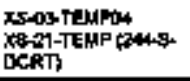 & CCkT tonement & 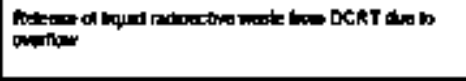 & 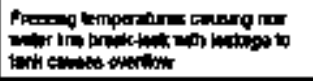 & 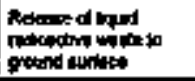 \\
\hline XS-OBSTEMPOS & 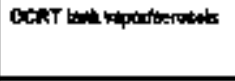 & 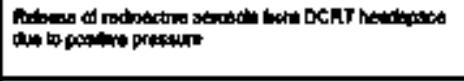 & 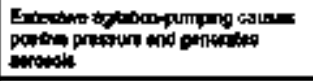 & 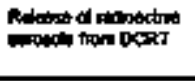 \\
\hline XE-AS-TEHWOA & 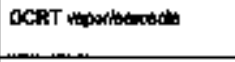 & 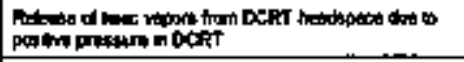 & 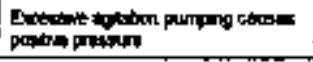 & 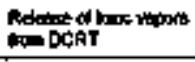 \\
\hline Xg-OSTEMPOP & 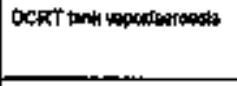 & 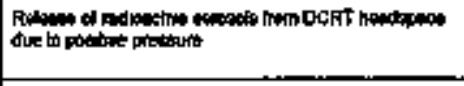 & 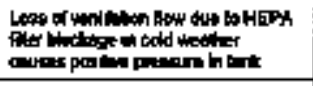 & 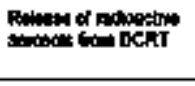 \\
\hline 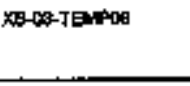 & 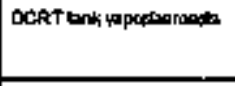 & 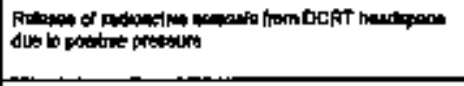 & 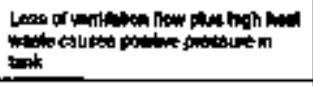 & 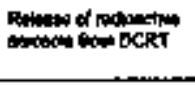 \\
\hline XE-0s-TENon & 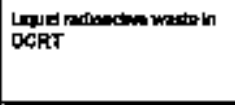 & 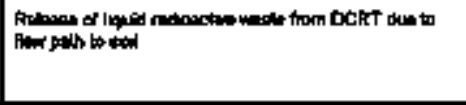 & 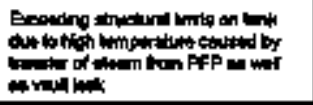 & 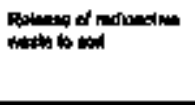 \\
\hline 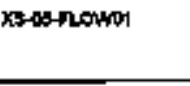 & 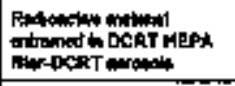 & 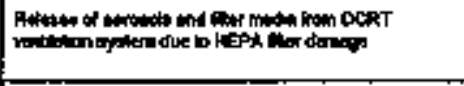 & 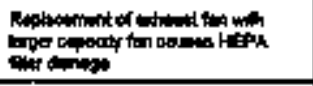 & 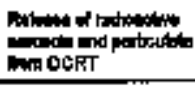 \\
\hline$x=-\infty \operatorname{now} 2$ & 管 & 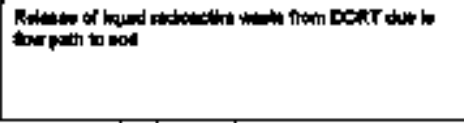 & 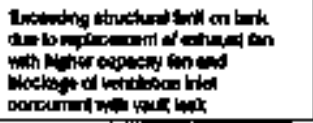 & 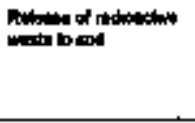 \\
\hline
\end{tabular}




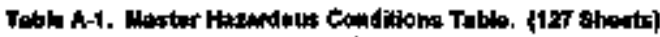

\begin{tabular}{|c|c|c|c|c|}
\hline ID & 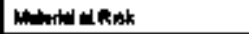 & 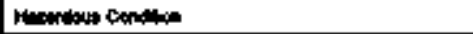 & مشاب & Conaceanken \\
\hline XE-60-Tuted & 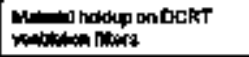 & 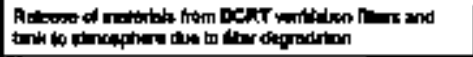 & 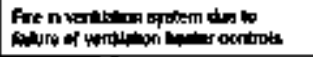 & 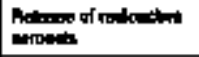 \\
\hline X\$-0S-TENPQ2 & 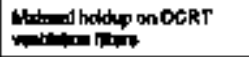 & 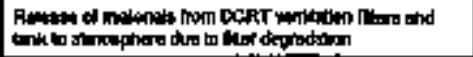 & 货 $n$ & 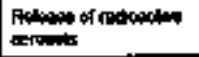 \\
\hline XSAOFLOMUI & DCFT tant & 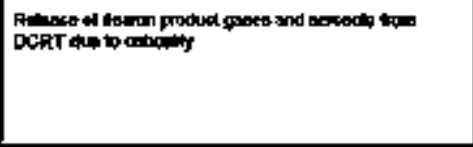 & 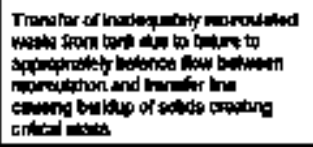 & 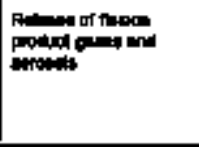 \\
\hline Xs-0s-FLovines & OCAT nots beng & 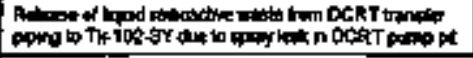 & 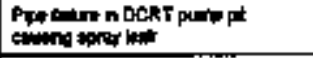 & 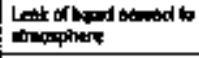 \\
\hline X5-00-FLOW03 & 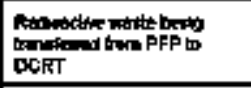 & 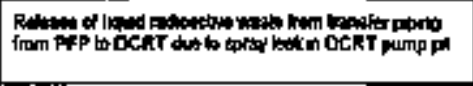 & Potefidit n DCRE pump pt & 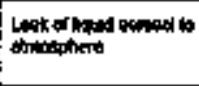 \\
\hline xभ-09-7LWM & 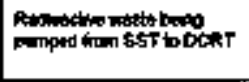 & 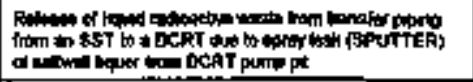 & Fostion & 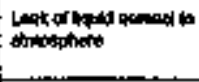 \\
\hline xg-03ptowns & 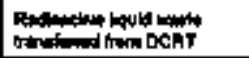 & 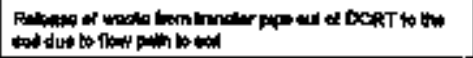 & 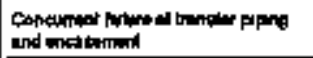 & 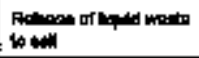 \\
\hline Xs-0s-atownd & GCRT tonk conterita & 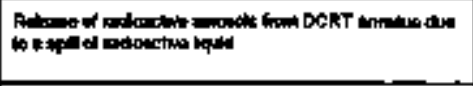 & 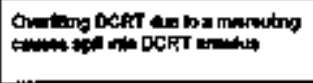 & 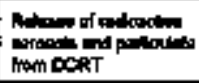 \\
\hline Xtannowner & 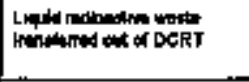 & 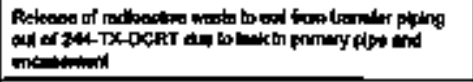 & 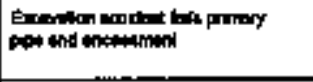 & 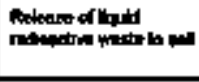 \\
\hline Xstofottenting & 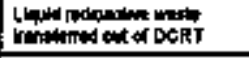 & 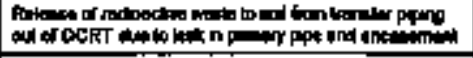 & 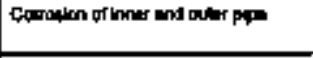 & 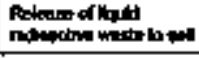 \\
\hline 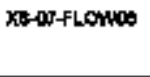 & 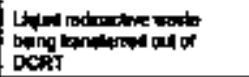 & 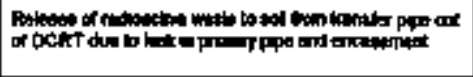 & 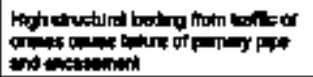 & 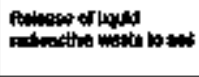 \\
\hline
\end{tabular}




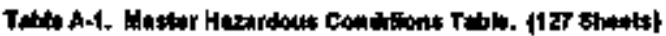

\begin{tabular}{|c|c|c|c|c|}
\hline ID & Hatenol at Rour & Hogedous Candion & Cowat & Corabopintion \\
\hline xs=07 tolotinat & 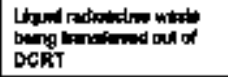 & 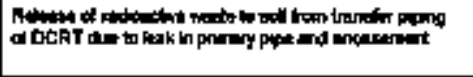 & 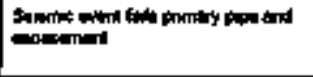 & 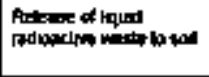 \\
\hline xs-uTfatound & 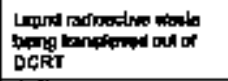 & 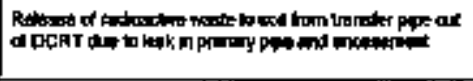 & 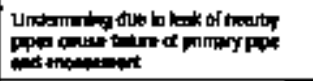 & 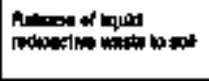 \\
\hline xS-or flound & 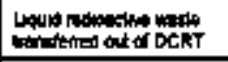 & 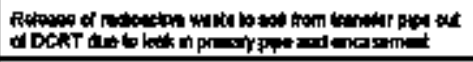 & 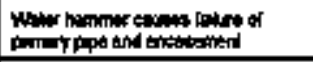 & 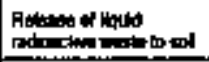 \\
\hline xg-11 LEYEM & 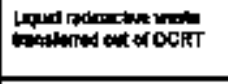 & 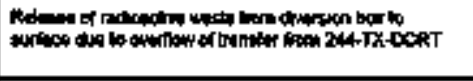 & 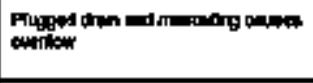 & 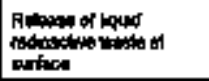 \\
\hline 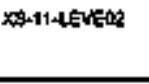 & 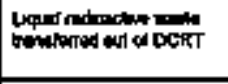 & 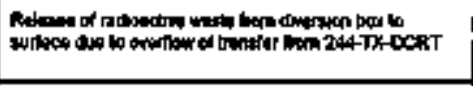 & 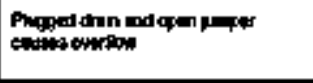 & 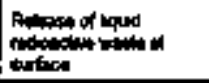 \\
\hline 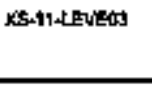 & 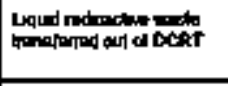 & 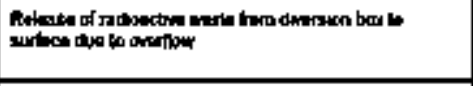 & 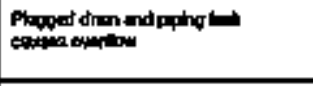 & 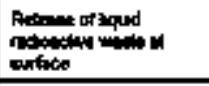 \\
\hline X9.42nochno: & 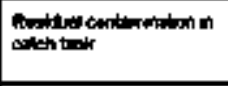 & 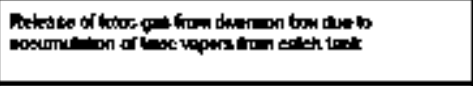 & 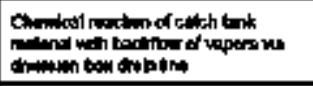 & 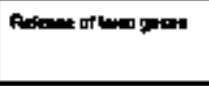 \\
\hline$x=12$ MONnos & 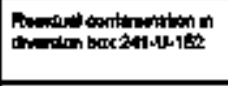 & 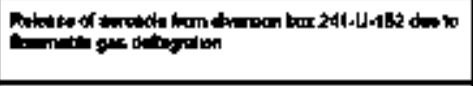 & 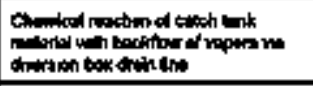 & 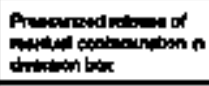 \\
\hline$x=12$,Plowes & 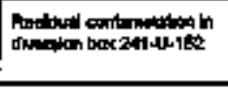 & 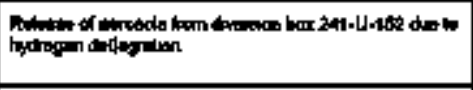 & 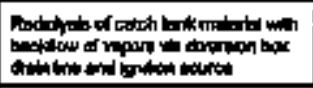 & 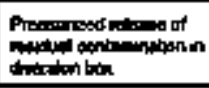 \\
\hline 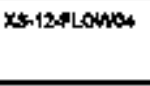 & 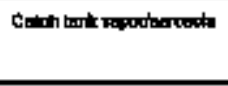 & 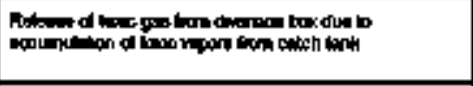 & 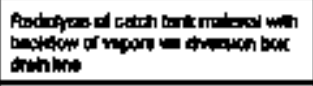 & 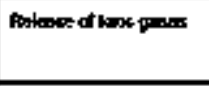 \\
\hline xstizenowits & 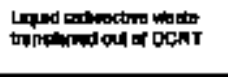 & 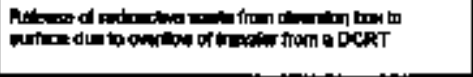 & 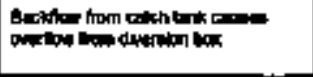 & 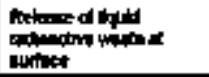 \\
\hline
\end{tabular}




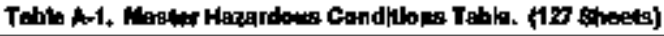

\begin{tabular}{|c|c|c|c|c|}
\hline $\boldsymbol{\varphi}$ & Numal at Risk & 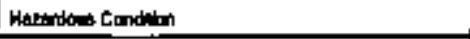 & cour & Cantenguthet \\
\hline XS-12-FLDMNOS & 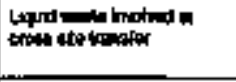 & 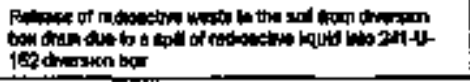 & 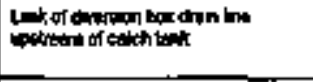 & 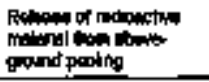 \\
\hline YB-13LEVE & 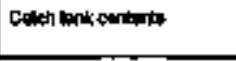 & 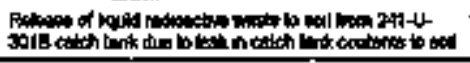 & 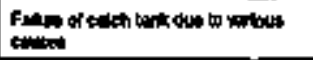 & 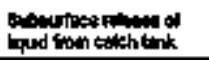 \\
\hline Xts-13-RESPO1 & 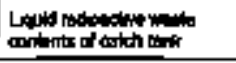 & 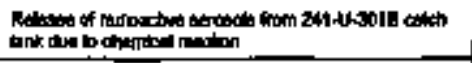 & 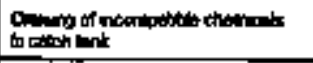 & 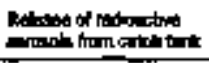 \\
\hline XS.13-PFGSOQ & 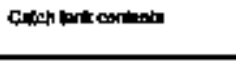 & 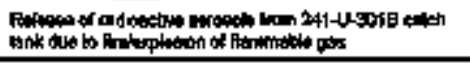 & 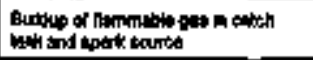 & 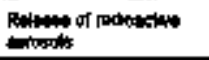 \\
\hline xa-13pindsos & 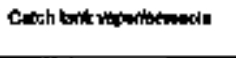 & 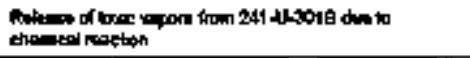 & 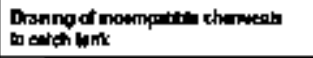 & 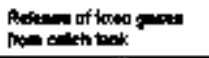 \\
\hline xs-19-PRaspor & 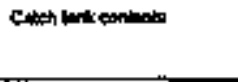 & 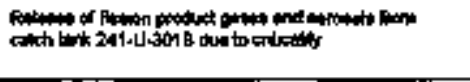 & 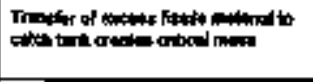 & 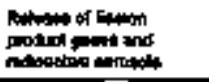 \\
\hline X6-20月1:DWOH & 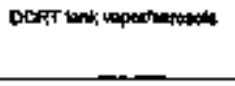 & 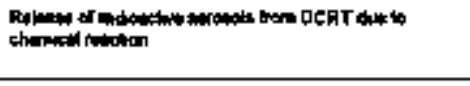 & 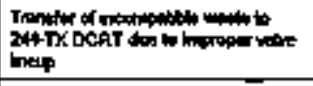 & 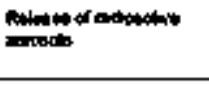 \\
\hline 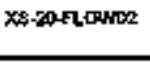 & 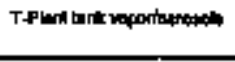 & 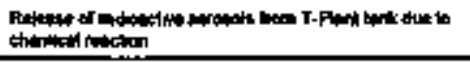 & 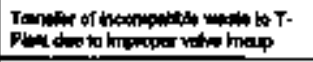 & 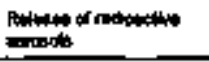 \\
\hline$x \operatorname{senthouns}$ & 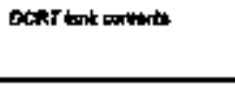 & 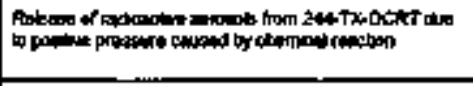 & 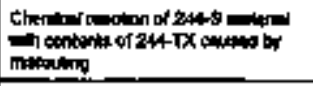 & 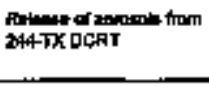 \\
\hline 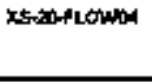 & CCAT & 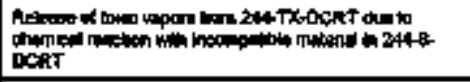 & 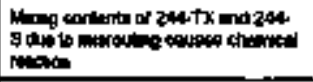 & 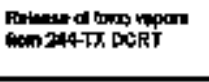 \\
\hline 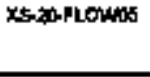 & 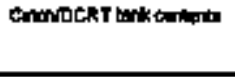 & 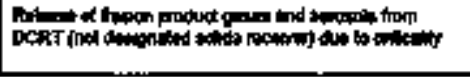 & 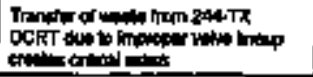 & 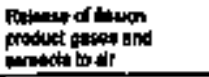 \\
\hline
\end{tabular}




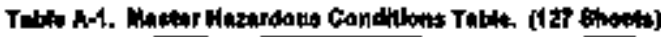

\begin{tabular}{|c|c|c|c|c|}
\hline$\omega$ & 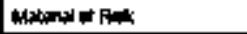 & Mandeus Condion & couse & Cenumpence \\
\hline 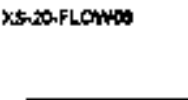 & 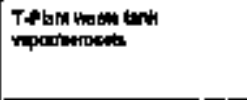 & 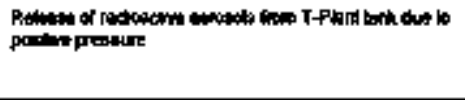 & 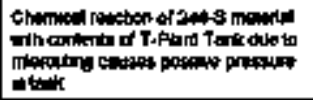 & 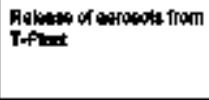 \\
\hline 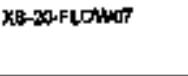 & 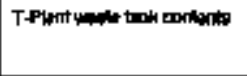 & 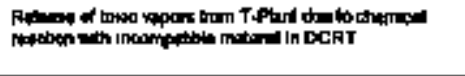 & 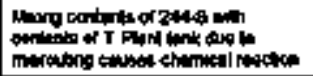 & 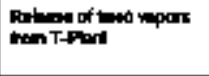 \\
\hline 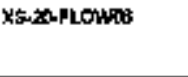 & 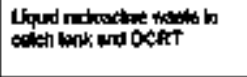 & 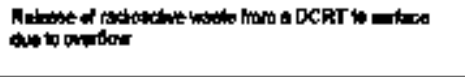 & 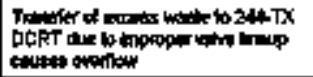 & 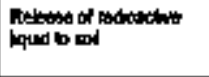 \\
\hline xtsarlonke & 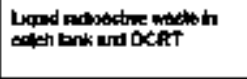 & 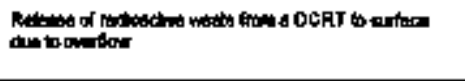 & 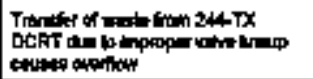 & 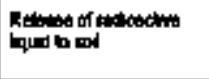 \\
\hline 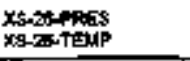 & 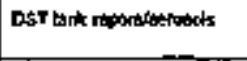 & 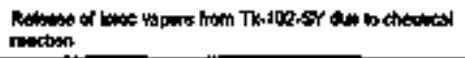 & 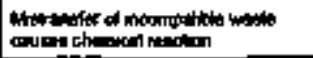 & 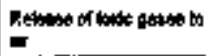 \\
\hline 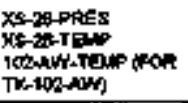 & DST Larikentarits & 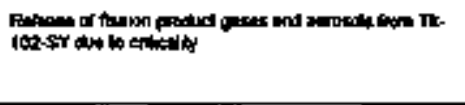 & 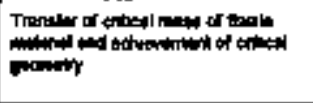 & 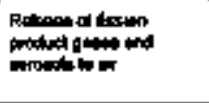 \\
\hline XE-2A-PREESOI & 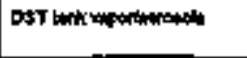 & 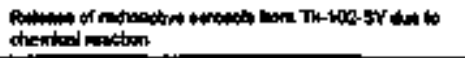 & 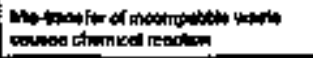 & 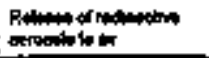 \\
\hline 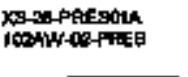 & 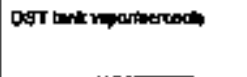 & 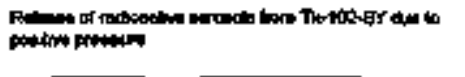 & 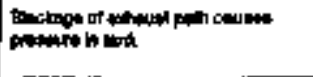 & 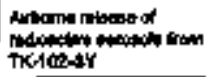 \\
\hline XEOAOAFSLC & DQT Ink conturits & 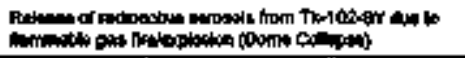 & 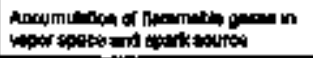 & 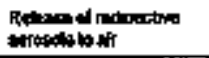 \\
\hline 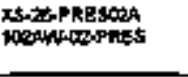 & 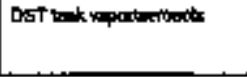 & 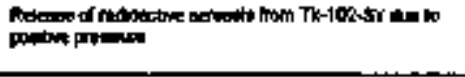 & 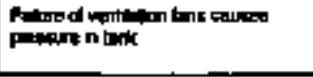 & 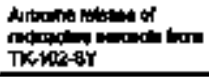 \\
\hline
\end{tabular}




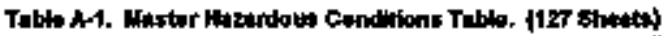

\begin{tabular}{|c|c|c|c|c|}
\hline $\boldsymbol{D}$ & Mumoer of Rak & Havolua Gendibon & cantin & Conoweunes \\
\hline 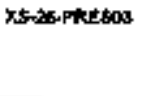 & 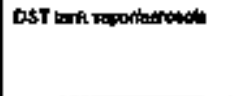 & 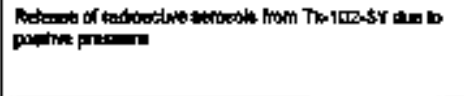 & 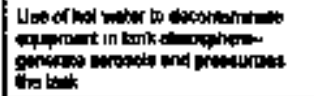 & 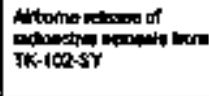 \\
\hline XE-30-PRT906 & 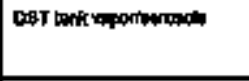 & 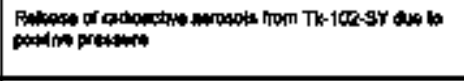 & 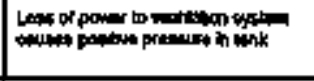 & 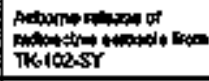 \\
\hline $\begin{array}{l}\text { XS.2ATELP } \\
\text { 102AW-OBMTEMP }\end{array}$ & 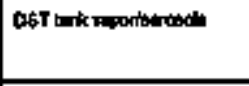 & 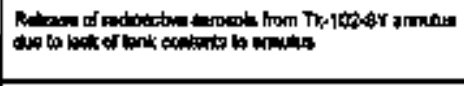 & 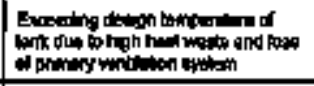 & 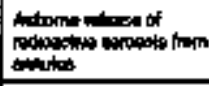 \\
\hline 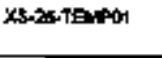 & 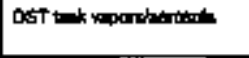 & 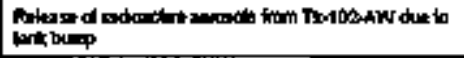 & 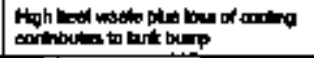 & 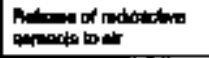 \\
\hline$x$ stit-Tentios & 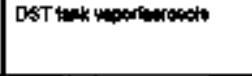 & 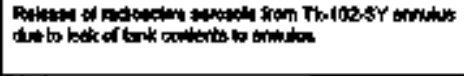 & 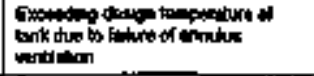 & 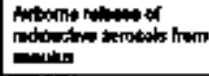 \\
\hline xs-34nowit & 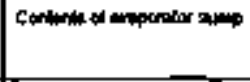 & 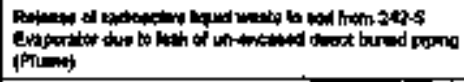 & 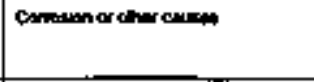 & 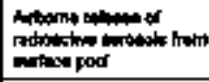 \\
\hline$x \cos +100 \mathrm{nin}$ & 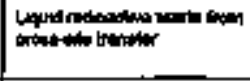 & 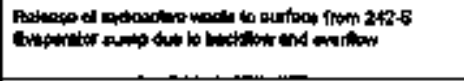 & 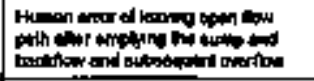 & 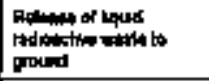 \\
\hline XE-37-FLOWNO1 & 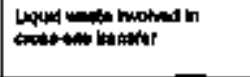 & 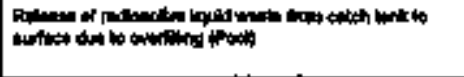 & 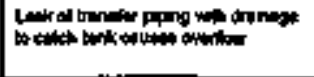 & 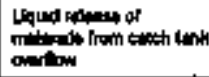 \\
\hline xBS-sy-fLorw02 & 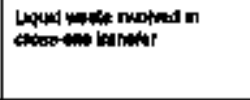 & 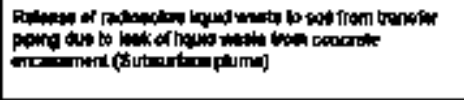 & 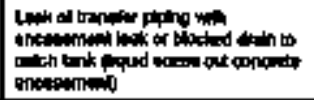 & 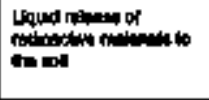 \\
\hline XE-41-10MAM & 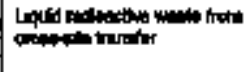 & 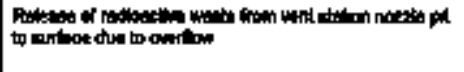 & 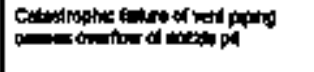 & 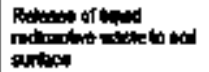 \\
\hline
\end{tabular}




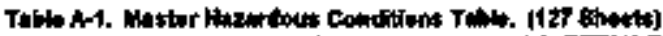

\begin{tabular}{|c|c|c|c|c|}
\hline I & 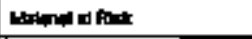 & Hansording cendibon & Cenas & 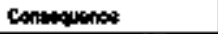 \\
\hline Xstinnothite & 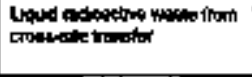 & 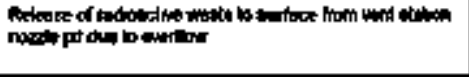 & 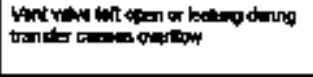 & 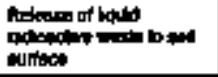 \\
\hline 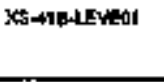 & 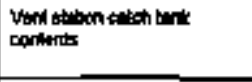 & 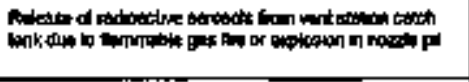 & 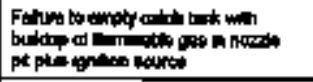 & 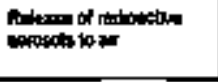 \\
\hline XS-41B-FYAR2 & 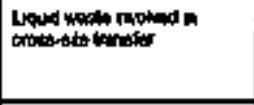 & 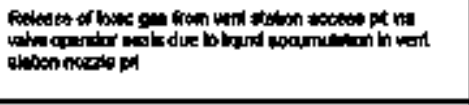 & 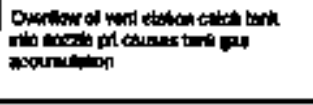 & 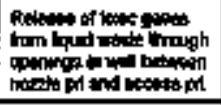 \\
\hline Xst41B-LErens & 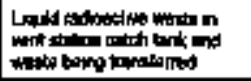 & 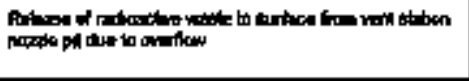 & 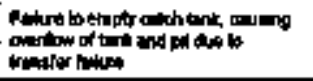 & 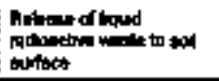 \\
\hline Xs-41 B-LEVEC4 & 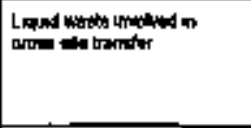 & 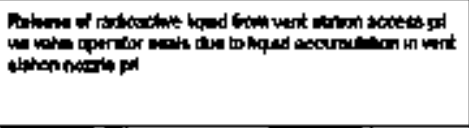 & 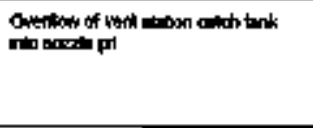 & 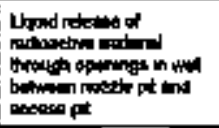 \\
\hline XB-AIC-LEKE & 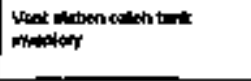 & 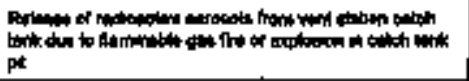 & 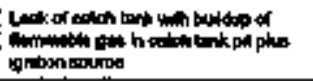 & 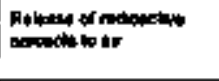 \\
\hline$x 8$-NC-LEVEC & 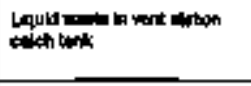 & 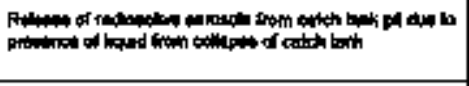 & 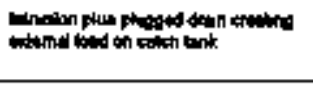 & 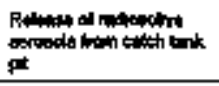 \\
\hline X8-N)C-LEVEC9 & 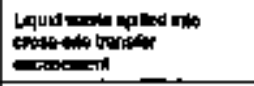 & 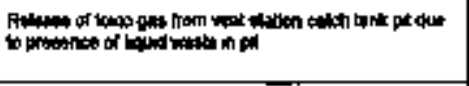 & 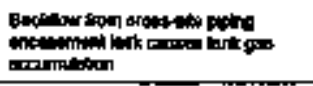 & 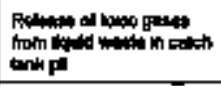 \\
\hline X89-flon & 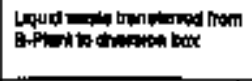 & 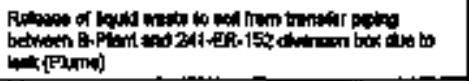 & 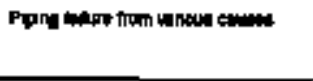 & 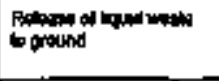 \\
\hline 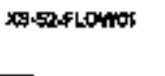 & 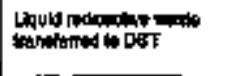 & 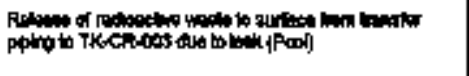 & 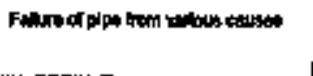 & 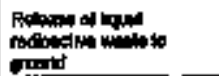 \\
\hline
\end{tabular}




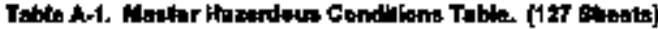

\begin{tabular}{|c|c|c|c|c|}
\hline ID & Whated of th & Hoceodons tontulat & couser & Goptapequares \\
\hline XEA & 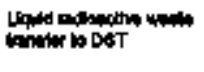 & 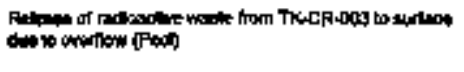 & 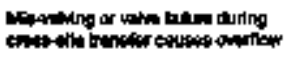 & 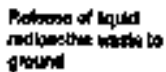 \\
\hline
\end{tabular}


HNF-SD-WHATI.764, Rev. 1

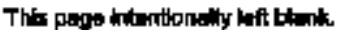




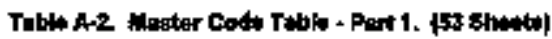

\begin{tabular}{|c|c|c|c|c|c|c|c|c|c|c|c|c|c|c|c|}
\hline ID & $\begin{array}{l}\text { Intiod } \\
\text { Sthit } \\
\text { Car } \\
\text { NC }\end{array}$ & $\underset{n=6}{n=1}$ & $\operatorname{ent}_{\cos }$ & Bay & 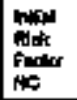 & 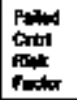 & condy & Con & Rer & 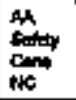 & $\underset{f \times C}{M}$ & the & Fing & 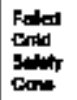 & $\begin{array}{l}\text { fatilud } \\
\text { Find } \\
\text { Find }\end{array}$ \\
\hline 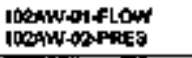 & $\mathbf{w}$ & 70 & Es & $\ln =19$ & 10 & s & $\mathbf{Y}$ & 800 & $\tan$ & st2 & Fo & Aanentied & Properted & 82 & fa \\
\hline 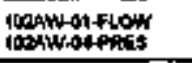 & \$3 & Fo & $\mathbf{E}$ & e-14 & 10 & 15 & $\mathbf{m o}$ & $\infty$ & $15 x$ & & & $\Leftrightarrow$ & *9 & $\$$ & $\mathbf{n}$ \\
\hline 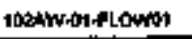 & ss & $n$ & nz & t-10 & 19 & 16 & $\mathrm{FQ}$ & Don & $15 x$ & $s$ & rs & 92 & $\mathbf{F a}$ & 6 & $\mathbf{r z}$ \\
\hline 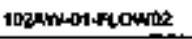 & $\$ z$ & $\mathbf{n}$ & 라 & - $-2-4$ & 14 & 13 & F & 022 & $21 x$ & $\mathbf{5 3}$ & $\mathbf{F u}$ & Promantiod & Propions & \$3 & $\mathbf{r 1}$ \\
\hline nazAn-01 flowns & 8 & $\mathrm{~F}$ & 다 & D.3 b & 14 & 13 & $\boldsymbol{p}$ & D16 & $21 x$ & $\$ 3$ & $\mathrm{FP}$ & 81 & $\mathbf{F}$ & $\$ 3$ & $\mathbf{F 1}$ \\
\hline WOAM-OHFAOW04 & $\$ 7$ & $F$ & E2 & asb & 14 & 10 & PO & Der & $\sin$ & $\$$ & $\mathbf{P r}$ & 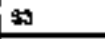 & $\mathbf{F o}$ & $\$ 3$ & ro \\
\hline 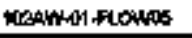 & 82 & $\mathbf{F 2}$ & E2 & intb & 13 & 13 & $\mathrm{PO}$ & Dat & $31 x$ & 59 & $\mathbf{F}$ & $\$ 1$ & $\mathbf{F}$ & ss & n \\
\hline 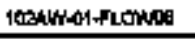 & s2 & rs & e2 & thes & 14 & 13 & $P D$ & Mi & $21 x$ & 8 & Ps & si & $\mathbf{F}$ & 93 & $n$ \\
\hline 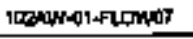 & az & $B$ & 12 & $0-3-p$ & 14 & 13 & Pt & D17 & $21 x$ & \$9 & $F 3$ & B1 & F & 8 & $\mathbf{F 1}$ \\
\hline 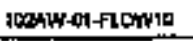 & $\mathbf{g}$ & $\mathbf{F z}$ & $\underline{E 2}$ & $c-2 b$ & 12 & 19 & $\mathrm{~T}$ & Ex & $20 x$ & $\mathbf{6 9}$ & F3 & 81 & P\$ & 50 & F1 \\
\hline 10arw-01+Row11 & 92 & F3 & Ex & 020 & 14 & 13 & $T$ & 100 & $2 x$ & 59 & $\mathbf{F s}$ & 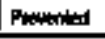 & Pronumad & $\$ 3$ & $\mathbf{F 1}$ \\
\hline Joshw-ol-Tenp & \$ & $\mathbf{r 1}$ & 53 & 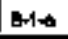 & 13 & 13 & $\mathbf{P D}$ & DoI & $16 x$ & ss & ps & Punented & Prominted & $\mathbf{s}$ & $\mathbf{m}$ \\
\hline 10\%AW-A1-TEMART & ss & F. & E2 & $+2+b$ & 12 & 13 & Wo & DO1 & $2 \pi x$ & $\$$ & F3. & \$1 & $\mathrm{FB}$ & 8 & F1 \\
\hline 102НHИ-O1-TEMPO1A & 8 & F2 & E2 & C-2 & 12 & 19 & mo & Dot & $20 x$ & 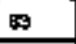 & $\mathbf{F 3}$ & 81 & $F$ & 6 & F1 \\
\hline 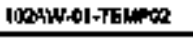 & 52 & $\mathbf{F}$ & E2 & 524 & 12 & 13 & wo & DaI & $2 \operatorname{sex}$ & sa & $\mathbf{F s}$ & si & rs & 6 & $\mathbf{F i}$ \\
\hline 103AwOBLEVE & si & $\mathbf{P}$ & E1 & & 11 & 6 & T.05 & D12 & & & & & & $\mathbf{s 1}$ & $\mathbf{H 1}$ \\
\hline 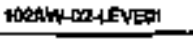 & sh & F1 & E1 & C.1- & 0 & & $T$ & $\mathrm{mat}$ & $\max$ & E1 & $F 1$ & 81 & F3 & & \\
\hline 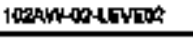 & B1 & $\mathbf{F I}$ & $\mathbf{E}$ & & 5 & & ros & DI2 & & & & & & & \\
\hline 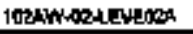 & \$1 & $\mathbf{f s}$ & EI & & 11 & $s$ & T.cos & EDd & & & & & & s1 & $\mathbf{f 1}$ \\
\hline
\end{tabular}




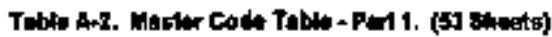

\begin{tabular}{|c|c|c|c|c|c|c|c|c|c|c|c|c|c|c|c|}
\hline 10 & 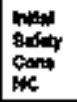 & $\begin{array}{l}\text { nom } \\
\text { finq } \\
N C\end{array}$ & En & and & 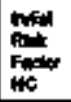 & 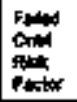 & $\begin{array}{l}\text { Fonty } \\
\text { codt } \\
-\end{array}$ & Give & $\operatorname{Rip}_{\log }$ & $\begin{array}{l}\text { Mh } \\
\text { Enty } \\
\text { Nent }\end{array}$ & $\begin{array}{l}\operatorname{Mh}_{\mathrm{F}} \\
\mathrm{NG}\end{array}$ & sepcint & $A B C$ & $\begin{array}{l}\text { Fand } \\
\text { Cont } \\
\text { golkty } \\
\text { Conte }\end{array}$ & الה: \\
\hline 10shitarueves & sh & $\mathrm{Fa}$ & $\theta$ & $c-2-0$ & 14 & 10 & Tots & $E 0$ & $\cos x$ & 6 & $\mathrm{~F} / \mathrm{B}$ & Provemed & 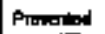 & 83 & f1 \\
\hline 100\%W-GR-UEVEMA & an & $F$ & E & $c-2-4$ & 14 & 19 & $\mathbf{T}$ & ED? & $2 x$ & 8 & $\mathrm{~F}$ & Panwertied & Pronderild & $\$$ & $\mathbf{F 1}$ \\
\hline 10DNW-GHEVEDS & 92 & $\mathbf{F}$ & E2 & $6-2-b$ & 14 & 19 & $\mathbf{T}$ & EDQ & $2 x$ & 8 & $F$ & Penswithed & Proveried & 8 & $\mathbf{F 1}$ \\
\hline 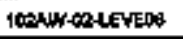 & $\$ 1$ & $\mathrm{Hs}$ & es: & & 6 & & 108 & 012 & & & & & & & \\
\hline 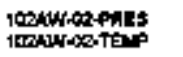 & $\$$ & $\mathbf{P s}$ & 12 & cines & 54 & 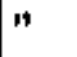 & $T \cos r$ & $\infty$ & 22 & \$1 & $\boldsymbol{P}$ & $\mathbf{s 1}$ & $\mathbf{F s}$ & I1 & $\mathbf{m}$ \\
\hline 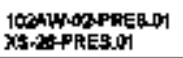 & 91 & NaA & $m$ & $\infty$ & & & 9 & For & & & & & & & \\
\hline 1000urtatengsor & $\mathbf{s}$ & $\mathbf{P 2}$ & e2 & $4-10$ & 15 & is & T.SD.H & 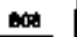 & $\tan x$ & st & $\boldsymbol{A}$ & $\mathbf{s}$ & $P_{1}$ & ss & 12 \\
\hline 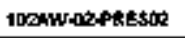 & 뇨 & $\mathbf{x}$ & E2. & colat & 14 & 11 & Thes & Eor & $\operatorname{sax}$ & 81 & $\mathbf{F 3}$ & st & A & 8 & is \\
\hline 10DAN WOPEEBOX & Eg & fo & E3 & $B-1 \rightarrow$ & 10 & 1 & T.\$D.Y & $\mathrm{spT}$ & $13 x$ & $\$ 0$ & fo & 8 & FQ & 90 & $\mathbf{p r o}$ \\
\hline 1003in-Deprespos & 8 & fo & ES & B-1-t & 10 & 1 & T.SD & cor1 & $13 x$ & s & 60 & 50 & $\mathrm{FQ}$ & 80 & ro \\
\hline 108AWH-O2PRESOS & 89 & fot & $\mathbf{E}$ & an-th & 10 & 1 & T.DS & 데1 & tos & so & $\mathbf{m}$ & so & $\mathrm{Fb}$ & 50 & Fo \\
\hline 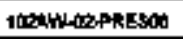 & $\mathbf{s}$ & po & Es & Bas & 10 & 1 & T\$D.Y & $\cos$ & $13 x$ & 50 & Mo & so & $P_{0}$ & 20 & Po \\
\hline 1039-08-PFE90 & в9 & Fo & E & B-14 & 10 & 1 & T.sp.v & 513 & $13 x$ & $\$$ & Fo & 80 & $\mathrm{FW}$ & 90 & ro \\
\hline 102AWL-02-PRESOP & 52 & $\mathbf{r g}$ & E1 & C-1- & 14 & 11 & T.DSE & DO1 & $22 x$ & $s_{1}$ & Fy & 91 & F & $\$ 1$ & $\mathrm{~F} 3$ \\
\hline 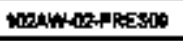 & $\$ 2$ & $\mathbf{F s}$ & $E 1$ & 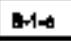 & 14 & 11 & T.Dser & Don & $2 x$ & 82 & $\mathbf{F u}$ & st & $\mathbf{P y}$ & $\$ 1$ & F3 \\
\hline 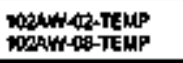 & $\$ 2$ & 12 & E1 & E.1 4 & 12 & $\Leftrightarrow$ & T.Dosh & E16 & odx & $\$ 2$ & $\mathbf{F}$ & 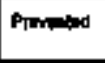 & Pheneted & $s$ & $\mathbf{F 1}$ \\
\hline 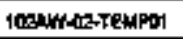 & 32 & $\mathbf{F}$ & E2 & B.14a & 12 & 自 & T.\$0.L & Dot & $\cos x$ & $\$ 2$ & $\mathbf{F a}$ & Pinnidd & Pronindad & 5 & $\mathbf{F 1}$ \\
\hline 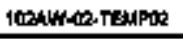 & 踥 & $\mathbf{m}$ & 妌 & B.14a & 12 & 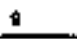 & T.so.t & Do1 & 1 ath & & & PTrmatid & Prontiod & 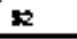 & $\mathbf{H}$ \\
\hline 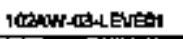 & stz & $\mathrm{F}_{2}$ & 血 & A-1-4 & 12 & 13 & Т,०白,H & 悳 & $\cos$ & 83 & $\mathbf{F g}$ & $\$ \$$ & F1 & $\mathbf{s}$ & $\mathbf{F 1}$ \\
\hline
\end{tabular}




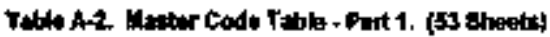

\begin{tabular}{|c|c|c|c|c|c|c|c|c|c|c|c|c|c|c|c|}
\hline $\mathbf{D}$ & 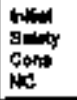 & 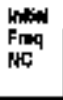 & $\underset{\operatorname{Con}}{\operatorname{En}}$ & aw & 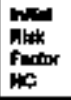 & 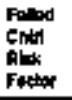 & Foctitir & ctang & $\operatorname{Rip}_{\infty}$ & 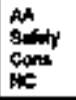 & $\underset{N \infty}{N_{\infty}}$ & Aptitu & App Dition & 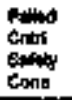 & $\begin{array}{l}\text { Fillat } \\
\text { Find } \\
\text { Find }\end{array}$ \\
\hline 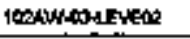 & 32 & Fi2 & 6 & A.1. & 17 & 13 & T.DE.H & $\mathbf{B x S}$ & $04 x$ & so & $\mathbf{F s}$ & $\Leftrightarrow$ & $\mathbf{F 1}$ & 83 & F1 \\
\hline 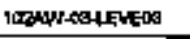 & $\$$ & F1 & $\mathbf{E g}$ & B-1-4 & 13 & 1 & T.øa & $\cos$ & $18 x$ & 90 & $\mathbf{F 1}$ & 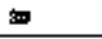 & Fo & 90 & Fo \\
\hline 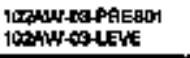 & \$1 & $\mathrm{Fz}$ & E1 & & 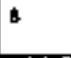 & & T.Ds. & $\mathrm{O}_{12}$ & & & & & & & \\
\hline 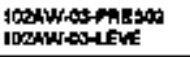 & sı & $\mathbf{N 2}$ & e1 & & 4 & & T.DA.V & a12 & & & & & & & \\
\hline 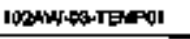 & $\Leftrightarrow$ & $\mathrm{F} 2$ & eq & $A=14$ & 16 & 13 & T.DQ & $8 \infty$ & $\cos$ & & & Puprented & PTweried & 63 & $\mathrm{Fi}$ \\
\hline 1003in-03-T日uptos & 52 & F & 62 & A $-1-8$ & 12 & 13 & T.DE & Bxis & aex & s. & $\mathbf{F} \mathbf{2}$ & Pomornand & Prowation & st & P \\
\hline 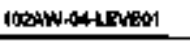 & si & $\mathbf{P}$ & EI & & 11 & 11 & WD & בום & & & & & & $\$ 1$ & is \\
\hline 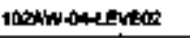 & st & $\mathbf{B}$ & el & & 11 & 13 & wo & Eस: & & & & & & $\$ 1$ & $\mathbf{F B}$ \\
\hline 10001W04LESEOS & 81 & $\mathrm{F3}$ & E1 & & 11 & 11 & $w$ & Ees & & & & & & 61 & $\mathrm{~F} 3$ \\
\hline 1029 & 81 & F3 & EI & & 11 & 11 & wo & Dos & & & & & & 61 & $\mathbf{F 3}$ \\
\hline 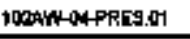 & s1 & Ho & NA & $\leftrightarrow$ & & & $\theta$ & P11 & & & & & & & \\
\hline 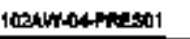 & 61 & $\mathbf{m}$ & E1 & & 11 & 11 & Mo & |खा & & & & & & 91 & $\mathbf{F}$ \\
\hline 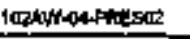 & 81 & $\mathbf{F}$ & E1 & & 11 & 11 & 40 & EN & & & & & & E1 & $\mathbf{F}$ \\
\hline 108XM-048TाRU.01 & si & Hh & $\mathbf{H H}$ & $\infty$ & & & 0 & F10 & & & & & & & \\
\hline 100WWA4.8TRU.02 & $s t$ & $\mathbf{r 2}$ & E1 & & 8 & & 40 & b14 & & & & & & & \\
\hline 10DLUHOASTRLLOA & 多 & $\mathbf{f a g}$ & Es & B-14 & 16 & $\theta$ & $\mathbf{T}$ & c10 & $12 x$ & az & $\mathbf{F}$ & Prindad & Armated & 62 & f: \\
\hline 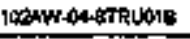 & $\$ 1$ & $\mathbf{F}$ & E1 & & 5 & & $m$ & DI8 & & & & & & & \\
\hline I02AW-04-STRLOR & ss & $\mathbf{F}$ & ES & E-4 & 98 & 6 & $\mathbf{T}$ & C10 & $13 x$ & $\mathbf{s z}$ & $\mathbf{F}$ & Prinnind & Prononted & 52 & po \\
\hline 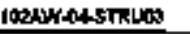 & 82 & $\mathbf{F}$ & E1 & Btod & 12 & 0 & mo & ONo & $\operatorname{con} x$ & sh & $\mathbf{F}$ & 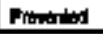 & 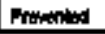 & 的 & $\mathbf{F I}$ \\
\hline
\end{tabular}


Tabte A-2. Wable Code Table - Part 1. (53 theets)

\begin{tabular}{|c|c|c|c|c|c|c|c|c|c|c|c|c|c|c|c|}
\hline ID & 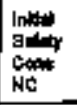 & 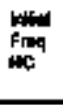 & $\begin{array}{l}\text { Emt } \\
\text { thoms }\end{array}$ & Ban & 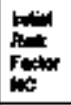 & 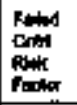 & Fotion & CNm & $n_{\infty}$ & $\begin{array}{l}\text { Mh } \\
\text { enter } \\
\text { cons } \\
\text { Wh }\end{array}$ & 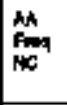 & $\begin{array}{l}\text { Nop Chat } \\
\text { Bonly }\end{array}$ & 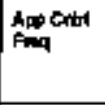 & 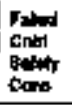 & $\begin{array}{l}\text { Fond } \\
\text { Cont } \\
\text { Fond }\end{array}$ \\
\hline 1024W-OA-8TRLOA & 82 & 17 & $E z$ & c-3b & tz & E & $m p$ & OAO & $20 x$ & si & $\mathrm{FB}$ & $\$ 1$ & Fu & B1 & P1 \\
\hline 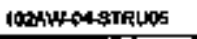 & $\mathbf{s z}$ & $\mathbf{f I}$ & en & $\cos$ & 3 & 6 & wo & Ditg & $20 x$ & s1 & $\mathbf{r s}$ & $\$ 1$ & p2 & \$1 & $\mathbf{F}$ \\
\hline 190hLA4TEP & $\Rightarrow$ & $\mathbf{f I}$ & EI & AHA & 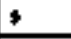 & 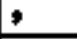 & wo & 800 & $97 x$ & st & F\$ & 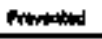 & Pinmold & $\mathbf{6}$ & F1 \\
\hline 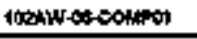 & 11 & 12 & $\mathbf{c}$ & & $B$ & & $\tau$ & Do1 & & & & & & & \\
\hline 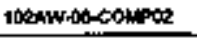 & 32 & 11 & e & A-12s & 8 & 3 & $T$ & $\operatorname{mon}$ & an $x$ & 81 & F1 & Frmated & Anumited & s1 & $\infty$ \\
\hline 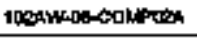 & 82 & $\mathbf{F 1}$ & EP & A.1-10: & 9 & 3 & TF\$ 18 & not & 81x & $\$ 1$ & F1 & Franonted & Pning & 81 & $\mathbf{F u}$ \\
\hline 102AK-06-COMPRS & 92 & F1 & $\mathbf{E}$ & AHA & 5 & 3 & $T$ & $\mathbf{m o r}$ & $01 x$ & 81 & F1 & Frouintod & Panumiad & 81 & Fo \\
\hline 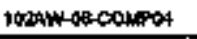 & 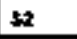 & FI & 62 & Are & 0 & 5 & Tos & 1002 & $01 x$ & $\$ 1$ & $\mathbf{F 1}$ & 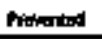 & 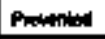 & $\$$ & $\mathbf{F o}$ \\
\hline 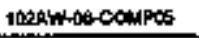 & 52 & ro & $e$ & Arma & A' & 3 & Tos & Nos & Do1X & $\$ 1$ & $\mathbf{P}_{1}$ & Anom & Provented & s1 & 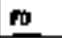 \\
\hline 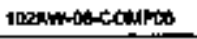 & 61 & A & e1 & & 11 & & $\tau$ & OOA & & & & & & & \\
\hline 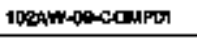 & \$9 & $\boldsymbol{F}$ & es & $4+14$ & 15 & 12 & TAD:D & BXE & $\operatorname{ces} x$ & $\mathbf{s z}$ & $\mathrm{F} 2$ & $\$ 2$ & $\mathbf{F 2}$ & 82 & $\mathbf{F}$ \\
\hline 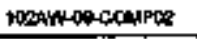 & $\$ 3$ & $\mathbf{F}$ & Es & $A-1 \rightarrow$ & 15 & 13 & $T$ & BP & $\cos x$ & & & nawintid & de. & 93 & $\mathbf{F}$ \\
\hline 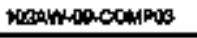 & st & $\mathbf{F 3}$ & c1 & & 11 & 8 & $\mathrm{~T}$ & DOA & & & & & & s1 & $\mathbf{F}$ \\
\hline 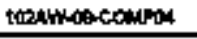 & 61 & As & e1 & & 11 & & $T . \$ 0.0$ & Don & & & & & & & \\
\hline 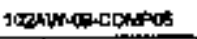 & 51 & $\mathrm{F3}$ & E1 & & 11 & 4 & ס.ק. & ED & & & & & & \$1 & $\mathbf{F z}$ \\
\hline 1004W-0:-TEAP & 80 & F3 & E1 & $\Leftrightarrow-1 \rightarrow$ & 14 & 11 & T.D8 & Di & $2 \pi x$ & \$I & $\mathbf{m}$ & s & $\mathbf{F 3}$ & 51 & $\boldsymbol{F}$ \\
\hline 102AW $\angle 0-T D M P 01$ & $s$ & $\mathbf{n}$ & E2 & 0 & 12 & 8 & T.05 P & $\infty$ & $1+4 x$ & & & Pheningal & Prminitod & $s$ & $\boldsymbol{M}$ \\
\hline 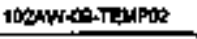 & $\$ 1$ & F1. & E1 & & $\$$ & & T.D.9 & Cont & & & & & & & \\
\hline 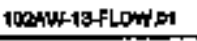 & Ba & AA & H & Pep & & & 0 & Fos & & & & & & & \\
\hline 1024W-13-FLOMYO1 & $\mathbf{s}$ & F1 & Ea & 6-1. & 13 & 13 & mp & olz & $\tan$ & ss & $\mathbf{F a}$ & 32 & $\mathbf{F 1}$ & 5 & Fa \\
\hline
\end{tabular}




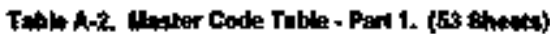

\begin{tabular}{|c|c|c|c|c|c|c|c|c|c|c|c|c|c|c|c|}
\hline $\mathbf{0}$ & 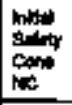 & $\begin{array}{l}\text { Whll } \\
\text { Froq } \\
\text { NG }\end{array}$ & ent & GIN & 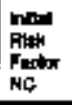 & $\begin{array}{l}\text { Find } \\
\text { crid } \\
\text { Finder } \\
\text { Fand }\end{array}$ & $\begin{array}{l}\text { facitr } \\
\text { code }\end{array}$ & Dom & Pop & $\begin{array}{l}\text { Mh } \\
\text { Exant } \\
\text { Com } \\
\text { NC }\end{array}$ & $\begin{array}{l}\text { AA } \\
\text { FNA } \\
\text { NEC }\end{array}$ & Aptonth & foptint & 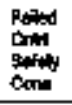 & $\begin{array}{l}\text { Poldod } \\
\text { Com } \\
\text { Find }\end{array}$ \\
\hline 100NW-13flowhe & ag & $\mathbf{F g}$ & E1 & $4-20$ & 14 & 13 & $P Q$ & EDe & $29 x$ & 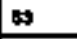 & $m$ & 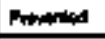 & Prowerited & 83 & F1 \\
\hline 10z:-0|at & 82 & $n$ & a & $c-2 b$ & 12 & is & mo & 100 & $20 x$ & s) & $\mathbf{F s}$ & su & $\mathbf{F a}$ & s5 & F1 \\
\hline $10<t+04 b 2$ & 요 & 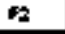 & a & 0.25 & 12 & 13 & wo & D:2 & 200 & s & $\mathbf{P a}$ & s1 & $\mathbf{F}$ & 73 & $\mathbf{M 1}$ \\
\hline 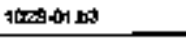 & $\Leftrightarrow$ & $\mathbf{P}$ & E2 & Bab & 14 & 8 & Ps & olz & 为 & 이 & Fu & 31 & $\boldsymbol{m}$ & st. & F1 \\
\hline $1029-02 \mathrm{~m}$ & 的 & $\mathrm{F} 2$ & E1 & $+1-$ & 12 & 9 & wo & Bat & orf & 87 & $\mathbf{F a}$ & Proneriled & Promented & 82 & F1 \\
\hline $1008-02 \mathrm{b2}$ & 87 & $F_{2}$ & E? & $A-1-$ & 12 & 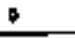 & wr & 809 & $97 x$ & $\mathbf{m 2}$ & $\mathbf{m}$ & Panoted & PTiminted & 82 & f1 \\
\hline $1005-02$. & 82 & $\mathbf{r s}$ & Eto & AN1-1- & 14 & 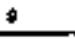 & $T$ & 825 & orx & & & Prominas & Primotion & \$ & 1 \\
\hline 1000 in & si & $\mathbf{r s}$ & E1 & & 11 & 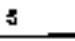 & $T$ & as & & & & & & ง1 & $\mathbf{F I}$ \\
\hline noesconbs & $3 z$ & $\mu_{2}$ & $E$ & A.1. & 17 & 13 & T.\$o & ax & nex & ss & $\mathrm{E}_{2}$ & PTprosted & Arom tod & st & F1 \\
\hline $10280 \mathrm{ab} 4$ & As & Fs & $\mathbf{E}$ & Aul 4 & 4 & 13 & T.8.8. & $\mathrm{EF}$ & $\log x$ & $\$ 3$ & $\mathbf{F}$ & Protienend & Pronengind & 93 & F1 \\
\hline $1028-04.016$ & 30 & $\mathrm{FP}$ & E2 & & 7 & & T.99 & 012 & & & & & & & \\
\hline torsedatsos & 80 & Fs & $\mathbf{r 2}$ & & 7 & & T.98 & Dos & & & & & & & \\
\hline votsol & 82 & As & E1 & B-10 & 14 & 11 & $T .00$ & on & 立x & 5 & $\mathbf{F a}$ & B1 & is & $\$ 1$ & $\mathbf{N}$ \\
\hline 1028040 & ss & $\mathbf{P 1}$ & $\mathbf{E X}$ & Ad4 4 & 13 & 12 & T.מ口n & ex & $00 x$ & 89 & $F_{3}$ & 82 & F1 & 92 & $\mathbf{F} 2$ \\
\hline 1025 ond & 83 & $\mathrm{FO}$ & $\mathbf{E 3}$ & 6-1-4 & 10 & 1 & $T, 894$ & $\mathrm{COR}$ & $13 x$ & 80 & $\mathrm{Fo}$ & so & $\mathrm{FO}$ & 90 & Fo \\
\hline $1055-94 a 1 b$ & 87 & $\mathrm{~F} 2$ & es & nolde & 12 & 12 & T.60\% & 806 & $\operatorname{tax}$ & 52 & $\mathbf{P s}$ & 些 & $F_{2}$ & sto & $\mathbf{F}$ \\
\hline 10000040. & 31 & $\mathbf{F 2}$ & E1 & & 4 & & $T$ & bes & & & & & & & \\
\hline $1500-0409$ & $\$ 3$. & Fo & E3 & A- & 10 & 6 & $T$ & 80 & Dos & 8 & $F$ & Pawnos & Plonented & 9 & Fo \\
\hline 10025-04.4 & 92 & $\mathbf{F 1}$ & [1 & ANA & 8 & 3 & $\mathrm{~T}$ & $A 0 B$ & Dax $x$ & si & $\mathbf{r 1}$ & AMt & Prominiod & EI & Fo \\
\hline 10060003 & 5 & $\mathbf{P}$ & est & B-14 & 10 & 5 & $T$ & G10 & $12 x$ & 02 & P3 & Aancind & 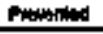 & 82 & $\mathbf{P B}$ \\
\hline
\end{tabular}




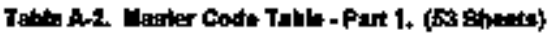

\begin{tabular}{|c|c|c|c|c|c|c|c|c|c|c|c|c|c|c|c|}
\hline as & $\begin{array}{l}\text { hes } \\
\text { seind } \\
\text { cont } \\
\text { HC }\end{array}$ & 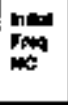 & $\begin{array}{l}\text { Enr } \\
\text { conit: }\end{array}$ & En & 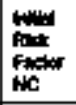 & 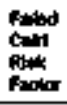 & Conthy & $\begin{array}{l}\text { Coun } \\
\text { arp }\end{array}$ & Am & $\begin{array}{l}\text { An } \\
\text { sority } \\
\text { Cont } \\
\text { NEE }\end{array}$ & 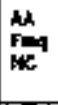 & Appcid & 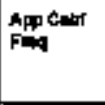 & 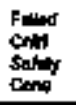 & 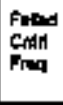 \\
\hline $1025-05$ alda & $\theta$ & $\mathbf{F o}$ & U & $E-1 \rightarrow$ & 19 & 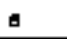 & $\mathbf{T}$ & 610 & $12 x$ & s? & F\$ & 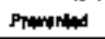 & Prominisud & $\mathbf{m}$ & $\mathbf{m o}$ \\
\hline $1028-60=2$ & 8 & f1 & Ed & B.1- & 13 & & $T$ & MI & $14 x$ & s & Ps & Is & $\mathbf{m}$ & & \\
\hline $1008+4000$ & 女s & f1 & Es & B-1-1 & 13 & 1 & $\boldsymbol{T}$ & 012 & 1Fx & so & $\mathbf{F 1}$ & $\mathbf{0 0}$ & $\mathbf{m o}$ & so & $\mathrm{FD}$ \\
\hline $1029-06=$ & $\mathbf{9}$ & F1 & $\mathrm{E}$ & \&-1- & 13 & 1 & $\mathbf{T}$ & c12 & $1 \hat{x}$ & $\mathrm{sot}$ & F1 & so & Fo & 80 & $\mathrm{FO}$ \\
\hline $1028-064$ & Bat & $\mathrm{F3}$ & E1 & & 11 & 11 & T.\$p.Y & $D N$ & & & & & & $\$ 1$ & $F$ \\
\hline $1000-01 h$ & $\infty$ & mo & os & D-1-4 & 10 & $\mathbf{I}$ & 1 ge. $V$ & $\mathrm{cos}$ & $13 x$ & $\Leftrightarrow$ & $\mathbf{F}$ & 80 & Fo & $\infty$ & $\mathbf{m o}$ \\
\hline $100 \times-010$ & $\mathbf{s}$ & H & es & $B-14$ & 10 & 1 & T.10. & $\cos$ & 15: & 4 & no & 0 & ro & so & $\omega$ \\
\hline $100 \times-01 c$ & $\mathbf{x}$ & fo & Es & E-14 & 10 & 1 & T.sa.r & $\cos$ & $13 \times$ & so & $\infty$ & so & Fo & so & fo \\
\hline $160 x-110$ & $\mathbf{s}$ & Fo & ES & EN120 & 10 & 1 & tsa.r & c13 & $13 x$ & 80 & $F O$ & so & Fo & E & FO \\
\hline 1005-01E & $\infty$ & F2 & Es & $e-14$ & 1s & 1 & T.\$9.4 & 611 & $13 x$ & so & $\mathrm{Fo}$ & 80 & Fo & 80 & FQ \\
\hline 1015-6015 & 32 & $\mathbf{P 2}$ & $\mathrm{B2}$ & 0.14 & 12 & 12 & T,\$D.L.Y & DON & $\cos x$ & $\$ 8$ & Fs & 8: & Fs & s & $P 2$ \\
\hline 106:2015 & sa & $\mathbf{k z}$ & E1 & 6-1: & 12 & 0 & $\mathbf{T}$ & eor & $\operatorname{mx}$ & 52 & $\mathbf{F s}$ & Prentated & Promenter & stz & 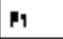 \\
\hline 1060-14 & 5.2 & $\mathrm{Fz}$ & E1 & cotes & 12 & a & tso.t. & Dot & $\mathbf{\infty x x}$ & 52 & A & Pinmated & 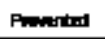 & 8 & $\mathbf{F 1}$ \\
\hline 10ec-011 & 㫙 & $\mathbf{F 2}$ & $\mathrm{E}$ & A-14 & to & to & T.\$O.H & Bax & $\cos x$ & 33 & $F$ & 83 & $\mathrm{Fz}$ & ss & $\mathrm{Fz}$ \\
\hline 1098-01 & 83 & $f 2$ & 8 & A.1-n & 15 & H & T.6D.H & 100 & $\infty 6 x$ & 69 & Fi & $\$ 3$ & $\mathbf{F 2}$ & $\$ 3$ & P2 \\
\hline $1000-01 k$ & $\$ 1$ & $\mathbf{P}$ & EI & & 11 & 日 & T.SD.L & $D_{02}$ & & & & & & $\$ 1$ & 13 \\
\hline toeca-pise & \$2 & F2 & E1 & B-1a & 2 & 12 & $T$ & EDY & $003 x$ & 92 & $F$ & Frimcind & 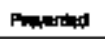 & 92 & $\mathbf{F z}$ \\
\hline poectilum & 93 & $\mathrm{Fa}$ & 62 & Ant & 16 & 13 & T.60.L & 8001 & $05 x$ & 69 & 87 & ss & Fo & ss & $F 1$ \\
\hline notann & $\$ 2$ & F1 & es & M-1- & 9 & 3 & T.SD.L. & $\operatorname{ADS}$ & and & 51 & P1 & 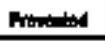 & Pranuind & 51 & $\mathbf{N O}$ \\
\hline 10sconthe & 80 & $\mathbf{p}$ & Es & & 4 & & T.stel & Ind & & & & & & & \\
\hline
\end{tabular}


Tabl M-2. Mrogter Code Tabla - Fart 1. (53 shetit)

\begin{tabular}{|c|c|c|c|c|c|c|c|c|c|c|c|c|c|c|c|}
\hline 10 & 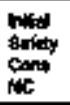 & 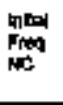 & $e^{\infty}$ & EIN & 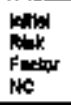 & 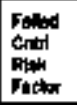 & $\begin{array}{l}\text { Fociwity } \\
\text { Doll }\end{array}$ & $\begin{array}{l}\text { Ctast } \\
\text { Gip }\end{array}$ & $R_{\infty \infty}$ & $\begin{array}{l}\text { Nh } \\
\text { stint } \\
\text { cans } \\
\text { iK }\end{array}$ & Pring & Aepcis & $\operatorname{mon}_{\operatorname{mos}}$ & 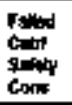 & $\begin{array}{l}\text { Pod } \\
\text { Find } \\
\text { Find }\end{array}$ \\
\hline 1 1x-eas & $\$$ & $\mathbf{P s}$ & el & & 11 & 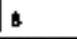 & T.s8_ & ote & & & & & & si & $\mathbf{F} \mathbf{2}$ \\
\hline $1490-015 *$ & 81 & $\mathrm{Fg}$ & 自 & & 11 & & $\mathrm{~T} \$ 8$ & O12 & & & & & & & $F 3$ \\
\hline $1090-091$ & $8 t$ & $\mathrm{Fa}$ & E1 & & 19 & B & TE\& l & $E 02$ & & & & & & 91 & $F_{2}$ \\
\hline 100 -014 & $\$ 0$ & $\boldsymbol{M}$ & EO & 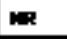 & & & $T, 48 \mathrm{R}$ & 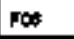 & & & & & & & \\
\hline 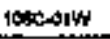 & $\$ 1$ & $\mathbf{F 1}$ & EI & & 5 & & $T$ & 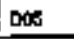 & & & & & & & \\
\hline $1060 \mathrm{CATx}$ & ss & Ps & $\mathbf{c s}$ & into & 15 & 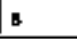 & $T$ & cNo & 13 & s2 & $\mathbf{F}$ & Arranited & Proverited & 82 & $\mathrm{Fo}$ \\
\hline 1000-01Y & $\$ 3$ & $\mathrm{~F}$ & 6 & B.r. & 15 & $*$ & $T$ & 54 & $12 x$ & \$2 & $\mathbf{F}$ & Provented & 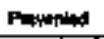 & 92 & $\mathbf{F}_{1}$ \\
\hline $100-02 x$ & $\Leftrightarrow$ & $\mathbf{F z}$ & E3 & $A-1 \rightarrow$ & 96 & 13 & T.8DNA & 暗 & $\cos$ & $\Leftrightarrow$ & $\boldsymbol{n}$ & $\Leftrightarrow$ & Po & s3 & 1 \\
\hline $1000-025$ & $\Leftrightarrow$ & $\mathbf{F z}$ & ES & $A-1-$ & 15 & 15 & T.STOMV & mor & $\cos x$ & $\mathbf{s}$ & $\boldsymbol{N}$ & $\Leftrightarrow$ & $n$ & s & 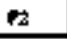 \\
\hline 1000-0025 & $\mathbf{s}$ & $\mathbf{2}$ & Es & Ant 1 & is & 15 & T.sth & axt & $\cos x$ & ss & $\mathbf{f}$ & $\mathbf{s}$ & F & $\mathbf{s}$ & F2 \\
\hline $1000-0-4 \alpha$ & $\mathbf{s}$ & 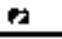 & D & An 120 & 16 & 15 & T.尹קн & BM & $\operatorname{Des} x$ & as & $\mathbf{F}$ & $\mathbf{s}$ & $\mathbf{F} 2$ & 99 & $\mathbf{F} 2$ \\
\hline 1000-0<14. & $\$$ & $\mathbf{F}$ & $E_{1}$ & A.14. & 12 & 15 & $\mathrm{~T}$, BDH & 808 & $05 x$ & Bs & $\mathrm{F} \mathbf{3}$ & 89 & $\mathbf{F} 2$ & $\theta$ & $\mathbf{F} 2$ \\
\hline 1000048 & $\Leftrightarrow$ & $\mathbf{F} 2$ & Es & A-1-A & 15 & 15 & T.SDH & 500 & $65 x$ & ss & is & 昰 & $\boldsymbol{r}$ & $\Leftrightarrow$ & $\boldsymbol{n}$ \\
\hline $10 \cos \theta 0$ & s1 & $\mathrm{Fg}$ & E1 & & 11 & 11 & 40 & e14 & & & & & & 5I & $\mathbf{s}$ \\
\hline $10 x-0=3$ & so & $\mathbf{m}$ & Eo & $m$ & & & mo & FOT & & & & & & & \\
\hline $1004-\cos 0$ & 82 & 12 & 51 & M14 & 4 & $\vartheta$ & mo & 605 & wx & 52 & $\mathbf{F 3}$ & Prowiond & Promind & 82 & $\mathrm{Fi}$ \\
\hline $1000 x-06$ & st & $\mathbf{F}$ & E1 & & 11 & & no & oos & & & & & & & \\
\hline 10000 - & $\$ 1$ & $\mathbf{r g}$ & Ex & & 11 & $\theta$ & wp & 0,0 & & & & & & 51 & $\mathbf{F}$ \\
\hline 1000,004 & 81 & Is & EI & & 11 & & wo & Dos & & & & & & & \\
\hline $1090-09$ & sp & $\mathrm{MHA}$ & $E$ & nz & & & wo & F10 & & & & & & & \\
\hline
\end{tabular}




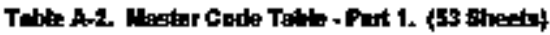

\begin{tabular}{|c|c|c|c|c|c|c|c|c|c|c|c|c|c|c|c|}
\hline ט & 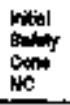 & $\begin{array}{l}\text { mod } \\
\text { Fimd } \\
\text { inc }\end{array}$ & $\begin{array}{l}\text { Ew } \\
\text { Coma }\end{array}$ & UN & |lle & 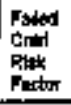 & $\begin{array}{l}\text { Polvey } \\
\text { Cafl }\end{array}$ & Coust & 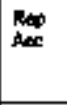 & 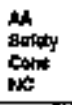 & $\begin{array}{l}\text { Nh } \\
\text { Fin } \\
\text { NC }\end{array}$ & ippon & ing chil & 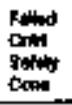 & 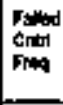 \\
\hline $109 C-06 K$ & B1 & $\mathbf{F a}$ & E1 & & 11 & 8 & wh & ont & & & & & & B1 & f2 \\
\hline $1060-0 \%$ & $E 2$ & $\mathbf{F}$ & EI & $A-1-n$ & 12 & 9 & no & $\operatorname{sen}$ & orx & $\$ 2$ & m & Prevarined & 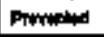 & 52 & $\mathbf{F 1}$ \\
\hline $100 x-094$ & 80 & wh & EO & m & & & wo & F14 & & & & & & & \\
\hline $1000-100$ & $\infty$ & nin & 5 & m & & & T.\$O.W & ror & & & & & & & \\
\hline $100 \mathrm{C}-10 \mathrm{C}$ & Bo & Hh & Ed & $m$ & & & T.so.v & $P 05$ & & & & & & & \\
\hline 109c-100 & 80 & Nh & Eo & $\mathbf{m}$ & & & $\mathrm{T} z \boldsymbol{B D} . \mathrm{V}$ & $\mathrm{Fot}$ & & & & & & & \\
\hline NOQC-10E & 180 & Nh & ED & $\mathbf{m i}$ & & & $T, \exp V$ & $\mathrm{FP} F$ & & & & & & & \\
\hline tooc-10 $\mathrm{r}$ & 51 & $\mathbf{r g}$ & eI & & tt & 11 & $T, 600 . \mathrm{K}$ & Dos & & & & & & 51 & rs \\
\hline $1000-10 \mathrm{~N}$ & st & $\mathbf{m}$ & 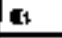 & & 3 & & 7.54 & $\operatorname{mos}$ & & & & & & & \\
\hline $1000-101$ & 82 & $\boldsymbol{F} 2$ & Es & 410 & 12 & 12 & $\mathbf{T}$ & 60 & De & $\$ 2$ & $\mathrm{Fs}$ & $\$ 1$ & $\mathrm{Fo}$ & st2 & $F$ \\
\hline $100019 x$ & $\$ 1$ & $\mathbf{F a}$ & E,1 & & 11 & 11 & $\mathbf{T}$ & DOF & & & & & & 81 & $F$ \\
\hline $100 x-10$ & $\$ 1$ & $\mathrm{~F} 3$ & E1 & & 11 & 11 & $\mathbf{T}$ & DOT & & & & & & $\$ 1$ & $\mathbf{F 3}$ \\
\hline $100 \times-11 \mathrm{~A}$ & $\$ 1$ & Ps & [1 & & 11 & 11 & $\mathbf{I}$ & $\infty 07$ & & & & & & $\$ 1$ & $\mathbf{P B}$ \\
\hline 1005 & 80 & NA & $\theta$ & Nh & & & E.sta.y & Ros & & & & & & & \\
\hline $1060-116$ & 90 & Wh & EO & NA & & & Tsp.4 & For & & & & & & & \\
\hline $1000 \mathrm{~s}$ & $\infty 0$ & NA & EO & Mr & & & T.9D.Y & 607 & & & & & & & \\
\hline $1000-11 E$ & $s$ & $\mathbf{F}$ & ⿷2 & B-10 & 14 & 7 & $T$ & cor & $1 \operatorname{sen} x$ & $8 x$ & $F$ & 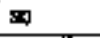 & FI & sto & $\omega$ \\
\hline $1000-1 \% F$ & 8 & ma & Eo & 해 & & & T.9p., 4 & Folr & & & & & & & \\
\hline $100 \mathrm{C}-110$ & B1 & $\mathbf{F g}$ & E1 & & 31 & 8 & $T, \mathbf{B 8}$ & Dor & & & & & & 91 & $\mathbf{F}$ \\
\hline Noc-11H & s1 & $\mathbf{r a}$ & E1 & & 11 & 11 & T.5. & Dors & & & & & & E1 & F \\
\hline
\end{tabular}




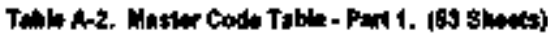

\begin{tabular}{|c|c|c|c|c|c|c|c|c|c|c|c|c|c|c|c|}
\hline ID & 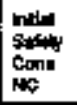 & 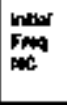 & Ex. & 自秋 & 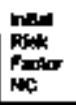 & 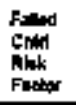 & Fuchy & Par. & $\sum_{n=0}$ & 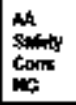 & $\begin{array}{l}M \\
\text { No }\end{array}$ & 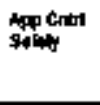 & $\begin{array}{l}\text { Appcrt } \\
\text { Find }\end{array}$ & $\begin{array}{l}\text { falwa } \\
\text { chat } \\
\text { salisy } \\
\text { Canso }\end{array}$ & 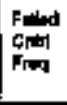 \\
\hline $1000 \times 111$ & $\$ 1$ & $\mathbf{F g}$ & [1 & & 11 & 1 & TSD.Y & $D 07$ & & & & & & $\$ 1$ & $\mathbf{7 2}$ \\
\hline $17011 \mathrm{~J}$ & 8 & $\mathrm{Fa}$ & E1 & onds & 14 & 12 & T.AD.V & Dor & $\operatorname{tos} x$ & & & 84 & Ps & $\$ 2$ & $\mathbf{p z}$ \\
\hline $111 \mathrm{~K}$ & 뵤 & p & E1 & Avto & 12 & 12 & $\mathbf{T}$ & 100 & $\cos x$ & & & \&1 & $\mathbf{P s}$ & $\$ 2$ & Fz \\
\hline 1609-116 & en & $\mathbf{F 3}$ & E1 & $\mathrm{B}-1-$ & 14 & 12 & $\mathbf{I}$ & $\operatorname{En}$ & 18:8x & & & 81 & $F$ & 82 & 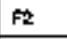 \\
\hline $1000-11 \mathrm{M}$ & 82 & $\mathbf{F 3}$ & E1 & B-1- & 14 & 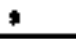 & $\tau$ & mor & $D \in x$ & & & $\$ 1$ & Fạ & 89 & F1 \\
\hline $1000-11 \mathrm{~N}$ & BI & rs & $\mathbf{1}$ & & 11 & $*$ & $T$ & Dor & & & & & & $\$ 1$ & $\mathrm{Pz}$ \\
\hline $100 \mathrm{C}-4 \mathrm{fD}$ & $\$ 2$ & $\mathbf{p}$ & er & $\theta-1=$ & 12 & B & $T$ & Dor & $\operatorname{tat} x$ & 요 & A & s1 & $\mathbf{P}$ & a) & $M$ \\
\hline $1090-12 k$ & s1 & $\mathrm{F}$ & E1 & & 11 & $B$ & T.88.6 & $\operatorname{\theta ec}$ & & & & & & 91 & $\mathbf{F 2}$ \\
\hline $1000-12 B$ & B1 & $\mathrm{FB}$ & E1 & & 11 & 8 & T,ES,L & Dog & & & & & & 81 & $\mathbf{f 2}$ \\
\hline $\cos x-12 x$ & \$1 & F3 & E1 & & 11 & 5 & T.6\%. & 000 & & & & & & $\$ 1$ & f2 \\
\hline $100 \times-1100$ & si & Ps & E1 & & 11 & 1 & T.SS.L. & $D 00$ & & & & & & $\$ 1$ & $P 2$ \\
\hline 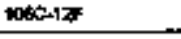 & 32 & F2 & 1 & $\mathrm{C}=10$ & 12 & 12 & T.S.6. & ext & ats & 효 & $\mathbf{p}$ & 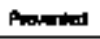 & Prnsend & $\underline{s}$ & F2 \\
\hline $1000-120$ & 60 & $F$ & Es & & 7 & & T.986 & Ex & & & & & & & \\
\hline wod-13h & 89 & $\mathrm{Fz}$ & E & $A-1 \rightarrow$ & 15 & 15 & T.60. $\mathrm{W}$ & 609 & $\cos x$ & 6 & FI & $\Leftrightarrow$ & $\mathbf{f 1}$ & 93 & $\mathrm{~F} 2$ \\
\hline $1000-135$ & 90 & $\mathrm{Nh}$ & en & NA & & & T.SSL & 500 & & & & & & & \\
\hline 10TANADIAE WEDA & b2 & $\mathbf{F 3}$ & ez & Q.14t & 14 & $\pm z$ & T.os. & $\cos$ & $17 x$ & & & 51 & $\mathbf{F}$ & 点 & $\$ 2$ \\
\hline 107ANGHEYED & 97 & $\mathbf{F}$ & E? & B-1-1 & 12 & 12 & T.DB.C & DN2 & $17 x$ & $\mathbf{B Z}$ & $\mathbf{F}$ & Bt & Fy & 78 & $\mathbf{F}$ \\
\hline 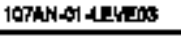 & 81 & f & EO & & 11 & 11 & T.125.6 & $5 \%$ & & & & & & 51 & $\mathbf{F g}$ \\
\hline 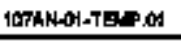 & $\$ 1$ & Mn & $\mathbf{m}$ & $\cos$ & & & T.Dect & Pos & & & & & & & \\
\hline 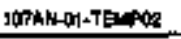 & $\$ 2$ & P1 & e & $201=$ & 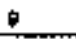 & 18 & T,Dsc & 00 & $17 x$ & 的 & fa & 81 & $\mathbf{F}$ & 8 & 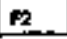 \\
\hline
\end{tabular}




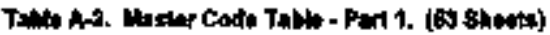

\begin{tabular}{|c|c|c|c|c|c|c|c|c|c|c|c|c|c|c|c|}
\hline 口ו & 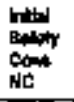 & Frad & $\begin{array}{l}\text { Errs } \\
\text { canis }\end{array}$ & $E$ & 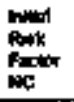 & $\begin{array}{l}\text { Fuled } \\
\text { Cont } \\
\text { Pint } \\
\text { Findor }\end{array}$ & fandery & Cane. & Mep & $\begin{array}{l}\text { Mh } \\
\text { Sariny } \\
\text { Cond } \\
\text { Nic }\end{array}$ & ${ }_{i=1}^{\text {Nhe }}$ & 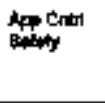 & - & 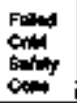 & $F_{\mathrm{F}}^{\mathrm{F}}$ \\
\hline 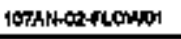 & 82 & $\mathbf{F a}$ & E2 & c-1- & 14 & 12 & TES.c & $\mathbf{m}$ & $\operatorname{sex}$ & & & 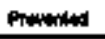 & Awninded & s & 2 \\
\hline 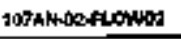 & $\$ 2$ & $\boldsymbol{m}$ & 도 & $\cos$ & 14 & 12 & Tract & Eor & dots & 船 & $\mathbf{F g}$ & Provertad & Prowited & 8 & P2 \\
\hline 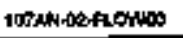 & st & $\boldsymbol{w}$ & $\mathbf{L}$ & & $\mathbf{s}$ & & Ps & Daz & & & & & & & \\
\hline 1OFANORPRES & az & $\mathbf{m a}$ & E2 & Borad & 14 & 12 & $\mathrm{Pg}$ & the & 17 & & & 81 & $\mathrm{~F} \mathbf{3}$ & 8 & $\mathbf{F} 2$ \\
\hline 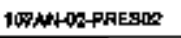 & 8 & $\mathbf{F a}$ & EI & B-1* & 14 & 12 & $\mathrm{PS}$ & 800 & $17 x$ & & & $\$ 1$ & P\$ & $\mathbf{s z}$ & $\mathbf{F}$ \\
\hline 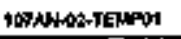 & $\$ 1$ & $\mathbf{F}$ & $\mathbf{E 1}$ & & 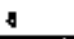 & & 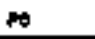 & DOM & & & & & & & \\
\hline HTHAOOSPLOW & $\$ 1$ & $\mathbf{P 3}$ & 4 & & 11 & 11 & po & 0204 & & & & & & a1 & F \\
\hline $1148 \times 02,6$ & st2 & $M$ & 12 & ext & 自 & is & wo & Ens & $\mathbf{a n x}$ & $\mathbf{s x}$ & $\mathbf{n}$ & a1 & $\mathbf{F}$ & $\$ 5$ & $\mathbf{F 1}$ \\
\hline 1148x-04,1.1s & 31 & es & 6 & & 11 & B & T.gp.V & pow & & & & & & s1 & $\mathbf{F} \mathbf{2}$ \\
\hline $1149 \times-04$ d.1b & 81 & $\mathbf{F} 3$ & ED & & 11 & 5 & $\mathrm{~T}$ & DOF & & & & & & $\$ 1$ & $\mathbf{n}$ \\
\hline $1145 \times 104 \pi 2$ & os & Fo & Es & Q-1- & 10 & 1 & T.\$\$ & $\cos$ & $13 x$ & so & fo & 6 & $\mu$ & so & $\infty$ \\
\hline 1145:04ns & ss & $F 1$ & es & Bara & is & 1 & T.SoL's & G12 & $1 \mathbf{4}$ & $\mathbf{s}$ & F1 & so & $\mathbf{R}$ & so & $\mathbf{r o}$ \\
\hline $1148 \times 0404,4$ & 51 & ks & E1 & & 11 & 11 & $\mathbf{T}$ & DOT & & & & & & 81 & $\mathrm{Fa}$ \\
\hline 114Sx-04b.1-1 & 91 & F 3 & E1 & & 11 & 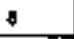 & $T .89$ & EDQ & & & & & & 61 & $\mathbf{F 2}$ \\
\hline 11: & 6 & f1 & ES & $D-1 \bullet$ & 13 & 1 & ISS.L.V & $\cos$ & 133 & 80 & $\mathbf{F} 1$ & $\$$ & $\mathbf{N}$ & $s$ & $\mathbf{m o}$ \\
\hline I19axashists & $\infty$ & $\mathbf{F}$ & $\mathbf{z}$ & & 4 & & TSALV & ther & & & & & & & \\
\hline 144Ex-00, 1 hin & si & $\mathbf{R}$ & 토 & & 11 & 11 & $\mathbf{I}$ & DOT & & & & & & B1 & $\mathrm{F}^{3}$ \\
\hline It 45x) adb.1 A.1b & 82 & F1 & E1 & E-1-1 & 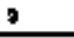 & 7 & $\mathbf{I}$ & DAB & $189 x$ & 80 & $\mathrm{Fa}$ & \$0 & $\mathbf{r s}$ & $s$ & 63 \\
\hline 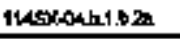 & 93 & $\mathbf{m}$ & $E$ & 614 & 13 & 1 & T.s.t. & 012 & $13 x$ & $\mathbf{s}$ & $\mathbf{F}$ & so & Fo & so & 40 \\
\hline 1145x-00h1.hy & $\mathbf{s}$ & $\mathbf{F 1}$ & $\mathbf{E d}$ & $B-1$ & 13 & 16 & $\mathbf{T}$ & M1:̣ & $14 x$ & 町 & $\underline{F_{2}}$ & sa & $\mathrm{F}_{\mathbf{2}}$ & $\$$ & $\mathbf{p}$ \\
\hline
\end{tabular}




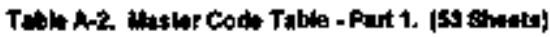

\begin{tabular}{|c|c|c|c|c|c|c|c|c|c|c|c|c|c|c|c|}
\hline $\mathbf{p}$ & 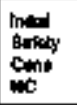 & $\begin{array}{l}\text { Fram } \\
\text { NC }\end{array}$ & $\begin{array}{l}\text { Enw } \\
\text { Cyante }\end{array}$ & and & 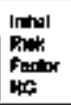 & $\begin{array}{l}\text { Fund } \\
\text { chidil } \\
\text { Posk } \\
\text { Fantor }\end{array}$ & Fanty & $\begin{array}{l}\text { Ct: } \\
\text { arp }\end{array}$ & $\operatorname{limp}_{\text {fex }}$ & Che & 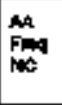 & 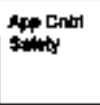 & app Cor & $\begin{array}{l}\text { Faved } \\
\text { Cait } \\
\text { Sifind } \\
\text { Cont }\end{array}$ & Fid \\
\hline $114 x-0, b 1 b=$ & 90 & 82 & $\mathrm{E}$ & & 4 & & T tos L & c112 & & & & & & & \\
\hline 11 devat b i b tb & $\$ 0$ & 11 & 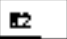 & & 2 & & $T$ & DI: & & & & & & & \\
\hline 11 atsos b 101 & $\$ 1$ & $\boldsymbol{n}$ & $\mathbf{I} 1$ & & 11 & 11 & $T S D V$ & $\mathbf{D O}$ & & & & & & $\$ 1$ & N \\
\hline 1148X-04 b 1 a & 80 & mo & Ea & B-1-A & 10 & 1 & T 1884 & $\cos$ & 13k & an & Fo & $3: a$ & Fo & 80 & Fo \\
\hline $11493-04$ is 1 o 3 & so & $\mathbf{F}$ & E & & 4 & & 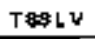 & COR & & & & & & & \\
\hline 1148kot b I d 1 & $\$ 1$ & $\mathbf{F}$ & E1 & & 11 & 11 & $T=0$ & oor & & & & & & $\$ 1$ & P \\
\hline $1145 \times 100461$ d2 & $\infty$ & $m$ & es & beat & is & 1 & $\mathbf{T} \$ L \mathbf{V}$ & क्षा & $13 x$ & so & $\$ 1$ & so & for & $\infty$ & no \\
\hline $1148 x$ 어 b 1 d d & $\infty$ & $n$ & es & & 4 & & 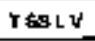 & Dor & & & & & & & \\
\hline $1109 \times 10460$ & $\mathbf{s}$ & F1 & ER & $+14 *$ & 13 & 16 & 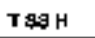 & ado & $\operatorname{sex}$ & कs & $\mathbf{f a}$ & B9 & $\mathbf{F} \mathbf{2}$ & $\mathbf{8 0}$ & $\mathbf{F}$ \\
\hline $1145 x-0005$ & s1 & $\mathrm{F} 3$ & E1 & & 11 & 8 & I ss L $\boldsymbol{V}$ & DOT & & & & & & E1 & $\mathbf{F}$ \\
\hline 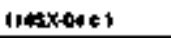 & 82 & F2 & E2 & Drata & 12 & ? & $\mathbf{T}$ & OOT & $180 x$ & 52 & ps & $\$ 1$ & Ps & 玤 & $\mathbf{F 1}$ \\
\hline $1145 \times 000=20$ & $\$ 2$ & Fs & E & Am & 14 & 12 & $\mathbf{t}$ & $\infty$ & 1 to & & & $\$ 1$ & $\mathbf{B}$ & $\Delta$ & $n$ \\
\hline $114 \$ \times 0402 b$ & siz & $\mathbf{p s}$ & es & 0.14 & 14 & 12 & $\mathbf{I}$ & bot & $188 x$ & & & 51 & $\mathbf{F u}$ & 뇨 & $\mathbf{F}$ \\
\hline $1149 x-04020$ & 52 & $\mathbf{E F}$ & E2 & $B-1-9$ & 14 & 9 & $\mathbf{T}$ & DOT & $D$ & & & $\$ 1$ & F3 & 82 & $\mathbf{F I}$ \\
\hline 1145xode2d & 82 & F2 & EF & B-1.t & 12 & 12 & $\mathbf{T}$ & $E 00$ & $198 x$ & & & si & $\mathbf{r}$ & $\mathbf{s}$ & $\mathbf{F}$ \\
\hline 114 4axatio b 1 & 53 & $\mathbf{\mu 2}$ & Es & $4-1=$ & 18 & 18 & $\mathbf{Y \$ O H}$ & bos. & $\operatorname{tax}$ & ga & $\mathbf{F}$ & 89 & $\mathrm{Fz}$ & st & $\mathbf{F 2}$ \\
\hline $1146 \times-65$ : 2 & $\$ 2$ & $k 0$ & ER & $B-1+4$ & $\theta$ & 6 & T\$ & Do1 & $1 \operatorname{sen} x$ & & & 82 & Fo & $\$ 2$ & FP \\
\hline $1143 x-05 b 3$ & 83 & $\mathrm{FO}$ & ES & D-1 1 & 10 & 1 & T SSL & c11 & $1=x$ & 80 & $\mathbf{m}$ & 30 & $\mathbf{F o}$ & 50 & Fo \\
\hline 1145x-e0 t72 & 53 & $\mathbf{F 1}$ & E & 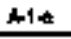 & 13 & 1 & $\mathbf{T}$ & $\cos$ & $13 x$ & so & $\mathbf{F 1}$ & 如 & Fo & 90 & Fo \\
\hline 11460.060000 & 32 & 71 & E1 & $+1=$ & 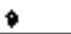 & ; & $\boldsymbol{T}$ & wad & atx & 的 & $m$ & Preponing & Pranatiod & s1 & $\mathbf{m o}$ \\
\hline
\end{tabular}




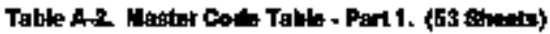

\begin{tabular}{|c|c|c|c|c|c|c|c|c|c|c|c|c|c|c|c|}
\hline $\mathbf{N 0}$ & 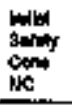 & 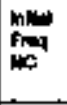 & Etw & DIN & 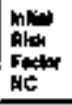 & 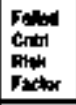 & $\begin{array}{l}\text { Fasily } \\
\text { coode }\end{array}$ & $\operatorname{com}_{\text {cimp }}$ & Rer & 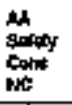 & 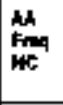 & ing & imatil & 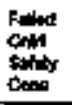 & Fid \\
\hline $1+46 \times-06, d$ & 82 & $\mathbf{F a}$ & EI & A-1 & 14 & 18 & т.вمبر & 800 & $05 x$ & \$3 & Fa & 83 & $\mathbf{F} 2$ & 83 & $\mathbf{F} 2$ \\
\hline $1148 \times 008+10$ & 63 & P3 & est & B-r-n & 16 & 8 & $\mathbf{T}$ & 510 & $12 x$ & sa & $\boldsymbol{m}$ & Prontitiod & Prowitud & $\mathbf{k}$ & $\infty$ \\
\hline $1148 x-09 * 16$ & 83 & F1 & es & $A-1-\oplus$ & 19 & 15 & T\&O.H & $\mathbf{m o n}$ & $\cos x$ & 53 & $\mathbf{F s}$ & $\Leftrightarrow$ & $F_{2}$ & 6 & $r$ \\
\hline 114000102 & 50 & Ps & 2 & & 7 & & $\mathbf{T}$ & Doo. & & & & & & & \\
\hline $1146 \times 106013$ & As & $\mathrm{Ft}$ & $E B$ & Bing & 13 & 1 & $\mathbf{T}$ & C12 & 19 & an & F1 & 80 & Fo & 80 & $\mathbf{F a}$ \\
\hline 114 dxacte & 81 & NA & Nk & FP & & & $r$ & $F 06$ & & & & & & & \\
\hline SOHRALA & 81 & $\mathrm{F3}$ & $\mathbf{E}$ & & 11 & 8 & $\mathrm{FOON}$ & Do1 & & & & & & 4 & $\mathbf{n}$ \\
\hline 304AR-018 & $\$ 1$ & $\mathbf{P 3}$ & 1 & & 11 & 11 & rand & $D 00$ & & & & & & si & $r s$ \\
\hline sounthole & ง1 & Bs & E1 & & 11 & 11 & Pad & Don & & & & & & \$ & $\mathbf{f a}$ \\
\hline 20ankin 0 & 81 & 53 & E1 & & 11 & 11 & $\mathrm{FaOA}$ & Dos & & & & & & 81 & $\mathbf{F}$ \\
\hline Zounade & si & $\mathbf{F}$ & E1 & & 4 & & $\mathrm{Fand}$ & Baps & & & & & & & \\
\hline OWAR-OHF & $\$ 1$ & f1 & E1 & & 5 & & F204 & Don & & & & & & & \\
\hline 204R-016 & $\$ 1$ & 62 & $\mathbf{E 1}$ & & : & & for & Doo & & & & & & & \\
\hline $2004 k$ on & $\boldsymbol{g}$ & $\mathbf{F}$ & 2 & 6 & 12 & 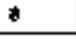 & HaA & a & 25 & 51 & $\mathbf{F z}$ & 51 & $\boldsymbol{r z}$ & g्ष & $\mathrm{Fz}$ \\
\hline Z04شFAOII & 8 & $\mathbf{F} 2$ & EI & c-2ob & 12 & $\square$ & Fan & 012 & $25 x$ & 61 & f2 & 81 & $\mathrm{Fz}$ & 81 & $\mathbf{F z}$ \\
\hline 2044R-01] & $\$ 1$ & $\mathbf{F}$ & $\mathbf{E} 1$ & & 11 & 11 & f2014 & 608 & & & & & & s1 & $\mathbf{r 3}$ \\
\hline 21URAdik & s1 & $\mathbf{s}$ & E1 & & 15 & 11 & $\operatorname{mon}$ & ene & & & & & & al & $B$ \\
\hline 204trast & s? & $\mathbf{F}$ & E1 & 8.26 & 12 & 므. & FAT & $\mathrm{cos}$ & $25 x$ & B1 & F2 & 81 & $F$ & $\$ 1$ & $\mathbf{F z}$ \\
\hline $204 A R+068$ & 82 & $\mathbf{F}$ & E1 & $c-2 b$ & 12 & $\bullet$ & Fan & D12 & $25 x$ & $\$ 1$ & F & $\$ 1$ & $\mathrm{Fz}$ & 51 & $\boldsymbol{F z}$ \\
\hline 204AR-0AC & 91 & $\mathbf{2}$ & E1 & & B & & For & oos & & & & & & & \\
\hline
\end{tabular}


Table A-2 Mastep Cod Tablo - Part 1. (53 sheets)

\begin{tabular}{|c|c|c|c|c|c|c|c|c|c|c|c|c|c|c|c|}
\hline $\mathbf{0}$ & 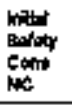 & 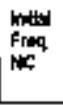 & Cun & EN & 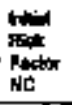 & $\begin{array}{l}\text { Fut } \\
\text { End } \\
\text { Rlat } \\
\text { Feofor }\end{array}$ & ${ }_{0 \rightarrow 4}$ & Con & $\ln _{n \rightarrow \infty}$ & 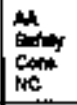 & 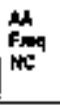 & Liptin & م=9 & $\begin{array}{l}\text { fand } \\
\text { chind } \\
\text { sinty } \\
\text { comp }\end{array}$ & $\begin{array}{l}F=0 \\
\text { Find } \\
\text { find }\end{array}$ \\
\hline 204R-CD & 51 & $\mathbf{F 2}$ & E1 & & 8 & & Fast & Dos & & & & & & & \\
\hline $30 A R-13 x$ & 51 & F3 & EI & & 11 & 11 & nod & no & & & & & & a1 & Fs \\
\hline 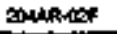 & 81 & F\$ & $E 1$ & & 11 & 11 & rast & Ext & & & & & & $\$ 1$ & $\mathbf{B}$ \\
\hline govateras & 51 & Ps & t & & 11 & 11 & $\operatorname{tax}$ & pos & & & & & & 81 & $\mathrm{FB}$ \\
\hline $204 A \mathrm{P}-0<8$ & ह1 & $\mathbf{F}$ & E1 & & 91 & 11 & FoP & EQ8 & & & & & & B1 & F3 \\
\hline$x \Delta A-\cos$ & 盎 & $\mathbf{F z}$ & E1 & & $E$ & & rast & 012 & & & & & & & \\
\hline 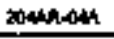 & 51 & $\mathrm{~F}^{3}$ & $\mathbf{e}_{1}$ & & 11 & 11 & rast & Dot & & & & & & 31 & $P$ \\
\hline Xuenfacont & 1) & $\boldsymbol{\theta}$ & $\mathbf{n 1}$ & & $1 \tau$ & 11 & $\mathrm{Fan}_{2}$ & Enot & & & & & & s1 & $\mathbf{F}$ \\
\hline 20041046 & s1 & $\mathbf{P B}$ & et & & t1 & 11 & $F 2004$ & EDY & & & & & & 81 & $\mathbf{f 4}$ \\
\hline 2041 RO40 & 81 & $\mathbf{F}$ & E1 & & 11 & 11 & F204 & EOA & & & & & & B1 & fo \\
\hline DOAR-Q4E & \$1 & $\mathbf{F u}$ & E1 & & 11 & 11 & Fand & cos & & & & & & 11 & $\mathbf{P a}$ \\
\hline Dank-04k & $\$ 1$ & $\mathbf{F}$ & E1 & & 8 & & Pan & bat & & & & & & & \\
\hline Totaraton & $\$ 1$ & $\mathbf{r}$ & $E 1$ & & 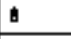 & & F 204 & D12. & & & & & & & \\
\hline 2044R-058 & g1 & $\mathbf{A s}$ & E1 & & 11 & 11 & FaO4 & $E 06$ & & & & & & 81 & $\mathbf{F a}$ \\
\hline 304 A.cosc & $\$ 1$ & $\mathbf{F}$ & $\mathbf{E 1}$ & & 8 & & F20.4 & ENo & & & & & & & \\
\hline zomphosh & $\$ 1$ & F & $\mathbf{E} 1$ & & 11 & 11 & Pan & $\cos$ & & & & & & $\$ 1$ & k \\
\hline 204AR-069 & 31 & $\mathbf{s}$ & E1 & & 11 & 11 & $\mathrm{FaOC}$ & out & & & & & & 81 & $\mathbf{F}$ \\
\hline 204AR-00C & 91 & $F$ & E1 & & 11 & 11 & Fod & $E=09$ & & & & & & 91 & $\mathbf{n}$ \\
\hline 20ukAoA & 57 & $\boldsymbol{F z}$ & E? & e-1- & 12 & 10 & Pad & Eos & $15 x$ & 59 & $\mathbf{F 3}$ & 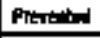 & 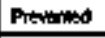 & 53 & ro \\
\hline 2upeners. & 51 & $\mathbf{r y}$ & E1 & & 11 & 11. & 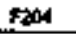 & E01 & & & & & & $8 t$ & rs \\
\hline
\end{tabular}


Table 42. Master Cade Tuble - Purt 1. [5] Ahest:;

\begin{tabular}{|c|c|c|c|c|c|c|c|c|c|c|c|c|c|c|c|}
\hline 10 & 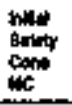 & $\begin{array}{l}\text { Whidal } \\
\text { Fray } \\
\text { NG }\end{array}$ & En & UIN & 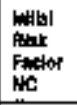 & 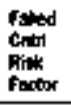 & fontin & $\begin{array}{l}\text { Covose } \\
\text { app }\end{array}$ & $\operatorname{lip}_{n \in 0}$ & $\begin{array}{l}\text { Nh } \\
\text { Extr } \\
\text { Cint } \\
\text { NC }\end{array}$ & $\begin{array}{l}\mathrm{Mh} \\
\text { Frog } \\
\text { NC }\end{array}$ & App Crit & frop & 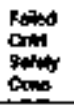 & 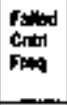 \\
\hline 204AF-DFC & 81 & $\mathrm{Fz}$ & E1 & & 8 & & Fon & Eoa & & & & & & & \\
\hline SOARR-6an & 52 & $\mathbf{z}$ & $\mathbf{E 2}$ & 4.26 & 12 & 4 & rost & on: & $26 x$ & 51 & $\mathbf{P}$ & 91 & $\mathbf{P 2}$ & 21 & $\mathbf{F}$ \\
\hline 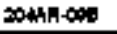 & 62 & $\mathbf{P z}$ & EP & $\cos$ & 12 & $\bullet$ & fast & $\mathrm{DI} 2$ & $25 x$ & $\$ 1$ & $\mathbf{m}$ & s1 & $\mathbf{F r}$ & 81 & $\mathbf{f 2}$ \\
\hline Tbunpact & 52 & $\mathbf{F}$ & 2 & $c-2 b$ & 12 & 4 & ran & $\infty$ & $20 x$ & 01 & $\mathrm{~F} 2$ & 81 & $\mathrm{~F}_{2}$ & st & 2 \\
\hline DMANF-DeO & A2 & $\mathbf{F} \mathbf{2}$ & e1. & 0,20 & 12 & 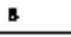 & fres & bis & $25 x$ & a1 & $\mathbf{F 2}$ & 81 & $F_{2}$ & s1 & $F 2$ \\
\hline ZDAR-00E & 81 & $\mathbf{F 2}$ & E1 & & $g$ & & Fon & Dor & & & & & & & \\
\hline 204an-0or & $\$ 1$ & F & E1 & & 11 & 11 & Fat4 & ow & & & & & & 81 & $\mathbf{F a}$ \\
\hline 2014kisos & sto & $r$ & E1 & & 4 & & rat & Dt: & & & & & & & \\
\hline DUARAOAH & $\$ 1$ & $\mathbf{m}$ & n1 & & 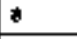 & & $\mathrm{Hat}$ & $\theta=$ & & & & & & & \\
\hline $20 M A R-114$ & g: & $\mathbf{F u}$ & e1 & & II & 11 & FOM4 & Dor & & & & & & $8 x$ & E3 \\
\hline 204AR-11日 & 9l & $\mathbf{F g}$ & E1 & & 11 & 11 & $F 204$ & Dor & & & & & & 81 & $\mathbf{F}$ \\
\hline 204AR-11c & $\$ 1$ & $\mathbf{F}$ & E1 & & 11 & 11 & F2014 & DOT & & & & & & 81 & $\mathrm{F3}$ \\
\hline zockst110 & $\$ 1$ & 13 & E1 & & 11 & 11 & Pad & Exs & & & & & & $\$ 1$ & $\mathbf{P 3}$ \\
\hline zounpolic & si & $\mathbf{b}$ & E1 & & 11 & 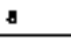 & Fand & Dour & & & & & & 51 & $\mathbf{7}$ \\
\hline 2014R-11F & 32 & $\boldsymbol{m}$ & E1 & And 14 & 12 & 12 & F204 & 80 & $\cos$ & 97 & $\boldsymbol{r}$ & si & $\mathrm{FF}$ & 92 & $\mathrm{Fz}$ \\
\hline $243 w-41$ & 51 & 17 & $\mathbf{E} 1$ & & 11 & 11 & 7213 & 528 & & & & & & 61 & $\mathrm{Fs}$ \\
\hline 2131406 & s1 & Ps & et & & 11 & 11 & $\mathbf{F 2 1 3}$ & D14 & & & & & & $\mathbf{s i}$ & P \\
\hline $213 w 0$ & 81 & $\mathrm{FZ}$ & 5 & & 4 & & $\mathbf{F} 13$ & 809 & & & & & & & \\
\hline $213 w-04$ & 81 & $\mathbf{r}$ & E1 & & $\theta$ & & Fal & 8000 & & & & & & & \\
\hline 213wes & st & $\mathbf{r 2}$ & E1 & & 8 & & p21s & Bo: & & & & & & & \\
\hline
\end{tabular}




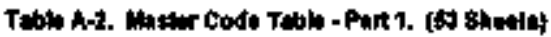

\begin{tabular}{|c|c|c|c|c|c|c|c|c|c|c|c|c|c|c|c|}
\hline 10 & $\begin{array}{l}\text { mold } \\
\text { batisty } \\
\text { Cong } \\
\text { WC }\end{array}$ & 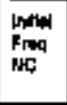 & CNen & Bes & 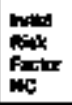 & 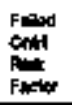 & $\begin{array}{l}\text { Foenty } \\
\text { cosd }\end{array}$ & $\begin{array}{l}\text { Cand } \\
\text { GT }\end{array}$ & $\operatorname{mep}_{n \in c}$ & 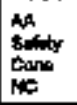 & 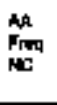 & AppCH & Hpon & 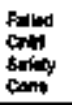 & $\begin{array}{l}\text { fuld } \\
\text { Cmind } \\
\text { froq }\end{array}$ \\
\hline 213 wat & a1 & F2 & E1 & & $\bullet$ & & F러거 & וا & & & & & & & \\
\hline 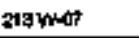 & s1 & $F_{1}$ & E1 & & 6 & & F213 & and & & & & & & & \\
\hline 219 पndes & 91 & $\mathbf{F} 2$ & E1 & & 1 & & $F_{213}$ & Das & & & & & & & \\
\hline 213w- & $\$ 1$ & $\mathbf{f g}$ & E1 & & 11 & 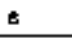 & f213 & 505 & & & & & & $\$ 1$ & $\mathrm{Fz}$ \\
\hline 2444 -01 403 & 31 & $\mathbf{m}$ & E1 & & * & & P244 & DOF & & & & & & & \\
\hline $2404 n-9.6 .01$ & ง1 & $F$ & E1 & & 5 & & 124 & Dor & & & & & & & \\
\hline 24anpor the & 81 & $\mathbf{F} 0$ & E1 & & 3 & & $F_{244}$ & Dor & & & & & & & \\
\hline 24AMR-01 hess & $\$ 1$ & fo & E1 & & 3 & & F244 & घहा & & & & & & & \\
\hline $244 \mathrm{R}-01$ hD & 52 & f* & $\mathbf{E} 1$ & B-1-4 & 14 & 5 & f24d & DOT & Dex & BA & Fo & st & $\mathbf{F} 2$ & 91 & $F 1$ \\
\hline 2mant-01 e01 & $s 1$ & As & Bo & & 11 & 5 & F344 & $\infty$ & & & & & & $\$ 1$ & $\mathbf{n}$ \\
\hline 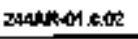 & si & n & 50 & & 11 & 11 & 124 & cort & & & & & & s1 & $\mathbf{R}$ \\
\hline $2 A+A P=M=03$ & s1 & $\mathbf{F a}$ & $E$ & & 11 & t1 & Fo4 & (x) & & & & & & $\$ 1$ & $\mathbf{P}$ \\
\hline 244AR-01_RO & B1 & $\mathbf{F}$ & 10 & & 4 & & F244 & OA1 & & & & & & & \\
\hline 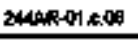 & 81 & $\mathbf{F a}$ & E0 & & 11 & 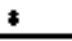 & 024 & Dof & & & & & & $\$ 1$ & $\mathbf{N 2}$ \\
\hline 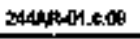 & s & m & 보 & $M$ & 12 & $\mathrm{~B}$ & 1244 & bot. & $\tan$ & 노 & $\mathrm{F}$ & $\mathbf{6 3}$ & $\mathbf{n}$ & 83 & 12 \\
\hline 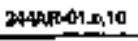 & E1 & $\mathbf{F}$ & EO & & 11 & s & Fa4 & torr & & & & & & 91 & $f 2$ \\
\hline 24AMR-01,s11 & s1 & 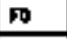 & EO & & 9 & & B44 & Ext & & & & & & & \\
\hline 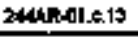 & 31 & $\mathbf{r}$ & te & & $d$ & & 1244 & 007 & & & & & & & \\
\hline 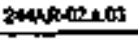 & 帛 & F1 & $\theta$ & A.1. $1=$ & 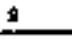 & 18 & Pan & sod & (6) & \$ & H & $\mathbf{5}$ & $\mathbf{F} 2$ & $\mathbf{s}$ & $\mathbf{m}$ \\
\hline 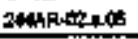 & \$0 & $F$ & co & & 7 & & F24 & 001 & & & & & & & \\
\hline
\end{tabular}


Table A-2. Master Code Table - Part 1. (5s theetsy

\begin{tabular}{|c|c|c|c|c|c|c|c|c|c|c|c|c|c|c|c|}
\hline ID & 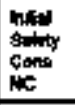 & $\begin{array}{l}\min _{\mathrm{N}} \\
\mathrm{NC} \\
\mathrm{NC}\end{array}$ & EN & 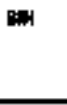 & $\begin{array}{l}\text { Wiss } \\
\text { Rht } \\
\text { Fodter } \\
\text { NC }\end{array}$ & 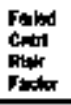 & $\begin{array}{l}\text { Rocinut } \\
\text { Dodt }\end{array}$ & $\begin{array}{l}\text { Gondo } \\
\text { orp }\end{array}$ & 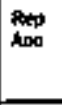 & 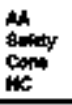 & $\begin{array}{l}\text { Mh } \\
\text { Fing } \\
\text { Nic }\end{array}$ & pont & fop $\mathrm{Cn}$ & $\begin{array}{l}\text { find } \\
\text { Cotit } \\
\text { stinty } \\
\text { cons }\end{array}$ & 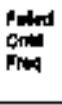 \\
\hline $24001000=00$ & 80 & F3 & Er & & $y$ & & Fa44 & D1 & & & & & & & \\
\hline 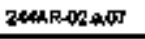 & 50 & F1 & $\mathrm{E}$ & a-1- & $\vartheta$ & 13 & $\mathrm{~F} 241$ & $\infty$ & $2 \operatorname{ax}$ & 69 & F1 & $\Leftrightarrow$ & 51 & 10 & $\mathbf{F}$ \\
\hline 244AR-08 20100 & s! & $\mathbf{F 3}$ & EO & & 11 & 8 & F2H & 008 & & & & & & $\$ 1$ & $\mathbf{H 2}$ \\
\hline $2+1+802000$ & $\$ 1$ & $\mathrm{~F} 3$ & e? & & 11 & 11 & 724 & Dos & & & & & & a1 & $\mathrm{PB}$ \\
\hline $240 \mathrm{kn}-10.10$ & $\$ 1$ & $\mathbf{F}$ & 16 & & 11 & $\Delta$ & P241 & DGS & & & & & & 81 & F2 \\
\hline 24APC02n-11 & s & $\mathbf{F}$ & E2 & & 11 & 11 & Fot & Dog & $\cdot$ & & & & & $\$ 1$ & $\mathrm{~F}$ \\
\hline 244AR-02-12 & 31 & $\mathbf{F}$ & EP & & 31 & 11 & $f_{244}$ & D12 & & & & & & $\$ 1$ & $n$ \\
\hline 24SAR-024,13 & 电 & $\mathbf{F}$ & $\mathbf{E 2}$ & & 7 & & 124 & Di2 & & & & & & & \\
\hline $244002+14$ & s5 & fa & Es & $B-1-$ & 15 & 15 & 224 & 에: & $14 x$ & $\$$ & F & s3. & $P 2$ & $\mathbf{s}$ & F \\
\hline $244 / 40000$ & \$1 & $m$ & e1 & & $\underline{z}$ & & fat4 & 口x1 & & & & & & & \\
\hline 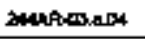 & $\mathbf{3 2}$ & $\mathbf{F}$ & E2 & Alilan & 12 & 15 & F244 & Bxo & $05 x$ & E & $\mathbf{F s}$ & 83 & $\mathbf{F 2}$ & $\Leftrightarrow$ & $\mathbf{f}$ \\
\hline $2040 \times 103,106$ & 82 & $\boldsymbol{r}$ & E2 & C-14 & 12 & 3 & Fat4 & Dor & $20 x$ & 81 & $\mathbf{m}$ & 81 & $\mathbf{F o}$ & $\$ 1$ & $\omega$ \\
\hline 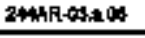 & B1 & Fo & E1 & & 3 & & 1244 & Don & & & & & & & \\
\hline 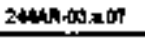 & $\$ 1$ & $\mathbf{r}$ & E1 & & $s$ & & 1244 & Dor & & & & & & & \\
\hline 2404 ind03n00 & 82 & $\mathbf{s}$ & E2 & \& & 14 & $\mathbf{a}$ & $\mathrm{F} 240$ & Don & $23 x$ & 81 & $\mathrm{FP}$ & $\$ 1$ & Fo & 87 & $\omega$ \\
\hline 244AR+09 909 & 92 & $\mathbf{F 1}$ & E2 & A-1- & 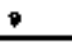 & 9 & F244 & 405 & $01 x$ & s) & $F$ & Prower & Pavented & s1 & Fo \\
\hline 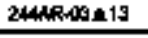 & $\$ 1$ & FO & EO & & 3 & & P244 & tot & & & & & & & \\
\hline 244keda14 & a1 & $\boldsymbol{M}$ & Eo & & $\mathbf{a}$ & & F2A4 & Dog & & & & & & & \\
\hline 24AAR-03016 & 90 & $\mathbf{F}$ & Eo & & 7 & & F244 & D:2 & & & & & & & \\
\hline 244AR-0010 19 & $\infty$ & $\mathbf{F 3}$ & Eo & & 7 & & P24 & Dit & & & & & & & \\
\hline
\end{tabular}


Table A-2. Ungtar Code Table - Pard 1. (E3 Shents)

\begin{tabular}{|c|c|c|c|c|c|c|c|c|c|c|c|c|c|c|c|}
\hline ID & $\begin{array}{l}\text { intal } \\
\text { sith } \\
\text { Cona } \\
\text { NC }\end{array}$ & $\begin{array}{l}\text { Mral } \\
\text { Freq } \\
\text { NFE }\end{array}$ & $\begin{array}{l}\text { Enrr } \\
\text { Cons }\end{array}$ & Bus & 象 & $\begin{array}{l}\text { Fald } \\
\text { Cmirt } \\
\text { Ret } \\
\text { Futor }\end{array}$ & $\begin{array}{l}\text { Fucey } \\
\text { Eotis }\end{array}$ & $\begin{array}{l}\text { Cum } \\
\text { orp }\end{array}$ & 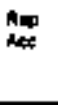 & 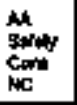 & 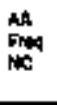 & $\begin{array}{l}\text { Apptot } \\
\text { Saliny }\end{array}$ & Apponts & 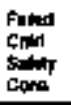 & did \\
\hline 2MAR_D3:17 & stt & F2 & EO & & 4 & & F241 & D12 & & & & & & & \\
\hline $246 \mathrm{AR}-03: 18$ & 81 & $\mathrm{~F} 2$ & EN & & 日 & & P2:44 & DAt & & & & & & & \\
\hline 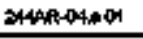 & B1 & $\mathbf{F} 2$ & 50 & & 日 & & P>A & O14 & & & & & & & \\
\hline 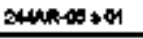 & Es & Wh & $\mathrm{Mm}$ & oce & & & $\mathbf{F} 24$ & f12 & & & & & & & \\
\hline $24440060=02$ & to & $\mathbf{F}$ & $\underline{B}$ & B-14= & 12 & 10 & Pan & D18 & $10 x$ & 8 & $\mathrm{FQ}$ & $\$ 3$ & $\mathrm{Fa}$ & 旳 & $\mathbf{F q}$ \\
\hline 2A4AR-0A I 62 & 81 & $\boldsymbol{w h}$ & $\mathbf{H}$ & $\mathbf{F P}$ & & & Fan & ror & & & & & & & \\
\hline 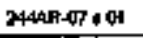 & 81 & FO & E! & & 3 & & Pzit & $D Q$ & & & & & & & \\
\hline 244 ther 4,02 & $\$ 1$ & $\mathrm{FO}$ & EI & & 3 & & 2 & 000 & & & & & & & \\
\hline 240 hatat o os & 91 & $\mathbf{r o}$ & II & & ع. & & 124 & poo & & & & & & & \\
\hline 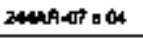 & s1 & $\infty$ & Nod & AP & $\mathbf{s}$ & & F244 & $\mathrm{FPQ}$ & & & & & & & \\
\hline 24ARPPEOI & 81 & $\mathrm{~kg}$ & Non & क्ट्प & 3 & & F2:4 & FOr & & & & & & & \\
\hline 244AFPHD2 & 31 & Nar & Mh & $\infty$ & & & F214 & mos & & & & & & & \\
\hline 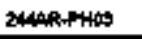 & $\$ 1$ & NA & $m$ & $\mathbf{R P}$ & & & 1204 & rod & & & & & & & \\
\hline 244kephou & si & $\mathrm{Nh}$ & $\mathbf{m}$ & oct & & & $\mathbf{2 4 4}$ & $\mathrm{F} 12$ & & & & & & & \\
\hline 24AAR-PHTE & s1 & was & $\mathbf{m}$ & 0 & & & F244 & Fos & & & & & & & \\
\hline 24AAR-PHNQ & B1 & NA & HW & 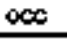 & & & F244 & $\mathbf{F} 12$ & & & & & & & \\
\hline 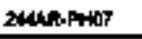 & s & 哺 & $\mathrm{m}$ & Qces & & & F2:4 & $\mathbf{F o s}$ & & & & & & & \\
\hline 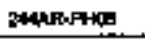 & \$1 & $\mathbf{m}$ & $\ln$ & ops, & & & F244 & $\omega s$ & & & & & & & \\
\hline 244AR-9709 & 81 & $\mathbf{m}$ & m & ocs. & & & F344 & ress & & & & & & & \\
\hline 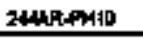 & B1 & $\mathrm{m}$ & H & ogc & & & 704 & ras. & & & & & & & \\
\hline
\end{tabular}


Table A-2 vaster Coda Tobla + Part 1. (53 Aheta)

\begin{tabular}{|c|c|c|c|c|c|c|c|c|c|c|c|c|c|c|c|}
\hline 0 & 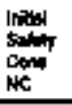 & $\begin{array}{l}\text { Voillor } \\
\text { Freq } \\
\text { NGC }\end{array}$ & Enena & UN & 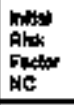 & 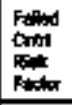 & $\begin{array}{l}\text { Fodili } \\
\text { code }\end{array}$ & $\begin{array}{l}\text { Catsos } \\
\text { Brp }\end{array}$ & $\operatorname{lip}_{n \infty}$ & 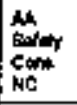 & $\underset{N \rightarrow C}{\cos }$ & 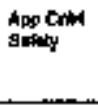 & Fineq & 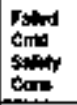 & $\begin{array}{l}\text { Adled } \\
\text { Oxin } \\
\text { ind }\end{array}$ \\
\hline 20AAAAPIFI 1 & 的 & Ma & $\mathbf{m a}$ & occ & & & 1244 & Fos & & & & & & & \\
\hline $244 \mathrm{FP}+\mathrm{H} 2$ & 81 & NA & $m$ & oce & & & $\mathbf{F} 2 \mathbf{s}$ & $\mathbf{F O S}$ & & & & & & & \\
\hline 24HAPH & B1 & Nh & an & osc & & & Fo4s & Fos & & & & & & & \\
\hline $244 \mathrm{R}-\mathrm{H} 11$ & 5 & wh & $M$ & $\infty$ & & & P24d & $\operatorname{mos}$ & & & & & & & \\
\hline 2464APPW15 & tot & hin & $\operatorname{men}$ & $\operatorname{coc}$ & & & F24 & ex & & & & & & & \\
\hline 24MAPHIA & B1 & $\mathbf{m}$ & $\mathrm{ruh}$ & $\operatorname{coc}$ & & & F244 & fot & & & & & & & \\
\hline 244R-PH17 & 81 & $\mathbf{m}$ & $\mathrm{MH}$ & 0,0 & & & Fo:44 & $\mathbf{F} 12$ & & & & & & & \\
\hline 244HPFH10 & 81 & $\mathrm{~m}$ & nom & $\infty$ & & & P241 & res & & & & & & & \\
\hline 244Ak-110 & 11 & $\mathbf{m}$ & in & $0<0$ & & & 24 & ms & & & & & & & \\
\hline $2720 \times-01$ & $\$$ & 62 & E1 & & a & & $\mathbf{s t 3}$ & bor & & & & & & & \\
\hline ZTPN-12 & 82 & $\mathbf{F 1}$ & E1 & No1. & $s$ & D & ss & 5 & $\operatorname{sox}$ & \$2 & $\mathrm{F}_{3}$ & 82 & $\mathbf{F} 1$ & 6 & F1 \\
\hline 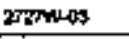 & $\mathbf{9 2}$ & $\mathbf{F}$ & E1 & $A-1-1$ & 12 & . & Bs & 021 & $50 x$ & st & F\$ & $\mathbf{5 z}$ & $F 1$ & 82 & n \\
\hline 2rrmad & 52 & 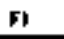 & c1 & 14 & 9 & 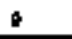 & 58 & M & $\operatorname{six}$ & 32 & A & $\leq 2$ & 1 & siz & fI \\
\hline ZLTHAd & $\$ 2$ & As & E1 & A. A. & 14 & $\theta$ & $\$ 8$ & Bos & $\sin$ & \$2 & $\mathbf{F}$ & 50 & k1 & $s$ & $\mathbf{F 1}$ \\
\hline grermas & 52 & $F_{2}$ & E & And & 12 & 8 & 69 & Dox & 30 & & & 62 & $\mathbf{F} 1$ & $E$ & $\mathbf{F 1}$ \\
\hline soon & $\$ 1$ & F3 & 61 & & 11 & 11 & $\$ 90$ & $\mathrm{DN} 2$ & & & & & & B1 & $\mathbf{F 3}$ \\
\hline $\cos 2$ & 81 & $\mathbf{B s}$ & e1 & & 11 & 11 & $\mathrm{sen}$ & Da & & & & & & $s$ & $\mathbf{r 3}$ \\
\hline scipos & 51 & $\mathrm{F3}$ & E1 & & 11 & 11 & 80 & pos & & & & & & $\$ 1$ & 13 \\
\hline s000 & 91 & F 3 & E1 & & 11 & 11 & $\$ 90$ & BXP & & & & & & 81 & $F 3$ \\
\hline 905 & 51 & $F 1$ & E1 & & 5 & & 590 & 80 & & & & & & & \\
\hline
\end{tabular}




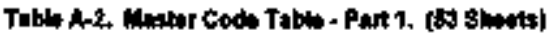

\begin{tabular}{|c|c|c|c|c|c|c|c|c|c|c|c|c|c|c|c|}
\hline סו & 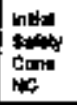 & 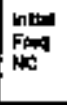 & Ex & and & 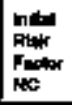 & 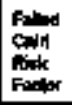 & finery & $\underset{\operatorname{Com}}{C=1}$ & mes & 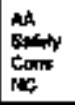 & $\begin{array}{l}\text { Ah } \\
\text { Froq } \\
\text { NGE }\end{array}$ & spp Cot & Fing & 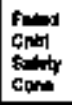 & 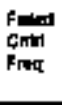 \\
\hline $\operatorname{son} 0$ & $\$ 1$ & $\mathbf{r s}$ & 4 & & 11 & is & $\operatorname{sen}$ & 012 & & & & & & 61 & $\mathbf{1 3}$ \\
\hline E00-4t? & a1 & $\mathrm{Fa}$ & $e_{1}$ & & 11 & 11 & 800 & $\cos$ & & & & & & by & Fs \\
\hline Eooss & sy & $\mathrm{Fa}$ & et & & 11 & 11 & 800 & Dot & & & & & & bu & $\mathbf{F}$ \\
\hline $200-19$ & Bg & $\mathrm{Fz}$ & E1 & & 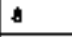 & & $\sin$ & 1000 & & & & & & & \\
\hline $100-10$ & $\mathbf{B 1}$ & $\mathbf{F 1}$ & E1 & & 6 & & $s p 0$ & 809 & & & & & & & \\
\hline explt & 31 & Ps & $\mathbf{E} 1$ & & 11 & 11 & 90 & Did & & & & & & $\$ 1$ & $\mathbf{s}$ \\
\hline ANOA & s & Nh & $\mathbf{M H}$ & $\infty 00$ & & & 0 & $\boldsymbol{N o s}$ & & & & & & & \\
\hline END.014 & B1 & Ma & $E_{1}$ & $\infty x$ & & & Con & FES & & & & & & & \\
\hline$B=02$ & 82 & $\mathbf{F Z}$ & E1 & $A=1=$ & 12 & 16 & $\mathbf{I}$ & 804 & $05 x$ & $\mathrm{Ba}$ & $\mathbf{F S}$ & 83 & $\mathbf{F} 2$ & 8 & $\mathbf{F}$ \\
\hline$B N D-\infty A+1$ & 31 & F2 & E1 & $\infty<0$ & 4 & & con & 804 & & & & & & & \\
\hline cersat & 32 & 80 & 52 & B-1-1 & $\bullet$ & & $M$ & Dos? & $\operatorname{tax}$ & si & ro & $\$ 1$ & $\infty$ & & \\
\hline 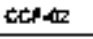 & \$1 & $\mathbf{s}$ & E1 & & 11 & 11 & $M$ & octs & & & & & & s1 & $\mathbf{b}$ \\
\hline 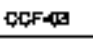 & 81 & $\mathbf{F} 3$ & EO & $k p$ & 11 & 11 & $\boldsymbol{M}$ & & & & & & & B1 & 63 \\
\hline CCF-0 & si & P3 & EO & RP & 11 & 11 & M & & & & & & & B & $\mathrm{fg}$ \\
\hline ceptes & so & He & EO & NA & & & $M$ & & & & & & & & \\
\hline CCFAt & 80 & wh & $\mathbf{E}$ & NR & & & $M$ & & & & & & & & \\
\hline CCF-G & 32 & . & $E$ & $c-2 a b$ & 14 & 15 & H & E00 & $2 x$ & $\$ 3$ & $F$ & Pranerind & Parninid & 83 & $\boldsymbol{F}$ \\
\hline$C C F-C B$ & 50 & Mh & ED & NR & & & H & & & & & & & & \\
\hline $\cos -\infty \theta$ & 80 & $\mathrm{HA}$ & E0 & $\mathbf{H R}$ & & & $\mathbf{G}$ & & & & & & & & \\
\hline$c(c=10$ & 90 & $\mathbf{H e h}$ & $\mathbf{m}$ & GR & & & 19 & & & & & & & & \\
\hline
\end{tabular}




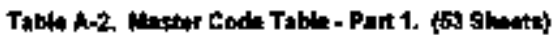

\begin{tabular}{|c|c|c|c|c|c|c|c|c|c|c|c|c|c|c|c|}
\hline $\mathbf{0}$ & 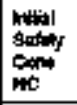 & 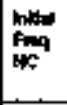 & Gir & Ben & $\begin{array}{l}\text { With } \\
\text { Peik } \\
\text { Foctor } \\
\text { WC }\end{array}$ & $\begin{array}{l}\text { Fadid } \\
\text { Chilit } \\
\text { Addef } \\
\text { Fiter }\end{array}$ & Fechy & Cap & $\operatorname{Rep}_{A \infty}$ & 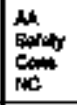 & Fin: & 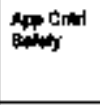 & Mppcis & 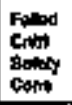 & 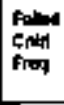 \\
\hline $\cos -11$ & $\$ 0$ & m & es & $m$ & & & 6 & & & & & & & & \\
\hline $\cos -12$ & 00 & Mh & et & his & & & 0 & & & & & & & & \\
\hline der.19 & $\$ 0$ & $M$ & $E 0$ & $\min$ & & & $\theta$ & & & & & & & & \\
\hline LCcF.14 & $\$ 1$ & $M$ & $m$ & $\operatorname{coc}$ & & & G & Fis & & & & & & & \\
\hline ACF.15 & s1 & $\mathrm{NH}$ & ra & $\infty$ & & & 0 & $\mathbf{F 1 2}$ & & & & & & & \\
\hline $\operatorname{cec}$ - 10 & Q1 & $F$ & Ex & & 11 & 11 & $M$ & DN & & & & & & $\mathbf{s 1}$ & Fs \\
\hline CEF.17 & 8 & F1 & Es & Arac & 13 & 1 & T.t.5 & $\cos$ & $13 x$ & so & F1 & so & ro & sa & No \\
\hline Dep.1s. & so & Fo & os & B-14 & 10 & 1 & tso.y & an & $\operatorname{lan}$ & so & Po & $\boldsymbol{m}$ & NA & 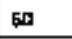 & Fo \\
\hline CCF-1D & so & . & $\mathrm{Eg}$ & Borda & 10 & 1 & 1 sp.4 & c51 & $14 x$ & 50 & $\mathrm{FO}$ & $\mathbf{m}$ & Nh & m & Fo \\
\hline $\cos -20$ & En & 12 & EI & B-1-8 & 12 & 9 & $\mathbf{T}$ & DOH & $03 x$ & $\$ 2$ & f9 & Propotid & Fwionted & 52 & F1 \\
\hline $\cos 01$ & Bat & $\mathrm{Fu}$ & E) & & 14 & 11 & IR & 014 & & & & & & s1 & $\oplus$ \\
\hline copdes & 50 & $\mathrm{~F}$ & Eo & & $I$ & & ER & DIA & & & & & & & \\
\hline $\cos \theta$ & 51 & $n$ & EI & & 11 & 11 & ER & 며옹 & & & & & & B1 & F \\
\hline CPP-OA & 50 & $\mathbf{N}$ & 툐 & & 7 & & ER & 미 & & & & & & & \\
\hline $\cos -65$ & \$1 & $\mathbf{F}$ & 51 & & a & & ER & 00 & & & & & & & \\
\hline $\cos 20$ & s1 & $\mathbf{F y}$ & eb & & 11 & 11 & es & 020 & & & & & & \$1 & $\mathbf{A}$ \\
\hline copsp & so & $\mathbf{s}$ & e & & 7 & & 皇 & $D 06$ & & & & & & & \\
\hline CDP-DS & $\$ 1$ & $\mathbf{F}$ & E1 & & 11 & 11 & $\mathrm{gR}$ & $\$ 00$ & & & & & & 31 & Fo \\
\hline $\cos \phi$ & \$1 & Bh & Nh & هce & & & 0 & .2x & & & & & & & \\
\hline CDP.10 & $\mathbf{3 2}$ & $\mathbf{F 3}$ & 18 & $c \rightarrow 0$ & 14 & 11 & ER & t站 & 2 & a1 & $\mathbf{P}$ & sa & 0 & si & $\mathbf{r s}$ \\
\hline
\end{tabular}




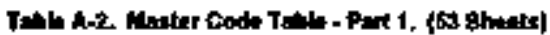

\begin{tabular}{|c|c|c|c|c|c|c|c|c|c|c|c|c|c|c|c|}
\hline$D$ & $\begin{array}{l}\text { Intal } \\
\text { Bantrir } \\
\text { Cans } \\
\text { NC }\end{array}$ & & Ear & 6an & 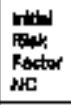 & $\begin{array}{l}\text { Falod } \\
\text { chand } \\
\text { itho } \\
\text { factor }\end{array}$ & $\begin{array}{l}\text { Pechiny } \\
\text { Dode }\end{array}$ & Gav & Af & 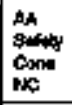 & $\mathrm{Fin}_{\mathrm{N}}$ & spentirl & Apportil & 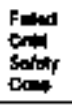 & $\begin{array}{l}\text { ralld } \\
\text { colis } \\
\text { Find }\end{array}$ \\
\hline $\cos 01$ & $\Leftrightarrow$ & $\boldsymbol{p}$ & $\mathbf{E}$ & e-1- & 10 & 15 & WB & 베2 & $15 x$ & & & 32 & $\mathbf{P}$ & $\Leftrightarrow$ & $\mathbf{F}$ \\
\hline $\operatorname{coth} \theta 2$ & $\mathbf{s 1}$ & $\mathbf{P a}$ & Er & & 11 & 11 & wo & Dnd & & & & & & $\mathbf{1 1}$ & $\mathbf{r o s}$ \\
\hline chostas & $\mathbf{s 1}$ & $\mathbf{f s}$ & $\theta$ & & 11 & 11 & WE & Dor & & & & & & \$1 & $\mathbf{r s}$ \\
\hline COBA4 & 32 & $\mathrm{~F}$ & F & $6-2 b$ & 14 & is & WB & D12 & sex & ss & $\mathrm{Fs}$ & $\mathbf{3 1}$ & $\mathbf{F 3}$ & 30 & $m$ \\
\hline $\cos 0 \sin ^{\circ}$ & 31 & $\mathrm{~F} 9$ & Es & & 11 & 11 & WE & E14 & & & & & & 81 & $\mathbf{F}$ \\
\hline $\cos \theta \theta^{\circ}$ & 31 & $\mathbf{P a}$ & EI & & 11 & 15 & WE & 014 & & & & & & $\$ 1$ & $\mathbf{F g}$ \\
\hline cots-est & s1 & $\mathbf{P s}$ & EI & & 11 & 6 & WB & Die & & & & & & 51 & $\mathbf{p}$ \\
\hline colluatd & $\mathbf{s 1}$ & $\mathbf{n}$ & E1 & & 11 & $B$ & Ws & $\infty$ & & & & & & s1 & 12 \\
\hline COQB & B1 & $\mathrm{Fa}$ & E1 & & 11 & 11 & ws & Dats & & & & & & 61 & $\mathbf{n}$ \\
\hline cos- -4 & B1 & fy & c1 & & 11 & 6 & NB & E14 & & & & & & B1 & $\mathbf{F}$ \\
\hline $\cos \theta$ & si & $\mathbf{r s}$ & 4 & & 11 & 11 & NB & DW & & & & & & si & $\mathbf{F a}$ \\
\hline CGo-45 & 51 & ss & $\mathbf{H}_{1}$ & & 11 & 11 & WB & ons & & & & & & $\$ 1$ & bs \\
\hline C,Ce-s] & 81 & $\boldsymbol{F z}$ & E1 & & $B$ & & WB & Ex & & & & & & & \\
\hline $000-06$ & 92 & $\mathrm{FP}$ & F & a्qb & 14 & 13 & nas & D12 & 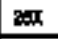 & 59 & $F$ & s1 & $\mathbf{F s}$ & 93 & F1 \\
\hline ERNAOH & st & Fs & E1 & & 11 & 8 & $\mathrm{~T}$ & 108 & & & & & & 31 & $\boldsymbol{F} 2$ \\
\hline CRN-OA + & bt & $\mathbf{F t}$ & E1 & & $1 \%$ & b & $\mathrm{OM}$ & E* & & & & & & $\$ 1$ & $\mathbf{r 2}$ \\
\hline CARAOS & $\$ 1$ & F3 & E1 & & 11 & 4 & $T$ & 00 & & & & & & $s 1$ & $P$ \\
\hline CRAN02+1 & 31 & $F 2$ & E1 & & 8 & & con & 020 & & & & & & & \\
\hline chandos & 83 & P3 & Es & and & 16 & d & $\mathbf{T}$ & E10 & $12 \alpha$ & sh & $\mathbf{P a}$ & Prmented & Prmated & a2 & FD \\
\hline ctistould & 然 & A & $E_{1}$ & B-14: & 14. & 6 & ton & E10 & $12 x$ & & & Pramolind & Fromednd & 点 & Po \\
\hline
\end{tabular}




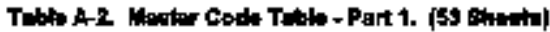

\begin{tabular}{|c|c|c|c|c|c|c|c|c|c|c|c|c|c|c|c|}
\hline 10 & 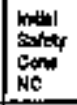 & 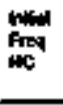 & $\begin{array}{l}\text { Enw } \\
\text { Cons }\end{array}$ & 61\% & finter & 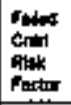 & follies & Otop & $\operatorname{lip}_{n \rightarrow \infty}$ & $\begin{array}{l}\text { Mh } \\
\text { atetr } \\
\text { const } \\
\text { NC }\end{array}$ & $\operatorname{Preq}_{N C}^{M}$ & Soscint & fincidit & 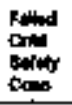 & tid \\
\hline CRR-03-18 & 50 & $\mathbf{A}$ & Ez & 1084 & 14 & 13 & cen & E10 & $21 x$ & 83 & $\mathbf{P B}$ & PTncitad & Prmated & 39 & FI \\
\hline Cow 04 & 8 & $\mathbf{F}$ & E\$ & Borta & 18 & 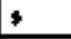 & $T$ & C10 & 120 & 80 & $F 3$ & PTPut & PTimand & 82 & FI \\
\hline CPWOHA & 82 & $\mathbf{F}$ & E1 & Sol-1 & 12 & $\$$ & can & 010 & $12 x$ & 82 & $\mathbf{F B}$ & PTented & PTimonad & 82 & FI \\
\hline cratodin & 22 & $\mathbf{F}$ & E2 & $8-26$ & 12 & 4 & ctu & D10 & $21 x$ & & & PTonotid & Printad & $B 2$ & fo \\
\hline chondos & siz & $r$ & Bt & A-14a & 14 & 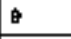 & $\mathbf{T}$ & 복 & of & 뵤 & $\mathbf{N}$ & Protut & Aratated & $5 z$ & p \\
\hline CFMMed & 81 & $\mathbf{H}$ & E1 & & 11 & . & Des & 83 & & & & & & 81 & $r z$ \\
\hline CRNAC & 92 & $F$ & E & $41-0$ & 14 & 9 & $T$ & 029 & wx & $\$ 8$ & $\mathbf{F a}$ & Pewiontiand & Pranoritad & $\$ 2$ & F1 \\
\hline CRN-QH & $\$ 1$ & F3 & E1 & & 11 & 8 & 에 & 00 & & & & & & 81 & $\mathbf{F}$ \\
\hline ERN-OS & 52 & F3 & $\mathbf{2}$ & $A-1-6$ & 14 & 9 & $T$ & t & $\operatorname{tar} x$ & $\$ 2$ & F3 & Pawnonives & Powintald & sis & $\mathbf{F i}$ \\
\hline CENAOAd & 51 & $\mathbf{H 2}$ & EI & & 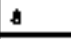 & & 매 & 5 & & & & & & & \\
\hline CFNDOS & 93 & $\mathbf{F}$ & 노 & 수1-10 & 14 & $\mathbf{s}$ & $T$ & E21 & OTx & $\$ 2$ & $\mathbf{r a}$ & Provernd & Preverted & 92 & $\mathbf{F 1}$ \\
\hline GR:DQH & $\$ 1$ & F1 & E & & 5 & & 우 & 821 & & & & & & & \\
\hline CRHAO & 82 & Fs & E2 & $B-1=$ & 14 & 5 & $T$ & Eas & $12 x$ & & & 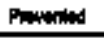 & Prewarited & $\$ 1$ & $\mathbf{F r}$ \\
\hline CRIDOAN & s & $\mathbf{B}$ & EI & B-14. & 14 & 8 & 에 & 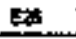 & 1: & & & & & $\$ 1$ & $\mathbf{M}$ \\
\hline Cotop-10 & 如 & $F$ & 4 & B-1-1 & 14 & $B$ & $T$ & box & $12 \pi$ & & & Pranoring & Prantion & $\$ 1$ & $\mathrm{~F} 1$ \\
\hline cran-10 & 82 & $\mathrm{~F} 2$ & E1 & E-1-1 & 12 & 5 & 에 & Das & $16 \mathrm{Cx}$ & 57 & $\mathrm{F3}$ & Pronumind & Prowinined & 89 & F1 \\
\hline Corth-11 & $\mathbf{s}$ & $\mathbf{A}$ & E3 & C:1-10 & 18 & 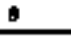 & $T$ & CNo & $12 x$ & $\$ 2$ & $\mathbf{F s}$ & Proverined & Plowerital & $\Leftrightarrow 2$ & $\mathbf{m}$ \\
\hline Cfan 11 - & $\$$ & $\mathbf{P}$ & E1 & 6-14 & 14 & 5 & an & E10 & 18 & & & Proverted & Provided & sa & $\mathbf{M}$ \\
\hline chan-11-B & 80 & P & E1 & B-1-1 & 14 & 11 & OM & 510 & $M x$ & 51 & F & 81 & $\mathbf{P S}$ & 91 & $\mathbf{F}$ \\
\hline cosh11.K & 92 & fg & F & $c-2 b$ & 14 & $\bullet$ & OM & E10 & $205 x$ & 82 & $\mathbf{F 3}$ & 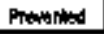 & Praninged & $E$ & Fo \\
\hline
\end{tabular}


Table A-2, Matur Code Table. Part 1. (53 sheota)

\begin{tabular}{|c|c|c|c|c|c|c|c|c|c|c|c|c|c|c|c|}
\hline D & 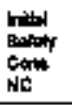 & 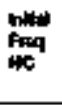 & End & M & 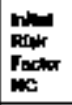 & $\begin{array}{l}\text { fuld } \\
\text { chntil } \\
\text { inth } \\
\text { Fuctor }\end{array}$ & Fadit & Cip & $\lim _{\infty}$ & 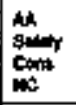 & $\underset{N \in C}{\operatorname{Mn}}$ & App Crit & Fip & 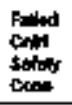 & id \\
\hline cee사-12 & 89 & $\mathbf{F a}$ & EF & $\theta-1-n$ & to & 7 & $T$ & c10 & $\tan$ & 92 & $\mathbf{F a}$ & Prtoritind & Pindind & 82 & FI \\
\hline CA:A-12+4h & 32 & $\mathbf{r}$ & 61 & entan & 12 & 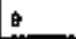 & 에 & D10 & tak & 92 & $\boldsymbol{n}$ & Prosoted & Provented & $\$ 2$ & 11 \\
\hline 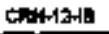 & 52 & $\mathbf{r} \mathbf{2}$ & 1 & e.1- & 12 & 11 & 에서 & 010 & $M K$ & a1 & $\mathbf{p}$ & 91 & $\mathbf{F s}$ & 31 & rs \\
\hline $6 \cos ^{2}-12+\mathrm{c}$ & $\$ 2$ & 12 & 12 & $c+2 b$ & 12 & 0 & OHe & Dי & $\operatorname{anx}$ & & & Proming & 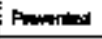 & sz & fo \\
\hline CPRA-13-1A & 82 & Fz & E1 & Bo-14 & 12 & $\checkmark$ & ONC & No & $10 x$ & & & Promentid & 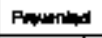 & 82 & F1 \\
\hline CRon-130 & $\$ 2$ & $\mathbf{F} 2$ & E1 & B-1-8 & 12 & 14 & 에 & 005 & $31 x$ & 81 & $\mathbf{F}$ & $\$ 1$ & fa & 91 & $\mathbf{F q}$ \\
\hline CALP13.1C & 52 & $\mathbf{F}$ & E2 & D-1-4 & 12 & 8 & OM & $\cos$ & $10 \times$ & & & Pronoted & Preverted & $s z$ & 51 \\
\hline chnor 13a & ss & Ps & Es & Ada & 10 & 自 & $T$ & $\infty$ & 10 & $\underline{z 2}$ & $z$ & Arringed & Preveritad & sz & 1 \\
\hline Caxp-134 & 63 & P & es & 1.-14: & 13 & $\mathbf{s}$ & $\mathbf{T}$ & pas & $18 x$ & ธี & $\boldsymbol{z}$ & $\$ 2$ & $F_{2}$ & 92 & $\mathbf{r 1}$ \\
\hline CARr-14 & $\$ 1$ & $\mathrm{NA}$ & $\mathbf{m}$ & RP & & & 0 & F10 & & & & & & & \\
\hline GRN-14A & $s 1$ & Nh & E1 & $\$ 00$ & & & on & F10 & & & & & & & \\
\hline CRN-15 & $\$ 1$ & M & M & $R F$ & & & $G$ & F10 & & & & & & & \\
\hline CRN-1BH & 51 & $\mathrm{NH}$ & E1 & $\infty$ & & & an & $\mathbf{1 0}$ & & & & & & & \\
\hline CRN-15 & $\$ 1$ & Nh & in & RP & & & 0 & $F(x$ & & & & & & & \\
\hline CRNA1BA & 51 & NA & E1 & 000 & & & coll & Fo5 & & & & & & & \\
\hline cantily & $\$ 1$ & $\mathrm{~F}$ & E1 & & 11 & 11 & $\mathbf{T}$ & Ex & & & & & & $\$ 1$ & $\mathbf{F 3}$ \\
\hline chathertal & s1 & 点 & E1 & 0 & & & ben & fats & & & & & & & \\
\hline crowas & 요 & 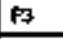 & Eq & $B-1-1$ & 14 & 11 & $\mathbf{T}$ & D10 & $\operatorname{six}$ & 81 & $F$ & 31 & $\boldsymbol{r}$ & 59 & $\boldsymbol{F 3}$ \\
\hline Cont-14 & $\mathbf{s 2}$ & f & EI & B.10 & 14 & 11 & ar & E10 & $M x$ & si & Ps & $\mathbf{s 1}$ & $\mathbf{F}$ & 81 & $\mathbf{F}$ \\
\hline Entwh & 요 & $\mathbf{P}$ & EI & E-14 & 14 & 0 & $\mathbf{T}$ & Q10 & tax & & & Provertend & Prmated & s & $\mathbf{P 1}$ \\
\hline
\end{tabular}




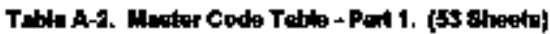

\begin{tabular}{|c|c|c|c|c|c|c|c|c|c|c|c|c|c|c|c|}
\hline$\omega$ & $\begin{array}{l}\text { Intifl } \\
\text { sataty } \\
\text { Dand } \\
\text { NiC }\end{array}$ & 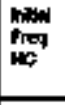 & En & BNA & 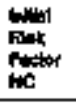 & 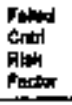 & $\begin{array}{l}\text { Fotitir } \\
\text { cot }\end{array}$ & con & $R_{\infty}$ & $\begin{array}{l}\text { M } \\
\text { sondy } \\
\text { Cont } \\
\text { int }\end{array}$ & $\begin{array}{l}\text { Nh } \\
\text { Fich } \\
\text { WC }\end{array}$ & $\begin{array}{l}\text { Apt cintil } \\
\text { dofly }\end{array}$ & Aroq & 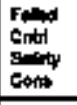 & 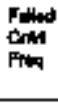 \\
\hline $\operatorname{crop}-1 \theta$ & $* 2$ & $\mathbf{f z}$ & Es & BN-1-n & 12 & 11 & DM & Doso & six & 81 & $\mathbf{F}$ & si & $\mathbf{A}$ & $\$ 1$ & $\mathrm{~F} 3$ \\
\hline $\cos 20$ & 32 & $\mathbf{F}$ & EI & 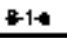 & 14 & . & $\mathrm{T}$ & 50 & aed & & & Prementipd & Propurind & 92 & $\mathrm{F1}$ \\
\hline Crow-20-1 & 32 & $\boldsymbol{F} 2$ & EI & e-1. & 12 & 11 & pN & 105 & XIX & $\$ 1$ & $\mathbf{F a}$ & 81 & $\mathbf{F}$ & $\$ 1$ & $\mathrm{~F}$ \\
\hline GPin-21 & 90 & $\mathbf{F B}$ & Et & & 3 & & $\mathrm{~T}$ & Eto & & & & & & & \\
\hline 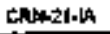 & 50 & $\mathrm{PA}$ & 12 & & $y$ & & Ga & E10 & & & & & & & \\
\hline 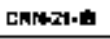 & st & $\mathrm{B}$ & 12 & 8.2b & 14 & $\mathbf{1 a}$ & ond & E & $21 x$ & $\mathbf{s 3}$ & $\mathrm{PB}$ & Proverter & Prowinad & s & $\mathbf{F I}$ \\
\hline CARras & $\mathbf{8 0}$ & $\mathbf{F}$ & $\mathrm{E3}$ & & 4 & & $\mathbf{I}$ & DNo & & & & & & & \\
\hline CRN-ZZ-SA & $\mathbf{9 0}$ & $\mathbf{f 2}$ & E2 & & 4 & & on & Dow & & & & & & & \\
\hline CRNAORG & 62 & $f$ & $\mathbf{E}$ & $B-2 b$ & 12 & 6 & 에 & DNo & $21 x$ & $\$ 1$ & Ps & Proventud & Prumint & 82 & $\mathbf{p}$ \\
\hline CRNAB & st & $\mathbf{n}$ & es & $8-3, b$ & 4 & 由 & $\mathbf{T}$ & DOA & $n x$ & $s$ & $y_{2}$ & Powation & 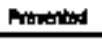 & sz & $\mathbf{F I}$ \\
\hline CRN-CSH & 点 & $\mathbf{F}$ & $E$ & B-9b & 12 & 9 & at & Dos & $\operatorname{tax}$ & & & Plaperted & Primetild & $s$ & $\mathbf{F 1}$ \\
\hline CRN-206-8 & $\$ 2$ & $\mathbf{F}$ & E2 & Q1-1* & 12 & 11 & can & Dos & $31 x$ & & & 81 & $\mathrm{FO}$ & 61 & $\mathbf{F}$ \\
\hline CRNASA & 51 & $\mathbf{N M}$ & m. & ot: & & & 0 & $\mathrm{FW}$ & & & & & & & \\
\hline CRN-244 & $\$ 1$ & 此 & E1 & $\$ \infty C$ & & & cen & $\mathbf{F O X}$ & & & & & & & \\
\hline chonds. & si & $\mathbf{k n}$ & $m$ & DCC & & & (6) & $F$ & & & & & & & \\
\hline Gork-25-1A & s1 & $\mathrm{man}$ & E1 & oos & & & con & $\mathrm{Fz}$ & & & & & & & \\
\hline 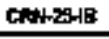 & 8 & $\mathbf{F}$ & E1 & cost & 14 & 11 & an & Ex & $31 x$ & 31 & $\mathbf{F}$ & s1 & $\mathbf{F 3}$ & 81 & $\mathbf{F s}$ \\
\hline Coths: & $\$ 1$ & $\mathbf{h k}$ & Hat & $\infty$ & & & 由. & $\mathrm{FzO}$ & & & & & & & \\
\hline cton-2)- & si & $\mathbf{m}$ & E1 & $\operatorname{coc}$ & & & CNM & 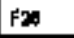 & & & & & & & \\
\hline copar-2B-1B & 62 & $\mathbf{F 2}$ & E1 & $\cos b$ & 12 & 11 & cat & Dos & $31 x$ & s1 & $\mathbf{m}$ & sit & F3 & \$1 & 13 \\
\hline
\end{tabular}




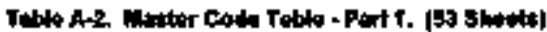

\begin{tabular}{|c|c|c|c|c|c|c|c|c|c|c|c|c|c|c|c|}
\hline 10 & 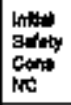 & 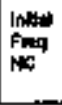 & Em & 16 & 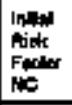 & 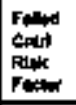 & Found & Gip & Ros & $\begin{array}{l}\text { An } \\
\text { selinty } \\
\text { Cone } \\
\text { ic }\end{array}$ & $\prod_{\omega}^{\infty}$ & 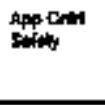 & App Crtat & 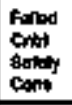 & 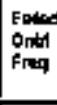 \\
\hline CRNWG & \$1 & $\mathbf{M}$ & $\mathrm{nch}$ & octc & & & 白 & Pot & & & & & & & \\
\hline CRN-2TH & 31 & H & EI & occ & & & con & Da & & & & & & & \\
\hline CRN-27-3 & 32 & $\mathbf{F}$ & EI & cose & 12 & 11 & con & D1ధ & $31 x$ & $\$ 1$ & $\mathrm{Fa}$ & 31 & $\mathbf{F s}$ & 81 & $\mathbf{F 3}$ \\
\hline GRN-2AH & $\$$ & Fs & 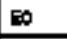 & & 7 & & con & 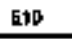 & & & & & & & \\
\hline EMtar & $\mathbf{5 1}$ & As & EI & & 11 & 11 & in & E14 & & & & & & $\$ 1$ & $\mathbf{F 3}$ \\
\hline Evede1 & \$1 & $\mathrm{Fa}$ & E1 & & 11 & 11 & cen & E14 & & & & & & $\$ 1$ & 15 \\
\hline cyatoz & Bat & F\$ & E1 & & 11 & 11 & wo & D12 & & & & & & 81 & $\mathbf{F 3}$ \\
\hline $\cos -\infty$ & 5 & F3 & E1 & & 11 & 11 & con & D12 & & & & & & $\$ 1$ & $\mathrm{~F}^{3}$ \\
\hline curtes & 5 & Ps & EI & & 11 & 11 & Ho & {$[0,0$} & & & & & & 61 & $\mathbf{F 3}$ \\
\hline Chintors & s1 & $\mathrm{F}$ & $\mathbf{E}$ & & 11 & 11 & an & Dos & & & & & & $\leqslant 1$ & $\mathbf{n s}$ \\
\hline CYRTOA & B1 & F1 & E1 & & 8 & & wo & Dists & & & & & & & \\
\hline CWR-OA & B1 & $\mathrm{Fz}$ & E1 & & 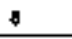 & & De & DIB & & & & & & & \\
\hline CURAS & 82 & F3 & 61 & A.l. & 14 & 9 & wo & 000 & $\sin$ & & & PTonitid & PTantiod & 5 & $\mathbf{F 1}$ \\
\hline CVRASA & \$1 & F1 & EI & & 5 & & ow & $\mathrm{BOA}$ & & & & & & & \\
\hline G) & \$1 & 3at & Nh & PP & & & 0 & F14 & & & & & & & \\
\hline CVR-6B & s1 & WA & E1 & & & & Dan & F14 & & & & & & & \\
\hline CrRar & s1 & $\mathrm{m}$ & in & $\boldsymbol{n}$ & & & 6 & F14 & & & & & & & \\
\hline EvR-05s & B1 & wh & E1 & Ap & & & ons & F14 & & & & & & & \\
\hline CVRR-08 & 91 & HA & Ah & QP & & & a & F14 & & & & & & & \\
\hline CVR=16-1 & st & He & E1 & RP & & & 에 & 514 & & & & & & & \\
\hline
\end{tabular}




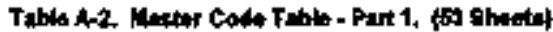

\begin{tabular}{|c|c|c|c|c|c|c|c|c|c|c|c|c|c|c|c|}
\hline I & 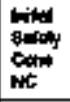 & 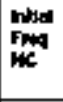 & $\begin{array}{l}\text { Enr } \\
\cos \end{array}$ & Bnx & 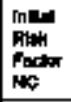 & 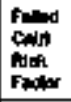 & Fander & $\begin{array}{l}\text { Gour } \\
\text { OT }\end{array}$ & $R_{\rightarrow \infty}^{R}$ & 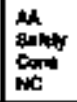 & 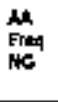 & App cing & ApD CNor & 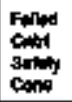 & 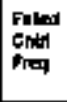 \\
\hline CYR-00 & $\$ 1$ & Wh & MA & $\stackrel{p}{P}$ & & & 0 & Fs: & & & & & & & \\
\hline cuncos & $\$ 1$ & Na & e1 & $k e$ & & & oes & Pod & & & & & & & \\
\hline cyk-10 & $\$ 1$ & NA & 跙 & oct & & & 6 & PO6 & & & & & & & \\
\hline CVIR-IDH & $\$ 1$ & $\mathrm{Na}$ & et & $\infty$ & & & ond & Pod & & & & & & & \\
\hline CLA:11 & 91 & Na & in & $\infty \times c$ & & & 4 & $F \mathbb{R}$ & & & & & & & \\
\hline CVR-11+ & 91 & Wh & E1 & 000 & & & 에 & $\mathrm{FW}$ & & & & & & & \\
\hline CWR.12H & 81 & $\mathrm{F3}$ & E1 & & 11 & 11 & ow & DOA & & & & & & 51 & $\mathbf{B}$ \\
\hline Chrk-15r & 81 & $\mathbf{B}$ & e1 & & 11 & 11 & ON & D4 & & & & & & 51 & 53 \\
\hline 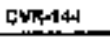 & 51 & $F$ & E1 & $0<C$ & 11 & 11 & ON & Fu & & & & & & 61 & $F 3$ \\
\hline CWR-15Y & 8 & $\mathrm{FB}$ & E1 & B-1- & 14 & $s$ & 에 & D10 & DPX & & & Prenuntind & 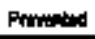 & 88 & f1 \\
\hline DCHAOI & B1 & NA & Mh & RP & & & 6 & Fos & & & & & & & \\
\hline DCPMO1] & s1 & Ne & E1 & $R P$ & & & on & mos & & & & & & & \\
\hline Deneses & 51 & 8 & E1 & & 11 & 11 & 0 & 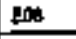 & & & & & & 61 & $\mathbf{F}$ \\
\hline DCN-6OA & 的 & $\boldsymbol{F}$ & $E_{1}$ & & 6 & & ON & Dos & & & & & & & \\
\hline DCN-cen & 81 & f2 & $\mathbf{E} 1$ & & $*$ & & ON & 100 & & & & & & & \\
\hline$D C N=05$ & s1 & $F 1$ & E1 & & $\mathbf{s}$ & & 0 & Dis & & & & & & & \\
\hline OCN-BaH & $\mathbf{b 1}$ & P2 & E1 & Pap & $B$ & & aln & DIt: & & & & & & & \\
\hline OCHAC & 81 & $\mathbf{r}$ & E1 & & 5 & & 0 & 200 & & & & & & & \\
\hline OON-04H & 91 & 12 & $E 2$ & 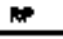 & 5 & & can & $\infty 0$ & & & & & & & \\
\hline OCN 00 & s & $\mathrm{m}$ & M & OCE & & & $a$ & Fos & & & & & & & \\
\hline
\end{tabular}




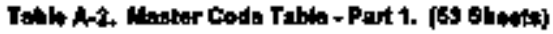

\begin{tabular}{|c|c|c|c|c|c|c|c|c|c|c|c|c|c|c|c|}
\hline 10 & 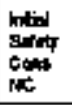 & $\begin{array}{l}\text { Fitheq } \\
\text { Freq } \\
\text { He }\end{array}$ & $\cos$ & 是A & 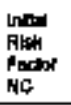 & 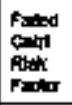 & $\begin{array}{l}\text { Padtir } \\
\text { code }\end{array}$ & Fip & $\lim _{n \rightarrow \infty}$ & 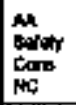 & $\operatorname{Fin}_{i \in \mathbb{R}}$ & Apinterit & Frieg & 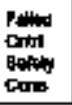 & 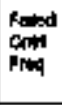 \\
\hline DCNAOA & 91 & Wh & E1 & $\operatorname{coc}$ & & & Con & Fos & & & & & & & \\
\hline DCNOS & $\$ 1$ & WA & Mh & RP & & & 0 & $\mathrm{FOS}$ & & & & & & & \\
\hline DCHast & $\$ 1$ & HA & [1 & RP & & & on & $\mathrm{Fos}$ & & & & & & & \\
\hline DCh-or & $s 1$ & $\mathbf{m}$ & $\mathbf{m}$ & $\cos$ & & & 白 & POA & & & & & & & \\
\hline DCNAIt & s1. & wh & E1 & $\operatorname{coc}$ & & & an & kot & & & & & & & \\
\hline EE-B & 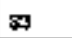 & F1 & ES & $A-1 \rightarrow$ & 13 & 10 & $\boldsymbol{M}$ & 823 & $11 x$ & & & $\Leftrightarrow$ & $\mathrm{FQ}$ & B9 & $\mathbf{F}$ \\
\hline$I=-\infty$ & 31 & $\boldsymbol{B}$ & 51 & & 11 & 11 & $\omega$ & $B=0$ & & & & & & 31 & $\mathbf{H s}$ \\
\hline EESAS & s1 & $\mathbf{r 2}$ & E1 & & む & & M & Dis & & & & & & & \\
\hline EE-04do & st & $\mathrm{m}_{2}$ & Es & heis & 18 & 13 & $T$ & ה & 自为 & & & Parertiod & Amertal & 3s & F1 \\
\hline$E=-04 b$ & 的 & $\mathbf{F z}$ & 白 & MA4 & 15 & 13 & $T$ & 685 & $02 x$ & & & Prawied & Prenertied & \$3 & F1 \\
\hline EE-DF- & 89 & $\mathrm{FB}$ & $\mathrm{Eg}$ & $n-1-1$ & 10 & 19 & $T$ & B2s & 09 & ss & Pz & Proverined & Prowinind & 83 & $F 1$ \\
\hline E[Dos: & $\mathbf{s 3}$ & $\mathbf{F P}$ & E9 & +16 & 16 & 13 & $T$ & cos & 098 & $\mathbf{s}$ & $\mathbf{F}$ & Prowerted & Promented & $\$ 3$ & $\mathbf{F 1}$ \\
\hline Eedod & s1 & $\mathbf{m} 2$ & E1 & & . & & $M$ & 12as & & & & & & & \\
\hline 的禹 & Bo & $\mathrm{man}$ & $\mathrm{Ea}$ & at & & & M & & & & & & & & \\
\hline 压一国 & 90 & m & $E$ & NRR & & & i & & & & & & & & \\
\hline Exas & st & $\mathbf{F y}$ & E & & 11 & 11 & 胡 & $F 12$ & & & & & & $\$ 1$ & $\mathbf{A}$ \\
\hline Be-10 & B1 & $\mathrm{FO}$ & Ba & & 3 & & ti & Diz & & & & & & & \\
\hline EE-11 & $\$ 2$ & $\mathrm{Fz}$ & 6 & cosb & 12 & 12 & 0 & $0+2$ & $20 x$ & & & $s t$ & $F 2$ & 50 & $n$ \\
\hline 드-12 & $\$ 1$ & M & eq & OCC & & & 6 & F12 & & & & & & & \\
\hline exts & A1 & $\mathrm{Na}$ & Na & 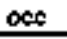 & & & 0 & $\mathbf{F 1 2}$ & & & & & & & \\
\hline
\end{tabular}


Tabbe A-2. Macier Code Tablo - Part 1. (5s Bheete)

\begin{tabular}{|c|c|c|c|c|c|c|c|c|c|c|c|c|c|c|c|}
\hline 10 & 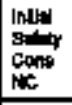 & $\sum_{\substack{n+1 \\
n=0}}$ & tom & end & 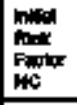 & 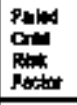 & $\begin{array}{l}\text { Fank } \\
\text { coll }\end{array}$ & מק & $\operatorname{Rip}_{\operatorname{mos}}$ & cont & $\underset{H C}{M}$ & sepcont & Preq & 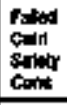 & $\begin{array}{l}\text { Fall } \\
\text { ond } \\
\text { Friag }\end{array}$ \\
\hline EE-14 & 91 & N4 & NA & क्षद्र & & & (19 & $\mathbf{F Z}$ & & & & & & & \\
\hline EE.15 & 51 & ra & HA & occ & & & 0 & $\mathbf{F 2 2}$ & & & & & & & \\
\hline EE.16 & 89 & $\mathrm{FJ}$ & 5 & $B-1-9$ & 16 & 13 & $\boldsymbol{\omega}$ & DO & 10 & 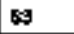 & $F$ & Prapiotid & Prexontad & $\$ s$ & F1 \\
\hline 61) & 32 & $\mathbf{r a}$ & e1 & C-14 & 14 & 11 & A & De2 & $28 x$ & $\$ 1$ & $\mathbf{P}$ & $\$ 1$ & $\mathrm{Fs}$ & $\$ 1$ & fg \\
\hline Efasol & s1 & Nh & $m$ & $\operatorname{loc}$ & & & 6 & res & & & & & & & \\
\hline thyols & 91 & NA & $\mathbf{E}_{1}$ & DCC & & & ON & fisto & & & & & & & \\
\hline APH02 & 81 & MA & in & occ & & & 8 & F65 & & & & & & & \\
\hline 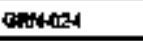 & $\$ 1$ & $\mathrm{NH}$ & e1 & oos & & & om & 505 & & & & & & & \\
\hline Entures & 31 & Nh & e1 & $\infty$ & & & ont & mors & & & & & & & \\
\hline kzostholend & \$s & $\mathbf{k g}$ & E: & & 11 & $B$ & $\mathrm{pt}$ & Eot & & & & & & si & 12 \\
\hline 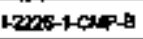 & so & $\mathbf{F}_{\mathbf{Z}}$ & EO & & 4 & & T.ct & EO1 & & & & & & & \\
\hline 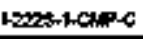 & st & $\mathbf{F}$ & 52 & $a-1 \rightarrow$ & 14 & 15 & Wo & EOI & $15 x$ & $\mathbf{5 3}$ & $\mathbf{F g}$ & $\approx 2$ & $\mathbf{F g}$ & 8 & $\mathbf{F}$ \\
\hline 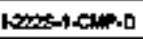 & so & $\mathbf{m}$ & $\mathbf{E}$ & & 4 & & T.CD & Eot & & & & & & & \\
\hline 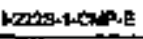 & 63 & $\mathbf{N}$ & Et & N14 & 10 & 12 & T.CD & Bor & 0 & 的 & $\mathbf{F}$ & ss & Fi & ts & $\mathbf{n}$ \\
\hline LOZE-1MA-A & st2 & fo & E2 & B-1- & 14 & 11 & T.cb & EOS & Inx & 61 & P & a1 & $\boldsymbol{F}$ & Bt & fa \\
\hline $12 m+4-12 x+4$ & st & $\mathbf{m}$ & E & $D-1-n$ & 14 & 8 & T. D. & Do1 & $\operatorname{sg}$ & $\mathbf{s}$ & cas & Arusented & Prowand & $\Leftrightarrow$ & $n$ \\
\hline 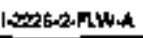 & 81 & $\mathrm{Fa}$ & E1 & & 11 & 11 & 0 & Ees & & & & & & st & F\$ \\
\hline 1.2023-2-FLW-6 & so & $\mathrm{Fa}$ & E1 & & 7 & & $T c t$ & Exs & & & & & & & \\
\hline $1-2 \pi 05-2-F, W-C$ & 62 & $\mathbf{F}$ & E: & B-1-1 & 12 & $\theta$ & 509 & Exa & $\cos$ & 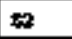 & $\mathbf{F}$ & Proverted & Propended & 87 & Fo \\
\hline I-200s-2NWD & 0 & $\mathbf{F}$ & L1 & & 7 & & $T \cdot \infty$ & $\cos$ & & & & & & & \\
\hline
\end{tabular}




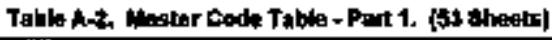

\begin{tabular}{|c|c|c|c|c|c|c|c|c|c|c|c|c|c|c|c|}
\hline Dו & 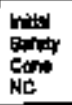 & 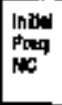 & EN & BIN & 1. & 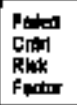 & Pouts & $\begin{array}{l}\text { Cunter } \\
\text { cis }\end{array}$ & Repp & 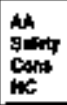 & AN & Sipint & fincent & $\begin{array}{l}\text { Raled } \\
\text { Crit| } \\
\text { Batsty } \\
\text { Coom }\end{array}$ & $\begin{array}{l}\text { Fand } \\
\text { Ond } \\
\text { Fion }\end{array}$ \\
\hline HOZSZUEKA & 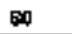 & F1 & E1 & & 2 & & Ac & D10 & & & & & & & \\
\hline $12025-24$ EKGB & 82 & $\mathbf{F I}$ & E2 & D-1 b & 8 & 15 & $\mathrm{mo}$ & O18 & $15 x$ & s3 & P & s2 & Fy & 5 & $\mathbf{7 2}$ \\
\hline H210-2HEK- & st2 & $\mathbf{F z}$ & E2 & $\theta-1 \rightarrow$ & 12 & 13 & $P C$ & 12 & $15 x$ & 6 & $\mathbf{F g}$ & 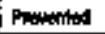 & Printald & $\mathbf{s}$ & F1 \\
\hline 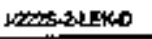 & so & $\mathbf{M 1}_{1}$ & E1 & & 2 & & $\boldsymbol{c}$ & Die & & & & & & & \\
\hline HFos-2ukce & 102 & $\boldsymbol{N z}$ & 酌 & B-qab & 32 & is & wo & Dוt & $15 x$ & s3 & $\mathbf{n}$ & 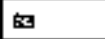 & F2 & 80 & Fz \\
\hline H2MS-2PREA & 82 & $\mathrm{F3}$ & $\mathbf{E}$ & B-1-1 & 14 & 19 & T,CD & Do1 & $2 x$ & $\$ 1$ & f & BI & $\mathbf{F a}$ & 81 & F3 \\
\hline $13 \times 24-1+F \mid N-A$ & $g$ & $\mathbf{F z}$ & 87 & $4-1=$ & 76 & 15 & T.DSH. & Bale & Dax & $\$ 3$ & $\mathbf{F}$ & $\mathbf{s o}$ & FI & so & $\mathbf{F}$ \\
\hline 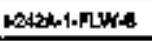 & ss & $\boldsymbol{n} 2$ & es & melat & 1t & 95 & T.OS.H.P & Bot & $D a x$ & 8 & F3 & 50 & $\mathbf{F I}$ & \$s & $\mathbf{F 2}$ \\
\hline 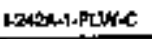 & ஆ3 & $\mathbf{F}$ & es & Anelat & 16 & It & T.tog $\mathrm{He}$ & ocs & ast & so & $\mathbf{n}$ & 的 & P1 & 的 & 2 \\
\hline 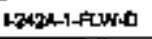 & 83 & $F_{2}$ & 边 & Antal & 15 & 15 & 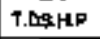 & Bons & acx & 8 & $\mathbf{f a}$ & 89 & F1 & 8 & F2 \\
\hline 1-2+24-1-FUW-E & $\$ 9$ & $F x$ & 5 & $A-1-B$ & 15 & 15 & T.DF.H.P. & Dos & $\log$ & 59 & $\mathbf{F a}$ & $\mathbf{9 9}$ & 51 & $\boldsymbol{\omega}$ & 72 \\
\hline 1-2004-1-81wF & $\$ 0$ & 59 & 16 & Hor & 7 & & TOS.E & $E$ & & & & & & & \\
\hline 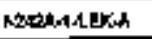 & 80 & $* 1$ & e1 & & 2 & & $P D$ & DIt & & & & & & & \\
\hline 1-200A-1-LEKA & st & F1 & 62 & B-14 & D & 15 & $m$ & Dis & $14 x$ & $\boldsymbol{\theta}$ & $\mathrm{Fo}$ & 82 & $\mathrm{F3}$ & 83 & F 2 \\
\hline 12:20-9-Lex-C & $\$ 2$ & $\mathrm{~F} 2$ & E2 & B-1-b & 12 & 13 & סק & 022 & 140 & 89 & $F 9$ & Prontind & 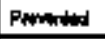 & is & f1 \\
\hline Retel-1-LKCD & $\$ 0$ & $\mathbf{m}$ & 1 & & 2 & & $\mathbf{p r}$ & Drs & & & & & & & \\
\hline 1.20BM-14EK,E & and & $m$ & E2 & 0-1t & 12 & 16 & wo & Dre & $10 x$ & $\$$ & Ps & $\$ 3$ & fz & 83 & $f 2$ \\
\hline 190A-2CAPA & $\infty$ & $\mathbf{F a}$ & E1 & $\sin$ & 3 & 3 & ros.E & $E 00$ & & & & & & $\$ 1$ & $\mathbf{F r}$ \\
\hline 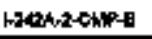 & \$ & $\mathbf{F}$ & Es & $B-1-4$ & 15 & +1 & Tos & $\mathbf{m 1}$ & $3=x$ & b & $\mathrm{FH}$ & 34 & $\mathbf{P}$ & ง1 & $\mathbf{F r}$ \\
\hline 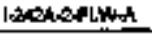 & 92 & n & 2 & b.1.t. & 14 & 11 & T.0.8 & E1: & $m x$ & $\mathbf{s 1}$ & $\mathbf{F}$ & 61 & Fs & ง1 & ro \\
\hline
\end{tabular}




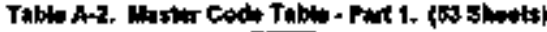

\begin{tabular}{|c|c|c|c|c|c|c|c|c|c|c|c|c|c|c|c|}
\hline 0 & 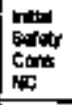 & 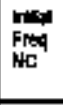 & En & and & 售 & $\begin{array}{l}\text { Folnod } \\
\text { Cind } \\
\text { Fhe } \\
\text { Frestor }\end{array}$ & fosin & Gomp & App & 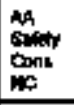 & $\begin{array}{l}\text { As } \\
\text { Prtiq } \\
\text { NC }\end{array}$ & App Cot & Fip & $\begin{array}{l}\text { finnd } \\
\text { contid } \\
\text { solity } \\
\text { Conit }\end{array}$ & 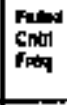 \\
\hline 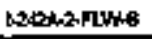 & 92 & $\mathbf{n}$ & $\mathbf{E}$ & C14 & 14 & 11 & T.cos & E15 & $20 x$ & 31 & $\mathbf{F}$ & $\$ 1$ & $\mathrm{PH}$ & 31 & F3 \\
\hline Lesoldenth & 50 & F1 & E1 & & 2 & & PD & 018 & & & & & & & \\
\hline 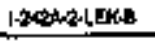 & 32 & Fis & E & Pold & 0 & 16 & no & Olis & $16 x$ & 93 & 63 & 48 & $\mathrm{Ps}$ & $\$ 3$ & $\mathbf{F z}$ \\
\hline 1-26042-Lex< & 64 & f2 & Ez & 0-16 & 12 & 13 & PO & $\infty$ & 1Ex & 83 & 59 & Prapolor & Prearilad & $\$ 3$ & E1 \\
\hline 1.2420.2.LEK-D & 6 & $F 1$ & $\mathbf{E} 1$ & & 2 & & PO & DIB & & & & & & & \\
\hline 12004,210KE & 92 & $\mathbf{F 2}$ & $\mathbf{E 2}$ & $M+$ & 12 & 15 & no & Dit: & $15 x$ & $\mathbf{s}$ & $\mathbf{F a}$ & as & $\mathbf{r} 2$ & sts & $\mathbf{2}$ \\
\hline 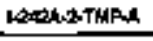 & so & m & 6 & NN & $?$ & & ins & Dor & & & & & & & \\
\hline 12ARA-2-1/Ach & $6 z$ & F2 & $\mathbf{E z}$ & C-14: & 12 & 11 & Ths.h & E़्ञ & $\mathbf{z x}$ & 努 & $\mathrm{F} 2$ & 51 & $\mathbf{f a}$ & 81 & Fs \\
\hline $12040-2-6956$ & so & $\mathbf{F 2}$ & 10 & NR & 4 & & PO & E03 & & & & & & & \\
\hline $1206 h_{3}-F L W A$ & 37 & $\mathbf{F s}$ & 12 & B.1. th & 14 & 11 & I.0s. & Do1 & $22 x$ & si & $\mathbf{F s}$ & 51 & A & $\$ 1$ & $\mathbf{P J}$ \\
\hline 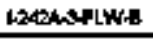 & $\$ 2$ & $\mathbf{r s}$ & 4 & $0-44$ & 14 & 11 & T.cos.E & Dor & $2 x$ & si & A & 91 & fa & $\$ 1$ & $\mathbf{m}$ \\
\hline 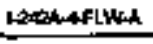 & st & $\mathrm{Ft}$ & E1 & & 11 & : & TNEE & O12 & & & & & & si & $\mathrm{Fz}$ \\
\hline 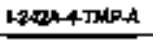 & s3 & $\mathrm{FQ}$ & Es & A-1- & 10 & & T.COSE & Eng & $\cos x$ & & & $\leqslant$ & Fq & & \\
\hline 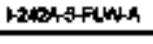 & 80 & $\mathrm{Fz}$ & mo & 嘴 & 4 & & T.WS & Eos & & & & & & & \\
\hline 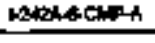 & $\Leftrightarrow$ & $\mathbf{P s}$ & Es & Solnt & 16 & 11 & T.DE & EoI & $\sqrt{2}$ & s1 & Fo & 51 & $\mathbf{6}$ & 94 & $\mathbf{6}$ \\
\hline 120036-findid & 51 & $\mathrm{Fa}$ & $E_{1}$ & & 21 & $\mathrm{~B}$ & TNE & th12 & & & & & & Et & p2 \\
\hline 12046-7LA-B & 路 & $\mathrm{FB}$ & E2 & $c-2 p$ & 14 & 13 & T.DS,E & 012 & $28 x$ & $\$$ & Fs & $\$ 1$ & $\boldsymbol{m}$ & $\infty$ & $\mathbf{F 1}$ \\
\hline Metakdrunto & 51 & $\mathbf{P}$ & w & & 11 & & 0 & Ess & & & & & & & \\
\hline Bherligh & 61 & Fi & E1 & & 5 & & $T+\infty$ & Dot & & & & & & & \\
\hline $13+24+7+20144$ & 62 & 51 & $\mathrm{E} 2$ & Cols & P & 11 & T.中g.E & Do5 & $20 x$ & $\$ 1$ & Ps & 8 & $\mathrm{Fo}$ & 81 & fa \\
\hline
\end{tabular}




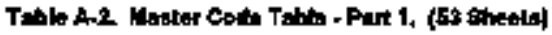

\begin{tabular}{|c|c|c|c|c|c|c|c|c|c|c|c|c|c|c|c|}
\hline I & 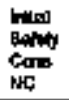 & $\underset{m}{n=0}$ & Em & Ban & 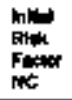 & The & Pach & $\begin{array}{l}\text { caurs } \\
\text { Cip }\end{array}$ & Rep & 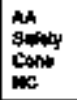 & $\begin{array}{l}\mu \\
F_{m=4} \\
M\end{array}$ & Anth & fing & 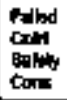 & 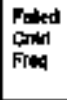 \\
\hline 1-24:AA-7-FLYYB & 82 & $\mathbf{F i}$ & 52 & $c-1 \rightarrow$ & 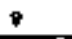 & 11 & Tose & Eos & $2 x$ & s1 & F3 & st & $\mathbf{F} 3$ & $8 t$ & $F$ \\
\hline 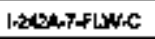 & $\$$ & $\mathbf{m}$ & E2 & an-10 & 0 & & T.08. $\mathrm{F}$ & DOS: & $22 x$ & & & & & & \\
\hline $1-20307-9 R 5-\lambda$ & s1 & $\mathbf{r}$ & es & ocs & B & & 0 & DO5 & & & & & & & \\
\hline Latato-1fents & $\$ 1$ & es & el & & 11 & 21 & 0 & Dof & & & & & & 31 & $\mathbf{F 3}$ \\
\hline 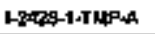 & 81 & $F$ & E1 & & 11 & 11 & 0 & Dos & & & & & & s1 & $\mathrm{B}_{3}$ \\
\hline 1-2009-2FLINA & 82 & $\mathbf{F 1}$ & $\mathbf{F}$ & $c-2-b$ & $\$$ & 13 & Thes & D12 & $29 x$ & 89 & $\mathrm{F}$ & 61 & $\mathbf{n}$ & $\$$ & $F 1$ \\
\hline $1-2 \cos 2 \pi$. & st & F2 & est & & 8 & & 0 & Ios & & & & & & & \\
\hline 1200-2rin.e & so & $\mathbf{F}$ & et & & 4 & & T.CD & Eot & & so & $\mathbf{H s}$ & & & & \\
\hline 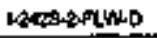 & so & F 2 & es & & 4 & & 0 & ens & & & & & & & \\
\hline LOQ8-2-A hite & $\$ 2$ & $\mathbf{F z}$ & E? & $6-20$ & 12 & 13 & $D$ & Ens & $29 x$ & 由 & 5 & $\$ 1$ & Pa & $\$ 3$ & F1 \\
\hline L2RS-2LEX-A & so & F1 & E1 & & 2 & & $\mathbf{F o}$ & 016 & & & & & & & \\
\hline 1201245K. & st & $\mathbf{M}$ & 12 & E-1b & 8 & 15 & wo & 018 & ISX & $\mathbf{s}$ & py & 52 & $\mathbf{F 3}$ & s9 & $F 2$ \\
\hline MatsinakC & az & F & $E 2$ & B-1t & 12 & 13 & $P Q$ & $\cos$ & $18 x$ & sa & $\mathbf{F a}$ & Provented & Prisented & 80 & $M 1$ \\
\hline [-20EE-R-LEKL & 80 & F1 & $\mathbf{E}_{1}$ & & 2 & & PE & Dse & & & & & & & \\
\hline 12403-2-LEK-I & 50 & F & 52 & $D-1-b$ & 12 & 15 & wo & DI: & $15 x$ & gs & $F$ & 89 & $\mathrm{Fz}$ & $\mathbf{B a}$ & F2 \\
\hline $1.2425-40 \mathrm{NH}$ & to & $\mathbf{3}$ & $\mathbf{E 1}$ & & 7 & & T.CD & eas & & & & & & & \\
\hline 1.3420-4fure & 91 & F2 & Eo & & $\theta$ & & 0 & Eod & & & & & & & \\
\hline $1-2428-474 \times-C$ & $5-2$ & $n$ & $E 2$ & c-24 & 12 & 13 & 0 & 506 & $29 x$ & 98 & $\mathbf{F}$ & 81 & $\mathrm{F3}$ & 8 & F1 \\
\hline $1-245-1+140$ & $\$ 2$ & F1 & $\mathbf{E}$ & $c-2 b$ & 0 & 15 & $T . D 8$ & o12 & $20 x$ & \%5 & 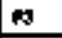 & $\$ 1$ & F\$ & $\mathrm{Bg}$ & FI \\
\hline $12426-4.18 \times 4$ & $\$$ & $\mathbf{P a}$ & E1 & & 7 & & PD & oit: & & & & & & & \\
\hline
\end{tabular}




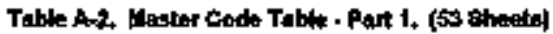

\begin{tabular}{|c|c|c|c|c|c|c|c|c|c|c|c|c|c|c|c|}
\hline 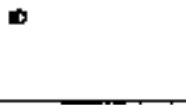 & $\begin{array}{l}\text { mitsl } \\
\text { semplot } \\
\text { Cons } \\
\text { WC }\end{array}$ & 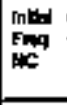 & EN & UM & 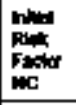 & 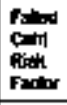 & Poat) & cor & $\operatorname{lop}_{\operatorname{sed}}$ & 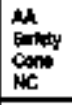 & $\begin{array}{l}\text { Nh } \\
\text { Pniq } \\
\text { NC }\end{array}$ & Aqp cint & ippotid & 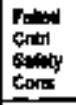 & $\begin{array}{l}\text { Fousd } \\
\text { Crint } \\
\text { Fron }\end{array}$ \\
\hline 120S-HEKS & $\$ 1$ & $\mathbf{F O}$ & g & & 11 & & 0 & $0+6$ & & & & & & & \\
\hline 1.205-41EKC & 31 & $\mathbf{F 2}$ & 的 & & 5 & & PD & $m$ & & & & & & & \\
\hline 1203-4HKa & so & $\mathbf{F 1}$ & EI & & 2 & & PD & ㅁ․ & & & & & & & \\
\hline HELA-4LKE & 62 & $\mathbf{F 2}$ & E2 & D-14 & 12 & is & wo & Dน & $15 x$ & $\mathbf{s s}$ & $\mathbf{r s}$ & ง3 & $F_{4}$ & st. & $\mathbf{F}$ \\
\hline BQ42E-6-CMPA & 91 & $\mathrm{~F}_{2}$ & e1 & & 4 & & 708 & cos & & & & & & & \\
\hline 12+254-CMPE & 51 & F2 & $\mathrm{E}_{1}$ & & 8 & & T.0\$ & cos & & & & & & & \\
\hline 1.24JS-CAMPC & $\$ 1$ & $\mathrm{Fr}$ & E1 & & 8 & & T.0s & coss & & & & & & & \\
\hline 1.24258-CMRO & \$1 & $\mathbf{z}$ & E1 & & 8 & & T.0s & Lx: & & & & & & & \\
\hline 1024285 fund & s1 & Pz & E1 & & 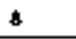 & & TOE & tats & & & & & & & \\
\hline 1-242t-5-7w6-8 & 51 & $F 2$ & et & & 8 & & ths & 605 & & & & & & & \\
\hline 1-2428-45LWC & 81 & $\mathbf{F r}$ & E1 & & 8 & & Tos & $\cos$ & & & & & & & \\
\hline 1.2608-AnsA & $\$ 1$ & $\mathbf{F z}$ & 60 & $\infty$ & d & & $\mathbf{G}$ & ent & & & & & & & \\
\hline 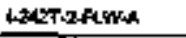 & 60 & $\mathrm{AB}$ & E1 & & 7 & & T.ss & EOC & & & & & & & \\
\hline LEAT-3FinLA & B1 & $\mathbf{F}$ & $E 1$ & & 11 & 11 & wo & 600 & & & & & & 81 & $\mathbf{F}$ \\
\hline L-24THAFWA & $\$ 1$ & FI & E1 & & 21 & 11 & wo & D12 & & & & & & $\$ 1$ & F \\
\hline 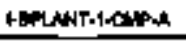 & so & $\infty$ & $\mathbf{E} 1$ & & 7 & & $\mathrm{Ac}$ & Eon & & & & & & & \\
\hline HEPLANT-1-DPP & 82 & $\mathbf{P}$ & $E 2$ & B.16 & 14 & 18 & wo & EOT & $16 x$ & so & $\mathbf{r}$ & 82 & $\mathrm{FB}$ & 8s & $\mathbf{F 2}$ \\
\hline 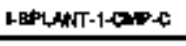 & 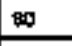 & $\mathbf{F a}$ & En & & 7 & & T.DS & EOS & & & & & & & \\
\hline HEPLANT:1-OWPOO & 50 & F & EO & & 7 & & T.DS & ext & & & & & & & \\
\hline HELNT-1-Gape & bo & 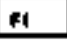 & $\boldsymbol{b}$ & An1s & 10 & 12 & $T$ & Bon & Desex & 纪 & $n$ & in & $F_{2}$ & 32 & 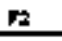 \\
\hline
\end{tabular}




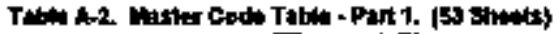

\begin{tabular}{|c|c|c|c|c|c|c|c|c|c|c|c|c|c|c|c|}
\hline $\mathbf{0}$ & $\begin{array}{l}\text { new } \\
\text { stetr } \\
\text { Cons } \\
\text { NC }\end{array}$ & $\operatorname{lin}_{\substack{\min \\
\text { ix }}}$ & Ew & Ben & 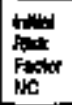 & 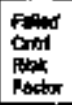 & Feorits & con & Rom & 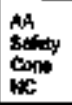 & 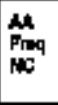 & Appent & finct & 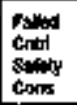 & $\begin{array}{l}\text { Faled } \\
\text { Oand } \\
\text { Find }\end{array}$ \\
\hline LEPLNT-1 TRPA & 91 & P2 & $\mathbf{E 1}$ & & 8 & & $T .00$ & Eot & & & & & & & \\
\hline 1-PРLAHT-2FLWA & 800 & $\mathrm{Bg}$ & EO & 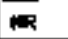 & 7 & & $a$ & Fon & & & & & & & \\
\hline 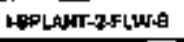 & $\mathrm{B}$ & EI & Eo & & 2 & & $\mathrm{~T}, \mathrm{pB}$ & EIS & & & & & & & \\
\hline PEPLAMT-2+LLWEC & $\$ 1$ & $\mathrm{Fi}$ & E: & & 5 & & $\pi, 08$ & 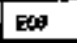 & & & & & & & \\
\hline IARLAMTZELWDO & si & $\mathbf{r s}$ & $\mathbf{E}$ & 6-10. & 14 & 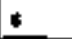 & T.DS & Eon & $\operatorname{tos} x$ & 81 & $\mathbf{F a}$ & PTrmotid & Pronetid & $g$ & $\mathbf{P O}$ \\
\hline 1-GALANT-ZLEKMA & at & F1 & E1 & & 2 & & $\mathrm{PC}$ & Dot & & & & & & & \\
\hline मEYANT-2-UEK-B & B2 & $\mathbf{F 1}$ & Ex & E-1- & 6 & 16 & 40 & D18 & $15 x$ & E3 & $\mathrm{Fg}$ & 57 & $\mathrm{~F}$ & 0 & F \\
\hline HPQUIM-2-LEK-C & 82 & $\mathbf{P 2}$ & E & $0-1+6$ & 12 & 13 & PC & bas & $100 x$ & 59 & is & 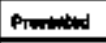 & Promenter & $\Leftrightarrow$ & fI \\
\hline HPLAN-2UNKED & $\infty$ & $\mathbf{F i}$ & Eq & & 2 & & DC & D10 & & & & & & & \\
\hline HEPLANT:ALEKT & $6 z$ & Fz & $4 z$ & 1.1t & 12 & 14 & wo & ons & $16 x$ & so & Po & 93 & $\mathbf{F z}$ & $\mathrm{Ea}$ & F \\
\hline LFFA-1-ACT Ah & as & $\mathrm{F3}$ & Ez & A-1- & 14 & 3 & $\tau+8$ & ADS & 91 & a1 & F1 & PTowited & Fwandind & $\$ 1$ & fo \\
\hline HAFP-1-GFPA & 80 & $\mathbf{F 3}$ & EI & & $T$ & & 1,00 & 래 & & & & & & & \\
\hline HFA.1.CKM-g & t2 & rs & e & Ant & 14 & 3 & TDS & No1 & $01 x$ & 51 & 1 & 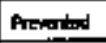 & Provented & s1 & ro \\
\hline HPP.g-chPA & so & $\mathbf{B}$ & 1 & & 7 & & T.CO & $\mathrm{ED}$ & & & & & & & \\
\hline 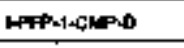 & 82 & $\mathbf{F s}$ & $\theta$ & A-14: & 14 & 3 & 506 & NOS & olx & si & F1 & Pranted & Prominged & 91 & $\mathbf{F} \mathbf{G}$ \\
\hline MPF,I-CHP-E & 93 & $\mathbf{F}$ & EB & $A-1 \rightarrow$ & 15 & 12 & Tos & EOA & $\cos$ & & & $\Leftrightarrow$ & 2 & 32 & $\mathbf{F} 2$ \\
\hline HAPA-Litas & $\$ 1$ & ps & [1 & & 11 & $B$ & mo & ens & & & & & & s1 & $\mathbf{r}$ \\
\hline 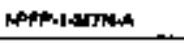 & so & K & et & & 3 & & PO & Els & & & & & & & \\
\hline |LPFP-2fLW/A & 90 & $\mathbf{f 3}$ & E1 & & 7 & & $T, C D$ & E00 & & & & & & & \\
\hline IPFP 24 IIK-h & 50 & $\$ 1$ & E1 & & 2 & & PO & D 18 & & & & & & & \\
\hline
\end{tabular}




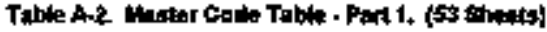

\begin{tabular}{|c|c|c|c|c|c|c|c|c|c|c|c|c|c|c|c|}
\hline ס & 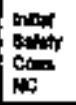 & 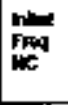 & Em & on & 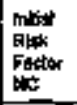 & 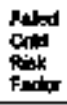 & codis & $C_{\text {G.m. }}$ & Rop & 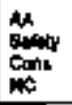 & $\begin{array}{l}\boldsymbol{M A} \\
\mathrm{F} \\
\boldsymbol{\omega} \boldsymbol{\omega}\end{array}$ & Apat & Fin & 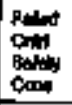 & 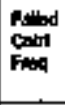 \\
\hline IAPPFZLEK-B & $\$ 2$ & FI & $E 2$ & E-1t & 9 & 15 & no & 018 & $15 x$ & s. & $\mathbf{F}$ & $\$ 2$ & $\mathrm{Fg}$ & 83 & $\mathbf{F}$ \\
\hline IAPPALEKGE & 92 & $n$ & E2 & D-1t & 12 & 1s & PQ & oses & $16 x$ & 5 & $\mathbf{b}$ & Proututed & Prownods & $\mathbf{s}$ & $\mathbf{P 1}$ \\
\hline 1APPA2LEKGD & 90 & fI & E1 & & 2 & & PO & Dis & & & & & & & \\
\hline HAfPulake & 6 & E & $\mathbb{E}$ & B-14te & 12 & $1 \mathrm{~s}$ & Wo & OAt & $16 x$ & ss & $\mathbf{F s}$ & sa & F2 & $\$$ & $\mathbf{F} 2$ \\
\hline 1PFP-2:-TाP-A & BI & $\boldsymbol{F}$ & E1 & & 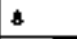 & & fot & Dor & & & & & & & \\
\hline 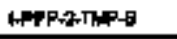 & $\$ 2$ & $\mathbf{n} 2$ & s? & D.1-1 & 12 & 11 & T.Cp & DOH & $\infty x$ & $\$ 1$ & $\mathbf{F 3}$ & $\mathbf{E 1}$ & FI & 81 & F \\
\hline LPFPMCLAPA & so & $\mathbf{m}$ & E1 & & 7 & & $\mathrm{TCD}$ & ext & & & & & & & \\
\hline LPFA3FLWES & 的 & $\mathbf{F s}$ & E1 & & , & & 1.00 & tes & & & & & & & \\
\hline 1-PFPALIWLS & B1 & F3 & 61 & & 11 & - & t.to & Eos & & & & & & 8 & $\mathrm{Fz}$ \\
\hline HFP\$DKA & $\$$ & $\mathbf{F 1}$ & et & & 2 & & Dh & Dot & & & & & & & \\
\hline LPNe-HLext & s & $\mathbf{m i}$ & 2 & $b-1 b$ & 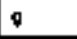 & 19 & wo & Dit & $15 x$ & os & P3 & 8 & $\mathbf{b}$ & 3) & $\mathbf{m}$ \\
\hline LPFPALLLCXt & so & $\boldsymbol{F}$ & 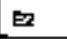 & A.4b & 12 & 15 & mo & Des & $10 x$ & as & rs & Punded & Pronimud & st & $\mathbf{F}_{1}$ \\
\hline IFF-gueto & 80 & F1 & E1 & & 2 & & $\mathrm{Fb}$ & the & & & & & & & \\
\hline IPFPЗLEKE & $\$ 2$ & $\mathrm{~F} 2$ & ez & $B-1-b$ & 12 & 15 & wo & 019 & $15 x$ & 5 & F3 & 89 & $\mathbf{r}$ & $\$$ & $\mathrm{Fz}$ \\
\hline IPUKIX-1-AGT-h & $\$ 0$ & $\mathbf{p y}$ & et & & 7 & & $\mathrm{PC}$ & DOS & & & & & & & \\
\hline HAREXINATR & 82 & $\mathbf{F}$ & E & and & 14 & 16 & Wo & 006 & $\tan x$ & Ba & $\mathrm{Fa}$ & $\infty$ & $\boldsymbol{n}$ & 83 & $P_{2}$ \\
\hline LPUREX-1-ABT-C, & $\$ 0$ & $\mathrm{~F}$ & es & NR & 7 & & 108 & 0006 & & & & & & & \\
\hline Heunex-1-chipa & $\infty$ & m & 81 & & 7 & & P4 & Dor & & & & & & & \\
\hline 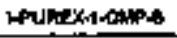 & 32 & a & E2 & 6-16 & 14 & 25 & WD & שיק & 15M & $s$ & Ps & 12 & $\mathbf{F g}$ & a & $\mathbf{F}$ \\
\hline HFUPEX & 90 & Fs. & EQ & NF & $t$ & & T.DS & | & & & & & & & \\
\hline
\end{tabular}




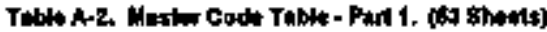

\begin{tabular}{|c|c|c|c|c|c|c|c|c|c|c|c|c|c|c|c|}
\hline $\mathbf{0}$ & 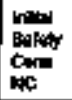 & 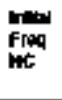 & Ems & 睡 & $\begin{array}{l}\text { molim } \\
\text { Oilox } \\
\text { Fucter } \\
\text { NC }\end{array}$ & 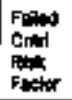 & Fection & Goup & Fep & $\begin{array}{l}\text { Man } \\
\text { gefinty } \\
\text { cane } \\
\text { NG }\end{array}$ & 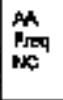 & Apo Cor & Apperint & 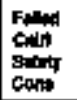 & $\lim _{\ln }^{x}$ \\
\hline 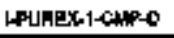 & 93 & F & EO & $\boldsymbol{m}$ & 7 & & T.DS & EDA & & & & & & & \\
\hline LPUAEYX-1-LUL-h & $\$ 2$ & $\mathbf{F}$ & 5 & Bo-14: & 14 & 11 & T, ps & en: & $\tan$ & $\$ 1$ & $\mathbf{F}$ & 51 & 6 & $\$ 1$ & $\mathbf{P a}$ \\
\hline LPUNEX-1-TIMPAh & si & $\mathbf{m}$ & E1 & & 11 & t & T.tos & en: & & & & & & si & $\mathbf{F 2}$ \\
\hline HPUREXRPLWh & $\$ 0$ & $\mathbf{m k}$ & gan & $2 R_{R}$ & & & $\theta$ & Fos & & & & & & & \\
\hline IPUPEXZUEKSA & 80 & $\mathbf{F 1}$ & E1 & & 2 & & $\mathrm{PC}$ & D19 & & & & & & & \\
\hline HMHEXX24EK-B & 5 & F1 & q2 & Both & 8 & 15 & mo & 머 & $15 x$ & $\mathrm{sa}$ & $\mathbf{p s}$ & ss & Bd & 80 & f \\
\hline DAUESWAEKC & $\$ 2$ & $\mathbf{F 2}$ & E2 & Actb & 12 & 13 & $\mathrm{AC}$ & De2 & $18 x$ & st & F & Anded & Prowalud & 约 & 11 \\
\hline IFUPESAEKGD & 90 & $\mathbf{F 1}$ & E1 & & 2 & & Pc & 머난 & & & & & & & \\
\hline HPLFEXZLEKE & 92 & $F$ & E & B-H & 12 & 15 & wo & O18 & $15 x$ & $\mathrm{Eg}$ & $\mathbf{F}$ & 59 & $\mathbf{F} 2$ & 53 & $\mathbf{F}_{2}$ \\
\hline IPUPAX-2PRE-A & 82 & $\mathrm{Fz}$ & E & Q $=1$ - & 12 & 11 & TDS & 001 & $3 \pi x$ & si & Fs & sI & F5 & 81 & $\mathbf{F}$ \\
\hline 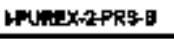 & 81 & $\mathbf{F 3}$ & 51 & & I1 & $B$ & Tns & Dos & & & & & & si & $\mathbf{F 2}$ \\
\hline 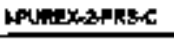 & si & $\mathbf{r}$ & es & AP & t] & & 0 & Des & & & & & & & \\
\hline 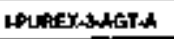 & 的 & $\mathbf{F}$ & es & & 7 & & $\mathbf{P C}$ & Diss & & & & & & & \\
\hline HYPQDX-3-10T-6 & 82 & F3 & Et & D-1+b & 14 & ss & 40 & 0,5 & $15 x$ & $\mathbf{5 3}$ & $\mathbf{E s}$ & $\mathbf{3 2}$ & m & 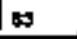 & $\mathbf{F 2}$ \\
\hline |.PUREXAACT-C & 50 & $\boldsymbol{r}$ & EO & NR & $T$ & & T.DS & $\cos$ & & & & & & & \\
\hline I.PUNEXX3CLP, & 60 & $\mathbf{F}$ & $\mathbf{E}$ & & 7 & & $P C$ & $\mathrm{en}$ & & & & & & & \\
\hline LLUREX-3CLPA & s7 & $\mathbf{P}$ & et & E-14 & 14 & 15 & WD & 터1 & $15 x$ & $\$$ & $\mathbf{F} \xi$ & $\$ 2$ & $\mathbf{F}$ & $\mathrm{Bg}$ & f2 \\
\hline 1.PURFX-SCLPCC & ક요 & ra & ED & NR & $T$ & & T.DF & (19) & & & & & & & \\
\hline HPUREXOSCKNO & so & $\mathbf{\omega}$ & $\mathbf{D}$ & WR & 7 & & T.DQ & In & & & & & & & \\
\hline HuAExBarge & so & $\mathbf{m}$ & 로 & Q-14: & 14 & a & J.68 & and & os $x$ & sis & $F 1$ & Promend & Pherited & 5 & $\mathbf{P}$ \\
\hline
\end{tabular}




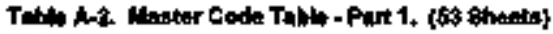

\begin{tabular}{|c|c|c|c|c|c|c|c|c|c|c|c|c|c|c|c|}
\hline D & 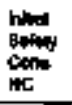 & 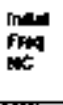 & Gor & EN & $\begin{array}{l}\text { Inted } \\
\text { Rlan } \\
\text { Pactor } \\
\text { NC }\end{array}$ & 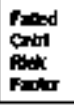 & $\begin{array}{l}\text { Rociloy } \\
\text { Codt }\end{array}$ & Con & Aer & $\begin{array}{l}\text { in } \\
\text { sakn } \\
\text { NCE } \\
\text { NC }\end{array}$ & $\operatorname{Fin}_{\mathbf{m}}^{M}$ & Apiptith & Apporit & 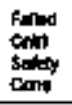 & $\begin{array}{l}\text { Fund } \\
\text { Cnoth } \\
\text { freld }\end{array}$ \\
\hline IPUREX-HULA & 92 & $\mathbf{F 3}$ & $\boldsymbol{n}$ & B-1-4 & 14 & 11 & T.DO & I01 & $* 2 x$ & 81 & $\mathbf{F 3}$ & 81 & $\mathbf{F}$ & 81 & $\mathbf{F 3}$ \\
\hline HPUn: & $\$ 1$ & Ps & el & & 11 & $B$ & T.DS & EII & & & & & & 51 & $\mathbf{H 2}$ \\
\hline Munex-4fLine & $\$ 7$ & $\boldsymbol{\mu s}$ & $\boldsymbol{c}$ & $c 2 b$ & 14 & 13 & T.DS & Dod & $20 x$ & s3 & rs & st & $\mathbf{F}$ & s3 & $\mathbf{F t}$ \\
\hline HPUFESXHEKA & so & $\mathbf{F 1}$ & EI & & 2 & & $m$ & Dne & & & & & & & \\
\hline HPUFEX-AUEK & 82 & $\mathbf{F 1}$ & E & EN16. & $s$ & 15 & no & Den & $15 x$ & $\$ 3$ & F3 & 82 & $\mathbf{n}$ & 83 & $F z$ \\
\hline PPUREX-YUEKC & 92 & $\mathbf{F 2}$ & $\boldsymbol{n}$ & $B-10$ & 12 & 13 & $P C$ & Dor & $16 x$ & ss & F3 & Prawiond & Prwnented & 83 & $\mathbf{F 1}$ \\
\hline 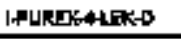 & $\infty$ & $\mathbf{F 1}$ & E1 & & 2 & & $P C$ & Dide & & & & & & & \\
\hline |PUAEK+HEKE & 52 & $\mathbf{F}$ & 12 & B-1tb & 12 & 15 & no & Q16 & $16 x$ & so & $\mathrm{FH}$ & ss & $F \boldsymbol{z}$ & s3. & $\mathbf{r z}$ \\
\hline |PUREXAPRSAh & ss & $\mathrm{Fz}$ & E2 & pola & 12 & 11 & T.0S & Do1 & $30 x$ & $\$ 1$ & F 3 & $\$ 1$ & $\mathbf{F}^{2}$ & \$1 & $\mathbf{m}$ \\
\hline PUREX СPA3 6 & si & $\mathrm{FF}$ & $\mathbf{E} 1$ & & 11 & 4 & T.中田 & Dos & & & & & & $\$ 1$ & $\mathrm{~F}$ \\
\hline IPURTX-APRE-C & $\$ 1$ & $\mathbf{F} 2$ & E0 & FP & 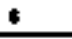 & & 0 & FDE & & & & & & & \\
\hline 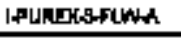 & $\$ 1$ & R3 & E1 & & 11 & d & T.DS & Eest & & & & & & 31 & $\mathbf{F 2}$ \\
\hline HAREXeNLHA & s1 & $\mathbf{F 2}$ & E1 & & 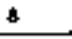 & & Tos & 601 & & & & & & & \\
\hline HPREXKAFUMAA & so & $\mathbf{F} 3$ & E1 & & 7 & & Ton & EDQ & & & & & & & \\
\hline HYREX-67WB & B1 & f2 & E1 & & 4 & & $\mathrm{I}, \mathrm{CN}$ & $\mathrm{eDB}$ & & & & & & & \\
\hline HuREXANuHC & s1 & 22 & E1 & & 4 & & T.COA & ente & & & & & & & \\
\hline 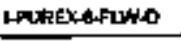 & so & F1 & EO & $\mathbf{m}$ & 2 & & 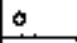 & $\mathbf{m a}$ & & & & & & & \\
\hline 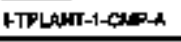 & 8 & fa & E1 & & 7 & & WC & BDor & & & & & & & \\
\hline DTPLAMT-1-CAM-B & $\$ 7$ & $\mathbf{m}$ & $\mathbf{E}$ & $D-1-\infty$ & 14 & 18 & wo & EMI & $\operatorname{tg} x$ & 5 & $\mathbf{A}$ & $s$ & $n$ & $\mathbf{m}$ & $\mathbf{F}$ \\
\hline 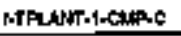 & so & $\mathbf{f r a}$ & $\infty$ & & 7 & & T.DE & Eon & & & & & & & \\
\hline
\end{tabular}




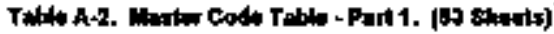

\begin{tabular}{|c|c|c|c|c|c|c|c|c|c|c|c|c|c|c|c|}
\hline 0 & 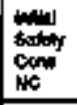 & 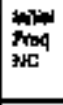 & EN & $\theta=x$ & 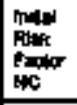 & 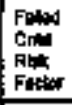 & Farits & Gwa & $\begin{array}{l}\text { Rep } \\
\text { ind }\end{array}$ & $\begin{array}{l}\text { MA } \\
\text { SW } \\
\text { MC } \\
\text { NC }\end{array}$ & $\underset{N=}{M}$ & sactor & fop contr & 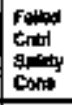 & 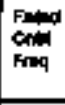 \\
\hline BTPUNT-1-CAtPA & 曲 & $\mathbf{B}$ & $\infty$ & & $T$ & & Tos & 妙 & & & & & & & \\
\hline 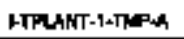 & s1 & $F$ & E1 & & $\mathbf{t}$ & & 109 & EN1 & & & & & & & \\
\hline 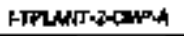 & 39 & F & E2 & a & 14 & 11 & T.क्ष & ER & $\operatorname{anx}$ & E1 & rt & si & $\mathrm{A}$ & 81 & F \\
\hline |.TPLANT-2ALWA & so & F1 & E & & 2 & & T.DO & 700 & & & & & & & \\
\hline 1.TPLWHT.2.FIWA & s1 & F1 & EI & & 5 & & Tns & ext & & & & & & & \\
\hline I.TPLWWT-2-1EKA & so & $\mathbf{F 1}$ & EI & & 2 & & $\mathrm{PC}$ & Dis & & & & & & & \\
\hline HPUNTQLEKE & $\$ 2$ & F1 & e & Bolt & 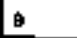 & 15 & wo & D1: & 150 & $\theta$ & $\mathbf{F s}$ & 影 & $F$ & $\$ 3$ & Fz \\
\hline 1TPLڤИT-2-LEKK-C & 67 & F 2 & E2 & $\theta \times 1+b$ & 12 & 19 & $p$ & $\infty$ & $1 \mathrm{gak}$ & 83 & $F$ & Prowiond & PTowationt & 5 & F1 \\
\hline HTPLAMT-ZUEK-D & 80 & FI & E1 & & 2 & & $p$ & DAB & & & & & & & \\
\hline ITPLAKT \&AEK.E & $s 2$ & $\mathbf{r 2}$ & 포 & ateb & 12 & 15 & Wo & Dis & $15 x$ & so & F3 & $\mathbf{s t}$ & $\mathbf{F 2}$ & sa & 22 \\
\hline 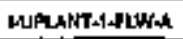 & 80 & $\mathbf{F o}$ & E1 & & 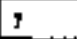 & & PC & Dot & & & & & & & \\
\hline HWFUNT-14 EkAh & 90 & $\mathrm{~F}$ & E1 & & 9 & & $\mathrm{AC}$ & ग18 & & & & & & & \\
\hline HLPLANTI-1EKK & 52 & F1 & (7) & $6-24$ & $\mathbf{s}$ & 13 & $P C$ & Den & $23 x$ & $\$ 3$ & f3 & Provininded & Drewerited & 5 & $\mathbf{F I}$ \\
\hline MUPLANT:1.LEXSC & 80 & $\mathbf{F 1}$ & et & & 2 & & $m$ & DAs & & & & & & & \\
\hline 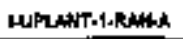 & a1 & $\mathbf{m}$ & e1 & & 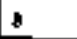 & & Wo & OHe & & & & & & & \\
\hline mu-01 & 51 & F & E1 & & 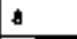 & & $t$ & $m$ & & & & & & & \\
\hline ITx-9t & 51 & $F$ & $\mathbf{E 1}$ & & 21 & 11 & Can & Dor & & & & & & $\mathbf{5}$ & $\mathbf{m}$ \\
\hline ITK- & Bd & $\mathbf{r}$ & E1 & & $*$ & & $T$ & oOs & & & & & & & \\
\hline mK-024 & B1 & F1 & E1 & & 6 & & ots & Don & & & & & & & \\
\hline $1 T K-03$ & 81 & $\mathbf{F} 3$ & E1 & & 11 & 11 & $T$ & E14 & & & & & & B1 & F \\
\hline
\end{tabular}


Toble A-2. Ungtar Code Tuble + Fait 1. (53 9leats)

\begin{tabular}{|c|c|c|c|c|c|c|c|c|c|c|c|c|c|c|c|}
\hline 10 & 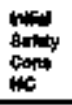 & $\begin{array}{l}\text { midu } \\
\text { freq } \\
\text { NCC }\end{array}$ & Enr & 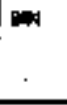 & 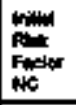 & 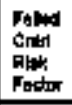 & Catb & SNp & $\underset{100}{R \bullet 0}$ & $\begin{array}{l}\text { Ah } \\
\text { exther } \\
\text { Cond } \\
\text { NC }\end{array}$ & $\begin{array}{l}M \\
\text { Fivq } \\
\text { NGC }\end{array}$ & 我 & Emp & 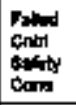 & 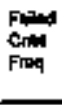 \\
\hline mKas & 81 & $\mathrm{Fa}$ & E1 & & 11 & 11 & ont & E14 & & & & & & 9 & $\mathbf{A}$ \\
\hline molet & $\$ 1$ & F3 & E1 & & 11 & 11 & $T$ & DOS & & & & & & 01 & $F$ \\
\hline IMK-OH & $\$ 1$ & $\mathrm{Fz}$ & E1 & & 0 & & O4 & DOS & & & & & & & \\
\hline hThes & $t$ & $\mathbf{z}$ & E1 & e-1. & 12 & 11 & $T$ & ext & 20 & $\mathrm{~K}$ & As & s. & A & ง1 & F3 \\
\hline MKksts & sz & $r z$ & e1 & D.1. & 12 & & ONA & Eor & $20 x$ & $\$ 1$ & $p_{2}$ & st & Fs & & \\
\hline mises & 32 & F1 & ER & N10 & 8 & 3 & $T$ & 104 & alx & aI & $\mathbf{F 1}$ & FT T) & DTowirim & 81 & Fot \\
\hline ITK-⿻ & 92 & F1 & E? & Antell & 9 & 3 & 에 & $A$ & $\cos x$ & 81 & F1 & 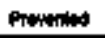 & Prowand & $\$ 1$ & $\mathrm{FV}$ \\
\hline IIn-0\% & *1 & F3 & Es & & 11 & 8 & $T$ & D10 & & & & & & st & $\mathrm{F} 2$ \\
\hline Ithasth & $\$ 1$ & $\mathrm{~F}$ & $\mathbf{E 1}$ & & 6 & & on & הבט & & & & & & & \\
\hline 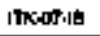 & 51 & $\mathbf{F}$ & $\mathbf{E}$ & & s & & an & $\theta$ & & 5 & $F R$ & & & & \\
\hline mқ:06 & 요 & $\mathbf{~} \mathbf{3}$ & E? & M-1- & 18 & 13 & $\mathbf{T}$ & Do4 & $\operatorname{sex}$ & & & $\$$ & Fo & 6 & 51 \\
\hline ITKOSH & $\mathbf{s a}$ & $\mathbf{p z}$ & $E$ & Anter. & 12 & 19 & and & 601 & $\cos x$ & 89 & $\mathrm{Fg}$ & $\$ 3$ & Po & $\mathbf{9}$ & F1 \\
\hline mKeng & 60 & 6 & 72 & $A-10$ & \$ & 15 & $\mathbf{I}$ & $\mathrm{cos}$ & $\infty x$ & & & $\mathbf{s 3}$ & $P_{2}$ & ss & $f$ \\
\hline ITKOSA & $s 2$ & $\mathbf{F 2}$ & 12 & tide & 12 & 15 & on & on: & $\alpha x$ & ss & $\mathrm{kJ}$ & 83 & $F$ & 由 & $\mathbf{A 2}$ \\
\hline ТК-10 & 50 & $\mathbf{F a}$ & 8 & A.1- & 14 & 15 & 매 & Bo4 & $06 x$ & 53 & $\mathrm{~F} 3$ & ss & $\boldsymbol{F}$ & 89 & $\boldsymbol{F}$ \\
\hline$m k-10-$ & $\mathrm{Bg}$ & $\mathbf{F}$ & 62 & $A-1-n$ & 10 & 15 & I.SD.H & BOA & $05 x$ & & & sa & $\mathbf{r 2}$ & $\mathbf{s}$ & $\mathbf{F} 2$ \\
\hline mK-10t & $\mathbf{s o}$ & $\mathbf{F}$ & 12 & Atrat & 18 & 15 & $\mathbf{T}$ & EOA & 04 & st & 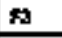 & 89 & F1 & 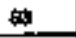 & $\mathbf{F}$ \\
\hline ITK-11 & $\mathbf{s t}$ & 12 & $\mathbf{E 2}$ & HAd & 18 & 14 & $\mathbf{T}$ & on & $\tan x$ & gs & A & $\mathbf{8 g}$ & $\mathbf{F 1}$ & 89 & $\mathbf{F 2}$ \\
\hline$|\pi k-41|$ & B2 & $\mathbf{F 1}$ & E2 & +14 & $\$$ & 15 & ond & Do9 & $11 x$ & 63 & $\sqrt{2}$ & $\mathbf{s}$ & $\mathbf{F 2}$ & 93 & $q$ \\
\hline IK-FR-01 & 91 & $\mathrm{F3}$ & Et & & 19 & 11 & $T$ & Dof & & & & & & si & $\mathbf{p a}$ \\
\hline
\end{tabular}


Tabla A-2. Master toda Tabla - Part 1. (59 sheats]

\begin{tabular}{|c|c|c|c|c|c|c|c|c|c|c|c|c|c|c|c|}
\hline 10 & 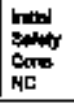 & foid & Ent & EW & 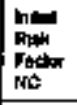 & $\begin{array}{l}\text { fasod } \\
\text { Gatiol } \\
\text { futh } \\
\text { fardor }\end{array}$ & Ractur & Cond & $\lim _{i \infty 0}$ & $\begin{array}{l}\text { in } \\
\text { sestivy } \\
\text { cond } \\
\text { NE }\end{array}$ & $\begin{array}{l}\mu \alpha \\
F_{M}\end{array}$ & App Crat & Fim & 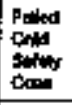 & Fald \\
\hline TKFA-G & \$1 & fo & $\mathbf{E} 1$ & & 11 & $t_{1}$ & $T$ & EOS & & & & & & 81 & $\mathbf{F g}$ \\
\hline IThithes & 32 & fi & E & ade & 6 & 11 & $T$ & ets & $\$ 1 x$ & $\$ 1$ & Ps & $\mathbf{\$ 1}$ & $\mathbf{m}$ & 5) & $m$ \\
\hline Ifkolhen & ss & f1 & 巴9 & Ex 1 & 19 & 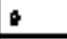 & $T$ & tast & $12 x$ & $s z$ & $\mathrm{Ps}$ & Providited & Prominas & $5 x$ & FI \\
\hline rTorn-6s & $\$ 1$ & $\mathbf{m}$ & E1 & & 11 & 11 & $T$ & 1 & & & & & & 6 & $\mathbf{N}$ \\
\hline TKKFF-EO & 81 & $\mathbf{F a}$ & E1 & & 11 & 11 & $T$ & DA & & & & & & 61 & $\mathbf{F}$ \\
\hline TKF+R-GT & 8 & $\mathrm{fg}$ & E1 & & 11 & 11 & $T$ & 012 & & & & & & 51 & $\mathbf{P P}$ \\
\hline ITKFEAS & 52 & $\mathbf{P S}$ & E2 & B-1 & 14 & 11 & $T$ & $\cos$ & 31 & $\$ 1$ & rs & 81 & $\mathbf{B}$ & 9) & $\mathbf{b}$ \\
\hline TKKNA -0 & s1 & 12 & te1 & & a & & $T$ & axs & & & & & & & \\
\hline ITK-FA-10 & $\$ 1$ & $\boldsymbol{F}$ & E1 & & a & & $T$ & 005 & & & & & & & \\
\hline MK-FR-11 & 81 & F1 & Ex & & 5 & & $T$ & DES & & & & & & & \\
\hline MKFFR-12 & s1 & $\mathbf{F 1}$ & e1 & & 5 & & $\mathbf{T}$ & 008 & & $\$$ & 69 & & & & \\
\hline ITKAeR-13 & s2 & $\mathbf{H}$ & 12 & M-1e & 0 & 15 & $\mathbf{I}$ & Bot & and & st & $\mathbf{0}$ & st & F1 & sง & $\mathbf{r}$ \\
\hline ItKakkit & 31 & $\mathbf{F}$ & $\mathbf{E}_{1}$ & & 11 & 11 & $\mathbf{T}$ & E14 & & & & & & $\$ 1$ & $\mathbf{F}$ \\
\hline ITKFFR-1 & की & $\mathrm{F} 3$ & E1 & & 11 & 11 & $\mathbf{T}$ & $E 00$ & & & & & & $\$ 1$ & $\mathrm{~F}$ \\
\hline mKFr-10 & 51 & $\mathbf{F}$ & E1 & & $\mathrm{E}$ & & $T$ & $\mathrm{Du}$ & & & & & & & \\
\hline ITKARAT & 31 & $\mathbf{F}$ & 도. & & 1 & & $\mathbf{T}$ & D12 & & & & & & & \\
\hline ITK-fento & 81 & $\mathbf{F}$ & E1 & & 11 & 11 & $\mathbf{T}$ & Ear & & & & & & 51 & P3 \\
\hline TKFr-1S & Eq & $F 1$ & $\mathbf{E}$ & B-1 & 13 & $s$ & $\mathbf{I}$ & $\mathrm{Cag}$ & $12 \pi$ & 52 & $\boldsymbol{F}$ & Presterived & Proming & 82 & F1 \\
\hline mKethex & 51 & 0 & E1 & & 11 & 11 & $\boldsymbol{T}$ & E14 & & & & & & EI & $\mathbf{F 3}$ \\
\hline TTKPARII & 51 & $\mathbf{P B}$ & E1 & & 11 & 11 & $\mathbf{T}$ & $\Delta$ & & & & & & s! & $\mathbf{P 3}$ \\
\hline
\end{tabular}




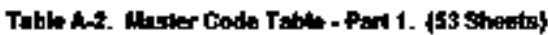

\begin{tabular}{|c|c|c|c|c|c|c|c|c|c|c|c|c|c|c|c|}
\hline b & 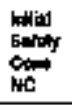 & $\lim _{i \rightarrow \infty}$ & $\begin{array}{l}\text { Eimr } \\
\text { Comat }\end{array}$ & Bw & 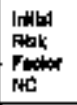 & $\begin{array}{l}\text { Fuld } \\
\text { Cond } \\
\text { Mlak } \\
\text { Factir }\end{array}$ & $\begin{array}{l}\text { Rasity } \\
\text { cods }\end{array}$ & con & $\lim _{\text {tor }}$ & 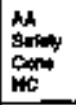 & $\begin{array}{l}\text { Nh } \\
\text { Fiqq } \\
\text { M. }\end{array}$ & 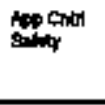 & Fip ctin & 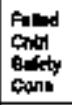 & $\begin{array}{l}\text { Fined } \\
\text { ctint } \\
\text { Find }\end{array}$ \\
\hline |IK-FR-2Z & 82 & F1 & E2 & $6-1-4$ & 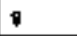 & 11 & $\mathbf{I}$ & Dol & $3 x$ & $\$ 1$ & $\mathrm{Fa}$ & 91 & $\mathbf{F B}$ & $\$ 1$ & $\mathrm{F3}$ \\
\hline ITKज: & s) & fo & $\mathbf{E 1}$ & & 11 & 41 & $T$ & D12 & & & & & & 01 & $\mathbf{F s}$ \\
\hline TKkTh-24 & $\$ 1$ & $\mathbf{2}$ & ro & & 0 & & $\mathbf{T}$ & Dos & & & & & & & \\
\hline trkethos & ง1 & $\mathbf{r} 2$ & 的 & & a & & $\tau$ & $D \infty$ & & & & & & & \\
\hline IthGFas & s1 & $\mathbf{F}$ & E1 & & 11 & 11 & $\tau$ & 006 & & & & & & 的 & $\mathbf{F 3}$ \\
\hline TKSFRET & $\$ 1$ & $\mathbf{F}$ & E1 & & 6 & & $T$ & Das & & & & & & & \\
\hline ITK-FR-2 & 91 & $\mathrm{~F}$ & EI & & 11 & 11 & $T$ & Da & & & & & & s1 & $\mathbf{f g}$ \\
\hline NPAI & $\$ 1$ & $\mathbf{F 3}$ & eI & & 11 & 11 & 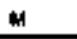 & D21 & & & & & & $\$ 1$ & a \\
\hline APAB & 㕸 & $\mathbf{z}$ & Z1 & Nold & 12 & 12 & $M$ & 1821 & $\mathbf{\omega} \mathbf{x}$ & & & st & 22 & $8 z$ & 12 \\
\hline IPas & 52 & $\mathbf{r}$ & $E z$ & A-14 & 12 & 18 & $\mathbf{M}$ & B21 & 11 & 80 & $\$ 2$ & $s$ & p & 80 & $\mathbf{F 2}$ \\
\hline $10-\infty$ & 92 & F1 & E2 & A-1-A & 9 & 11 & H & 020 & आx & 81 & $\mathbf{F a}$ & 81 & $\mathbf{f 3}$ & 51 & $\mathrm{Fg}$ \\
\hline$\omega P-\infty$ & $\$ 1$ & $\mathrm{~F}$ & E1 & & 11 & 11 & H & D20 & & & & & & si & N \\
\hline$m-4$ & sa & $\mathbf{P 1}$ & es & b-14t & 13 & & $\omega$ & D16 & 14 & $\mathbf{s}$ & Fo & s3 & Fo & & \\
\hline respes & s & $\mathbf{P}$ & E2 & $c-3 b$ & 15 & 13 & $M$ & 매 & $\operatorname{lax}$ & 5 & F1 & $\mathbf{s}$ & 1 & 89 & F1 \\
\hline $10-0$ & 여 & F1 & 13 & & 5 & & $M$ & 018 & & & & & & & \\
\hline$M^{2}+10$ & 59 & $\mathbf{F}$ & E1 & & B & & H & Dit & & & & & & & \\
\hline $\operatorname{mos} 1$ & s & $\mathbf{n}$ & e & M.1- & 10 & & $M$ & 014 & $11 x$ & & & $s$ & mo & & \\
\hline-12 & 的 & 5 & E1 & & 11 & 11 & $M$ & 000 & & & & & & 81 & F \\
\hline MP-19 & 69 & $\mathrm{~F}$ & es & E-1.4 & 15 & $\theta$ & $M$ & 810 & $14 x$ & 20 & Fo & st & Fo & 98 & Fv \\
\hline$n=-14$ & 31 & $\boldsymbol{\theta}$ & E1 & & 11 & 11 & $\mathbf{M}$ & m & & & & & & st & $\mathbf{m}$ \\
\hline
\end{tabular}




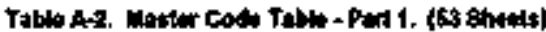

\begin{tabular}{|c|c|c|c|c|c|c|c|c|c|c|c|c|c|c|c|}
\hline ID & 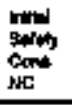 & Fipd & Ew & ger & 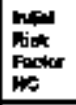 & 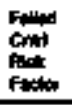 & Code & Exp & inte & $\begin{array}{l}\text { Ad } \\
\text { sints } \\
\text { cons } \\
\text { Not }\end{array}$ & $\underset{N C}{N_{N}^{M}}$ & App Gut & 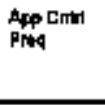 & 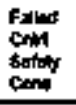 & $\begin{array}{l}\text { Fund } \\
\text { Crist } \\
\text { Frimq }\end{array}$ \\
\hline NP-1 & $\$ 2$ & $\mathbf{F g}$ & E1 & Ha & 14 & 16 & 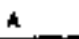 & 000 & $\cos x$ & $\$$ & $\mathbf{F s}$ & ss & $\mathbf{F}$ & 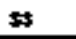 & $\mathbf{F}$ \\
\hline DorT-EXT3HA & \$5 & $\boldsymbol{n}$ & $\mathbf{5}$ & B-1- & 18 & 13 & Ps. & $\cos$ & $18 x$ & & & 80 & Fo & 83 & F1 \\
\hline OKTEXT301B & $\$ 2$ & $r$ & $\mathbf{E}$ & anct & 12 & 13 & $\mathbf{P a}$ & exs & $20+x$ & 8d & $\mathrm{Fa}$ & 81 & $\mathbf{F}$ & 63 & F1 \\
\hline OCTI-FLOMII-O & st & m & $E$ & AP & ta & it & $P A$ & $\mathbf{F a s}$ & & & & & & st & $\mathbf{P s}$ \\
\hline 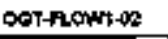 & 92 & $F 2$ & E2 & $e-2 t$ & 12 & to & WD & E19 & 征 & Es & $\mathbf{F}$ & $\$ 1$ & $\mathrm{PF}$ & 83 & $\mathbf{r i}$ \\
\hline DGThoorAy-oss & 03 & $\mathbf{F 1}$ & es. & $B-1=$ & 13 & ns & $\mathbf{P k}$ & Eoc & $15 x$ & $\$$ & $\boldsymbol{n}$ & Marditad & Provorid & 中 & $\boldsymbol{F}_{1}$ \\
\hline 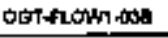 & $\$ 2$ & $\boldsymbol{r}$ & mi & $\Delta \infty \mathrm{b}$ & $\mathbf{a}$ & ta & $\mathbf{F i n}$ & EOS & $2 \pi x$ & s & $\mathbf{P}$ & g1 & F & so & F1 \\
\hline 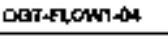 & 92 & 11 & 2 & $8-2 \mathrm{~b}$ & $\$$ & 13 & $\mathrm{Fm}$ & ㅁot & $2 \pi x$ & $\mathbf{s 0}$ & $\mathbf{F s}$ & $\$ 1$ & $\mathbf{F G}$ & sa & F1 \\
\hline ODT+ROAN1+06 & 80 & F & E1 & & 7 & & $T, \oplus 8$ & Dog & & & & & & & \\
\hline OoT-nlow1-06 & 62 & $\mathrm{Fz}$ & E2 & $0-26$ & 12 & 13 & T.ED & Iot & $20 x$ & 85 & $\boldsymbol{F}$ & $\$ 1$ & Fo & $\infty$ & FI \\
\hline DGTPLON2HO1A & $\$ 1$ & $\mathbf{F 2}$ & E2 & & $\Delta$ & $\Delta$ & $\mathbf{P a}$ & E1s & & & & & & $\$ 1$ & $\mathbf{z}$ \\
\hline CX6T- & $\$ 2$ & $\mathbf{p}$ & 트 & D.26 & 12 & 13 & $\mathbf{P A}$ & EN3 & $\operatorname{mix}$ & s & s & s1 & $\mathbf{r a}$ & $\mathbf{s g}$ & $\mathbf{F} \mathbf{1}$ \\
\hline DOT+FLAR:0R & $\mathbf{s}$ & $\mathbf{n}$ & $\mathbf{E}$ & B-1-4 & 15 & 13 & An & E13. & $15 x$ & 83 & $\mathbf{F 3}$ & 89 & $\mathrm{Fo}$ & 33 & Fi \\
\hline COT-FLON2-03A & E1 & $\mathrm{F} 1$ & ER & & 5 & 5 & Ph & E13 & & & & & & 51 & $\mathbf{F I}$ \\
\hline CST.PLONZOAB & 82 & F1 & E2 & B.20 & 自 & 13 & $\mathbf{P h}$ & Eis & $\mathbf{m x}$ & ss & $\mathbf{r}$ & si & $\mathbf{F} \mathbf{s}$ & $\mathbf{s}$ & $\$ 1$ \\
\hline Dort-INTEeson & s! & P1 & E1 & & 5 & & סאו & D12 & & & & & & & \\
\hline OAT-NसBOA & 8 & $* 1$ & 臨 & B-1-1 & 13 & 19 & Ph & D1: & $15 x$ & 88 & $\mathbf{F}$ & 69 & $\mathbf{F 1}$ & E9 & $\mathbf{F 1}$ \\
\hline OCT.PHPS-O2 & 8 & F1 & EN & B-1. & 15 & 13 & Ph & 020 & $\mathrm{fFr}$ & & & st & $\mathbf{r i}$ & 3 & $\mathbf{F 1}$ \\
\hline OGTOPSIAM & st & $\mathrm{fa}$ & $\mathbf{E} 1$ & & 11 & 4 & $\mathbf{P M}$ & D10 & & & & & & $\$ 1$. & $F$ \\
\hline oporstates1-01 & $s$ & $\mathbf{F 1}$ & Ez & $c-2-b$ & ఫ & 13 & ס & Dots & $20 x$ & 蚂 & $\mathrm{Fo}$ & 51 & $\mathrm{Fa}$ & Ð & $F 1$ \\
\hline
\end{tabular}




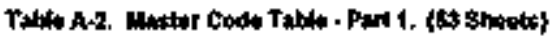

\begin{tabular}{|c|c|c|c|c|c|c|c|c|c|c|c|c|c|c|c|}
\hline $\mathbf{D}$ & 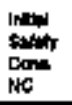 & 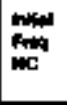 & Em & gan & 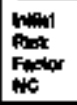 & 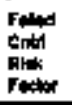 & Foentir & Tomp & Fap & $\begin{array}{l}\text { Ma } \\
\text { colosy } \\
\text { We }\end{array}$ & $\bmod _{\text {Mod }}^{M}$ & Appot & Mppt & 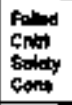 & $\begin{array}{l}\text { Find } \\
\text { Onte } \\
\text { Frim }\end{array}$ \\
\hline DGTHEAC1-AM & $\$ 2$ & 11 & $\boldsymbol{z}$ & $c-2 b$ & 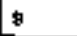 & 13 & wo & Di) & \pm 0 & औ & $\mathbf{p}$ & ba & $\boldsymbol{n}$ & 4 & $P$ \\
\hline Ot-GPECA1AI & si & $\mathbf{F}$ & EO & RP & 31 & 11 & $P_{n}$ & Fos & & & & & & s1 & $\mathrm{F}$ \\
\hline 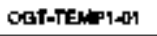 & si & FI & EO & RP & 5 & & $P$ ph & $\cos 4$ & & & & & & & \\
\hline 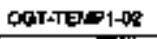 & 82 & fI & E? & B-1- & $\bullet$ & 13 & $P A$ & 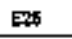 & $10 x$ & s4 & $\mathbf{F}$ & Paymindos & PTrmailed & $\$ 3$ & F1 \\
\hline 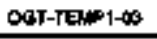 & $\Leftrightarrow$ & fi & E? & $c-2 b$ & 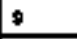 & 13 & wo & EEM & $20 x$ & \$s & $\boldsymbol{m}$ & 61 & F* & 93 & $F 1$ \\
\hline MAD-OI & si & M & m & RP & & & 6 & Fos & & & & & & & \\
\hline MA口何士 & s1 & $\mathbf{m}$ & E1 & "R & & & ONA & Ros & & & & & & & \\
\hline Fapdes & 81 & पू & NA & FP & & & 6 & Fos & & & & & & & \\
\hline PAD-024 & $\$ 1$ & MA & EI & $\operatorname{occ}$ & & & 에 & Fos & & & & & & & \\
\hline Rop-0a & $\$ 1$ & AA & Nh & PP & & & $\theta$ & $\omega \infty$ & & & & & & & \\
\hline pencos & $\$ 1$ & un & et & $\mathbf{m P}$ & & & OM & res & & & & & & & \\
\hline Rtstert & 81 & $\mathbf{r 3}$ & Er & & 11 & 11 & + & E14 & & & & & & s1 & $\mathbf{r g}$ \\
\hline F\$PQ⿻11 & $\$ 1$ & F 3 & Ef & & 11 & 11 & 매 & E14 & & & & & & B1 & $\mathbf{F}$ \\
\hline$R$ Rin-02 & s1 & AR & wh & occ & & & 0 & $F \% 5$ & & & & & & & \\
\hline 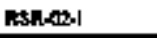 & s1 & NA & E1 & oct & & & on & exs & & & & & & & \\
\hline F⿻. & 81 & Nh & ath & $\Leftrightarrow C$ & & & 0 & 100 & & & & & & & \\
\hline 져가마 & $\$ 1$ & NA & E1 & occ & & & OM & Fos & & & & & & & \\
\hline REROA & $\mathbf{s}$ & No & mat & $\mathbf{R P}$ & & & $\mathbf{T}$ & POS & & & & & & & \\
\hline nsteat4 & $\mathbf{s t}$ & 粠 & E1 & AP & & & ctol & $\mathrm{mos}$ & & & & & & & \\
\hline A: $R 06$ & son & 功 & ES & & 7 & & $\mathbf{T}$ & DOA & & & & & & & \\
\hline
\end{tabular}




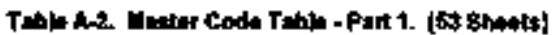

\begin{tabular}{|c|c|c|c|c|c|c|c|c|c|c|c|c|c|c|c|}
\hline 10 & $\begin{array}{l}\text { mal } \\
\text { teifing } \\
\text { cond } \\
\text { NC }\end{array}$ & 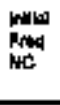 & $\operatorname{Enw}_{\text {ent }}$ & en & 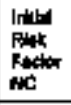 & $\begin{array}{l}\text { Fald } \\
\text { Cont } \\
\text { Fador }\end{array}$ & $\begin{array}{l}\text { Prond } \\
\text { coste }\end{array}$ & con & $\lim _{n \rightarrow 0}$ & 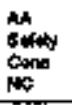 & Wh & 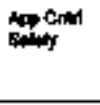 & Fipe & $\begin{array}{l}\text { fund } \\
\text { cist } \\
\text { consty }\end{array}$ & 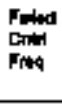 \\
\hline 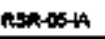 & $\$ 1$ & $n$ & $\mathbf{E 1}$ & & $\theta$ & & 에 & DOH & & & & & & & \\
\hline Rsposeris & 81 & F2 & 62 & & 0 & & on & DOA & & & & & & & \\
\hline Rstivotil & $\mathbf{s}$ & $\mathbf{F}$ & $E 2$ & M.10 & 12 & H & 에 & 0 & $\cos$ & so & $\mathbf{P s}$ & ss & $n$ & $\omega$ & $n$ \\
\hline |Fegr-07-1 & 32 & $\mathbf{F a}$ & ER & A.lad & 14 & $\theta$ & ond & Pres & $\operatorname{tex}$ & $a_{2}$ & $\mathrm{Fz}$ & $\mathbf{s}$ & F2 & 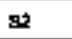 & F1 \\
\hline 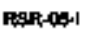 & 31 & Nh & E1 & Occ & & & ond & F14 & & & & & & & \\
\hline sF-0 & b1 & fo & $\mathbf{E} 1$ & & 11 & 11 & $T$ & E14 & & & & & & 51 & fo \\
\hline SPPणना & s1 & $\mathrm{m}$ & e1 & otc & & & 에 & Fo5 & & & & & & & \\
\hline TFS-1 & 58 & $\mathbf{F}$ & $E 2$ & $c-2 b$ & 14 & $\mathbf{R}$ & T.CO & 10 & $\operatorname{tax}$ & 8 & $\mathrm{MJ}_{3}$ & s1 & $\mathbf{P}$ & 8 & F1 \\
\hline V田H01 & 32 & $\mathbf{F g}$ & E? & A.14: & 14 & 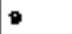 & $T$ & 860 & $m x$ & & & Prented & PTrmelent & 92 & F1 \\
\hline v타-01- & 82 & $\mathbf{F}$ & E2 & $A-1-9$ & 12 & 9 & out & 809 & $\cos x$ & 80 & f3 & Prenertipd & PTrapiond & 82 & $\mathbf{f 1}$ \\
\hline VExtor & B2 & f1 & E1 & Q1- & 9 & & $T$ & D1* & $\operatorname{dex}$ & & & Pronter & Pronenind & & \\
\hline VEHot & siz & F1 & E1 & b-14 & $\theta$ & & on & D1* & oegr & & & 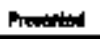 & Mtrutined & & \\
\hline velas & 32 & $\mathbf{F}$ & $E$ & Mate & 12 & & $T$ & tos & tot & & & Phemat & Phrmeled & & \\
\hline VEF+03 & 82 & $\mathbf{n}$ & E2 & $A-1-$ & 12 & & on & एक & Dot $x$ & & & 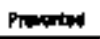 & Prarielad & & \\
\hline Vato4 & st & $\mathbf{m h}$ & wh & $\mathbf{P P}^{p}$ & & & $\mathbf{T}$ & Fos & & & & & & & \\
\hline VI:404 & $\$ 1$ & $m$ & 51 & $\mathbf{P P}$ & & & on & $\operatorname{ros}$ & & & & & & & \\
\hline Yetsots & $\$ 1$ & NA & Nh & $\infty$ & & & $\mathbf{T}$ & Fos & & & & & & & \\
\hline VEF-OS & 31 & Na & [1 & $\infty$ & & & cor & $\boldsymbol{W}$ & & & & & & & \\
\hline vet: & $\$ 1$ & Nin & Ha & $\infty$ & & & $\mathbf{T}$ & ris & & & & & & & \\
\hline verLost & 81 & NA & E1 & $\infty x$ & & & os & Has & & & & & & & \\
\hline
\end{tabular}




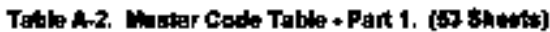

\begin{tabular}{|c|c|c|c|c|c|c|c|c|c|c|c|c|c|c|c|}
\hline 6 & $\begin{array}{l}\text { med } \\
\text { stinty } \\
\text { conx } \\
\text { mic }\end{array}$ & 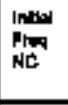 & Evin & eIN & $\begin{array}{l}\text { Inithl } \\
\text { Auck } \\
\text { Fistor } \\
\text { Ne }\end{array}$ & 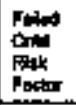 & Cods & अp & $\sum_{n \infty 0}$ & 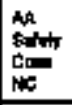 & $\operatorname{mox}_{\mathrm{wC}}$ & Apcind & Apol & 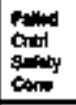 & 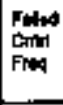 \\
\hline 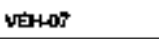 & 80 & is & 10 & & $y$ & & $\mathrm{~T}$ & tost & & & & & & & \\
\hline vators & 92 & $\mathrm{Fa}$ & E1 & $A-1-4$ & 11 & 19 & on & Bक6 & $\cos x$ & 时 & $\mathbf{F}$ & Papmertion & Prominied & $\$$ & $\mathbf{r 1}$ \\
\hline ฟפम06 & 80 & $\mathbf{F}$ & $\mathrm{ED}$ & & 7 & & on & E10 & & & & & & & \\
\hline veror & $\$ 1$ & 59 & ET & & 11 & 11 & or & etd & & & & & & sa & e \\
\hline Volpos & 3 & fo & E1 & & 11 & 11 & ov & E14 & & & & & & st & $\mathbf{F a}$ \\
\hline vaunas & s1 & m & E1 & & 11 & 11 & $8 y$ & D14 & & & & & & gI & Fy \\
\hline valuan & 31 & a & E1 & & 11 & 11 & EN & 000 & & & & & & 91 & fs \\
\hline veluos & 81 & $\mathbf{m}$ & res & $\infty$ & & & ov & $\mathbf{F o s}$ & & & & & & & \\
\hline vistos & 81 & $\operatorname{man}$ & $\mathrm{Man}$ & 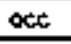 & & & $\mathbf{s w}$ & 514 & & & & & & & \\
\hline MESBRLOHOH & $s z$ & $\boldsymbol{F}$ & $E_{2}$ & $\mathrm{c}$ & 14 & 13 & wo & tot & atin & st & $\mathrm{Ps}$ & $\$ 1$ & $\mathbf{F}$ & s & F1 \\
\hline 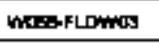 & $s z$ & 12 & E2 & $\mathrm{c} \approx \mathrm{b}$ & 12 & 13 & WD & 어2 & $\operatorname{sex}$ & $\mathbf{s 3}$ & $\mathbf{P 3}$ & 81 & $\mathbf{F B}$ & 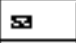 & fi \\
\hline 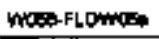 & $\$ 2$ & $\boldsymbol{N}$ & $\Leftrightarrow 2$ & $c-2-b$ & 14 & 13 & WD & EO4 & $20 x$ & $\$ 3$ & $F$ & s1 & $\mathrm{F} 3$ & 69 & F1 \\
\hline Museflomos & 92 & $\mathbf{F z}$ & $\mathrm{E}$ & $\mathrm{cob}$ & 12 & 13 & WD & and & $20 x$ & $\$ 9$ & $F 3$ & s1 & F3 & 53 & $\mathbf{F 1}$ \\
\hline mext-Plontis: & s2 & $\mathbf{m}$ & E & $c_{-2} b$ & 12 & 13 & WD & D2 & ant & s) & Bs & $\$ 1$ & $\mathbf{F}$ & ss & F1 \\
\hline 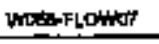 & 媇 & $\mathbf{F s}$ & $\underline{E}$ & $\mathrm{cos}$ & 14 & 13 & WD & E90 & 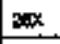 & $\$$ & $\mathrm{~F} 3$ & $\$ 1$ & $\mathbf{F}$ & 8 & F1 \\
\hline MOAPALOMTR & 92 & 72 & 早 & c-2s & 12 & 13 & WD & 012 & $20 x$ & 39 & $\mathrm{Fr}$ & 81 & $\mathrm{F3}$ & $\mathbf{E 3}$ & $\mathbf{H}$ \\
\hline MoFf-fowl1a & 6 & $\mathrm{F3}$ & E & 0.20 & 14 & 13 & WD & Eet & $\operatorname{xax}$ & ss & $\mathbf{H}$ & เ1 & $\mathbf{f a}$ & 53 & $\mathbf{F !}$ \\
\hline Whosflowrtib & $\leq 2$ & $\mathbf{r}$ & $\underline{E}$ & $c_{20}$ & 12 & 13 & WD & D12 & 2 & ss & $\mathbf{F s}$ & $\$ 1$ & $\mathbf{s}$ & st & F1 \\
\hline 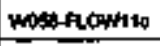 & $\$ 2$ & 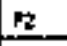 & $\mathbf{E}$ & cats & 12 & 13 & WD & $\mathrm{D} 12$ & $200 x$ & so & F & 81 & $\mathbf{B}$ & 好 & FI \\
\hline MOSP-1LOH13 & 82 & $\mathrm{~F} \rightarrow$ & E & $\cos 20$ & 11 & 13 & WD & Ex & $200 \mathrm{~K}$ & 5 & $F 3$ & $\mathbf{E}$ & F & 89 & FI \\
\hline
\end{tabular}




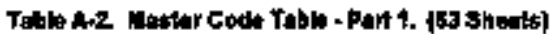

\begin{tabular}{|c|c|c|c|c|c|c|c|c|c|c|c|c|c|c|c|}
\hline ID & 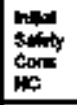 & $\begin{array}{l}\text { Intiol } \\
\text { Proq } \\
\text { HEC }\end{array}$ & Cond & DIN & 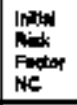 & 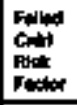 & contin & Genth & $\min _{\ln }$ & 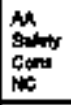 & ${\underset{\mathrm{Fr}}{\mathrm{Mr}}}_{\mathrm{MC}}$ & Alat & Aיה & 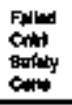 & \\
\hline Wh6:PLCON1S & s & F2 & Es & 0.18 & 15 & 10 & Wo & Eos & $12 x$ & s & P & son & $\mathbf{F a}$ & 6 & 12 \\
\hline H069-710mi: & $\mathbf{8}$ & $\mathbf{F z}$ & Es & E-ret & 15 & 15 & pa & 口12 & $16 x$ & $E$ & $\mathbf{E s}$ & $E 2$ & $\mathbf{F P}$ & $\$$ & F2 \\
\hline 4068-Funvi7g & $\mathbf{s a}$ & $\mathbf{F}$ & ES & E-1-1 & 15 & 16 & סוm & ene & $16 x$ & $E$ & $\mathbf{F a}$ & $E$ & $\mathbf{F a}$ & 83 & $F$ \\
\hline WOSSFr: & $\Rightarrow$ & F & e3 & B-1- & 15 & 15 & no & 012 & $15 x$ & 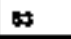 & PF & $\mathbf{2}$ & $P 3$ & 83 & f2 \\
\hline Hosentow17s & $\infty$ & $\mathbf{n}$ & es & B.120 & 15 & 媇 & no & D16 & $1 \neq x$ & $\Leftrightarrow$ & Fs & $\Leftrightarrow$ & $\mathbf{F s}$ & $\mathbf{3}$ & $n$ \\
\hline nost-10N16 & 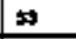 & 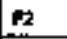 & $\mathbf{E S}$ & B.1.0 & 15 & t & סו & ent & $10 \mathrm{x}$ & 5 & Fo & $5 z$ & $\mathbf{F 3}$ & $\mathbf{s}$ & F 2 \\
\hline Wo: ELOWH & so & F & Eg & Q.14 & 15 & ts & $m o$ & $\mathrm{DII}_{2}$ & $1 \mathbf{E x}$ & Ee & $\mathrm{Fa}$ & 6 & $F$ & $\mathbf{9 3}$ & $\mathbf{F}$ \\
\hline 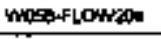 & 5 & $\mathbf{F}_{2}$ & Eq & B-1- & 15 & 15 & mo & $E D$ & $15 x$ & 83 & $\mathrm{Fa}$ & 82 & F3 & $\$ 3$ & $\mathbf{F}$ \\
\hline MESFFlowrob & 果 & $\mathbf{F}$ & E9 & $9-1$ & 15 & 15 & mo & O12 & $15 x$ & s & Ps & $s$ & Fs & s & $\mathbf{n}$ \\
\hline nescelownix & ss & $\mathbf{F}$ & Es & ela & is & 15 & wo & 016 & $15 x$ & st & Ps & $s$ & $\mathbf{H}$ & \$ & $n$ \\
\hline molserfectala & $3 z$ & n & es & $\mathbf{c}-2 b$ & 12 & 13 & Po & on 2 & $21 x$ & 6 & $\mathbf{F o}$ & 61 & $\mathbf{n}$ & $\mathbf{s}$ & *I \\
\hline 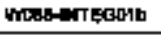 & 52 & $\boldsymbol{F}$ & $E$ & cosb & 12 & 13 & $F$ & O12 & $21 x$ & $\$ 8$ & $\mathrm{~F} 3$ & 81 & $\mathbf{F} 3$ & $\$ 3$ & F1 \\
\hline 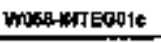 & 8 & $\mathbf{F}$ & E2 & $c-2 b$ & 12 & 19 & wo & on 2 & $2 a x$ & $\$ 3$ & F3 & \$) & F & 89 & 5 \\
\hline WOSEAHTECDIA & 92 & $\mathbf{m}$ & 52 & $c=0$ & 12 & 19 & wo & On2 & $20 x$ & st & is & \$1 & $\mathbf{n}$ & 80 & $\mathbf{F I}$ \\
\hline Woot-InTECSAst & 32 & 12 & s2 & 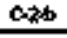 & 12 & 13 & PU & Dod & 动 & $\$ 3$ & $\mathrm{FQ}$ & $8 \pi$ & $\mathbf{F}$ & $\$ 3$ & $\mathbf{F 1}$ \\
\hline 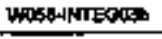 & 89 & $\mathbf{F}$ & 표 & $\cos$ & 12 & 10 & wo & 005 & 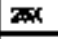 & $\$ 9$ & $\mathrm{F3}$ & 81 & $\mathbf{F g}$ & $\mathbf{p a}$ & FQ \\
\hline WMSPANTECOSA & 97 & $\boldsymbol{F 2}$ & $\mathbf{2}$ & 0.20 & 12 & 13 & $\mathbf{F D}$ & on: & $2 \operatorname{six}$ & s) & $\mathbf{B}$ & Pitstinted & Prenteried & 5 & $F 1$ \\
\hline wo6t-nkTestob & 52 & $\mathbf{F}$ & $\mathbf{s}$ & 0.26 & 44 & 15 & wo & Des & $2+x$ & ss & $\mathrm{Fa}$ & Armented & Premeleod & s9 & $\mathbf{m}$ \\
\hline Whosintescorn & 新 & 13 & e. & 000 & 14 & 13 & $P G$ & ont & $2 \pi x$ & so & $\mathrm{Fg}$ & 91 & $\mathrm{FP}$ & क力 & P1. \\
\hline wos-nitector & 安 & $\mathrm{F} 2$ & $\underline{E}$ & $\mathrm{cos}$ & 12 & 13 & WD & D12 & 269 & 5 & $n$ & s1 & Fy & 由 & F1 \\
\hline
\end{tabular}




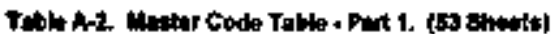

\begin{tabular}{|c|c|c|c|c|c|c|c|c|c|c|c|c|c|c|c|}
\hline $\mathbf{0}$ & 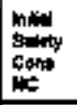 & $\begin{array}{l}m=1 \\
\text { five } \\
m\end{array}$ & $\begin{array}{l}\text { En } \\
\text { toms } \\
\text { tom }\end{array}$ & gas & 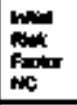 & 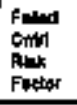 & $\begin{array}{l}\text { Futh } \\
\text { Cofia }\end{array}$ & Comp & $P_{000}$ & $\begin{array}{l}\text { Ah } \\
\text { Sent } \\
\text { NCE } \\
\text { NC }\end{array}$ & $M_{m \in d}^{M}$ & Apctition & 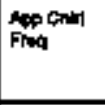 & 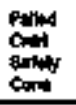 & $\begin{array}{l}\text { Fald } \\
\text { Erith } \\
\text { Fraq }\end{array}$ \\
\hline 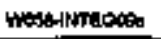 & 82 & f1 & E2 & $a b$ & ? & 13 & $P D$ & $\infty$ & $21 x$ & 的 & $\mathbf{N}$ & $\$ 1$ & $\mathbf{F s}$ & as & F1 \\
\hline Mastintifesceb & $\Leftrightarrow$ & $\mathbf{F 1}$ & E? & cab & $\mathbf{\theta}$ & 13 & WD & Dos & $29 x$ & 8 & $\mathbf{F}$ & क1 & $\mathrm{Fg}$ & $\$$ & $\mathbf{F r}$ \\
\hline УOSAINTES11. & 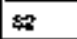 & $\mathbf{F 1}$ & $\mathrm{E} 2$ & $c-2 b$ & H & 13 & Po & DHP & $21 x$ & 89 & $\mathbf{F}$ & gu & $m$ & $\$$ & $\mathbf{F r}$ \\
\hline 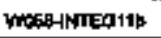 & $\$ 2$ & $\mathbf{r}$ & ㅌ2 & $a-2 b$ & $\$$ & 13 & wo & Dw & $\operatorname{2ts}$ & 89 & $\mathbf{F} 2$ & $\mathbf{8}$ & $\mathrm{F} 2$ & 8 & FH \\
\hline 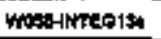 & 할 & 12 & 82 & $a-2 b$ & 12 & 13 & PD & 000 & $21 x$ & \$5 & $\boldsymbol{p}$ & $\$ 1$ & $\mathrm{Fa}$ & $s$ & $\boldsymbol{F}$ \\
\hline mistolinesist & gap & k2 & E2 & $a-\infty$ & 12 & 19 & PD & oti & $21 x$ & $\mathbf{s}$ & $\boldsymbol{P}$ & $\$ 1$ & $\mathbf{m s}$ & s3 & H \\
\hline Vmotalntedisc & $\$ 2$ & $\mathbf{F} 2$ & E2 & a.2. b & Iz & 13 & wo & Do6 & $20 x$ & 50 & P & EI & Ps & $\$$ & $\mathbf{F E}$ \\
\hline MD68-INTEG130 & 02 & $\mathbf{F 2}$ & E2 & $\cos _{2-p}$ & 12 & 13 & WD & Dol & 20 & 89 & $\mathbf{F} \mathbf{3}$ & $\mathbf{3}$ & Fa & $\$ 8$ & $\mathbf{F r}$ \\
\hline WOSPHNTEQ140 & 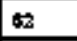 & $\mathbf{F}$ & E.2 & $c-2 b$ & 12 & 10 & PU & DOS & $21 x$ & 95 & $\mathbf{F}$ & $\$ 1$ & F3 & 93 & 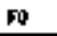 \\
\hline MDSANTCOSA & $s$ & $\mu z$ & $\rightleftarrows$ & $c-z b$ & 12 & 10 & Wo & 000 & تل & $\$$ & $P 2$ & $\$ 1$ & Fs & so & $\omega$ \\
\hline 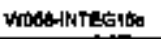 & 8 & $\mathbf{P a}$ & $\mathbf{E 2}$ & cosib & 14 & 13 & po & $D Q$ & exs & $\mathbf{s}$ & $\mathbf{A}$ & Proveting & Primitad & 53 & $\mathbf{F r}$ \\
\hline 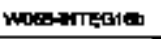 & 82 & $\mathrm{~F} 3$ & E & $\mathrm{c}_{\mathbf{b}}$ & 14 & 13 & wo & 02 & $26 x$ & $\$ 3$ & $\mathrm{FB}$ & 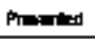 & Pravertad & $\$ 3$ & F1 \\
\hline W0SP-NTECA & 52 & $\mathbf{F}$ & $\mathbf{2}$ & $\mathrm{c}-20$ & 12 & 15 & $\mathbf{P D}$ & $D 12$ & $21 x$ & 83 & $\mathrm{F3}$ & 61 & $\mathrm{Fu}$ & ss & $\mathbf{F z}$ \\
\hline 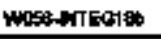 & s2 & 72 & E & 020 & 12 & 13 & No & Q12 & $\tan$ & st & $\mathbf{F 3}$ & 5) & $\mathrm{Fg}$ & * & $\boldsymbol{H} 1$ \\
\hline MLSAETEGa: & 62 & F1 & EF & $c-2 b$ & e & is & Pp & 吸4 & $21 x$ & $\$ 3$ & ps & s & $\mathbf{n}$ & $\$ 3$ & $\mathbf{F}$ \\
\hline 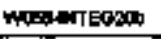 & 82 & f1 & Ex & $5-26$ & D & 13 & mo & 며 & $28 x$ & so & $\mathrm{Fos}$ & 51 & 的 & $\$$ & F1 \\
\hline Nox-MTEC?2 & 52 & 1 & $\mathbf{E}$ & c.26 & D & 13 & PO & D1: & $21 x$ & st & $\mathbf{F 3}$ & 81 & F2 & 59 & F1 \\
\hline Wost-MTEGTA & $\$ 2$ & $\mathbf{F}$ & $p$ & $c-2 b$ & B & 10 & no & 車 & $\Delta x$ & \$3. & $\mathbf{m}$ & ss & PO & 8 & $\mathbf{H}$ \\
\hline 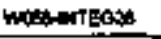 & 82 & F1 & $\theta$ & 60 & 0 & 13 & 40 & D10 & $20 x$ & 83 & F & s3. & $\mathbf{F 2}$ & $\$ 3$ & $F 1$ \\
\hline 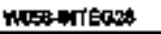 & 22 & F1 & $\mathbf{2}$ & $c-2 b$ & D & 10 & wo & 029 & $2 x$ & ES. & Fo & 暗 & $\mathbf{F o}$ & $\$$ & $F 0$ \\
\hline
\end{tabular}




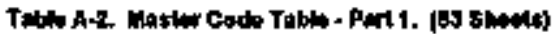

\begin{tabular}{|c|c|c|c|c|c|c|c|c|c|c|c|c|c|c|c|}
\hline ID & 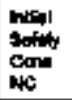 & $\min _{m}^{m}$ & 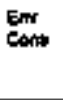 & Bes & 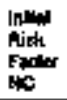 & $\begin{array}{l}\text { Follod } \\
\text { forts } \\
\text { fastor }\end{array}$ & tode & Orp: & Rec & 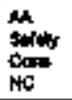 & 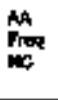 & apponit & 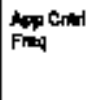 & 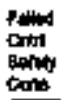 & 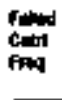 \\
\hline MHSOANT EGSO & $\mathbf{s 2}$ & 51 & e & $c-2 b$ & $\mathbf{B}$ & 13 & mo & bits & and & $s$ & $\mathbf{F}$ & $\mathbf{s}$ & $F_{7}$ & 50 & $\mathbf{F 1}$ \\
\hline WMOSO-1NTECCS & $\Leftrightarrow$ & Fi & $\mathbb{B}$ & 10 & 18 & 18 & mo & Diz & $15 x$ & $\mathbf{s}$ & $\mathbf{F}$ & ह⿴囗十 & FI & 89 & fo \\
\hline 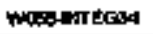 & $\mathbf{s 3}$ & 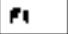 & $\mathbf{s}$ & :1a & 13 & to & mo & DES & 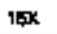 & $s$ & FP & 63 & $\mathrm{FQ}$ & 83 & $\mathbf{F o}$ \\
\hline WWO-WTECक\% & $\$ 3$ & $\mathbf{F}$ & $\theta$ & 6-14:0 & 13 & 15 & mo & Dors & $15 x$ & $\mathrm{E}$ & $P 3$ & $\$ 9$ & $\mathrm{Pz}$ & 83 & 72 \\
\hline НК유. & 53 & $\mathbf{F 1}$ & Ea & $8-1-4$ & 13 & 10 & 40 & $\mathbf{0 2 4}$ & $15 x$ & 63 & Fo & $\$ 3$ & $\mathbf{F b}$ & 35 & mo \\
\hline HOSHATEG3? & 83 & $\mathbf{F} 1$ & Es & 8.1. & 13 & 15 & $n$ & Dis & $19 x$ & 83 & rs & st & $F 2$ & sto & $\mathbf{m}$ \\
\hline MASHPRESB & t5 & $\mathbf{F y}$ & 로 & 2020 & 14 & 13 & wo & ma & 政 & $\$$ & F 3 & 81 & $\mathbf{F g}$ & 83 & $\mathbf{F 1}$ \\
\hline MHSQPREEAT & 92 & $\mathbf{P}$ & E & cost & 14 & 13 & wo & DA17 & $\sin$ & 8 & $F$ & st & $F$ & $\$ 3$ & $\mathbf{F 1}$ \\
\hline xolfounos & 67 & $\mathbf{F 2}$ & 브 & $A+1 *$ & 12 & 15 & T.co & BOI & $\cos x$ & 89 & F & s3 & $\mathbf{m}$ & $\$ 3$ & $\mathbf{F}$ \\
\hline 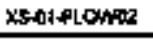 & 59 & $\boldsymbol{F Z}$ & $\mathrm{EA}$ & $A-1-$ & 15 & 15 & T.SD.H & $\mathbf{B 0 0}$ & $\cos x$ & 93 & $\mathbf{r s}$ & \$3 & $\mathbf{F}$ & s3 & $\mathbf{r} 2$ \\
\hline 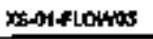 & sa & $\mathbf{F 2}$ & es & A-1 & 16 & 10 & 4 & $B \times$ & $\mathbf{0 6}$ & ss & $\mathbf{m}$ & 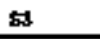 & $\mathbf{F t}$ & as & F \\
\hline Cothiflown & $\$ 1$ & $\mathbf{F}$ & et & & 4 & & $T .40$ & Ees & & & & & & & \\
\hline XSG Floums & 82 & $\mathrm{~F} 2$ & 应 & Q 14 & 12 & 13 & $w \bar{r}$ & EAS & $2 \mathrm{zx}$ & $\$ 3$ & F & 81 & FH & s3 & n1 \\
\hline XSAIFLONA & 97 & $\mathrm{Fa}$ & 62 & $a-2-b$ & 14 & 13 & WO & $\mathbf{F \omega}$ & $204 x$ & $\$$ & $\mathbf{P J}$ & $s 1$ & $\mathbf{m}$ & $\$ 3$ & F1 \\
\hline X5-A1/FLONA7 & s & $\mathbf{F g}$ & 표 & Estb & 14 & 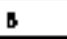 & P白 & D12 & $2 x$ & 31 & $\mathrm{P3}$ & bi & fo & 31 & $\boldsymbol{H}$ \\
\hline X四-OHPAEEd1 & 的 & $\mathbf{F z}$ & Es & Babb & 12 & 13 & PO & E13 & $21 x$ & st & $\boldsymbol{F}$ & si & $\mathbf{F}$ & $\mathbf{s}$ & F1 \\
\hline 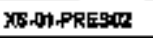 & $\$ 1$ & F3 & 67 & & 11 & 11 & $\mathbf{H}$ & D12 & & & & & & 51 & P3 \\
\hline 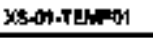 & $\mathbf{s}$ & $\mathbf{F 3}$ & 22 & B-10 & 14 & 13 & wo & Dod & $13 x$ & $\mathbf{s}$ & $\mathbf{F}$ & Ald & Phentiond & 8 & $\boldsymbol{M}$ \\
\hline 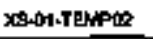 & 的 & Ps & et & & 11 & 8 & wo & EAs: & & & & & & 91 & E1 \\
\hline XE-0\&-F它心 & $\$ 1$ & F2 & E1 & & $\mathrm{E}$ & & 0 & EDe & & & & & & & \\
\hline
\end{tabular}




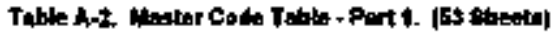

\begin{tabular}{|c|c|c|c|c|c|c|c|c|c|c|c|c|c|c|c|}
\hline $\mathbf{D}$ & 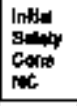 & $\begin{array}{l}\text { Hined } \\
\text { Freq } \\
\text { NGE }\end{array}$ & Ens & ENN & 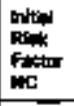 & 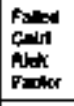 & Pand & $\begin{array}{l}\text { Eautse } \\
\text { आP }\end{array}$ & fipos & 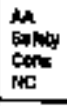 & $\operatorname{Fin}_{\text {MC }}$ & eppont & Appon & $\begin{array}{l}\text { falind } \\
\text { coint } \\
\text { solites } \\
\text { cond }\end{array}$ & Find \\
\hline 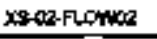 & $E$ & $\mathrm{~F} 3$ & EI & D-14 & 14 & 11 & T. & EO1 & $32 x$ & 81 & $F$ & s1 & $\mathbf{f 3}$ & 61 & 63 \\
\hline 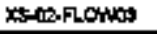 & 82 & $\mathbf{F} 2$ & EI & $c-1=0$ & 12 & 6 & T.100 & ED: & $\cos x$ & 32 & $\mathbf{F s}$ & Prevented & Prowingud & 52 & fo \\
\hline 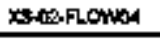 & 52 & $\mathrm{Fa}$ & $\mathbf{E}$ & 1.5.th & 12 & $\mathbf{s}$ & PO & ENoA & $2 \mathrm{Tx}$ & si & $\mathbf{F 3}$ & si & er & $\$ 1$ & F1 \\
\hline Xstandontos & 82 & $\mathrm{Bd}$ & 를 & tow & 14 & 13 & op & Di2 & $27 x$ & st & $\mathbf{F}$ & $s t$ & H & ss & $\mu$ \\
\hline 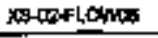 & $\mathbf{s 1}$ & $\mathbf{F}$ & E1 & & 11 & 8 & I.CD & 草咀 & & & & & & s1 & $\mathbf{F}$ \\
\hline XOSOSEVE & 81 & $\mathbf{F}$ & $E$ & & 11 & 11 & T.CD & 머2 & & & & & & 51 & $\mathbf{F}$ \\
\hline 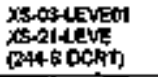 & 82 & $\boldsymbol{E} 2$ & 6 & $A-1 \cdot a$ & 12 & 15 & T.CD & 800 & DEX & 93 & $\mathbf{B}$ & s3 & $P 1$ & 5 & 2 \\
\hline 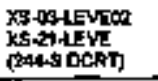 & $\$ 8$ & $\mathrm{FO}$ & $\mathbf{E}$ & $A-1 *$ & 14 & 9 & T.CD & $A \in O$ & $01 x$ & ง1 & 1 & Protitined & Pronietid & 31 & Fo \\
\hline XE-DS-PREEOO1 & B1 & $\mathrm{F}$ & E1 & & 11 & 11 & $t .00$ & Don & & & & & & 51 & $\mathbf{F q}$ \\
\hline 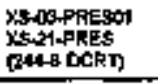 & 97 & $\boldsymbol{F 2}$ & $\boldsymbol{\nabla}$ & N10 & 12 & 15 & T.CD & papr & $\cos x$ & st & $\mathbf{0}$ & $\leqslant$ & FI & $\mathbf{s y}$ & $\boldsymbol{m}$ \\
\hline XS-DS-PAEgOAN & $\$ 1$ & $\mathbf{F 3}$ & 51 & & 11 & 4 & r.co & $\mathrm{DNT}$ & & & & & & 51 & $\boldsymbol{m}$ \\
\hline 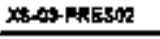 & $\$ 1$ & $\mathbf{P 3}$ & E1 & & 14 & 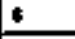 & T.CD & $e m$ & & & & & & 51 & $\mathbf{F}$ \\
\hline 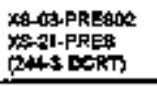 & 92 & $\mathbf{F S}$ & e1 & E-1. & 14 & a & T.Cक & Dot & $20 x$ & 91 & $\mathbf{F a}$ & EA & $\mathrm{FB}$ & 81 & $\mathrm{~F} 2$ \\
\hline X6-asPREtand & 81 & $\mathrm{Fa}$ & E1 & & 11 & 11 & T.दि & Dats & & & & & & B1 & Pa \\
\hline$x B-69+$ ripsops & 81 & $\mathrm{F}$ & E1 & & 11 & 8 & $T, 60$ & Dow & & & & & & 81 & $\mathrm{~F}$ \\
\hline Xo-opopisis & 8 & $\mathbf{F z}$ & E2 & $4-1$ & 12 & 11 & T.CD & Do1 & $20 x$ & 01 & $\mathbf{m}$ & s1 & $\mathbf{F s}$ & का & $\mathrm{F3}$ \\
\hline
\end{tabular}




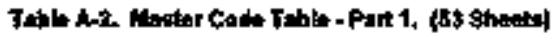

\begin{tabular}{|c|c|c|c|c|c|c|c|c|c|c|c|c|c|c|c|}
\hline $\mathbf{0}$ & 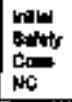 & mat & Es & $=1$ & 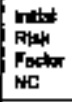 & 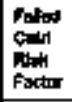 & $\rightarrow$ & $\begin{array}{l}\text { cones } \\
\cos \end{array}$ & Rat & 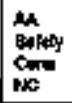 & $\begin{array}{l}\text { Mh } \\
\text { Frata } \\
\text { NG }\end{array}$ & 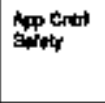 & $\operatorname{lop}_{\mathrm{F}}$ & 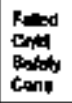 & $\begin{array}{l}\text { Fuled } \\
\text { chil } \\
\text { Ind }\end{array}$ \\
\hline Xs-0s-TEMPO1 & 82 & $\mathbf{F 2}$ & E1 & s.14 & 12 & 8 & $T, \infty$ & Dop1 & $\cos x$ & 87 & Fi & Pronenotid & Prenertad & 89 & Fo \\
\hline 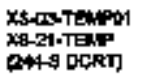 & 50 & $\mathbf{A s}$ & E & & 7 & & T.100 & Dor & & & & & & & \\
\hline HSOS-TEMPVI & sq & $\mathbf{F 2}$ & E1 & $c-1-$ & 12 & 11 & $T, \infty$ & DO1 & $22 x$ & $\$ 1$ & $F$ & $\$ 1$ & $\mathbf{F a}$ & 81 & $\mathrm{F3}$ \\
\hline 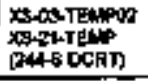 & $s$ & $\mathbf{r y}$ & EO & ه1:10 & 14 & 3 & T.CO & 100 & $01 x$ & $s 1$ & F1 & Powiontid & Prouptod & 81 & Fo \\
\hline 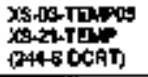 & 32 & $\mathbf{F 2}$ & $\boldsymbol{n}$ & M.lat & 12 & 15 & TCD & Bar & $\cos x$ & sa & $F$ & 83 & FI & 93 & F \\
\hline xक-03-тв-194 & si & $\mathbf{F 1}$ & E1 & & 5 & & $\mathbf{T . C 0}$ & $\sec 8$ & & & & & & & \\
\hline 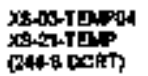 & $\$ 2$ & Ps & E2 & $a+b$ & $1 d$ & 19 & r.co & D2d & $20 x$ & 53 & P & 91 & F3 & as & P1 \\
\hline K3-08.T17405 & $\$ 1$ & $\mathrm{~F}$ & E1 & & 11 & 8 & T.CD & [15 & & & & & & $s_{1}$ & 22 \\
\hline xsos perings & 31 & B3 & es & & 11 & 8 & T.CD & ENS & & & & & & 61 & 62 \\
\hline Xeos- Terifor & $\mathbf{s 1}$ & $\mathbf{F s}$ & et & & 11 & . & T.C.0 & Dor & & & & & & \$1 & $\mathbf{P 2}$ \\
\hline XB-OB-TEHODE & $\$ 1$ & $\mathrm{~F} 3$ & E1 & & 11 & 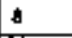 & $\mathbf{I} . \mathbf{c p}$ & bur & & & & & & 81 & $\mathbf{F} 2$ \\
\hline 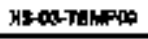 & 80 & $\mathbf{M}$ & 63 & & 2 & & T.cb & DoI & & & & & & & \\
\hline 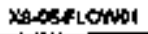 & sin & f2 & E1 & B-14a & 12 & 5 & T.cD & $\mathbf{E} \boldsymbol{\theta}$ & $\cos$ & 自 & $\mathbf{F s}$ & $\$ 1$ & $\varphi_{2}$ & 31 & $\mathbf{F 1}$ \\
\hline 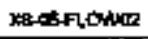 & 80 & $\mathbf{F} 2$ & Es & & 4 & & T.ec & E1] & & & & & & & \\
\hline XR-OS-TENPM1 & 82 & fo & E? & A A-1 & 14 & 5 & T.CD & 608 & $D \rightarrow x$ & 51 & $\mathbf{F}$ & $\$ 1$ & F2 & a & F1 \\
\hline recositures & $\Leftrightarrow$ & a & $\mathbf{n}$ & $4-1-1$ & 14 & 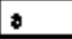 & T.CD & $\cos$ & Dex & 81 & $\boldsymbol{n}$ & 32 & $\mathbf{F 1}$ & Ei & $\mathbf{F}$ \\
\hline
\end{tabular}




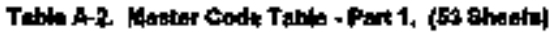

\begin{tabular}{|c|c|c|c|c|c|c|c|c|c|c|c|c|c|c|c|}
\hline 10 & $\begin{array}{l}\text { nhal } \\
\text { ganty } \\
\text { Cont } \\
\text { mi }\end{array}$ & $\begin{array}{l}\text { mbid } \\
\text { Find } \\
\text { inc }\end{array}$ & EN & BM & 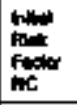 & 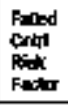 & $\begin{array}{l}\text { Farth } \\
\text { cots }\end{array}$ & $\begin{array}{l}\operatorname{con}{ }^{2} \\
\text { orp }\end{array}$ & Rep & $\begin{array}{l}\text { Mh } \\
\text { \&anty } \\
\text { Cons } \\
\text { NC }\end{array}$ & 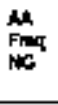 & mpe ont & $\begin{array}{l}\text { Apptond } \\
\text { Finq }\end{array}$ & 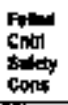 & $\begin{array}{l}\text { Fold } \\
\text { Cnot } \\
\text { nos }\end{array}$ \\
\hline X6-08-7chnor & \$2 & $\mathrm{Fg}$ & E & $A-1-n$ & 14 & 3 & $T, C 0$ & No9 & $01 x$ & $\$ 1$ & F1 & 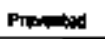 & Ponvolut & 61 & $\mathrm{FP}$ \\
\hline Xs-ot-nownit & $\$ 3$ & $\mathbf{F} 2$ & E & at- & 15 & 13 & $w \sigma$ & DN12 & 15 & ss & F3 & $s \mathbf{s}$ & P & 8 & $\mathbf{F}$ \\
\hline Xs-ob-flortits & $\$ 3$ & $\mathbf{F} \mathbf{2}$ & $\mathrm{Ea}$ & B-1- & 15 & 15 & $\omega 0$ & DN12 & $15 x$ & s8 & $\mathbf{F 3}$ & 82 & Fu & $\mathbf{s a}$ & $\mathbf{F}$ \\
\hline 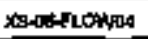 & $\$ 2$ & 12 & ES & D-14 & 12 & is & 40 & 012 & $\operatorname{tax}$ & $\mathbf{3 3}$ & $\mathbf{F s}$ & 52 & $\mathbf{m}$ & E3. & $\mathbf{r 2}$ \\
\hline XB-00.FLThos & 묘 & $F 2$ & 12 & B.3b & 12 & 13 & $\mathrm{PC}$ & D12 & $21 x$ & $\mathbf{s}$ & $\mathbf{F 3}$ & si & $\mathbf{F u}$ & 国 & $\mathrm{Pl}$ \\
\hline xB-09-7loning & $\$ 1$ & $\mathrm{Fg}$ & E1 & & 11 & 5 & $T, 90$ & $E 00$ & & & & & & 51 & $\mathbf{F z}$ \\
\hline KE-bi.forial & s7 & $\mathbf{F 3}$ & $\boldsymbol{\theta}$ & 020 & 14 & 13 & PC & $D m$ & $21 x$ & 93 & F & $s 1$ & PO & 8 & $\mathbf{F 1}$ \\
\hline 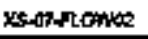 & $s z$ & $\mathbf{r o}$ & $m$ & asb & $\mathbf{M}$ & is & 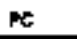 & and & $21 \mathrm{~h}$ & ss & $\mathbf{N}$ & $s 1$ & $\mathbf{P}$ & 5 & $\boldsymbol{F} t$ \\
\hline 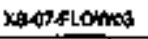 & $s$ & $\mathbf{F 3}$ & $e 2$ & Bsta & 14 & 10 & $P C$ & pow & $21 x$ & sas & $\mathbf{F}$ & \& & Fo & 的 & $\mathbf{m o}$ \\
\hline XB-OT-FLothod & 如 & $\mathrm{Fz}$ & 1 & b- & 12 & 13 & $P C$ & Dens & $21 x$ & $\$ 3$ & $F$ & $\$ 1$ & $\mathbf{F} 2$ & 69 & f \\
\hline Xas-fomis & 62 & $\mathbf{F 3}$ & e? & B-30 & 14 & 15 & $\mathbf{P C}$ & De12 & $21 x$ & 83 & F 7 & 81 & $\mathbf{F g}$ & s3 & $\mathbf{F 2}$ \\
\hline XESAPFLOWM6 & 92 & $\mathbf{B}$ & $\mathbf{E P}$ & $\mathrm{B}-3+\mathrm{b}$ & 14 & 13 & $p$ & D17 & 21: & 59 & n & 51 & $\mathbf{F}$ & so & 1 \\
\hline Xto114Fver & ss & $\mathbf{F}$ & ez & $0-26$ & 14 & 13 & wo & ent & $20 x$ & so & $\mathbf{P a}$ & \$1 & $\mathbf{F}$ & 息 & $\mathbf{H}$ \\
\hline ces-11-1Fvitg & 竹 & $\mathrm{Fa}$ & 12 & $a+b$ & 14 & 13 & wo & EOA & $\mathbf{2 a x}$ & 93 & $\mathbf{F}$ & a & $\mathbf{F}$ & 69 & F \\
\hline 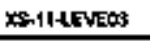 & 82 & $\mathbf{F 3}$ & E? & c.20 & 14 & 13 & wD & $\mathrm{Dm}$ & $20 x$ & 83 & F3 & $\$ 1$ & $\mathbf{f}$ & s & F1 \\
\hline Xs-12.TLOWH & st & 73 & 51 & & 11 & 11 & ב & ent & & & & & & s & $\boldsymbol{n}$ \\
\hline 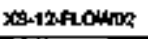 & $\$ 2$ & F1 & E1 & A-14: & 1 & 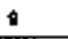 & Wo & 瞩 & ortx & st & $\mathbf{P}$ & Pantiold & Promoted & 的 & $\mathbf{F 1}$ \\
\hline xs-12floums: & 89 & $F 1$ & E1 & A-10 & 1 & D & $w p$ & 略 & or $x$ & 62 & $m$ & Brpanind & Prumatod & 82 & $\mathbf{F} 1$ \\
\hline 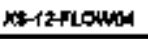 & $\$ 1$ & $\boldsymbol{0}$ & 51 & & 11 & & wo & $\infty$ & & $\$ 1$ & 1 & & & & \\
\hline 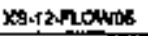 & $\$ 2$ & $\mathbf{p}$ & E2 & cot & 14 & 13 & 40 & Dot & $2+x$ & $s$ & $\mathbf{p}$ & st & $\mathbf{r g}$ & s3 & 11 \\
\hline
\end{tabular}


Tobto A-2. Mactor Cots Table - Part 1. (59 Stheota)

\begin{tabular}{|c|c|c|c|c|c|c|c|c|c|c|c|c|c|c|c|}
\hline ID & 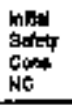 & 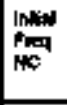 & Ew & BN & 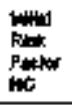 & 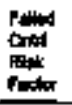 & Code & Gmp & $m_{\infty}$ & ind & 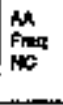 & 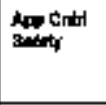 & Fip Cont & 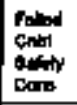 & 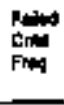 \\
\hline X8-12-Rlownto & si & $\mathbf{F B}$ & ex & & 11 & $\mathbf{a}$ & WD & bus & & & & & & 91 & F2 \\
\hline XS-13LEVE & 80 & $\mathrm{~F} \mathbf{3}$ & EF & & 7 & & T.CN & D12 & & & & & & & \\
\hline XB-13PPEQGO1 & 82 & $\mathbf{F} \mathbf{3}$ & EF & s-1-1 & 15 & 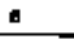 & R.CN & DOI & DSS & & & Pomining & Promonitad & 48 & $\mathrm{FQ}$ \\
\hline 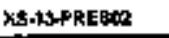 & 9 & $\mathbf{F} 2$ & E2 & A.i-i= & 15 & 15 & T.CN & 8008 & DAK & 4 & F & s & $\mathbf{F I}$ & 8 & $\mathbf{F}$ \\
\hline XE-13-PREsus & $\$$ & $\boldsymbol{n}$ & $\mathbf{E}$ & Cor14 & $\mathbf{i z}$ & $\mathrm{ir}$ & T.EN & Di1 & $21 x$ & sl & $\mathrm{AS}$ & 61 & H & si & $\mathbf{P}$ \\
\hline 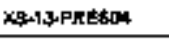 & 67 & F1 & 12 & A.14: & $p$ & 3 & T.C.M & an & 01X & 91 & F1 & PToronterd & Panverind & 61 & Fo \\
\hline 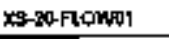 & $\$ 2$ & $\mathrm{~F} 3$ & E2 & G-1-1 & 14 & 8 & $T, C D$ & EDS & $\operatorname{sen} x$ & & & PToranum & Prwornent & $8 \mathbf{2}$ & fo \\
\hline xesanforme & $\$ 1$ & $\mathrm{F2}$ & E2 & & 8 & & 0 & 504 & & & & & & & \\
\hline xs-xonorns & $\$ 2$ & $\mathbf{m}$ & 5r & 1.14 & 12 & 6 & T.CD & 800 & $\cos x$ & sz & $\mathbf{A s}$ & Premented & Proventat & $\$ 2$ & No \\
\hline x5-30-PLOWHA & 52 & $\mathbf{H z}$ & 12 & cons & 12 & 11 & T.CE & ens & $\cos$ & 51 & $\mathbf{m}$ & 81 & $P$ & 51 & Bs \\
\hline 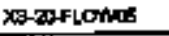 & 92 & FI & $\underline{5}$ & A-4 & 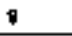 & 3 & T.CD & $\mathbf{m 0 6}$ & $91 x$ & 81 & F1 & Prorontod & Proninged & 81 & $\mathbf{F v}$ \\
\hline XS-XSFLCMnne & $s 1$ & $\mathbf{F}$ & E2 & & 8 & & 0 & EOQ & & & & & & & \\
\hline Xastoflomo? & $\$ 1$ & $\mathbf{F}$ & Ex & & ] & & 0 & E00 & & & & & & & \\
\hline xastontonis. & 82 & $\mathbf{F}$ & E2 & $0-26$ & 14 & 13 & T,Co & E00 & 落 & $s$ & $\mathbf{F}$ & 91 & $\mathbf{F}$ & 亩 & FI \\
\hline 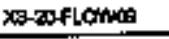 & ss & $\mathbf{F}$ & 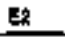 & $5-20$ & 14 & 13 & t.co & 600 & 20⿱亠䒑⿱亠幺⿲丶丶丶 & 63 & $\mathrm{Fu}$ & $\$ 1$ & $\mathbf{F G}$ & 83 & F1 \\
\hline 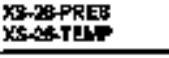 & 80 & $\mathbf{F}$ & $E$ & c-1e & 14 & 11 & $\mathbf{T}$ & $E 00$ & 23 & $\$ 1$ & $\boldsymbol{F 3}$ & $\$ 1$ & $\mathbf{F}$ & s1 & B3 \\
\hline 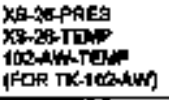 & 的 & f1 & 虹 & $A-1=4$ & 1 & $\mathbf{a}$ & 108 & Mo1 & $\cos x$ & s1 & F1 & Prenumid & Prand & 91 & fo \\
\hline XF-2:-PREF01 & 8 & $\mathbf{F a}$ & E? & G-1-A & 14 & 6 & $\boldsymbol{T}$ & 600 & $203 x$ & & & Prondt & Prominald & $\$$ & $\mathbf{m}$ \\
\hline
\end{tabular}




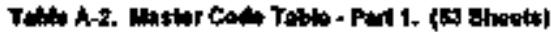

\begin{tabular}{|c|c|c|c|c|c|c|c|c|c|c|c|c|c|c|c|}
\hline$b$ & 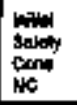 & 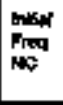 & Con & Ba & 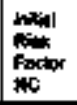 & 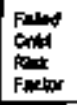 & fentip & Gmp & 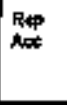 & 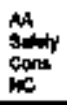 & $\underset{N}{M}$ & top Cint & Fpp & 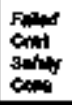 & لبn \\
\hline 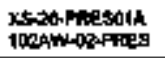 & s1 & ps & FI & & 11 & 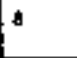 & $\boldsymbol{T}$ & $\infty$ & & & & & & $\mathbf{s i}$ & p2 \\
\hline 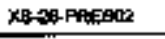 & pes & $\mathbf{F}$ & [a & A-1- & 15 & 15 & T.80. & ONo & $00 x$ & $\$ 9$ & $\mathbf{F 9}$ & $\$ 9$ & F1 & 6 & $\mathbf{F}$ \\
\hline 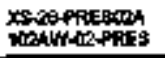 & $s_{1}$ & F3 & 61 & & 11 & 4 & Tsp.Y & DW & & & & & & 81 & $\mathbf{F 2}$ \\
\hline 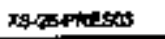 & Bt & is & Et & & $\mathbf{1 1}$ & 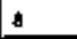 & $\mathbf{T}$ & Don & & & & & & s1 & $p_{2}$ \\
\hline 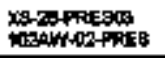 & 91 & $\mathrm{~F}$ & E1 & & 11 & 11 & t.sb.y & 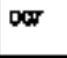 & & & & & & 61 & $\mathbf{F}$ \\
\hline 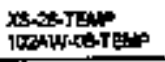 & st & $\mathbf{m}$ & e! & & 5 & & T.so.v & $D Q ⿻$ & & & & & & & \\
\hline X5-28-1 & \$2 & $\mathrm{F} 2$ & 52 & $B-1-0$ & 12 & 9 & 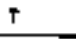 & DOM & $1 \beta 2 x$ & & & Pranionsed & Pripentited & 82 & E1 \\
\hline Xs-2a-TIMPan & औ1 & F1 & E1 & & 5 & & $\tau, \cos , 4$ & DOF & & & & & & & \\
\hline 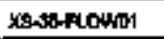 & Bo & $\mathbf{B}$ & et & & 7 & 7 & $\boldsymbol{\infty}$ & Dt2 & & & & & & 30 & $\mathbf{F s}$ \\
\hline 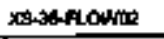 & st & $\mathbf{F s}$ & Ez & $a-2 b b$ & 14 & 13 & 0 & EOS & 部 & sy & $\mathrm{P3}$ & 버 & $\mathbf{F}$ & 63 & F1 \\
\hline 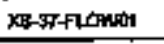 & so & F3 & E2 & $c-2 b$ & 14 & 13 & T.CW & ㅁ12 & $2 \pi x$ & 83 & F3 & \$1 & $\mathrm{Fg}$ & $\$ 3$ & $\mathbf{F 1}$ \\
\hline XB-3P+10Na2 & 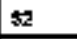 & $\mathrm{Fg}$ & E2 & cosso & 14 & 5 & PE & $\mathrm{D}+2$ & $20 x$ & si & F3 & s1 & ro & $\$ 1$ & F1 \\
\hline XS-419.PLOMnO & s & H3 & E2 & 0.26 & 14 & 13 & $\omega \sigma$ & Ot2 & $203 x$ & s) & $\mathbf{p s}$ & s1 & is & st & $\mathbf{m}$ \\
\hline 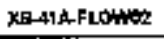 & s? & $\mathbf{F s}$ & E2 & $0-2 b$ & t4 & 13 & we & EOC: & $2+x$ & $\mathbf{8}$ & $\mathrm{F3}$ & B1 & $\mathbf{r}$ & as & F1. \\
\hline XF-11P-LEFEM & $\mathbf{6 2}$ & $\mathbf{2}$ & E2 & A-4 & 12 & 15 & T.CH & Dops & $\cos x$ & 5 & FY & $\$ 3$ & $\mathrm{Ft}$ & 59 & $F 2$ \\
\hline X5-418-4eVtexh & $\$ 1$ & $\mathbf{0}$ & E1 & & 11 & $\$ 1$ & WD & Do* & & & & & & $\$ 1$ & $\mathbf{P 3}$ \\
\hline 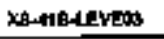 & $\mathbf{s}$ & $\mathbf{A}$ & E? & 024 & 14 & 13 & Wo & ext & $2+x$ & 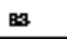 & $\mathbf{f s}$ & 81 & $\mathbf{F I}$ & 勻 & $\boldsymbol{M}$ \\
\hline XS-11B-LEVEOH & 51 & 为 & E1 & & 11 & 11 & wo & pos & & & & & & a1 & $\mathbf{P}$ \\
\hline
\end{tabular}




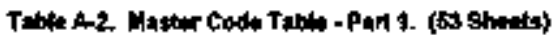

\begin{tabular}{|c|c|c|c|c|c|c|c|c|c|c|c|c|c|c|c|}
\hline 旧 & 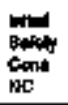 & 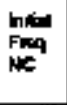 & 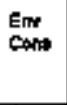 & $\tan$ & 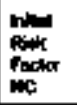 & 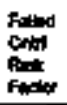 & Farmir & con & $\operatorname{Pap}_{\text {Aes }}$ & 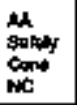 & Fich & $\underset{\text { Baplaty }}{\operatorname{loph}}$ & Find & 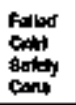 & $\begin{array}{l}\text { futad } \\
\text { Cownt } \\
\text { freq }\end{array}$ \\
\hline$x$ ticheried & $\$$ & $\mathbf{F 1}$ & E1 & & $\$$ & & T.tem & QOA & & & & & & & \\
\hline 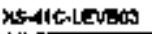 & s1 & $\mathbf{r}$ & EI & & 11 & 11 & T.ch & Dי⿻三丨 & & & & & & s1 & $\mathbf{F}$ \\
\hline 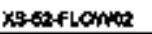 & $\$ 2$ & 6.3 & $\mathbf{q}$ & $\cos 20$ & 14 & 13 & $\mathrm{~T}, \mathrm{GN}$ & 600 & 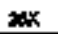 & 89 & $F$ & 91 & F\$ & 8 & $\mathbf{P 1}$ \\
\hline
\end{tabular}


HWF.8DWh-TR-784, Rer. 1

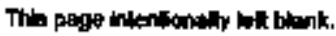




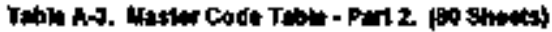

\begin{tabular}{|c|c|c|}
\hline D & 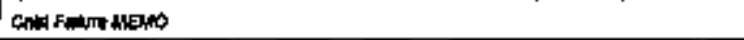 & 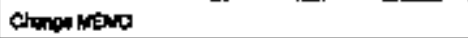 \\
\hline 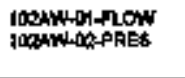 & & 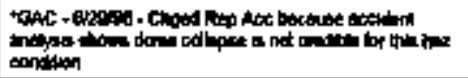 \\
\hline 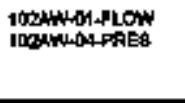 & 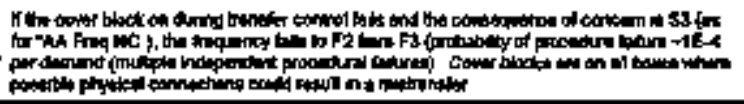 & 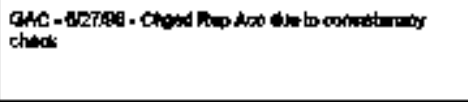 \\
\hline 10exhNOA-PLONOS & 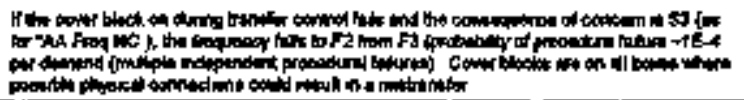 & 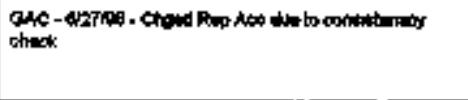 \\
\hline 10zAlyos fectroz & 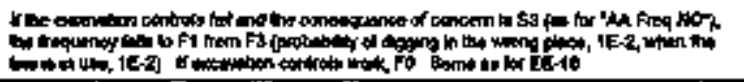 & 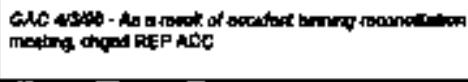 \\
\hline 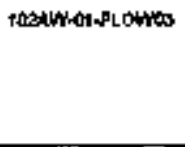 & 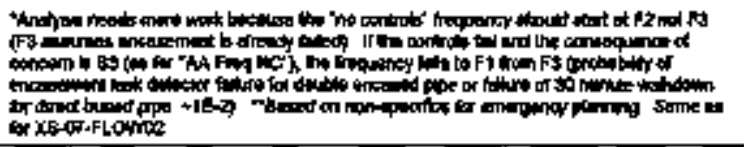 & \\
\hline 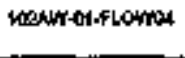 & 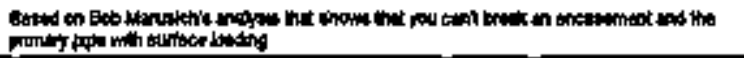 & \\
\hline 1004H-Difloums & 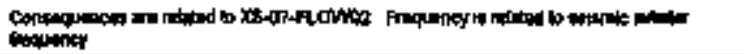 & \\
\hline 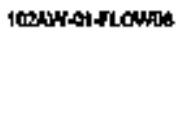 & 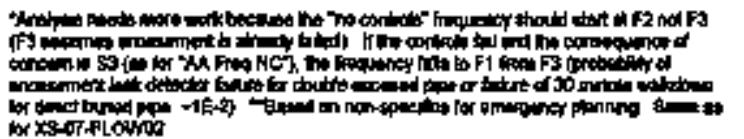 & \\
\hline
\end{tabular}




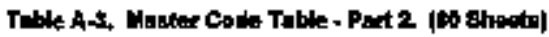

\begin{tabular}{|c|c|c|}
\hline 10 & 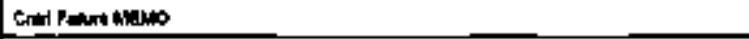 & entromeno \\
\hline 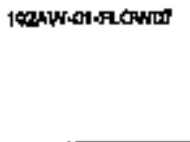 & 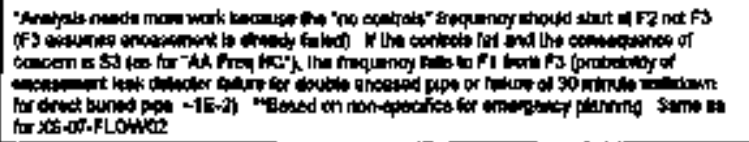 & \\
\hline 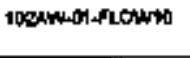 & bunded by wosefassor & 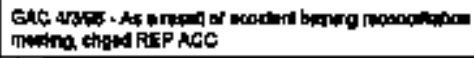 \\
\hline 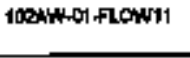 & 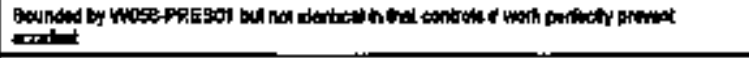 & 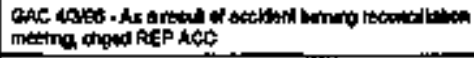 \\
\hline 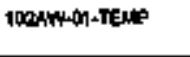 & 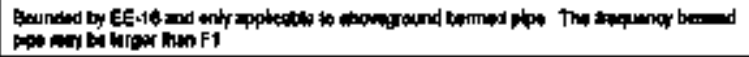 & \\
\hline NESANAT-TEKAPOS & Bourild by WOSBPRESOH & 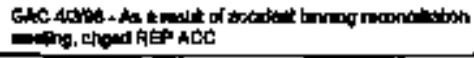 \\
\hline WOZMAOA-TEMPLSA & 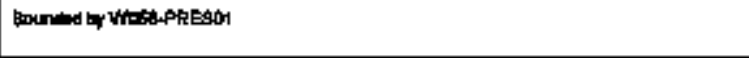 & 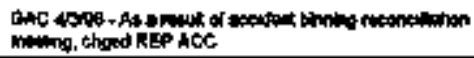 \\
\hline 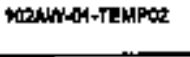 & 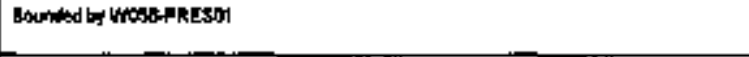 & 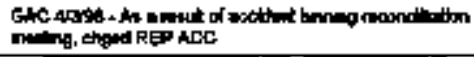 \\
\hline WO201N-DELEVE & 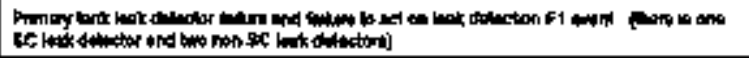 & \\
\hline \multicolumn{3}{|l|}{ 106nurter. IFined } \\
\hline \multicolumn{3}{|l|}{ 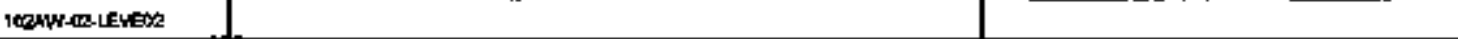 } \\
\hline 102AW-02-LESERS & 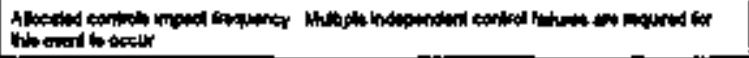 & \\
\hline 10QMW-GR-LELEA & 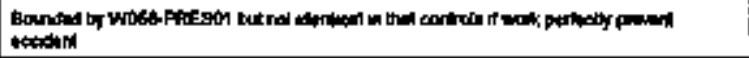 & 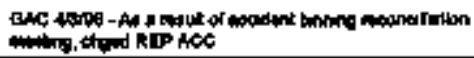 \\
\hline 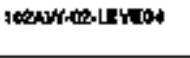 & 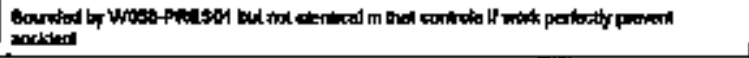 & 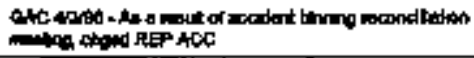 \\
\hline 10034h-cougreds & 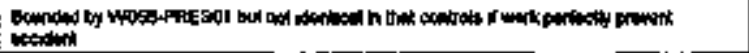 & \\
\hline
\end{tabular}




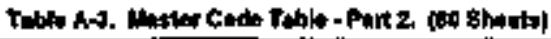

\begin{tabular}{|c|c|c|}
\hline ID & 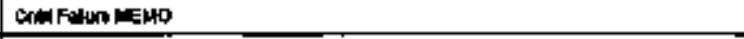 & 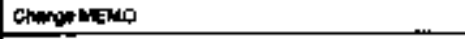 \\
\hline \multicolumn{3}{|l|}{ MESWW-GE-LEVEOE } \\
\hline 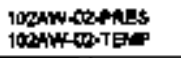 & 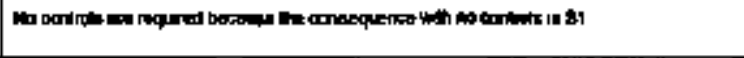 & \\
\hline \multicolumn{3}{|l|}{ 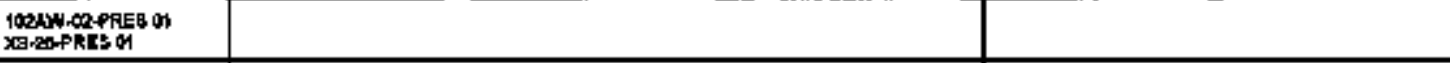 } \\
\hline 102AN oremesor & 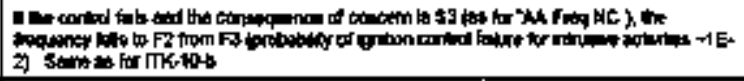 & 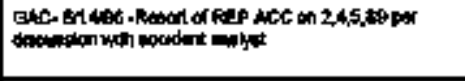 \\
\hline 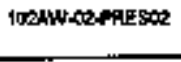 & 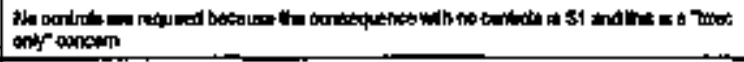 & \\
\hline 102An-00-PABO03 & 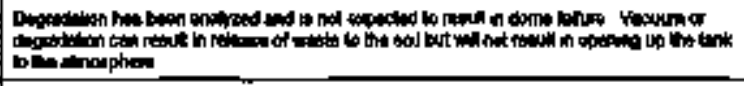 & 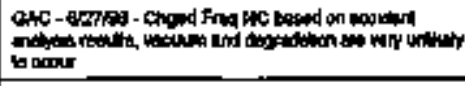 \\
\hline 102AW-OSPRE804 & 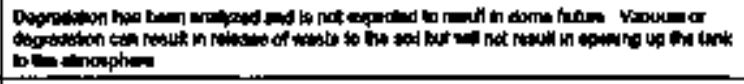 & 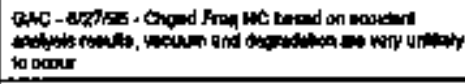 \\
\hline 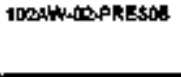 & 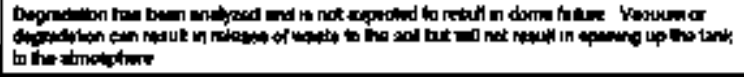 & 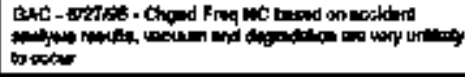 \\
\hline 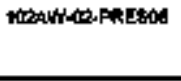 & 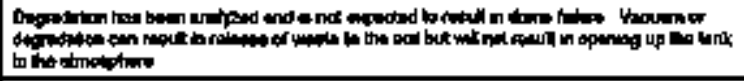 & 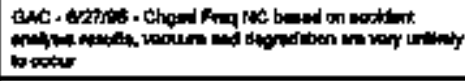 \\
\hline 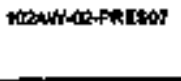 & 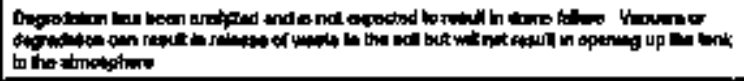 & 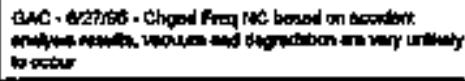 \\
\hline 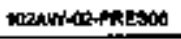 & 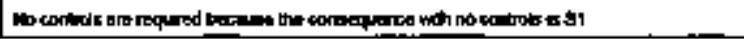 & \\
\hline $100 \mathrm{NW}-02 \mathrm{PFA}=90$ & 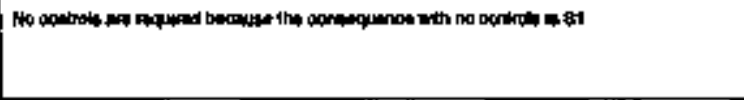 & 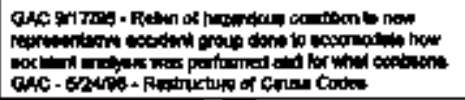 \\
\hline
\end{tabular}




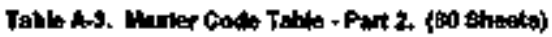

\begin{tabular}{|c|c|c|}
\hline 由 & 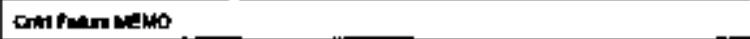 & Gongtions \\
\hline 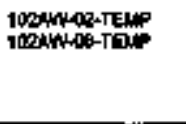 & 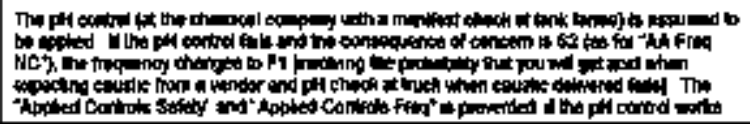 & 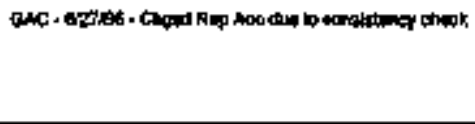 \\
\hline 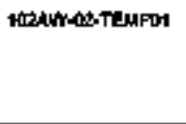 & 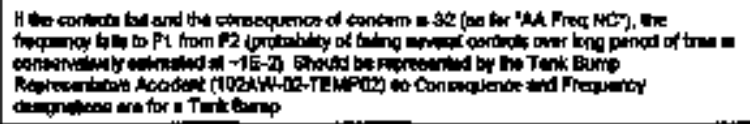 & \\
\hline 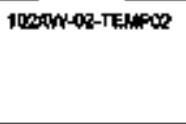 & 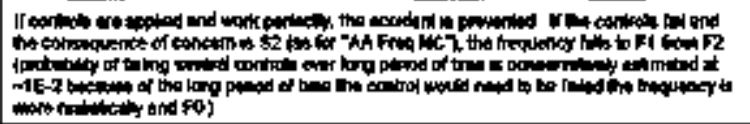 & 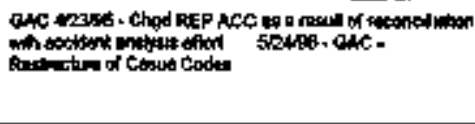 \\
\hline 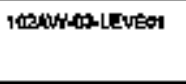 & 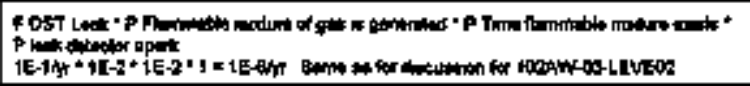 & 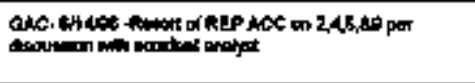 \\
\hline 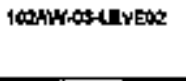 & 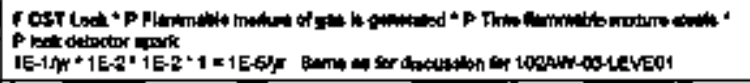 & 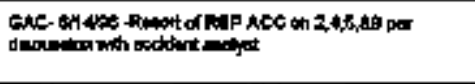 \\
\hline 16ank-os-centeos & 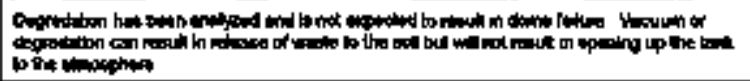 & 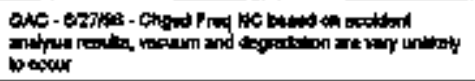 \\
\hline \multicolumn{3}{|l|}{ 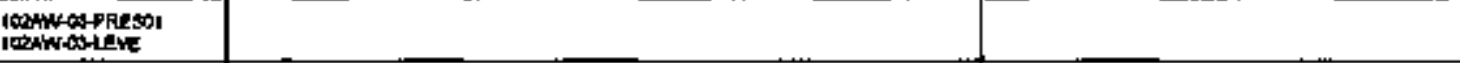 } \\
\hline \multicolumn{3}{|l|}{ 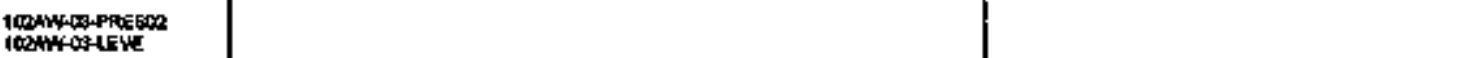 } \\
\hline 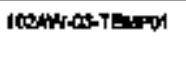 & 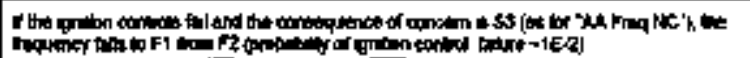 & 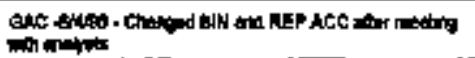 \\
\hline 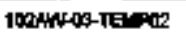 & 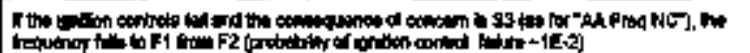 & \\
\hline
\end{tabular}




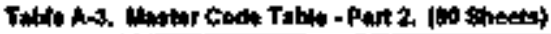

\begin{tabular}{|c|c|c|}
\hline סו & 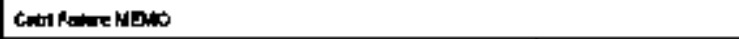 & Genqe MUN \\
\hline 102AW-ONLEEOI & 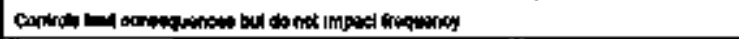 & \\
\hline 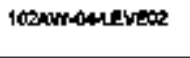 & 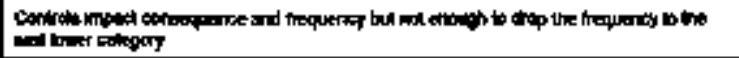 & \\
\hline 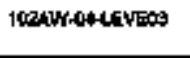 & 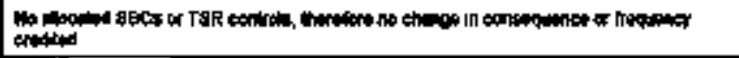 & \\
\hline 10stwhouterton & 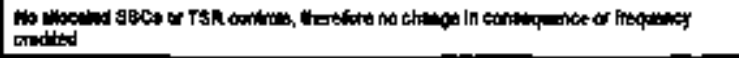 & \\
\hline 100OW-OMPRE 6 o1 & & 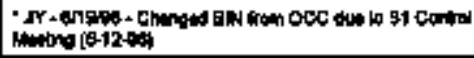 \\
\hline 1002AWUAMPNESO1 & 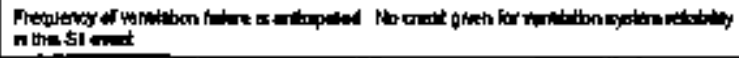 & \\
\hline 1020N4-04-PRE & 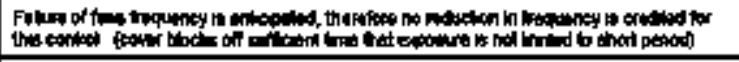 & \\
\hline \multicolumn{3}{|l|}{ 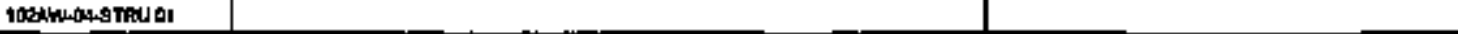 } \\
\hline \multicolumn{3}{|l|}{ 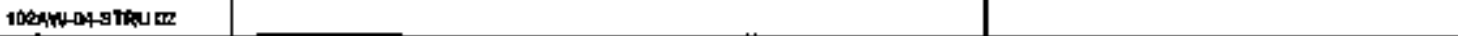 } \\
\hline 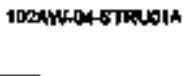 & 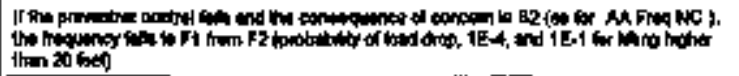 & \\
\hline \multicolumn{3}{|l|}{ 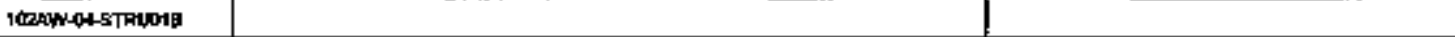 } \\
\hline 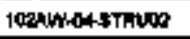 & 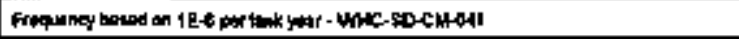 & \\
\hline 10034hodstivios & 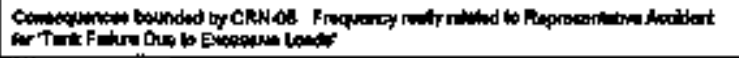 & \\
\hline 102AN-04-5ThIOH & 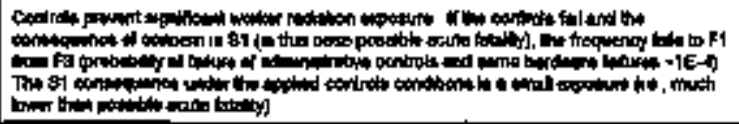 & 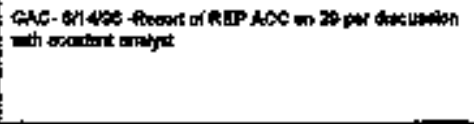 \\
\hline IGBAWMOA-BTRMPOS & 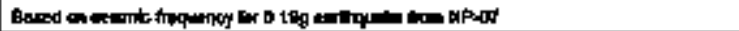 & \\
\hline
\end{tabular}




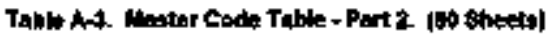

\begin{tabular}{|c|c|c|}
\hline סו & 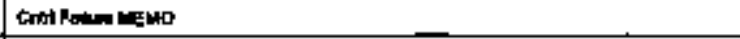 & CHE MEND \\
\hline 102AMGo-TEN & 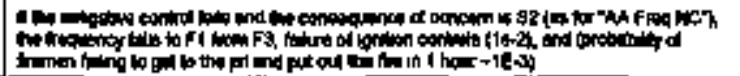 & \\
\hline \multicolumn{3}{|l|}{ 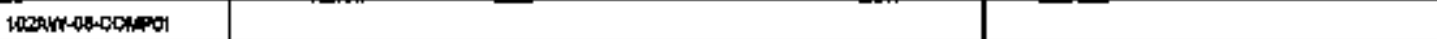 } \\
\hline 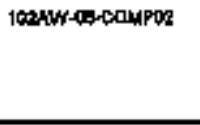 & 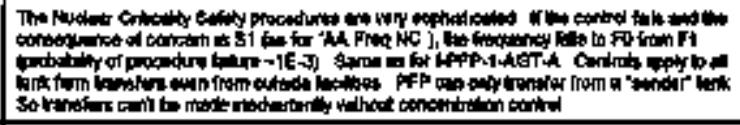 & \\
\hline 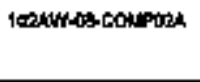 & 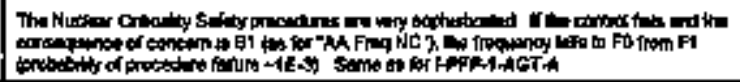 & \\
\hline 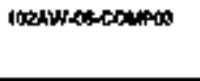 & 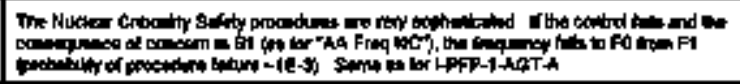 & \\
\hline 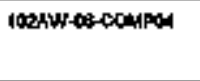 & 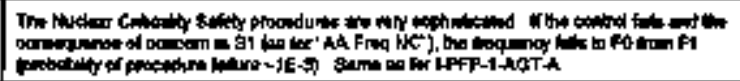 & \\
\hline 10024\%-08-schings & 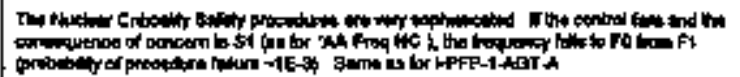 & \\
\hline 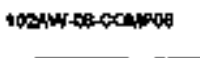 & 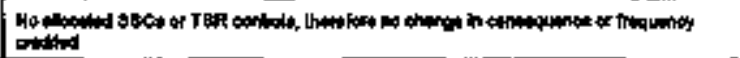 & 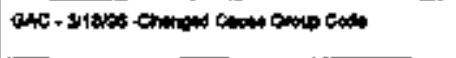 \\
\hline topantedecter & 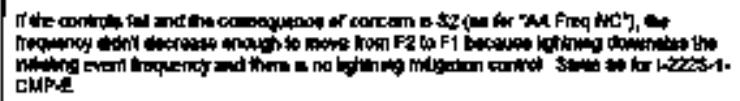 & 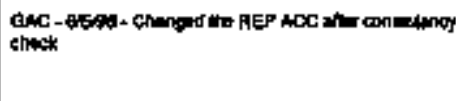 \\
\hline 102an-co-co4pas & 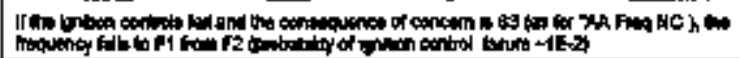 & \\
\hline 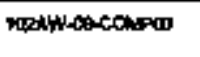 & 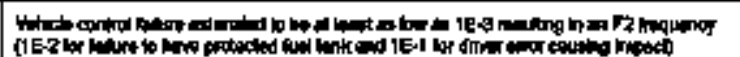 & \\
\hline
\end{tabular}




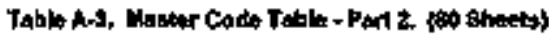

\begin{tabular}{|c|c|c|}
\hline I & Coti Fandin meno & להת \\
\hline 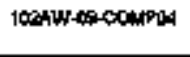 & 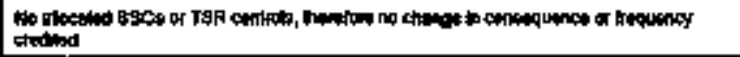 & \\
\hline 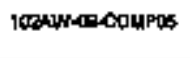 & 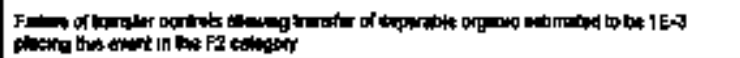 & \\
\hline 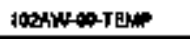 & 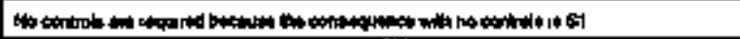 & \\
\hline 1004W-caterpor & 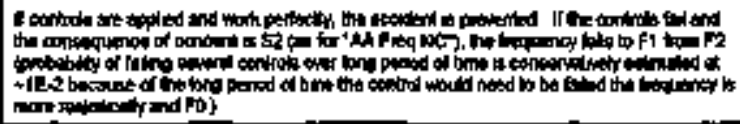 & 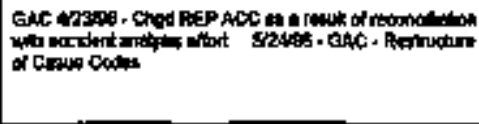 \\
\hline \multicolumn{3}{|l|}{ 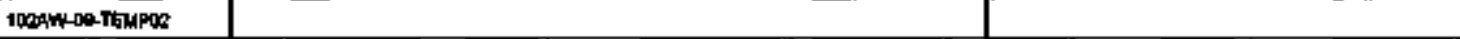 } \\
\hline 108ank-13rtow on & & 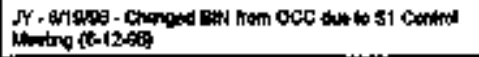 \\
\hline 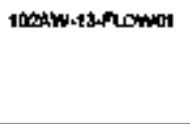 & 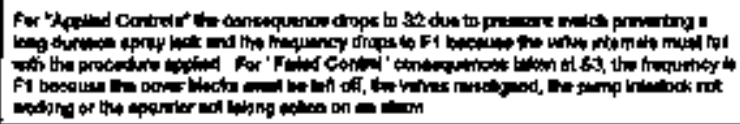 & \\
\hline 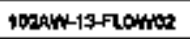 & 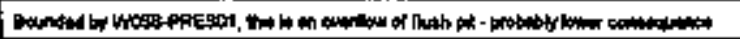 & \\
\hline 10250101 & 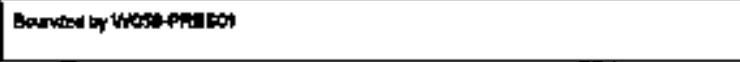 & 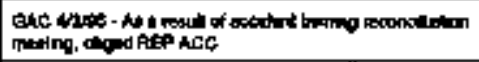 \\
\hline 1009016 & 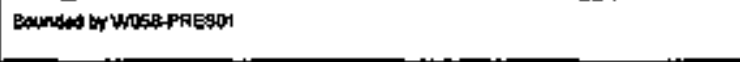 & 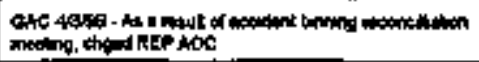 \\
\hline $\cos 2801$ bs & 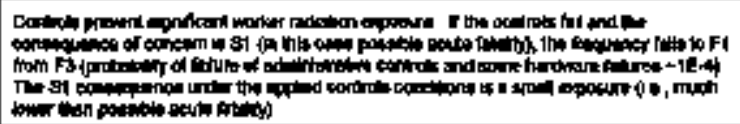 & 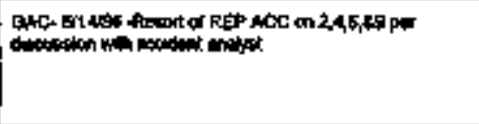 \\
\hline $1002-00201$ & 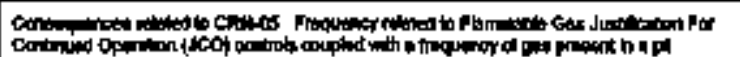 & \\
\hline
\end{tabular}




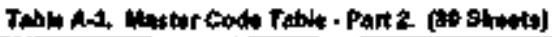

\begin{tabular}{|c|c|c|}
\hline ID & 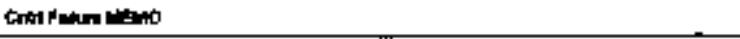 & 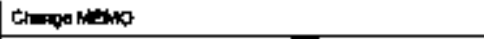 \\
\hline t0250 $\mathrm{p} 2$ & 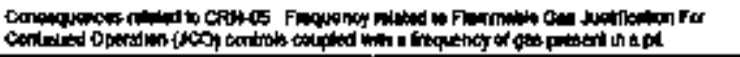 & \\
\hline NoRg-QR 吸 & 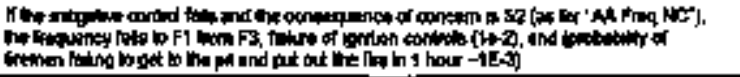 & 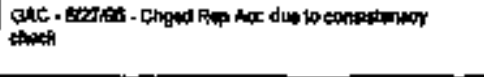 \\
\hline $1025-62 \mathrm{ds}$ & 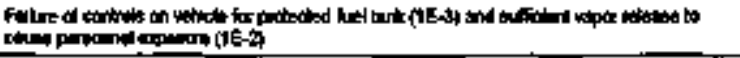 & \\
\hline $1025-036$ & 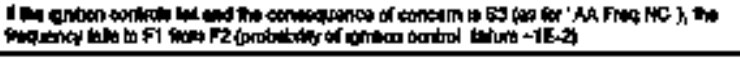 & \\
\hline $10 \cos 00 \mathrm{bat}$ & 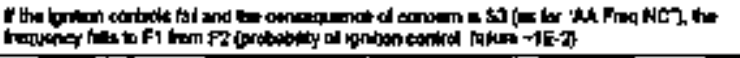 & 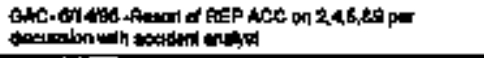 \\
\hline 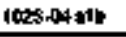 & & \\
\hline $1023.04=110$ & & \\
\hline 100004 & 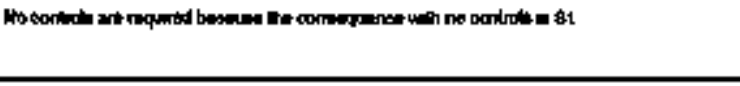 & 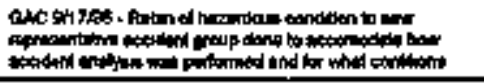 \\
\hline $1604 \mathrm{c}$ & 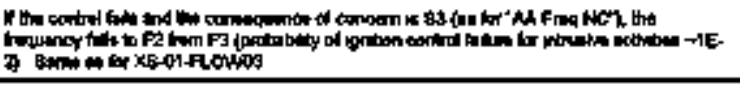 & \\
\hline 102500 क & 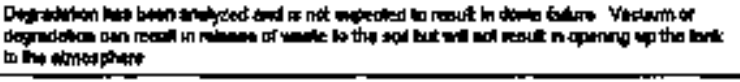 & 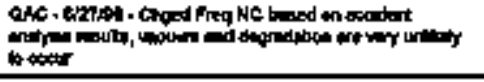 \\
\hline $1025+0.4616$ & 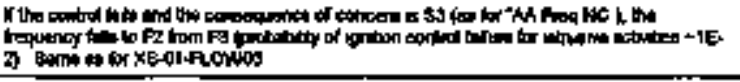 & \\
\hline $1025-040$ & & \\
\hline $1025-64$ at & & 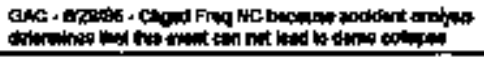 \\
\hline
\end{tabular}




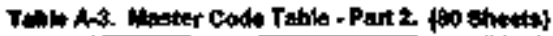

\begin{tabular}{|c|c|c|}
\hline$\omega$ & 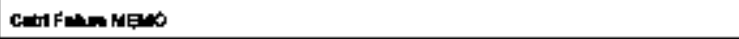 & 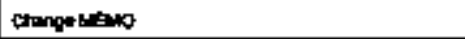 \\
\hline $1000-0404$ & 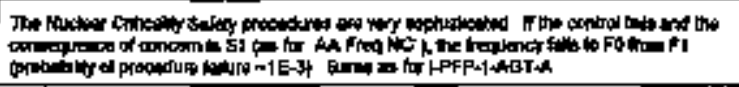 & \\
\hline 102905810 & 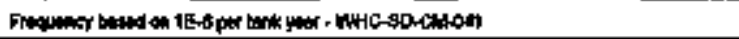 & \\
\hline $102=050114$ & 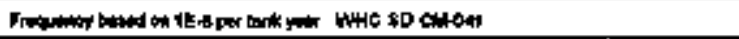 & \\
\hline \multicolumn{3}{|l|}{1002060} \\
\hline 1025) & 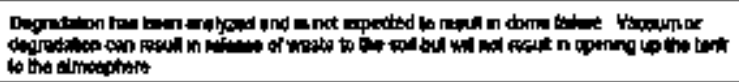 & 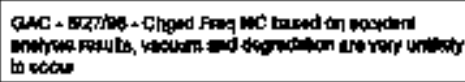 \\
\hline 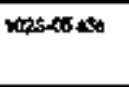 & 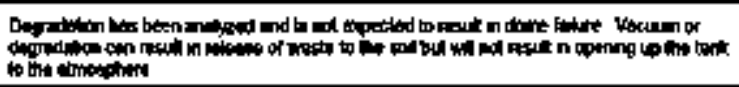 & 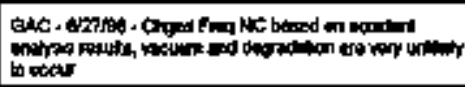 \\
\hline 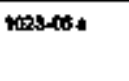 & 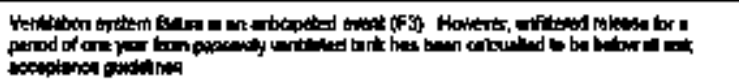 & 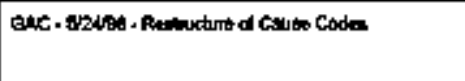 \\
\hline 1006-01A & 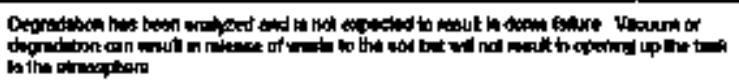 & 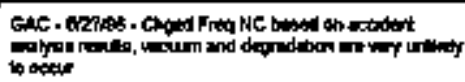 \\
\hline $1600-010$ & 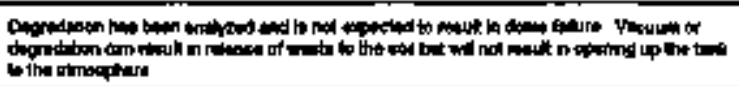 & 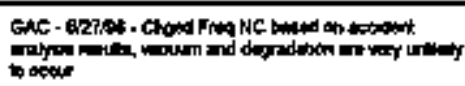 \\
\hline $1000-016$ & 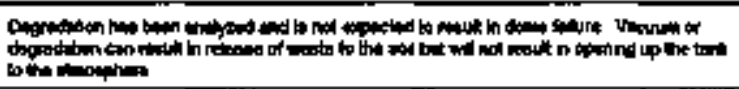 & 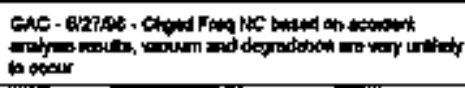 \\
\hline 100000 & 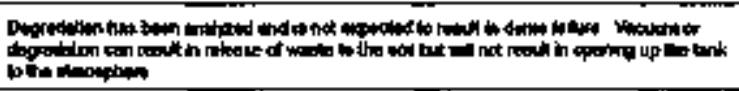 & 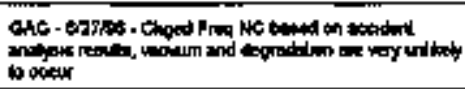 \\
\hline IOt:-O1E & 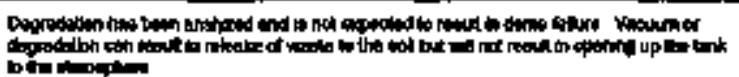 & \\
\hline
\end{tabular}




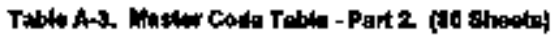

\begin{tabular}{|c|c|c|}
\hline $\mathbf{0}$ & Can Pan Mento & 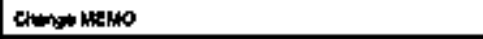 \\
\hline $100 x$ 에 $\mathrm{F}$ & 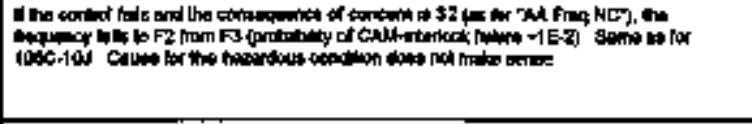 & 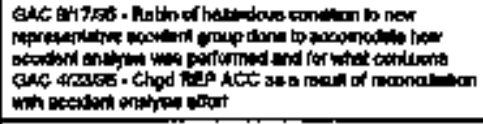 \\
\hline 1069.010 & 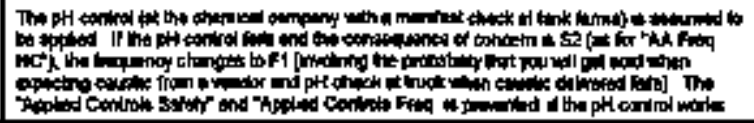 & \\
\hline 100C-01H & 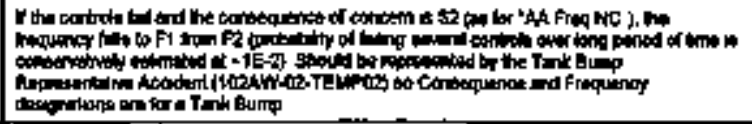 & 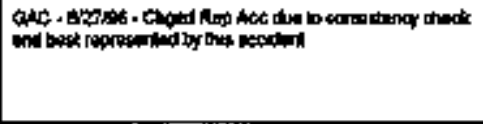 \\
\hline $1000-01$ & 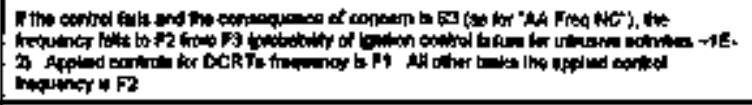 & \\
\hline 100e-01 & 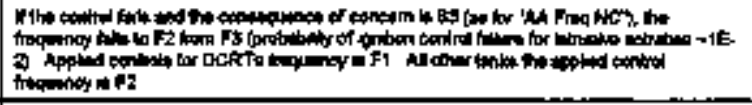 & \\
\hline $1000-0 \mathrm{kK}$ & 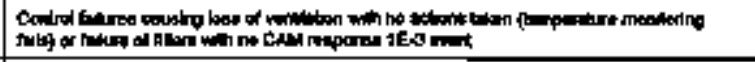 & \\
\hline $1000-914$ & 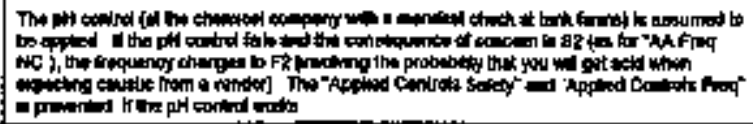 & \\
\hline $1000-0104$ & 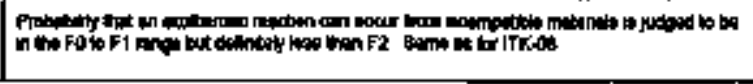 & 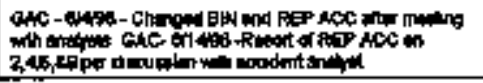 \\
\hline
\end{tabular}


Table Ans, Hastar Gode Table - Part 2, (800 theets)

\begin{tabular}{|c|c|c|}
\hline D & 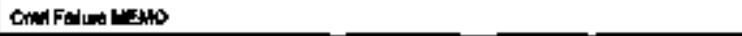 & 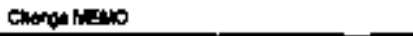 \\
\hline 1000-0In & 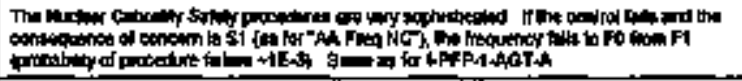 & \\
\hline \multicolumn{3}{|l|}{ 100C-01Ra } \\
\hline 100 -01t & 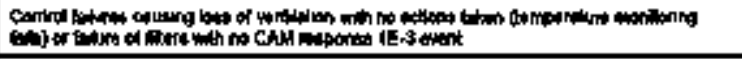 & \\
\hline vosiote. & 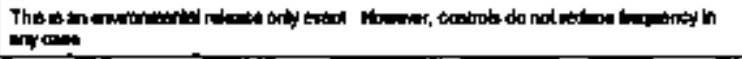 & \\
\hline $10 x$ 에 & 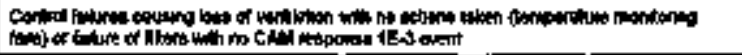 & \\
\hline 10tetentu & & \\
\hline \multicolumn{3}{|l|}{$10 \mathrm{xc}-01 \%$} \\
\hline $1090-01 x$ & 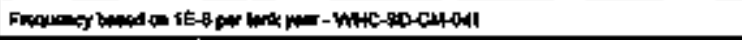 & \\
\hline צt & 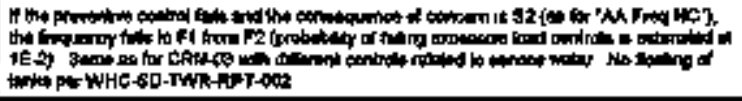 & \\
\hline 10000000 & 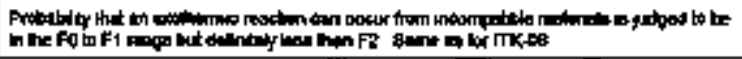 & \\
\hline $1090-\infty$ & 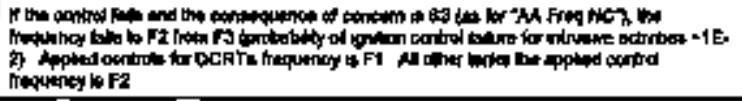 & \\
\hline $100 c-02 x$ & 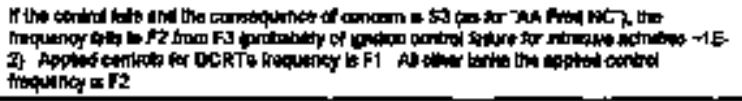 & \\
\hline vocola & 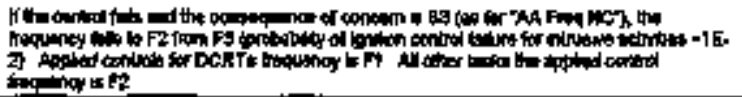 & 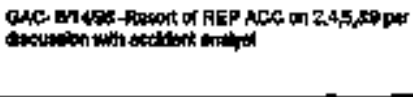 \\
\hline
\end{tabular}




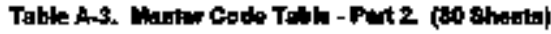

\begin{tabular}{|c|c|c|}
\hline 10 & 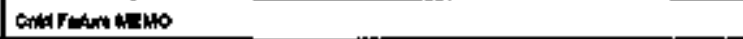 & Sherpo kewo \\
\hline $100 x-040$ & 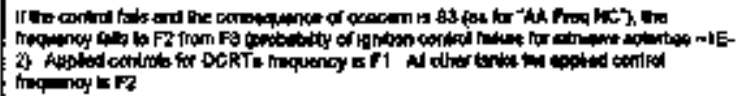 & 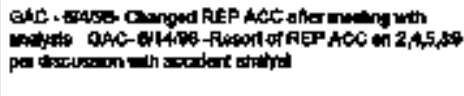 \\
\hline I0X-04: & 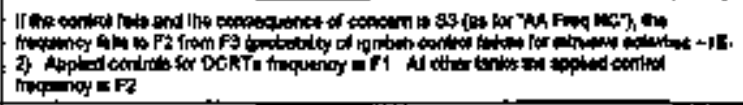 & 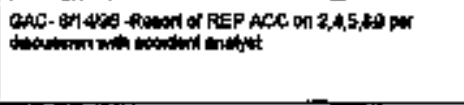 \\
\hline $1096-034$ & 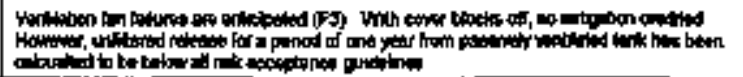 & \\
\hline \multicolumn{3}{|l|}{1000469} \\
\hline $1000-090$ & 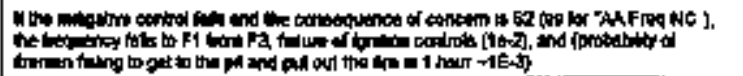 & \\
\hline $100 \mathrm{C}-0 \mathrm{BF}$ & 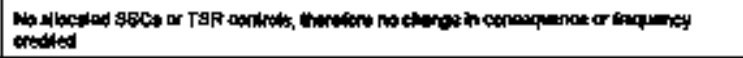 & \\
\hline 1000000 & 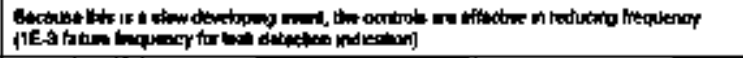 & \\
\hline $1000-09 \mathrm{H}$ & 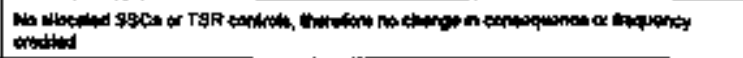 & \\
\hline \multicolumn{3}{|l|}{105000} \\
\hline $1000-09 \mathrm{~K}$ & 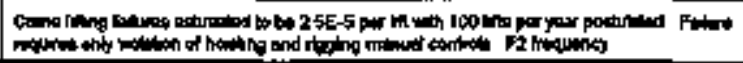 & 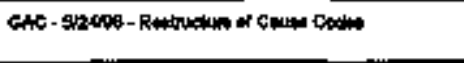 \\
\hline 10000 & 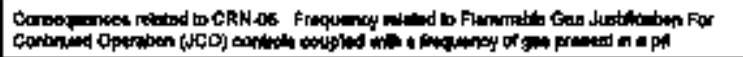 & \\
\hline \multicolumn{3}{|l|}{108 - } \\
\hline \multicolumn{3}{|l|}{$1000-100$} \\
\hline 1000 & & \\
\hline
\end{tabular}




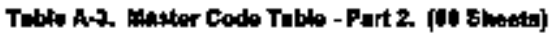

\begin{tabular}{|c|c|c|}
\hline 10 & Cour Fatro bento & Chomentero \\
\hline \multicolumn{3}{|l|}{$1000-100$} \\
\hline \multicolumn{3}{|l|}{ 1090-10e } \\
\hline $100 x-10 F$ & 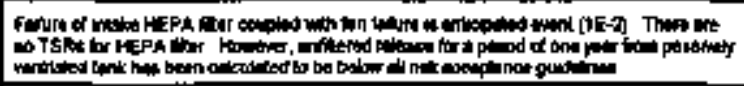 & \\
\hline 109-10H & & 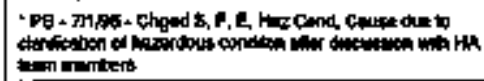 \\
\hline $1006-16$ & 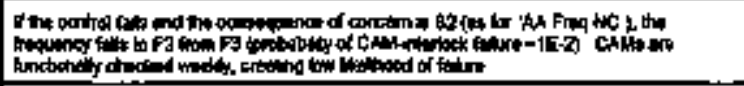 & \\
\hline ขoce-10 $x$ & 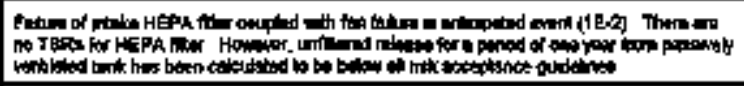 & 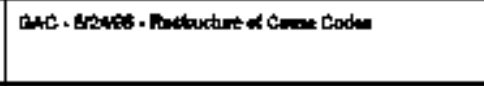 \\
\hline vosc-10t & 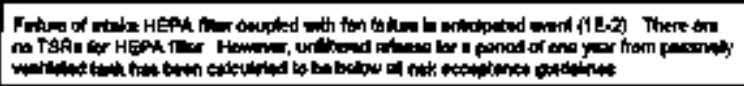 & \\
\hline $1000-114$ & 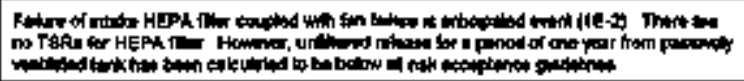 & \\
\hline \multicolumn{3}{|l|}{$100<-110$} \\
\hline \multicolumn{3}{|l|}{$1000-11 c$} \\
\hline \multicolumn{3}{|l|}{$109 x-110$} \\
\hline 100 -11E & 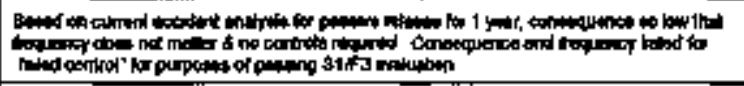 & 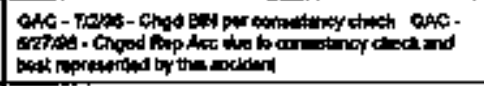 \\
\hline \multicolumn{3}{|l|}{$1006-11 F$} \\
\hline IDocestis & 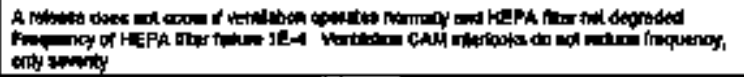 & 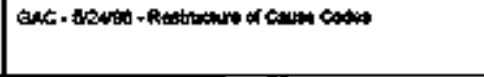 \\
\hline
\end{tabular}




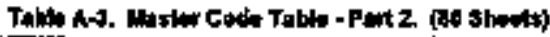

\begin{tabular}{|c|c|c|}
\hline 10 & 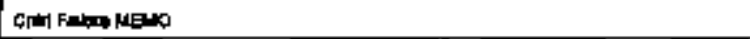 & 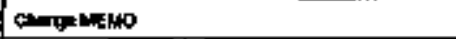 \\
\hline 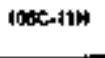 & 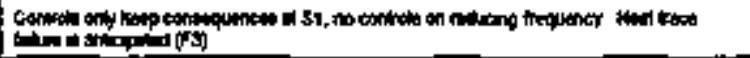 & \\
\hline $1008-111$ & 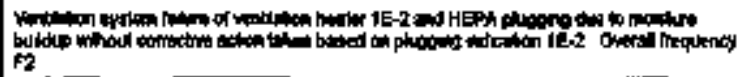 & 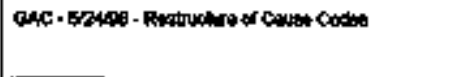 \\
\hline $1000-[1 \downarrow$ & 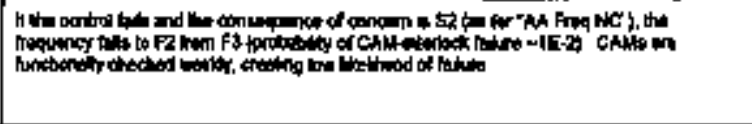 & 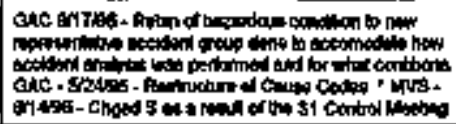 \\
\hline $1000-11 k$ & 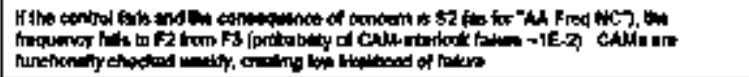 & \\
\hline $\operatorname{cosec-116}$ & 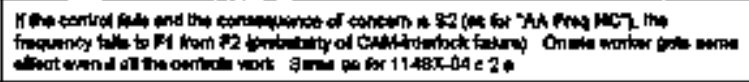 & 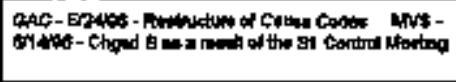 \\
\hline 2006-11 & 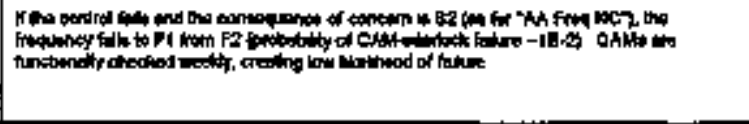 & 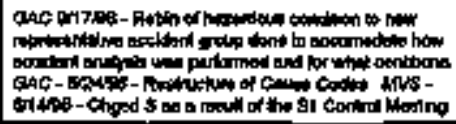 \\
\hline 1000-194 & 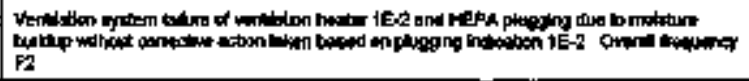 & 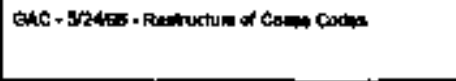 \\
\hline $100 t-110$ & 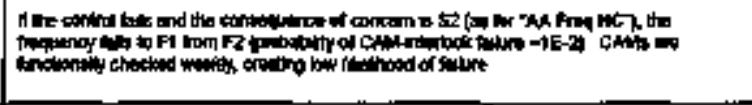 & 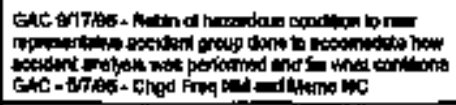 \\
\hline $1006-12 A$ & 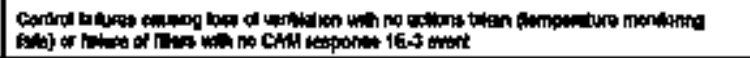 & \\
\hline $16 x-128$ & 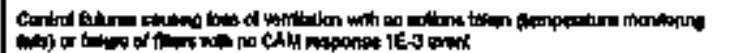 & \\
\hline
\end{tabular}




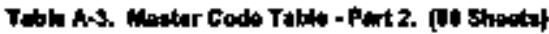

\begin{tabular}{|c|c|c|}
\hline ID & 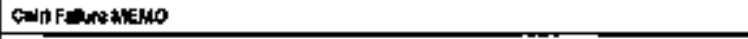 & Crencena \\
\hline $10 \Delta x-12 C$ & 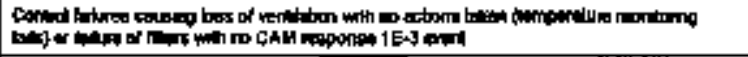 & \\
\hline $1000-120$ & 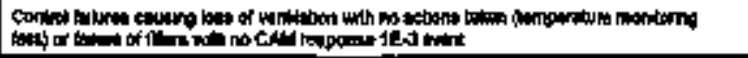 & \\
\hline 10ECATF & 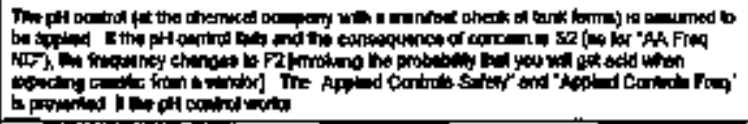 & 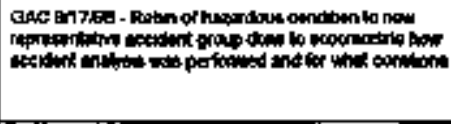 \\
\hline \multicolumn{3}{|l|}{$1000-120$} \\
\hline $100 \times 213 x$ & 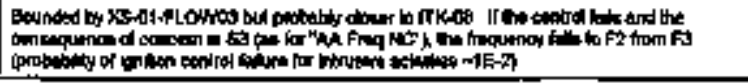 & \\
\hline \multicolumn{3}{|l|}{$\operatorname{coc}-13$} \\
\hline 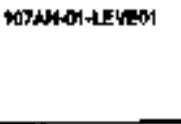 & 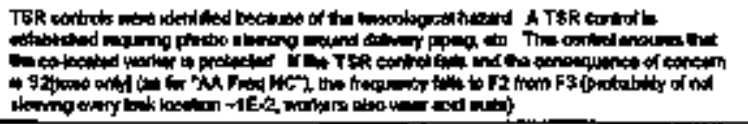 & \\
\hline 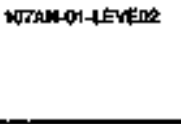 & 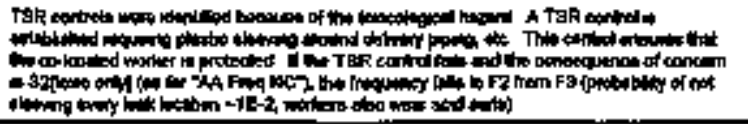 & \\
\hline IOTANHCA-LETEA & 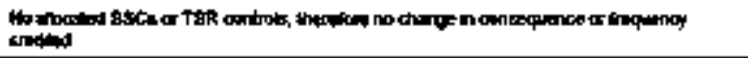 & \\
\hline \multicolumn{3}{|l|}{ A07AHOA-IEMP O1 } \\
\hline 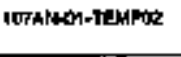 & 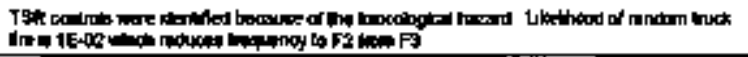 & 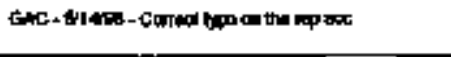 \\
\hline
\end{tabular}




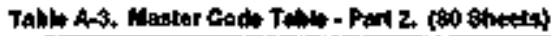

\begin{tabular}{|c|c|c|}
\hline 它 & 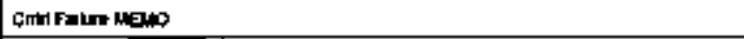 & Gange rexpr \\
\hline 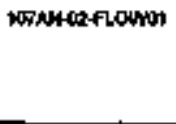 & 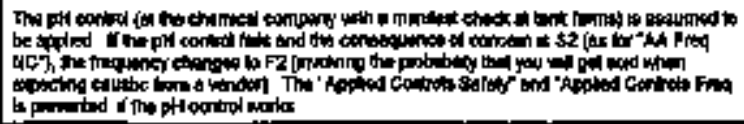 & 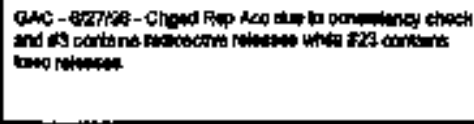 \\
\hline 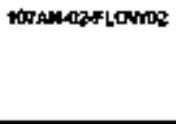 & 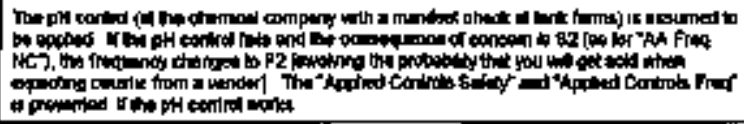 & 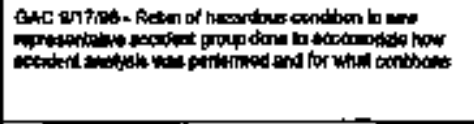 \\
\hline \multicolumn{3}{|l|}{ 107ANOEFLOCHAS } \\
\hline 107AWMBPEES & 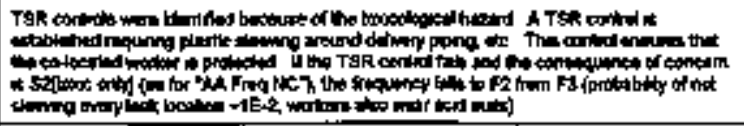 & \\
\hline 107ANHCOPFEBOZ & 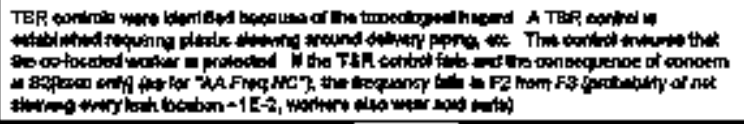 & \\
\hline \multicolumn{3}{|l|}{ 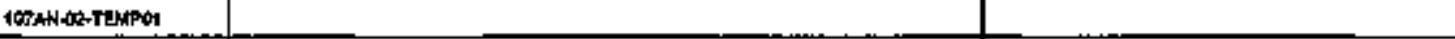 } \\
\hline IOSANABRFLON & 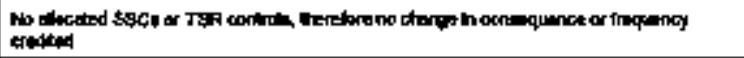 & \\
\hline $1145 x+00$ & 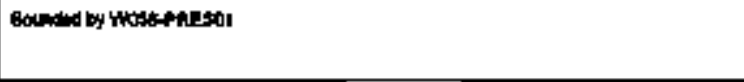 & 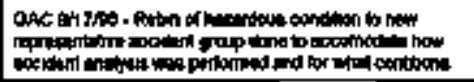 \\
\hline 114sicont ita & 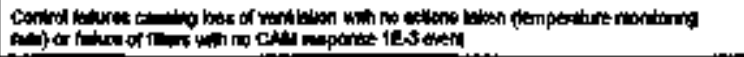 & \\
\hline $1145 x-001010$ & 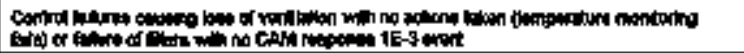 & \\
\hline
\end{tabular}




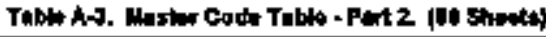

\begin{tabular}{|c|c|c|}
\hline 9 & 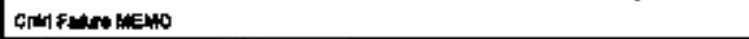 & Cripnop kakr \\
\hline $1149 \times 00132$ & 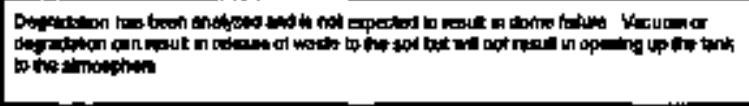 & 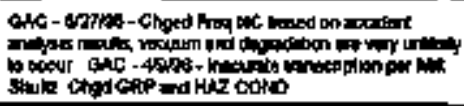 \\
\hline $1148 x-0 f$ og & 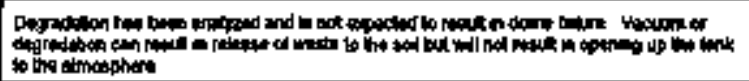 & \\
\hline $114 \sin x+04=4$ & 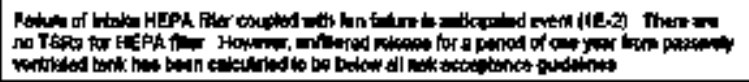 & 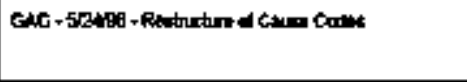 \\
\hline $1445 \times 100$ b 1 - 1 & 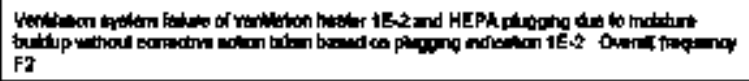 & \\
\hline $1145 x \cos$ b $1 \leqslant 2$ & 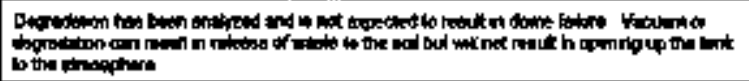 & \\
\hline \multicolumn{3}{|l|}{$5145 \times 010103$} \\
\hline $1145 \times 046$ i b $1=$ & 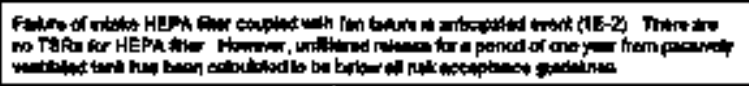 & \\
\hline $1145 \times 04616.10$ & 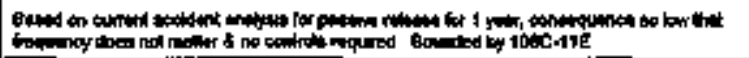 & \\
\hline $1148 \times 04 \mathrm{~b} I \mathrm{bm}$ & 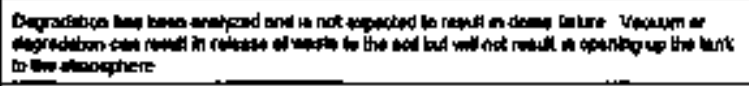 & \\
\hline \multicolumn{3}{|l|}{ 1: $145 x-0401$ b $7 \mathrm{~h}$} \\
\hline \multicolumn{3}{|l|}{$114 \times 600$ b 1 b s } \\
\hline \multicolumn{3}{|l|}{$116 x$ and b 1 b t } \\
\hline $1149 x-04$ b 1 o I & 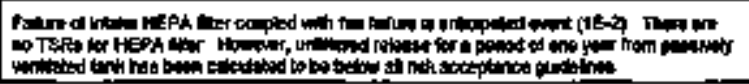 & 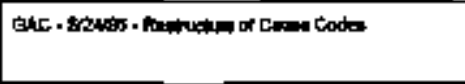 \\
\hline
\end{tabular}




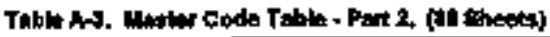

\begin{tabular}{|c|c|c|}
\hline 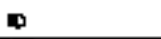 & 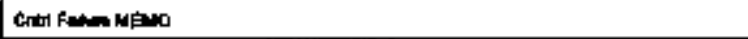 & Crunge \\
\hline $1145 x-0<b \mid<2$ & 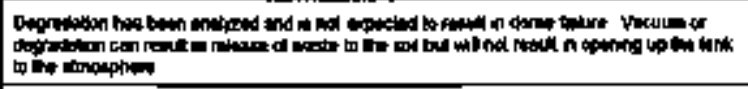 & 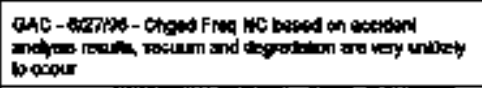 \\
\hline \multicolumn{3}{|l|}{$1148 K-0401$ \& 9} \\
\hline 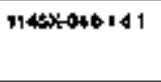 & 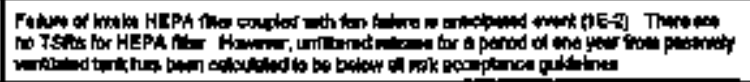 & \\
\hline $11493-0401$ \& 2 & 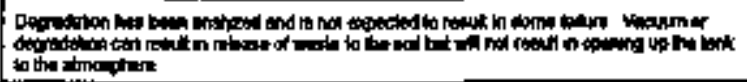 & \\
\hline \multicolumn{3}{|l|}{$11+8 x-04 b 103$} \\
\hline 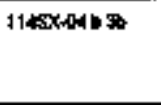 & 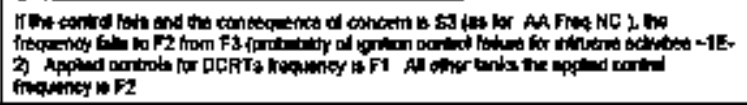 & 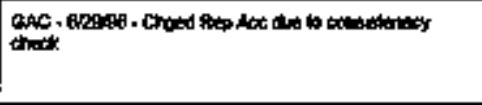 \\
\hline 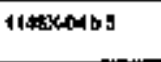 & 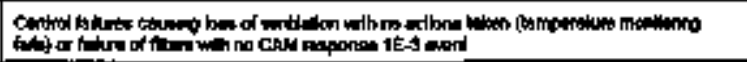 & \\
\hline $11 \operatorname{seg} x-04=1$ & 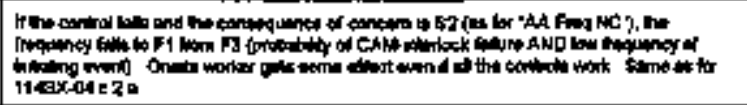 & 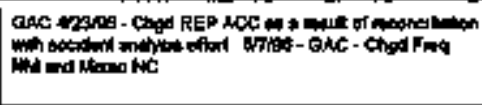 \\
\hline $11+4 x-04<20$ & 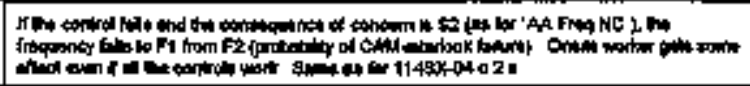 & 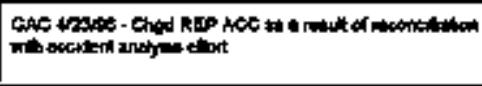 \\
\hline $1446 x-04$ t $2 b$ & 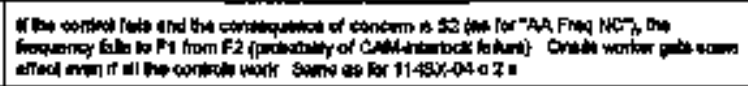 & 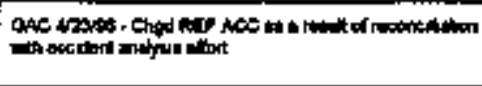 \\
\hline $114 \$ 5-0 d<26$ & 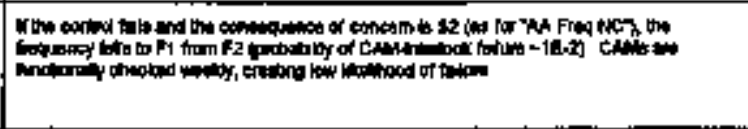 & 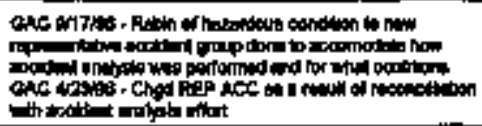 \\
\hline
\end{tabular}




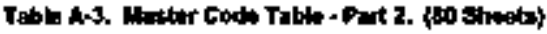

\begin{tabular}{|c|c|c|}
\hline $\mathbf{0}$ & 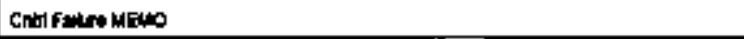 & 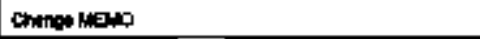 \\
\hline $114560 \mathrm{c}$ < 24 & 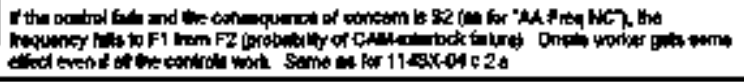 & 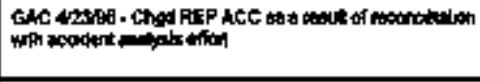 \\
\hline $1146 \times 606$ b 1 & 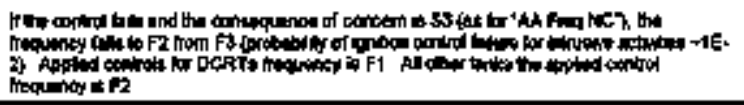 & \\
\hline $1146 \times 606$ t 2 & & 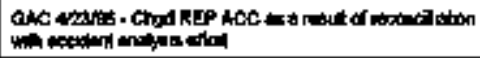 \\
\hline $1145 \times 605 \mathrm{bs}$ & 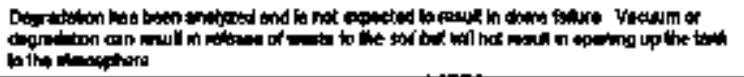 & 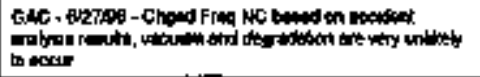 \\
\hline 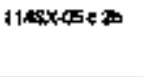 & 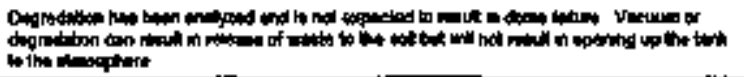 & 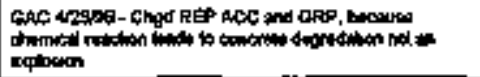 \\
\hline 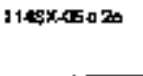 & 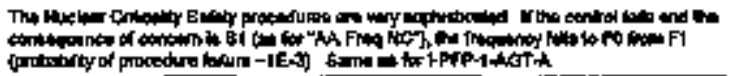 & \\
\hline 2148x-ab d & 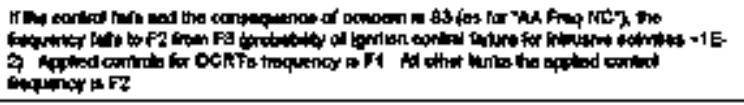 & 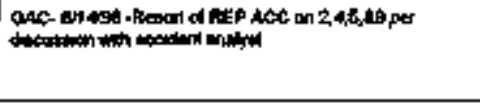 \\
\hline 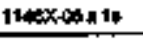 & 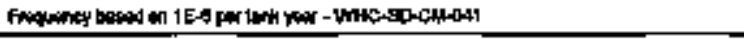 & \\
\hline 114sticosa ib & 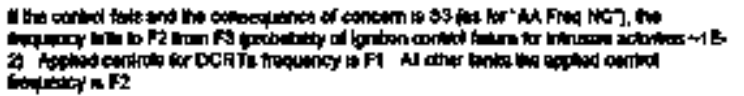 & \\
\hline \multicolumn{3}{|l|}{$1146 \times-00=2$} \\
\hline $1149 X_{-100}+3$ & 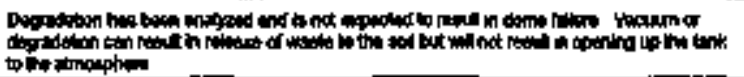 & 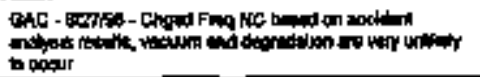 \\
\hline
\end{tabular}




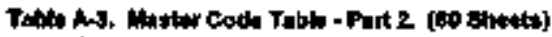

\begin{tabular}{|c|c|c|}
\hline ID & 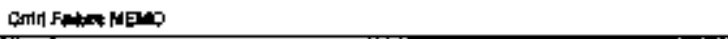 & Chengengen \\
\hline $1148 x-\infty$ & & 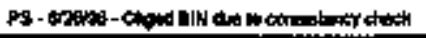 \\
\hline zodikardin & 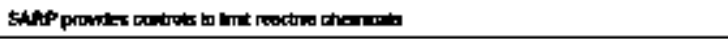 & 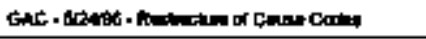 \\
\hline 204AF-01 B & 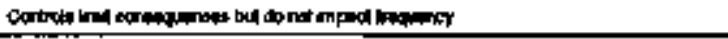 & 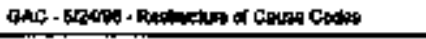 \\
\hline 3omanalc & 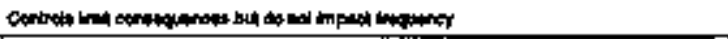 & 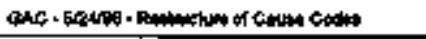 \\
\hline 304R-DND & 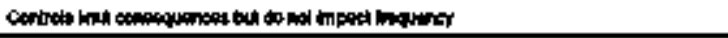 & \\
\hline \multicolumn{3}{|l|}{ xaminare } \\
\hline \multicolumn{3}{|l|}{ ZuAk:01F } \\
\hline \multicolumn{3}{|l|}{ SOART-010 } \\
\hline XOR-014 & 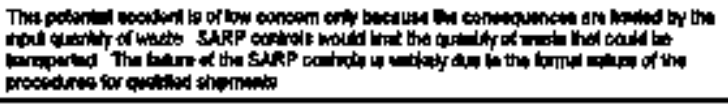 & 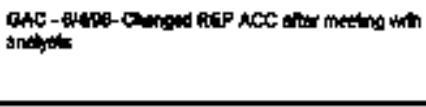 \\
\hline 20uspoll & 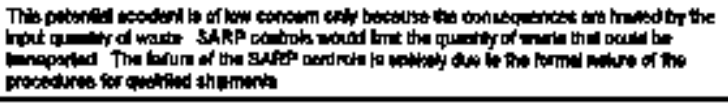 & \\
\hline bunsors & 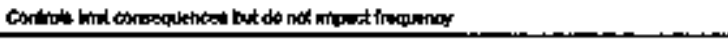 & \\
\hline mathaton & 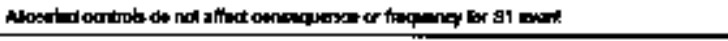 & \\
\hline 204AR-003 & 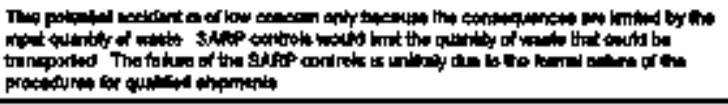 & \\
\hline 2044k-06is & 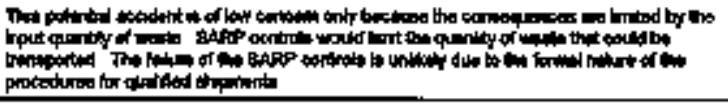 & \\
\hline ZOARAoec & & 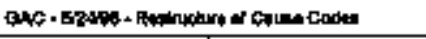 \\
\hline ZUAR.0RQ & & \\
\hline
\end{tabular}




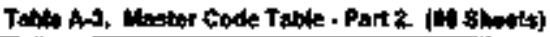

\begin{tabular}{|c|c|c|}
\hline ID & 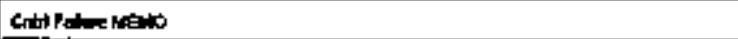 & Chenge Mteres \\
\hline SOAFR-DEE & 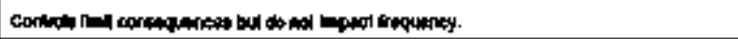 & \\
\hline $\tan R \cdot \cos$ & 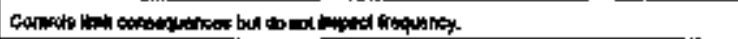 & \\
\hline 2004R-004 & 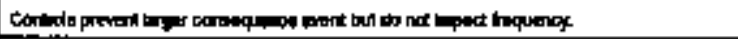 & \\
\hline zonfindose & 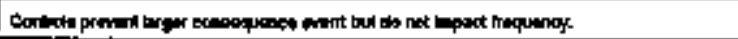 & \\
\hline \multicolumn{3}{|l|}{ 204AR-03C } \\
\hline 204R-104h & 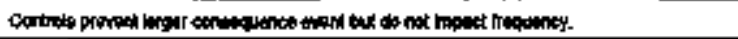 & \\
\hline Dounch & 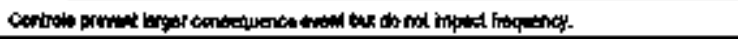 & \\
\hline MOARACE & 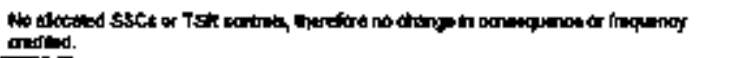 & \\
\hline 20ARRAT & 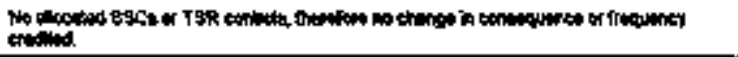 & 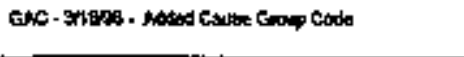 \\
\hline 20uafioen & 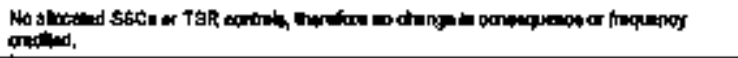 & 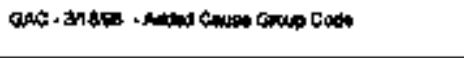 \\
\hline \multicolumn{3}{|l|}{ zanters } \\
\hline \multicolumn{3}{|l|}{ zodikesh } \\
\hline 2004istats & 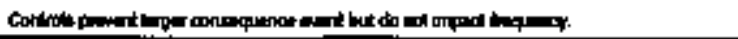 & \\
\hline \multicolumn{3}{|l|}{$\cos R \cos$} \\
\hline zontengesh & 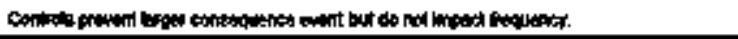 & \\
\hline $\cos 2006$ & 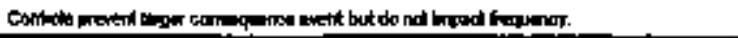 & \\
\hline TOAAR-0\% & 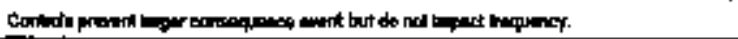 & \\
\hline 204AR-6Th & 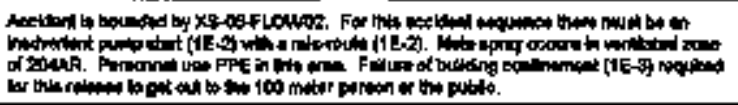 & 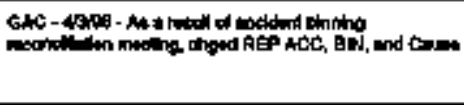 \\
\hline 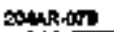 & 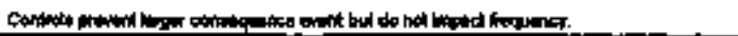 & \\
\hline
\end{tabular}




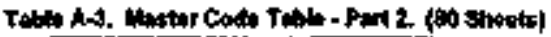

\begin{tabular}{|c|c|c|}
\hline $\mathbf{0}$ & 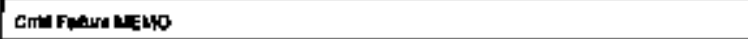 & Ching ravo \\
\hline \multicolumn{3}{|l|}{ 204AR-OTC } \\
\hline antectan & 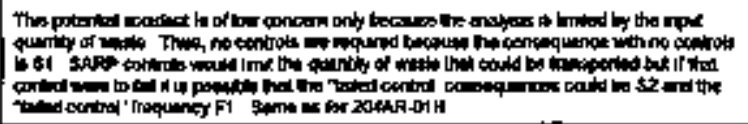 & 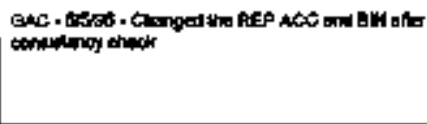 \\
\hline 204artere & 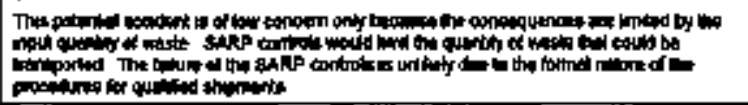 & 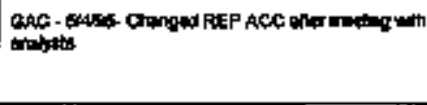 \\
\hline $204 \mathrm{R}-00 \mathrm{C}$ & 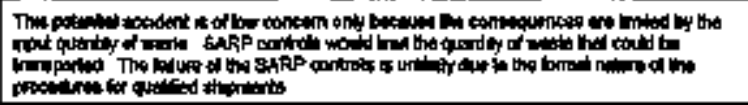 & \\
\hline 2044RAOS & 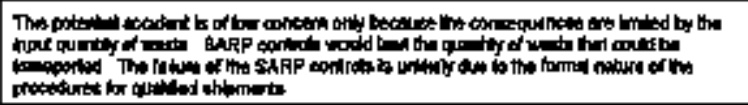 & \\
\hline \multicolumn{3}{|l|}{ XunRuse } \\
\hline Toundes & 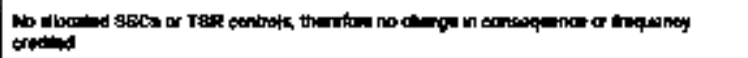 & \\
\hline \multicolumn{3}{|l|}{ Xo4hr-40G } \\
\hline \multicolumn{3}{|l|}{ SOARAOH } \\
\hline TDER-11A & 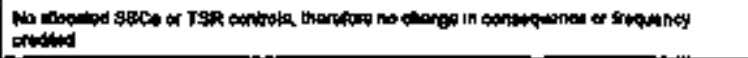 & \\
\hline DOWR-118 & 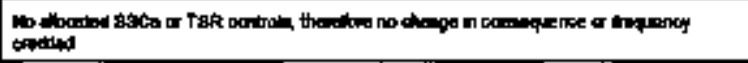 & \\
\hline $2040-416$ & 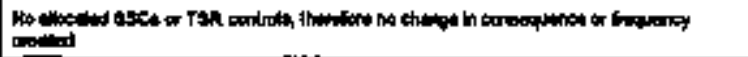 & \\
\hline 204AR & 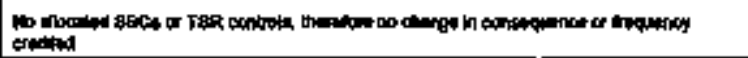 & \\
\hline
\end{tabular}


Tobla A-3. Warker Codt Tah' - Furt 2. (100 Etheetsy

\begin{tabular}{|c|c|c|}
\hline 10 & Com Tasum wemo & Charesticho \\
\hline Mear-11E & 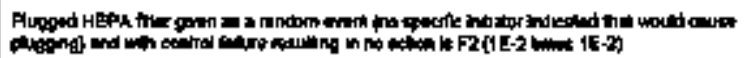 & \\
\hline 204kpi11 & 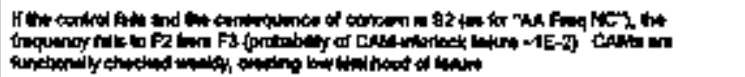 & \\
\hline $215 \mid m-01$ & 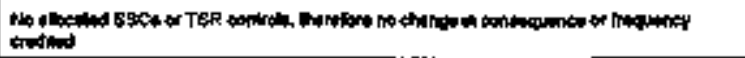 & \\
\hline $213 w-6$ & 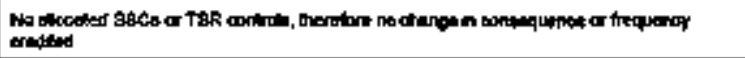 & \\
\hline \multicolumn{3}{|l|}{ 213 Whas } \\
\hline \multicolumn{3}{|l|}{ 2taw was } \\
\hline \multicolumn{3}{|l|}{263 was } \\
\hline 213 wo & & 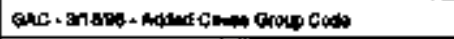 \\
\hline $213 \%-0 T$ & & 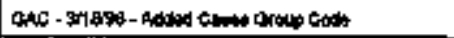 \\
\hline $213 m-00$ & & 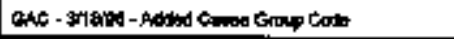 \\
\hline 213 wed & 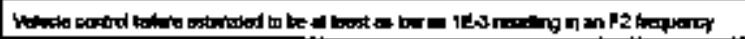 & \\
\hline 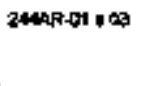 & & 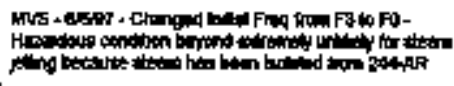 \\
\hline 244kRol b01 & & 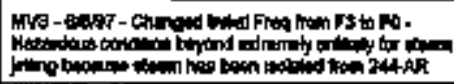 \\
\hline 2HARtol bot2 & & 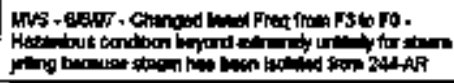 \\
\hline 244AR-01 bes & & 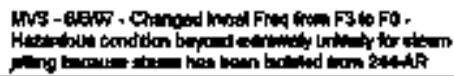 \\
\hline
\end{tabular}


Tald M-3. Hoater Code Toble - Pat 2. (30 shootol)

\begin{tabular}{|c|c|c|}
\hline 10 & 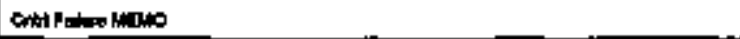 & Chengentwo \\
\hline 240ARAB bod & 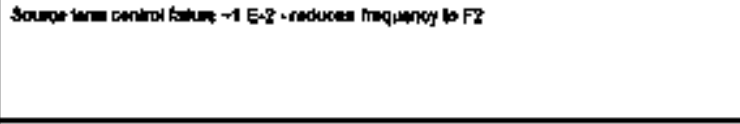 & 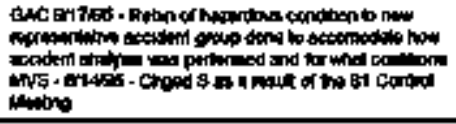 \\
\hline $2400 k-01<01$ & 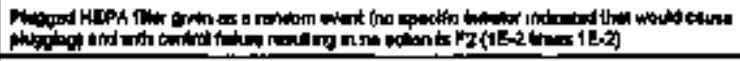 & \\
\hline $244 \mathrm{~F}-01+00$ & 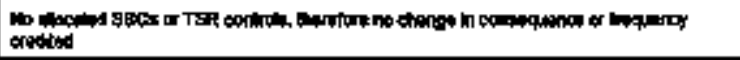 & \\
\hline 24under ed & 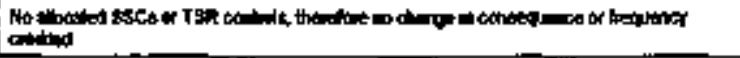 & \\
\hline \multicolumn{3}{|l|}{$2444001 \div 07$} \\
\hline 240Ras e od & 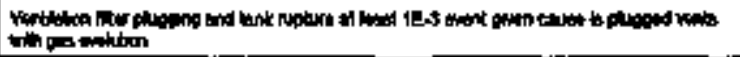 & \\
\hline 24Atatos dod & 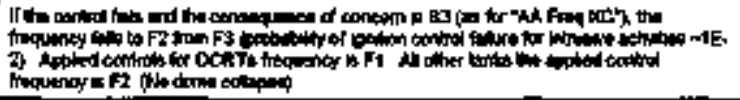 & 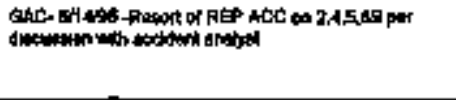 \\
\hline $24 A R-910$ & 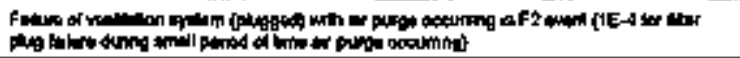 & \\
\hline 2AdRol t I1 & & 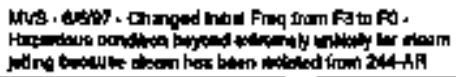 \\
\hline \multicolumn{3}{|l|}{ 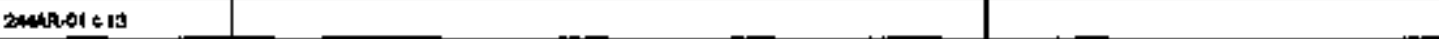 } \\
\hline $\operatorname{2an} p-\sin =0$ & 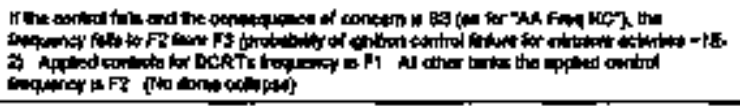 & 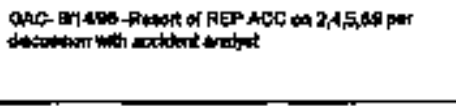 \\
\hline \multicolumn{3}{|l|}{$24+16-02000$} \\
\hline 24kk-02ubs & & \\
\hline
\end{tabular}


Table A-3. Master Code Table - Part 2 (40 Shets)

\begin{tabular}{|c|c|c|}
\hline 10 & 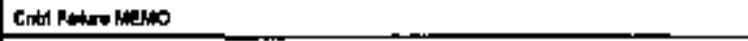 & Grenge kato \\
\hline $24 M R-02=6$ & 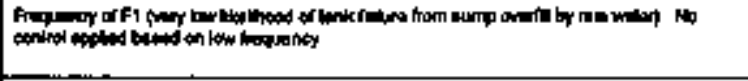 & 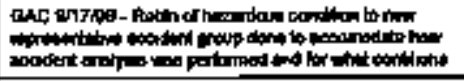 \\
\hline $2+4 A R-02=0$ OC & 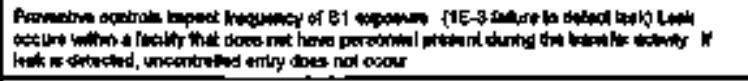 & \\
\hline ZUNRAR DOt & 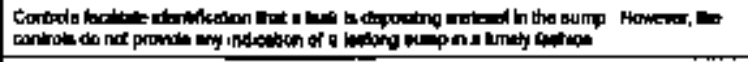 & \\
\hline 2400,02 in 19 & 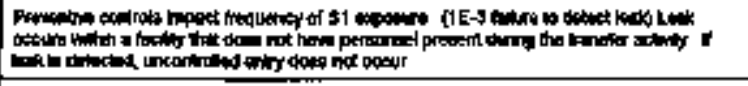 & \\
\hline 244 R $-02=11$ & 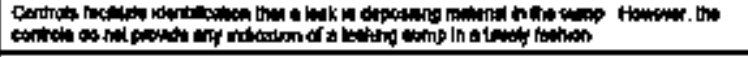 & \\
\hline $244 \ln -02 a 12$ & 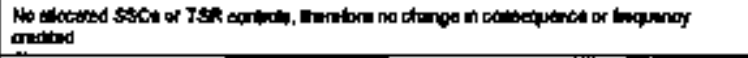 & \\
\hline \multicolumn{3}{|l|}{24401809013} \\
\hline $2444 R-07014$ & Sanot: For NA-OT & \\
\hline \multicolumn{3}{|l|}{$244 \pi \cdot 100$ a 08} \\
\hline $2004 A-100=04$ & 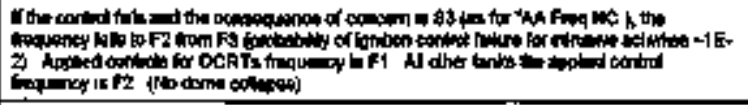 & 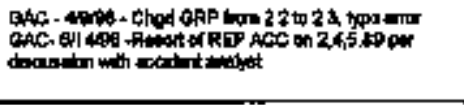 \\
\hline 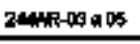 & 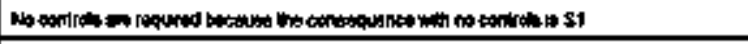 & \\
\hline $24416=00$ a 06 & & 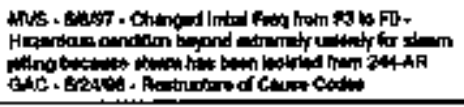 \\
\hline $24440-00$ o in & & 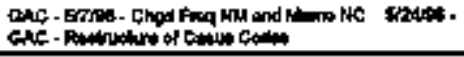 \\
\hline
\end{tabular}




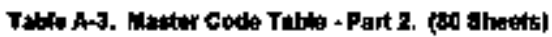

\begin{tabular}{|c|c|c|}
\hline 10 & 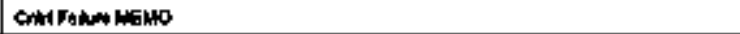 & 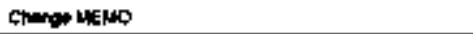 \\
\hline 24UACOS a OC & 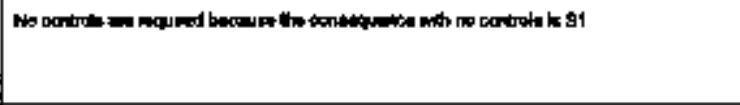 & 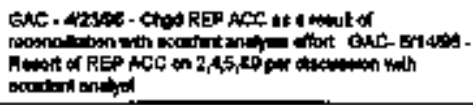 \\
\hline 244Rtos a 09 & 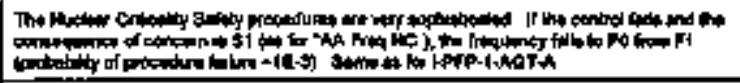 & \\
\hline 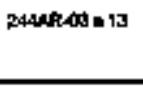 & & 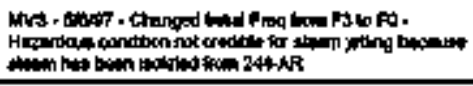 \\
\hline $2404 t a d s=14$ & & 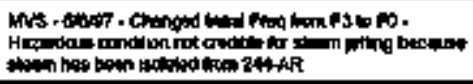 \\
\hline \multicolumn{3}{|l|}{ 240trats o is } \\
\hline \multicolumn{3}{|l|}{ 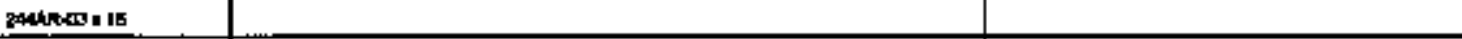 } \\
\hline \multicolumn{3}{|l|}{$2040 \sin 17$} \\
\hline \multicolumn{3}{|l|}{ 244AR-O9. I8 } \\
\hline \multicolumn{3}{|l|}{ 24therota or } \\
\hline \multicolumn{3}{|l|}{$244 \ln 400+01$} \\
\hline $20 \operatorname{sen}-0402$ & 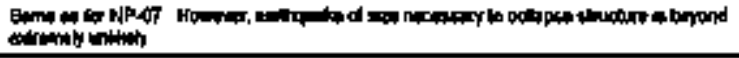 & \\
\hline 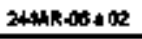 & & 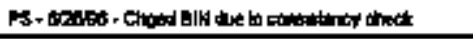 \\
\hline 2444A- $00 \approx 01$ & & 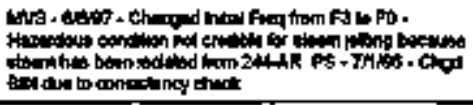 \\
\hline 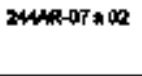 & & 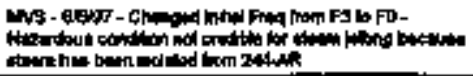 \\
\hline
\end{tabular}




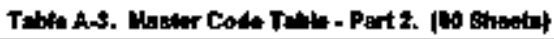

\begin{tabular}{|c|c|c|}
\hline D & 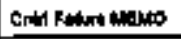 & Conghno \\
\hline $244 a+0 \pi=18$ & & 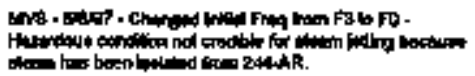 \\
\hline 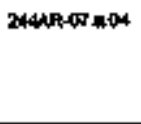 & & 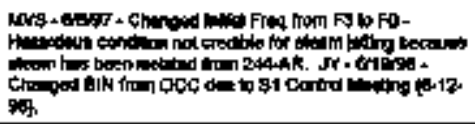 \\
\hline 240R.PHOA & & 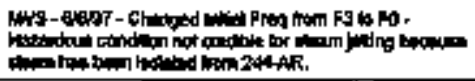 \\
\hline \multicolumn{3}{|l|}{ 24WRPFER } \\
\hline $244 \pi n+m k 3$ & & 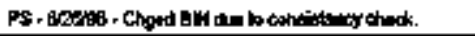 \\
\hline \multicolumn{3}{|l|}{ zankfondid } \\
\hline \multicolumn{3}{|l|}{ 2MPRfAits } \\
\hline \multicolumn{3}{|l|}{ 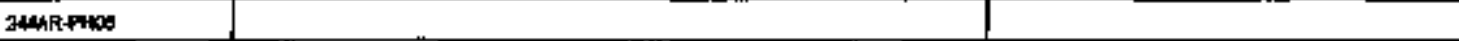 } \\
\hline \multicolumn{3}{|l|}{ 244RRPWDT } \\
\hline \multicolumn{3}{|l|}{ 2esukentes } \\
\hline \multicolumn{3}{|l|}{ Zenapents. } \\
\hline \multicolumn{3}{|l|}{ ZLMRPHID } \\
\hline \multicolumn{3}{|l|}{ 24antemitis } \\
\hline \multicolumn{3}{|l|}{ 244AR.PHT2 } \\
\hline \multicolumn{3}{|l|}{ 2MANRPH3 } \\
\hline \multicolumn{3}{|l|}{ 2aunePH1d } \\
\hline \multicolumn{3}{|l|}{ 244Ak,PH49 } \\
\hline Z4SeA.PHth & & \\
\hline
\end{tabular}


Tahle A.s. Waster Code Table - Fat 2 [at Sheats]

\begin{tabular}{|c|c|c|}
\hline $\mathbf{0}$ & 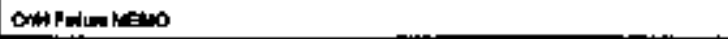 & chepretres \\
\hline \multicolumn{3}{|c|}{ 24LRPHI } \\
\hline \multicolumn{3}{|c|}{ 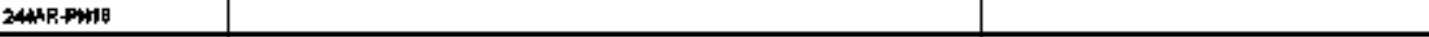 } \\
\hline \multicolumn{3}{|c|}{ 24Athatho } \\
\hline \multicolumn{3}{|l|}{ ZPJWA-91 } \\
\hline 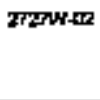 & 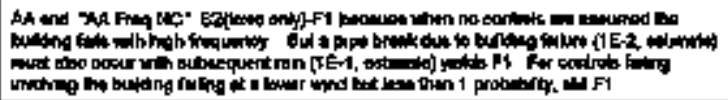 & \\
\hline 27randes & 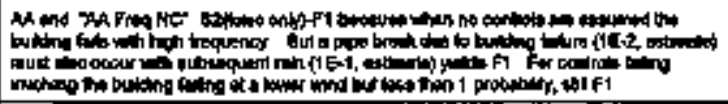 & 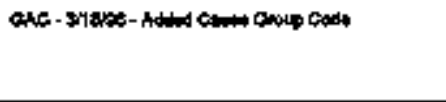 \\
\hline $27 \pi N-04$ & 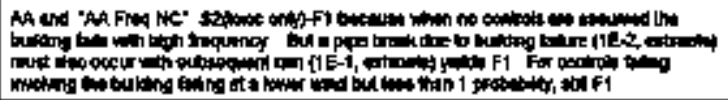 & \\
\hline 2P201405 & 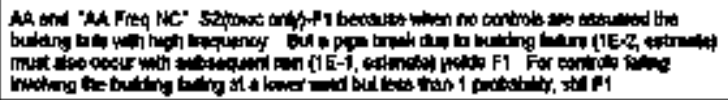 & 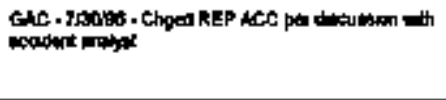 \\
\hline totintos & 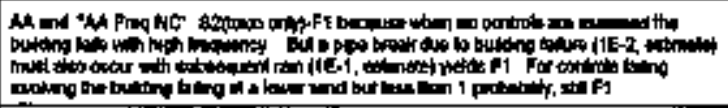 & 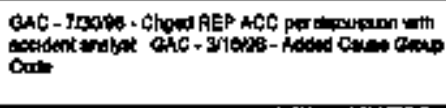 \\
\hline 90-01 & 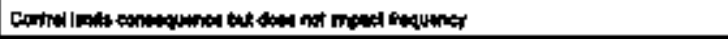 & \\
\hline $\sec 0$ & 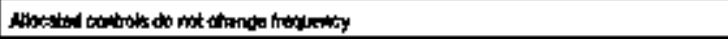 & \\
\hline Datbos & 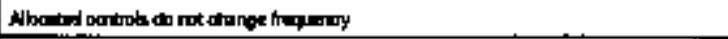 & 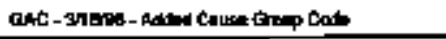 \\
\hline spos & 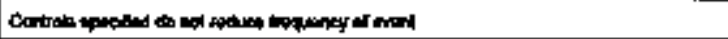 & \\
\hline $\operatorname{sos}$ & & 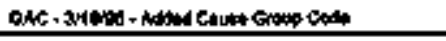 \\
\hline
\end{tabular}


Table A-3. Matar Cods Table - Part 2. 190 Sheets]

\begin{tabular}{|c|c|c|}
\hline 冓 & Cont Ruten kento & chergon MENo \\
\hline most & 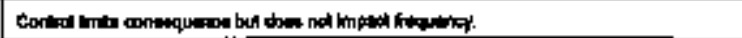 & \\
\hline motot & 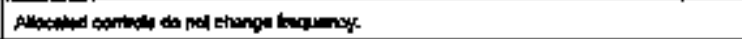 & \\
\hline $900-06$ & 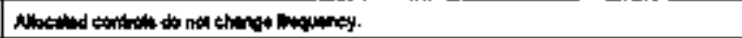 & 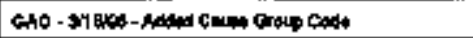 \\
\hline \multicolumn{3}{|l|}{$900-00$} \\
\hline $100-10$ & & 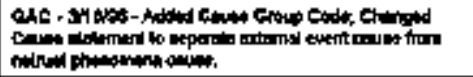 \\
\hline no-11 & 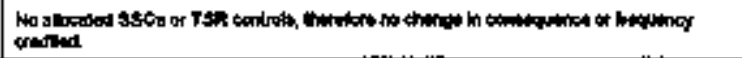 & \\
\hline \multicolumn{3}{|l|}{ Expor } \\
\hline \multicolumn{3}{|c|}{ Buspold } \\
\hline $8 m 002$ & 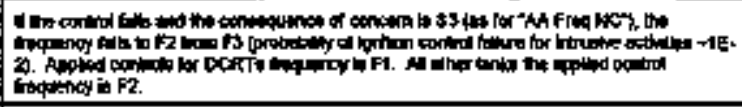 & 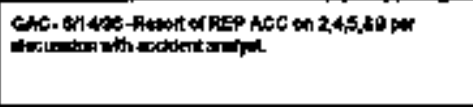 \\
\hline \multicolumn{3}{|l|}{ 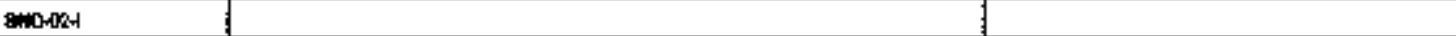 } \\
\hline $\cos a$ & & 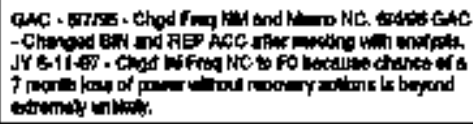 \\
\hline CCFAS & 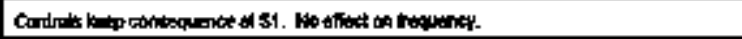 & \\
\hline tofos & 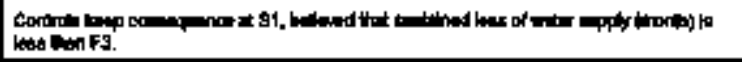 & \\
\hline $\cos 104$ & 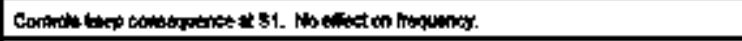 & \\
\hline \multicolumn{3}{|l|}{$\cos 0}$. \\
\hline cefot & & \\
\hline
\end{tabular}


Tabb A-3. Mestar Coda Teblo - Fant 2. (190 ahoeta)

\begin{tabular}{|c|c|c|}
\hline$\Delta$ & Solt Facum Mento & Chmoukans \\
\hline $\operatorname{cosen}$ & 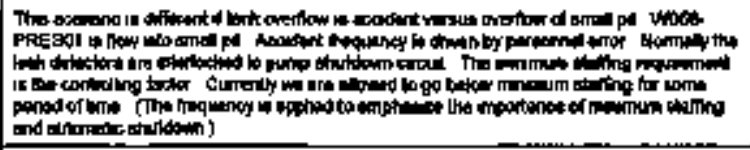 & 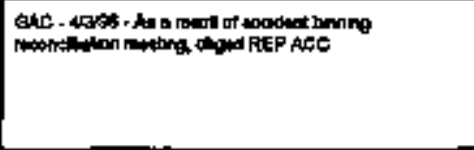 \\
\hline \multicolumn{3}{|l|}{$\mathrm{CCF}-\mathrm{OH}$} \\
\hline \multicolumn{3}{|l|}{ LCبA- } \\
\hline \multicolumn{3}{|l|}{$\cos A \theta$} \\
\hline \multicolumn{3}{|l|}{ CCF-11 } \\
\hline \multicolumn{3}{|l|}{ CCEF-12 } \\
\hline \multicolumn{3}{|l|}{ Cc:F-13 } \\
\hline \multicolumn{3}{|l|}{ Cc.p.14 } \\
\hline \multicolumn{3}{|l|}{$\cos -15$} \\
\hline CCA -19 & 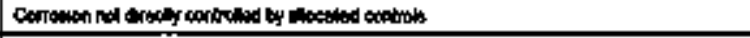 & \\
\hline tef-17 & 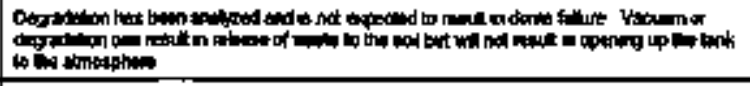 & 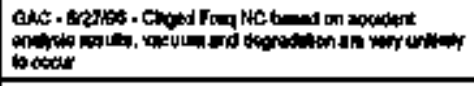 \\
\hline $\cos \theta$ & 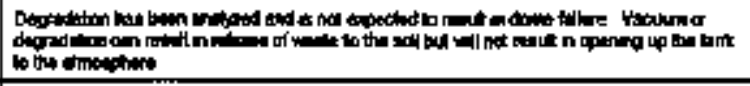 & 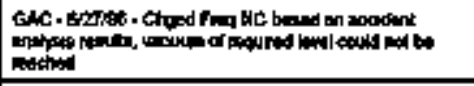 \\
\hline Cetr.19 & 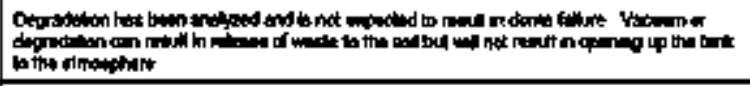 & 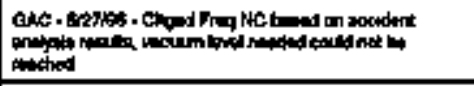 \\
\hline cetr-20 & 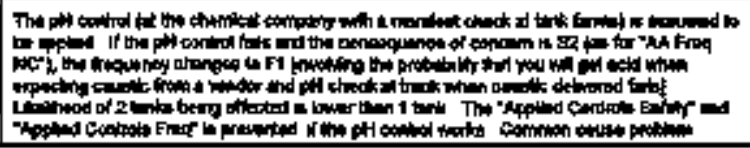 & 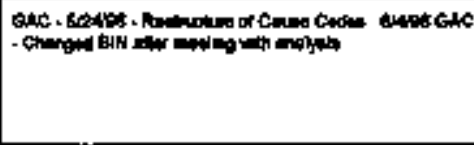 \\
\hline
\end{tabular}




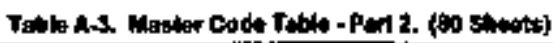

\begin{tabular}{|c|c|c|}
\hline $\mathbf{m}$ & Can Fan MEN & 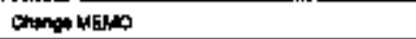 \\
\hline $\cos 201$ & 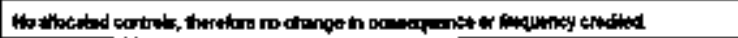 & \\
\hline \multicolumn{3}{|l|}{ CDP-AR } \\
\hline $\operatorname{cospos}$ & 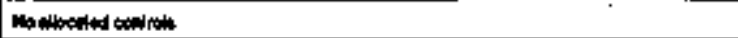 & \\
\hline \multicolumn{3}{|l|}{ ctopiot } \\
\hline \multicolumn{3}{|l|}{$\operatorname{cophos}$} \\
\hline copentes & 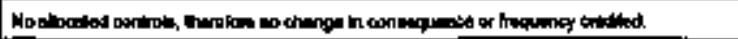 & \\
\hline \multicolumn{3}{|l|}{ COPPDOP } \\
\hline $\operatorname{cop} 00$ & 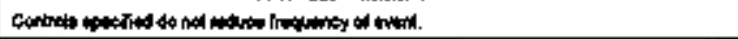 & \\
\hline \multicolumn{3}{|l|}{$\cos \theta$} \\
\hline $\cos \rightarrow 10$ & 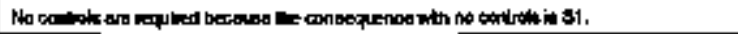 & \\
\hline coteror & 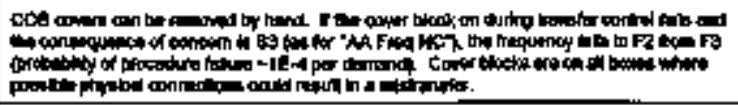 & \\
\hline 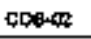 & 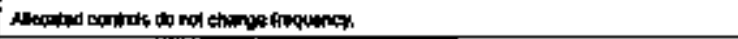 & \\
\hline $\cos \theta$ & 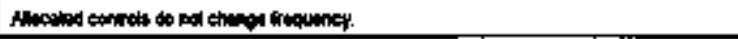 & 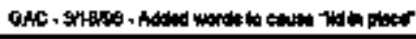 \\
\hline $\cos -04$ & 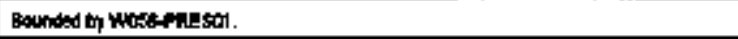 & \\
\hline $\cos \theta-40$ & 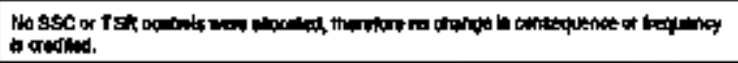 & \\
\hline contost & 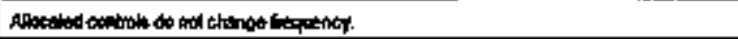 & \\
\hline cotedos & 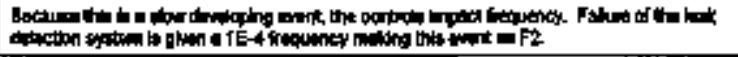 & \\
\hline $\cos 05 \mathrm{~s}$ & 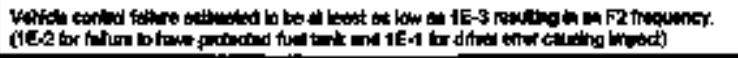 & \\
\hline coses. & 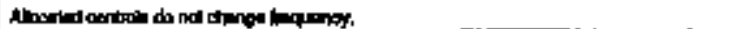 & \\
\hline
\end{tabular}




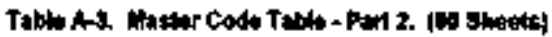

\begin{tabular}{|c|c|c|}
\hline$D$ & Coid Foum & 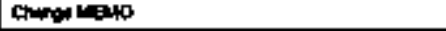 \\
\hline 60p-0G & 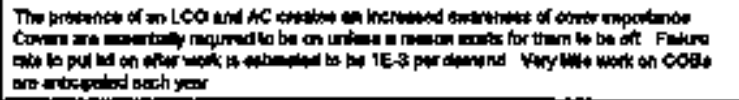 & \\
\hline cobota & 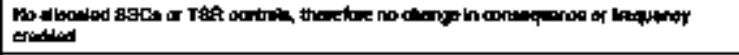 & \\
\hline 60908 & 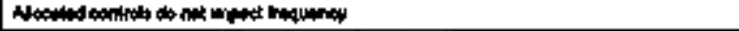 & \\
\hline \multicolumn{3}{|l|}{ ctcos:07 } \\
\hline ctobs & Banded by Modit PREsor & \\
\hline CRHOT & 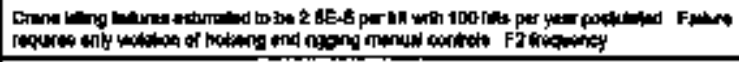 & \\
\hline CPRH-OI- & 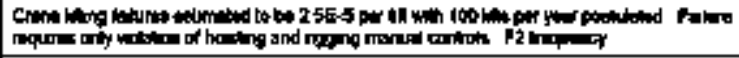 & \\
\hline CRN4DR & 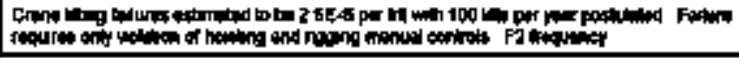 & 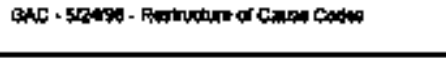 \\
\hline \multicolumn{3}{|l|}{ CRNAORH } \\
\hline Conned & 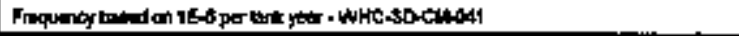 & \\
\hline Controsula & 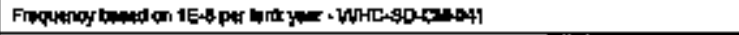 & \\
\hline cristogrs & 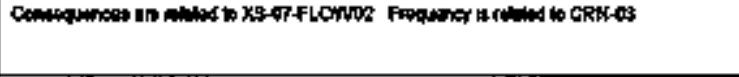 & 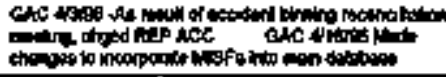 \\
\hline critod & 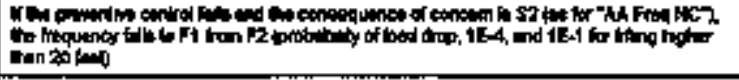 & \\
\hline conatosid & 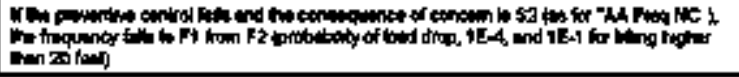 & \\
\hline
\end{tabular}




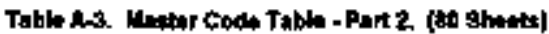

\begin{tabular}{|c|c|c|}
\hline D & 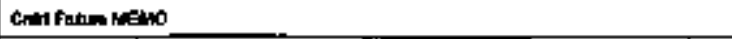 & Chenge \\
\hline eninosis & 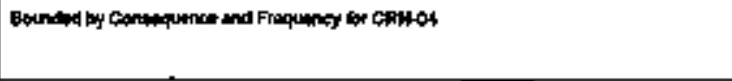 & 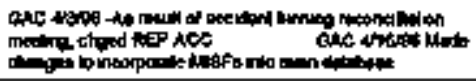 \\
\hline GRNOS & 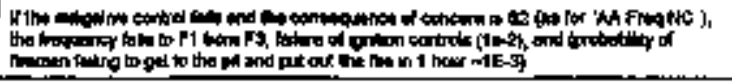 & \\
\hline CRRAOCH & 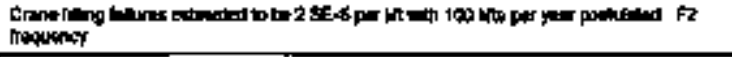 & \\
\hline ENNot & 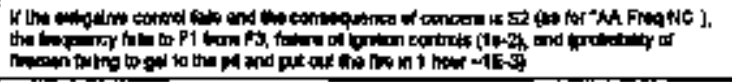 & \\
\hline ERN-DH & 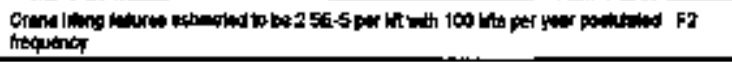 & \\
\hline CRNAT & 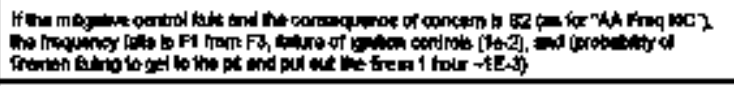 & \\
\hline \multicolumn{3}{|l|}{ Cortarlol } \\
\hline cravod & 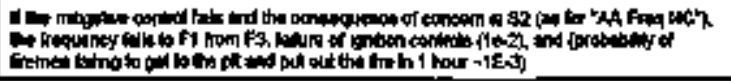 & \\
\hline \multicolumn{3}{|l|}{ CFoboti } \\
\hline centos & 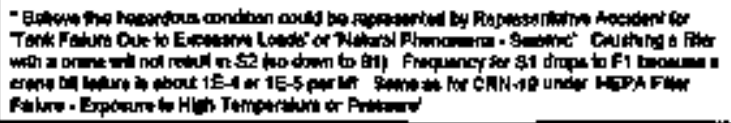 & 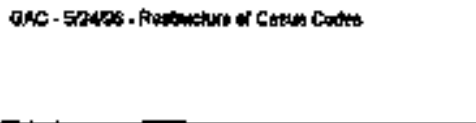 \\
\hline CRPWATI & 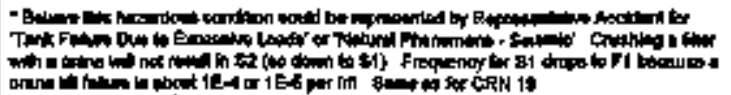 & \\
\hline
\end{tabular}




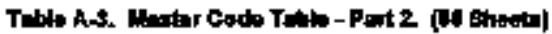

\begin{tabular}{|c|c|c|}
\hline 10 & 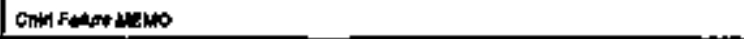 & chare Matas \\
\hline СRИ.10 & 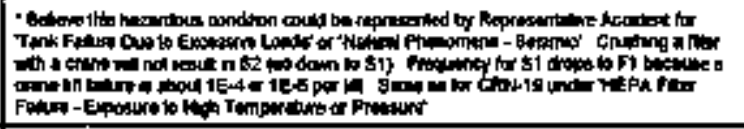 & 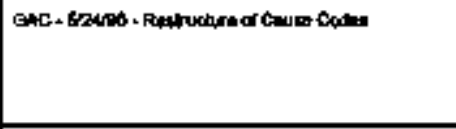 \\
\hline OAN-10 & 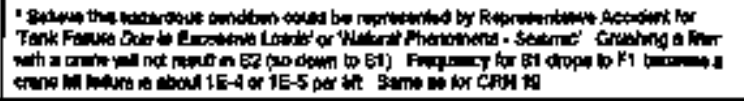 & \\
\hline CRN-11 & 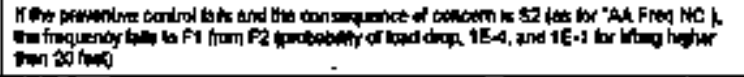 & \\
\hline CRN-11-h & 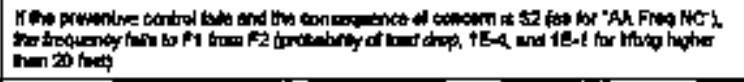 & \\
\hline CRAN-1:-18 & 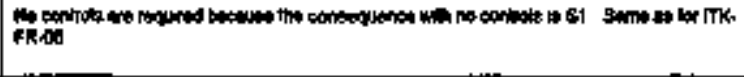 & 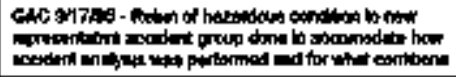 \\
\hline colos-11+ct & 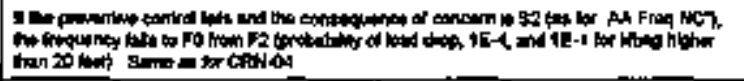 & \\
\hline tora-12 & 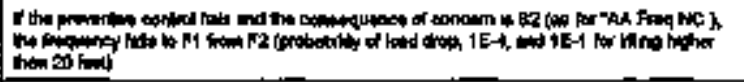 & \\
\hline CRan-12-1A & 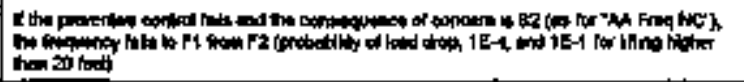 & \\
\hline CRN-12\% & 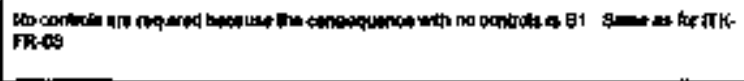 & 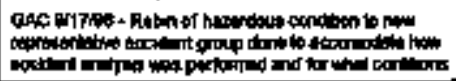 \\
\hline CAN $-12 \leadsto C$ & 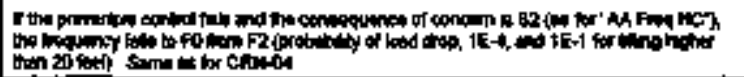 & \\
\hline
\end{tabular}




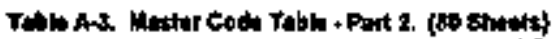

\begin{tabular}{|c|c|c|}
\hline ID & 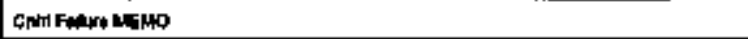 & 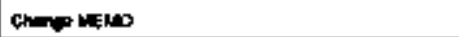 \\
\hline CNal $154 h$ & 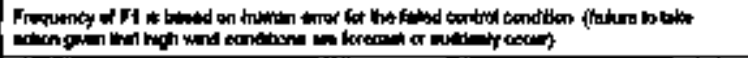 & \\
\hline Cowat-13-18 & 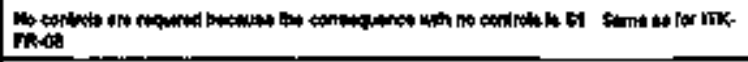 & \\
\hline cantiasc & 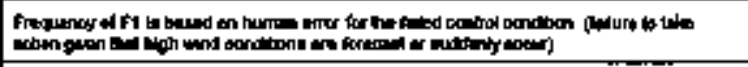 & \\
\hline entise & 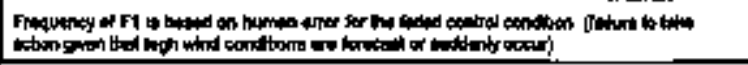 & \\
\hline CREM 13b & 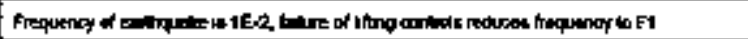 & \\
\hline CAKK14 & & 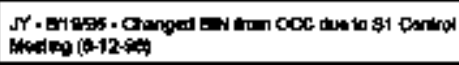 \\
\hline \multicolumn{3}{|l|}{ GRN-14-1 } \\
\hline Ckrik18 & & 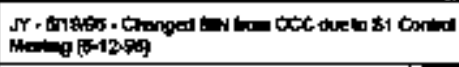 \\
\hline \multicolumn{3}{|l|}{ CRN-15-1 } \\
\hline coratiti & & 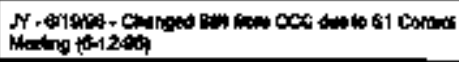 \\
\hline \multicolumn{3}{|l|}{ 두만-1어 } \\
\hline chant17 & 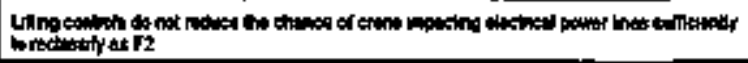 & \\
\hline \multicolumn{3}{|l|}{ tomatit-1 } \\
\hline contrats & 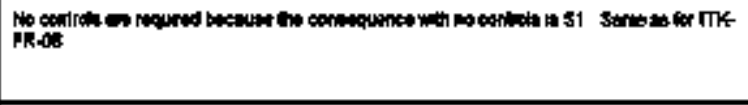 & 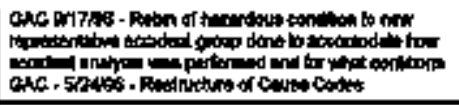 \\
\hline crathist & 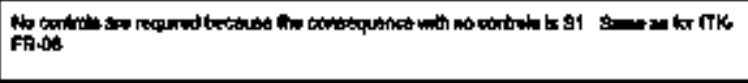 & 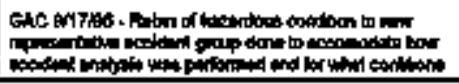 \\
\hline
\end{tabular}




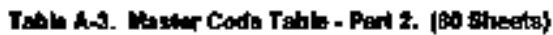

\begin{tabular}{|c|c|c|}
\hline 旧 & 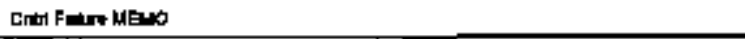 & Chinas MFA \\
\hline CRRH & 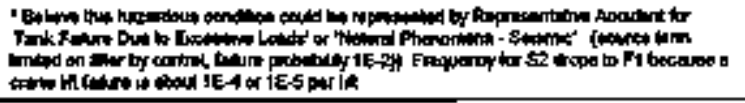 & 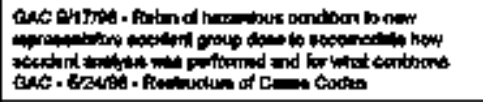 \\
\hline CRk+Non & 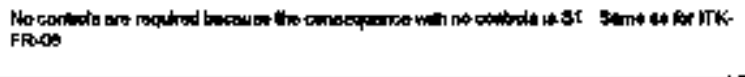 & 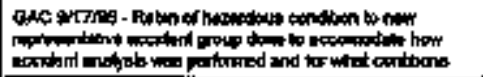 \\
\hline GRA+20 & 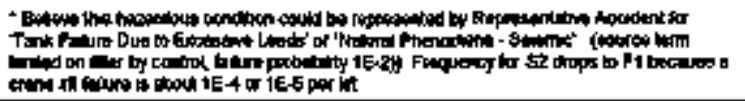 & 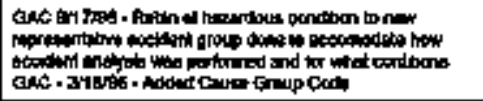 \\
\hline $\cos (x)=1$ & 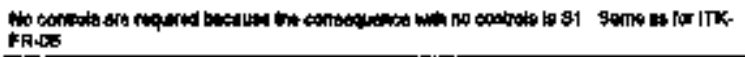 & \\
\hline cosesis & & 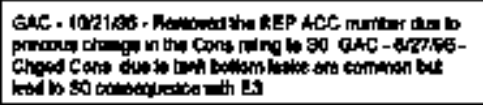 \\
\hline crim214h & & 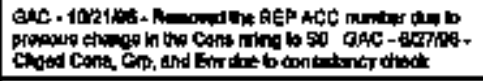 \\
\hline CPan-21-1B & 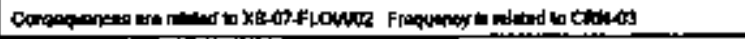 & \\
\hline Contr22 & & 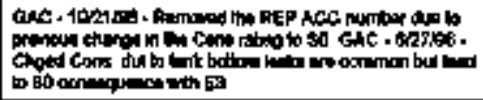 \\
\hline chackisin & & 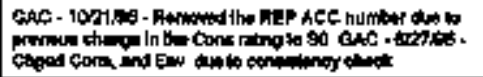 \\
\hline CANOZn & 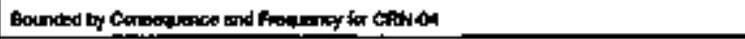 & \\
\hline CARN-ZZ & 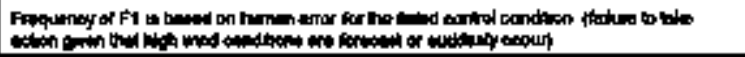 & 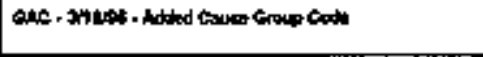 \\
\hline
\end{tabular}




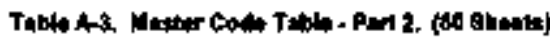

\begin{tabular}{|c|c|c|}
\hline 10 & Cnit fran Nato & Chinge knuto \\
\hline$C h \mathrm{CH}+23 \mathrm{M}$ & 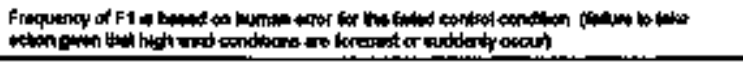 & \\
\hline CFan-236 & 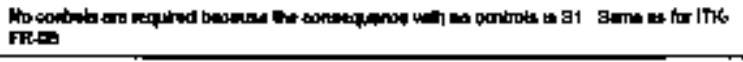 & \\
\hline \multicolumn{3}{|l|}{ ceantid } \\
\hline \multicolumn{3}{|l|}{ CAN4t24 } \\
\hline \multicolumn{3}{|l|}{ cromats } \\
\hline \multicolumn{3}{|l|}{ CRMOSIA } \\
\hline GRMESHB & 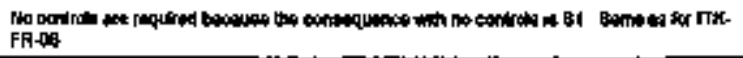 & 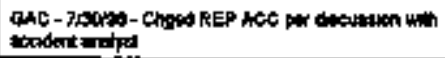 \\
\hline \multicolumn{3}{|l|}{ CRTHEs } \\
\hline \multicolumn{3}{|l|}{ CRN-ZS-1R } \\
\hline CRN-2A-48 & 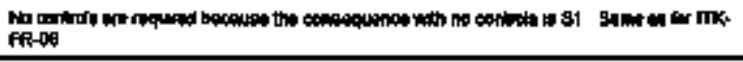 & 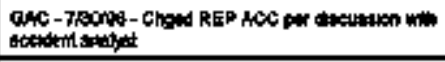 \\
\hline \multicolumn{3}{|l|}{ CkNND } \\
\hline \multicolumn{3}{|l|}{ Gripknth } \\
\hline Centexta & 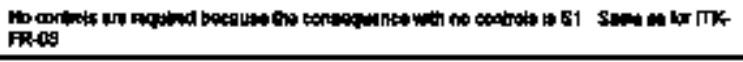 & 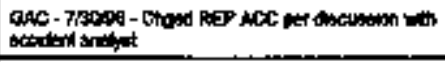 \\
\hline \multicolumn{3}{|l|}{ 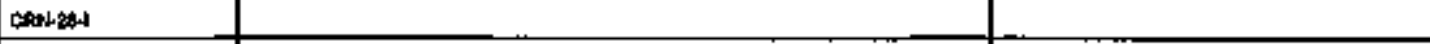 } \\
\hline GVR-91 & 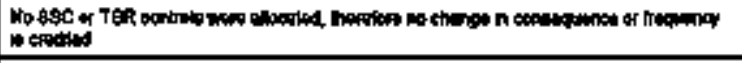 & \\
\hline Guktos.1 & 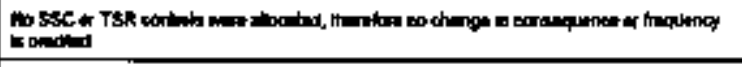 & \\
\hline conos & 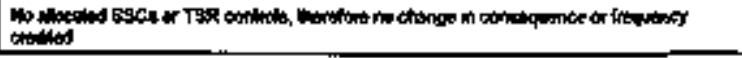 & \\
\hline
\end{tabular}




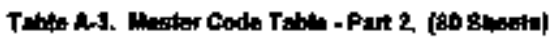

\begin{tabular}{|c|c|c|}
\hline D & 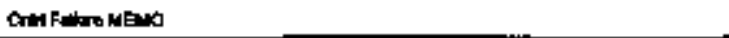 & Cherg tivito \\
\hline CQR-ORH & 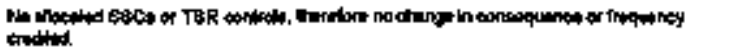 & \\
\hline GWHOd & 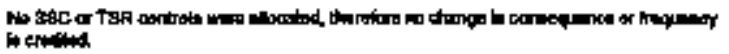 & \\
\hline criksol & 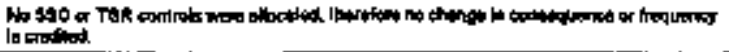 & \\
\hline GVR-04 & & 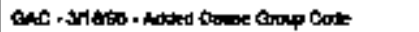 \\
\hline \multicolumn{3}{|l|}{ CVR-04 } \\
\hline CWRTS & 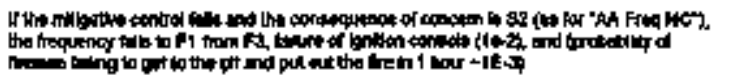 & \\
\hline \multicolumn{3}{|l|}{ CWR-05-1 } \\
\hline $\cos \theta$ & & 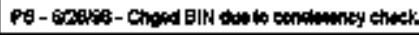 \\
\hline \multicolumn{3}{|l|}{ CWhate-1 } \\
\hline chred? & & 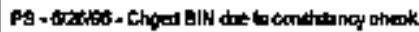 \\
\hline \multicolumn{3}{|l|}{ CथR-णrd } \\
\hline CWR-D8 & & 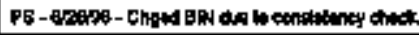 \\
\hline \multicolumn{3}{|l|}{ CHWrat } \\
\hline CHAdo & & 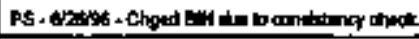 \\
\hline \multicolumn{3}{|l|}{ CMR-OP } \\
\hline \multicolumn{3}{|l|}{ charto } \\
\hline \multicolumn{3}{|l|}{ Chatov } \\
\hline \multicolumn{3}{|l|}{ CAk-11 } \\
\hline CSR-111 & & \\
\hline
\end{tabular}


Tolo A-3. Mindter Code Toblo - Payt 2. (tod shents)

\begin{tabular}{|c|c|c|}
\hline $\mathbf{m}$ & 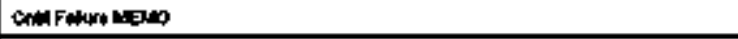 & Ceang:LENHO \\
\hline chancis & 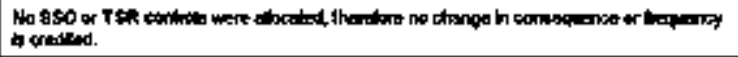 & \\
\hline cont.13.1 & 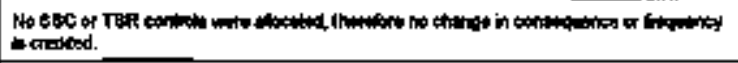 & \\
\hline CNR-14-1 & 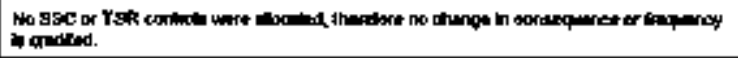 & \\
\hline CuR.15.1 & 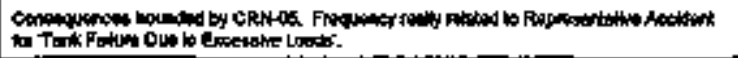 & \\
\hline DCHO1 & & 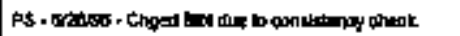 \\
\hline DCNㅓㄱㄱ & & 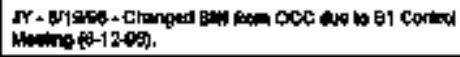 \\
\hline Dengar & 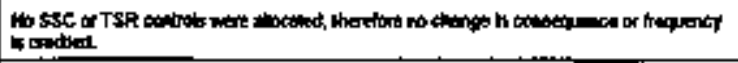 & \\
\hline \multicolumn{3}{|l|}{ Demor-k } \\
\hline \multicolumn{3}{|l|}{ DCHOS.18 } \\
\hline DCHAOA & & 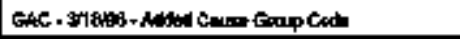 \\
\hline \multicolumn{3}{|l|}{ DCNon-1 } \\
\hline ocmon & & 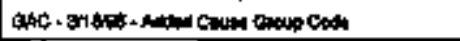 \\
\hline \multicolumn{3}{|l|}{ OCNACH } \\
\hline \multicolumn{3}{|l|}{ Denat } \\
\hline \multicolumn{3}{|l|}{ OCN-OS- } \\
\hline DCNAOB & & 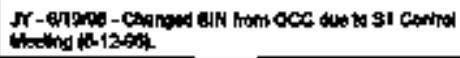 \\
\hline DCWLOH & & 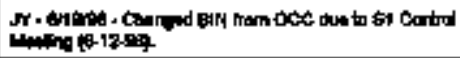 \\
\hline DCand & & \\
\hline
\end{tabular}




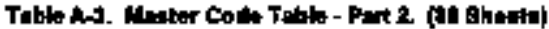

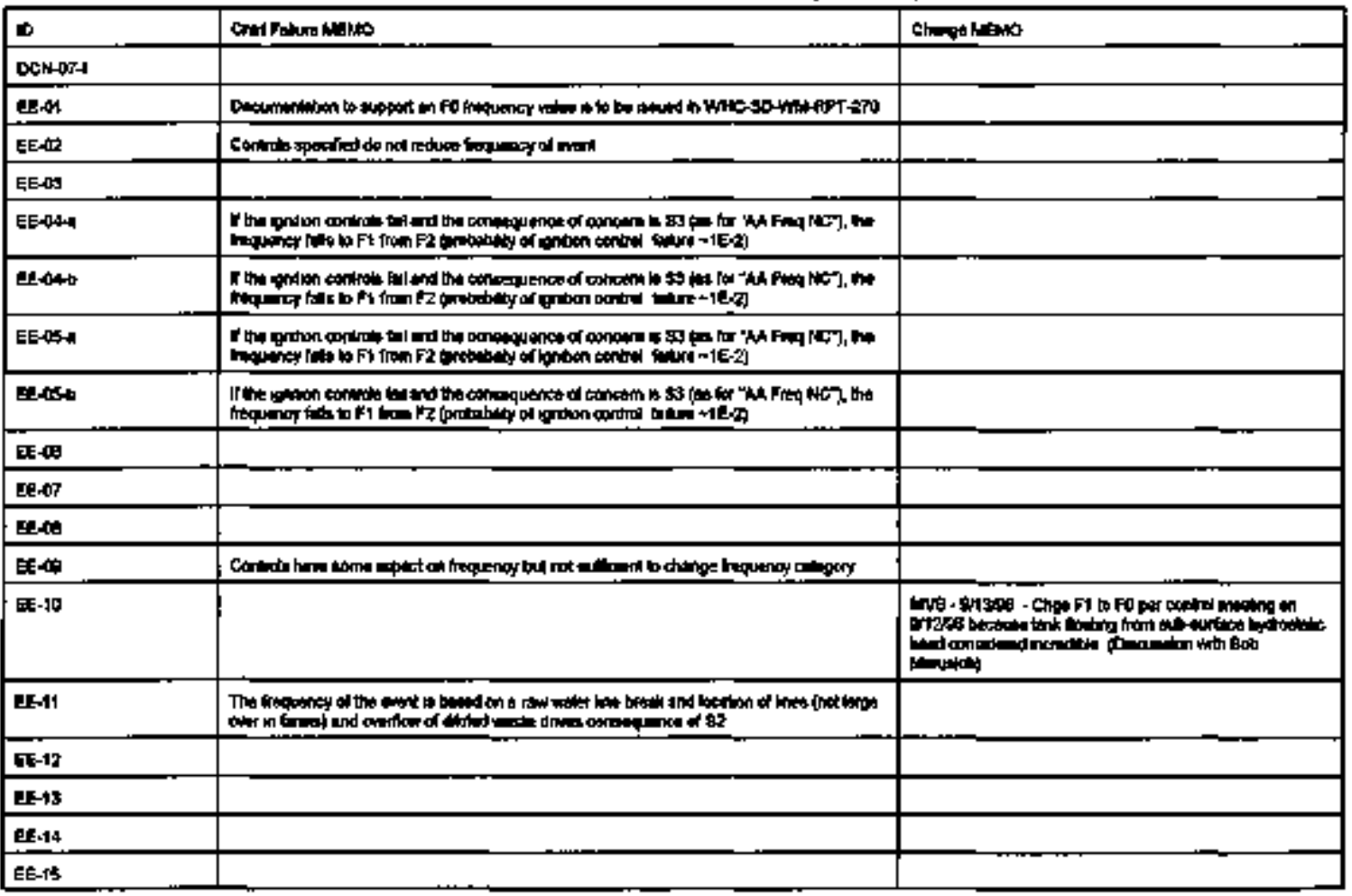


Totble A-S. Master Cots Tobla + Part 2. (100 shente)

\begin{tabular}{|c|c|c|}
\hline 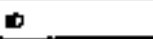 & 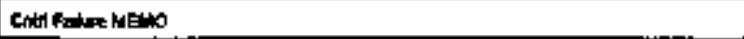 & Gropen kilas \\
\hline E-1B & 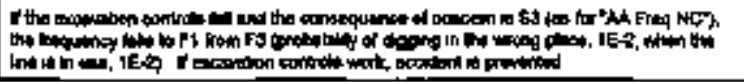 & \\
\hline EE-T & 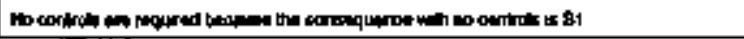 & \\
\hline \multicolumn{3}{|l|}{ QRNOH } \\
\hline \multicolumn{3}{|l|}{ ORNAOA- } \\
\hline \multicolumn{3}{|l|}{ GRN-OS } \\
\hline \multicolumn{3}{|l|}{ GRNA你- } \\
\hline \multicolumn{3}{|l|}{ DRFN-034 } \\
\hline $1-2026-1-G N a A h$ & 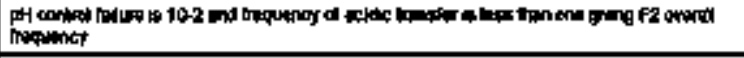 & \\
\hline \multicolumn{3}{|l|}{ hens-1-chest } \\
\hline 1-2226-1-ctips & 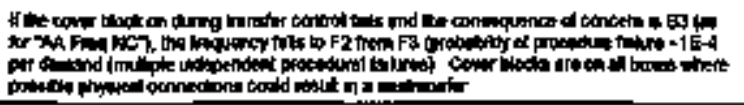 & \\
\hline \multicolumn{3}{|l|}{$1-2029-1-0 \times 0$} \\
\hline HO2S-1-CâE & 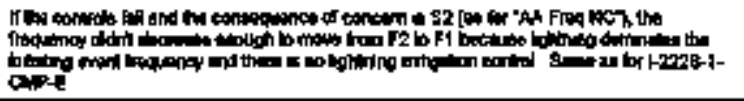 & 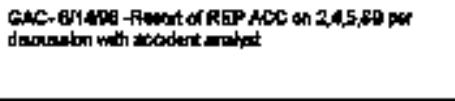 \\
\hline 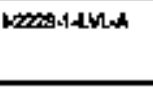 & 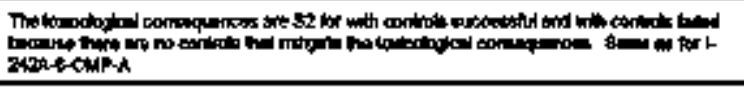 & \\
\hline 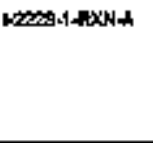 & 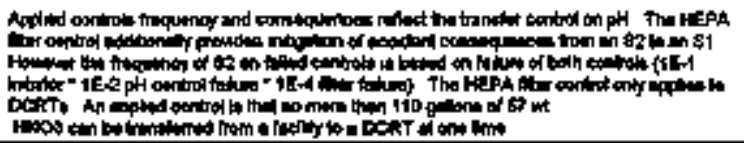 & 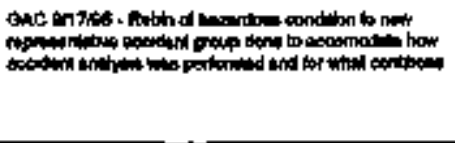 \\
\hline
\end{tabular}




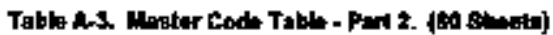

\begin{tabular}{|c|c|c|}
\hline ID & Drtil fairs MDAs & שחנא \\
\hline L2:28-2.FUWA & 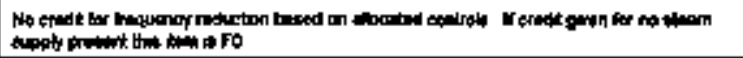 & \\
\hline \multicolumn{3}{|l|}{ 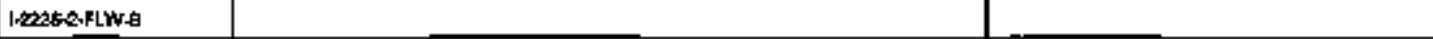 } \\
\hline 12028-2-FLW-G & 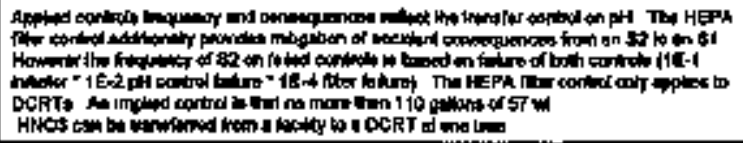 & 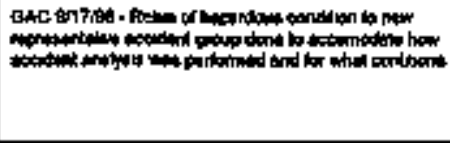 \\
\hline \multicolumn{3}{|l|}{ 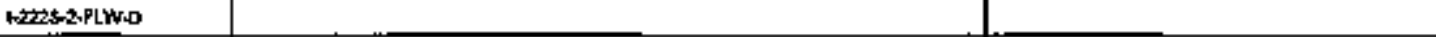 } \\
\hline \multicolumn{3}{|l|}{ 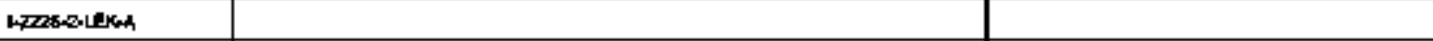 } \\
\hline 1-2029-2-1EK-9 & 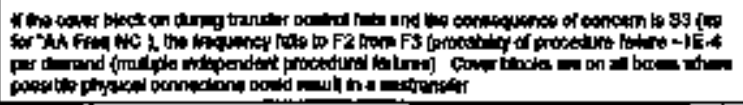 & \\
\hline LORS-2AEK-C & 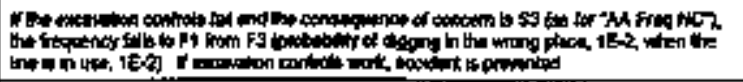 & 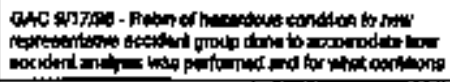 \\
\hline \multicolumn{3}{|l|}{$102289-24$ EK-D } \\
\hline FOOS-ZHEK-E & 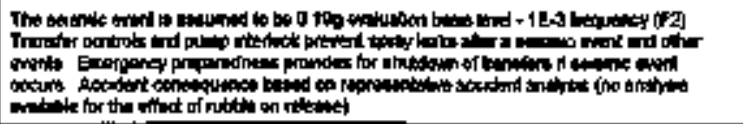 & \\
\hline 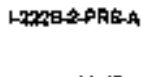 & 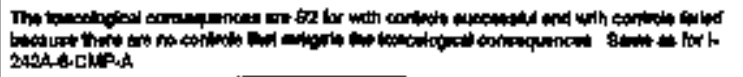 & \\
\hline 1-243-1+flW-A & 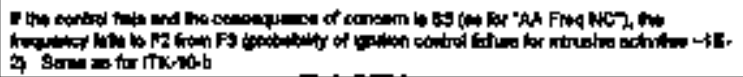 & 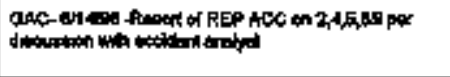 \\
\hline
\end{tabular}




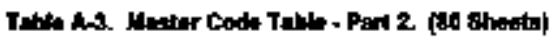

\begin{tabular}{|c|c|c|}
\hline 10 & 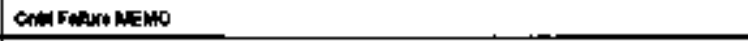 & Conge Mulu \\
\hline $1-2424-1+4 L W 6$ & 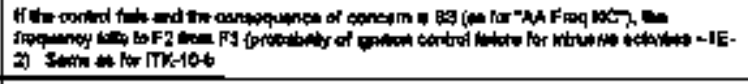 & 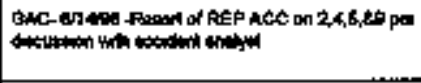 \\
\hline 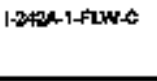 & 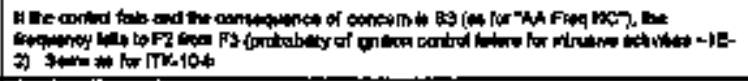 & 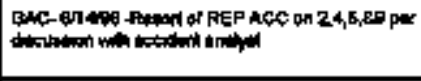 \\
\hline 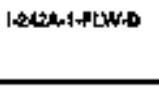 & 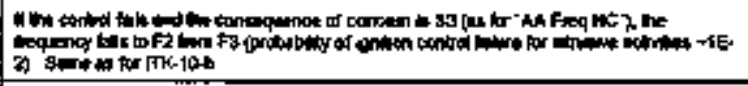 & 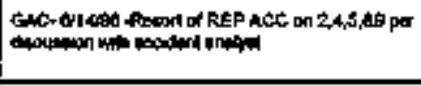 \\
\hline 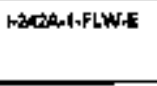 & 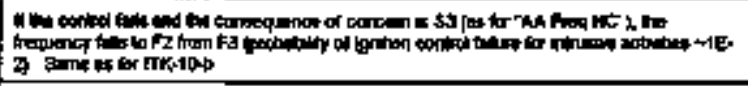 & 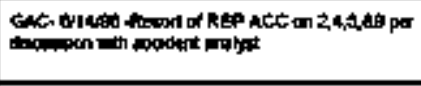 \\
\hline \multicolumn{3}{|l|}{ 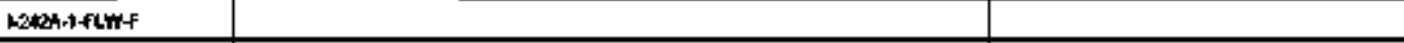 } \\
\hline \multicolumn{3}{|l|}{ 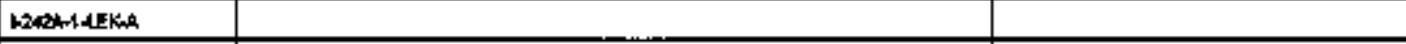 } \\
\hline WZWZM-1 +EKS & 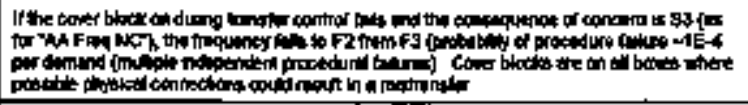 & \\
\hline 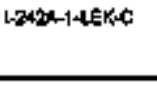 & 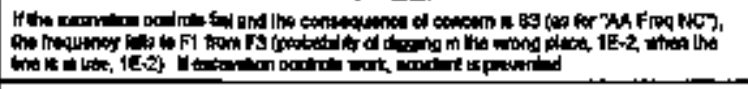 & \\
\hline \multicolumn{3}{|l|}{$1-2424+140 \times 0$} \\
\hline $1-2,24-1-1 E X E$ & 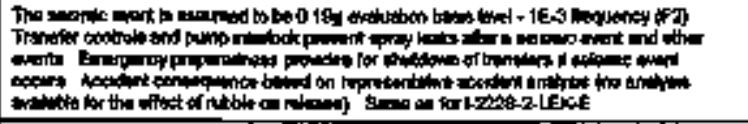 & \\
\hline Laten-2-chath & 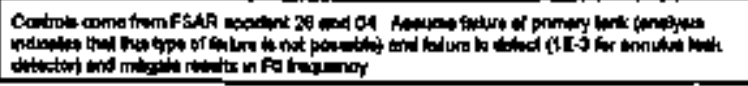 & \\
\hline
\end{tabular}




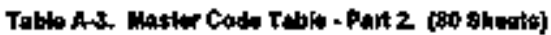

\begin{tabular}{|c|c|c|}
\hline 10 & 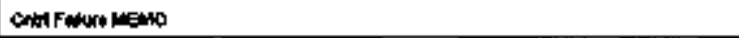 & 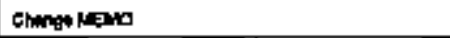 \\
\hline $1242 \operatorname{sen} 200$ & 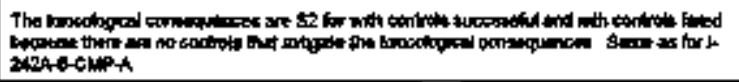 & \\
\hline loconizanth & 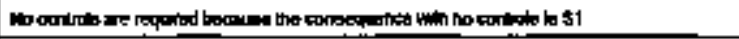 & \\
\hline $12 \operatorname{sen} 2$ knt: & 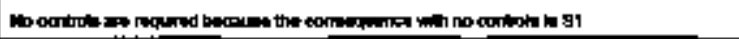 & \\
\hline \multicolumn{3}{|l|}{ 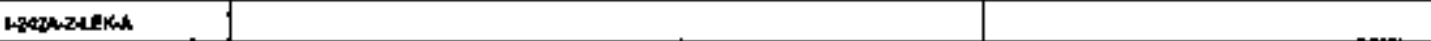 } \\
\hline HZ2-2AZZEKB & 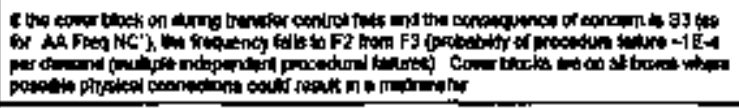 & \\
\hline N2OH2-LEKC & 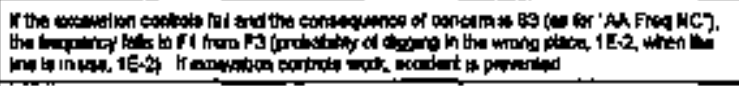 & \\
\hline \multicolumn{3}{|l|}{ 1204-2-LEKO } \\
\hline W2WRHZLEE & 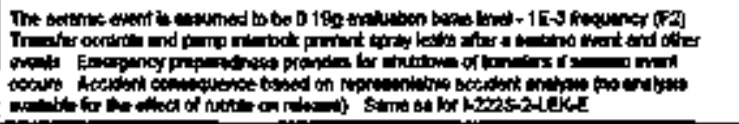 & \\
\hline \multicolumn{3}{|l|}{ 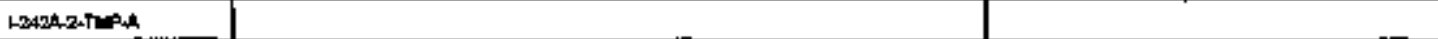 } \\
\hline $1-2424+2+28-4$ & 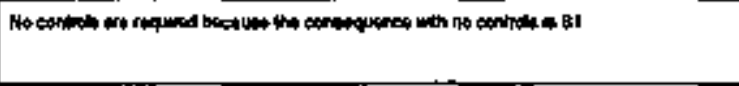 & 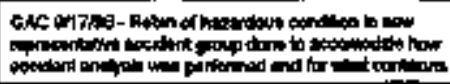 \\
\hline \multicolumn{3}{|l|}{$1-242+\sqrt{2}+2806$} \\
\hline Hedestrinth & 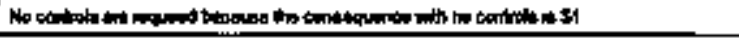 & \\
\hline 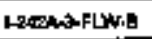 & 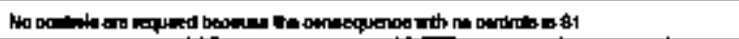 & \\
\hline Hash-FINA & 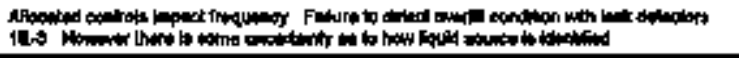 & \\
\hline NACHATMPA & & \\
\hline
\end{tabular}




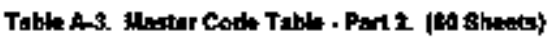

\begin{tabular}{|c|c|c|}
\hline $\mathbf{I D}$ & Crtaf Fabrotua & Clonge hath \\
\hline \multicolumn{3}{|l|}{ 1-2423-F-FLA } \\
\hline HA2A-6-CAPA & 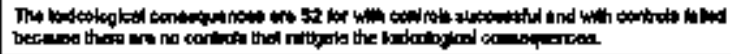 & \\
\hline -20+NA-B-FLW-A & 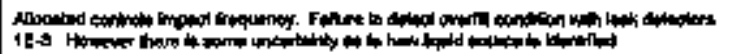 & \\
\hline Mat-6hH-s & 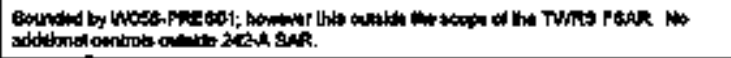 & \\
\hline \multicolumn{3}{|l|}{$100040 \mathrm{HLH}$} \\
\hline \multicolumn{3}{|l|}{ 1-2004-J-CMPA } \\
\hline 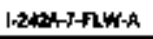 & 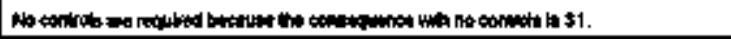 & \\
\hline 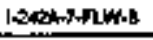 & 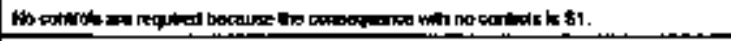 & \\
\hline 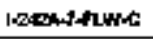 & 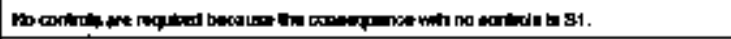 & \\
\hline \multicolumn{3}{|l|}{ 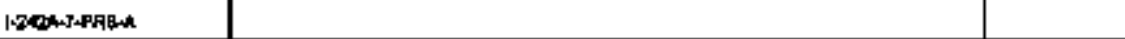 } \\
\hline 1-2048-1-PRBA & 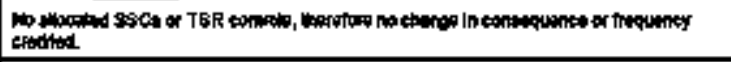 & \\
\hline 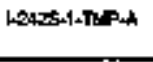 & 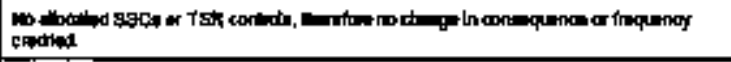 & \\
\hline $1-2420-2+7 w-h$ & 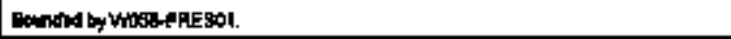 & \\
\hline \multicolumn{2}{|l|}{ L2425-2nLwe } & \\
\hline \multicolumn{2}{|l|}{$1-24200+464<$} & \\
\hline \multicolumn{2}{|l|}{$1-2098-2-140$} & \\
\hline maser-2+lite & Bainded by Vhasprestor. & \\
\hline 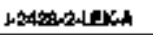 & & \\
\hline
\end{tabular}




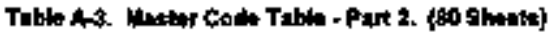

\begin{tabular}{|c|c|c|}
\hline IP & 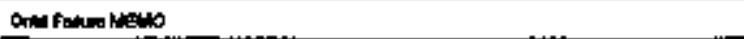 & Chanpalteres \\
\hline $1-2688-6 \times x-8$ & 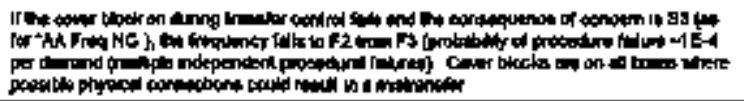 & \\
\hline $23025-2.10 k-c$ & 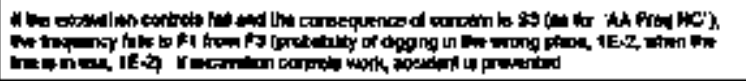 & \\
\hline \multicolumn{3}{|l|}{$1-2429-2-46 \times 0$} \\
\hline $1-2005-2, L 6 K-1$ & 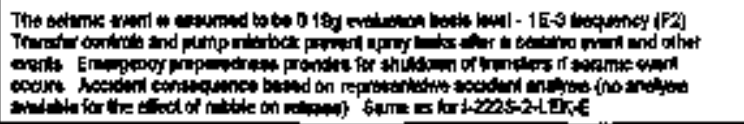 & \\
\hline \multicolumn{3}{|l|}{ 12008-4fiwh } \\
\hline \multicolumn{3}{|l|}{ L2C6-4FHM-B } \\
\hline Wess-4finct & 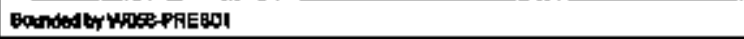 & \\
\hline 12006+fLWD & Bound-dtr Whoseqfesol & \\
\hline \multicolumn{3}{|l|}{ 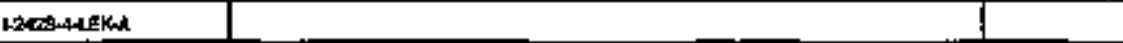 } \\
\hline LZCE-4UEK-B & 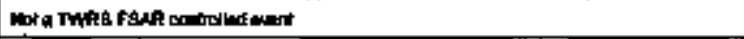 & \\
\hline \multicolumn{3}{|l|}{$1.2625 .418 K-C$} \\
\hline \multicolumn{3}{|l|}{$1.24250410 \times 0$} \\
\hline L7428-4HEXE & 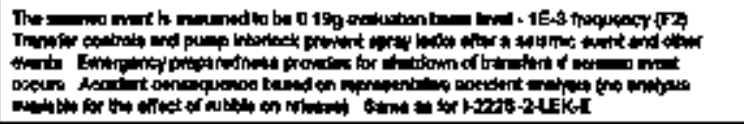 & \\
\hline \multicolumn{3}{|l|}{ lowedectiph } \\
\hline 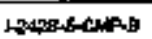 & & \\
\hline
\end{tabular}


Toble A-3. Mnotor Code Tablo + Part 2. (W Bhootey

\begin{tabular}{|c|c|c|}
\hline $\mathbf{D}$ & 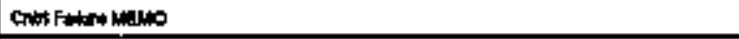 & thenso \\
\hline \multicolumn{3}{|l|}{ 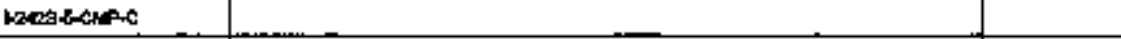 } \\
\hline \multicolumn{3}{|l|}{ L2BE-6-GAP-D } \\
\hline \multicolumn{3}{|l|}{ 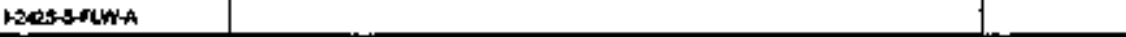 } \\
\hline \multicolumn{3}{|l|}{ 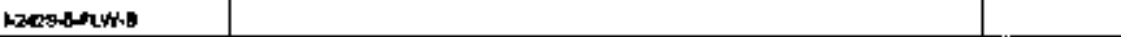 } \\
\hline \multicolumn{3}{|l|}{ 1.242368-PLWCA } \\
\hline \multicolumn{3}{|l|}{ 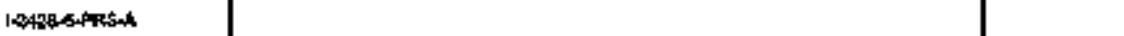 } \\
\hline \multicolumn{3}{|l|}{ NEATR-2-FLWWA } \\
\hline HOW:THFIWHA & 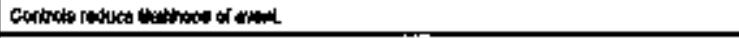 & \\
\hline HELT-PLWA & 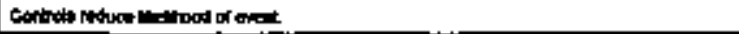 & \\
\hline \multicolumn{3}{|l|}{ 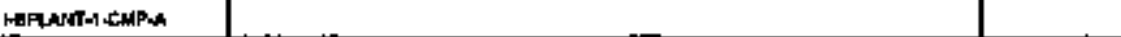 } \\
\hline 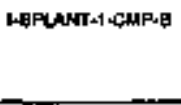 & 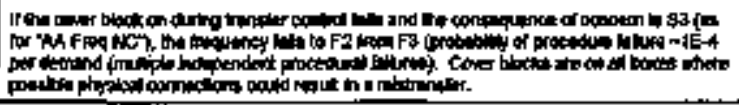 & \\
\hline \multicolumn{3}{|l|}{ IBPLANT-1-CNP-C } \\
\hline \multicolumn{3}{|l|}{ FELANT-1-CHPDO } \\
\hline HEPLANT-1-CLPE & 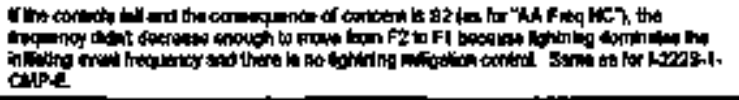 & \\
\hline \multicolumn{3}{|l|}{ HPYANT-9-TTP-A } \\
\hline \multicolumn{3}{|l|}{ FEMLANTERLWWA } \\
\hline \multicolumn{2}{|l|}{ IOPLATRARINB } & \\
\hline 1.BPLANTLAAMNC & & \\
\hline
\end{tabular}




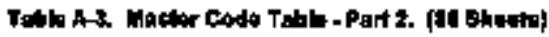

\begin{tabular}{|c|c|c|}
\hline 10 & 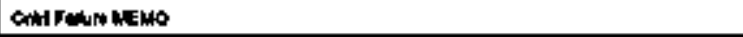 & 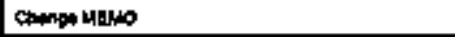 \\
\hline HEANTRALLWO & 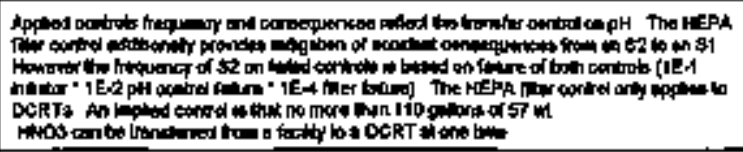 & 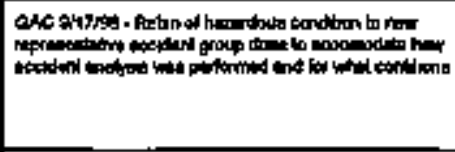 \\
\hline \multicolumn{3}{|l|}{ 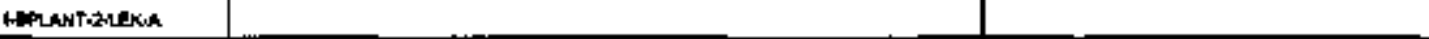 } \\
\hline HEPANT-ZCLEKB & 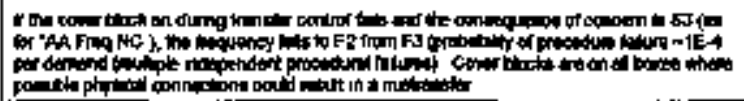 & \\
\hline HPLANT-2HEKS-C & 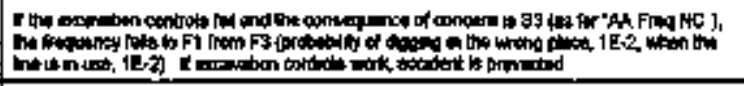 & \\
\hline \multicolumn{3}{|l|}{ 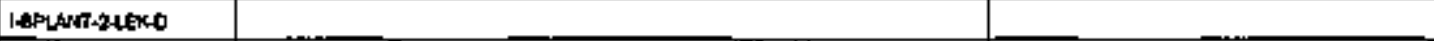 } \\
\hline 1. PPLANT-2LEKE & 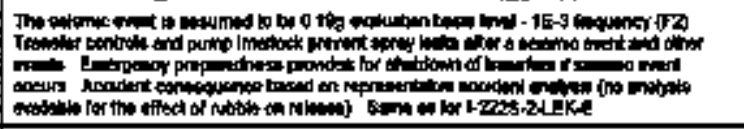 & \\
\hline 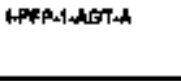 & 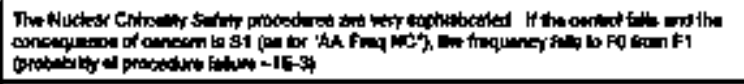 & 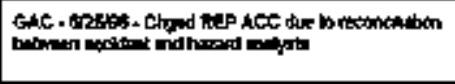 \\
\hline \multicolumn{3}{|l|}{ HPP, 1,chend } \\
\hline 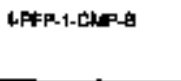 & 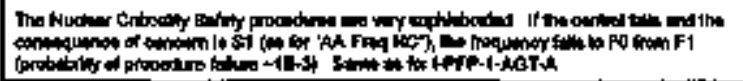 & \\
\hline \multicolumn{3}{|l|}{ APFP-1-CXP-C } \\
\hline LFPP-1-CINP-D & 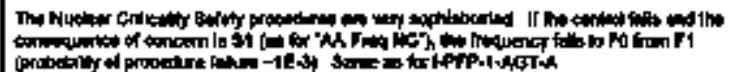 & \\
\hline
\end{tabular}


Tabte A-s. Master Cods Tably - Part 2. (Bo Ethoda)

\begin{tabular}{|c|c|c|}
\hline 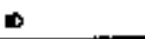 & Smran Fan wento & chepontulo \\
\hline |PPP.|-CAPP.E & 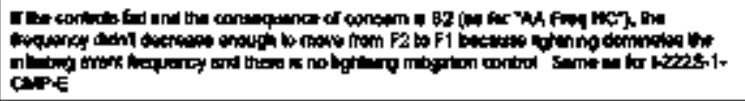 & \\
\hline |-mp.|.เทn-A & 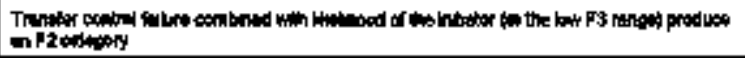 & \\
\hline \multicolumn{3}{|l|}{ 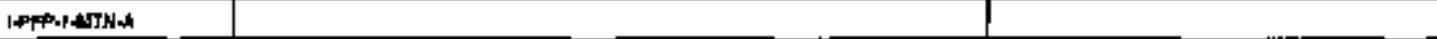 } \\
\hline \multicolumn{3}{|l|}{ HPF-2ARWh } \\
\hline \multicolumn{3}{|l|}{ IPFP-ZUEKA } \\
\hline IPPR-ZUEK- & 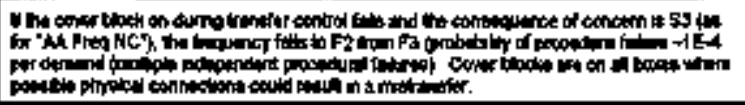 & \\
\hline IPPPAZENAC & 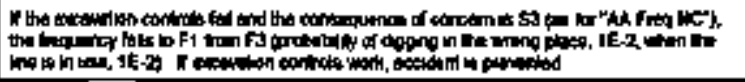 & 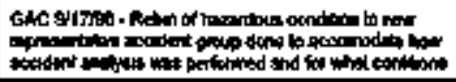 \\
\hline \multicolumn{3}{|l|}{ LPAP,ZIEKGD } \\
\hline LPFPUZLEKKE & 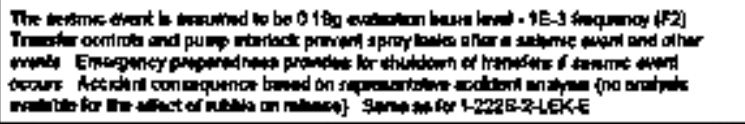 & \\
\hline \multicolumn{3}{|l|}{ LPPP-ITPA } \\
\hline MFPdTh: & 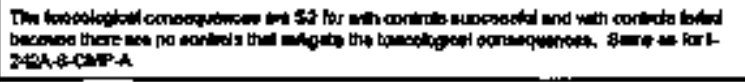 & \\
\hline \multicolumn{3}{|l|}{ 1efpuscanpin } \\
\hline |APFB3fLWA & & \\
\hline
\end{tabular}


Toble A-s. Maser Coda Table - Pand 2. k30 aheotal

\begin{tabular}{|c|c|c|}
\hline 10 & 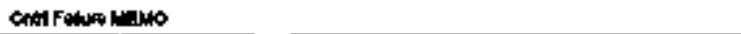 & 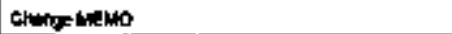 \\
\hline LAPesphyth & 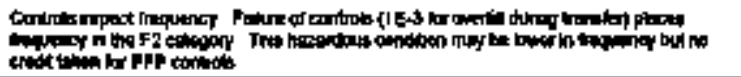 & \\
\hline \multicolumn{3}{|l|}{ LFPPAstexkath } \\
\hline HFF-WLEKGB & 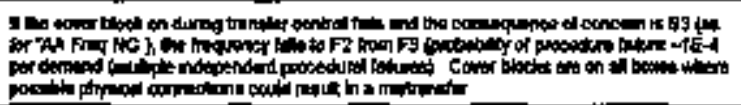 & \\
\hline HFP-HEXC & 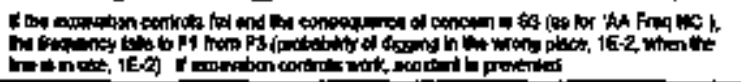 & 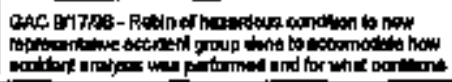 \\
\hline \multicolumn{3}{|l|}{ LPFP-HExo } \\
\hline PPFP-HEKE & 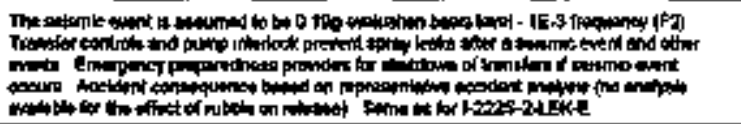 & \\
\hline \multicolumn{3}{|l|}{ 1PUMEX,1 AGTN } \\
\hline 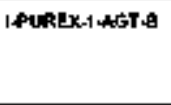 & 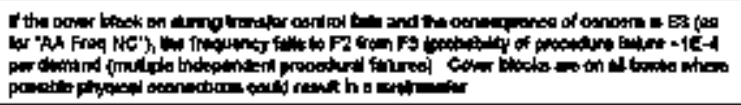 & \\
\hline \multicolumn{3}{|l|}{ |PUVEE-1-ASTT-C } \\
\hline \multicolumn{3}{|l|}{ 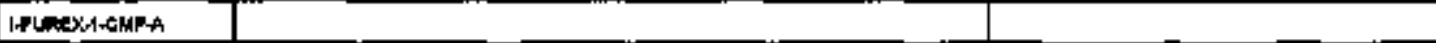 } \\
\hline 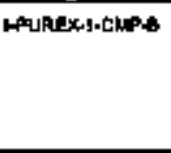 & 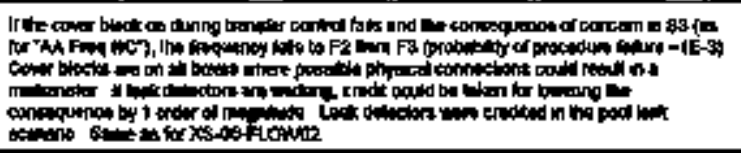 & \\
\hline LPUREXX-1-CAPAC & & \\
\hline
\end{tabular}




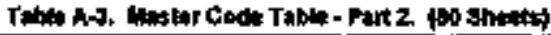

\begin{tabular}{|c|c|c|}
\hline $\mathbf{0}$ & 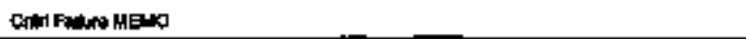 & קטבאו \\
\hline \multicolumn{3}{|l|}{ 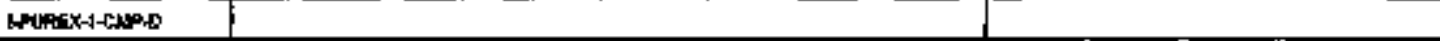 } \\
\hline Lever: & 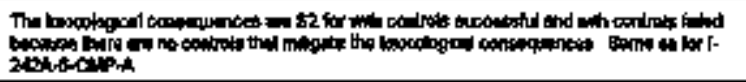 & \\
\hline IPUREX-1,Th=-A & 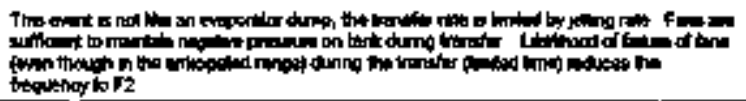 & \\
\hline \multicolumn{3}{|l|}{ 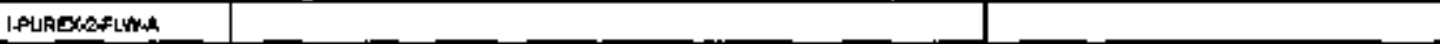 } \\
\hline \multicolumn{3}{|l|}{ 1PUREX-2AEK-A } \\
\hline PPUREK-2HEK-8 & 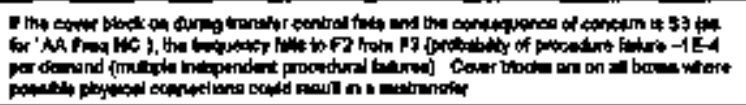 & \\
\hline 1puex-2urxect & 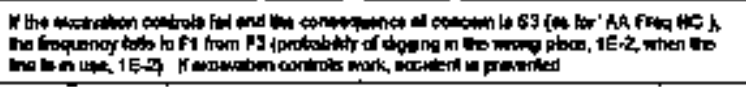 & \\
\hline \multicolumn{3}{|l|}{ HMREXN-LEX-D } \\
\hline 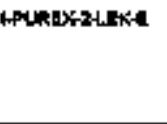 & 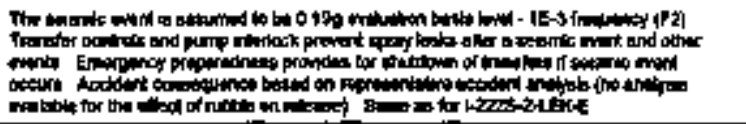 & \\
\hline HPUPEXWPRBA & 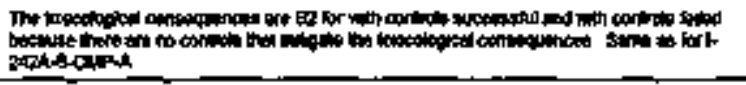 & \\
\hline 1-PIDEx-2PPA-B & 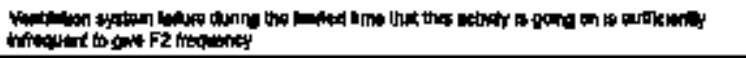 & \\
\hline 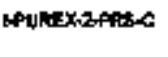 & & 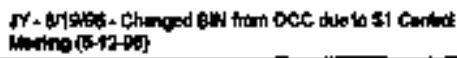 \\
\hline IPUATX-3ACTA & & \\
\hline
\end{tabular}




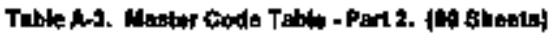

\begin{tabular}{|c|c|c|}
\hline $\boldsymbol{q}$ & 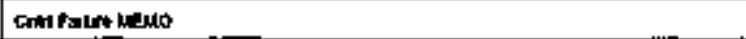 & 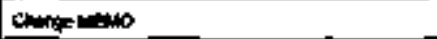 \\
\hline HPUREX-3AGT-B & 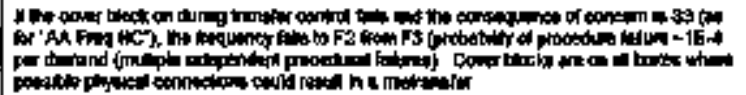 & \\
\hline \multicolumn{3}{|l|}{ APUL:AXAGT-C } \\
\hline \multicolumn{3}{|l|}{ 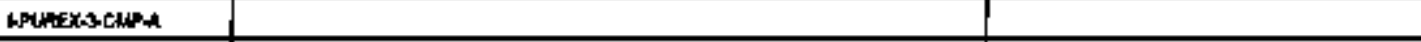 } \\
\hline 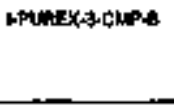 & 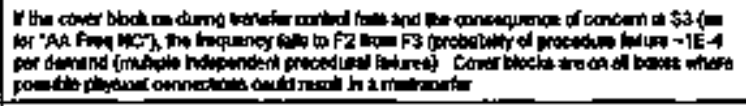 & \\
\hline \multicolumn{3}{|l|}{ 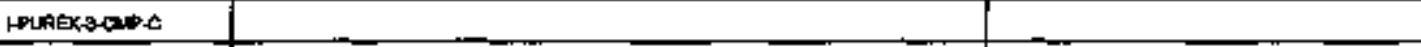 } \\
\hline \multicolumn{3}{|l|}{ 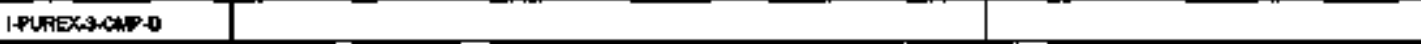 } \\
\hline Ietragkstane & 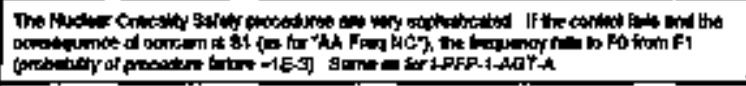 & \\
\hline IDUREX-S-ALA-A & 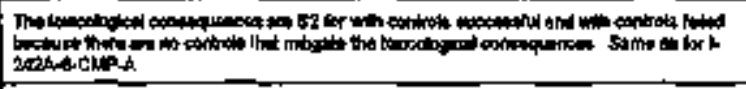 & \\
\hline |PLREXE-TRPA & 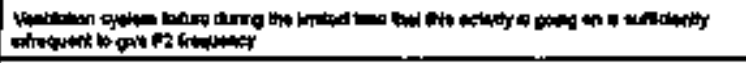 & \\
\hline LFUREX_AfLYH-日 & Boundod by Vhetepactes 1 & \\
\hline \multicolumn{3}{|l|}{ LEUREX,4LEKA } \\
\hline HPAREX-1RK-0 & 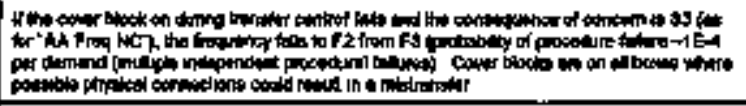 & \\
\hline 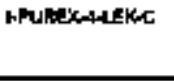 & 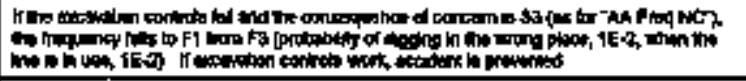 & 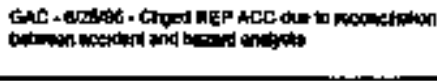 \\
\hline HPUNTX-1EKO & & \\
\hline
\end{tabular}




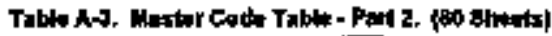

\begin{tabular}{|c|c|c|}
\hline 10 & 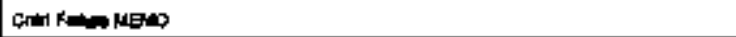 & CWongukEus \\
\hline IPUREGHEKEE & 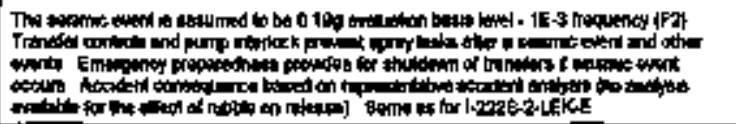 & \\
\hline LPLREX-PPAg & 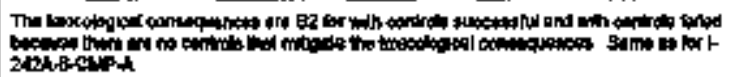 & \\
\hline 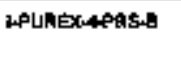 & 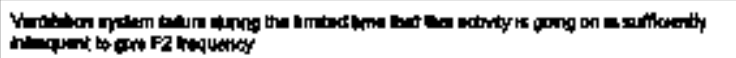 & \\
\hline HPLRBXAPRS-C & & 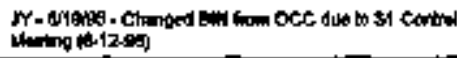 \\
\hline HHREXS-Fum & 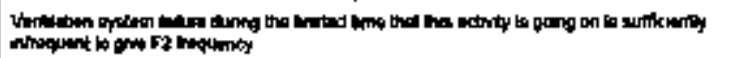 & \\
\hline \multicolumn{3}{|l|}{ Hmpexerturs } \\
\hline \multicolumn{3}{|l|}{ MPUNEXANUYA } \\
\hline \multicolumn{3}{|l|}{ IPUPEX E-FUHA } \\
\hline \multicolumn{3}{|l|}{ IPUREX-FEWW } \\
\hline \multicolumn{3}{|l|}{ 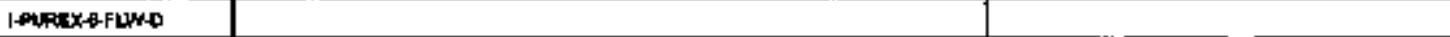 } \\
\hline \multicolumn{3}{|l|}{ 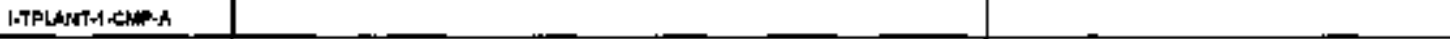 } \\
\hline LTALANT1-CHAPS9 & 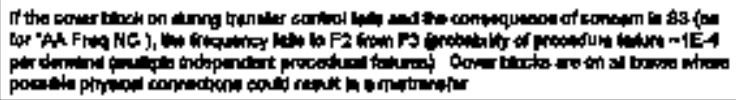 & \\
\hline \multicolumn{3}{|l|}{ 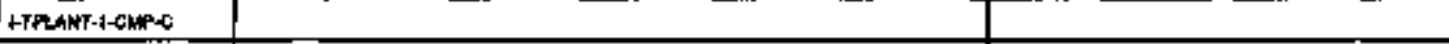 } \\
\hline \multicolumn{3}{|l|}{ 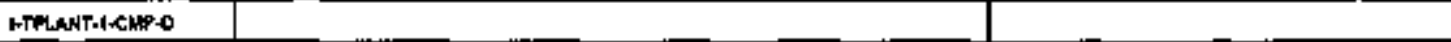 } \\
\hline 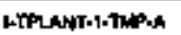 & & \\
\hline
\end{tabular}


Table A-3. Masker Godk Table - Pert 2. (CN slands)

\begin{tabular}{|c|c|c|}
\hline ID & 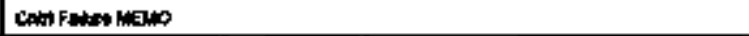 & 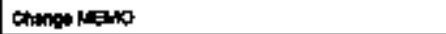 \\
\hline 1.TPLAMUACMPAA & 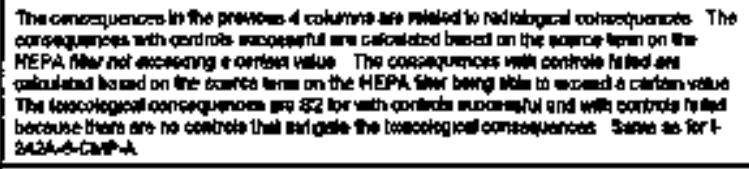 & \\
\hline \multicolumn{3}{|l|}{ LTFUANTERLLLA } \\
\hline \multicolumn{3}{|l|}{ 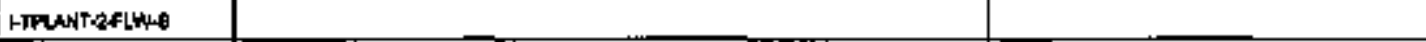 } \\
\hline \multicolumn{3}{|l|}{ HPLART-ZUEKA } \\
\hline HTPLANTIHFKA & 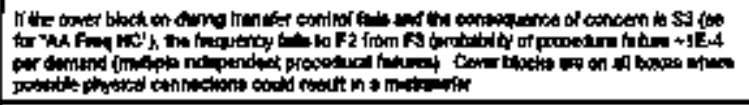 & \\
\hline คTPLAT-ZHEKC & 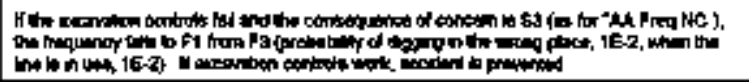 & 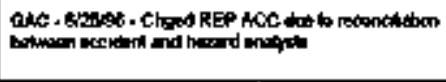 \\
\hline \multicolumn{3}{|l|}{ LTMUNT-ZUEKED } \\
\hline ITPLATRALEKE & 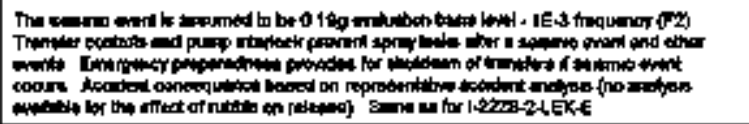 & \\
\hline \multicolumn{3}{|l|}{ |MELNIT-1FLWA } \\
\hline \multicolumn{3}{|l|}{ IAPLANT-1AEKA } \\
\hline LWWWNTIAESA & 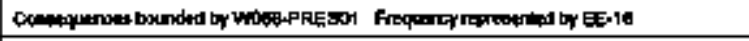 & \\
\hline \multicolumn{3}{|l|}{ HuPANT-1-Lekc } \\
\hline \multicolumn{3}{|l|}{ HQPLAT-1-RWAA } \\
\hline ITka1 & & 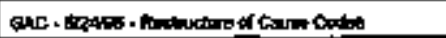 \\
\hline
\end{tabular}




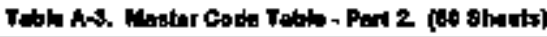

\begin{tabular}{|c|c|c|}
\hline $\mathbf{\Delta}$ & Cont Fann MESTo & 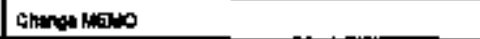 \\
\hline $\mid \pi<001+1$ & 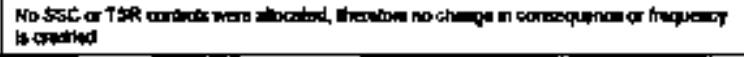 & \\
\hline ITKala & & 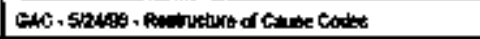 \\
\hline \multicolumn{3}{|l|}{$|\pi k 02|$} \\
\hline 1 I & 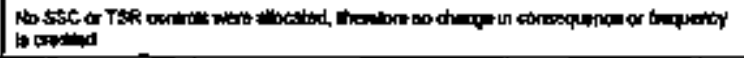 & \\
\hline rTKoss & 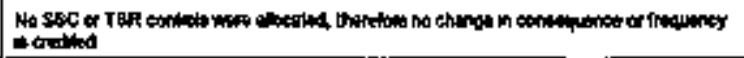 & \\
\hline तार-OQ & 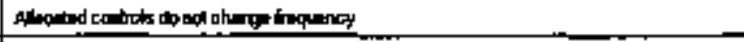 & 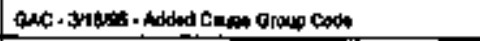 \\
\hline \multicolumn{3}{|l|}{$\pi \mathrm{k}-041$} \\
\hline ITKas & 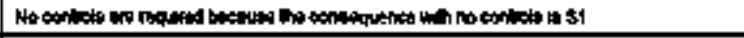 & \\
\hline \multicolumn{3}{|l|}{ moses } \\
\hline nxts & 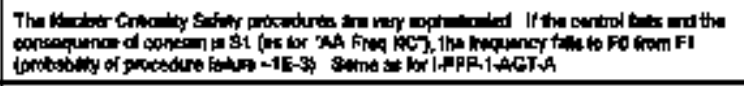 & \\
\hline moser & 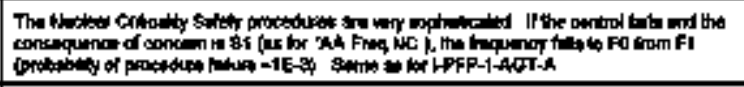 & 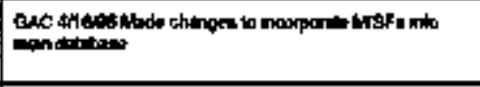 \\
\hline Incor & 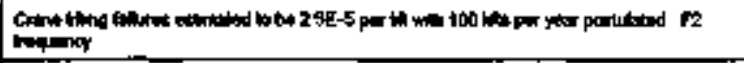 & \\
\hline \multicolumn{3}{|l|}{ ITK-0\% \# } \\
\hline \multicolumn{3}{|l|}{ In<orts } \\
\hline mK-06 & 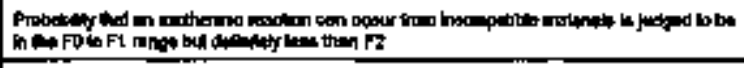 & 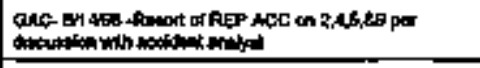 \\
\hline rTKos-I & 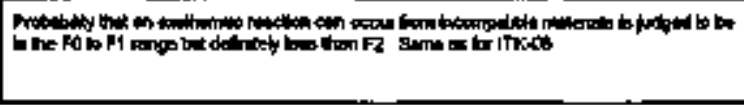 & 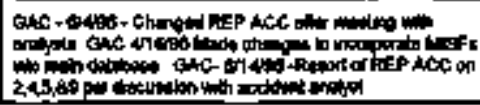 \\
\hline
\end{tabular}




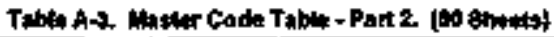

\begin{tabular}{|c|c|c|}
\hline D & 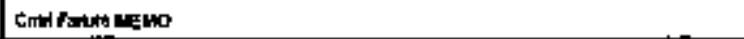 & chrof mand \\
\hline$\pi k-00$ & 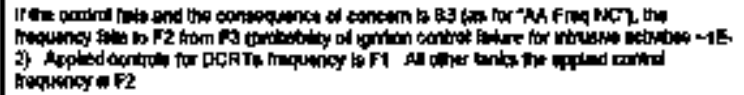 & 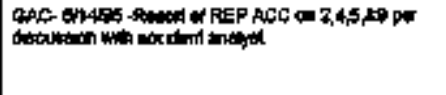 \\
\hline mKDool & 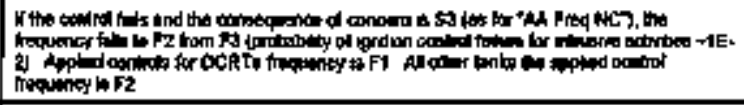 & 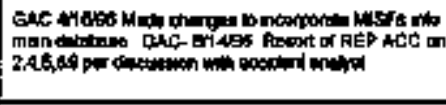 \\
\hline ITK-10 & 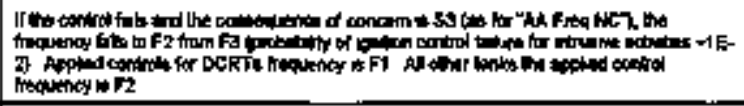 & 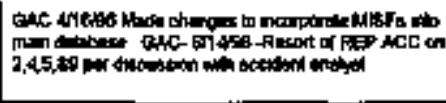 \\
\hline Ithe-100 & 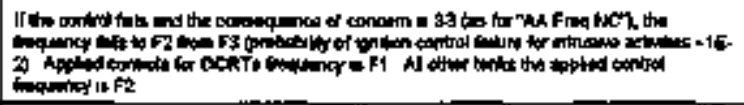 & 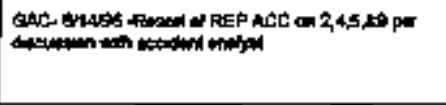 \\
\hline mK-10b & 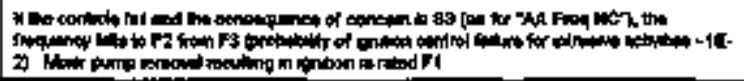 & 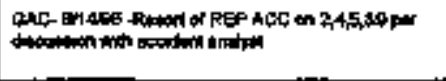 \\
\hline ImKa11 & 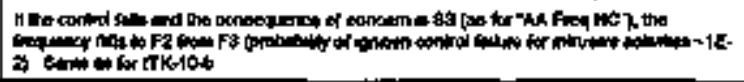 & 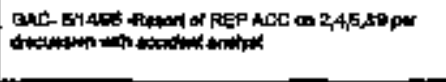 \\
\hline sthosid & 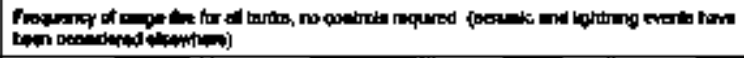 & 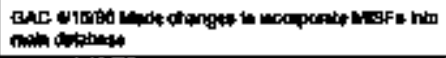 \\
\hline TKKFR-MI & 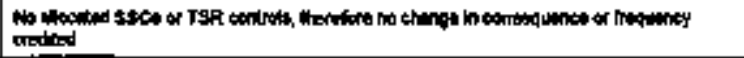 & \\
\hline TKEFR-02 & 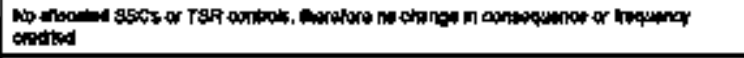 & \\
\hline Thuspos & 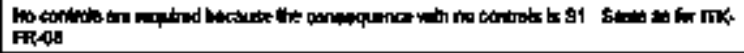 & 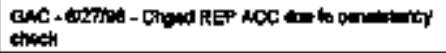 \\
\hline
\end{tabular}


Tabla A-s. Muster Cods Table - Part 2 (W Atrete)

\begin{tabular}{|c|c|c|}
\hline b & 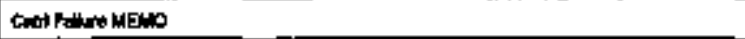 & 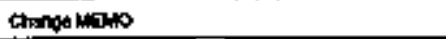 \\
\hline mathor & 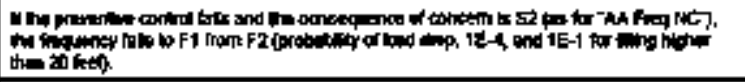 & \\
\hline ImK-Fand & 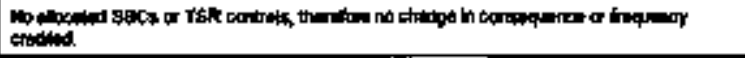 & \\
\hline TK-FR-QB & 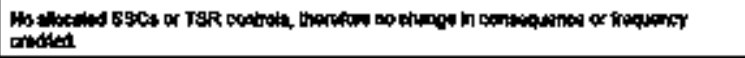 & \\
\hline ITK-FA-0S & 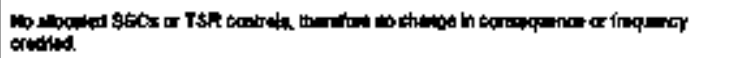 & \\
\hline InKSRER & 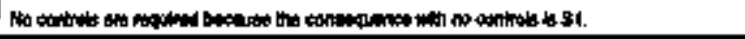 & \\
\hline \multicolumn{3}{|l|}{ mKNRAO } \\
\hline \multicolumn{3}{|l|}{ TTKAR-10 } \\
\hline \multicolumn{3}{|l|}{ TKKFR11 } \\
\hline \multicolumn{3}{|l|}{ mK+R-12 } \\
\hline mKroks 13 & 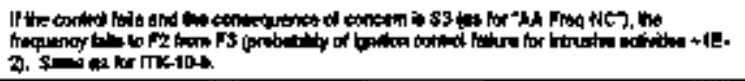 & 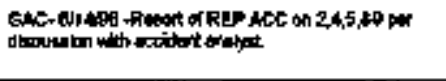 \\
\hline mKefR-14 & 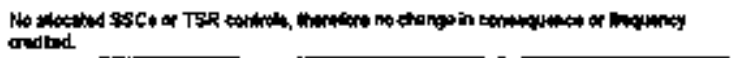 & \\
\hline MK-FR-15 & 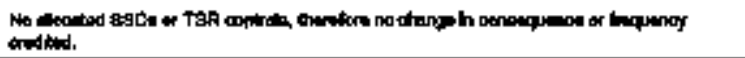 & \\
\hline \multicolumn{3}{|l|}{ ITKFR+16 } \\
\hline \multicolumn{3}{|l|}{ IIKFFAt } \\
\hline ITK-FR-18 & 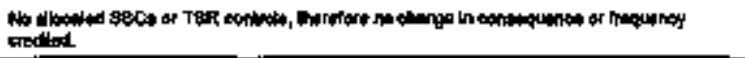 & \\
\hline mo.FR-16 & 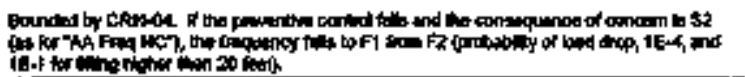 & 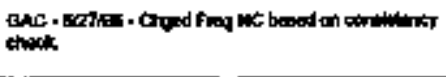 \\
\hline
\end{tabular}




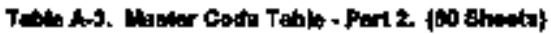

\begin{tabular}{|c|c|c|}
\hline $\boldsymbol{b}$ & 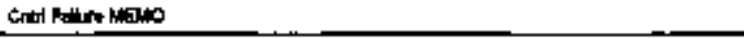 & Chenge MERO \\
\hline Therag & 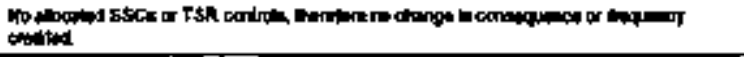 & \\
\hline mask-21 & 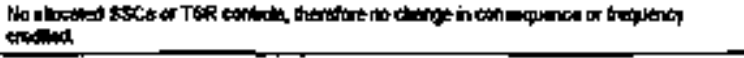 & \\
\hline MKK-fit-22 & 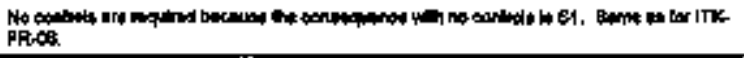 & 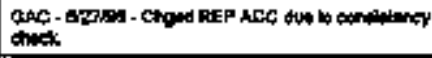 \\
\hline Thaskis & 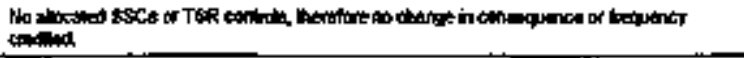 & \\
\hline \multicolumn{3}{|l|}{ ITK-FR-24 } \\
\hline \multicolumn{3}{|l|}{ Internzs } \\
\hline InKatrat & 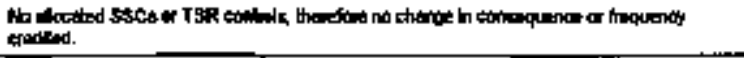 & \\
\hline \multicolumn{3}{|l|}{ TKFR-Z7 } \\
\hline ITKGTREA & 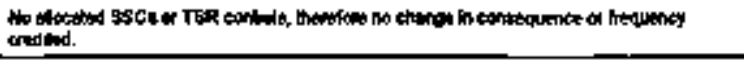 & \\
\hline NRAI & 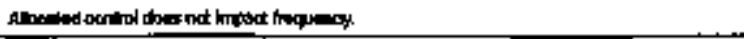 & \\
\hline NPan & 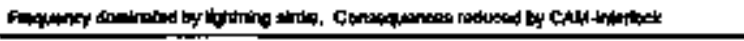 & \\
\hline NP-OS & 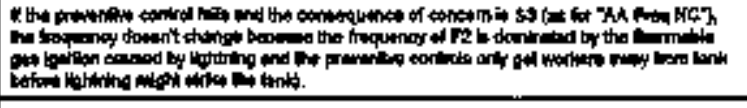 & \\
\hline prats & 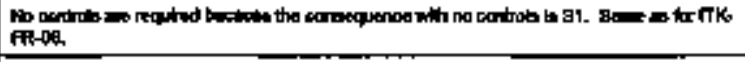 & \\
\hline mos & 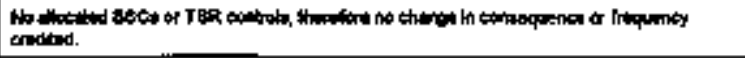 & \\
\hline \multicolumn{3}{|l|}{ Patw } \\
\hline $10-68$ & 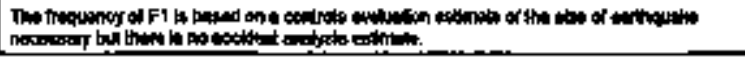 & \\
\hline
\end{tabular}




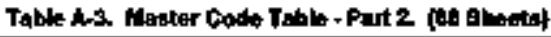

\begin{tabular}{|c|c|c|}
\hline ID & Cort Filan Man & ing \\
\hline \multicolumn{3}{|l|}{$19-00$} \\
\hline \multicolumn{3}{|l|}{$N P-10$} \\
\hline \multicolumn{3}{|l|}{$N p-11$} \\
\hline NP=12 & 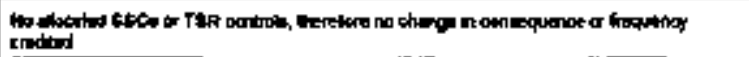 & \\
\hline NP-13 & 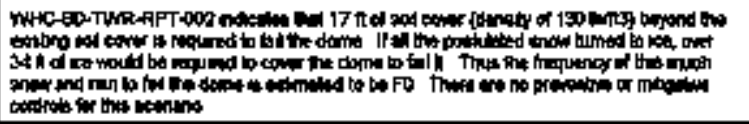 & \\
\hline NP.14 & 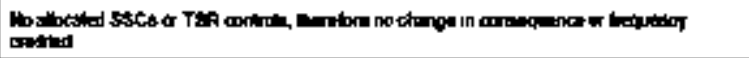 & \\
\hline NP,15 & 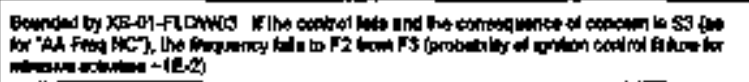 & 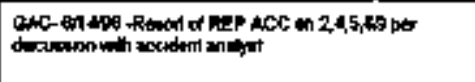 \\
\hline DQPT-EXT3-O1A & 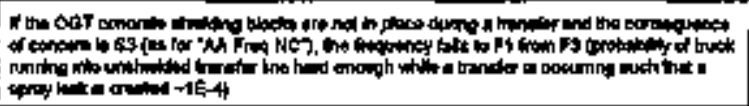 & 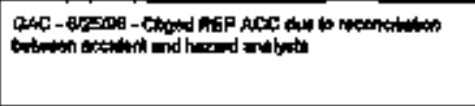 \\
\hline OET-EXTß-019 & 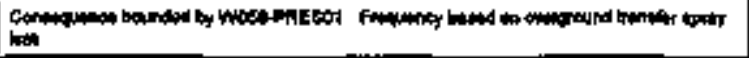 & 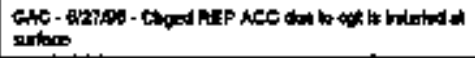 \\
\hline oparflowhis & 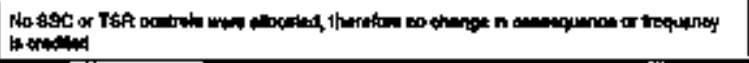 & 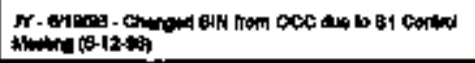 \\
\hline MGT.PLONHEZ & 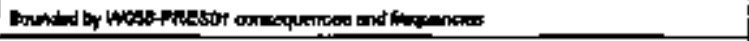 & \\
\hline ORT-FLOWridos & 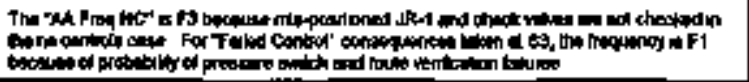 & 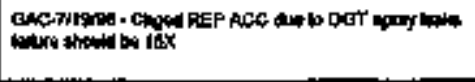 \\
\hline CXT-rowride & 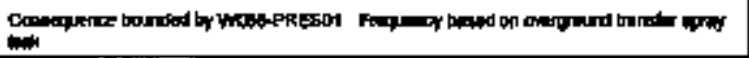 & 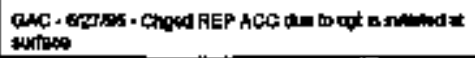 \\
\hline
\end{tabular}




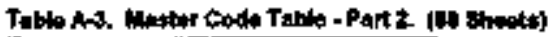

\begin{tabular}{|c|c|c|}
\hline $\mathbf{D}$ & 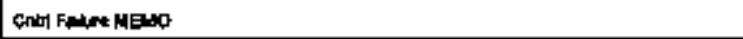 & Chwoph naw \\
\hline ootfroun-04 & 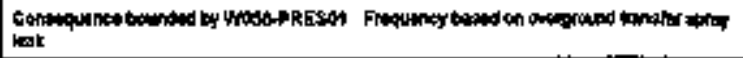 & 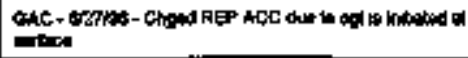 \\
\hline \multicolumn{3}{|l|}{ OGTा HOWW1-0S } \\
\hline DOT-Rormas & 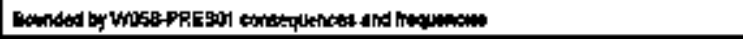 & \\
\hline OOTROOW-O1A & 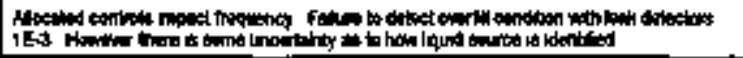 & \\
\hline ogTfledw2018 & 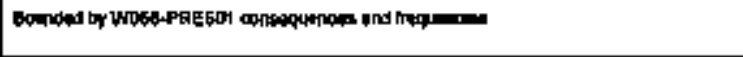 & 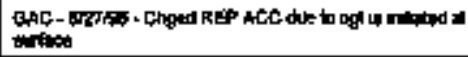 \\
\hline DeTflotring & 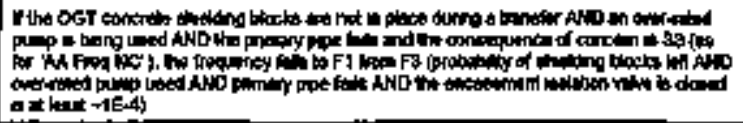 & 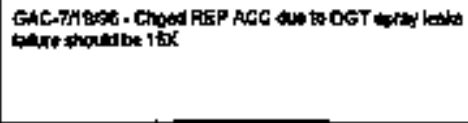 \\
\hline DGT-PLDNZTEM & 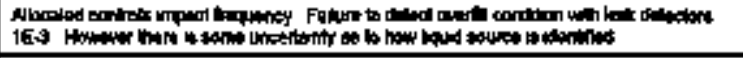 & \\
\hline OCT.FLON203: & 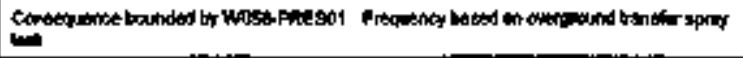 & 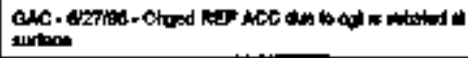 \\
\hline \multicolumn{3}{|l|}{ 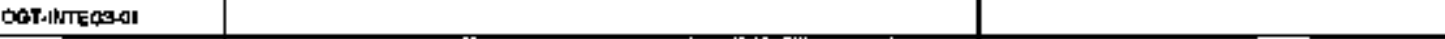 } \\
\hline ORT-NPSOA & 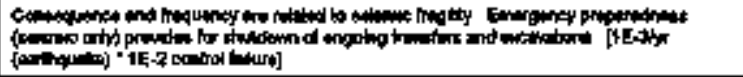 & 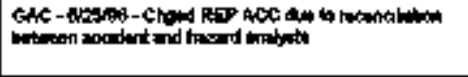 \\
\hline Cotr-rasos & 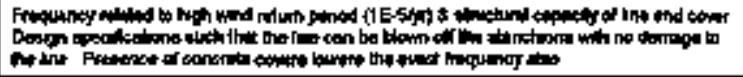 & 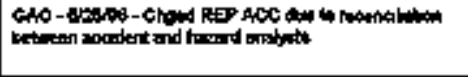 \\
\hline OOT-OPST-O1 & 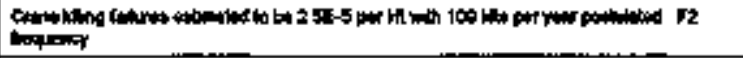 & \\
\hline OGTPREG1QI & 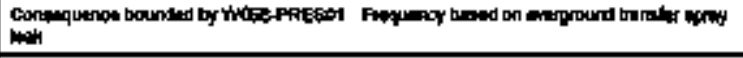 & \\
\hline OOTFEAC1-OM & 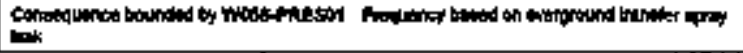 & \\
\hline
\end{tabular}




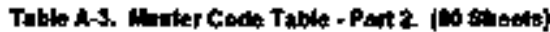

\begin{tabular}{|c|c|c|}
\hline ID & Ertat fahre Mano & 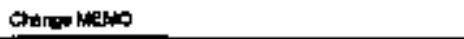 \\
\hline LXT-SPEC, & 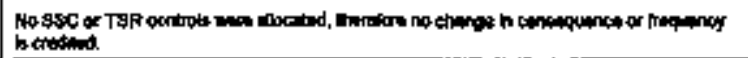 & 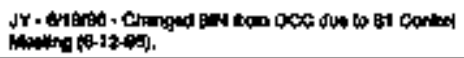 \\
\hline QX3T-TEMP1401 & & 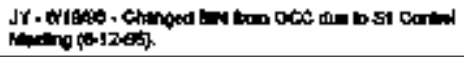 \\
\hline ФकT-TEMPT- & 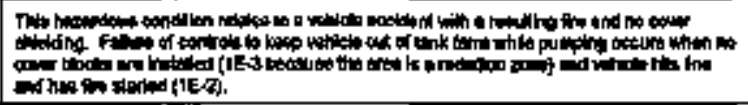 & 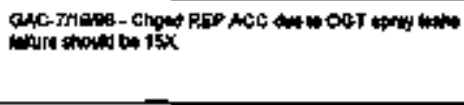 \\
\hline OST,TERFIOS & 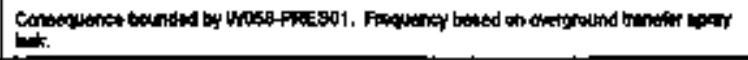 & \\
\hline Aato-OH & & 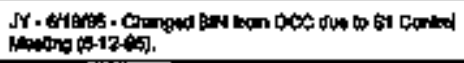 \\
\hline 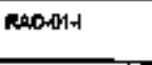 & & 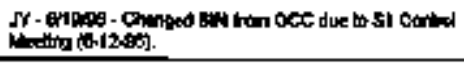 \\
\hline paspos & & 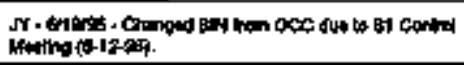 \\
\hline \multicolumn{3}{|l|}{ Danas.1 } \\
\hline nowas & & 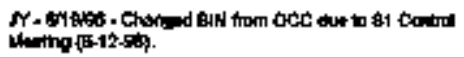 \\
\hline PAD-19-1 & & $\cdot$ \\
\hline ntan-or & 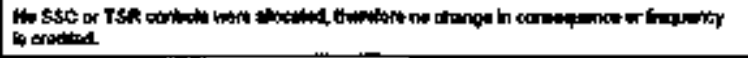 & 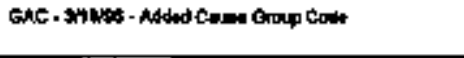 \\
\hline R\$R-01-I & 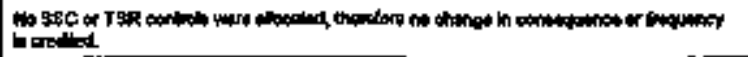 & \\
\hline \multicolumn{3}{|l|}{ 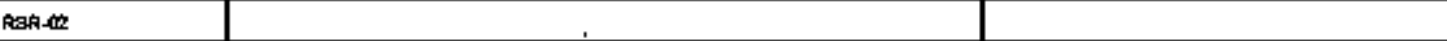 } \\
\hline Ras-an-1 & & 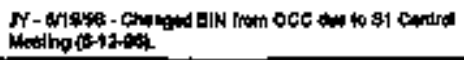 \\
\hline NGALS & & \\
\hline
\end{tabular}




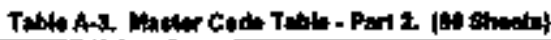

\begin{tabular}{|c|c|c|}
\hline 田 & 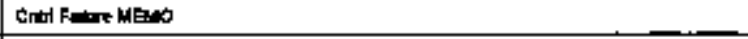 & charqu wetho \\
\hline RER-03 & & 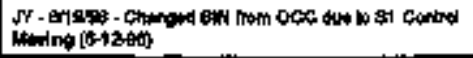 \\
\hline FERTOA & & 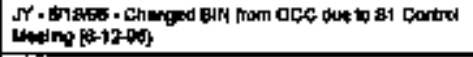 \\
\hline RAsta-1 & & 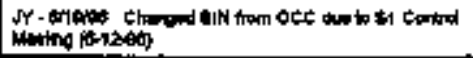 \\
\hline \multicolumn{3}{|l|}{ RqBuntos } \\
\hline \multicolumn{3}{|l|}{ PFPR-65-LA } \\
\hline \multicolumn{3}{|l|}{ RSP-CSAB } \\
\hline nsthot & 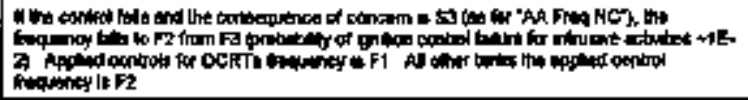 & 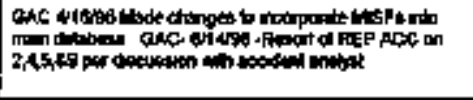 \\
\hline nereofd & 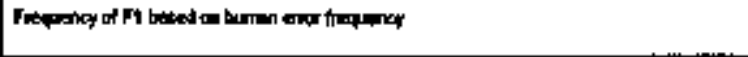 & 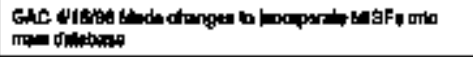 \\
\hline \multicolumn{3}{|l|}{ RSR-0A } \\
\hline SArot & 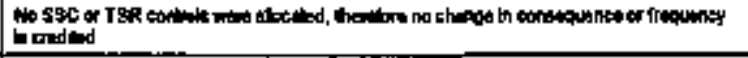 & 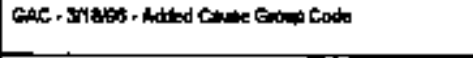 \\
\hline \multicolumn{3}{|l|}{ EFF-01-4 } \\
\hline nes.1 & 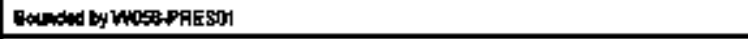 & 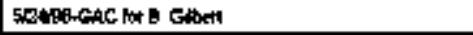 \\
\hline verar & 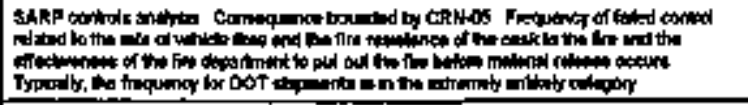 & \\
\hline VEH-QII-I & 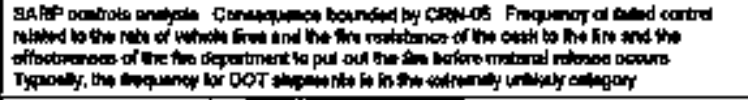 & 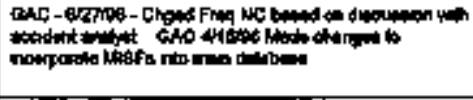 \\
\hline Wertor & 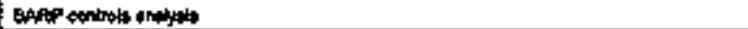 & 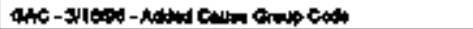 \\
\hline
\end{tabular}




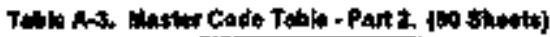

\begin{tabular}{|c|c|c|}
\hline ט & 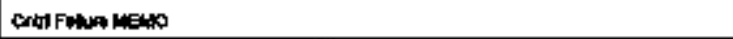 & Chanes Nans \\
\hline VEH-OWA & 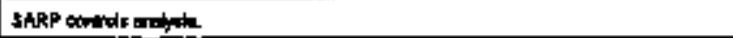 & \\
\hline Vem-cos & SNkP contoda freptit. & \\
\hline Vetwo3- & SAAP anitios metols. & 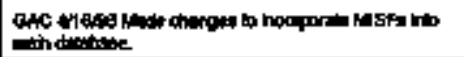 \\
\hline Vertod & & 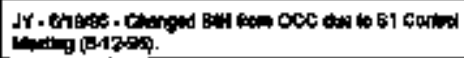 \\
\hline vaHod| & & 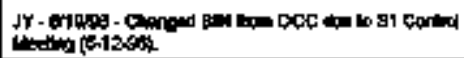 \\
\hline V2H05 & $\cdot$ & \\
\hline \multicolumn{3}{|l|}{ VENos. } \\
\hline \multicolumn{3}{|l|}{ vêtat } \\
\hline \multicolumn{3}{|l|}{ พ타여이이 } \\
\hline \multicolumn{3}{|l|}{ พघ40 } \\
\hline Yerofal & 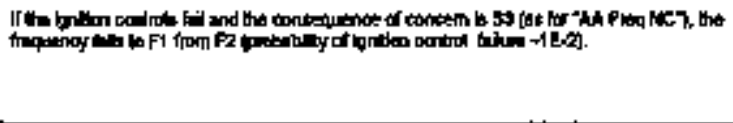 & 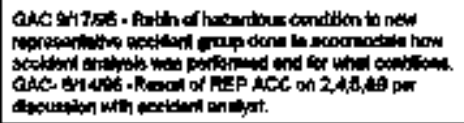 \\
\hline \multicolumn{3}{|l|}{ WEHOSH } \\
\hline Ysuar & 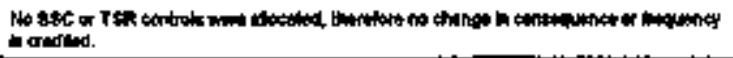 & \\
\hline WMLसम & 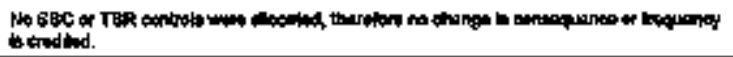 & \\
\hline vellow & 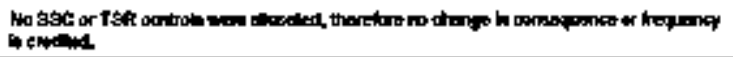 & \\
\hline veror & 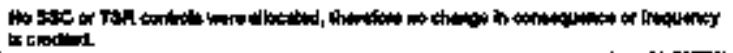 & \\
\hline
\end{tabular}




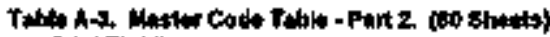

\begin{tabular}{|c|c|c|}
\hline tro & Cmir Fatro MGes & Crenge Mex \\
\hline \multicolumn{3}{|l|}{ Yoves } \\
\hline \multicolumn{3}{|l|}{ vsumbs } \\
\hline WDSPflower & 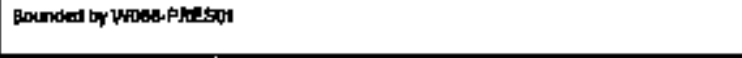 & 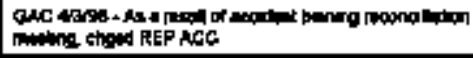 \\
\hline WNSSFLOWOS & 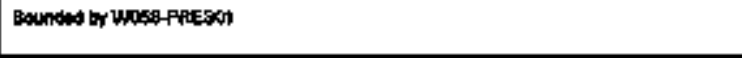 & 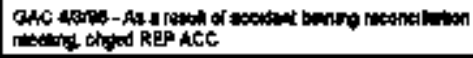 \\
\hline wocesfounted & 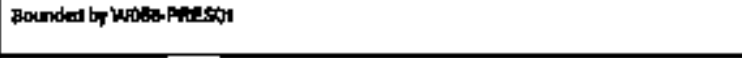 & 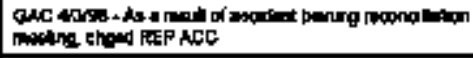 \\
\hline mosiflotws & 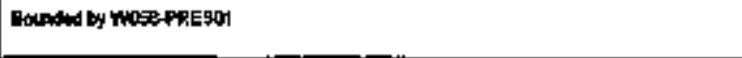 & 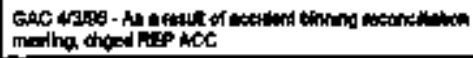 \\
\hline 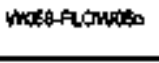 & Goundad by whoteretor & 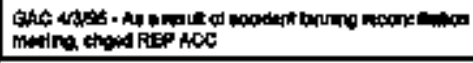 \\
\hline incesplotudr & Bouldod by Wess-PREFy & 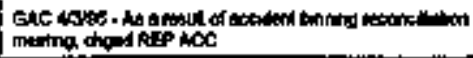 \\
\hline Mrse-flomos & Banded by hesapaEgor & 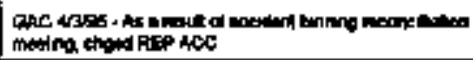 \\
\hline Woosnow1s & Bounted by nese-PRESOM & 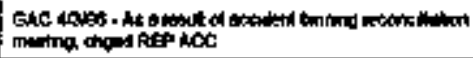 \\
\hline Mosp-Rowils & 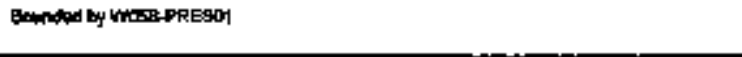 & 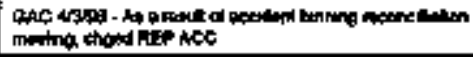 \\
\hline wostFLotw11a & 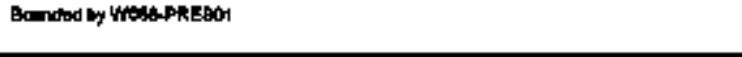 & 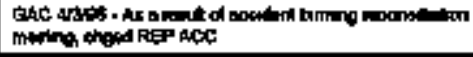 \\
\hline 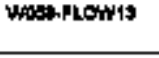 & 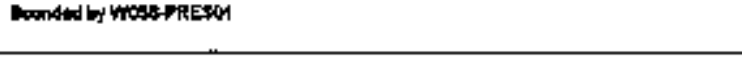 & 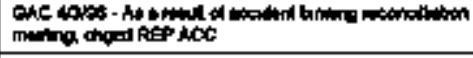 \\
\hline WOSE-FLONaG & 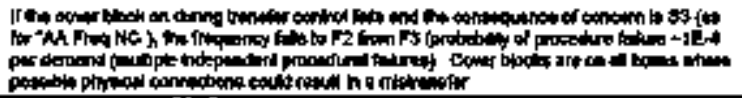 & \\
\hline
\end{tabular}




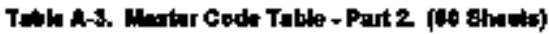

\begin{tabular}{|c|c|c|}
\hline ID & 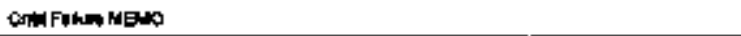 & Chanos MENO \\
\hline 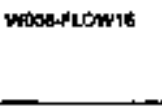 & 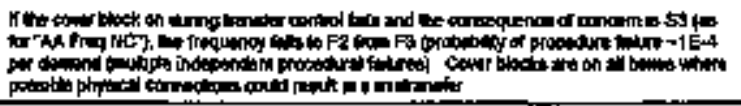 & \\
\hline Woss-row170 & 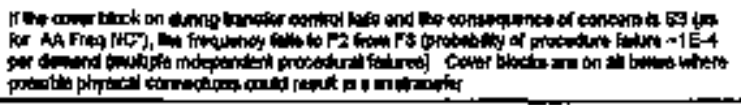 & \\
\hline WOE-FLOWITb & 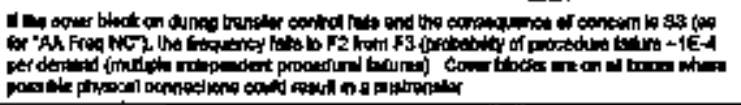 & \\
\hline nNostFLowITS & 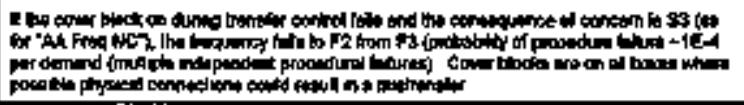 & \\
\hline nosteflow1: & 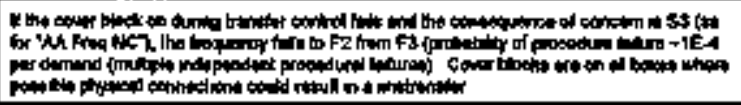 & \\
\hline westadowite & 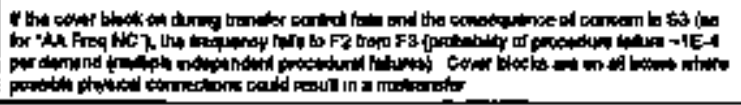 & \\
\hline motgarlowpan & 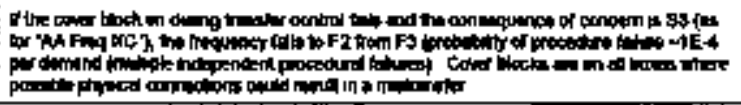 & \\
\hline mose+towne & 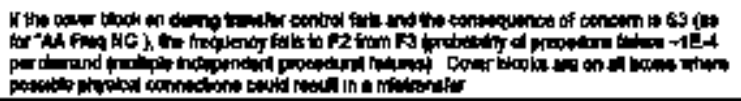 & 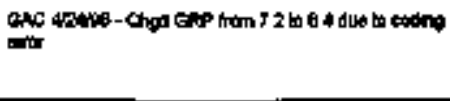 \\
\hline
\end{tabular}




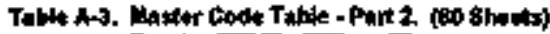

\begin{tabular}{|c|c|c|}
\hline $\mathbf{p}$ & 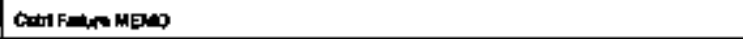 & Congatiento \\
\hline ntseflownot & 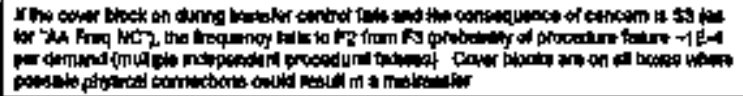 & 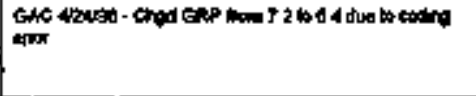 \\
\hline Whadountecols & 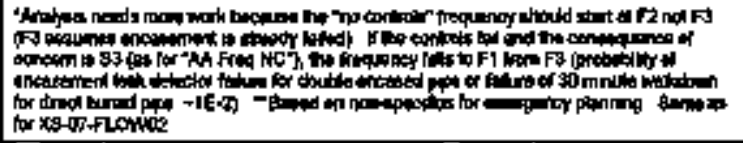 & 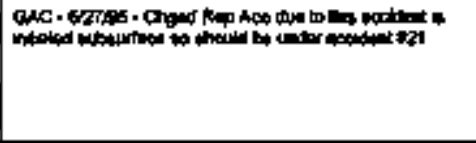 \\
\hline WA68-ANTECOIL & 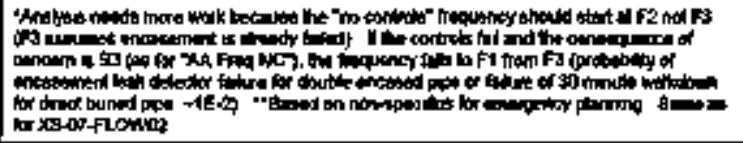 & 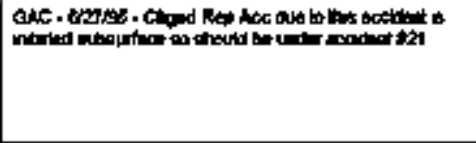 \\
\hline wosg-numerote & 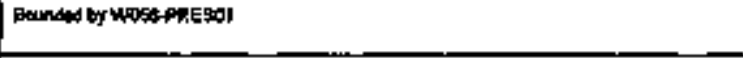 & 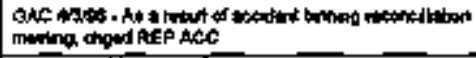 \\
\hline WHEWWTEEMd & 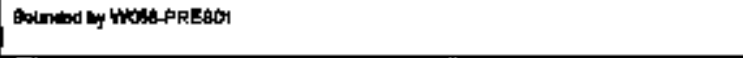 & 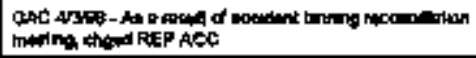 \\
\hline MOSO-WTELP3: & 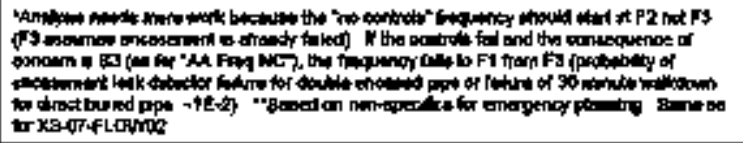 & 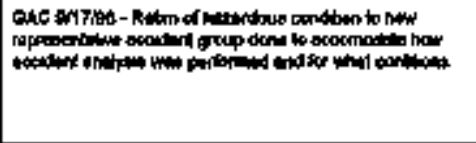 \\
\hline Mose-WTEODSt & 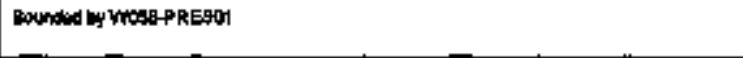 & 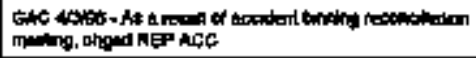 \\
\hline Mropenteisosa & 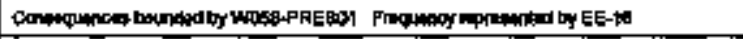 & \\
\hline WUSPHИTECOS: & 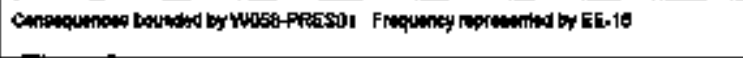 & 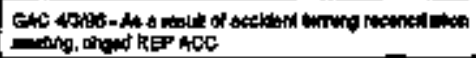 \\
\hline
\end{tabular}


Tuble A-3, Metter Cods Tablo - Part 2 (at sheds)

\begin{tabular}{|c|c|c|}
\hline $\boldsymbol{\omega}$ & 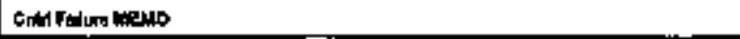 & Cherenterso \\
\hline WoktwTEGoh & 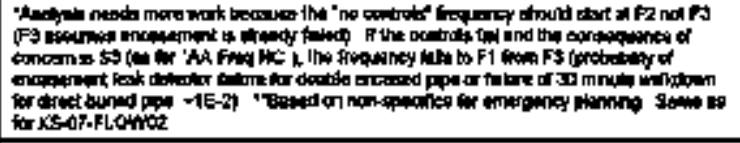 & 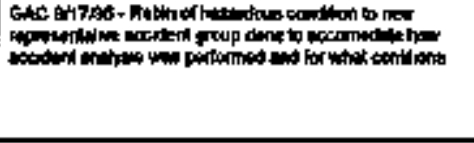 \\
\hline WosB-NHTEGOTh & 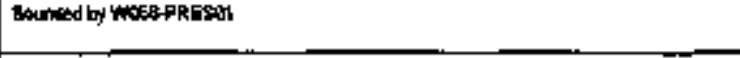 & 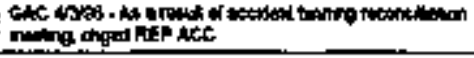 \\
\hline MOSB-ATECO:A & 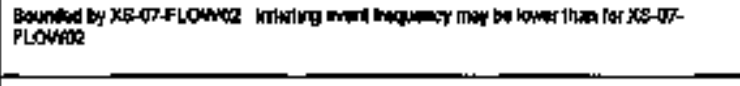 & 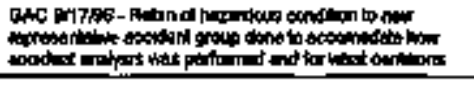 \\
\hline 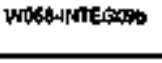 & 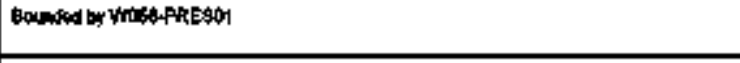 & 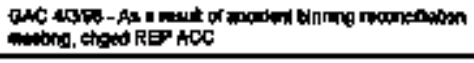 \\
\hline MOSSAMTEEI1: & 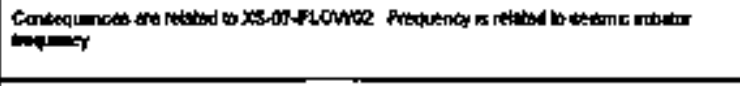 & 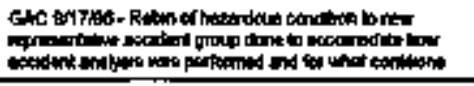 \\
\hline W0SB-1nTeGitb & 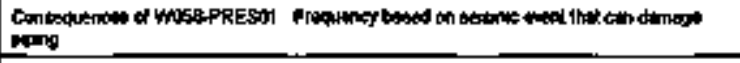 & 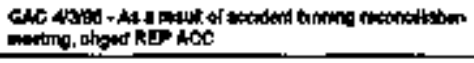 \\
\hline WOLGWTER13 & 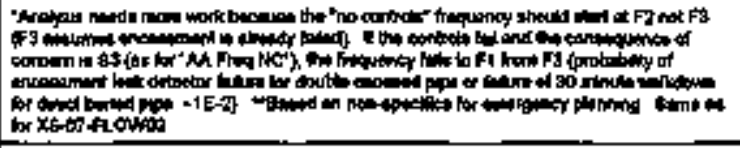 & 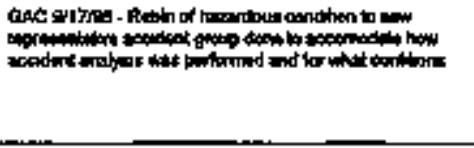 \\
\hline WOKOWTEBAB & 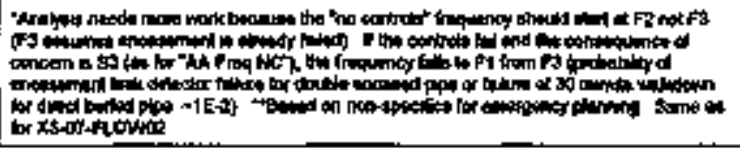 & 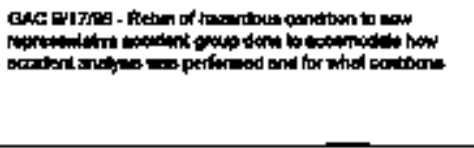 \\
\hline HetsenTEC130 & Bondied by mociofiesph & 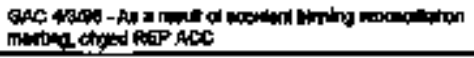 \\
\hline
\end{tabular}




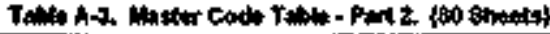

\begin{tabular}{|c|c|c|}
\hline ID & 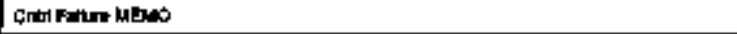 & Ganf ravo \\
\hline W0E- WTEO13: & Benninctor wosg fatesol & 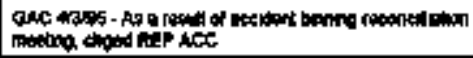 \\
\hline MOP-NIEGA4 & 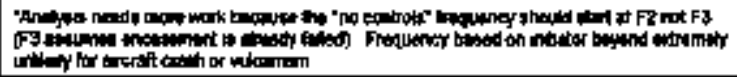 & 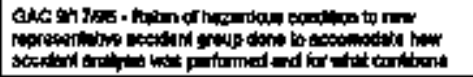 \\
\hline 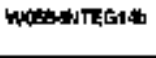 & 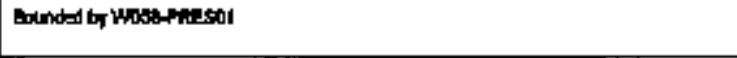 & 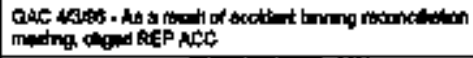 \\
\hline HESB-NTEGAB & 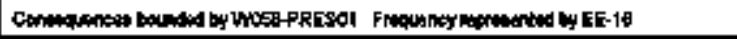 & \\
\hline HESE-ATTESIII & 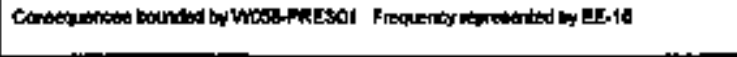 & 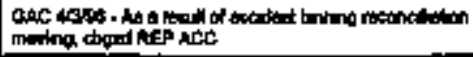 \\
\hline 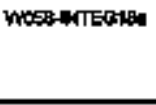 & 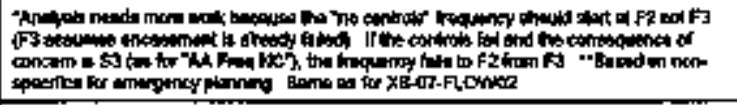 & 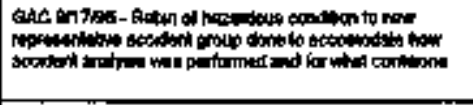 \\
\hline WEPHNTEGM & Counded by W0OPREQPI & 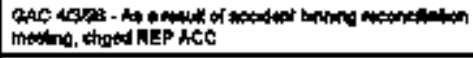 \\
\hline WDESARTEOSO- & 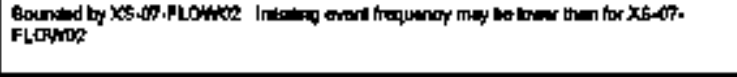 & 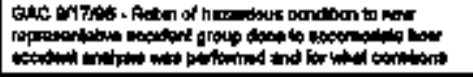 \\
\hline WOCHATERAG & 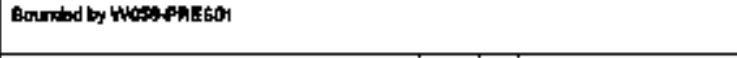 & 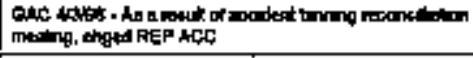 \\
\hline WDS-HTEAR & Conton & 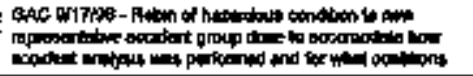 \\
\hline Woss-1wTro2d & 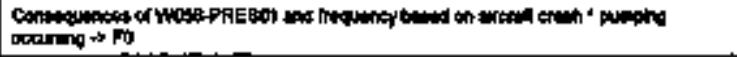 & 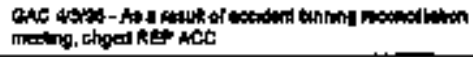 \\
\hline WOSSHNTERa & 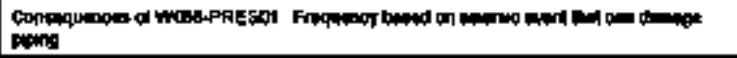 & 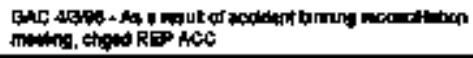 \\
\hline Wistinteack & 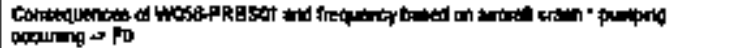 & 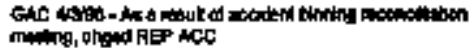 \\
\hline
\end{tabular}




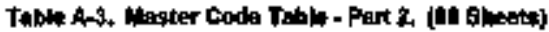

\begin{tabular}{|c|c|c|}
\hline $\mathbf{0}$ & 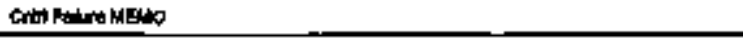 & Aronolingen \\
\hline MOPBATECOSO & 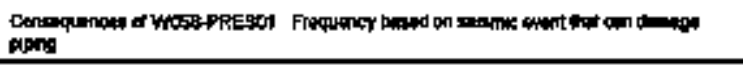 & 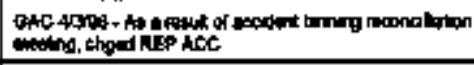 \\
\hline 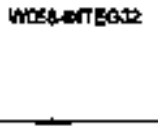 & 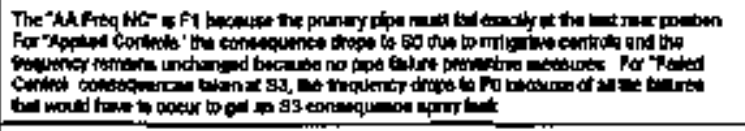 & 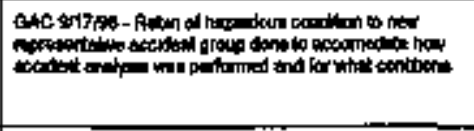 \\
\hline WMSPHNEOMA & 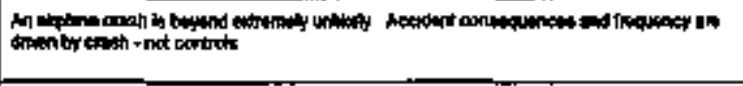 & 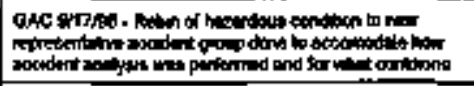 \\
\hline wasphrmeast & 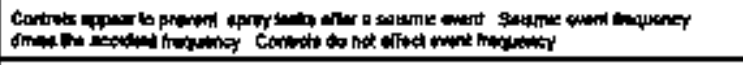 & \\
\hline 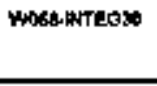 & 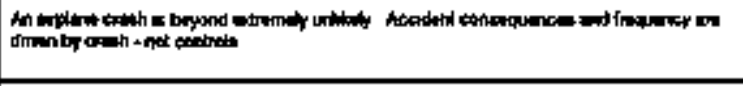 & 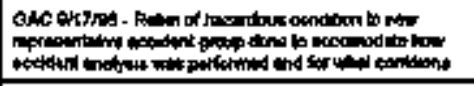 \\
\hline Mosentuos? & 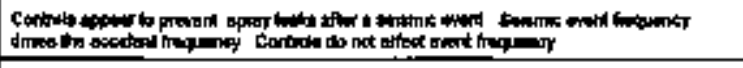 & \\
\hline WOEAFFE: & 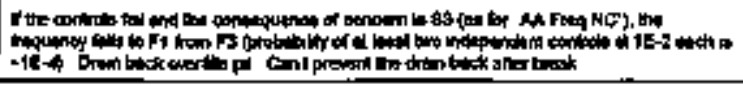 & \\
\hline 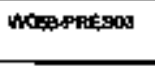 & 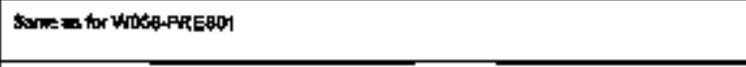 & 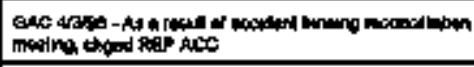 \\
\hline XE:-A1PLOCNOH & 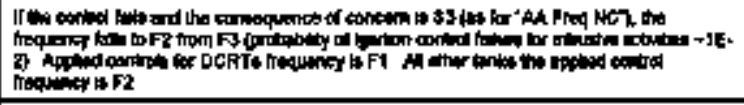 & 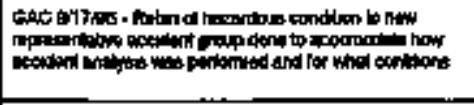 \\
\hline 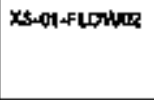 & 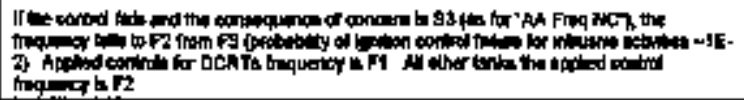 & \\
\hline
\end{tabular}




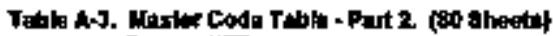

\begin{tabular}{|c|c|c|}
\hline 10 & 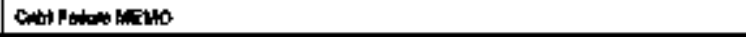 & 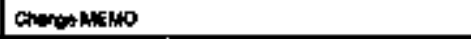 \\
\hline xts-on plotonts & 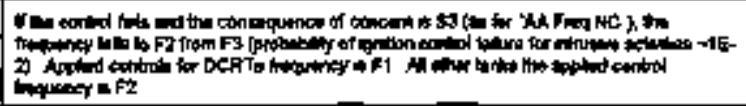 & \\
\hline \multicolumn{3}{|l|}{ 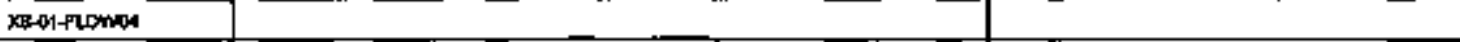 } \\
\hline XE-01-Funias & 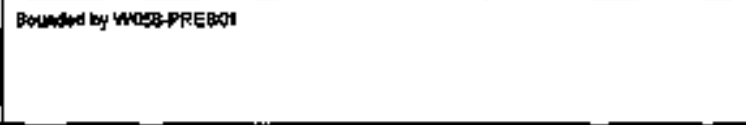 & 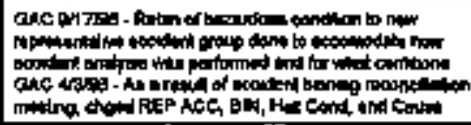 \\
\hline xis-alflovins & 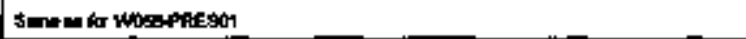 & \\
\hline xts-01+20wist & 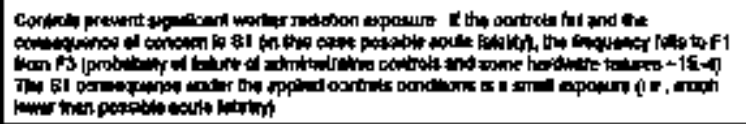 & \\
\hline 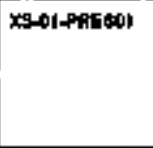 & 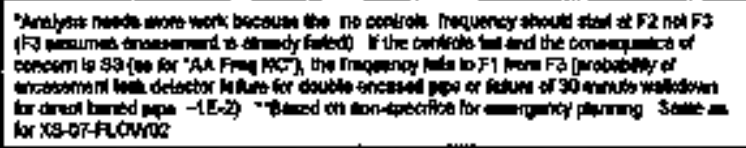 & \\
\hline 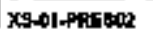 & 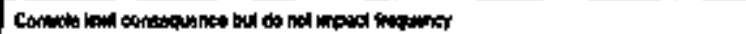 & \\
\hline 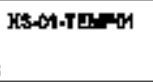 & 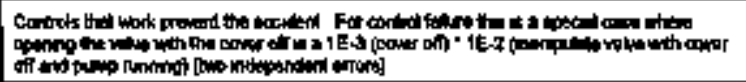 & \\
\hline ron-Tukne & 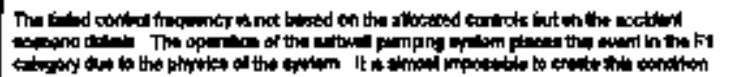 & \\
\hline Ko.tactown & & \\
\hline
\end{tabular}




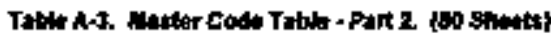

\begin{tabular}{|c|c|c|}
\hline 10 & 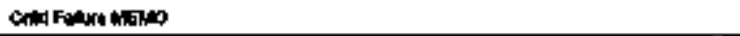 & 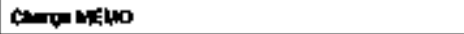 \\
\hline 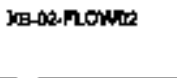 & 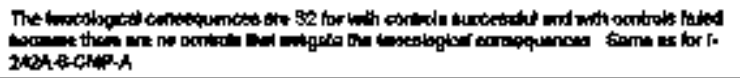 & \\
\hline XE-DLFLONWE & 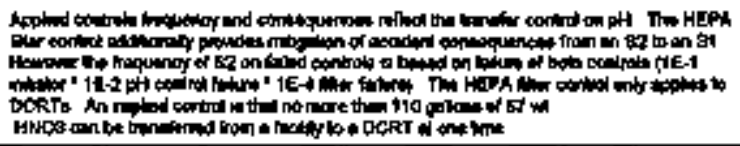 & 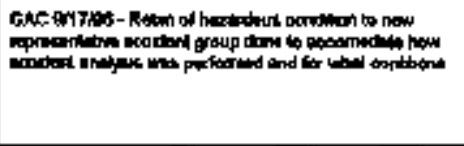 \\
\hline 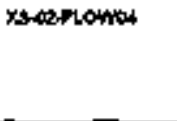 & 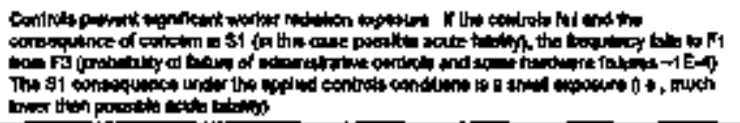 & \\
\hline xg-ogelowtes & 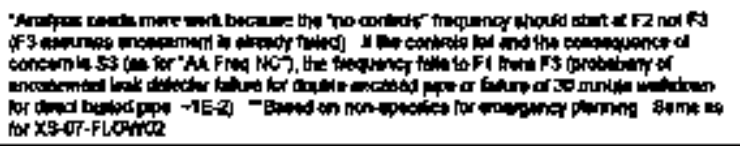 & 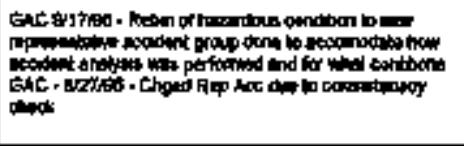 \\
\hline 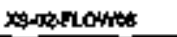 & 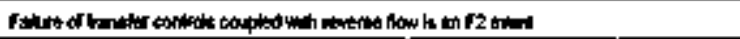 & \\
\hline Xothence & 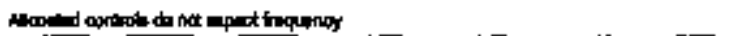 & \\
\hline 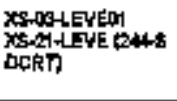 & 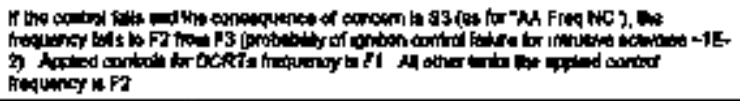 & 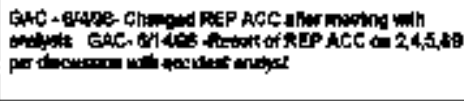 \\
\hline 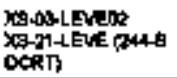 & 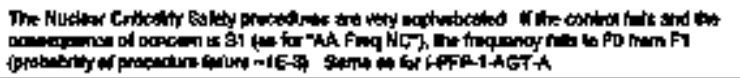 & 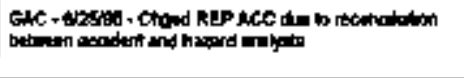 \\
\hline 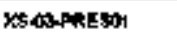 & 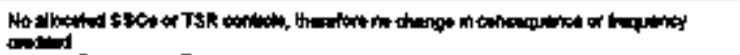 & \\
\hline
\end{tabular}




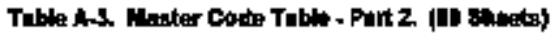

\begin{tabular}{|c|c|c|}
\hline 10 & 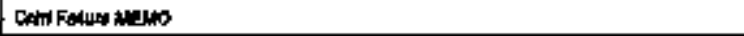 & 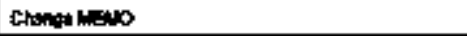 \\
\hline 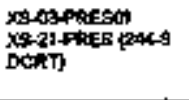 & 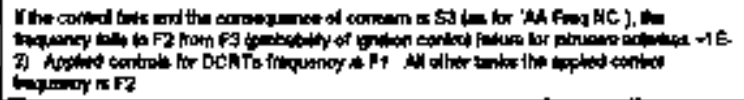 & 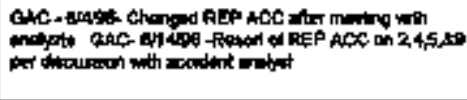 \\
\hline Xs-o3pREBoth & 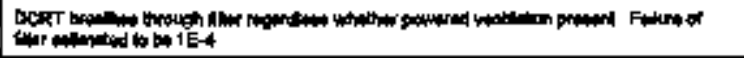 & \\
\hline xoratingesen & 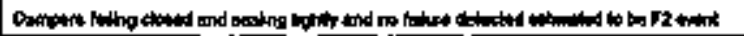 & \\
\hline 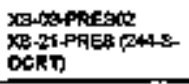 & 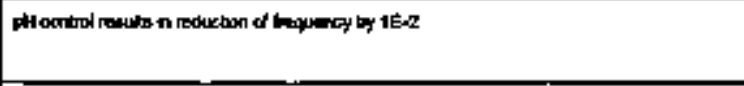 & 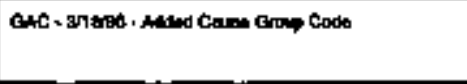 \\
\hline xabopinests & 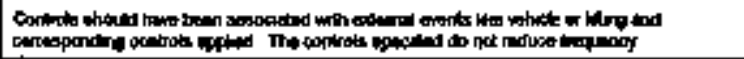 & \\
\hline XB-09-PRESPD4 & 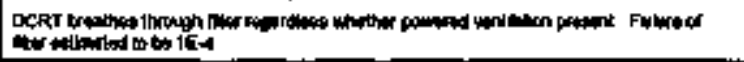 & \\
\hline xtod-PREstid & 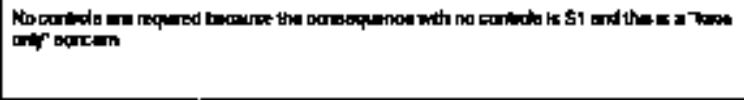 & 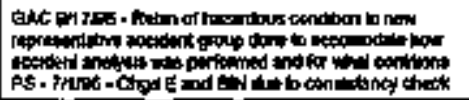 \\
\hline XpO03TtEMPN & 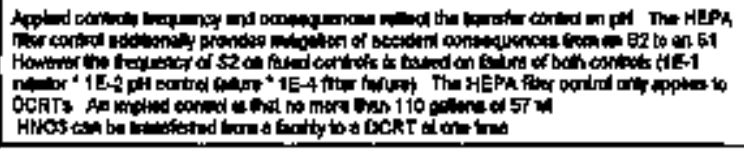 & 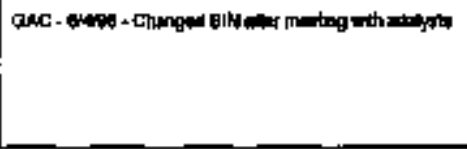 \\
\hline 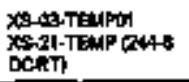 & & 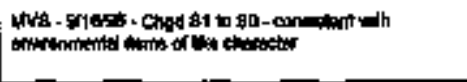 \\
\hline 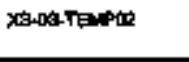 & 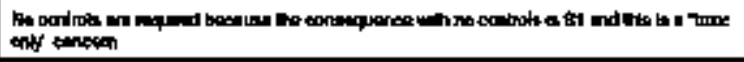 & \\
\hline 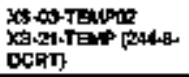 & 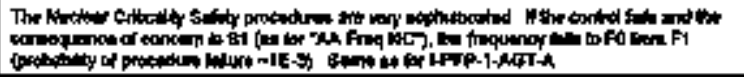 & \\
\hline
\end{tabular}




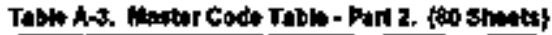

\begin{tabular}{|c|c|c|}
\hline $\boldsymbol{D}$ & 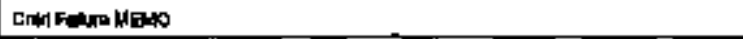 & Chonen Nan \\
\hline 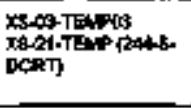 & 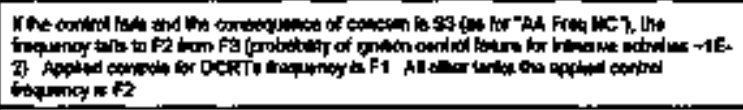 & 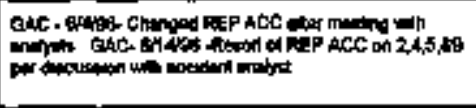 \\
\hline \multicolumn{3}{|l|}{ 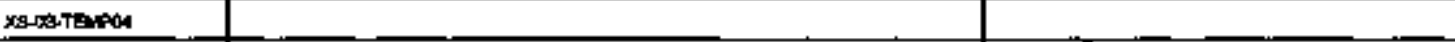 } \\
\hline 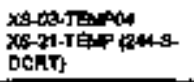 & Bouneof by host-piassus & 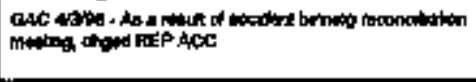 \\
\hline xom-TIMets & 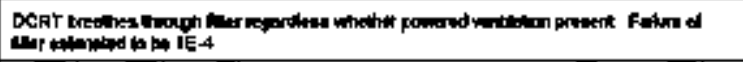 & \\
\hline Y0-0\%-TEP0s & 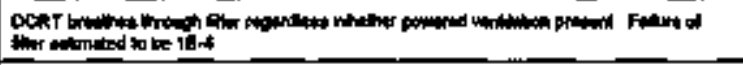 & \\
\hline 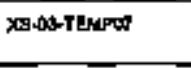 & 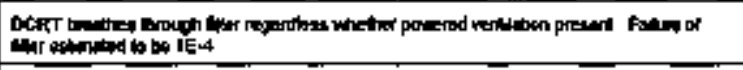 & \\
\hline xs-as-тетір & 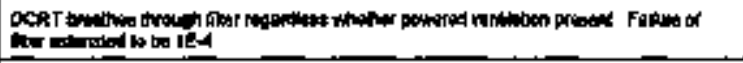 & \\
\hline 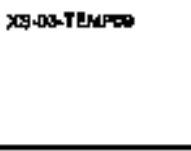 & & 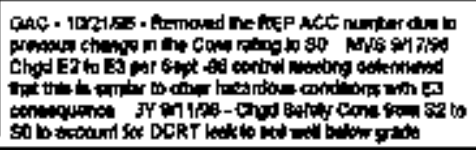 \\
\hline 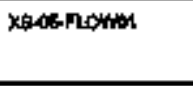 & 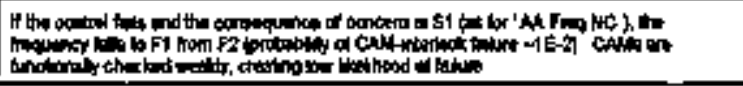 & 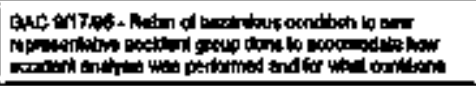 \\
\hline Xerasslowhe & & 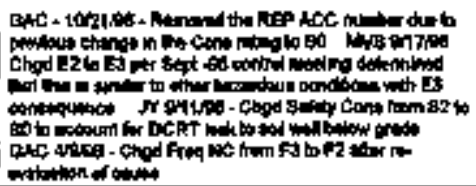 \\
\hline
\end{tabular}




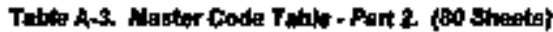

\begin{tabular}{|c|c|c|}
\hline$\omega$ & 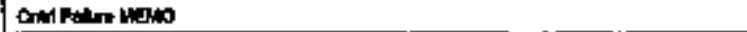 & 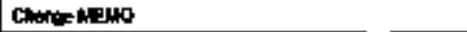 \\
\hline x马-05-TEUPO & 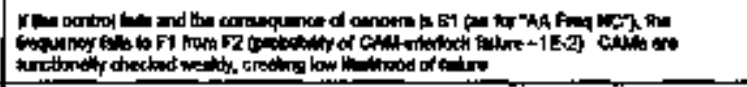 & \\
\hline XF-05-Tentag & 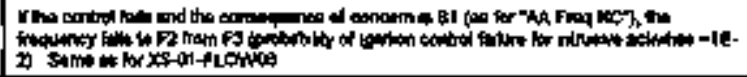 & \\
\hline XB-OSFLONHA & 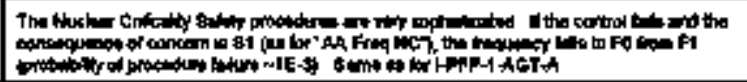 & \\
\hline xaseffuther & 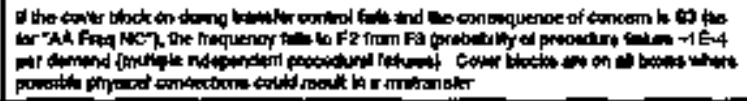 & \\
\hline 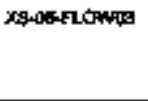 & 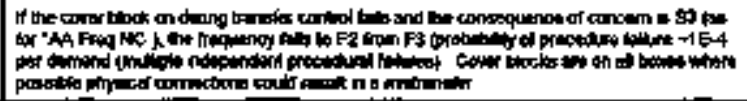 & \\
\hline 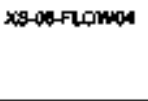 & 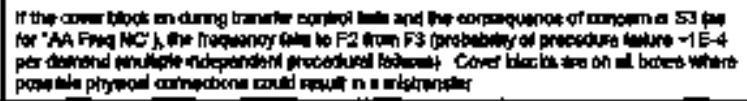 & 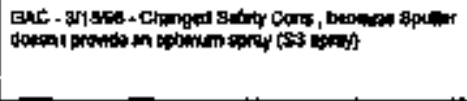 \\
\hline xtrosicomins & 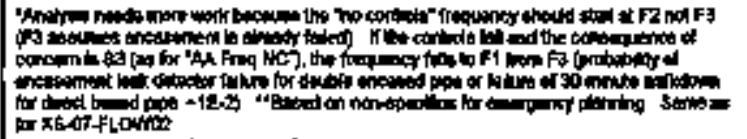 & \\
\hline xstofifomits & 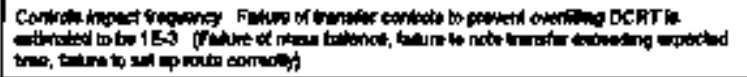 & \\
\hline$x=0, n+10 m 61$ & 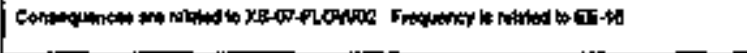 & 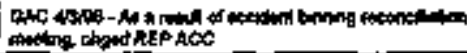 \\
\hline
\end{tabular}




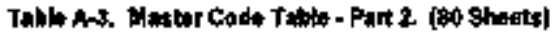

\begin{tabular}{|c|c|c|}
\hline ID & 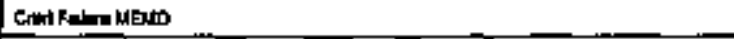 & Chengentho \\
\hline 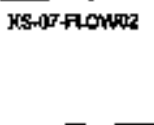 & 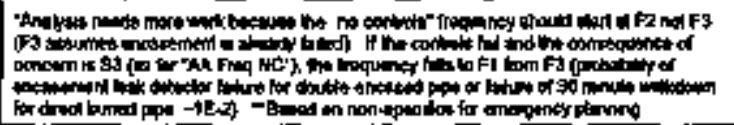 & \\
\hline xasos.rocintes & 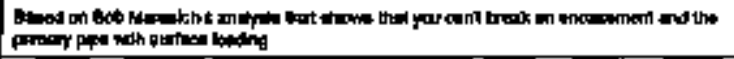 & \\
\hline$x \in-6$-rloning & 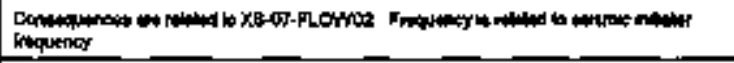 & \\
\hline XE-OF, FLOWKO & 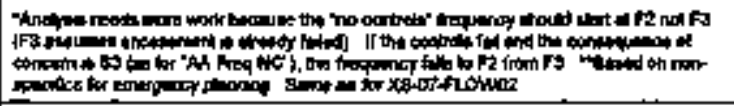 & \\
\hline$X B-O T$ FLONKS & 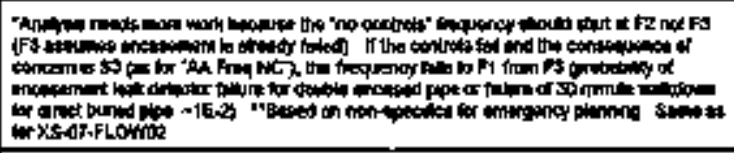 & \\
\hline 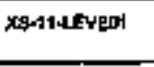 & 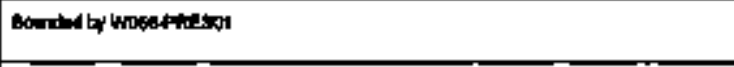 & 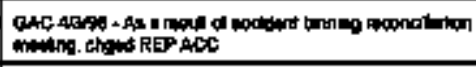 \\
\hline X5-114EVET2 & 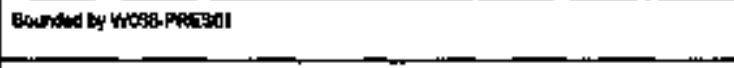 & 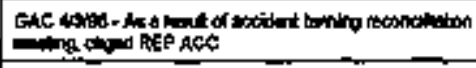 \\
\hline XS-11-LEVEDS & 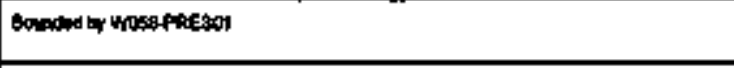 & 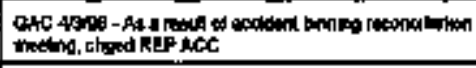 \\
\hline xastizandrar & 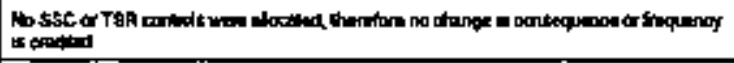 & \\
\hline 36.12+Flown & 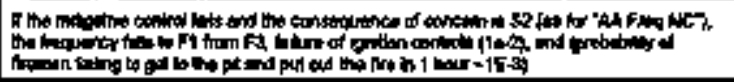 & \\
\hline
\end{tabular}




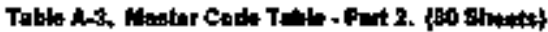

\begin{tabular}{|c|c|c|}
\hline 10 & 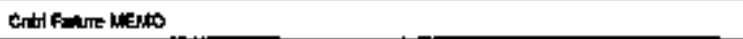 & Gonga luters \\
\hline 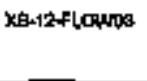 & 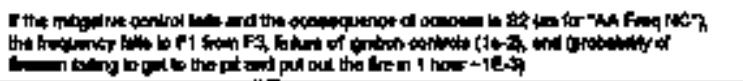 & \\
\hline \multicolumn{3}{|l|}{ Xs-12flown:4 } \\
\hline XB-12 FownS & 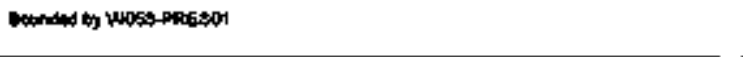 & 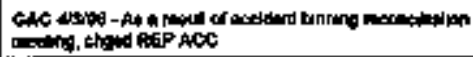 \\
\hline Xs-12+fLGuphos & 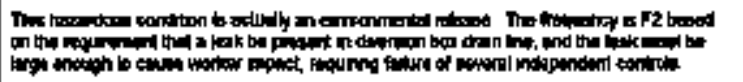 & \\
\hline \multicolumn{3}{|l|}{ XE-13HEYE } \\
\hline XS-13PRESOH & 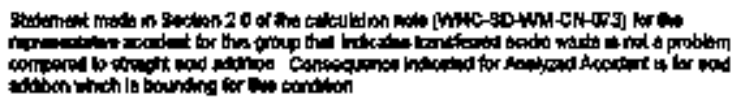 & 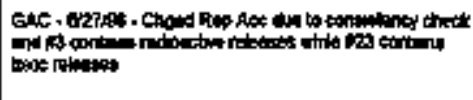 \\
\hline Ks:13PREsos & 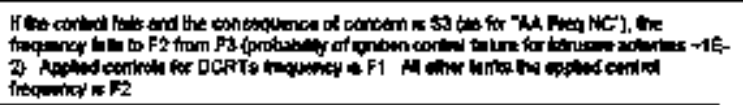 & 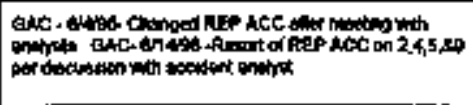 \\
\hline C6s-43-PREgod & 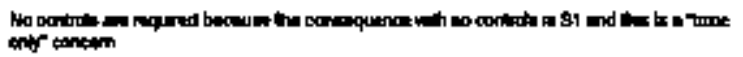 & \\
\hline XS-13-PRESOA & 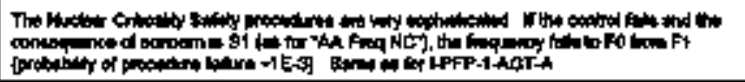 & \\
\hline Xo-70-rlokvi & 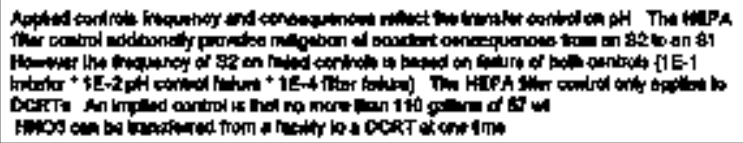 & 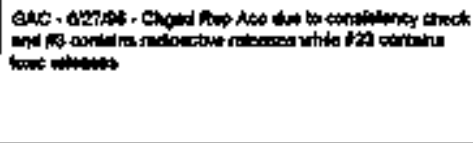 \\
\hline$x \in-\infty$ flowina & & \\
\hline
\end{tabular}


Tath A-3. Waster Code Table - Furt 2. 190 ghnetc|

\begin{tabular}{|c|c|c|}
\hline 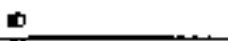 & Confram tonso & 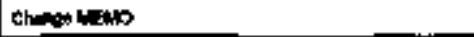 \\
\hline 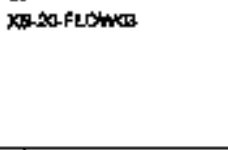 & 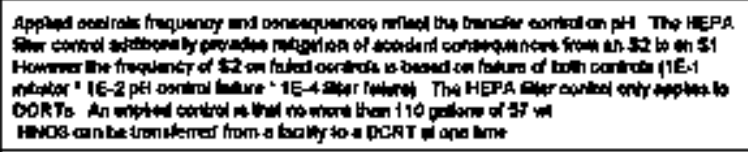 & 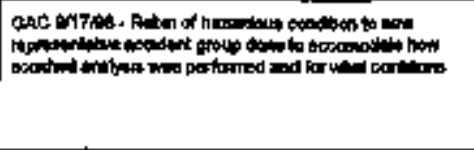 \\
\hline XB-Daflownar & 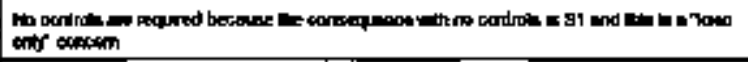 & \\
\hline xs-30-FLones & 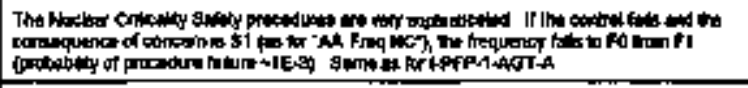 & \\
\hline \multicolumn{3}{|l|}{ 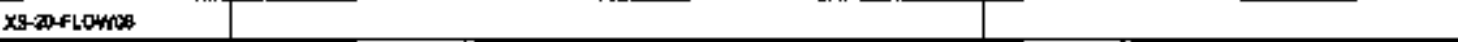 } \\
\hline \multicolumn{3}{|l|}{ xt-z0-flowns } \\
\hline x6-ällown: & Bounded by Who58-PRESOA & 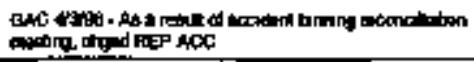 \\
\hline Xs-20-7Lown: & 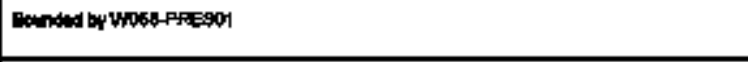 & 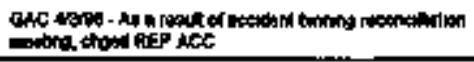 \\
\hline 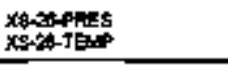 & 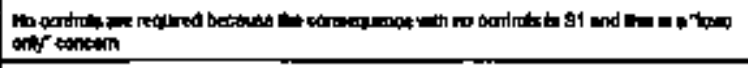 & \\
\hline 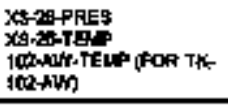 & 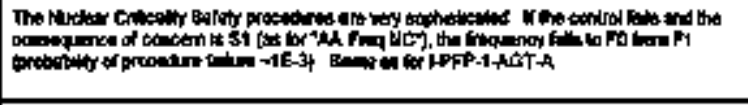 & \\
\hline XE-XERESEM & 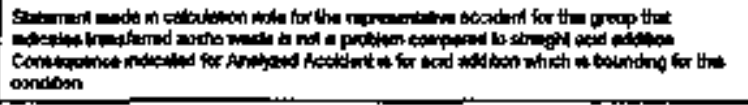 & 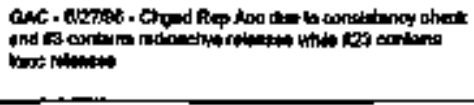 \\
\hline 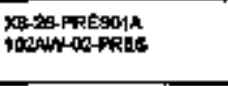 & 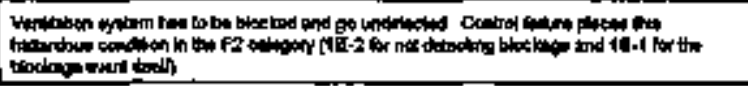 & \\
\hline
\end{tabular}


Tublt A-3, Mater Cods Teble - Part 2., 380 thests]

\begin{tabular}{|c|c|c|}
\hline $\mathbf{n}$ & CNA Faina MEAN & Chtroe undo \\
\hline 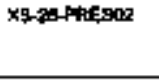 & 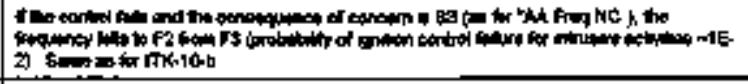 & 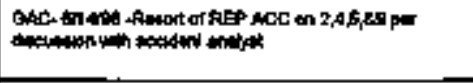 \\
\hline 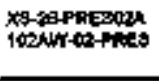 & 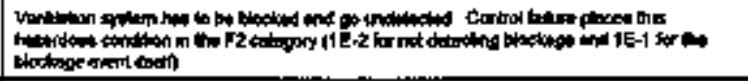 & \\
\hline 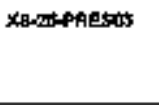 & 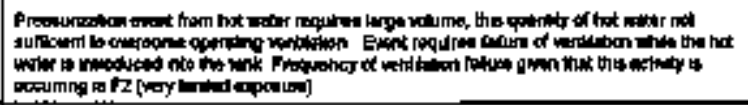 & \\
\hline 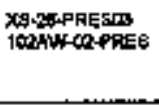 & 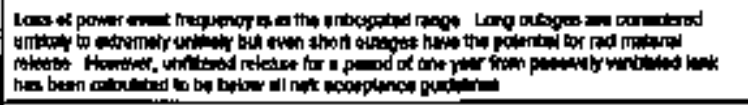 & \\
\hline \multicolumn{3}{|l|}{ 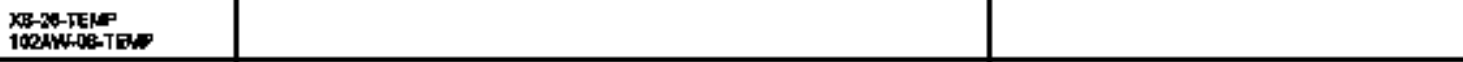 } \\
\hline Xs-xa-Tention & 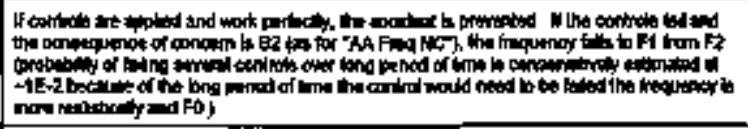 & 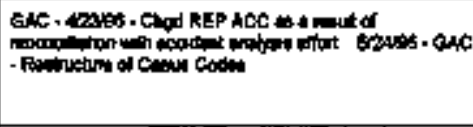 \\
\hline \multicolumn{3}{|l|}{ XB-2B-T6חP02 } \\
\hline Xswentortor & 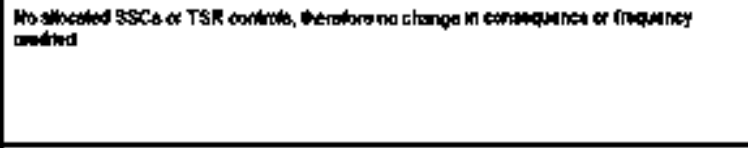 & 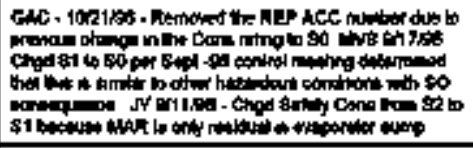 \\
\hline xtstiplowne & 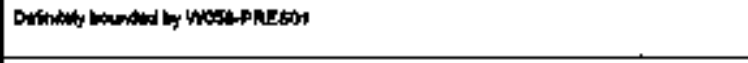 & 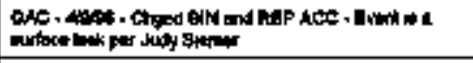 \\
\hline X8-37-FLOMOY & 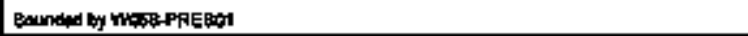 & \\
\hline
\end{tabular}




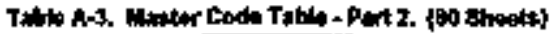

\begin{tabular}{|c|c|c|}
\hline $\mathbf{p}$ & 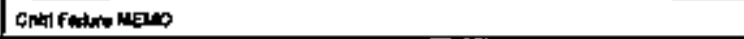 & 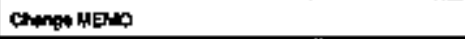 \\
\hline$X B-37-F L O W D 2$ & 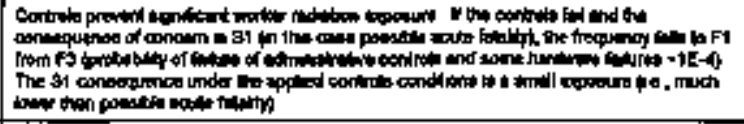 & \\
\hline 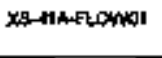 & 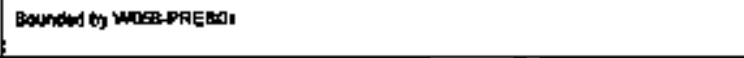 & 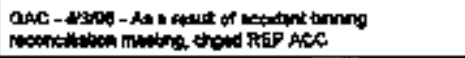 \\
\hline 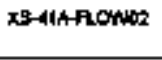 & 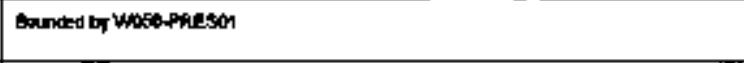 & 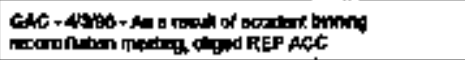 \\
\hline 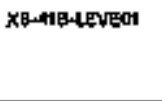 & 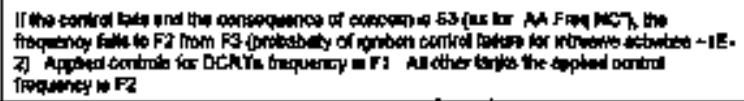 & 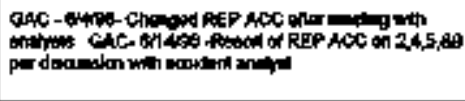 \\
\hline XSATR-LEVER & 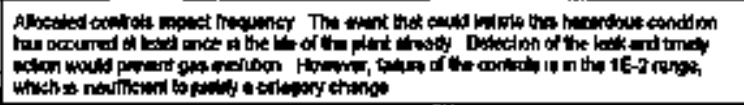 & \\
\hline X5-41B-15VTO3 & Sound d by MESBPRESAT & 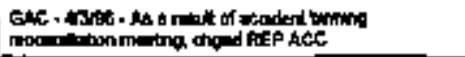 \\
\hline 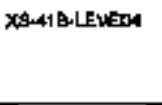 & 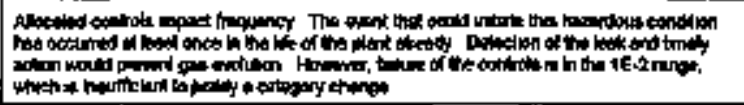 & \\
\hline XS-41C-CEVEOI & 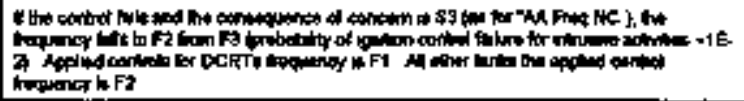 & 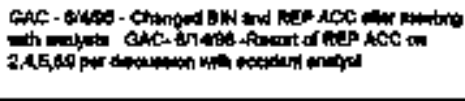 \\
\hline \multicolumn{3}{|l|}{$x=-d i c .11$ iter } \\
\hline 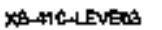 & 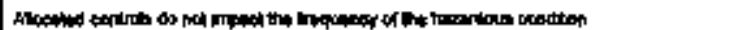 & \\
\hline
\end{tabular}




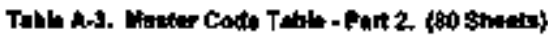

\begin{tabular}{|c|c|c|}
\hline ID & 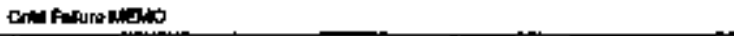 & chendind \\
\hline Xو-FFLCW & 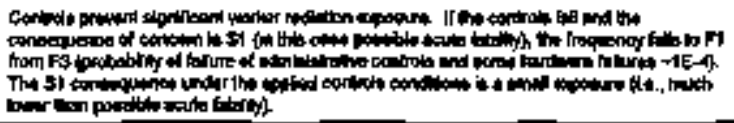 & \\
\hline 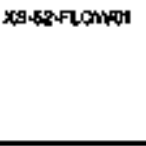 & 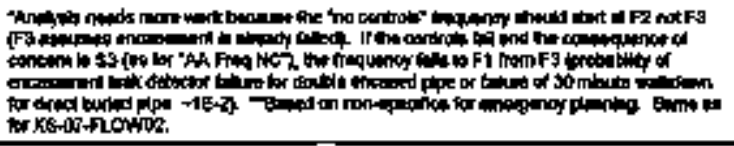 & \\
\hline 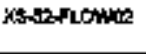 & 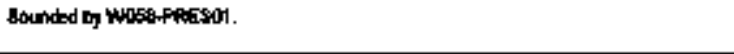 & 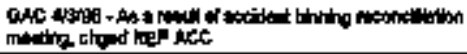 \\
\hline
\end{tabular}

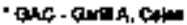

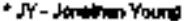

- Mrst - inlan v. Shutz

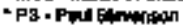




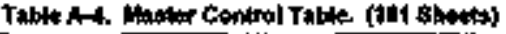

\begin{tabular}{|c|c|c|c|c|}
\hline 由 & Prew stst & $M * 8 B C$ & Phen TsR & 山TSR \\
\hline 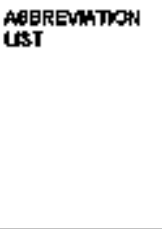 & 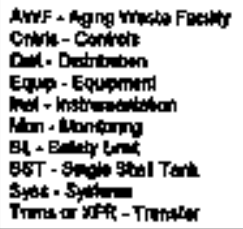 & 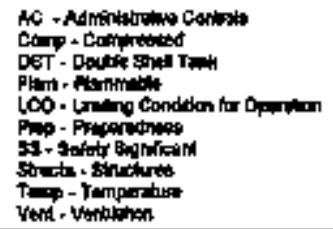 & 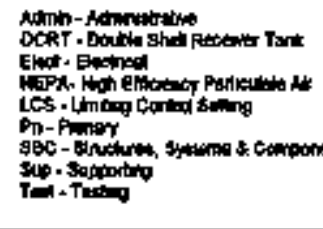 & 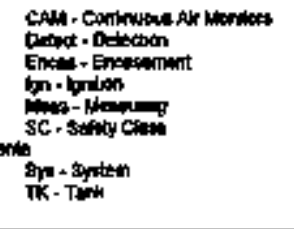 \\
\hline 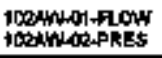 & Meno parand & Nom & Nond mired & Hont meaped \\
\hline 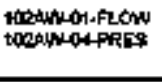 & Mone ragired & 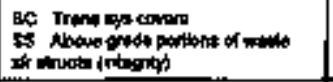 & 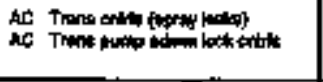 & 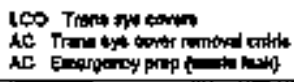 \\
\hline 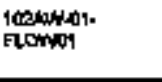 & Nowne requard & 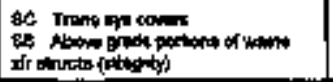 & 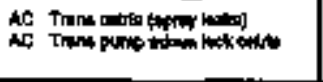 & 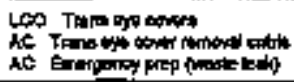 \\
\hline 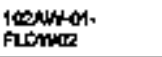 & 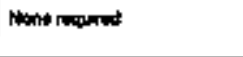 & Hone pequered & te exoction antit & 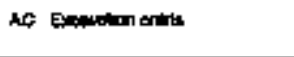 \\
\hline 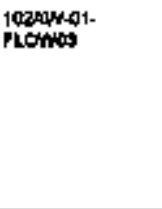 & 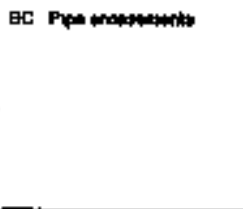 & 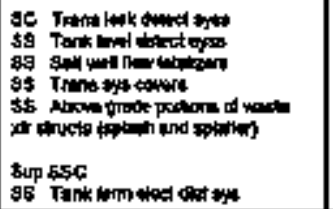 & 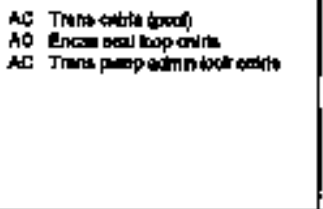 & 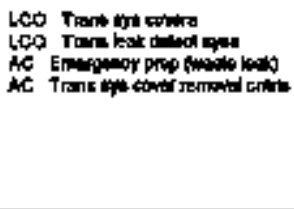 \\
\hline 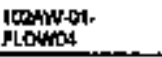 & Nenereroured & Mone roquind & Mon & Natien magutid \\
\hline
\end{tabular}




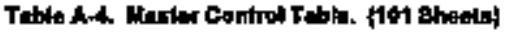

\begin{tabular}{|c|c|c|c|c|}
\hline 10 & Putr sste & Massc & Pm TSk & $\operatorname{Min}$ \\
\hline 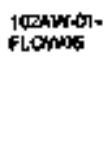 & 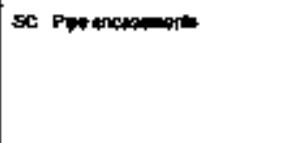 & 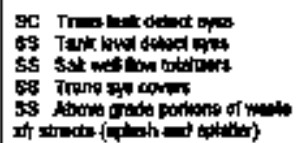 & 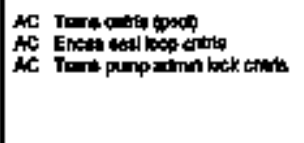 & 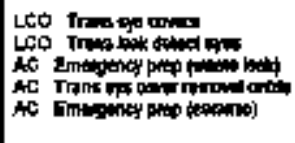 \\
\hline $\begin{array}{l}1 \Rightarrow \text { andort- } \\
\text { flithat }\end{array}$ & 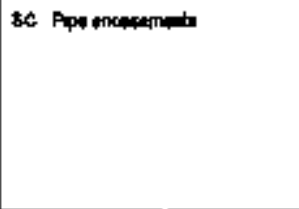 & 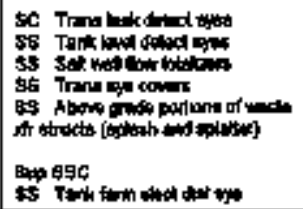 & 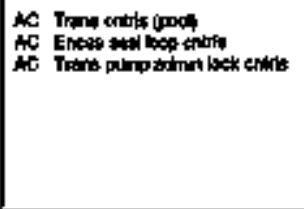 & 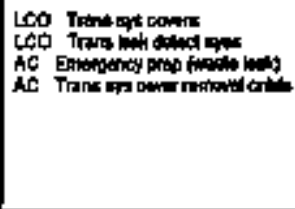 \\
\hline $\begin{array}{l}\text { lorentin-m- } \\
\text { Flowns }\end{array}$ & 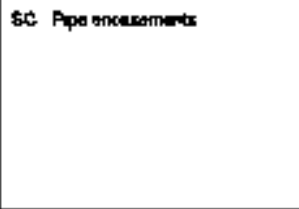 & 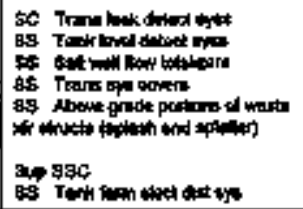 & 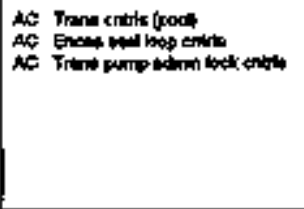 & 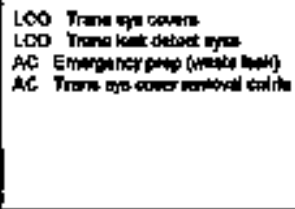 \\
\hline 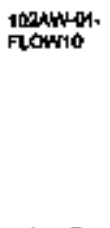 & 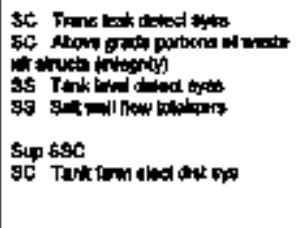 & 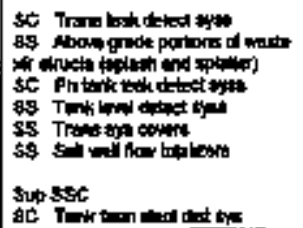 & 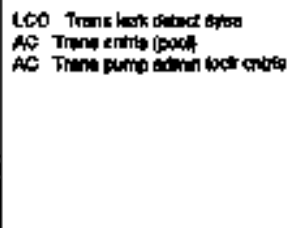 & 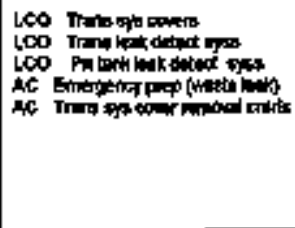 \\
\hline
\end{tabular}




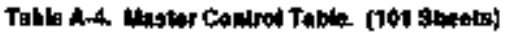

\begin{tabular}{|c|c|c|c|c|}
\hline 10 & Phw 95C & Mssc & Prew Tat & 山 Tsk \\
\hline 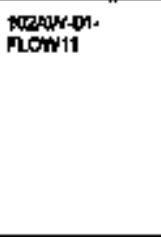 & 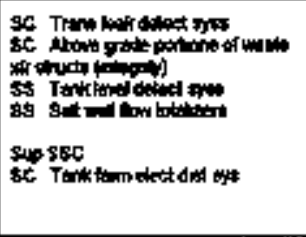 & 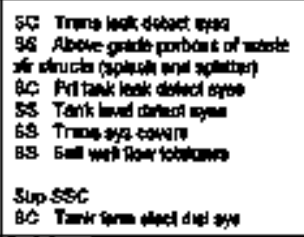 & 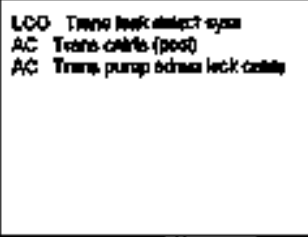 & 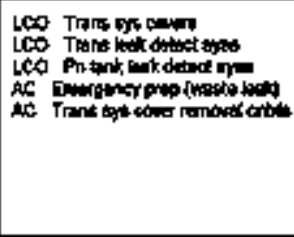 \\
\hline 1002NW-OS-TEKP & Anotions & 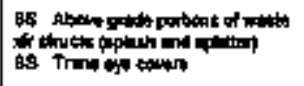 & Act The The & 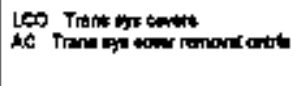 \\
\hline 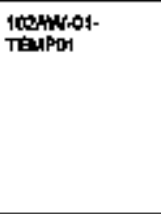 & 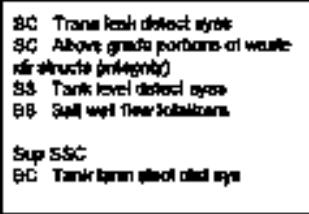 & 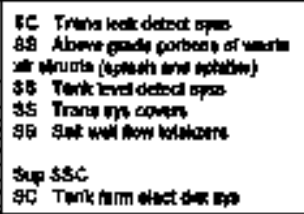 & 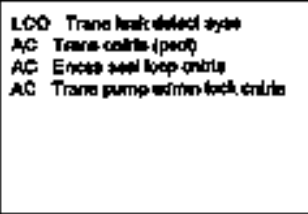 & 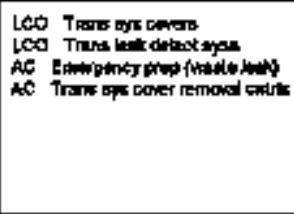 \\
\hline pozank-101- & 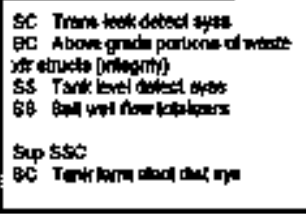 & 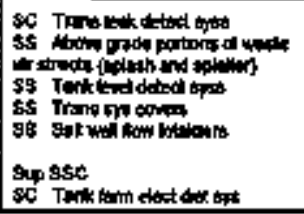 & 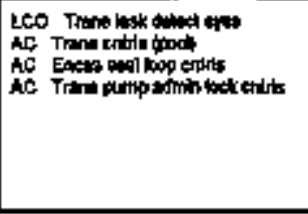 & 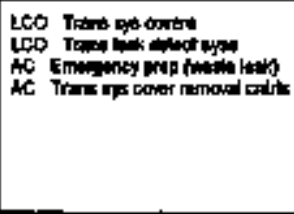 \\
\hline
\end{tabular}




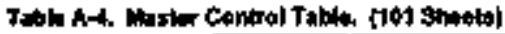

\begin{tabular}{|c|c|c|c|c|}
\hline I & PTw ESC & M & Pwr TzR & He TsR \\
\hline 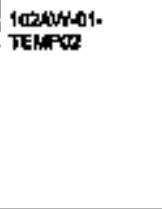 & 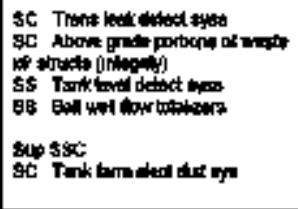 & 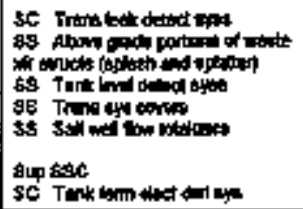 & 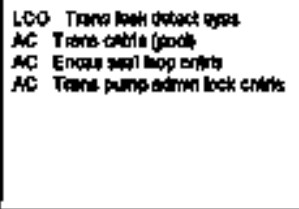 & 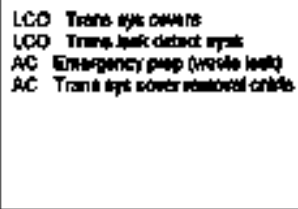 \\
\hline 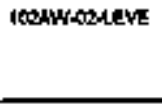 & 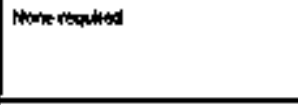 & 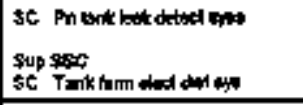 & Nom rapurid & 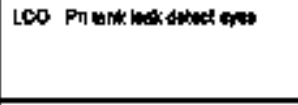 \\
\hline $\begin{array}{l}\text { 10osturats. } \\
\text { LEver }\end{array}$ & Now nowas & Wone reaprost & Nom & 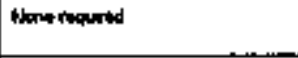 \\
\hline 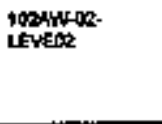 & $\operatorname{sichandand~}$ & 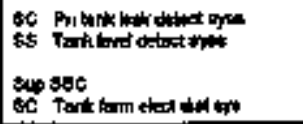 & 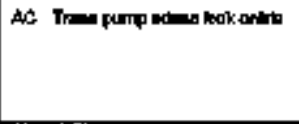 & 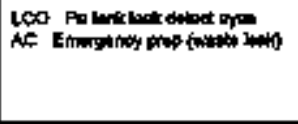 \\
\hline 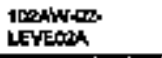 & Pane requad & sc Pn bit lentedind ofos & 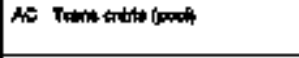 & 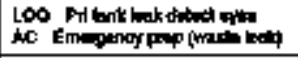 \\
\hline 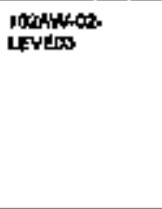 & 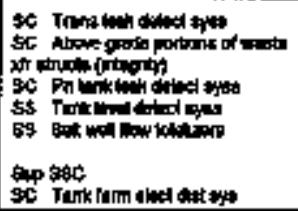 & 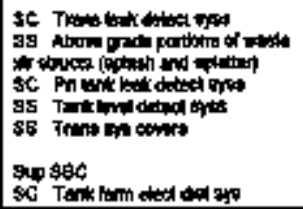 & 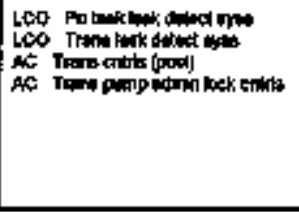 & 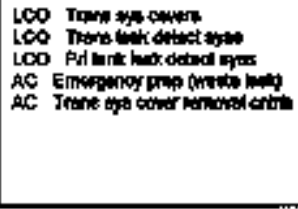 \\
\hline
\end{tabular}


Tabio A-4. Master Controt Tahis. (191 Shents)

\begin{tabular}{|c|c|c|c|c|}
\hline 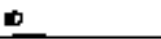 & Anw \&G & ingser & PMrTtBR & $\mathbf{m T a R}$ \\
\hline 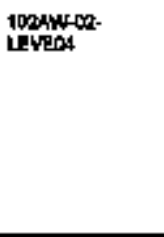 & 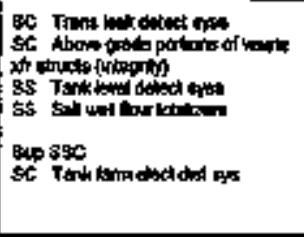 & 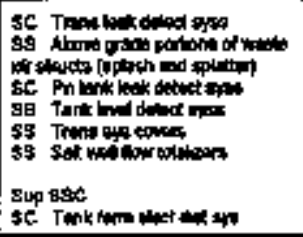 & 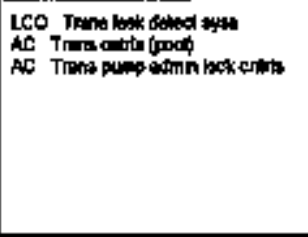 & 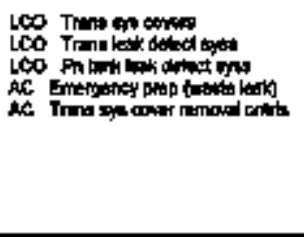 \\
\hline 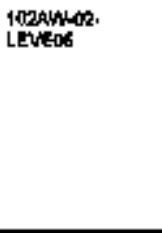 & 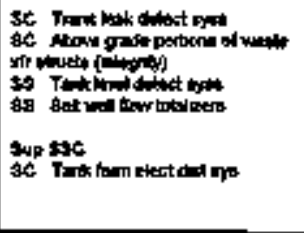 & 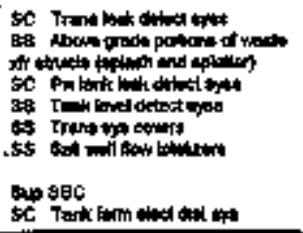 & 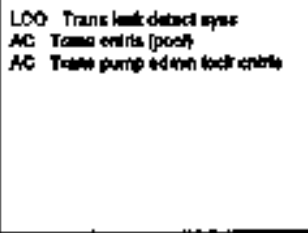 & 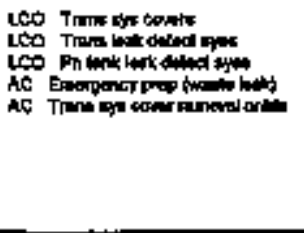 \\
\hline 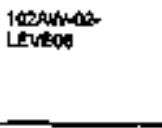 & Mene mapoed & 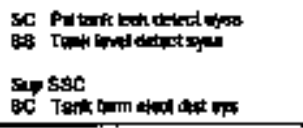 & 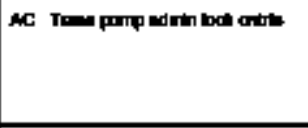 & 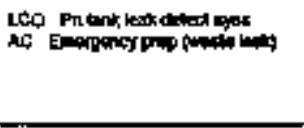 \\
\hline $\begin{array}{l}\text { IOEAWNOEPRTS } \\
\text { ICONWLE-TEAP }\end{array}$ & Neme nowines & Nanonowases & 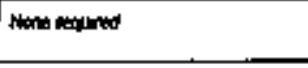 & 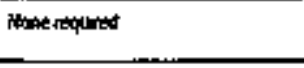 \\
\hline 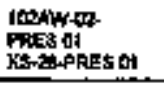 & $\lim$ nimurad & Nene ragumad & Plano nowned & Nomerepurod \\
\hline 19tenw-gat & 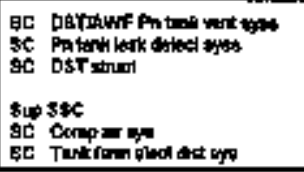 & Mons wepurad & 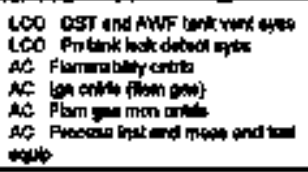 & 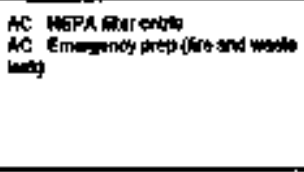 \\
\hline
\end{tabular}




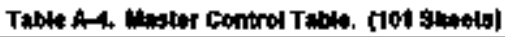

\begin{tabular}{|c|c|c|c|c|}
\hline $\mathbf{D}$ & Pre ssc & $\mu \neq \operatorname{ssc}$ & RMN T:FR & MUTSR \\
\hline 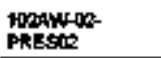 & Mom naperd & Nom renurwa & 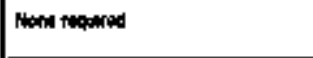 & 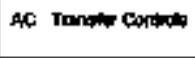 \\
\hline NoAnLos & Ss & Nom ropured & 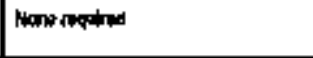 & Hant \\
\hline 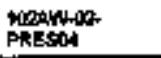 & St & 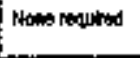 & 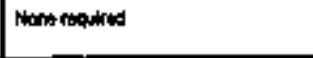 & Nond malos \\
\hline $\begin{array}{l}\text { pootunton } \\
\text { perasios }\end{array}$ & 日B OôT atrut & rene napard & Nome maqued & Whou requited \\
\hline 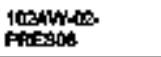 & SS DST thut: & dentritand & Bbou minged & Wone roputed \\
\hline 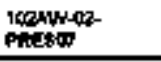 & E9 DET Druak & 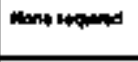 & 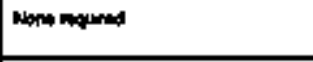 & Hone requited \\
\hline 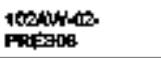 & How nophed & Hone reaparal & Bbne mopend & Home topurad \\
\hline $\begin{array}{l}\text { Iopunpos } \\
\text { phistos }\end{array}$ & 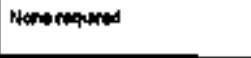 & 14 & 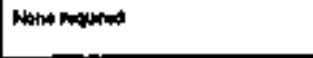 & Home required \\
\hline 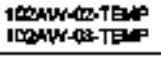 & Howerequired & Hone roper & 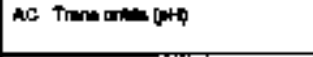 & Atone requirad \\
\hline 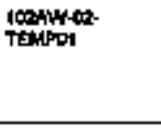 & 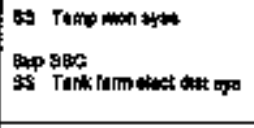 & 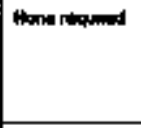 & 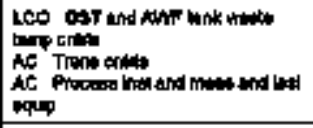 & 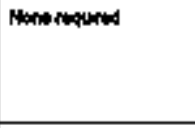 \\
\hline $\begin{array}{l}100 \mu w-\infty 2 \\
17002\end{array}$ & 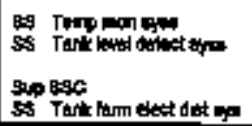 & mono nopirad & 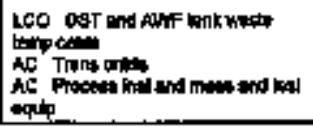 & 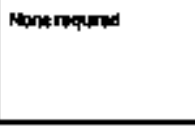 \\
\hline
\end{tabular}




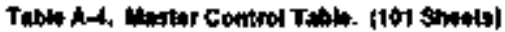

\begin{tabular}{|c|c|c|c|c|}
\hline Ip & Pan ssc & M\$sc & Pm TEk & Mar I \\
\hline 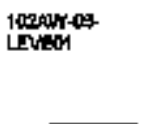 & 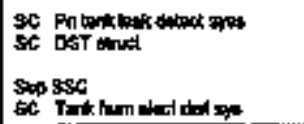 & Bens roperod & 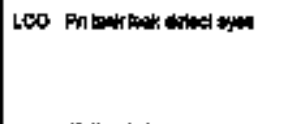 & A \\
\hline 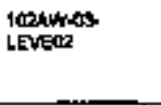 & 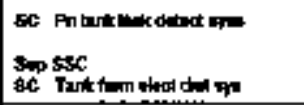 & 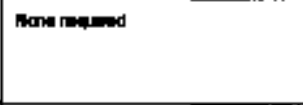 & 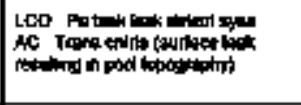 & Rene nequarios \\
\hline 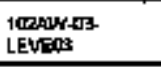 & \$9 рвт truct & Nene royerod & Herso riquad & Non requind \\
\hline 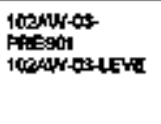 & Hene nomed & 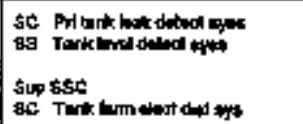 & Nom naphed & 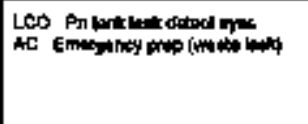 \\
\hline 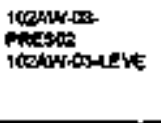 & 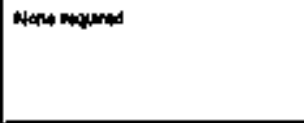 & 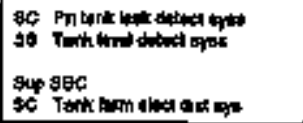 & 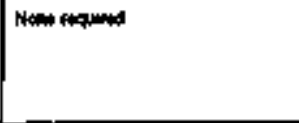 & 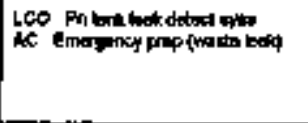 \\
\hline 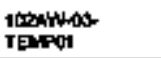 & 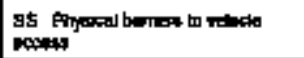 & Wore rapured & 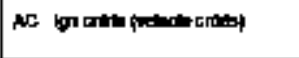 & 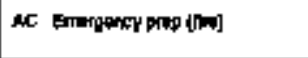 \\
\hline $\begin{array}{l}\text { topentos- } \\
\text { Tupios }\end{array}$ & 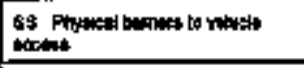 & Wone repures & 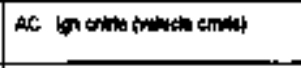 & 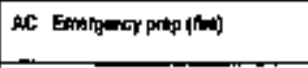 \\
\hline 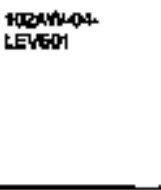 & 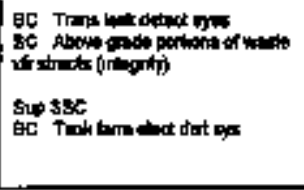 & 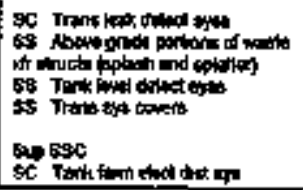 & 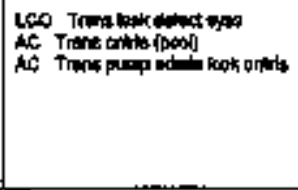 & 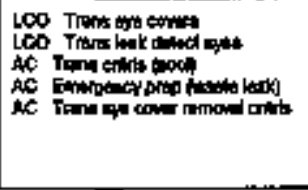 \\
\hline
\end{tabular}


Tabis A-4. Master Conirod Teble, MO1 Shatat)

\begin{tabular}{|c|c|c|c|c|}
\hline $\boldsymbol{\omega}$ & 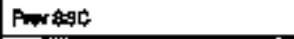 & NAEBC & PNT TSP & HMT TFR \\
\hline $\begin{array}{l}\text { 1eshiwot } \\
\text { ufvetes }\end{array}$ & Nown mopird & 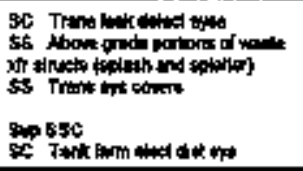 & 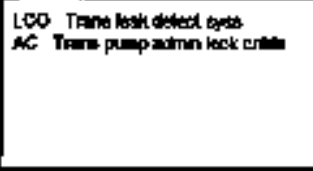 & 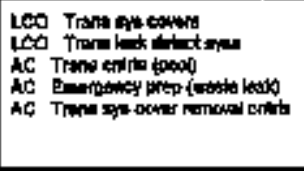 \\
\hline 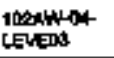 & Nententisted & Hene roputeod & 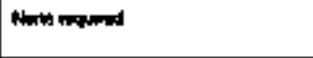 & 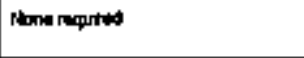 \\
\hline 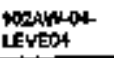 & 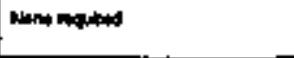 & 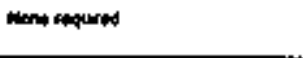 & A & (n) \\
\hline $\begin{array}{l}\text { 100andat. } \\
\text { PREB of }\end{array}$ & Nene reapad & Hone requared & Now roper & Uno rempled \\
\hline 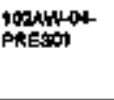 & 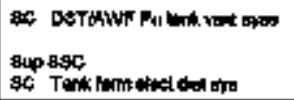 & 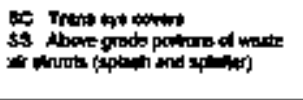 & 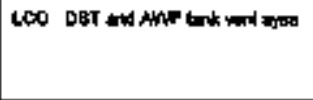 & 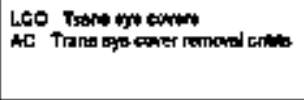 \\
\hline 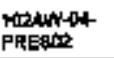 & Whated & 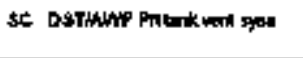 & 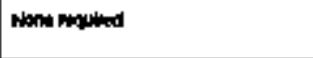 & Lect OST mA AWN unk with of \\
\hline 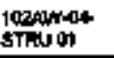 & 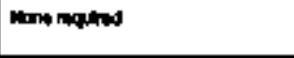 & 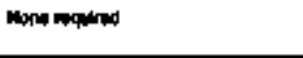 & Mone mapend & Non requend \\
\hline 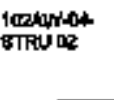 & 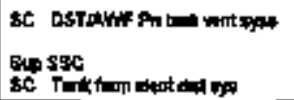 & Wone requed & 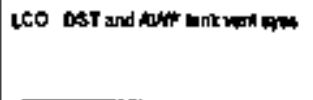 & 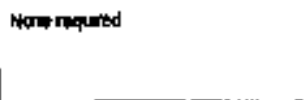 \\
\hline $\begin{array}{l}\text { 100anN-04 } \\
\text { strivels }\end{array}$ & 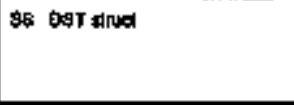 & Mone napios & 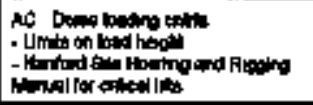 & Nonorequated \\
\hline 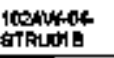 & Pind requared & Wone requend & Whe reculared & 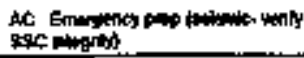 \\
\hline
\end{tabular}




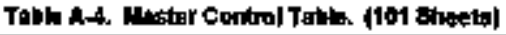

\begin{tabular}{|c|c|c|c|c|}
\hline ID & Pin tsse & im sect & Fom TSR & MTSR \\
\hline $\begin{array}{l}\text { 10Pawder } \\
\text { STRUDB }\end{array}$ & 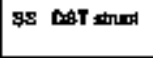 & Utone nopumed & 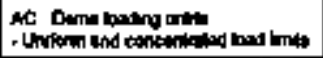 & 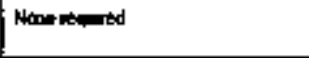 \\
\hline 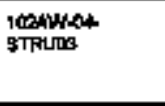 & st Cost ithuet & Hine requariod & 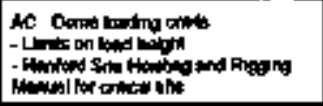 & 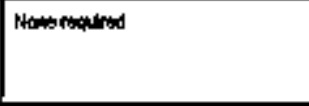 \\
\hline 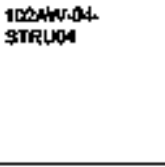 & Nane roquere & Mon reaglnd & 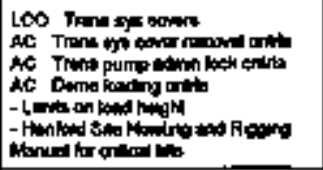 & 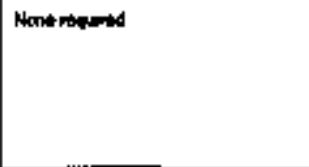 \\
\hline 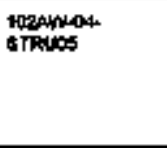 & Nonerequired & 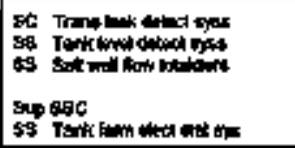 & 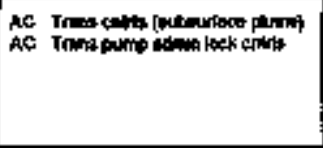 & 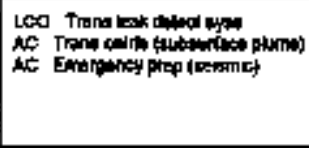 \\
\hline Norenko4TeMP & Nans requined & Nom maped & How mourd & 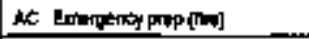 \\
\hline 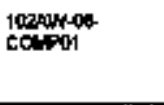 & 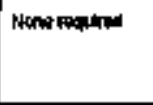 & 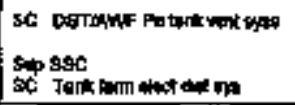 & Mand rapuad & 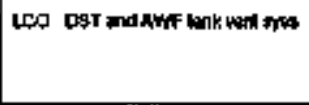 \\
\hline $\begin{array}{l}\text { losentok } \\
\text { contos }\end{array}$ & Nentertaditad & Howe nesuld & 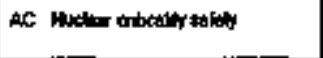 & Hene resured \\
\hline 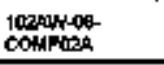 & None ropuros & iloma ropulat & 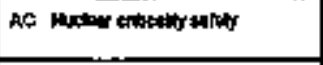 & 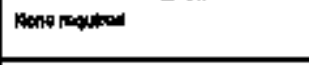 \\
\hline 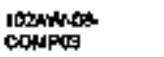 & 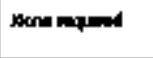 & Hene anquned & 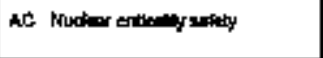 & None reaguted \\
\hline logentest & Mand istom & Nomention & 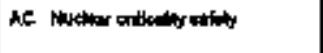 & 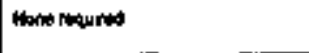 \\
\hline
\end{tabular}




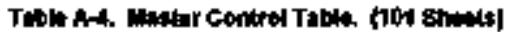

\begin{tabular}{|c|c|c|c|c|}
\hline ID & Pww sec & 粗的 & PMT TER & In TER \\
\hline 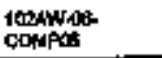 & Whated res & hone requad & 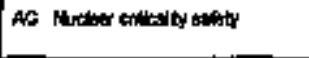 & 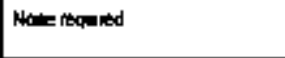 \\
\hline 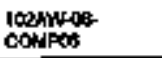 & Mone pewres & Nomo nematris & Nent ropured & Alate \\
\hline 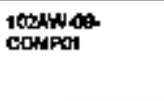 & 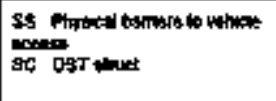 & Hothe loupurod & 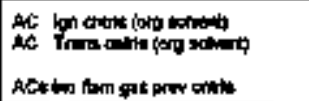 & 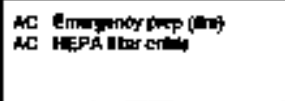 \\
\hline $\begin{array}{l}\text { 100andos } \\
\text { Compos }\end{array}$ & 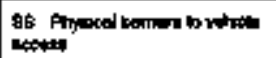 & Mone rang & 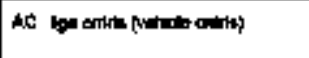 & 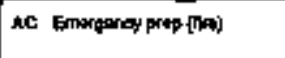 \\
\hline 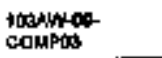 & 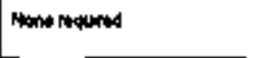 & 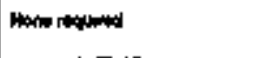 & 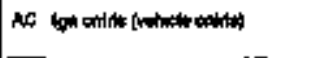 & Nowe napingd \\
\hline $\begin{array}{l}\text { poparos } \\
\text { conpot }\end{array}$ & repme requered & 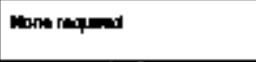 & Mant & 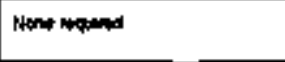 \\
\hline noturisos & 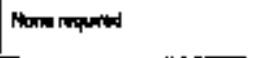 & 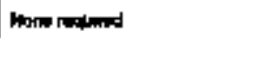 & 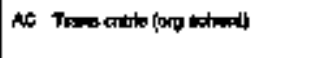 & Hane repard \\
\hline 10OAK-0OTENIP & Nonesaped & 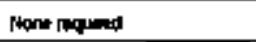 & 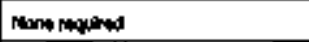 & man mons \\
\hline 10RAN-69- & 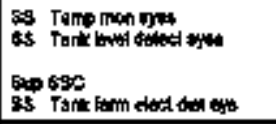 & 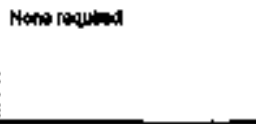 & 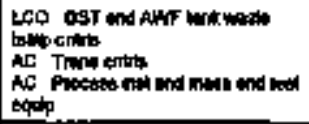 & 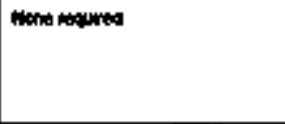 \\
\hline 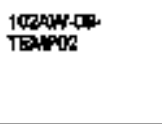 & Monnmed & 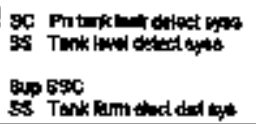 & 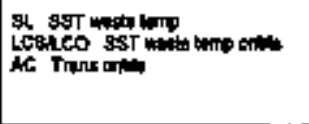 & 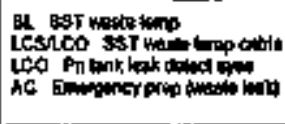 \\
\hline 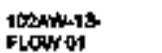 & Hanterented & Na. matind & 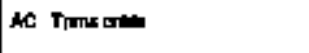 & Mane requend \\
\hline
\end{tabular}




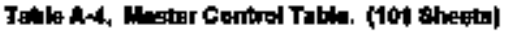

\begin{tabular}{|c|c|c|c|c|}
\hline ID & PNen Eg: & ursse & Pma TSR & Mtsk \\
\hline $\begin{array}{l}1025 \text { thts } \\
\text { PLOWO1 }\end{array}$ & 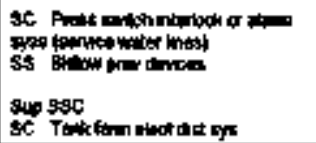 & 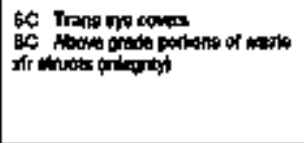 & 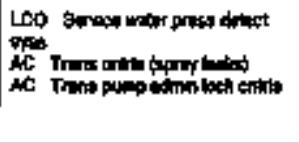 & 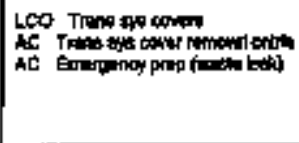 \\
\hline 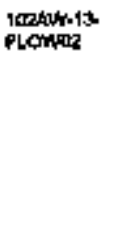 & 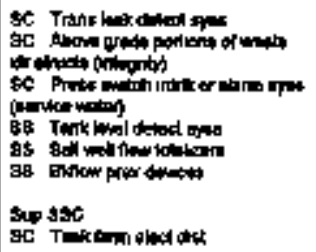 & 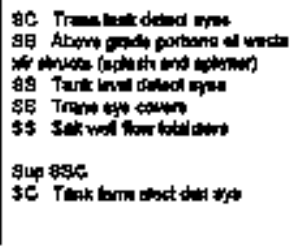 & 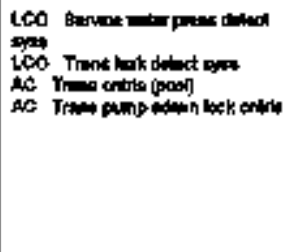 & 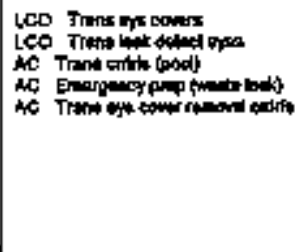 \\
\hline $100|50|$ at & 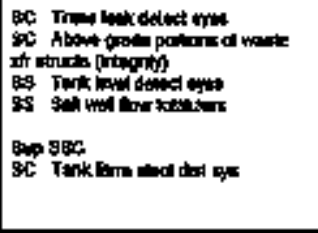 & 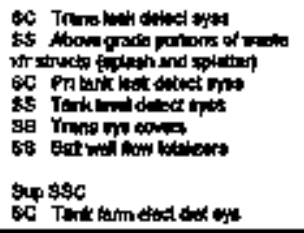 & 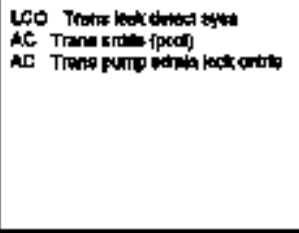 & 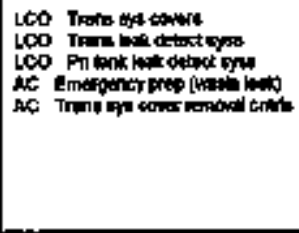 \\
\hline totes the & 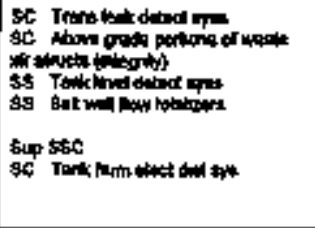 & 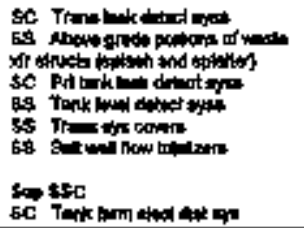 & 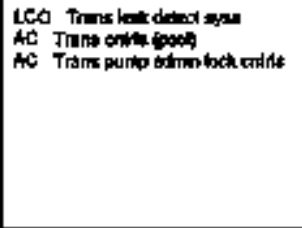 & 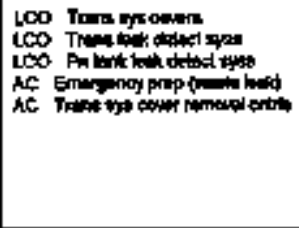 \\
\hline
\end{tabular}




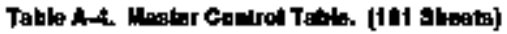

\begin{tabular}{|c|c|c|c|c|}
\hline 10 & Pron $84 x$ & Antesc & PTt TSA & MT: \\
\hline Co2B-at bas & \& Ppequamints & 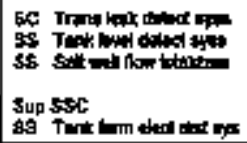 & 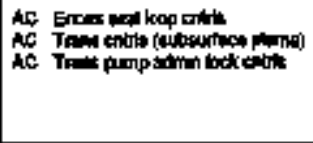 & 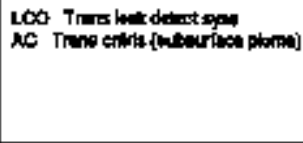 \\
\hline $1 \cos 00 \mathrm{col}$ & 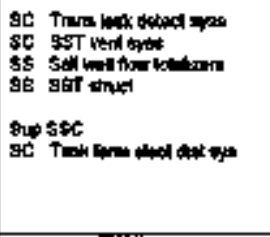 & Fone mourned & 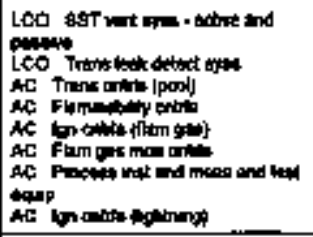 & 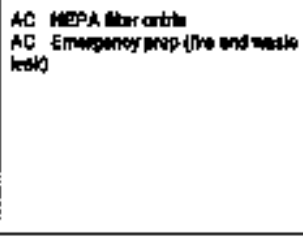 \\
\hline $1028-0242$ & 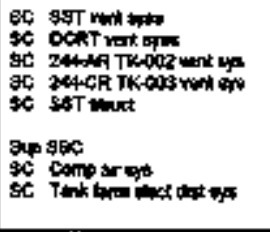 & 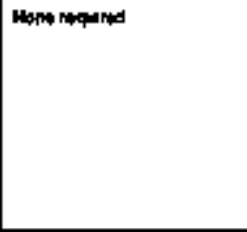 & 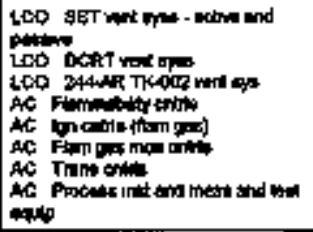 & 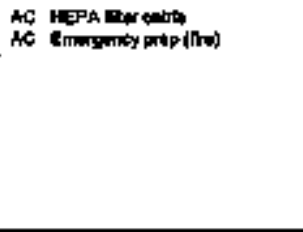 \\
\hline 1009020 & 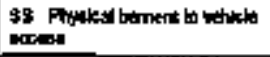 & Mone requad & 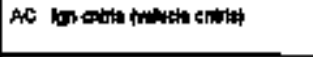 & AC Emerponey prap ines \\
\hline 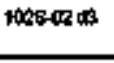 & 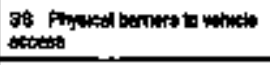 & man ropand & 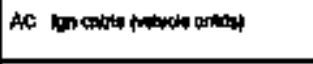 & AC Emmeney proptins \\
\hline noessut bo & 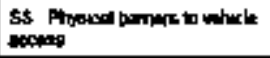 & Btanow reaphed & 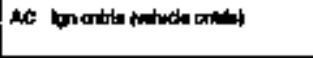 & AC Emetpenoy praptifin \\
\hline w0350s b4 & 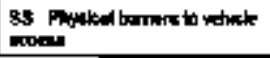 & How roptind & 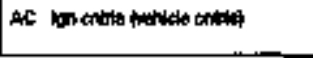 & ic efrationey protines \\
\hline
\end{tabular}


Tabto A-4. Hagter Control Table. (141 shuols)

\begin{tabular}{|c|c|c|c|c|}
\hline ID & Prw StS & M ssc & PTw $\mathrm{T}$ 睌 & NASR TS \\
\hline $1029-04$ alb & 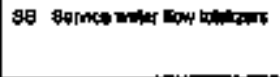 & 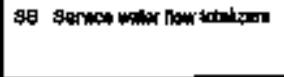 & 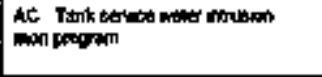 & 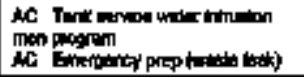 \\
\hline 100s-0atelat & 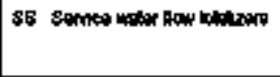 & 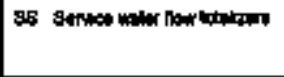 & 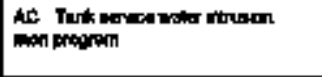 & 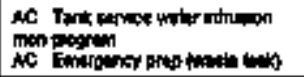 \\
\hline $1000-04=3$ & None riqumater & Mane benqund & Hone & Narted \\
\hline 1008040 & 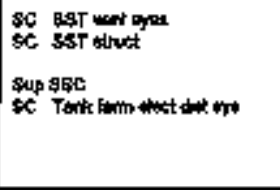 & Mone nowarid & 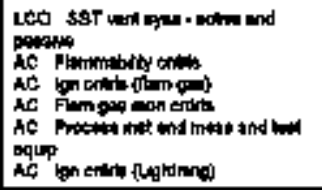 & 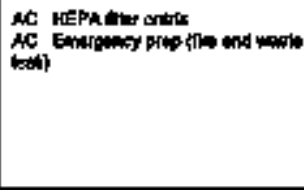 \\
\hline 10004 सा & 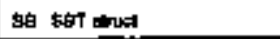 & Pone requmed & Bont & Ntend \\
\hline $1028-04$ w1 & 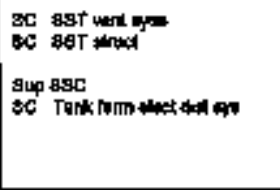 & Nome rapianded & 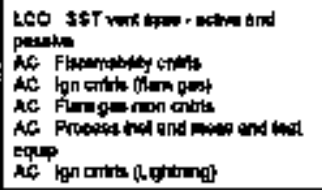 & 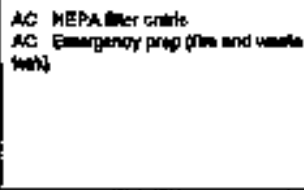 \\
\hline 10050 ad ex & None refultes & 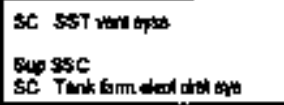 & 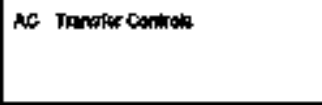 & 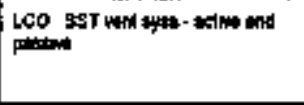 \\
\hline $1028-0403$ & Nonow repuned & Rane magnim & Aes Thend edth (pod) & 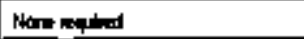 \\
\hline $10080.04 \%$ & 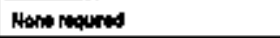 & Hone rapiat & 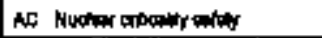 & 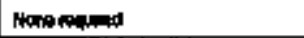 \\
\hline 100sers ald & 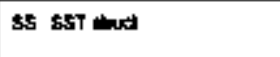 & 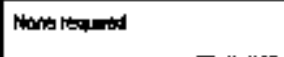 & 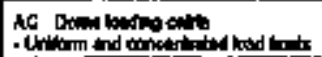 & 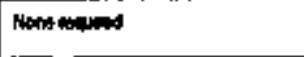 \\
\hline
\end{tabular}


Tahto A-4, Hastar Control Table (101 shect:)

\begin{tabular}{|c|c|c|c|c|}
\hline ID & Pan sec & Nem sec & PW TSR & W YaR \\
\hline 102006 wion & 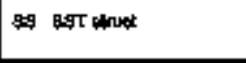 & Wont intion & 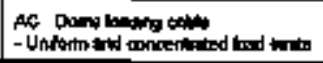 & How nowired \\
\hline 100945 a & fac ast atrol & Hom nompand & 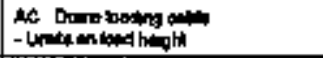 & 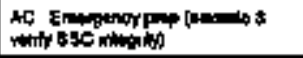 \\
\hline $10 \mathrm{stan}$ - 0 & 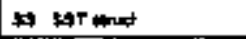 & Hone requed & 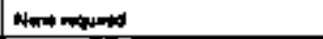 & None requned \\
\hline 16025 & S5 & Whene roqued & Nation & None requted \\
\hline $1025-00$. & Hone ropared & Mone nequened & Nomengured & 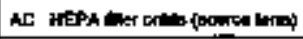 \\
\hline Ioscond & \&s क्रा मnt & Hon neapinat & Neno indiped & None repured \\
\hline 1900018 & \$s \$sT Huest & 190 & 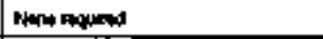 & Hone repures \\
\hline 10atere & \$\$ \$\$T : trin: & Nane rapued & What nearad & 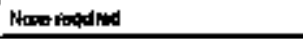 \\
\hline notcolo & st stst atrex & Nane mequated & Hone requltied & Nowerequerd \\
\hline NoCHE & as st exthet & 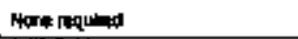 & Nane nequited & Hon rapend \\
\hline Nopact F & Nome requind & 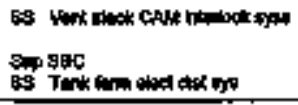 & Wime noqured & 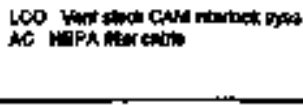 \\
\hline 1090-015 & Hone reaured & 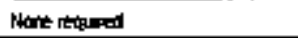 & AS Thes onts iptir & 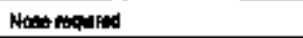 \\
\hline 1006-01H & 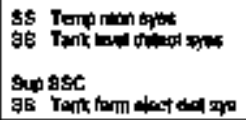 & Nome nequared & 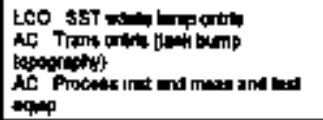 & Hanengand \\
\hline
\end{tabular}


Table A-A, Maske Condrol Takin. [106 smats]

\begin{tabular}{|c|c|c|c|c|}
\hline I & 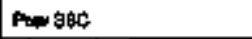 & unsect & Aw T得 & WTSR \\
\hline 1000-011 & 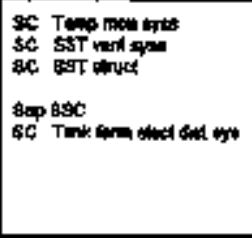 & None rapand & 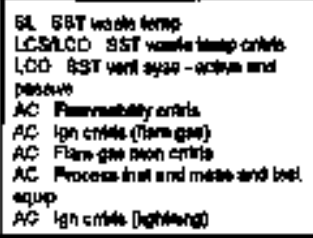 & 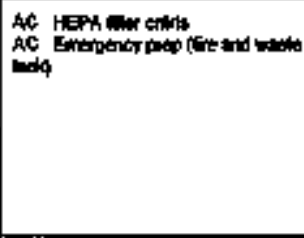 \\
\hline 1605-01] & 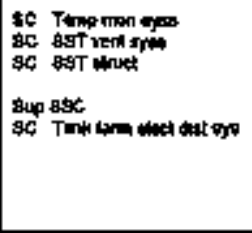 & Nomen ronited & 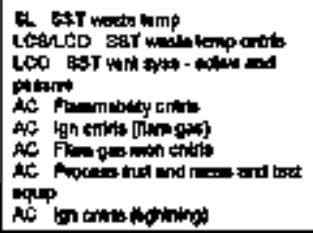 & 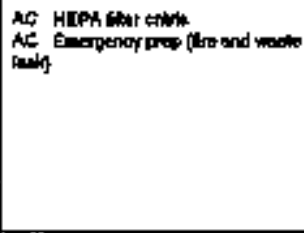 \\
\hline IDet-ork & 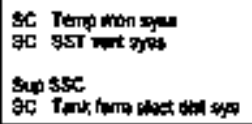 & 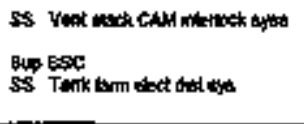 & 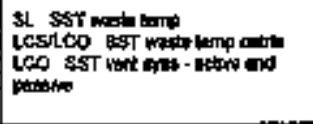 & 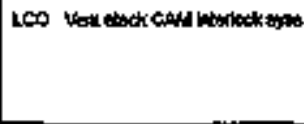 \\
\hline 108C.MI M & Noneraperad & 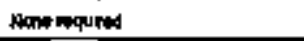 & AC Thenseren [DH] & Mona nopord \\
\hline 1006- 에나 & 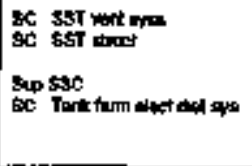 & Nenw atoured & 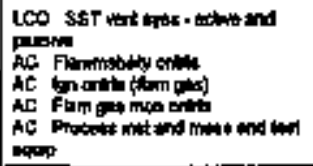 & 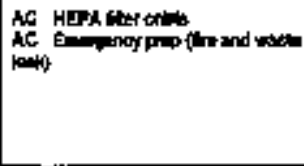 \\
\hline 1096-01n & Nanempond & Mone rogured & 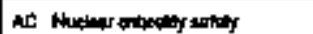 & Nene requent \\
\hline
\end{tabular}


Table A-4. Waster Control Table (101 thent:)

\begin{tabular}{|c|c|c|c|c|}
\hline $\mathbf{D}$ & Pow Ssc & mssc & Pm T\$R & Ane TSR \\
\hline $100 \cos \theta$ & Nomitramal & 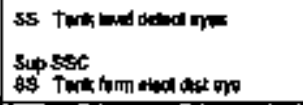 & Hen requal & None nequend \\
\hline $1600-018$ & 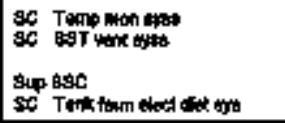 & 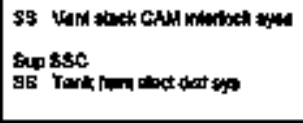 & 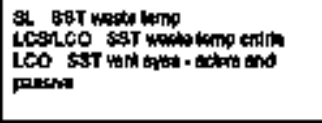 & 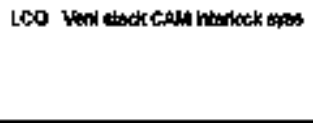 \\
\hline 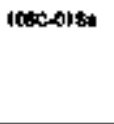 & 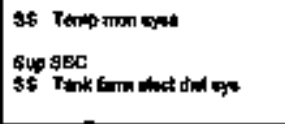 & 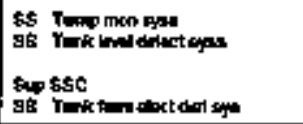 & 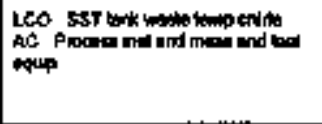 & 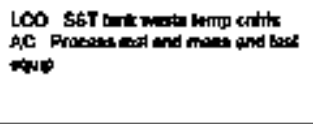 \\
\hline ICBç-01T & 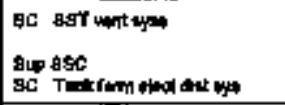 & 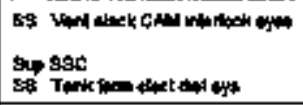 & Leo bst vort mox - wdime and & 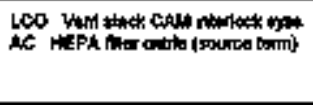 \\
\hline 168e-alu & 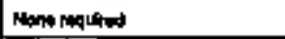 & 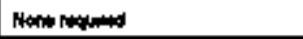 & 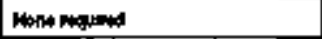 & 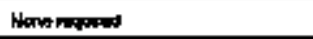 \\
\hline tootc-olw & Hone rimaned & None reatiod & Hon mand & None reyanes \\
\hline $100 \mathrm{cos}-01 x$ & 8s stT stact & Plane requed & 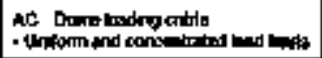 & 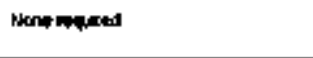 \\
\hline 100-01Y & 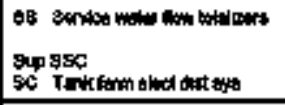 & 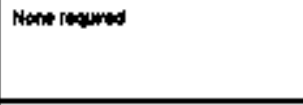 & 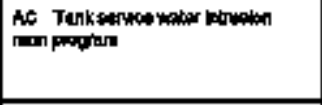 & None loopred \\
\hline notcos & 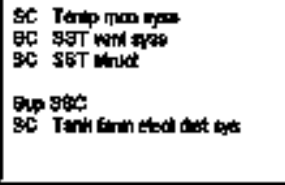 & 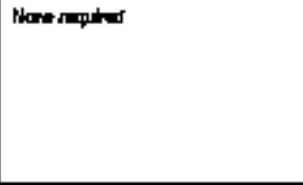 & 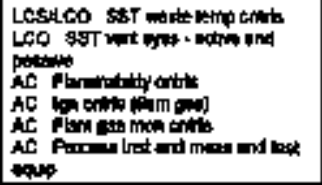 & 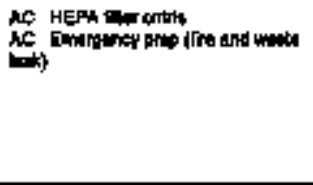 \\
\hline
\end{tabular}


Table A-4. Master Control Tellb. [101 obenits]

\begin{tabular}{|c|c|c|c|c|}
\hline $\mathbf{D}$ & PTrer BSC & $45 s c$ & Pm $T \$ R$ & MITSR \\
\hline 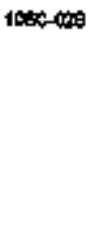 & 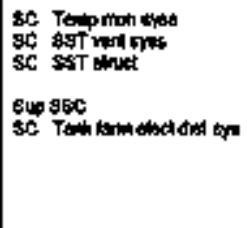 & Nkan reapurned & 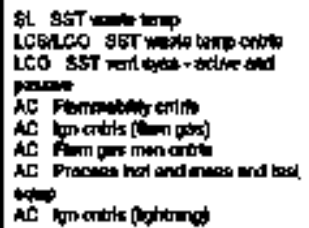 & 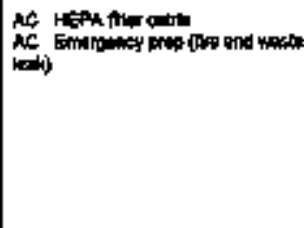 \\
\hline $100 \mathrm{C}-\operatorname{Dac}$ & 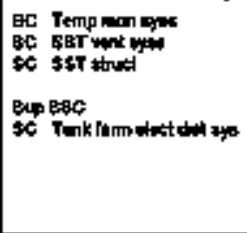 & Mans roqurad & 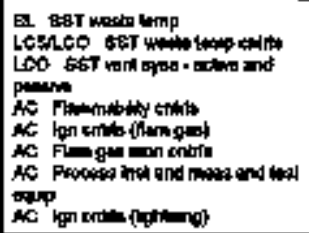 & 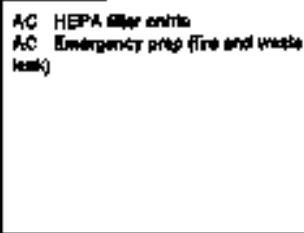 \\
\hline $1060-04 A$ & 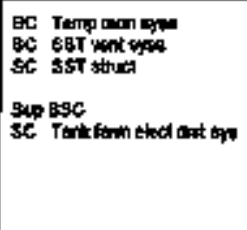 & 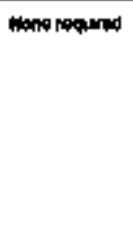 & 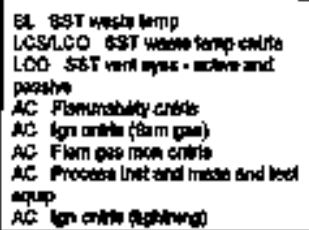 & 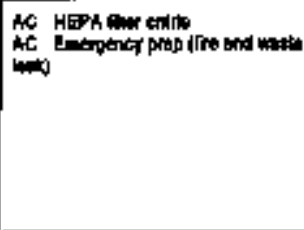 \\
\hline
\end{tabular}


Table A-A. Whater Comirol Fobla. (101 Sbatt)

\begin{tabular}{|c|c|c|c|c|}
\hline ID & Prorsec & MAN 5SC & HMW TBR & $\mu<T s \mathbb{R}_{R}$ \\
\hline logecoux & 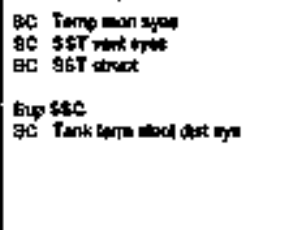 & thore reaperd & 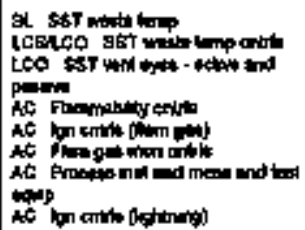 & 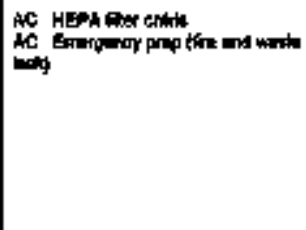 \\
\hline 100000 & 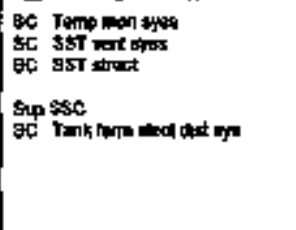 & Han & 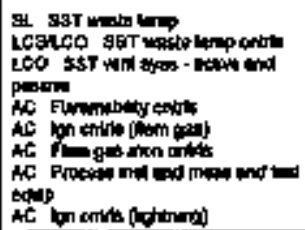 & 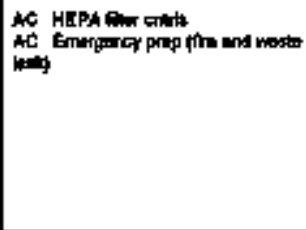 \\
\hline $1000-203$ & 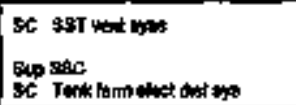 & 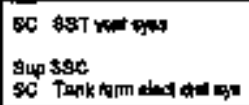 & 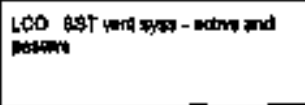 & 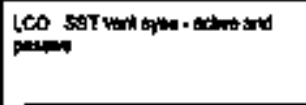 \\
\hline 1ascates & 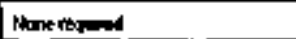 & Hon nowed & Momernated & Bton:mąned \\
\hline $1 \cos -960$ & 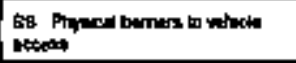 & Work rasend & 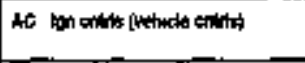 & 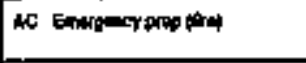 \\
\hline $1000-000$ & 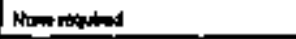 & 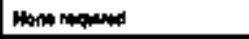 & Mintrumaneid & Mom mapend \\
\hline $1600-0<0$ & 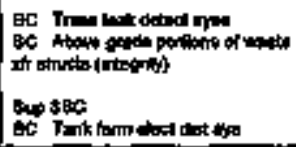 & 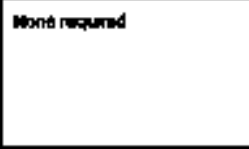 & 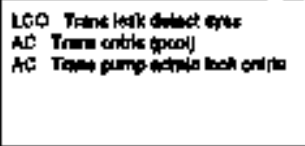 & 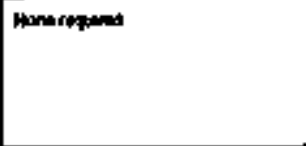 \\
\hline $10 x-006$ & Naptroup a & nom thatiod & Hom the pingd & Nontminumerf \\
\hline
\end{tabular}




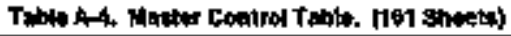

\begin{tabular}{|c|c|c|c|c|}
\hline $\mathbf{p}$ & $\mathrm{Pnn} 890 \mathrm{C}$ & Nat & Pap T\$P & Mrg \\
\hline nec-0a & Honereasurial & Along nepuread & 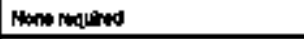 & Hone repured \\
\hline $\cos -0 \cos$ & None & Nonesequard & 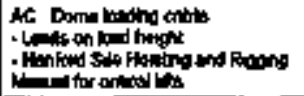 & Mone requind \\
\hline vocotal & 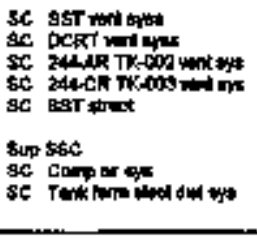 & Nons atomited & 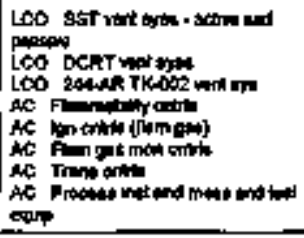 & 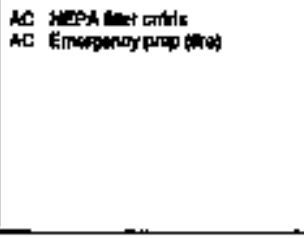 \\
\hline $10 x-001$ & Pane rapired & Hone requarod & Nenn mainged & 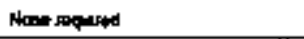 \\
\hline $1096-168$ & Hon & Heme & Nom realind & 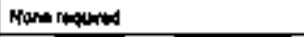 \\
\hline $100=10$ & Hont & 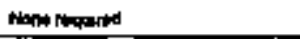 & itam & 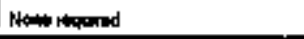 \\
\hline $100=100$ & Monteratid & Hom & Hone revined & Noneropened \\
\hline IDocos-10E & Nane requirad & kbre reaphad & None mapuld & 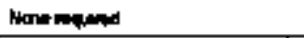 \\
\hline 10ex-ief & 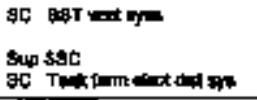 & Hone nequend & 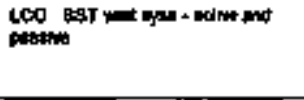 & 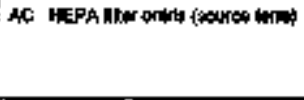 \\
\hline 108G-10H & Nomerepured & 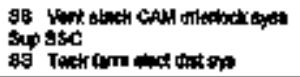 & What ropulat & 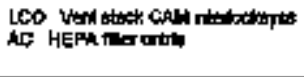 \\
\hline $100 x-10 y$ & None required & 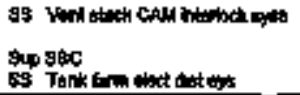 & Fond & 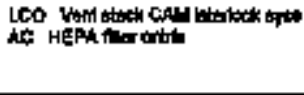 \\
\hline
\end{tabular}




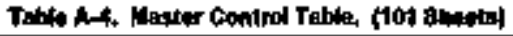

\begin{tabular}{|c|c|c|c|c|}
\hline it & Pिursse & ustc & Pur Tak & Wrat \\
\hline $109 \mathrm{C}-10 \%$ & 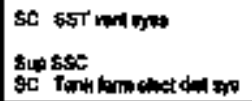 & None rapand & 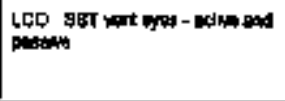 & 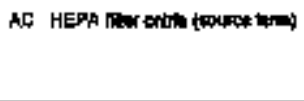 \\
\hline iosicia & 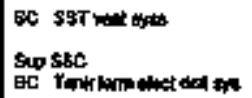 & Btome requer & 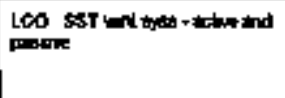 & 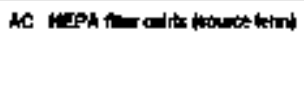 \\
\hline $\sec x-111$ & 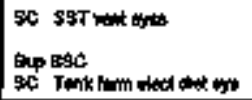 & Mone nequed & 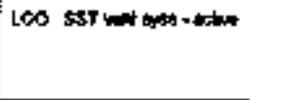 & 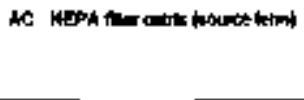 \\
\hline 10000 & Nand ingind & Nare rewathed & Alenen minged & None magures \\
\hline 10 ㅅ-11C & Ninem & Nome noqured & 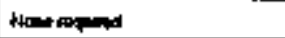 & Mand repured \\
\hline 106c-a10 & None mapread & Hons nequared & Nom andind & 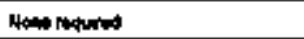 \\
\hline $109 C-110$ & Hons nexpind & 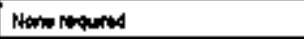 & Naminimid & Nemen maputer \\
\hline$+\infty 00-41 F$ & Nent ind & Mare hapilrod & Mone napred & None reapund \\
\hline 100C-11白 & Wene raguited & 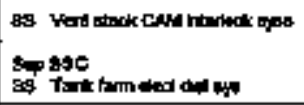 & Mone nopured & 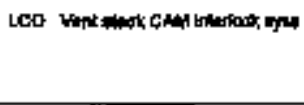 \\
\hline $100 \mathrm{C}-11 \mathrm{H}$ & Alone requared & 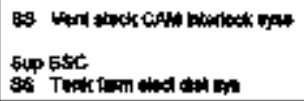 & 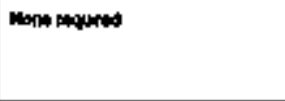 & 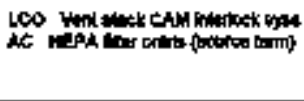 \\
\hline $1000-114$ & 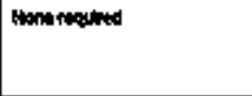 & 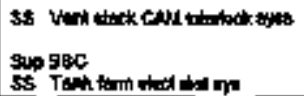 & Who tequed & 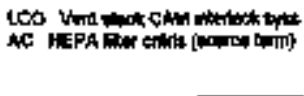 \\
\hline
\end{tabular}




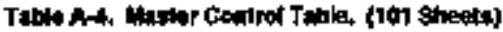

\begin{tabular}{|c|c|c|c|c|}
\hline 1 & Prw & 녕요 & PTw TAR & Im ISR \\
\hline $1000-11 \mathrm{~J}$ & 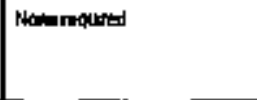 & 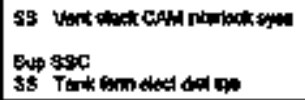 & Ithromond & 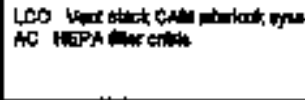 \\
\hline $100 \mathrm{ec}-11 \mathrm{~K}$ & Nonerequared & 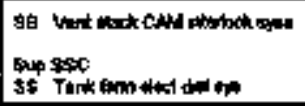 & Hone mapred & 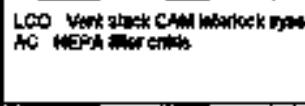 \\
\hline $109 \mathrm{C}-11 \mathrm{~L}$ & Moperequared & 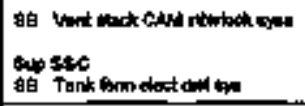 & 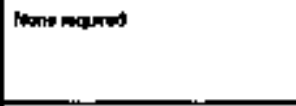 & 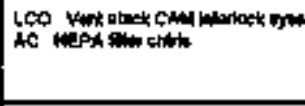 \\
\hline $\cos -14$ & 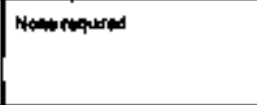 & 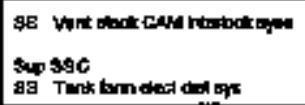 & Thene nequted & 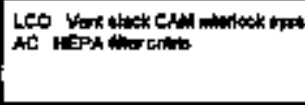 \\
\hline $\operatorname{noc}-114$ & 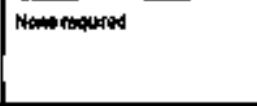 & 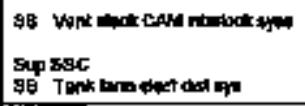 & Pane noward & 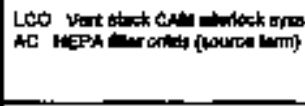 \\
\hline NoSt-110 & Nontroquted & 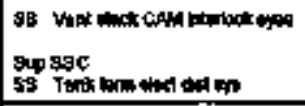 & Ctano mandind & 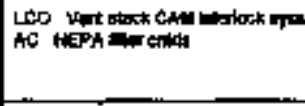 \\
\hline Nooc-13h & 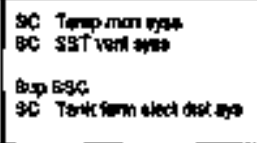 & 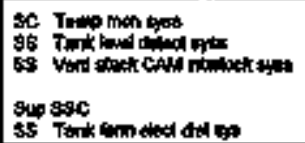 & 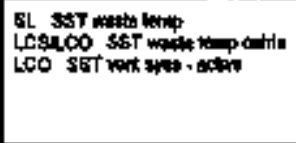 & 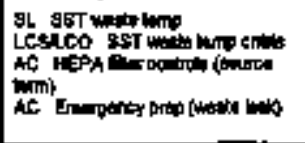 \\
\hline
\end{tabular}




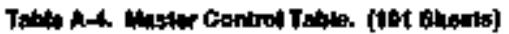

\begin{tabular}{|c|c|c|c|c|}
\hline ID & PAN 日BC & bysec & Prev Ts: & It ISh \\
\hline $10 \operatorname{coc}+29$ & 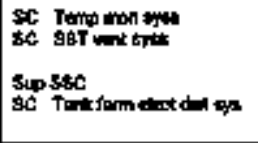 & 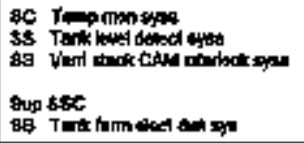 & 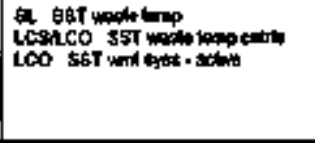 & 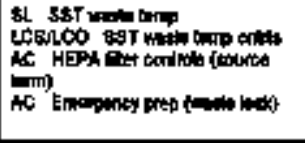 \\
\hline $\cos -130$ & 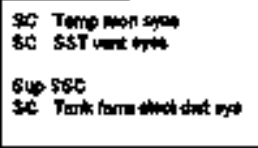 & 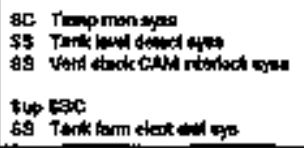 & 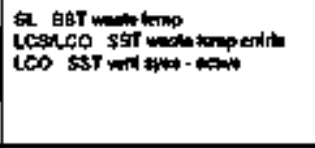 & 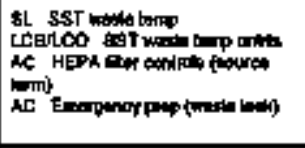 \\
\hline $1000-120$ & 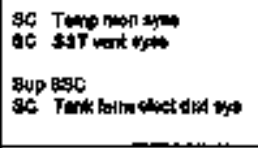 & 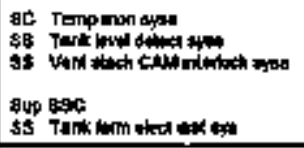 & 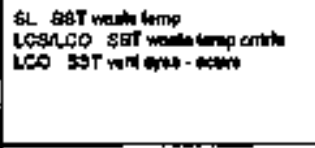 & 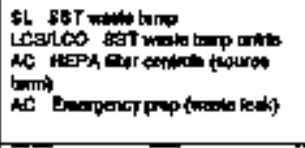 \\
\hline $1090-17$ & Mone rapanded & None roinglod & 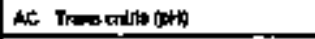 & None repurod \\
\hline $1000-120$ & Anen mantad & 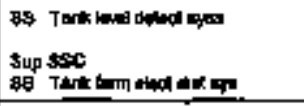 & Nanempinad & 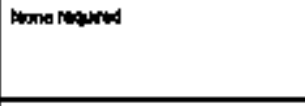 \\
\hline $\operatorname{tasc}-134$ & 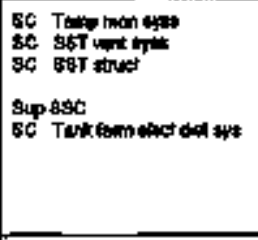 & Home nowed & 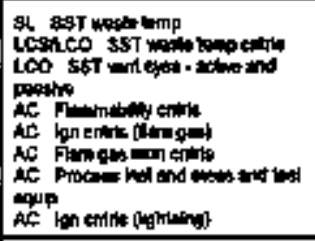 & 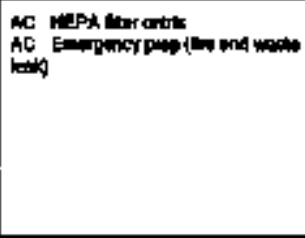 \\
\hline Iaxe-13: & Neme mation & 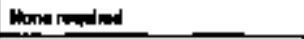 & None nowhed & 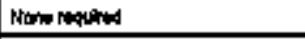 \\
\hline 197ANOA-LEVEY & 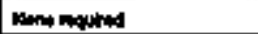 & Nome neprod & 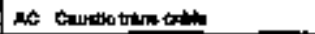 & Ptone ropits \\
\hline
\end{tabular}


Table A-4 Hastif Gonirod Table. (101 theety)

\begin{tabular}{|c|c|c|c|c|}
\hline $\mathbf{0}$ & Pren sex & Nessc & Pher Tst & 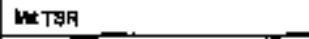 \\
\hline 1DAHLOM-LEVECS & 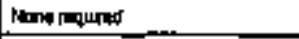 & 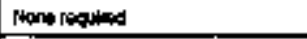 & Af & Nent Fiaplod \\
\hline 10FAHWO1-LEVEDS & Nome nowared & Nons & 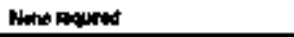 & Ant \\
\hline 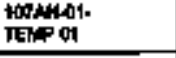 & Nomerequired & Notit megrad & 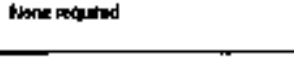 & 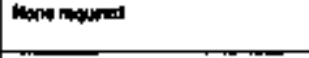 \\
\hline 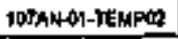 & Nown ropured & Nown rapiand & 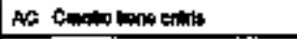 & 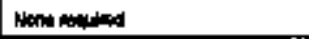 \\
\hline 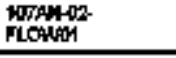 & Nome roquared & 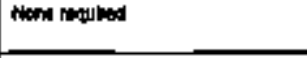 & 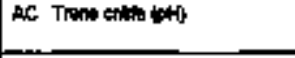 & Sind \\
\hline 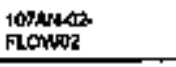 & Hom mased & han maned & mone nogund & 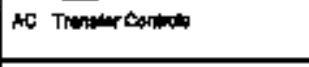 \\
\hline 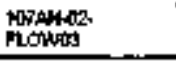 & 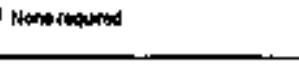 & nems lowed & 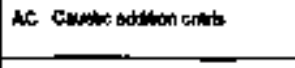 & How raphed \\
\hline 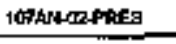 & Malement & Nbene ropquad & 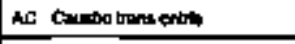 & Hon \\
\hline 1UTANCQPREFGOZ & Mnomąarad & 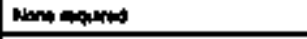 & 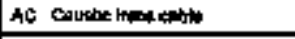 & Mono rogunat \\
\hline 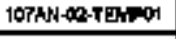 & Nons nopind & Nonit mand & 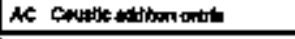 & Btome noquend \\
\hline 10TANGOSFLON & 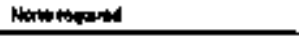 & Norit nangrod & 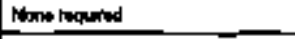 & None nequited \\
\hline 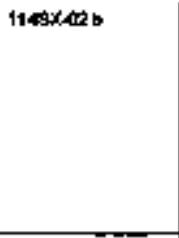 & 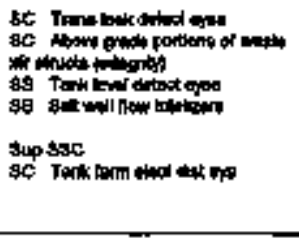 & 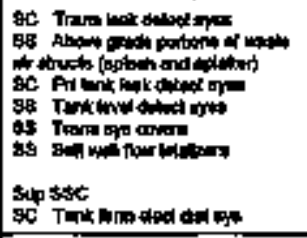 & 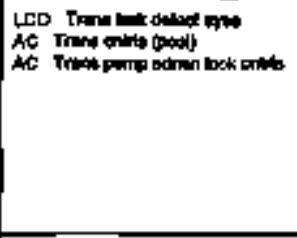 & 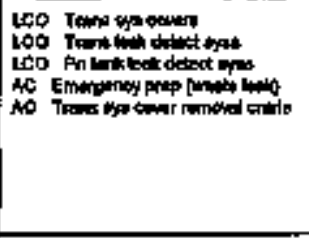 \\
\hline 1145x-OF i it & 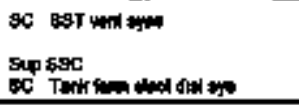 & 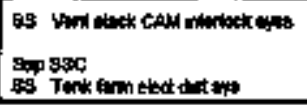 & 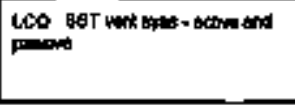 & 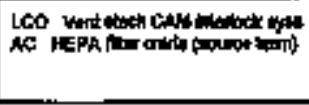 \\
\hline
\end{tabular}




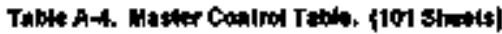

\begin{tabular}{|c|c|c|c|c|}
\hline $\mathbf{E}$ & Prw\$s & $n=98 c$ & 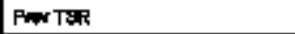 & METSR \\
\hline $17+48 X 04+16$ & 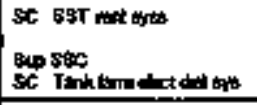 & 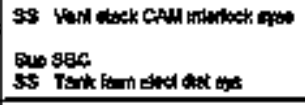 & 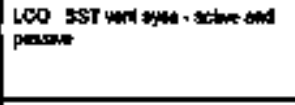 & 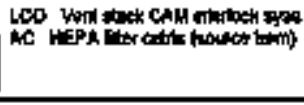 \\
\hline r14Ex-OAd 2 & AS SET strud & Hane manger & Nomanod & Hantengod \\
\hline $1146 b_{0}-04 a+3$ & sos bST hud & Nowe nay & $\mathrm{NH}_{0}=\mathrm{n}$ & 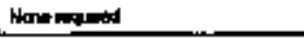 \\
\hline $1145 x-04=4$ & 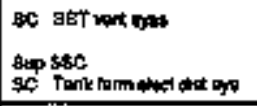 & Home raparas & LCD 68T & 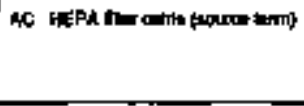 \\
\hline 11 asx-od b 1 : 1 & Hint & Nomatapunas & 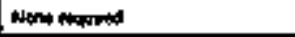 & 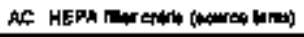 \\
\hline $1145 x+0 d$ b $1 \in 2$ & 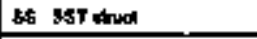 & Netheramed & Nhim requar & Nonumatem \\
\hline $11460.04 b 1=3$ & 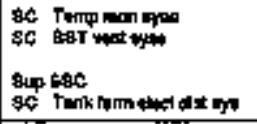 & Hone rimpor & 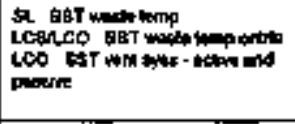 & 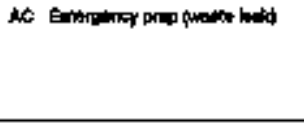 \\
\hline 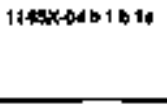 & 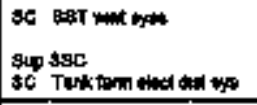 & Nomponimes & 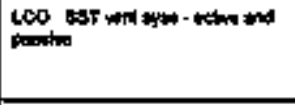 & 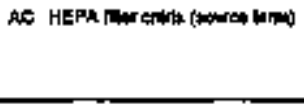 \\
\hline $1 \mathrm{As} 5 \mathrm{~L} 0 \mathrm{db} 1 \mathrm{~b}$ is & 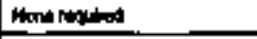 & St & 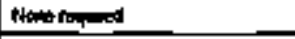 & None \\
\hline $1145 \times 100$ b 1 b. & s9 stt burt & Nonempung & Flon miperd & Nonentiphed \\
\hline $1148 \times-04$ b 1 b 2 th & FC ह\$का जhug & Manes napured & 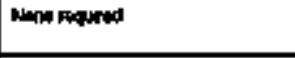 & 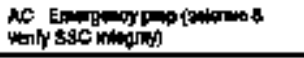 \\
\hline $11=9 x-904$ b 1 b 5 ta & 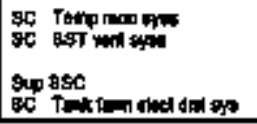 & horentquher & 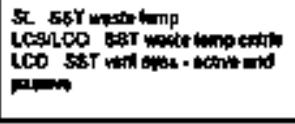 & 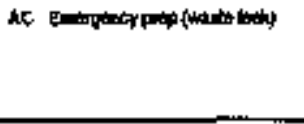 \\
\hline
\end{tabular}




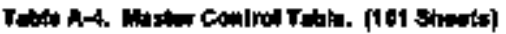

\begin{tabular}{|c|c|c|c|c|}
\hline D & Pew $5: 9 c$ & in sogc & DTow Tझ & NEF FR \\
\hline $1445 \times 04 b 103 b$ & 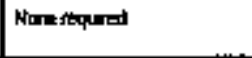 & mone radiad & What inged & 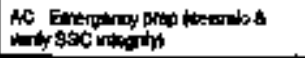 \\
\hline $1148 x, 04 b 1 \leqslant 1$ & 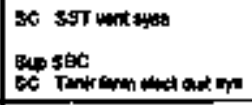 & Hon popurad & 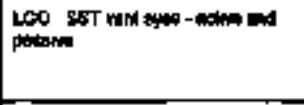 & 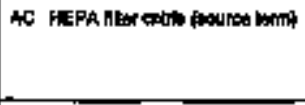 \\
\hline $1145 x-046<1<2$ & 53 s9T witel & Hone rapand & Nente nondind & 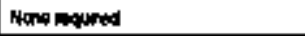 \\
\hline $1145 \times 0461$ ca & 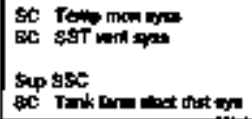 & dand & 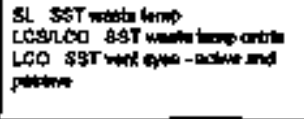 & 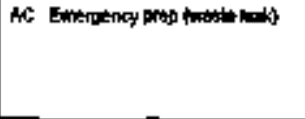 \\
\hline $11493 \times-04 b 1$ a 1 & 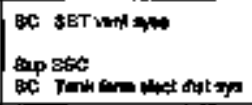 & Maped & 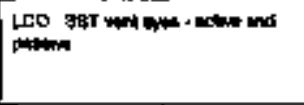 & 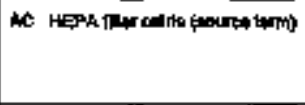 \\
\hline $11+0 x-04 b 142$ & 3) 2\$T औint & 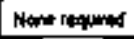 & Non & 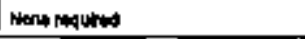 \\
\hline 11 axcoub 1 d & 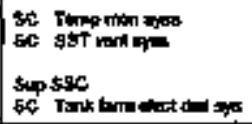 & dithon & 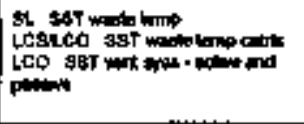 & 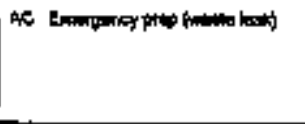 \\
\hline $114 \Rightarrow x-04 \mid b 3 b$ & 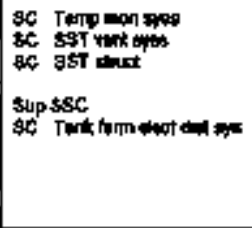 & 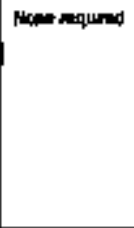 & 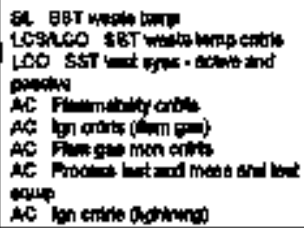 & 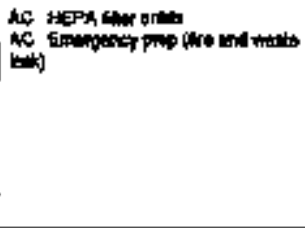 \\
\hline
\end{tabular}


Tatole At Howter Control Tehlo. (101 theats)

\begin{tabular}{|c|c|c|c|c|}
\hline ID & Pror SSC & 새 ssect & Phar TSR & m Tse \\
\hline $11485-0405$ & 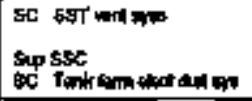 & Hom nandud & 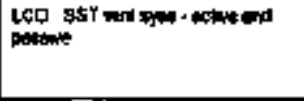 & 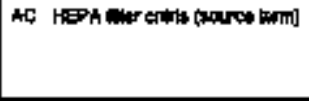 \\
\hline $1145 x+04$ t 1 & 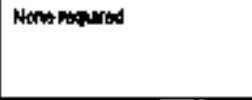 & 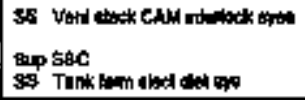 & 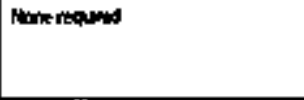 & 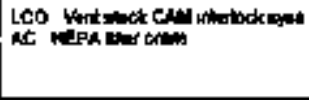 \\
\hline $1145 x=4$ ta & 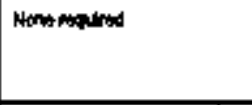 & 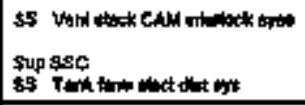 & 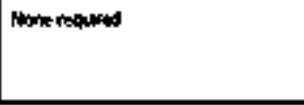 & 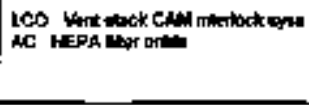 \\
\hline $11495 \times 401026$ & thentinamad & 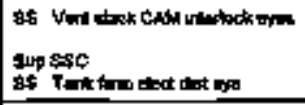 & Nown nasid & 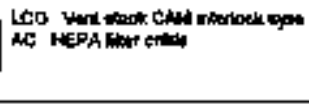 \\
\hline $11496-04$ a 20 & Mone requad & 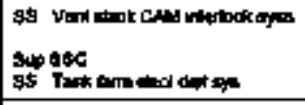 & Nom mapors & 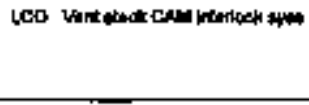 \\
\hline $11495-04$ o 24 & $\operatorname{mon}$ andand & 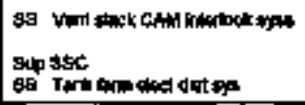 & Nonsaped & 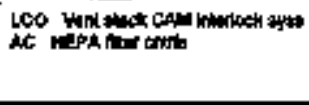 \\
\hline $1149 x-05+1$ & 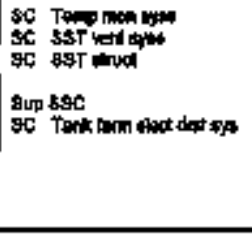 & 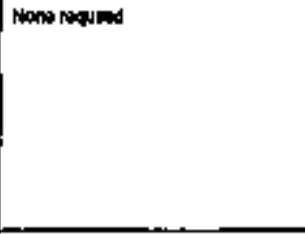 & 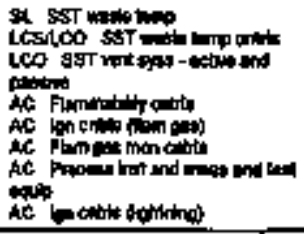 & 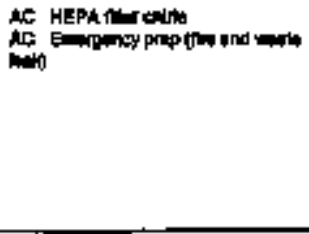 \\
\hline 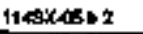 & Now rephed & Nomenamed & Mene raquind & nomp naped \\
\hline
\end{tabular}




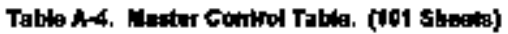

\begin{tabular}{|c|c|c|c|c|}
\hline ID & Pnw S6C & Ant $89 \mathrm{C}$ & Prow TPR & im rag \\
\hline $1145006 \mathrm{~b}$ & S\& BsT thent & 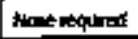 & Whine mingred & None ingum \\
\hline $1246 x-060$ 当 & 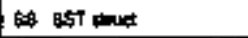 & 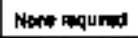 & 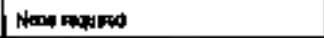 & 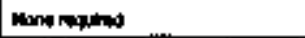 \\
\hline $1143 \% 0502 x$ & Non mons & Nhes mared & 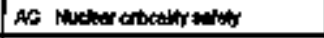 & Home renured \\
\hline $1145 \times 5014$ & 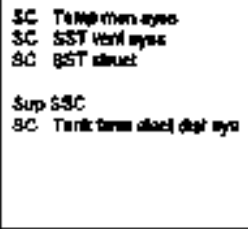 & Hom thand & 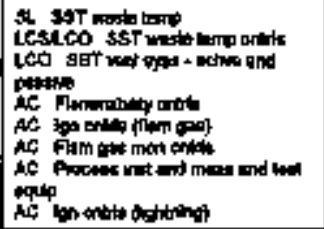 & 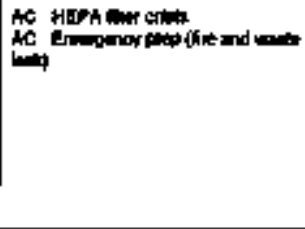 \\
\hline $11<5 x \sec n 1$ & \$\$ \$BT ftw & Hone maperod & 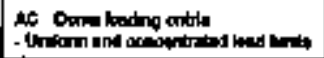 & None roputed \\
\hline $1149 x-09$ a $1 b$ & 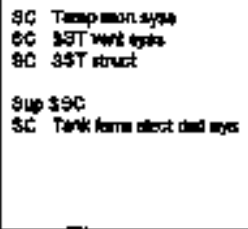 & Napon nequind & 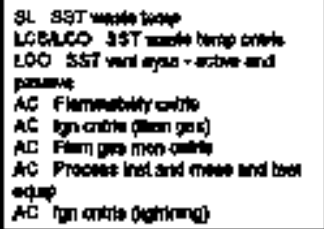 & 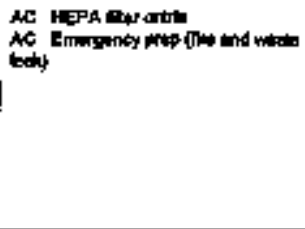 \\
\hline $1145 x-00 * 2$ & Hone napend & Hone naparid & Amo repured & 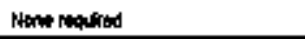 \\
\hline 1145xkest 13 & Ss SET thud & Btente ralund & Honterequided & Nant staplad \\
\hline 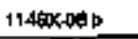 & Homplapd & Itome nowand & Molnoming & 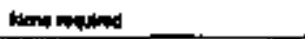 \\
\hline 20MAROLA & Nowongended & 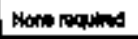 & Hone riogind & 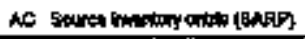 \\
\hline auknong & 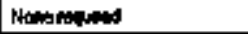 & Nom & Nontropurted & 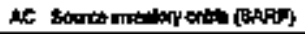 \\
\hline xuentedt & 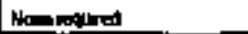 & Nant mogled & 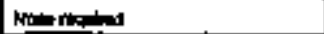 & 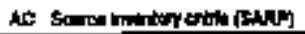 \\
\hline
\end{tabular}




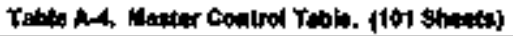

\begin{tabular}{|c|c|c|c|c|}
\hline $\mathbf{D}$ & Prer B\$C & Hst5 & PT: T\&R & uㅡ Trg̣k \\
\hline 204AR-010 & 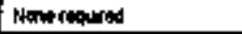 & 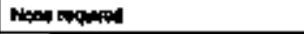 & 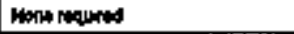 & 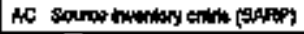 \\
\hline GUAR-ME & None wey now & 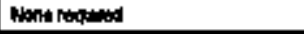 & Wone ropured & Not sewed intitiony ants. \\
\hline HANAROAF & Nonim mangand & Hon maphed & Home naquind & 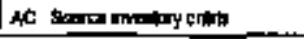 \\
\hline TOAR-010 & Honerequeread & 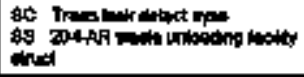 & 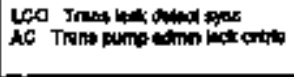 & 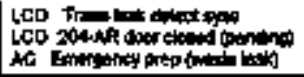 \\
\hline ZOMRRAH & 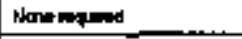 & Itbone requed & Pane nquared & 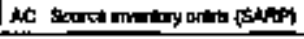 \\
\hline 204R-0|I & 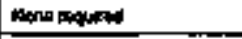 & Mone rapand & Moneraparas & 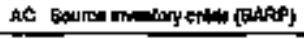 \\
\hline 20AARAIJ & 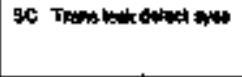 & 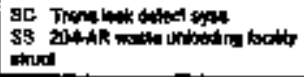 & 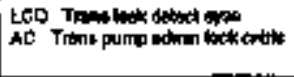 & 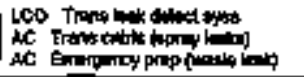 \\
\hline POAAR-OIK & 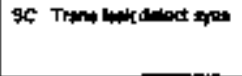 & 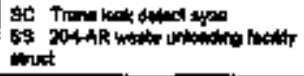 & 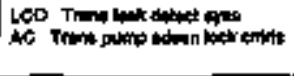 & 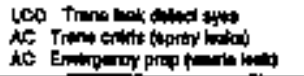 \\
\hline acentrase & Nome nempunad & Hare rapined & 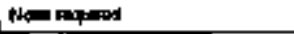 & 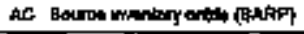 \\
\hline 304AR-GaA & Hons napand & Bron rapand & rentrequered & 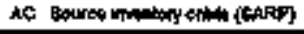 \\
\hline sonventes & 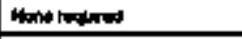 & Nons & 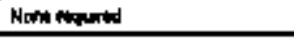 & 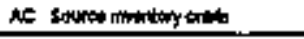 \\
\hline Buthroses & None required & Hetion ropured & Nomeres & 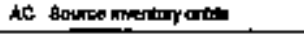 \\
\hline 2OAAR-DEE & Nove reparay & EC Trans int defied apen & Ntend meparad & 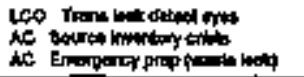 \\
\hline XUARRARF & 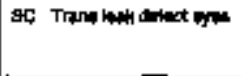 & 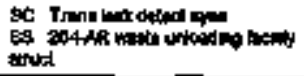 & 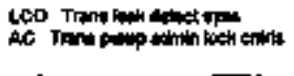 & 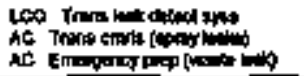 \\
\hline 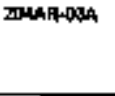 & Nom noqurod & 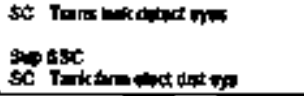 & 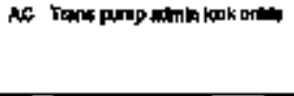 & 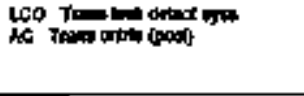 \\
\hline
\end{tabular}




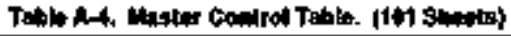

\begin{tabular}{|c|c|c|c|c|}
\hline 10 & Mre bste & masc & Pitu ton. & MTap \\
\hline 204AR-038 & 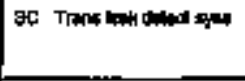 & 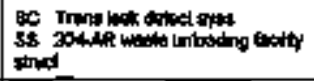 & 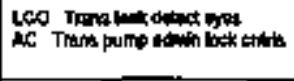 & 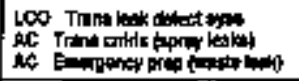 \\
\hline 204ARox & None reapured & Nono apourid & Nane reauriod & Nenos resured \\
\hline xatroun & Nont rimpind & 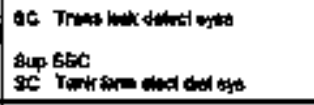 & 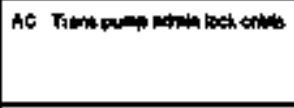 & 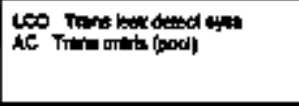 \\
\hline ritingons & 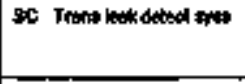 & 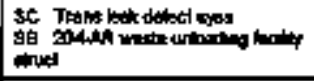 & 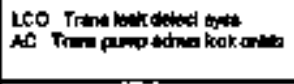 & 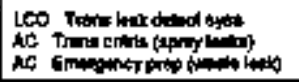 \\
\hline $2004 \pi-0+c$ & 1 & 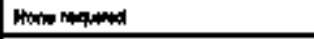 & 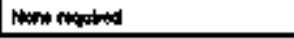 & 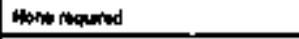 \\
\hline$x+4=00$ & Norm & Hone inand & None ropind & Hime requerod \\
\hline Xodipape & Hene nowered & Bone naperd & Ntent & None ropitiod \\
\hline ZOAAR-DGF & Mone ropen & 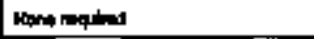 & Nantrapumad & Hane requered \\
\hline OOAR-DSA & Mang maped & 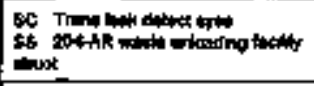 & 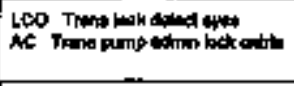 & 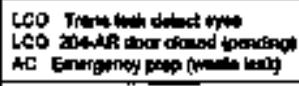 \\
\hline 204AR-DC & 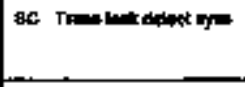 & 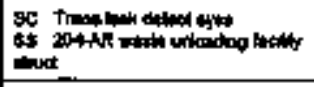 & 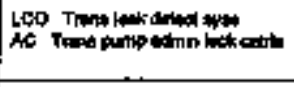 & 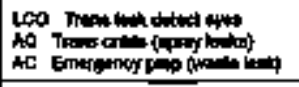 \\
\hline DMAR-DEC & Mone roped & 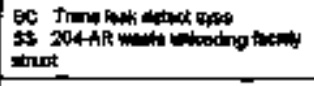 & 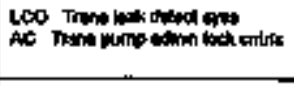 & 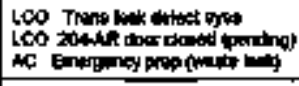 \\
\hline 200R:04 & Nons requind & 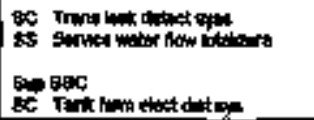 & Nonn nowired & 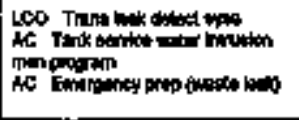 \\
\hline
\end{tabular}




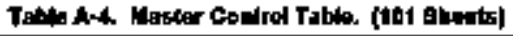

\begin{tabular}{|c|c|c|c|c|}
\hline ID & Paw soc & $\mathrm{HASC}$ & Paw Ts & METSR \\
\hline 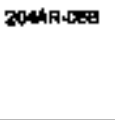 & 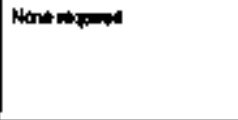 & 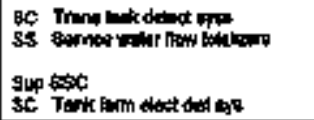 & Henenguried & 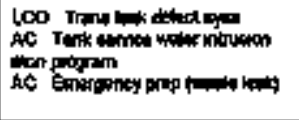 \\
\hline LANR-040 & 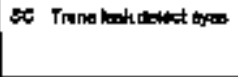 & 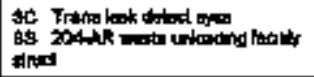 & 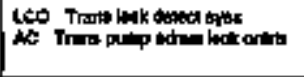 & 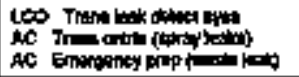 \\
\hline $2046905 A$ & Nonen anured & 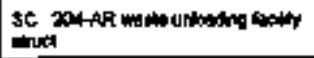 & 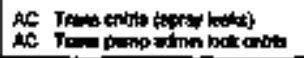 & 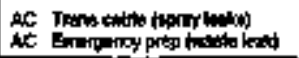 \\
\hline molations & Mone requind & 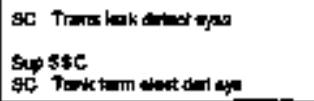 & 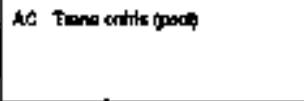 & 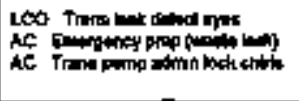 \\
\hline 20SAR-OTC & Bone noquired & 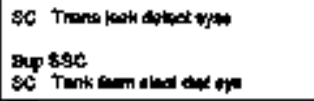 & 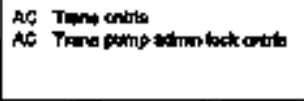 & 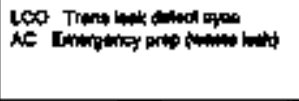 \\
\hline 204AR-604 & Monersepution & 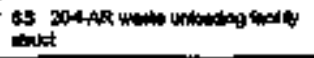 & $\operatorname{lom} 10$ & 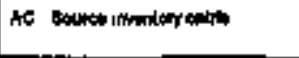 \\
\hline adufioss & Wine rapured & Mepre requmed & Hone nowpel & 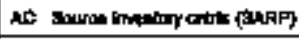 \\
\hline 204AR-0\% & Dene repures & Hare requered & Monp numet & 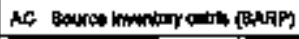 \\
\hline 204AR-0\%0 & rapereasures & Nowis reperes & 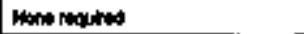 & 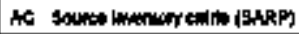 \\
\hline Tueth-tac & Manted & Noit trilanad & Mane nquired & Norre napumed \\
\hline TUARAF & 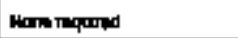 & 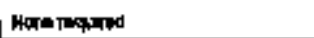 & 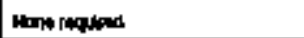 & Lomen naphes \\
\hline 204AR-696 & 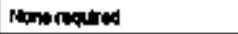 & Nane rapulad & 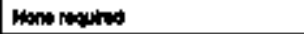 & Hone nesures \\
\hline 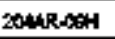 & Non reped & 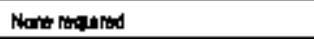 & Hithe required & 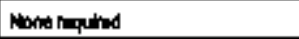 \\
\hline Xuntk-11k & d & 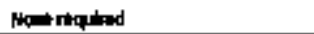 & the magumat & Monen roqurod \\
\hline
\end{tabular}


Tubte A-A. Wabter Control Table. (10t Bhoebs)

\begin{tabular}{|c|c|c|c|c|}
\hline 10 & Pлw sste & $\omega 35 c$ & Put TSR & WTSR \\
\hline Z04Ak-11日 & Nane nowind & lone ropand & Non reguned & Honentephad \\
\hline 2OAH-11C & None required & Mone rapuad & thenen repurad & 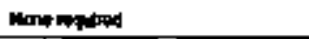 \\
\hline $20418-110$ & 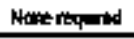 & 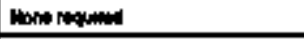 & Neme nenquined & Alon \\
\hline zount ate & Nomentianed & 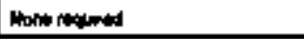 & Neme roquind & 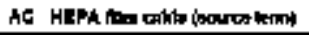 \\
\hline 2otisentis & Noterengumad & 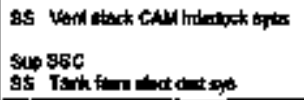 & Nonernquited & 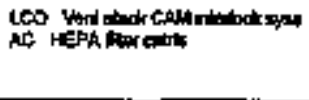 \\
\hline 213 wh & thone manerad & 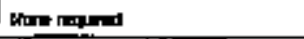 & Nbran manted & None nimped \\
\hline $29+402$ & Nom manned & Nome raquing & Nane & 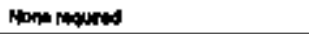 \\
\hline $2+3 \times-13$ & Nom & 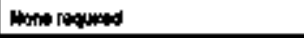 & Nente rocuind & 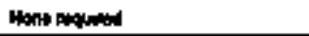 \\
\hline $26 \mathrm{n}$ nod & Herne may & 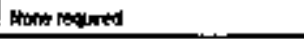 & Blene napinged & Hone reglated \\
\hline 213 Was & mone mand & Notot thuhed & Mone den & None riqued \\
\hline 219 mat & Mene monead & Hartemqures & Mons maphad & None requined \\
\hline 219 wes & 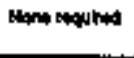 & 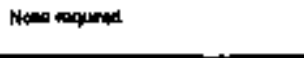 & 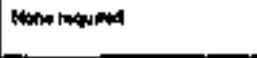 & 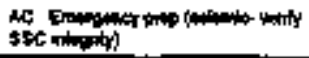 \\
\hline 213 wod & Monem mated & Norempquant & Mare mapirod & None requared \\
\hline 219 was & Lome nepuras & Nener monumad & 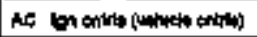 & 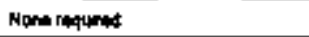 \\
\hline $244 R-\theta 4=0 s$ & Nont raspod & 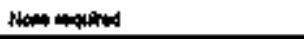 & 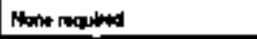 & 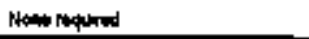 \\
\hline 240kAM b प & None regirad & Neren maphad & Nane requited & Nave roqured \\
\hline $24001-01$ bat & 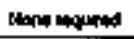 & Heno roopured & 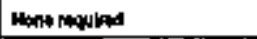 & AC HFA \\
\hline 24सR-91 B & 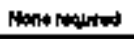 & 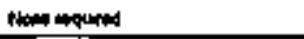 & 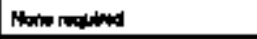 & 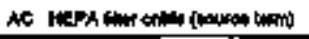 \\
\hline 24AkRor b t on & 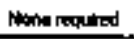 & 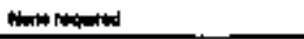 & Nantrequined & 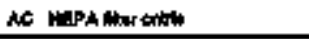 \\
\hline 2uleded at & Nonne requened & 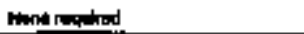 & Nenerequared & 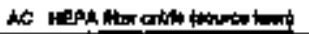 \\
\hline
\end{tabular}




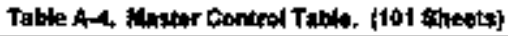

\begin{tabular}{|c|c|c|c|c|}
\hline 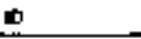 & Primstas & Masse & Pion Ts: & m T宓 \\
\hline $244 \mathrm{R}-01 \in \mathrm{DD}$ & Nonom naphind & Hane loparad & 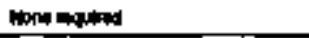 & 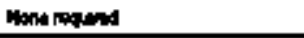 \\
\hline 240Red ton & Nene requars & Howe loplat & Kane mulest & 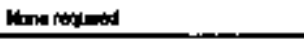 \\
\hline 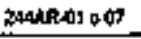 & He raquend & 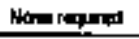 & AC HEPhy & (a) \\
\hline 24AR-O1 $=09$ & 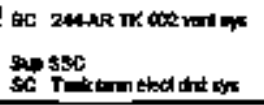 & Nanu reaperal & 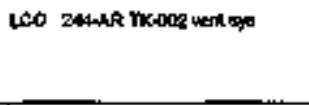 & 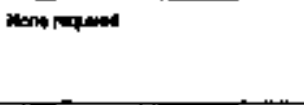 \\
\hline 2440 P. & 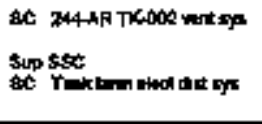 & nane rewand & 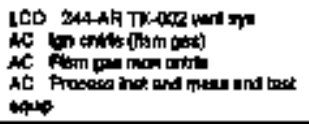 & 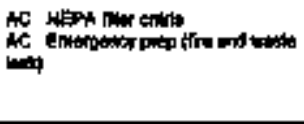 \\
\hline $244 k+01$ a 10 & Nont heaprod & int inurod & 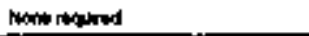 & 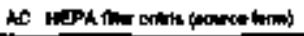 \\
\hline 2404R.01 o 11 & Mene noqurod & Herem anquand & Nome roquered & 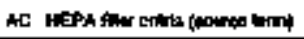 \\
\hline 240mR-01 \& 13 & Mone maptad & Montrapumad & Nare requarid & Nangropured \\
\hline $2404 \pi-\infty$ & 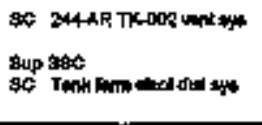 & Went resures & 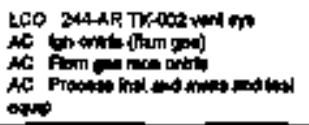 & 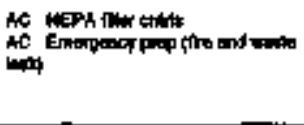 \\
\hline 244AR-0R a 06 & 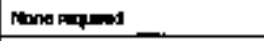 & Wone requland & Nacerequered & Nonq ampured \\
\hline 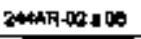 & 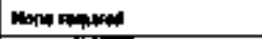 & Nent nopures & Nank requareq & Nonereguines \\
\hline $244+02 a$ of & Minn misid & Hont mantod & 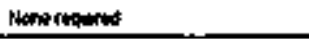 & Ptom rinquired \\
\hline 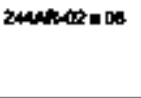 & 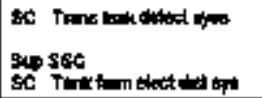 & Whe requend & 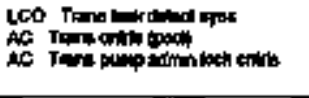 & 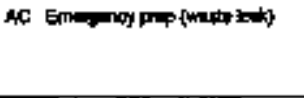 \\
\hline
\end{tabular}


Table A-4 Mustar Gonirol Table (jo1 Gheats)

\begin{tabular}{|c|c|c|c|c|}
\hline ID & Pisu ssc & Ma sst: & Pm Ts:R & MTs \\
\hline 244REA प10 & 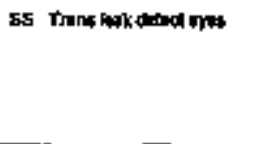 & 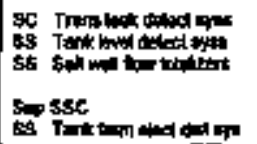 & 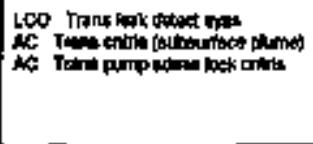 & 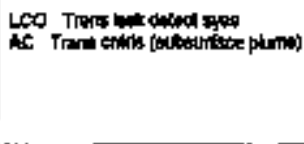 \\
\hline 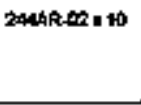 & 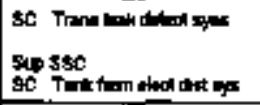 & Mane nemperad & 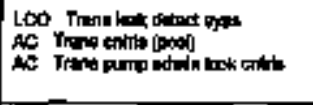 & 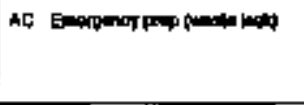 \\
\hline 2WAR-DR=11 & 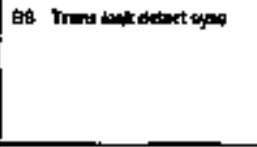 & 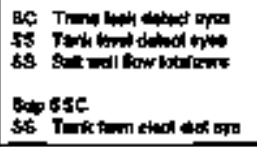 & 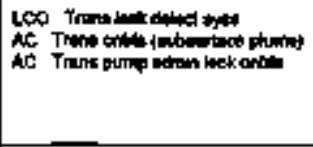 & 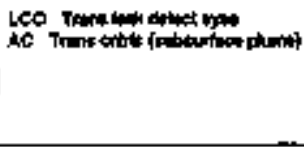 \\
\hline 24 Yaras $0<=12$ & Nare roquared & Nene ramped & Nones roqured & Honompured \\
\hline 24SAR-0R \& 15 & None repaured & 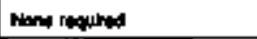 & None nequened & 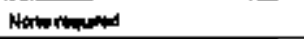 \\
\hline $2 \operatorname{man}-1014$ & Neanomind & 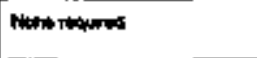 & 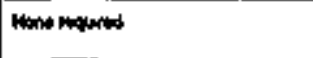 & 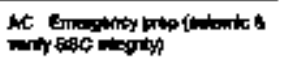 \\
\hline 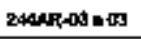 & Nore moquired & Nent rayumed & acf Thers aristis & Nomercopurad \\
\hline $24 \operatorname{an} 03$ ing & 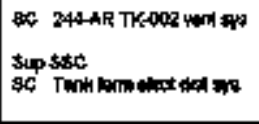 & Nomentented & 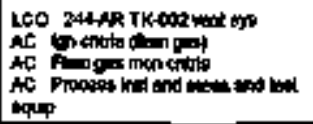 & 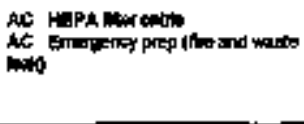 \\
\hline 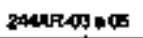 & Hent mathent & Nbonempurat & Mins noquind & Haw \\
\hline 24AnR- $\alpha=0$ & Man mand & 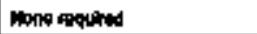 & Hone reapirod & atent \\
\hline 24inkes a of & 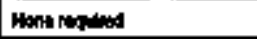 & Hon wopured & 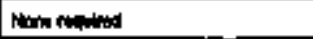 & d \\
\hline $240 \mathrm{n}-\mathrm{cos}+0 \mathrm{~s}$ & Wine lated & Nomedonghed & 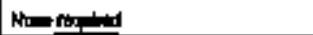 & 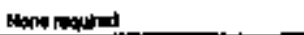 \\
\hline
\end{tabular}


Tablo A-4. Master Controd Tabla. \{101 stereic|

\begin{tabular}{|c|c|c|c|c|}
\hline I & PTw 5 Sac & 녕 \$c & Praw 139 & M TER \\
\hline 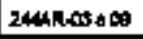 & Nime noquad & Hone requitid & 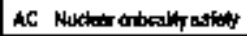 & None \\
\hline $2 \operatorname{sen} A-6013$ & Moneropurad & Mones romparad & 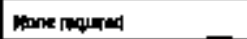 & 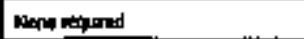 \\
\hline 244 R-03:14 & Nown ropotad & None raped & Bone nequind & Heno nopuread \\
\hline $204 n-0$ as 18 & None required & Hont nitarid & 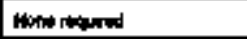 & Nenw nowned \\
\hline 2athresto 16 & Motionequated & Wone ropalmad & 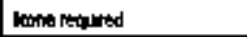 & 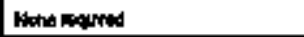 \\
\hline 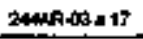 & Nomapinar & 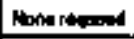 & Wentanguted & Anom \\
\hline 244AR-03a Y8 & NHow & None reapen & Hare requand & 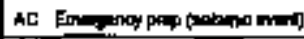 \\
\hline $240, R-04=01$ & 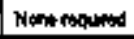 & Lano ponent & 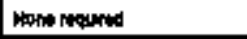 & 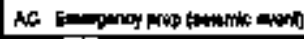 \\
\hline 24 andosat or & 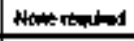 & 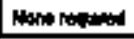 & Nate notionod & Alone rounded \\
\hline 24tureded a of & Hore maped & Note lagend & rowe naginad & 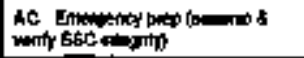 \\
\hline $24 A A R-09 \oplus 02$ & 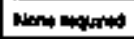 & Non requined & 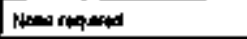 & Name requard \\
\hline $264 a-m$ an & NAt & 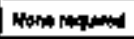 & 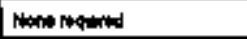 & Wowo rouread \\
\hline 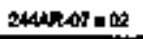 & Atone ropers & Noto & 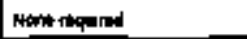 & Nom \\
\hline 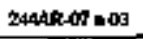 & 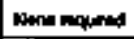 & Nan replod & How roquand & Nome rovidud \\
\hline 2ASAR-GT +04 & 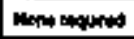 & 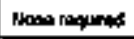 & $\operatorname{lng}$ & 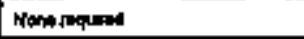 \\
\hline $2446 \times-4+41$ & 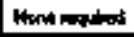 & 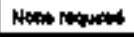 & 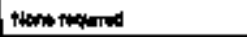 & 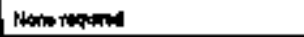 \\
\hline zuatifitice & Hone reqped & Nom ropitod & Noseriogared & None roquind \\
\hline 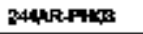 & 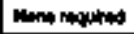 & None requind & 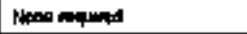 & Nomarapen \\
\hline $244 \times+404$ & Wone & 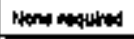 & Nan & 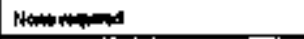 \\
\hline 344kRPus & Hone requed & thine niphod & None ropilend & Has \\
\hline julafexs & Mene raptred & 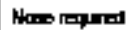 & 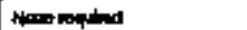 & Normerequad \\
\hline
\end{tabular}




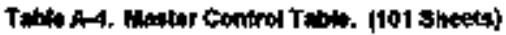

\begin{tabular}{|c|c|c|c|c|}
\hline 0 & PIw 日8: & Nta gac & PTw T\& & MTPR \\
\hline 24A4R+Tior & Hene neaped & Nowe renured & Botos rowined & Bent nopind \\
\hline 240Rewh & Neme requand & Nos mated & Nate nquard & Atone befpred \\
\hline 244tiaphete & Mone requariad & Nomerapures & None rpquared & Uanes naphad \\
\hline 24SURPAHD & 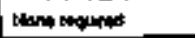 & Nonn rapinas & 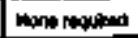 & acm mapher \\
\hline 244he+m1 & Home reagured & None reapures & Bone ranured & Ment \\
\hline 244RPW12 & None Nowurtited & 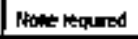 & 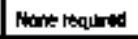 & Wone nigned \\
\hline zachentists & Norit noquend & Hanempund & Namengumed & Anon lapted \\
\hline 24ARAPH14 & Mtrinematment & Nonemained & Hannerequared & Hane payuand \\
\hline $204 A+P H 15$ & Nesen raminat & Nong mpationd & Noen ropired & Hoine requarias \\
\hline 24AR+PH16 & Nom rim & Nion rephed & S & Note roquarid \\
\hline 244RPPH17 & 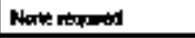 & thene roipland & 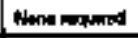 & Nominguled \\
\hline 24MARPHA & Now nequad & None nequind & Nonempored & None mationd \\
\hline 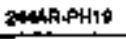 & Nom maned & Alane maped & Neew repured & 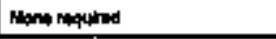 \\
\hline 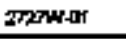 & 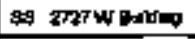 & Noma & Anow frapins & 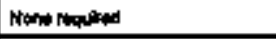 \\
\hline $27 \pi N W 00$ & st 272TWAMnd & 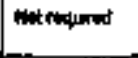 & 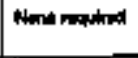 & 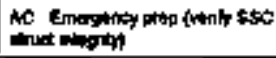 \\
\hline 2HENAOA & 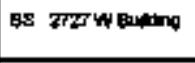 & Motequared & 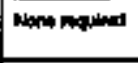 & 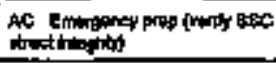 \\
\hline 27arritad & 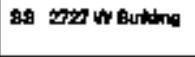 & Watropind & Mane rapued & 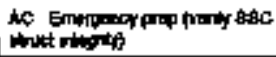 \\
\hline 27rTn+os & es $27 \%$ w onding & 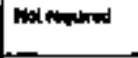 & 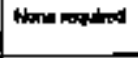 & 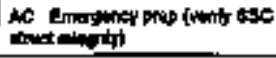 \\
\hline $2+2 N=00$ & 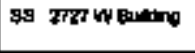 & witmporied & Nonom minged & 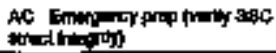 \\
\hline
\end{tabular}




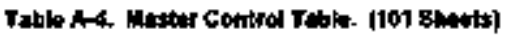

\begin{tabular}{|c|c|c|c|c|}
\hline $\boldsymbol{D}$ & Prw 589 & int $\mathbf{5 B C}$ & Pâr T: & MH T\&R \\
\hline $\operatorname{san}$ & None requed & None foldured & 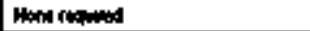 & 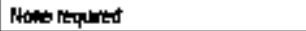 \\
\hline othen & Namerequitud & Nesep noputed & Ptented & 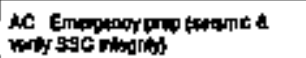 \\
\hline $\sin 103$ & rwine requrad & Hare lesurt & Whot topest & 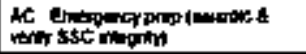 \\
\hline $\cos 04$ & Nore requitad & Nome itcquthef & Bhereded & MC Em-garpmp thal \\
\hline con-0s & Nowerniphed & Now 4 enquined & Rempend & AC Emenimsong finel \\
\hline 90000 & Hoporequed & Whot anowited & Mond monted & Nons lequed \\
\hline 00007 & Nan & Wone tounted & Nand monstod & 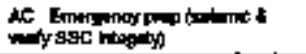 \\
\hline $900-00$ & Nownopind & New moqured & Rempond & 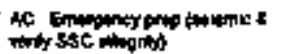 \\
\hline $600-0$ & Ham rewand & Hone noquertod & Nowh mord & 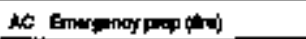 \\
\hline $\cos -10$ & Baneropiad & Wone roquned & Nixu nopred & 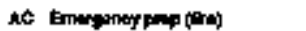 \\
\hline $\cot -11$ & Crtte antanod m SARP & 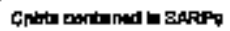 & 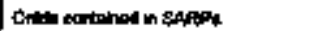 & 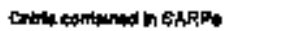 \\
\hline END & Mani mand & Noten Naparad & 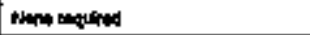 & Wind int \\
\hline BNo-01t & Bont & None Hanted & 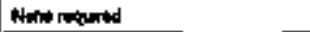 & Hone resplod \\
\hline GND-as & 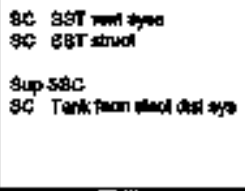 & None ropred & 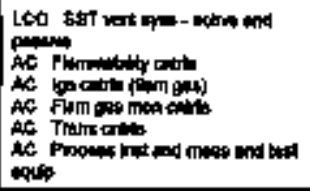 & 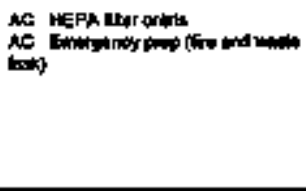 \\
\hline PAD Dar & Nonde rapulad & الה & dane raquind & 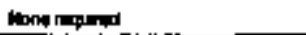 \\
\hline $\operatorname{ces} A 1$ & 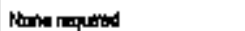 & 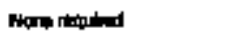 & Hon roghted & Wone reaured \\
\hline
\end{tabular}




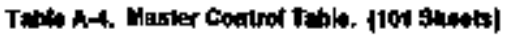

\begin{tabular}{|c|c|c|c|c|}
\hline 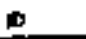 & Privs & Nas soc & PMT TE: & Mt TSM \\
\hline $\cos -02$ & 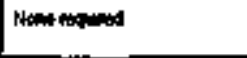 & don & 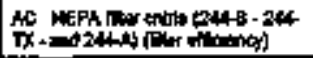 & 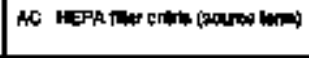 \\
\hline $\cos =0$ & Hons and and & 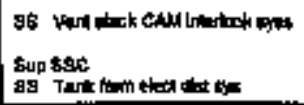 & 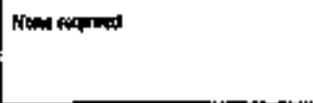 & 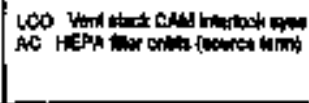 \\
\hline cotedat & Now ateand & 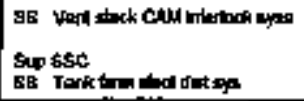 & 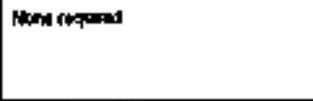 & 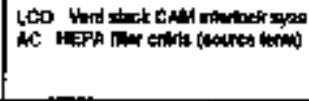 \\
\hline CCGF-0S & Nona & 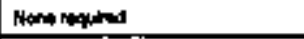 & Nom & Nanem raphed \\
\hline CCFF- 09 & Hone nangerd & Nhon & 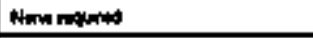 & Nend mainat \\
\hline CeFAP & 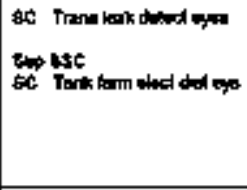 & 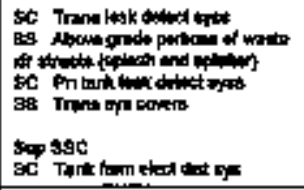 & 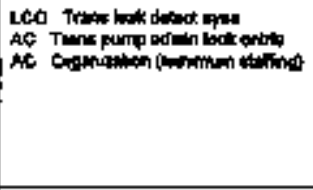 & 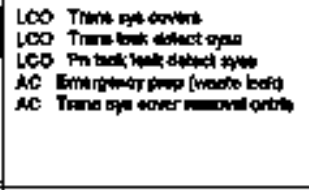 \\
\hline CCFF-A & 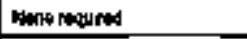 & Nome reapured & Hom naymed & Nenene requared \\
\hline $\cos -\infty$ & None requated & Nom ronuted & 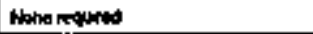 & Hon inures \\
\hline Cerr.10 & When napurad & 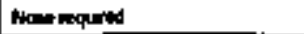 & Hone ropquined & Hon reapurad \\
\hline gact.11 & 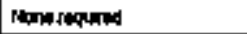 & Nentomphrod & Mone nequed & 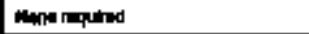 \\
\hline Cof-12 & Nom roparad & Nene ropurad & Dand & Hent ingured \\
\hline COF,13 & Winte ingurind & 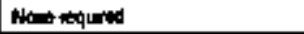 & Herom aryines: & Hand rounded \\
\hline CCAP-14 & 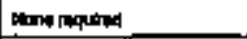 & Non mpuntad & Mons nophed & Mentrayud \\
\hline$\phi x=18$ & Nome reputred & Nimporaphed & Now requtiod & Hens rapurad \\
\hline
\end{tabular}




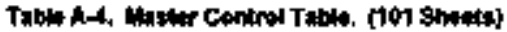

\begin{tabular}{|c|c|c|c|c|}
\hline $\mathbf{D}$ & Prer asc & Wats & Ane Ttre & TS: \\
\hline $\cos 16$ & 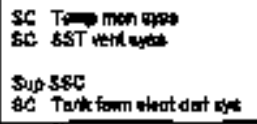 & 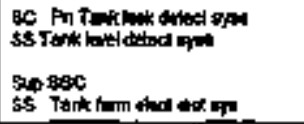 & 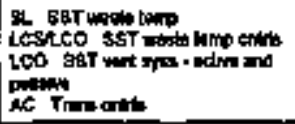 & 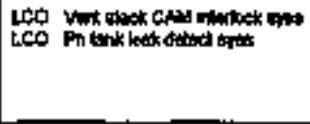 \\
\hline 005.17 & sa sor urot & mone roengend & Horte requnded & pone rapured \\
\hline CCFF-IS & 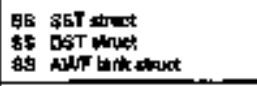 & Mone rapay & Mbone raparat & None nomured \\
\hline ECF-19 & 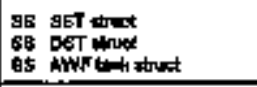 & Hene roped & ibne roquend & None rapurest \\
\hline cefos & Nameropiod & More bopred & 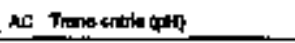 & Nonerequired \\
\hline copfor & Hene requand & 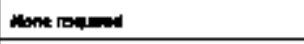 & 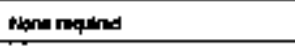 & None requares \\
\hline $\operatorname{cop} \theta$ & Nonerapureat & Hor & 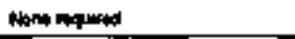 & Nom nopuras \\
\hline CDPG & Mont & 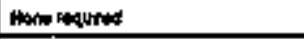 & Whin mand & 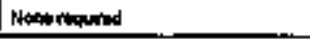 \\
\hline CLPAN & Btine ropurd & Hore & 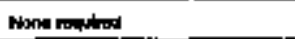 & 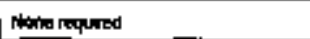 \\
\hline Capes & Phanerequmed & Mone raginged & Mapre & None requered \\
\hline $\cos \theta$ & 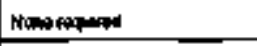 & Mons requant & mine nompund & Nomerequires \\
\hline cop-or & 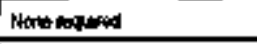 & Hore rapunas & 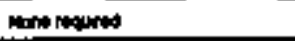 & 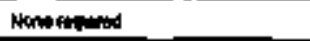 \\
\hline Gopad & Nonted & 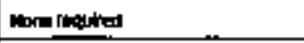 & 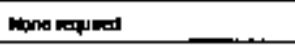 & 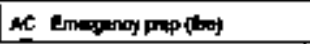 \\
\hline copos & 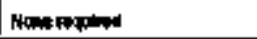 & 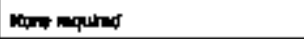 & Wone onged & 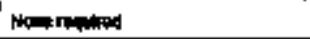 \\
\hline $\cos -10$ & 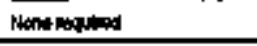 & Hown mapures & 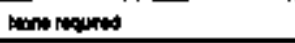 & 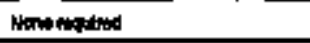 \\
\hline cosen & 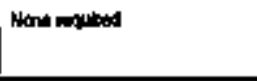 & 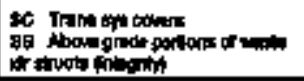 & 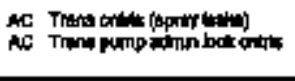 & 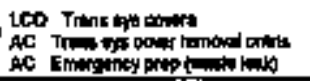 \\
\hline
\end{tabular}


Tablo A-4. Master Coptral Takb. [101 Shatis]

\begin{tabular}{|c|c|c|c|c|}
\hline $\boldsymbol{\omega}$ & Pä & Mt Bgc & Phw T\$P & WTSA \\
\hline $\cos -\infty$ & Nene reaplad & 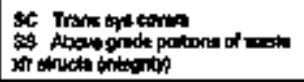 & 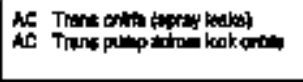 & 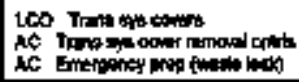 \\
\hline COEAOS & Wone requald & 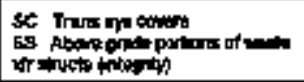 & 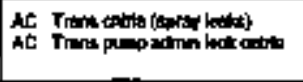 & 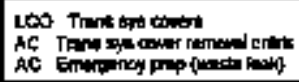 \\
\hline copero4 & 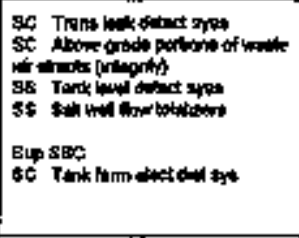 & 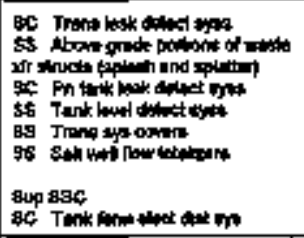 & 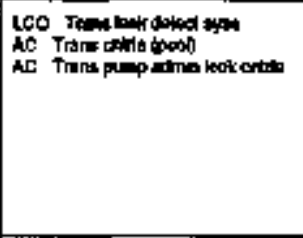 & 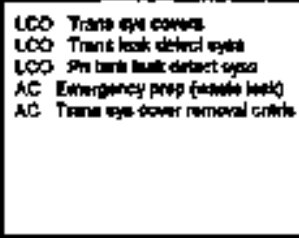 \\
\hline copose & Nom Mand & Nom romatiod & Ntaw ming & None requard \\
\hline $\cos 00$ & 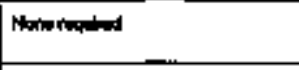 & sct tron mpons & Mant rogumad & 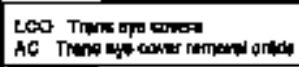 \\
\hline CDasto & 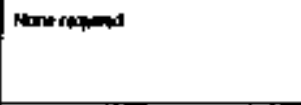 & 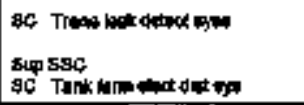 & 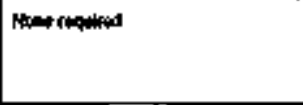 & 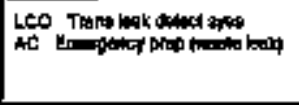 \\
\hline $\cos -\cos \theta$ & Non Nownes & Mone requall & 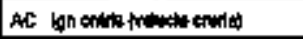 & Nowien requared \\
\hline $\cos 050$ & Nent mistifid & 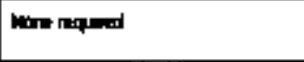 & 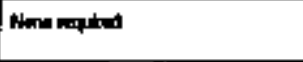 & 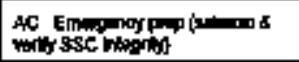 \\
\hline $\cos -0 s^{4}$ & 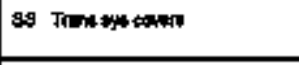 & 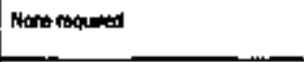 & 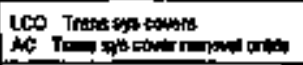 & None naputiod \\
\hline $\cos -0$ & 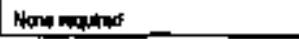 & 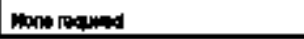 & Non popind & 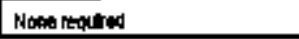 \\
\hline
\end{tabular}




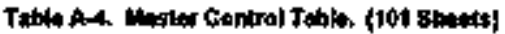

\begin{tabular}{|c|c|c|c|c|}
\hline ID & Pryr Basc & Nestc & ParT TE: & utTơR \\
\hline $\operatorname{coB}-0 \mathrm{~B}$ & 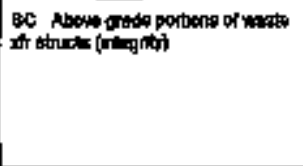 & 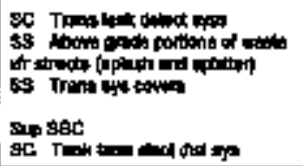 & A & 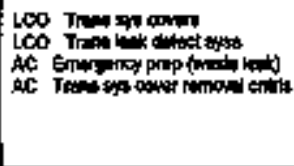 \\
\hline COBAT & Mote roquad & 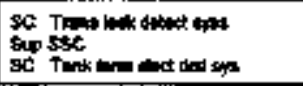 & 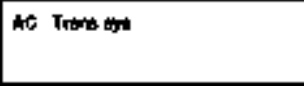 & 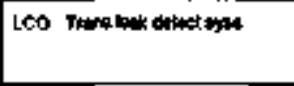 \\
\hline 60903 & 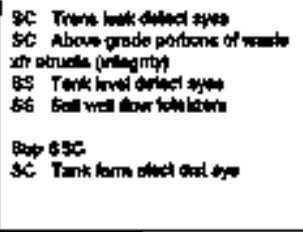 & 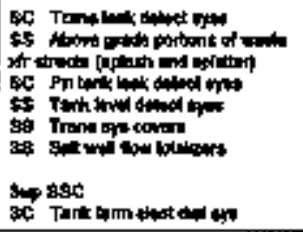 & 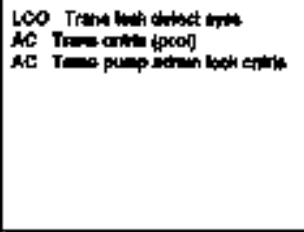 & 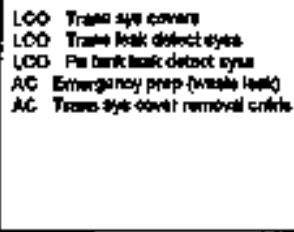 \\
\hline CRttor & Hent & Nates renumad & 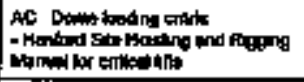 & 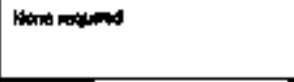 \\
\hline contort & הen & Nome anumar & 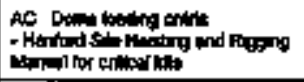 & 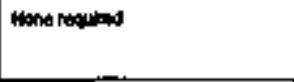 \\
\hline craton & Bont penpos & Nemoreputed & 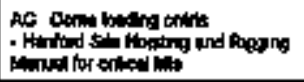 & Hond resulat \\
\hline CAmogil & Hone resulues & Hone opures & 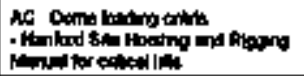 & Monatelunes \\
\hline
\end{tabular}




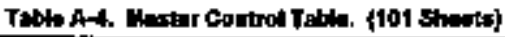

\begin{tabular}{|c|c|c|c|c|}
\hline ID & Pawssc & me 68c & Prertsh & M TPR \\
\hline CANAOS & 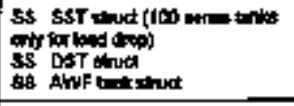 & 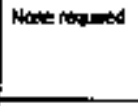 & 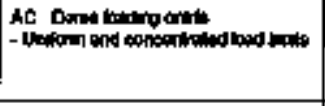 & Nand \\
\hline ContoLra & 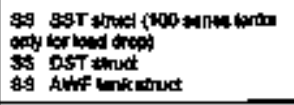 & Hane rempinas & AC Domm loodire & Nond niming \\
\hline $600203-18$ & Deng & Mne repured & 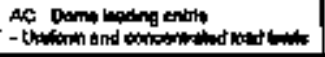 & temen mopurad \\
\hline Ctanou & 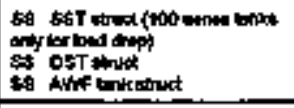 & Nont lonoted & 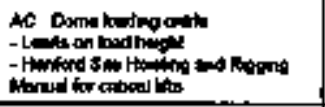 & Nethe riogred \\
\hline 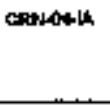 & Bene naprad & Honter required & 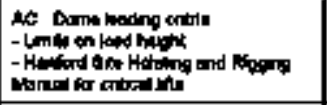 & Mone requared \\
\hline CRMOAS & Hont reqpend & Mone requared & 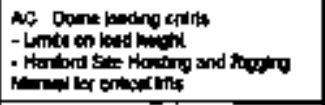 & Monerequared \\
\hline GRNA & 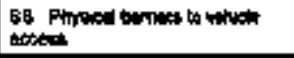 & 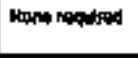 & 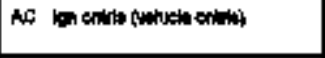 & 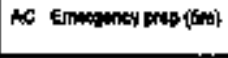 \\
\hline CFAntosi & 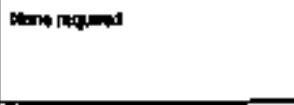 & Itone requend & 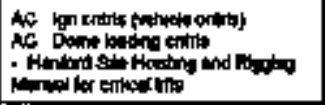 & 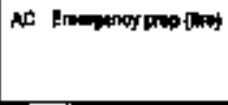 \\
\hline GRNOS & 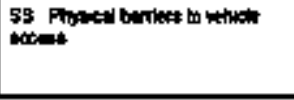 & Mono reafied & 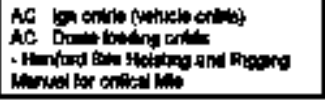 & NCEmmoney mop [fied \\
\hline
\end{tabular}




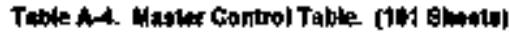

\begin{tabular}{|c|c|c|c|c|}
\hline 由 & Pitroster & wesec & Praw Ts: & MTER \\
\hline CRHEOH & Mono nomind & 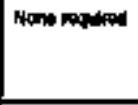 & 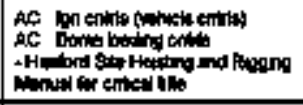 & 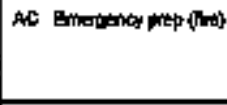 \\
\hline CRHOT & 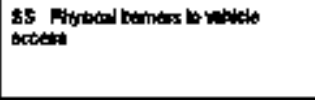 & Noter & 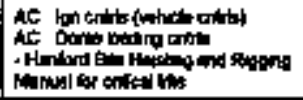 & 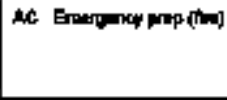 \\
\hline CRNAOTA & 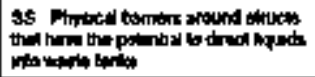 & Noter woputed & 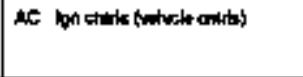 & AC Emergenoy prep (fri) \\
\hline GRHAd & What & Nom & 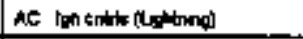 & 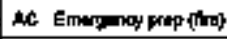 \\
\hline CRNAOAOA & Hone roquhad & Hom rengers & 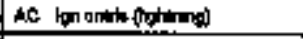 & AC Emmorney wap (hros) \\
\hline $\operatorname{ctan} 00$ & 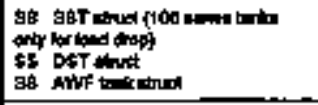 & Haneropenen & 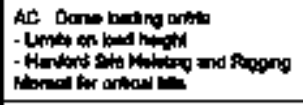 & 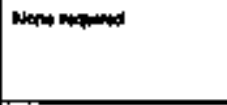 \\
\hline CPan-0er & Nenerequired & Monerimpined & 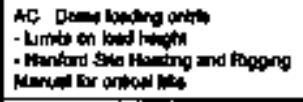 & 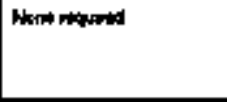 \\
\hline CRA-10 & 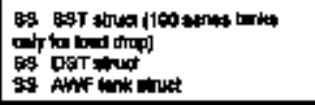 & Won rewures & 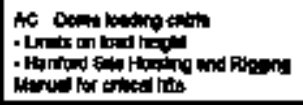 & Hone penpod \\
\hline CRM-1021 & 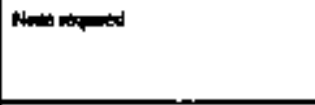 & Whate roquind & 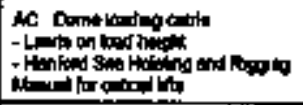 & ition nopand \\
\hline CÁR-11 & 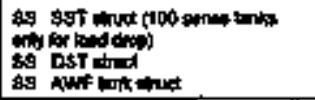 & bonow napred & 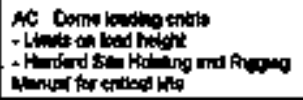 & None natived \\
\hline
\end{tabular}




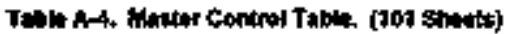

\begin{tabular}{|c|c|c|c|c|}
\hline $\mathbf{D}$ & Pon 590 & $\omega \mathrm{H}$ & PIN TBR & NASR TSR \\
\hline 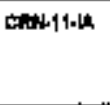 & Hond mapted & Hene resures & 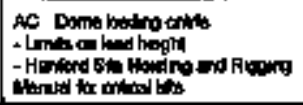 & Manimingat \\
\hline CRN-11」E & 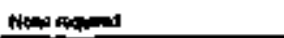 & 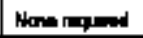 & Nane naphed & Nentratanted \\
\hline CRN-11CL & Natem mond & 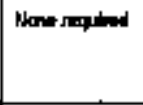 & 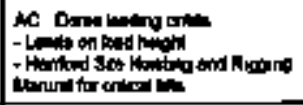 & 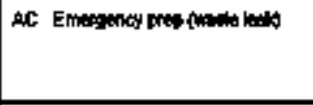 \\
\hline $\cos 12$ & 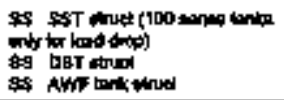 & 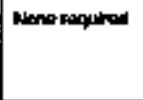 & 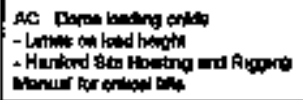 & Mone andined \\
\hline cotositis & 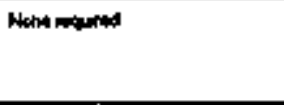 & 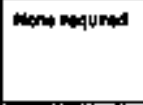 & 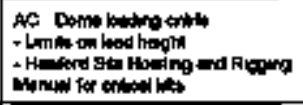 & Mone bapand \\
\hline chamizat & Mene Pexplad & 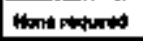 & Nonim thand & 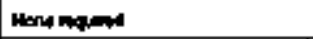 \\
\hline CRANI2+S & neno angived & Hone reaputiod & 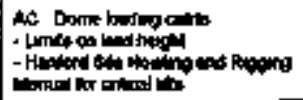 & AC Entaricy \\
\hline CAR-134 & Amos nopurned & Mone requiter & 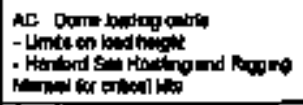 & 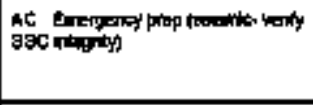 \\
\hline CRNG13HE & Fine requared & 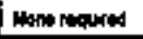 & Hone rapand & Nomen inganded \\
\hline CAR $+13-13$ & None nopurid & Atod & 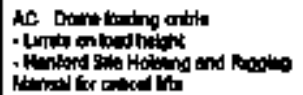 & 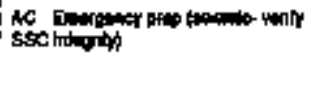 \\
\hline
\end{tabular}


Tahin A-4. Mastw Comtrol Table, (101 theats)

\begin{tabular}{|c|c|c|c|c|}
\hline 10 & Prw & Lasc & Pin TSR & MUTSR \\
\hline CRK-13-A & Mone rodurad & Hon & 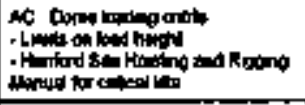 & dant \\
\hline CRN-A3 & 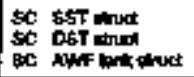 & Whine roperal & 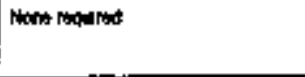 & 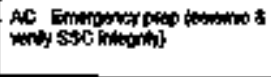 \\
\hline CRN-14 & NHon & Mono Nipand & Nens rapunod & Hene maped \\
\hline CRND-14 & Hection roquind & None tapred & 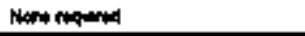 & Nens \\
\hline CRN-16 & Now requind & Nones ropited & 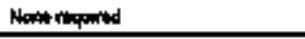 & Nothen \\
\hline CRN-15- & Neene ropurad & None reaphed & Now ropined & Henew nonger \\
\hline 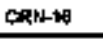 & Nons fiparied & Nown Naquars: & ileme ragatid & Mene mapend \\
\hline CRNATH & Nonw did & Nono & nemp a aquind & 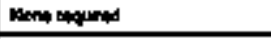 \\
\hline cototit & 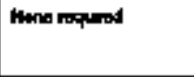 & Nhom ropind & 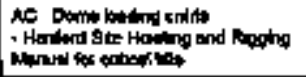 & Imotions \\
\hline ERNANH & Hown roputud & d & 然 & 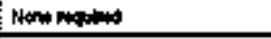 \\
\hline GRtt-1: & Ho requed & None ropapor & Nown roudind & Nond nam \\
\hline CPRN-184 & Nenerequared & None ropulad & Htare mapered & None noplond \\
\hline CRNA 10 & Nom ropured & 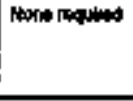 & 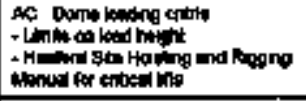 & 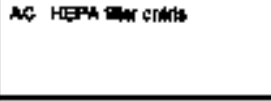 \\
\hline Cetwital & 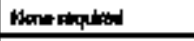 & 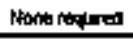 & Nom maind & tion nesured \\
\hline crata & Mona rantiped & Hone raphad & 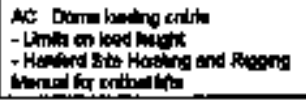 & 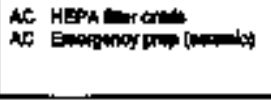 \\
\hline
\end{tabular}


Table A-4. Masier Control Table, (101 ganeta)

\begin{tabular}{|c|c|c|c|c|}
\hline סו & 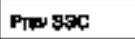 & MAEsC & Pan TER & AKTgR \\
\hline CRN-20H & Minn Man & 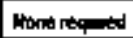 & Hone nowined & NHA \\
\hline CRPLEI & 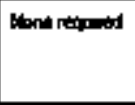 & 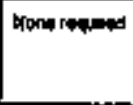 & 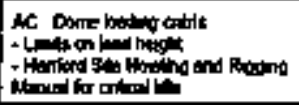 & Nor \\
\hline CRaknter & 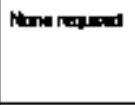 & 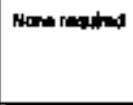 & 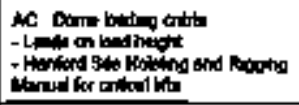 & Hanempirad \\
\hline cras-21-1B & Narne requesed & None rapunes & 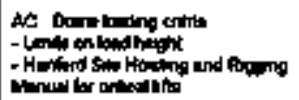 & Nens mened \\
\hline $\operatorname{cosin} 22$ & 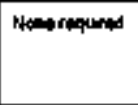 & Hand Vidured & 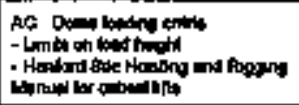 & A \\
\hline entatik & Now & Hone required & 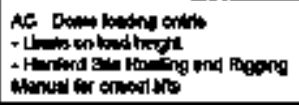 & Hons rocuted \\
\hline chntzes.18 & Nanerequmet & Mane requares & 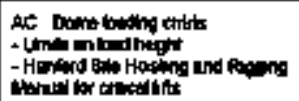 & Nane maphed \\
\hline c्मptwa & Nompinquind & Nowa fopures & 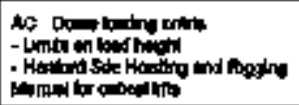 & 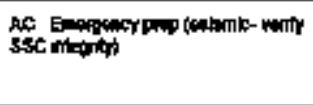 \\
\hline enkzath & Hent mopined & 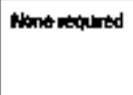 & 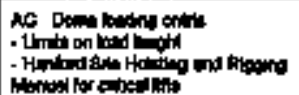 & 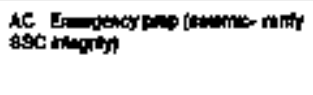 \\
\hline
\end{tabular}




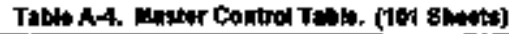

\begin{tabular}{|c|c|c|c|c|}
\hline 10 & PWN & Uाt & 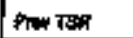 & nat \\
\hline CRin-Z3-B & 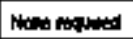 & 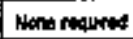 & Whand & None nopiond \\
\hline continged & Nhe manger & Hone nequrent & 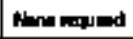 & 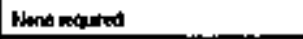 \\
\hline ctancal & 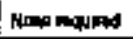 & None required & Hond and and & Mbore mqund \\
\hline crantis & Nand & 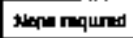 & 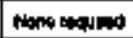 & Mbon nqumed \\
\hline CRH-2) & 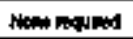 & thon requred & diand & Mone nophed \\
\hline CRARESIB & 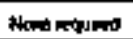 & Whethe roputed & thon anded & 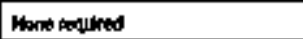 \\
\hline Chunst & Han mequed & Mens rmathow & Hone napd & Bane maquhd \\
\hline CARג植 & Deen moyred & Merne noquad & Name namped & Mone nquard \\
\hline CRN-Z6 & Hon & Man requared & 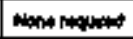 & Hon nequared \\
\hline CRN-2T & 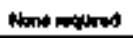 & 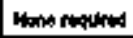 & Honem mond & Atint \\
\hline CRN-ZPH & None maphod & None ropinod & bone magurod & Alone roquand \\
\hline CRN-ZTS & Nbon maped & anne roquind & Hone magrod & Alore roquand \\
\hline CRN-Z3-4 & 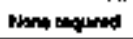 & Mane mequined & 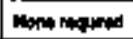 & Hone noquired \\
\hline Gentes & Alons & 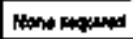 & Maris & Nons ranumed \\
\hline em-0l-1 & Hons rophiod & dan & Hare roquiod & 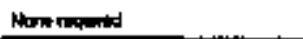 \\
\hline cva & Phontroguad & Nure repaprod & Nare mogered & Nartinaped \\
\hline 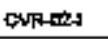 & 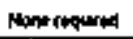 & Mand & Noph & 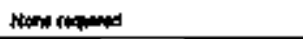 \\
\hline chirea & d & 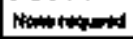 & 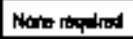 & 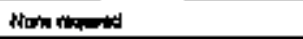 \\
\hline critost & 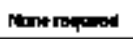 & $\mathrm{HF}=\mathrm{rep}$ - & Mate nopuded & 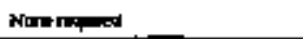 \\
\hline CMFan & 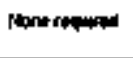 & 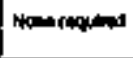 & 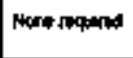 & 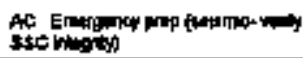 \\
\hline GWR-OH & 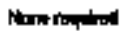 & 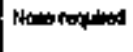 & Mare rophitad & 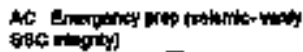 \\
\hline
\end{tabular}


Tubte A-d. Mastur Control Tuble, (101 Btreets

\begin{tabular}{|c|c|c|c|c|}
\hline $\mathbf{D}$ & Putrsst & MA 69S & P䄳 TSR & M TBR \\
\hline 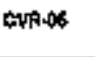 & 自 & 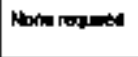 & 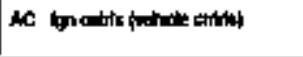 & 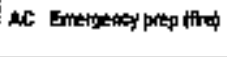 \\
\hline crmater & 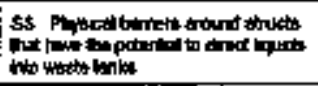 & Mone resped & 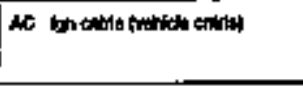 & 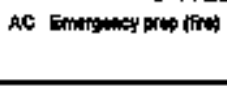 \\
\hline CVR:09 & Nond matand & Nom rapipad & 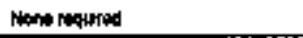 & Mane maned \\
\hline CURAOH & Norw monged & Now requind & 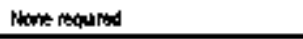 & Nons noming \\
\hline CURAOA & Hern manded & Nom toptred & Nore mantid & Hethe nownod \\
\hline EMR-0)- & tiene mapted & 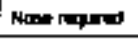 & Nant requent & Hene nanged \\
\hline CWR-09 & 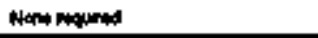 & How requares & thengrequeried & Bane minged \\
\hline stmon-1 & 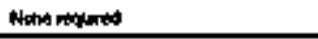 & Nons numed & d & Bons minind \\
\hline $\operatorname{cthac}$ & 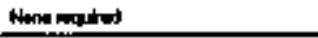 & Nonationurwa & How ropend & 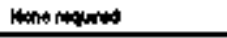 \\
\hline Chanal & 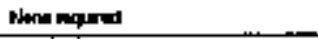 & None requered & Nare roperad & Anit ragurad \\
\hline CWR:-10 & Nema sapend & Nkene required & Neenorrogend & Mene rapled \\
\hline Che $10-1$ & 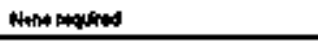 & Nons rempurat & | & 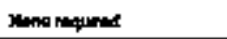 \\
\hline chasent & Hent requand & 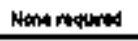 & Nant thaphad & 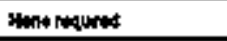 \\
\hline Cht:11.1 & Nenou requeress & Nonerequted & Noner ropared & Hone roguthed \\
\hline CWR-12-4 & 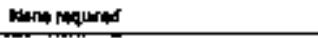 & None moquined & 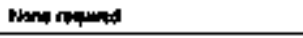 & Mone nequired \\
\hline chn-15 & 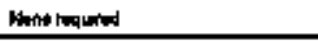 & Nonom mopored & A & 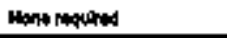 \\
\hline Crtsil4 & Hene ropured & Hond poptind & 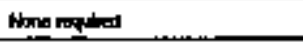 & 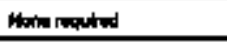 \\
\hline CMRISA & 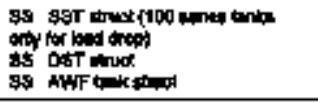 & Bane required & 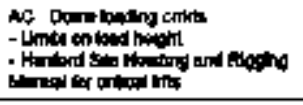 & Wine requard \\
\hline Datant & Mene ropurod & 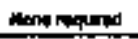 & 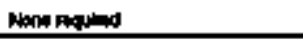 & Man naquend \\
\hline
\end{tabular}


Tabti A4. Waster Control Tithe [101 Showls]

\begin{tabular}{|c|c|c|c|c|}
\hline 0 & Prew spec & in stc & Frw T\$R & AN TSR \\
\hline DCNAOI & 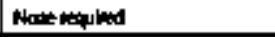 & 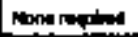 & 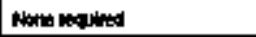 & 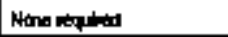 \\
\hline Dorntar & 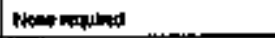 & 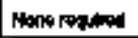 & 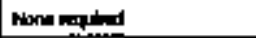 & 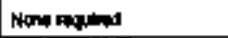 \\
\hline OCH-CR-M & Nam & Wine rapend & Plona manimad & 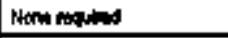 \\
\hline 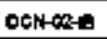 & Now & None ropted & 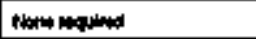 & 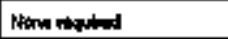 \\
\hline DENAOS & Nothed maped & None raped & 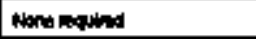 & 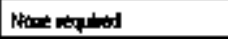 \\
\hline DCNASH & 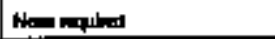 & 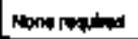 & Hond male & 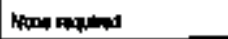 \\
\hline $\mathrm{OCH}+64$ & Nomin napind & Home raphis & Nern maplat & Nocew roptods \\
\hline DCHOH & Honn napiod & Nine roplatid & Ploma nowition & Nent \\
\hline Dow+05 & Whot in whend & Nane raphed & 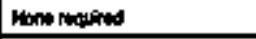 & Hond raghted \\
\hline Dewhos. & Vhon requined & Hon manger & Hine bovied & Senen raphered \\
\hline $0 x+106$ & Bone majhed & Hom rempuread & Wane nophed & vine peipuray \\
\hline Despost & Whom nopirend & 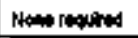 & $\operatorname{lng}$ mand & 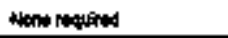 \\
\hline Dentor & Bons rapiond & 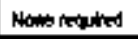 & Mino reposind & thenestont \\
\hline Deftaril & Atone requitiod & Nom reaghteod & White bayind & Jlone ragated \\
\hline EEA1 & Done rogphod & None rapured & Nine nophed & AC: Emapency Arap this \\
\hline EE-OR & Hene mogured & 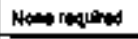 & Mane nopited & 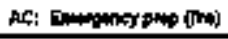 \\
\hline EE-19) & Bone nusured & Nom inotion & 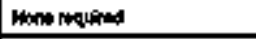 & Hon lowyind \\
\hline EE-OAd: & 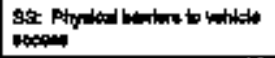 & Non required & 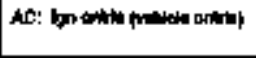 & 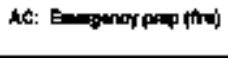 \\
\hline EE-OH & 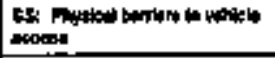 & 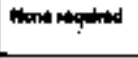 & 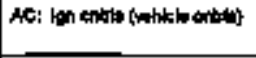 & 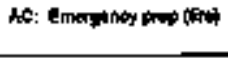 \\
\hline eEtase & 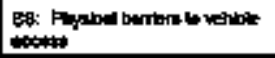 & Mons & 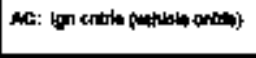 & 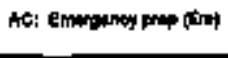 \\
\hline
\end{tabular}




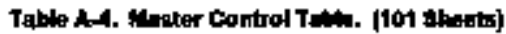

\begin{tabular}{|c|c|c|c|c|}
\hline It. & mons & $M 1350$ & PTh TSR & MTSR \\
\hline EE-bS & 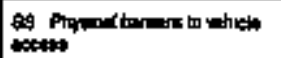 & Mone noquhed & 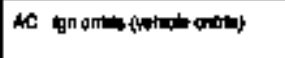 & 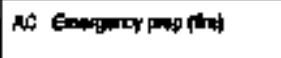 \\
\hline expst & 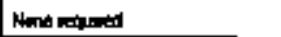 & Hantroinged & 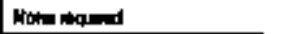 & AC \\
\hline EEE-GP & Hene maper & Monererapend & 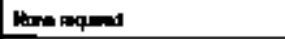 & Moru anpard \\
\hline EE- & Hant in a d & Nener rapipd & Mn & Mbon manad \\
\hline TE-OP & 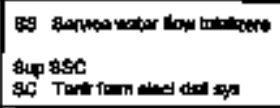 & Nenoprequered & 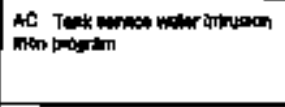 & Nomen mintion \\
\hline EE-to & 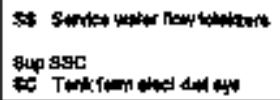 & 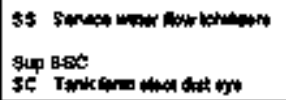 & Nemandind & Wint rosumat \\
\hline ets-5t & 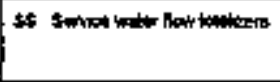 & 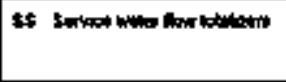 & 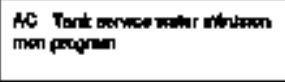 & 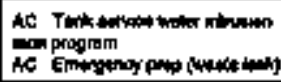 \\
\hline EE-12 & 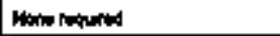 & 每 & 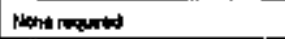 & 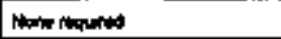 \\
\hline EE-1] & Who rowitind & Nome ropand & Noneralod & Nane maqued \\
\hline EE 14 & Wone noqued & Mone requent & 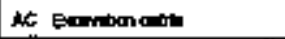 & Uane requered \\
\hline EE-15 & Wone requared & Paרp & 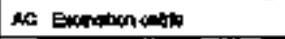 & mono repurarad \\
\hline EE-14 & 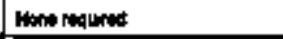 & Nono requesos & AC Detritangente & 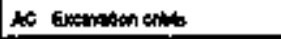 \\
\hline 71. & Mingroped & Nan & itc berombenctits & 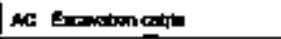 \\
\hline Geapar & Nitengand & 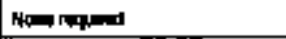 & None reapond & How Faquind \\
\hline QRan- & 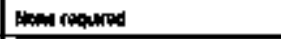 & 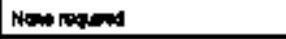 & thane rapurad & Ntom fopedrod \\
\hline Getator & 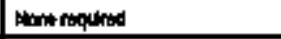 & 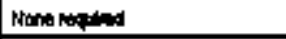 & Whe ritipind & Nowentopund \\
\hline 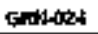 & Anemplined & Nom & 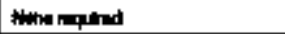 & 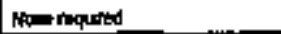 \\
\hline
\end{tabular}




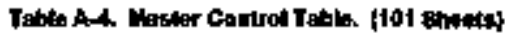

\begin{tabular}{|c|c|c|c|c|}
\hline 10 & Prw क्षै & Mssc & Pur T’力a & M TSR \\
\hline (5Fan-63-1 & Itom repured & Mones requand & 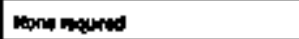 & Nene noguted \\
\hline 1TRE-1-cavp-A & Atare & Nown reapred & 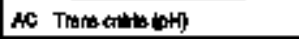 & Nonerexperd \\
\hline 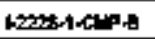 & Hon mapand & 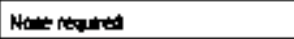 & Howe locandad & 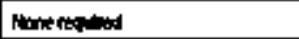 \\
\hline 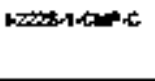 & 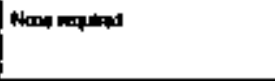 & 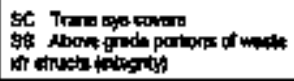 & 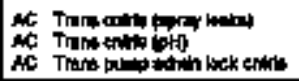 & 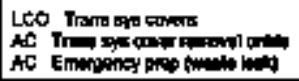 \\
\hline nesestitano & 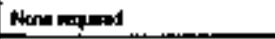 & 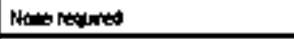 & 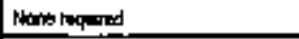 & 10 \\
\hline 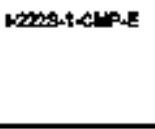 & 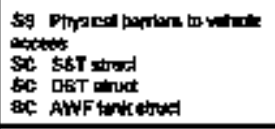 & None randed & 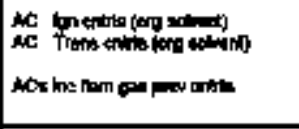 & 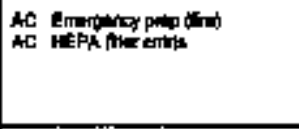 \\
\hline - $2029-1+2$ - -1 & 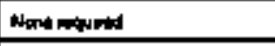 & 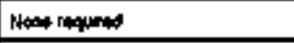 & Nonot Mand & 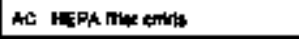 \\
\hline$n m+1-0 \times N A$ & Nene napired & 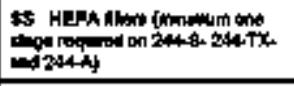 & No Truenth bith & Te mePh min dith (244-244 \\
\hline mant-2ruh-h & Nene peopost & 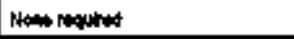 & 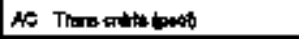 & 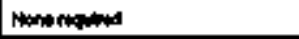 \\
\hline 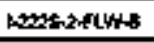 & 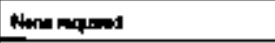 & Nane rowiod & 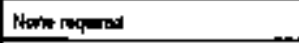 & 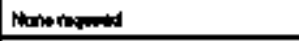 \\
\hline andsenthe & Nent & None reqpered & 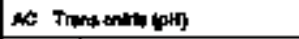 & Nenereapod \\
\hline 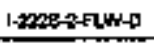 & 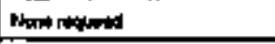 & 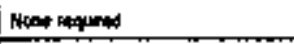 & Nent lloming & Nontromiand \\
\hline H-mS-HEk- & Nene renger od & Ntem n & Non to requad & 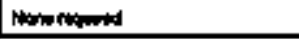 \\
\hline Hassorex & Dhene requeread & 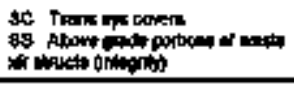 & 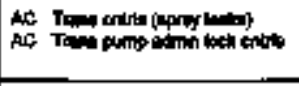 & 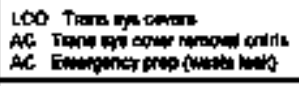 \\
\hline $2 \cos 5-3 \mid 1 \times x$ & 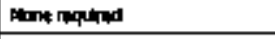 & Hone required & 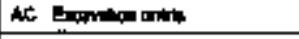 & 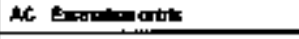 \\
\hline$+2028060 \times 0$ & Denoropund & 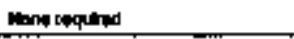 & Nbon andind & Nons nophed \\
\hline
\end{tabular}




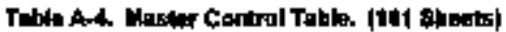

\begin{tabular}{|c|c|c|c|c|}
\hline 1 & Prox s5x & in ssc & Pren Ts: & ME T:H \\
\hline 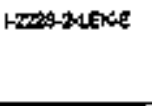 & 1400\% maquired & 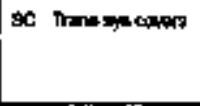 & 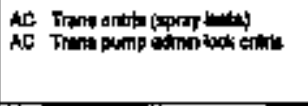 & 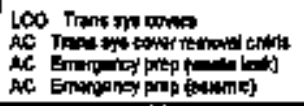 \\
\hline 1.22:5-2.PR6-A & Hone ropuras & 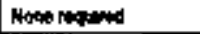 & 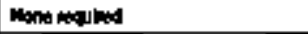 & 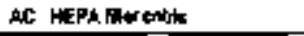 \\
\hline 1.262A-1.FLWA & 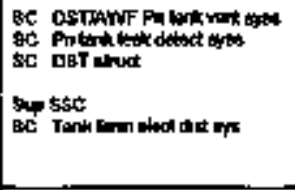 & 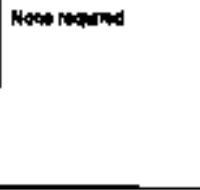 & 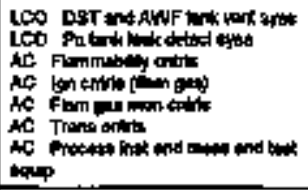 & 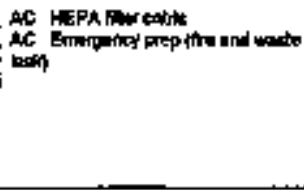 \\
\hline 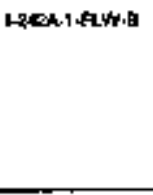 & 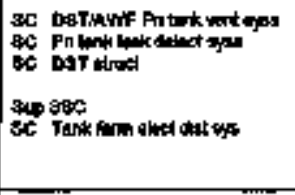 & Hane repprod & 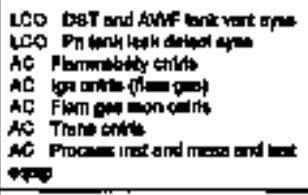 & 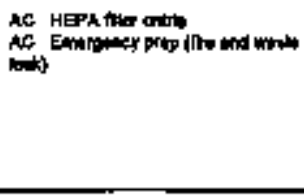 \\
\hline$\omega 20+1+1+2$ & 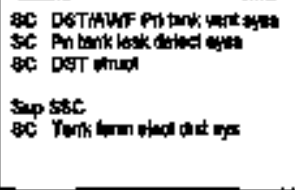 & Hend rowured & 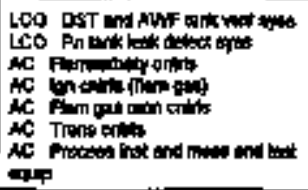 & 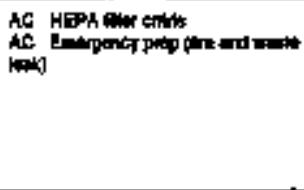 \\
\hline 1-242A-1-FLU10 & 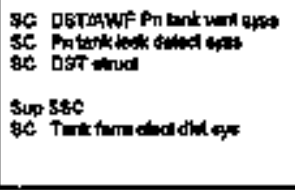 & Mne matured & 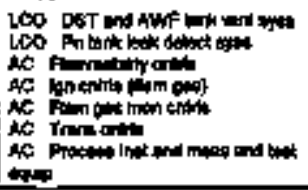 & 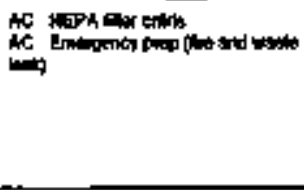 \\
\hline
\end{tabular}


Taban A-4 Mnoter Control Tabla, f101 thataly

\begin{tabular}{|c|c|c|c|c|}
\hline 10 & Pwr stec & wsec & Fur TSR & MTBR \\
\hline 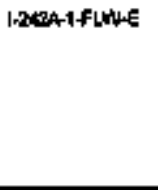 & 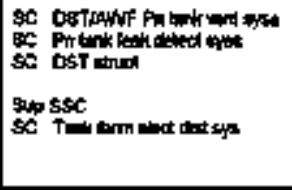 & 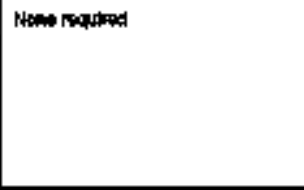 & 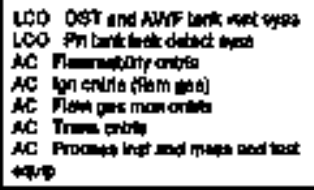 & 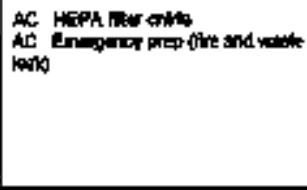 \\
\hline 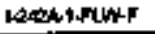 & Nowe requend & Nenes rogated & Hont maplad & Nene noped \\
\hline 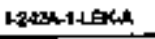 & Now roquend & Nene requpod & Nore roqurad & Nonu rapeed \\
\hline Webl-1_EK-B & 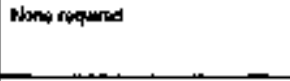 & 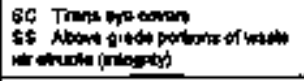 & 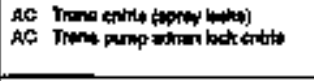 & 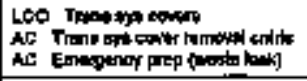 \\
\hline boek1-LExa & 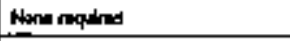 & Nons requered & 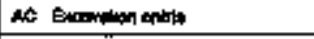 & Not Empupben entrits \\
\hline $1-2 * 041-10 \times-0$ & Nom ropumial & Neme rimpuned & 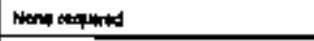 & 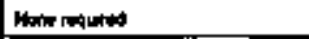 \\
\hline 1.2WU-1-LERE & 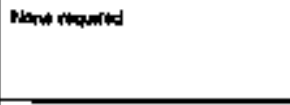 & 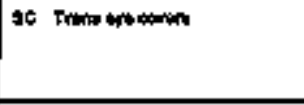 & 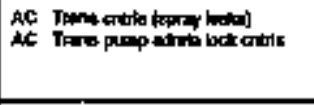 & 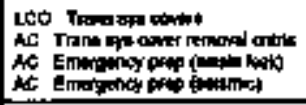 \\
\hline 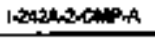 & Mine resuld & Nane ropatiod & 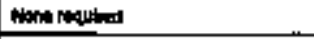 & Nom maquind \\
\hline $108434+201048$ & mon nequid & Nonos requand & None requand & M MEPA \\
\hline 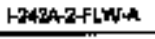 & Hon hopud & Mon rapard & 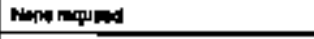 & Now required \\
\hline $1-264-2+F L+15 B$ & Aano nang & Hone reaved & 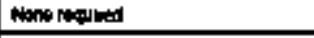 & Neterempuited \\
\hline 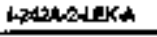 & Mand nequerd & Morsertidid & Whe rripund & Atow requend \\
\hline Heavuexs & rane now & 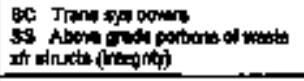 & 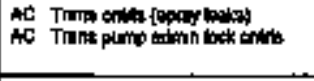 & 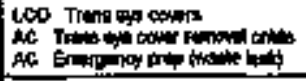 \\
\hline Hoghtuect & Mine nownd & 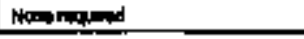 & 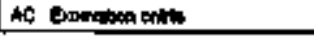 & 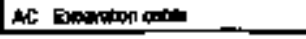 \\
\hline
\end{tabular}




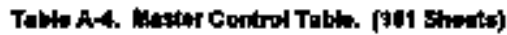

\begin{tabular}{|c|c|c|c|c|}
\hline $\mathbf{m}$ & Pren BgC & M Bgc & PTw 198 & MTRR \\
\hline 130162texp & 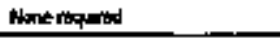 & 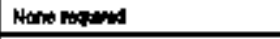 & Noneranued & Alom \\
\hline Wob-2Lére & Hane napand & 的 Trespons & 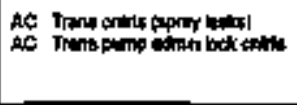 & 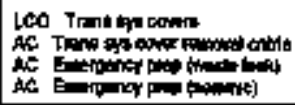 \\
\hline Lakn-2-17np-A & 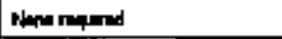 & Wont farpind & 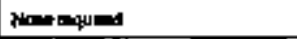 & Hene menpod \\
\hline $1-21042+15 C A$ & Hons & Aenominum & 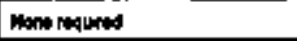 & ano rapurnd \\
\hline 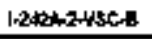 & Wone arigned & stonoresyons & Wonterequatsod & Whe ronumed \\
\hline l.zedustovin & Hone maphed & Moneteland & Wone nequend & Hone requared \\
\hline 1.2004-3fLW- & Mone naphed & Morn: rengref & Mone nequind & Wone roqured \\
\hline $1-2904-4$ rush & 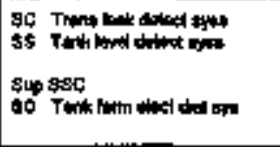 & 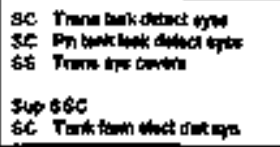 & 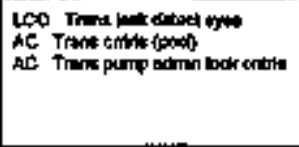 & 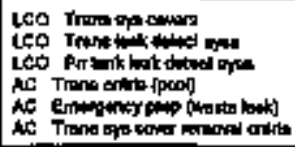 \\
\hline $1.242 A-7 \pi P A$ & 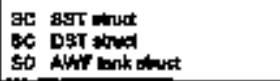 & Rone requirad & Nore ropened & AC Emegenof wap (thi) \\
\hline $1-24246+564$ & Nene noquind & Nenerequed & Nore nopalned & Wimenoqured \\
\hline $1242 A-6 \cos ^{-1}$ & Nene requared & thene requeread & Mane requand & 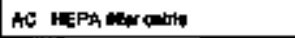 \\
\hline 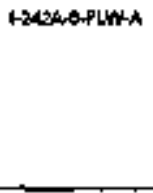 & 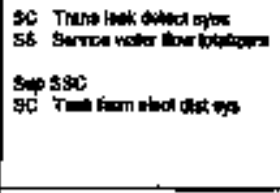 & 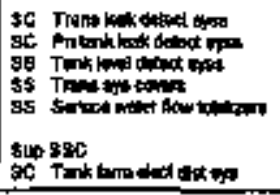 & 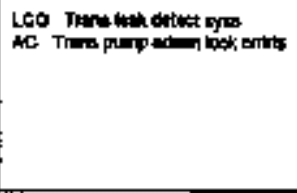 & 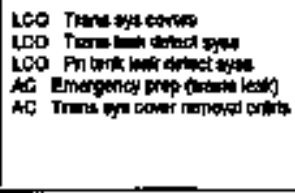 \\
\hline 1203нFune & 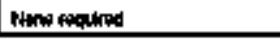 & Nones maptat & Nomoneaninged & Nom namiod \\
\hline
\end{tabular}


Tabla A-4. Mapter Copimd Talla. (109 thests)

\begin{tabular}{|c|c|c|c|c|}
\hline ID & Prt str & $m \operatorname{ssc} c$ & PTw TSR & ML Iss \\
\hline 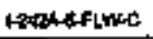 & Kano naquand & 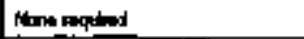 & 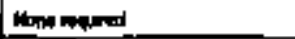 & Homeraped \\
\hline 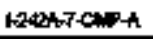 & dים & Hons rapalipd & None nopid & Nom rominged \\
\hline 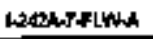 & Horne rogared & 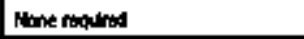 & 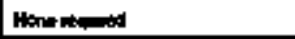 & 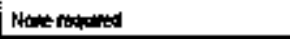 \\
\hline Latzanterthe & weine itogitad & 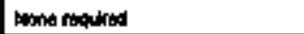 & Herterepor & Nom nopiod \\
\hline 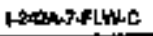 & Dhe noperd & 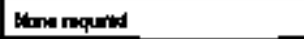 & 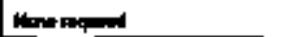 & 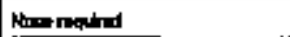 \\
\hline 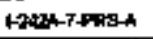 & 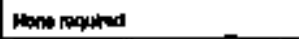 & Mane noqum & Mons & Morre (requind \\
\hline KOQRS1PRSA & 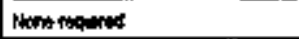 & Mons & Whone requres & An \\
\hline L2atsi-Thenh & Noth tequated & 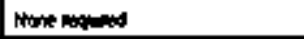 & 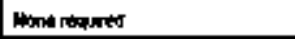 & d \\
\hline Letsominth & 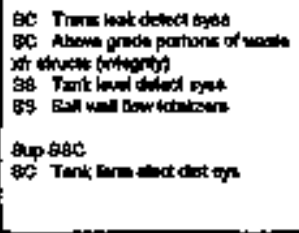 & 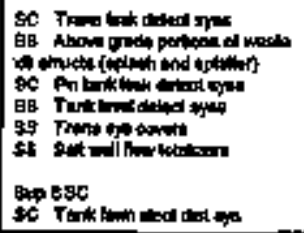 & 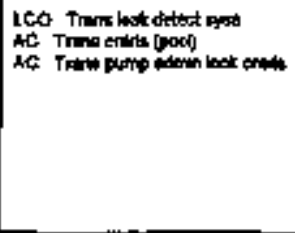 & 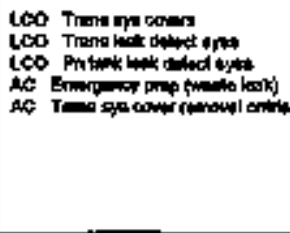 \\
\hline 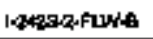 & Heaproptred & Non ranged & None rapured & Nenu nquated \\
\hline 1-2428-2-rumc & Now rapanded & 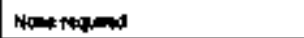 & Rome ranumed & 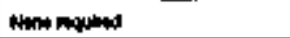 \\
\hline $1.24020+1640$ & 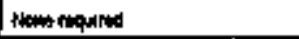 & 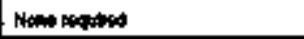 & Hane ropted & 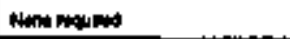 \\
\hline 1.P425-2-10we & 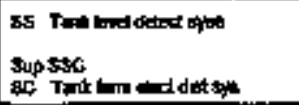 & 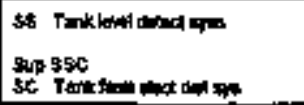 & 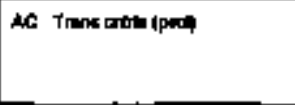 & 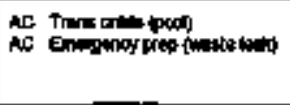 \\
\hline $1-2428-2-10 x-4$ & Nono nowion & 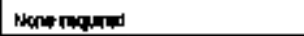 & Howe reapurad & nome nounerd \\
\hline
\end{tabular}




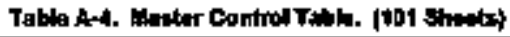

\begin{tabular}{|c|c|c|c|c|}
\hline 10 & PTew gac & we $5 \%$ & PTw TSA & MU TER \\
\hline Hact-24 EKe & Who peyinted & 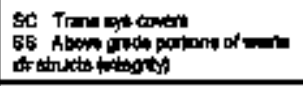 & 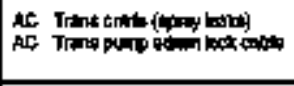 & 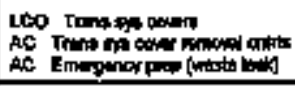 \\
\hline 1206201EKC & What redured & 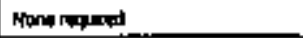 & NCE Empan nith & 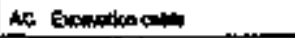 \\
\hline $13 \cos 2 x \cdot 160$ & Hone requred & Nonerapled & Nome rangund & Dentrepurad \\
\hline 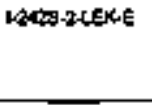 & Wone anpind & EC Trom was & 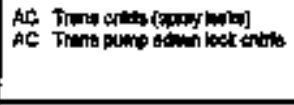 & 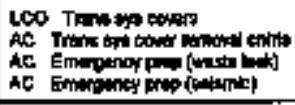 \\
\hline $1-2429-4+1 W-A$ & 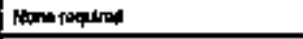 & Nens mopumes & Hane & Stond \\
\hline 1.2425-4nLH4 & Nomenapial & Hone requriod & Honte ropunted & Nomentid \\
\hline $12425-41 \mathrm{LWWC}$ & 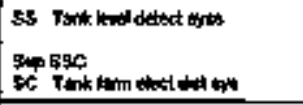 & 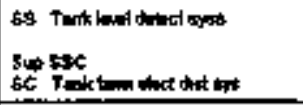 & 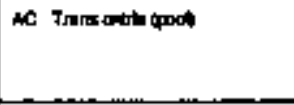 & 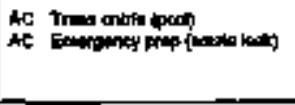 \\
\hline 1.242B-4-Funt & 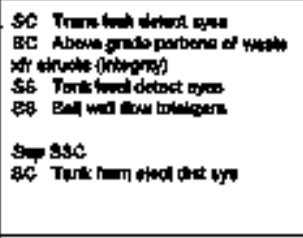 & 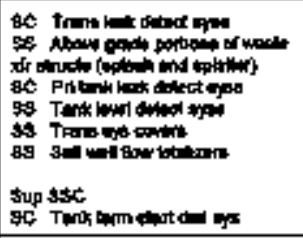 & 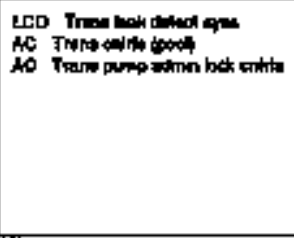 & 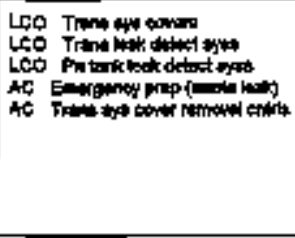 \\
\hline HELS-AEKA-A & Berip nopyend & din & 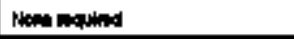 & 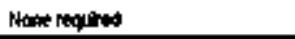 \\
\hline rect-4 eke & Non nonupes & None neped & Now & $\mathrm{Nom}=$ mipited \\
\hline $120 \log 44 \mathrm{AKC}$ & 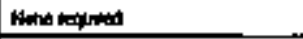 & 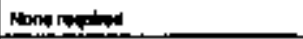 & 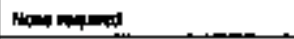 & AC Ecrititan mothe \\
\hline LOAS-AUEK-O & Menes mated & 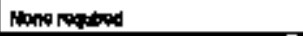 & 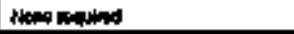 & 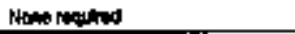 \\
\hline
\end{tabular}




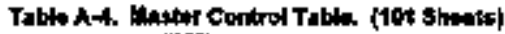

\begin{tabular}{|c|c|c|c|c|}
\hline 10 & Pren 690 & Hit $\theta 90$ & PTw TSR & $m$ tsh \\
\hline lowag-4hate & Mond 19 & 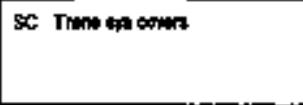 & 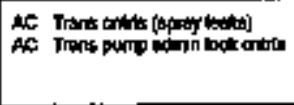 & 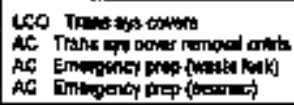 \\
\hline No4296-CIPA & monom nam & Han nappind & Noep nouked & Hent requerad \\
\hline 00425sClup: & Mono nowad & Hone nopphed & Nomen noured & Nene requirat \\
\hline 12428-5-ChPC-C & Hond nopid & Nenaronurned & 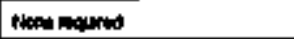 & 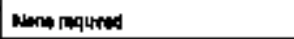 \\
\hline 10425-S-CNPDD & 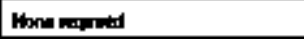 & Nonstapinges & 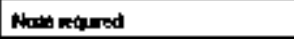 & Nene neatred \\
\hline 1.2425 s.plWh & Monem ingend & Hane reation & Honu nqured & thene requined \\
\hline 1.242ESEFLNA & Mona naprod & Hane reganded & Nene Inquired & Henes noqured \\
\hline 1-2438-s-fundt & Amon mand & Nenerequared & Nom nownos & nere requind \\
\hline 1.76295 ingh & Nond & Nens nowions & 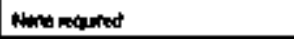 & Name rapuned \\
\hline 1.2ETTZELWHA & 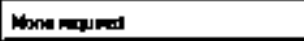 & 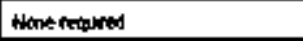 & Nonem mathed & Whane requared \\
\hline 1.242T \$सthath & 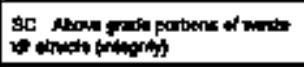 & 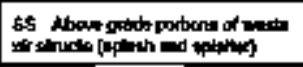 & Nern mpend & 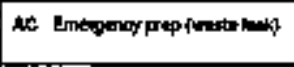 \\
\hline 1-24T-4नIWA & 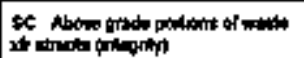 & 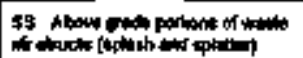 & Noton & 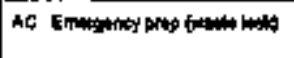 \\
\hline A LPLNT1-AT- & Nane named & None requeros & 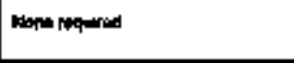 & Mine nopurpat \\
\hline 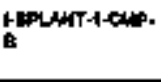 & Nane raqued & 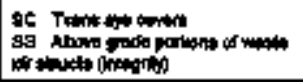 & 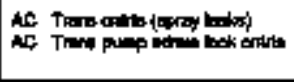 & 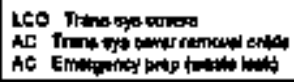 \\
\hline 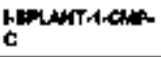 & Nonne noquered & None & Hone roperad & 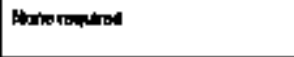 \\
\hline 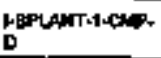 & Nono nand & None requatis & 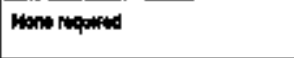 & 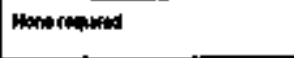 \\
\hline
\end{tabular}




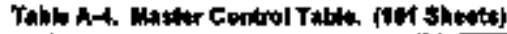

\begin{tabular}{|c|c|c|c|c|}
\hline סו & Phr gsc & M\$s & Pिए Tर्ष & Nats \\
\hline IFANAT-1-CWP- & 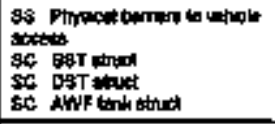 & Hent reapriad & 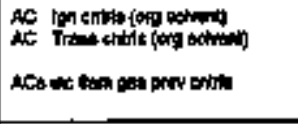 & 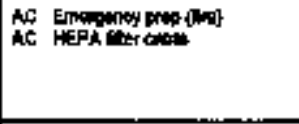 \\
\hline L.ERANT.1.THE. & 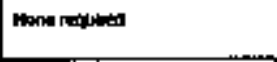 & 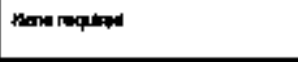 & Mane mated & Atime noquind \\
\hline HELANTAFIN & Aant & Whe requed & 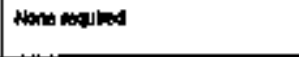 & Hone reanid \\
\hline E & Maneraquinot & Man mas & 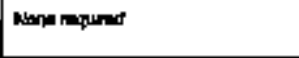 & Maneapod \\
\hline LOPLNAT-2FLW. & Nomenodined & Nowe teansed & 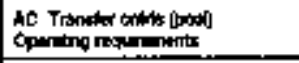 & 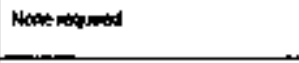 \\
\hline $\begin{array}{l}\text { LBPLANT-2ALW- } \\
\text { D }\end{array}$ & Noneraquind & Now rampars & AC Trara utef(pH) & 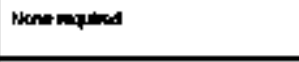 \\
\hline NePLANM-2+EK-X & NAtim & Nowe torpond & 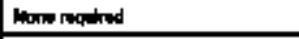 & Nent inimad \\
\hline 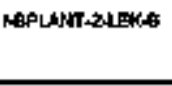 & Nom nopurad & 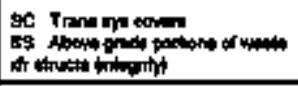 & 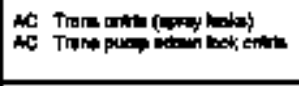 & 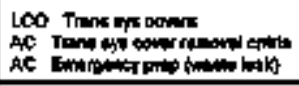 \\
\hline LEPLAM-ZLEKC & Nemerlopand & Nhanerequred & AC Erevedion atits & AC Exonch onts \\
\hline 1-BPLANT-2LEKA & Honerapured & Nonerequared & Hape noperend & 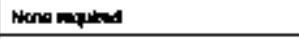 \\
\hline HERUNT-LEKE & 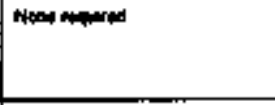 & 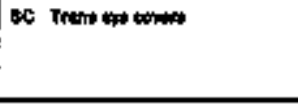 & 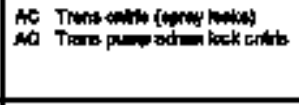 & 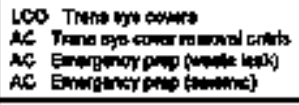 \\
\hline IAPA-1 AGTH & 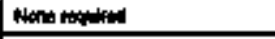 & Nane ray unted & 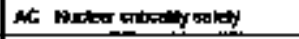 & Hene nopired \\
\hline LPFPA1 & Nontherat & Nani nengumed & 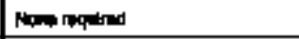 & 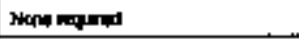 \\
\hline HEFP-1 $\angle C A P A B$ & 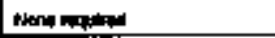 & Nono ripenter & 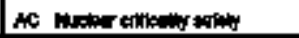 & Nong monited \\
\hline
\end{tabular}


Table A-4. Master Condrol Tabtu. f10t aheats]

\begin{tabular}{|c|c|c|c|c|}
\hline 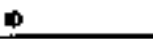 & Prer $\leftrightarrow 9$ & Mstc & $\mathbf{~}$ & uㅏ Tst \\
\hline HPF-1-CWPAC & ime roquend & Haninapropd & None nopand & Morat maphand \\
\hline LPP,|-CHP-D & Nons noqured & Nonerequind & 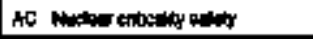 & Home nowad \\
\hline lopp.jotares & 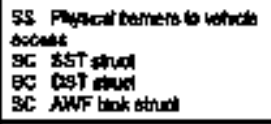 & Nene nequmed & 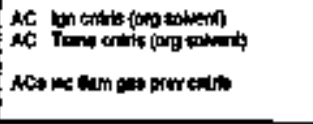 & 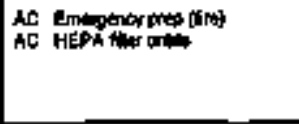 \\
\hline IPFP-1Lith & 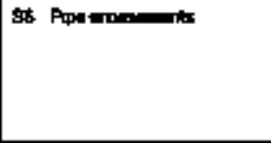 & 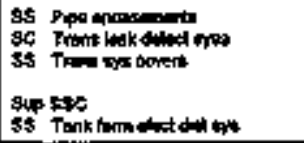 & 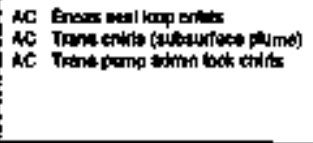 & 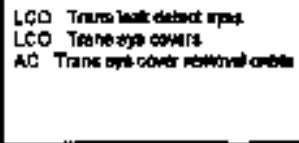 \\
\hline I-PFestathath & Hone nimurod. & Home reperat & Hane nagurew & Nonernogured \\
\hline IPFP-PLW-A & None minged & Non & Heene minited & Noes requared \\
\hline LPFP-ZIEK-A & Bron mapead & 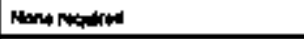 & 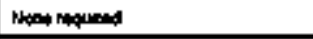 & 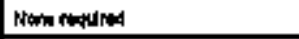 \\
\hline APRP-2-HEK-B & 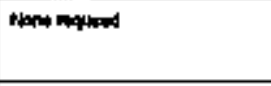 & 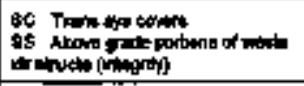 & 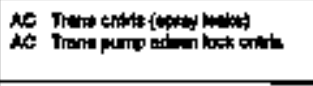 & 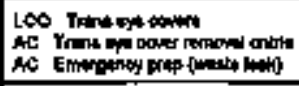 \\
\hline APrP-2HEK-C & Nom nompent & Nomantion & 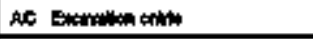 & 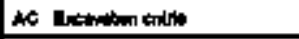 \\
\hline LPFP-2AEKD & photan nowed & Noinde required & None raguterd & Pantrequed \\
\hline |PFPQLExe & Wine roquited & 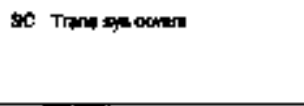 & 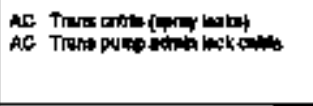 & 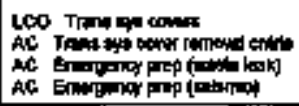 \\
\hline IPFP/2-TMPA & Mine noqured & 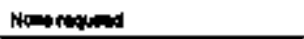 & Hono raparod & Nont maped \\
\hline Here.t.then & Wone nowhed & 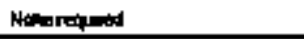 & 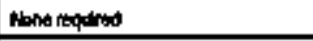 & 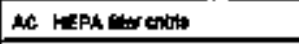 \\
\hline LPFPanciten & 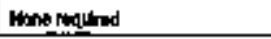 & 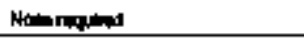 & Nopen nompined & 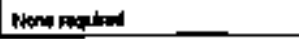 \\
\hline
\end{tabular}




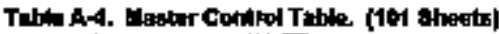

\begin{tabular}{|c|c|c|c|c|}
\hline ID & Prow ssc & m 950 & Pww TBR & MITSR \\
\hline 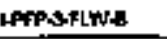 & Nom moperod & 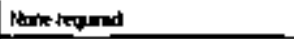 & Nonemened & 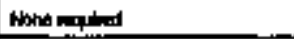 \\
\hline HAPS-FLWS & 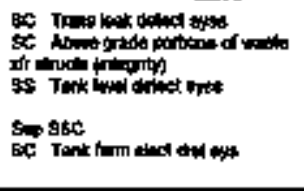 & 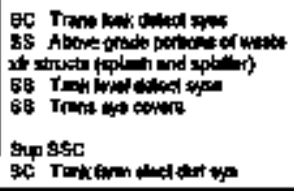 & 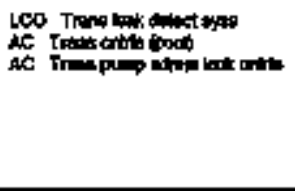 & 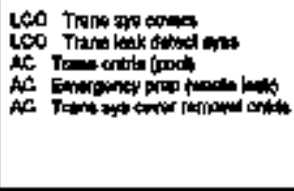 \\
\hline 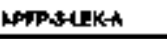 & Norem mingod & 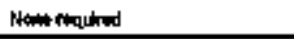 & Hone racind & Non mint \\
\hline LPFP,HEKC: & 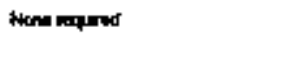 & 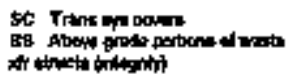 & 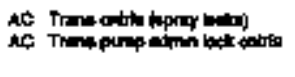 & 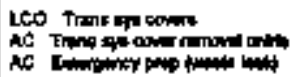 \\
\hline nPpisukec & Noree roputad & Nown tequred & 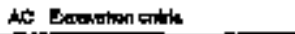 & 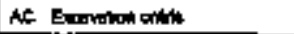 \\
\hline IPFPUS LEKD & mone nequend & Nene roquind & Wone requared & Deno roqued \\
\hline IPFP-अ,EK-E & 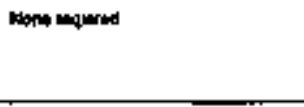 & 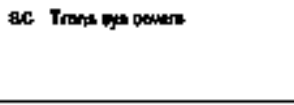 & 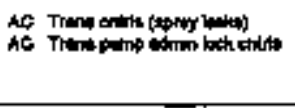 & 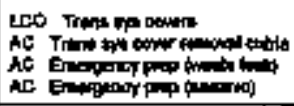 \\
\hline IPUREX-1_ACTAA & 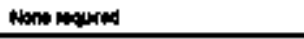 & phom sepures & then & Mon pand \\
\hline HNURDSTASTE & 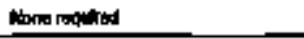 & 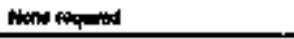 & Mone mather & Nant requer \\
\hline LFUREX-1-AGT-C & Winu noment & 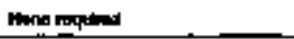 & Vorm nequmed & Mane nqumod \\
\hline HAREEX-1CG - & 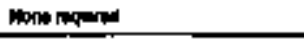 & Benplapilat & None repunand & 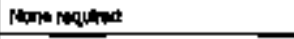 \\
\hline APUREX-1-CAMPB & Nene гериты & tions fopulis & Mone resured & Pand \\
\hline Apurexilchec & 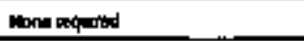 & 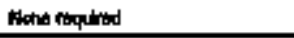 & Vone trapured & 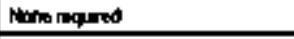 \\
\hline 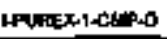 & daph & Manu & How rapuratd & Hal requind \\
\hline 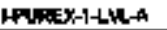 & Bone napkad & $\operatorname{man} 4$ & None paphod & Nome repund \\
\hline
\end{tabular}


Tuble A-4. Wenter Conkrod Table, \{101 theets;

\begin{tabular}{|c|c|c|c|c|}
\hline$\omega$ & Prew ssc & $M \operatorname{Bsc}$ & MWT T\$R & Nat \\
\hline Letexes-Then & Mine nempind & Menenoumed & Mone mopard & 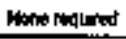 \\
\hline HMREX-3PUWA & 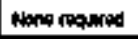 & Nones requires & Nome naphis & Wine noqund \\
\hline 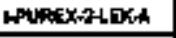 & 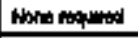 & Nens requist & Nons folantid & Hone repured \\
\hline IPtmax a LUKE & Anne notid & Neth ringind & 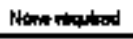 & 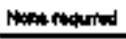 \\
\hline 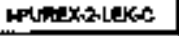 & Wone maped & Nhene requird & Nanot & Nown requitad \\
\hline Henex-34, & 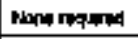 & None nequined & Nom anpand & Winte noquer \\
\hline HPUTEXXALEKE & Meno nayuad & Mones reaperod & Nomo pranind & Rime requines \\
\hline HOPEXX-2PRSA & 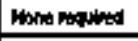 & Nenn requista & Nonefolation & Nont repoured \\
\hline 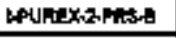 & Mone maped & bine ritarid & Nhant is & Henerequitad \\
\hline 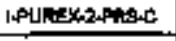 & Dane requad & 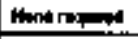 & Nhor & Monemaqued \\
\hline HUAREX-AAGTA & None nowind & 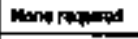 & And & 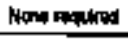 \\
\hline IPUREX-3AST日 & Ded & Ame ropens & Hom & 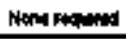 \\
\hline HAREKSAETA & 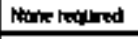 & Hond & 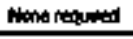 & Nons \\
\hline 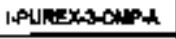 & Whou maper & 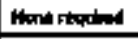 & Nonempled & Nhe noquand \\
\hline 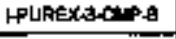 & 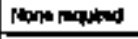 & Mons raped & Hom maphad & Nome raperpod \\
\hline IPUREX-3GM-G & 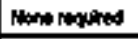 & Hone roming & Nom & Nopes napiod \\
\hline 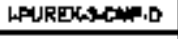 & Phone rowared & 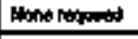 & Hint & None topond \\
\hline 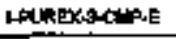 & Mant nquered & Mone renged & Nene mined & Hene mequed \\
\hline 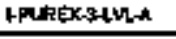 & 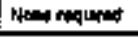 & Nonempined & Nomo napinat & ㅊens \\
\hline HPLREX3-TM A & Nomin requird & 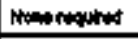 & 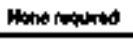 & Alene \\
\hline LPUR DX 4 TLWA & Nenen nogund & Nomoropited & lone noperod & Nlene reqperd \\
\hline HWAGEx-1ESh & Nane requend & Mone roped & 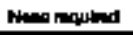 & Non maphat \\
\hline
\end{tabular}




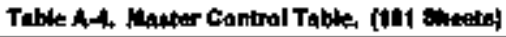

\begin{tabular}{|c|c|c|c|c|}
\hline b & Anir $\$ S C$ & Itstsc & Mr T Tan & m Tg̣p \\
\hline LPQFEX-AEKB & Arom requitid & Noneronumes & Hone nopuld & 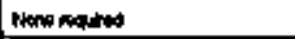 \\
\hline IPUREX-4Hak-C & Hons nonitad & 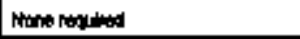 & 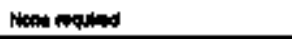 & 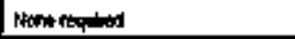 \\
\hline Helkeratero & Who raquerod & $\mathrm{Na}$ ingled & 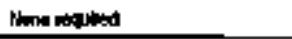 & Homene \\
\hline LPMFEX,HESE & moneropitod & Nonernquived & Hone ropiled & Nenen \\
\hline 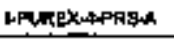 & whe required & None repoured & Nonen raghed & Deren paphad \\
\hline HPWFEX-APES & Man maplad & Nene reavirud & Nome nopitad & Denu \\
\hline HPURDXAPAE.G & Mome napures & Natentrenuled & 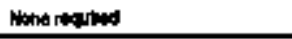 & None \\
\hline 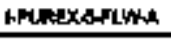 & 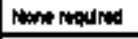 & Hone renquind & thon ranulad & Hon mater \\
\hline 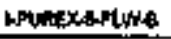 & materapind & 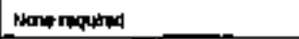 & ripno nenched & 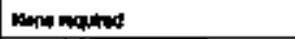 \\
\hline 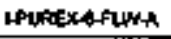 & Hant nepid & Aono propuras & 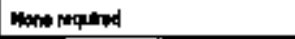 & Wone roperat \\
\hline |PUREX-6RLWE & 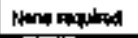 & Hona & Mons napirad & Whote requitad \\
\hline IPUREX-6.PLWA & Hann & Wholderad & Alant & Mtron rogedind \\
\hline 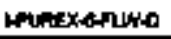 & 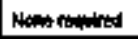 & Hone ingured & Mhe requifed & 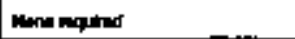 \\
\hline 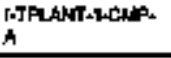 & Hen axpled & Mane naptipd & dim & Mano reound \\
\hline $\begin{array}{l}\text { I-THLWT-1-CAP. } \\
\text { B }\end{array}$ & 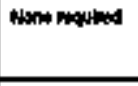 & 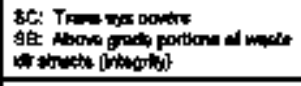 & 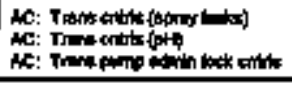 & 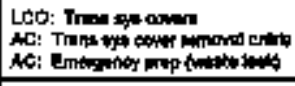 \\
\hline $\begin{array}{l}\text { HTELANT-1AKNP. } \\
\text { C }\end{array}$ & Hond and & 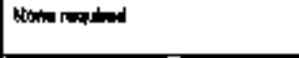 & 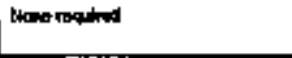 & None roppland \\
\hline $\begin{array}{l}\text { |-TPLANT-1-CMP. } \\
\text { D }\end{array}$ & Alene and & 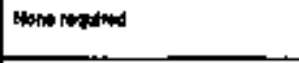 & Aloth & 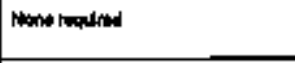 \\
\hline $\begin{array}{l}\text { ATPLNT-A.THA } \\
\text { A }\end{array}$ & Wone nopled & 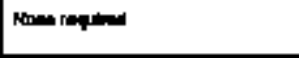 & Nem reaghed & Mone requitian \\
\hline
\end{tabular}




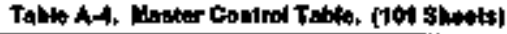

\begin{tabular}{|c|c|c|c|c|}
\hline סו & An ws & watst & 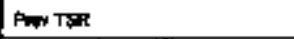 & MI T\$P \\
\hline HTPLAIT-2-OR & thon and & 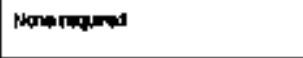 & Hewroparas & 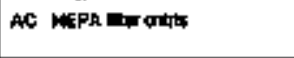 \\
\hline 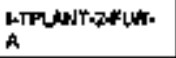 & Bbou nopuad & Hentrinted & Ham nomand & 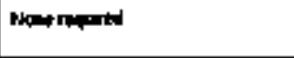 \\
\hline 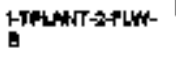 & Haw mand & Hotom ropisid & 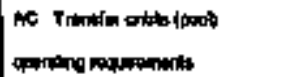 & 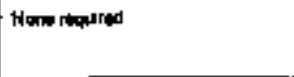 \\
\hline HPLANT-2LEKA & 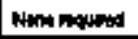 & Nowisapina4 & 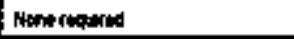 & None rapund \\
\hline HTPLATRTLEF & 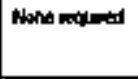 & 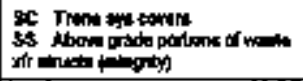 & 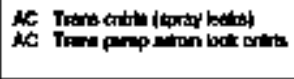 & 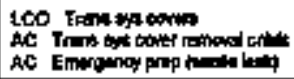 \\
\hline LTPLANTR-2LE-C & Nons relly & Nons newures & 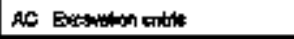 & 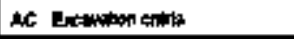 \\
\hline RTILLATALAKO & Whe requard & Hens requesed & Nent & 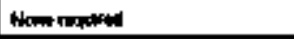 \\
\hline HTFLARALEKE & Whe roqured & 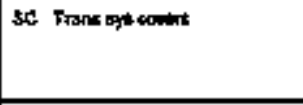 & 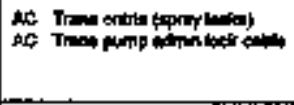 & 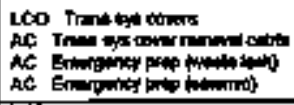 \\
\hline HELANTI-FLLW & Ulone nequered & Mone required & 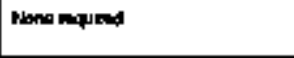 & Mens mapiond \\
\hline IELPLANAT-1,LEK-K & None roquind & Honte rapuatud & Hone rowned & Mene napinat \\
\hline |RPYNTT-1.LEKA & None requared & None roquered & ic Brenon orte & 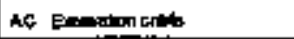 \\
\hline $\begin{array}{l}\text { ILFUNT-1-2EK } \\
\text { G }\end{array}$ & 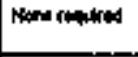 & Nome popured & Mand anding & 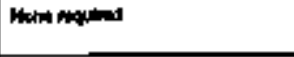 \\
\hline $\begin{array}{l}\text { Hoplatilanth } \\
\text { A }\end{array}$ & NGonempind & None reapred & Whom roquned & Dena nopred \\
\hline ITK- & 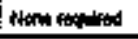 & 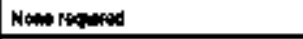 & 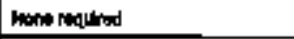 & Nane rapiras \\
\hline rik-OHन & Nanem & 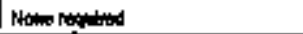 & Mane roquined & Nene napros: \\
\hline
\end{tabular}




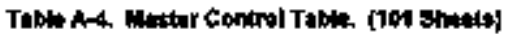

\begin{tabular}{|c|c|c|c|c|}
\hline $\mathbf{D}$ & Pur 8sc & HABC & Ph JFा & Notsh \\
\hline motes & Hoterequitad & Nons required & 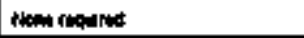 & Home noures \\
\hline motay & How mapurad & Nane fangures & Naternond & Bund requited \\
\hline $\mid+x-\infty$ & Nenw ruased & Nene ropuched & Nene maparind & Dhen moquered \\
\hline$|\pi k-\theta 3|$ & Honw fapurod & Feno rapures & Noni hagend & Vheneroqured \\
\hline ITK-A4 & 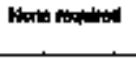 & tionu ropured & Nom nowiond & 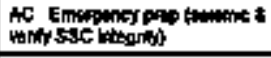 \\
\hline Itrk-044 & 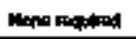 & Hene maperd & None minged & Nerenenqumed \\
\hline mK-05 & Moni nipand & 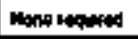 & 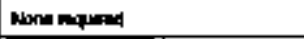 & Nonenapored \\
\hline mKKos H & 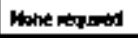 & ithe isopind & 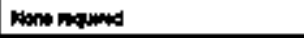 & Nomefequired \\
\hline mosot & Hone minged & Wone ropand & 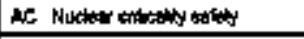 & 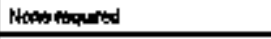 \\
\hline Itrotoder & None mengerd & Inone requand & 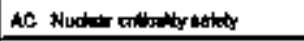 & Nam \\
\hline mKo & Nans moper & Wone noquesed & 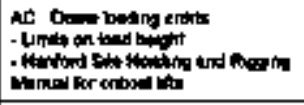 & Alenemented \\
\hline TTK-0R-14 & Non and & Mnom & Nomin nequind & Napererequind \\
\hline rthosis-ig & Mone mand & Hone moperied & 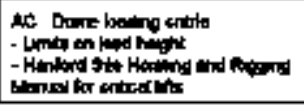 & Namerequeded \\
\hline
\end{tabular}




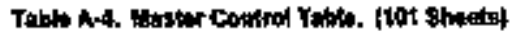

\begin{tabular}{|c|c|c|c|c|}
\hline$\infty$ & Mot Essc & No6C & Phertak & M Ts: \\
\hline TRK-0 & 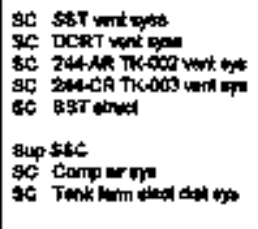 & 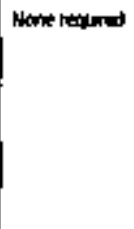 & 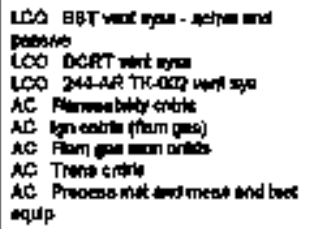 & 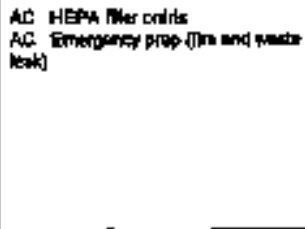 \\
\hline $17 K 001$ & Napen unquered & None repar & 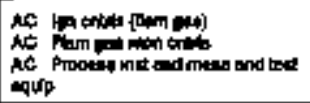 & 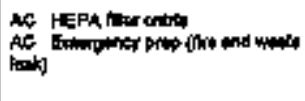 \\
\hline rkeas & 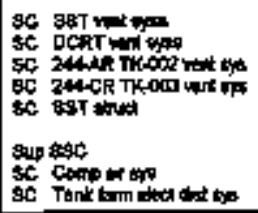 & 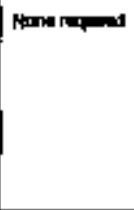 & 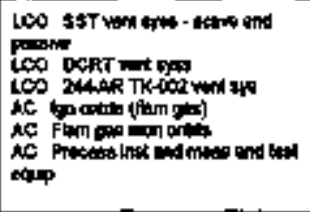 & 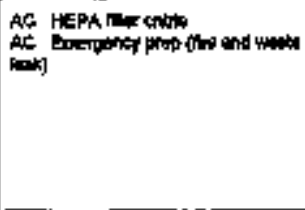 \\
\hline mkeO & 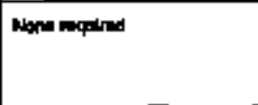 & 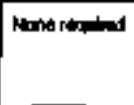 & 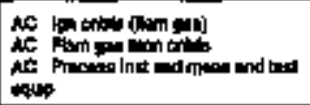 & 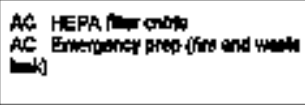 \\
\hline$\pi / 6.10 .1$ & 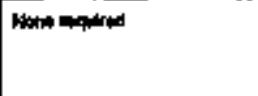 & Manem maped & 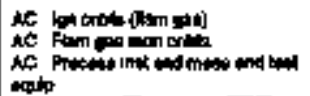 & AC \\
\hline
\end{tabular}




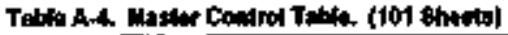

\begin{tabular}{|c|c|c|c|c|}
\hline 17 & Pnw bst & 14* 586 & Aurtsa & M T5R \\
\hline ITK-10-4 & 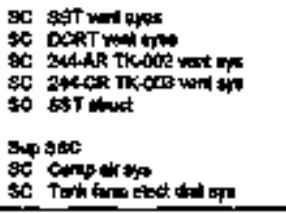 & 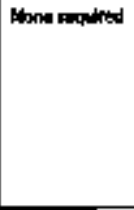 & 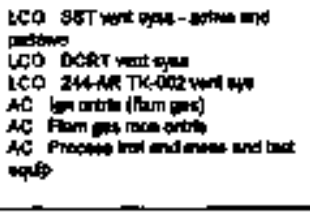 & 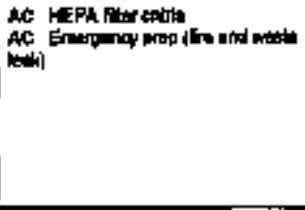 \\
\hline ITK-10-6 & 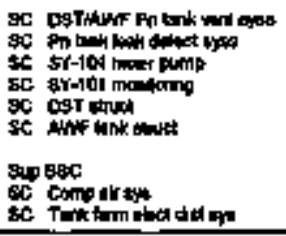 & Home ropodind & 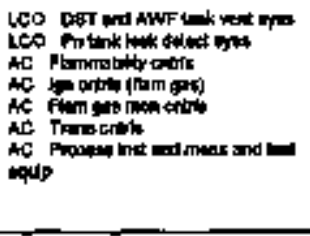 & 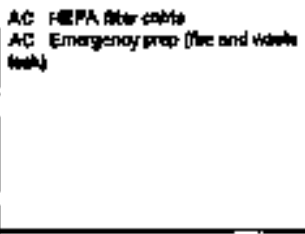 \\
\hline ITK-11 & 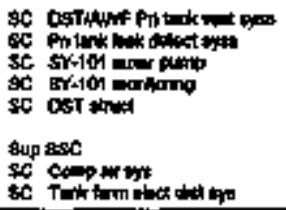 & Hont requind & 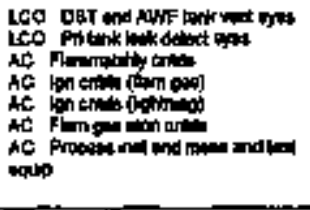 & 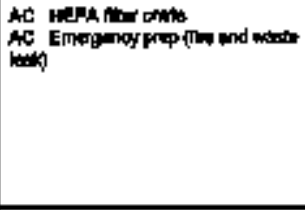 \\
\hline ITK-11- & Nbond mipund & 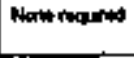 & 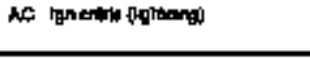 & 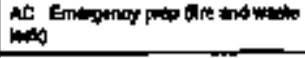 \\
\hline ItKFran & Hone roparad & 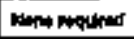 & Mone reapter & 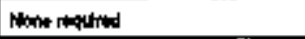 \\
\hline ITKPR-OR & None mpined & 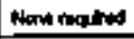 & 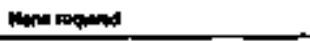 & Mane nopitod \\
\hline ITKFR-03 & 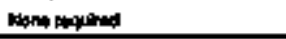 & thenes moputed & 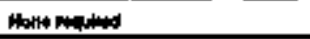 & Nome maquirad \\
\hline
\end{tabular}




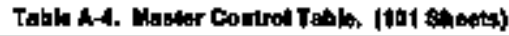

\begin{tabular}{|c|c|c|c|c|}
\hline D & ParrsBC & inssc & Pre tst: & Ht Tst \\
\hline TKसमR-0S & 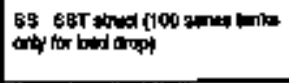 & 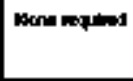 & 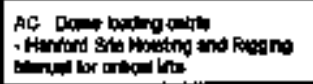 & Nane nopherd \\
\hline TK-FR'S & 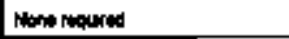 & Mens mapred & Mane & 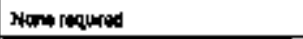 \\
\hline MK-PR-OB & Now & Lane monered & 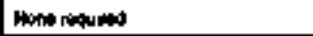 & 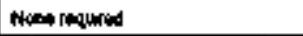 \\
\hline ImKNR-OST & 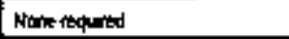 & Hone maned & Whine requined & 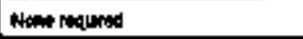 \\
\hline rikmats & Pane roqumad & 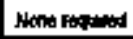 & 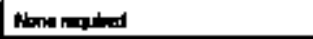 & 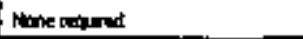 \\
\hline ITKAFA- & Mone napured & SNon & 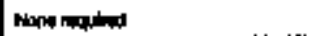 & Nane raphed \\
\hline ПTK-FR-10 & 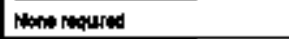 & 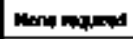 & 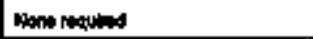 & 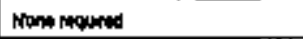 \\
\hline ITK-PR-1t & 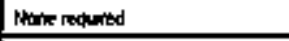 & 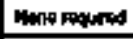 & Hone recting & Name raqued \\
\hline$m K+R-12$ & None nogend & 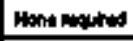 & horre rminild & Nhatide requad \\
\hline ITKafA-13 & Nanerequmed & 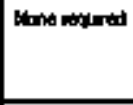 & 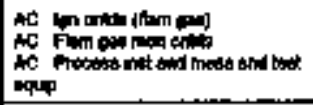 & 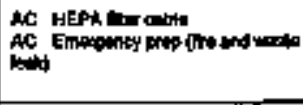 \\
\hline InKFp-14 & 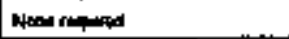 & 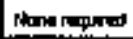 & Wure requind & Neene requeroud \\
\hline TKKER-15 & 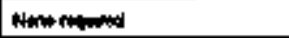 & Nonempurmet & Bow & 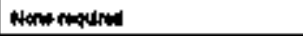 \\
\hline ITKNTK10 & How riperd & 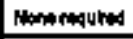 & Nome requlined & How rowphist \\
\hline ITGSP:17 & 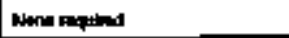 & 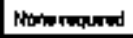 & Rtare noptowd & neone noquad \\
\hline TKKFR-18 & Nhan mapind & Noneraqured & 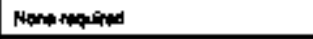 & 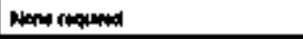 \\
\hline TTK-TR-19 & 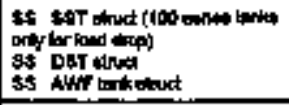 & Nompropuras & 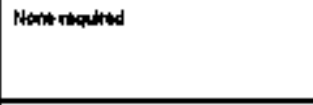 & 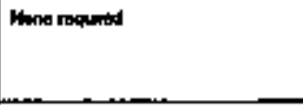 \\
\hline$m x+A x$ & Mone nquand & Nhen reaplod & Promeraptined & 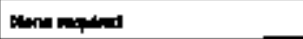 \\
\hline 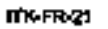 & Won rowind & Nowe neqund & Now repadind & 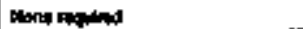 \\
\hline
\end{tabular}


Toblo A-4. Wnetar Cortroi Tablo. (101 bhela)

\begin{tabular}{|c|c|c|c|c|}
\hline 10 & Prorste & Mussc & PMW TSR & int Tsa \\
\hline rikek-Z2Z & Alow & Mine requitiod & 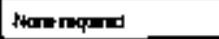 & Dine napuned \\
\hline MKPRAZ & Non raplend & Mand & 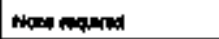 & Nomerequined \\
\hline ITKFR $\Rightarrow$ & 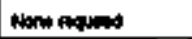 & Hond nowlod & 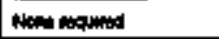 & Nonerequitod \\
\hline TIKARES & 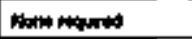 & Mone ropest & d & Monerapered \\
\hline ITKFR-29 & 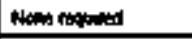 & Wha roped & Nantertqua & Bune noquned \\
\hline TKGPRLE & 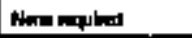 & 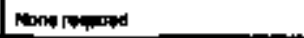 & Hon mand & Panerepared \\
\hline TKGFR: & Nbon mapad & 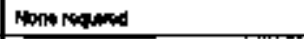 & Rnep nawad & Menerisumad \\
\hline 1901 & Wone rapures & 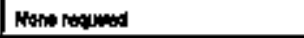 & 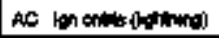 & 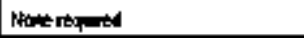 \\
\hline 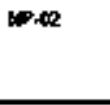 & Mone manted & 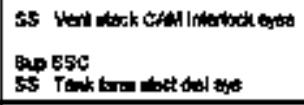 & Whot ringend & 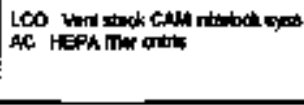 \\
\hline$N=0 s$ & Nane requited & How mequant & 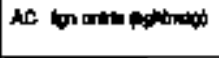 & 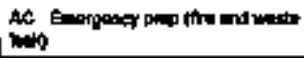 \\
\hline SA- & Mane nopures & Hor & 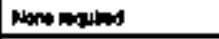 & Plons \\
\hline Nos & thate requard & Now trowhed & Whore peapond & Hon man \\
\hline$M$ & 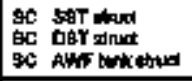 & Hon nequand & Atone nopurow & 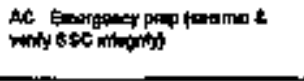 \\
\hline NPAd & 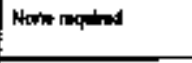 & thonemequad & Nome roquand & 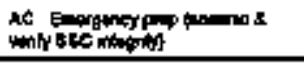 \\
\hline WP-09 & 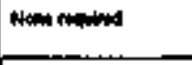 & 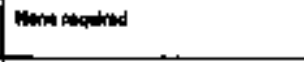 & Now rapand & 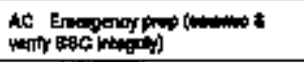 \\
\hline$N P=10$ & Nune requilad & 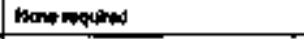 & Ptone reagurad & 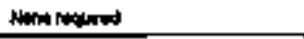 \\
\hline$N P-11$ & A & Imon & 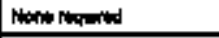 & Hone rapy red \\
\hline$n-12$ & 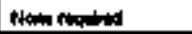 & 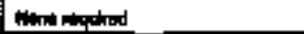 & 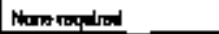 & Hine requind \\
\hline
\end{tabular}




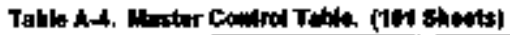

\begin{tabular}{|c|c|c|c|c|}
\hline 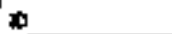 & Pit SSE & MHsso & PArTER & MTER \\
\hline NP-1ד & 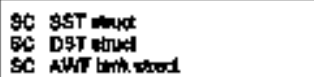 & Non: nampler & Nant lequinis & 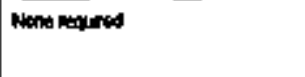 \\
\hline NP-14 & Llone requared & Nonentiated & Mant motiond & 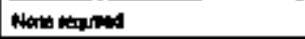 \\
\hline WP.45 & Noperopured & Nene cequ red & 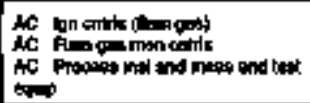 & 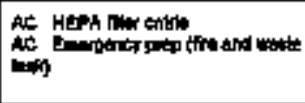 \\
\hline OGTEXTIMA & 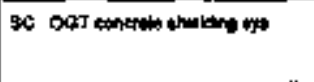 & Nonenequared & 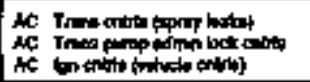 & 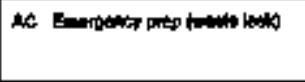 \\
\hline OOTEXTঙ, & 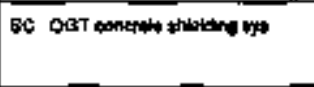 & Honerequref & 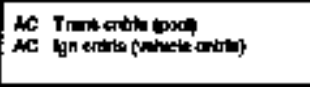 & 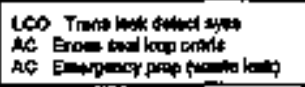 \\
\hline OवानLOWH-01 & Nome mouired & Naneraquined & Hontor roquand & Non \\
\hline OETFLOWH- & 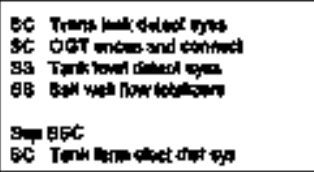 & 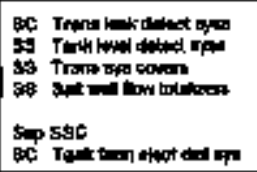 & 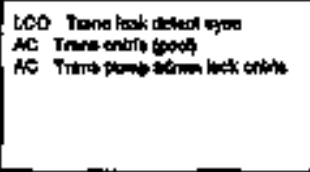 & 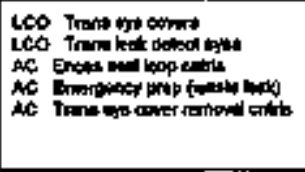 \\
\hline Oढ़T flowmats & 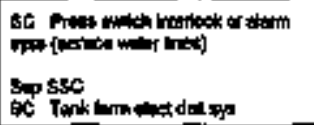 & Nemerenum & 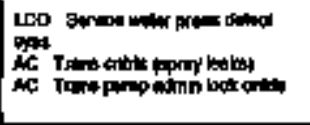 & 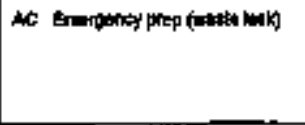 \\
\hline OGTPLOWno39 & 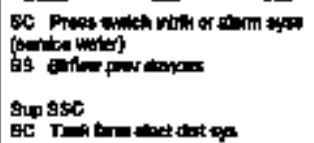 & Now repunded & 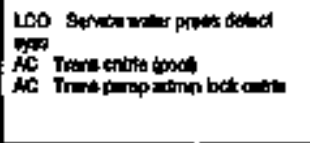 & Nene \\
\hline
\end{tabular}




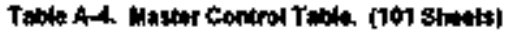

\begin{tabular}{|c|c|c|c|c|}
\hline $\boldsymbol{D}$ & Par \$9sc & Megsc & Pur TSRR & W TrR \\
\hline OGTFLOWH-04 & 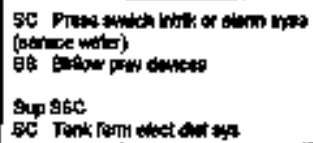 & lomo raciand & 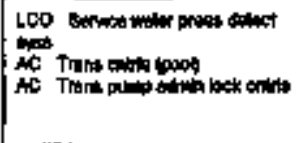 & Nam ropens \\
\hline OCT-1ㅇN1-05 & Ntom anpinded & Nom & 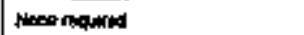 & Han explas \\
\hline OGTMON1-DA & 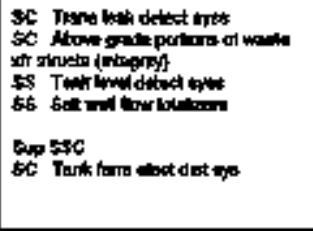 & 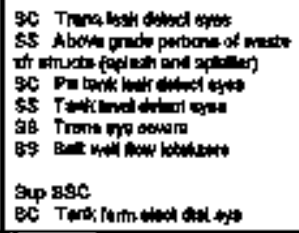 & 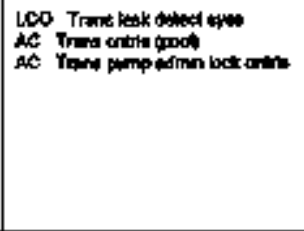 & 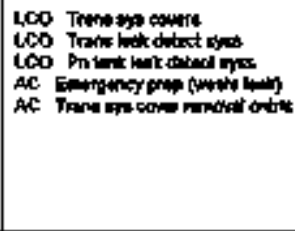 \\
\hline OATHLONB-o1A & 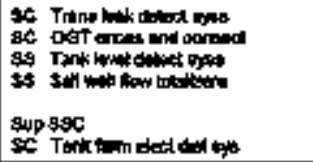 & 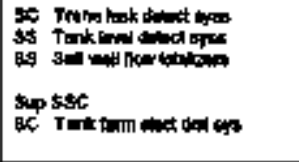 & 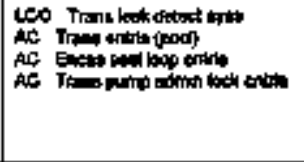 & 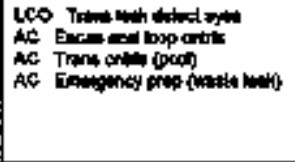 \\
\hline dotflowzals & 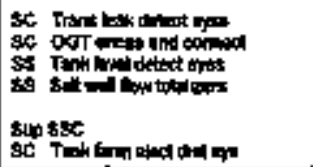 & 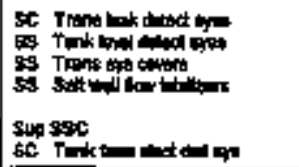 & 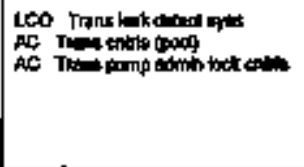 & 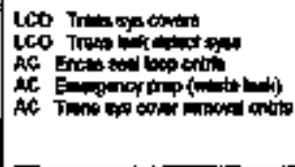 \\
\hline ootrlown-n & 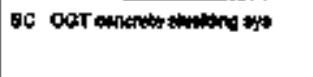 & 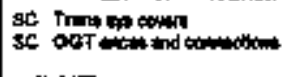 & 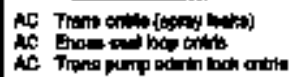 & 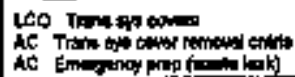 \\
\hline
\end{tabular}


Toblo A-4. Marer Control Table. [16 shotts]

\begin{tabular}{|c|c|c|c|c|}
\hline 10 & Pren GBC & Mssc & PNa TER & Het Ts \\
\hline 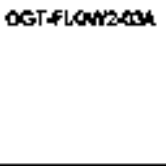 & 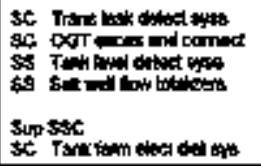 & 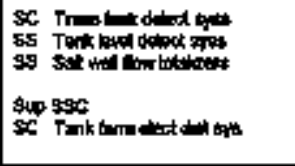 & 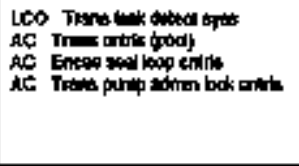 & 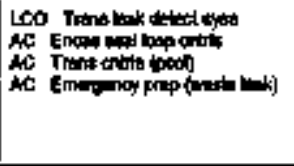 \\
\hline OCTALOMnttes & 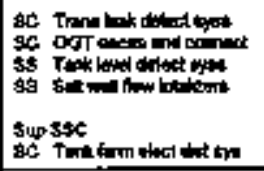 & 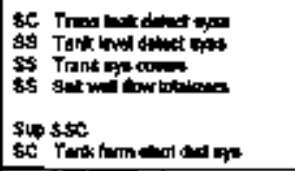 & 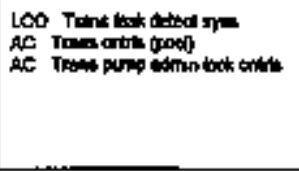 & 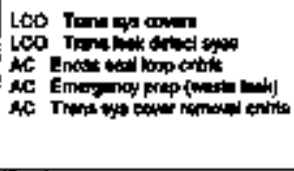 \\
\hline 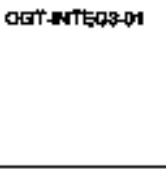 & 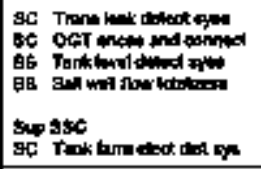 & 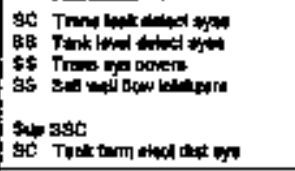 & 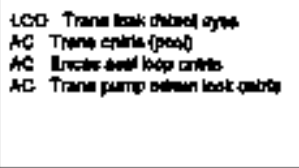 & 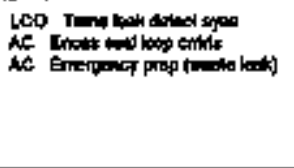 \\
\hline DOTSAPßOM & Narrerequened & 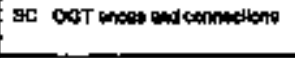 & 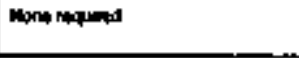 & 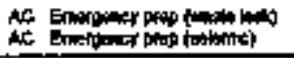 \\
\hline OGTNAPSOR & Nanin requered & 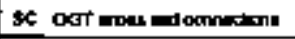 & More relonged & MC ting \\
\hline Cxit-Dergi-01 & Nant nquand & Nenenongutrect & 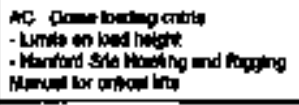 & Mand and \\
\hline OSTFRES1M & 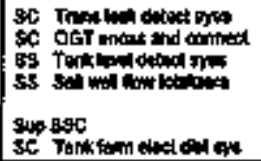 & 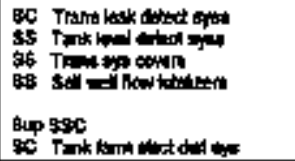 & 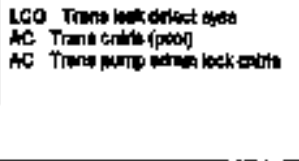 & 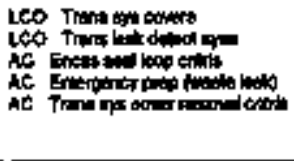 \\
\hline
\end{tabular}




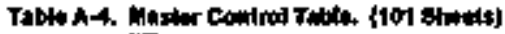

\begin{tabular}{|c|c|c|c|c|}
\hline $\boldsymbol{D}$ & Pmo \$sc & Ner Bs:C & PAN TER & 摬 TSh \\
\hline OOT fuEAC1-11 & 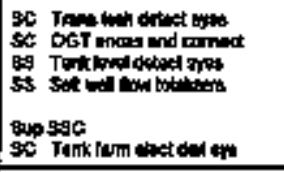 & 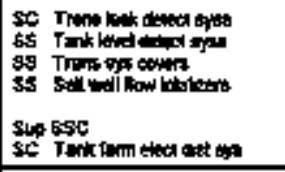 & 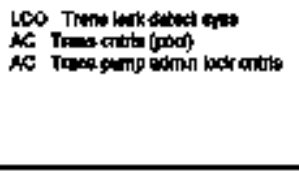 & 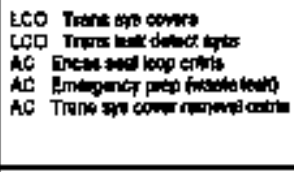 \\
\hline OGTSPRACIN & 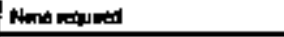 & 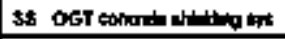 & 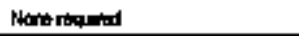 & Whe nangted \\
\hline DGT.TEF-191 & Anen & 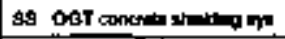 & Nonemanged & Nane requared \\
\hline adt+TE-R & 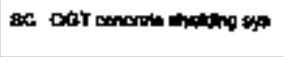 & Mene requard & 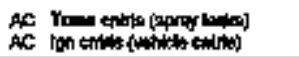 & 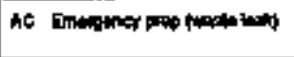 \\
\hline OGT-TELPIAS & 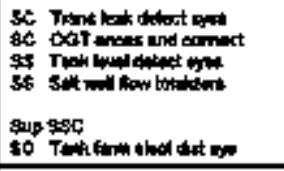 & 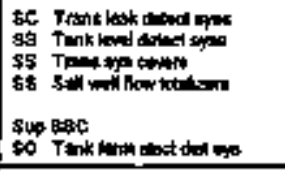 & 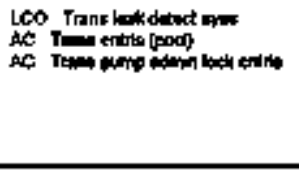 & 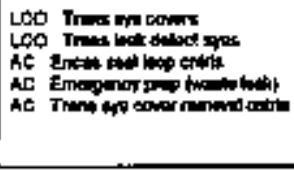 \\
\hline Ruto-0r & Phine required & None roaphod & Hone roqued & Nontapalined \\
\hline Adthort & Unen required & None resperod & Mone mopend & 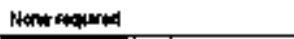 \\
\hline RAODOE & None nopunat & Nam Nom & Man & 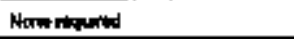 \\
\hline Now- & 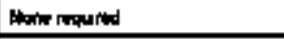 & 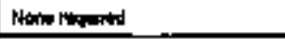 & 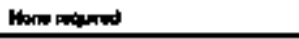 & Nane mopured \\
\hline Prons & None roquired & Nome ro, lind & Wone anquad & Nan: manered \\
\hline Fuposed & Itane requeread & 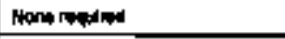 & Mona nowaref & Nont fipurew \\
\hline RAR-O1 & 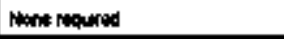 & 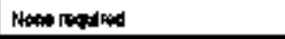 & Nanom & 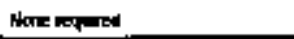 \\
\hline Rsend & Nowe tapulad & 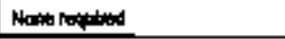 & Mand roptind & 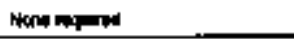 \\
\hline nemen & 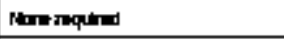 & 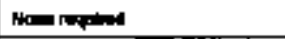 & 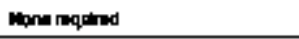 & Nend niperat \\
\hline RQgR-004 & Nomenopalind & Nom & ind & Nent \\
\hline
\end{tabular}




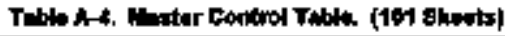

\begin{tabular}{|c|c|c|c|c|}
\hline 10 & Prew 650 & Mn 896 & FWW TR: & Mt TsR \\
\hline Rtstas & Horm malperd & Norrerequired & 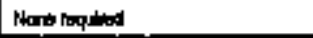 & 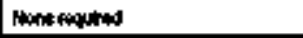 \\
\hline 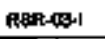 & 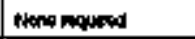 & Hon: raquind & Nare maperd & 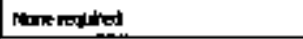 \\
\hline Reston & 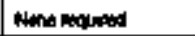 & Ptentreanures & Nonespaquad & | \\
\hline etstow & Hone napred & 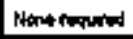 & 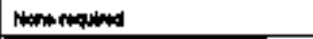 & 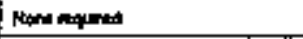 \\
\hline estitas & Hene regared & None reapulad & 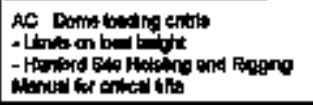 & Man \\
\hline 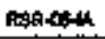 & Mones mapurapd & None noquend & Henenged & Nathen \\
\hline 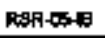 & Mino napurad & 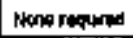 & 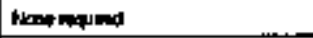 & Non mand \\
\hline RSA-W & Who nopured & Nene reapured & 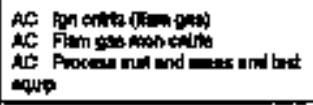 & 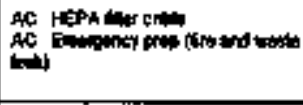 \\
\hline mathrofit & 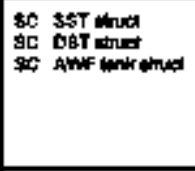 & 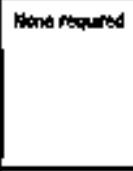 & 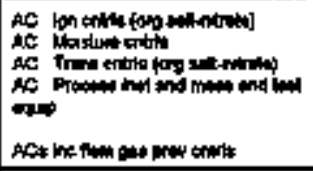 & 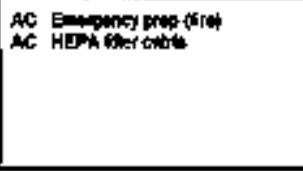 \\
\hline 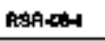 & Mene requared & Hane required & Now rophed & 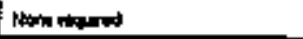 \\
\hline \$F-O & 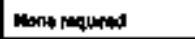 & Hens required & 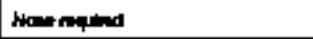 & Nuremalfed \\
\hline Spr-o1-4 & None & 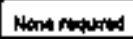 & 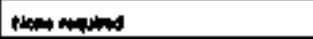 & 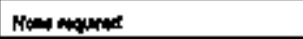 \\
\hline
\end{tabular}


Tabke A-4, Mattor Control Table. (101 sheats)

\begin{tabular}{|c|c|c|c|c|}
\hline ID & Phere & Mt \$झ्द & Pre Tsh & whok \\
\hline TE:1 & 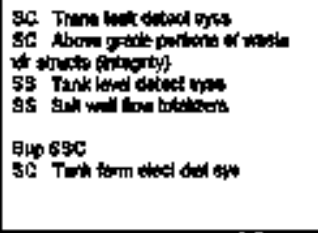 & 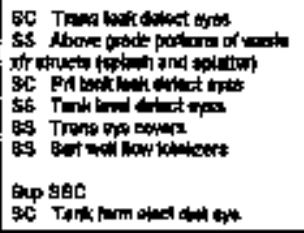 & 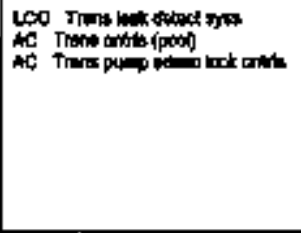 & 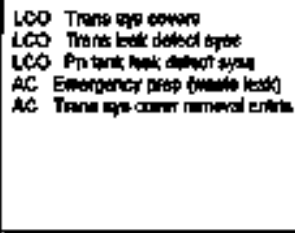 \\
\hline WENका & Nome rogund & 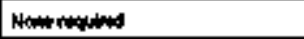 & None naphend & Henis \\
\hline VEH 0 OHA & None roquind & thoneringuted & Nothe ropened & Eone rowand \\
\hline VEH-DF & Crothererterned in GAAA & thth oobned In GARP. & 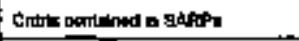 & 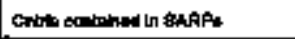 \\
\hline VEH-ORA & 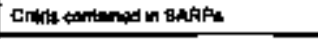 & 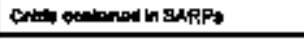 & 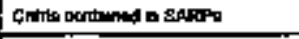 & 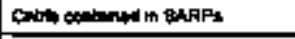 \\
\hline$\sqrt{2}+\infty$ & 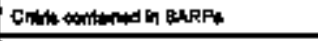 & 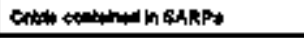 & 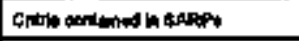 & Ctho \\
\hline VEH & 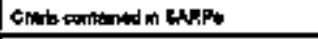 & 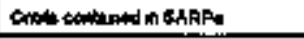 & 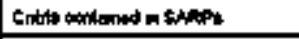 & Gotte withod in thapt \\
\hline VEHWON & 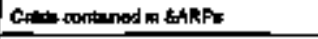 & Coto contuned a SNAP. & 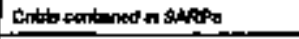 & 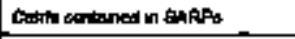 \\
\hline พอน०+1 & 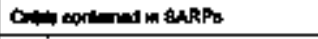 & 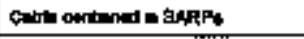 & 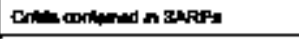 & 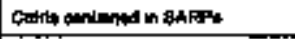 \\
\hline vettos & 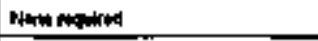 & Hont Nequread & Nons notiond & 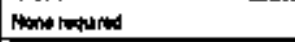 \\
\hline Vthosil & 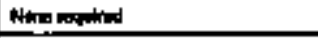 & Hint r row & Nath natiod & Whe requirod \\
\hline vaphot & 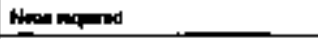 & Wone requtied & Mane roxided & Hone nopured \\
\hline VEHOOH & 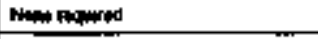 & Mane aspourted & 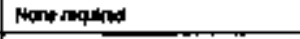 & mono nopureat \\
\hline wero? & 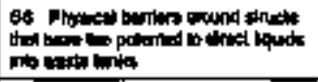 & 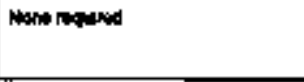 & diman & 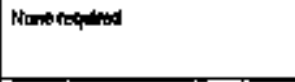 \\
\hline Hemerd & 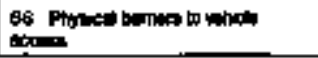 & 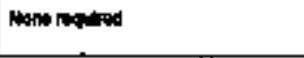 & 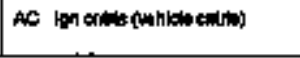 & 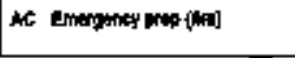 \\
\hline
\end{tabular}




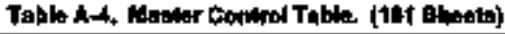

\begin{tabular}{|c|c|c|c|c|}
\hline 10 & Pron $55 t$ & masct & Pra Tsh & 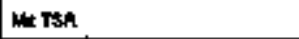 \\
\hline VEHOSI & 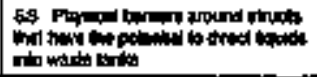 & Mane requm & 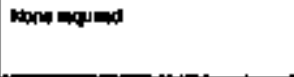 & Honompurod \\
\hline vapos & 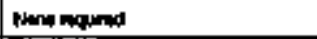 & 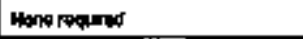 & 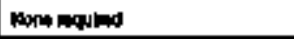 & Nomeraparas \\
\hline vovas. & then nowared & Shon ramered & 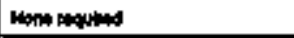 & Nomerapund \\
\hline veros & Nomin nowned & Nome rienreds & Hom & Nemorequired \\
\hline tstuped & Hand & Nonte iteried & Itone ratipend & Not: requind \\
\hline VEtuts & Mene mathed & None repad & Mone requend & Honomand \\
\hline vosucts & Monal maphand & 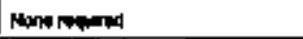 & Nophoping & 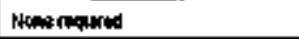 \\
\hline W0os-FLDM1 & 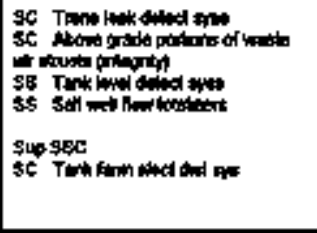 & 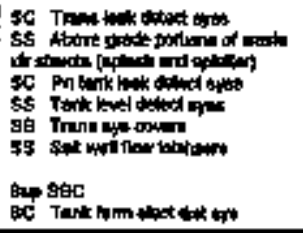 & 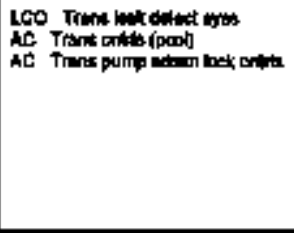 & 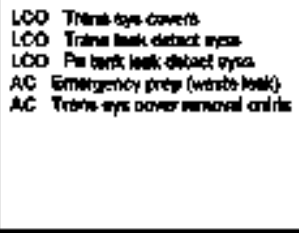 \\
\hline WOSPALOHDS & 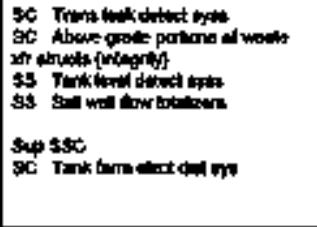 & 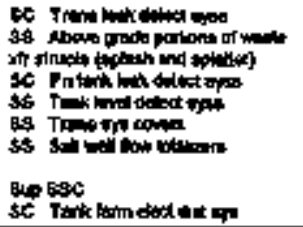 & 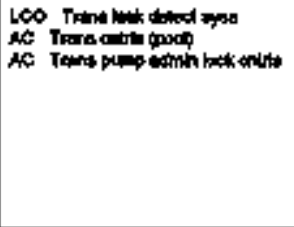 & 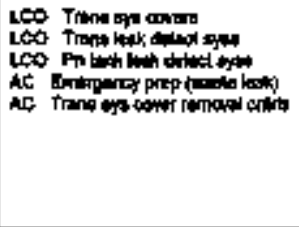 \\
\hline
\end{tabular}




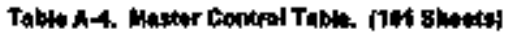

\begin{tabular}{|c|c|c|c|c|}
\hline m & Ane ssc & us sec & Pr. TER & Nin TSt \\
\hline mostrownos & 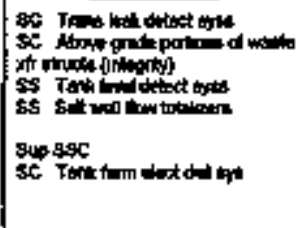 & 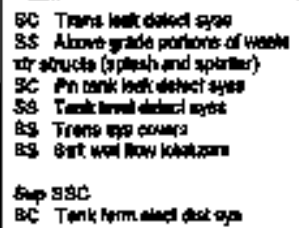 & 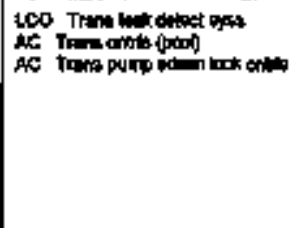 & 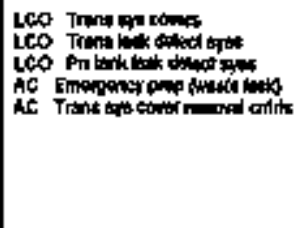 \\
\hline ness Fuchioss & 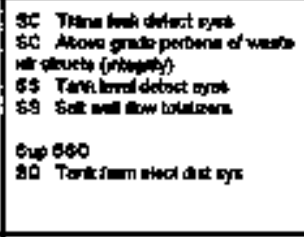 & 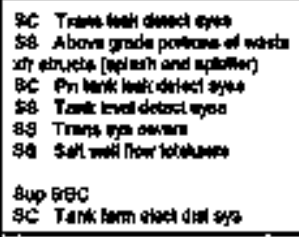 & 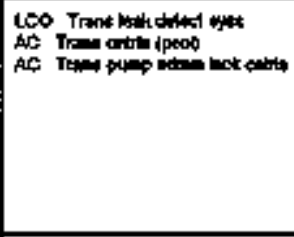 & 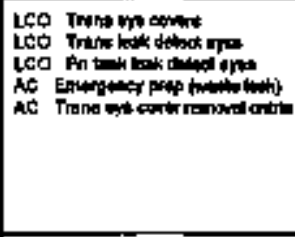 \\
\hline Wridorl LOMres & 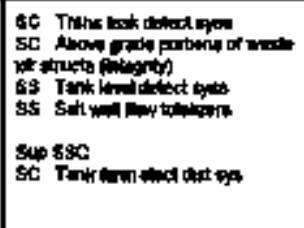 & 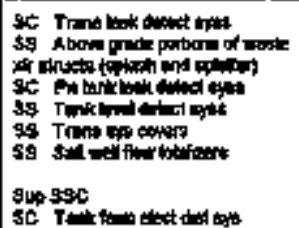 & 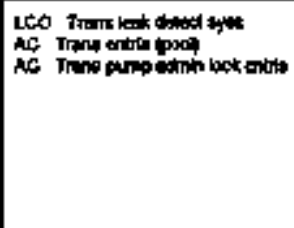 & 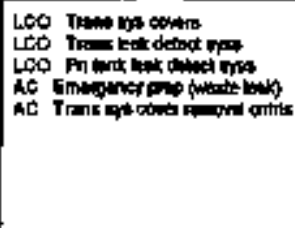 \\
\hline
\end{tabular}


Talk A-4 inatior Control Trable. (101 thentu)

\begin{tabular}{|c|c|c|c|c|}
\hline D & Paw SSC & masc & PNw TER & 네 r 198 \\
\hline 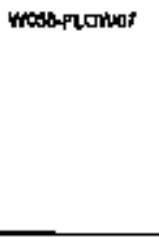 & 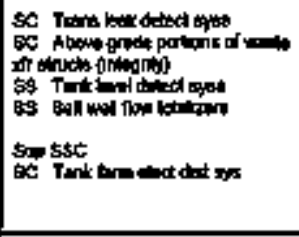 & 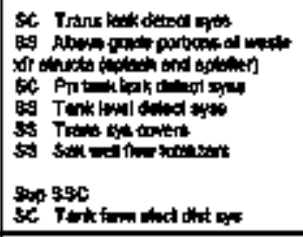 & 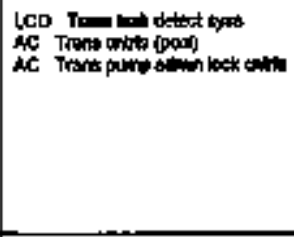 & 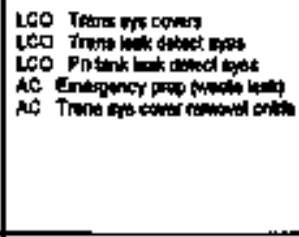 \\
\hline Inte-flomos & 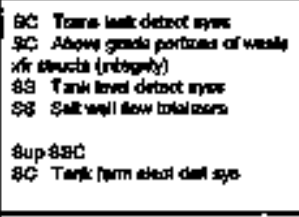 & 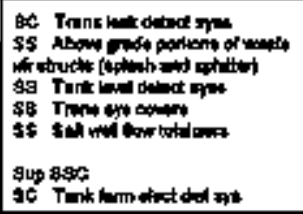 & 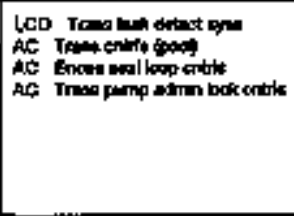 & 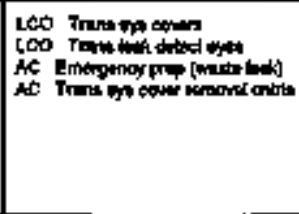 \\
\hline WoSAfLOW'I10 & 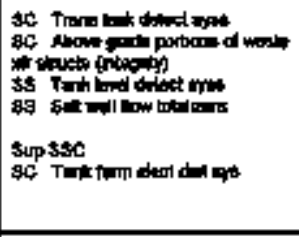 & 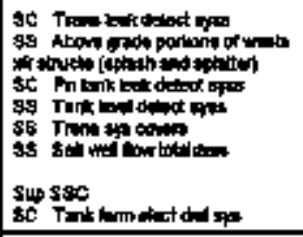 & 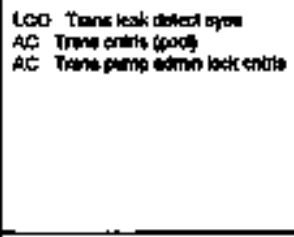 & 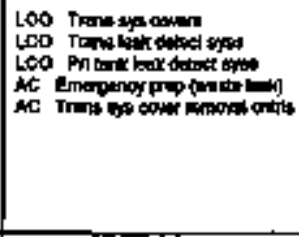 \\
\hline Modr-fow110 & 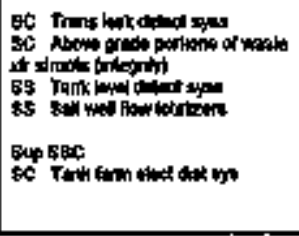 & 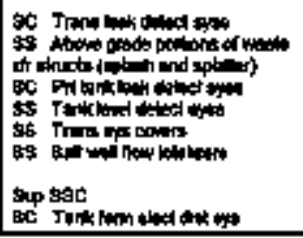 & 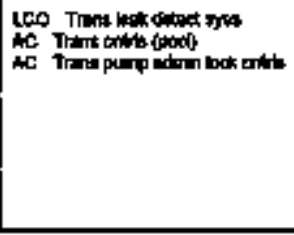 & 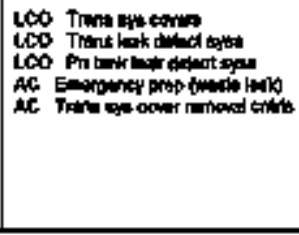 \\
\hline
\end{tabular}




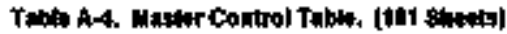

\begin{tabular}{|c|c|c|c|c|}
\hline $\mathbf{D}$ & Prod 85 & $\mu$ ssc & Pren TbR & Wte Tto \\
\hline WOSP-10N11e & 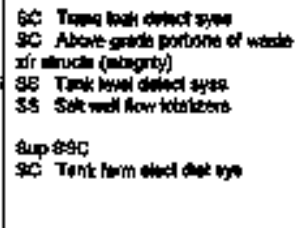 & 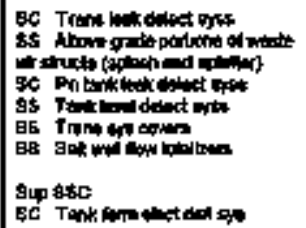 & 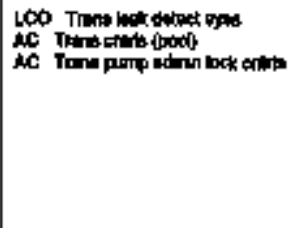 & 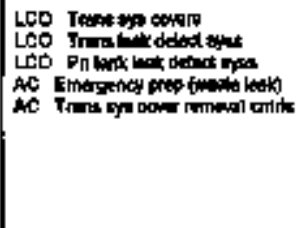 \\
\hline wostorlow1s & 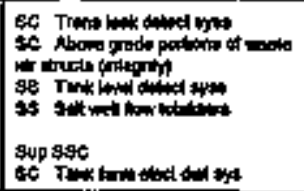 & 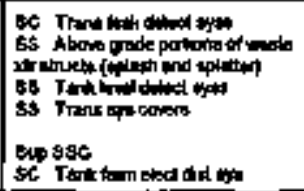 & 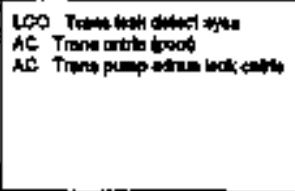 & 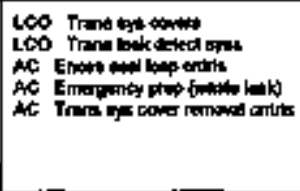 \\
\hline mosertomis & Hens roquined & 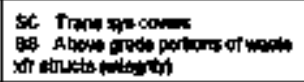 & 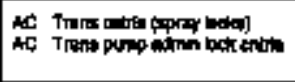 & 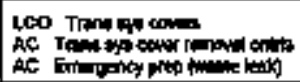 \\
\hline WoSPLOWIS & Whe ropred & 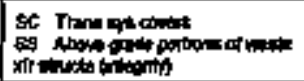 & 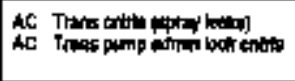 & 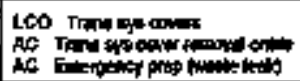 \\
\hline unserplowillo & 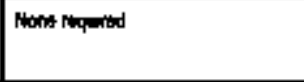 & 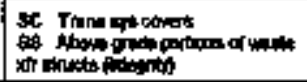 & 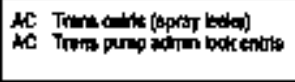 & 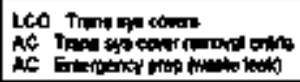 \\
\hline Mnseplowks & 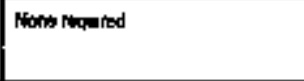 & 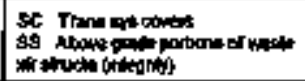 & 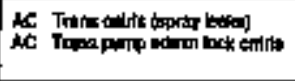 & 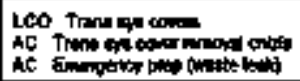 \\
\hline Wnasmountre & Retentrophed & 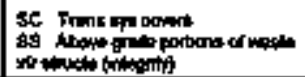 & 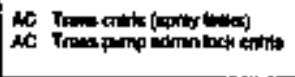 & 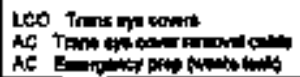 \\
\hline
\end{tabular}




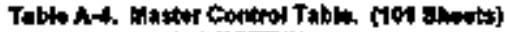

\begin{tabular}{|c|c|c|c|c|}
\hline $\boldsymbol{D}$ & PA \$SCC & NA 吿 & Pant TER & mTgk \\
\hline MosfFonts & Htom & 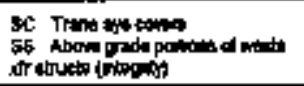 & 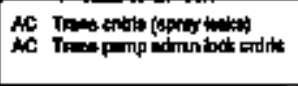 & 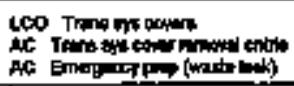 \\
\hline MOSPRLONA & 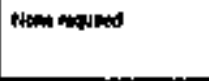 & 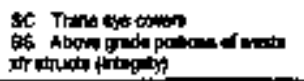 & 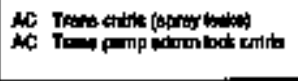 & 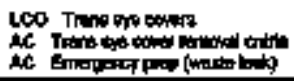 \\
\hline 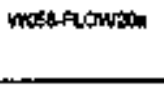 & Hon landind & 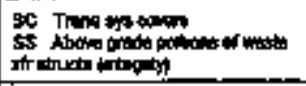 & 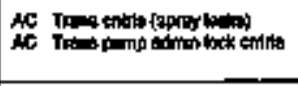 & 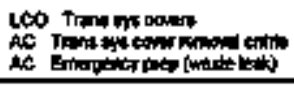 \\
\hline Wobsteftwich & 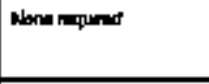 & 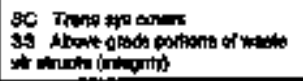 & 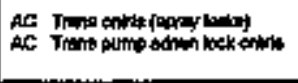 & 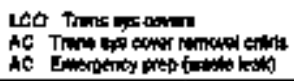 \\
\hline Wo6s.FLOWHAD & Abone requard & 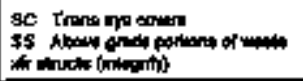 & 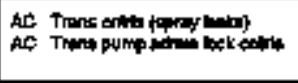 & 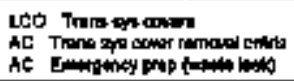 \\
\hline WOES WTECSOII & 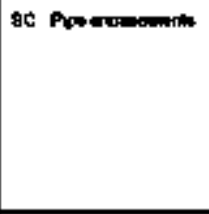 & 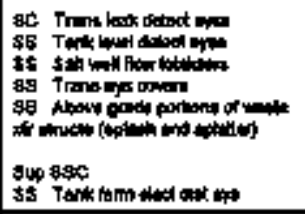 & 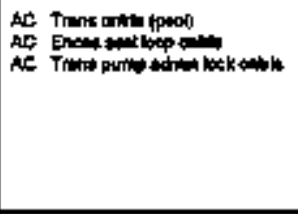 & 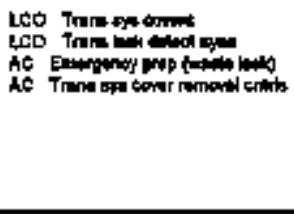 \\
\hline WOEOWTEEOND & \&C Macalonosists & 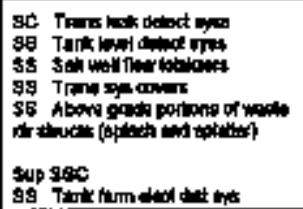 & 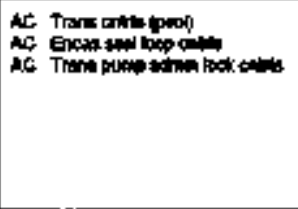 & 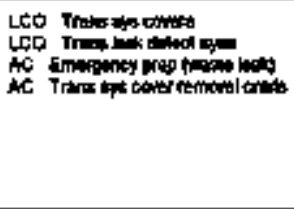 \\
\hline
\end{tabular}




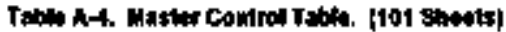

\begin{tabular}{|c|c|c|c|c|}
\hline סا & Prorse & 새 \$9心 & DTw ras & 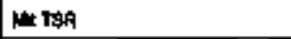 \\
\hline HOS\%-NTEGME & 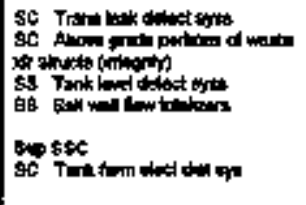 & 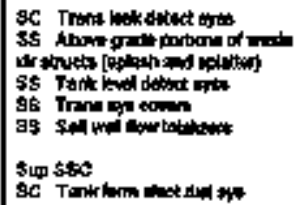 & 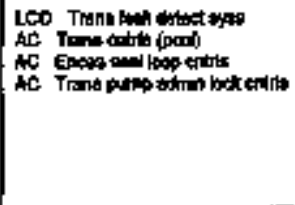 & 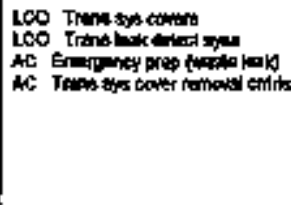 \\
\hline Mesp-witeold & 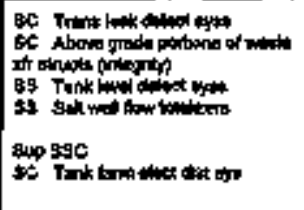 & 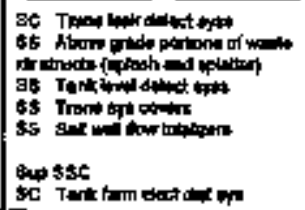 & 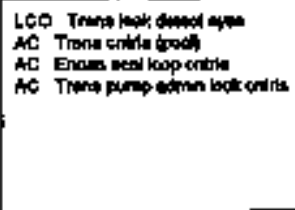 & 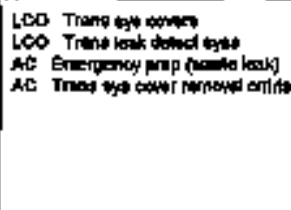 \\
\hline mosolntecoson & 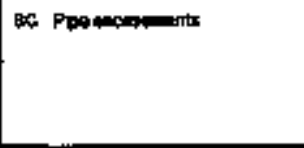 & 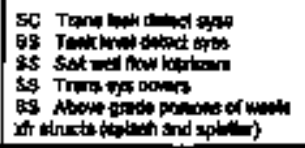 & 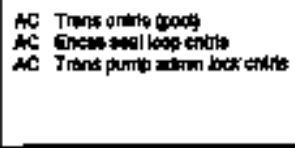 & 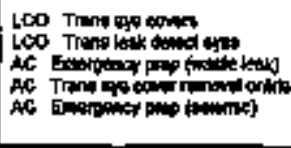 \\
\hline 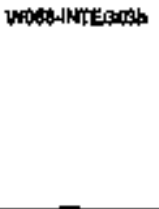 & 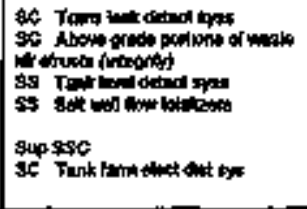 & 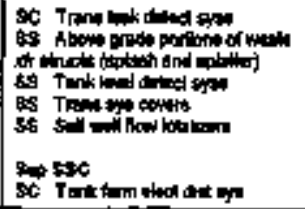 & 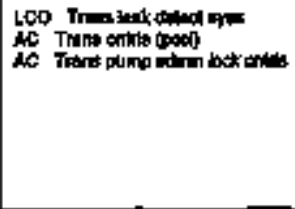 & 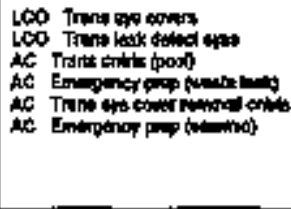 \\
\hline MQR-WTEDOFA & Shapen & Mand requod & 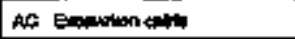 & 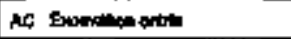 \\
\hline Mose-1NTEAs: & Lint & 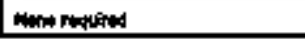 & 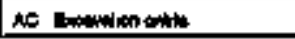 & 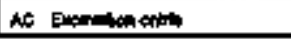 \\
\hline
\end{tabular}


Tubla A-4. Mastor Cortrol Table. (100 sheats)

\begin{tabular}{|c|c|c|c|c|}
\hline ID & Pnew SSC & Marsect & Pw TSR & Mt TSE \\
\hline norewtesofi & to Fpethomentit & 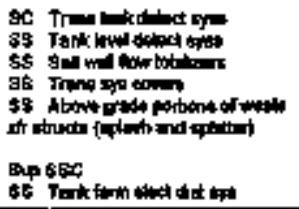 & 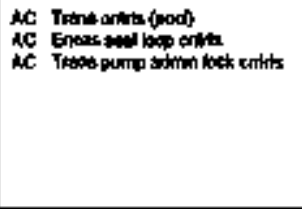 & 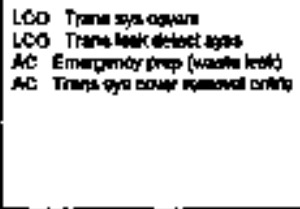 \\
\hline 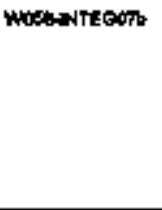 & 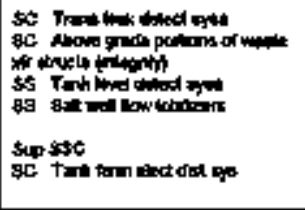 & 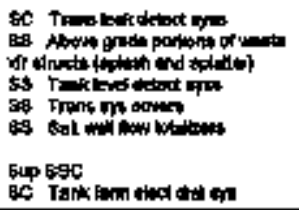 & 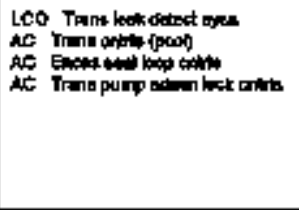 & 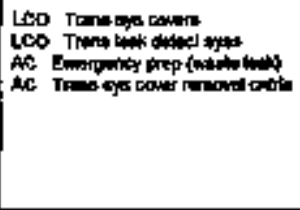 \\
\hline 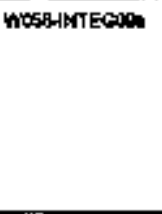 & SC Plpo prestammis & 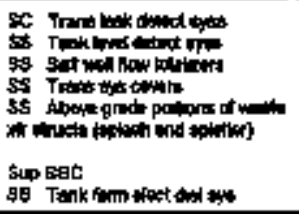 & 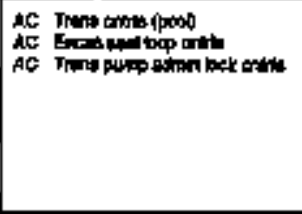 & 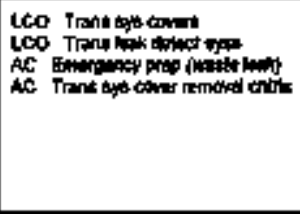 \\
\hline wostinneens & 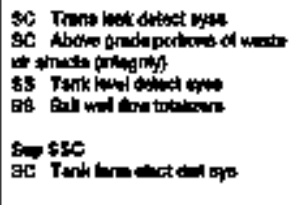 & 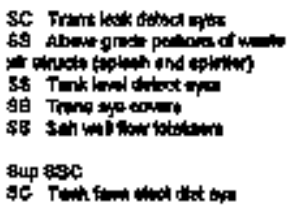 & 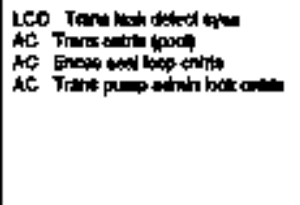 & 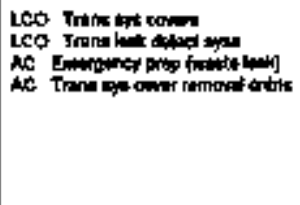 \\
\hline
\end{tabular}


Talo A-4. Woste Control Tabls. [101 Shats]

\begin{tabular}{|c|c|c|c|c|}
\hline ID & PTer BgC & Na \&OC & Praw TSR & MTt㫙 \\
\hline 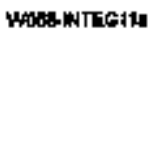 & 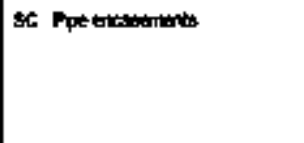 & 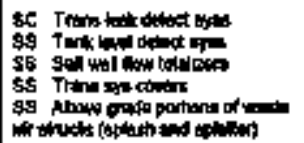 & 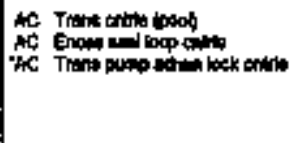 & 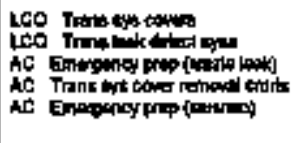 \\
\hline 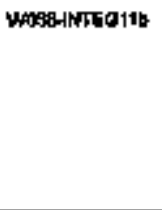 & 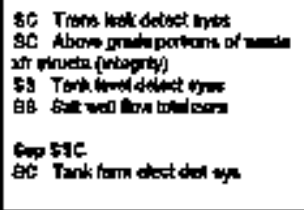 & 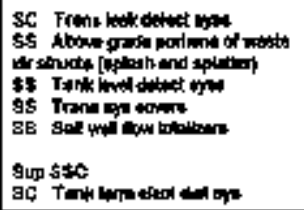 & 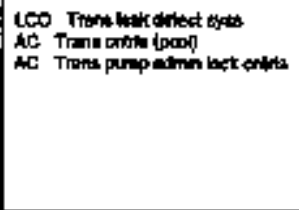 & 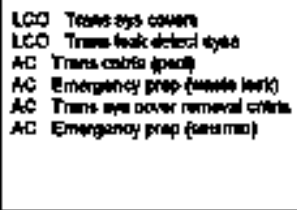 \\
\hline WOSSWIEAIF & 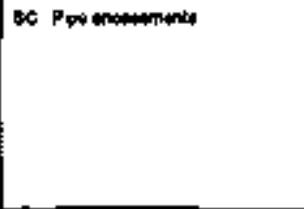 & 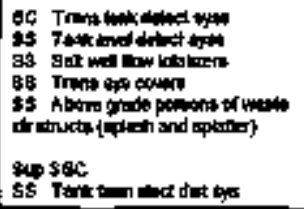 & 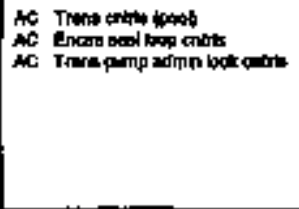 & 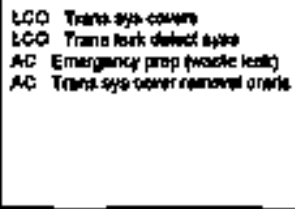 \\
\hline 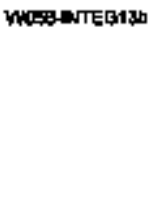 & 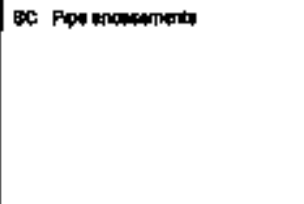 & 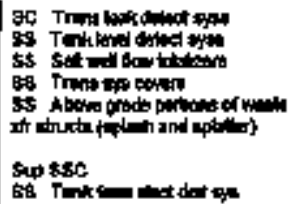 & 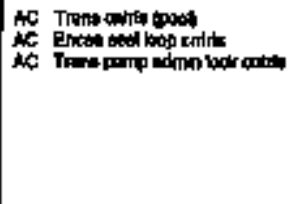 & 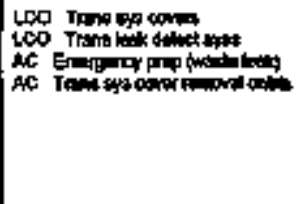 \\
\hline
\end{tabular}




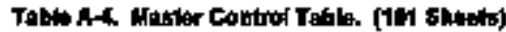

\begin{tabular}{|c|c|c|c|c|}
\hline $\mathbf{0}$ & Prow 500 & ate & PWu TRR & MAT TER \\
\hline whatelnTetion & 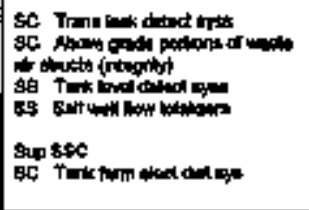 & 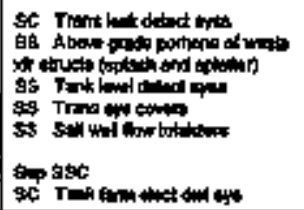 & 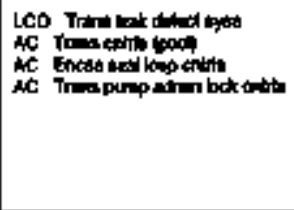 & 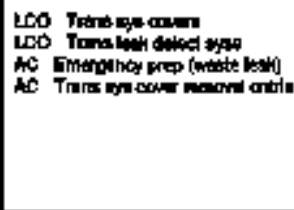 \\
\hline 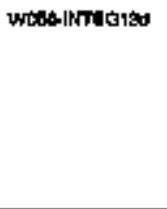 & 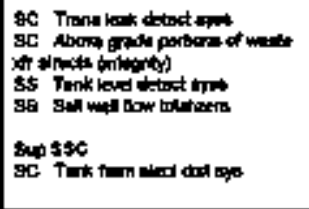 & 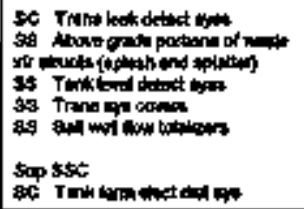 & 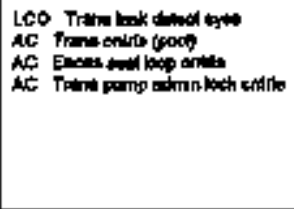 & 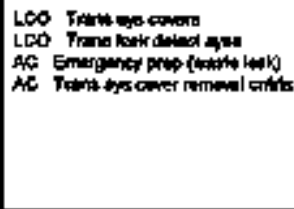 \\
\hline WOSP-WTEG| 4 & 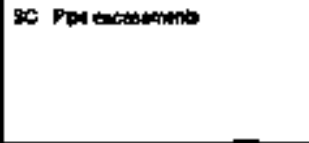 & 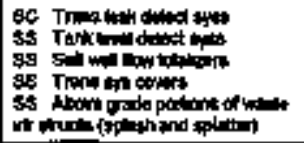 & 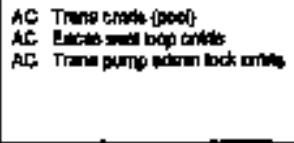 & 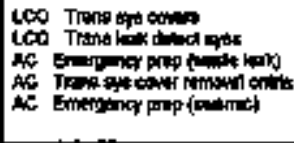 \\
\hline МОSP-MIEG & 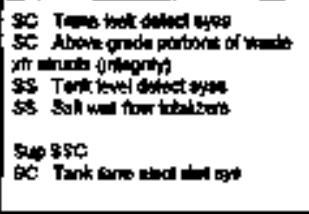 & 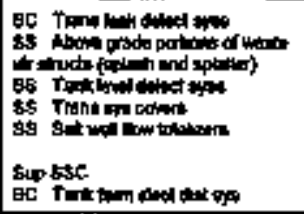 & 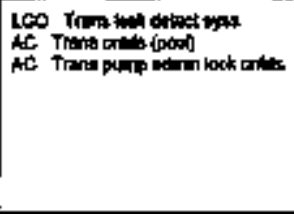 & 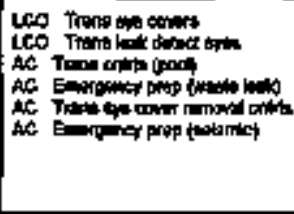 \\
\hline Whese-1NTEO10. & 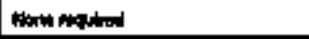 & 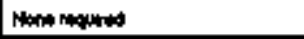 & to denaber & 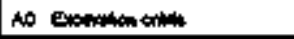 \\
\hline Whatentrose & 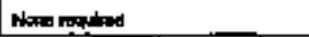 & 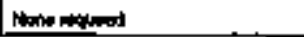 & 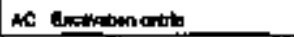 & NC \\
\hline
\end{tabular}




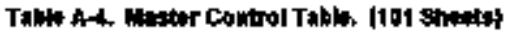

\begin{tabular}{|c|c|c|c|c|}
\hline 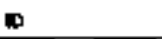 & Prur sqC & $4 \$ \$ 9 C$ & PMn T\$SA & M T甘木k \\
\hline W0se-NTEG & 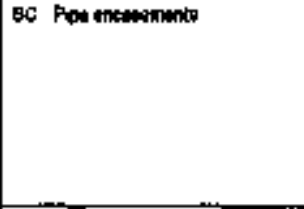 & 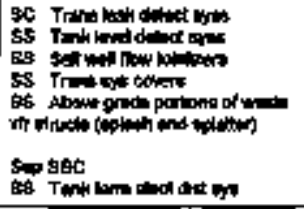 & 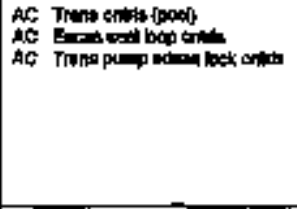 & 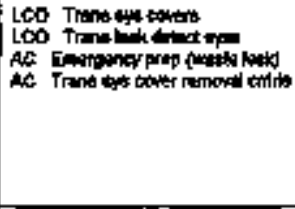 \\
\hline MOSPHNTEG16 & 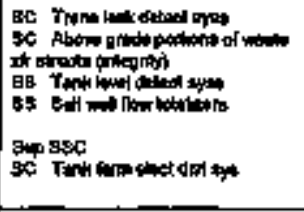 & 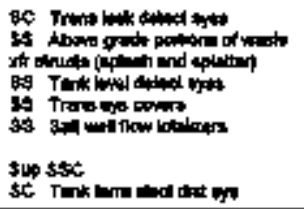 & 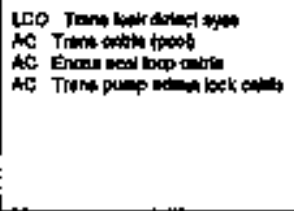 & 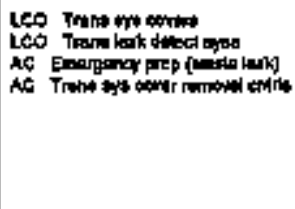 \\
\hline WOSPINTEGCAR & 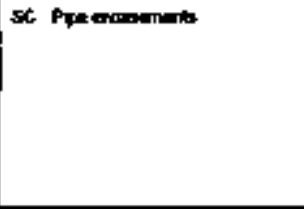 & 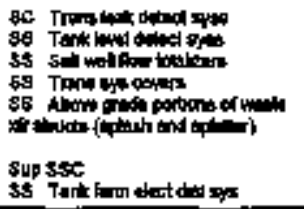 & 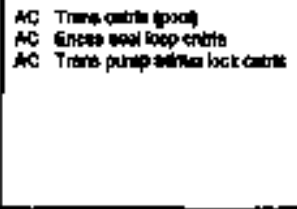 & 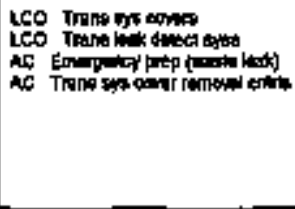 \\
\hline wots NTESTS & 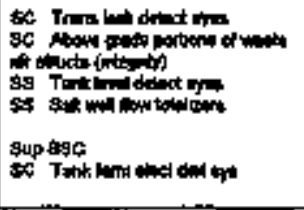 & 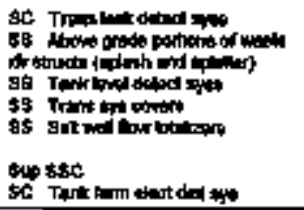 & 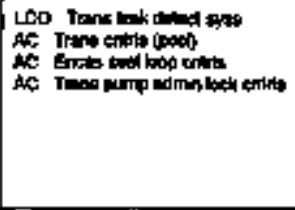 & 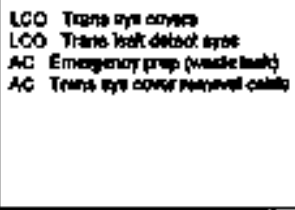 \\
\hline
\end{tabular}




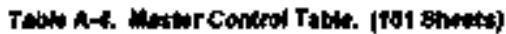

\begin{tabular}{|c|c|c|c|c|}
\hline $\mathbf{D}$ & Prar 的的 & in sqs & PTE TSR & in Tap \\
\hline nOSP-MTEC:2 & Hom now & 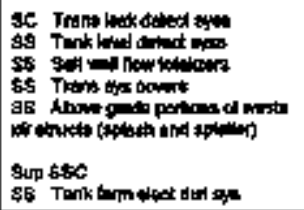 & 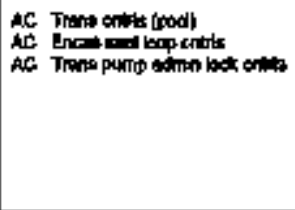 & 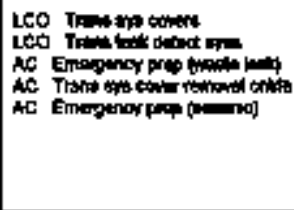 \\
\hline 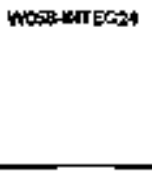 & 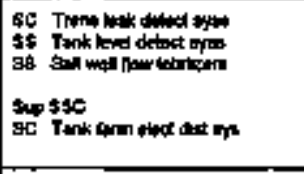 & 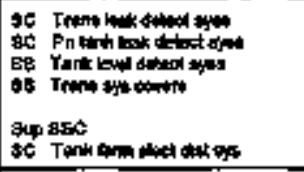 & 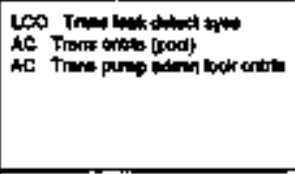 & 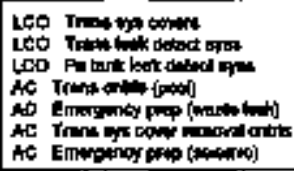 \\
\hline WosaHNITECNS & 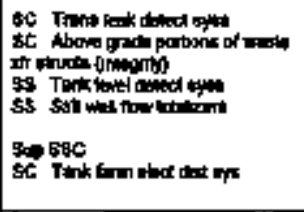 & 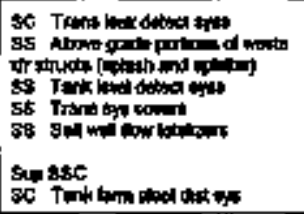 & 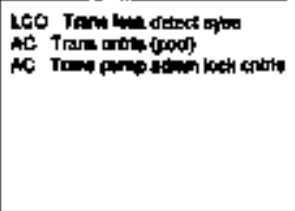 & 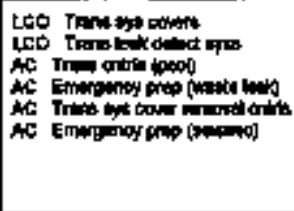 \\
\hline WOSS-NTEC?: & 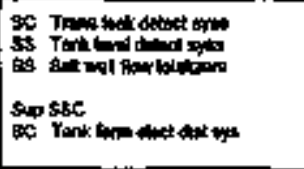 & 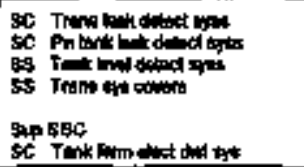 & 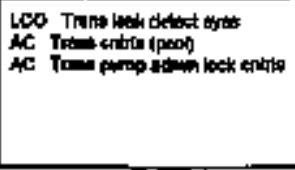 & 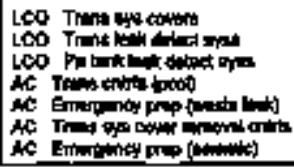 \\
\hline
\end{tabular}




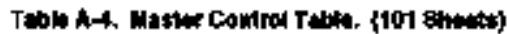

\begin{tabular}{|c|c|c|c|c|}
\hline D & Prop 日s & 네 多积 & Pror tisa & LW The \\
\hline WASB-WTIEESO & 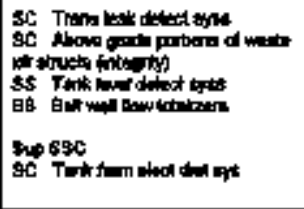 & 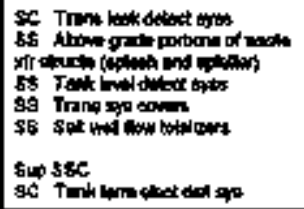 & 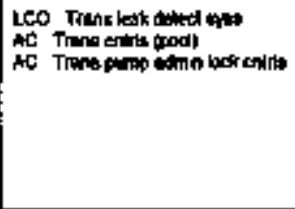 & 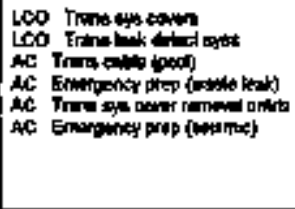 \\
\hline WOSS ANTECST & Nant & 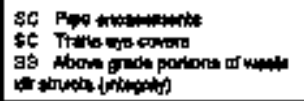 & 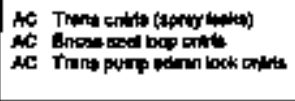 & 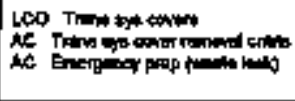 \\
\hline HESAHNTESSA & Nterin rapured & d & None rapured & NAth mand \\
\hline 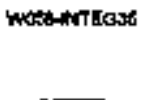 & None roqured & sc Thereseromers & 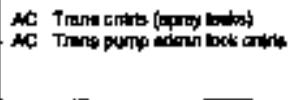 & 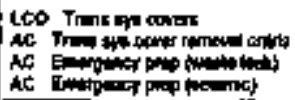 \\
\hline modearfiegsa & Nom noqumpd & Romeremperd & Nans moquind & Nene mant \\
\hline mos:-1Nmeas? & ntomingured & 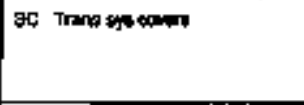 & 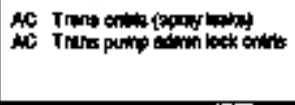 & 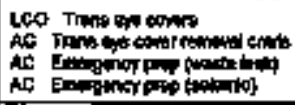 \\
\hline WASBPfaEsor & 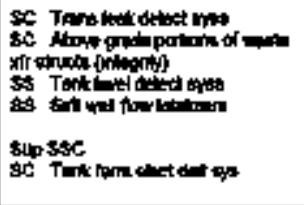 & 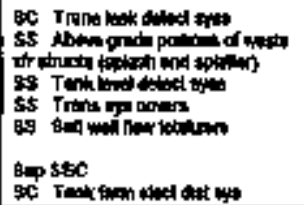 & 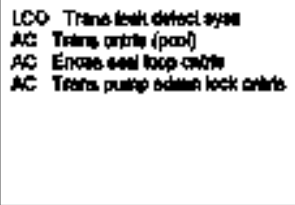 & 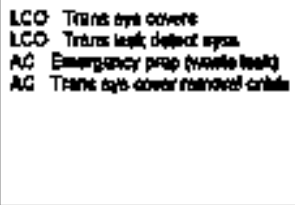 \\
\hline
\end{tabular}




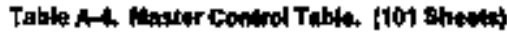

\begin{tabular}{|c|c|c|c|c|}
\hline 1 & Pur sse & ustect & Prm TSK & 情拍的 \\
\hline WTSBPRESO3 & 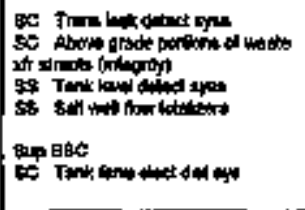 & 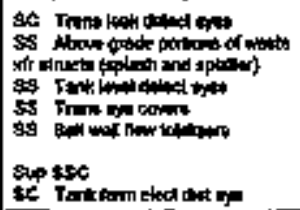 & 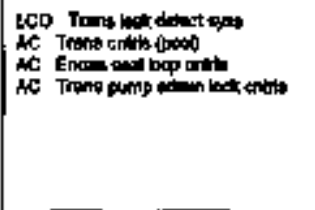 & 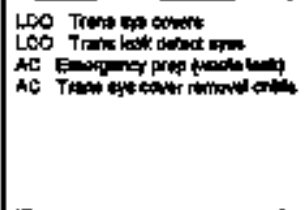 \\
\hline X\&-O1FLOWO1 & 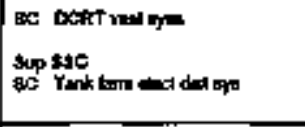 & Neneserapursed & 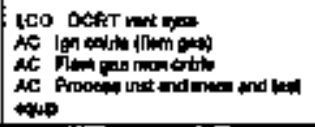 & 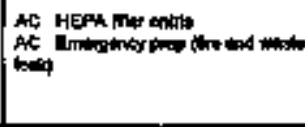 \\
\hline$x \leq-\infty n+10 w a x$ & 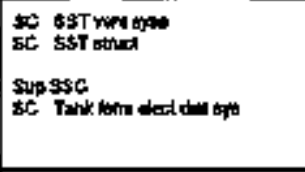 & 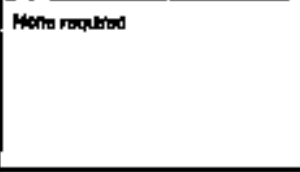 & 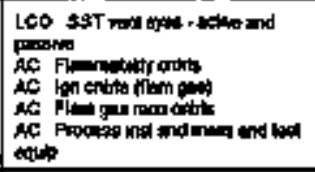 & 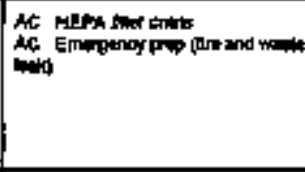 \\
\hline Xsaldnonits & 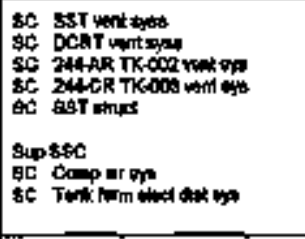 & 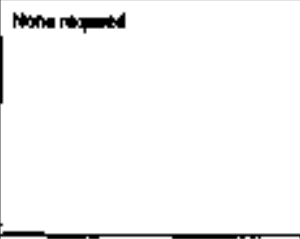 & 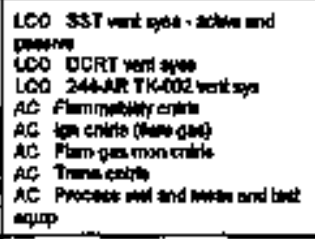 & 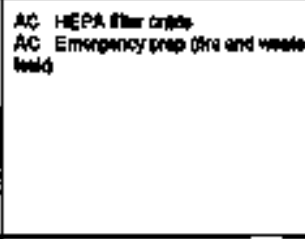 \\
\hline X8-OA-FLOWNOA & Nome molinet & 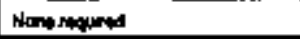 & 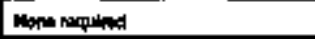 & M \\
\hline
\end{tabular}




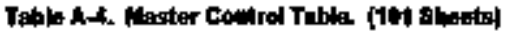

\begin{tabular}{|c|c|c|c|c|}
\hline $\mathbf{n}$ & 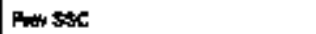 & Usse & Plw Tsin & MTsk \\
\hline$x$ obtflowhs & 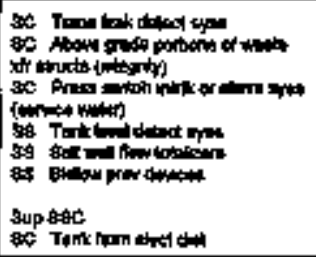 & 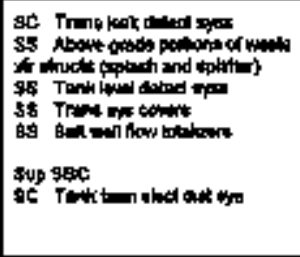 & 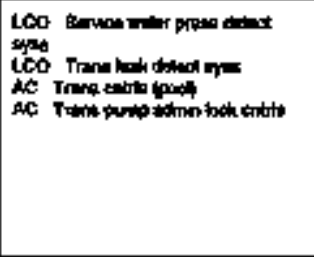 & 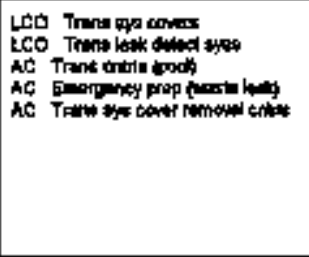 \\
\hline 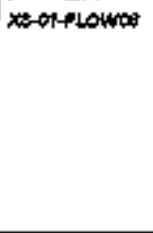 & 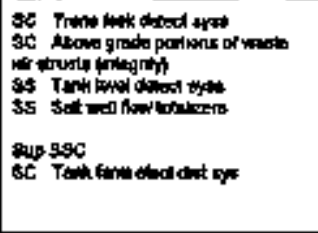 & 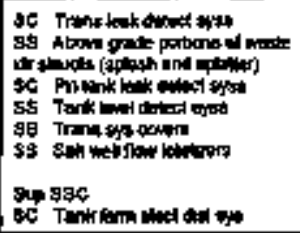 & 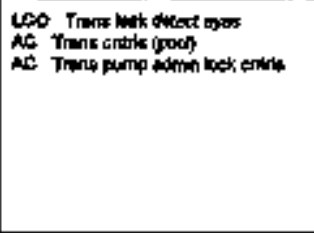 & 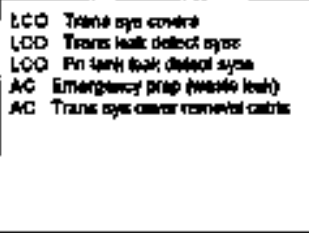 \\
\hline XB-A1-RLOURF & 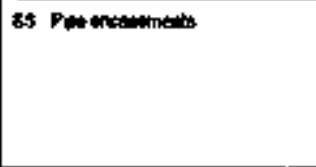 & 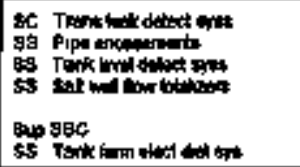 & 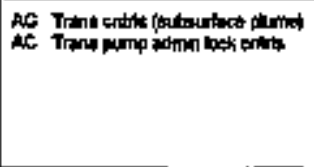 & 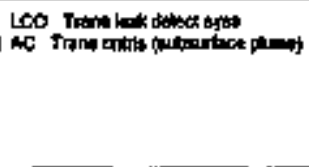 \\
\hline 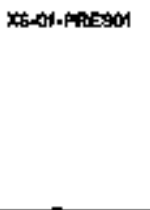 & 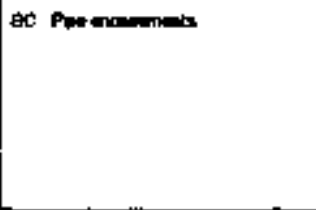 & 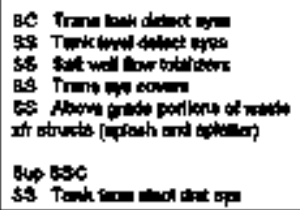 & 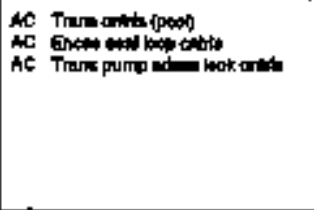 & 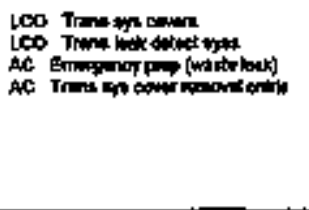 \\
\hline
\end{tabular}


Tahla A-A, Master Control Table. (10t Shopts)

\begin{tabular}{|c|c|c|c|c|}
\hline ID & Ann S9c & n & Daw Tak & MA TSR \\
\hline 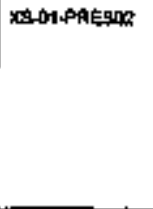 & Nane repuned & 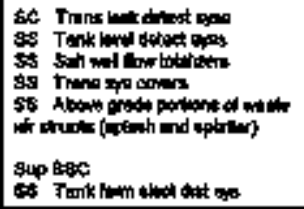 & 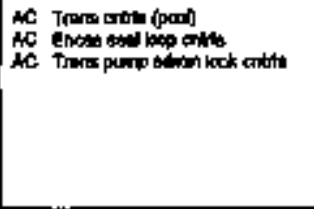 & 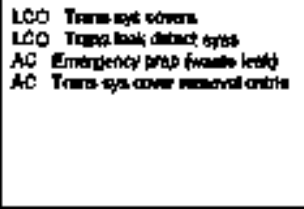 \\
\hline 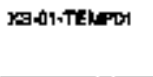 & Woes requined & 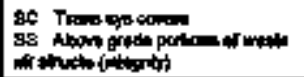 & 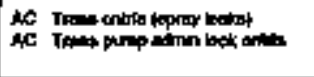 & 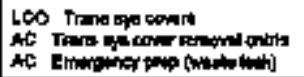 \\
\hline 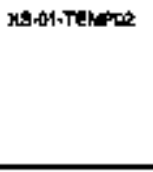 & Nower requand & 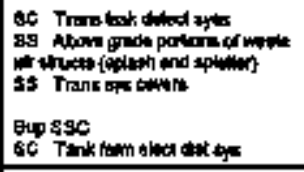 & AC Fon crite kposh & 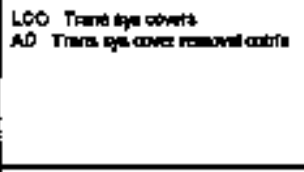 \\
\hline Xsomelome1 & Bhemanded & 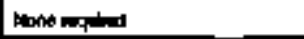 & Nomit angmed & 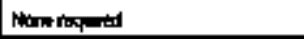 \\
\hline XB-LePIDnm & 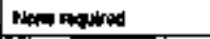 & Anne papand & Nhas rapiand & 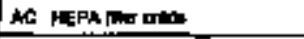 \\
\hline$x$ Xagflombs & 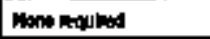 & 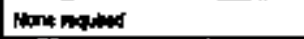 & 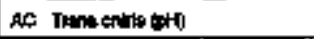 & Now \\
\hline X5:02FLOHAS & 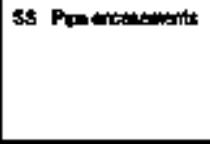 & 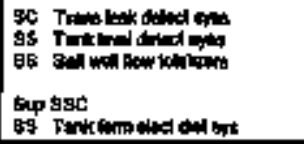 & 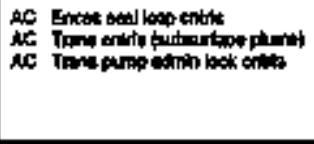 & 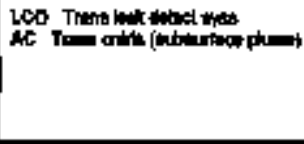 \\
\hline
\end{tabular}




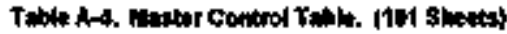

\begin{tabular}{|c|c|c|c|c|}
\hline फ & Pre 白的 & Mt $38 x$ & Prevtsin & 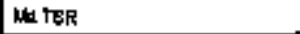 \\
\hline XS-02AlOANOS & 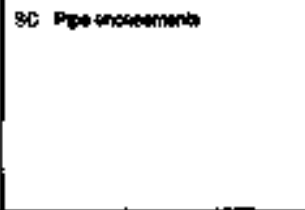 & 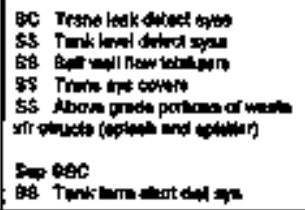 & 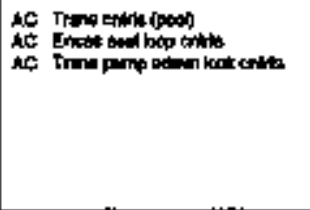 & 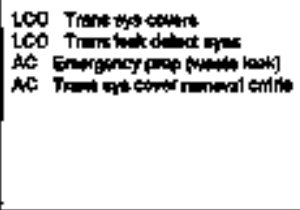 \\
\hline XB-0athonose & nowared & 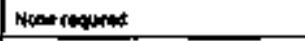 & 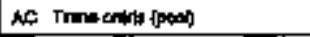 & 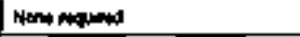 \\
\hline coshester & 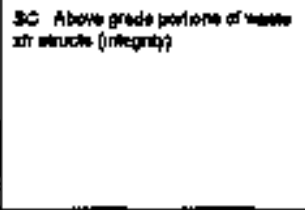 & 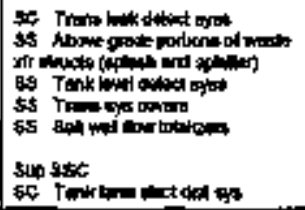 & Notion inunat & 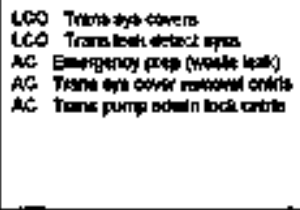 \\
\hline 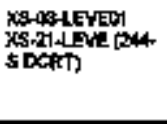 & 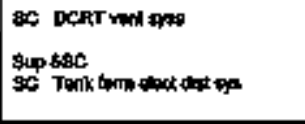 & Bones repures & 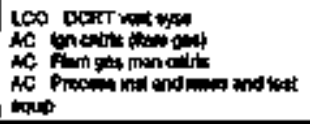 & 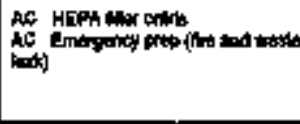 \\
\hline 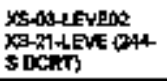 & 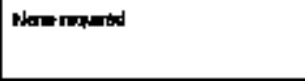 & Stane rapumed & 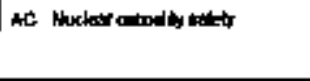 & Hone anperd \\
\hline X9.03-PAEEDOH & Monem inged & Wind rapent & Nane nequinted & Won mequard \\
\hline 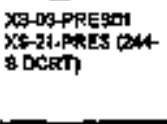 & 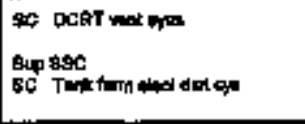 & 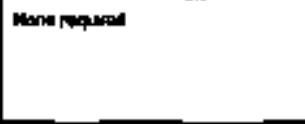 & 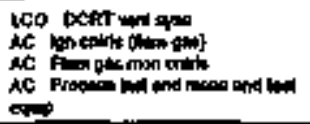 & AC HDa \\
\hline
\end{tabular}




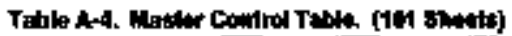

\begin{tabular}{|c|c|c|c|c|}
\hline ID & Prop ogec & $\cos \operatorname{sis} c$ & Prew Tsp & Mt TER \\
\hline 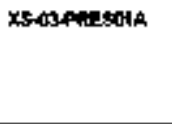 & Nom & 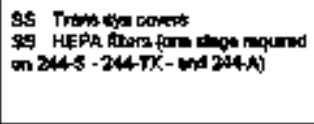 & 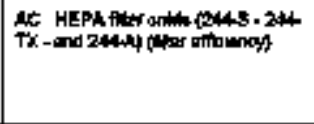 & 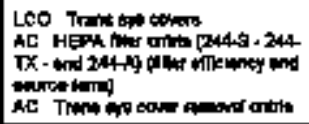 \\
\hline 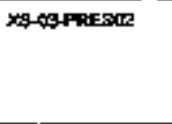 & None requendid & 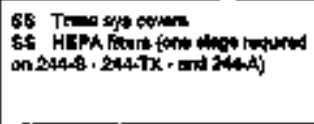 & 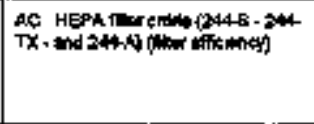 & 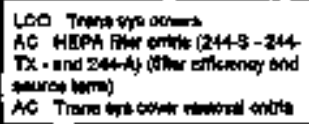 \\
\hline 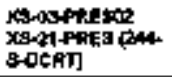 & 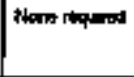 & rene required & Ac Transqude(pH) & thene requed \\
\hline Xs-00-10Fios & Honn raperd & 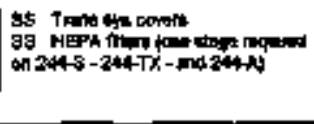 & 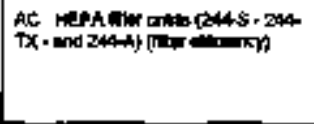 & 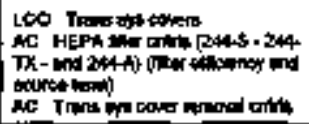 \\
\hline 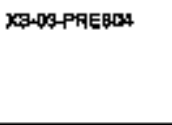 & 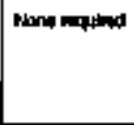 & 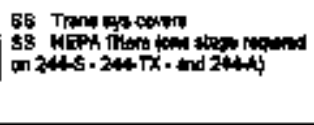 & 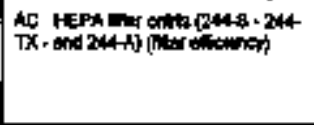 & 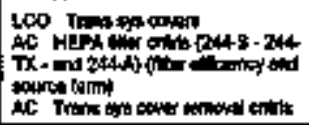 \\
\hline XSAOAPRESOS & 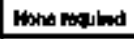 & Nand repand & Pore nacions & Hallempind \\
\hline )(t5-03-TEKAOA & monengend & 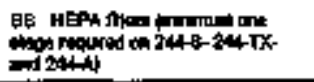 & 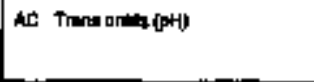 & AC HEPA \\
\hline 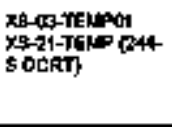 & Mone nequind & 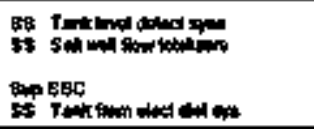 & Mane noqued & 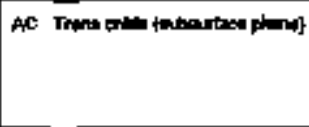 \\
\hline 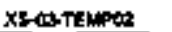 & Wone matw & Nane ropaten & Mum roquitod & 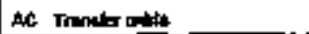 \\
\hline
\end{tabular}




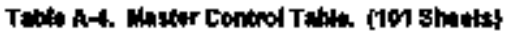

\begin{tabular}{|c|c|c|c|c|}
\hline $\mathbf{0}$ & Parssc & Msgc & Partop & At TgR \\
\hline 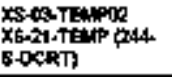 & 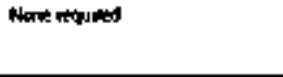 & | & 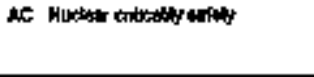 & 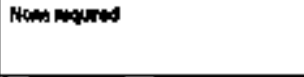 \\
\hline 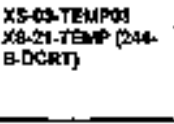 & 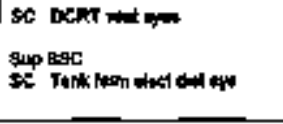 & Mand ingand & 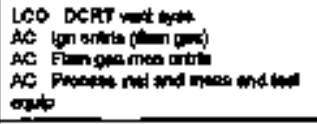 & 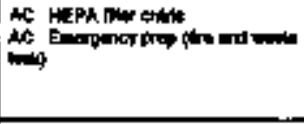 \\
\hline xh-m-TEAPQ4 & 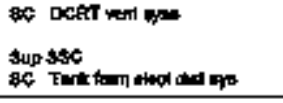 & 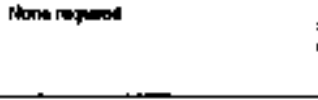 & 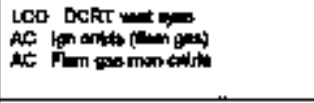 & Non maped \\
\hline 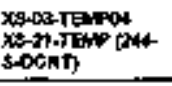 & 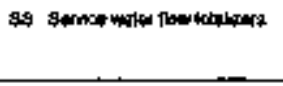 & 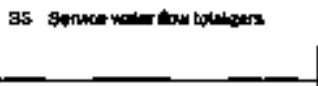 & 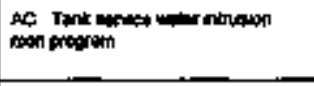 & 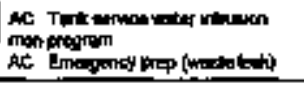 \\
\hline XS-03-Tgeros & Mone roqumed & 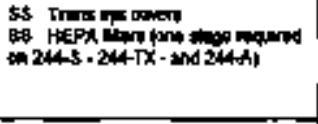 & 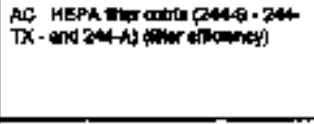 & 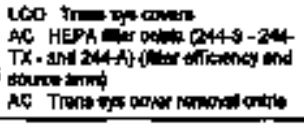 \\
\hline 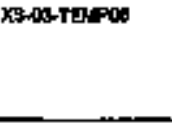 & 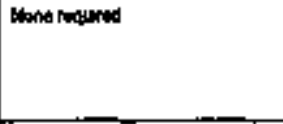 & 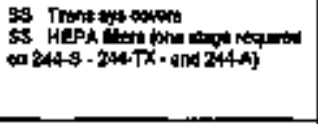 & 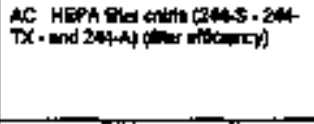 & 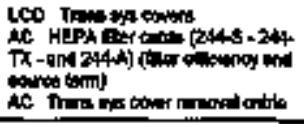 \\
\hline 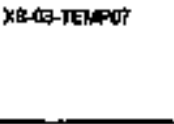 & Home repurad & 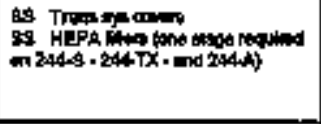 & 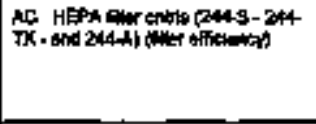 & 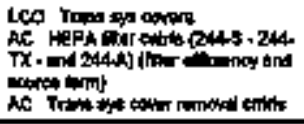 \\
\hline 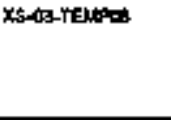 & None requind & 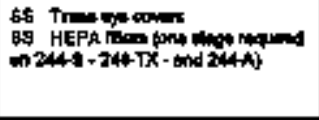 & 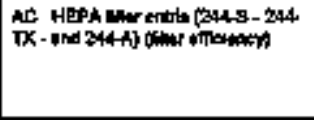 & 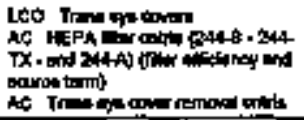 \\
\hline
\end{tabular}




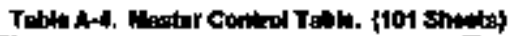

\begin{tabular}{|c|c|c|c|c|}
\hline $\mathbf{0 0}$ & Pmen \$sc & $m$ Fec & PMw TER & s TSA \\
\hline MES-01-TELPES & Ntation requend & 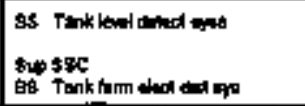 & Howe requitiod & 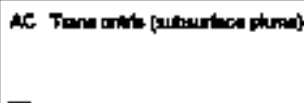 \\
\hline XB-OSF, Dyner & 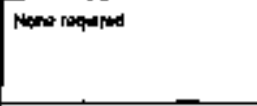 & 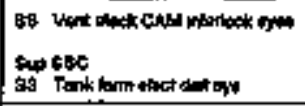 & 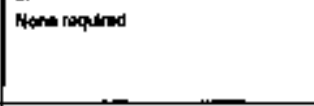 & 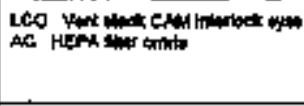 \\
\hline XB-05FLOWN2 & Nemen manum & 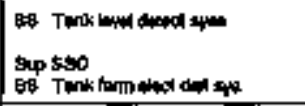 & None requerd & 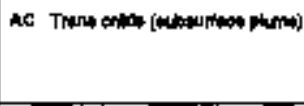 \\
\hline \% & nomo animiod & 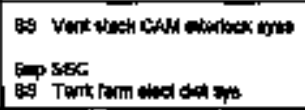 & 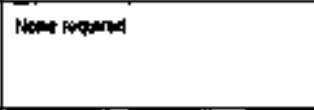 & 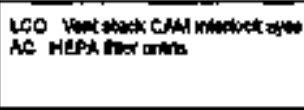 \\
\hline Xs-as-TenPan & 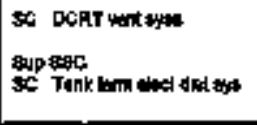 & 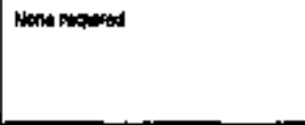 & 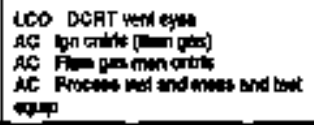 & 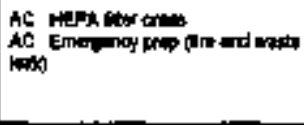 \\
\hline 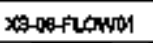 & Mons nouind & 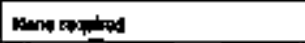 & 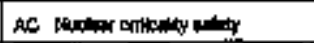 & lane ropurad \\
\hline Xo-46-704020 & Nono nownd & 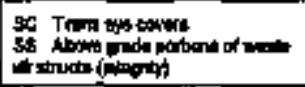 & 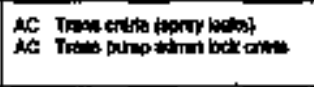 & 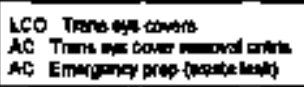 \\
\hline X6.06-7lown: & 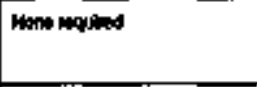 & 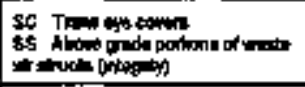 & 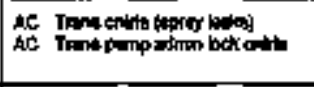 & 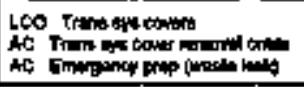 \\
\hline 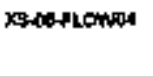 & Mino inumed & 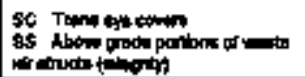 & 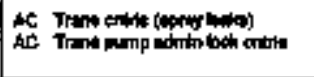 & 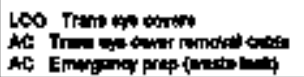 \\
\hline
\end{tabular}




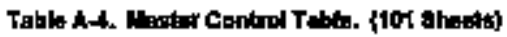

\begin{tabular}{|c|c|c|c|c|}
\hline ID & Prw SSC & im stc & Pow Tg: & wh Ts: \\
\hline xodeplonins & 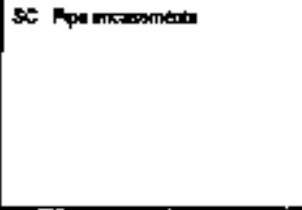 & 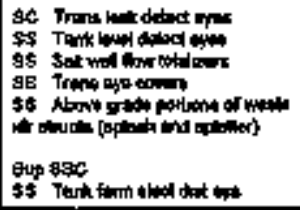 & 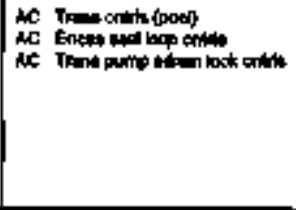 & 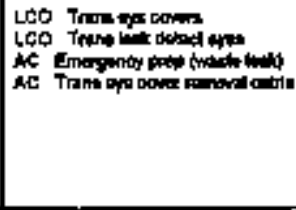 \\
\hline 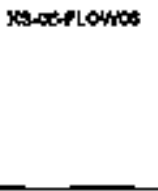 & 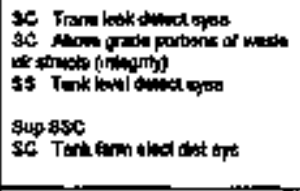 & 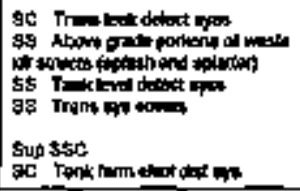 & 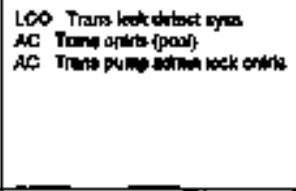 & 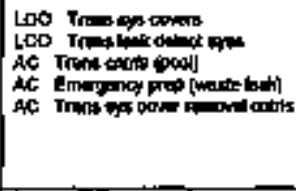 \\
\hline XB-0T-7LONDI & 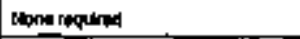 & Nom repurad & 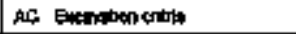 & AC Eucunown orith \\
\hline xB-07-7and & 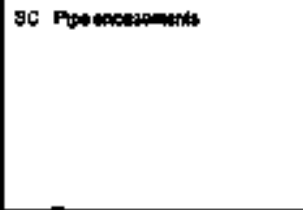 & 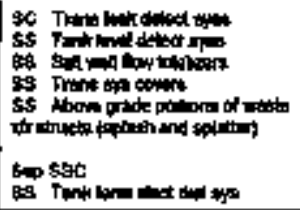 & 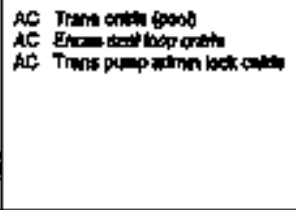 & 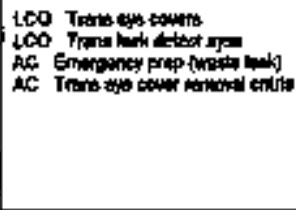 \\
\hline$x \&-6+700$ & Named & 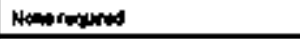 & 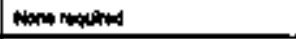 & 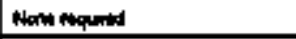 \\
\hline NESAr-LOHOA & 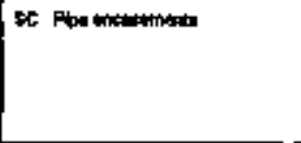 & 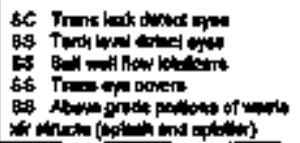 & 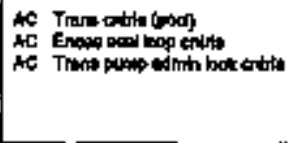 & 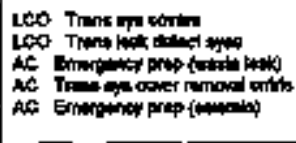 \\
\hline
\end{tabular}




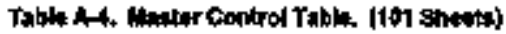

\begin{tabular}{|c|c|c|c|c|}
\hline is & Prw stc & $w \operatorname{ssc}$ & Phe Tsa & NMtst \\
\hline 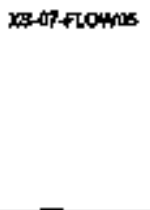 & scic Ppoweasmenth & 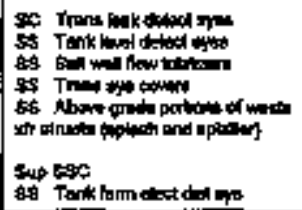 & 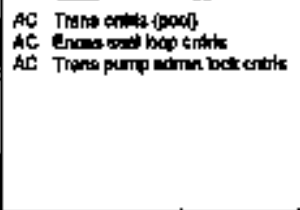 & 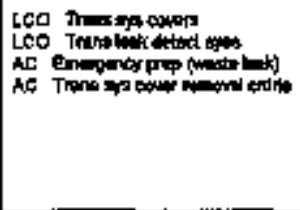 \\
\hline X8-ot Flownos & 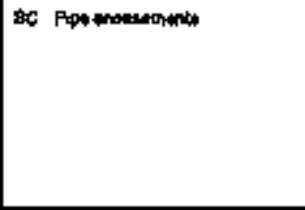 & 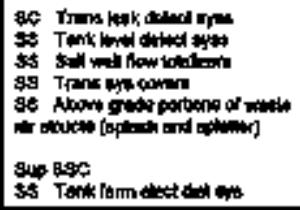 & 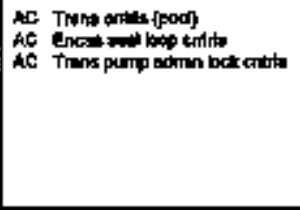 & 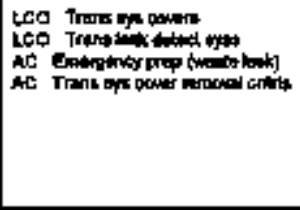 \\
\hline 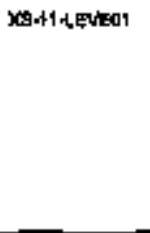 & 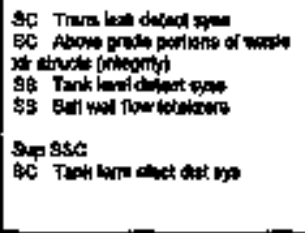 & 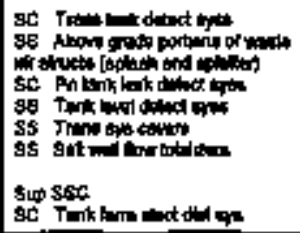 & 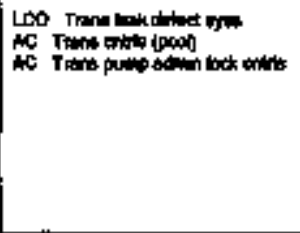 & 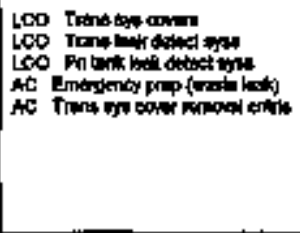 \\
\hline XB-11-LEMEO2 & 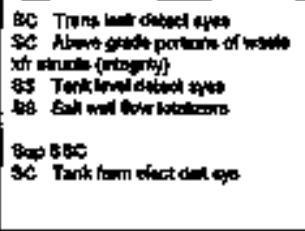 & 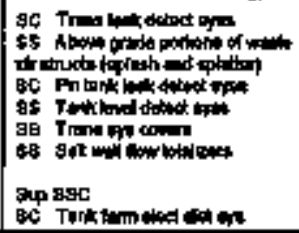 & 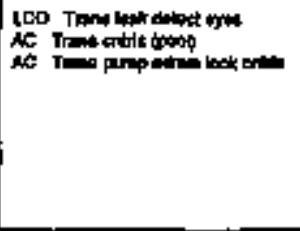 & 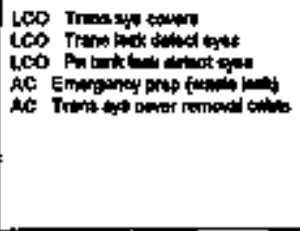 \\
\hline
\end{tabular}




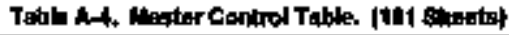

\begin{tabular}{|c|c|c|c|c|}
\hline 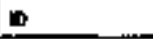 & Prew SSC & MSSC & Pin Tst & IE TSR \\
\hline XE-11-LEVEOG & 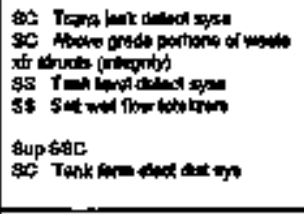 & 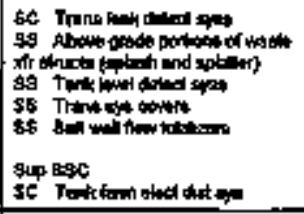 & 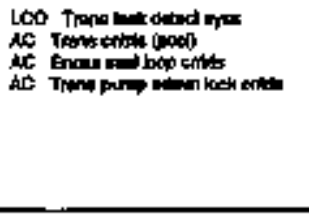 & 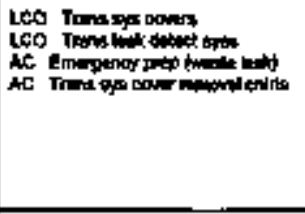 \\
\hline Xs-12flochuat & Done requared & 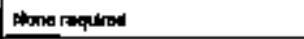 & 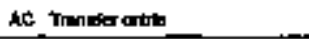 & Nontanged \\
\hline$x$ S-12-7LCHAR & Mana rapurad & Utwe requered & 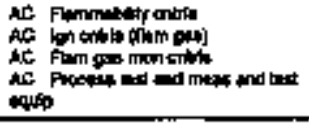 & 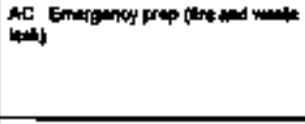 \\
\hline Ms.l2fFlonts & None requed & Nowe reaped & 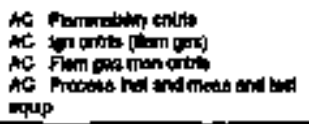 & 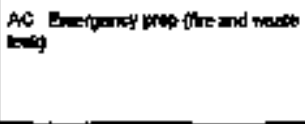 \\
\hline$x$ X3-12.7umat & mone noquarad & Alone rapinges & 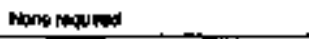 & 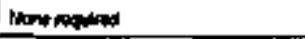 \\
\hline XF-12-rlones & 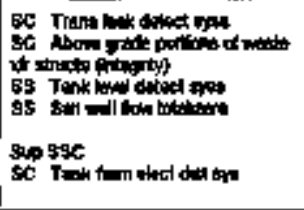 & 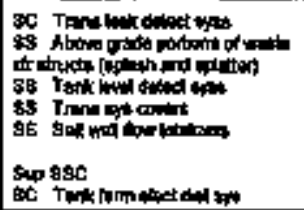 & 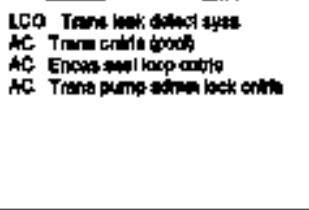 & 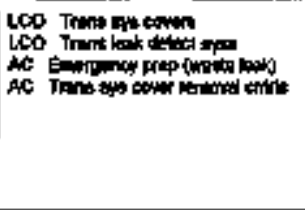 \\
\hline
\end{tabular}




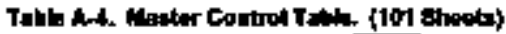

\begin{tabular}{|c|c|c|c|c|}
\hline IQ & Puor sBC & in ssec & PAn TBR & 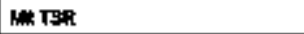 \\
\hline 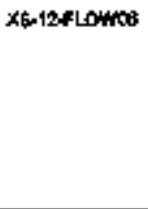 & 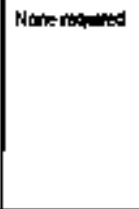 & 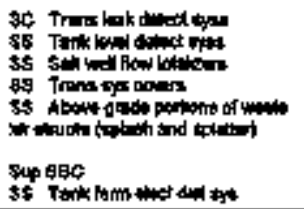 & 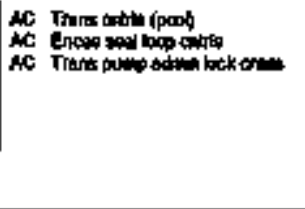 & 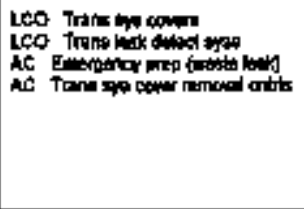 \\
\hline 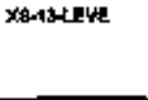 & S & 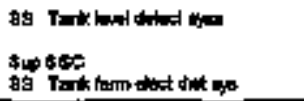 & 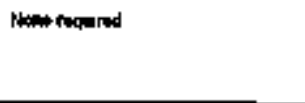 & 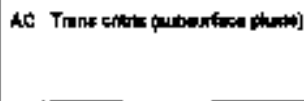 \\
\hline XS-TSPAESOT & None pequted & Non raparent & Ac Trematratith & Mantenqumed \\
\hline$X E$-13-PRE 302 & mone mqured & Non raperad & 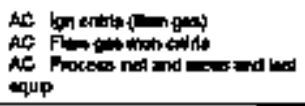 & 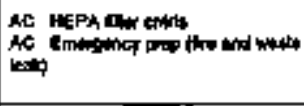 \\
\hline$X:$-13-PREBC3 & Mant anpand & 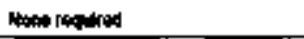 & AC Thonator ention & Nono rapured \\
\hline 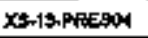 & nom reponted & Aant resul & 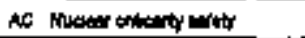 & 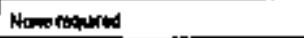 \\
\hline 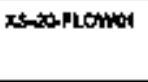 & Atone folduras & 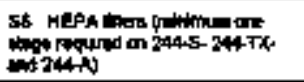 & 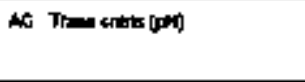 & 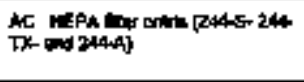 \\
\hline$x \leq x$-PLONIR & 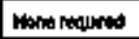 & None raped & act Natestits & Nanermquitiod \\
\hline 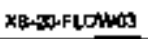 & Mone rocket & 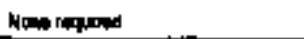 & 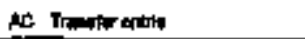 & 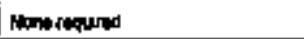 \\
\hline XSsoflomos & Nomine requinat & Nen newures & AC Trumain oflo & 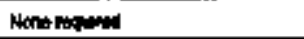 \\
\hline xaraponos & Hone nequified & Nant & 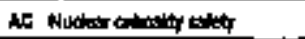 & 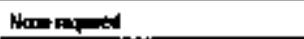 \\
\hline 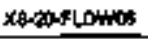 & Nomenompitied & Nomatomand & AC. Trana artis $(0 \mathrm{pod})$ & 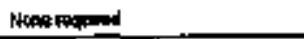 \\
\hline 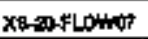 & Minin napured & Heng reavion & 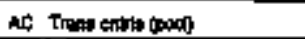 & Nomercaptiad \\
\hline
\end{tabular}




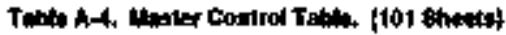

\begin{tabular}{|c|c|c|c|c|}
\hline ID & Pinstser & Nowso & Prer Tot & Mt Ts: \\
\hline XO-XFLOWD:8 & 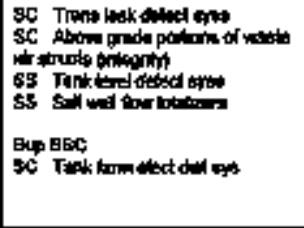 & 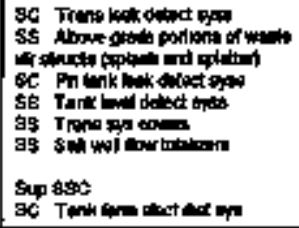 & 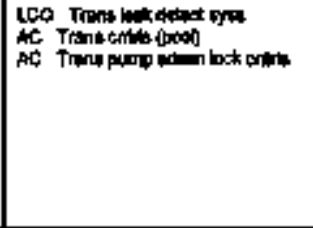 & 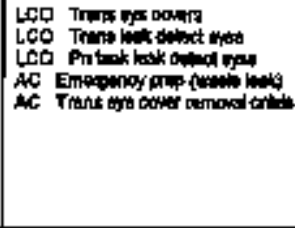 \\
\hline xtot-3rocomos & 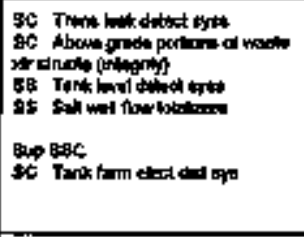 & 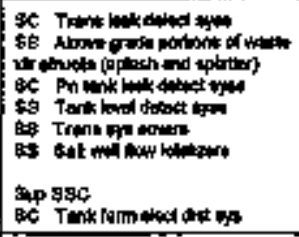 & 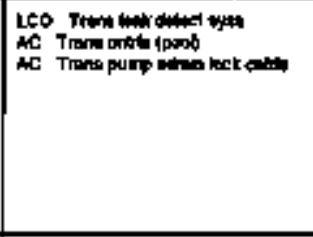 & 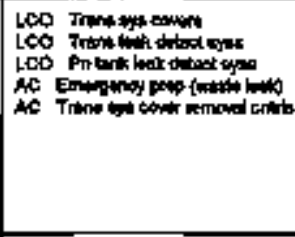 \\
\hline 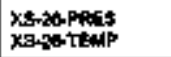 & d & 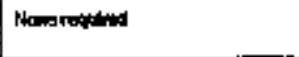 & 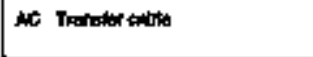 & 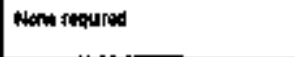 \\
\hline 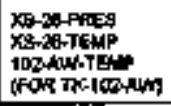 & 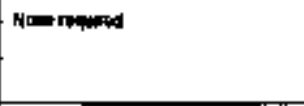 & 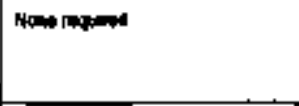 & 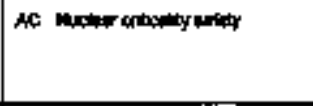 & Nacen maquid \\
\hline$x=\angle \theta P A=1001$ & Amo panded & Hont resulad & 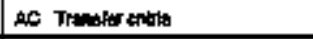 & nim foposd \\
\hline 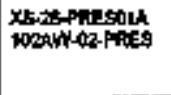 & 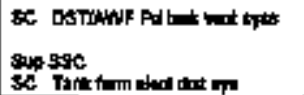 & 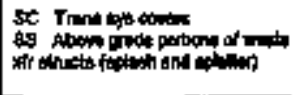 & 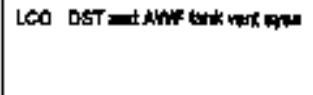 & 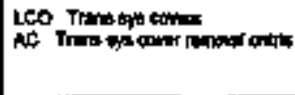 \\
\hline
\end{tabular}




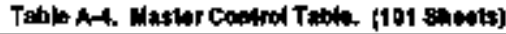

\begin{tabular}{|c|c|c|c|c|}
\hline ID & Phe \$sc & $\ln \leqslant 0$ & Dren ragk & Mtt TEA \\
\hline LS-26PAEF:OA & 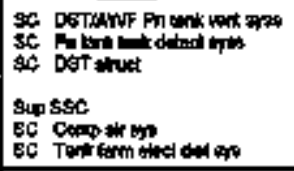 & Mans reapired & 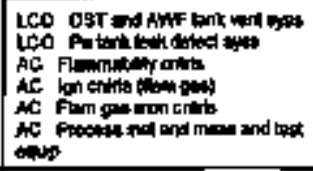 & 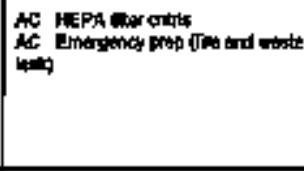 \\
\hline 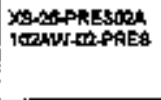 & 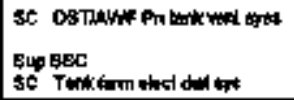 & 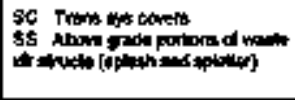 & 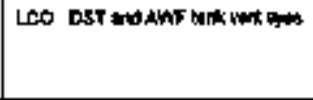 & 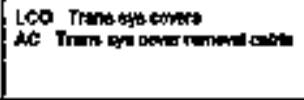 \\
\hline 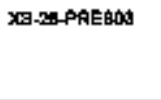 & 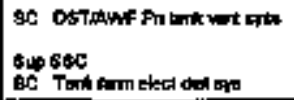 & 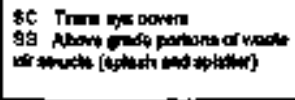 & 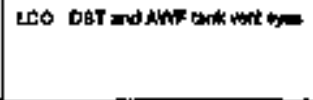 & 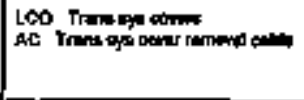 \\
\hline 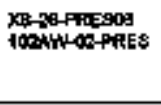 & 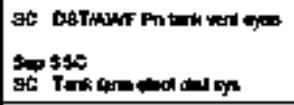 & 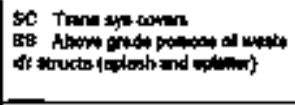 & 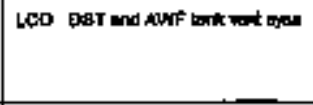 & 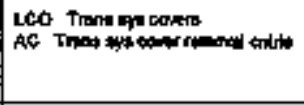 \\
\hline 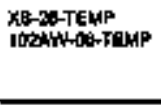 & 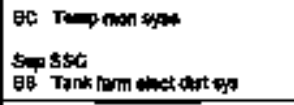 & Non rapead & 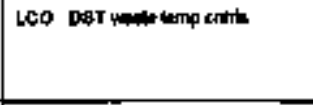 & 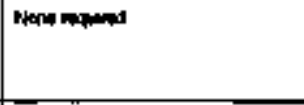 \\
\hline XEAATEMPG & 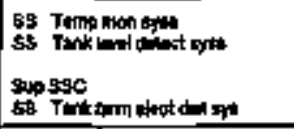 & 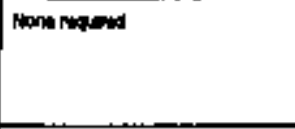 & 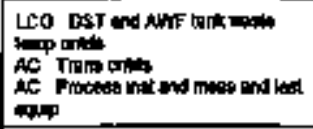 & 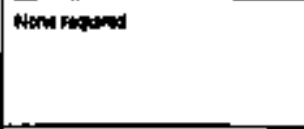 \\
\hline$x \cos \theta-\tan 20$ & 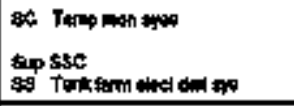 & Hom rowures & 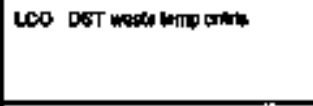 & Meno nowked \\
\hline xto-so- & 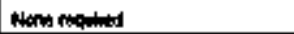 & 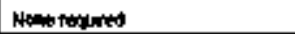 & 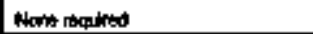 & Naind \\
\hline
\end{tabular}




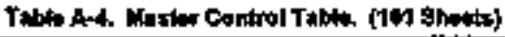

\begin{tabular}{|c|c|c|c|c|}
\hline $\mathbf{p}$ & Prep \$sc & $\ln 68 \mathrm{c}$ & Pan T\$ฺ & MEN TSF \\
\hline XS-5FFLOAHE & 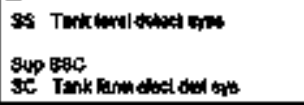 & 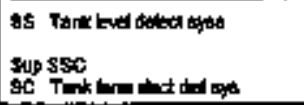 & 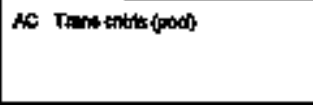 & 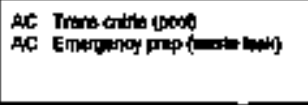 \\
\hline X6afr+exchups & 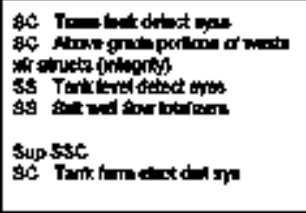 & 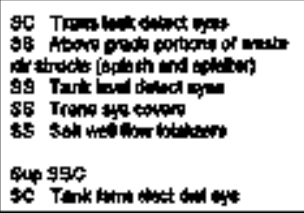 & 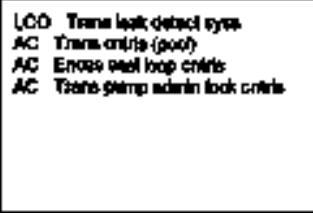 & 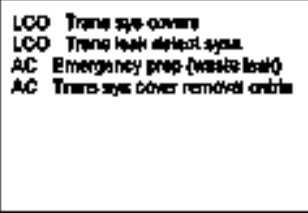 \\
\hline no-s7flowns & S\$ & 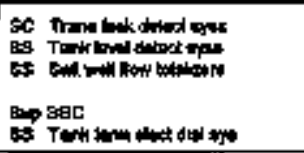 & 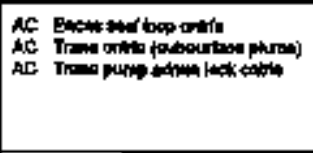 & 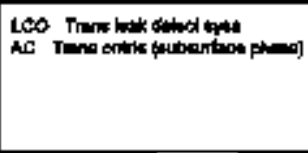 \\
\hline XB-A1A-mony & 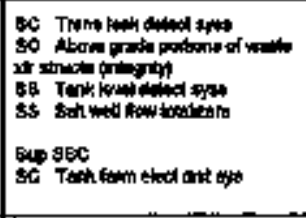 & 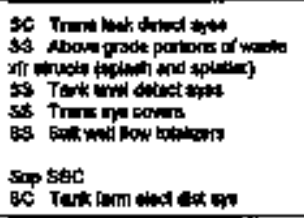 & 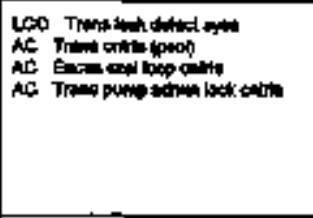 & 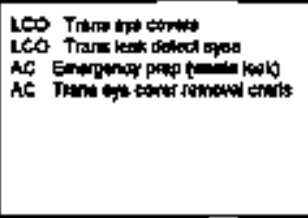 \\
\hline XB-A1A-FLOMDO & 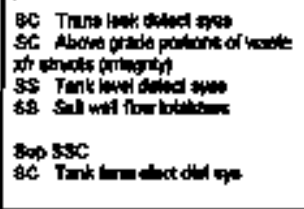 & 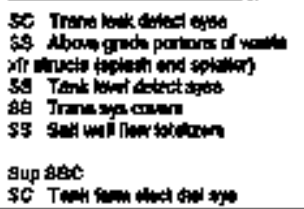 & 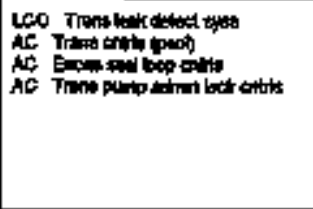 & 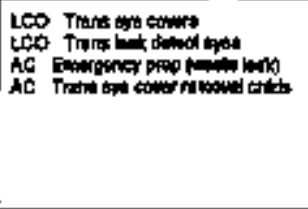 \\
\hline
\end{tabular}


Table A-4. Hatter Cortrol Table. (101 Shats)

\begin{tabular}{|c|c|c|c|c|}
\hline 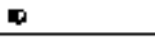 & Prar \$क्ष & ut \$sc & PTer TSR & netsin \\
\hline XS-A1BHELEDI & 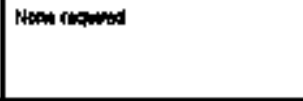 & Nenoropurad & 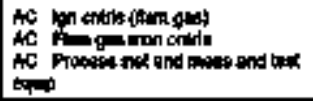 & 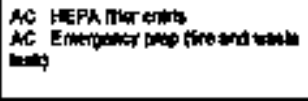 \\
\hline 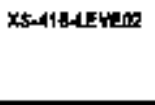 & 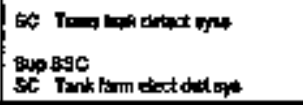 & Haneringred & 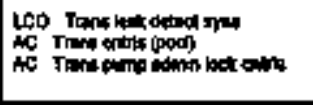 & 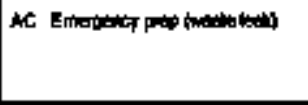 \\
\hline 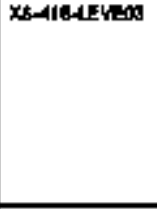 & 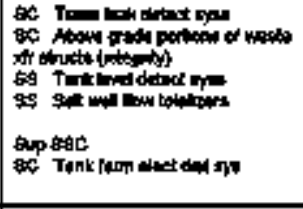 & 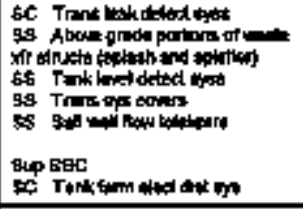 & 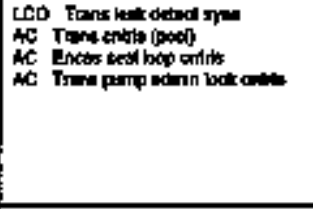 & 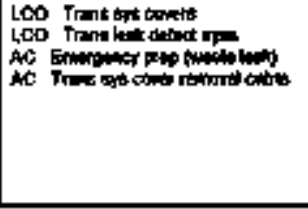 \\
\hline$X s-410+1 E 5 E-4$ & 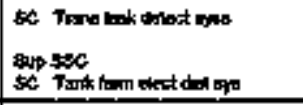 & Hom nowion & 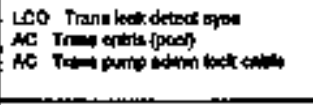 & 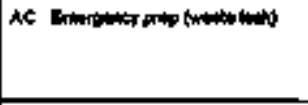 \\
\hline 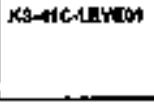 & Neno naphed & Mone requped & 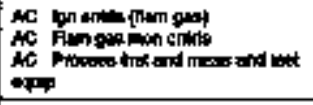 & 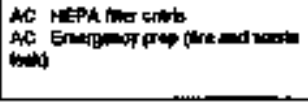 \\
\hline XSA41CHEVERI & Him noqured & Nonen raquind & 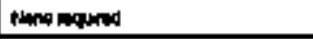 & mon raperd \\
\hline$X B-41 C-1 E Y=03$ & 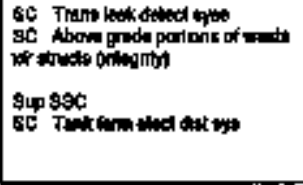 & 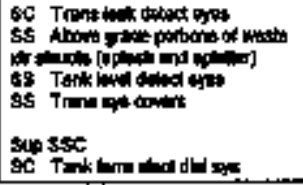 & 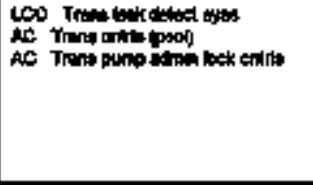 & 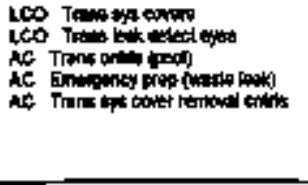 \\
\hline
\end{tabular}




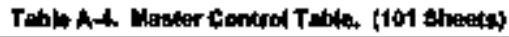

\begin{tabular}{|c|c|c|c|c|}
\hline ID & Prm घБट & Bis ssc & Ht TSR & Mt ISR \\
\hline x\$-46-rion & 58 Ppomaniminata & 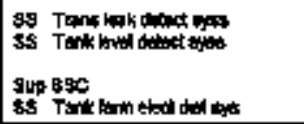 & 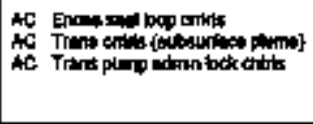 & 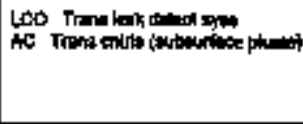 \\
\hline istostrumal & 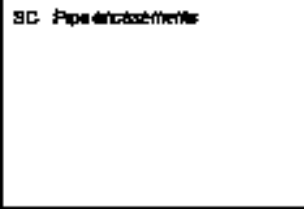 & 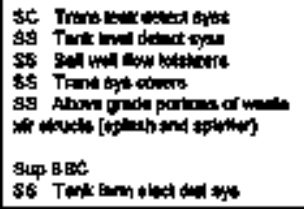 & 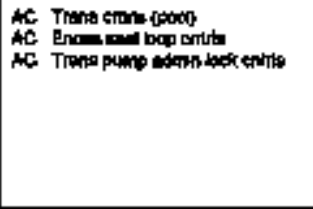 & 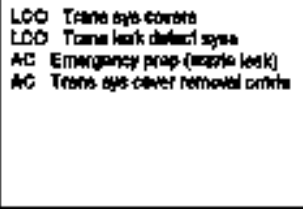 \\
\hline XV- & 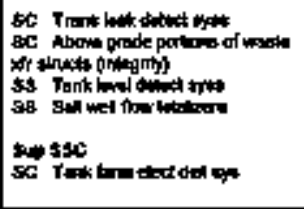 & 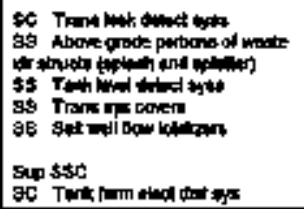 & 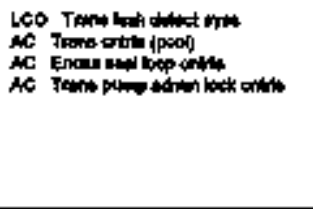 & 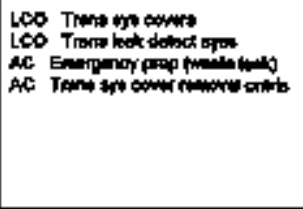 \\
\hline
\end{tabular}


HNF-SC-MAS-TT-7BA, REV. 1

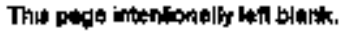




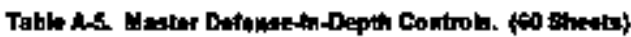

\begin{tabular}{|c|c|c|}
\hline 由 & Doropminitin & 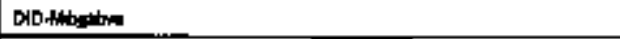 \\
\hline 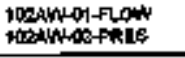 & 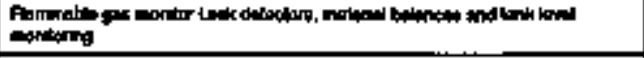 & Nare \\
\hline 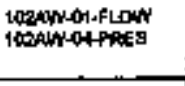 & Leth intho of ment & 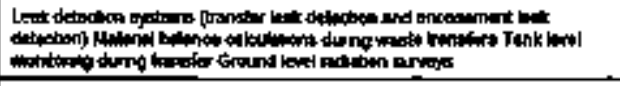 \\
\hline 1GEUM-OM.PLONOH & 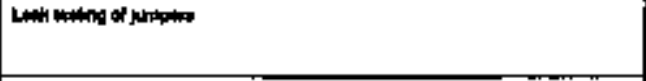 & 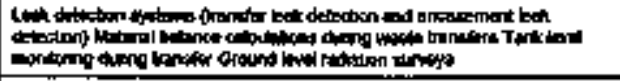 \\
\hline 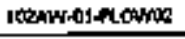 & Hors: & 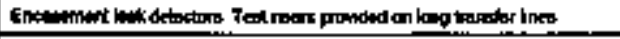 \\
\hline ILGHNAI+LOWHE & Nente & 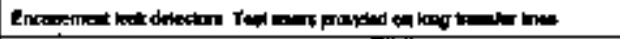 \\
\hline 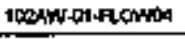 & Mare & 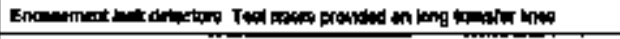 \\
\hline 1004h-OHARON06 & Nare & 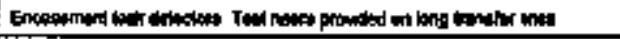 \\
\hline mednstolnoway & Noing & 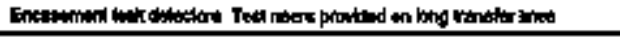 \\
\hline 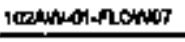 & Nions & 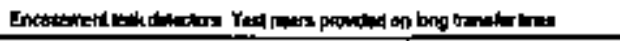 \\
\hline $102 \times 4 \times 01+F[$ LWN10 & 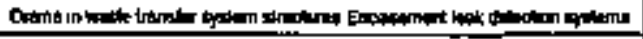 & 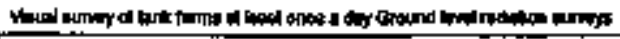 \\
\hline 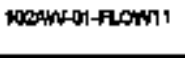 & 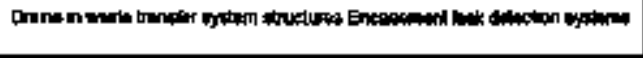 & 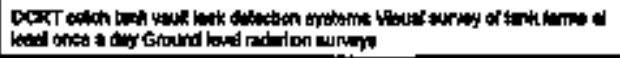 \\
\hline 1QDANDOH-TEMP & Not & 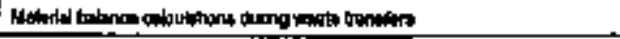 \\
\hline 102AW-O1-TÉMPOS & 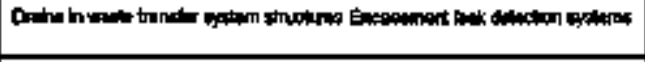 & 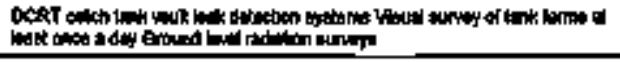 \\
\hline 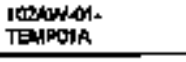 & 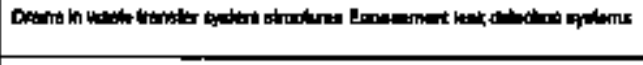 & 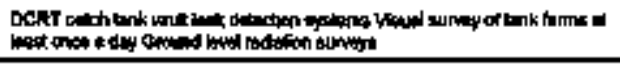 \\
\hline 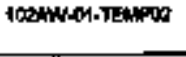 & 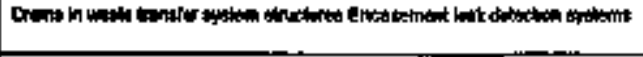 & 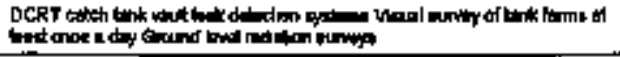 \\
\hline 1DOWNAZUERE & 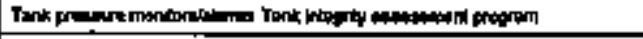 & $\operatorname{mon}$ \\
\hline 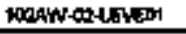 & mane & N \\
\hline
\end{tabular}




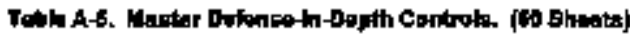

\begin{tabular}{|c|c|c|}
\hline 10 & 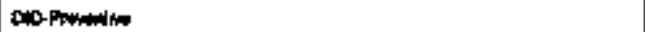 & 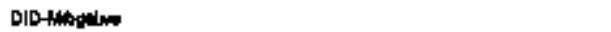 \\
\hline 10burstebriten & 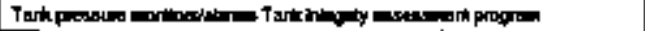 & Nans \\
\hline 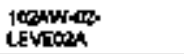 & $\operatorname{Hom}$ & Nort \\
\hline 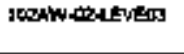 & 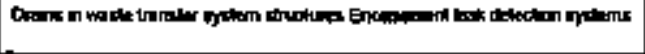 & 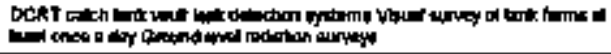 \\
\hline 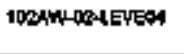 & 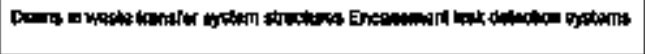 & 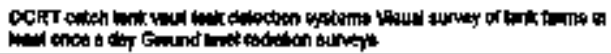 \\
\hline 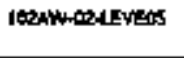 & 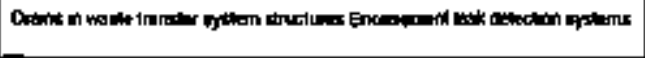 & 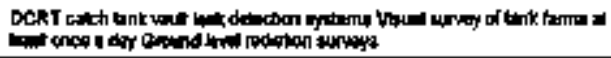 \\
\hline 10DNWDOR-1EVEQ & 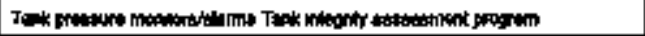 & Hean \\
\hline 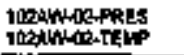 & 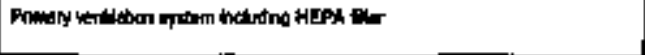 & Denti bleckt \\
\hline 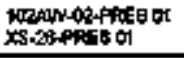 & Mpon & Who \\
\hline 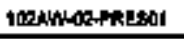 & Ft:metile per monter & Hond \\
\hline 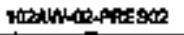 & Wure. & thone \\
\hline 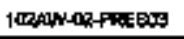 & 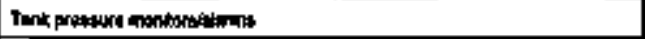 & Wone \\
\hline 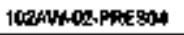 & 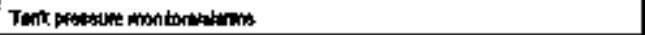 & What \\
\hline 10enyos Prisesos. & 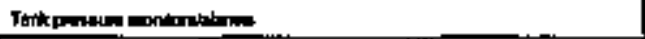 & Honn. \\
\hline 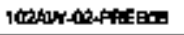 & 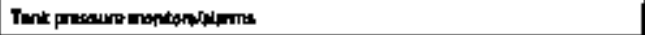 & Wone \\
\hline 1004W-00-PREF? & 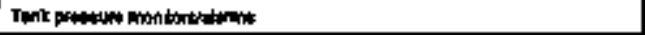 & How \\
\hline lonhwerpinizsos & $\mathrm{Nanh}$ & 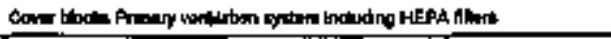 \\
\hline 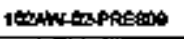 & None & 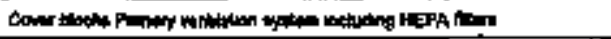 \\
\hline 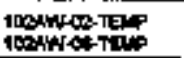 & Nant & Nomb \\
\hline
\end{tabular}




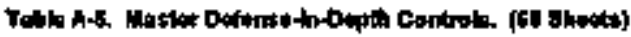

\begin{tabular}{|c|c|c|}
\hline 冏 & Conmentith & 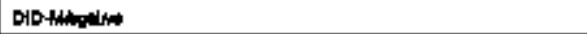 \\
\hline 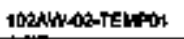 & Nond & Mare \\
\hline 10QWHLORTEMPOZ & 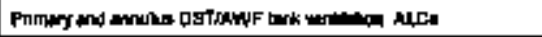 & 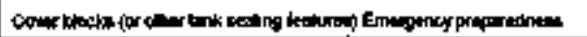 \\
\hline 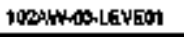 & 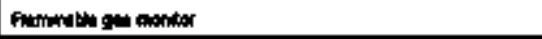 & Nons \\
\hline Itocklv oplatiog & 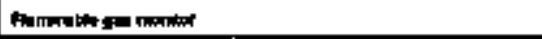 & Nons \\
\hline 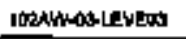 & 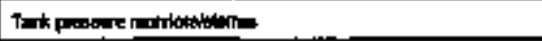 & Nom \\
\hline 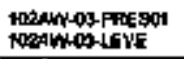 & What & How \\
\hline 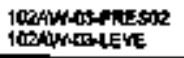 & Anatio & hent \\
\hline 10OAW-AS-TEROA & 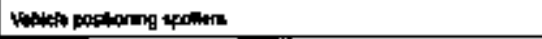 & Nong \\
\hline 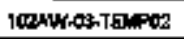 & 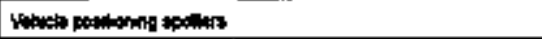 & Hent \\
\hline 100HWOAELAM & 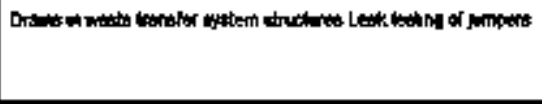 & 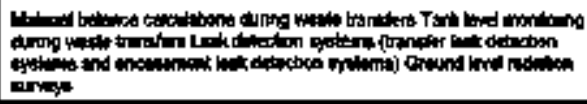 \\
\hline 1002hwoulevent & On & 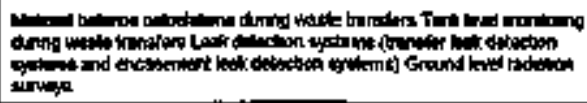 \\
\hline 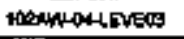 & 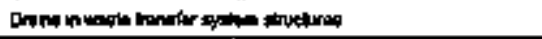 & 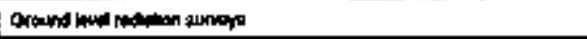 \\
\hline 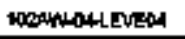 & 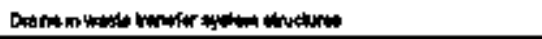 & 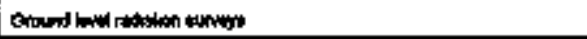 \\
\hline Wothathass on & Nore & Wone \\
\hline 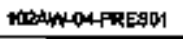 & Nisen & 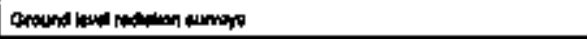 \\
\hline 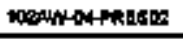 & Nhin & 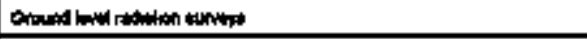 \\
\hline 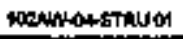 & Matr & Hond \\
\hline vandin-04staves & Nere & Nom \\
\hline
\end{tabular}




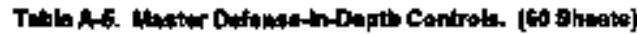

\begin{tabular}{|c|c|c|}
\hline $\mathbf{0}$ & 0.0.Promedred & 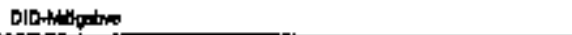 \\
\hline $\begin{array}{l}\text { Icainy-put } \\
\text { strivank }\end{array}$ & 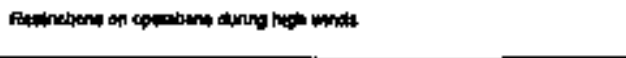 & 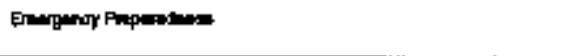 \\
\hline 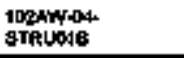 & None & $\operatorname{lan}$ \\
\hline Itranton-\$Thith & 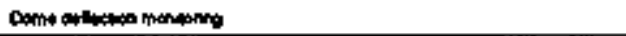 & 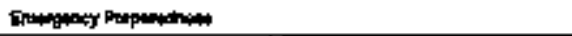 \\
\hline 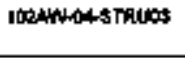 & 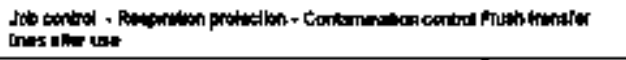 & Nhent \\
\hline 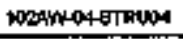 & Hon: & 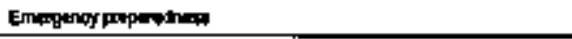 \\
\hline WOALALOA-STRUNS & hane & 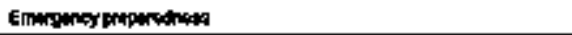 \\
\hline 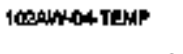 & 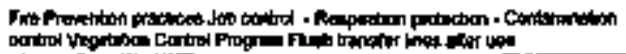 & What \\
\hline 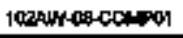 & $\tan 0$ & What \\
\hline 102antegeconog & Hete & Whon \\
\hline 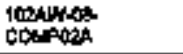 & Notits & Whid \\
\hline 102AW-06-Conpos & hane & $\operatorname{lona}$ \\
\hline 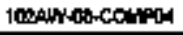 & Who & Hon \\
\hline 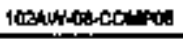 & Bene & hbon \\
\hline 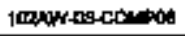 & 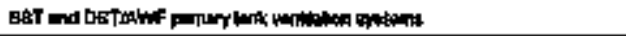 & Wra \\
\hline 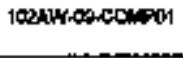 & 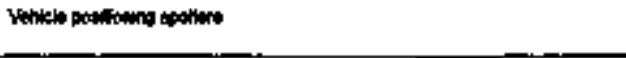 & 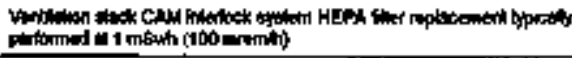 \\
\hline 1EOHW-tracot:xh & 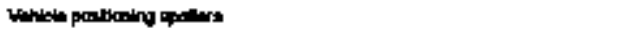 & Wone \\
\hline 1004n-co-conimas & 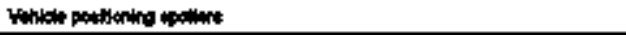 & Who \\
\hline 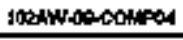 & Sampingtoritute & WhP tratidin lork permd \\
\hline 10 Mnd & No & Wond \\
\hline
\end{tabular}




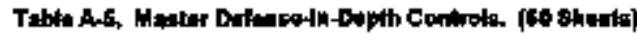

\begin{tabular}{|c|c|c|}
\hline $\mathbf{n}$ & DIDPhintiter & 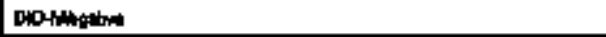 \\
\hline 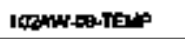 & Nare & 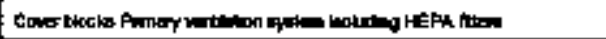 \\
\hline 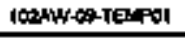 & 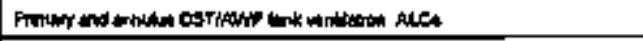 & 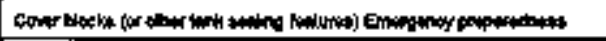 \\
\hline 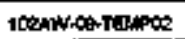 & Plone & Hent \\
\hline $\begin{array}{l}\text { 10Bawisk } \\
\text { FLow od }\end{array}$ & Nem & Anom \\
\hline 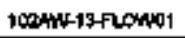 & lones & 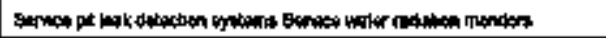 \\
\hline noshin:15Fonos & 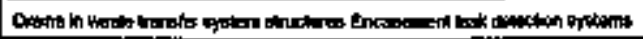 & 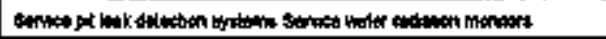 \\
\hline $\operatorname{trasedn~at~}$ & 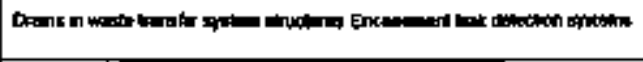 & 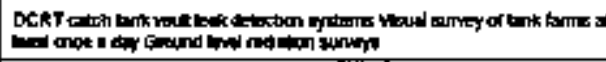 \\
\hline $102901 \mathrm{bz}$ & 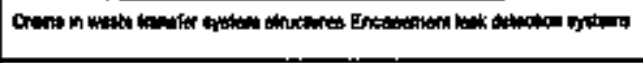 & 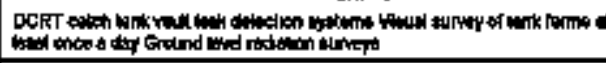 \\
\hline 10050163 & 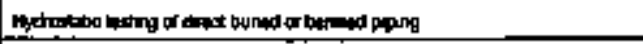 & 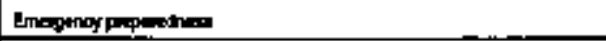 \\
\hline $1028-48 \mathrm{~b}$ & 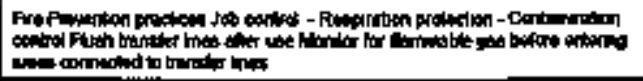 & Nintin \\
\hline pose-0s to & 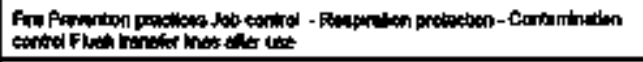 & Para \\
\hline 1meter ar & 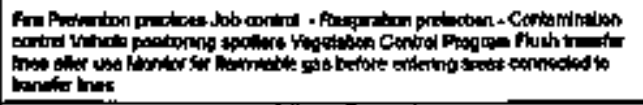 & Nort \\
\hline $1002-10$ ats & 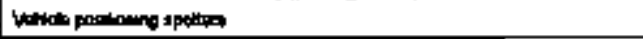 & Hent \\
\hline $1008-03$ b & 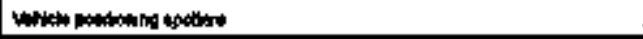 & ing \\
\hline iectasts bed & 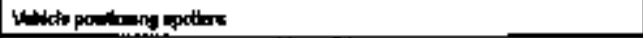 & $\ln i \operatorname{lin}$ \\
\hline Itras - ac als & 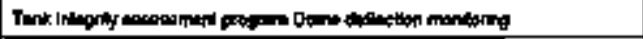 & Hone \\
\hline 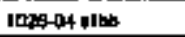 & 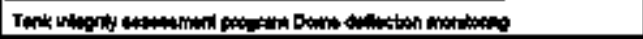 & Nons \\
\hline
\end{tabular}




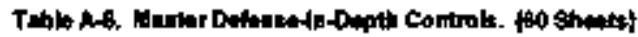

\begin{tabular}{|c|c|c|}
\hline 10 & Copsteveritue & 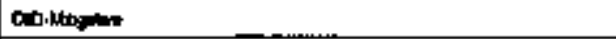 \\
\hline to28-04 10 & Noter & 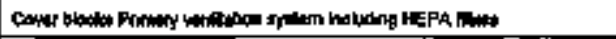 \\
\hline $1005-04 t$ & 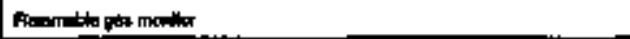 & None \\
\hline 1025-04 dif & 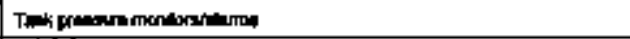 & Nato \\
\hline toge- 04 *16 & 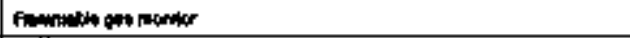 & thene \\
\hline $102804 \times 2$ & now & $\operatorname{lng}$ \\
\hline 1025040 & 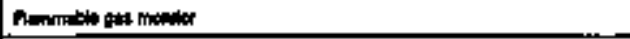 & Anom \\
\hline 1etstout od & $\operatorname{mon}$ & Horre \\
\hline I0s-00ate. & 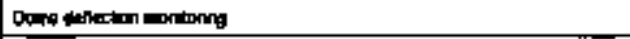 & 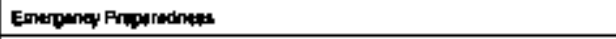 \\
\hline 1600000010 & Hans & Emmoney Properosmen. \\
\hline $100 \cos \frac{2}{2}$ & 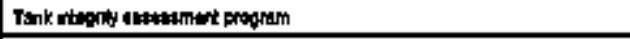 & anne \\
\hline 1025-106 क & 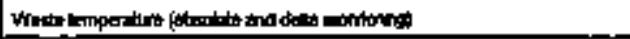 & mons \\
\hline 100000 ats & 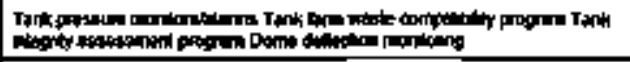 & Dtons \\
\hline 100500 & 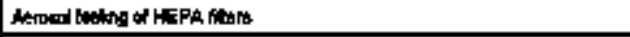 & Nonte \\
\hline $1000-014$ & 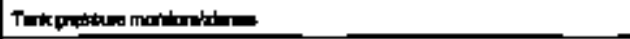 & None \\
\hline Noteng & 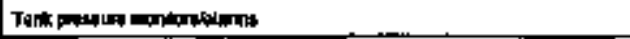 & $\operatorname{mon}$ \\
\hline 109-01c & 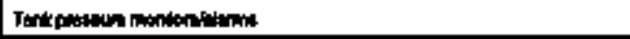 & none \\
\hline DOACOND & 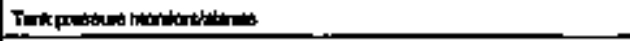 & Nome \\
\hline toecon E & 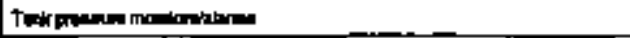 & Name \\
\hline 1000-01F & 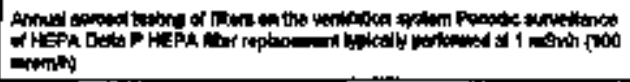 & 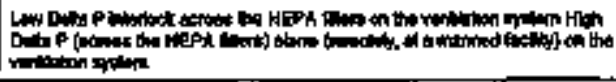 \\
\hline $100 \mathrm{c}-014$ & Nonte & Shonst \\
\hline
\end{tabular}




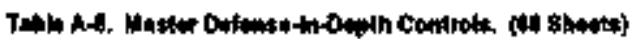

\begin{tabular}{|c|c|c|}
\hline ID & Dוק & 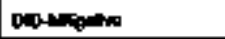 \\
\hline $1006-44$ & Ntime & Aroter \\
\hline 100c-011 & 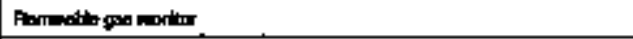 & Wone \\
\hline $1600-0 \mathrm{~d}$ & 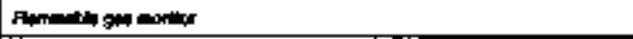 & mans \\
\hline $100<-01 k$ & 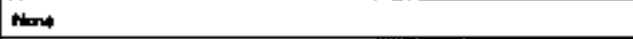 & None \\
\hline $1602-016$ & nom & None \\
\hline 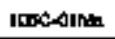 & 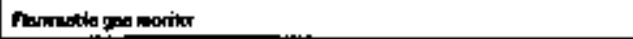 & Nare \\
\hline 1coctan & None & Nant \\
\hline 10ac-onta & 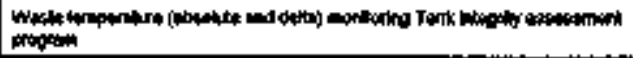 & 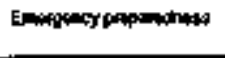 \\
\hline 1Dec-ont & 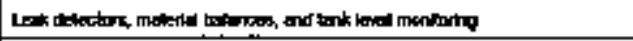 & $\mathrm{Han}$ \\
\hline Nopc-019 & 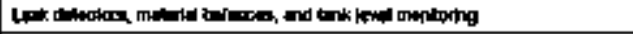 & $\operatorname{lng}(0)$ \\
\hline $100 \mathrm{C}-01 \mathrm{~T}$ & 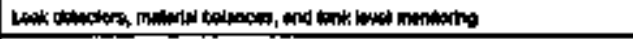 & Nomed \\
\hline 1000010 & Nont & nome \\
\hline 100c-ofur & 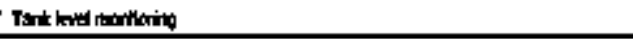 & Hant \\
\hline $10 \cos -01 x$ & 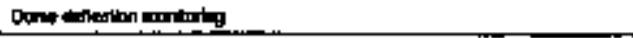 & 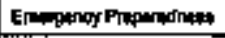 \\
\hline $1000-01 Y$ & 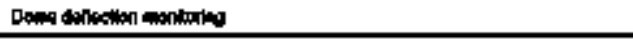 & 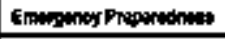 \\
\hline $10 \cos -004$ & 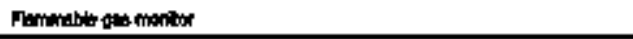 & thente \\
\hline 1000 & 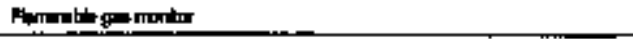 & mons \\
\hline $100 x-0 x$ & 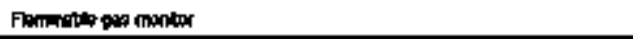 & $\min$ \\
\hline 1000004 & 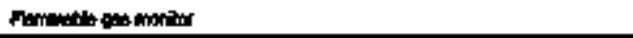 & Hond \\
\hline nocesen. & 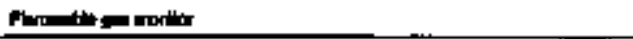 & Mon \\
\hline $1000-198$ & 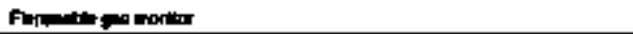 & nom \\
\hline
\end{tabular}




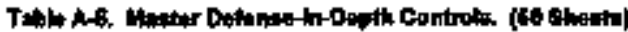

\begin{tabular}{|c|c|c|}
\hline$\omega$ & 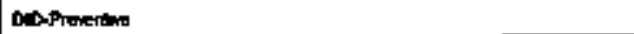 & 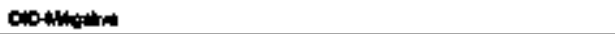 \\
\hline $1000-09 h$ & Hom & Grourdined ndmin wurnep \\
\hline $10 x-106$ & Hone & Nans \\
\hline $1000-0=0$ & 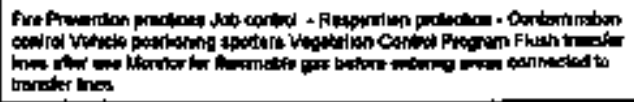 & Hinn \\
\hline 16acarar & 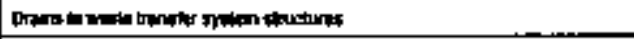 & 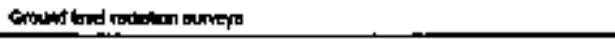 \\
\hline $1000-00$ & 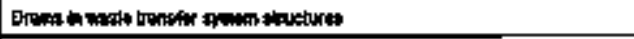 & 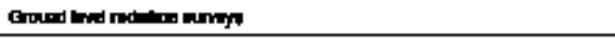 \\
\hline 1000-0in & 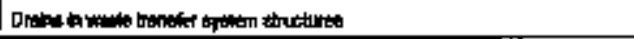 & 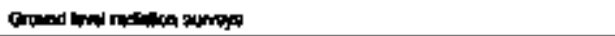 \\
\hline 1005-A. & Nare & mose \\
\hline 1006-gix & 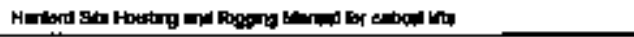 & nons \\
\hline noc-on & 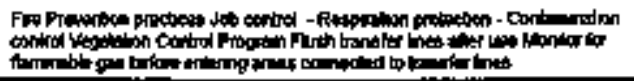 & Hone \\
\hline $100 \mathrm{C}-\mathrm{QBA}$ & 14ans & mon \\
\hline $100 x-108$ & How & hane \\
\hline 1000 & Motic & Nom \\
\hline $10 \mathrm{sec}-100$ & Hane & thene \\
\hline $100 C-105$ & None & Hone \\
\hline $\cos -10 \%$ & Nonto & 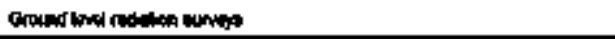 \\
\hline $100 \mathrm{c} 10 \mathrm{H}$ & 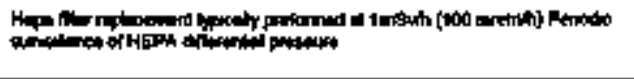 & 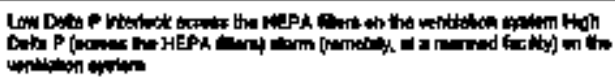 \\
\hline $10 \cos -10 \mathrm{~d}$ & 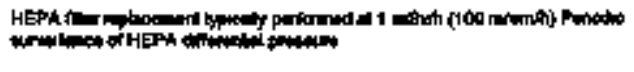 & 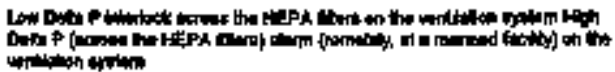 \\
\hline
\end{tabular}




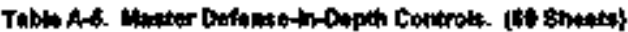

\begin{tabular}{|c|c|c|}
\hline 10 & ONOPAnima & 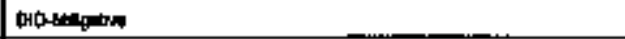 \\
\hline $1000-10 x$ & Nom & 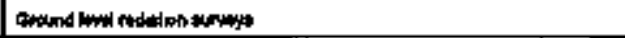 \\
\hline $100 \cos 10$ & Nam & 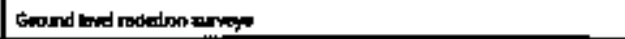 \\
\hline $1096-11 \mathrm{~A}$ & 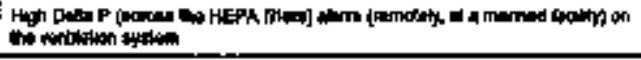 & 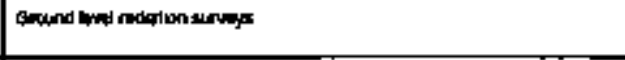 \\
\hline $100-118$ & Nem & Nate \\
\hline 10001F & Hone & Nene \\
\hline $1000-110$ & none & Nan \\
\hline 100C-11E & 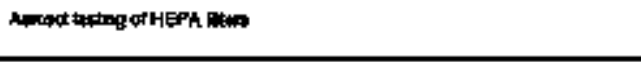 & 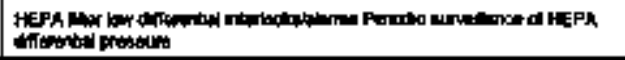 \\
\hline $1000-11 \mathrm{~F}$ & Imo & thon \\
\hline $1090-119$ & $\tan$ & 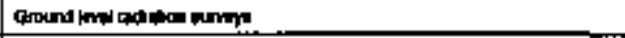 \\
\hline $\operatorname{moc}-11 \mathrm{H}$ & $\min$ & 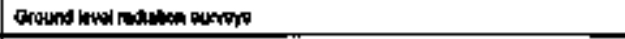 \\
\hline noge-111 & Hom & 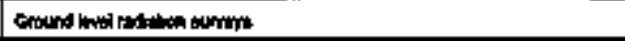 \\
\hline $\operatorname{noc}-11$ & 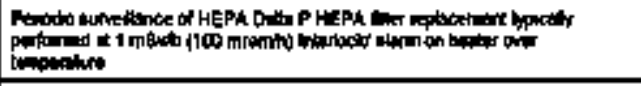 & 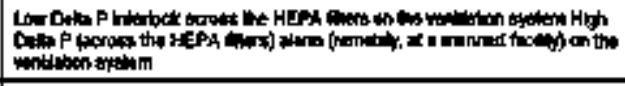 \\
\hline $100 \mathrm{C}-11 \mathrm{~K}$ & 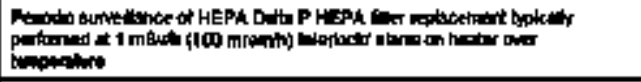 & 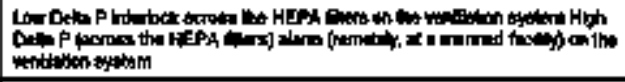 \\
\hline 100C-11L & Arived lesting of &  \\
\hline 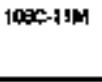 & 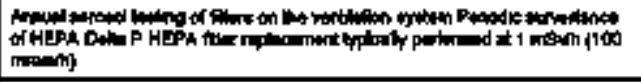 & 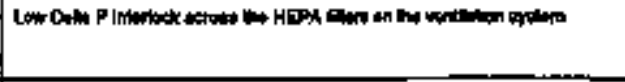 \\
\hline $100 \%-11 \mathrm{~A}$ & Nimin & 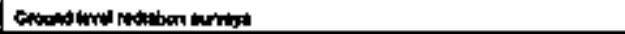 \\
\hline
\end{tabular}




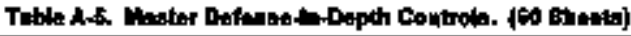

\begin{tabular}{|c|c|c|}
\hline 10 & Do-Phonation & 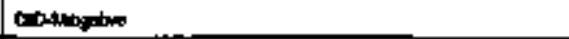 \\
\hline $6000-110$ & 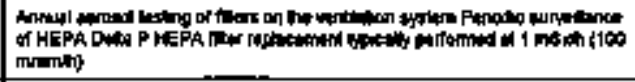 & 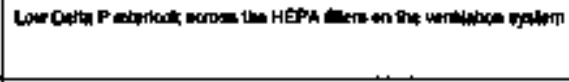 \\
\hline $1000-12 A$ & 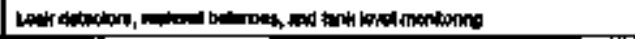 & Hione \\
\hline $1090-120$ & 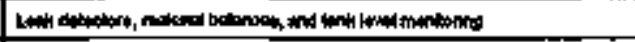 & Ninn \\
\hline $100 \mathrm{c}-12 \mathrm{C}$ & 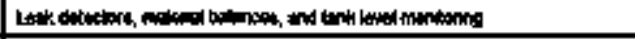 & thene \\
\hline 1000-170 & 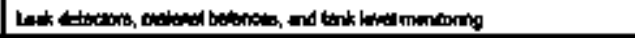 & Nkand \\
\hline $105 c 18$ & mon & nens \\
\hline $1000-120$ & 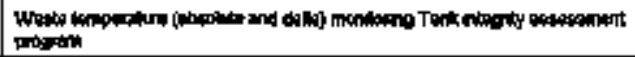 & 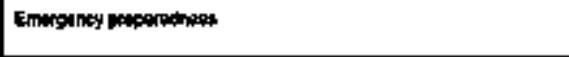 \\
\hline 1000 13. & 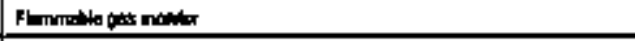 & Wone \\
\hline 10000 & 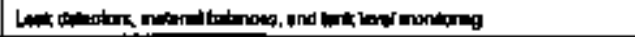 & Hone \\
\hline 107ANAM-LIVIEDI & Nano & 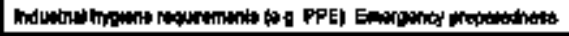 \\
\hline 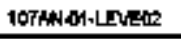 & Nant & 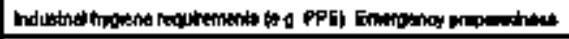 \\
\hline 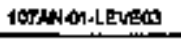 & Nore & 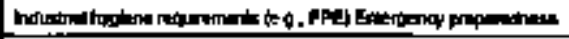 \\
\hline 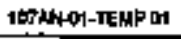 & Hore & Wone \\
\hline 10TANAMTTEMPOR & Nans & 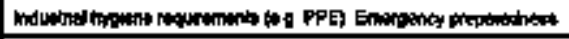 \\
\hline 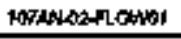 & Hente & None \\
\hline 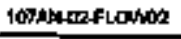 & Nene & Nome \\
\hline 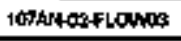 & Nems & 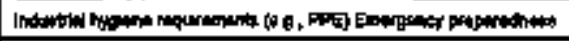 \\
\hline 107ANAOAPTES & Hone & 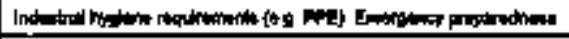 \\
\hline 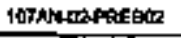 & None & 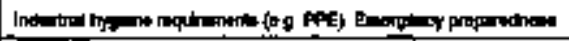 \\
\hline 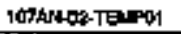 & Nemp & 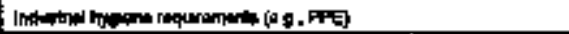 \\
\hline
\end{tabular}




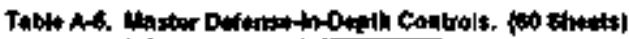

\begin{tabular}{|c|c|c|}
\hline $\mathbf{0}$ & 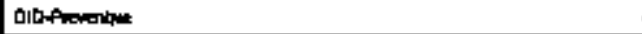 & 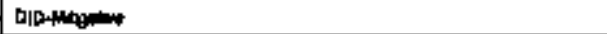 \\
\hline 10jutios now & $\tan$ & 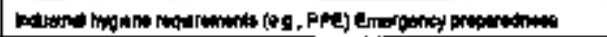 \\
\hline $114 \$ 3 x, 02 b$ & 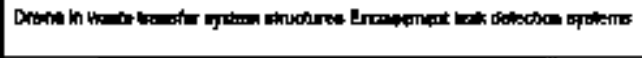 & 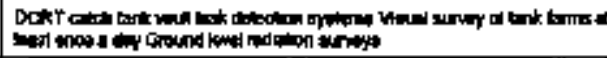 \\
\hline \$145: & 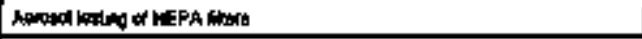 & 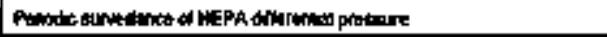 \\
\hline 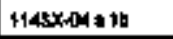 & 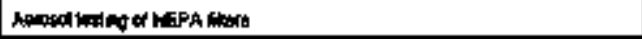 & 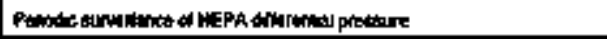 \\
\hline$T 145 x+04$ a 2 & 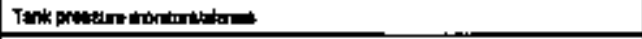 & Hone \\
\hline $11+4 \times-04$ a 3 & 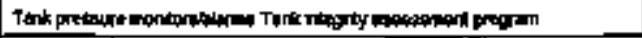 & $\ln$ \\
\hline $1145 \times-0444$ & 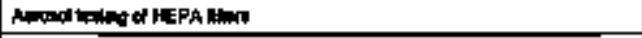 & 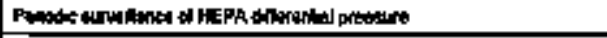 \\
\hline $1145 \times 0010111$ & 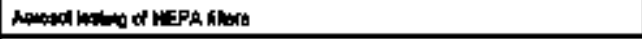 & 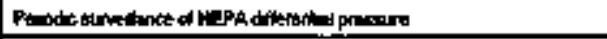 \\
\hline $1149 \times 0 \mathrm{~d} b 1 \mathrm{~B} 2$ & 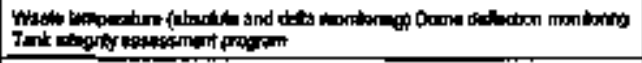 & Hon \\
\hline $1149 x-04 b 1+3$ & 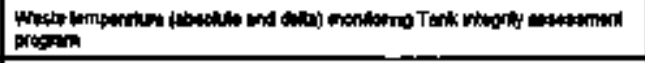 & 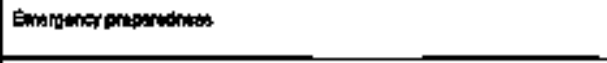 \\
\hline 1145xos b 1 b it & 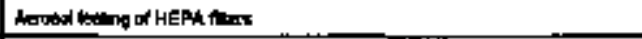 & 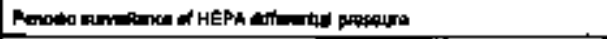 \\
\hline 1190x-OA 1 b 1b & $\mathrm{Non}$ & 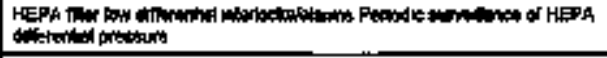 \\
\hline $1145 \times 004$ b 1620 & 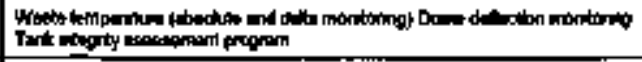 & Nome \\
\hline 1140X-OKb1 1 : & 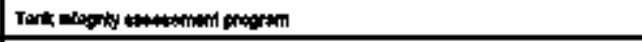 & Noter \\
\hline$F 146 \times 0461 b 34$ & 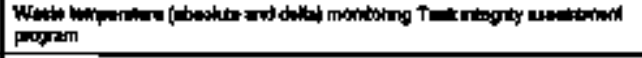 & 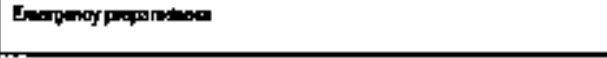 \\
\hline $114 \times-0401$ b 35 & Plent & 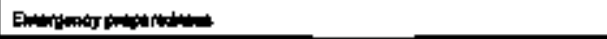 \\
\hline $1146 \times 04 b 1=1$ & 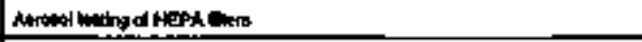 & 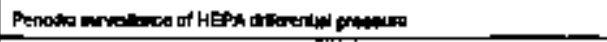 \\
\hline $1148 \times 04 b 1$ a 2 & 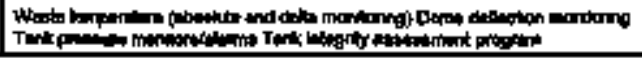 & Hons \\
\hline
\end{tabular}




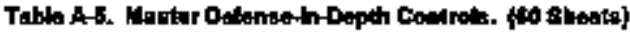

\begin{tabular}{|c|c|c|}
\hline ID & DOPTomatint & DADAmpare \\
\hline $1148 \times(3)$ b 1 o 3 & 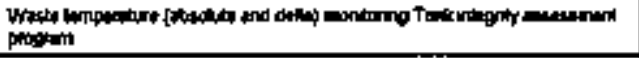 & Emerganey prepenation: \\
\hline $1145 x=4 b 1 \mathrm{~d} 1$ & 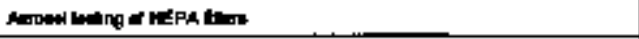 & 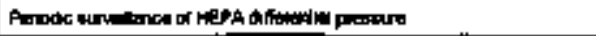 \\
\hline $1148 \times c 004$ b $1 \mathrm{~d} 2$ & 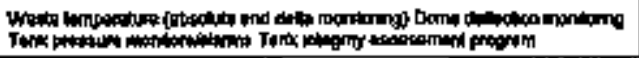 & Non \\
\hline $11+4 x-04 b 1 \mathrm{~d} 3$ & 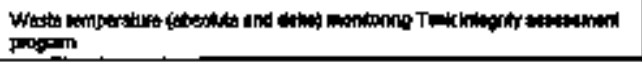 & 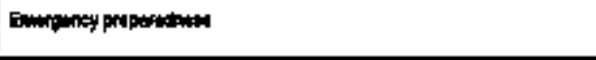 \\
\hline $1149 \times 50 \mathrm{~b}$ b 3 & Flammoblo parmantar & Namile \\
\hline $1148 x-04$ b 5 & 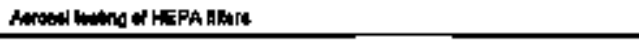 & 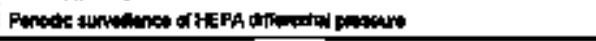 \\
\hline $1145 x-0 \mid c 1$ & Nante & 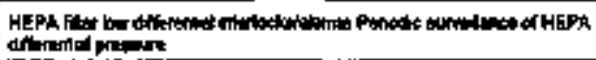 \\
\hline 110400028 & 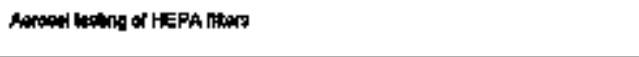 & 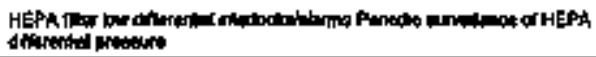 \\
\hline 114 KOAd $2 b$ & 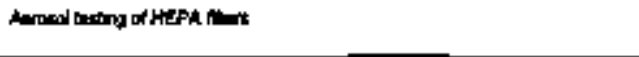 & 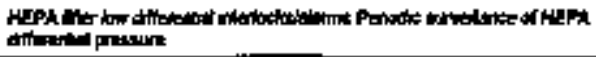 \\
\hline $11452-0 \times 027$ & 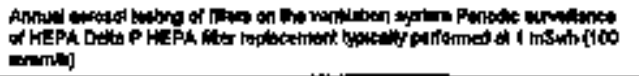 & 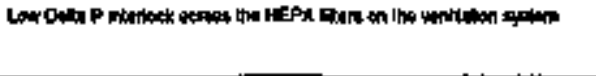 \\
\hline $1246 \times-0<024$ & 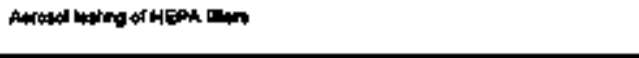 & 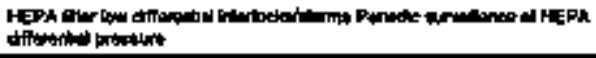 \\
\hline $1145 \times 0064$ & 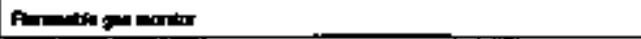 & Nones \\
\hline 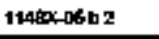 & 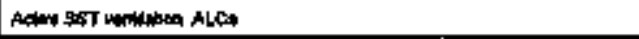 & 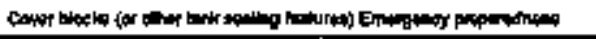 \\
\hline $149 x^{2}-0503$ & 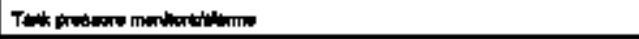 & Notitu \\
\hline $1645 \times 10003$ & 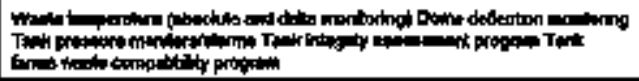 & rane \\
\hline$\$ 144 x-10 \times x$ & None & now \\
\hline
\end{tabular}




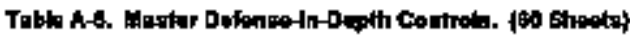

\begin{tabular}{|c|c|c|}
\hline 10 & DIDPtavalang & 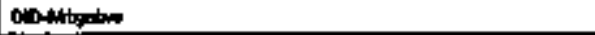 \\
\hline $1143 \times 600 d$ & Fummaterganom & thand \\
\hline $1148 \times-09 \cdot 14$ & 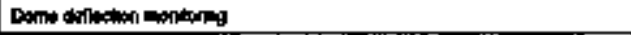 & 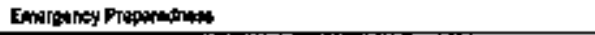 \\
\hline $1145 \times 60 \mathrm{~d} \cdot 10$ & 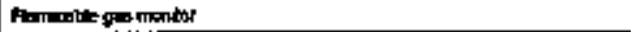 & Mare \\
\hline $1145 x 00$ b 2 & Whe & 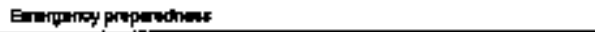 \\
\hline $1145 x-0 d a t$ & 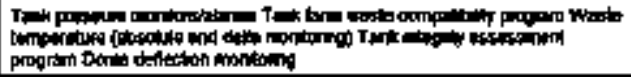 & Hore \\
\hline $1145 \times 600$ & Bane & Nom \\
\hline 201AR-pish & 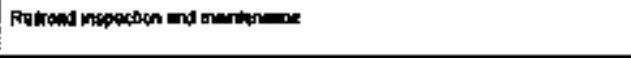 & 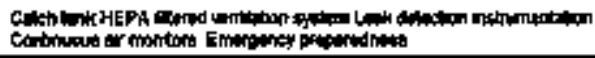 \\
\hline 2044R-onB & 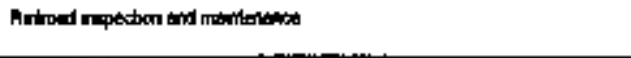 & 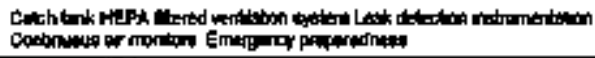 \\
\hline 3OAnR-01C & 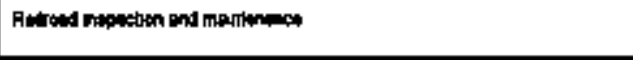 & 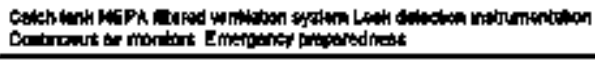 \\
\hline 2OAthR-OHD & 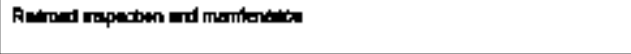 & 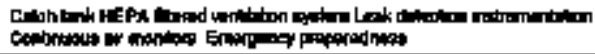 \\
\hline 20\%1R.015 & Nonte & 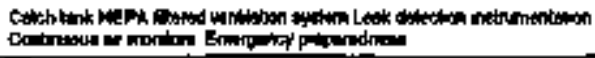 \\
\hline TaAR-OnF & 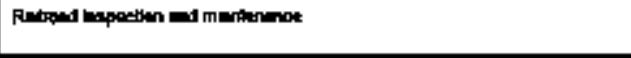 & 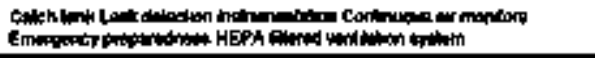 \\
\hline adinois & Non & 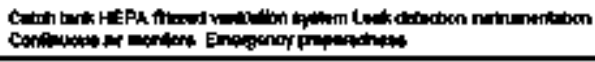 \\
\hline 204AR-0IH & 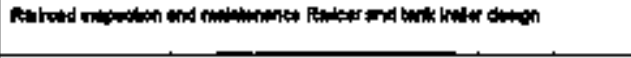 & 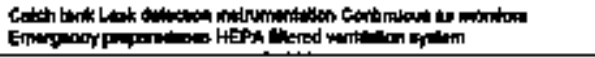 \\
\hline 2004kitai: & 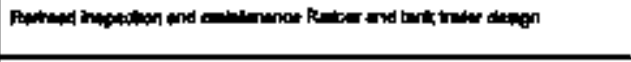 & 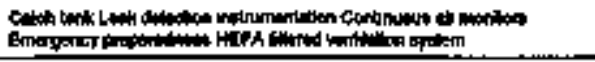 \\
\hline 204kroHJ & Powead the & 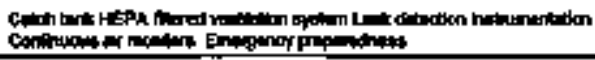 \\
\hline
\end{tabular}




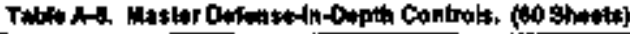

\begin{tabular}{|c|c|c|}
\hline$\omega$ & 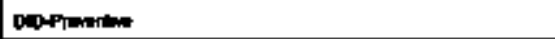 & bituringmen \\
\hline âR-0rk & 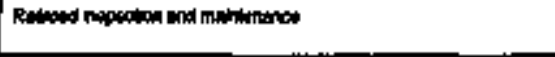 & 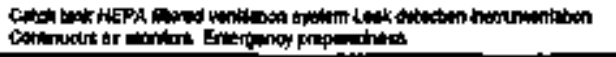 \\
\hline 204ART-00A & 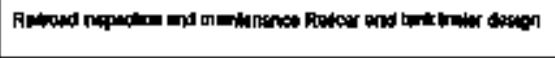 & 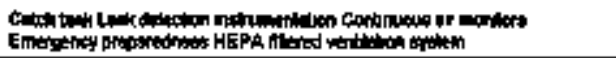 \\
\hline DuRness & 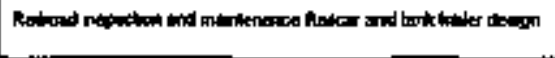 & 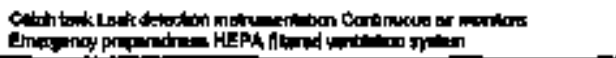 \\
\hline 204AR-00C & Howe & 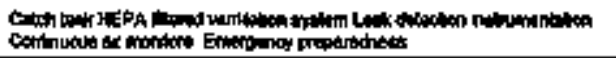 \\
\hline actrestos & Han & 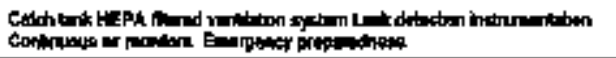 \\
\hline 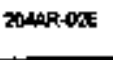 & 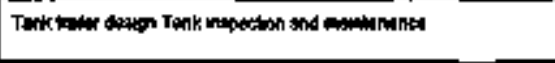 & 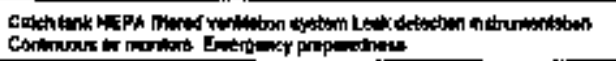 \\
\hline 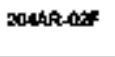 & 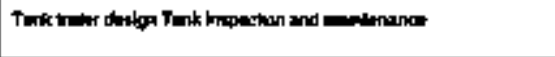 & 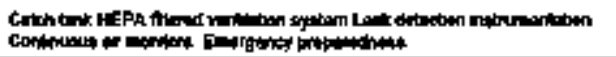 \\
\hline 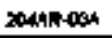 & 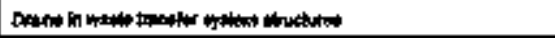 & 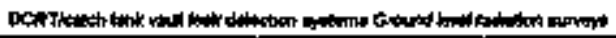 \\
\hline Xuthosing & 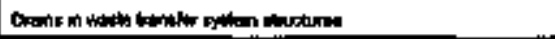 & 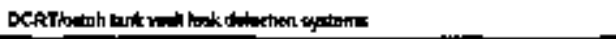 \\
\hline XUAR_RE & None & 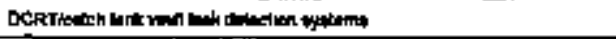 \\
\hline Botharach & 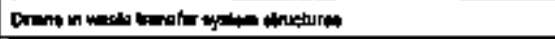 & 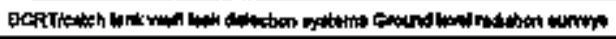 \\
\hline $20+4 n-00$ & 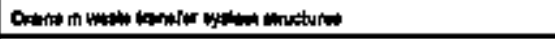 & 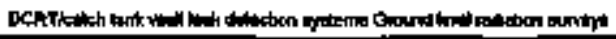 \\
\hline CORARAL & Hone & 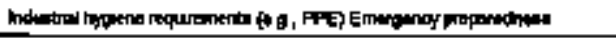 \\
\hline 204artoutp & Hon: & It) \\
\hline 204AN-OAE & nom & 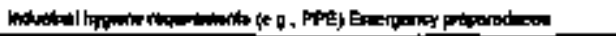 \\
\hline 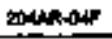 & Hone & 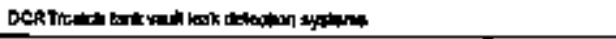 \\
\hline 20arkach & Wone & 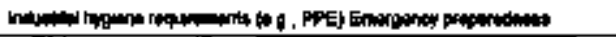 \\
\hline 2044R-0. & Mons & | \\
\hline
\end{tabular}




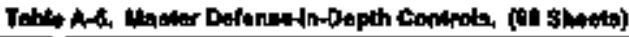

\begin{tabular}{|c|c|c|}
\hline $\mathbf{p}$ & 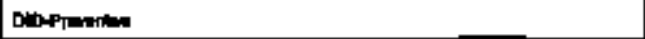 & Oanterpatice \\
\hline $2044 \times-4=4$ & None & 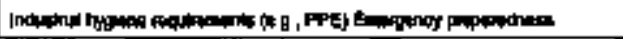 \\
\hline DonRegh & 施 & 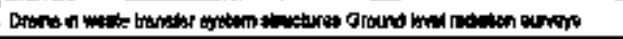 \\
\hline 200hR-0ote & 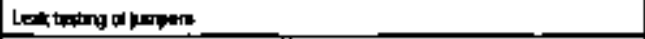 & 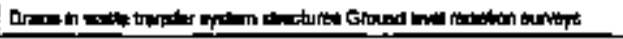 \\
\hline 304R-000 & 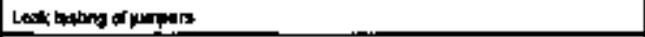 & 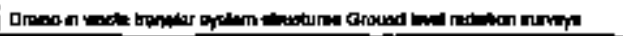 \\
\hline 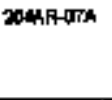 & Hom & 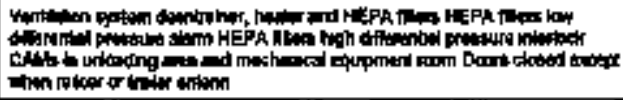 \\
\hline 200R-0rs & 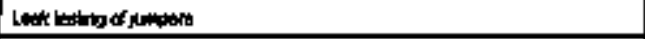 & 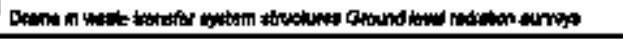 \\
\hline Buserorc & hine & Hant \\
\hline Dasapioder & 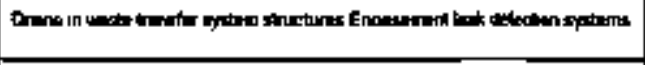 & 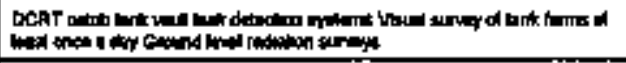 \\
\hline 2014k0es & 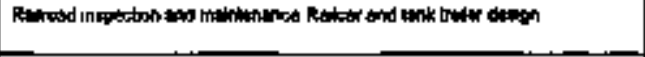 & 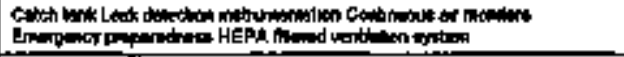 \\
\hline PMar-oenc & Ban & 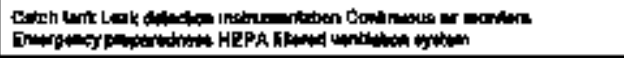 \\
\hline 204kntoso & 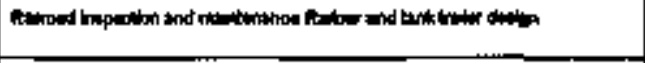 & 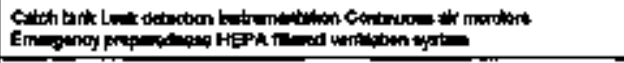 \\
\hline 204RR-00E & 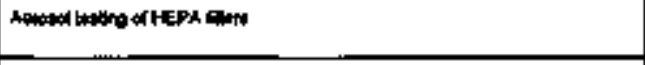 & 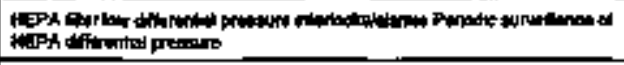 \\
\hline Zourtafe & 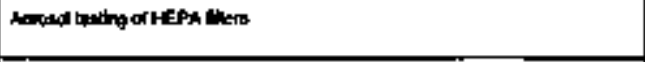 & 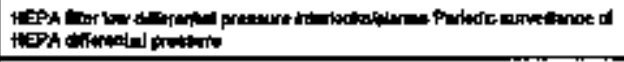 \\
\hline $2044 n \cos$ & Hine & 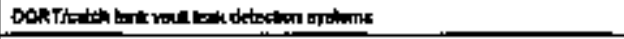 \\
\hline 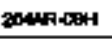 & thons & 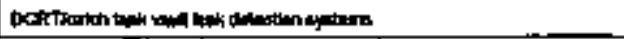 \\
\hline 204tat-11h & 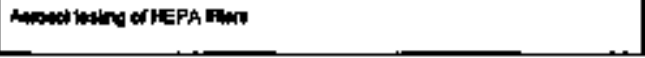 & 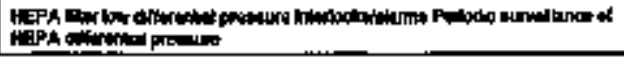 \\
\hline
\end{tabular}




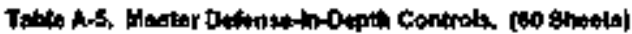

\begin{tabular}{|c|c|c|}
\hline ID & 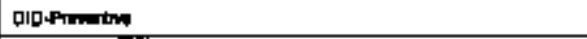 & pitokention \\
\hline 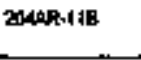 & 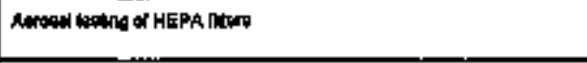 & 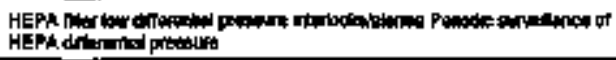 \\
\hline $204 A R-216$ & 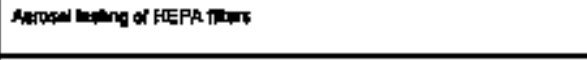 & 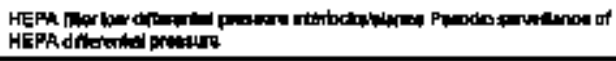 \\
\hline 2004k-11D & 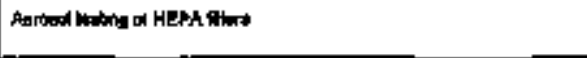 & 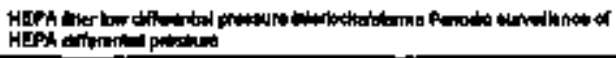 \\
\hline 2044R-11E & 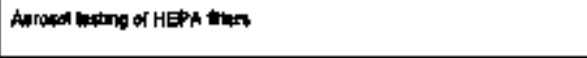 & 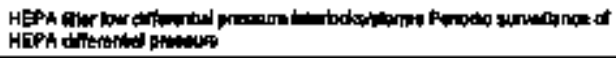 \\
\hline 20.4A-11f & 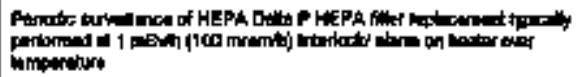 & 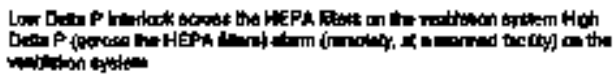 \\
\hline 213401 & 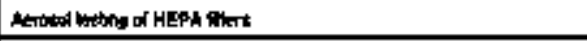 & 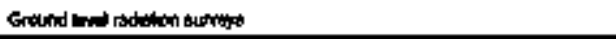 \\
\hline 219 wer & terowd bitong of HEPA tiver & Ground lined raoming murnot \\
\hline 213น-女 & Nane & 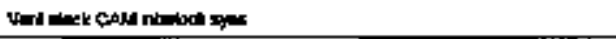 \\
\hline 213 won & Hom & 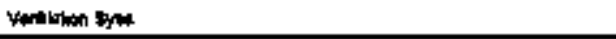 \\
\hline 213wask & None & Whintion sin \\
\hline 213 w-xad & Hone & 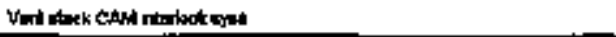 \\
\hline 213w-क? & none & 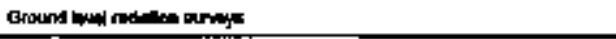 \\
\hline 203w-4 & Wont & 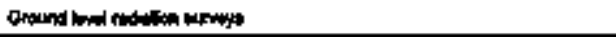 \\
\hline Z13 Wus & 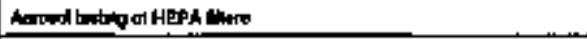 & 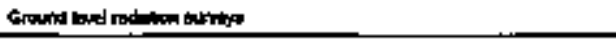 \\
\hline $24012+01 \cdot 03$ & 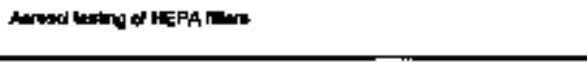 & 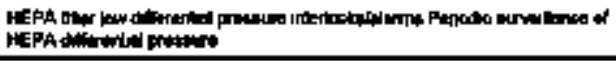 \\
\hline 2emented a on & 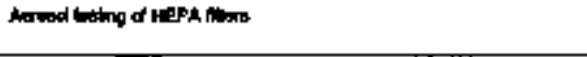 & 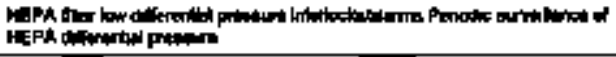 \\
\hline 244AR01 b02 & 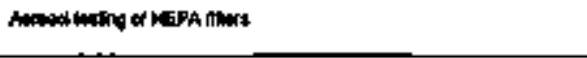 & 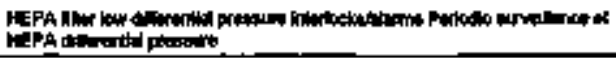 \\
\hline
\end{tabular}




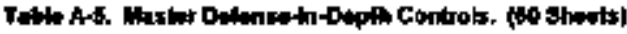

\begin{tabular}{|c|c|c|}
\hline$\omega$ & 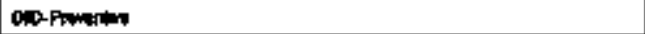 & 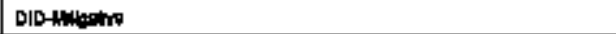 \\
\hline 2Aderen bes & 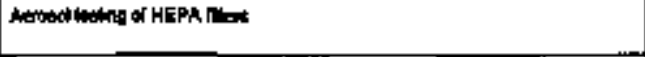 & 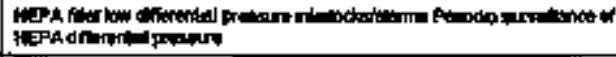 \\
\hline 240RR-1 OD & 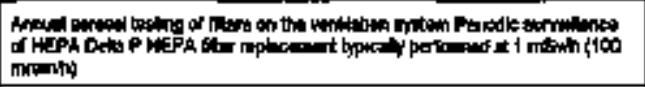 & 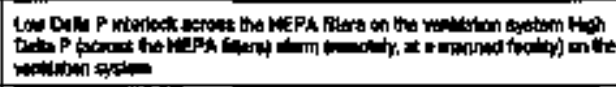 \\
\hline 244AR-O1 o On & 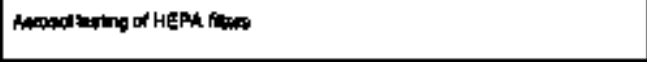 & 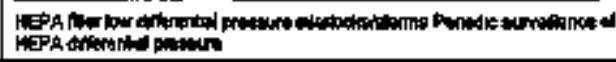 \\
\hline 2ALARAII EM & 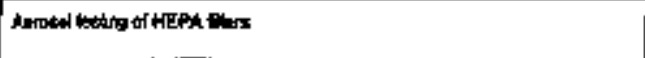 & 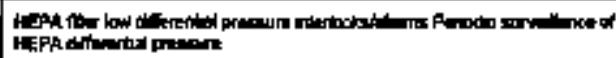 \\
\hline $244 \mathrm{R}-01$ e og & 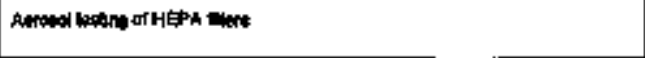 & 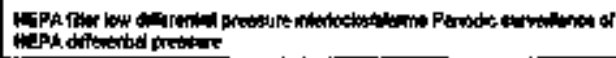 \\
\hline 24MARAI DO & 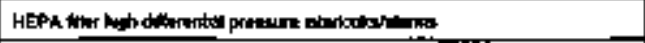 & Wone \\
\hline 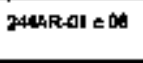 & 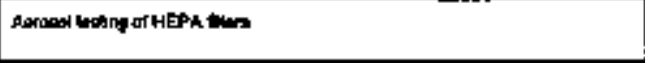 & 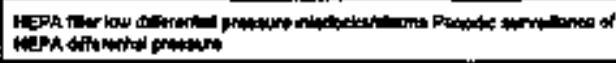 \\
\hline $244 k-01+\infty$ & 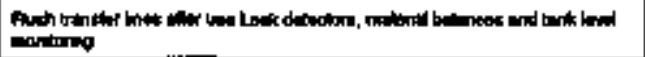 & Wom \\
\hline 2MAR-DH \& IO & 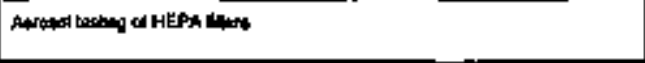 & 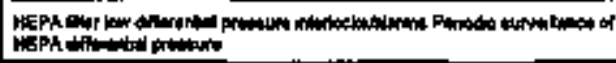 \\
\hline 24AA-O1 Q.11 & 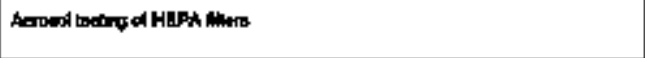 & 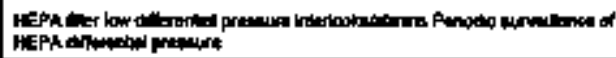 \\
\hline $204 x+01<19$ & 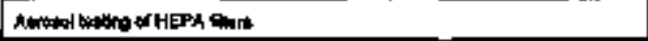 & 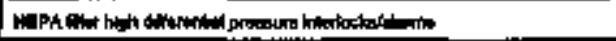 \\
\hline zathen a tos & 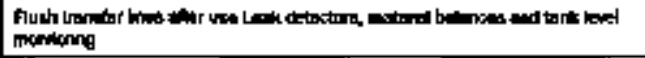 & Rare \\
\hline $244 A R=05$ & 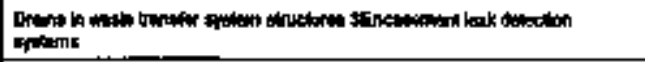 & 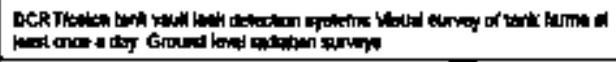 \\
\hline 2404 R 08 ज्ञ 06 & monom & 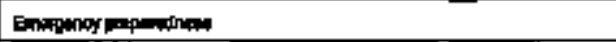 \\
\hline 244AR-0r a or & 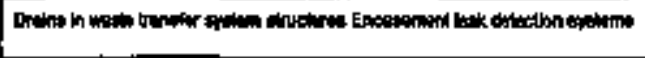 & 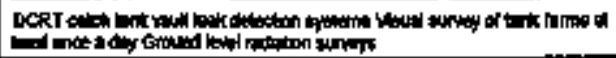 \\
\hline
\end{tabular}




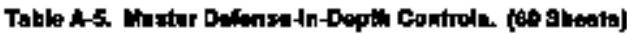

\begin{tabular}{|c|c|c|}
\hline 10 & Daphonition & 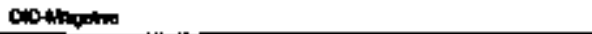 \\
\hline 24AARATR DOA & Lot the of pmpar & 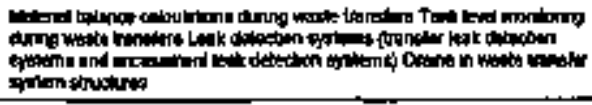 \\
\hline $240 \mathrm{R}-02 \div 00$ & 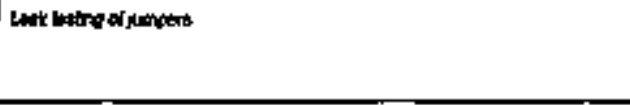 & 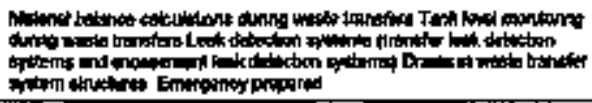 \\
\hline $344 \mathrm{R}-02$ औ 10 & 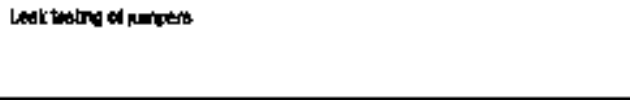 & 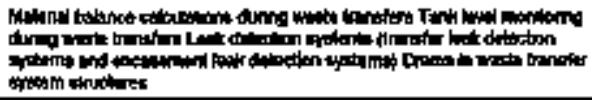 \\
\hline $2404 A 40211$ & Leat twotro ad pumpert & 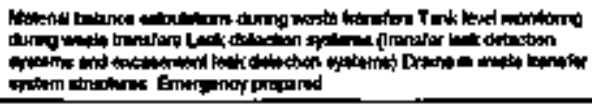 \\
\hline 24GAR-002a 12 & 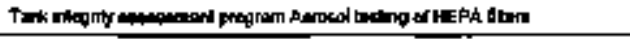 & 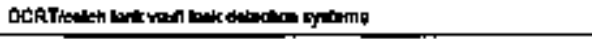 \\
\hline $24-4 x-02 * 13$ & Nans & 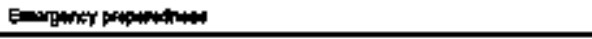 \\
\hline $244 \times-12+14$ & 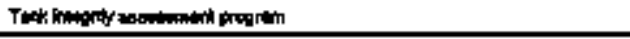 & Nont \\
\hline 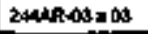 & 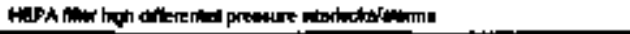 & Hent \\
\hline P4SAR-03. 04 & 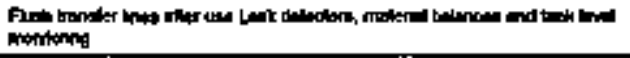 & К⿴囗十 \\
\hline 24uthosand & Nom & 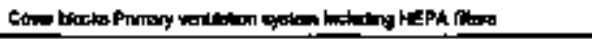 \\
\hline tempros nos & 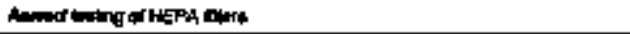 & 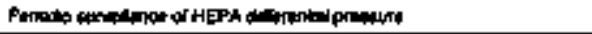 \\
\hline 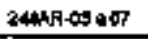 & 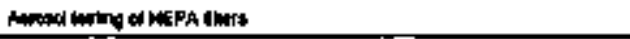 & 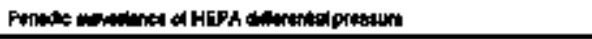 \\
\hline 2404hos a & late & 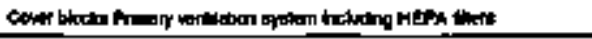 \\
\hline 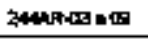 & Mon & Nom \\
\hline $244 \times-63=13$ & Apread tering of MEPA whes & 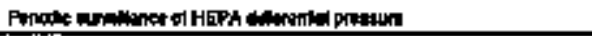 \\
\hline
\end{tabular}




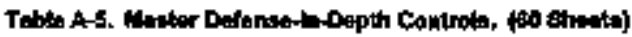

\begin{tabular}{|c|c|c|}
\hline $\mathbf{0}$ & 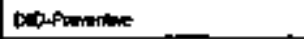 & 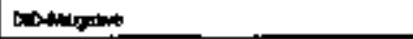 \\
\hline $244 \times R-63=14$ & 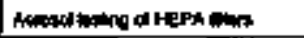 & 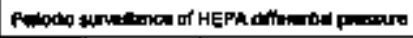 \\
\hline $240 \mathrm{R}$ os $* 15$ & Hone & 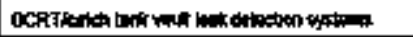 \\
\hline 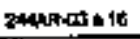 & Mons & 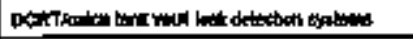 \\
\hline 244सRAs: 17 & Hom & 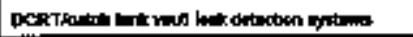 \\
\hline 244kP-60 14 14 & $\operatorname{lon} 9$ & Hon \\
\hline 244RR0400 & Natifo & Monse \\
\hline 244kRobol ol & Name & Mats \\
\hline 244ARAd in & 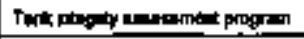 & $M_{\varphi \rightarrow m}$ \\
\hline $244 R-08$ is & Nomb & 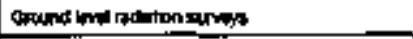 \\
\hline 244LR-07 I01 & None & monse \\
\hline 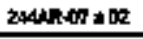 & Hare & 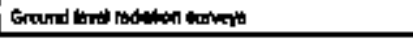 \\
\hline TAMARAOA a As & Hare & 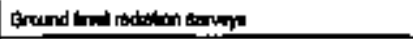 \\
\hline raAAR-OT a OM & Nenes: & Hone \\
\hline 24MRPFHO1 & ntans & Mont \\
\hline $2440 \mathrm{~m}+\mathrm{mos}$ & Han & Mont: \\
\hline 2antaratios & Nene & $\operatorname{lng}$ \\
\hline 34arfanch & Namen & Ningen \\
\hline 244 Newos & Nont & Nomo \\
\hline 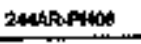 & Nons. & $\mathrm{Hon}$ \\
\hline 2HAPFFit? & Nonen & 사에교 \\
\hline 24Aikfmits & monos & Nint \\
\hline 244ikfows & Hone & Hone \\
\hline
\end{tabular}




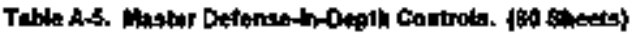

\begin{tabular}{|c|c|c|}
\hline $\boldsymbol{~}$ & 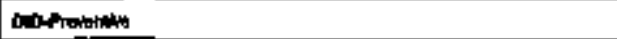 & 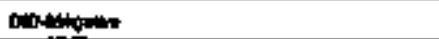 \\
\hline 2MARPIHO & $\ln 4$ & Wone \\
\hline 24ARRPMI & now & nomo \\
\hline 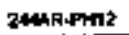 & Hone & Whone \\
\hline 2401R.PH13 & Mone & Mone \\
\hline 244ARPHIS & Mon: & thent: \\
\hline 24UR.PH15 & Mons & Nonse \\
\hline 244hepH10 & Morke & Bhont \\
\hline 244AR:PH17 & When & Vane \\
\hline 244RPP्P19 & $\operatorname{man}$ & $\operatorname{man}$ \\
\hline $240 \mathrm{AP}+419$ & Nam & Nan \\
\hline Z2rintar & Nant & Nant \\
\hline atranos & 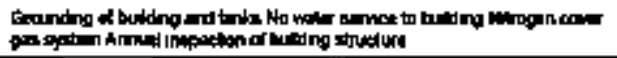 & 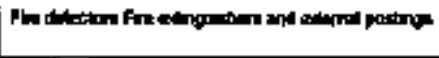 \\
\hline 272rntos & 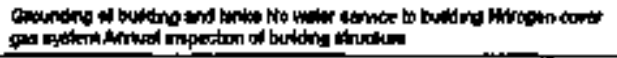 & 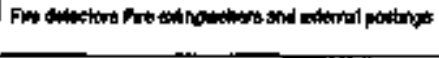 \\
\hline zTrTh-OA & 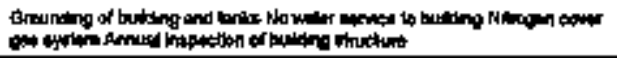 & 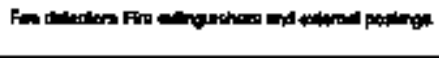 \\
\hline merumal & 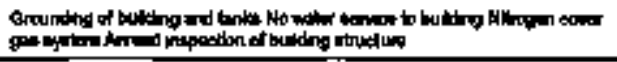 & 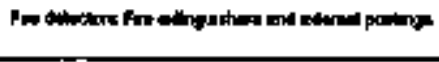 \\
\hline manog & 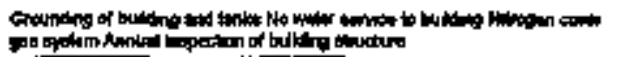 & 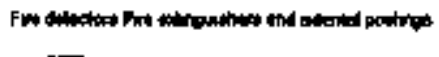 \\
\hline 5001 & Nane & 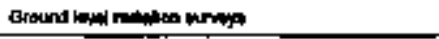 \\
\hline 20002 & (5) & 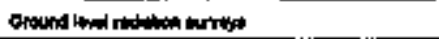 \\
\hline 0004 & شسب & 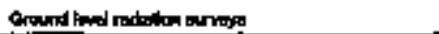 \\
\hline
\end{tabular}




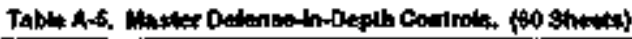

\begin{tabular}{|c|c|c|}
\hline 10 & 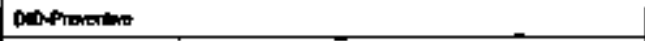 & 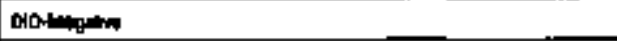 \\
\hline 90004 & 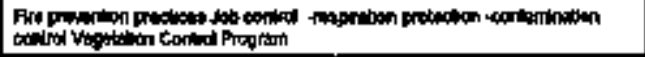 & 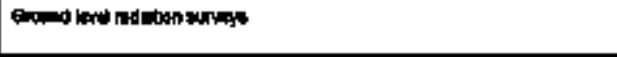 \\
\hline $\operatorname{cop} 04$ & 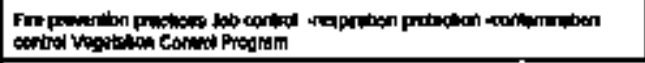 & 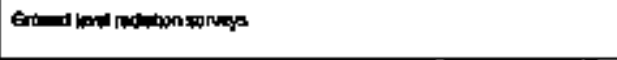 \\
\hline otones & Hon & 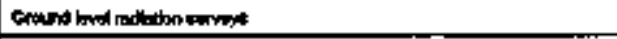 \\
\hline $\operatorname{sen} \theta$ & 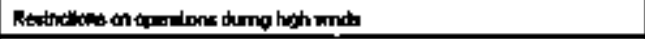 & 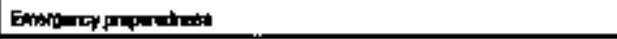 \\
\hline$\$ 0000$ & 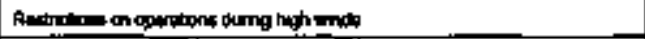 & 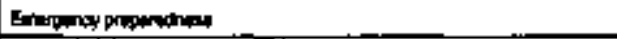 \\
\hline 90009 & 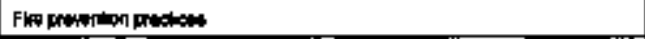 & Hons: \\
\hline 900.10 & 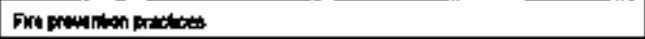 & Nonos \\
\hline eso-11 & Nome & 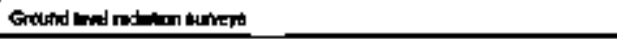 \\
\hline expas & $\mathrm{Han}=$ & Nore \\
\hline Bapalt & Nare & Hane \\
\hline expos & 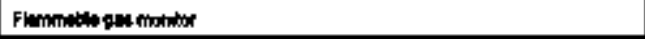 & Nons \\
\hline expost & Nanter & Hents \\
\hline $\cos$ & Hone & Hon: \\
\hline $\cos =02$ & 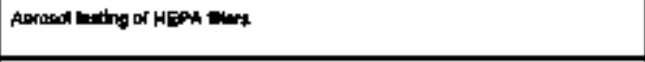 & 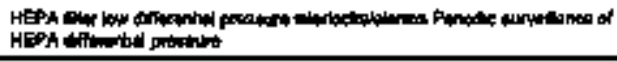 \\
\hline $\cos$ & 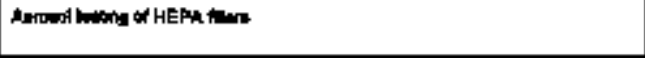 & 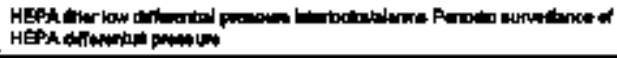 \\
\hline eqr-04 & 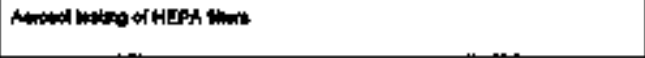 & 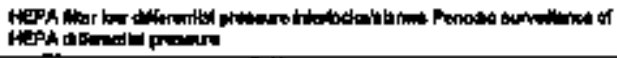 \\
\hline cद्वF्06 & nom & Nent \\
\hline estar & mons & then \\
\hline $000 \mathrm{~s}$ & 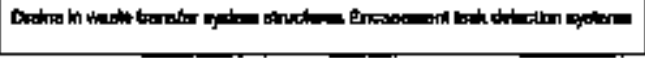 & 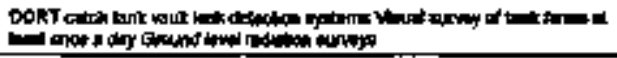 \\
\hline
\end{tabular}




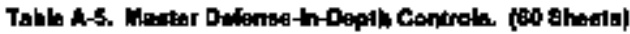

\begin{tabular}{|c|c|c|}
\hline 旧 & Dothenation & 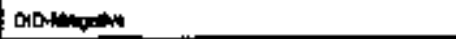 \\
\hline CCF $\triangle 9$ & $\operatorname{mon}$ & Mons \\
\hline COF- -0 & Whe & Nons \\
\hline CCF-10 & Lon & Honst \\
\hline CCF-11 & Mone & None \\
\hline CCFF-12 & none & Nant \\
\hline $\cos -13$ & Whos & $\tan 9$ \\
\hline $\cos -14$ & Mone & henct \\
\hline Qcos-15 & Mone & Mne \\
\hline $\cos =-10$ & 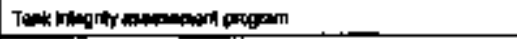 & 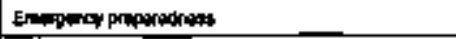 \\
\hline cos -17 & 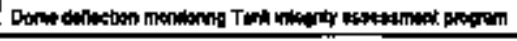 & $\operatorname{man}$ \\
\hline CEF.1\% & 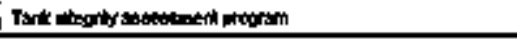 & Hone \\
\hline cestat & 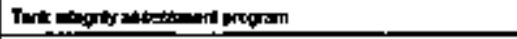 & Won \\
\hline cosf-20 & Hones & Mane \\
\hline $\cos -01$ & Who & 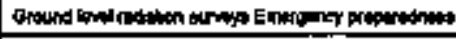 \\
\hline CDPan & Nonet & Wime \\
\hline CDepd & Rare & 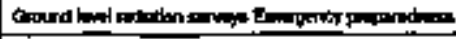 \\
\hline $\mathrm{copoON}$ & Nonst & 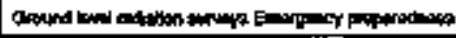 \\
\hline CDPOS & 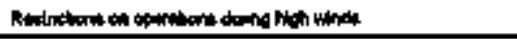 & 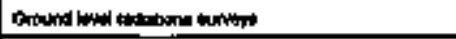 \\
\hline $\operatorname{cop} 00$ & 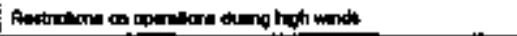 & 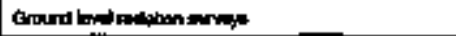 \\
\hline CDPRP & 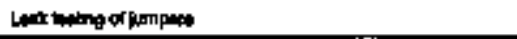 & 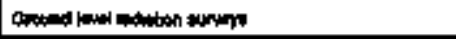 \\
\hline copos & 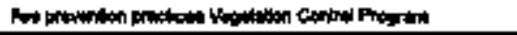 & Bow \\
\hline ODPDS & Nomes & Wune \\
\hline
\end{tabular}




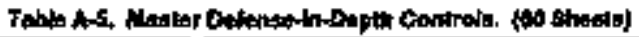

\begin{tabular}{|c|c|c|}
\hline $\mathbf{0}$ & 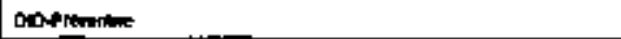 & Dothome \\
\hline COP-10 & Expmingn Cortipts & 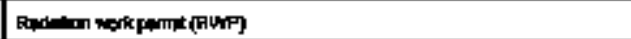 \\
\hline $\cos \theta$ & 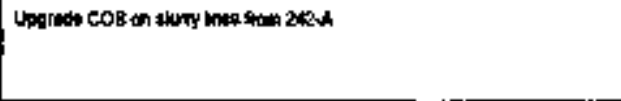 & 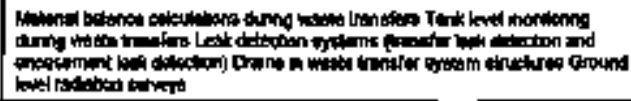 \\
\hline $\cos \theta 2$ & 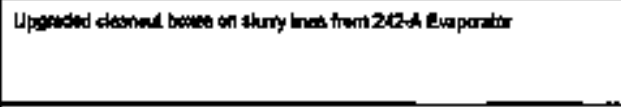 & 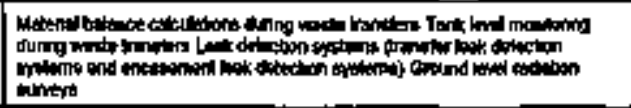 \\
\hline casing & 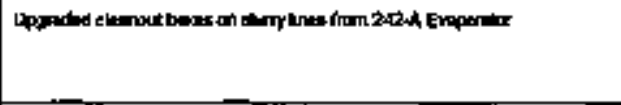 & 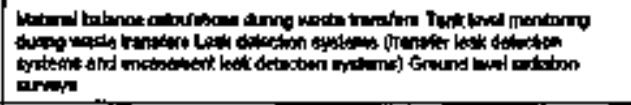 \\
\hline cospos & 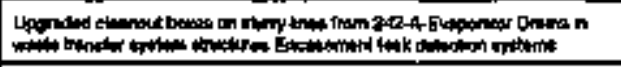 & 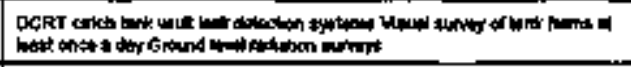 \\
\hline cotorita & 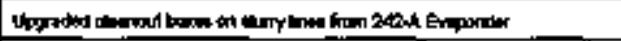 & Gound Whed rodton wrom \\
\hline COB-G & 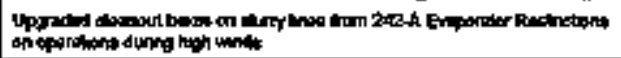 & Hone \\
\hline collose & thint & 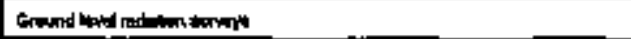 \\
\hline conbedot & 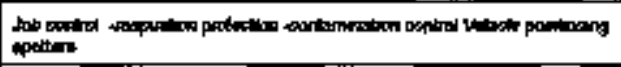 & 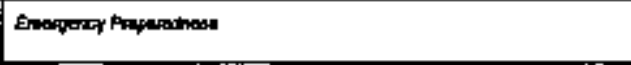 \\
\hline $\cos -\infty$ & Whot & 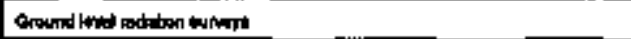 \\
\hline $\cos -\infty x$ & 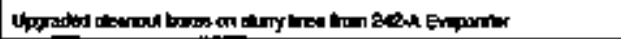 & 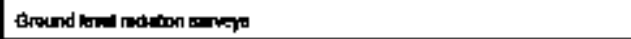 \\
\hline conosin & 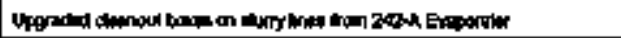 & 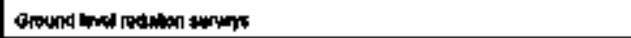 \\
\hline cosos & 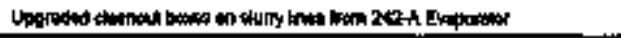 & 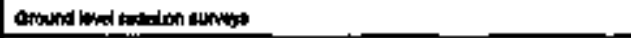 \\
\hline $\cos \theta$ & Nond reipurat & 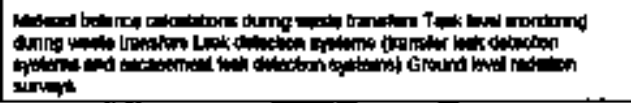 \\
\hline
\end{tabular}




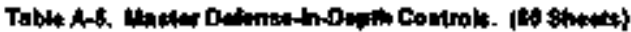

\begin{tabular}{|c|c|c|}
\hline 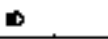 & Dapherestros & 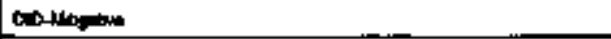 \\
\hline $600-08$ & 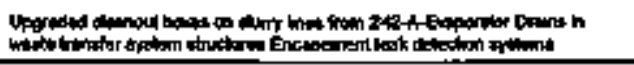 & 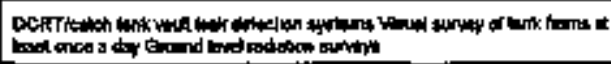 \\
\hline CFNat & Wone raped & 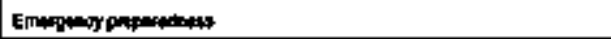 \\
\hline CPon-01-1 & Hon inyumad & 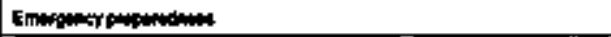 \\
\hline contas & Hono numper & 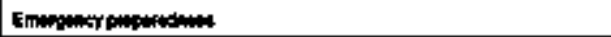 \\
\hline contint & 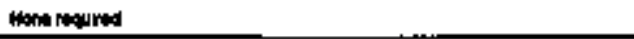 & 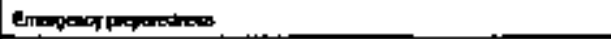 \\
\hline Ghardes & Dome dituctur manking & 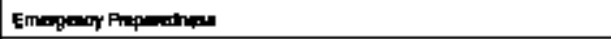 \\
\hline 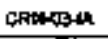 & 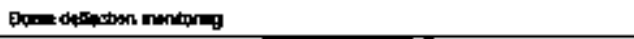 & 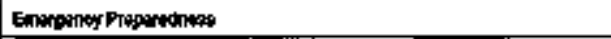 \\
\hline CARtoss & Hono & Wone \\
\hline CRN-OA & 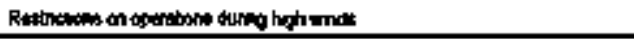 & ENerfency Plaptredineds \\
\hline ERrotouth & 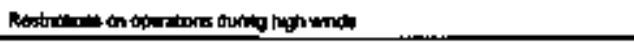 & 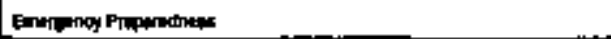 \\
\hline CAMdOAG & nare & Nome \\
\hline CRN-OS & 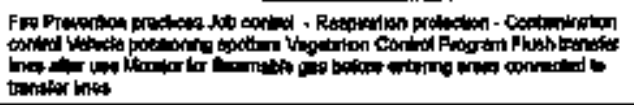 & Mone \\
\hline CRNASU & 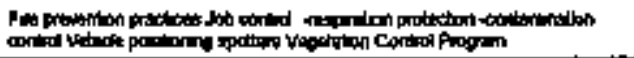 & 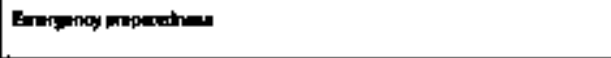 \\
\hline 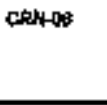 & 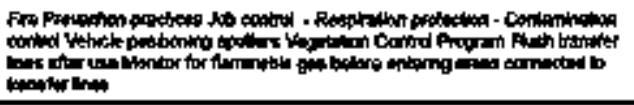 & Nons \\
\hline contand & 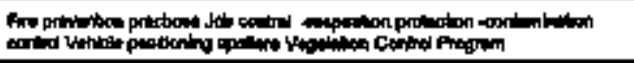 & 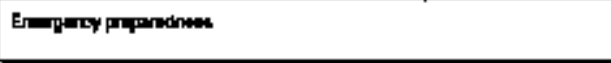 \\
\hline conas & 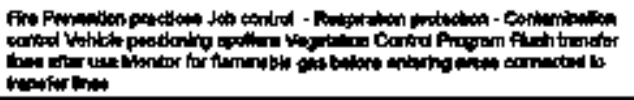 & Nont \\
\hline
\end{tabular}




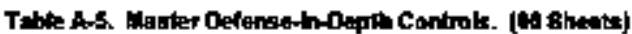

\begin{tabular}{|c|c|c|}
\hline $\mathbf{0}$ & 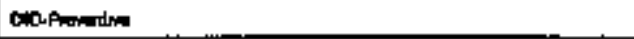 & 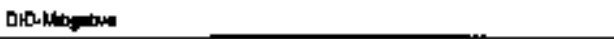 \\
\hline consutit-1 & 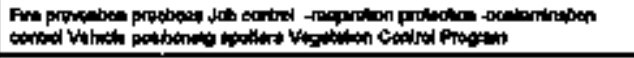 & 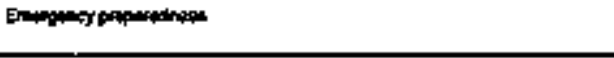 \\
\hline cfandos & 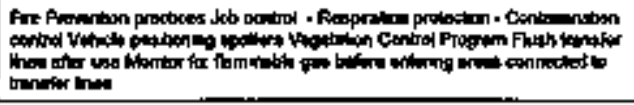 & Hean \\
\hline CAMATH & 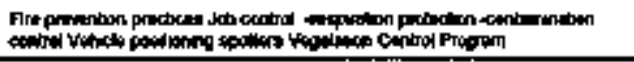 & 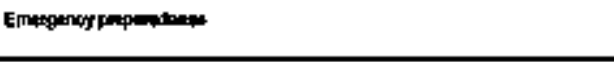 \\
\hline CRKFOS & Norne & 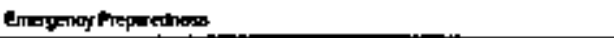 \\
\hline CRNACAM & Morn & 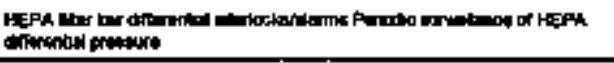 \\
\hline CRHAT & 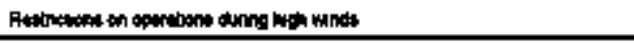 & 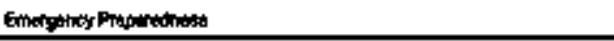 \\
\hline CRNAOH & Natere & 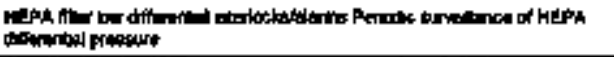 \\
\hline CCFin-11 & 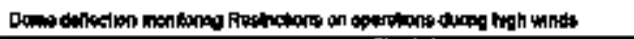 & 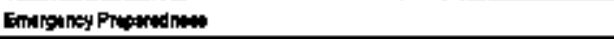 \\
\hline CRat-11-1 & 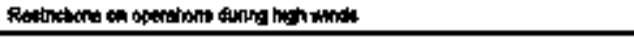 & 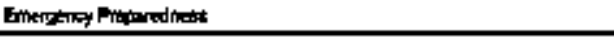 \\
\hline Cant 111B & 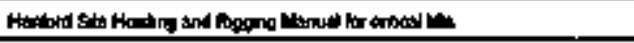 & Mone \\
\hline Cow11. 10 & 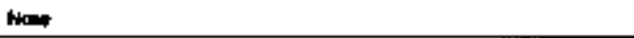 & 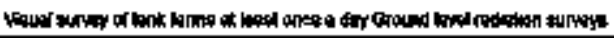 \\
\hline CAan 12 & 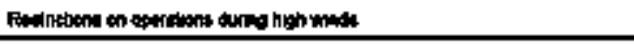 & 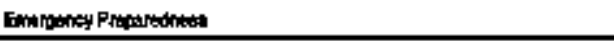 \\
\hline CEAH.12.4h & 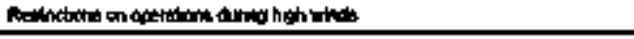 & 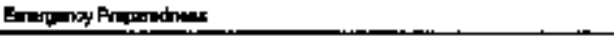 \\
\hline CRM-12: & 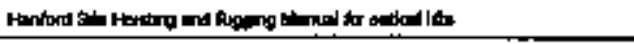 & Hanos \\
\hline CAR-12AC & Nonst & 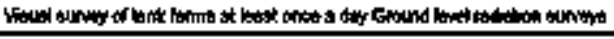 \\
\hline CRHA13H & 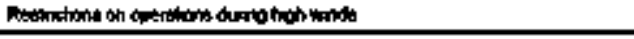 & Nane. \\
\hline CRNGHA & 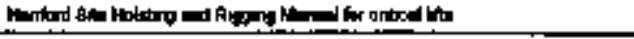 & Mane \\
\hline $\cos (4+13+16$ & 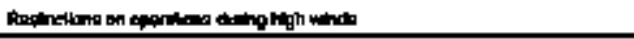 & Nont \\
\hline
\end{tabular}




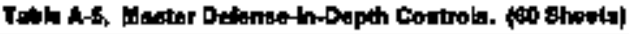

\begin{tabular}{|c|c|c|}
\hline $\mathbf{D}$ & Daphonerture & 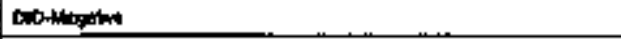 \\
\hline cont-13 & 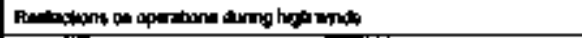 & mon \\
\hline chatista & 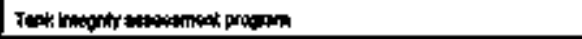 & then \\
\hline CRAN14 & Nane noquend & Done \\
\hline 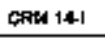 & Mone requind & Mon \\
\hline CRN-15 & Mare nagumed & $\min$ \\
\hline CFA-15-1 & Nome ropurad & $\operatorname{mon}$ \\
\hline CNow 16 & None requitid & Wone \\
\hline ChRM-16-1 & Natie nquand & Mone \\
\hline CRN-17 & 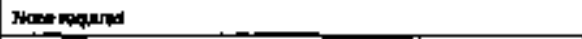 & 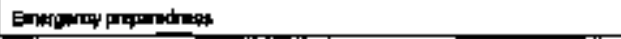 \\
\hline CRRL-1T-1 & Nen manat & Hown \\
\hline CRN-18 & 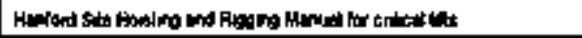 & Natonte \\
\hline CRR $1+16-1$ & 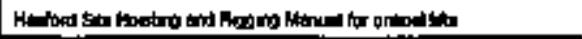 & Non \\
\hline CRH-19 & 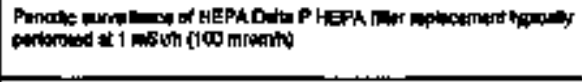 & 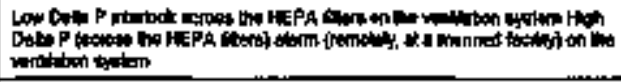 \\
\hline chating & 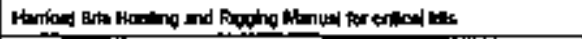 & Name \\
\hline Contros & 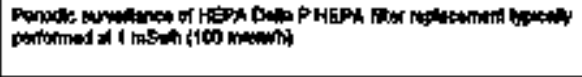 & 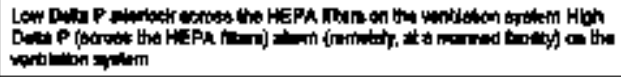 \\
\hline contenter & 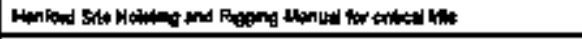 & Nhenes \\
\hline conth21 & Nhthe nater & 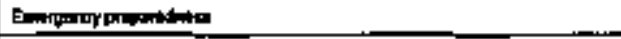 \\
\hline cheng-th & None matapd & Emporopopp \\
\hline CERH21-19 & Ime & Nom \\
\hline ORH $\approx$ & 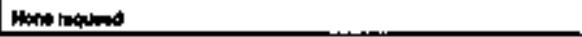 & 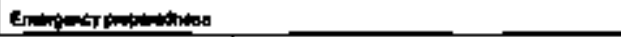 \\
\hline
\end{tabular}




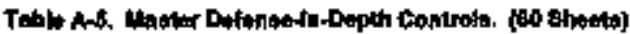

\begin{tabular}{|c|c|c|}
\hline $\mathbf{D}$ & Do-Protution & 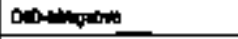 \\
\hline CRN-20-Sh & Mant rengum & Em⿻ \\
\hline CRN-22S: & time & None \\
\hline CRNGB & 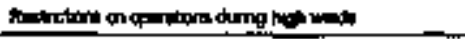 & Whon \\
\hline cravisuk & 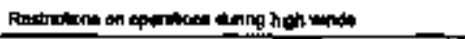 & Nane \\
\hline crat-2s- & 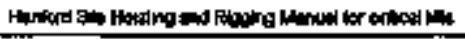 & Nono \\
\hline Chand 24 & blone ropaced & Nonte \\
\hline chans4 & Mote rotand & Non \\
\hline canto & Nane mapend & $\mathrm{Non}$ \\
\hline cring-a & Nom ragroud & Nons \\
\hline Chin-25-10 & 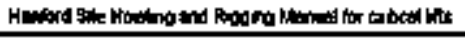 & Nons: \\
\hline cath: & 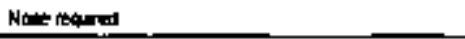 & How \\
\hline CAN會浊 & Nonemenead & lane \\
\hline CRN-23H & 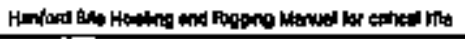 & Nent \\
\hline CRN-2T & Nons anowed & Henst \\
\hline CAN-27.4h & Hontialnow & Hont \\
\hline CRN-ZT-1 & 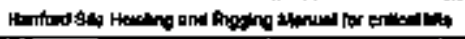 & $\operatorname{man}$ \\
\hline CRN-2\&-1 & $\operatorname{lom}$ & 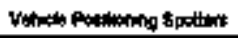 \\
\hline chener & 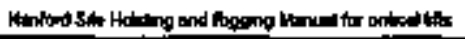 & 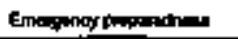 \\
\hline Cyte-1 & 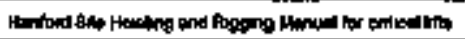 & 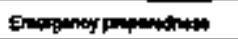 \\
\hline $\cos -\infty$ & 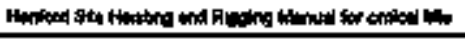 & 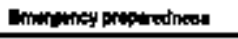 \\
\hline chenes-1 & 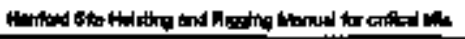 & Emaboingy propendren \\
\hline cutam & 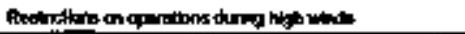 & 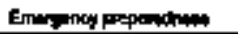 \\
\hline
\end{tabular}




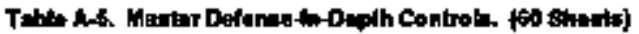

\begin{tabular}{|c|c|c|}
\hline I0 & 6ipsionvitur & 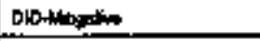 \\
\hline CWR-DSU & 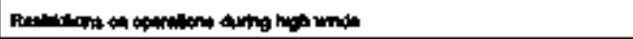 & Enegenop preperedrex \\
\hline CrR-04 & Whot & 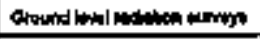 \\
\hline CUR-04 & Atane & 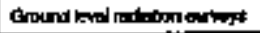 \\
\hline EQReOS & 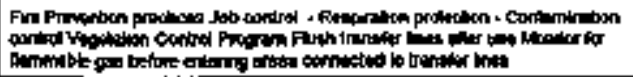 & Wone \\
\hline torintal & 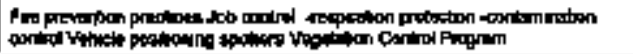 & Who \\
\hline CyR-09 & Nons: & nomo \\
\hline curtont & Nond & Whond \\
\hline Cutitor & None & Whone \\
\hline CyTi-0ra & rign: & Whon \\
\hline CYR-09 & Nong & Who \\
\hline curros & Nart & mond \\
\hline Cundo & Nant & Wone \\
\hline 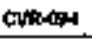 & Nomit & Lond \\
\hline c4R-10 & Hans & Want \\
\hline couplou & Nate & Whota \\
\hline CU/A1 & Mare & Whn \\
\hline CWA-11- & $\operatorname{Hopag}$ & Hane \\
\hline CWA-124 & 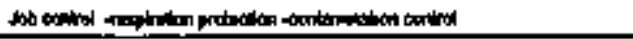 & 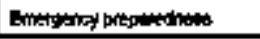 \\
\hline chindu & 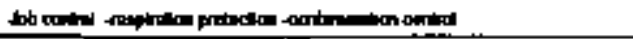 & Emerp rey propothe \\
\hline chit-14 & $\mathrm{How}$ & Wan \\
\hline
\end{tabular}




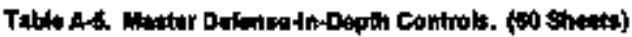

\begin{tabular}{|c|c|c|}
\hline I & oupheorin. & 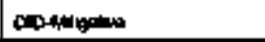 \\
\hline CWM-1E- & 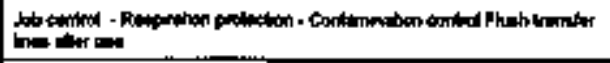 & $\lim$ \\
\hline OCHOI & Nons & inot \\
\hline Octword & Notho & Whone \\
\hline octlos & 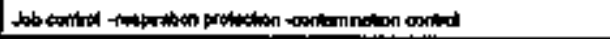 & 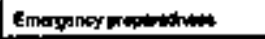 \\
\hline DCN-DQH & 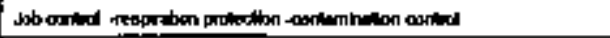 & None \\
\hline DEN-DOA & 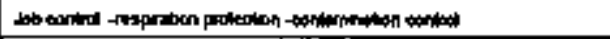 & than \\
\hline Dow & nonst & Nons \\
\hline OCtWat & 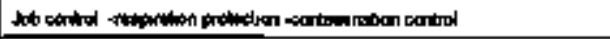 & Mono \\
\hline Demon & 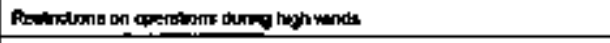 & 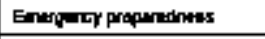 \\
\hline Dexpot & 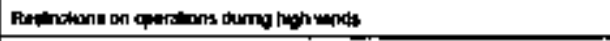 & 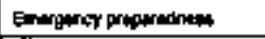 \\
\hline Denos & $\operatorname{lng} 0$ & Nonte \\
\hline Dencosi & Hone & Nane \\
\hline acturs & None & $\mathrm{NanO}$ \\
\hline Desas-1 & mons & Nom \\
\hline DCHOT & tions & Nont \\
\hline DCHOP.1 & ithe & $\mathrm{Nant}$ \\
\hline 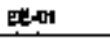 & Mon & 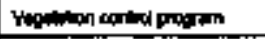 \\
\hline $\mathbf{E - \infty}$ & 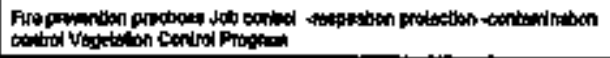 & Nant \\
\hline EE:As & $\lim$ & 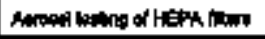 \\
\hline Ex-ata & 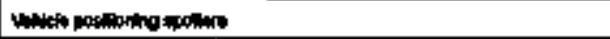 & Nant \\
\hline ES-O4t & 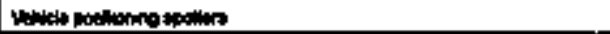 & $\mathrm{Nar}$ \\
\hline
\end{tabular}




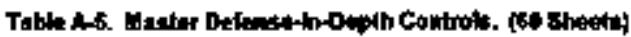

\begin{tabular}{|c|c|c|}
\hline 10 & Doplantions & contuman \\
\hline EE-4SA & 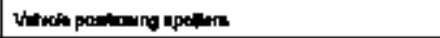 & Hon \\
\hline Eesto & 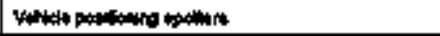 & Nom \\
\hline EE-16 & 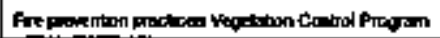 & thent \\
\hline ERAr & None & Whone \\
\hline EE- & hon: & Hone \\
\hline $\mathbf{a}-\infty$ & $\operatorname{lng}$ & 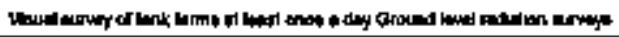 \\
\hline eE-16 & him & 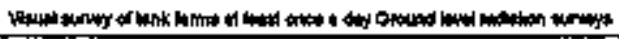 \\
\hline EE-11 & Forace woder mptem frowe probachn & 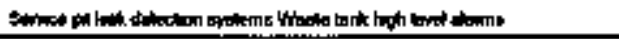 \\
\hline EE-12 & mons & Fone \\
\hline EE-W & Mans & Brone \\
\hline et-14 & Hant & Hons \\
\hline Et-15 & Atare & Hons \\
\hline EE-18 & mate & 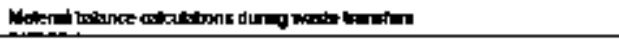 \\
\hline EE-17 & $\operatorname{mon}$ & 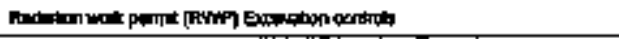 \\
\hline GRNGI & mons & Whon \\
\hline 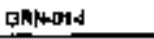 & ntit & mone \\
\hline DRH-D2 & Mut: & Nore \\
\hline GRN-DEA & Mant & $\operatorname{Man} 0$ \\
\hline ctotatos & Bant & Hone \\
\hline $1-2028-1-\cos \theta-A$ & 수엥 & 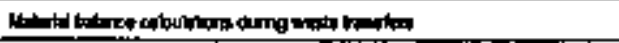 \\
\hline H2025-1-CAPr & Hom & Nonte \\
\hline
\end{tabular}


Tabtu As. Marker Oelengeth-Oepth Coirinota. (wo Bheeta)

\begin{tabular}{|c|c|c|}
\hline 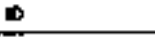 & Copplanontin. & 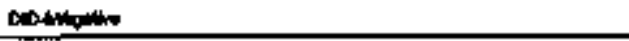 \\
\hline KOAS-1-CNAPCC & Len boting of jum & 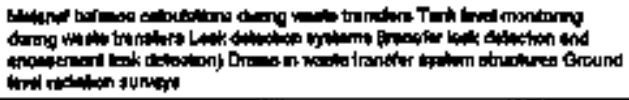 \\
\hline 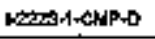 & Nint & Hone \\
\hline 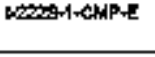 & Noner & 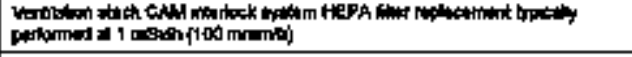 \\
\hline $1-2006-1-44-4$ & 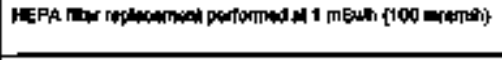 & 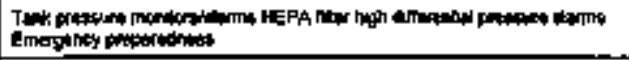 \\
\hline $12 \cos s-1+k \pi n$ & Mone & Pane \\
\hline $1-2208-2-F L W A$ & 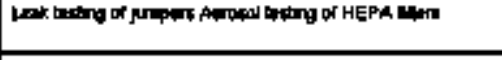 & 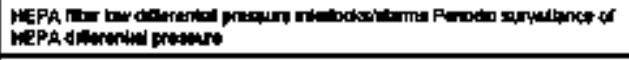 \\
\hline 1-2025-2.plwe & Nons & Mne \\
\hline 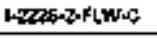 & Nate & Mun \\
\hline 1-22202-2-FLn-D & Naris & Hons \\
\hline $12220-2-15 K A$ & Nomi & Nats \\
\hline 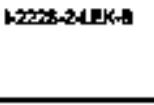 & 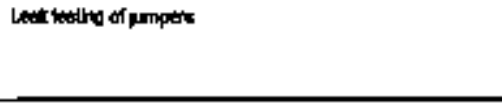 & 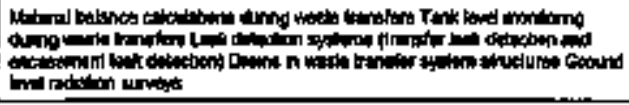 \\
\hline ILZOE-R-AEK-C & 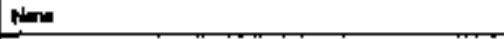 & 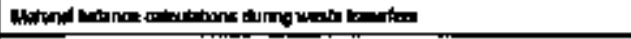 \\
\hline I.ZOPSAREKOD & Nons & Noters \\
\hline KOEOE-ZAEKE & Nom & 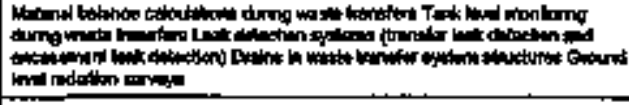 \\
\hline $1-Z \operatorname{Cos}-2-\operatorname{Pag} \alpha$ & 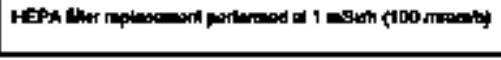 & 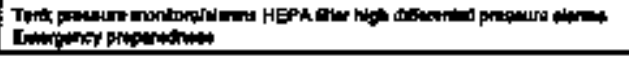 \\
\hline
\end{tabular}




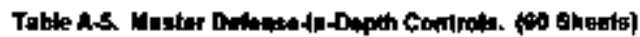

\begin{tabular}{|c|c|c|}
\hline p & DIDPraming & chandpare \\
\hline $1342+1-P u m-k$ & Fammitio pas morria & Hor \\
\hline heshentwa & 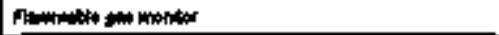 & hon: \\
\hline 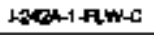 & 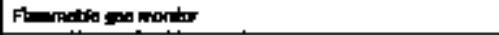 & Nand \\
\hline 1-7be-1-fitw-0 & 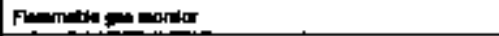 & Nhot \\
\hline 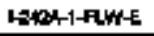 & Prenmetila Fardar & Mant \\
\hline 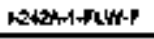 & Nom & Wone \\
\hline 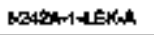 & Nom & Whon \\
\hline $10424+1+10 \times 6$ & Leek treting of Mraper & 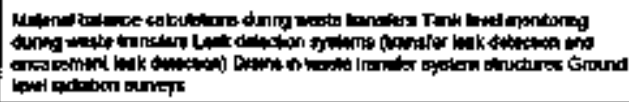 \\
\hline $1-2+23-1-\angle E K C$ & Horm & 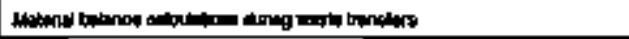 \\
\hline 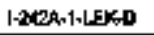 & Hang & Nate \\
\hline $\mid-200 x-1 \cdot 10 x=$ & land & 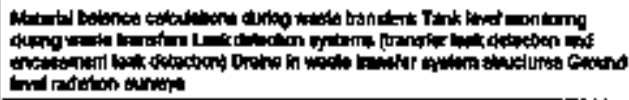 \\
\hline 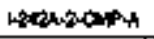 & Whan & Hon: \\
\hline $1304-2-c h a n$ & 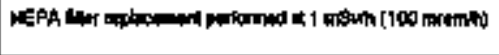 & 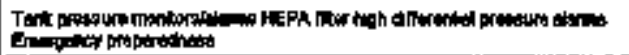 \\
\hline 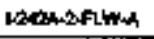 & Phan & 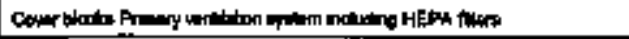 \\
\hline 1-201-2.FWH & Mond & 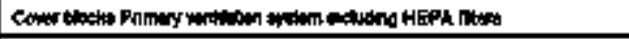 \\
\hline 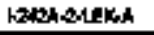 & Mans & Ho: \\
\hline WDALEREB & 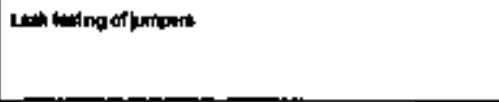 & 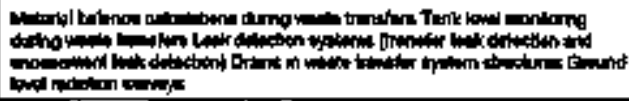 \\
\hline
\end{tabular}




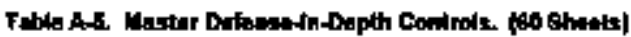

\begin{tabular}{|c|c|c|}
\hline $\mathbf{0}$ & DiDFmintow & 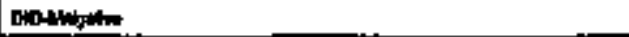 \\
\hline $1-2+24-210 \times-6$ & Utart & 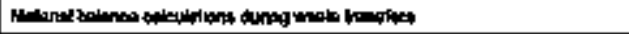 \\
\hline $\operatorname{latah-2-1ex-\theta }$ & Nom & Honte \\
\hline sedontelexe & Howe & 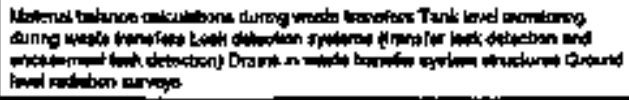 \\
\hline 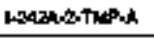 & Hare & Kone \\
\hline 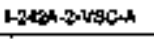 & Nom & 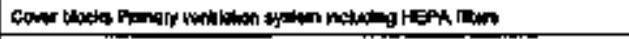 \\
\hline $1-200+2-490-0$ & Nenses & Hons \\
\hline 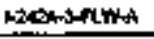 & 西 & 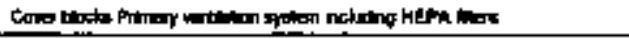 \\
\hline 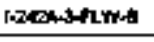 & None & 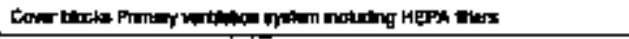 \\
\hline $1.2004-42 m$ & 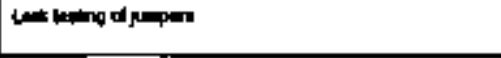 & 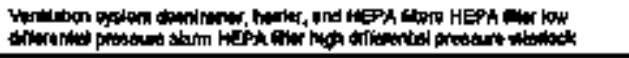 \\
\hline 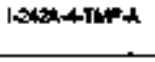 & Nons & 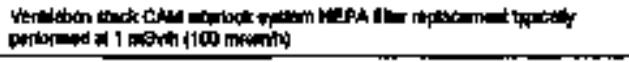 \\
\hline $1-2+2 k-5-\pi L W-\lambda$ & None & $\sin 0$ \\
\hline $1-2 * 26, \theta-C M P A n$ & 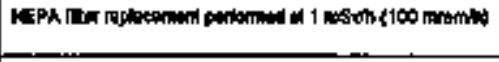 & 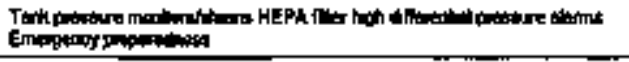 \\
\hline Hatertawh & 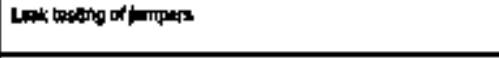 & 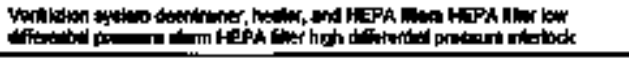 \\
\hline nedoutflive & 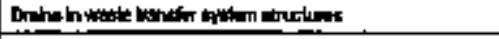 & 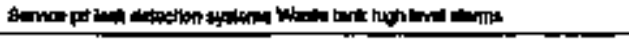 \\
\hline LECA-FLW-C & Kin & mono \\
\hline 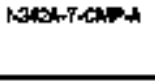 & thocontol & Mons \\
\hline $1-2 R+2-F \mid W A$ & Notent & 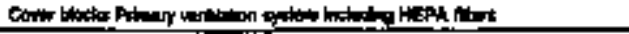 \\
\hline
\end{tabular}




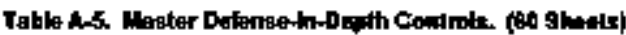

\begin{tabular}{|c|c|c|}
\hline 10 & Confongtive & 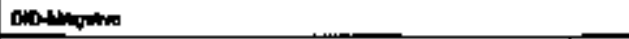 \\
\hline 1-20201-FLthe & Nen & 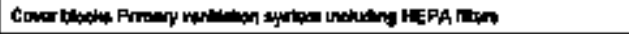 \\
\hline 1.242A-7-FLWLC & Hint & 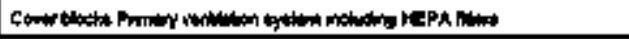 \\
\hline 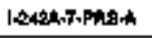 & None & Mare \\
\hline 1-2428-1-PRS-A & 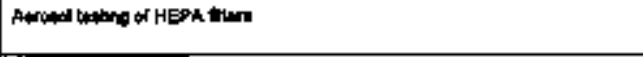 & 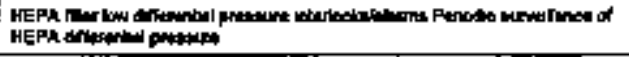 \\
\hline 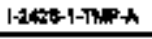 & mollowerg tord hrattits & 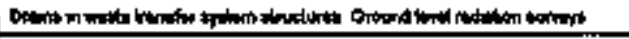 \\
\hline 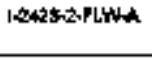 & 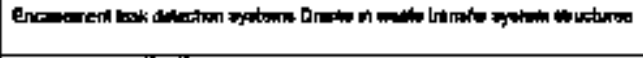 & 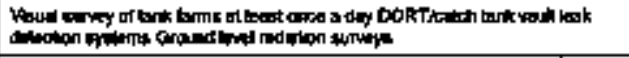 \\
\hline 1-242B-2-FLincB & 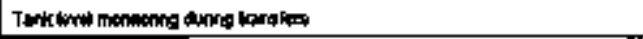 & mans \\
\hline $\log 425-2-F L i n-C$ & Hine & none \\
\hline $1+2025-2 \cdot \ln m-0$ & None & Mane \\
\hline 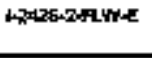 & Hon & 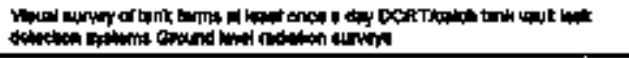 \\
\hline $1+2425-2+15 k-1$ & Hone & Nans \\
\hline 1-205-2AEKA & 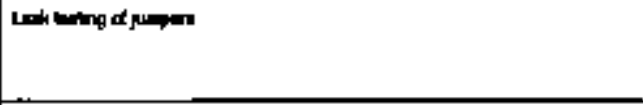 & 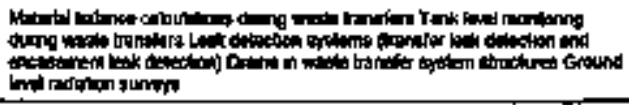 \\
\hline $1200-2 \cos c$ & Wante & 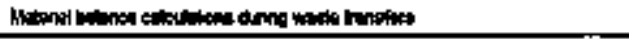 \\
\hline 12CS-2utaxo & Hond & Nate \\
\hline W2WOALER & Name & 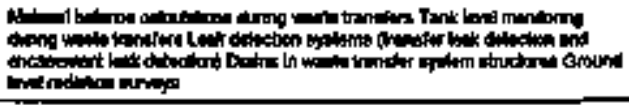 \\
\hline LOCES-ARWhA & Nente & Nom \\
\hline 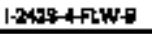 & 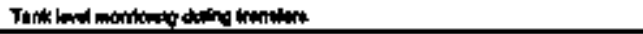 & Ning \\
\hline
\end{tabular}




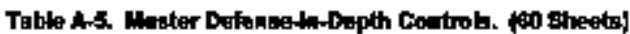

\begin{tabular}{|c|c|c|}
\hline 10 & Diventwom & 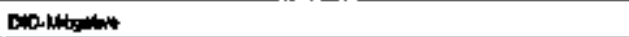 \\
\hline 1-20208-4ALthec & thane & 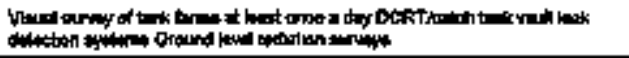 \\
\hline 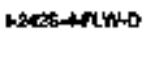 & 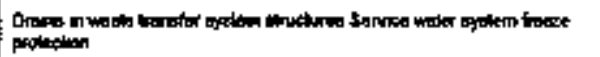 & 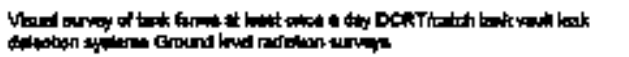 \\
\hline $1-25 \%-4 E k-\lambda$ & 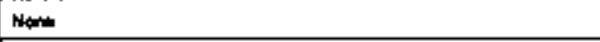 & $\operatorname{lom}_{4}$ \\
\hline $1-200-41 E K-B$ & 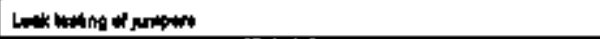 & 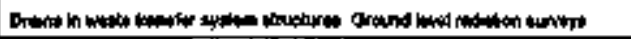 \\
\hline 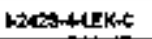 & Mare & Mone \\
\hline KaCS-4AEKOB & rater & Stone \\
\hline LOCS4HEK-E & Mant & 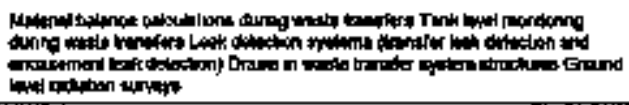 \\
\hline $1-2425-5-C M P A$ & 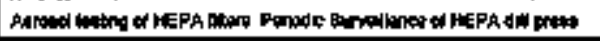 & 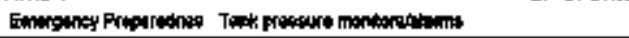 \\
\hline 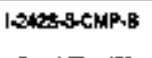 & 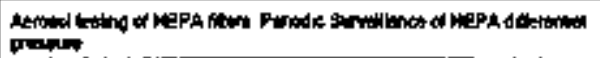 & 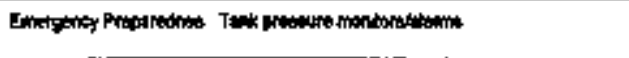 \\
\hline $1-2428-5-919 P-C$ & 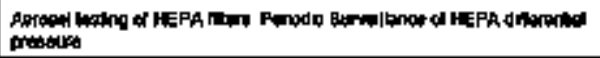 & 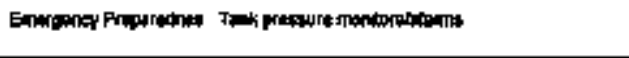 \\
\hline 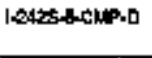 & 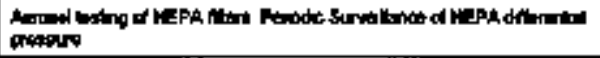 & 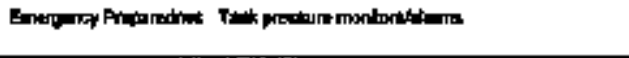 \\
\hline $1-26205+7 W h$ & 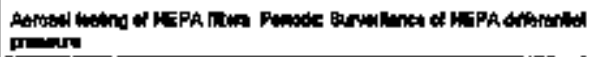 & 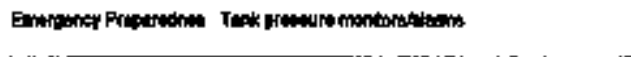 \\
\hline 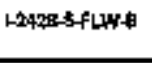 & 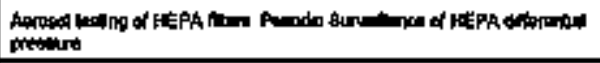 & 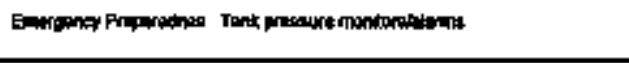 \\
\hline Latastrits & 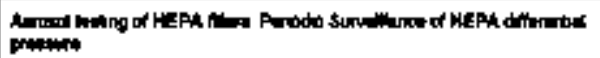 & 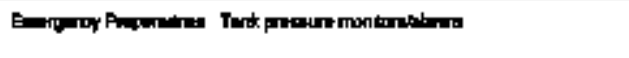 \\
\hline 120as-6hs-h & 舟 & Nath \\
\hline 1-20T-2Atwh & Hon & Hane \\
\hline
\end{tabular}




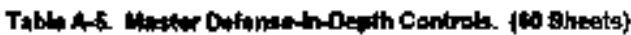

\begin{tabular}{|c|c|c|}
\hline 吅 & DIDPsanatia & 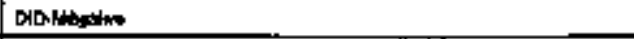 \\
\hline 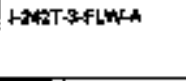 & 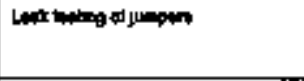 & 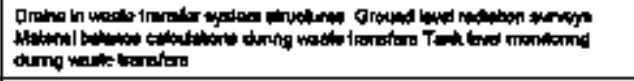 \\
\hline 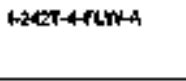 & 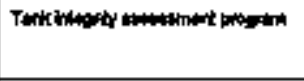 & 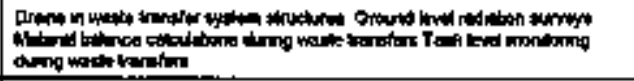 \\
\hline HPLLATII-CWPA & Nent & $\mathrm{Han}$ \\
\hline MBPLAWT-1-Canp-6 & Coch wing of jumpors & 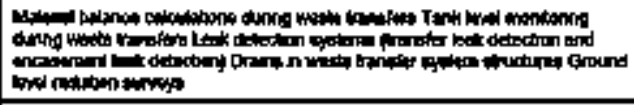 \\
\hline 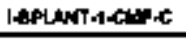 & Hone & tions \\
\hline 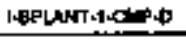 & Mone & Hond \\
\hline HBPLANT-1CGPEE & than & 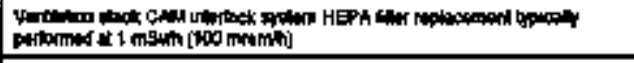 \\
\hline I.ARLANTH-THAPA & Notre & 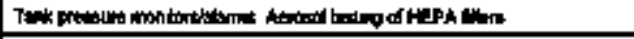 \\
\hline 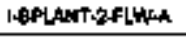 & ron & Hon \\
\hline 1-GPLANT-2FLพพ-日 & none & $\operatorname{lng}$ \\
\hline IERLANTRZPLWLC & Hone & nom \\
\hline IARLATTEFAWD & Mn & Hone \\
\hline HPANMT-2HEK-A & Kane & Nane \\
\hline 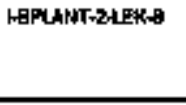 & 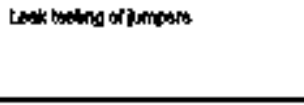 & 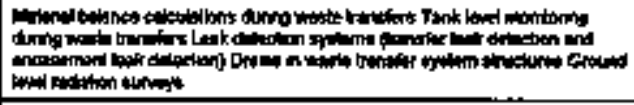 \\
\hline LEPANTALERC & Name & 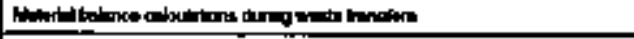 \\
\hline LEPUNT-ALExd & None & Mone \\
\hline
\end{tabular}




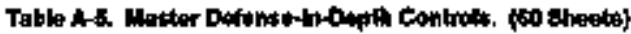

\begin{tabular}{|c|c|c|}
\hline ID & Depremoter: & 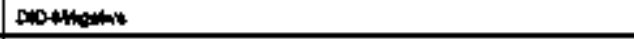 \\
\hline IAPLANT-2-LEXE & Now & 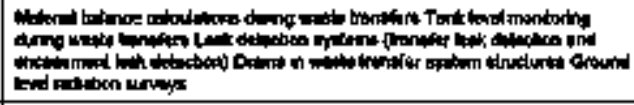 \\
\hline HPFP14AGT/4h & mant & $\lim$ \\
\hline LPFP.1-CoPrah & Nats & $\operatorname{man}$ \\
\hline LPFP-1-caip-6 & Nom & mons \\
\hline 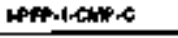 & Nenter & Mare \\
\hline IPAPMACA & here & $\cos =$ \\
\hline |PFP-14GPEE & Nans & 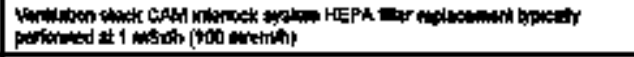 \\
\hline IAPA-1LVLA & 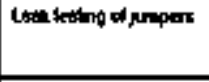 & 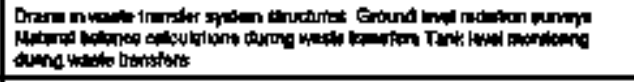 \\
\hline HPR-HATHA & Nhent & Nons \\
\hline LeppuzFind & None & $\mathrm{Hag} \rightarrow$ \\
\hline HFPADEKAM & abron & $\mathrm{Nans}$ \\
\hline HPFP-भLEK-A & Low ining of impere & 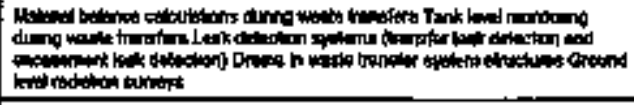 \\
\hline BFFP.ZLEKC & Min & 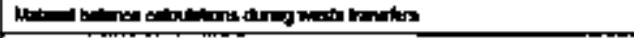 \\
\hline 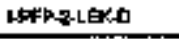 & thene & Hein \\
\hline HeFP-2Lax-E & Wim & 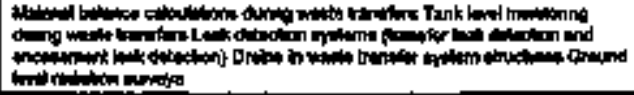 \\
\hline
\end{tabular}




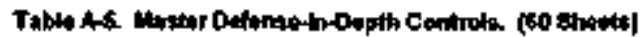

\begin{tabular}{|c|c|c|}
\hline $\mathbf{0}$ & DIDPTrumbin & 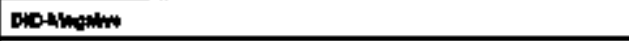 \\
\hline 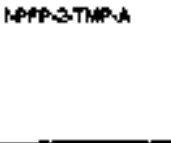 & How & 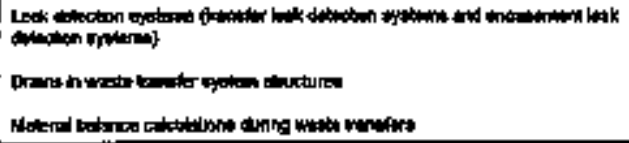 \\
\hline LPPP.2.TMP,B & 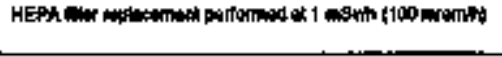 & 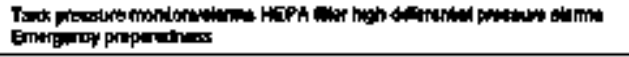 \\
\hline HPP-3GMPA & 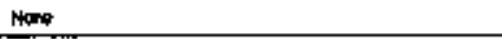 & Hone \\
\hline 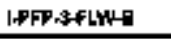 & Nam & Nons \\
\hline IPPR-SELWHEC & Nater & 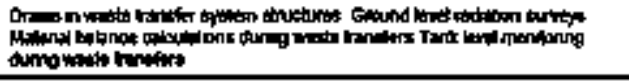 \\
\hline HEPPHHEKa & Nher & Nore \\
\hline 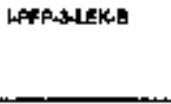 & 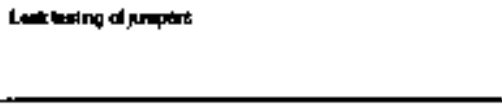 & 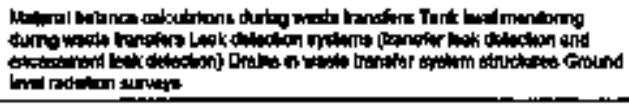 \\
\hline LPFALLEKCE & mone & 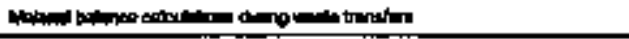 \\
\hline IPFP-3-LIK-0 & $\operatorname{lon}$ & Now \\
\hline IATP-3LLER & Whe & 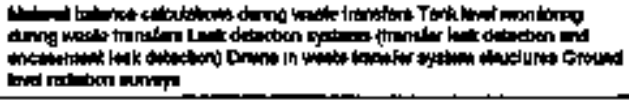 \\
\hline IPLTEEX-1 ABT-A & tonne & and \\
\hline If PUREX-1+46-1 & Hante & Nom \\
\hline IPUPAR-1 AGT-C & Nothe & now \\
\hline LEVREX & Nane & Nenes \\
\hline LPUREX-1-CFR-B & Nimo: & nom \\
\hline
\end{tabular}




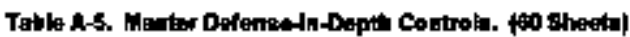

\begin{tabular}{|c|c|c|}
\hline$\omega$ & DAPTomthn & 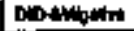 \\
\hline LPUREX-1 GaPAC & Hont & Wone \\
\hline MUAEEX-1-CNOD & Hom & Mons \\
\hline 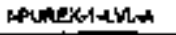 & Eare & Wone \\
\hline 1PUREX-1-thm & Bare & Wone \\
\hline 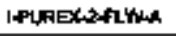 & Elore & Kone \\
\hline HPUEX-Z-CEK-A & Nont & Marin \\
\hline IHURDX-2-LEKK & Natid & Wothe \\
\hline 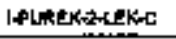 & Nowe & Prom \\
\hline |.PUAEX_ZL, EK-D & Now & Hpon: \\
\hline HPUREX-ZHEK-I & Nang & Hont \\
\hline IFURDX-2-PRBA & Nown & Harne \\
\hline HPURDGZPRSA & None & Han \\
\hline 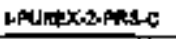 & None & Hos \\
\hline 1PWQ्रX-3ACT-A & None & Rom \\
\hline 1-Pugx $-3-467.4$ & Hone & Nhat \\
\hline 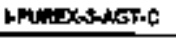 & libne & $\mathrm{Ho}=$ \\
\hline 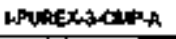 & Hone & Nong \\
\hline HPITIX-3-GMPE & lable & Nund \\
\hline 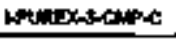 & thene & Han \\
\hline Ifthex-3-cter & none & Nowe \\
\hline 19yAIx-3-Gipe & wonte & Hond \\
\hline IPUnEx-3HWh & rore & Hant \\
\hline
\end{tabular}




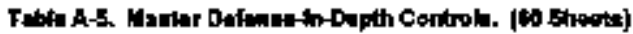

\begin{tabular}{|c|c|c|}
\hline 10 & 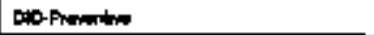 & 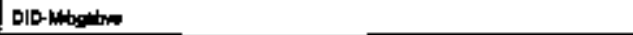 \\
\hline IFURE $x \rightarrow-T M P A$ & flone & Nong \\
\hline HPUREX-4FLH-B & Nown & $\mathrm{Nen}$ \\
\hline HPURAK-MUKCh & Nhon & Nome \\
\hline RPURDXALEKB & Narna & Noment \\
\hline IPUREXUAEKGE & Nime & $\mathrm{Nat}$ \\
\hline IPUREX,4UEK-D & Hinnt & Nomen \\
\hline IPUREX-4LFKL & Aland & Now \\
\hline HMUREX-4PASA & Nond & Mane \\
\hline 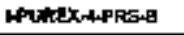 & None & $\mathrm{NaO}$ \\
\hline IPUTREX APREBC & Whot & Hornt \\
\hline HPUREX-5-FLWh & hane & Hane \\
\hline IPUPEX-5-PLWA & Note & What \\
\hline 1.PLinexofumah & None & Hbon \\
\hline IPLNESXAFUHB & 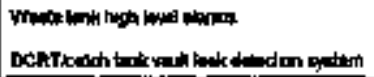 & 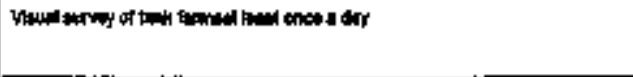 \\
\hline HPLAEX-HLW-C & 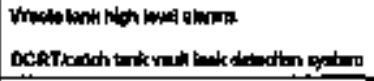 & Nonn \\
\hline LELAEX-4RW-D & Kinn & None \\
\hline HTPAAKT-A-CHPA & Btore & lowe \\
\hline ITPLANT-1-CMPAB & 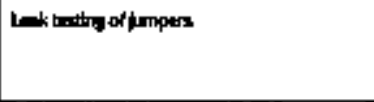 & 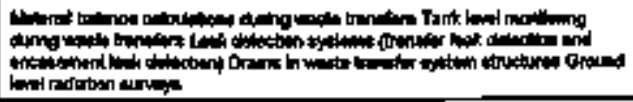 \\
\hline
\end{tabular}




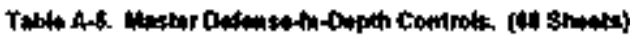

\begin{tabular}{|c|c|c|}
\hline 中 & Lophintin & 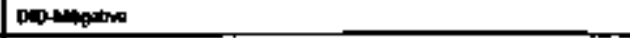 \\
\hline -EPLANT-1-CWP-C & Nions & monsto \\
\hline 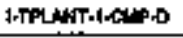 & Hone & Wone \\
\hline ATPUNT-I-TLPA & thens & 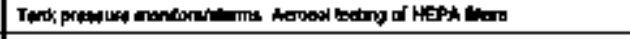 \\
\hline 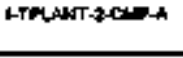 & 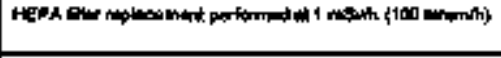 & 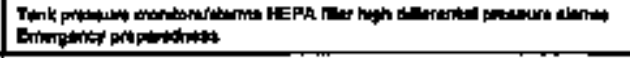 \\
\hline 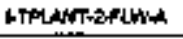 & Hone & Mare \\
\hline 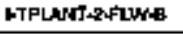 & None & Hore \\
\hline ITPLANT-2-LEXA & mans & Nons \\
\hline RTPLNAT-2LEX: & Letk wing of jumpors & 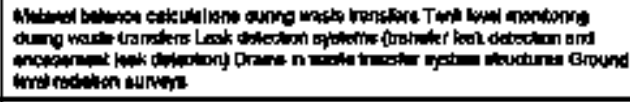 \\
\hline 1.TPLARTHLLEKC & Mant & 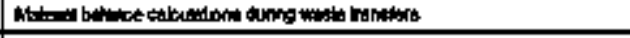 \\
\hline 1.TALANTALEKOO & xare & $\operatorname{Han}$ \\
\hline I.TPUAM-PLEKE & 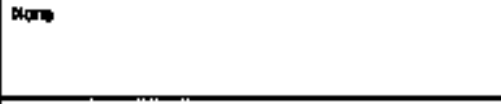 & 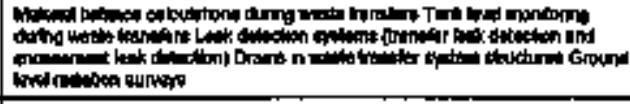 \\
\hline 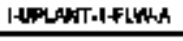 & Hone & Hane \\
\hline IAPLLAT.1AEKGh & Nate & Han \\
\hline |LPLAMT:1LEKG & Name & 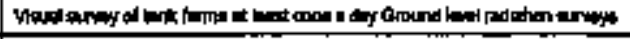 \\
\hline HOLAHT-1LEKC & Nant & the \\
\hline 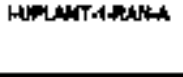 & 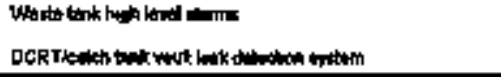 & Hon \\
\hline TTK-91 & $\mathrm{Nath}$ & 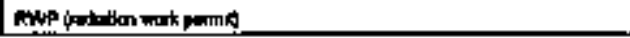 \\
\hline
\end{tabular}




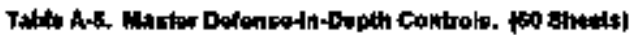

\begin{tabular}{|c|c|c|}
\hline 10 & 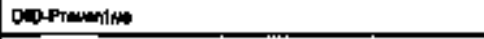 & 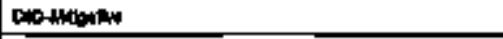 \\
\hline Trsor-1 & Mons & 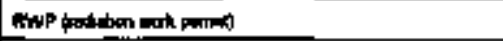 \\
\hline rTKo: & None & 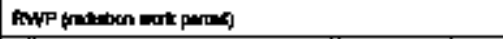 \\
\hline ITKA & Nonos & 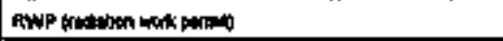 \\
\hline $\operatorname{mass}$ & Nom & 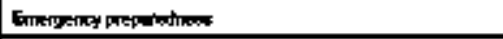 \\
\hline Imoras & How & Emerpency phepufudition \\
\hline ITK-DA & 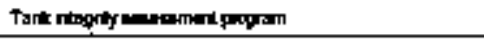 & $\operatorname{man}$ \\
\hline IIK-OA| & ronst & 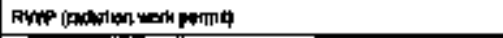 \\
\hline ITKAS & Nenes & 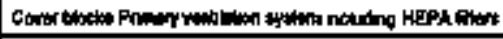 \\
\hline Ink-0ot & Han & Mone \\
\hline dTK-06 & Hone & mon \\
\hline$\pi k-0 B-1$ & Mons & Hom \\
\hline ITKEN & 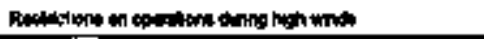 & 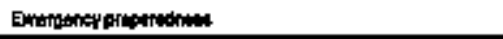 \\
\hline nkos.hen & Ions & RU/P (radstint work patinl) \\
\hline Ithotols & 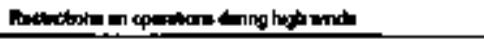 & 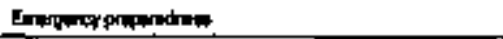 \\
\hline mKen & 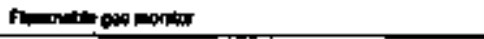 & Hon \\
\hline ImKasol & 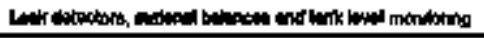 & Nonte \\
\hline ITKs & 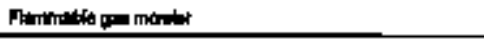 & blone \\
\hline TK-09H & 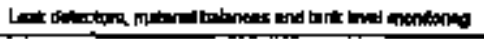 & then \\
\hline InK.10 & 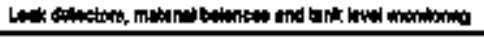 & Whene \\
\hline ITRoran & 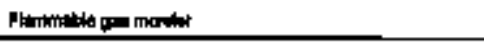 & Hone \\
\hline Thk-106 & Nan & kims \\
\hline trk-11 & 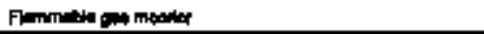 & nist \\
\hline
\end{tabular}




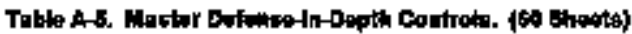

\begin{tabular}{|c|c|c|}
\hline $\mathbf{0}$ & 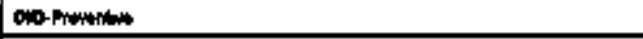 & 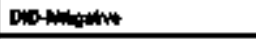 \\
\hline $17 k-114$ & 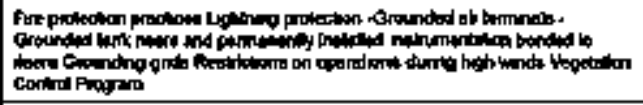 & Wone \\
\hline ITK-FR-DH & 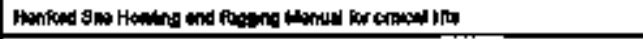 & 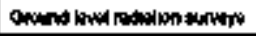 \\
\hline MK-FR-DZ & 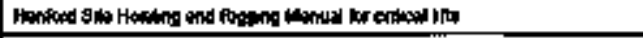 & 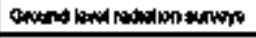 \\
\hline 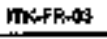 & 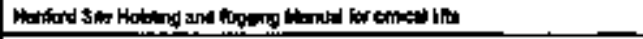 & Mons \\
\hline tmKark-04. & Monen & 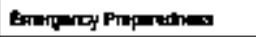 \\
\hline InKFrand & 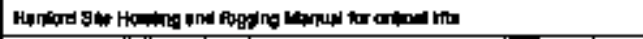 & 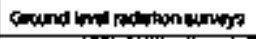 \\
\hline InK-FR-DQ & 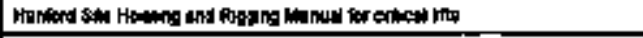 & 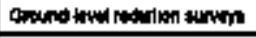 \\
\hline ITKFR-DS & 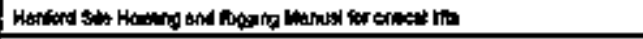 & 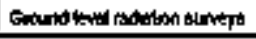 \\
\hline InKAROD & 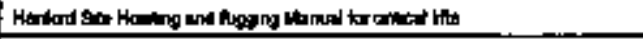 & 배 \\
\hline TKसमres & rant & 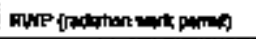 \\
\hline TKKFE-10 & Nome & 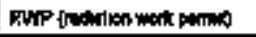 \\
\hline MKFR-19 & thenter & 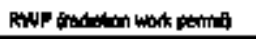 \\
\hline mKGPR:12 & bin & 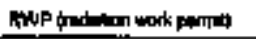 \\
\hline ITKARR-13 & 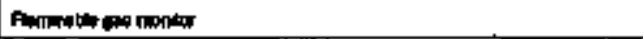 & Nons \\
\hline TKK-FR-14 & 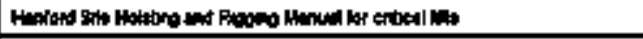 & 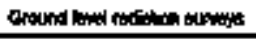 \\
\hline ITK-PM-15 & 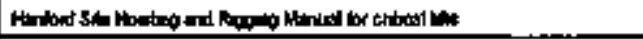 & 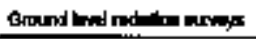 \\
\hline ITKSFA-16 & Nhne & 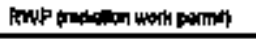 \\
\hline TKKFFR-1T & Ninent & 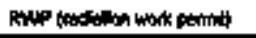 \\
\hline Ins-ar-15 & 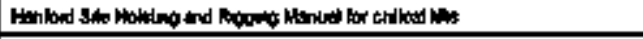 & 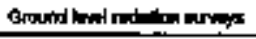 \\
\hline mequ-19 & Hone & 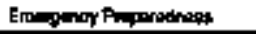 \\
\hline
\end{tabular}




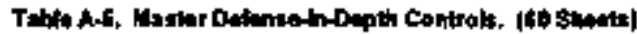

\begin{tabular}{|c|c|c|}
\hline 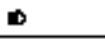 & Cab-Pwartive & 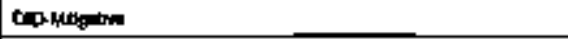 \\
\hline ITK-FR-20 & 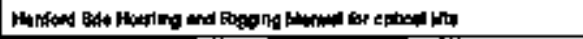 & 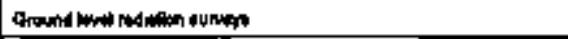 \\
\hline MKk-PR-Zi & 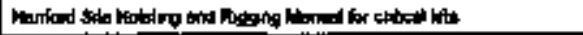 & 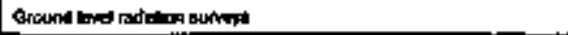 \\
\hline TKKFR-22 & 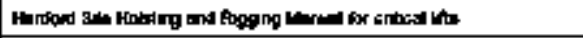 & then \\
\hline HTK.FR-23 & 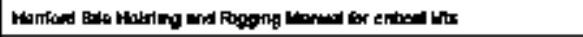 & 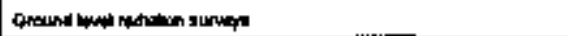 \\
\hline ITK-FR-2M & Won & 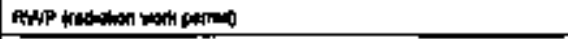 \\
\hline 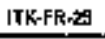 & mate & 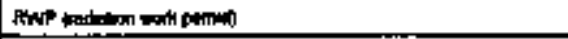 \\
\hline ITKaPR= & 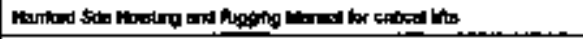 & 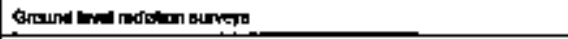 \\
\hline ratikzz & Nant: & 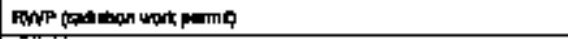 \\
\hline marta-29 & 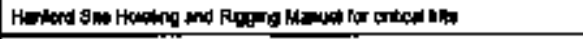 & 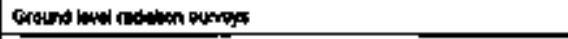 \\
\hline NPA-A & 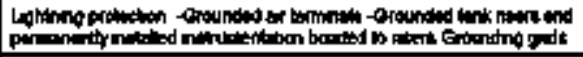 & mote \\
\hline NPQR & 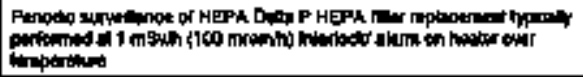 & 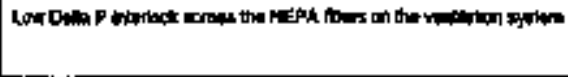 \\
\hline NPAOS & 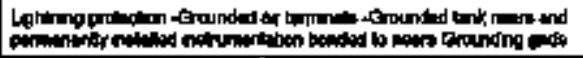 & $\operatorname{lom}$ \\
\hline NP-15 & 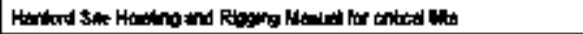 & Morre \\
\hline NPAD & 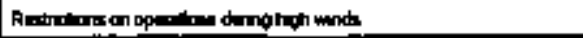 & bon \\
\hline mater & 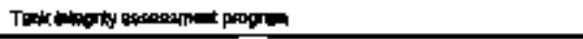 & 40 \\
\hline 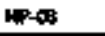 & Whent & 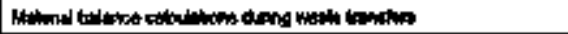 \\
\hline 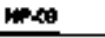 & 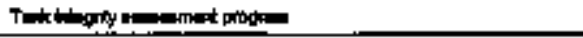 & Mane \\
\hline Mposo & Mang & Emaraney Dap \\
\hline UP-11 & Nons & How \\
\hline
\end{tabular}




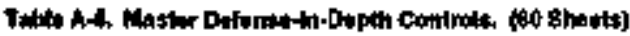

\begin{tabular}{|c|c|c|}
\hline 10 & 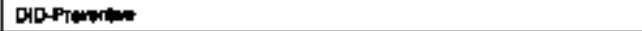 & Dovergen \\
\hline $\operatorname{ap} 12$ & 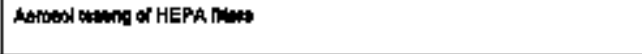 & 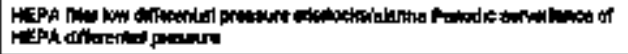 \\
\hline 0.13 & 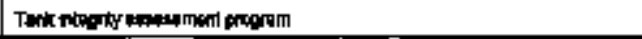 & Hons \\
\hline $\operatorname{men} 4$ & 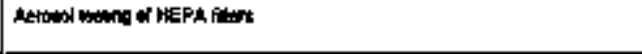 & 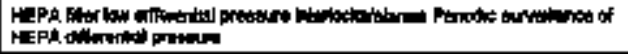 \\
\hline LP-15 & Hon & None \\
\hline OाЕXTअ्जीA & 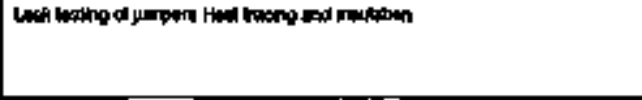 & 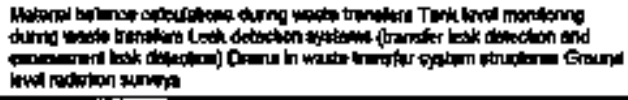 \\
\hline Cotext3ong & 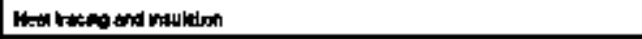 & 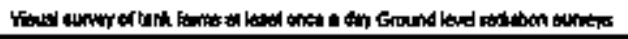 \\
\hline CATALONIAI & Whan & No \\
\hline QGT-ALOHADZ & fenet treng andmatice & 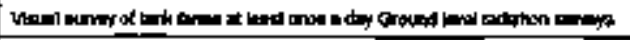 \\
\hline OOT-RLWH-DG4 & 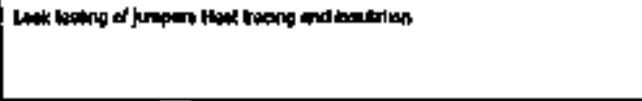 & 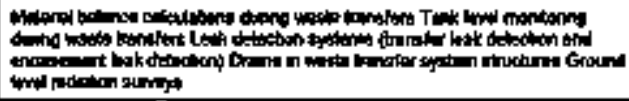 \\
\hline 06T-MOH- $0 \%$ & 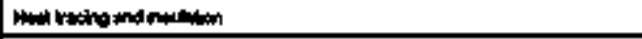 & 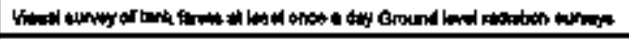 \\
\hline OGI HONHAt & 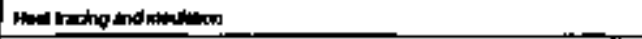 & 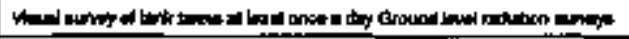 \\
\hline QధTT-FLONY-06 & Nons & Tark Lend litentorng \\
\hline 06-FمشN-06 & 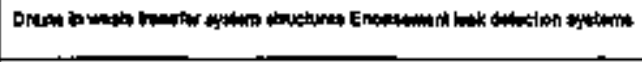 & 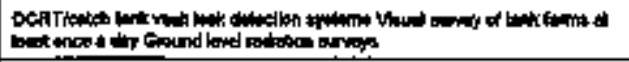 \\
\hline 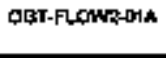 & 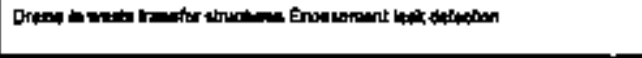 & 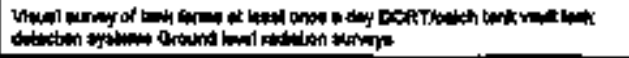 \\
\hline 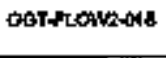 & 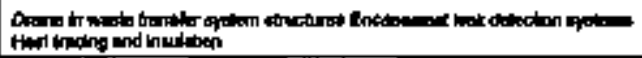 & 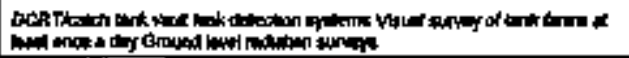 \\
\hline
\end{tabular}




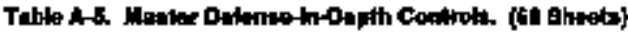

\begin{tabular}{|c|c|c|}
\hline 0 & DID Phon & 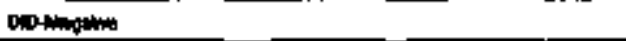 \\
\hline 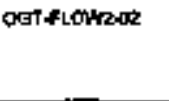 & 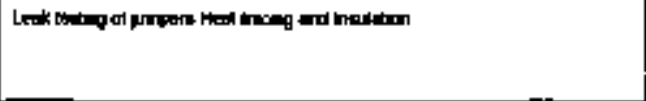 & 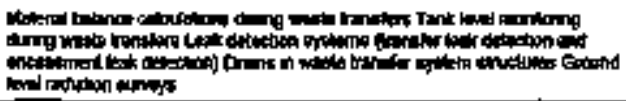 \\
\hline OOT-ROMN2-03A & 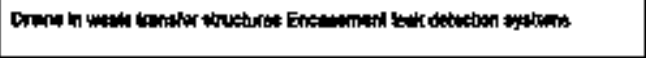 & 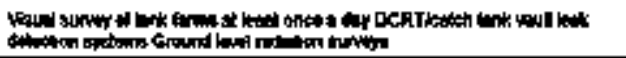 \\
\hline 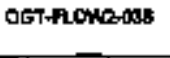 & 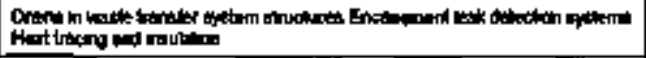 & 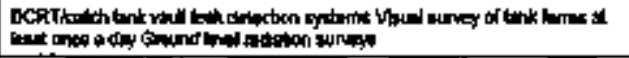 \\
\hline OCTANTEDSOI & 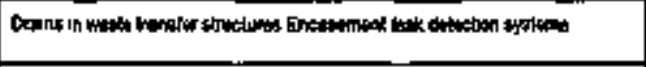 & 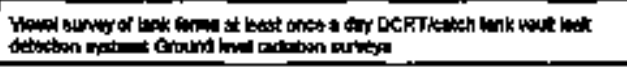 \\
\hline DBT-NPBOn & 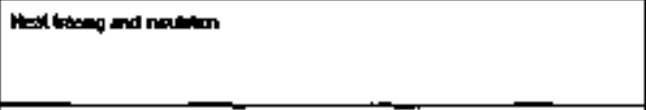 & 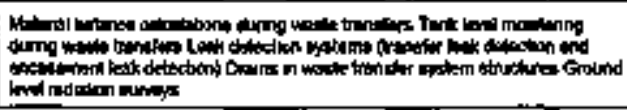 \\
\hline oxt-WPBon & 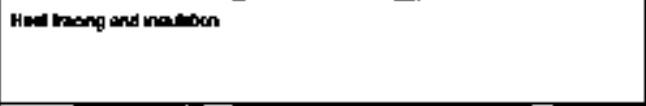 & 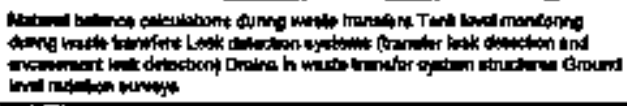 \\
\hline OCT-CPS1-01 & 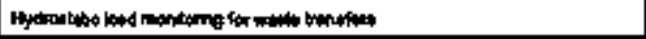 & 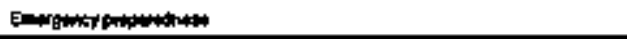 \\
\hline DETHPEBI-OA & 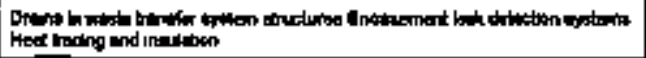 & 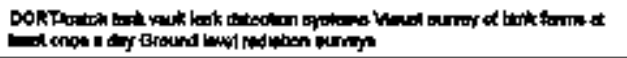 \\
\hline OAT FERC1-OM & 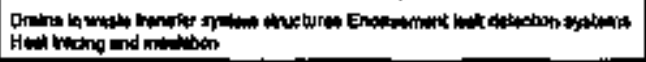 & 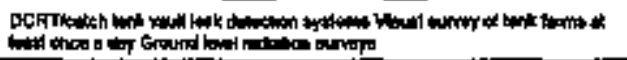 \\
\hline OAT-SPEC1401 & Horne. & Nowe \\
\hline 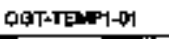 & Nane & nimon \\
\hline OOT-TENPHOA & 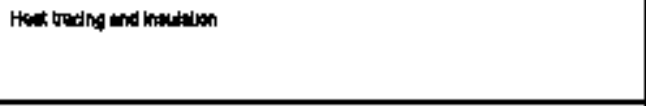 & 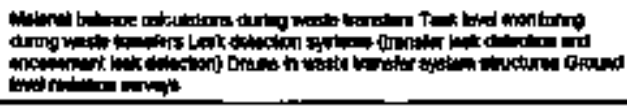 \\
\hline DGT-THWATH & 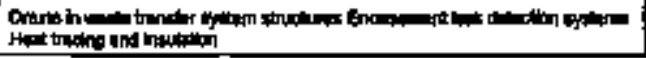 & 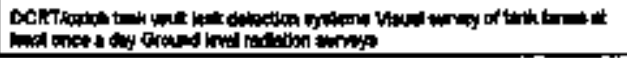 \\
\hline
\end{tabular}




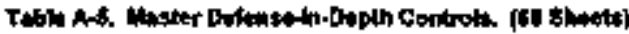

\begin{tabular}{|c|c|c|}
\hline$\omega$ & DIDPraxilin & 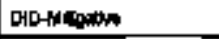 \\
\hline nABOb & $\mathrm{Nom}=$ & thent \\
\hline FADDIA & $\mathrm{Han}$ & Nort \\
\hline RNDO2 & Nom & Noma \\
\hline NoDOSA & Ntent & $\mathrm{Nlom}$ \\
\hline expos & Nomet & Nonet \\
\hline RADL-00- & Nenes & Hone \\
\hline PAPR-D1 & Nenes & 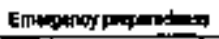 \\
\hline RSR-01-1 & Nenten & 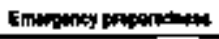 \\
\hline MSTR-DR & inne & Hone \\
\hline Ass & Hone & Hente \\
\hline$A B R 00$ & Mon & $\operatorname{mon}$ \\
\hline RRPR-03H & nome & Wons \\
\hline$R \& R$ RA & Nbond & Ethorgenty mopulatits \\
\hline Rssout & Hon & 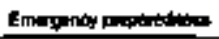 \\
\hline Aaros & Nome & 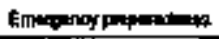 \\
\hline RgR-0S-n & None & Ewaraney proponthodt \\
\hline esines-18 & Btoren & 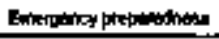 \\
\hline 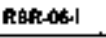 & 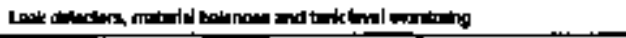 & Whon \\
\hline R日R-OP- & 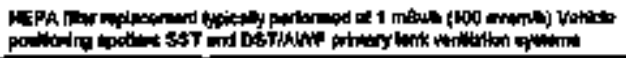 & min \\
\hline Agatoll & Wone & Whn \\
\hline EFF-01 & Nonter & 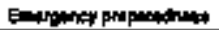 \\
\hline
\end{tabular}




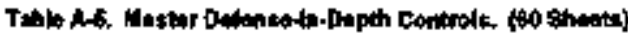

\begin{tabular}{|c|c|c|}
\hline H & phopreneme & 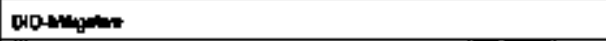 \\
\hline arFalt & Honto & Hon \\
\hline ThE-1 & 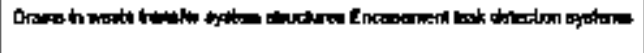 & 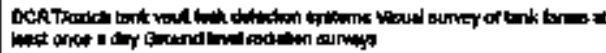 \\
\hline VEth & 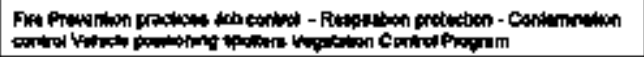 & Wons \\
\hline ventar-1 & 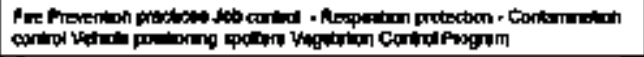 & Anom \\
\hline VEHeC & Nomente & rente \\
\hline VEHOLA & the & Natit \\
\hline 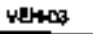 & Hone & Nans: \\
\hline МEHOSH & nonst & Nomen \\
\hline YEHमX & 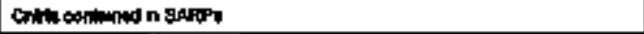 & Consentaned in SARPF \\
\hline YEHATA & 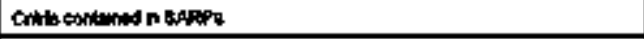 & 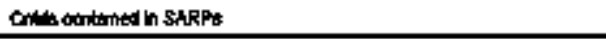 \\
\hline vethos & 散 & Nom \\
\hline VEHOSH & inn & $\operatorname{lnon}$ \\
\hline WEHAD & Noms & Nots \\
\hline VEHA-06-1 & thentio & Athente \\
\hline VItas & 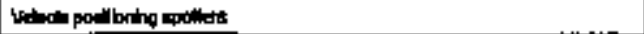 & $\mathrm{mann}$ \\
\hline 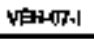 & 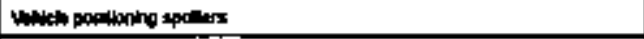 & Henos \\
\hline vatosed & 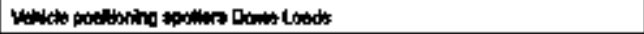 & Hore \\
\hline verutes & Hone & 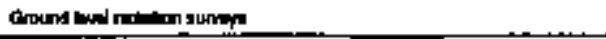 \\
\hline vicuen & 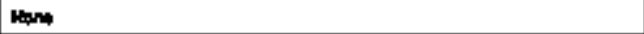 & Dinmency papdreofneas \\
\hline VI:400 & 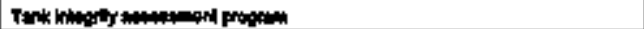 & 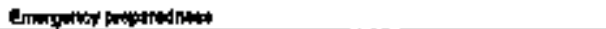 \\
\hline
\end{tabular}




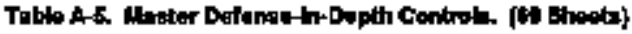

\begin{tabular}{|c|c|c|}
\hline 由 & DIDPRmitht & DID thborind \\
\hline W\$uad & 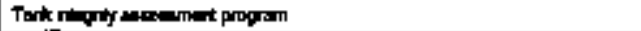 & 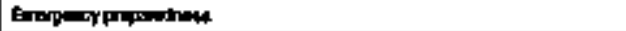 \\
\hline visures & More & Wown \\
\hline Nod 00 & $\operatorname{lin} \theta$ & Nond \\
\hline WREARomer & 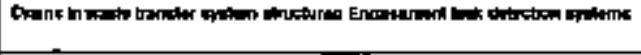 & 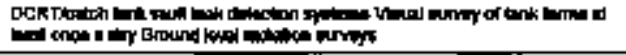 \\
\hline 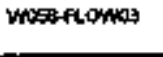 & 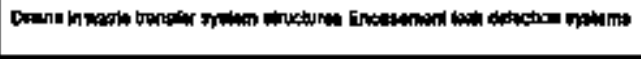 & 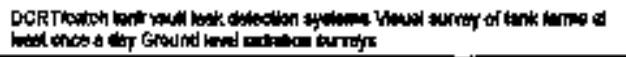 \\
\hline Wocipratontest & 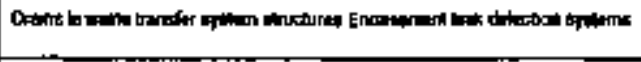 & 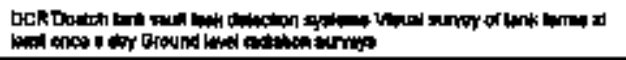 \\
\hline 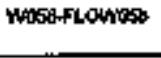 & 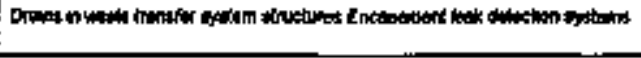 & 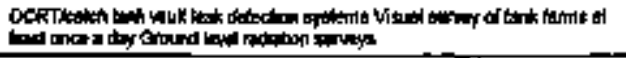 \\
\hline MOSB-FLônosa & 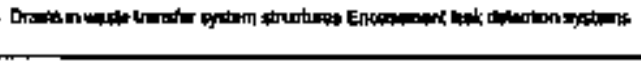 & 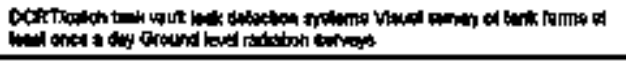 \\
\hline Whos nown? & 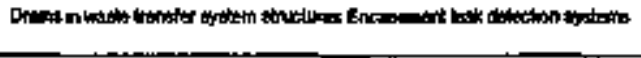 & 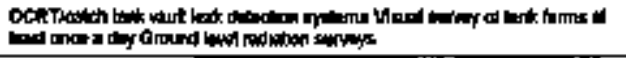 \\
\hline nosprlounto & 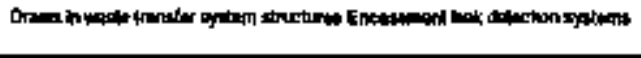 & 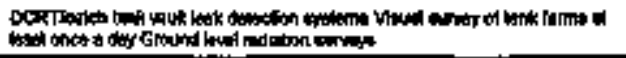 \\
\hline Whespleonita & 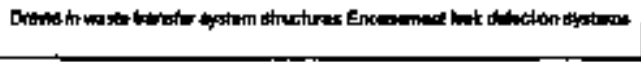 & 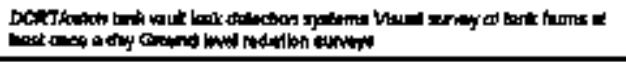 \\
\hline WH:PLCHN11b & 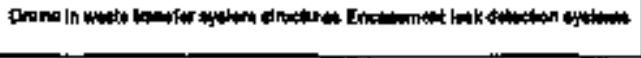 & 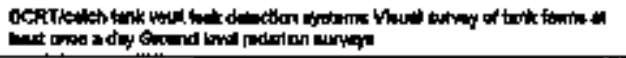 \\
\hline 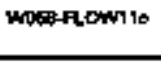 & 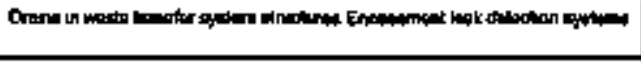 & 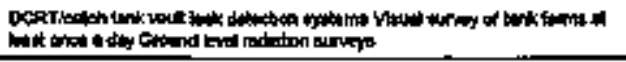 \\
\hline WOdtALOHAJ & 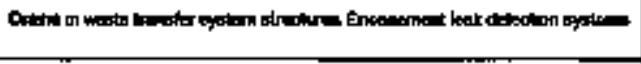 & 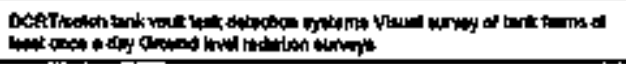 \\
\hline madrow15 & 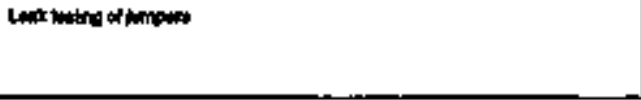 & 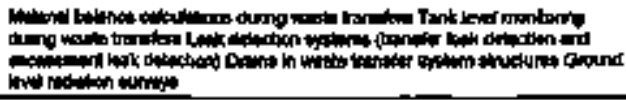 \\
\hline
\end{tabular}




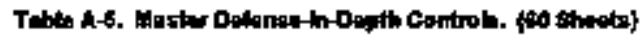

\begin{tabular}{|c|c|c|}
\hline 10 & 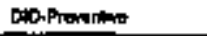 & DID PH: \\
\hline noxphomis & Lefrending of Mrepert & 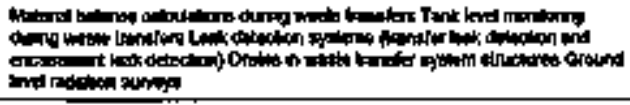 \\
\hline Mx:3-10WI7 & & 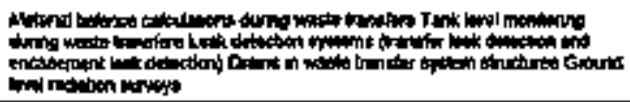 \\
\hline W"osonLWWI & Latk weing of pmptert & 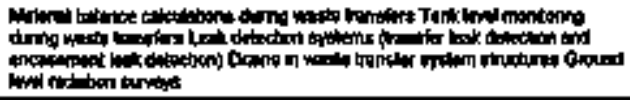 \\
\hline Wostrowatila & Ledk hath of pmpex & 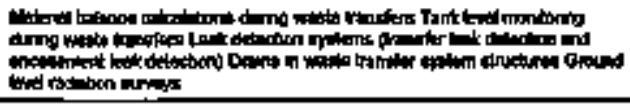 \\
\hline Wosefrow19 & Lat tang d Jumper & 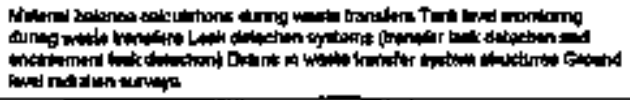 \\
\hline WOSA-FLOHAIs & 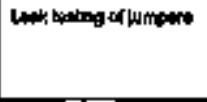 & 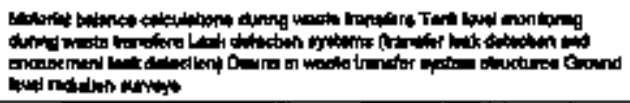 \\
\hline Hoserpotwis & 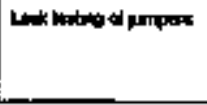 & 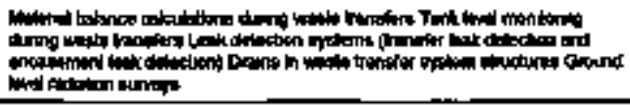 \\
\hline 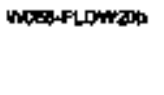 & Lept thing of Jumper & 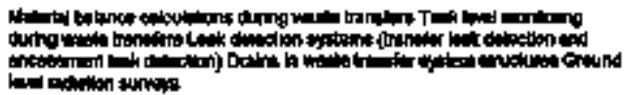 \\
\hline
\end{tabular}




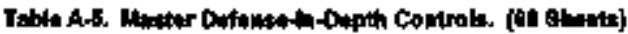

\begin{tabular}{|c|c|c|}
\hline $\mathbf{D}$ & Coptraneriten & Athilepong \\
\hline mosirtonsax & 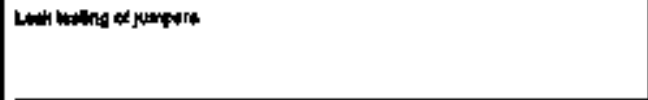 & 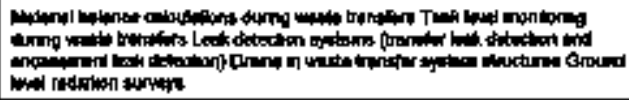 \\
\hline 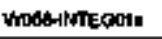 & Mone & 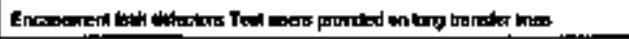 \\
\hline Whoterlifecootb & Mone & 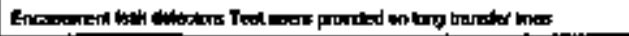 \\
\hline 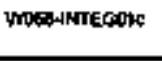 & 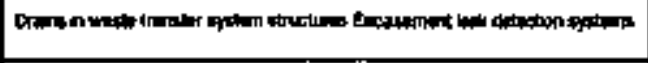 & 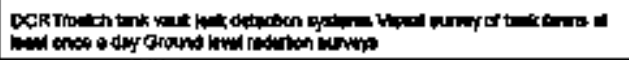 \\
\hline 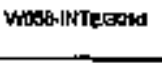 & 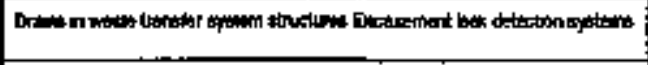 & 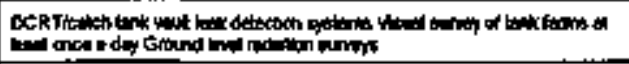 \\
\hline WOSSHNECCOS: & Hons & 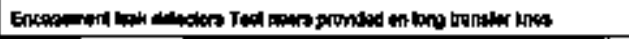 \\
\hline WosswTectin & 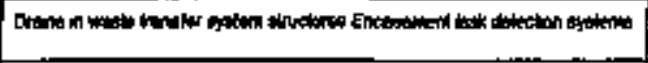 & 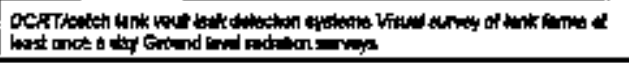 \\
\hline 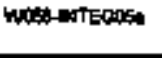 & Hent & 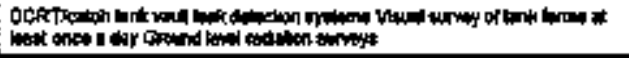 \\
\hline nosterTegoth & 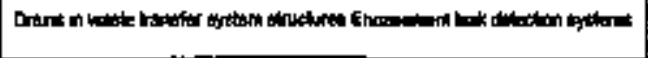 & 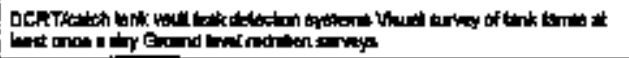 \\
\hline 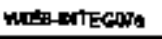 & Nem & 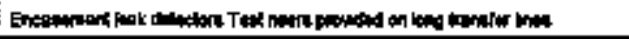 \\
\hline MOASTLCOD & 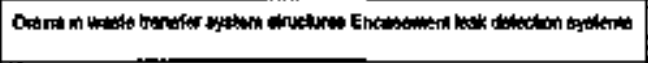 & 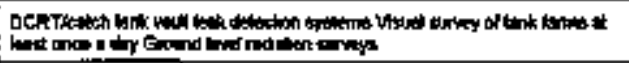 \\
\hline 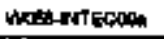 & theng & 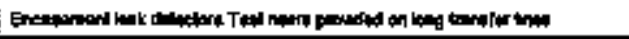 \\
\hline MOSA-NTECOH: & 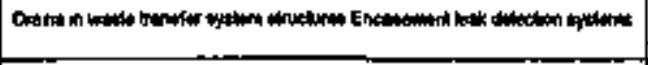 & 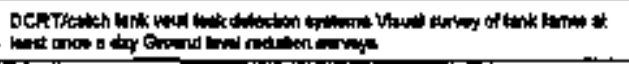 \\
\hline WOER-NTEO11 1 & ines & 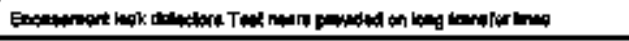 \\
\hline MOSB-WTTCS1 1 & 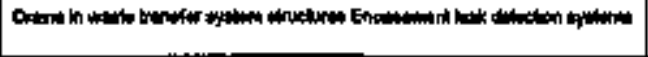 & 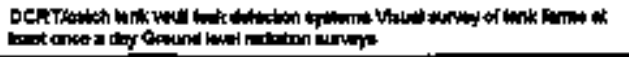 \\
\hline 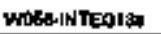 & Mons & 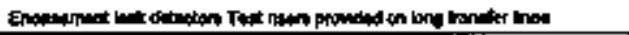 \\
\hline
\end{tabular}




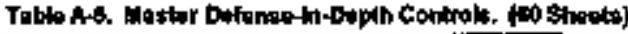

\begin{tabular}{|c|c|c|}
\hline ID & 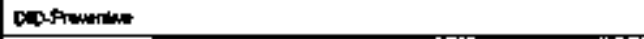 & 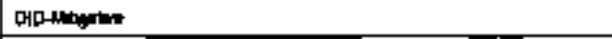 \\
\hline Voctintea1s: & mon & 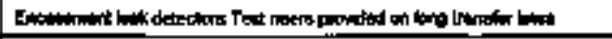 \\
\hline 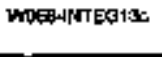 & 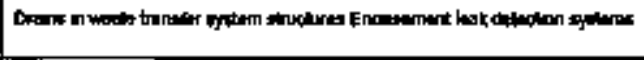 & 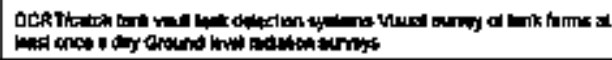 \\
\hline Woconintegisd & 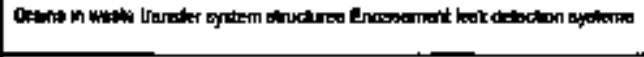 & 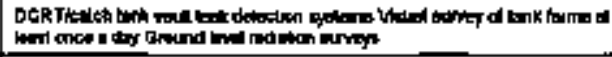 \\
\hline MOSHNEEGAO & Nore & 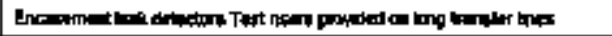 \\
\hline MASBINTEOI4b & 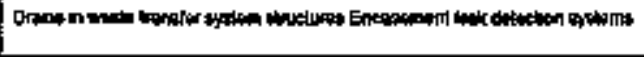 & 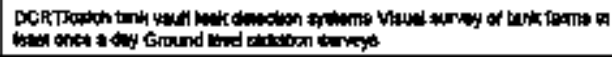 \\
\hline Hogerintdaism & 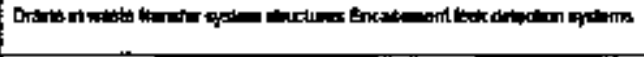 & 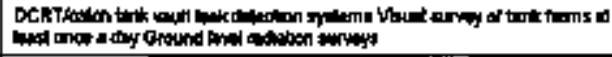 \\
\hline Mosolntcoleb & 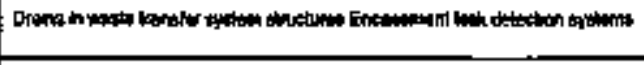 & 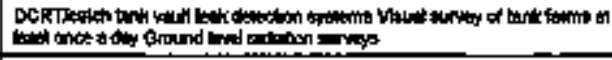 \\
\hline 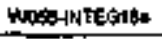 & Hare & 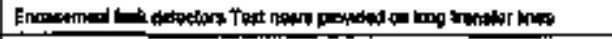 \\
\hline MOSS-INTEOIBD & 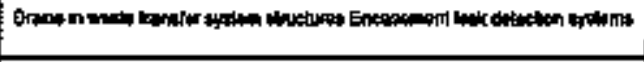 & 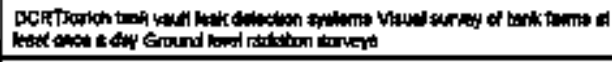 \\
\hline 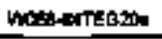 & Now & 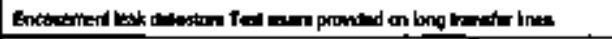 \\
\hline 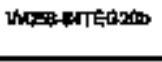 & 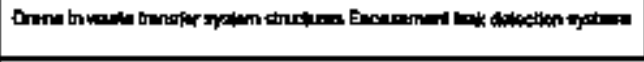 & 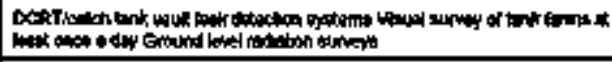 \\
\hline wastritan & Noth & 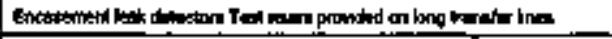 \\
\hline Whatentedis4 & 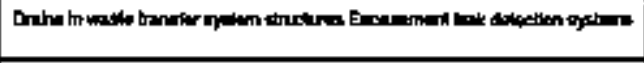 & 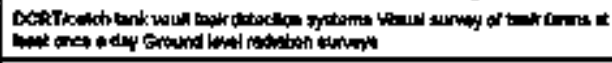 \\
\hline 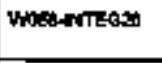 & 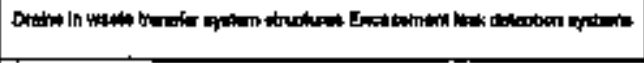 & 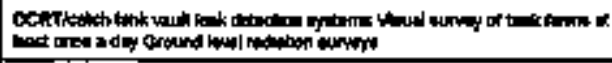 \\
\hline 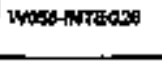 & 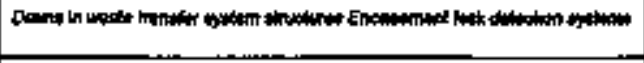 & 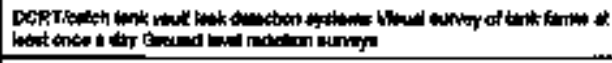 \\
\hline WOSA-INTECSO & 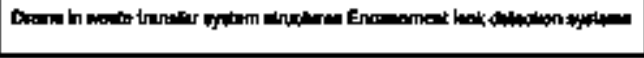 & 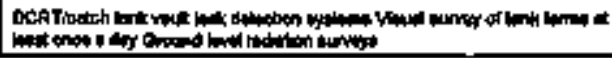 \\
\hline
\end{tabular}




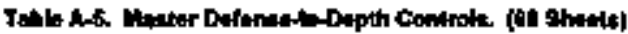

\begin{tabular}{|c|c|c|}
\hline ID & DID.Aknathad & 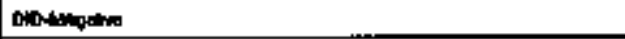 \\
\hline WNoSANTEQRS & Nom & 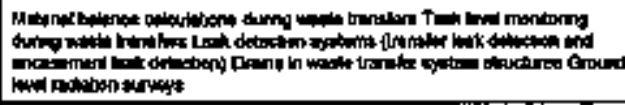 \\
\hline WHSBNNTEST4 & Nane & 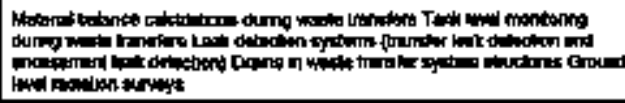 \\
\hline mostintest & Hant & 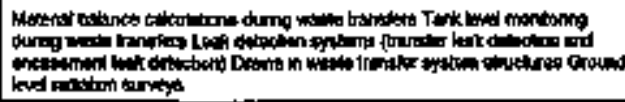 \\
\hline Whes HTEL & Han & 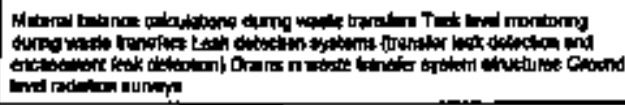 \\
\hline Motementers & Mont & 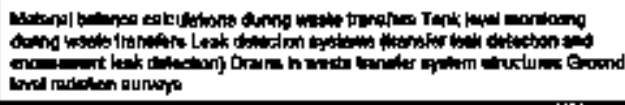 \\
\hline 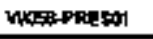 & 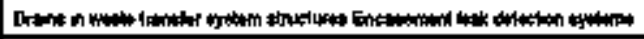 & 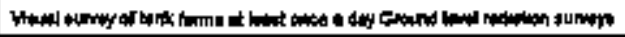 \\
\hline nest-nizstios & 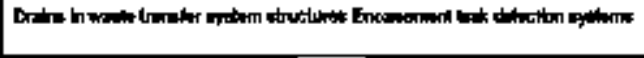 & 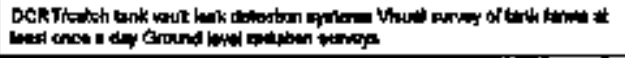 \\
\hline xR-01-Fionar & 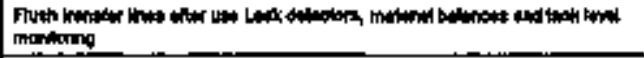 & Nem \\
\hline$X+01-F L O M O Z$ & 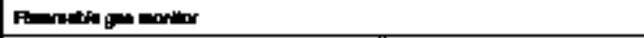 & $\mathrm{Hon}$ \\
\hline xB-01-Fonims & 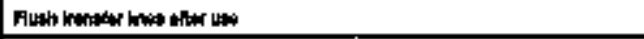 & Nin: \\
\hline XESOI-PLOHWH. & 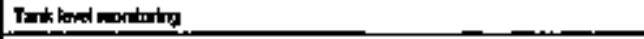 & Nont \\
\hline KS-O1flouns & 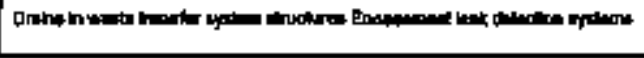 & 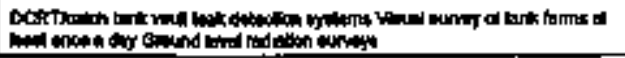 \\
\hline
\end{tabular}




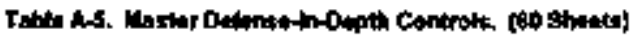

\begin{tabular}{|c|c|c|}
\hline (t) & DIDPranim & 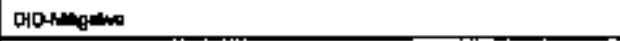 \\
\hline 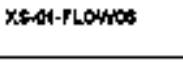 & 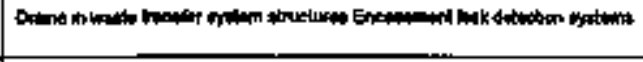 & 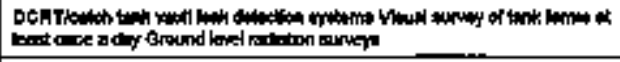 \\
\hline XB-0A-FLEMTOT & 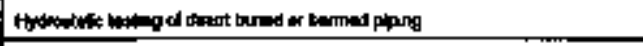 & 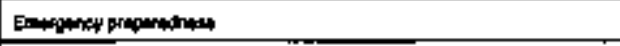 \\
\hline Xss-01.Pn: & None & 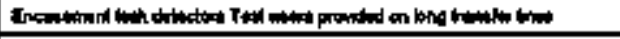 \\
\hline 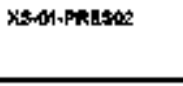 & 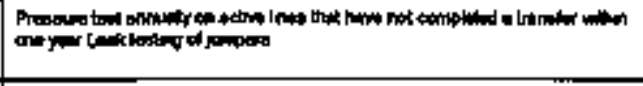 & 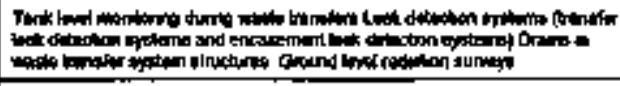 \\
\hline 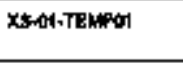 & 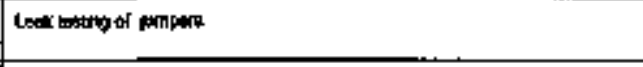 & 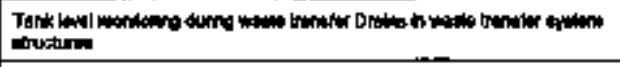 \\
\hline X\&-01-TEAPO2 & Lestioging of $\mu$ mpen & 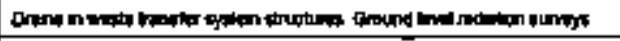 \\
\hline xisanflown & Home & 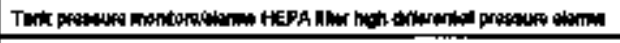 \\
\hline 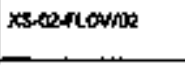 & 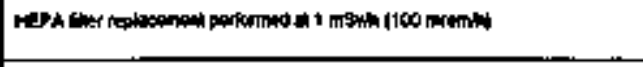 & 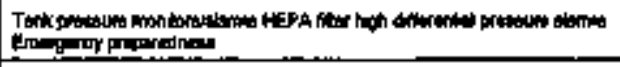 \\
\hline x6-OAFONAP3 & None & $\ln x$ \\
\hline Xa-grolotwan & 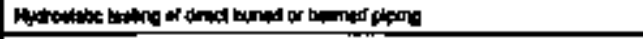 & 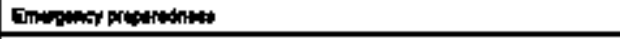 \\
\hline 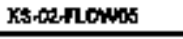 & thorie & 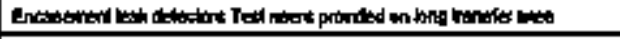 \\
\hline 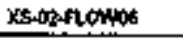 & ocon & bons \\
\hline Xß08-LEVE & 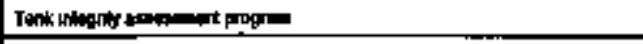 & 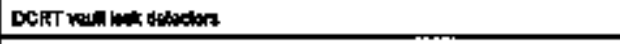 \\
\hline 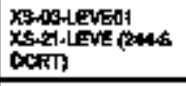 & 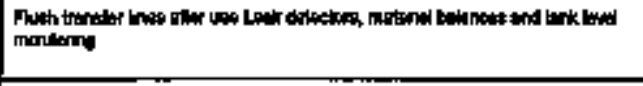 & What \\
\hline 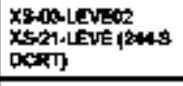 & $\mathrm{Nht}$ & Btone \\
\hline xalaphidsor & 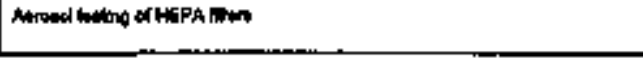 & 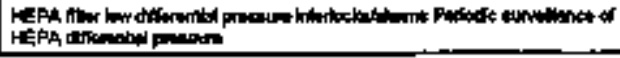 \\
\hline
\end{tabular}




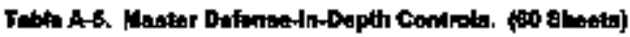

\begin{tabular}{|c|c|c|}
\hline I0 & 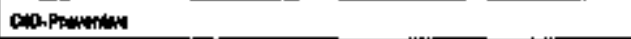 & Dampan \\
\hline 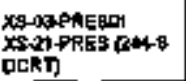 & 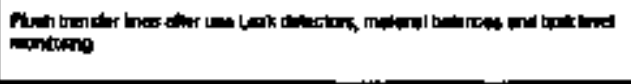 & Wone \\
\hline XE-03_PRESOTA & 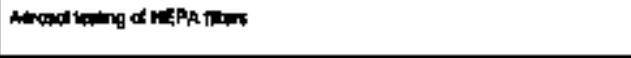 & 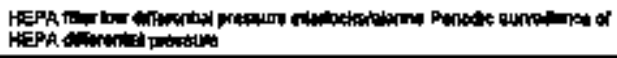 \\
\hline KSHABPRESNO & 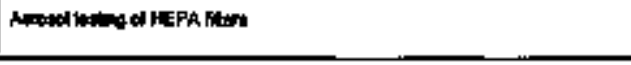 & 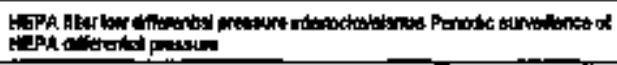 \\
\hline 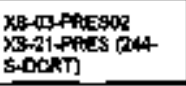 & Nan & Nanom \\
\hline 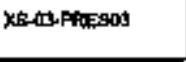 & 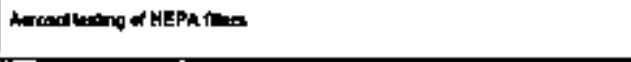 & 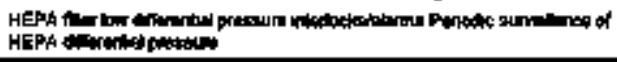 \\
\hline xs-osencsod & 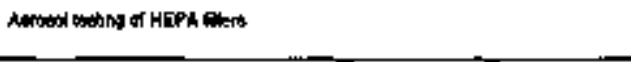 & 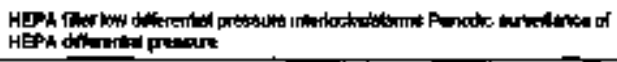 \\
\hline 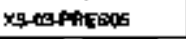 & 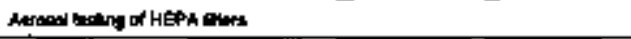 & $\operatorname{Nons}$ \\
\hline 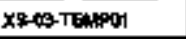 & Nom & Nats \\
\hline 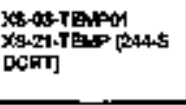 & 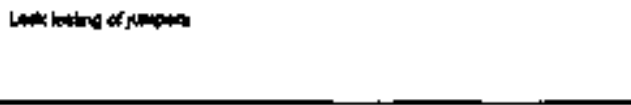 & 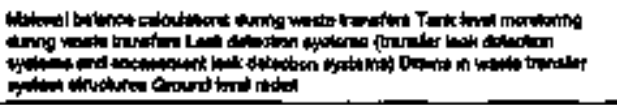 \\
\hline xom-texpen & None & 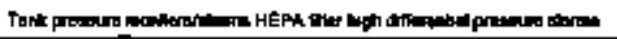 \\
\hline 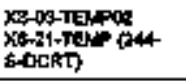 & $\ln 4$ & tons \\
\hline 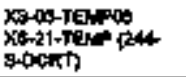 & 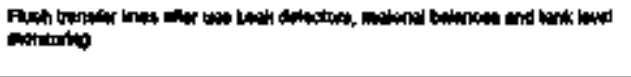 & $\operatorname{mon} \theta$ \\
\hline XE-0S-TEMOA4 & long & $\operatorname{lime}$ \\
\hline
\end{tabular}




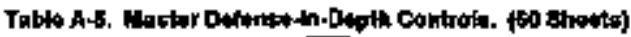

\begin{tabular}{|c|c|c|}
\hline 0 & Depravintimin & Donthentise \\
\hline 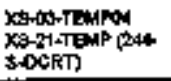 & 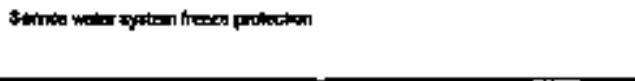 & 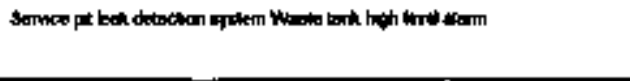 \\
\hline remastentos & 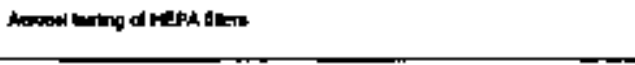 & 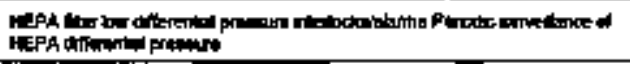 \\
\hline 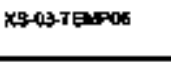 & 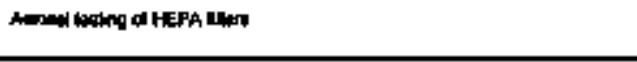 & 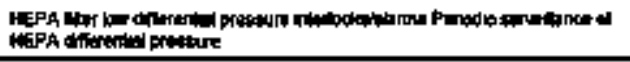 \\
\hline 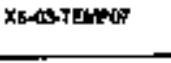 & 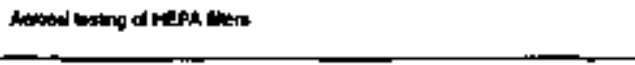 & 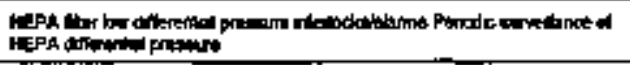 \\
\hline 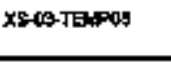 & 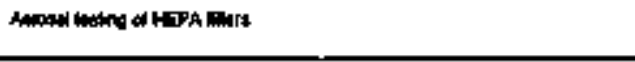 & 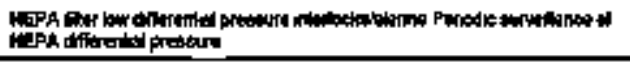 \\
\hline Xaterentes & 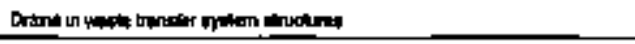 & 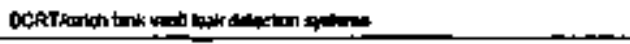 \\
\hline 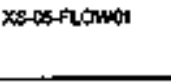 & 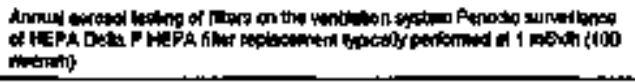 & 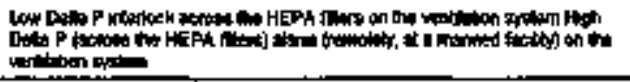 \\
\hline 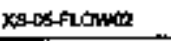 & 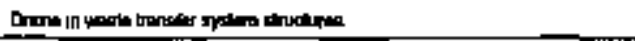 & 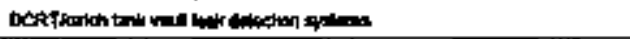 \\
\hline XS-05-Tampon & 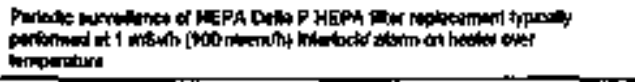 & 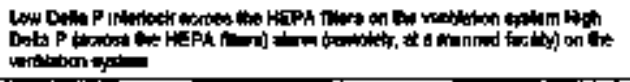 \\
\hline XS-05-TEup02 & 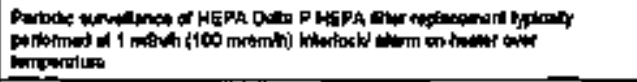 & 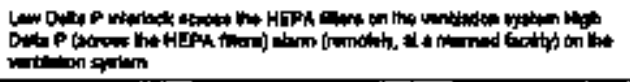 \\
\hline 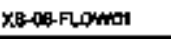 & Nome & Stonse \\
\hline xeAtrlowita & 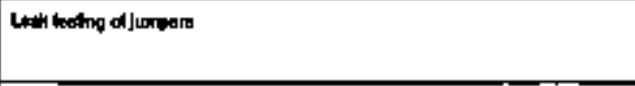 & 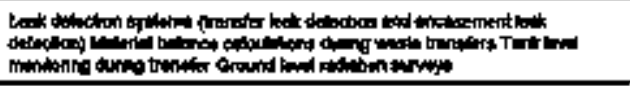 \\
\hline 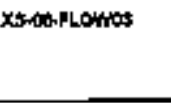 & Lath lowing of |limperd & 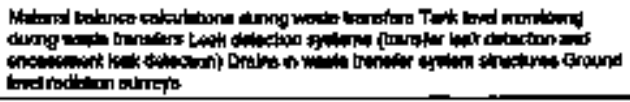 \\
\hline
\end{tabular}




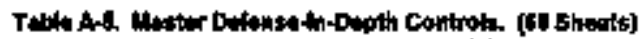

\begin{tabular}{|c|c|c|}
\hline a & 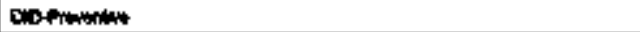 & 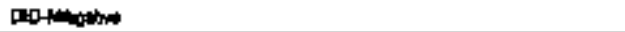 \\
\hline xs-otruotund & Len lecting of Junpen & 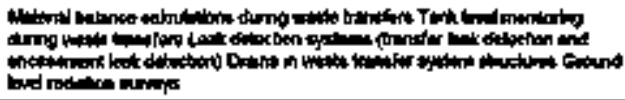 \\
\hline 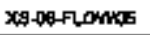 & 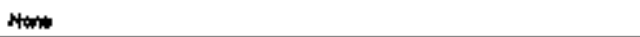 & 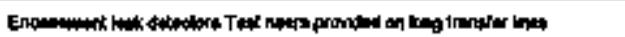 \\
\hline xp-06-fonks & 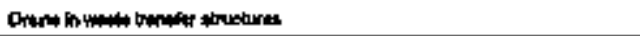 & 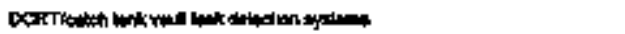 \\
\hline XE-OF-PLOHOI & Nan & 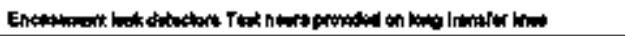 \\
\hline 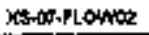 & Ptan & 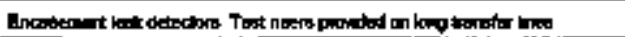 \\
\hline 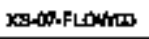 & Nłont & 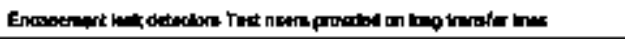 \\
\hline 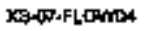 & Now & 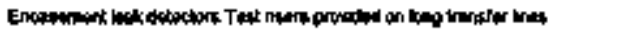 \\
\hline 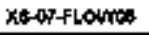 & Hom & 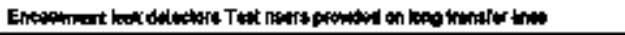 \\
\hline XSAP.PLOWres & Han & 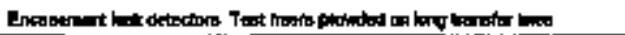 \\
\hline 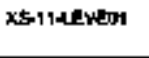 & 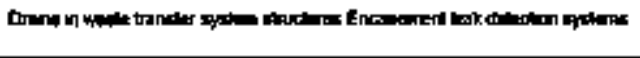 & 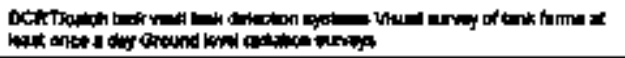 \\
\hline XE-11-LEWEOR & 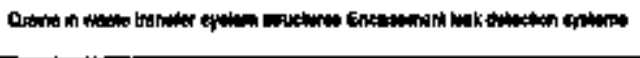 & 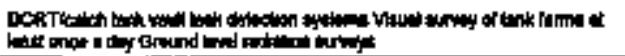 \\
\hline XS114EYERB & 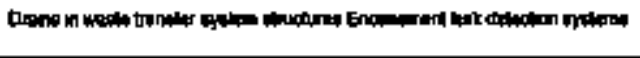 & 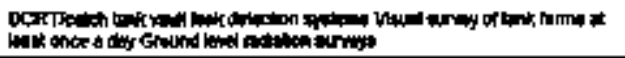 \\
\hline xS-12flown & Nare & What \\
\hline 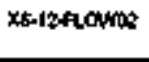 & 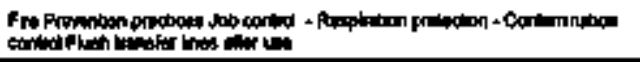 & Nome \\
\hline KS-12mothin & 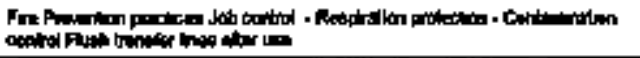 & Pat \\
\hline Xs-12+rochos & Nente & Nant \\
\hline KS-12NLOWTS & 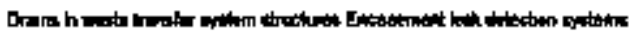 & 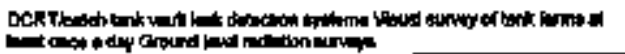 \\
\hline
\end{tabular}




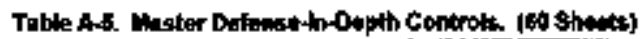

\begin{tabular}{|c|c|c|}
\hline ID & 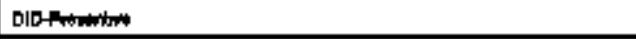 & 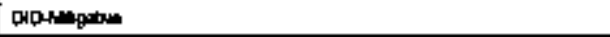 \\
\hline X8-12-ALUWB & 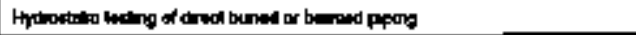 & 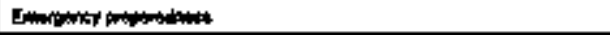 \\
\hline X:-13-UEYE & 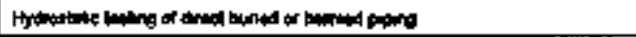 & 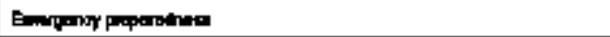 \\
\hline xastaphicsol & Noth & Nem \\
\hline x8-13-PREgrm & 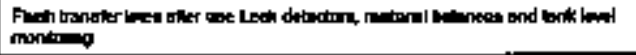 & $\operatorname{Nam}$ \\
\hline XS-13PREgas & Niper & Hare \\
\hline 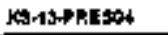 & thing & Nem \\
\hline 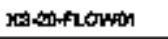 & Nkme & Nont \\
\hline 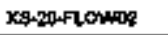 & None & Nowe \\
\hline XS-20-FLonto9 & Han: & Nane \\
\hline 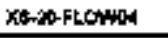 & Hane & Hone \\
\hline xstan-PLotwos & Hone & thens \\
\hline XSERTFLOMOS & None & Nand \\
\hline xS-20-1LOWR? & Hon & Mart \\
\hline x6-20-7ormo & 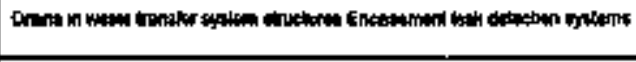 & 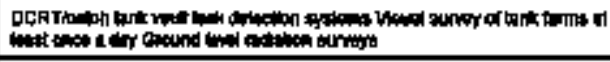 \\
\hline 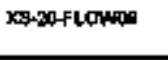 & 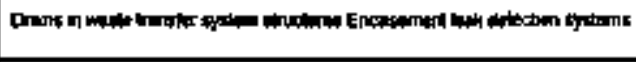 & 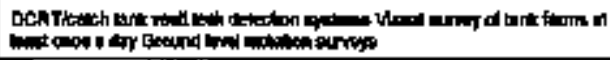 \\
\hline 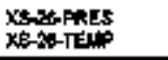 & ind & Nand \\
\hline 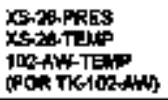 & Nante & Nant \\
\hline xSAt-Pineson & Hbon & Nhas \\
\hline
\end{tabular}




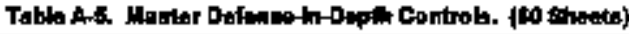

\begin{tabular}{|c|c|c|}
\hline 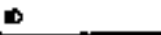 & Dio- Nimine & Dillongara \\
\hline 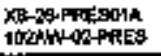 & 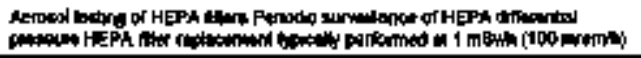 & 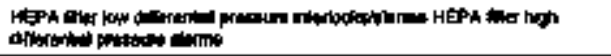 \\
\hline 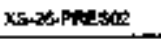 & 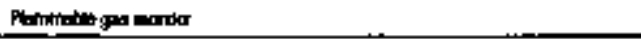 & hathe \\
\hline WO-FPPESTOWH & 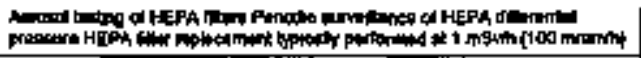 & 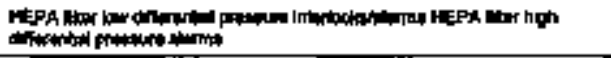 \\
\hline XS-2BPREETH & 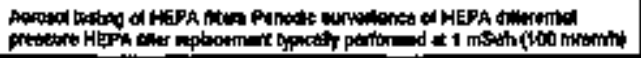 & 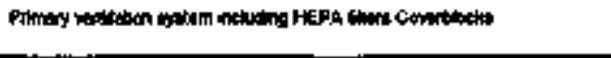 \\
\hline 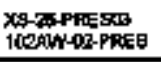 & 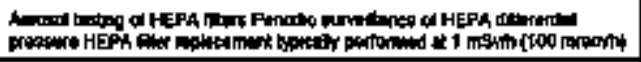 & 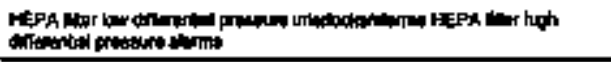 \\
\hline 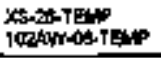 & What & Hine \\
\hline xe-za-tenay & 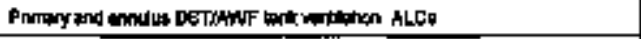 & 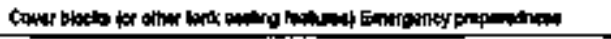 \\
\hline Xs-2a-Tampaz & Hons & Hotit \\
\hline 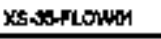 & 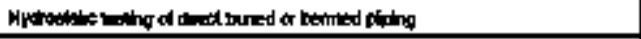 & 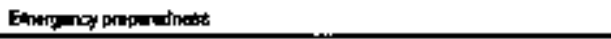 \\
\hline 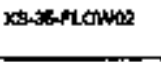 & 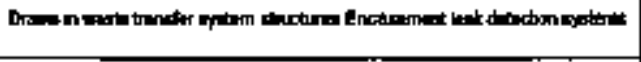 & 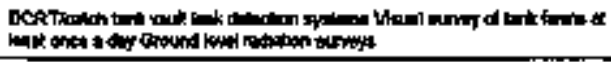 \\
\hline 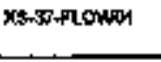 & 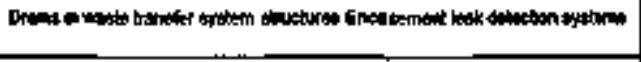 & 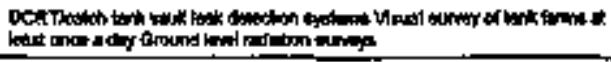 \\
\hline 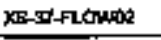 & 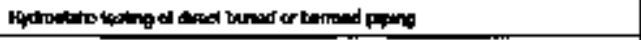 & 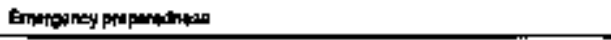 \\
\hline 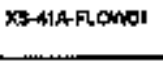 & 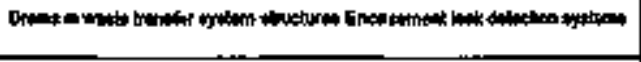 & 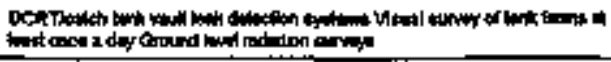 \\
\hline XIIAAFLOWhe & 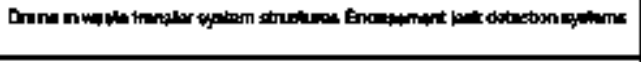 & 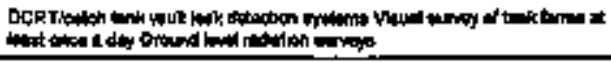 \\
\hline Xto-416-Latvig & 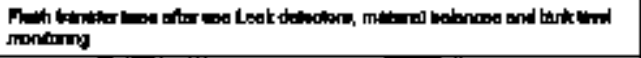 & Nare \\
\hline H8-AIE-UEVER & 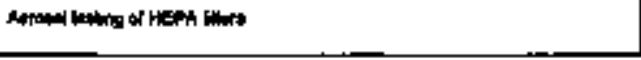 & 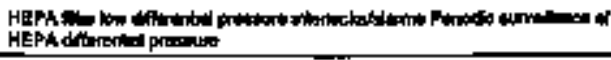 \\
\hline
\end{tabular}




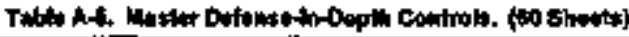

\begin{tabular}{|c|c|c|}
\hline I & 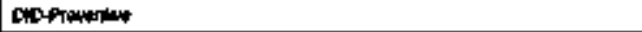 & 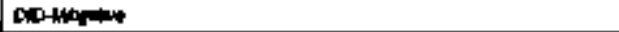 \\
\hline 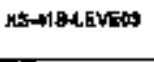 & 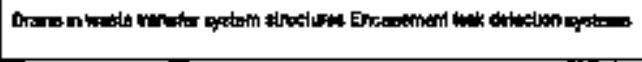 & 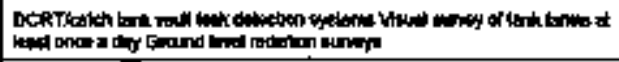 \\
\hline XS-41BLEVEOA & 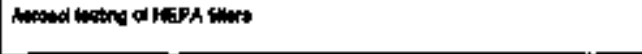 & 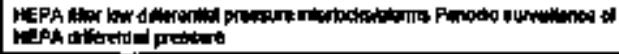 \\
\hline XQBSACHEYTEM & 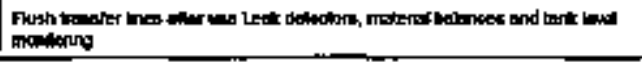 & Hone \\
\hline XF-dIC-LEYEOR & Nort & 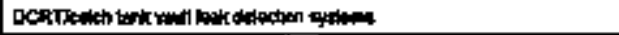 \\
\hline XS-4ICLYRA & 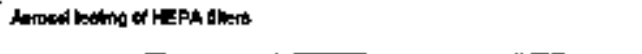 & 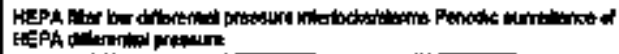 \\
\hline X3-45-FLOPN & 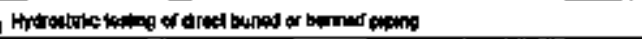 & 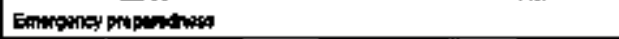 \\
\hline XEAS,PLONM & Namit & 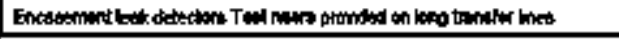 \\
\hline xs-stalomen & 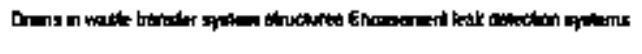 & 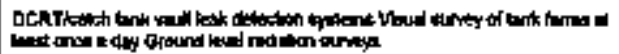 \\
\hline
\end{tabular}


Table A 6. Conirot Werno. (100 ahedte)

\begin{tabular}{|c|c|c|c|}
\hline 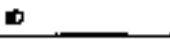 & P SGC MEMO & Masce MUn & Conitor twatho \\
\hline 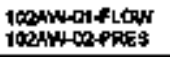 & & & 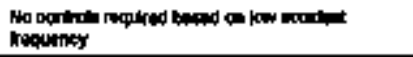 \\
\hline 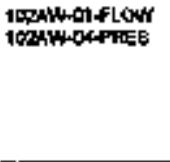 & & 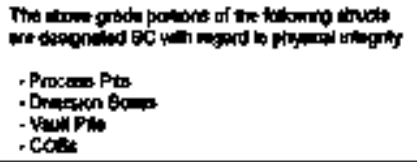 & 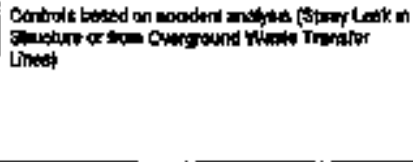 \\
\hline $\begin{array}{l}\text { 10zarwar. } \\
\text { Fownot }\end{array}$ & & 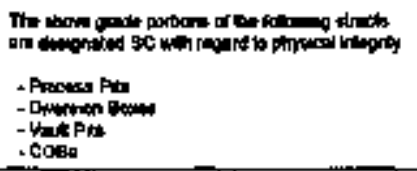 & 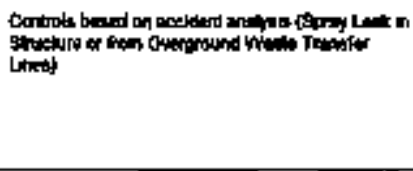 \\
\hline $\begin{array}{l}\text { 10pays-on- } \\
\text { nowne? }\end{array}$ & & & 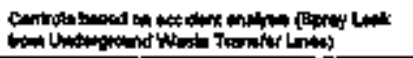 \\
\hline $\begin{array}{l}\text { 1004w-01- } \\
\text { FLones }\end{array}$ & & 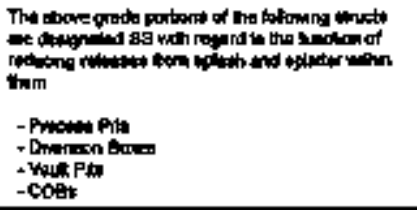 & 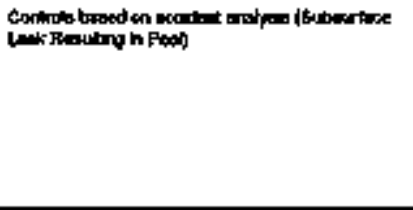 \\
\hline 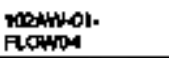 & & & 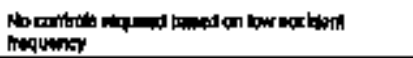 \\
\hline
\end{tabular}


Tablo Ant, controd Wano. (100, Shatts)

\begin{tabular}{|c|c|c|c|}
\hline 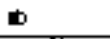 & P ascenteno & Mhesc Mand & Controll Mant \\
\hline $\begin{array}{l}\text { Iosenter-or- } \\
\text { FLothes }\end{array}$ & & 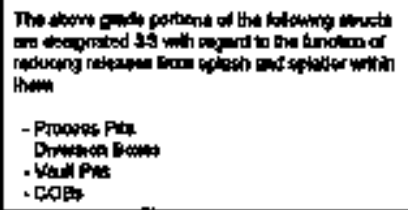 & 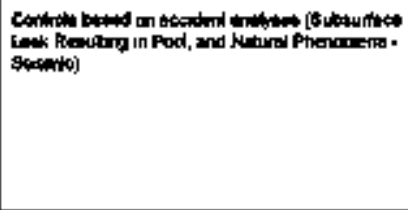 \\
\hline $\begin{array}{l}\text { 10pinw-01- } \\
\text { Fowiot }\end{array}$ & & 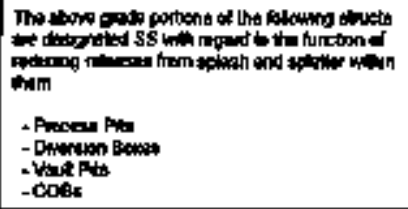 & 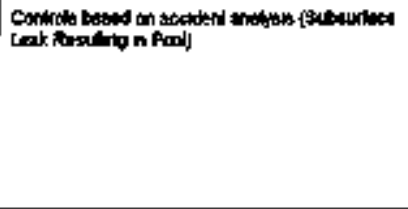 \\
\hline 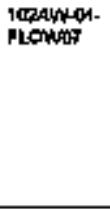 & & 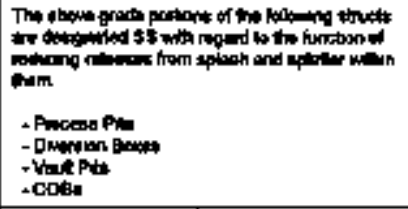 & 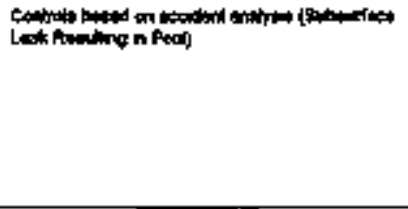 \\
\hline 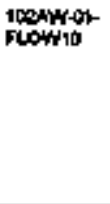 & 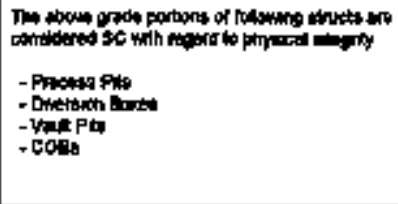 & 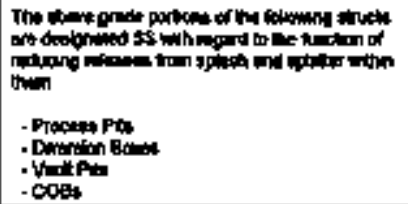 & 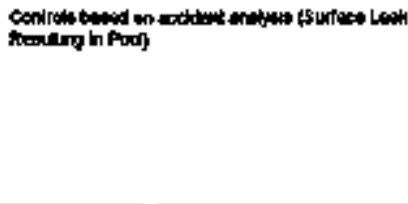 \\
\hline
\end{tabular}


Table A. Control Humb, \{100 ahowipl

\begin{tabular}{|c|c|c|c|}
\hline 10 & $P$ POC MENO & m sscin metap & 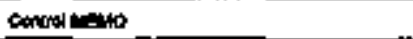 \\
\hline $\begin{array}{l}\text { 100\%W-G1- } \\
\text { Plowa1 }\end{array}$ & 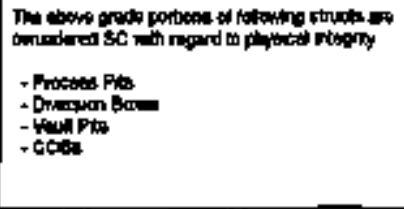 & 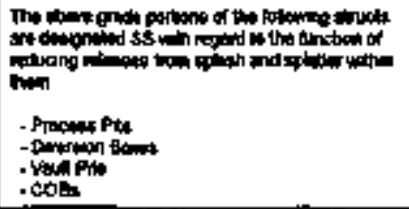 & 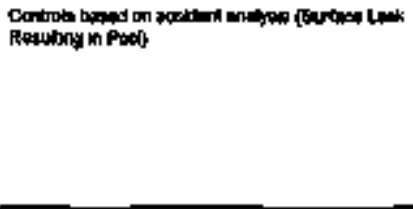 \\
\hline IC2شW-01-TEגP & & 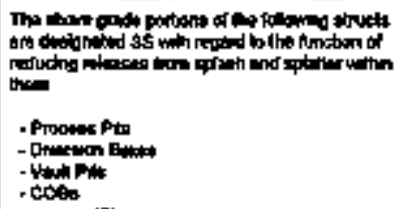 & 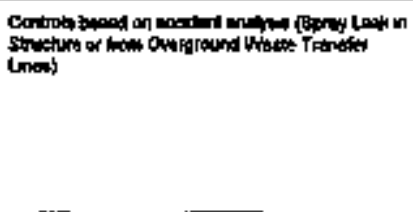 \\
\hline 1000indor- & 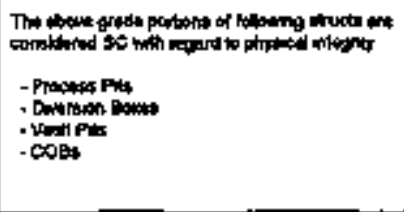 & 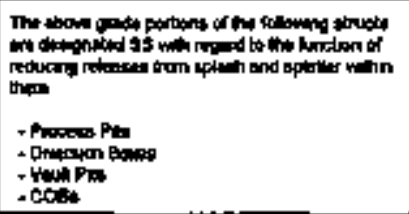 & 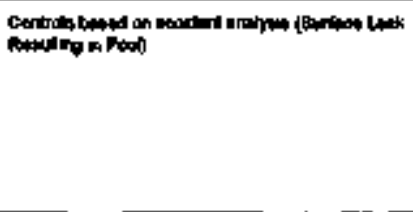 \\
\hline 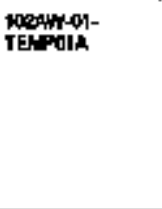 & 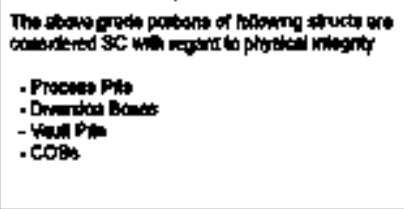 & 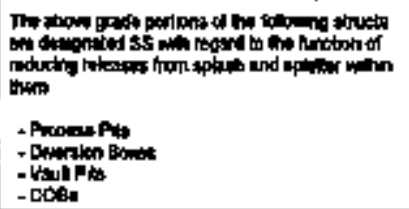 & 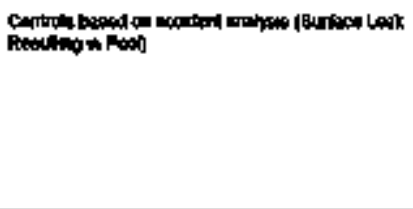 \\
\hline
\end{tabular}




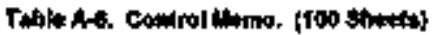

\begin{tabular}{|c|c|c|c|}
\hline 10 & 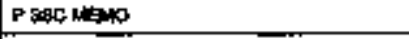 & HO5C HEND & Contwad hello \\
\hline 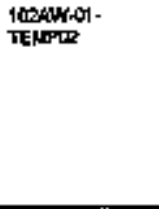 & 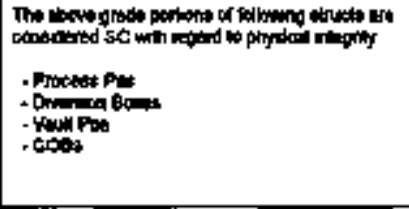 & 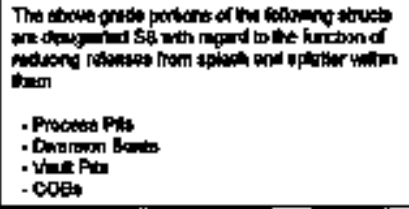 & 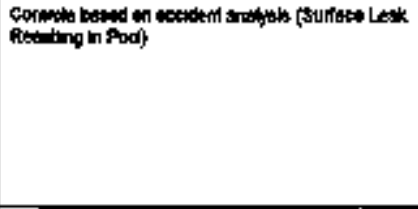 \\
\hline ItOHWDR-1EV & & & 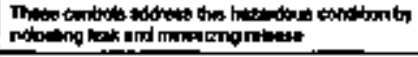 \\
\hline $\begin{array}{l}\text { 100andoR } \\
\text { LEVGOA }\end{array}$ & & & 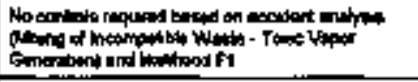 \\
\hline $\begin{array}{l}\text { 10BAwat } \\
\text { LEVED? }\end{array}$ & & & 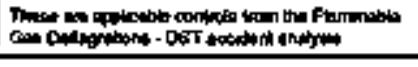 \\
\hline logntwer & & & 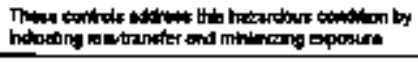 \\
\hline 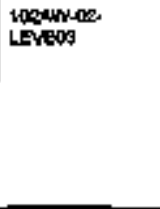 & 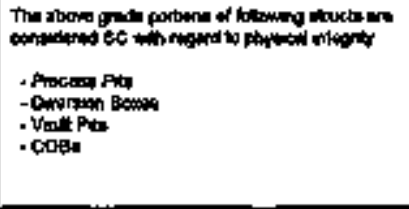 & 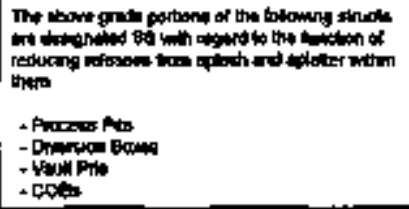 & 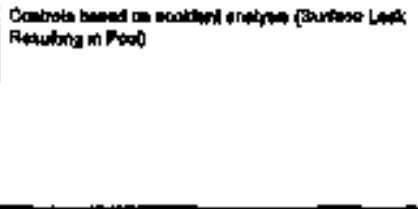 \\
\hline $\begin{array}{l}\text { lozaun-or } \\
\text { leveor }\end{array}$ & 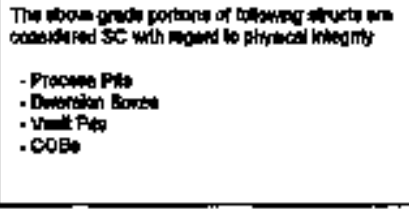 & 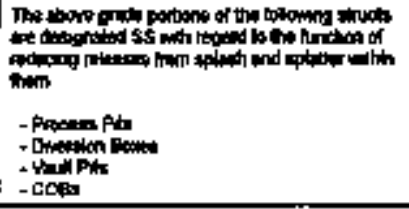 & 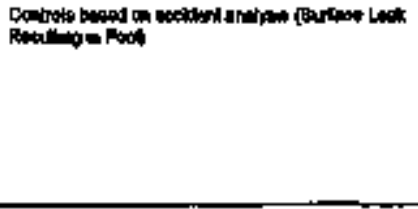 \\
\hline
\end{tabular}




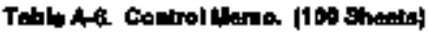

\begin{tabular}{|c|c|c|c|}
\hline 回 & م & M stc wavo & 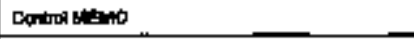 \\
\hline 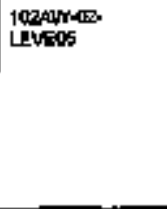 & 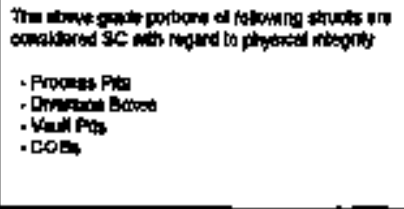 & 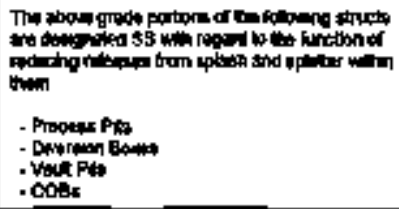 & 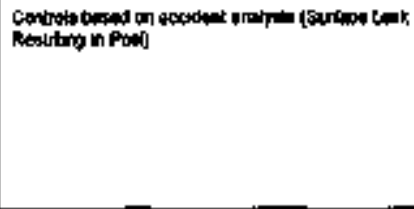 \\
\hline 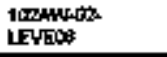 & & & 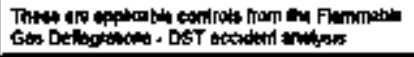 \\
\hline 102hw-0R+ARES & & & 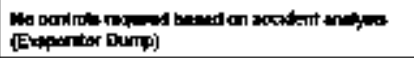 \\
\hline 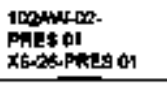 & & & \\
\hline 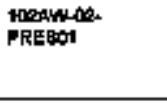 & & & 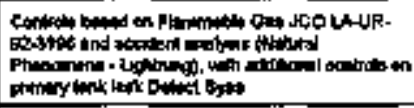 \\
\hline 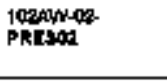 & & & 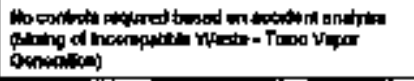 \\
\hline 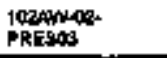 & & & 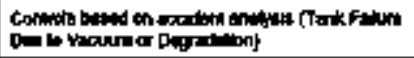 \\
\hline 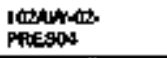 & & & 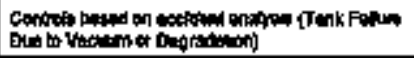 \\
\hline Iopungs. & & & 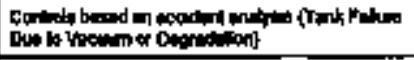 \\
\hline 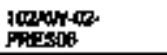 & & & 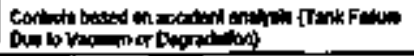 \\
\hline
\end{tabular}




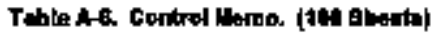

\begin{tabular}{|c|c|c|c|}
\hline 10 & P steindio & MSGC WENO & Conow Marios \\
\hline 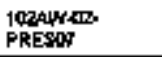 & & & 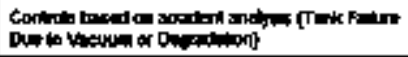 \\
\hline 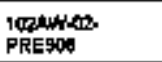 & & & 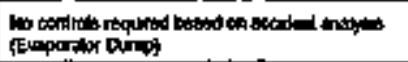 \\
\hline 1004anos. & & & 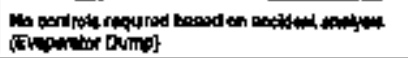 \\
\hline 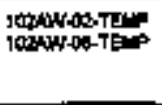 & & & 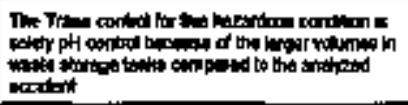 \\
\hline 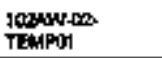 & & & 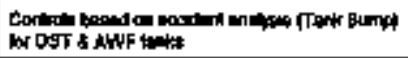 \\
\hline 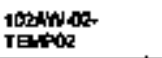 & & & 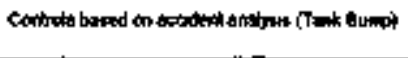 \\
\hline 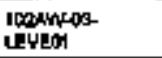 & & & 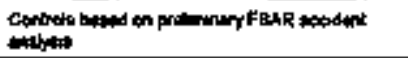 \\
\hline $\begin{array}{l}\text { logandus } \\
\text { LeVED? }\end{array}$ & & & 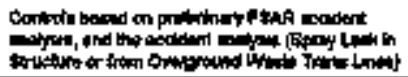 \\
\hline 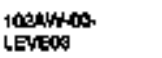 & & & 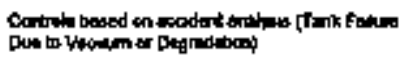 \\
\hline 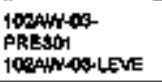 & & & \\
\hline 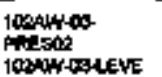 & & & \\
\hline
\end{tabular}




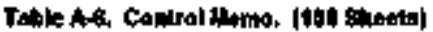

\begin{tabular}{|c|c|c|c|}
\hline ID & P StSC BEML & ussc nemb & 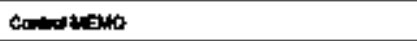 \\
\hline 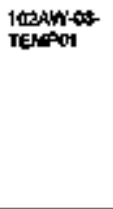 & 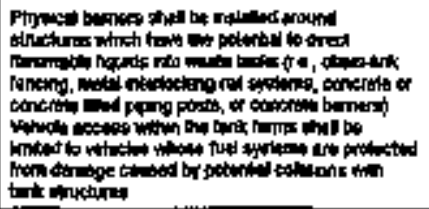 & & 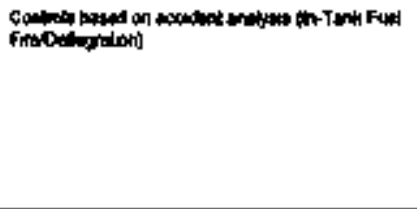 \\
\hline 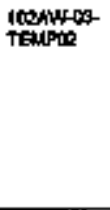 & 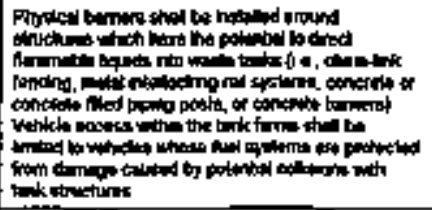 & & 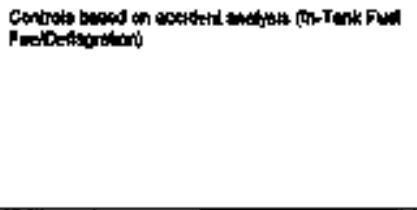 \\
\hline 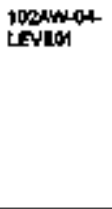 & 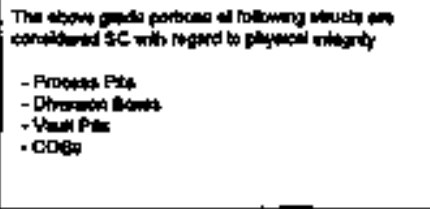 & 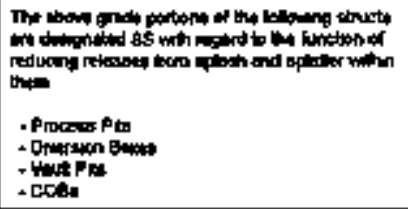 & 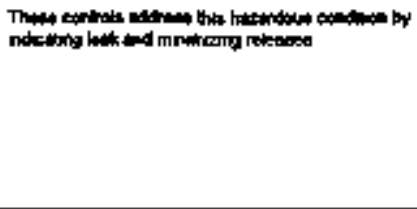 \\
\hline 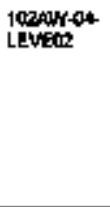 & & 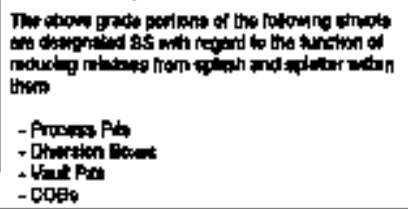 & 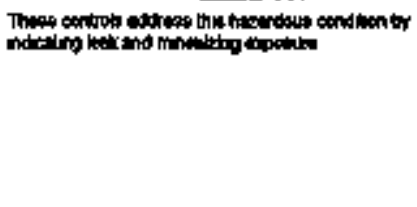 \\
\hline
\end{tabular}


Toble 4-6. Control Humo. 1109 shoots?

\begin{tabular}{|c|c|c|c|}
\hline 10 & P $3: 8 c, 109 \pi$ & MSSC HENAO & cenimed kivelo \\
\hline 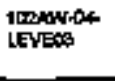 & & & 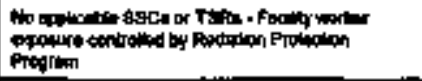 \\
\hline $\begin{array}{l}\text { popanka4 } \\
\text { LEVED4 }\end{array}$ & & & 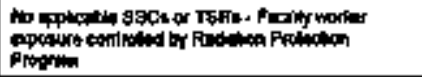 \\
\hline \multicolumn{4}{|l|}{$\begin{array}{l}\text { Tosainka4 } \\
\text { PREB on }\end{array}$} \\
\hline $\begin{array}{l}\text { 102anco4 } \\
\text { PRESol }\end{array}$ & & & 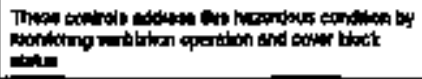 \\
\hline $\begin{array}{l}\text { 102AnLO4 } \\
\text { PRESOR }\end{array}$ & & & 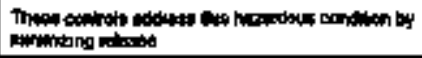 \\
\hline \multicolumn{4}{|l|}{ 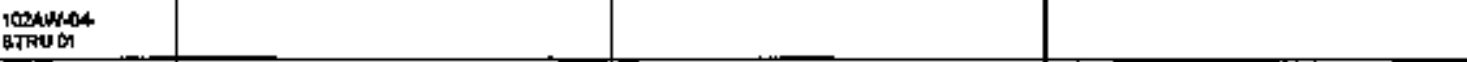 } \\
\hline \multicolumn{4}{|l|}{ 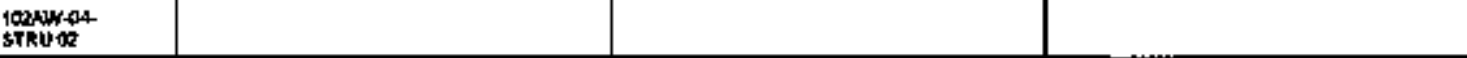 } \\
\hline 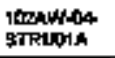 & & & 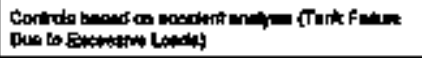 \\
\hline \multicolumn{4}{|l|}{$\begin{array}{l}\text { 100NH-O4 } \\
\text { BTAUTHE }\end{array}$} \\
\hline $\begin{array}{l}\text { KOgawac } \\
\text { \$Thuos }\end{array}$ & & & 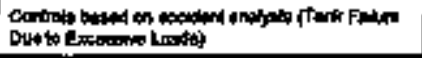 \\
\hline 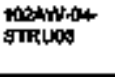 & & & 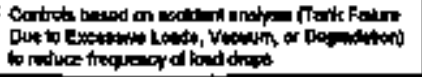 \\
\hline 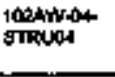 & & & 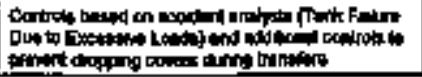 \\
\hline
\end{tabular}


Tabla A-6. Comirod Memo. t100 ehoeta)

\begin{tabular}{|c|c|c|c|}
\hline 由 & P sact bato & 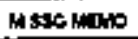 & cominoso \\
\hline $\begin{array}{l}\text { 1004n-04 } \\
\text { STRUas }\end{array}$ & & & 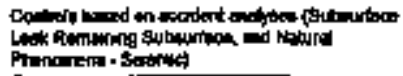 \\
\hline КОНH-OA-TELP & & & 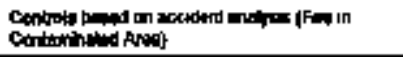 \\
\hline \multicolumn{4}{|l|}{ 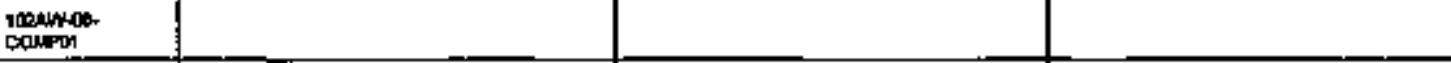 } \\
\hline 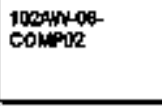 & & & 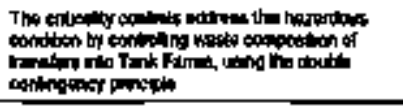 \\
\hline 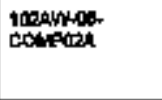 & & & 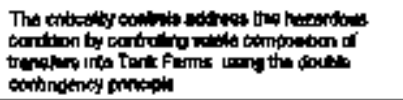 \\
\hline 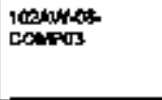 & & & 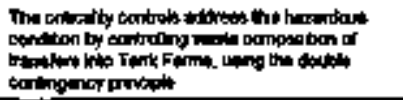 \\
\hline 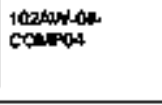 & & & 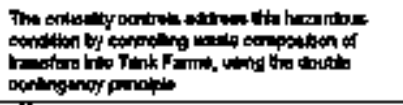 \\
\hline 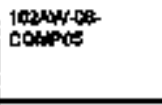 & & & 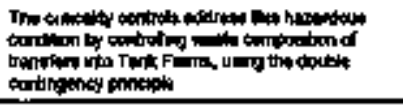 \\
\hline 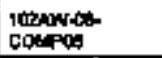 & & & 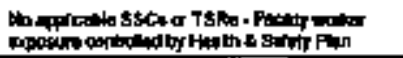 \\
\hline
\end{tabular}


Tuble A.6. Control thero. (11) shent)

\begin{tabular}{|c|c|c|c|}
\hline ID & P asc uzldo & H sec wan & 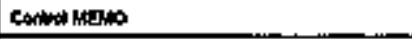 \\
\hline 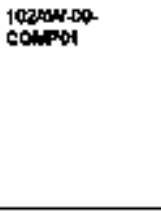 & 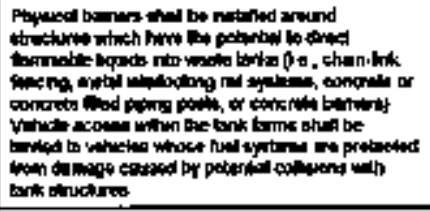 & & 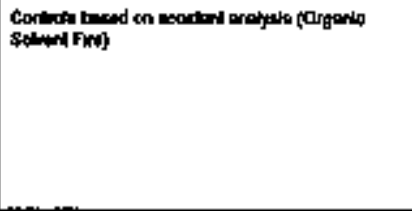 \\
\hline $\begin{array}{l}\text { IfPandpo } \\
\text { CONPR2 }\end{array}$ & 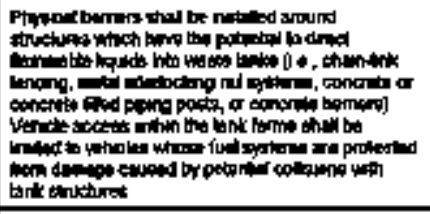 & & 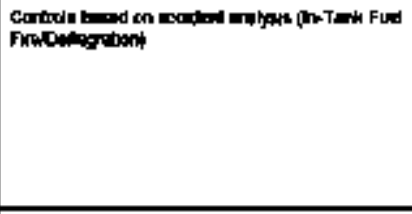 \\
\hline 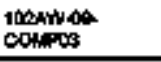 & & & 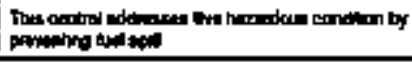 \\
\hline logentinger & & & 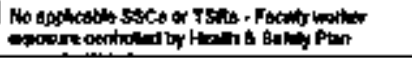 \\
\hline $\begin{array}{l}\text { 1024unas } \\
\text { confos }\end{array}$ & & & 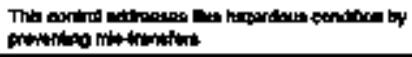 \\
\hline 1003HAotTELP & & & 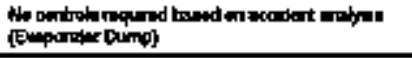 \\
\hline 1024W-os & & & 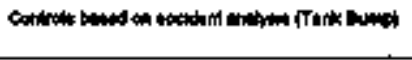 \\
\hline $\begin{array}{l}\text { Toghy-to } \\
\text { Tenpe }\end{array}$ & & & \\
\hline $\begin{array}{l}\text { loown-1s } \\
\text { Fhow on }\end{array}$ & & & \\
\hline
\end{tabular}




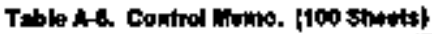

\begin{tabular}{|c|c|c|c|}
\hline 10 & 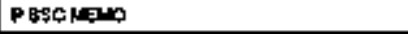 & 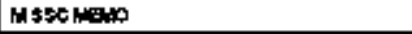 & 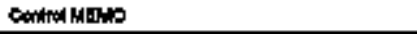 \\
\hline $\begin{array}{l}\text { 1004h-13 } \\
\text { FLowne1 }\end{array}$ & & 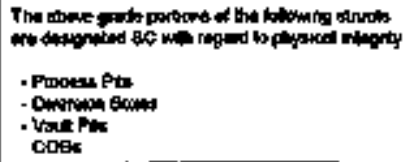 & 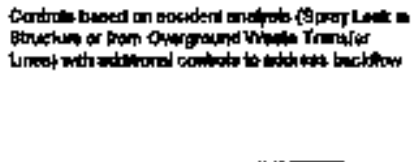 \\
\hline 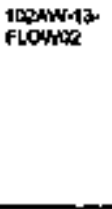 & 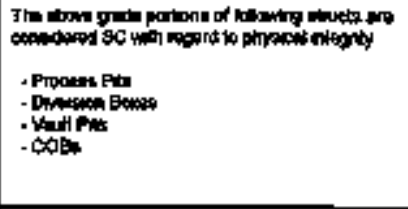 & 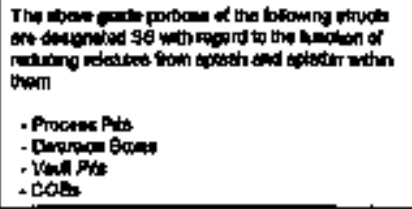 & 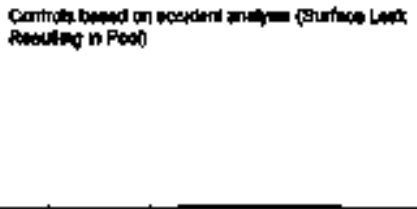 \\
\hline 1ezs-01 & 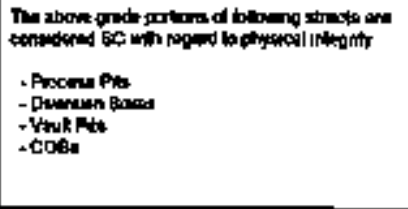 & 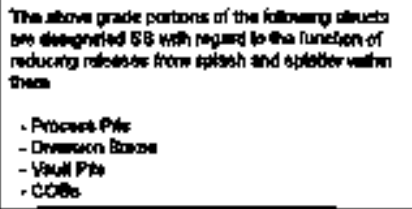 & 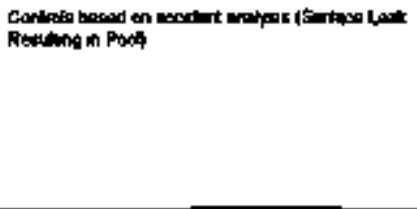 \\
\hline 1028.01 un & 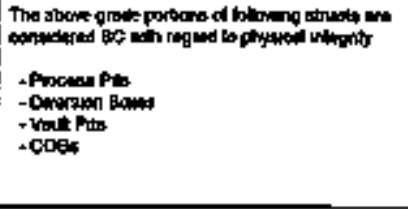 & 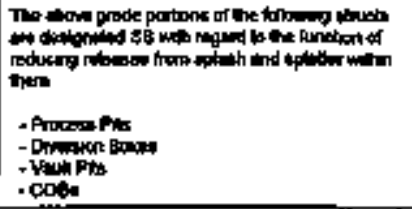 & 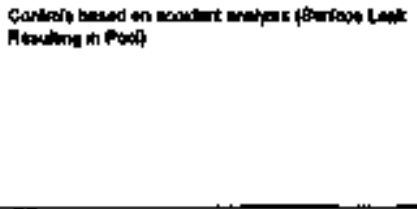 \\
\hline vogen $x$ & & & 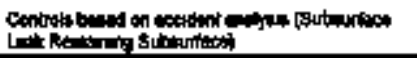 \\
\hline
\end{tabular}




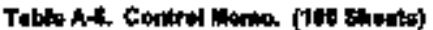

\begin{tabular}{|c|c|c|c|}
\hline $\boldsymbol{\omega}$ & 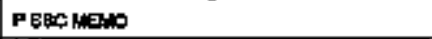 & M & 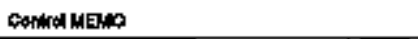 \\
\hline 10050.061 & & & 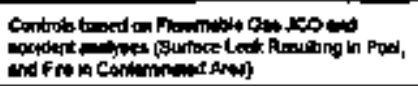 \\
\hline 10 正位比 & & & 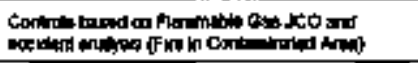 \\
\hline $1086-07 \times$ & 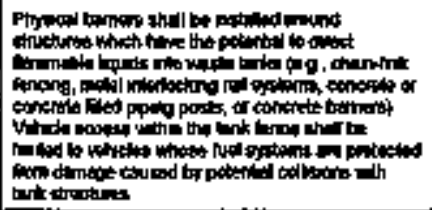 & & 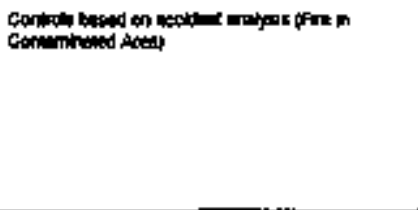 \\
\hline $1 \log 200$ d 3 & 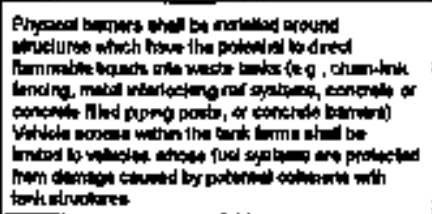 & & 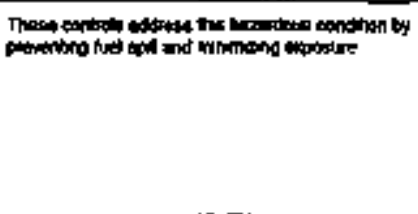 \\
\hline 16000360 & 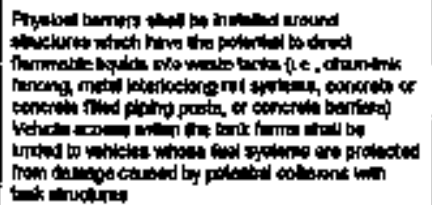 & & 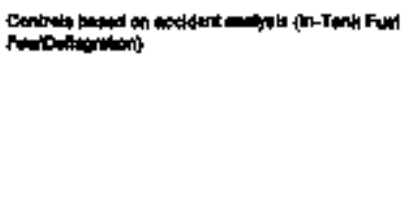 \\
\hline
\end{tabular}




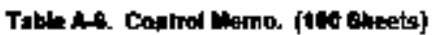

\begin{tabular}{|c|c|c|c|}
\hline $\mathbf{0}$ & 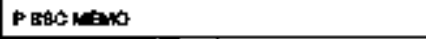 & 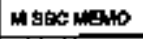 & 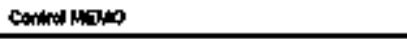 \\
\hline $1005-03$ b & 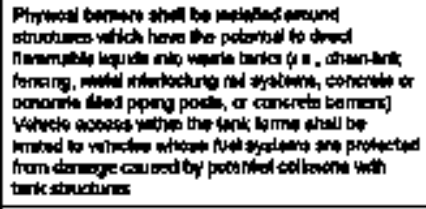 & & 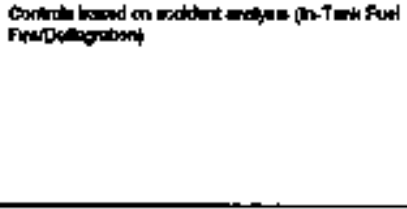 \\
\hline 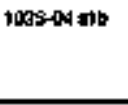 & & & 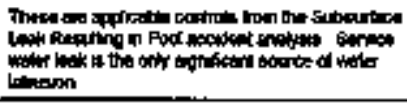 \\
\hline 1005004 an bot & & & 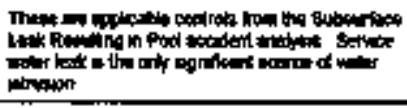 \\
\hline 1005040 & & & 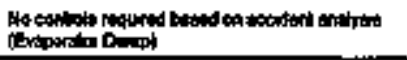 \\
\hline 10os-ode & & & 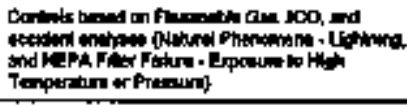 \\
\hline $10.504 \mathrm{~d}$ & & & 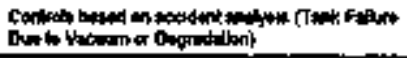 \\
\hline $100=04016$ & & & 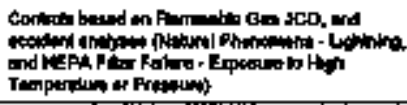 \\
\hline \multicolumn{4}{|l|}{$10 \cos \theta=0$} \\
\hline 10200060 & & & 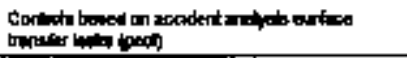 \\
\hline
\end{tabular}




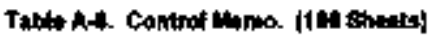

\begin{tabular}{|c|c|c|c|}
\hline $\mathbf{1 0}$ & 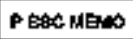 & usecticis & Control lax \\
\hline $1000-0404$ & & & 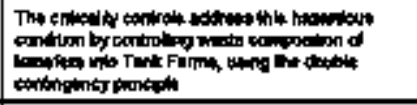 \\
\hline 100006 aln & & & 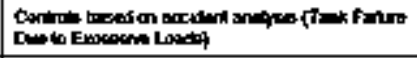 \\
\hline 10atsosolus & & & 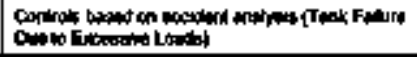 \\
\hline $102906=2$ & & & 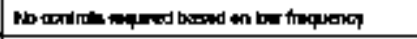 \\
\hline $1020-05$ as & & & 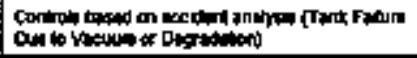 \\
\hline netsot tha & & & 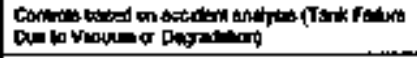 \\
\hline $1008-000$ & & & 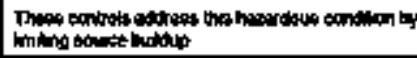 \\
\hline $106 \mathrm{con}$ & & & 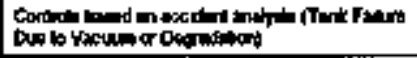 \\
\hline 1000 时 & & & 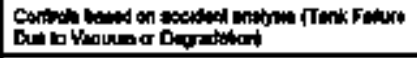 \\
\hline 1000 - & & & 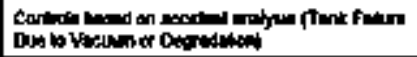 \\
\hline 100800 & & & 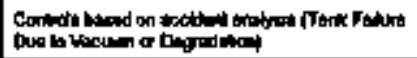 \\
\hline $1090-01 \mathrm{r}$ & & & 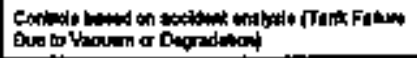 \\
\hline 10xA-01F & & & 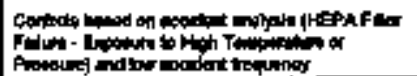 \\
\hline
\end{tabular}




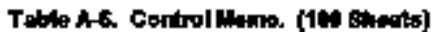

\begin{tabular}{|c|c|c|c|}
\hline ID & P \$\$ MINS & M Soc MNano & Oendod und \\
\hline 10acolo & & & 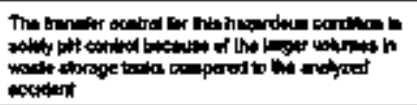 \\
\hline $1000.01 \mathrm{H}$ & & & 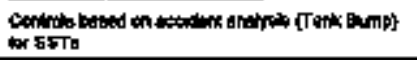 \\
\hline 106्त्वा। & & & 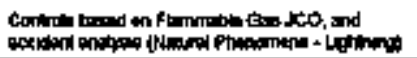 \\
\hline Tote-01] & & & 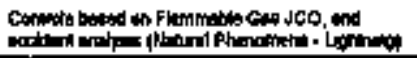 \\
\hline 10क्ष्म마 K & & & 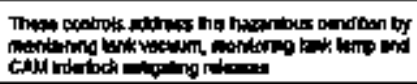 \\
\hline 1000어세 & & & 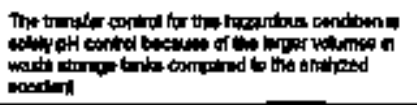 \\
\hline 1090-014 & & & 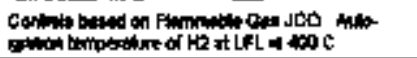 \\
\hline 10:001k & & & 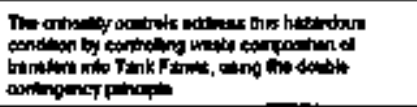 \\
\hline 1064-01Fan & & & 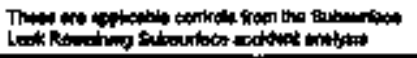 \\
\hline 160x-015 & & & 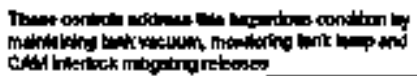 \\
\hline
\end{tabular}


This document was too large to scan as a whole document, therefore it required breaking into smaller sections.

Document number:_50-WM-TI- 264

\section{Section 2 of 2}

\section{Title: Ylanand Analges Natabase}

Date: $8 / 12 / 97$ _ Revision:

Originator: Oueni, Bg

Co: $\quad$ QQESH

Recipient:

Co:

References: $\quad \varepsilon Q L-629254$ 


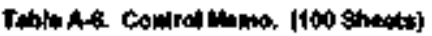

\begin{tabular}{|c|c|c|c|}
\hline D & P & 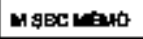 & Exhoud hento \\
\hline $100=0160$ & & & 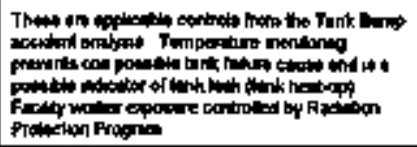 \\
\hline $100-01$ T & & & 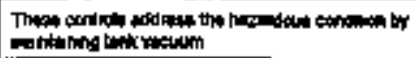 \\
\hline $10 x-014 y$ & & & \\
\hline 1008-014 & & & \\
\hline $10 \cos -01 x$ & & & 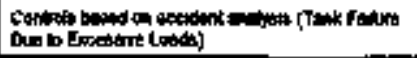 \\
\hline 1006-01Y & & & 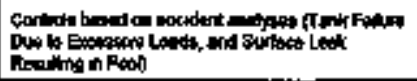 \\
\hline $10 \mathrm{coc}-0 \mathrm{es}$ & & & 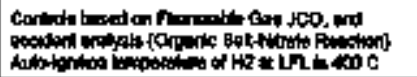 \\
\hline 1006-006 & & & 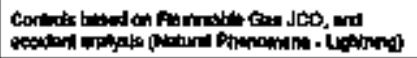 \\
\hline $1090-025$ & & & 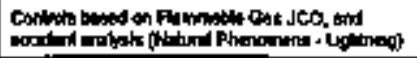 \\
\hline $1000-044$ & & & 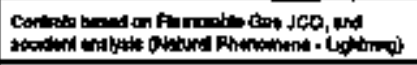 \\
\hline 10ecousa & & & 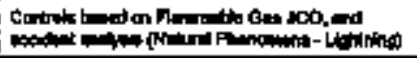 \\
\hline 100006 & & & 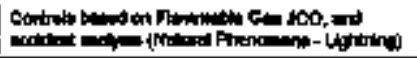 \\
\hline $100 x-004$ & & & 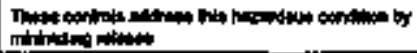 \\
\hline
\end{tabular}




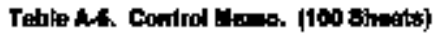

\begin{tabular}{|c|c|c|c|}
\hline ID & P 5sc Man & in s.s MDO & Comirad MESWO \\
\hline \multicolumn{4}{|l|}{ Noec-0ed } \\
\hline $1000-090$ & 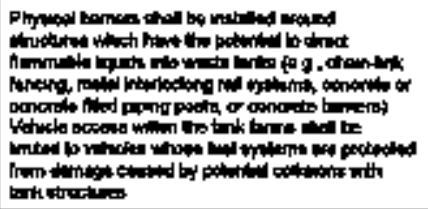 & & 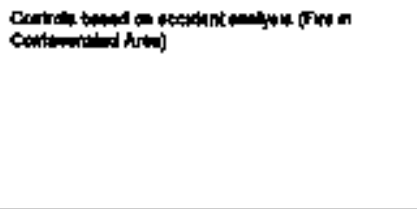 \\
\hline $1090-0 \%=$ & & & 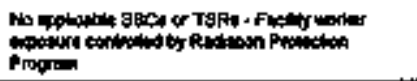 \\
\hline $1050-060$ & 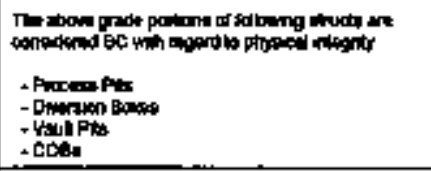 & & 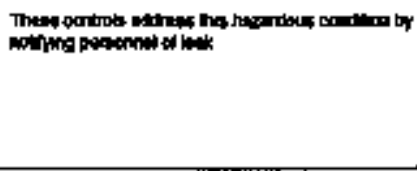 \\
\hline $1000-004$ & & & 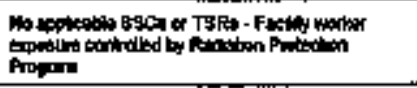 \\
\hline \multicolumn{4}{|l|}{100004} \\
\hline $1000000 x$ & & & 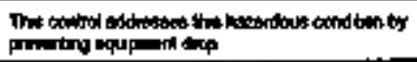 \\
\hline stacenan & & & 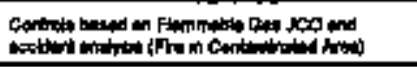 \\
\hline \multicolumn{4}{|l|}{$100 \mathrm{cos}$} \\
\hline \multicolumn{4}{|l|}{ IDEc-108 } \\
\hline $1096-10 C$ & & & \\
\hline
\end{tabular}




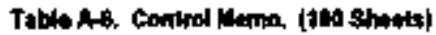

\begin{tabular}{|c|c|c|c|}
\hline 10 & P sse watub & 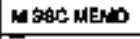 & Contral 140 \\
\hline \multicolumn{4}{|l|}{$106 \mathrm{C}-100$} \\
\hline \multicolumn{4}{|l|}{ 1006-10E } \\
\hline Noc.tor & & & 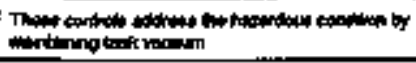 \\
\hline \multicolumn{4}{|l|}{$10 x-19+$} \\
\hline $100=10$ & & & 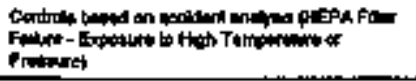 \\
\hline $1000-10 x$ & & & 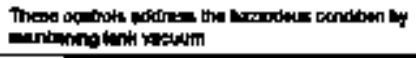 \\
\hline $10 \operatorname{coc}-10 \mathrm{~L}$ & & & 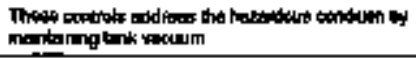 \\
\hline 1006-11A & & & 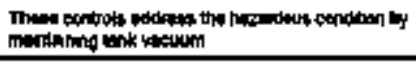 \\
\hline \multicolumn{4}{|l|}{$1006-118$} \\
\hline \multicolumn{4}{|l|}{ t00c-11c } \\
\hline \multicolumn{4}{|l|}{$1000-10$} \\
\hline $1060-11 E$ & & & 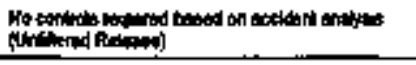 \\
\hline \multicolumn{4}{|l|}{ 10ac-11F } \\
\hline $106511 G$ & & & 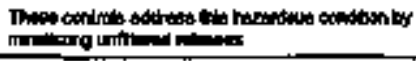 \\
\hline $100-11 \mathrm{H}$ & & & 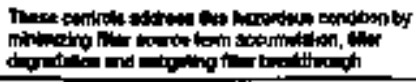 \\
\hline | & & & 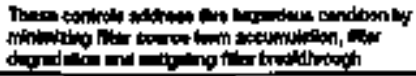 \\
\hline
\end{tabular}


Table 4.6. Comfol Lemo. (106 shane)

\begin{tabular}{|c|c|c|c|}
\hline 10 & P sac thato & 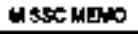 & Contw ineno \\
\hline $10: 2-11 \sqrt{1}$ & & & 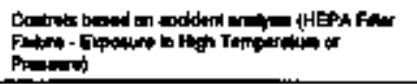 \\
\hline 109-11K & & & 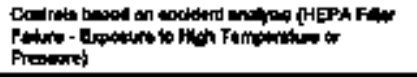 \\
\hline 100C-11L & & & 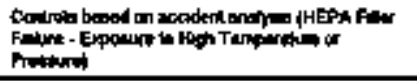 \\
\hline 1000-119 & & & 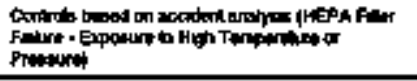 \\
\hline $1060-1$ IN & & & 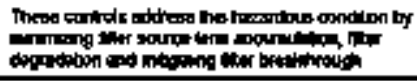 \\
\hline $10 \cos 110$ & & & 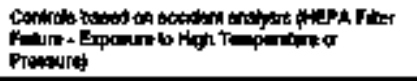 \\
\hline $1080-12 h$ & & & 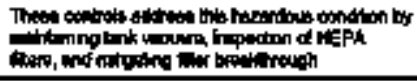 \\
\hline IDEG-12] & & & 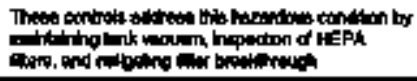 \\
\hline (106C-12C & & & 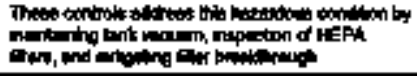 \\
\hline $1000-120$ & & & 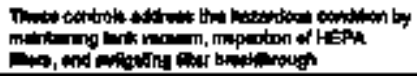 \\
\hline
\end{tabular}




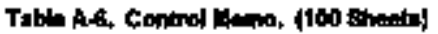

\begin{tabular}{|c|c|c|c|}
\hline $\boldsymbol{0}$ & P sasc Mand & M & 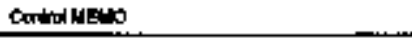 \\
\hline Woce-taf & & & 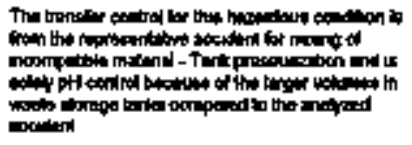 \\
\hline $10 \cos 126$ & & & 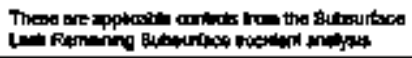 \\
\hline $10 \cos 13 \alpha$ & & & 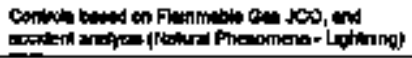 \\
\hline \multicolumn{4}{|l|}{$1050-138$} \\
\hline 107AN-OI-LENEO1 & & & 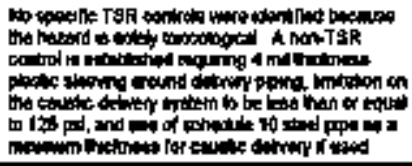 \\
\hline 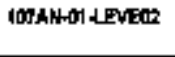 & & & 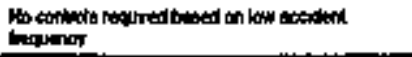 \\
\hline WOBAH-01-LEVEOS & & & 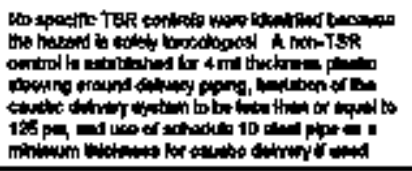 \\
\hline \multicolumn{4}{|l|}{ 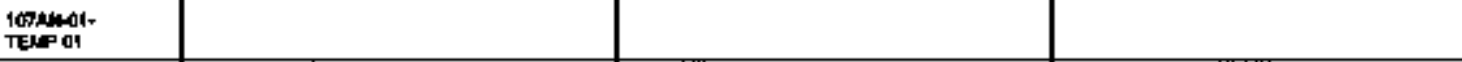 } \\
\hline 107AMO1-ГENPE & & & 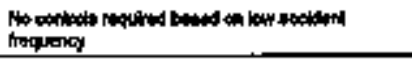 \\
\hline
\end{tabular}




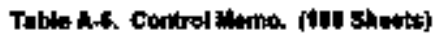

\begin{tabular}{|c|c|c|c|}
\hline 10 & A sec naw & 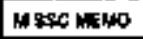 & contry \\
\hline $\begin{array}{l}\text { Dofdyoz } \\
\text { Florugr }\end{array}$ & & & 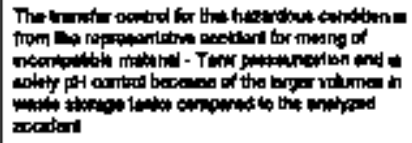 \\
\hline 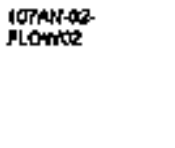 & & & 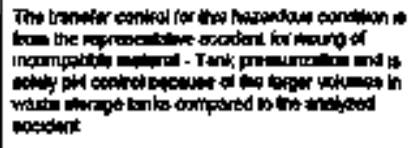 \\
\hline 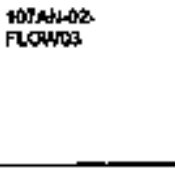 & & & 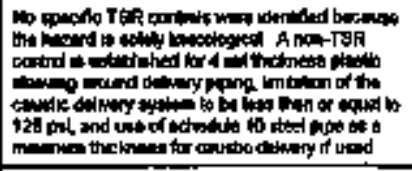 \\
\hline 107AKLOAPRES & & & 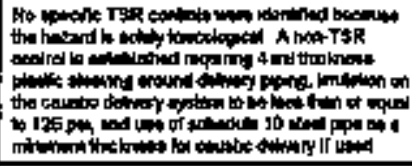 \\
\hline 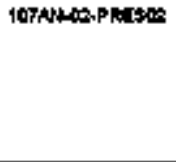 & & & 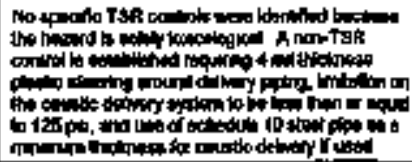 \\
\hline
\end{tabular}




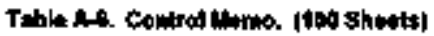

\begin{tabular}{|c|c|c|c|}
\hline 0 & $P$ P & M 980,100 & cortond heve \\
\hline \multicolumn{4}{|l|}{ 107AN-D2-TघAPO1 } \\
\hline IGtAN-AB-FLCON & & & 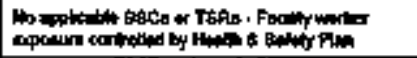 \\
\hline $11+\operatorname{tax}-620$ & 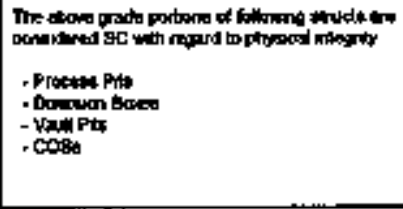 & 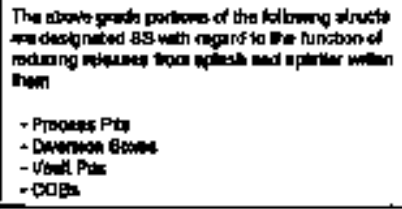 & 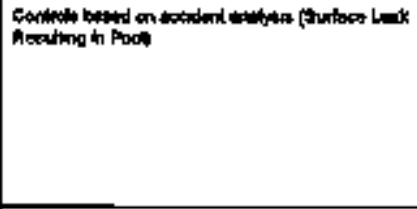 \\
\hline 1149x-04, 10 & & & 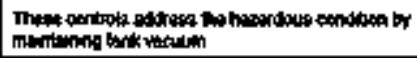 \\
\hline 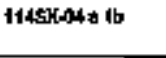 & & & 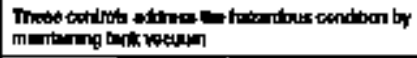 \\
\hline $1745 \mathrm{P}-0 \mathrm{~A} \in \mathrm{Z}$ & & & 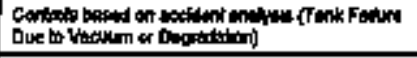 \\
\hline $1145604=1$ & & & 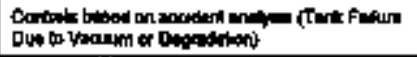 \\
\hline $1149+0404$ & & & 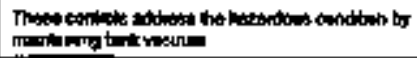 \\
\hline $11+8 x-04 b 1$ h 1 & & & 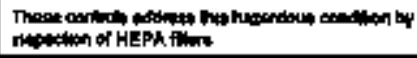 \\
\hline $1145 x=04 b 1=2$ & & & 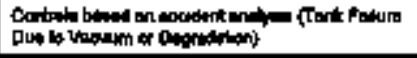 \\
\hline $114 x-04 b 1$ a 0 & & & 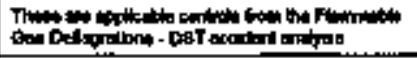 \\
\hline 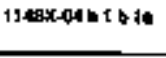 & & & 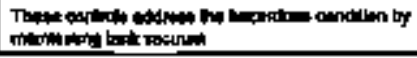 \\
\hline
\end{tabular}


Toble A-6. Controd Hong. (190 shortg)

\begin{tabular}{|c|c|c|c|}
\hline 10 & P & M c5x MEN & 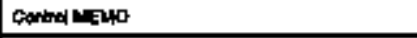 \\
\hline $1143 x-54$ b 1 b 16 & & & 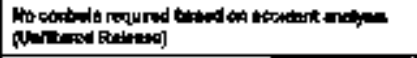 \\
\hline $1144 x-04$ b 1 b 20 & & & 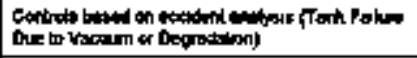 \\
\hline 114Ex-OA 1 b 20 & & & 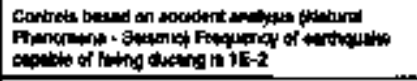 \\
\hline 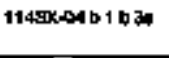 & & & 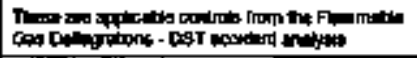 \\
\hline $1145 X-04$ b 1 b 35 & & & 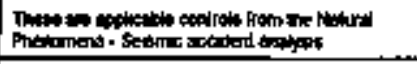 \\
\hline $11465-04$ b : 51 & & & 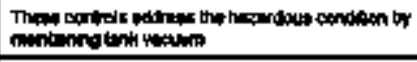 \\
\hline $1145 \times 04$ b $1<2$ & & & 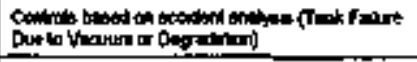 \\
\hline $1149 x-04 b 1=3$ & & & 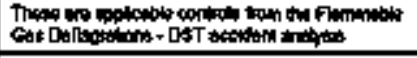 \\
\hline $1145 x-04$ b Id 1 & & & 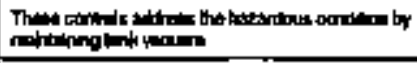 \\
\hline $11 \cos x-04$ o 102 & & & 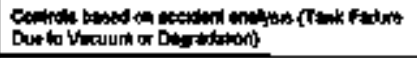 \\
\hline 11 क & & & 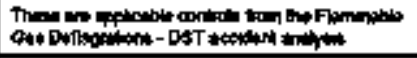 \\
\hline 41485, 04 b t5 & & & 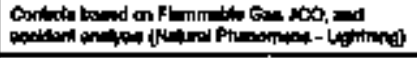 \\
\hline 11450-0d b : & & & 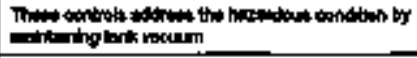 \\
\hline $1149 x=041$ & & & 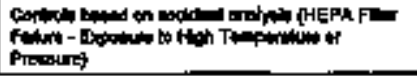 \\
\hline
\end{tabular}




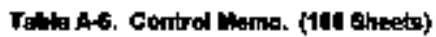

\begin{tabular}{|c|c|c|c|}
\hline $\mathbf{l a}$ & 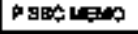 & H\$F & cortad hellos \\
\hline $1445 x-94 t 2$ & & & 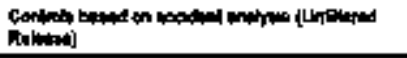 \\
\hline $1145 \times-04<2 b$ & & & 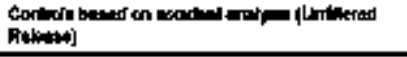 \\
\hline $11+8 x+04020$ & & & 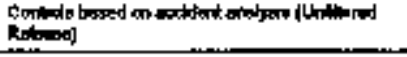 \\
\hline $11 \Delta-5 x-04024$ & & & 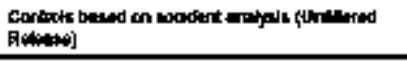 \\
\hline $1149 x-06 b 1$ & & & 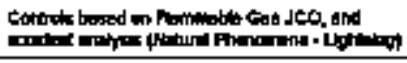 \\
\hline $110 \times 1 \times 0502$ & & & 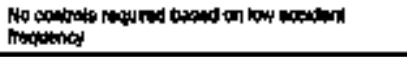 \\
\hline $1149 x-0$ b 13 & & & 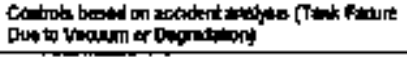 \\
\hline $11451(05$ t & & & 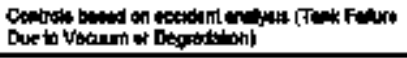 \\
\hline $1149 x-05 c 2$ & & & 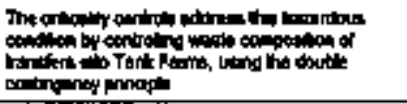 \\
\hline $1185 x-00 d$ & & & 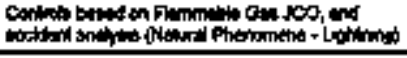 \\
\hline $1148 x-08 \cdot 14$ & & & 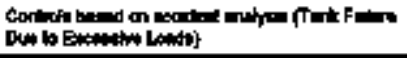 \\
\hline $114 \sec -0 \mathrm{at}=1 \mathrm{~b}$ & & & 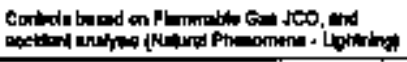 \\
\hline $1145 \mathrm{k}-0 \mid 1=2$ & & & 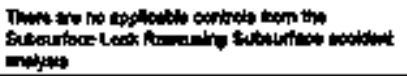 \\
\hline
\end{tabular}




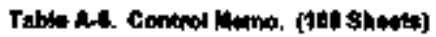

\begin{tabular}{|c|c|c|c|}
\hline $\mathbf{p}$ & 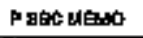 & 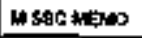 & Dathent \\
\hline $11493 x-09$ a 3 & & & 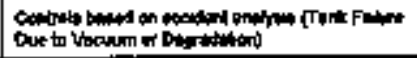 \\
\hline \multicolumn{4}{|l|}{$1148 \times-06$ b } \\
\hline xath-olk & & & 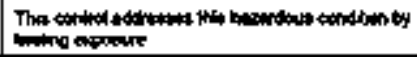 \\
\hline autusis & & & 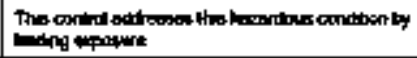 \\
\hline 204AF-91C & & & 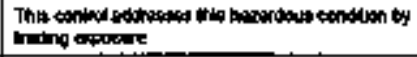 \\
\hline 2004R-910 & & & 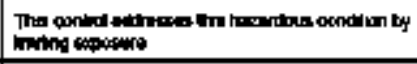 \\
\hline \multicolumn{4}{|l|}{ 2OAR-01E } \\
\hline \multicolumn{4}{|l|}{ 2OAkR-OHF } \\
\hline \multicolumn{4}{|l|}{ Doenk.01e } \\
\hline DAAR-OHH & & & 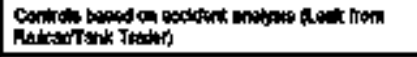 \\
\hline somedri & & & 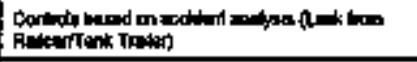 \\
\hline 204AR-01] & & & The \\
\hline DOLAROAK & & & 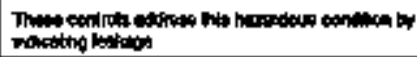 \\
\hline toatheren & & & 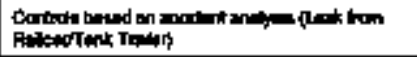 \\
\hline $20408-60$ & & & 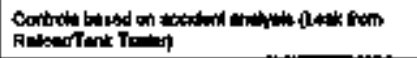 \\
\hline gomateact & & & \\
\hline
\end{tabular}




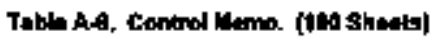

\begin{tabular}{|c|c|c|c|}
\hline מן & P géch vato & $M$ 自的 vano & 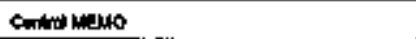 \\
\hline \multicolumn{4}{|l|}{$20+49-60$} \\
\hline Dour-oose & & & 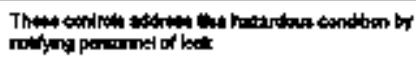 \\
\hline $204 k+0, p$ & & & 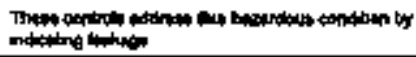 \\
\hline Xenk-ous & & & 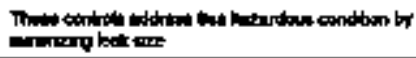 \\
\hline 204AR-QBB & & & 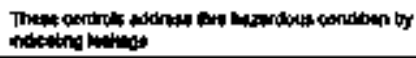 \\
\hline \multicolumn{4}{|l|}{ ZDAAR-003C } \\
\hline OMAR-OAR & & & 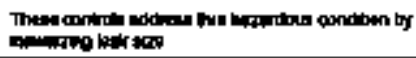 \\
\hline Dataroses. & & & 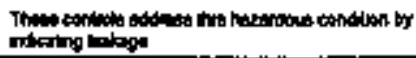 \\
\hline 204AR-04C & & & 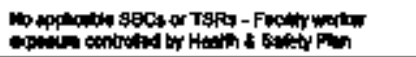 \\
\hline 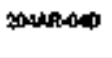 & & & 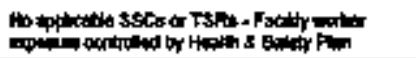 \\
\hline aputtonz & & & 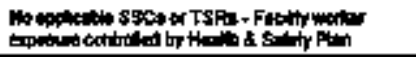 \\
\hline \multicolumn{4}{|l|}{ 204AR0F } \\
\hline \multicolumn{4}{|l|}{2046 RCSA } \\
\hline Dowher: & & & 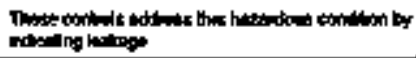 \\
\hline \multicolumn{4}{|l|}{ 204kR-cose } \\
\hline Dohinited & & & 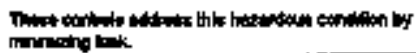 \\
\hline
\end{tabular}




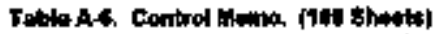

\begin{tabular}{|c|c|c|c|}
\hline ID & $P$ SEC wento & H sect Man & cother thintos \\
\hline SOAAR-06t & & & 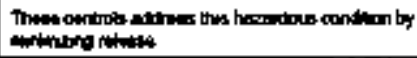 \\
\hline 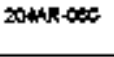 & & & 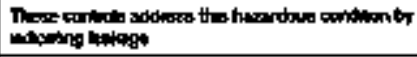 \\
\hline DOARAOTh & & & 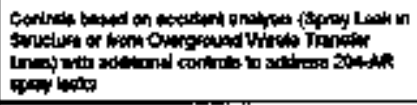 \\
\hline DAAF-0PrD & & & 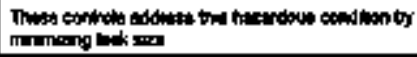 \\
\hline \multicolumn{4}{|l|}{ Irdak-0TC } \\
\hline TDARPRDOM & & & 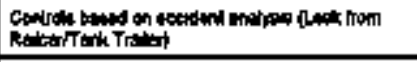 \\
\hline aUdk-098 & & & 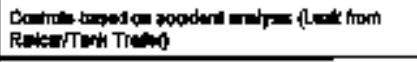 \\
\hline 204ati-0ec & & & 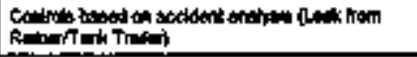 \\
\hline abseteco & & & 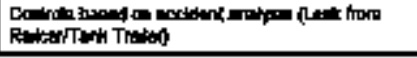 \\
\hline \multicolumn{4}{|l|}{ 204AR+Doc } \\
\hline aundor & & & 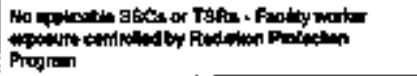 \\
\hline \multicolumn{4}{|l|}{ abdereats } \\
\hline \multicolumn{4}{|l|}{ OALAR-OAH } \\
\hline 20AAR-I1A & & & 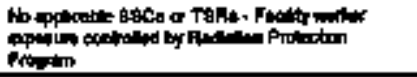 \\
\hline
\end{tabular}




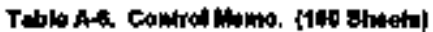

\begin{tabular}{|c|c|c|c|}
\hline ס & PLSC LIMO & is sec tonst & Controf lesto \\
\hline zoukntill & & & 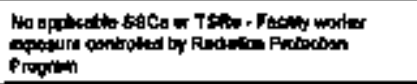 \\
\hline DO4AR+1 IC & & & 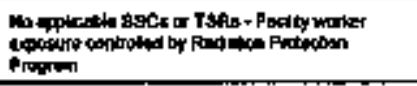 \\
\hline $204 R-110$ & & & 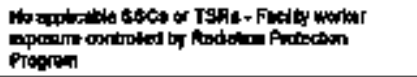 \\
\hline $204 \mathrm{R}-115$ & & & 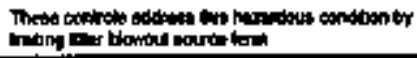 \\
\hline TDMRATIF & & & 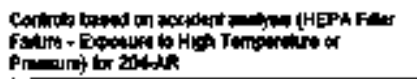 \\
\hline $213 w$ & & & 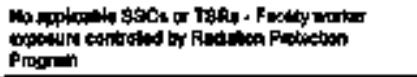 \\
\hline 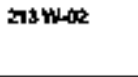 & & & 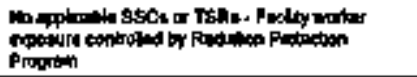 \\
\hline \multicolumn{4}{|l|}{$2+3 w_{03}$} \\
\hline \multicolumn{4}{|l|}{ 213 th-04 } \\
\hline \multicolumn{4}{|l|}{219 nर्S } \\
\hline \multicolumn{4}{|l|}{213 was } \\
\hline \multicolumn{4}{|l|}{ 213w-की } \\
\hline \multicolumn{4}{|l|}{$213 w-4$} \\
\hline $215 w-6$ & & & 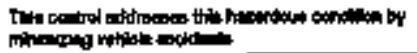 \\
\hline 244AF-O1 a D3 & & & 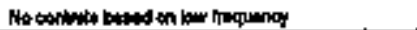 \\
\hline
\end{tabular}


Tabte Afe Control Wuna [160 sheotsy

\begin{tabular}{|c|c|c|c|}
\hline $\mathbf{0}$ & P gecc in 110 & Mbec WENo & 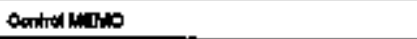 \\
\hline 240ke-01 b 어 & & & 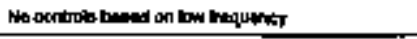 \\
\hline 2twhel bon & & & 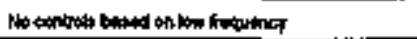 \\
\hline zuckter a do & & & He cuntrot treed on low frepuanor \\
\hline 244etedat b o4 & & & 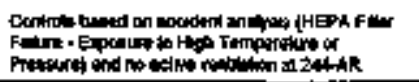 \\
\hline 2400009 c0s & & & 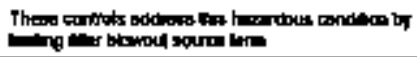 \\
\hline 2440R-O1 eOS & & & 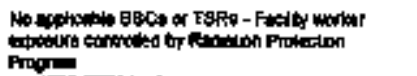 \\
\hline $24040.01 \div 03$ & & & 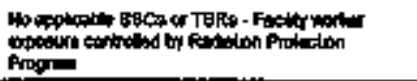 \\
\hline \multicolumn{4}{|l|}{$2 \operatorname{sen} A-01<$ of 7} \\
\hline $244 \mathrm{R}-01<08$ & & & 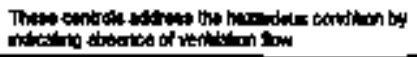 \\
\hline $244 k-01000$ & & & 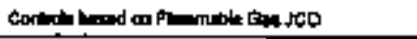 \\
\hline 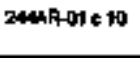 & & & 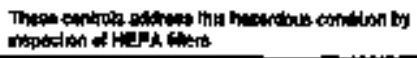 \\
\hline 244AR-O1 a 11 & & & 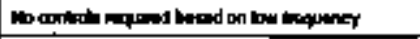 \\
\hline \multicolumn{4}{|l|}{$244 R-01$ \& 13} \\
\hline $2 \sin 02$ \& $x$ & & & 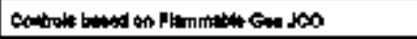 \\
\hline \multicolumn{4}{|l|}{2 avedizets } \\
\hline 24440.020 .09 & & & 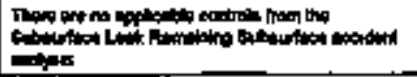 \\
\hline
\end{tabular}




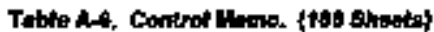

\begin{tabular}{|c|c|c|c|}
\hline ID & Psschas & 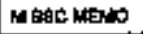 & Condert \\
\hline 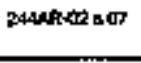 & & & 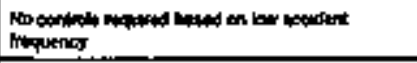 \\
\hline $2 m a n c 0200$ & & & 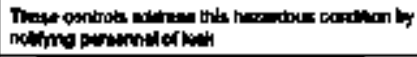 \\
\hline 244kR-CS I O & & & 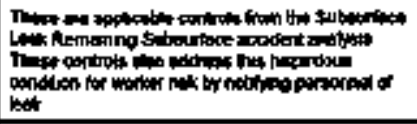 \\
\hline 24 kntor 10 & & & 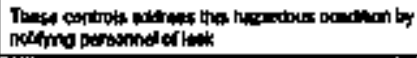 \\
\hline $2449 R-60=11$ & & & 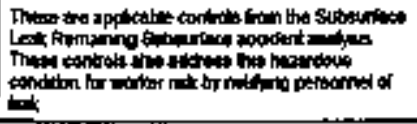 \\
\hline $244 k+02+12$ & & & 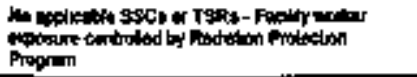 \\
\hline 244an-0. a 13 & & & 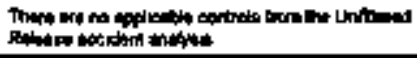 \\
\hline 2440200014 & & & 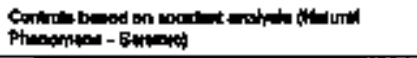 \\
\hline \multicolumn{4}{|l|}{$2444 R-00=03$} \\
\hline 24sap.09 on & & & 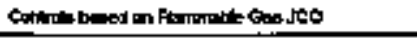 \\
\hline $240 \mathrm{hen}=06$ & & & 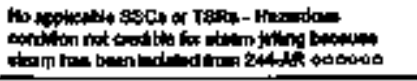 \\
\hline $244 \times 100000$ & & & 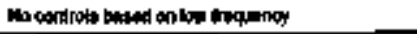 \\
\hline $2448 \Delta \mathrm{B}$ & & & \\
\hline
\end{tabular}




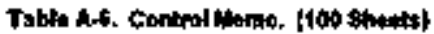

\begin{tabular}{|c|c|c|c|}
\hline $\mathbf{D}$ & P SEC LENONO & m sactant & Control tisen \\
\hline $244 R-03 * 00$ & & & 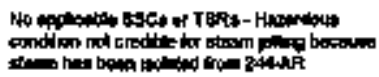 \\
\hline $244 \mathrm{R}-03 \cdot 09$ & & & 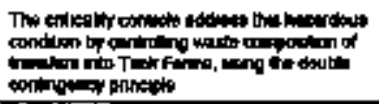 \\
\hline 244R.09: 13 & & & 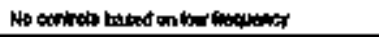 \\
\hline 2dake os a 14 & & & 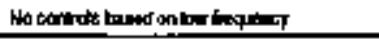 \\
\hline \multicolumn{4}{|l|}{ 24Maped a 15} \\
\hline \multicolumn{4}{|l|}{ 2446R-03 \& 19} \\
\hline \multicolumn{4}{|l|}{$24002-09 \cdot 17$} \\
\hline \multicolumn{4}{|l|}{ 24andos a 10} \\
\hline \multicolumn{4}{|l|}{ ZSARROA = OI } \\
\hline \multicolumn{4}{|l|}{ 2040R- 450101} \\
\hline $2444 R-05102$ & & & 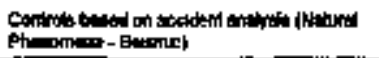 \\
\hline \multicolumn{4}{|l|}{ 2ankictos in } \\
\hline 2GARR-OT 100 & & & 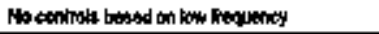 \\
\hline $244 \mathrm{~A} R-07$ a 028 & & & 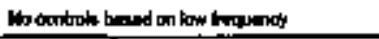 \\
\hline 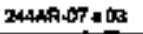 & & & 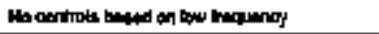 \\
\hline \multicolumn{4}{|l|}{ 2MAREDT I OM } \\
\hline \multicolumn{4}{|l|}{ 244AR-mor } \\
\hline \multicolumn{4}{|l|}{ 24AMAPNOK } \\
\hline 244ARPEDS & & & \\
\hline
\end{tabular}


Tabln A-4. Control Worno. [ate Sherite]

\begin{tabular}{|c|c|c|c|}
\hline 10 & pgex rato & H sec Mano & Contro וM \\
\hline \multicolumn{4}{|c|}{ 2Menf-PHON } \\
\hline \multicolumn{4}{|c|}{$244 \mathrm{ni}+105$} \\
\hline \multicolumn{4}{|c|}{$24018+1400$} \\
\hline \multicolumn{4}{|l|}{ 24OAFAPHOT } \\
\hline \multicolumn{4}{|l|}{ 2AGARPHOA } \\
\hline \multicolumn{4}{|c|}{ 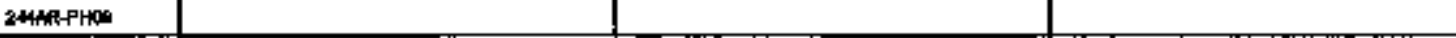 } \\
\hline \multicolumn{4}{|c|}{ 24WAPH10 } \\
\hline \multicolumn{4}{|c|}{ Z4MANPH11 } \\
\hline \multicolumn{4}{|c|}{ 24ACRPH12 } \\
\hline \multicolumn{4}{|c|}{ 24AR-PH13 } \\
\hline \multicolumn{4}{|c|}{$244 \mathrm{R}+\mathrm{H} 14$} \\
\hline \multicolumn{4}{|c|}{ 24cherents } \\
\hline \multicolumn{4}{|c|}{ 244kt+4146 } \\
\hline \multicolumn{4}{|c|}{ 244ak-7at } \\
\hline \multicolumn{4}{|c|}{$244 \mathrm{~A}+\mathrm{P}+15$} \\
\hline \multicolumn{4}{|l|}{ 24Mkeits } \\
\hline \multicolumn{4}{|l|}{ manwas } \\
\hline $27 r+402$ & & & 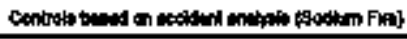 \\
\hline $2 \pi 2010$ & & & 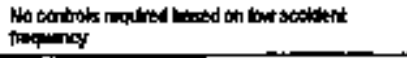 \\
\hline Thingan & & & 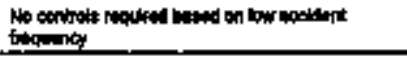 \\
\hline
\end{tabular}




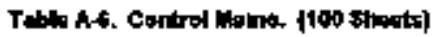

\begin{tabular}{|c|c|c|c|}
\hline $\mathbf{0}$ & P $\sec$ & MIsc MARO & Cemirat Men \\
\hline 2Tzentos & & & 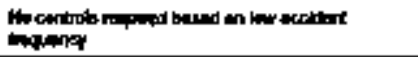 \\
\hline $2 \cos x$ & & & 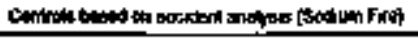 \\
\hline 00001 & & & 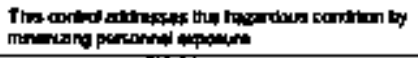 \\
\hline 90002 & & & 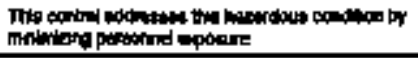 \\
\hline $900-\infty$ & & & 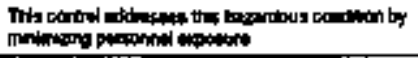 \\
\hline $800-04$ & & & 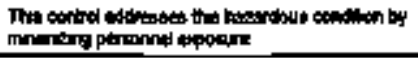 \\
\hline \multicolumn{4}{|l|}{9000} \\
\hline 9009 & & & 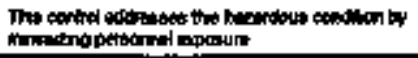 \\
\hline abot & & & 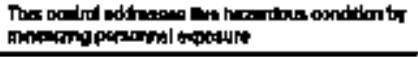 \\
\hline xabos & & & 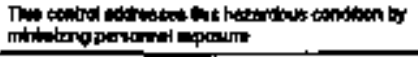 \\
\hline \multicolumn{4}{|l|}{ apos } \\
\hline \multicolumn{4}{|l|}{$\neq 0-10$} \\
\hline$\infty$ & & & 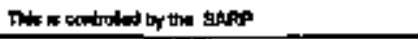 \\
\hline \multicolumn{4}{|l|}{ BNon } \\
\hline \multicolumn{4}{|l|}{ BND-OIt } \\
\hline DNDGR & & & 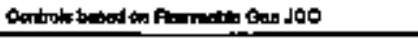 \\
\hline \multicolumn{4}{|l|}{ BNDEm } \\
\hline E्CF=-01 & & & 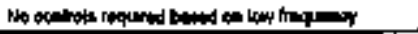 \\
\hline
\end{tabular}




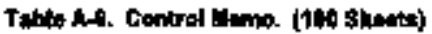

\begin{tabular}{|c|c|c|c|}
\hline 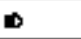 & P osc rate & M S5C MU & Control Ml|pes \\
\hline $\cos =0$ & & & 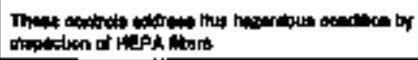 \\
\hline$c x=0$ & & & 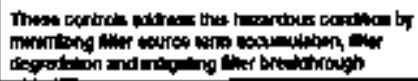 \\
\hline cossou & & & 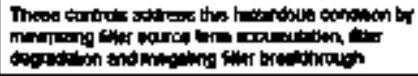 \\
\hline \multicolumn{4}{|l|}{$\operatorname{ccs} \theta$} \\
\hline \multicolumn{4}{|l|}{ ctcres } \\
\hline CCAFT & & 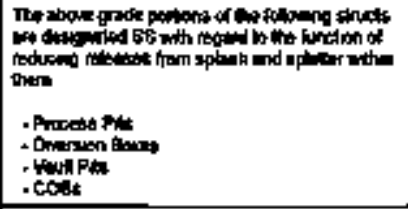 & 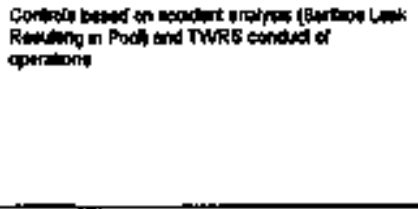 \\
\hline \multicolumn{4}{|l|}{ Cekas } \\
\hline \multicolumn{4}{|l|}{ Ccto $-\infty$} \\
\hline \multicolumn{4}{|l|}{ CEF.10 } \\
\hline \multicolumn{4}{|l|}{ CeF-11 } \\
\hline \multicolumn{4}{|l|}{ CCF-12 } \\
\hline \multicolumn{4}{|l|}{ CCFras } \\
\hline \multicolumn{4}{|l|}{ CCFi14 } \\
\hline $\cot =15$ & & & \\
\hline
\end{tabular}




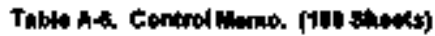

\begin{tabular}{|c|c|c|c|}
\hline 10 & P BAC MEP & $49 S C+3160$ & Contod lepes? \\
\hline 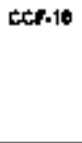 & & & 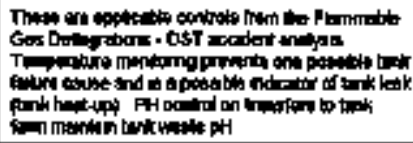 \\
\hline Cot.17 & & & 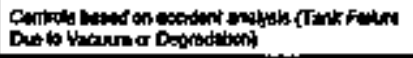 \\
\hline CCF-15 & & & 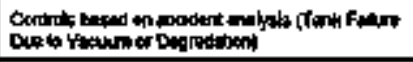 \\
\hline CCE.19 & & & 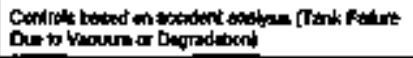 \\
\hline$C \mathrm{CF}-\infty \mathrm{O}$ & & & 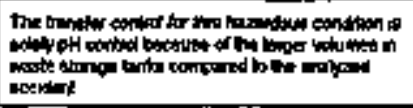 \\
\hline CDP-01 & & & 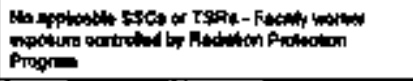 \\
\hline \multicolumn{4}{|l|}{$\operatorname{cop}-02$} \\
\hline copess & & & 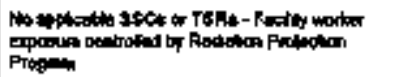 \\
\hline CDPAO & & & 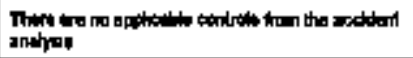 \\
\hline \multicolumn{4}{|l|}{ CDPES } \\
\hline totents & & & 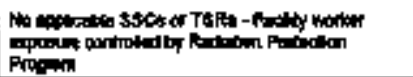 \\
\hline
\end{tabular}


Table A-6, Cankrod Lemo. (100 Shedto)

\begin{tabular}{|c|c|c|c|}
\hline 10 & P sto kn: & M soctintent & Contod ow \\
\hline copas & & & 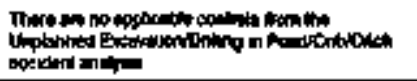 \\
\hline cDp-0日 & & & 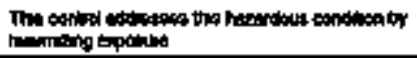 \\
\hline \multicolumn{4}{|l|}{$\cos 40$} \\
\hline CQPF-10 & & & 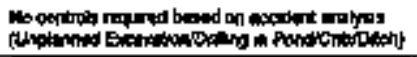 \\
\hline Coter & & 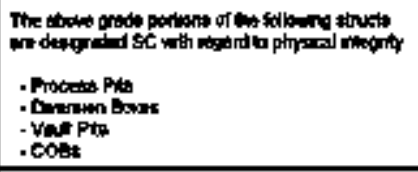 & 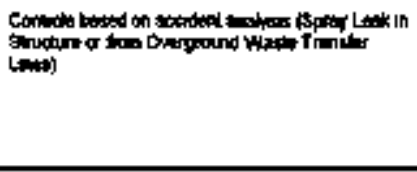 \\
\hline $\cos \theta$ & & 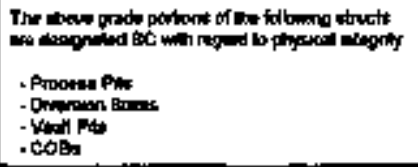 & 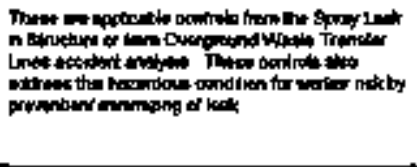 \\
\hline comas & & 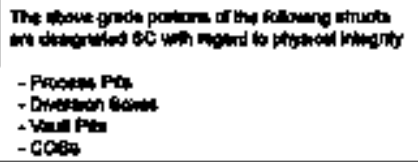 & 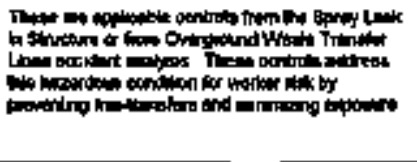 \\
\hline
\end{tabular}




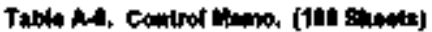

\begin{tabular}{|c|c|c|c|}
\hline 0 & P Ssc 2E & 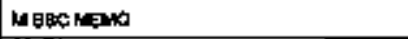 & Centrat Mbes \\
\hline cosent & 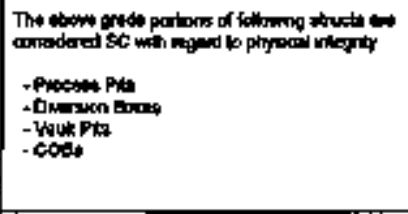 & 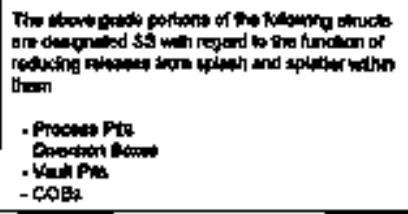 & 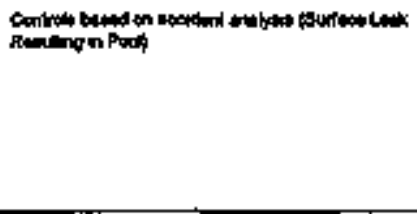 \\
\hline conasa & & & 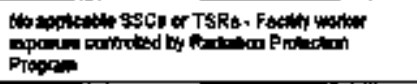 \\
\hline $\cos 05$ & & & 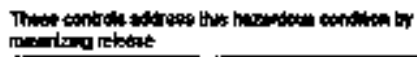 \\
\hline COB-OE & & & 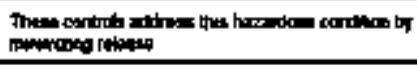 \\
\hline $\cos 205 d$ & & & 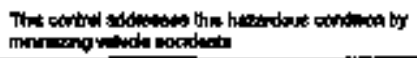 \\
\hline $\cos -0 s$ & & & 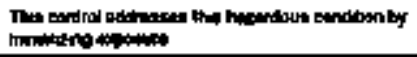 \\
\hline COQ.00r & & & 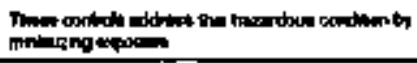 \\
\hline cos-0is & & & 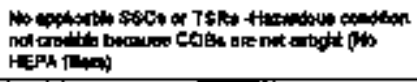 \\
\hline $\cos -000$ & 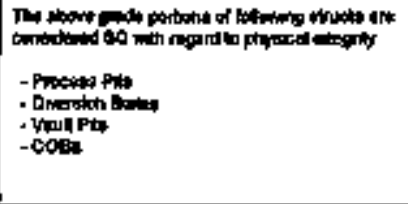 & 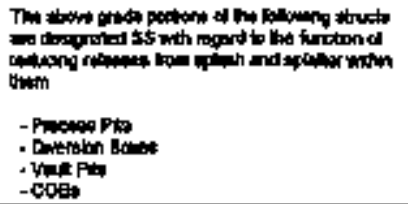 & 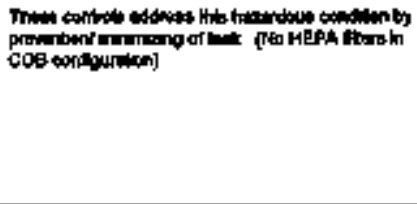 \\
\hline
\end{tabular}


Table A4, Centrol theno. (160 thoctpr

\begin{tabular}{|c|c|c|c|}
\hline 10 & 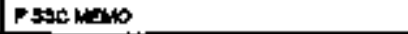 & M SOS MENO & 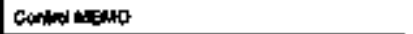 \\
\hline \multicolumn{4}{|l|}{ cogertr } \\
\hline COE- $0 B$ & 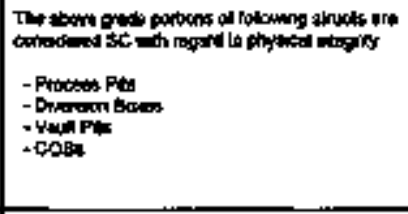 & 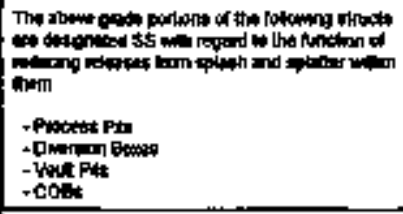 & 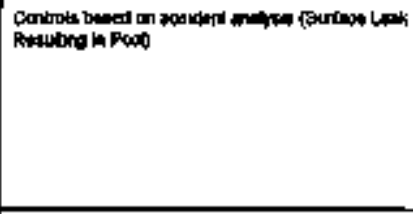 \\
\hline CRAtOr & & & 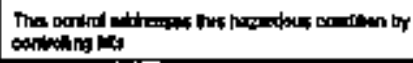 \\
\hline censord & & & 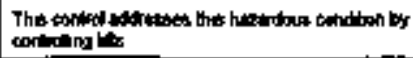 \\
\hline CROAOS & & & 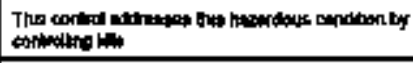 \\
\hline \multicolumn{4}{|l|}{ chaspos } \\
\hline CRasOS & & & 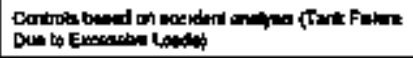 \\
\hline 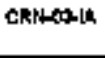 & & & 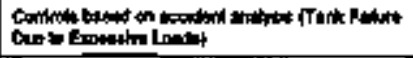 \\
\hline CRR-O9-1B & & & 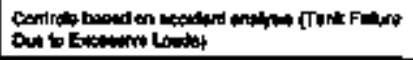 \\
\hline CANOAS & & & 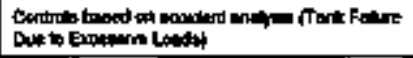 \\
\hline CRNOALIh & & & 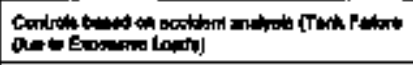 \\
\hline centorto & & & 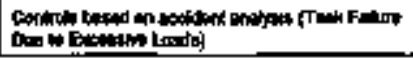 \\
\hline
\end{tabular}


Tobla A6. Contred Mimo. (100 binets)

\begin{tabular}{|c|c|c|c|}
\hline I & P BSE MEAO & M Baso Meno & 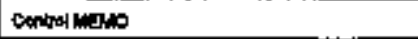 \\
\hline canded & 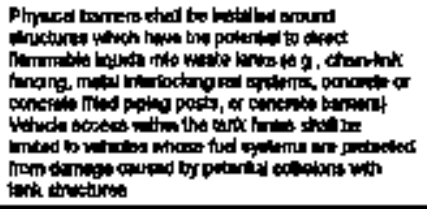 & & 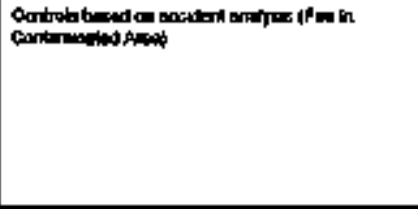 \\
\hline CAN 40 - & & & 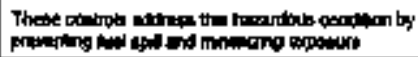 \\
\hline Contal 06 & 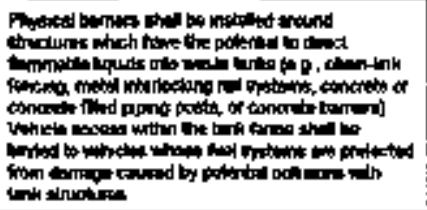 & & 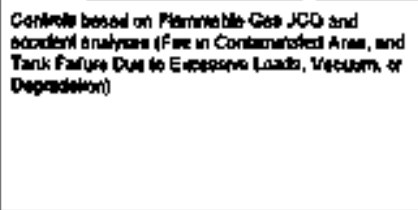 \\
\hline chator & & & 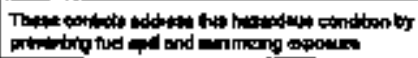 \\
\hline cenar & 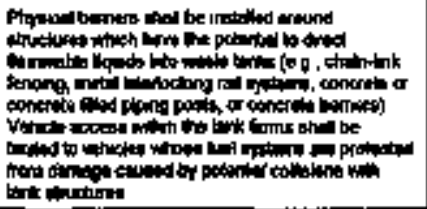 & & 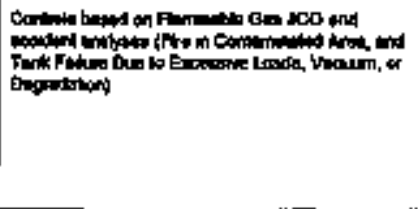 \\
\hline \multicolumn{4}{|l|}{ CRMOP } \\
\hline CRNOA & & & 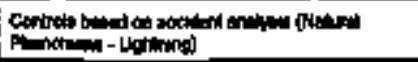 \\
\hline САNOAH & & & \\
\hline
\end{tabular}




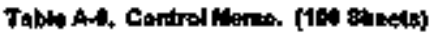

\begin{tabular}{|c|c|c|c|}
\hline $\mathbf{D}$ & A stsc Man & Mssch hus & 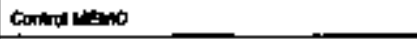 \\
\hline coston & & & 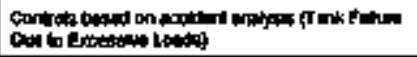 \\
\hline crandol & & & 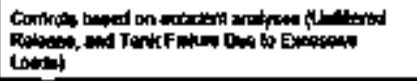 \\
\hline CNA 10 & & & 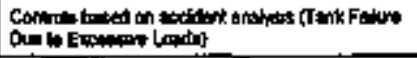 \\
\hline CRHAD & & & 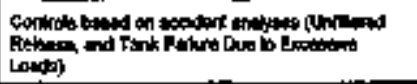 \\
\hline CRRHII & & & 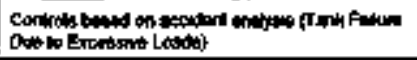 \\
\hline CRN-tI-H & & & 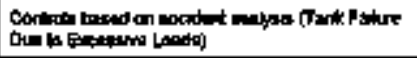 \\
\hline CRN-11-F & & & 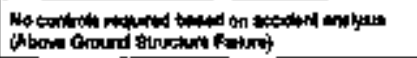 \\
\hline CRN-11 to & & & 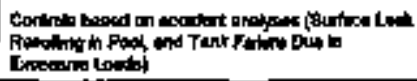 \\
\hline Cfant 12 & & & 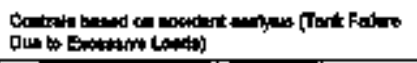 \\
\hline CHON-12HA & & & 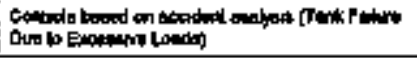 \\
\hline CRNA124B & & & 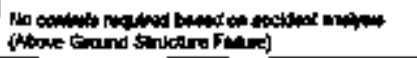 \\
\hline Ck1:12*t & & & 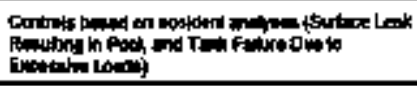 \\
\hline CR/A134h & & & 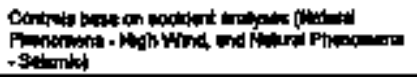 \\
\hline
\end{tabular}




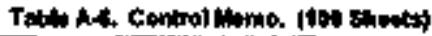

\begin{tabular}{|c|c|c|c|}
\hline $\mathbf{b}$ & P פBC REMO & 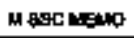 & Controd raw \\
\hline Chin-13/3 & & & 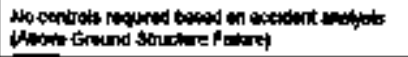 \\
\hline Cron-13- & & & 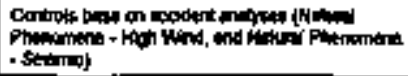 \\
\hline CRA. $13<4$ & & & 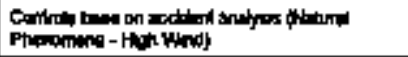 \\
\hline CRH.15t & & & 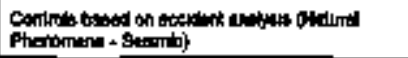 \\
\hline \multicolumn{4}{|l|}{ CARN-14 } \\
\hline \multicolumn{4}{|l|}{ ERH.14 } \\
\hline \multicolumn{4}{|l|}{ CRN-WU } \\
\hline \multicolumn{4}{|l|}{ CRIN-15-4 } \\
\hline \multicolumn{4}{|l|}{ ERN-W } \\
\hline \multicolumn{4}{|l|}{ CRNLWH } \\
\hline CRNA-1) & & & 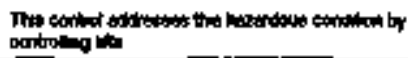 \\
\hline \multicolumn{4}{|l|}{ CRN-TI } \\
\hline centod & & & 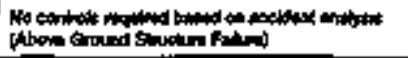 \\
\hline cenes-12-1 & & & 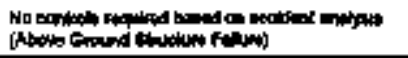 \\
\hline $\operatorname{ctan} 10$ & & & 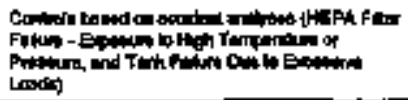 \\
\hline 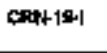 & & & 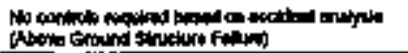 \\
\hline
\end{tabular}


Tabte M-6. Conked Hemo, f100 thouts

\begin{tabular}{|c|c|c|c|}
\hline$D$ & $P$ stsc nato & Msstc Mten & Control Latiot \\
\hline Cratso & & & 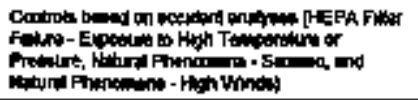 \\
\hline cterisxol & & & 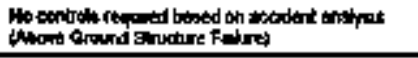 \\
\hline çandal & & & 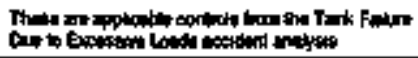 \\
\hline crintal-|k & & & 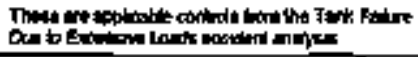 \\
\hline CAN-2x-3 & & & 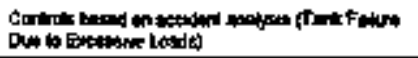 \\
\hline CRN-Z2 & & & 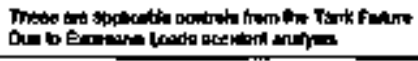 \\
\hline CRN-ZuL & & & 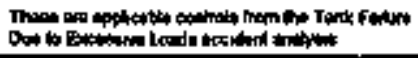 \\
\hline CAN 200 & & & 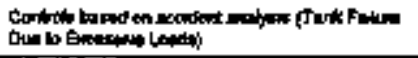 \\
\hline CRNL23 & & & 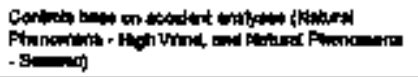 \\
\hline CRN-23-M & & & 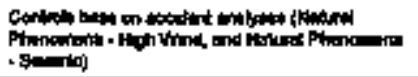 \\
\hline CRHL2S-G & & & 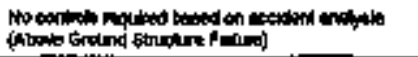 \\
\hline \multicolumn{4}{|l|}{ arengat } \\
\hline \multicolumn{4}{|l|}{ costant } \\
\hline CPitars & & & \\
\hline
\end{tabular}


Table AA, Control Memo. (164 atacks)

\begin{tabular}{|c|c|c|c|}
\hline 10 & P stsc Heino & W stsc & 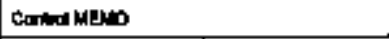 \\
\hline \multicolumn{4}{|l|}{ CANA } \\
\hline CRN-ZS-B & & & 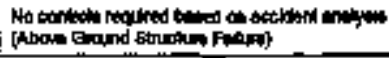 \\
\hline \multicolumn{4}{|l|}{ CAN-Z } \\
\hline \multicolumn{4}{|l|}{ CAN-Zsan } \\
\hline CRN-ZBE & & & 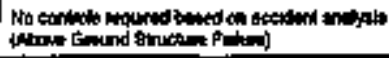 \\
\hline \multicolumn{4}{|l|}{ Cenk } \\
\hline \multicolumn{4}{|l|}{ cRrtzf-h } \\
\hline CRNHETE & & & 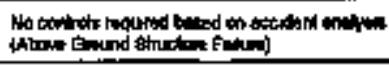 \\
\hline \multicolumn{4}{|l|}{ CRlN-204 } \\
\hline CAvTror & & & 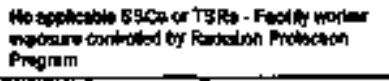 \\
\hline CURAT & & & 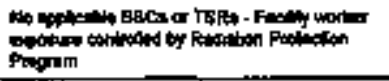 \\
\hline L4NA-m & & & 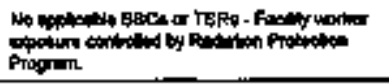 \\
\hline CWFOR- & & & 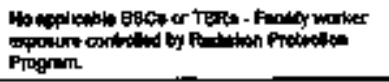 \\
\hline CRPAS & & & 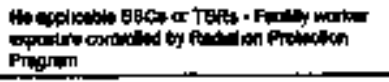 \\
\hline
\end{tabular}




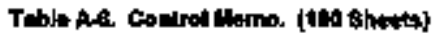

\begin{tabular}{|c|c|c|c|}
\hline $\mathbf{D}$ & PStS MER & in sst kato & 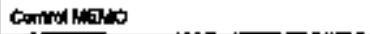 \\
\hline CIROSH & & & 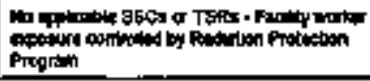 \\
\hline \multicolumn{4}{|l|}{ CLROM } \\
\hline \multicolumn{4}{|l|}{ chandit } \\
\hline COURAS & 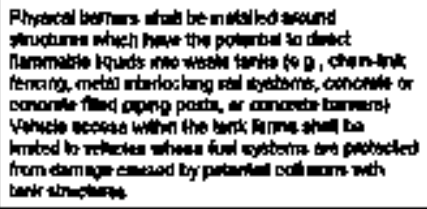 & & 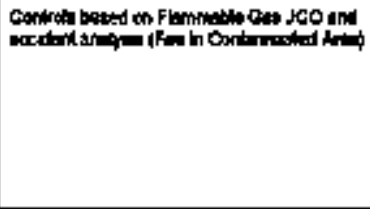 \\
\hline \multicolumn{4}{|l|}{ Chen-0s- } \\
\hline \multicolumn{4}{|l|}{ CWA-06 } \\
\hline \multicolumn{4}{|l|}{ CWR-DE-4 } \\
\hline \multicolumn{4}{|l|}{ CLR-DT } \\
\hline \multicolumn{4}{|l|}{ OLW-OF- } \\
\hline \multicolumn{4}{|l|}{ EWR-06 } \\
\hline \multicolumn{4}{|l|}{ Eकी06-1 } \\
\hline \multicolumn{4}{|l|}{$\cos \theta$} \\
\hline \multicolumn{4}{|l|}{ chatusal } \\
\hline \multicolumn{4}{|l|}{ GUR:10 } \\
\hline \multicolumn{4}{|l|}{ GVR-10 } \\
\hline \multicolumn{4}{|l|}{ chlt:.11 } \\
\hline chitoll.1 & & & \\
\hline
\end{tabular}




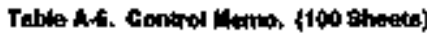

\begin{tabular}{|c|c|c|c|}
\hline 10 & P stsch nand & M LstC Nenos & 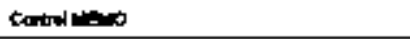 \\
\hline CWR-12H & & & 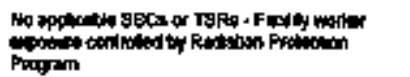 \\
\hline CNR-13 & & & 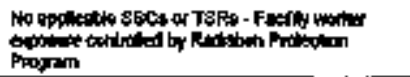 \\
\hline ALR-14 & & & 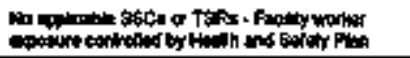 \\
\hline CuR181 & & & 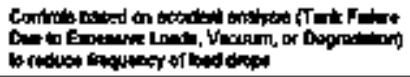 \\
\hline \multicolumn{4}{|l|}{ DCNAn } \\
\hline \multicolumn{4}{|l|}{ DCH.OAS } \\
\hline DCNDDO2 & & & 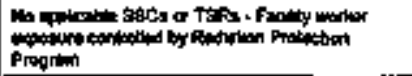 \\
\hline \multicolumn{4}{|c|}{ DCN-DREA } \\
\hline \multicolumn{4}{|c|}{ 0xin-00.18 } \\
\hline Deces & & & 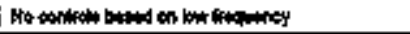 \\
\hline \multicolumn{4}{|l|}{ Destasl } \\
\hline \multicolumn{4}{|l|}{ Denos } \\
\hline DEWOH & & & 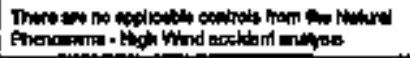 \\
\hline \multicolumn{4}{|l|}{ Octos } \\
\hline \multicolumn{4}{|l|}{ Dch0s-1 } \\
\hline \multicolumn{4}{|l|}{ DCNAS } \\
\hline DeNation & & & \\
\hline
\end{tabular}




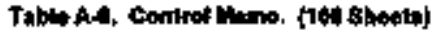

\begin{tabular}{|c|c|c|c|}
\hline 由 & P stac inato & 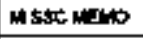 & 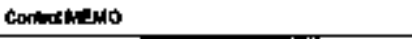 \\
\hline \multicolumn{4}{|l|}{ DCN 407} \\
\hline \multicolumn{4}{|l|}{ DCN-OPA } \\
\hline eter & & & 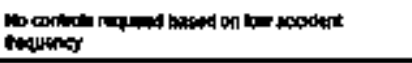 \\
\hline$E E-\infty R$ & & & 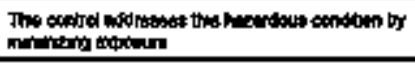 \\
\hline \multicolumn{4}{|l|}{ EE-10 } \\
\hline EE-04d & 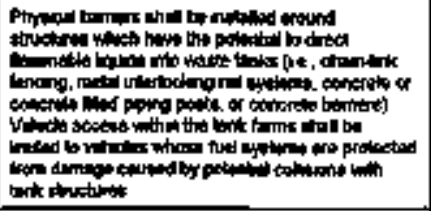 & & 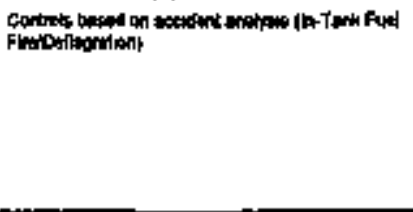 \\
\hline Ee-04t & 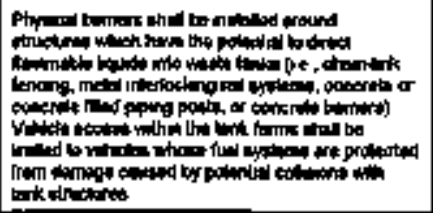 & & 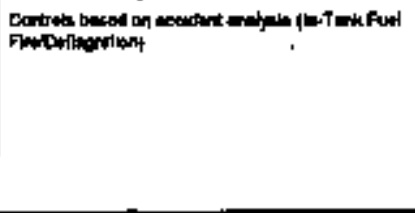 \\
\hline 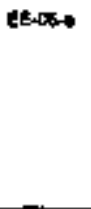 & 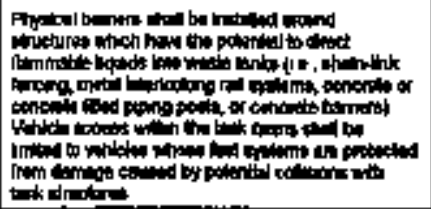 & & 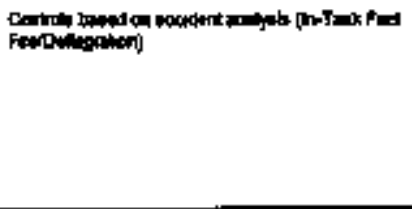 \\
\hline
\end{tabular}


Table Ath Conlred Limo. (10) Shevta)

\begin{tabular}{|c|c|c|c|}
\hline 回 & 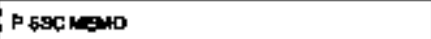 & Mtstrith & Catrod WEM \\
\hline EE-A & 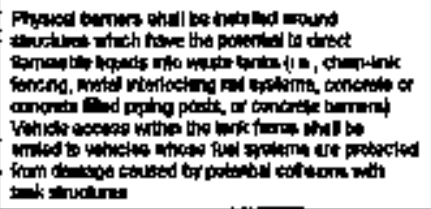 & & 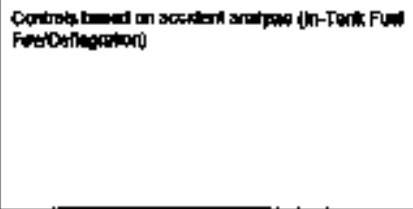 \\
\hline \multicolumn{4}{|l|}{ 舟-60 } \\
\hline \multicolumn{4}{|l|}{ IE-gr } \\
\hline \multicolumn{4}{|l|}{ EE -48} \\
\hline EE- $\theta$ & & & 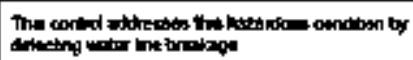 \\
\hline CE-10 & & & 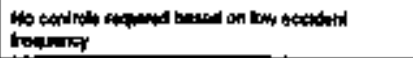 \\
\hline EE-11 & & & 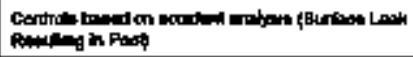 \\
\hline \multicolumn{4}{|l|}{ EE-12 } \\
\hline \multicolumn{4}{|l|}{ DEF } \\
\hline \multicolumn{4}{|l|}{ EE-4 } \\
\hline \multicolumn{4}{|l|}{ EE-15 } \\
\hline EI.+6 & & & 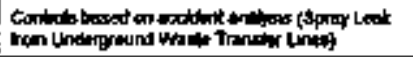 \\
\hline$E E-17$ & & & 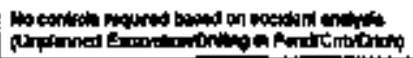 \\
\hline \multicolumn{4}{|l|}{ 它新价 } \\
\hline 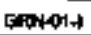 & & & \\
\hline
\end{tabular}


Tubla A-6. Conipol termo. (104 Aheats)

\begin{tabular}{|c|c|c|c|}
\hline$\Phi$ & P tse we & M sst & סק: \\
\hline \multicolumn{4}{|l|}{ QRMOQ } \\
\hline \multicolumn{4}{|l|}{ GRHARस्त } \\
\hline \multicolumn{4}{|l|}{ ofrosol } \\
\hline 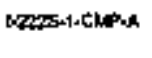 & & & 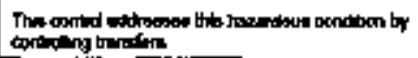 \\
\hline \multicolumn{4}{|l|}{ Banz-1-CAP-B } \\
\hline 1.2005-1-GAPCS & & 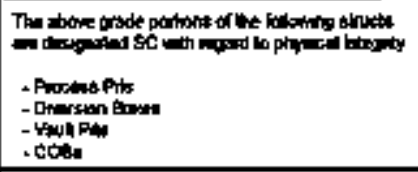 & 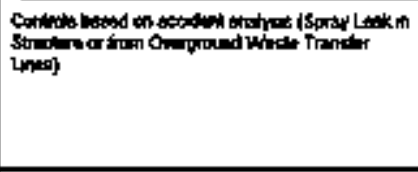 \\
\hline \multicolumn{4}{|l|}{ 1-2020-1-2014.0 } \\
\hline L2029-1-CAPE & 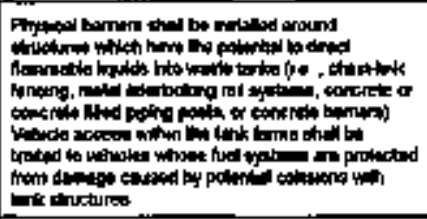 & & 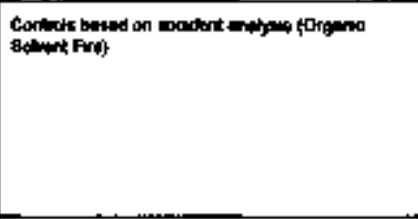 \\
\hline 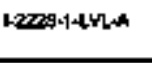 & & & 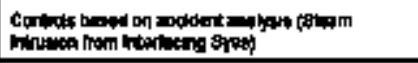 \\
\hline 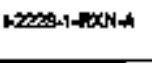 & & & 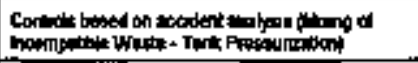 \\
\hline 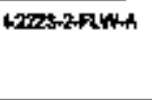 & & & 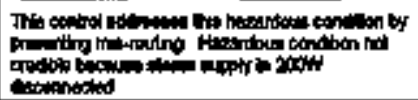 \\
\hline
\end{tabular}


Table At Conirol Mlowo. (100 Sheite)

\begin{tabular}{|c|c|c|c|}
\hline$D$ & P SSC MEKO & M ssct & 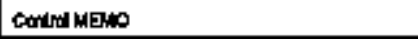 \\
\hline \multicolumn{4}{|l|}{ 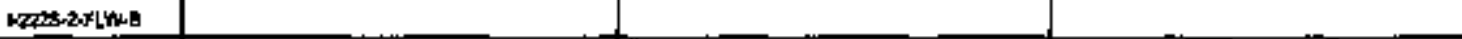 } \\
\hline 1-2028-2+7um-c & & & 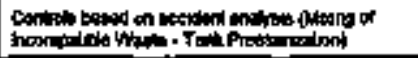 \\
\hline \multicolumn{4}{|l|}{$10 \cos 2 \pi+460$} \\
\hline \multicolumn{4}{|l|}{ 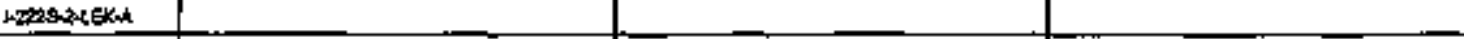 } \\
\hline 1-22200-LEK- & & 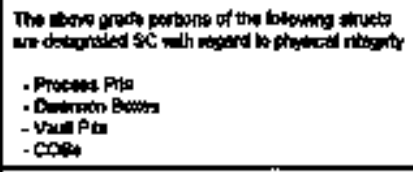 & 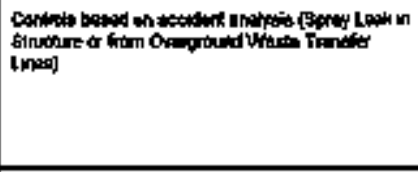 \\
\hline 1-2229-4 Ek-C & & & 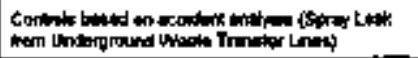 \\
\hline \multicolumn{4}{|l|}{ 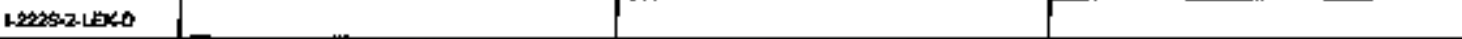 } \\
\hline 1-202-2-L0KE & & & 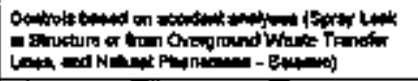 \\
\hline $1-2028-2-778-1$ & & & 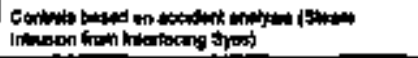 \\
\hline 120h-1.Futr-h & & & 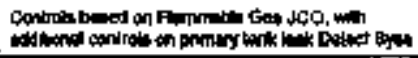 \\
\hline 12064-1.FLWE & & & 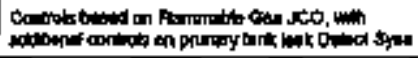 \\
\hline 1000/1-fintc & & & 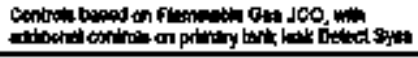 \\
\hline 1.20tentfinto & & & 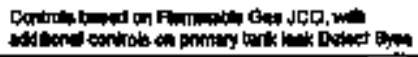 \\
\hline 1-2424-1FLW- & & & 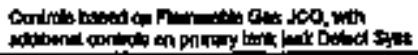 \\
\hline
\end{tabular}




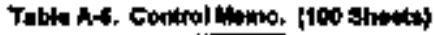

\begin{tabular}{|c|c|c|c|}
\hline ID & $P$ P & Magcheno & DAFA \\
\hline \multicolumn{4}{|l|}{ 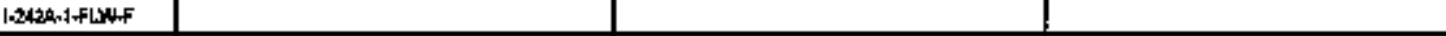 } \\
\hline \multicolumn{4}{|l|}{ 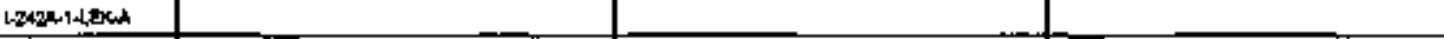 } \\
\hline HADA-1-LEK-B & & 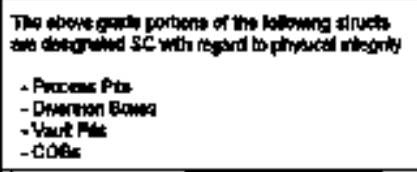 & 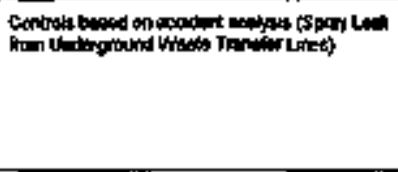 \\
\hline $1.242 A-1.407 K-C$ & & & 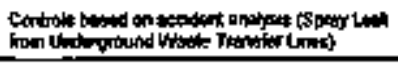 \\
\hline \multicolumn{4}{|l|}{ L-242A-1-LEKCD } \\
\hline 12OH-S-EKE & & & 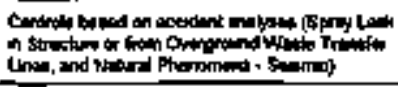 \\
\hline 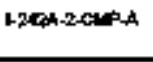 & & & 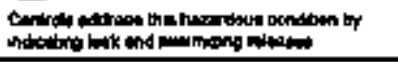 \\
\hline 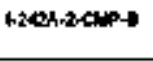 & & & 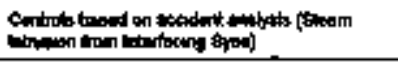 \\
\hline Hebs-2fLWA & & & 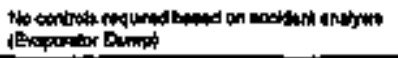 \\
\hline 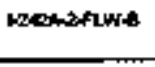 & & & 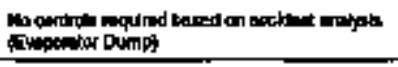 \\
\hline \multicolumn{4}{|l|}{ 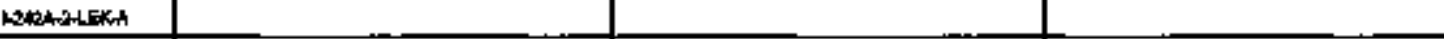 } \\
\hline 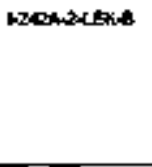 & & 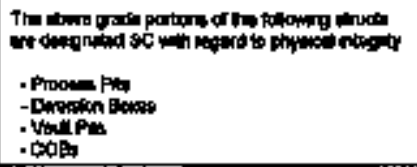 & 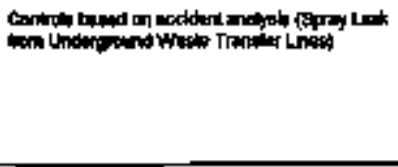 \\
\hline
\end{tabular}




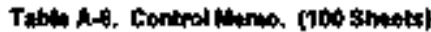

\begin{tabular}{|c|c|c|c|}
\hline $\mathbf{D}$ & PSScientop & MEgCNEND & Cantod Haxa \\
\hline H2BA2LEK-G & & & 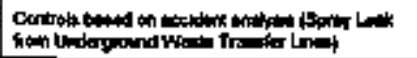 \\
\hline \multicolumn{4}{|l|}{ 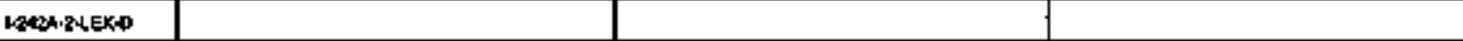 } \\
\hline $1-242+2-L e k E$ & & & 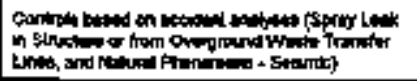 \\
\hline \multicolumn{4}{|l|}{$1.2424,2-\Gamma \mathrm{MPAh}$} \\
\hline $1-2+2+2-48 c-A$ & & & 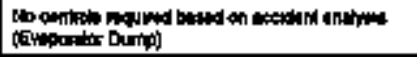 \\
\hline \multicolumn{4}{|l|}{ 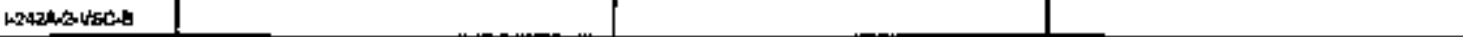 } \\
\hline H-242A-FLINKA & & & 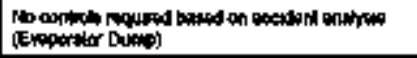 \\
\hline 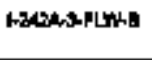 & & & 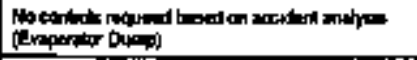 \\
\hline H-ALAAFINAA & & & 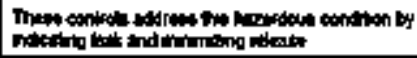 \\
\hline $1342 h+T H P A$ & & & 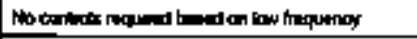 \\
\hline \multicolumn{4}{|l|}{$120048 f L$ L WA } \\
\hline 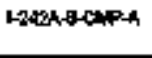 & & & 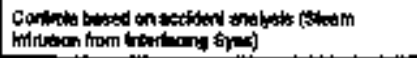 \\
\hline Mensfind & & & 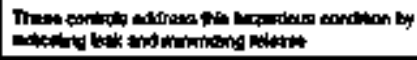 \\
\hline H20heAns & & & 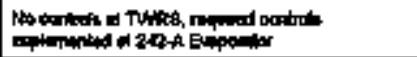 \\
\hline Hearefinte & & & 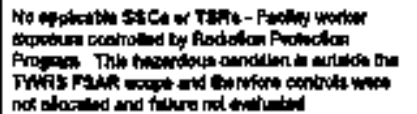 \\
\hline
\end{tabular}




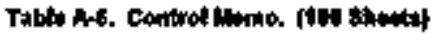

\begin{tabular}{|c|c|c|c|}
\hline 10 & P 68c⿻一𠃋十 & N B96 Haxp & 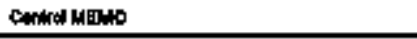 \\
\hline \multicolumn{4}{|l|}{ 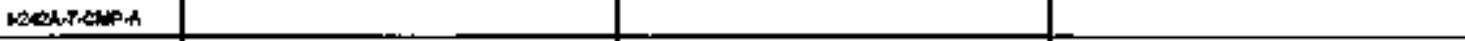 } \\
\hline 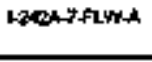 & & & 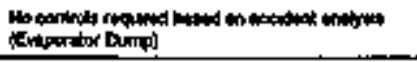 \\
\hline 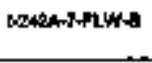 & & & 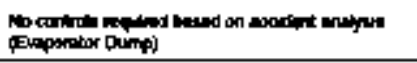 \\
\hline 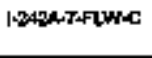 & & & 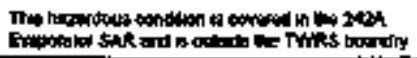 \\
\hline \multicolumn{4}{|l|}{ 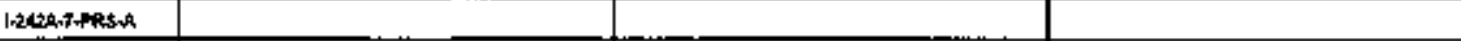 } \\
\hline 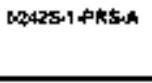 & & & 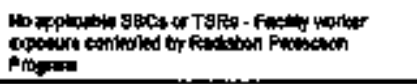 \\
\hline 1.2425-1-Tuph & & & 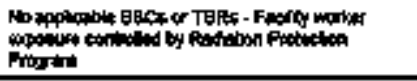 \\
\hline Hects-2PLWA & 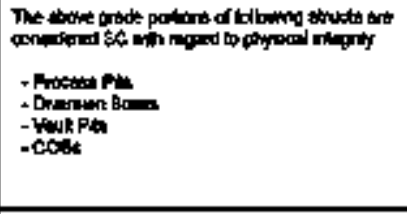 & 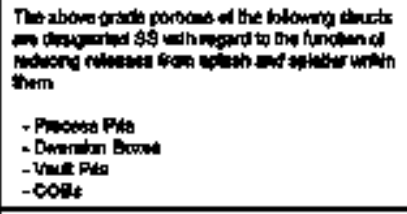 & 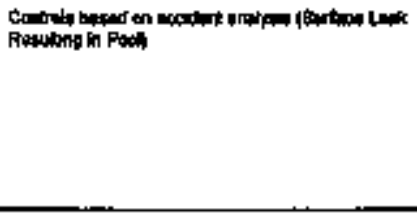 \\
\hline \multicolumn{4}{|l|}{ 12602fiw. } \\
\hline \multicolumn{4}{|l|}{ 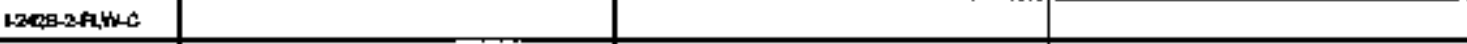 } \\
\hline \multicolumn{4}{|l|}{ 12428-2नLWD } \\
\hline 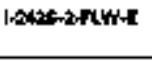 & & & 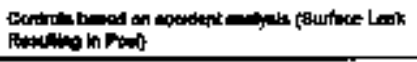 \\
\hline 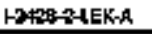 & & & \\
\hline
\end{tabular}




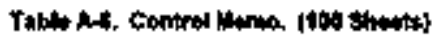

\begin{tabular}{|c|c|c|c|}
\hline 10 & paec pan & 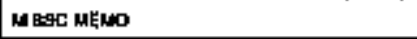 & 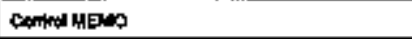 \\
\hline 1-2009-2Lext & & 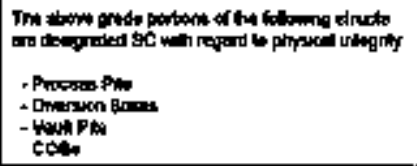 & 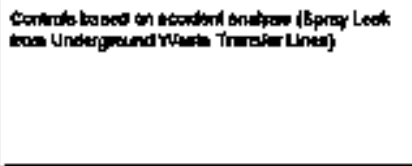 \\
\hline 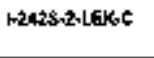 & & & 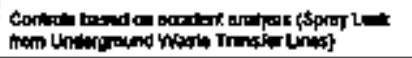 \\
\hline \multicolumn{4}{|l|}{ A-2429-2-LEK-D } \\
\hline HAdES-2LEK-L & & & 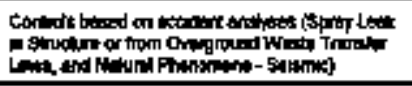 \\
\hline \multicolumn{4}{|l|}{ 1200-47Wh-A } \\
\hline \multicolumn{4}{|l|}{ 12425.4FIm.B } \\
\hline 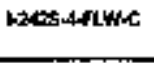 & & & 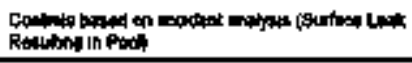 \\
\hline 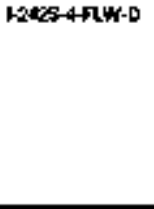 & 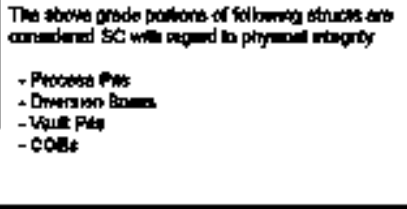 & 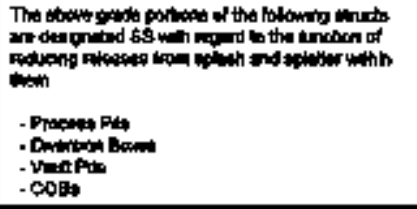 & 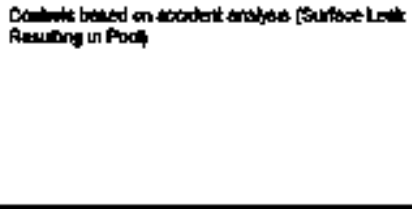 \\
\hline \multicolumn{4}{|l|}{$1-2425-46 E K+1$} \\
\hline $1.2428+4 \operatorname{Laxe}$ & & & 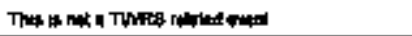 \\
\hline \multicolumn{4}{|l|}{$10+28-40 \times 6$} \\
\hline -2xzs-Hex-D & & & \\
\hline
\end{tabular}




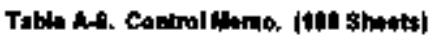

\begin{tabular}{|c|c|c|c|}
\hline ID & P gEC Lavo & Masc nowo & Controd natos \\
\hline HA2S-HEKE & & & 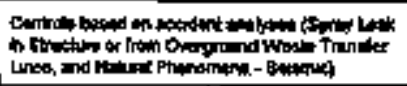 \\
\hline \multicolumn{4}{|l|}{$1-2425-6-0$ Mp $-x$} \\
\hline \multicolumn{4}{|l|}{ 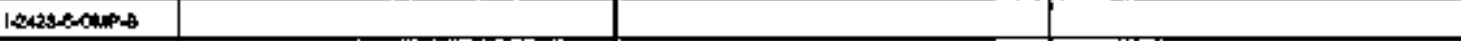 } \\
\hline \multicolumn{4}{|l|}{ |rotsoscanpach } \\
\hline \multicolumn{4}{|l|}{ 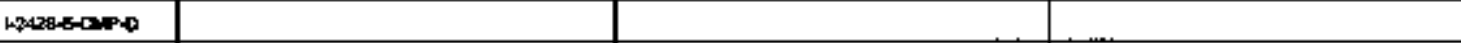 } \\
\hline \multicolumn{4}{|l|}{ 1-20489-5FLLAA } \\
\hline \multicolumn{4}{|l|}{ 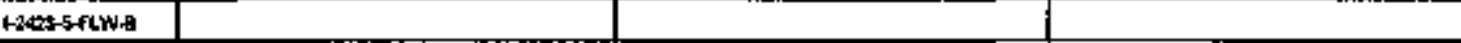 } \\
\hline \multicolumn{4}{|l|}{ neastanthe } \\
\hline \multicolumn{4}{|l|}{ 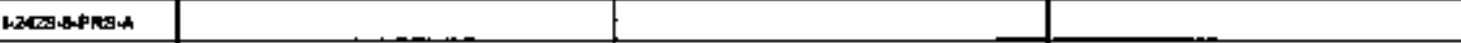 } \\
\hline \multicolumn{4}{|l|}{ 18GT-Zन्WMA } \\
\hline $1-2+21-3-3 \mathrm{~L}-\mathrm{k}$ & 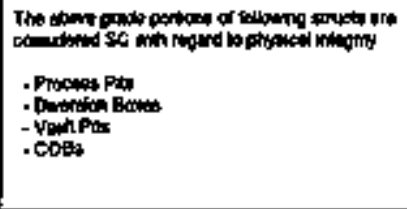 & 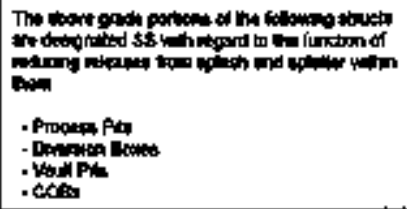 & 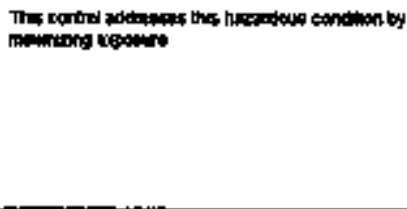 \\
\hline 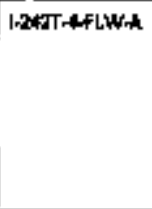 & 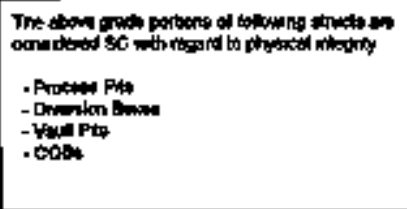 & 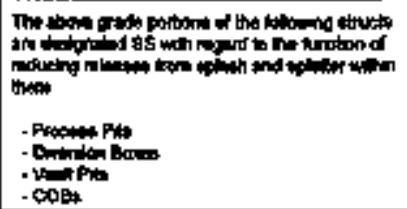 & 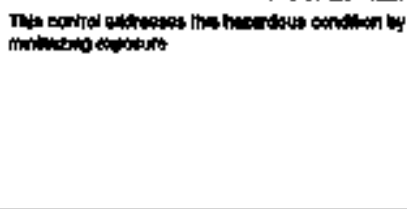 \\
\hline
\end{tabular}


Table A6. Contond Mero. (100 Shetto)

\begin{tabular}{|c|c|c|c|}
\hline$\omega$ & Psec newo & A sschentid & control kines \\
\hline \multicolumn{4}{|l|}{ 1-6PLART-1-C2:- } \\
\hline 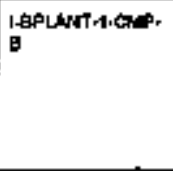 & & 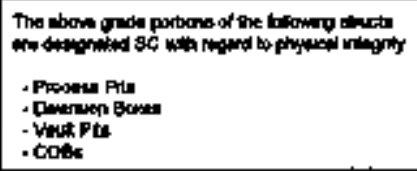 & 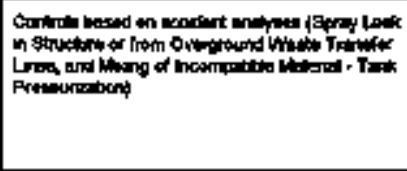 \\
\hline \multicolumn{4}{|l|}{ 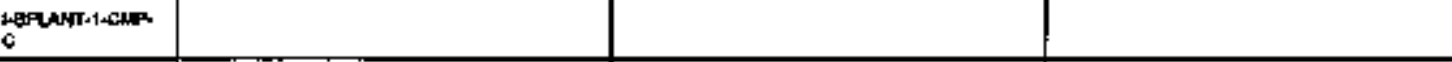 } \\
\hline \multicolumn{4}{|l|}{ 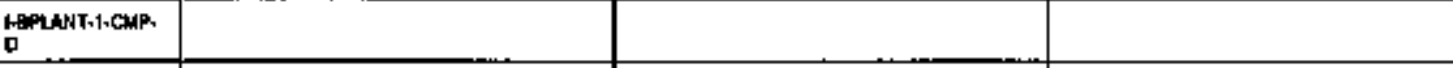 } \\
\hline 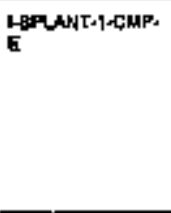 & 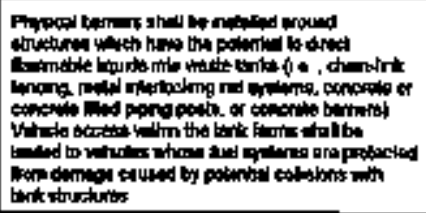 & & 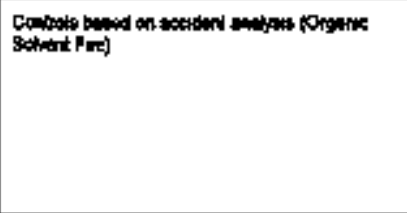 \\
\hline \multicolumn{4}{|l|}{ I-GPLAMT-1-THP. } \\
\hline \multicolumn{4}{|l|}{ IAPLATRELW. } \\
\hline \multicolumn{4}{|l|}{ |-EPLANT-2PLLW } \\
\hline \multicolumn{4}{|l|}{ 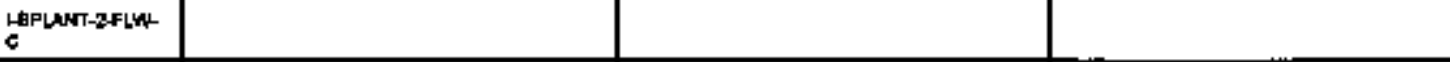 } \\
\hline 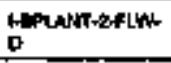 & & & 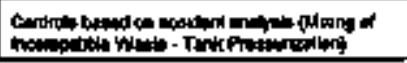 \\
\hline
\end{tabular}




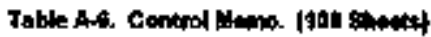

\begin{tabular}{|c|c|c|c|}
\hline L & Ps: MUNO & M SACHEM & Condalyes \\
\hline \multicolumn{4}{|l|}{ 1-BPLANTOAEKM } \\
\hline |-GPLANT-ZLEK-B & & 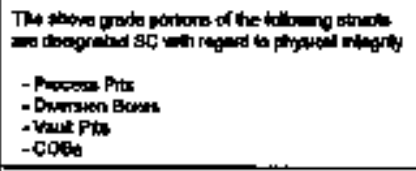 & 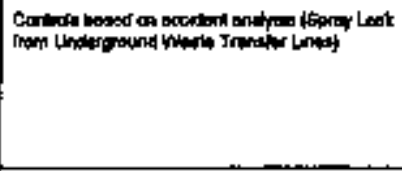 \\
\hline 1-PRANT-2HEK-C & & & 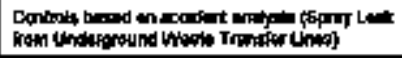 \\
\hline \multicolumn{4}{|l|}{ 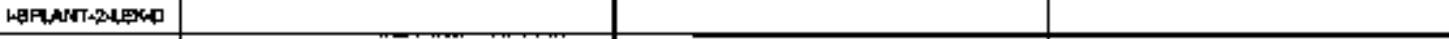 } \\
\hline HARUNT-ZTEKE & & & 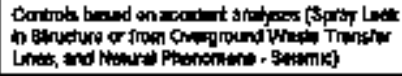 \\
\hline IFFP-1-Aप़T A & & & 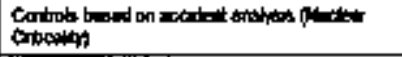 \\
\hline \multicolumn{4}{|l|}{ ANPP-AFEMA } \\
\hline LPFP.1.CLUta & & & 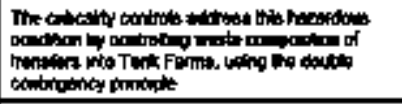 \\
\hline \multicolumn{4}{|l|}{ IPFD-1-GaApC } \\
\hline IFPP.|CAPAO & & & 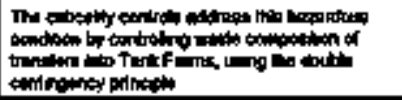 \\
\hline
\end{tabular}




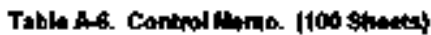

\begin{tabular}{|c|c|c|c|}
\hline D & P & H Stc wesub & Condrot MLKO \\
\hline |PPP-1-CAP-E & 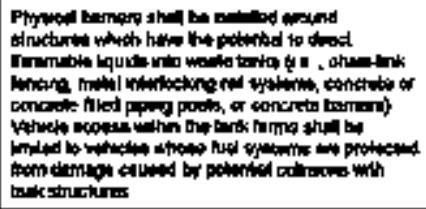 & & 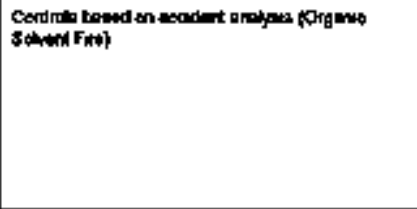 \\
\hline IPPPA14HA-A & & & 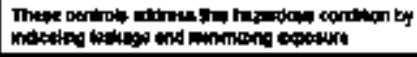 \\
\hline \multicolumn{4}{|l|}{ LPFP.14ATINA } \\
\hline \multicolumn{4}{|l|}{ LPFPAZPLWWA } \\
\hline \multicolumn{4}{|l|}{ IPFP-ZLEEKA } \\
\hline 1-PEP-2-1日X-日 & & 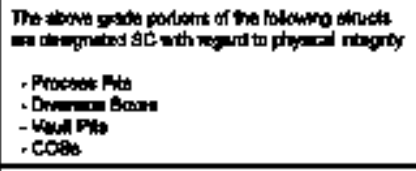 & 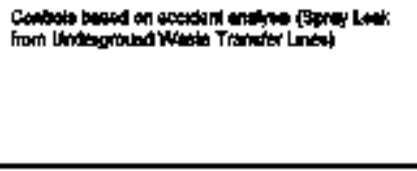 \\
\hline lappozdeket & & & 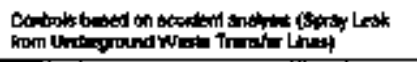 \\
\hline \multicolumn{4}{|l|}{ 1PF-2UEK-D } \\
\hline IAPPALEKE & & & 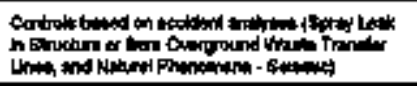 \\
\hline \multicolumn{4}{|l|}{ 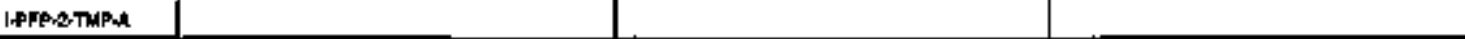 } \\
\hline 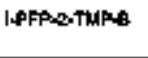 & & & 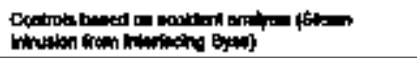 \\
\hline 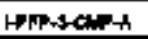 & & & \\
\hline
\end{tabular}




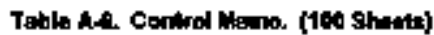

\begin{tabular}{|c|c|c|c|}
\hline ID & P SSTHEN & MS5E Mans & Contod rans \\
\hline \multicolumn{4}{|l|}{ LPFP-FLFHE } \\
\hline IPPP-A-PLW-G & 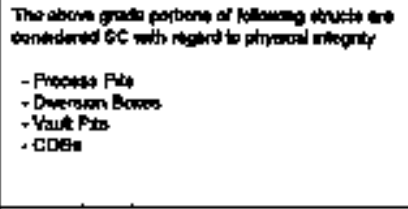 & 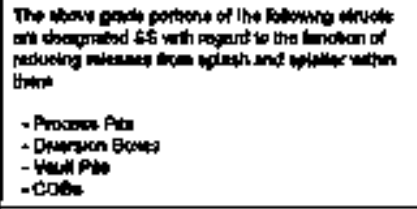 & 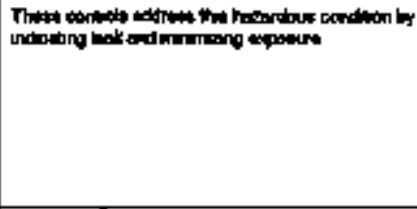 \\
\hline \multicolumn{4}{|l|}{ HOPP-ЗЕEК-A } \\
\hline IPFP-3HEK-B & & 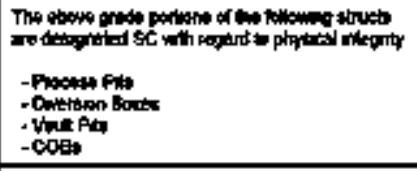 & 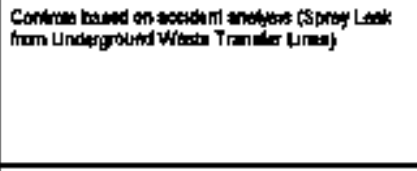 \\
\hline LEPPAHEKC & & & 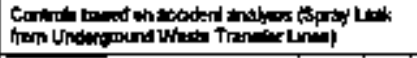 \\
\hline \multicolumn{4}{|l|}{ HPP-HEX } \\
\hline IFPPALEKA & & & 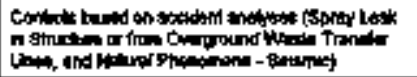 \\
\hline \multicolumn{4}{|l|}{ 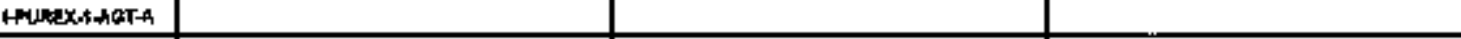 } \\
\hline 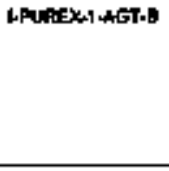 & & 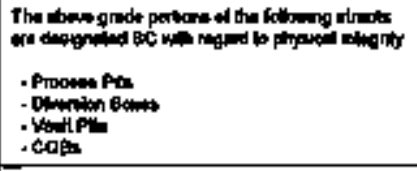 & 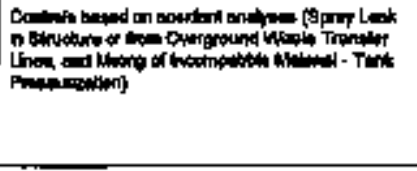 \\
\hline LPUFEX-1-NTR-LC & & & \\
\hline
\end{tabular}




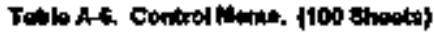

\begin{tabular}{|c|c|c|c|}
\hline 10 & P gact k4 & 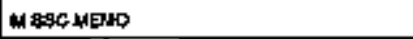 & Golvel kitemo \\
\hline \multicolumn{4}{|l|}{ LPQUREX-1-GKNA } \\
\hline HUREC-1नCHPA & & 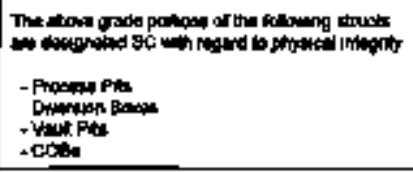 & 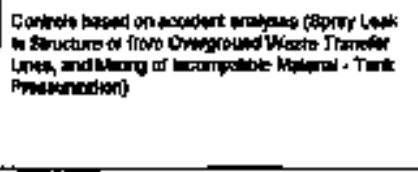 \\
\hline \multicolumn{4}{|l|}{ 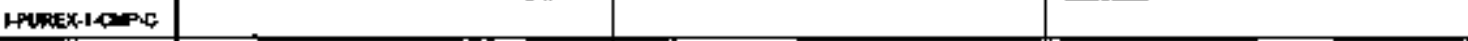 } \\
\hline \multicolumn{4}{|l|}{ Lentiax-1-capip } \\
\hline LPUnEX-1ULA & & & 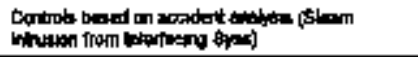 \\
\hline IPUREX-1-IMAM & & & 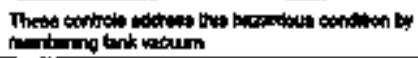 \\
\hline \multicolumn{4}{|l|}{ 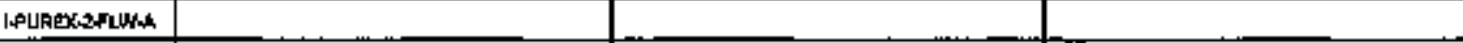 } \\
\hline \multicolumn{4}{|l|}{ 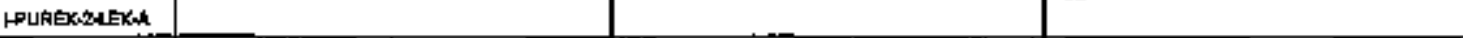 } \\
\hline IPUREX-24EK-B & & 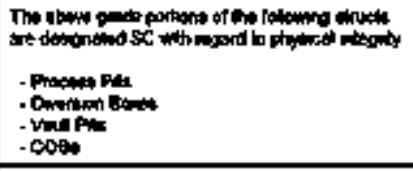 & 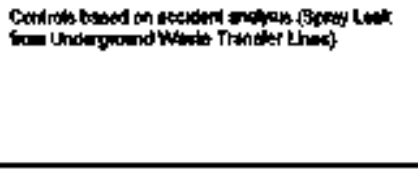 \\
\hline 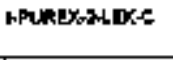 & & & 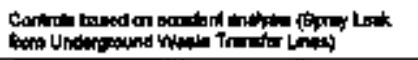 \\
\hline \multicolumn{4}{|l|}{ Hunex-2-40<-0 } \\
\hline 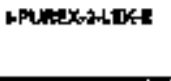 & & & 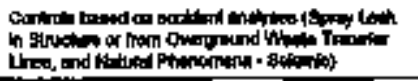 \\
\hline 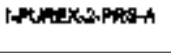 & & & 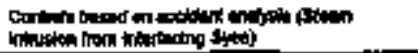 \\
\hline
\end{tabular}


Tabła N-6. Control Wemb. (100 shmets)

\begin{tabular}{|c|c|c|c|}
\hline 10 & ONA & H 6 St & Condorinews \\
\hline LURPX,ZPAS 由 & & & 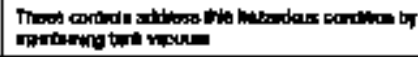 \\
\hline \multicolumn{4}{|l|}{ I.PURUX_ZPRSAC } \\
\hline \multicolumn{4}{|l|}{ HPUAEX 3 WhG TH } \\
\hline 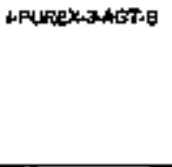 & & 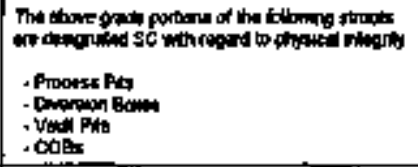 & 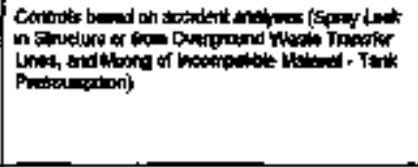 \\
\hline \multicolumn{4}{|l|}{ APUREX-3+QQT-C } \\
\hline \multicolumn{4}{|l|}{ HMPEX-3-SWP-A } \\
\hline HPUnexachape & & 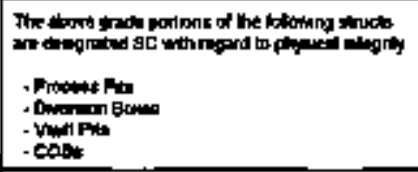 & 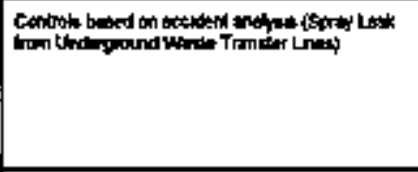 \\
\hline \multicolumn{4}{|l|}{ LPUREXSLCMPAC } \\
\hline \multicolumn{4}{|l|}{ HPUREXX-CHPO } \\
\hline levirizk-chere & & & 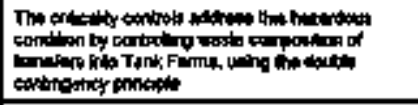 \\
\hline 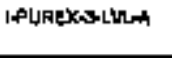 & & & 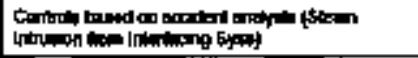 \\
\hline IPURERSTRAPA & & & 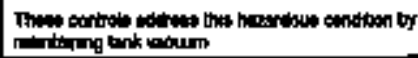 \\
\hline
\end{tabular}




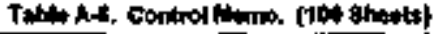

\begin{tabular}{|c|c|c|c|}
\hline 10 & P \& & M EЯC HEMD & Conind anders \\
\hline PPUPEX-4FLW-6 & 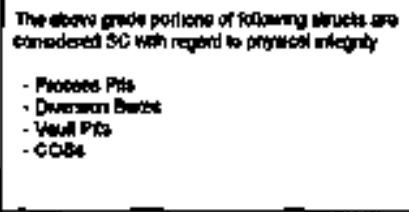 & 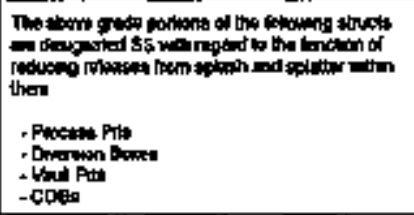 & 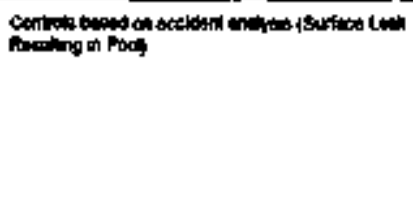 \\
\hline \multicolumn{4}{|l|}{ HPUREX } \\
\hline HFLREX-HEKS & & 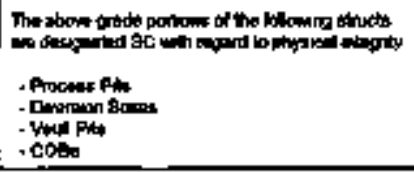 & 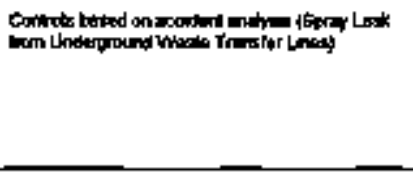 \\
\hline 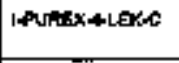 & & & 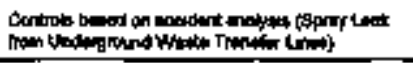 \\
\hline \multicolumn{4}{|l|}{ HOUREX-4LEX-D } \\
\hline 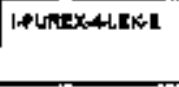 & & & 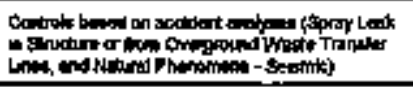 \\
\hline HPUREXKAPRSA & & & 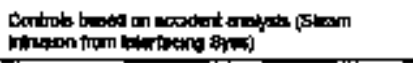 \\
\hline HPUREX-4PF-B & & & 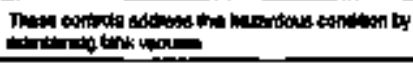 \\
\hline \multicolumn{4}{|l|}{ LPUREXYAPFA } \\
\hline HNUREKS-FLWA & & & 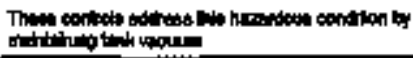 \\
\hline 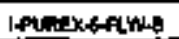 & & & \\
\hline
\end{tabular}




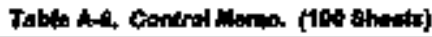

\begin{tabular}{|c|c|c|c|}
\hline $\mathbf{0}$ & Pstc have & M soc Muno & Contulne \\
\hline \multicolumn{4}{|l|}{ HPURESXERLWWH } \\
\hline \multicolumn{4}{|l|}{ 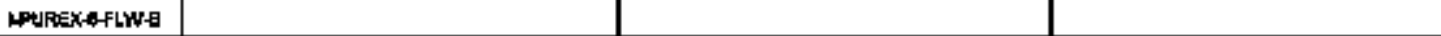 } \\
\hline \multicolumn{4}{|l|}{ IALREKCALITCC } \\
\hline \multicolumn{4}{|l|}{ IPUREKESTLLD } \\
\hline \multicolumn{4}{|l|}{ |-TPUANT-1-CWP } \\
\hline 1.TPLART.1-CWP. & & 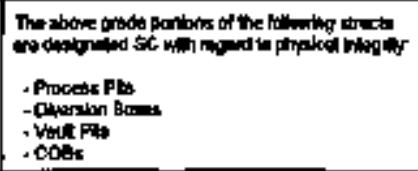 & 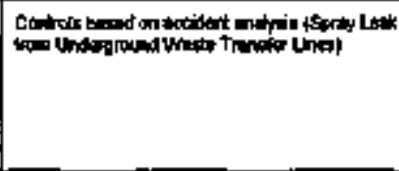 \\
\hline $\begin{array}{l}\text { I.TPLANT.1-GAP. } \\
\text { C }\end{array}$ & . & & \\
\hline \multicolumn{4}{|l|}{ HTLANT-LEAM- } \\
\hline \multicolumn{4}{|l|}{$\begin{array}{l}\text { LtPLENT-1.TuP. } \\
\text { A }\end{array}$} \\
\hline hTPLANIT-2 CHP. & & & 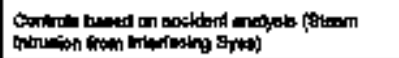 \\
\hline \multicolumn{4}{|l|}{ FTPLANT-2HUK- } \\
\hline \multicolumn{4}{|l|}{ 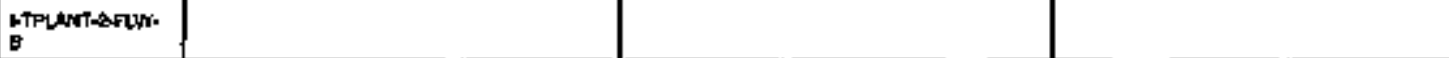 } \\
\hline 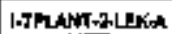 & & $\cdot$ & \\
\hline
\end{tabular}




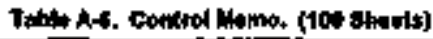

\begin{tabular}{|c|c|c|c|}
\hline $\boldsymbol{0}$ & P Sec nEANo & مא & Cortiolimento \\
\hline LTPLANT-2-LEK-B & & 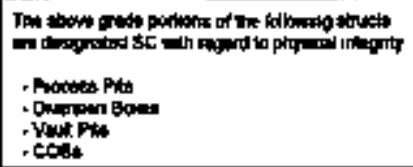 & 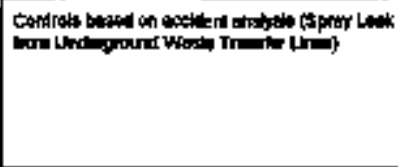 \\
\hline 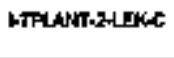 & & & 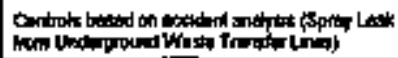 \\
\hline \multicolumn{4}{|l|}{ |.TPLANT-2-LEXD } \\
\hline I.TPLANT\&LEKE & & & 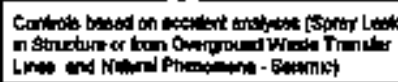 \\
\hline \multicolumn{4}{|l|}{ 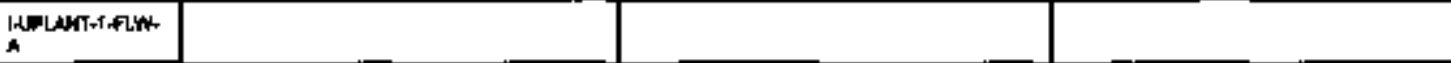 } \\
\hline \multicolumn{4}{|l|}{ HURLANT-1LEKA } \\
\hline MUPLANT-1-LEK-B & & & 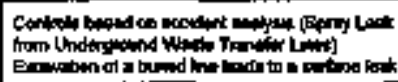 \\
\hline \multicolumn{4}{|l|}{ HUPLANH-1-UEK- } \\
\hline \multicolumn{4}{|l|}{ 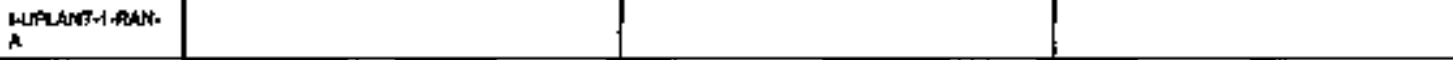 } \\
\hline \multicolumn{4}{|l|}{$\overrightarrow{r k O S}$} \\
\hline Txa-14 & & & 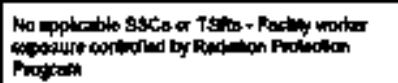 \\
\hline \multicolumn{4}{|l|}{ Inkel } \\
\hline InKGOA & & & \\
\hline
\end{tabular}




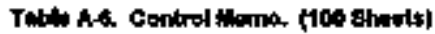

\begin{tabular}{|c|c|c|c|}
\hline as & P Ssct & H SSC WEMO & Sortapla \\
\hline ITKos & & & 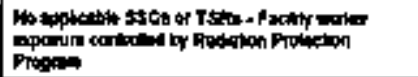 \\
\hline Iтқ-034| & & & 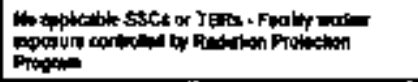 \\
\hline ITKKOM & & & 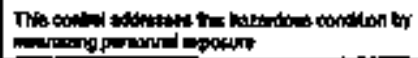 \\
\hline \multicolumn{4}{|l|}{ TK-0+1 } \\
\hline IFK0S & & & 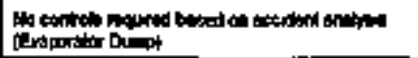 \\
\hline mx-0s-1 & & & 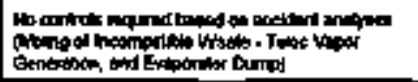 \\
\hline ITKCD & & & 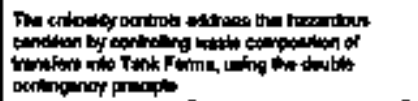 \\
\hline maxis & & & 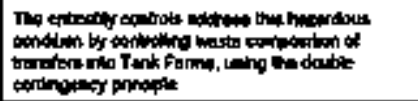 \\
\hline mk-or & & & 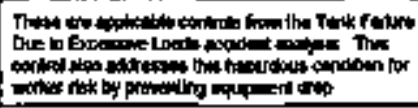 \\
\hline \multicolumn{4}{|l|}{ Tारण丁 मn } \\
\hline TTKOF-10 & & & 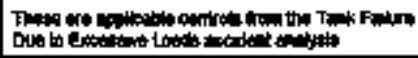 \\
\hline Trkas & & & 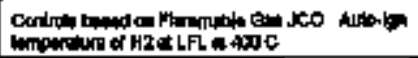 \\
\hline
\end{tabular}




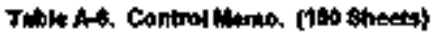

\begin{tabular}{|c|c|c|c|}
\hline $\mathbf{0}$ & P the & MSSC UENop & Comereng \\
\hline$\pi K=0.1$ & & & 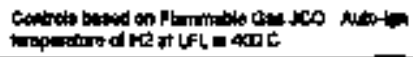 \\
\hline $1 \pi \mathbf{k}-\mathbf{A}$ & & & 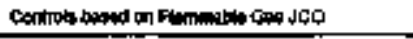 \\
\hline ITKم00| & & & 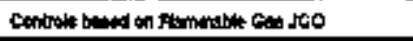 \\
\hline$|\pi \times-10|$ & & & 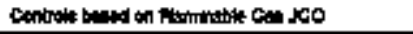 \\
\hline Imo.10 & & & 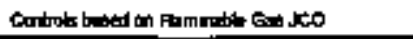 \\
\hline Ink-10मb & 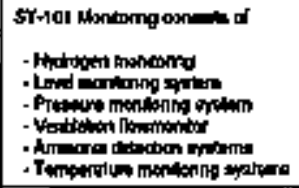 & & 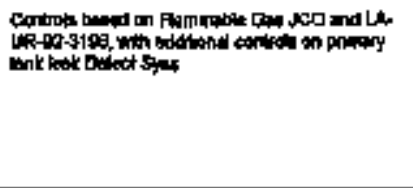 \\
\hline TTK-11 & 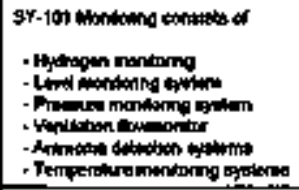 & & 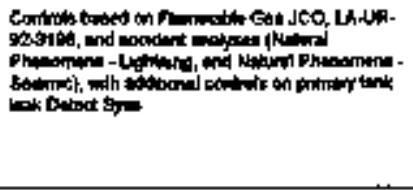 \\
\hline MRE-19-1 & & & 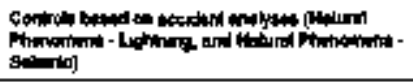 \\
\hline MKGFREM & & & 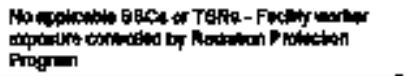 \\
\hline THEff-W & & & 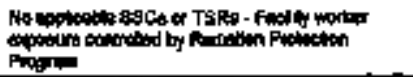 \\
\hline
\end{tabular}




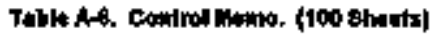

\begin{tabular}{|c|c|c|c|}
\hline ID & P SAC MENO & M spec nano & DEM \\
\hline rikethes & & & 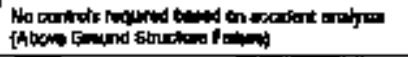 \\
\hline TKKFR-04 & & & 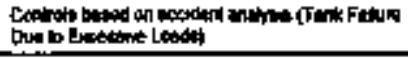 \\
\hline TKKFR-GS & & & 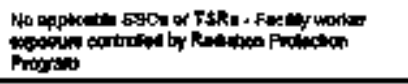 \\
\hline 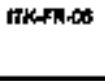 & & & 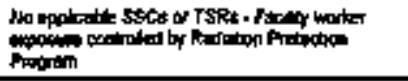 \\
\hline$m k-m+\infty]$ & & & 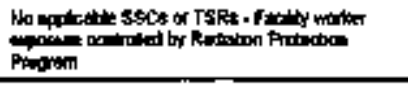 \\
\hline mk-1R-68 & & & 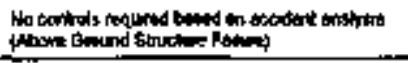 \\
\hline \multicolumn{4}{|l|}{ MK-FR-Ded } \\
\hline \multicolumn{4}{|l|}{ InK-FR-10 } \\
\hline \multicolumn{4}{|l|}{$1 m k+k-11$} \\
\hline \multicolumn{4}{|l|}{ ITKfR-12 } \\
\hline TKKFR-13 & & & 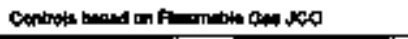 \\
\hline TKKFR-14 & & & 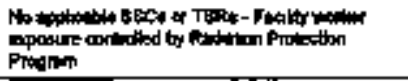 \\
\hline TRKFR-15 & & & 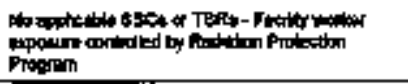 \\
\hline \multicolumn{4}{|l|}{ MKGFR-19 } \\
\hline ItKGPAs-17 & & & \\
\hline
\end{tabular}


Table A.6. Control tiemo, (104 ahesta)

\begin{tabular}{|c|c|c|c|}
\hline p & P stri nento & Msse mend & convest weros \\
\hline ITK-FR-16 & & & 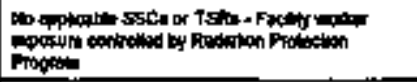 \\
\hline ITKFR-19 & & & 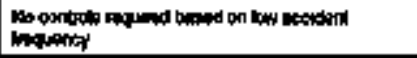 \\
\hline ITKFraxO & & & 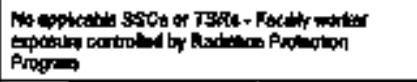 \\
\hline ITKFR21 & & & 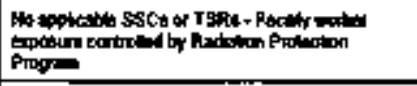 \\
\hline ITKK+FA-22 & & & 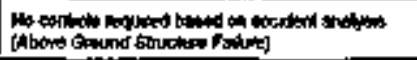 \\
\hline TKFA-Z & & & 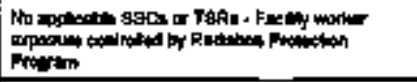 \\
\hline \multicolumn{4}{|l|}{ ITKGFACA } \\
\hline \multicolumn{4}{|l|}{ TKनPA } \\
\hline nK-PA-23 & & & 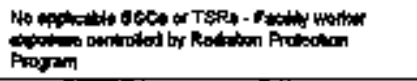 \\
\hline \multicolumn{4}{|l|}{ mKK-PR-Z7 } \\
\hline$m k+n=0$ & & & 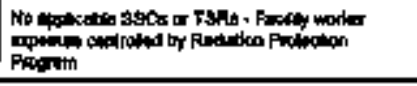 \\
\hline$n$ & & & 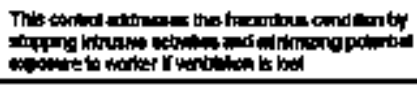 \\
\hline Nans & & & 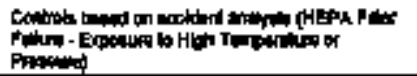 \\
\hline
\end{tabular}




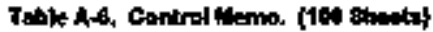

\begin{tabular}{|c|c|c|c|}
\hline Ib & ossin & $\Delta \operatorname{stc} m$ mo & Contos \\
\hline NPMOS & & & 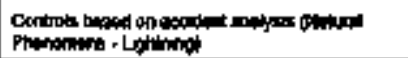 \\
\hline NPAB & & & 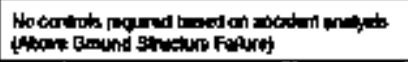 \\
\hline NAP-06 & & & 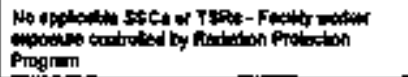 \\
\hline NPAT & & & 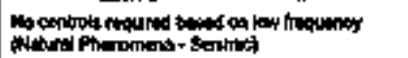 \\
\hline 19pad & & & 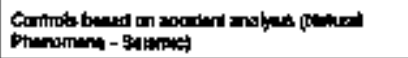 \\
\hline 100 & & & 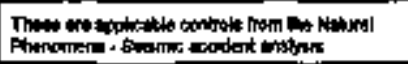 \\
\hline \multicolumn{4}{|l|}{ Peno } \\
\hline No-11 & & & 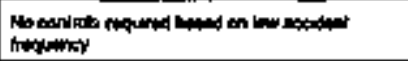 \\
\hline MP.12 & & & 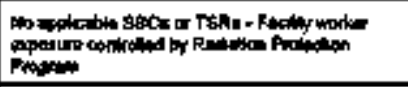 \\
\hline$N P-13$ & & & 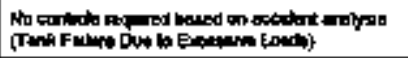 \\
\hline NP-14 & & & 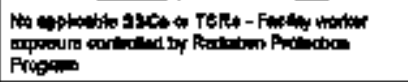 \\
\hline Ha-15 & & & 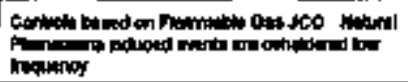 \\
\hline OОTEXTअOA & & & 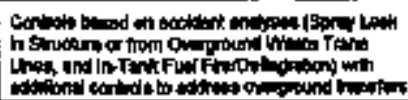 \\
\hline
\end{tabular}




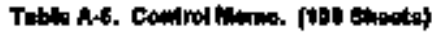

\begin{tabular}{|c|c|c|c|}
\hline 10 & F & MEBCG & Contiof \\
\hline DGTExT3AIB & & & 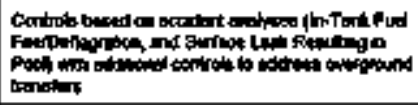 \\
\hline OST-RION1-0I & & & 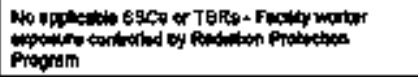 \\
\hline OGTrLOW1A & & & 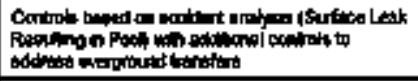 \\
\hline 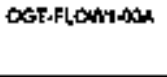 & & & 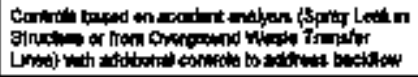 \\
\hline OST-FLONHAsta & & & 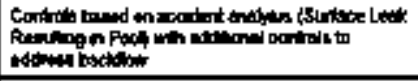 \\
\hline COT.PLOWHSA4 & & & 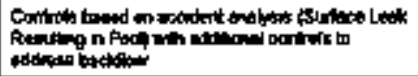 \\
\hline CorT.PLOMn-os & & & \\
\hline 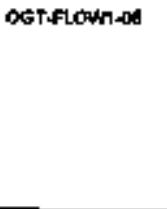 & 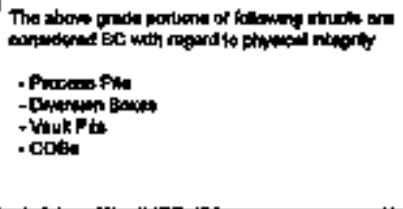 & 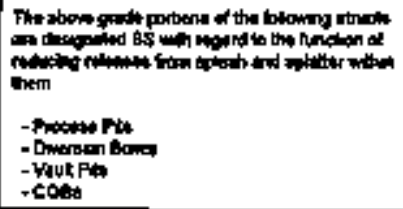 & 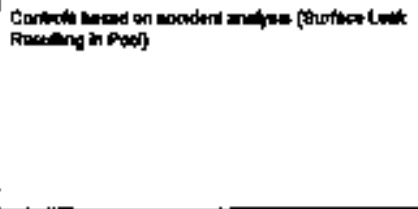 \\
\hline OGIFLOWR:MA & & & 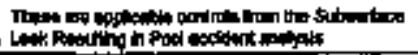 \\
\hline
\end{tabular}




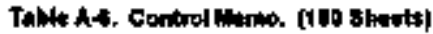

\begin{tabular}{|c|c|c|c|}
\hline $\boldsymbol{\omega}$ & P SBC MENO & $M$ \&9C MפखO & 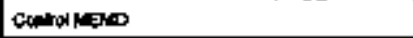 \\
\hline OGF.MCOrz-O1B & & & 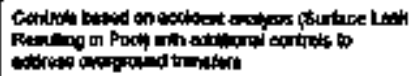 \\
\hline OBT-FLLWHZA2 & & & 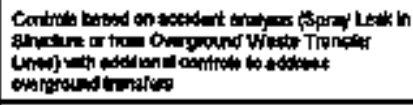 \\
\hline DGT.FLOU20Ad & & & 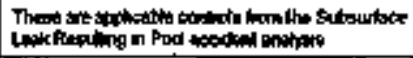 \\
\hline Cotflortr-pes & & & 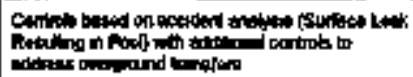 \\
\hline 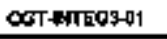 & & & \\
\hline Cottos & & & 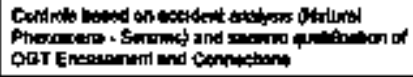 \\
\hline OGTHPSDE & & & 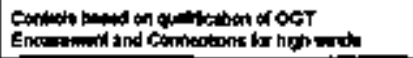 \\
\hline क्षा-क्pi in & & & 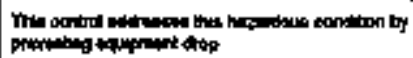 \\
\hline OGT Parrst-M & & & 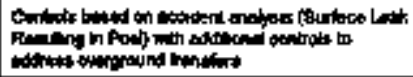 \\
\hline OGTREACIFI & & & 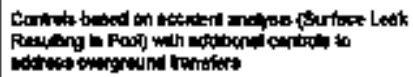 \\
\hline QGT-EtLS1-th & & & 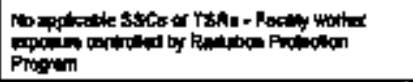 \\
\hline OAT-TEM-4M & & & \\
\hline
\end{tabular}


Table A-6. Contwol kema. (105 8knot:)

\begin{tabular}{|c|c|c|c|}
\hline 10 & P BsC WENO & M 640 ANENO & Conoror thentos \\
\hline Qtat-Teupldiz & & & 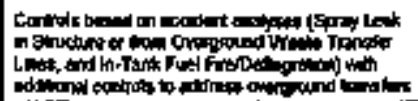 \\
\hline COT-ГघP1-(口) & & & 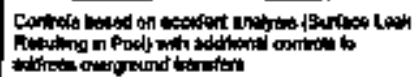 \\
\hline \multicolumn{4}{|l|}{ enaton } \\
\hline \multicolumn{4}{|l|}{ RAD-OIJ } \\
\hline \multicolumn{4}{|l|}{$\cos 200$} \\
\hline \multicolumn{4}{|l|}{ PADA- } \\
\hline \multicolumn{4}{|l|}{ atpats } \\
\hline \multicolumn{4}{|l|}{ PAD-O3Y } \\
\hline RSPROM & & & 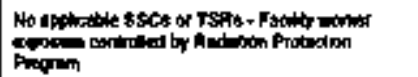 \\
\hline PSPA-04-1 & & & 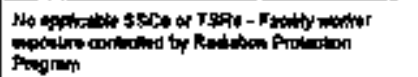 \\
\hline \multicolumn{4}{|l|}{ P6R-A } \\
\hline \multicolumn{4}{|l|}{ ntwh-n } \\
\hline \multicolumn{4}{|l|}{ ASA《E } \\
\hline \multicolumn{4}{|l|}{ AER-DSH } \\
\hline \multicolumn{4}{|l|}{$R \$ R-04$} \\
\hline \multicolumn{4}{|l|}{$R A R-04$} \\
\hline Rak-06 & & & 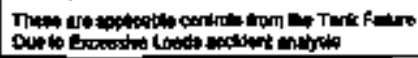 \\
\hline
\end{tabular}




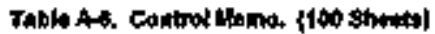

\begin{tabular}{|c|c|c|c|}
\hline o & 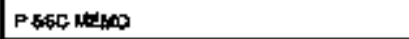 & M spcinax & Cantrd wexp \\
\hline \multicolumn{4}{|l|}{ RBR-OSHA } \\
\hline RERA5) & & & 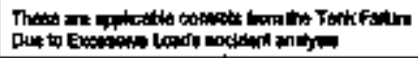 \\
\hline RER09H & & & 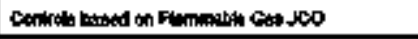 \\
\hline RSRTR-1 & & & 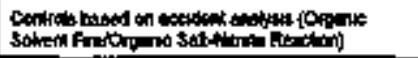 \\
\hline \multicolumn{4}{|l|}{ RPPR-0) } \\
\hline $6 F-01$ & & & 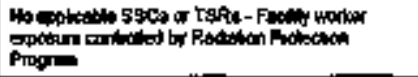 \\
\hline \multicolumn{4}{|l|}{ SFF-01- } \\
\hline TPB.1 & 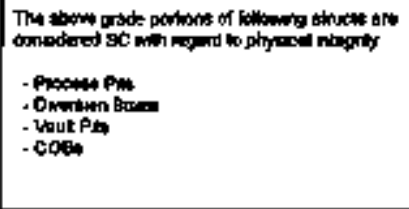 & 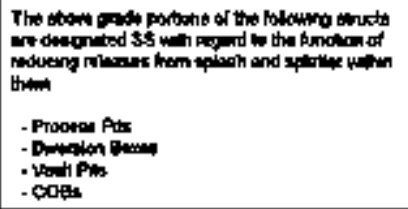 & 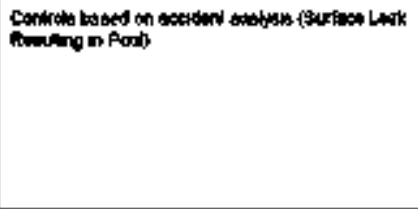 \\
\hline verto1 & & & 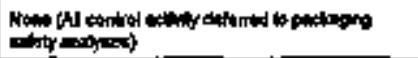 \\
\hline YEHAYII & & & 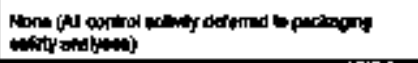 \\
\hline veter & & & 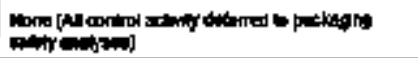 \\
\hline VEFOOH & & & 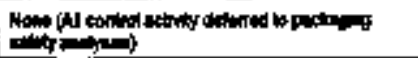 \\
\hline VEFtarB & & & 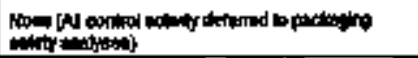 \\
\hline
\end{tabular}




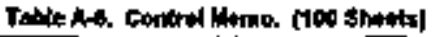

\begin{tabular}{|c|c|c|c|}
\hline D & P \$SC VEMD & M ESC, MEMO & Controp \\
\hline VEHas & & & 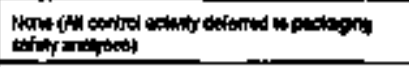 \\
\hline \multicolumn{4}{|l|}{ V바에 } \\
\hline \multicolumn{4}{|l|}{ Yemast } \\
\hline \multicolumn{4}{|l|}{ Vewas } \\
\hline \multicolumn{4}{|l|}{ Yemasel } \\
\hline \multicolumn{4}{|l|}{ VEHQS } \\
\hline \multicolumn{4}{|l|}{ VEHä } \\
\hline \multicolumn{4}{|l|}{ veltor } \\
\hline Yetatid & 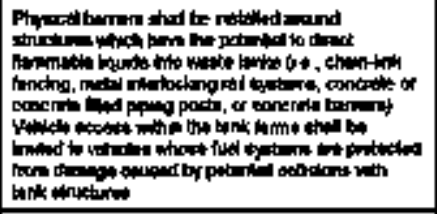 & & 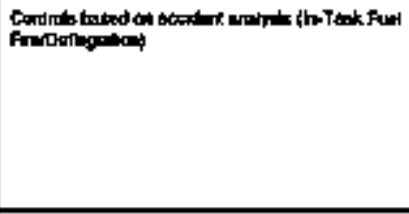 \\
\hline \multicolumn{4}{|l|}{ SaHot } \\
\hline vesur & & & 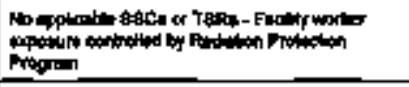 \\
\hline Vaddan & & & 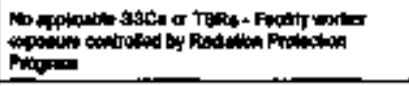 \\
\hline Y\$स-04 & & & 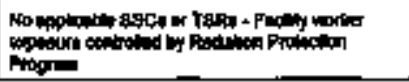 \\
\hline
\end{tabular}


Tibla AA. Controd Meno. (100 thentr)

\begin{tabular}{|c|c|c|c|}
\hline ID & P JEC NEWO & M SSC MEMO & 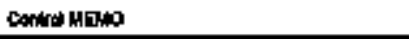 \\
\hline પSULOA & & & 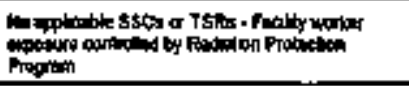 \\
\hline \multicolumn{4}{|l|}{ vilues } \\
\hline \multicolumn{4}{|l|}{ พ\$ب0s } \\
\hline mosstothivi & 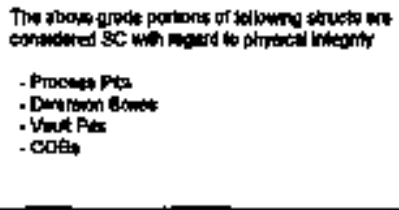 & 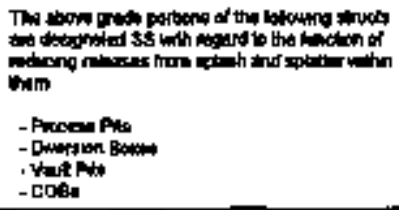 & 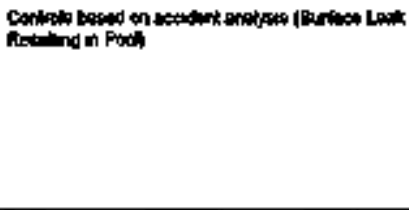 \\
\hline 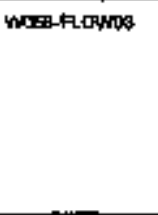 & 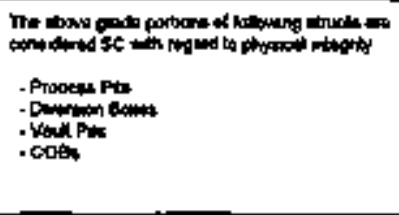 & 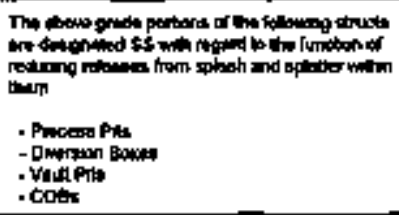 & 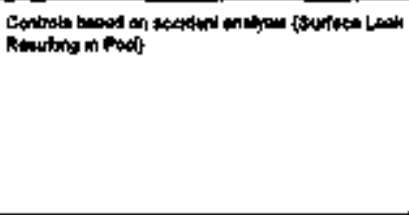 \\
\hline 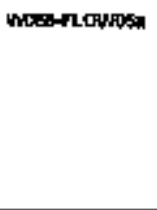 & 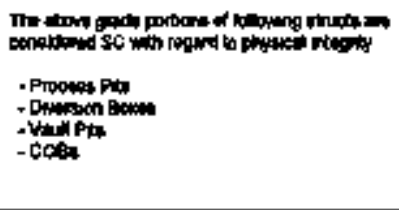 & 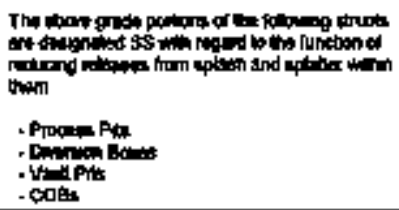 & 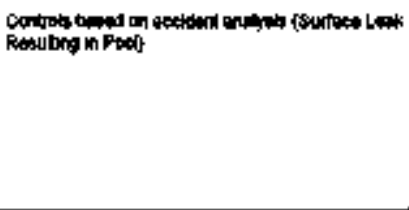 \\
\hline
\end{tabular}




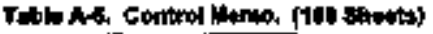

\begin{tabular}{|c|c|c|c|}
\hline 0 & P अGC मEND & M SBC MENONO & 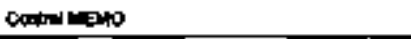 \\
\hline WNSERFLCANosb & 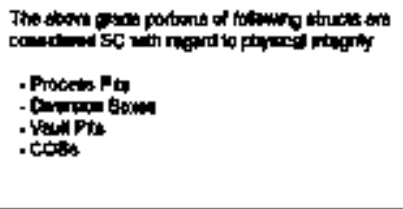 & 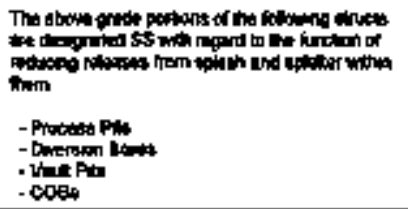 & 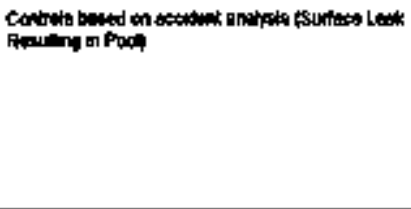 \\
\hline 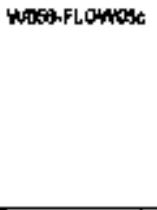 & 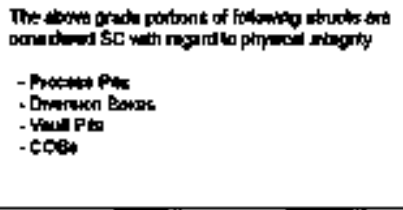 & 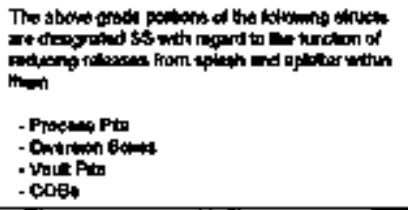 & 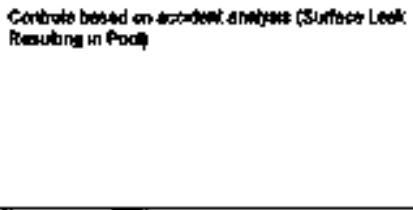 \\
\hline MOSAfLOMMT & 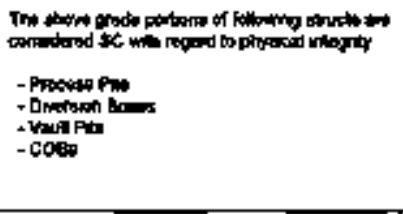 & 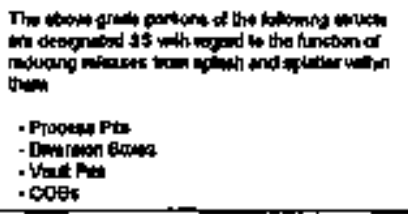 & 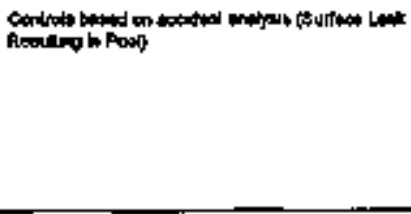 \\
\hline Wosp-rLCWANo & 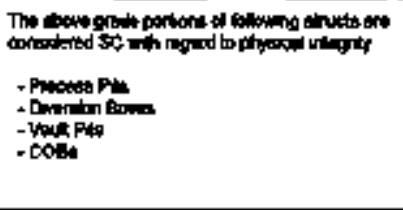 & 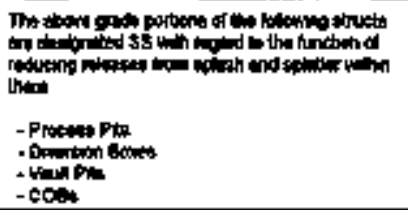 & 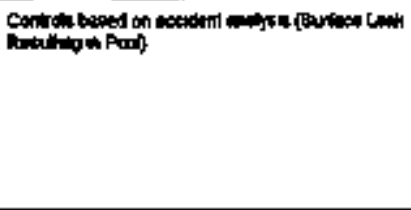 \\
\hline
\end{tabular}




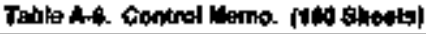

\begin{tabular}{|c|c|c|c|}
\hline $\mathbf{p}$ & $P$ \&sc & 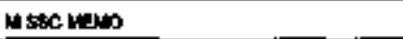 & Control betas \\
\hline nof-FLON11: & 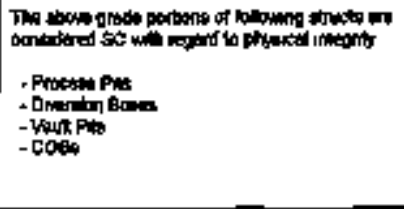 & 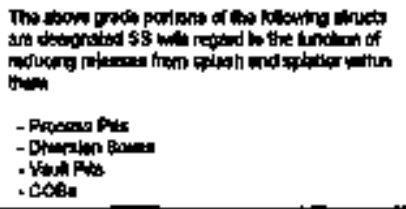 & 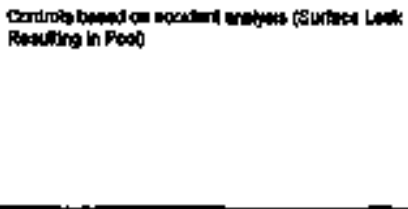 \\
\hline mesprourn 16 & 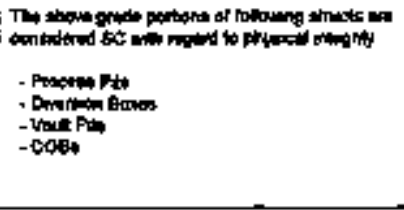 & 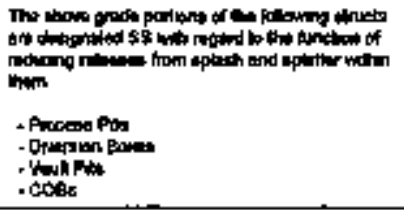 & 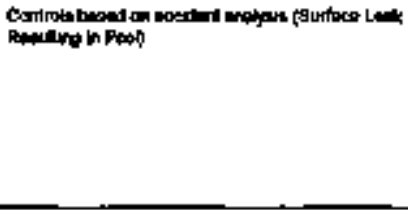 \\
\hline 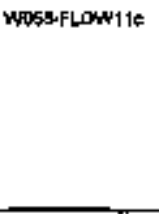 & 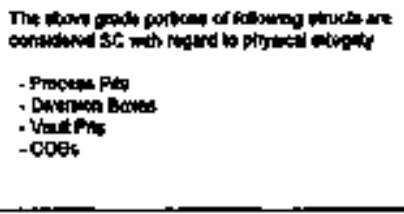 & 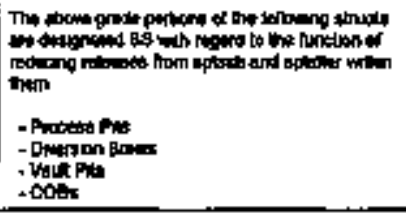 & 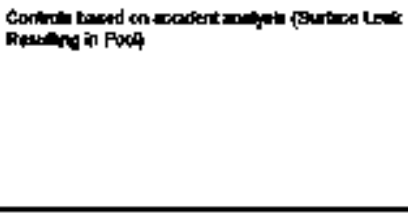 \\
\hline Wots-RLANis & 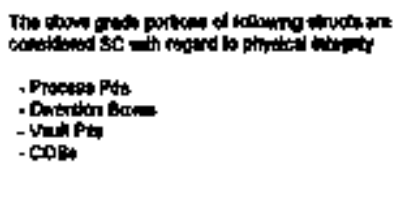 & 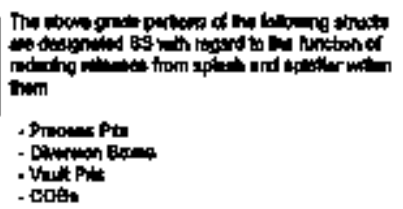 & 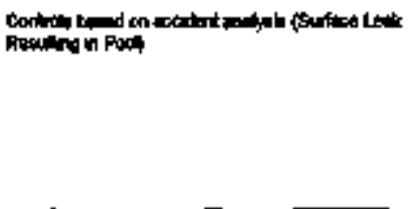 \\
\hline
\end{tabular}


Tatbs 4. Comitrel binmo. (100 Sherts)

\begin{tabular}{|c|c|c|c|}
\hline$D$ & P 380 & 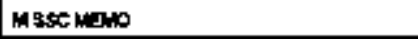 & Contw WGNO \\
\hline 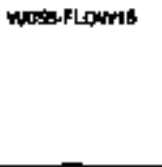 & & 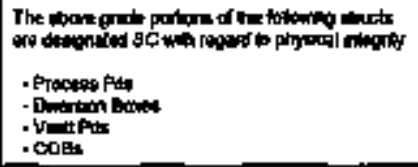 & 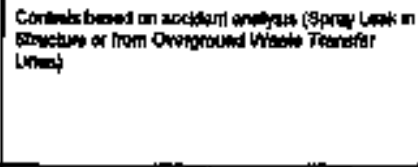 \\
\hline WOSB-ACOHTA & & 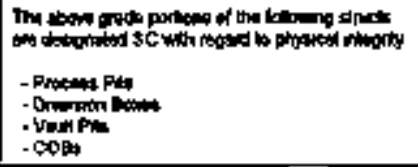 & 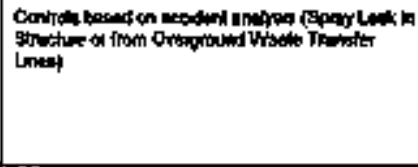 \\
\hline WhSPRLWN17n & & 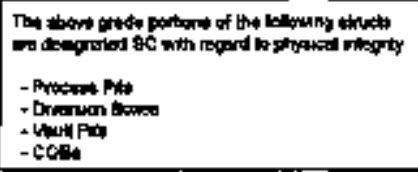 & 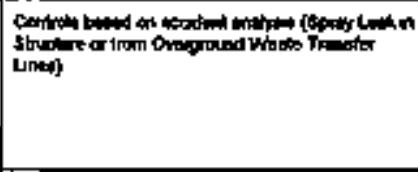 \\
\hline WOSAFLOH17b & & 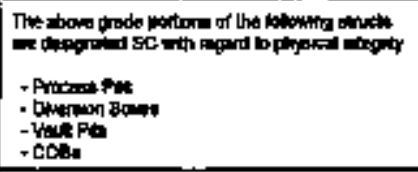 & 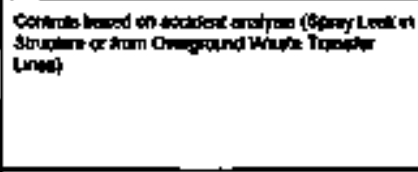 \\
\hline Hotofolowito & & 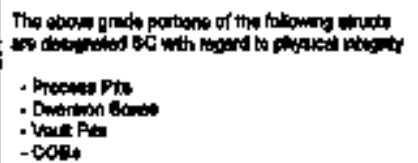 & 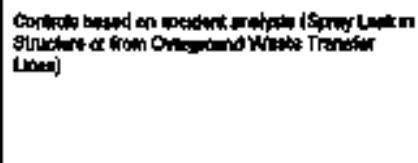 \\
\hline
\end{tabular}




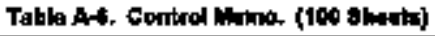

\begin{tabular}{|c|c|c|c|}
\hline$D$ & P 360 A & M Bgc MEANO & Contrat Mero \\
\hline MOSAPLOWH'. & & 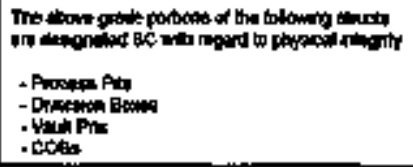 & 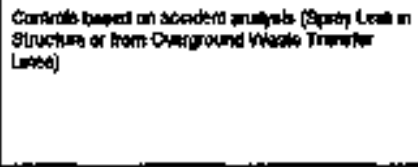 \\
\hline Whatsflawi & & 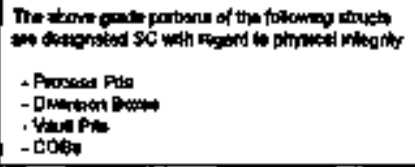 & 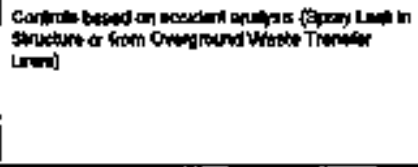 \\
\hline MOSBRLOW:MN & & 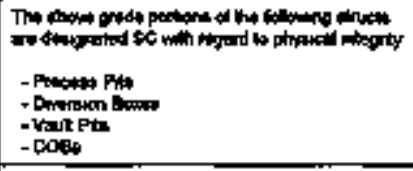 & 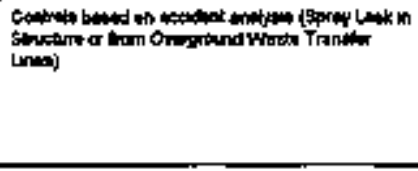 \\
\hline 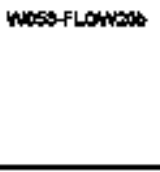 & & 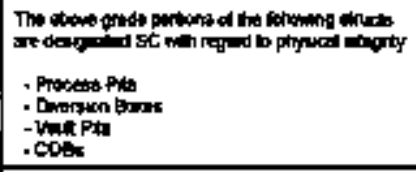 & 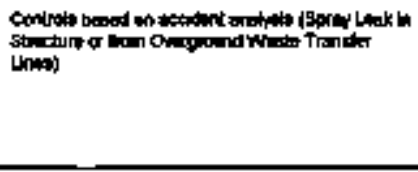 \\
\hline 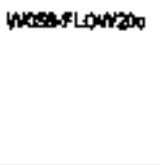 & & 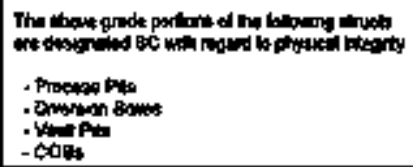 & 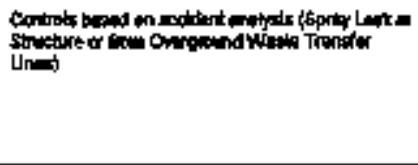 \\
\hline
\end{tabular}




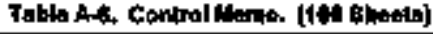

\begin{tabular}{|c|c|c|c|}
\hline $\mathbf{0}$ & P stst wen & 시 ssec wath & Catrol the \\
\hline WOSS-NTECPIA & & 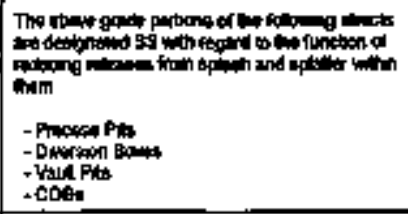 & 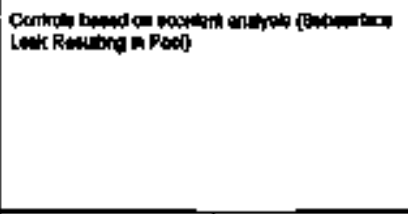 \\
\hline 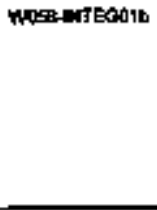 & & 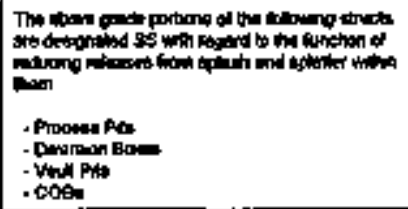 & 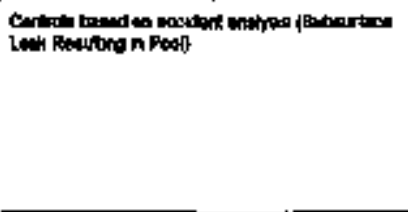 \\
\hline mosodrmesots & 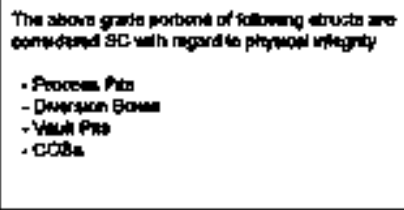 & 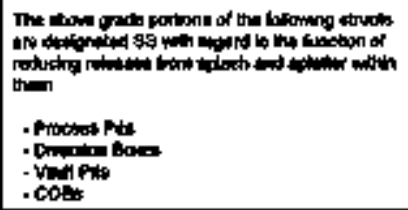 & 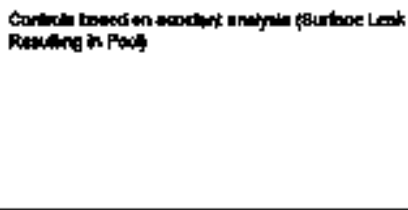 \\
\hline Woosuntegkind & 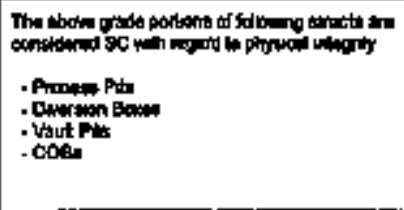 & 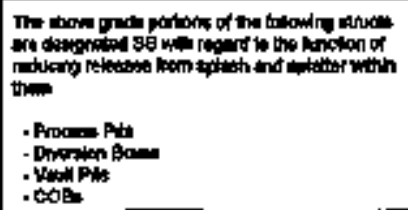 & 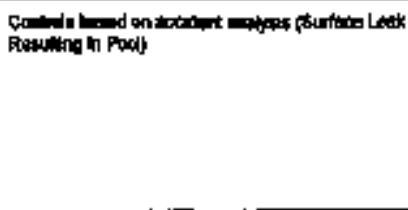 \\
\hline
\end{tabular}




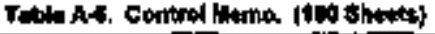

\begin{tabular}{|c|c|c|c|}
\hline ID & P : & HABC ME MAD & 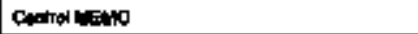 \\
\hline 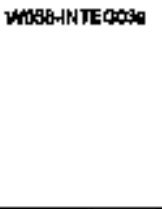 & & 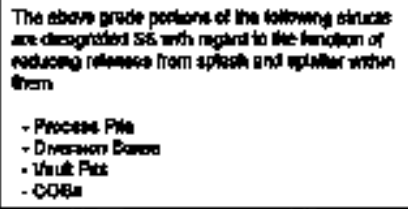 & 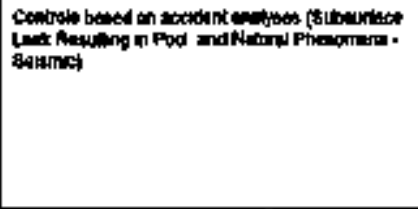 \\
\hline 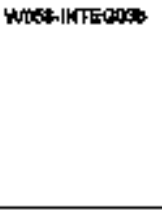 & 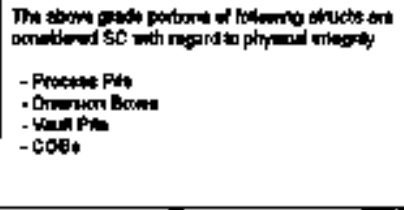 & 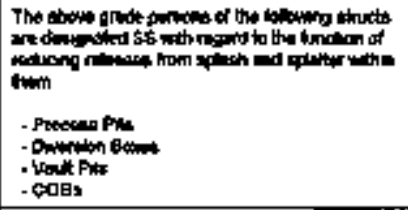 & 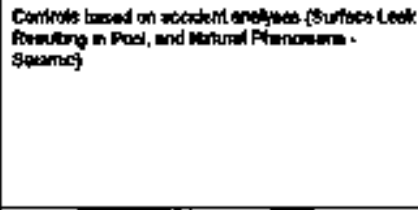 \\
\hline WWEOHTEBOE. & & & 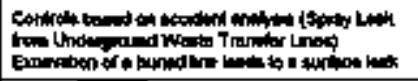 \\
\hline 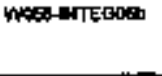 & & & 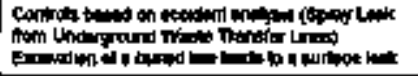 \\
\hline W0S:-wTECOTE & & 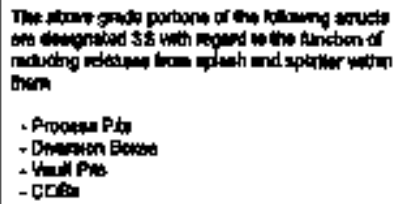 & 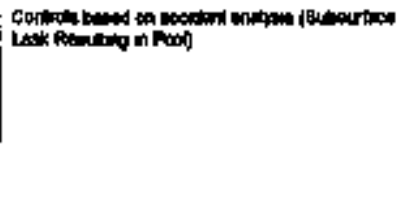 \\
\hline
\end{tabular}




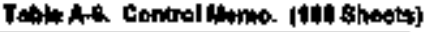

\begin{tabular}{|c|c|c|c|}
\hline D & $P$ gac Lagad & M HSC newh & 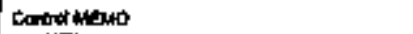 \\
\hline WOSSHNTEQDTb & 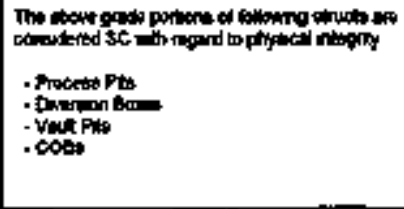 & 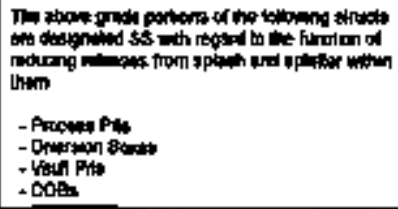 & 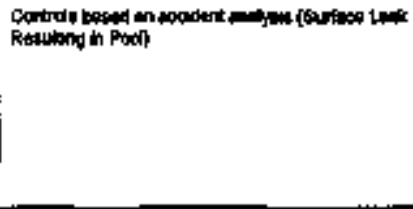 \\
\hline MNS3-WTECAQ & & 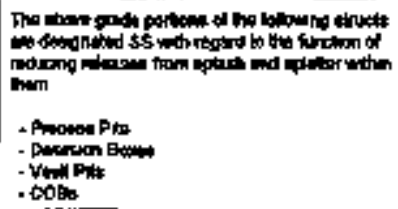 & 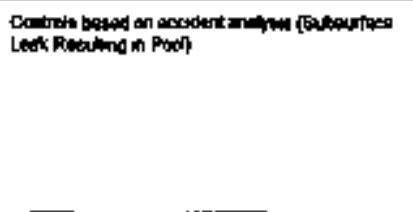 \\
\hline nNET-NIEGOS & 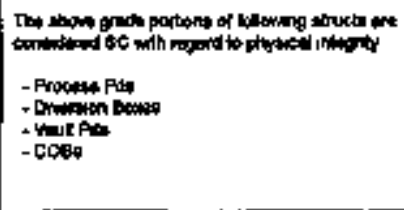 & 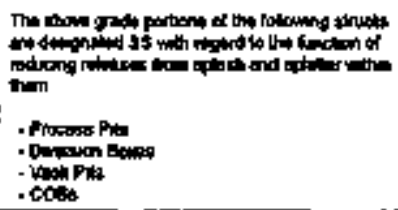 & 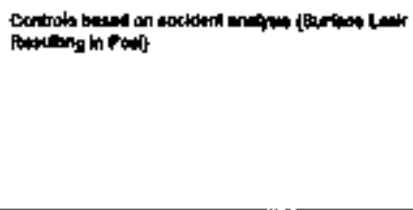 \\
\hline 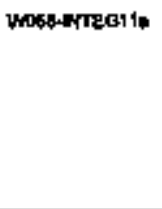 & & 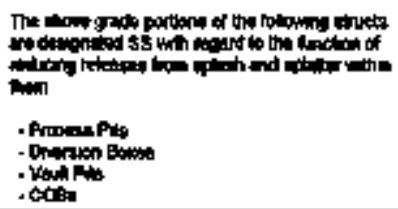 & 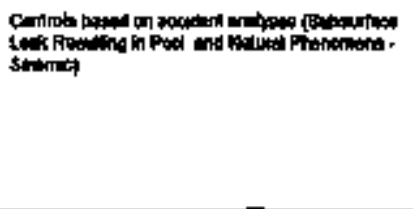 \\
\hline
\end{tabular}




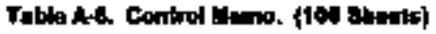

\begin{tabular}{|c|c|c|c|}
\hline ID & P gegc nean & M6و ME & Conted wene \\
\hline Wodstartegi ib & 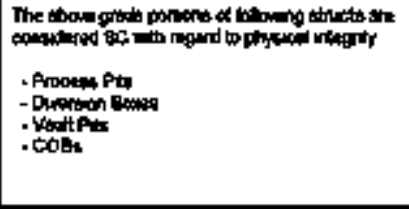 & 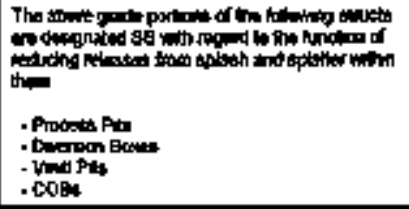 & 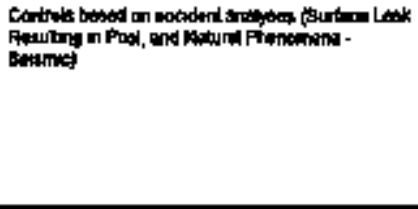 \\
\hline WhEA|NTE' 3- & & 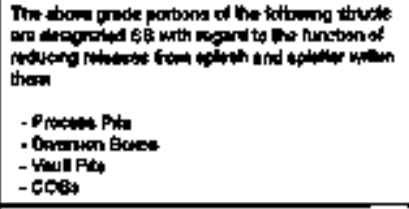 & 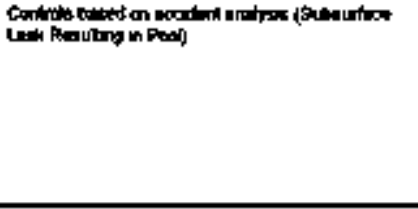 \\
\hline 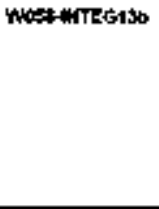 & & 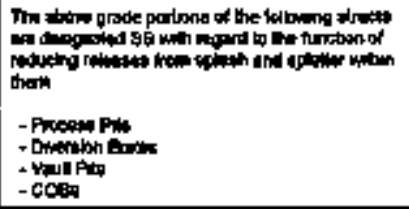 & 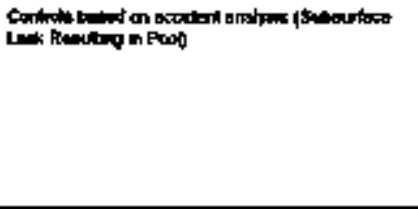 \\
\hline 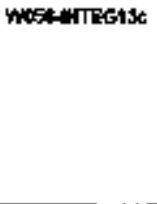 & 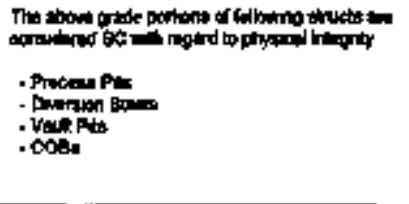 & 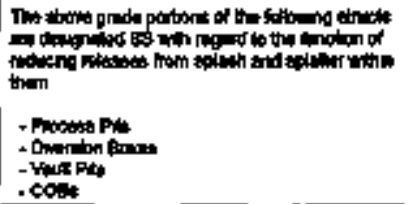 & 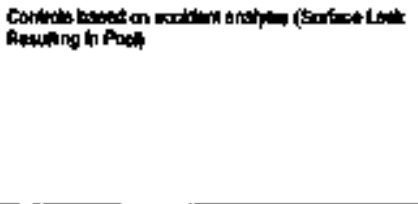 \\
\hline
\end{tabular}




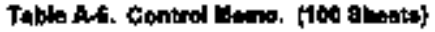

\begin{tabular}{|c|c|c|c|}
\hline 0 & Pstation & Hesctum & 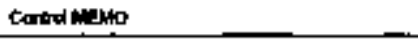 \\
\hline W05\%INTEOTIA & 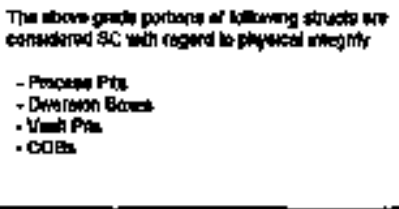 & 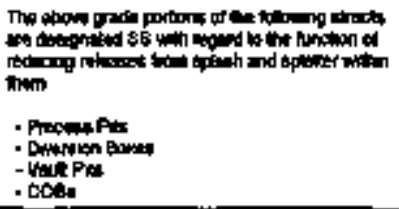 & 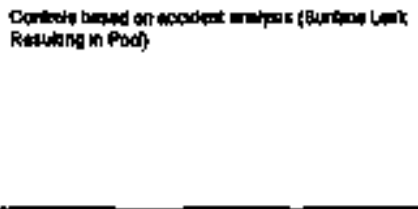 \\
\hline MRSSINTEO1 & & 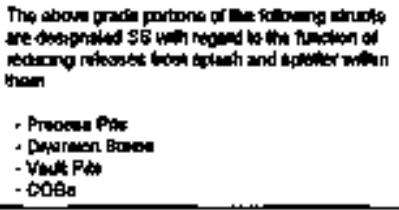 & 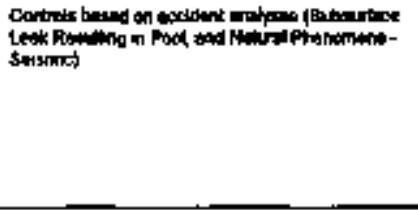 \\
\hline ndisentedi4b & 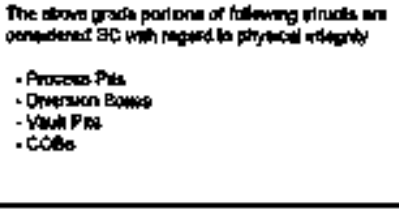 & 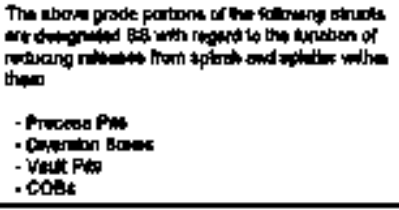 & 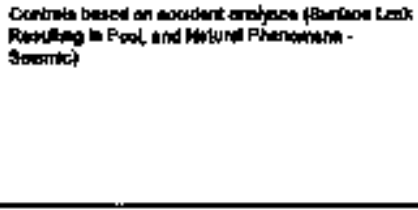 \\
\hline 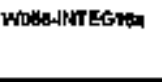 & & & 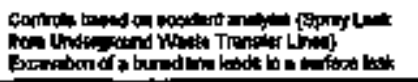 \\
\hline 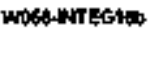 & & & 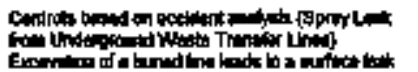 \\
\hline
\end{tabular}




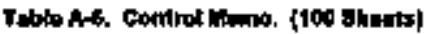

\begin{tabular}{|c|c|c|c|}
\hline $\mathbf{D}$ & $P \cos 111$ 19 & 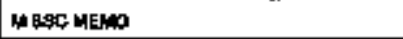 & 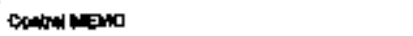 \\
\hline WNSHETEG1ha & & 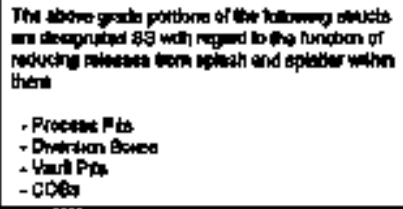 & 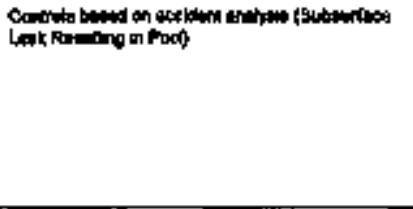 \\
\hline MESAHTEG1B & 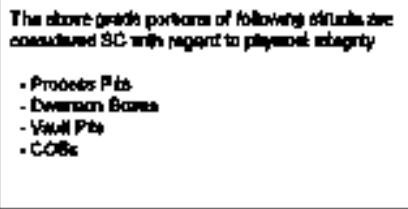 & 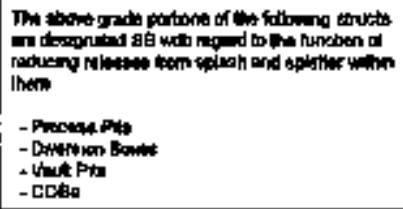 & 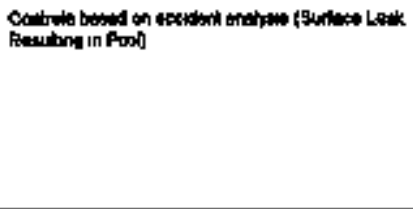 \\
\hline nogs-WITECSO & & 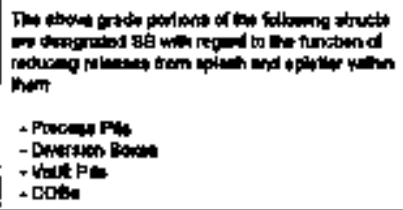 & 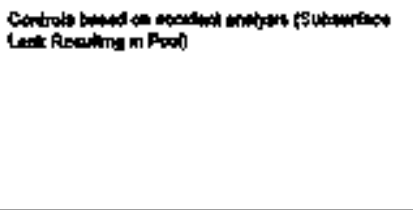 \\
\hline WOSB-WTEOSW & 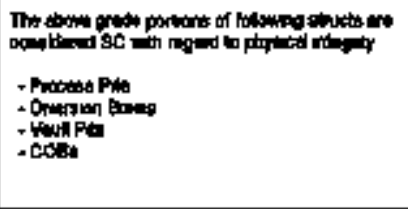 & 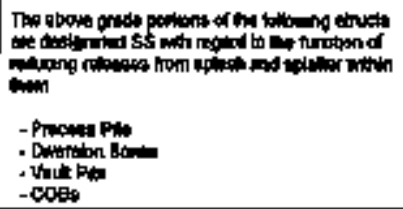 & 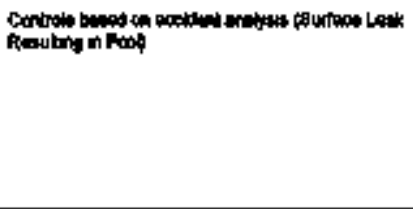 \\
\hline
\end{tabular}




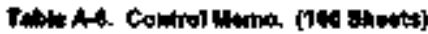

\begin{tabular}{|c|c|c|c|}
\hline 上D & $P$ p 영 & u specing & Contri \\
\hline Whotwiteat & & 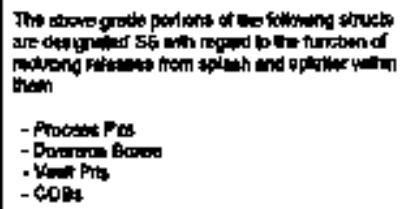 & 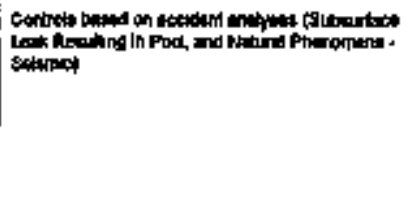 \\
\hline WOSOHTECO24 & & & 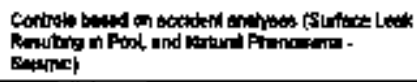 \\
\hline Wo56-1NTECS6 & 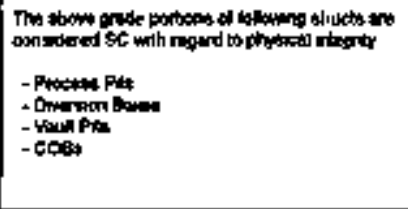 & 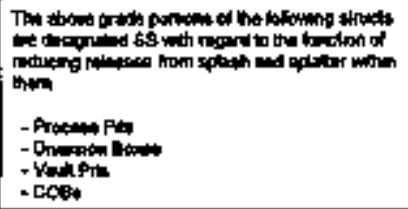 & 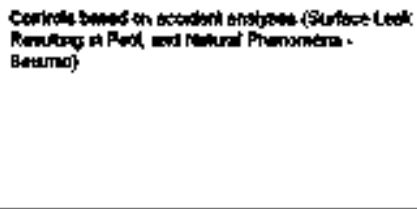 \\
\hline nost-INTECes & & & 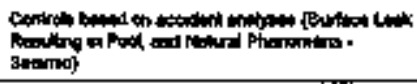 \\
\hline noss-1nTec630 & 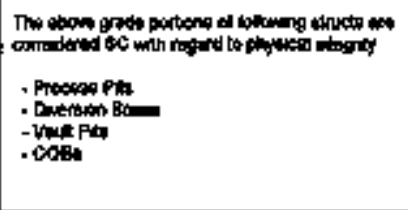 & 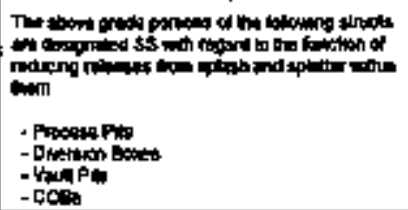 & 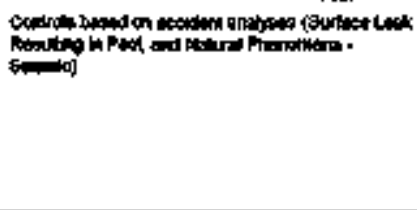 \\
\hline
\end{tabular}


Table A6. Control Mame. (106 abrits)

\begin{tabular}{|c|c|c|c|}
\hline$D$ & 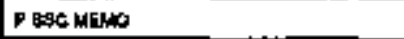 & M soc mate & 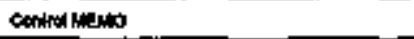 \\
\hline 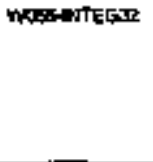 & & 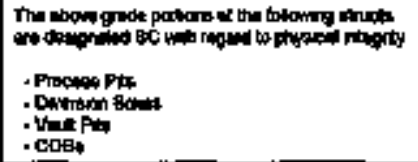 & 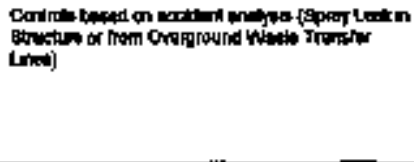 \\
\hline ncom-ntER34 & & & 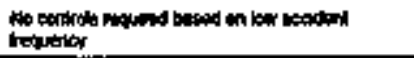 \\
\hline wobllnteess & & & 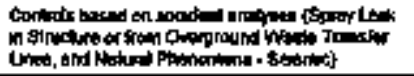 \\
\hline Whotharbest & & & 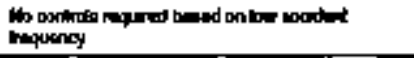 \\
\hline Mose-INTSagr & & & 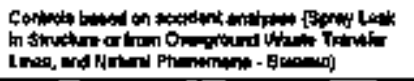 \\
\hline W05:-FAE9: & 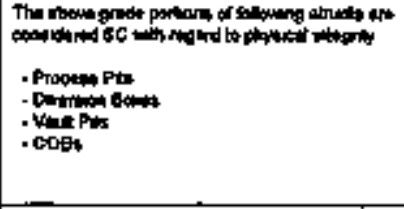 & 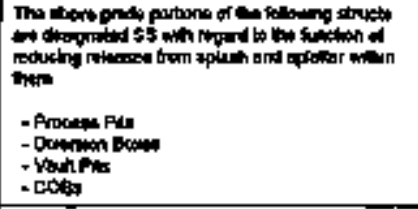 & 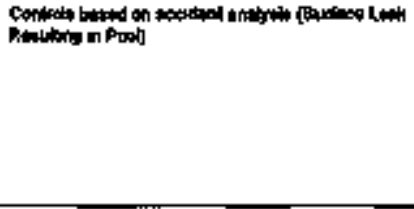 \\
\hline WOSP-PREEA3 & 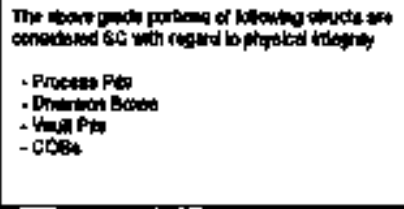 & 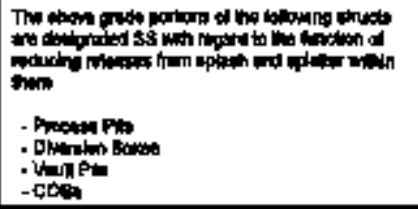 & 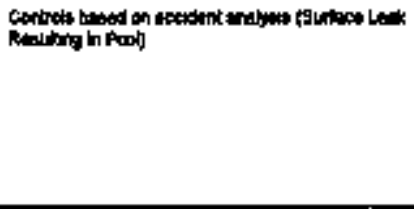 \\
\hline
\end{tabular}




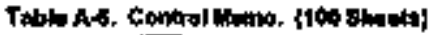

\begin{tabular}{|c|c|c|c|}
\hline ID & 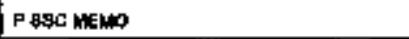 & M BSC WEWH & 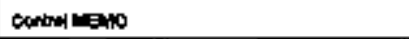 \\
\hline Xssonflorunt & & & 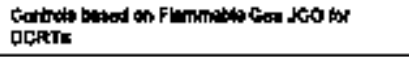 \\
\hline XB-OH-FLONAR & & & 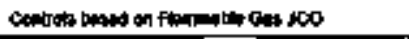 \\
\hline 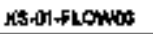 & & & 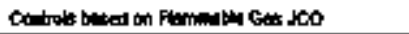 \\
\hline \multicolumn{4}{|l|}{ 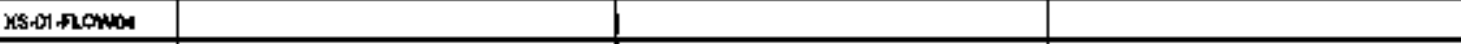 } \\
\hline 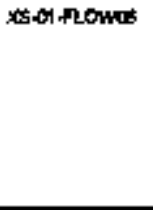 & 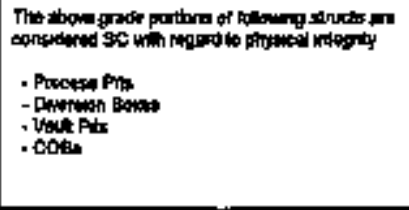 & 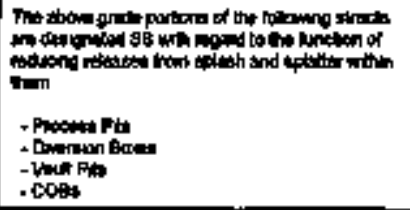 & 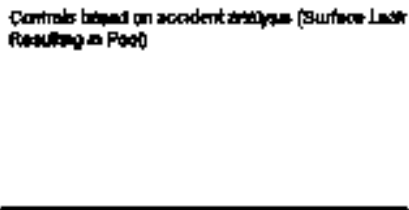 \\
\hline xe-01-FLOWH: & 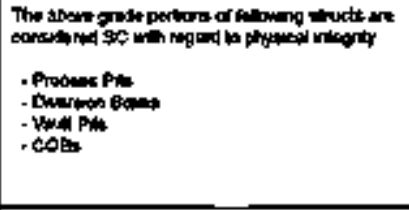 & 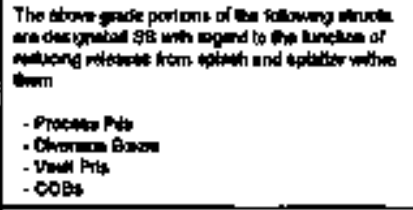 & 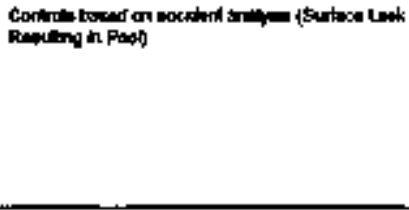 \\
\hline xsolrtomins & & & 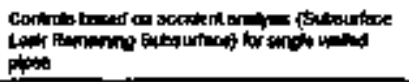 \\
\hline MSO-01+RESOI & & 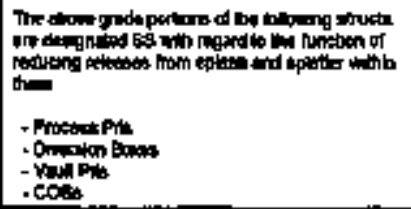 & 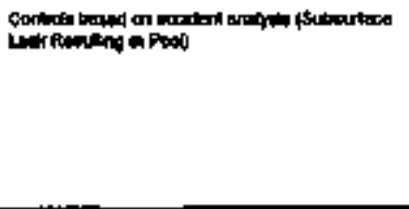 \\
\hline
\end{tabular}


Teble M-6. Contred Memo. [161 ahedes

\begin{tabular}{|c|c|c|c|}
\hline ID & 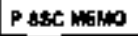 & HSSC MER & وست \\
\hline 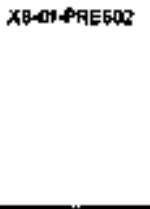 & & 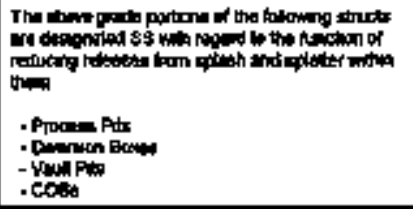 & 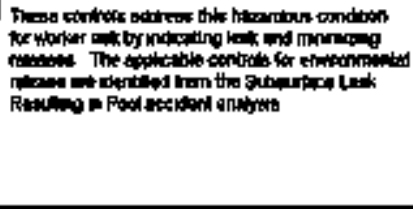 \\
\hline XEAOA-TEUFOH & & 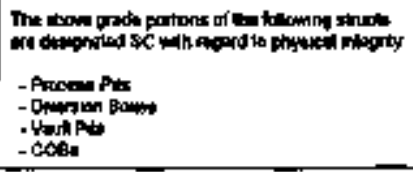 & 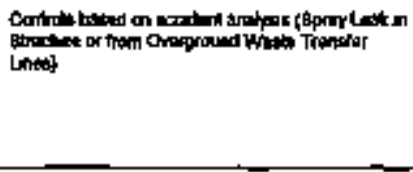 \\
\hline XE-01-TELPPO2 & & 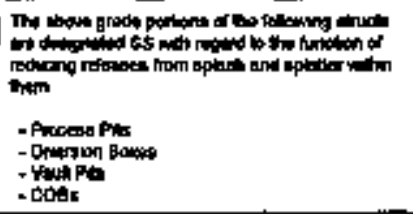 & 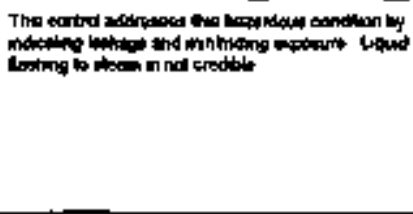 \\
\hline \multicolumn{4}{|l|}{ 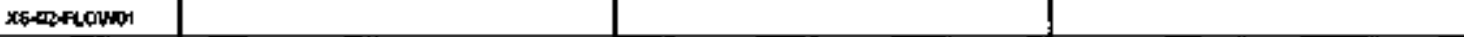 } \\
\hline xa-or-flown? & & & 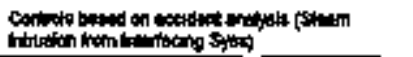 \\
\hline 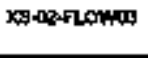 & & & 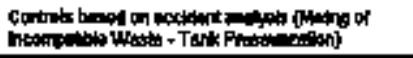 \\
\hline 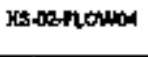 & & & 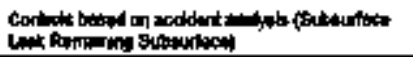 \\
\hline
\end{tabular}




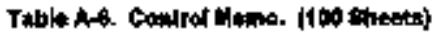

\begin{tabular}{|c|c|c|c|}
\hline $\boldsymbol{q}$ & P stchen & uste raxp & Gatrd mpes \\
\hline xe-0orforwas & & 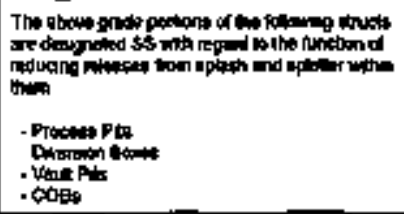 & 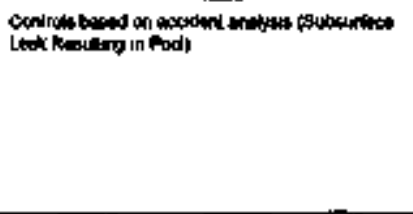 \\
\hline XHo2-flomas & & & 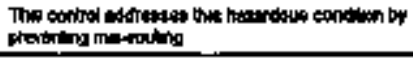 \\
\hline xis-a3.LEVE & 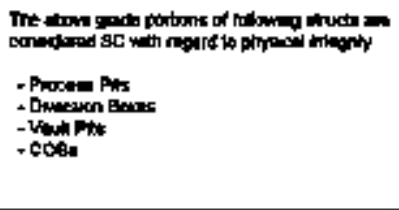 & 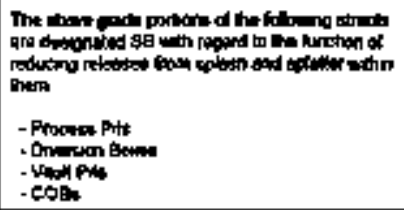 & 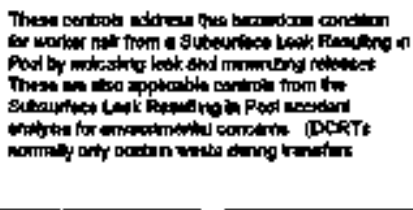 \\
\hline 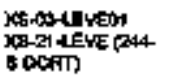 & & & 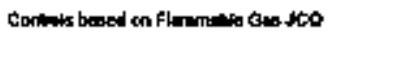 \\
\hline 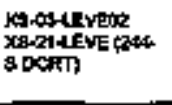 & & & 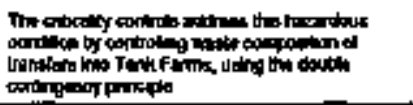 \\
\hline XE-03-PAEES & & & 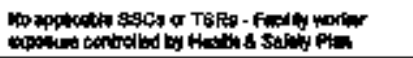 \\
\hline 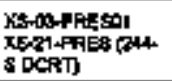 & & & 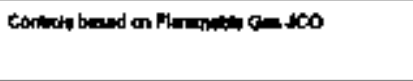 \\
\hline 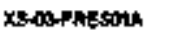 & & & 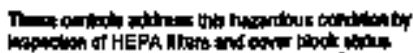 \\
\hline
\end{tabular}


Table A6. Contiol nerpa. (101 sivats)

\begin{tabular}{|c|c|c|c|}
\hline $\mathbf{b}$ & P SAC NEINo & هMeسו & Contrat Meneso \\
\hline 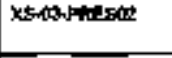 & & & 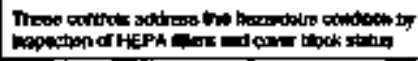 \\
\hline 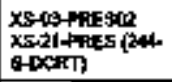 & & & 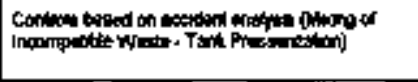 \\
\hline XY-OQP月E日OS & & & 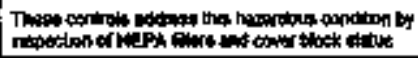 \\
\hline 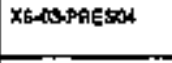 & & & 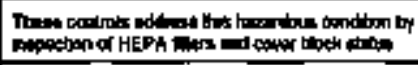 \\
\hline 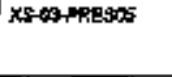 & & & 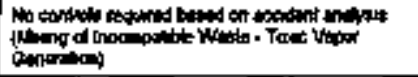 \\
\hline 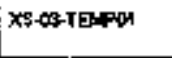 & & & 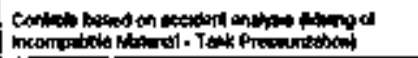 \\
\hline 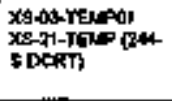 & & & 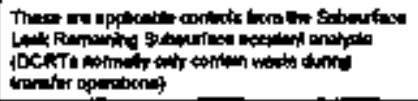 \\
\hline X8-03-TEस-0? & & & 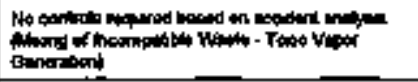 \\
\hline 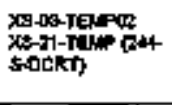 & & & 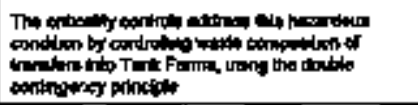 \\
\hline 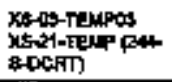 & & & 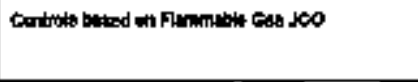 \\
\hline xos-os-remost & & & \\
\hline
\end{tabular}


Tabla 4-6. Control Mons. (14) shnats)

\begin{tabular}{|c|c|c|c|}
\hline D & 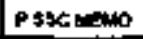 & M Bsc Meno & Doptod maxo \\
\hline 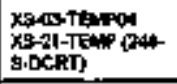 & & & 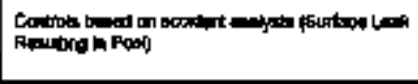 \\
\hline 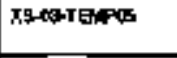 & & & 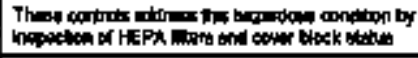 \\
\hline$x 5-09-10 \times 00$ & & & 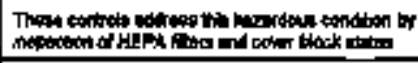 \\
\hline$x$ (S-0)-T & & & 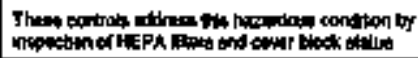 \\
\hline Xs-40-T Expess & & & 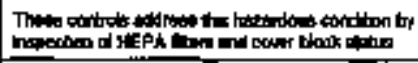 \\
\hline$X E-03-15$ MPOQ & & & 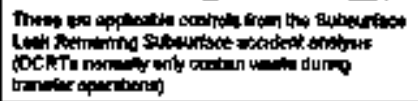 \\
\hline Xs-05-nomiol & & & 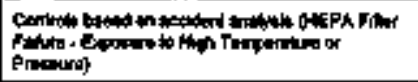 \\
\hline XB-05-Fown & & & 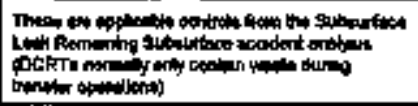 \\
\hline Xs-ostrapin & & & 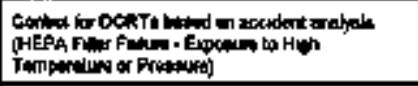 \\
\hline 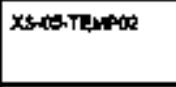 & & & 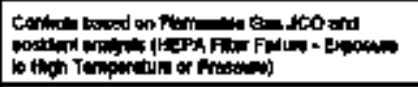 \\
\hline 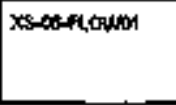 & & & 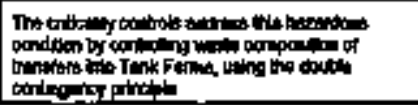 \\
\hline
\end{tabular}




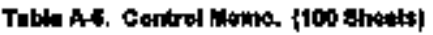

\begin{tabular}{|c|c|c|c|}
\hline ID & P Esc MER & MASC MELO & 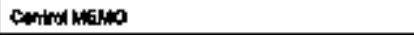 \\
\hline 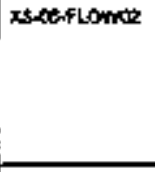 & & 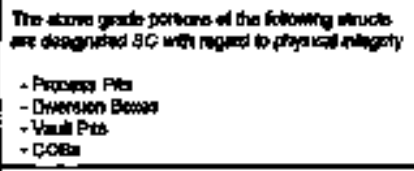 & 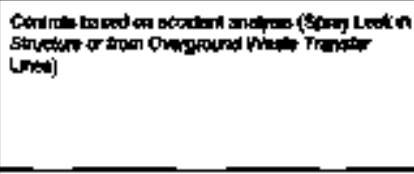 \\
\hline 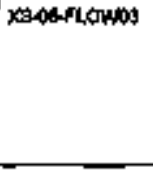 & & 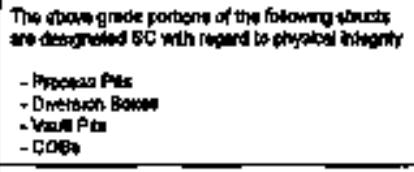 & 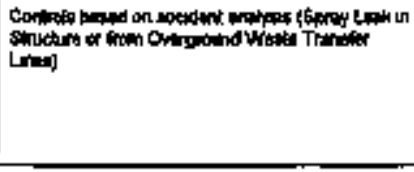 \\
\hline XS-03_fCOMF & & 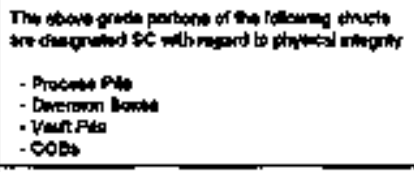 & 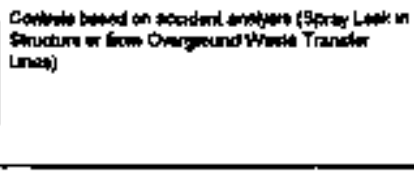 \\
\hline Xo-os-flonines & & 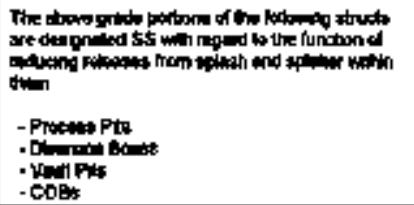 & 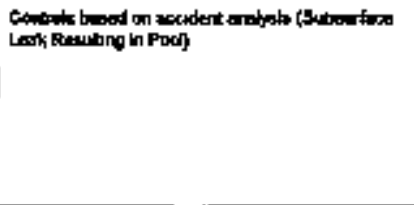 \\
\hline
\end{tabular}




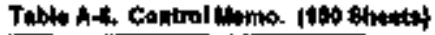

\begin{tabular}{|c|c|c|c|}
\hline ט & Pasc palpe & MSGC WEMO & Eontrod latat \\
\hline Xs-0.rformos & 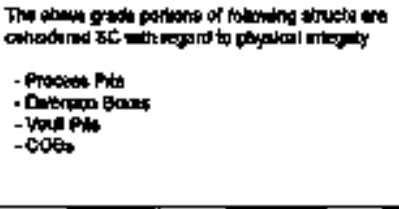 & 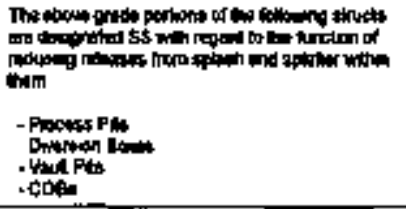 & 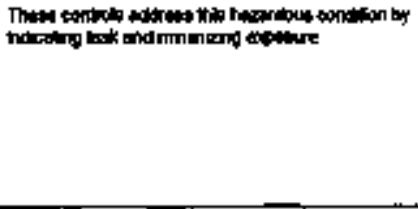 \\
\hline xS-OF-76hor & & & 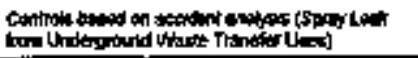 \\
\hline 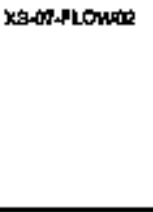 & & 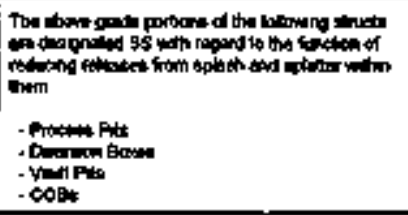 & 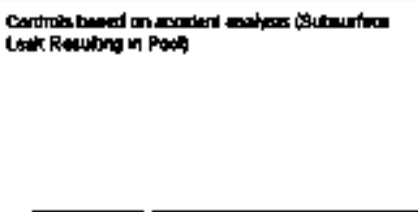 \\
\hline 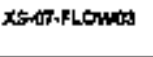 & & & 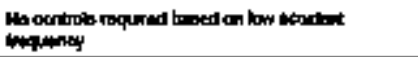 \\
\hline XP-07FLOWHA & & 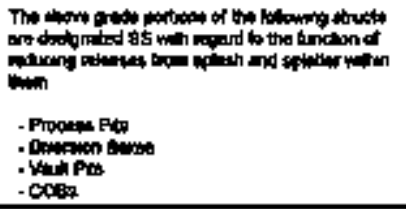 & 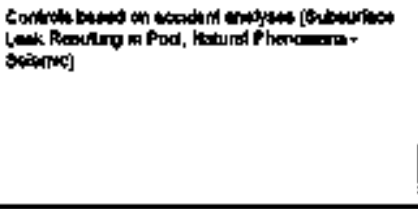 \\
\hline
\end{tabular}




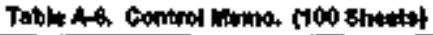

\begin{tabular}{|c|c|c|c|}
\hline 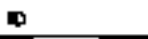 & $P$ PFC MENO & u sscich Men & conted hiento \\
\hline Xt-D-rLothis & & 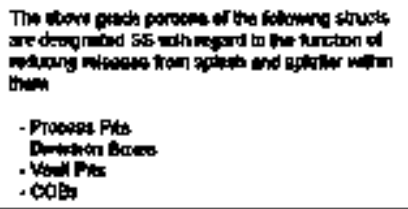 & 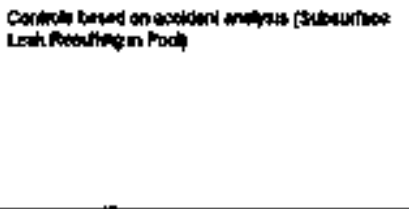 \\
\hline X5:07-flowhs & & 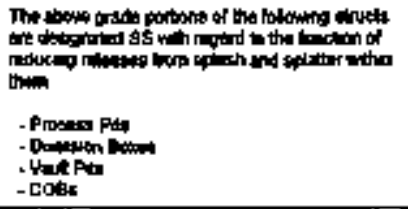 & 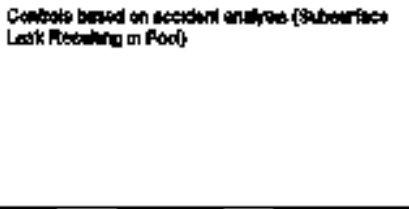 \\
\hline XSS-11-LEvith & 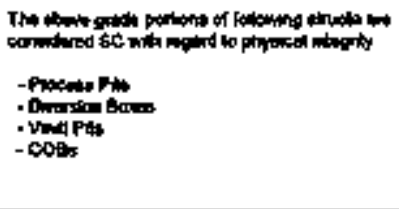 & 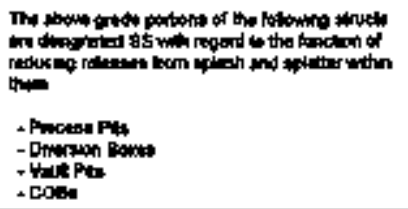 & 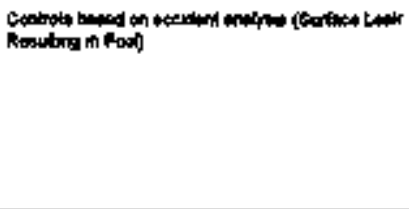 \\
\hline XY-1LCENED? & 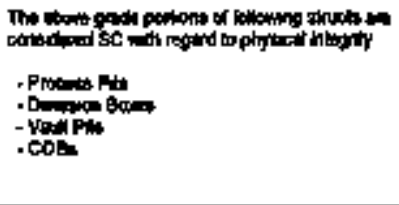 & 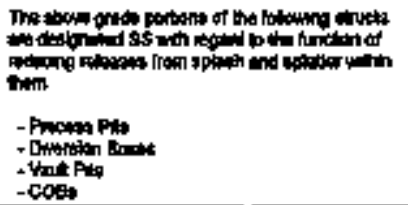 & 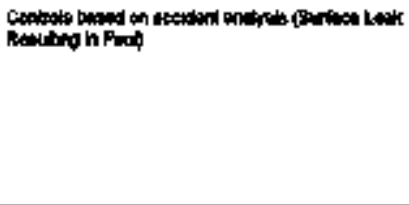 \\
\hline
\end{tabular}




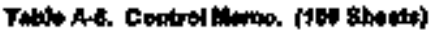

\begin{tabular}{|c|c|c|c|}
\hline 10 & 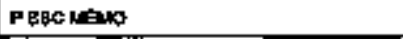 & HBBC MENO & 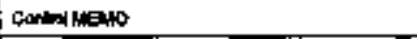 \\
\hline XS-11-ATVEOS & 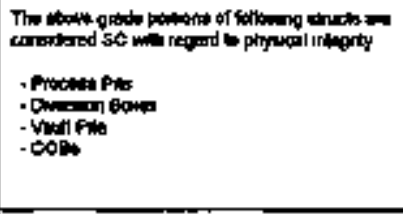 & 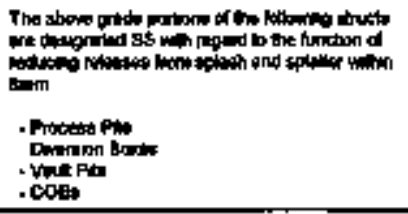 & 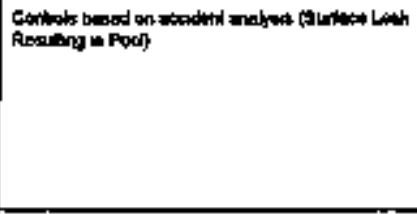 \\
\hline Xs-12flown & & & 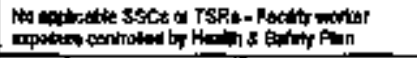 \\
\hline XE-12-fútuma & & & 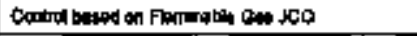 \\
\hline Xt-12-ruonwas & & & 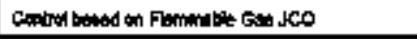 \\
\hline KEM-12NLONWA & & & 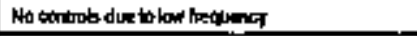 \\
\hline xts-12 MLONot & 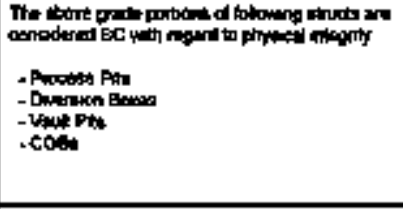 & 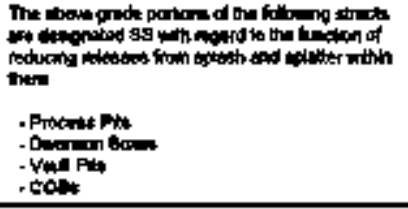 & 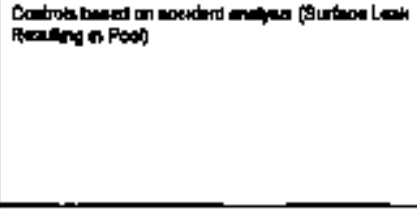 \\
\hline X6-:2:FLoHtits & & 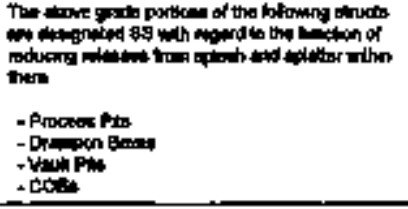 & 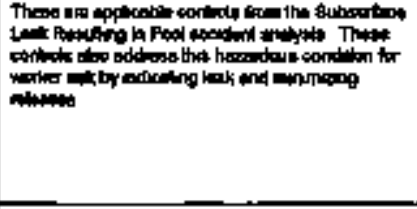 \\
\hline$x x^{3}-13-464=$ & & & 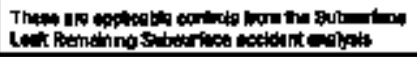 \\
\hline
\end{tabular}




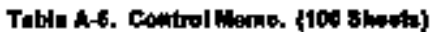

\begin{tabular}{|c|c|c|c|}
\hline 10 & P \$\$о кино & 由 & 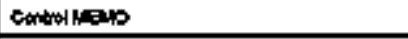 \\
\hline xes-13Pafét: & & & 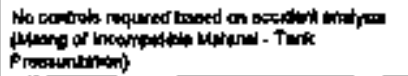 \\
\hline 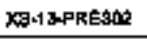 & & & 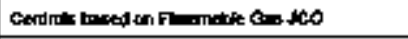 \\
\hline XS-1 S-PREBOS & & & 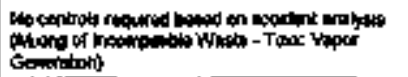 \\
\hline X9-1 3epRESto4 & & & 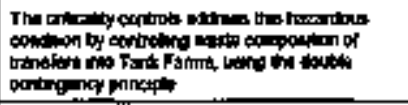 \\
\hline 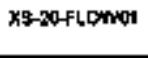 & & & 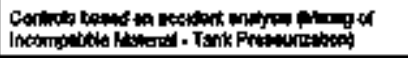 \\
\hline 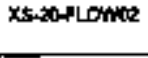 & & & 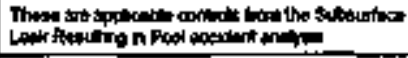 \\
\hline Xa-20-7omas & & & 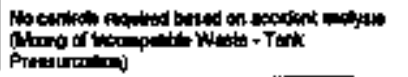 \\
\hline xS-2015LOWA4 & & & 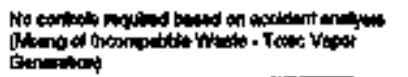 \\
\hline xh-ADFLown: & & & 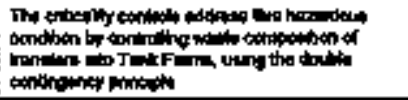 \\
\hline Xtowlown: & & & 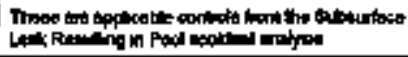 \\
\hline 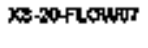 & & & 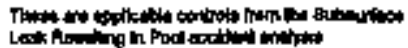 \\
\hline
\end{tabular}


Table A-6. Control Inomo. (10) Shatts

\begin{tabular}{|c|c|c|c|}
\hline D & $P$ F्व & 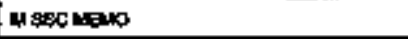 & Certer werts \\
\hline 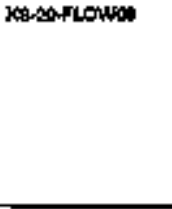 & 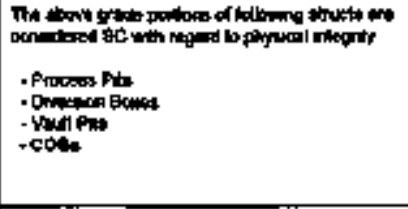 & 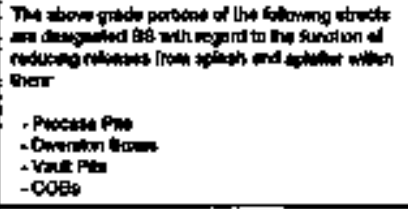 & 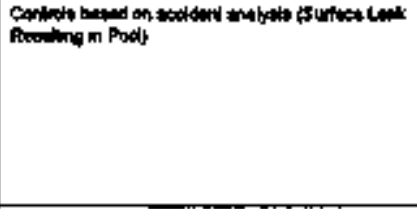 \\
\hline Xs-atiflones & 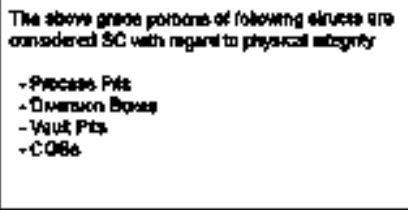 & 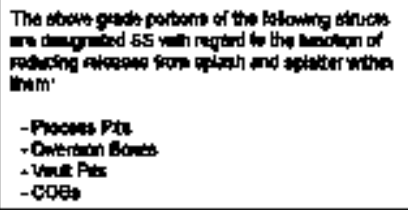 & 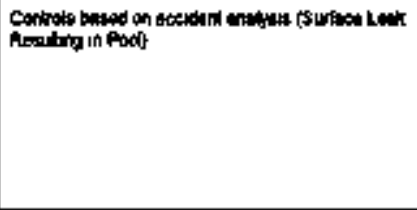 \\
\hline 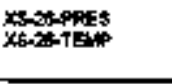 & & & 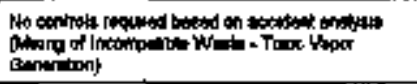 \\
\hline 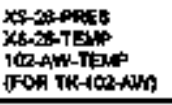 & & & 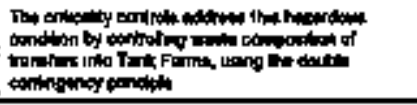 \\
\hline xtsestentest & & & 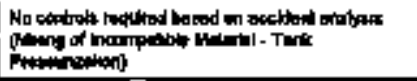 \\
\hline 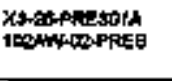 & & & 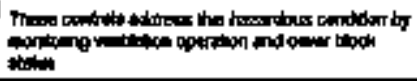 \\
\hline Ktotameston & & & 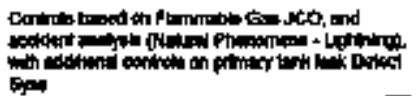 \\
\hline
\end{tabular}


Tahle A6, Condrod Mann, (100 Sheets)

\begin{tabular}{|c|c|c|c|}
\hline$\Delta$ & P stc MDNo & 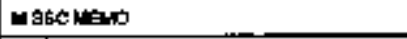 & Cantod unato \\
\hline 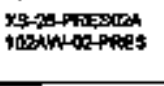 & & & 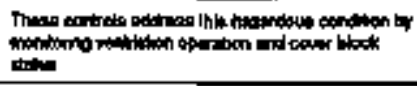 \\
\hline 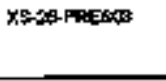 & & & 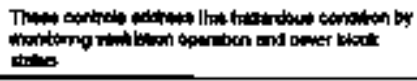 \\
\hline 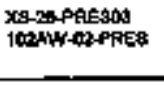 & & & 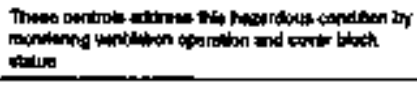 \\
\hline \multicolumn{4}{|l|}{ 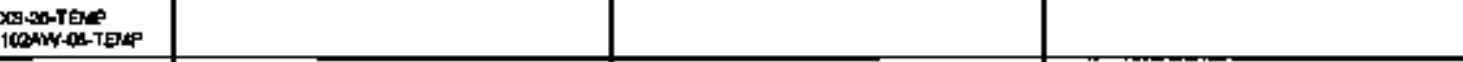 } \\
\hline Xs-*3-Tapor & & & 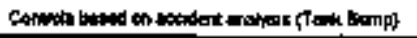 \\
\hline \multicolumn{4}{|l|}{ 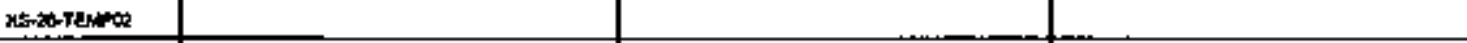 } \\
\hline 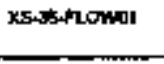 & & & 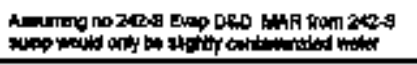 \\
\hline Xe-35-flown: & & & 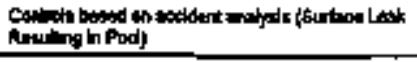 \\
\hline 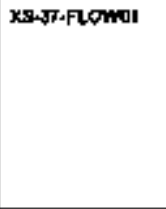 & 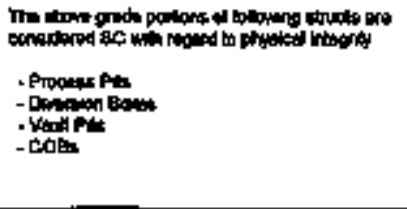 & 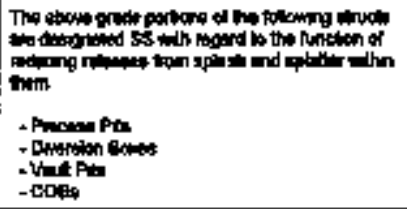 & 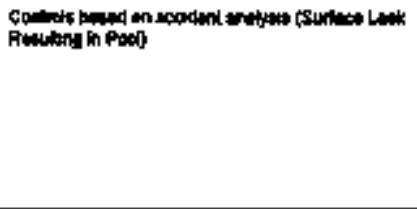 \\
\hline 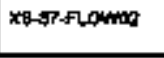 & & & 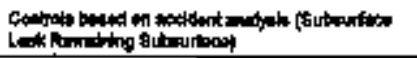 \\
\hline
\end{tabular}




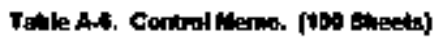

\begin{tabular}{|c|c|c|c|}
\hline ID & Pste nelus & H S\$C newo & control wats \\
\hline 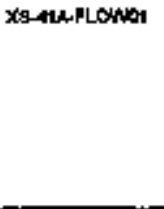 & 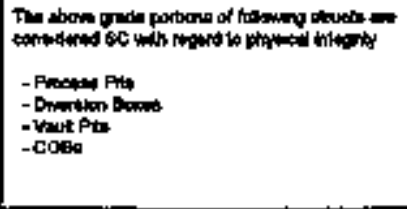 & 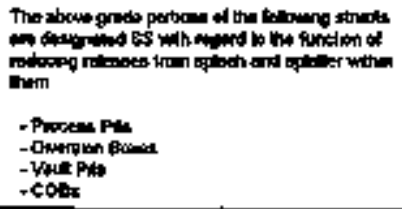 & 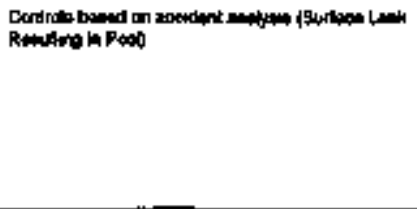 \\
\hline 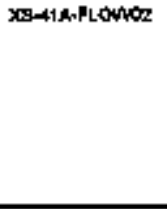 & 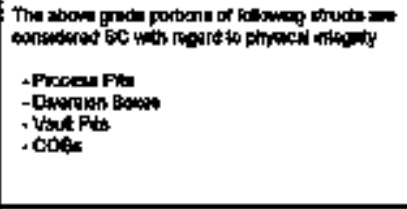 & 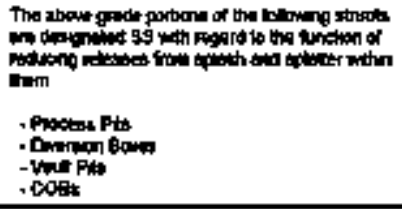 & 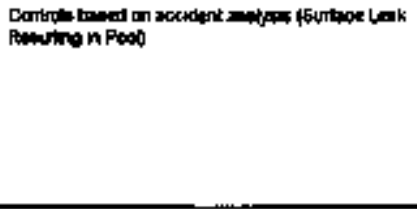 \\
\hline 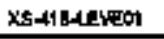 & & & 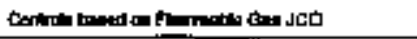 \\
\hline 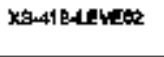 & & & 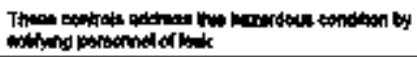 \\
\hline 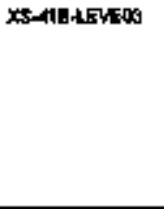 & 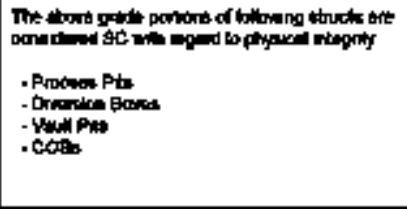 & 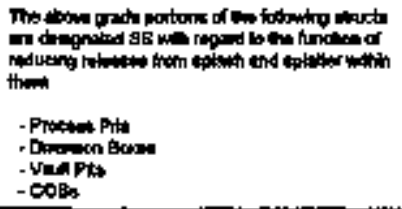 & 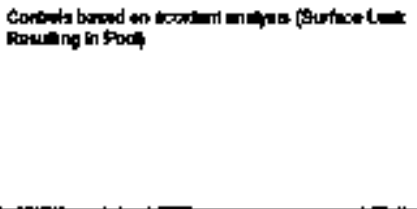 \\
\hline 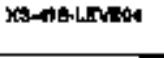 & & & 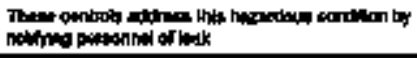 \\
\hline X6-4tC-LEYEA & & & 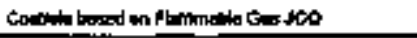 \\
\hline xt-4c-ifinion & & & \\
\hline
\end{tabular}




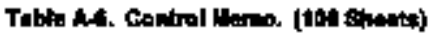

\begin{tabular}{|c|c|c|c|}
\hline$\Delta$ & 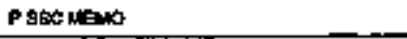 & M SSC MENO & Cortad ind \\
\hline XS-AIC-LELEDS & 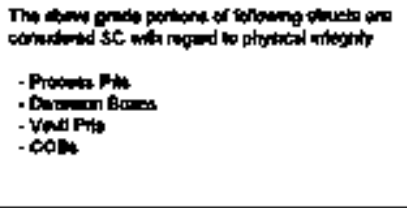 & 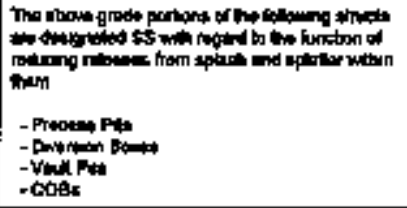 & 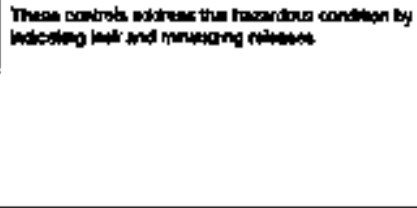 \\
\hline XB-aflony & & & 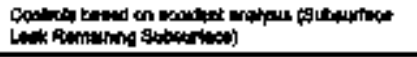 \\
\hline 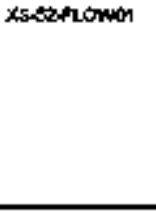 & & 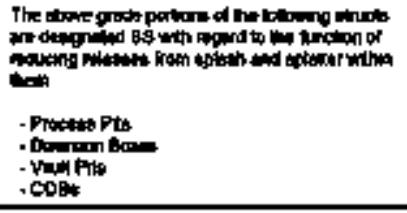 & 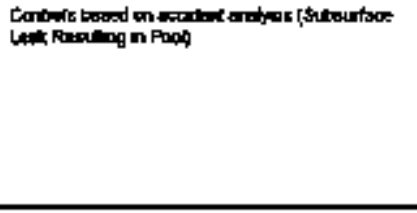 \\
\hline 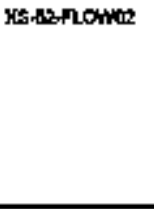 & 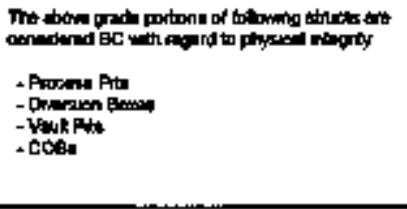 & 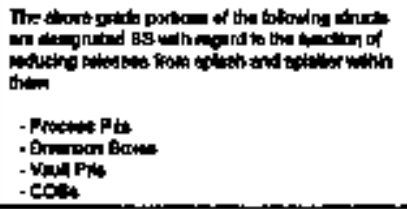 & 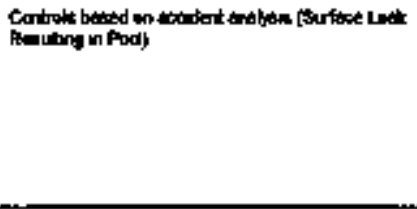 \\
\hline
\end{tabular}




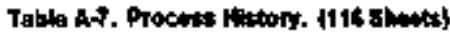

\begin{tabular}{|c|c|c|c|c|c|c|c|c|}
\hline 10 & Vterdi & finter & Fon & 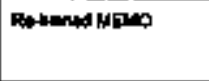 & ong & ing & sing & Beprinta \\
\hline 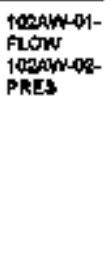 & 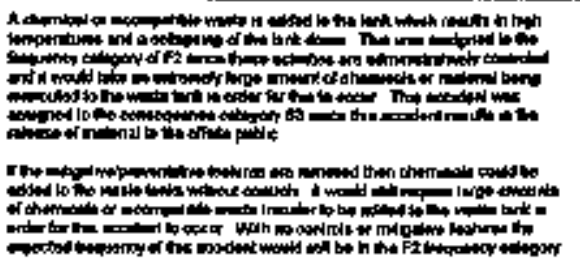 & & & & f2 & $\mathbf{r}$ & 93 & \\
\hline 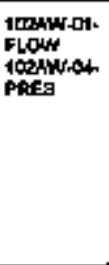 & 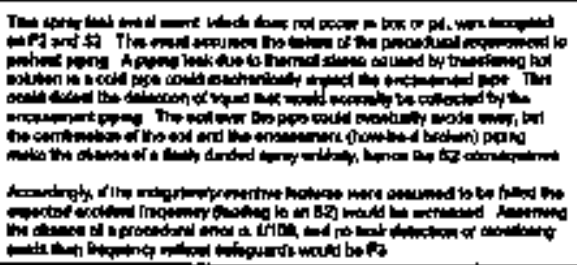 & so & & 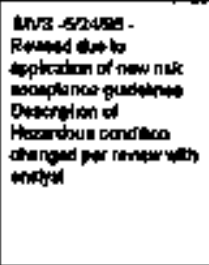 & $n$ & $\mathbf{F}$ & 82 & \\
\hline $\begin{array}{l}\text { 10anu-G1- } \\
\text { PLowity }\end{array}$ & 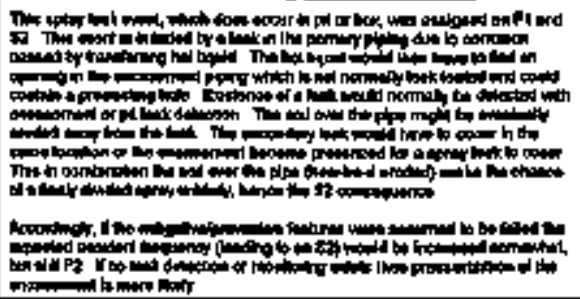 & 69 & & 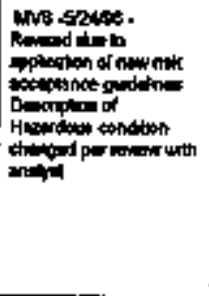 & $\mathbf{F 1}$ & 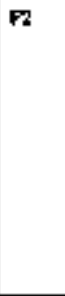 & 52 & \\
\hline
\end{tabular}


Tobla A-7. Proeks Hittory. (116 Swagts)

\begin{tabular}{|c|c|c|c|c|c|c|c|c|}
\hline I & MEMOA & $\begin{array}{l}\text { Fiv. } \\
\text { band } \\
\text { Eation }\end{array}$ & Fined & Rotimad Mero & $\lim _{\ln }$ & Pins & ontis & Romsinta \\
\hline 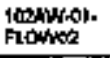 & 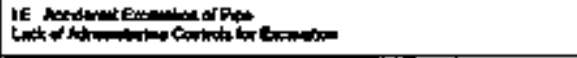 & & & & $\mathbf{m}$ & $\boldsymbol{F}$ & $\mathbf{s}$ & \\
\hline 1604w-or- & 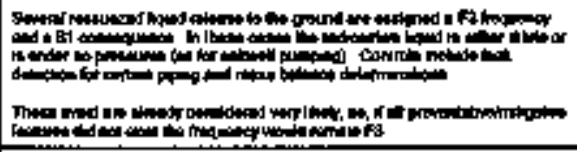 & & & & F3 & $\mathbf{F 3}$ & 82 & \\
\hline 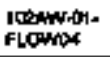 & 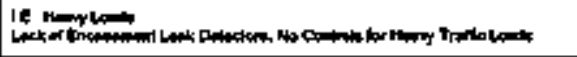 & & & & $\mathbf{F}$ & B & $\$ 2$ & \\
\hline 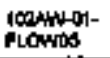 & IE tewne Ruent & & & & $\mathbf{f 2}$ & $\mathbf{F} \mathbf{z}$ & $\$ 2$ & \\
\hline $\begin{array}{l}\text { 10mandort } \\
\text { Flowpe }\end{array}$ & 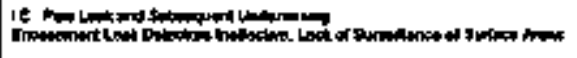 & & & & $\mathbf{r}$ & $\mathbf{H}$ & $\$ 2$ & \\
\hline 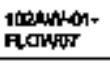 & 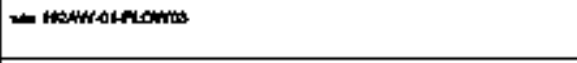 & & & & $\mathbf{A}$ & $\mathbf{P s}$ & ง2 & \\
\hline 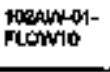 & 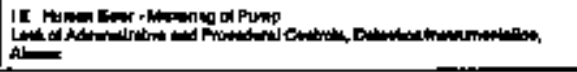 & & & & $\mathbf{F} \mathbf{A}$ & $\mathrm{Fz}$ & 92 & \\
\hline 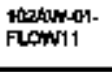 & 年 & & & & $\boldsymbol{r}$ & F3 & 鲸 & \\
\hline $\begin{array}{l}\text { 1GOUHAT. } \\
\text { TEMP }\end{array}$ & 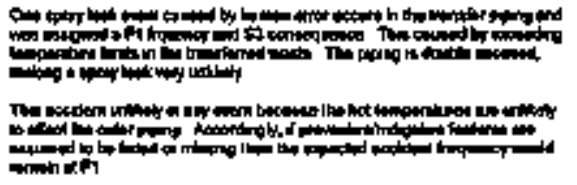 & & & & $\mathbf{n}$ & $\mathbf{m}$ & $\mathbf{8 3}$ & \\
\hline
\end{tabular}




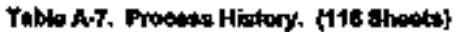

\begin{tabular}{|c|c|c|c|c|c|c|c|c|}
\hline $\mathbf{D}$ & Melto & intiod & 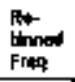 & 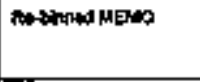 & Dng & 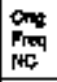 & $\operatorname{lin}_{\text {sath }}$ & 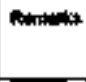 \\
\hline 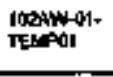 & 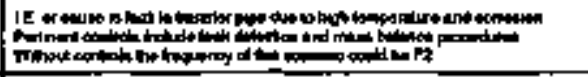 & & & & $\mathrm{Fr}$ & $\mathbf{F 2}$ & 82 & \\
\hline 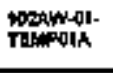 & 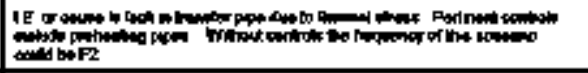 & & & & F1 & $F 2$ & 82 & \\
\hline $\begin{array}{l}\text { Dosenwa1. } \\
\text { TEMPM }\end{array}$ & 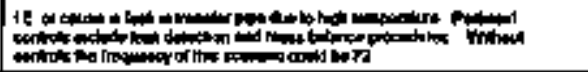 & & & & $\mathbf{H}$ & P2 & $s$ & \\
\hline $\begin{array}{l}\text { 102urres. } \\
\text { levt }\end{array}$ & 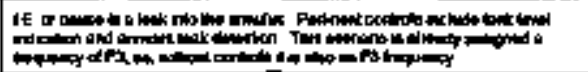 & & & & $\mathrm{Fg}$ & +9 & st & \\
\hline $\begin{array}{l}\text { logurnat } \\
\text { invion }\end{array}$ & 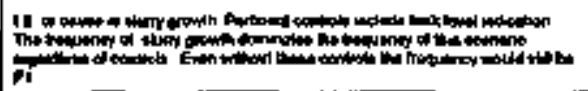 & 0 & & 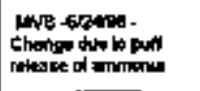 & F1 & P1 & 81 & \\
\hline $\begin{array}{l}\text { logaw-en } \\
\text { letrate }\end{array}$ & 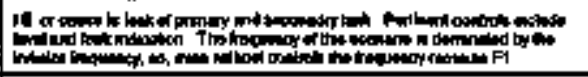 & & & & Fq & F1 & $\$ 1$ & \\
\hline 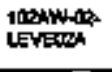 & 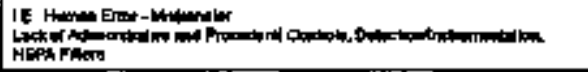 & & & & f1 & $\mathbf{F}$ & si & \\
\hline 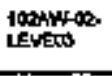 & 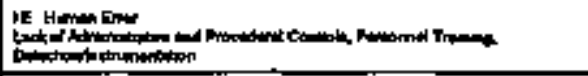 & & & & $n$ & $\mathbf{A}$ & $\$ 2$ & \\
\hline 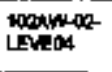 & 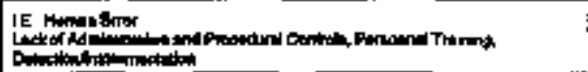 & & & & $\mathbf{F 2}$ & $\mathbf{m}$ & $\$ 2$ & \\
\hline Mandon & 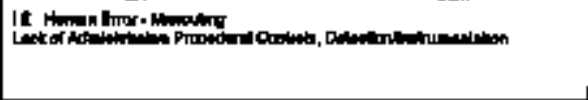 & \$2 & & 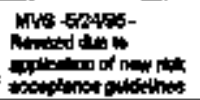 & F2 & $\mathbf{F}$ & s1 & \\
\hline
\end{tabular}


Table A-T. Procists Fislory. (116 sherts)

\begin{tabular}{|c|c|c|c|c|c|c|c|c|}
\hline $\boldsymbol{H}$ & MENONE & 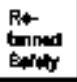 & $\lim _{\lim _{4}}$ & 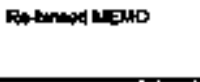 & ind & 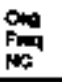 & ondity & Rempina \\
\hline 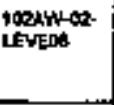 & 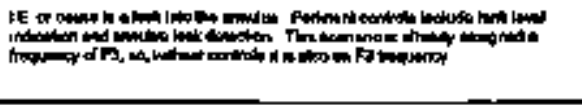 & & ri & 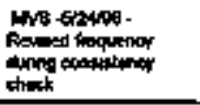 & $\mathbf{B}$ & $\mathbf{r s}$ & 61 & \\
\hline 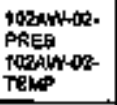 & 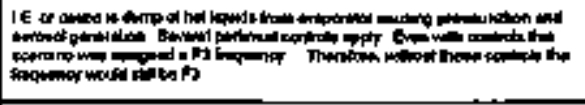 & & & & $\mathbf{A}$ & $\mathbf{F}$ & $\Leftrightarrow$ & \\
\hline 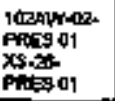 & & & & & $\mathbf{F r}$ & & 61 & \\
\hline $\begin{array}{l}\text { jogny-02- } \\
\text { pretsint }\end{array}$ & 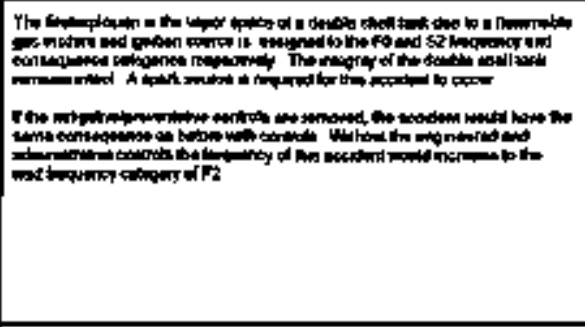 & $\$ 3$ & & 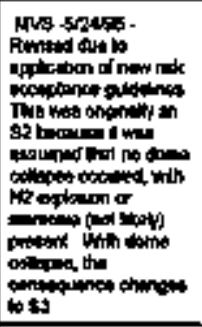 & $\mathbf{r}$ & $F 2$ & 82 & \\
\hline 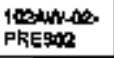 & 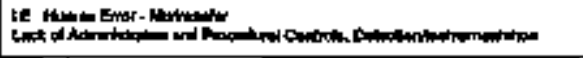 & & & & $\mathbf{F 3}$ & $\mathbf{F}$ & 82 & \\
\hline $\begin{array}{l}\text { 1024inotor } \\
\text { PREsos }\end{array}$ & 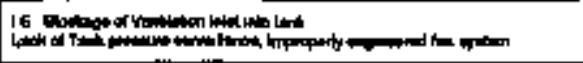 & & & & E1 & f1 & ss & \\
\hline 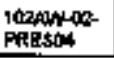 & 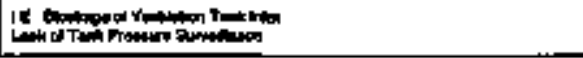 & & & & n & $\mathbf{n}$ & 93 & \\
\hline
\end{tabular}




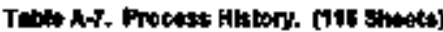

\begin{tabular}{|c|c|c|c|c|c|c|c|c|}
\hline $\mathbf{D}$ & MENON & 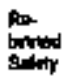 & 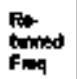 & Rotinned Hato & ong & 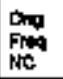 & Sols & Panorike \\
\hline $\begin{array}{l}\text { wownton- } \\
\text { PREStos }\end{array}$ & 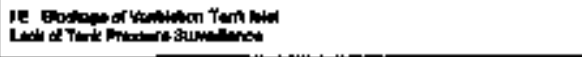 & & & & fI & F1 & 83 & \\
\hline 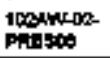 & 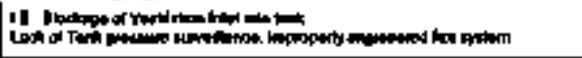 & & & & F1 & $n$ & se & \\
\hline 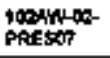 & IP & & & & 1 & $F$ & 89 & \\
\hline $\begin{array}{l}\text { noganop- } \\
\text { PHEPOS }\end{array}$ & 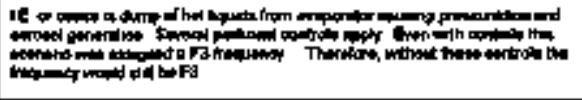 & 82 & & 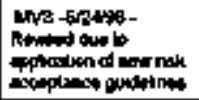 & $\mathbf{F}$ & ค & s1 & \\
\hline $\begin{array}{l}\text { 102Ain-D2 } \\
\text { PRESTOO }\end{array}$ & 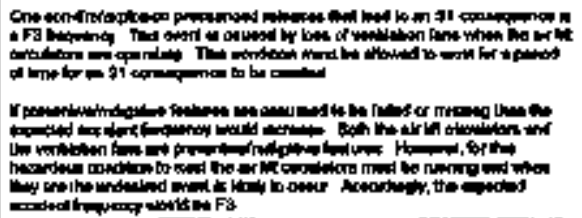 & 82 & & 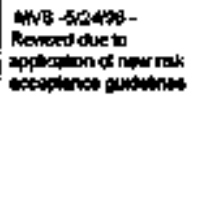 & fa & $\mathrm{FH}$ & 31 & \\
\hline 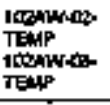 & 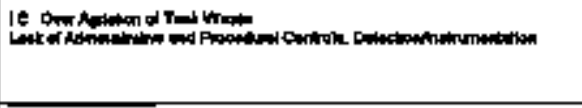 & 87 & $\mathbf{F}$ & 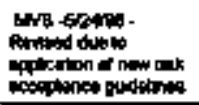 & p & $\mathrm{F}$ & \$ & \\
\hline $\begin{array}{l}\text { 1004w-os } \\
\text { TEtar }\end{array}$ & 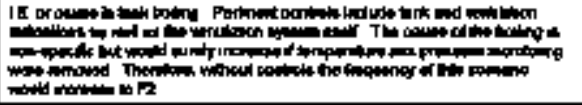 & & & & $F 1$ & $\mathbf{f 2}$ & 92 & \\
\hline 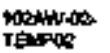 & 1 & & & & $\mathrm{Ft}$ & $\mathbf{F 2}$ & 82 & \\
\hline
\end{tabular}




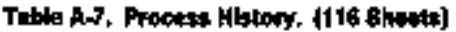

\begin{tabular}{|c|c|c|c|c|c|c|c|c|}
\hline b & Hello ne & singer & $\lim _{\text {innod }}$ & Rnbthed HDNO & Drog & Ond & 品 & Pommin \\
\hline 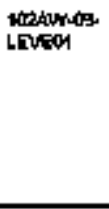 & 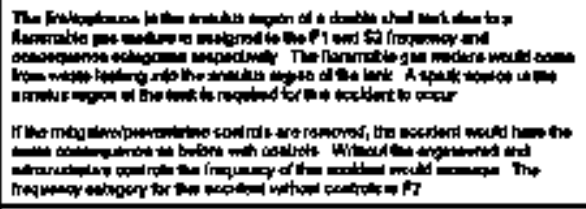 & & & & F1 & 87 & 82 & \\
\hline 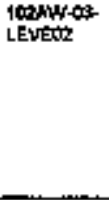 & 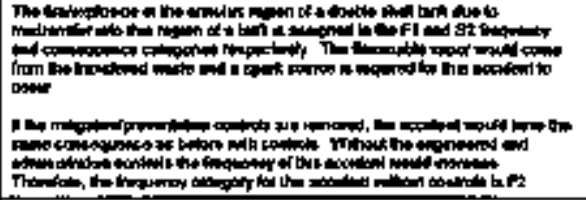 & & & & 1 & $\mathbf{F}$ & $\mathbf{s 2}$ & \\
\hline 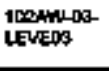 & 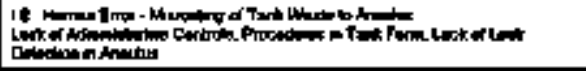 & & & & f1 & $\mathbf{F}$ & 83 & \\
\hline 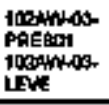 & 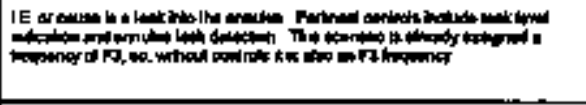 & & $\mathbf{F}$ & 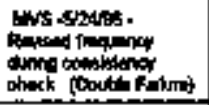 & $\mathbf{r}$ & Ps & 31 & \\
\hline 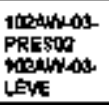 & 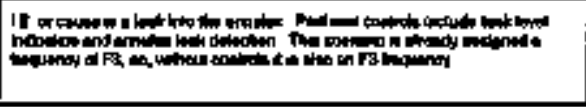 & & $\mathbf{F} 2$ & 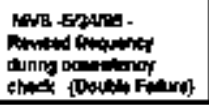 & fa & $\mathbf{F}$ & â & \\
\hline
\end{tabular}


Takla A-7. Procosts Halony. (116 Ghanta)

\begin{tabular}{|c|c|c|c|c|c|c|c|c|}
\hline 10 & סוסורו & $\lim _{\lim }$ & $\lim _{\lim }$ & Ratroned MESto & ond & 称 & ong & Aente \\
\hline 1000was & 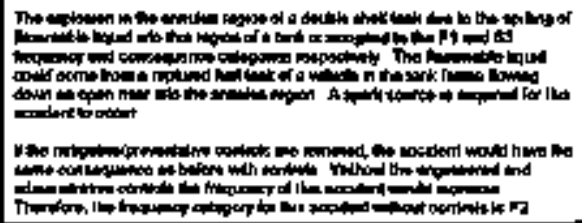 & & & & FI & $F 2$ & s3 & \\
\hline $\begin{array}{l}\text { togandos } \\
\text { Termat }\end{array}$ & 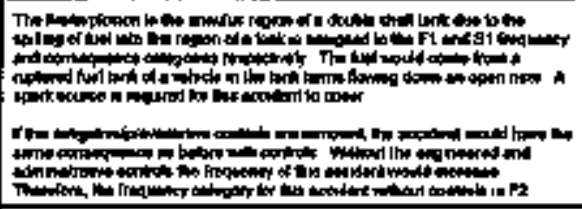 & & & & f1 & $\mathrm{F}_{2}$ & 92 & \\
\hline 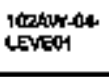 & 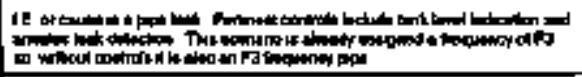 & & & & rs & es & $\$ 1$ & \\
\hline $\begin{array}{l}\text { lagentout } \\
\text { LEveos }\end{array}$ & 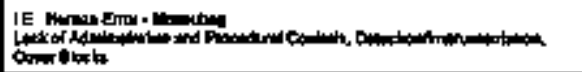 & & & & $\mathbf{F}$ & $\mathbf{F 3}$ & 91 & \\
\hline $\begin{array}{l}\text { 100Ain-04 } \\
\text { Liveos }\end{array}$ & 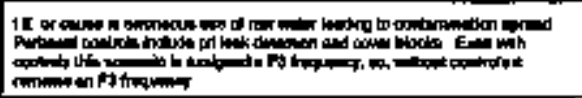 & & & & $\mathbf{F}$ & $\mathrm{FB}$ & $\$ 1$ & \\
\hline 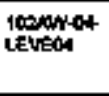 & 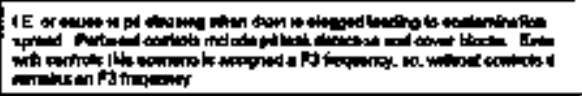 & & & & $\mathbf{F}$ & $\mathrm{FB}$ & 51 & \\
\hline $\begin{array}{l}\text { toenyou } \\
\text { preses of }\end{array}$ & & & & & $\boldsymbol{n}$ & & $s 1$ & \\
\hline
\end{tabular}


Table A7, Drockst Halony. (118 Sthats)

\begin{tabular}{|c|c|c|c|c|c|c|c|c|}
\hline$\infty$ & WENO WK & tom & $\lim _{\text {Fintag }}$ & Robmod LEAlt & Ondon & Orig & $\log _{\sin }$ & 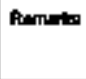 \\
\hline 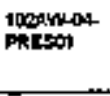 & 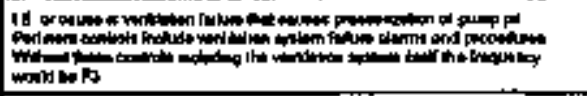 & & & & $\mathbf{F}$ & $\mathbf{F}$ & \$1 & \\
\hline $\begin{array}{l}\text { 10FAW04 } \\
\text { PRESOR }\end{array}$ & 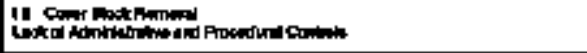 & & & & Ks & $\mathbf{F}$ & $\$ 1$ & \\
\hline $\begin{array}{l}\text { iogantont } \\
\text { strau of }\end{array}$ & & & & & $\mathrm{F}$ & & sı & \\
\hline $\begin{array}{l}\text { 102an-os } \\
\text { strat or }\end{array}$ & 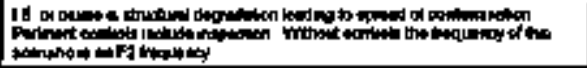 & & & & F1 & $\mathbf{n}$ & $\$ 1$ & \\
\hline 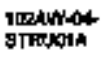 & 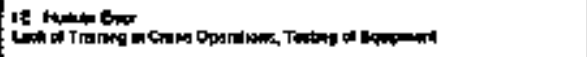 & & & & $\mathbf{F 1}$ & E & 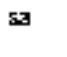 & \\
\hline 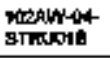 & IE & & & & $P 1$ & *I & s1 & \\
\hline $\begin{array}{l}\text { 10ONWOA } \\
\text { STRUD2 }\end{array}$ & IE Maran Tror & & & & $\mathbf{F 1}$ & Fs & 83 & \\
\hline 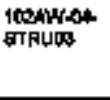 & 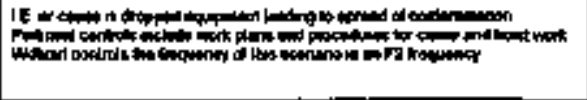 & sㄹ & & 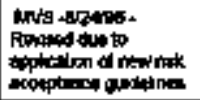 & f1 & $F 2$ & 51 & \\
\hline $\begin{array}{l}\text { 90601406. } \\
\text { STRO04 }\end{array}$ & 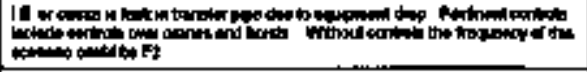 & & & & P1 & $\boldsymbol{F r}$ & 82 & \\
\hline $\begin{array}{l}\text { IIDAiNat } \\
\text { STRUOS }\end{array}$ & 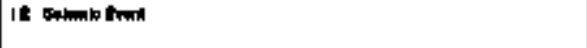 & & & & $\mathbf{F 1}$ & F1 & 82 & \\
\hline
\end{tabular}


Thble A-T. Procosd Hiatory. (11B Shental)

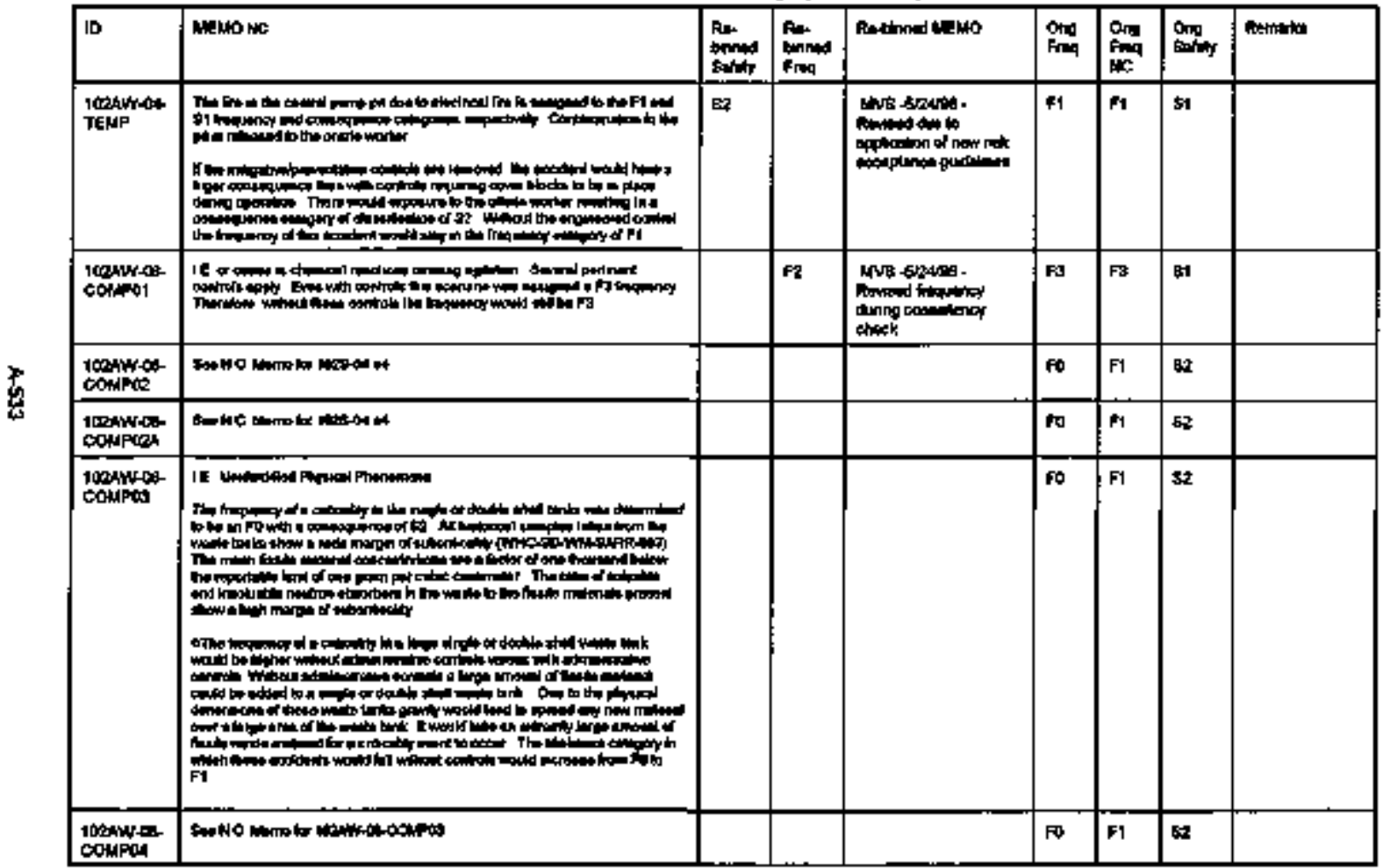


Tahb A-7. Process hitory. (116 atwit)

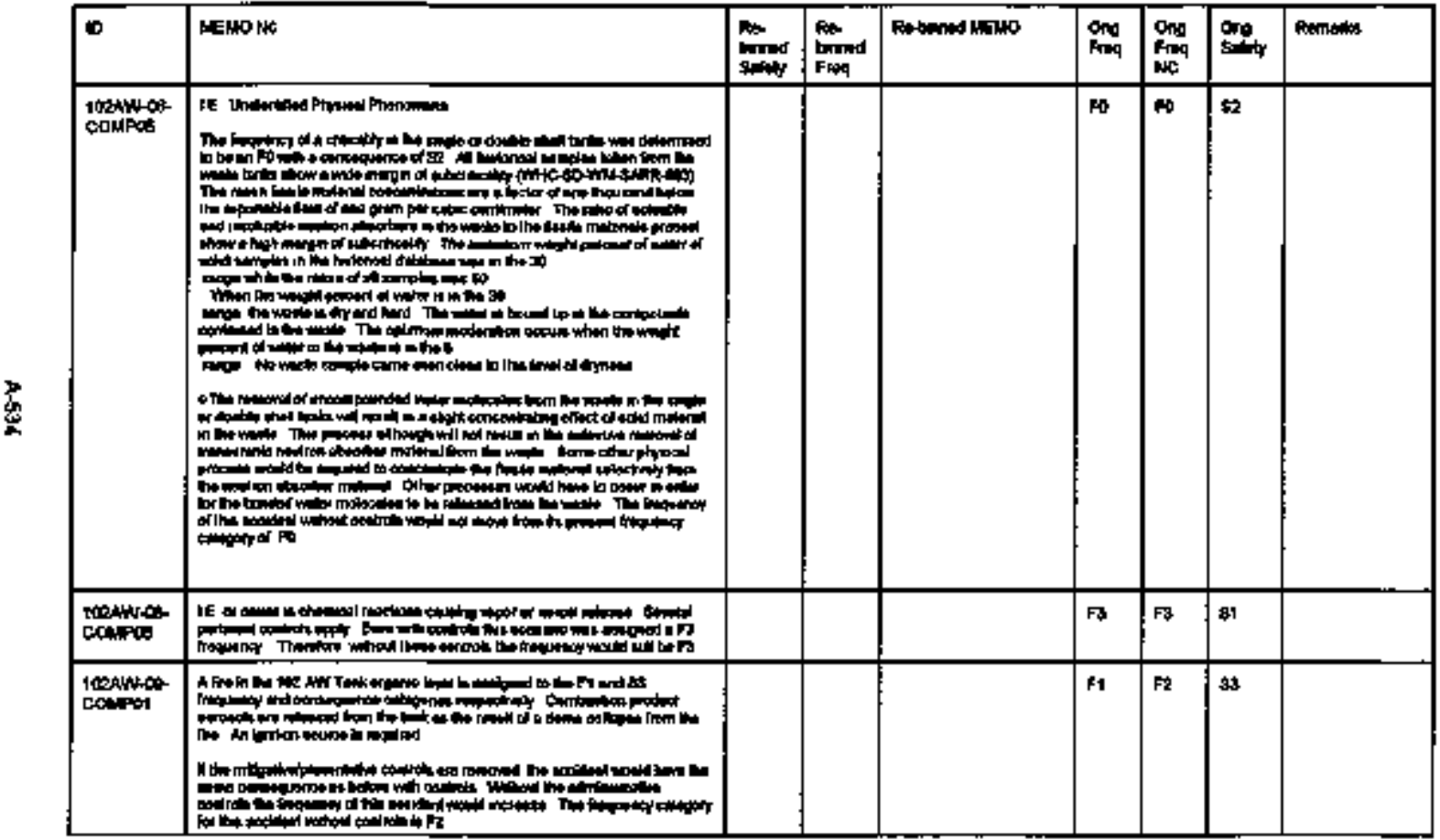


Table Ar. Proceds Hitery. (115 Sbets)

\begin{tabular}{|c|c|c|c|c|c|c|c|c|}
\hline ID & mewo ix & $\begin{array}{l}\text { Ror } \\
\text { bonned } \\
\text { Earing }\end{array}$ & Pes & 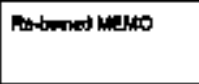 & on & $\begin{array}{l}\text { Pad } \\
\text { Nic }\end{array}$ & ong & Deminto \\
\hline lobatisen & 隹 & & & & F1 & $F 2$ & $\$ 3$ & \\
\hline losiwen & 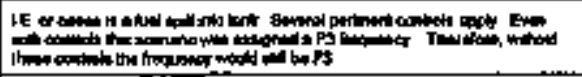 & & & & $\boldsymbol{n s}$ & $\mathbf{m}$ & $\$ 1$ & \\
\hline logintop & 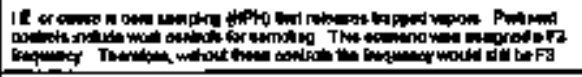 & & & & F3 & $\beta$ & $\$ 1$ & \\
\hline 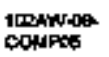 & 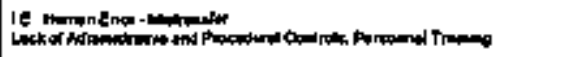 & & & & $F$ & $F$ & $b 1$ & \\
\hline $\begin{array}{l}\text { togant of } \\
\text { Tesp }\end{array}$ & 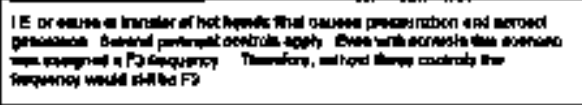 & 82 & & 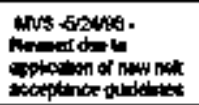 & $\mathbf{H 3}$ & 어 & 91 & \\
\hline 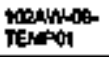 & $\omega x=-8$-Tanou & & & & F1 & $\mathbf{F} \mathbf{z}$ & $2 z$ & \\
\hline Teganog. & 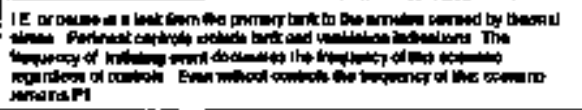 & & & & $\mathbf{n}$ & F1 & 91 & \\
\hline 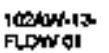 & & & & & $\mathbf{F} 2$ & & $\mathbf{s 1}$ & \\
\hline
\end{tabular}




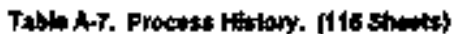

\begin{tabular}{|c|c|c|c|c|c|c|c|c|}
\hline $\mathbf{0}$ & 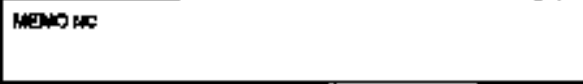 & Bribed & $\begin{array}{l}\text { Pl- } \\
\text { brined } \\
\text { Freq }\end{array}$ & 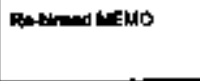 & Org & $\begin{array}{l}\text { ortg } \\
\text { Nited } \\
\text { NLt }\end{array}$ & 跑, & Aroming \\
\hline $\begin{array}{l}\text { losunt-13- } \\
\text { floutint }\end{array}$ & 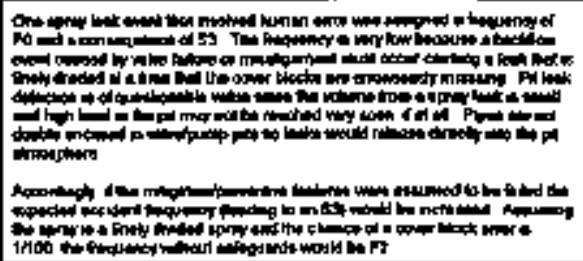 & & & & ro & F1 & 8 & \\
\hline 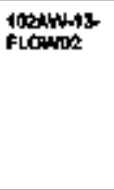 & 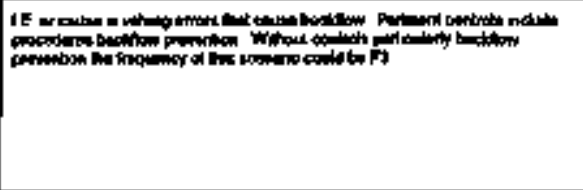 & 62 & & 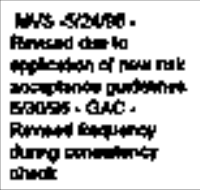 & $\mathbf{F}$ & $\neq$ & st & \\
\hline 1028-01 & 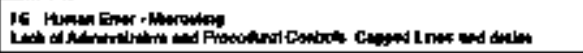 & & & & $\mathbf{F 1}$ & $\mathbf{F}_{\mathbf{2}}$ & 82 & \\
\hline $1025-016$ & 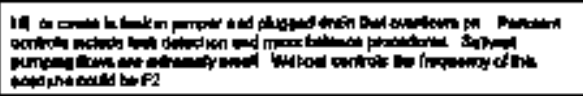 & & & & Ho & $\mathbf{F 2}$ & 52 & \\
\hline 10estal bu & 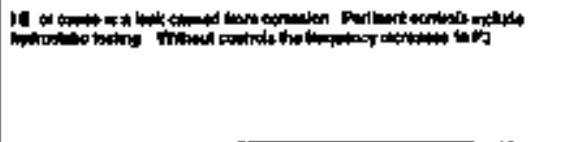 & 幽 & & 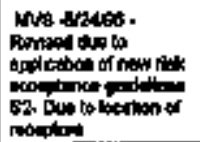 & $\mathbf{p s}$ & $\mathbf{m}$ & so & \\
\hline
\end{tabular}


Table Hit. Prodist Hitlony. (HA shents)

\begin{tabular}{|c|c|c|c|c|c|c|c|c|}
\hline 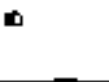 & MENOHC & $\begin{array}{l}\text { Ro } \\
\text { bentud } \\
\text { galdy }\end{array}$ & Ren & Arboned NEDo & ong & $\begin{array}{l}\text { Ong } \\
\text { ind } \\
\text { itc }\end{array}$ & ore & Raminto \\
\hline $1002-0064$ & 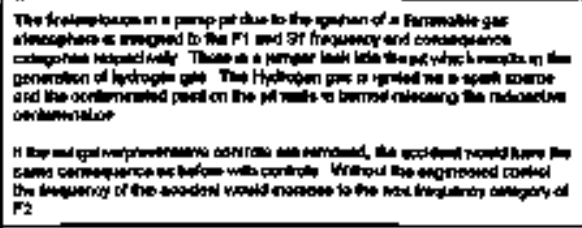 & 82 & & 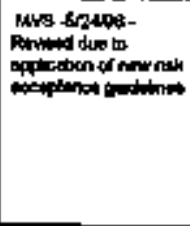 & $\mathbf{F I}$ & f2 & 61 & \\
\hline 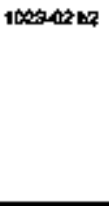 & 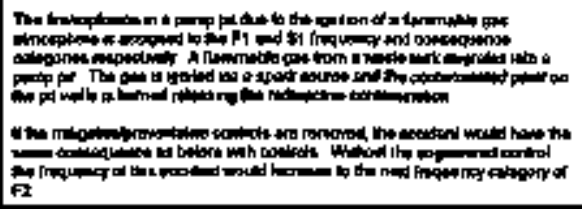 & ER & & 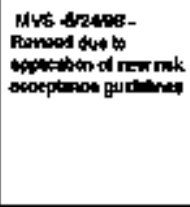 & $\mathbf{F 1}$ & $F 2$ & 61 & \\
\hline $1000-02$ 中 & 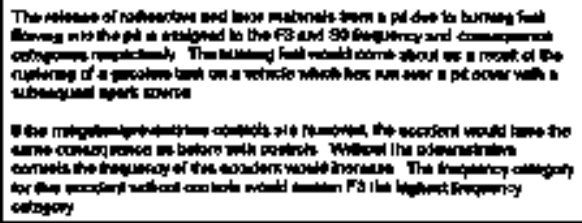 & $s$ & & 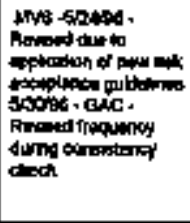 & is & $\boldsymbol{r s}$ & 36 & \\
\hline $1005-02 d t$ & 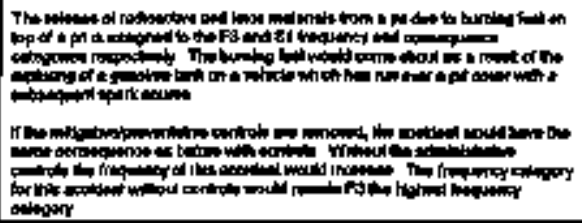 & & & & is & $\mathbf{m}$ & 31 & \\
\hline
\end{tabular}


Toble ArT. Prowats Hitiony. (116 strunts)

\begin{tabular}{|c|c|c|c|c|c|c|c|c|}
\hline$\omega$ & Manom & $\begin{array}{l}\text { Af- } \\
\text { bennod } \\
\text { Extory }\end{array}$ & Pond & 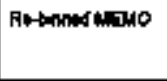 & Pron & $\begin{array}{l}\text { Ong } \\
\text { fing } \\
\text { NAC }\end{array}$ & Ond & Pemathe \\
\hline 100250340 & 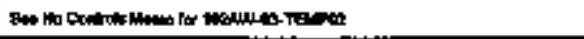 & & & & P1 & $\mathbf{F 2}$ & 52 & \\
\hline 10000004 & 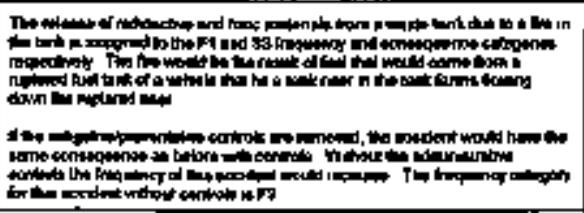 & & & & $F_{1}$ & $\mathbf{E s}$ & as & \\
\hline 0028 & 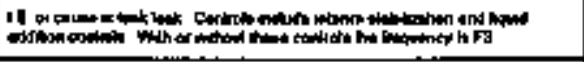 & & & & $\mathbf{F}$ & $\mathbf{r \$}$ & 4 & \\
\hline 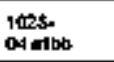 & 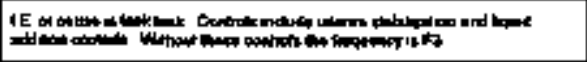 & & & & $\mathbf{f a}$ & $\mathrm{FB}$ & $\boldsymbol{x}$ & \\
\hline $1028-94$ a & 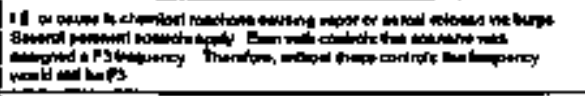 & 92 & & 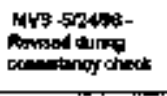 & $\mathbf{F}$ & rs & $\$ 1$ & \\
\hline 100505 & 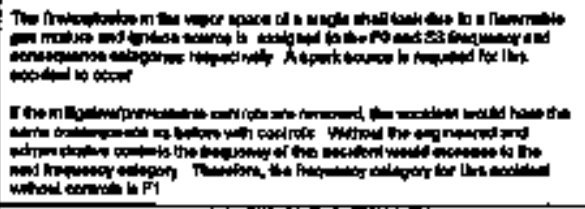 & & & & $n$ & $\mathbf{r}$ & 5 & \\
\hline 10his-at at & 19 & & & & $\mathbf{F o}$ & P1 & 9 & \\
\hline
\end{tabular}


Table Ar. Proosst Flation. (1 if shotst

\begin{tabular}{|c|c|c|c|c|c|c|c|c|}
\hline $\boldsymbol{D}$ & Nanemc & 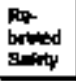 & Fond & Rabinand actolo & Ord & $\ln _{0.4}$ & $\begin{array}{l}\text { Drop } \\
\text { getios }\end{array}$ & 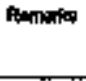 \\
\hline 2009- & 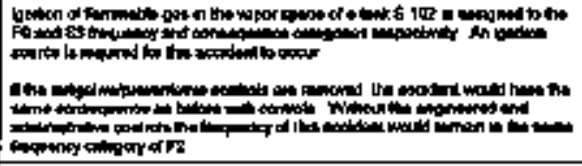 & & & & $\mathbf{F} 2$ & $\mathrm{~F}$ & 82 & \\
\hline $100-04 * 2$ & S & & & & $\mathbf{F}$ & $\mathbf{F z}$ & 81 & \\
\hline 10000 & 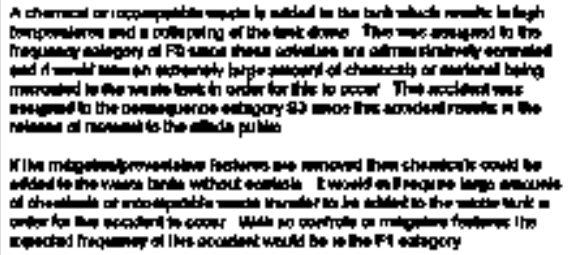 & & & & for & F1 & 83 & \\
\hline
\end{tabular}




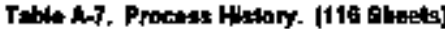

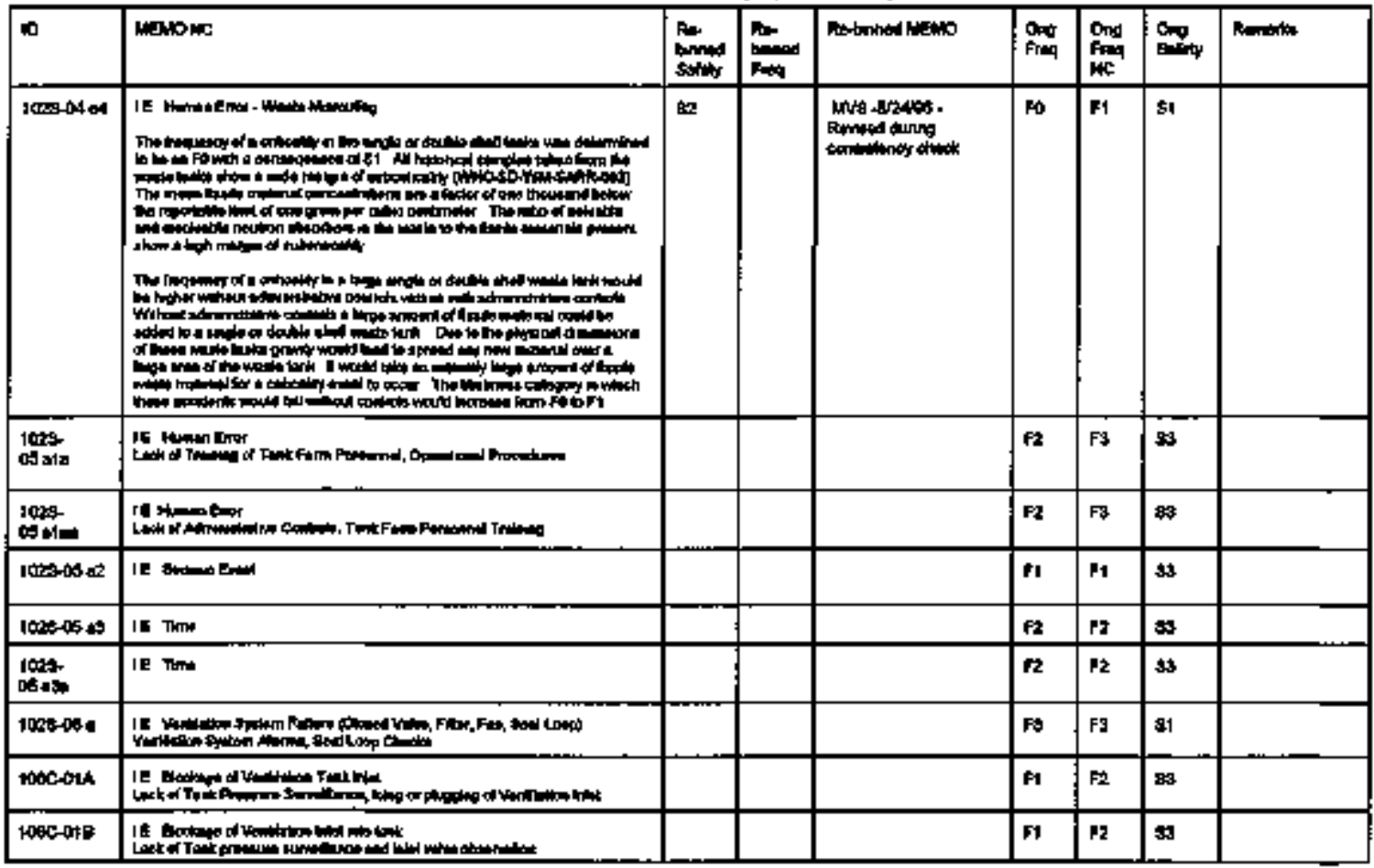


Tabio A-7. Procias Hirkary. (116 Sheta)

\begin{tabular}{|c|c|c|c|c|c|c|c|c|}
\hline $\boldsymbol{b}$ & NEAO AOC & Rimex & intion & Retinend izEO & orle & ing & org & kumata \\
\hline IOECक्षा & 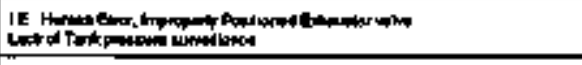 & & & & $\mathbf{F} 1$ & $\mathbf{m}$ & 89 & \\
\hline vote-110 & 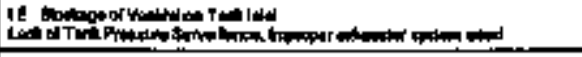 & & & & F1 & $p_{2}$ & so & \\
\hline tooc-a1e & 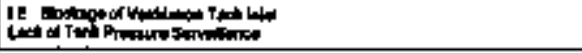 & & & & F1 & $\mathbf{F z}$ & 的 & \\
\hline $1060-0 \mathrm{fF}$ & 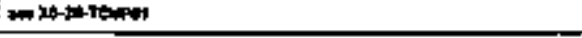 & & & & Fi & $\mathbf{m}$ & 52 & \\
\hline $1000-010$ & 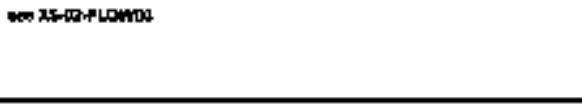 & 57 & & 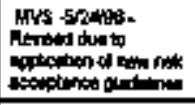 & $\mathbf{H 2}$ & $\mathbf{2}$ & $\$ 1$ & \\
\hline $100 \mathrm{coln}$ & 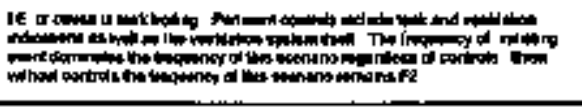 & 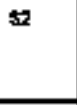 & & 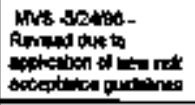 & $\mathrm{F} 2$ & $\mathbf{F}$ & b1 & \\
\hline 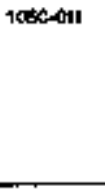 & 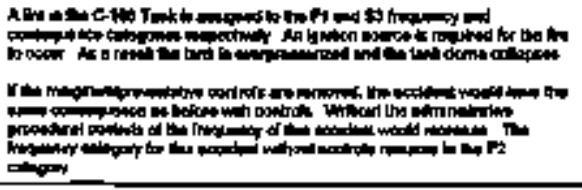 & & & & $\mathbf{F 1}$ & $\mathbf{F 2}$ & 83 & \\
\hline 100다에 & 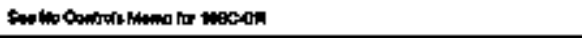 & & & & F* & $\mathbf{F}$ & $\mathbf{s t}$ & \\
\hline $10000 \mathrm{k}$ & 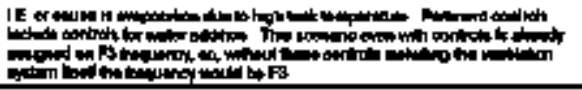 & & & & $\mathrm{F}$ & $\mathbf{F a}$ & 81 & \\
\hline
\end{tabular}




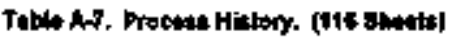

\begin{tabular}{|c|c|c|c|c|c|c|c|c|}
\hline ID & MUM & $\begin{array}{l}\text { Ro } \\
\text { binined } \\
\text { Bqhity }\end{array}$ & $\begin{array}{l}\text { Rit- } \\
\text { bhrod } \\
\text { Fing }\end{array}$ & 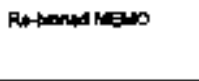 & $\operatorname{lng}$ & 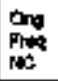 & senting & Fomorike \\
\hline $1000-01 M$ & 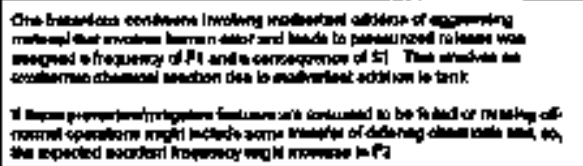 & $\mathbf{m}$ & & 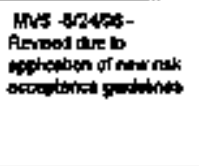 & $\mathbf{F 1}$ & $\boldsymbol{m}$ & B1 & \\
\hline $100 \mathrm{cos} / \mathrm{a}$ & 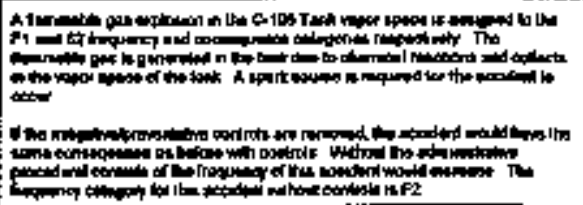 & ss & & 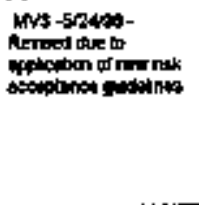 & FI & $\mathbf{F 2}$ & 82 & \\
\hline $10 \times 201 \mathrm{M}$ & 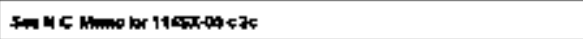 & & & & 巾 & FI & 58 & \\
\hline 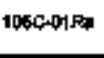 & 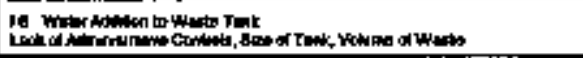 & & & & $F_{2}$ & F2 & 90 & \\
\hline $1000-915$ & 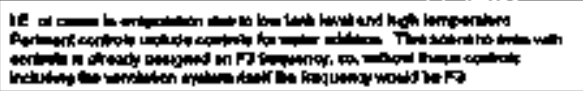 & & & & $\mathbf{P 3}$ & $\mathbf{P s}$ & $\$ 1$ & \\
\hline Now-0150 & If & & & & ps & $\mathbf{p s}$ & s1 & \\
\hline NOC-NT & 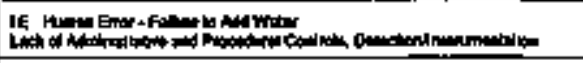 & & & & F3 & P3 & 31 & \\
\hline nocenu & & & & & FQ & & 80 & \\
\hline 1000-0aA & 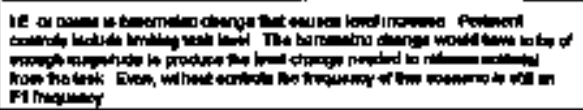 & & & & Fi & F1 & $\$ 1$ & \\
\hline
\end{tabular}


Table A-t. Prockss Hatory, (118 shoets)

\begin{tabular}{|c|c|c|c|c|c|c|c|c|}
\hline 10 & WENOH NE & Re. & Antion & Antmind tweno & ind & 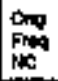 & ong & Amallat \\
\hline $100 \cos x$ & 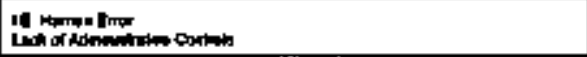 & & & & f1 & $\mathrm{FB}$ & 83 & \\
\hline 100c-aly & 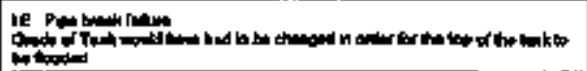 & & & & 11 & $\mathbf{m}$ & $\mathbf{s s}$ & \\
\hline $10 \times 0-1034$ & 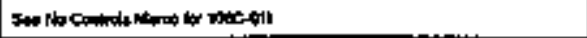 & & & & F1 & $\mathbf{F z}$ & 83 & \\
\hline $100 c-0 \mathrm{CO}$ & 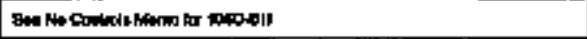 & & & & FI & $\boldsymbol{F z}$ & 93 & \\
\hline $100 x-\cos c$ & 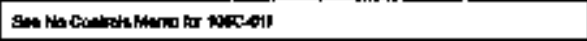 & & & & Fi & $\mathbf{F}$ & $\mathbf{3 5}$ & \\
\hline 10x-outh & 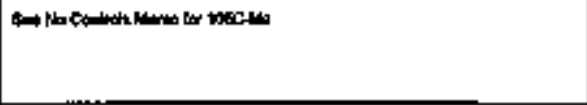 & 89 & & 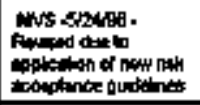 & $\mathbf{f 1}$ & $F$ & 62 & \\
\hline 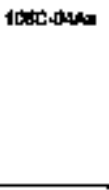 & 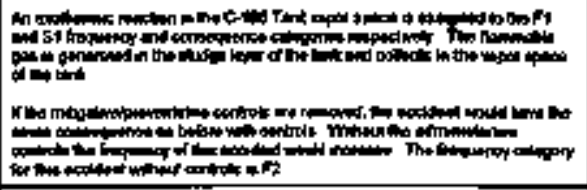 & 82 & & 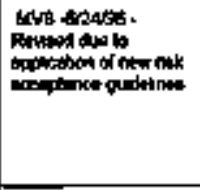 & F1 & $\mathbf{F}$ & $\$ 1$ & \\
\hline $1096-049$ & 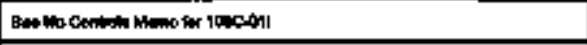 & & & & $\mathbf{m}$ & $\boldsymbol{H 2}$ & $\$$ & \\
\hline $1000-0 \mathrm{an}$ & IE Hutan Fnor & & & & 13 & $\mathbf{F}$ & \$1 & \\
\hline 10acosed & & & & & $\mathbf{F 3}$ & & $\$ 0$ & \\
\hline
\end{tabular}




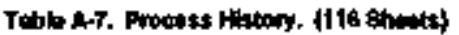

\begin{tabular}{|c|c|c|c|c|c|c|c|c|}
\hline (1) & 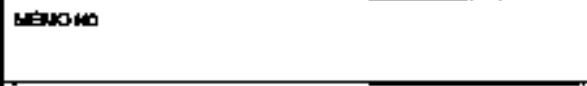 & Botidy & Penciad & 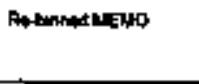 & Ong & ind & sistir & Remplestin \\
\hline $10,0<-000$ & 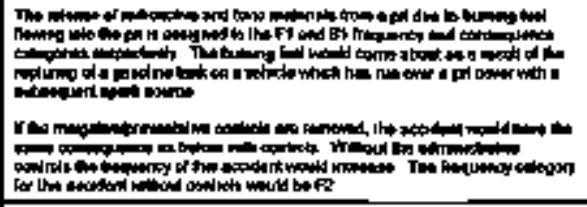 & 82 & & 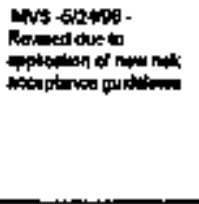 & $\mathbf{f 1}$ & $\mathbf{F}$ & $\$ 1$ & \\
\hline $100 x-60 \%$ & 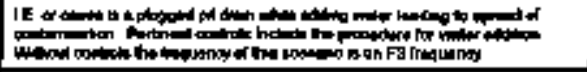 & & & & $\mathbf{F}$ & $\mathbf{F}$ & 6! & \\
\hline $1000-006$ & 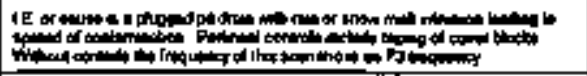 & & & & $\mathbf{r}$ & $\mathbf{r s}$ & sit & \\
\hline 1090004 & 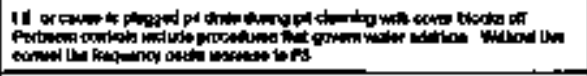 & & & & $\mathbf{F}$ & $F 3$ & B1 & \\
\hline $1000-100$ & & & & & $\mathbf{F 1}$ & & $\$ 0$ & \\
\hline $1000-00 x$ & 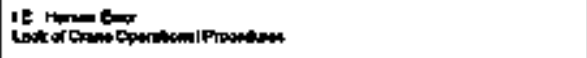 & & & & $\mathbf{F}$ & $F 3$ & B1 & \\
\hline 106000 & 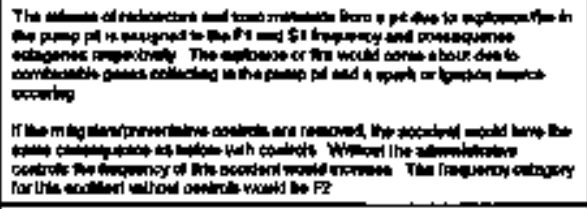 & a & & 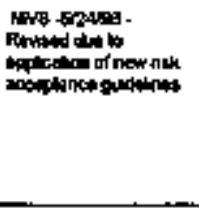 & $\mathbf{F 1}$ & $F \mathbf{2}$ & 31 & \\
\hline 1000 - & & & & & $\mathrm{F}_{2}$ & & so & \\
\hline 1000 & & & & & F3 & & 90 & \\
\hline
\end{tabular}




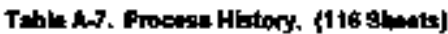

\begin{tabular}{|c|c|c|c|c|c|c|c|c|}
\hline סו & oted & him & 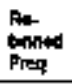 & Fumed bero & Prop & fing & 10 & Auminta \\
\hline 1090-10C & & & & & $\mathrm{FH}$ & & $\$$ & \\
\hline $1000-100$ & & & & & 13 & & 80 & \\
\hline $100 \times-102$ & & & & & $\mathbf{s}$ & & $\infty$ & \\
\hline $1004-100$ & 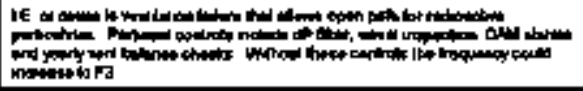 & & & & $n$ & Fs & 81 & \\
\hline $1600-104$ & & & & & $\mathbf{A 3}$ & & $\infty$ & \\
\hline 100C-10 & 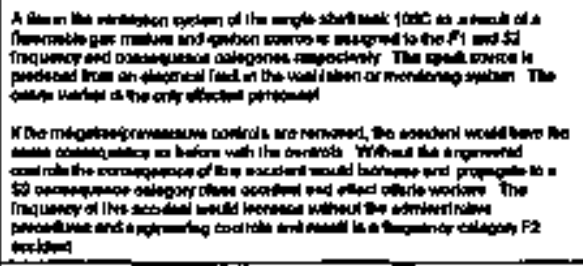 & & & & f1 & F2 & $\$ 2$ & \\
\hline Noc-10\% & 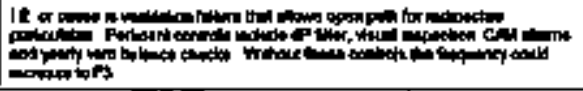 & & & & 2 & $F$ & 81 & \\
\hline $1000-104$ & 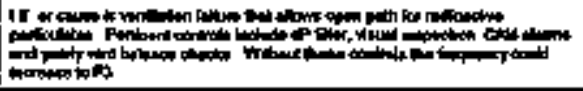 & & & & $\mathbf{F} \mathbf{2}$ & $F$ & $\$ 1$ & \\
\hline $100 x-11 A$ & 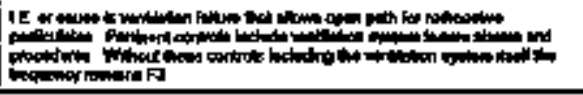 & & & & $\mathbf{r y}$ & $\mathbf{P H}$ & $\$ 1$ & \\
\hline $108 x-118$ & & & & & $\mathbf{P B}$ & & $\infty$ & \\
\hline
\end{tabular}




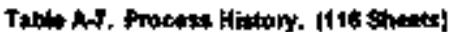

\begin{tabular}{|c|c|c|c|c|c|c|c|c|}
\hline 10 & Have & ar. & Pind & Ae-bined kive & Fon & $\begin{array}{l}\text { Ong } \\
\text { Fin } \\
\text { NC }\end{array}$ & com & Ramats \\
\hline $1000-116$ & & & & & $\mathbf{F F}$ & & 30 & \\
\hline $100-110$ & & & & & $\mathbf{B}$ & & $\$ 0$ & \\
\hline $1000-11 E$ & 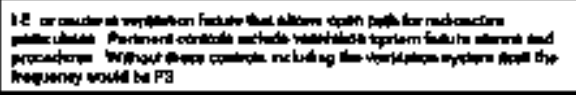 & & & & F1 & $\mathbf{A}$ & 38 & \\
\hline $10<-11 \%$ & & & & & $\mathbf{B}$ & & so & \\
\hline $105-110$ & 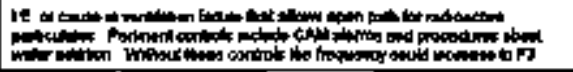 & & & & F & fy & 3 & \\
\hline $1000-16 H$ & 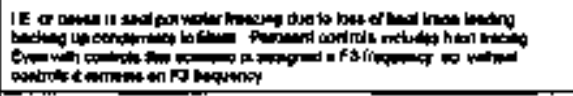 & & & & $\mathbf{r a}$ & P3 & st & \\
\hline $1000-1+1$ & 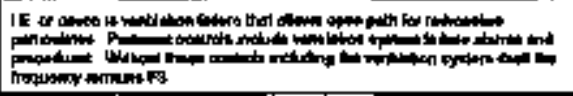 & & & & $\boldsymbol{m}$ & 15 & s1 & \\
\hline $1000-114$ & 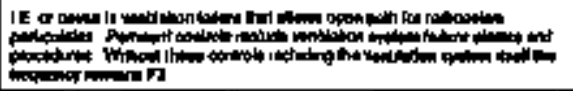 & & & & $\mathbf{m}$ & rs & st & \\
\hline $\cos -111 \%$ & 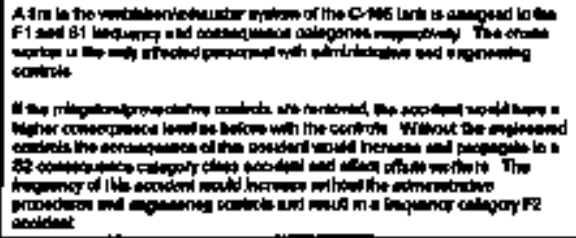 & 97 & & 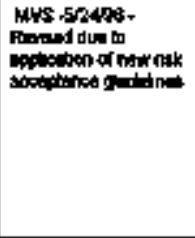 & $\mathbf{F 1}$ & $\mathbf{F} 2$ & 61 & \\
\hline
\end{tabular}




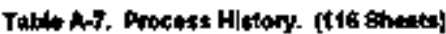

\begin{tabular}{|c|c|c|c|c|c|c|c|c|}
\hline $\mathbf{m}$ & Mano in & Rom & 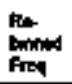 & 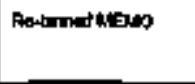 & im & Oridg & long & Rempios \\
\hline $1000-111$ & 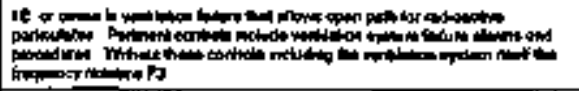 & & & & $\mathrm{Fa}$ & $\mathbf{F}$ & $\$ 1$ & \\
\hline N096-11서 & 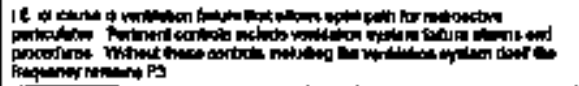 & & & & F3 & Fs & $\mathbf{3}$ & \\
\hline $000-11 \mathrm{~N}$ & 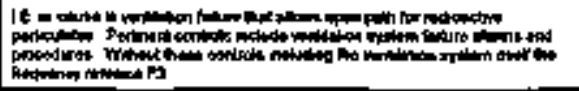 & & & & $F$ & $\mathbf{F a}$ & 3 & \\
\hline $1690-110$ & 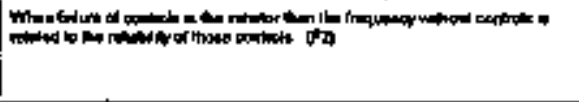 & az & & 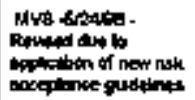 & fz & $F 2$ & 81 & \\
\hline $1 \cos -12 A$ & 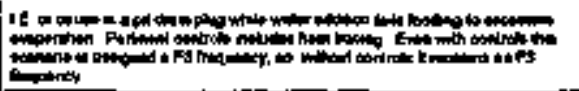 & & & & $\mathrm{F} 3$ & $F$ & 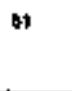 & \\
\hline $1006-128$ & 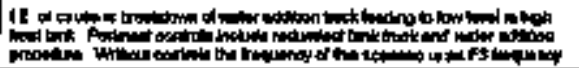 & & & & $\mathbf{F}$ & F\$ & G1 & \\
\hline 1000 & 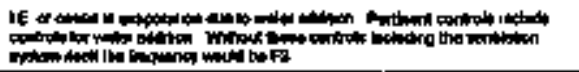 & & & & $\mathbf{F}$ & $F$ & $8 t$ & \\
\hline $1000-124$ & ק-1 & & & & $\mathbf{A}$ & $\mathbf{F}$ & 85 & \\
\hline $10 \mathrm{de}-125$ & 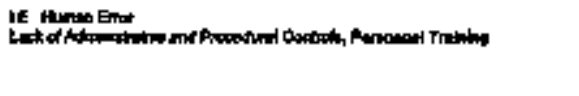 & s & $F_{2}$ & 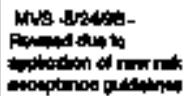 & F & $\mathrm{F}$ & 81 & \\
\hline
\end{tabular}


Table A-T. Procand Hotory, (116 aludta)

\begin{tabular}{|c|c|c|c|c|c|c|c|c|}
\hline 10 & 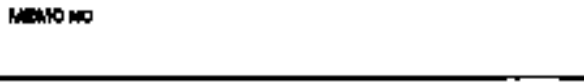 & toner & $\lim _{\lim }$ & Nobmind d NO & $\begin{array}{l}\text { Q40 } \\
\text { Freq }\end{array}$ & find & siper & Pismintion \\
\hline 1006 126 & IE & so & & 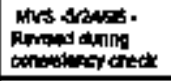 & 73 & $\boldsymbol{s}$ & $\$ 1$ & \\
\hline $1000-\pi 34$ & 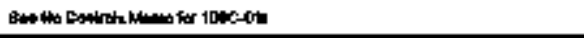 & & & & $\mathbf{P 1}$ & 62 & $s$ & \\
\hline $1000-139$ & & & & & $\boldsymbol{\mu}$ & & $\$ 0$ & \\
\hline 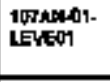 & 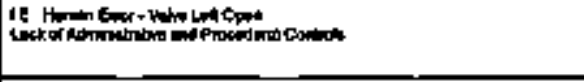 & $8 \pi$ & & 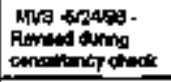 & $\mathbf{B}$ & $\mathbf{F}$ & $\mathbf{s 1}$ & \\
\hline $\begin{array}{l}\text { 1onkwoi- } \\
\text { LEMER }\end{array}$ & 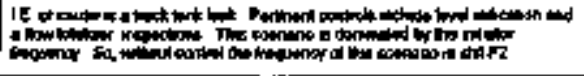 & 6z & & 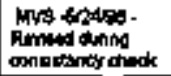 & $\mathbf{F}$ & f? & $\$ 1$ & \\
\hline $\begin{array}{l}\text { TATAHWOI. } \\
\text { LEVECOS }\end{array}$ & IE & & $\mathbf{N}$ & 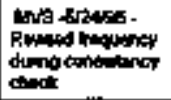 & $\mathbf{F 2}$ & $\mathrm{F} 2$ & s1 & \\
\hline 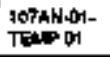 & & & & & F 2 & & B1 & \\
\hline $\begin{array}{l}\text { 107RN-Ot. } \\
\text { TEupice }\end{array}$ & 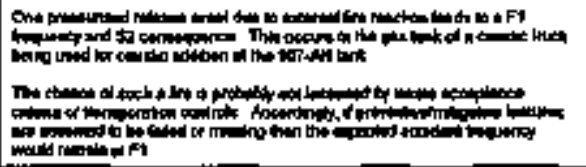 & & & & e1 & $\mathbf{F}_{1}$ & 52 & \\
\hline 1otrontap & 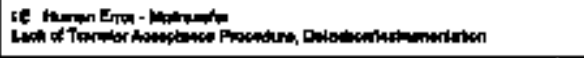 & & & & $\mathbf{F}$ & $F$ & $3 z$ & \\
\hline W7aHos & 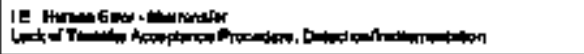 & & & & $\mathbf{F}$ & $\mathbf{P}$ & $\mathbf{s i}$ & \\
\hline
\end{tabular}


Tablo A7. Procies Ifitory. (115 ahetts)

\begin{tabular}{|c|c|c|c|c|c|c|c|c|}
\hline 10 & 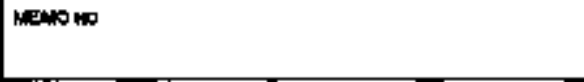 & intod & $\lim _{\text {inem }}$ & 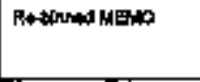 & Ing & $\underset{i m g}{\log }$ & $\log _{\pi \rightarrow 4}$ & Pumbos \\
\hline $\begin{array}{l}\text { banawos } \\
\text { rúniss }\end{array}$ & 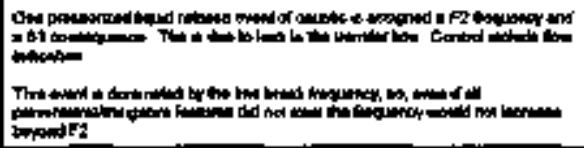 & & & & $F 2$ & $\mathbf{F} \mathbf{z}$ & 时 & \\
\hline $\begin{array}{l}\text { Totanda- } \\
\text { PRES }\end{array}$ & 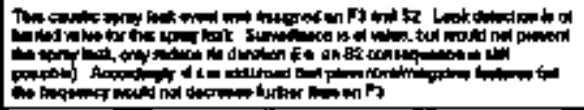 & & & & $\mathbf{F}$ & $\mathrm{F} /$ & 的 & \\
\hline $\begin{array}{l}\text { 107anos } \\
\text { PRestos }\end{array}$ & 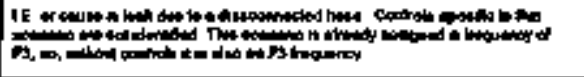 & st & & 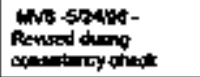 & $F$ & $\boldsymbol{F}$ & BI & \\
\hline Tentapion & " & & & & $\mathbf{F Z}$ & $\mathrm{Fz}$ & B1 & \\
\hline $\begin{array}{l}\text { 10TAN-L3- } \\
\text { PLON }\end{array}$ & 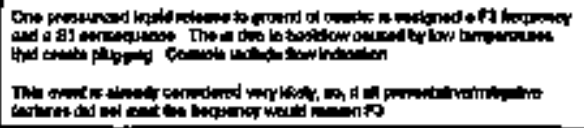 & & & & $\mathrm{F3}$ & 网 & \$1 & \\
\hline $110 x-40$ b & 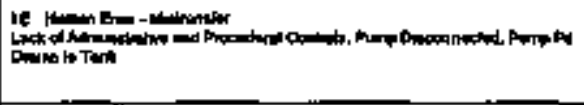 & 5 & & 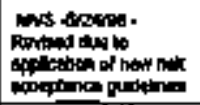 & $\boldsymbol{H}$ & 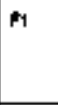 & s1 & \\
\hline $\begin{array}{l}\text { 1145x- } \\
\text { Deata }\end{array}$ & 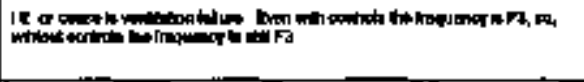 & S1 & & 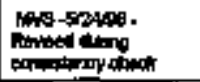 & P3 & F & $\$ 0$ & \\
\hline 1148x- & 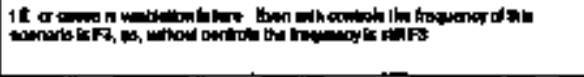 & s1 & & Mus-sonas. & $\mathbf{F}$ & $\mathbf{f a}$ & so & \\
\hline
\end{tabular}




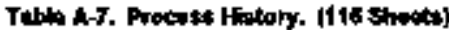

\begin{tabular}{|c|c|c|c|c|c|c|c|c|}
\hline 10 & KEAK n & tinked & Fim & 月0-6med Mexp & ond & ing & song & Rtom \\
\hline 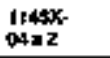 & 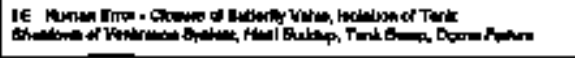 & & & & Po & F1 & ss & \\
\hline $1148 x$ & 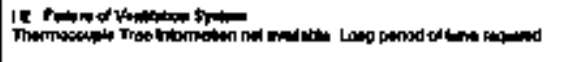 & & & & fo & $\mathbf{F 1}$ & $\$$ & \\
\hline $\begin{array}{l}114 \% \% \\
0404\end{array}$ & 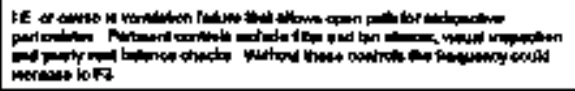 & & & & F1 & $\boldsymbol{F}$ & g1 & \\
\hline 04b1.1 & 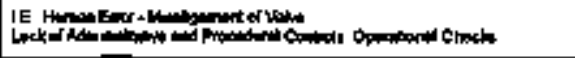 & & & & $\mathbf{m}$ & $\mathbf{F s}$ & st & \\
\hline $\operatorname{lit}_{0+1}+2$ & 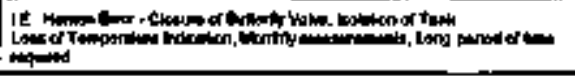 & & & & $\infty$ & $\mathbf{F 1}$ & \$ & \\
\hline 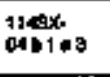 & 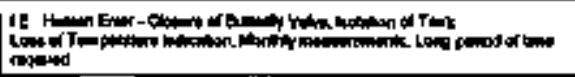 & & & & $\mathbf{F 1}$ & $F_{2}$ & 昰 & \\
\hline 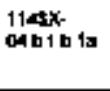 & 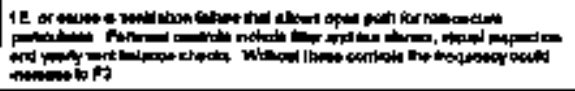 & & & & FI & Ps & sı & \\
\hline $\begin{array}{l}11454- \\
0 \times b 1016\end{array}$ & 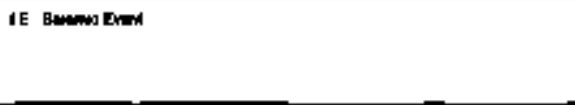 & 的 & & 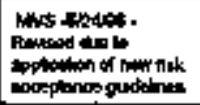 & $\mathbf{F}$ & $F 1$ & B1 & \\
\hline 0401629 & 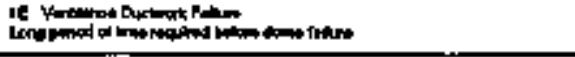 & & & & Fo & $\mathbf{F} 1$ & 83 & \\
\hline 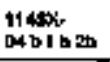 & 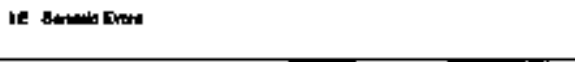 & & & & F1 & F1 & 89 & \\
\hline od $1148 x$ & 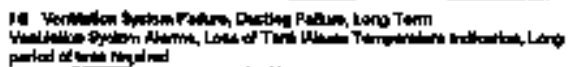 & & & & $\mathbf{r 1}$ & 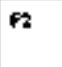 & so & \\
\hline
\end{tabular}




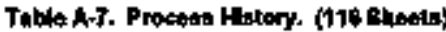

\begin{tabular}{|c|c|c|c|c|c|c|c|c|}
\hline I & Nanow & Ror & $\begin{array}{l}\text { Re. } \\
\text { tanod } \\
\text { Pran }\end{array}$ & 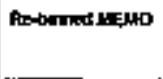 & Oriog & $\operatorname{com}_{\operatorname{mog}}$ & sily & Rntion \\
\hline 6401630 & 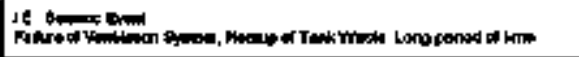 & & & & $\mathbf{F} 1$ & P1 & 90 & \\
\hline 114tix. & IE & & & & $\mathbf{F 1}$ & $\mathbf{m}$ & s: & \\
\hline $\lim _{0 \leqslant 1}<1<2$ & 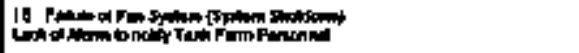 & & & & Fo & F & $\$ 3$ & \\
\hline I1458 & 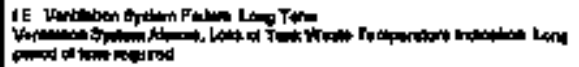 & & & & $\mathbf{F 1}$ & $\boldsymbol{n}$ & 50 & \\
\hline intex- 101 & 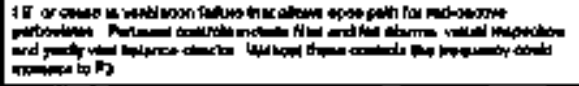 & & & & P1 & $\mathbf{F y}$ & $\$ 1$ & \\
\hline $\begin{array}{l}1149 x \\
04+1 d 2\end{array}$ & 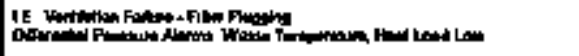 & & & & 10 & F1 & $\$ 3$ & \\
\hline 040143 & 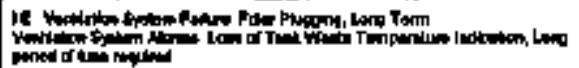 & & & & $\mathbf{F 1}$ & F 2 & $\$ 0$ & \\
\hline $\begin{array}{l}114565 \\
040 \%\end{array}$ & 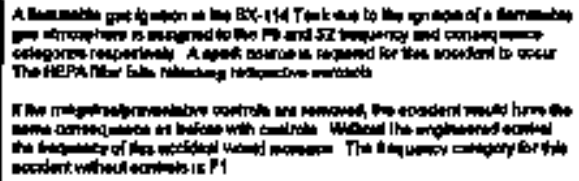 & $\mathbf{8}$ & & 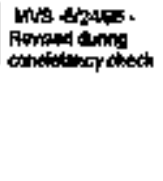 & Fit & $\mathbf{F 1}$ & 井 & \\
\hline $1140 x$ & 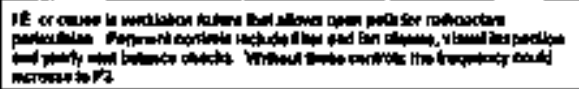 & & & & $\mathbf{F i}$ & $\mathbf{F 3}$ & s1 & \\
\hline
\end{tabular}




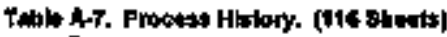

\begin{tabular}{|c|c|c|c|c|c|c|c|c|}
\hline 10 & MELOW & 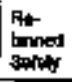 & $\begin{array}{l}\text { for } \\
\text { Finiod } \\
\text { Find }\end{array}$ & Rowinged werto & Prod & $\begin{array}{l}\mathrm{cog} \\
\mathrm{Fin}\end{array}$ & Ding & Romorla \\
\hline $\begin{array}{l}\$ 145 x \\
04=1\end{array}$ & 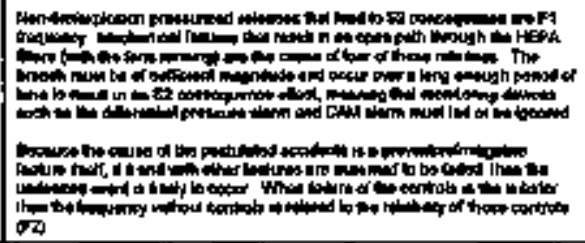 & & & & F1 & $F 2$ & 82 & \\
\hline $\begin{array}{l}1140 x- \\
0<020 .\end{array}$ & $\cos 1140 x+401$ & & & & Fi & f? & s2 & \\
\hline $\begin{array}{l}11+5 x- \\
0 \leqslant 2 b\end{array}$ & 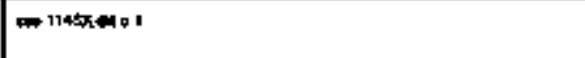 & & & & *1 & $\mathbf{F 3}$ & $\mathbf{8 2}$ & \\
\hline $\begin{array}{l}1149 x \\
04020\end{array}$ & H 1140:04: 1 & & & & F1 & fa & $\Leftrightarrow$ & \\
\hline $\begin{array}{l}\text { 1145x- } \\
\text { DHe 2d }\end{array}$ & 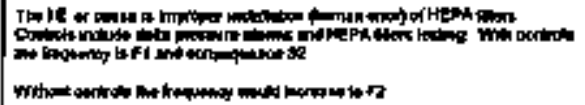 & & & & $\mathbf{F I}$ & $F_{2}$ & $\$ 7$ & \\
\hline $\begin{array}{l}1149 x- \\
00161\end{array}$ & 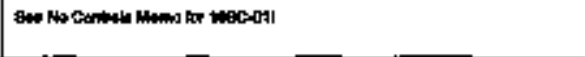 & & & & $\mathbf{F 1}$ & $n$ & $\theta$ & \\
\hline $\begin{array}{l}1146 x \\
0<b 2\end{array}$ & A & & & & fo & $\mathbf{F o}$ & $\$ 2$ & \\
\hline $\operatorname{lissx}_{0 \leqslant 3}$ & 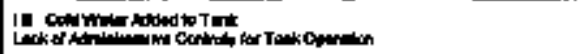 & & & & Ho & $\sqrt{2}$ & $\mathbf{s}$ & \\
\hline
\end{tabular}




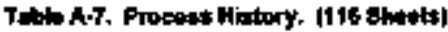

\begin{tabular}{|c|c|c|c|c|c|c|c|c|}
\hline 10 & 18 & Rot & 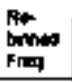 & Ao-owned MENo & ord & 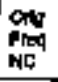 & ond & Aemotion \\
\hline $\begin{array}{l}1145 x \\
0 d=2 t\end{array}$ & A & & & & $F O$ & 81 & 35 & \\
\hline 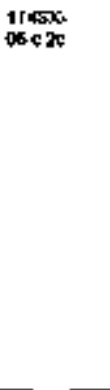 & IE A & $\$$ & & Hort Aracs. & Fo & $k_{1}$ & s1 & \\
\hline $1145 x-60 d$ & 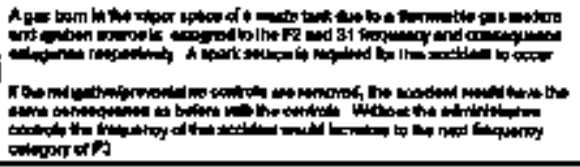 & $\$ 7$ & & 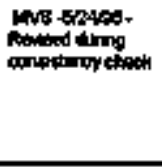 & F2 & F3 & s1 & \\
\hline
\end{tabular}




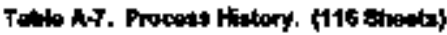

\begin{tabular}{|c|c|c|c|c|c|c|c|c|}
\hline ID & MEMT Mac & the & $\begin{array}{l}\text { For } \\
\text { trinod } \\
\text { Freag }\end{array}$ & Roriman MEMO & ond & $\begin{array}{l}\text { Ond } \\
\text { Pnd } \\
\text { NC }\end{array}$ & Ong & Ranorits \\
\hline $1140 x-$ & 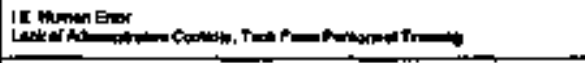 & & & & F2 & $\mathbf{n}$ & ss & \\
\hline $\lim _{t \rightarrow 10} 10$ & 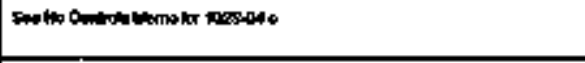 & & & & Fo & 51 & $\$ 3$ & \\
\hline $\begin{array}{l}1143 x- \\
0.52\end{array}$ & 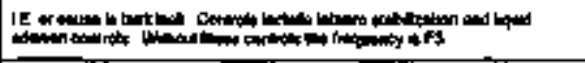 & & & & $\mathbf{1 2}$ & $\mathbf{F}$ & so & \\
\hline 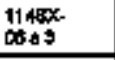 & It 7 m & & & & $\mathbf{F Z}$ & $\mathbf{F}$ & $\$ 3$ & \\
\hline $1145 \times 000$ b. & & & & & Fz & & 91 & \\
\hline TOAAREOAA & 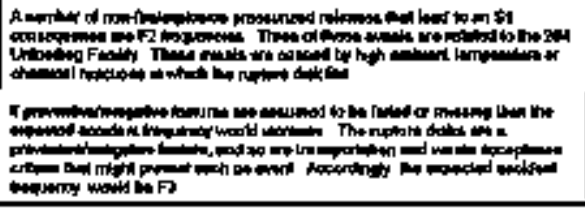 & & & & $F F$ & $\mathbf{F} 9$ & si & \\
\hline Xo4ARSOSE & stashosh & & & & 82 & a & $\$ 1$ & \\
\hline 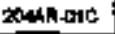 & H molnowh & & & & $\mathbf{F}$ & $\mathbf{F}$ & 的 & \\
\hline 2004R-010 & 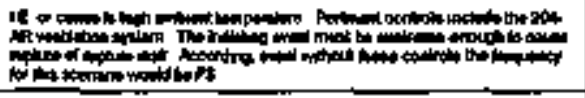 & & & & $\mathbf{n}$ & $\mathbf{m}$ & ह1 & \\
\hline 204AR-D纴 & 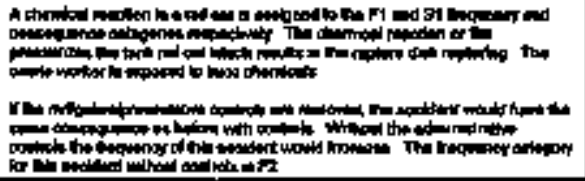 & & & & $\mathbf{F I}$ & $\mathbf{F}$ & BI & \\
\hline
\end{tabular}




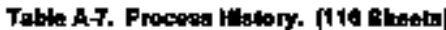

\begin{tabular}{|c|c|c|c|c|c|c|c|c|}
\hline 10 & wenth & $\begin{array}{l}\text { Roon } \\
\text { tominos } \\
\text { sting }\end{array}$ & $\lim _{\lim }$ & Nothind kexto & ing & $\begin{array}{l}\text { Ond } \\
\text { Frent } \\
\text { inc }\end{array}$ & ong & intorster \\
\hline $\operatorname{XoshR} R$ & 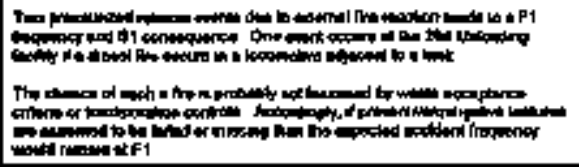 & & & & $F 1$ & Fi & \$1 & \\
\hline andrate & 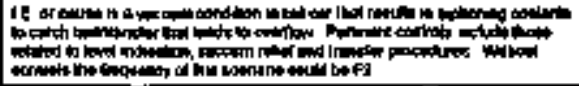 & & & & $\mathbf{F 1}$ & $\boldsymbol{P}$ & s1 & \\
\hline soment & 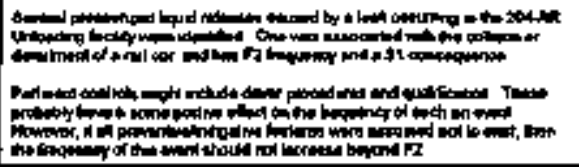 & s2 & & 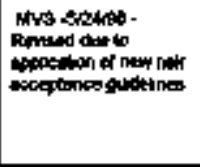 & 11 & $\mathbf{1 2}$ & $\$ 1$ & \\
\hline MARAo11 & 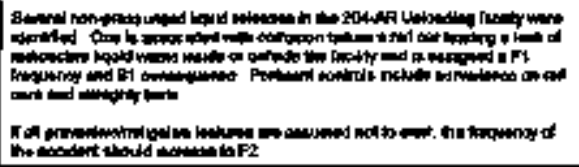 & $\Leftrightarrow$ & & 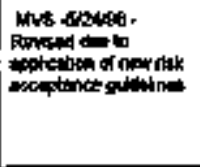 & $\mathbf{F}$ & $\boldsymbol{n}$ & $\$ 1$ & \\
\hline adrina1] & Ie & & & & $\mathbf{m}$ & $\mathbf{F a}$ & 81 & \\
\hline 204AR-01K & 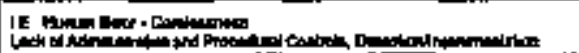 & & & & F1 & $\mathbf{r}$ & 51 & \\
\hline
\end{tabular}




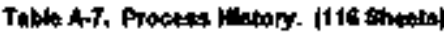

\begin{tabular}{|c|c|c|c|c|c|c|c|c|}
\hline 10 & Now & 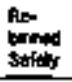 & 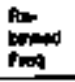 & Roximuted the & Prod & 点 & Dition & Fimne: \\
\hline sopringat & 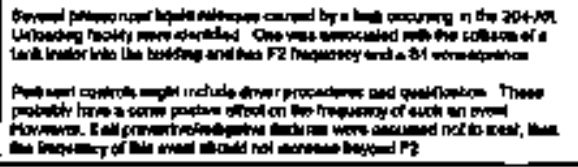 & 됴 & & 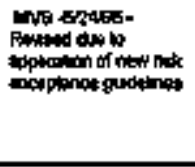 & $\mathbf{F} 2$ & $\mathbf{F}$ & B1 & \\
\hline $204 A+200$ & 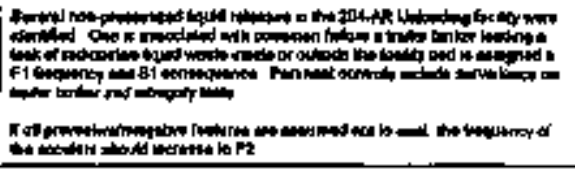 & s? & & 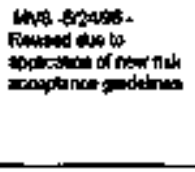 & f1 & $\mathrm{Fz}$ & B1 & \\
\hline TPAARAOCL & 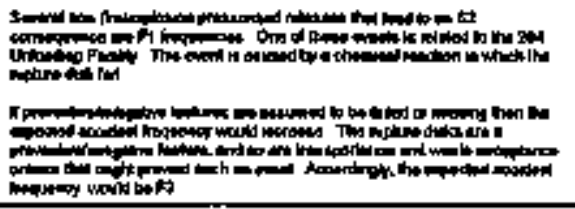 & & & & f 1 & $F_{2}$ & 81 & \\
\hline 204AR-exo & 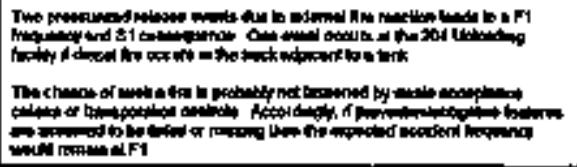 & & $\mathbf{F 2}$ & 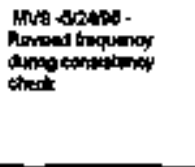 & $\boldsymbol{F}$ & F1 & \$1 & \\
\hline Xo4kAtraE & 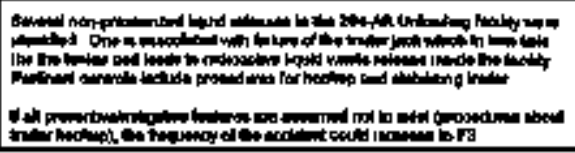 & & & & $\mathrm{Fz}$ & $\mathbf{F}$ & 91 & \\
\hline
\end{tabular}




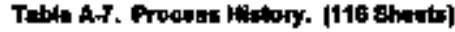

\begin{tabular}{|c|c|c|c|c|c|c|c|c|}
\hline 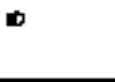 & MENOW & iming & Fim & Robumand waw & ing & $\operatorname{ling}_{\log }$ & ong & Penonint \\
\hline Dareperp & 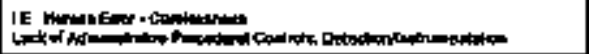 & & & & $\mathbf{F 1}$ & $\mathbf{F}$ & $s 1$ & \\
\hline 2044-03A & 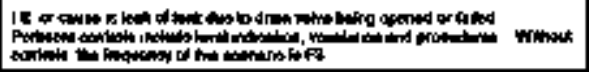 & & & & $\mathbf{f 2}$ & $\mathbf{F u}$ & $\$ 1$ & \\
\hline sancosa & 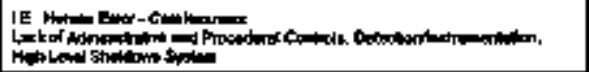 & & & & $\mathbf{P 2}$ & $\mathbf{N s}$ & $\$ 1$ & \\
\hline 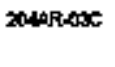 & 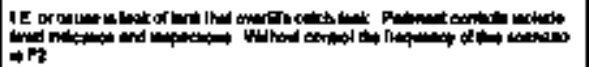 & & & & $\mathbf{H}$ & $\mathbf{F Z}$ & S1 & \\
\hline SOARROAA & 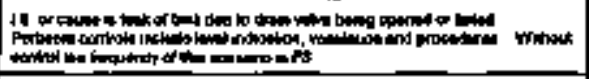 & & & & f2 & $\mathrm{Fl}_{3}$ & 51 & \\
\hline Xumpinas & 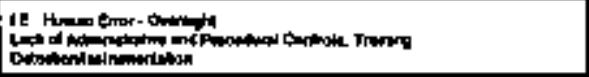 & & & & $\mathbf{F z}$ & $\mathrm{F}$ & s1 & \\
\hline Statitor: & IE & & & & $\mathbf{m}$ & $\mathbf{r s}$ & $\$ 1$ & \\
\hline 2044 - 000 & 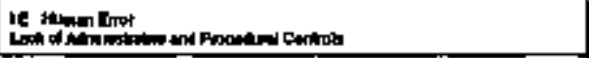 & & & & $\mathbf{F}$ & $F^{3}$ & 81 & \\
\hline 20MARQRE & 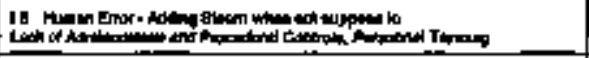 & & & & n & $\mathbf{F 3}$ & 51 & \\
\hline ZOAati-04F & 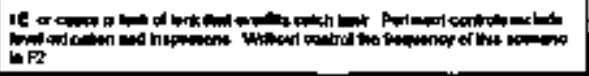 & & & & $\mathbf{F 1}$ & $\mathbf{F z}$ & 81 & \\
\hline 204kedon & Ie & & & & $\mathbf{r 2}$ & $F 2$ & B1 & \\
\hline
\end{tabular}




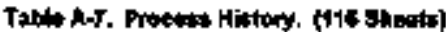

\begin{tabular}{|c|c|c|c|c|c|c|c|c|}
\hline ID & Mפ⿴囗十 & Pit & $\begin{array}{l}\text { Po- } \\
\text { Unned } \\
\text { Freeq }\end{array}$ & R*AMHA MENO & $P_{p \rightarrow p}$ & ing & orid & 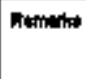 \\
\hline antinges & 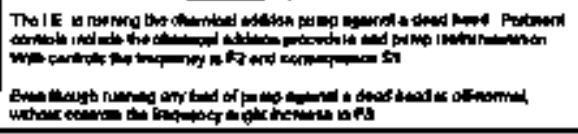 & & & & $F 2$ & $\mathbf{F a}$ & $\$$ & \\
\hline DNAT 04C & 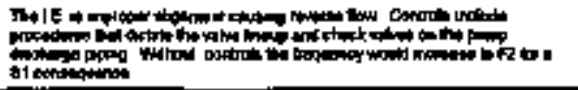 & & & & $\boldsymbol{M 1}$ & $\mathbf{m}$ & bi & \\
\hline DOWR-04A & 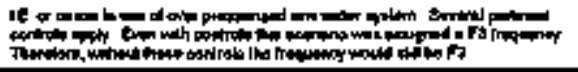 & & & & Ps & rs & s1 & \\
\hline 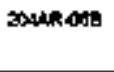 & 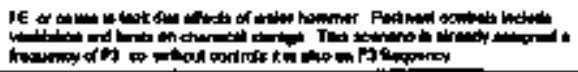 & & & & F3 & $\mathbf{r 3}$ & 5 & \\
\hline $204 \mathrm{AR} 00 \mathrm{C}$ & 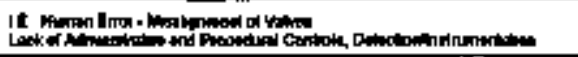 & & & & $\mathbf{F}_{1}$ & F & 81 & \\
\hline butross & 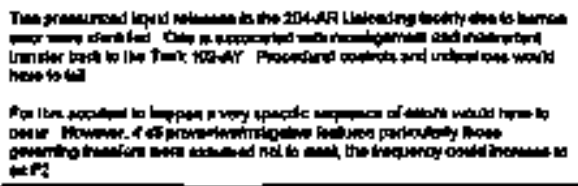 & & & & \$1 & $\mathrm{Fr}$ & 52 & \\
\hline MUAR-6is & 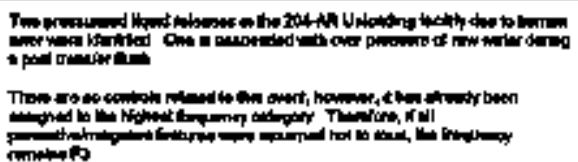 & & & & $F$ & $\mathbf{F s}$ & $\$ 1$ & \\
\hline
\end{tabular}


Table A-T. Frotede Hittery. (116 Shants)

\begin{tabular}{|c|c|c|c|c|c|c|c|c|}
\hline 10 & NFondo & 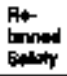 & $\begin{array}{l}\text { Bat } \\
\text { Fond } \\
\text { Fond }\end{array}$ & Robimod MENO & $\begin{array}{l}\text { Pro } \\
\text { fioq }\end{array}$ & $\begin{array}{l}\text { Ong } \\
\text { frid } \\
\text { nct }\end{array}$ & 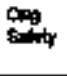 & Rantilit \\
\hline 20AR-0RC & 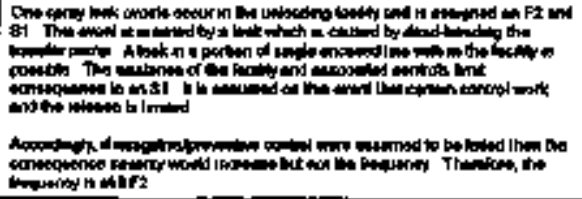 & & & & $\mathbf{F}$ & $\mathbf{2 2}$ & $\$ 1$ & \\
\hline TOMNRAEA & ה & & & & $\mathrm{Fz}$ & $F 2$ & 82 & \\
\hline TD4AF 069 & 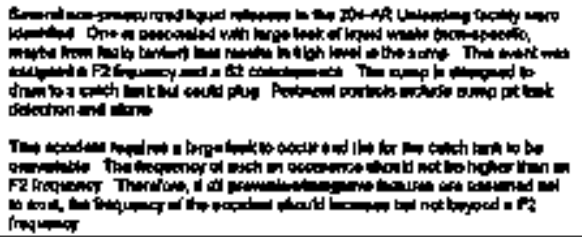 & & & & F2 & $F 2$ & 82 & \\
\hline
\end{tabular}




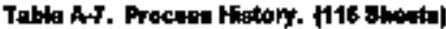

\begin{tabular}{|c|c|c|c|c|c|c|c|c|}
\hline 10 & 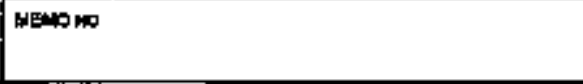 & $\begin{array}{l}\text { Bom } \\
\text { bined } \\
\text { Bativity }\end{array}$ & Pinted & Roblithed theno & ond & $\lim _{\substack{0 \\
\text { mat }}}$ & 的 & Aminta \\
\hline Zanaracs & 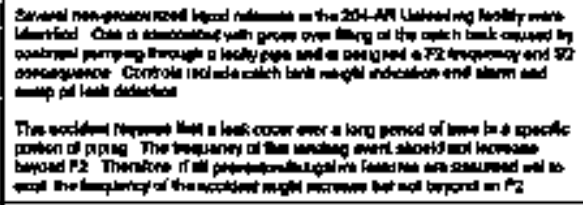 & & & & 2 & $\boldsymbol{r z}$ & 62 & \\
\hline 2040 toed & 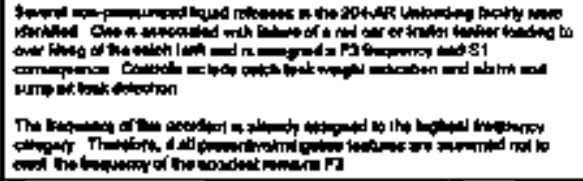 & 92 & $\mathbf{F Z}$ & 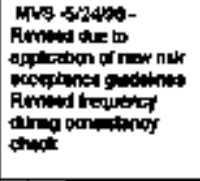 & $\boldsymbol{m}$ & $\mathbf{F s}$ & B) & \\
\hline 204NROAS & 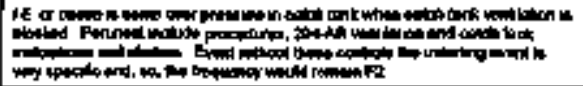 & & & & $\mathbf{F 2}$ & $n 2$ & s & \\
\hline Sounterer & 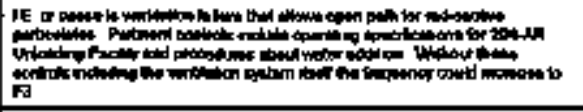 & & & & $\mathbf{r z}$ & $\mathbf{m}$ & as & \\
\hline $\operatorname{son} / 40003$ & 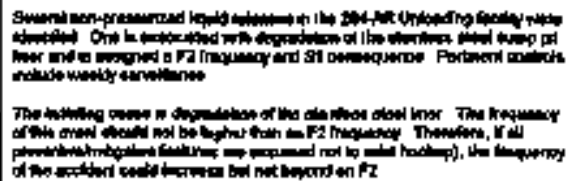 & 50 & & 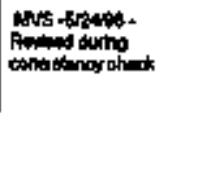 & $F_{2}$ & $\mathbf{p}_{2}$ & 81 & \\
\hline
\end{tabular}


Table Ant, Process Hetory. (116 shabt)

\begin{tabular}{|c|c|c|c|c|c|c|c|c|}
\hline 10 & MENOW W & ionim & Pond & 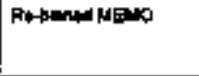 & ond & 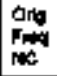 & Ong & Bumanten \\
\hline $204 n-\cos 1$ & 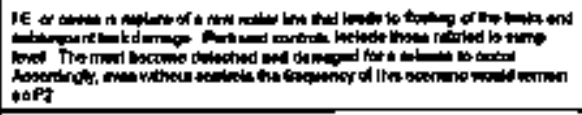 & & & & $\mathbf{F} 2$ & $\mathbf{F} \mathbf{2}$ & 81 & \\
\hline ACAR-A1h & 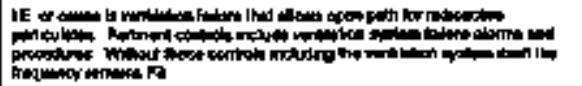 & & & & $\boldsymbol{F}$ & $\mathbf{F 3}$ & st & \\
\hline 204hk-11日 & 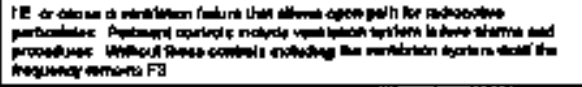 & & & & rs & F & 91 & \\
\hline zodinn-11C & 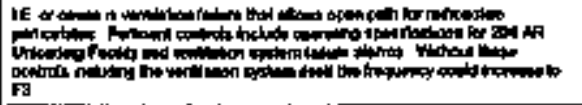 & & & & 72 & F & st & \\
\hline touks-110 & IE & & & & 12 & is & \$1 & \\
\hline Xoner-41 & 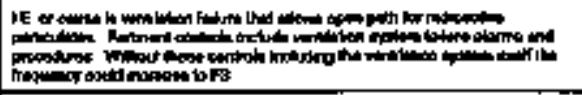 & & & & $\mathbf{r}$ & Fs & st & \\
\hline 304WR-1 i & 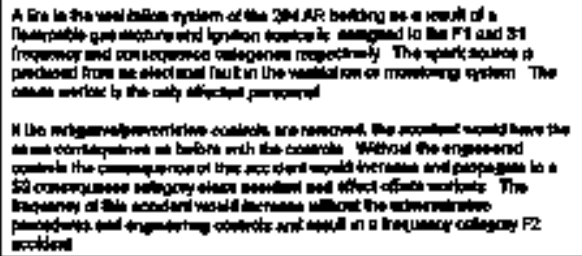 & 97 & & 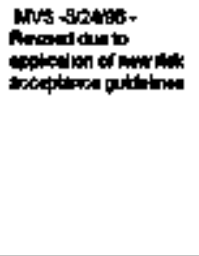 & $\mathbf{r 1}$ & $\mathbf{F 2}$ & s1 & \\
\hline
\end{tabular}




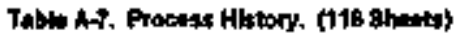

\begin{tabular}{|c|c|c|c|c|c|c|c|c|}
\hline $\mathbf{D}$ & WENONC & ofity & Primed & Retinned lusuto & ong & $\begin{array}{l}\text { orlo } \\
\text { Fidi }\end{array}$ & (n) & 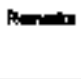 \\
\hline 213 Wh-01 & 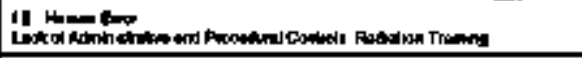 & & & & $\mathbf{f a}$ & fo & EI & \\
\hline 293 thate & 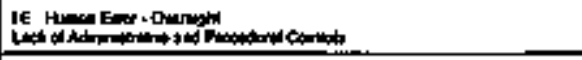 & & & & $\mathbf{2}$ & Fs & st & \\
\hline 213 th-09 & A & & & & $\mathbf{n}$ & $\mathbf{r 2}$ & $\mathbf{s 1}$ & \\
\hline $213 w-06$ & A & & & & $\mathbf{F 2}$ & $\mathbf{F} 2$ & $\$ 1$ & \\
\hline 213W-OS & 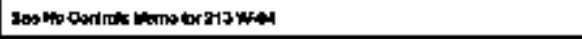 & & & & $p z$ & $\mathbf{f 2}$ & 91 & \\
\hline 2าง was & 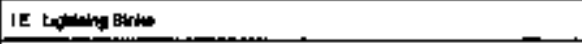 & & & & $\mathbf{R}$ & $\mathbf{n}$ & s1 & \\
\hline zuswor & IE & & & & F1 & $\mathbf{F} 1$ & $\$ 1$ & \\
\hline 213 wade & 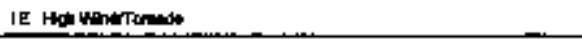 & & & & F1 & D2 & $\$ 1$ & \\
\hline
\end{tabular}


Table A-7, Procens History. (116 Streaty)

\begin{tabular}{|c|c|c|c|c|c|c|c|c|}
\hline פו & Mertowe & Rem & $\lim _{\lim }$ & Rostromideap & Ong & $\lim _{i=0}$ & ondy & Earrites \\
\hline $213 w-50$ & 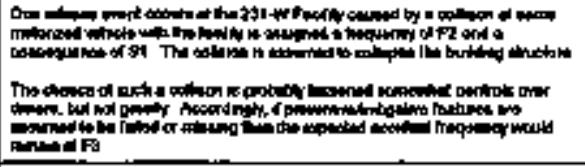 & & & & $\mathbf{F} 2$ & $\mathrm{FJ}$ & 51 & \\
\hline $\begin{array}{l}74405 . \\
\text { on } 103\end{array}$ & 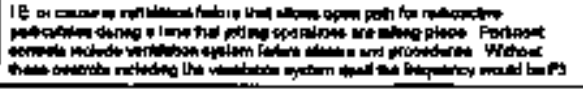 & & & & $\mathbf{F} 2$ & $\mathrm{Fg}$ & Bi & \\
\hline $\begin{array}{l}\text { 2404k } \\
\text { O1 bo1 }\end{array}$ & 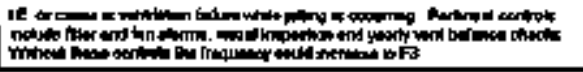 & & & & $\mathbf{F 1}$ & $\mathbf{F 3}$ & 91 & \\
\hline $\begin{array}{l}240 \text { th. } \\
\text { on t t to }\end{array}$ & 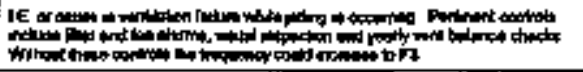 & & & & f1 & $\mathrm{Fa}$ & E1 & \\
\hline $\begin{array}{l}\text { 2404R- } \\
01 \text { D3 }\end{array}$ & 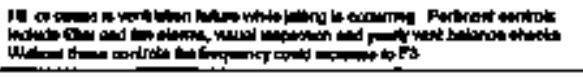 & & & & Fi & P3 & s & \\
\hline $\begin{array}{l}\text { 34akk. } \\
\text { al pod }\end{array}$ & 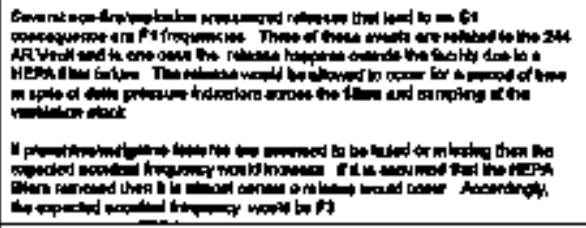 & & & & F1 & P3 & s & \\
\hline $\begin{array}{l}\text { 24yar- } \\
\text { Ot o }\end{array}$ & 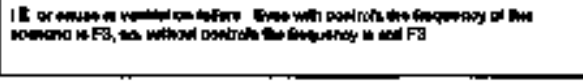 & 51 & & 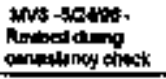 & r & $\boldsymbol{\theta}$ & so & \\
\hline
\end{tabular}


Tablo 47, Procans Hintory. \{118 8hatas\}

\begin{tabular}{|c|c|c|c|c|c|c|c|c|}
\hline $\boldsymbol{D}$ & MENONAC & salim & Ro. & 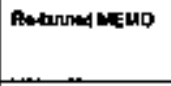 & Fon & $\begin{array}{l}\text { Ond } \\
\text { Friq } \\
\text { NCP }\end{array}$ & ong & Pinmitita \\
\hline 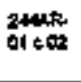 & 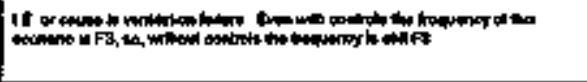 & 81 & & 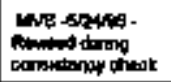 & $\mathrm{Fr}$ & $\mathbf{F}$ & on & \\
\hline 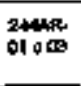 & 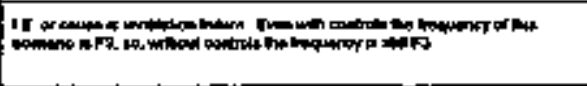 & 81 & & 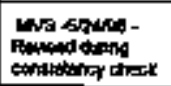 & $\mathrm{Fa}$ & Fs & 80 & \\
\hline $\begin{array}{l}\text { 2014k } \\
\text { Ot of }\end{array}$ & 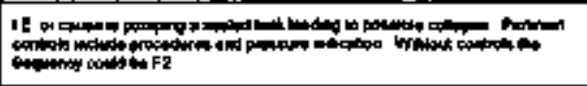 & 51 & & int apdos. & $\mathbf{m}$ & $\mathbf{m}$ & so & \\
\hline 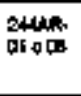 & 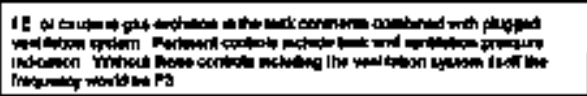 & 51 & & 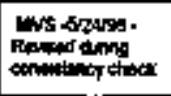 & $A 2$ & $\mathbf{P s}$ & so & \\
\hline of a de & 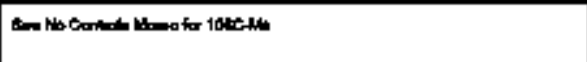 & & & & Fi & $n$ & $\Leftrightarrow$ & \\
\hline $24 \operatorname{ser}$ & 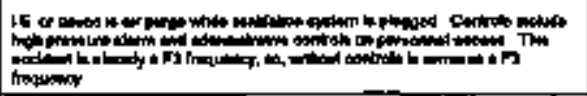 & 81 & & Mrs - fidus:- & F 3 & $\mathbf{F}$ & هs & \\
\hline 24ers- & 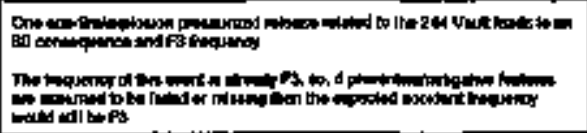 & $s 1$ & & MYS Endos: & $\mathbf{F 3}$ & $\mathbf{F}$ & $\infty$ & \\
\hline $\begin{array}{ll}344 R \\
010\end{array}$ & 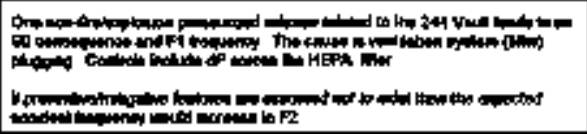 & à & & 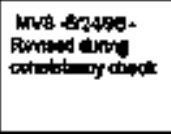 & F1 & $\mathbf{F} 2$ & 80 & \\
\hline 240R: & 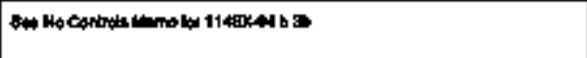 & & & & ro & $\mathbf{F I}$ & 32 & \\
\hline
\end{tabular}




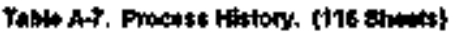

\begin{tabular}{|c|c|c|c|c|c|c|c|c|}
\hline 10 & MENONG & inimed & fim & 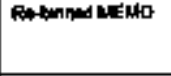 & fing & $\underset{100}{\operatorname{lom}}$ & Drator & Rembata \\
\hline 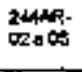 & 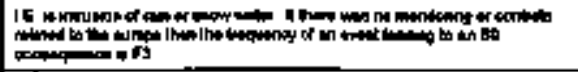 & & & & FI & FE & 80 & \\
\hline 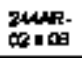 & 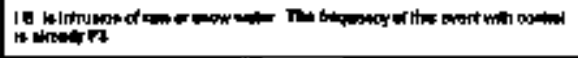 & & & & fa & F 3 & end & \\
\hline $\begin{array}{l}2448 . \\
02100\end{array}$ & 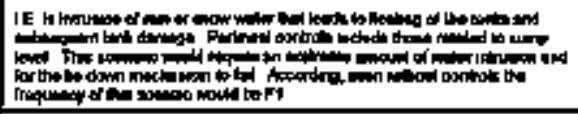 & & & & 61 & FI & 82 & \\
\hline $\begin{array}{l}240 \mathrm{~d} \text {. } \\
\text { DR a }\end{array}$ & 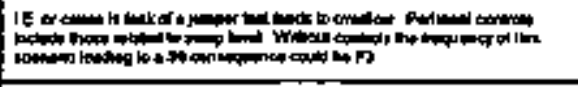 & $\mathbf{s 1}$ & & 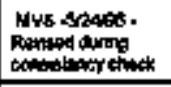 & Ps & rs & 30 & \\
\hline $\lim _{t \rightarrow 0}^{240}$ & IE & $s 1$ & & 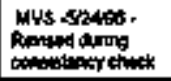 & P5 & PJ & so & \\
\hline 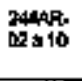 & 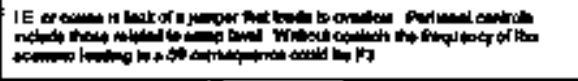 & s1 & & 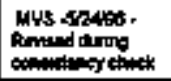 & $\mathbf{F 3}$ & Pl & so & \\
\hline 24apr. & 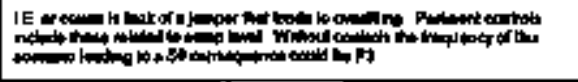 & s1 & & 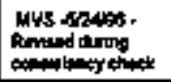 & $\mathbf{F 2}$ & $\mathrm{F}$ & so & \\
\hline 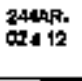 & 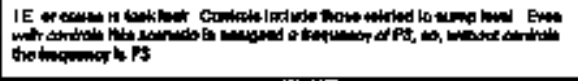 & $\$ 1$ & & 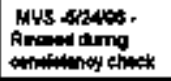 & 17 & 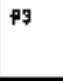 & so & \\
\hline 204ar. & 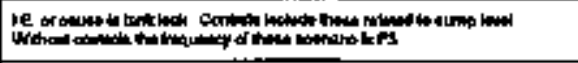 & & & & $\mathbf{F}$ & $\mathbf{n}$ & $\theta$ & \\
\hline 24017 & 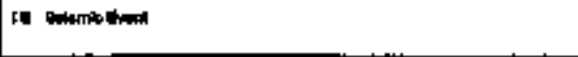 & & & & F1 & F1 & $\Rightarrow$ & \\
\hline
\end{tabular}




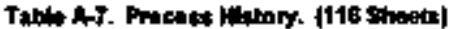

\begin{tabular}{|c|c|c|c|c|c|c|c|c|}
\hline 10 & 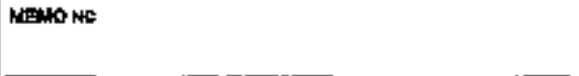 & Poinidy & Pand & Rolthord MENo & fon & ing & 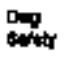 & 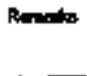 \\
\hline $\begin{array}{l}\text { 24der. } \\
03 \text { a d }\end{array}$ & 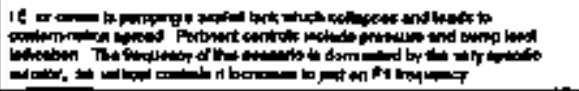 & & & & $\mathbf{F r}$ & $\mathrm{F} \mathbf{1}$ & B1 & \\
\hline 204ath & 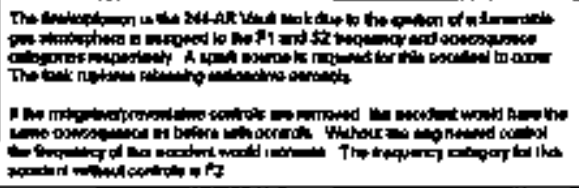 & & & & $\mathbf{E 1}$ & $F_{2}$ & 82 & \\
\hline $20448-$ & 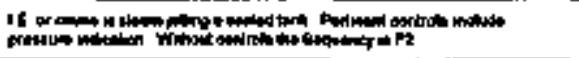 & & & & $\mathbf{F 1}$ & $\boldsymbol{F}$ & $\$ 2$ & \\
\hline $\operatorname{los}_{400}$ & 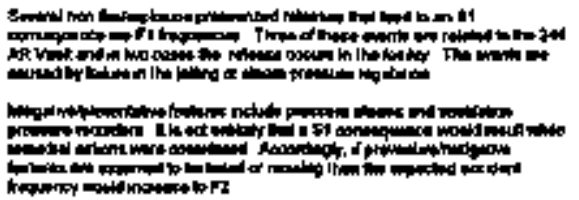 & & & & $\mathbf{F 1}$ & $F$ & 81 & \\
\hline 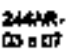 & 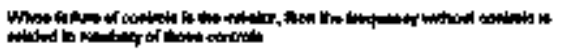 & & & & $m$ & $\mathbf{F} 2$ & st & \\
\hline 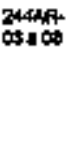 & " & & & & $\mathbf{F 1}$ & f3 & 32 & \\
\hline 2400 & 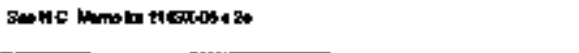 & & & & No & F1 & sin & \\
\hline
\end{tabular}


Tahl A-7. Procuct Hatory. 1118 sheets

\begin{tabular}{|c|c|c|c|c|c|c|c|c|}
\hline Dו & him & times & ling & 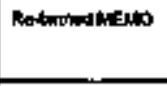 & Freq & $\begin{array}{l}\text { Ond } \\
\text { Fing } \\
\text { Ne }\end{array}$ & $\operatorname{lom}_{\text {sinty }}$ & Nomenthe \\
\hline $\begin{array}{l}\text { 2eine } \\
\text { os a } 13\end{array}$ & 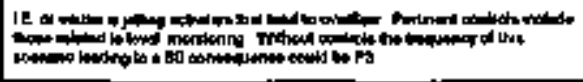 & a1 & & 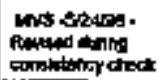 & $\mathbf{F 2}$ & $\boldsymbol{p}$ & 80 & \\
\hline $\begin{array}{l}2406 \\
031414\end{array}$ & 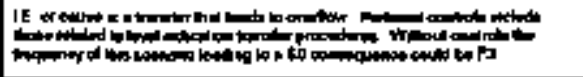 & 51 & & 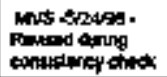 & F & $\boldsymbol{p s}$ & so & \\
\hline 24018 & 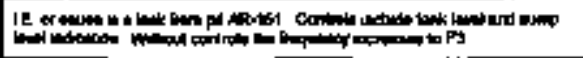 & & & & $\mathbf{F 2}$ & f3 & $\mathbf{s}$ & \\
\hline 2fur & 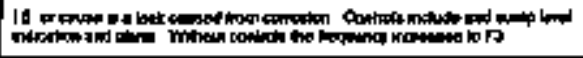 & & & & FE & $\mathbf{R}$ & $\$ 0$ & \\
\hline 2044: & 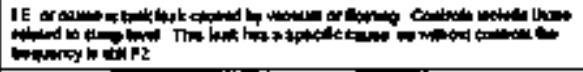 & & & & $\mathbf{F 2}$ & $\mathbf{F} 2$ & $s$ & \\
\hline 2440:- & 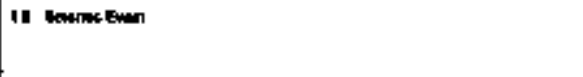 & $\$ 1$ & & lirs entase & 62 & $\mathbf{F} \mathbf{2}$ & $\infty$ & \\
\hline 244AR- & 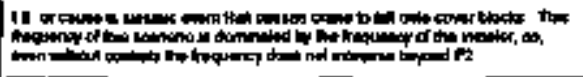 & $e_{1}$ & & 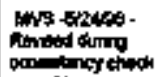 & F2 & $\mathbf{F}$ & $\$ 0$ & \\
\hline $\begin{array}{l}\text { 240kR- } \\
\text { os } 00101\end{array}$ & & & & & $\mathbf{F}$ & & 81 & \\
\hline $\begin{array}{ll}244 k . \\
0002\end{array}$ & If & & & & $\mathbf{P z}$ & $\mathbf{r}$ & 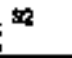 & \\
\hline $\operatorname{lom}_{0.02}$ & & & & & P2 & & s1 & \\
\hline $\begin{array}{l}\text { 244k- } \\
\text { of o o1 }\end{array}$ & 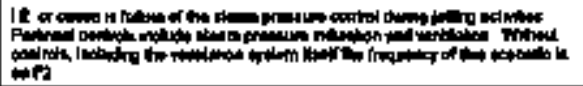 & & & & F1 & $\mathrm{Fz}$ & 5 & \\
\hline
\end{tabular}




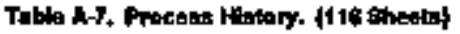

\begin{tabular}{|c|c|c|c|c|c|c|c|c|}
\hline $\mathbf{D}$ & شع & 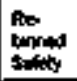 & Preq & 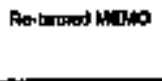 & fiop & 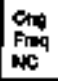 & 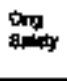 & Prematos \\
\hline $\begin{array}{l}244 \times R \% \\
0,002\end{array}$ & 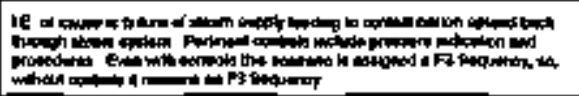 & & & & $\mathbf{B s}$ & $\mathbf{m}$ & si & \\
\hline 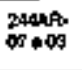 & 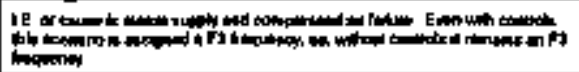 & & & & $\mathbf{F 3}$ & es & 51 & \\
\hline $\begin{array}{l}244 R \\
\text { o }\end{array}$ & & $s 1$ & $\mathrm{FH}$ & 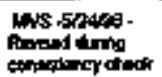 & f & & 50 & \\
\hline 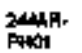 & & & & & F 3 & & 51 & \\
\hline PUar- & & & & & $\mathbf{F}$ & & 39 & \\
\hline $\begin{array}{l}\text { 2tink. } \\
\text { fend }\end{array}$ & & & & & 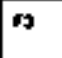 & & $\$ 1$ & \\
\hline 3NarR- & & & & & $\mathbf{F}$ & & 81 & \\
\hline imes & & & & & $\boldsymbol{n}$ & & B1 & \\
\hline PHoth & & & & & $\mathbf{F}$ & & $\$ 1$ & \\
\hline $\begin{array}{l}\text { 2440R } \\
\text { PHOP }\end{array}$ & & & & & $\mathbf{F}$ & & 81 & \\
\hline Proks & & & & & $\mathbf{A}$ & & 51 & \\
\hline PHAR- & & & & & f9 & & B & \\
\hline
\end{tabular}




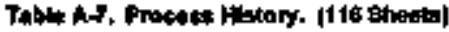

\begin{tabular}{|c|c|c|c|c|c|c|c|c|}
\hline ID & NH & thention & lim & 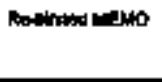 & $\begin{array}{l}\text { Preg } \\
\text { Fren }\end{array}$ & Oily & 盟 & Atermatre \\
\hline $\begin{array}{l}244 \text { A. } \\
\text { H }\end{array}$ & . & & & & $\mathbf{F}$ & & 81 & \\
\hline $\begin{array}{l}\text { 2ankn } \\
\text { Pis11 }\end{array}$ & & & & & $\mathbf{F}$ & & s1 & \\
\hline $\begin{array}{l}24+4 k \\
\text { PHiz }\end{array}$ & & & & & F & & 蜶 & \\
\hline $\begin{array}{l}\text { 24ker. } \\
\text { DHil3 }\end{array}$ & & & & & $\mathbf{f}$ & & $3 \pi$ & \\
\hline Panid & & & & & $\mathbf{r 3}$ & & si & \\
\hline $\begin{array}{l}\text { 2uster } \\
\text { PHis }\end{array}$ & & & & & $\mathrm{Fr}$ & & 81 & \\
\hline PAld & & & & & $\mathbf{r g}$ & & $\$ 1$ & \\
\hline $\begin{array}{l}\text { 24A4RA } \\
\text { PH17 }\end{array}$ & & & & & $\sqrt{3}$ & & $\$ 1$ & \\
\hline $\begin{array}{l}2+140 \\
\text { PHIA }\end{array}$ & & & & & $\mathbf{F g}$ & & ง1 & \\
\hline 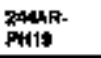 & & & & & H3 & & 31 & \\
\hline mERTHAr & 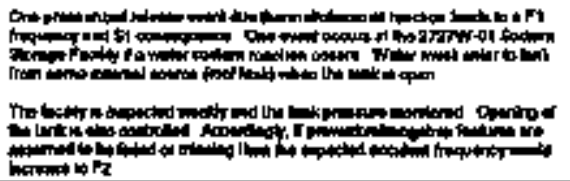 & & & & $\mathbf{F 1}$ & $\mathbf{F Z}$ & $\$ 1$ & \\
\hline
\end{tabular}


Tablo A-7, Procese Hatary. 1116 Shates)

\begin{tabular}{|c|c|c|c|c|c|c|c|c|}
\hline ID & 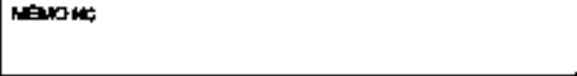 & $\lim _{\text {tomed }}$ & 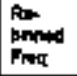 & As-binnedtuspep & 要 & $\begin{array}{l}\text { Don } \\
\text { Fin } \\
\text { NC }\end{array}$ & Ong & Mrroria \\
\hline manam & A & $E$ & & 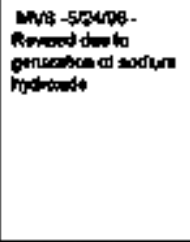 & F1 & F1 & â & \\
\hline $3727 \times 403$ & 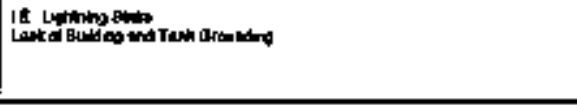 & 82 & & 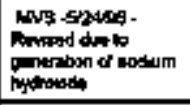 & F1 & $\mathbf{F} 2$ & E1 & \\
\hline 27randor & The & \$2 & & 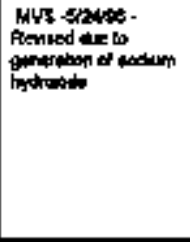 & P1 & $\mathbf{F 1}$ & $\$ 1$ & \\
\hline ZTRNAOA & 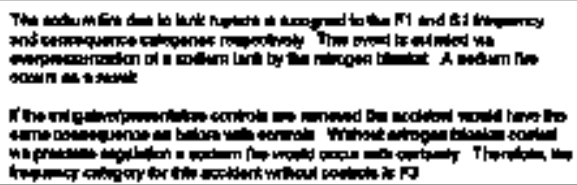 & $\mathbf{s z}$ & & 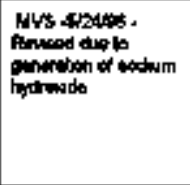 & F1 & $\mathbf{P}$ & 51 & \\
\hline
\end{tabular}




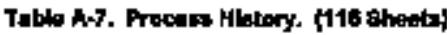

\begin{tabular}{|c|c|c|c|c|c|c|c|c|}
\hline $\mathbf{t}$ & Manore & Re & $\lim _{\lim }$ & Botinned MENo & ore & ond & 的 & Porantin \\
\hline $202 \pi N+00$ & 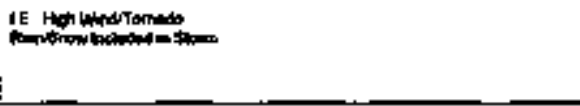 & s2 & & 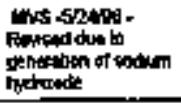 & $\mathbf{F i}$ & $\mathbf{r} 2$ & $\mathbf{s}$ & \\
\hline \$00 & If & & & & $\mathbf{H}$ & $\mathbf{m}$ & st & \\
\hline $\sin 0$ & 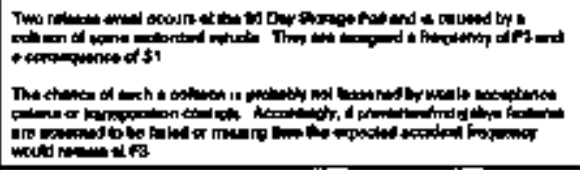 & & & & F3 & $\mathbf{5 3}$ & si & \\
\hline $\operatorname{scod} 0 \mathrm{~A}$ & 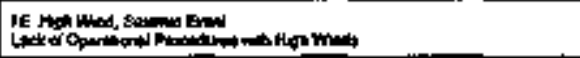 & & & & Fr & $\mathbf{F 3}$ & 91 & \\
\hline 50000 & 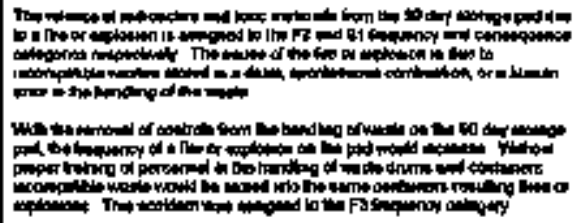 & & & & $\mathbf{F}$ & $\mathbf{H 3}$ & si & \\
\hline $\operatorname{sog} 05$ & 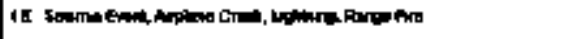 & & & & $\mathbf{F 1}$ & $\mathbf{F 1}$ & $\$ 1$ & \\
\hline phases & 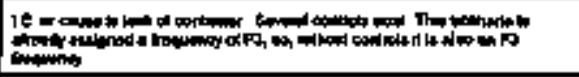 & & & & $\mathbf{A}$ & m & $\$ 1$ & \\
\hline atom & D象 & & & & $\mathbf{r s}$ & $\mathbf{p}$ & 81 & \\
\hline
\end{tabular}




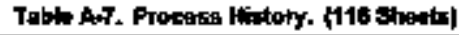

\begin{tabular}{|c|c|c|c|c|c|c|c|c|}
\hline I & HenONG & 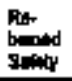 & $\begin{array}{l}\text { Ro } \\
\text { Row } \\
\text { Fing }\end{array}$ & 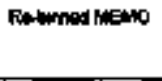 & ing & 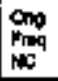 & ong & Petmina \\
\hline $\operatorname{sop} 00$ & 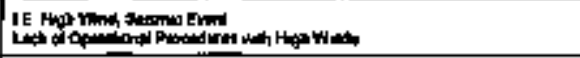 & & & & $\mathbf{F 2}$ & $\boldsymbol{s}$ & $s t$ & \\
\hline 90000 & 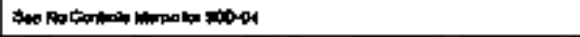 & & & & $\mathbf{F z}$ & $\mathbf{F 2}$ & \$1 & \\
\hline 000-10 & 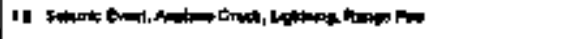 & & & & F1 & F1 & si & \\
\hline gan 11 & 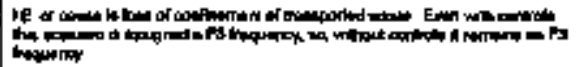 & & & & As & A & $\mathbf{8 1}$ & \\
\hline 60001 & & & & & P3 & & $\mathbf{5 1}$ & \\
\hline onatl & & & & & Fo & & si & 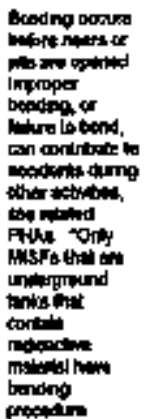 \\
\hline
\end{tabular}




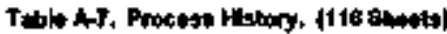

\begin{tabular}{|c|c|c|c|c|c|c|c|c|}
\hline מן & שו" & $\begin{array}{l}\text { her. } \\
\text { Stand }\end{array}$ & then & pallond & Ong & Fing & ang & $\ln x$ \\
\hline $\sin 02$ & 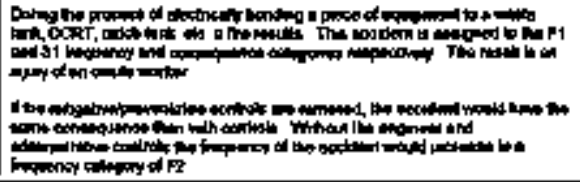 & 62 & & 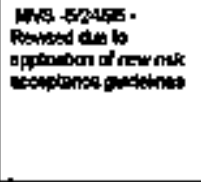 & $\mathbf{F 1}$ & $\mathbf{F Z}$ & si & \\
\hline Batorat & 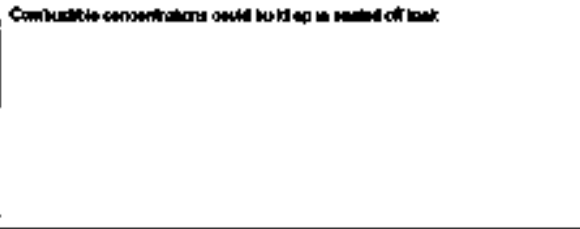 & & & & $\mathbf{F q}$ & & 31 & 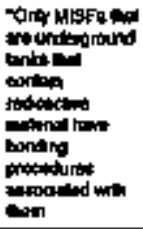 \\
\hline ectal & 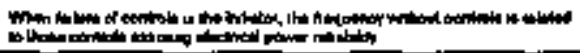 & & & & $\mathbf{r 2}$ & $\boldsymbol{\Gamma t}$ & $\mathbf{\omega}$ & \\
\hline coten & 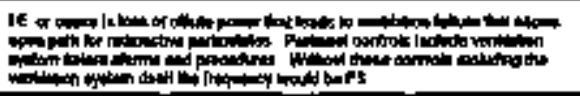 & & & & $\theta$ & F 3 & 的 & \\
\hline 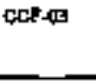 & & sı & $\mathbf{R}$ & 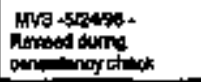 & $\mathbf{r}$ & & 80 & \\
\hline CCF-OH & & 的 & fo & 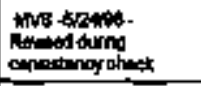 & Fa & & 的 & \\
\hline ctif-6s & & & & & $\mathrm{Fo}$ & & 30 & \\
\hline $\cos \cos ^{\circ}$ & & & & & $\mathbf{F 3}$ & & $\mathbf{m}$ & \\
\hline
\end{tabular}


Thele A.7. Proceds History, (116 thenct)

\begin{tabular}{|c|c|c|c|c|c|c|c|c|}
\hline ID & 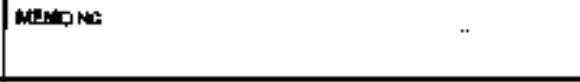 & $\sin _{\sin }$ & 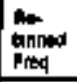 & 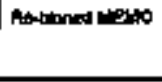 & Ong & $\lim _{\lim }$ & oing & Nonsos \\
\hline DeF-p & 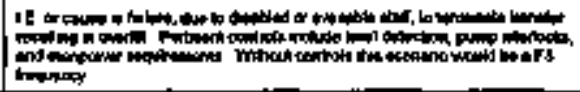 & & & & F1 & $\boldsymbol{m}$ & 82 & \\
\hline $\cos 0$ & & & & & $\mu_{2}$ & & $=0$ & \\
\hline CCF-08 & & & & & $\mathrm{Fo}$ & & of & \\
\hline cocf-10 & & & & & F\$ & & 30 & \\
\hline $\cos .11$ & & & & & F3 & & $\infty$ & \\
\hline $\cos -12$ & & & & & $\mathbf{F s}$ & & $\$$ & \\
\hline ceff-1a & & & & & F & & 心 & \\
\hline cक्cF-11 & & & & & $\mathrm{F3}$ & & sı & \\
\hline 004.15 & & & & & F\$ & & $\$ 1$ & \\
\hline CecF.16 & 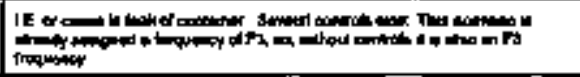 & & & & Fs & $\infty$ & $\$ 1$ & \\
\hline 도다-17 & IE TIm & & & & $F 2$ & $\mathbf{F} 2$ & $\Leftrightarrow$ & \\
\hline oorsts & 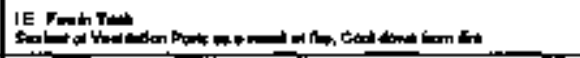 & & & & $\mathbf{F 1}$ & $\mathbf{F i}$ & w & \\
\hline cef-19 & 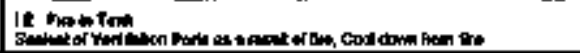 & & & & F1 & Fi & 89 & \\
\hline
\end{tabular}




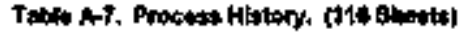

\begin{tabular}{|c|c|c|c|c|c|c|c|c|}
\hline סו & Dano & Bimition & Rinion & Notmed latwo & Five & Fing & orty & Pammina \\
\hline CCF-20 & 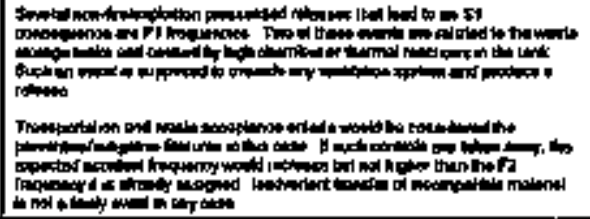 & E & & 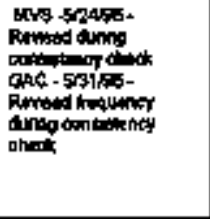 & $\mathbf{F 2}$ & $\mathbf{F} 2$ & 91 & \\
\hline GPP.01 & 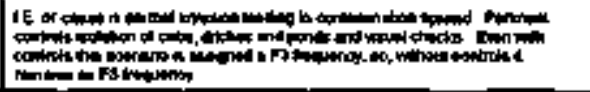 & & & & $\mathbf{F}$ & $\mathbf{F}$ & $\mathbf{s i}$ & \\
\hline copdes & 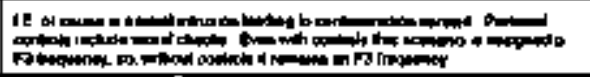 & & & & fa & $\mathrm{FB}$ & so & \\
\hline $\operatorname{cosen}$ & 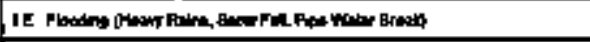 & & & & $\mathbf{F 3}$ & 13 & si & \\
\hline CDPOA & 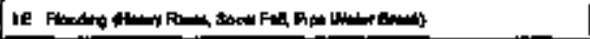 & & & & $\mathbf{B}$ & $\mathrm{F3}$ & 60 & \\
\hline $\cos -06$ & 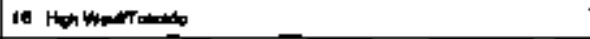 & & & & $\mathrm{F} 2$ & $\bar{F}$ & B1 & \\
\hline $\cos \theta$ & 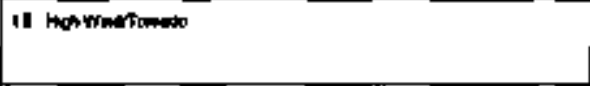 & $\$ 1$ & $\mathbf{B}$ & 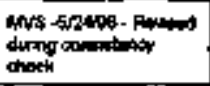 & $\mathbf{F}$ & $\mathbf{F}$ & \$ & \\
\hline cpp-or & 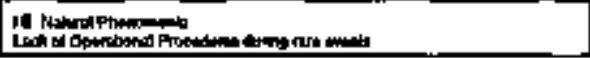 & & & & $f 9$ & $\mathbf{F}$ & 80 & \\
\hline
\end{tabular}


Table A.T. Process Hintary. (116 Bhatis)

\begin{tabular}{|c|c|c|c|c|c|c|c|c|}
\hline 10 & HENO NG & indit & 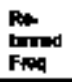 & 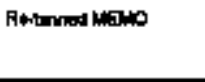 & Find & $\lim _{\substack{\infty \\
\text { ind }}}$ & $\operatorname{lng}_{1}$ & Patimatis \\
\hline $\cos 4$ & 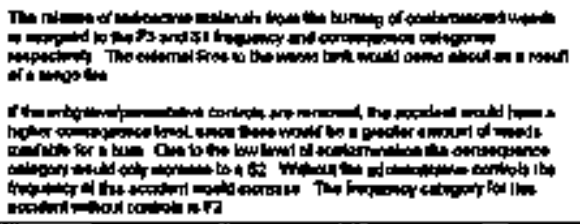 & & & & Ps & $\mathbf{N}$ & si & \\
\hline $\cos -\theta^{\circ}$ & & & & & $\mathbf{r 3}$ & & 81 & \\
\hline coento & 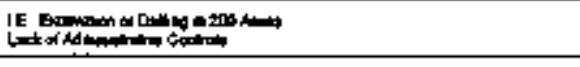 & & & & $\mathbf{k z}$ & $\mathbf{p}$ & $s z$ & \\
\hline coser & 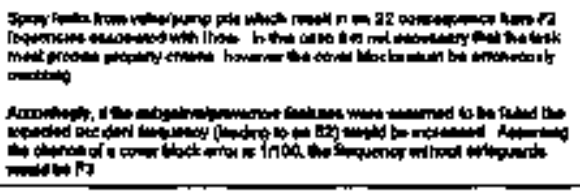 & $\mathbf{s u}$ & & 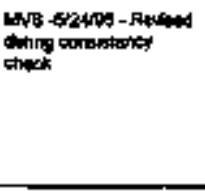 & F2 & Fo & 82 & \\
\hline $\cos -\infty$ & 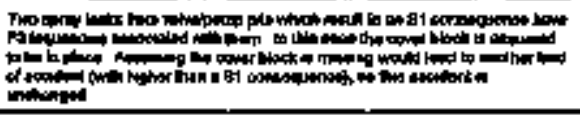 & & & & R3 & $\mathbf{F}$ & st & \\
\hline $\cos \theta-\infty$ & 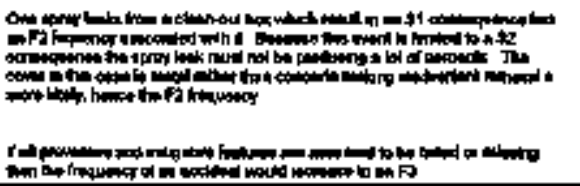 & & & & Fi & f & ar & \\
\hline
\end{tabular}




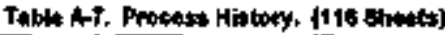

\begin{tabular}{|c|c|c|c|c|c|c|c|c|}
\hline 10 & MENONG & Prom & Bermid & Rorimead Hen & $\lim$ & $\begin{array}{l}\text { Drig } \\
\text { Fiod } \\
\text { NAC }\end{array}$ & and & Roming \\
\hline $\cos -0 \mathrm{~s}$ & 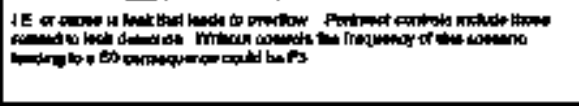 & $s$ & & 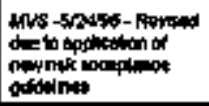 & $\mathbf{F 1}$ & R & $8 t$ & \\
\hline $\cos 0 \sin ^{-}$ & 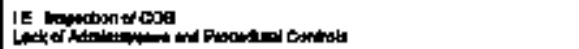 & & & & $\mathrm{FP}$ & $\mathrm{F}$ & $\$ 1$ & \\
\hline $\cos \cos a$ & 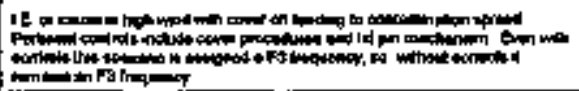 & & & & As & $\mathbf{s}$ & $\mathbf{s 1}$ & \\
\hline $\cos \theta 00^{\circ}$ & 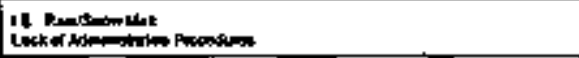 & & & & $\mathbf{r s}$ & $\mathbf{f 3}$ & 91 & \\
\hline $\cos -\alpha s$ & 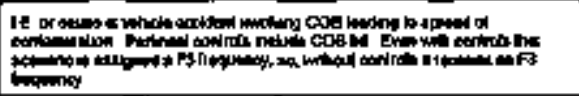 & & & & $\mathrm{F}$ & $\mathbf{F}$ & EI & \\
\hline chers. & II sometem & & & & $\mathbf{F}$ & $\mathbf{6}$ & $\$ 1$ & \\
\hline coeses & 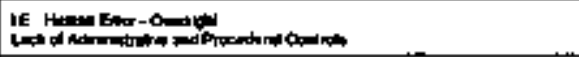 & & & & Fs & $\boldsymbol{P}$ & 51 & \\
\hline conorie & 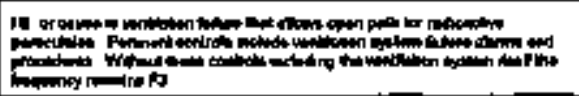 & & & & $\mathrm{FH}$ & $\mathbf{F}$ & 8I & \\
\hline coseng & 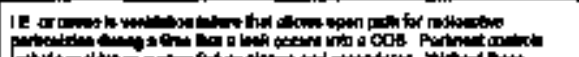 & & & & F & $\mathbf{r 3}$ & 61 & \\
\hline coser & 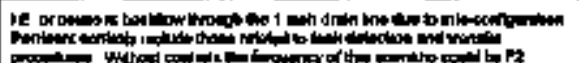 & & & & F1 & $\mathbf{n}$ & 51 & \\
\hline
\end{tabular}




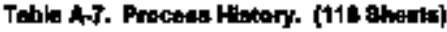

\begin{tabular}{|c|c|c|c|c|c|c|c|c|}
\hline 由 & Heno & Boridy & ing & 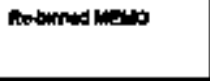 & ing & Prom & ory & Rontide \\
\hline costis & IE A & 62 & & 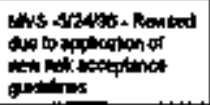 & $\mathbf{M}$ & $\mathbf{R s}$ & $\$ 1$ & \\
\hline chandor & 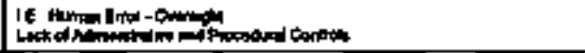 & & & & $\mathbf{F}$ & $\mathbf{F a}$ & $\$ 1$ & \\
\hline conat & 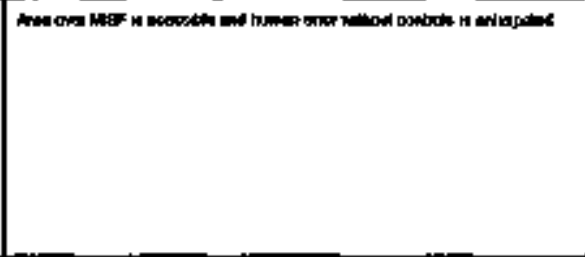 & & & & F2 & & s1 & 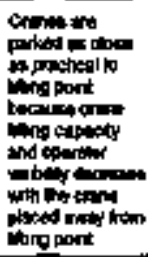 \\
\hline 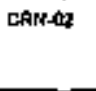 & 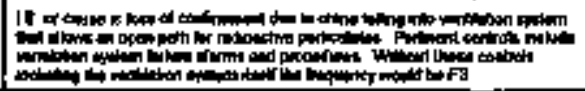 & & & & $\mathrm{Fa}$ & $\mathbf{F a}$ & $\mathbf{\$ 1}$ & \\
\hline CRNGMA & Anton & & & & $\mathbf{F}$ & & 31 & 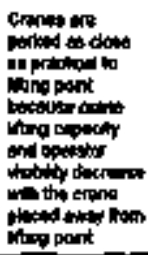 \\
\hline Grand03 & 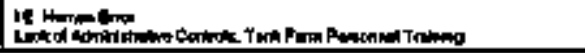 & & & & $\mathbf{2}$ & $F$ & 4 & \\
\hline
\end{tabular}




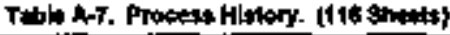

\begin{tabular}{|c|c|c|c|c|c|c|c|c|}
\hline I & או & 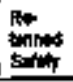 & for & 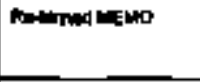 & Preg & $\begin{array}{l}\text { Ong } \\
\text { fod } \\
\text { NC }\end{array}$ & sing & hmoln \\
\hline CRHCOH & 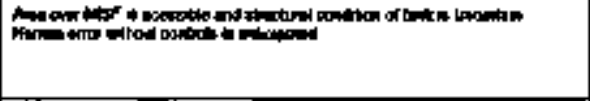 & 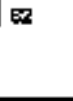 & & 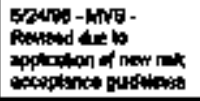 & $\mathbf{F 2}$ & & $\$ 1$ & \\
\hline CRHAOB & 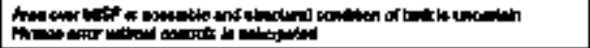 & & & & $\mathbf{F}$ & & 92 & \\
\hline CRNAD4 & 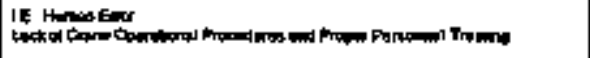 & & & & $\mathbf{F}$ & $\boldsymbol{F}$ & $\mathbf{s}$ & \\
\hline CRMOARA & 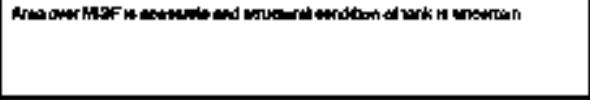 & $\$ 2$ & & 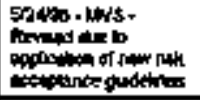 & 1 & & $\$ 1$ & \\
\hline 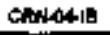 & 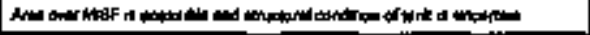 & & & & 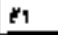 & & $s \mathbf{s}$ & \\
\hline 0,005 & 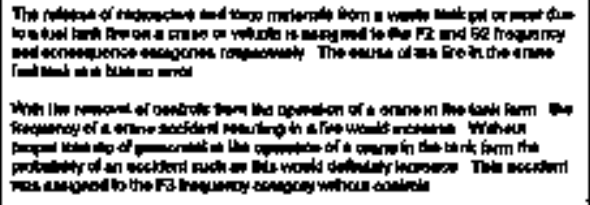 & & & & $\mathbf{F}$ & 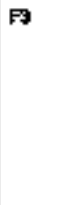 & 5 & \\
\hline 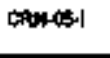 & All & & & & $\mathbf{F}$ & & \$1 & 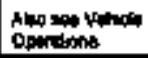 \\
\hline
\end{tabular}


Table 47. Procsas Hitery. (116 sheets)

\begin{tabular}{|c|c|c|c|c|c|c|c|c|}
\hline 10 & mENOL & 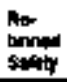 & fir & Nothinhat Mbid & ind & $\operatorname{lom}_{10}$ & orly & Rathe \\
\hline cakdes & 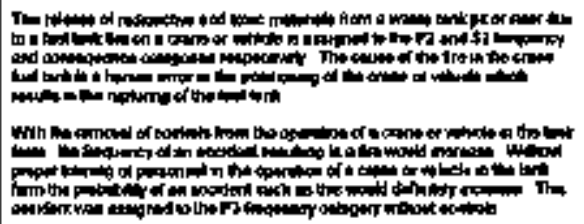 & & & & $\mathbf{F}$ & $\mathbf{F s}$ & 57 & \\
\hline CRNATEA & 管 & & & & r1 & & s1 & Not \\
\hline CoRAOT & 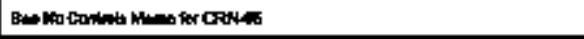 & & & & $\mathbf{F 1}$ & $\mathrm{F}$ & 8 & \\
\hline GAN-ONન & 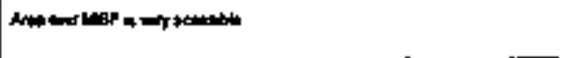 & & & & $\mathbf{F I}$ & & $\$ 1$ & 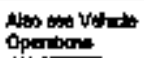 \\
\hline CRNAO & the & & & & $\mathbf{f}$ & $\mathrm{F}$ & 48 & \\
\hline Consolot & Dom & & & & $\mathrm{mo}$ & & $\$ 1$ & 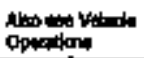 \\
\hline creves & IF & & & & $\mathbf{F 2}$ & $\mathbf{m}$ & 82 & \\
\hline
\end{tabular}




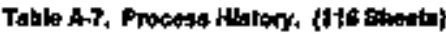

\begin{tabular}{|c|c|c|c|c|c|c|c|c|}
\hline म & 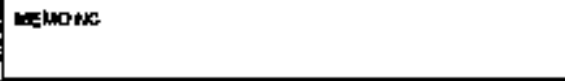 & 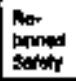 & Pinned & 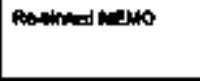 & $\operatorname{lom}_{0,9}$ & $\begin{array}{l}\infty \times 0 \\
\text { Fin } \\
0\end{array}$ & ond & Anomentos \\
\hline CANAOA & 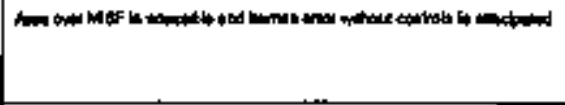 & $\square$ & & 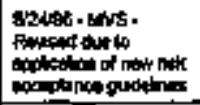 & $\mathbf{r}$ & & s1 & 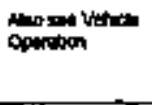 \\
\hline CRN-10 & 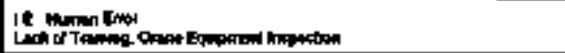 & & & & $\mathbf{F}$ & $\mathrm{Fa}$ & 安 & \\
\hline CRILWOM & 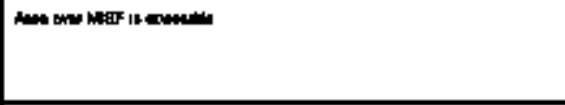 & $\mathbf{2 0}$ & & 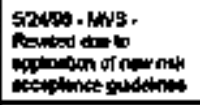 & F1 & & $\$ 1$ & 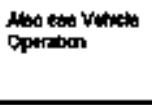 \\
\hline 6rat1 & IE & & & & 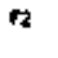 & $\mathbf{F a}$ & a & \\
\hline contintin & 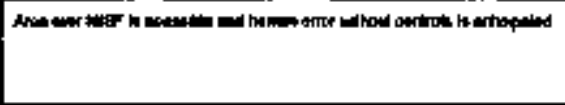 & 82 & & 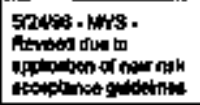 & f2 & & $\$ 1$ & 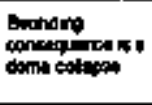 \\
\hline CNak11-10 & 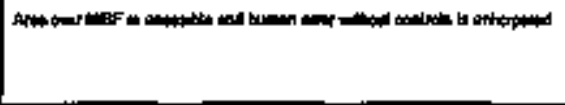 & $\$ 2$ & & 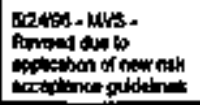 & $\mathbf{1 2}$ & & $\mathbf{s 1}$ & Eoundip \\
\hline CAN-1T-HC & 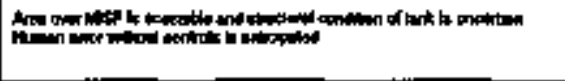 & & & & F & & 62 & 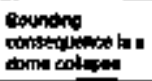 \\
\hline CAN-12 & 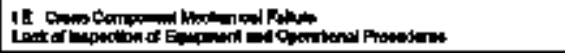 & & & & F1 & $\mathbf{F}$ & $\mathbf{E}$ & \\
\hline $\cos (124 \mathrm{~h}$ & 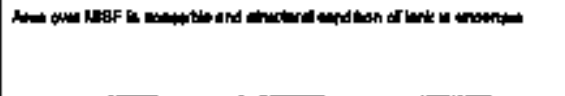 & $\mathbf{2}$ & & 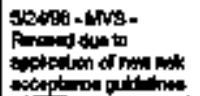 & $\mathbf{P}$ & & si & 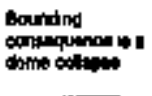 \\
\hline
\end{tabular}


Tehis A-7. Mrocens Hitory. (116 shests)

\begin{tabular}{|c|c|c|c|c|c|c|c|c|}
\hline 10 & MENONG & $\begin{array}{l}\text { Rot } \\
\text { sinned } \\
\text { sity }\end{array}$ & $\begin{array}{l}\text { for } \\
\text { tinted } \\
\text { Find }\end{array}$ & Bromod wato & $\underset{\text { Fing }}{\log }$ & $\begin{array}{l}\text { Orid } \\
\text { Notiq } \\
\text { NFC }\end{array}$ & ond & Remnedis \\
\hline Cret.13.18 & 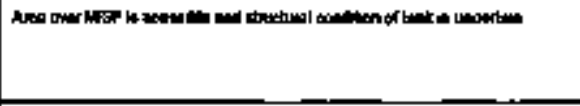 & $\mathbf{s t}$ & & 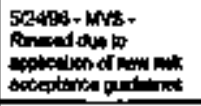 & $\mathbf{m}$ & & $\mathbf{s i}$ & 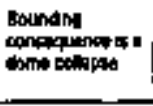 \\
\hline CRN-12tK & 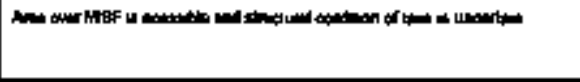 & & & & $\mathbf{F 1}$ & & $\$ 2$ & 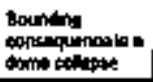 \\
\hline CRNA3HA & 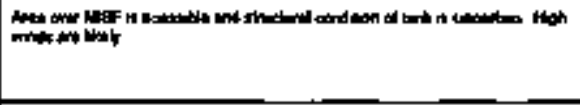 & 32 & & 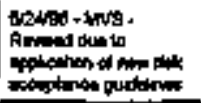 & Fo & & 81 & 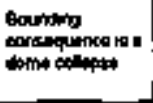 \\
\hline Cfat 13- 自 & 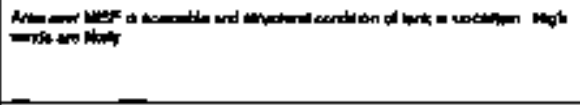 & $\mathbf{s z}$ & & 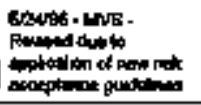 & Fo & & si & Eounding \\
\hline cans-13-1C & 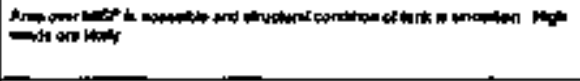 & & & & fo & & 82 & 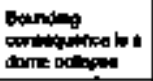 \\
\hline CRKGISA & IE rank & & & & f: & $\mathrm{F}^{3}$ & 80 & \\
\hline CAR 4130 & it townet ta & & & & $\bullet$ & $\mathbf{F 1}$ & $\Leftrightarrow$ & \\
\hline CANA 14 & & & & & $F$ & & gs & \\
\hline CANK-14 & & & & & $\mathbf{f 2}$ & & $\mathbf{8 1}$ & \\
\hline CRN-15 & & & & & $\mathbf{F}$ & & $\$ 1$ & \\
\hline 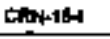 & & & & & $\mathbf{F}$ & & 81 & \\
\hline crapt-18 & & & & & $\mathbf{r}$ & & 81 & \\
\hline 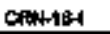 & & & & & $m$ & & s1 & \\
\hline
\end{tabular}




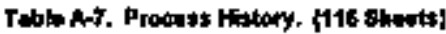

\begin{tabular}{|c|c|c|c|c|c|c|c|c|}
\hline $\mathbf{0}$ & MFHONE & ifindy & $\lim _{\lim }$ & Ro-benen LENo & Fin & $\lim _{\lim }^{\infty}$ & ו & Bomento \\
\hline Cantortit & M & & & & F3 & fo & $\$ 1$ & \\
\hline Conat-17-1 & & & & & F2 & & BI & \\
\hline conth-10 & 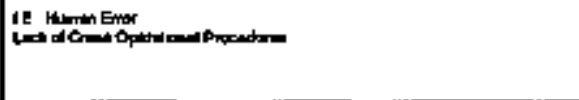 & $\mathbf{5 2}$ & & 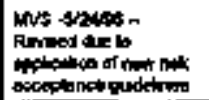 & $\mathbf{r 2}$ & rs & 81 & \\
\hline Cran 1 161 & 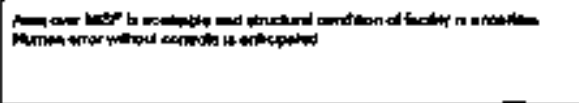 & 52 & & 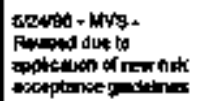 & $\mathbf{n}$ & & $\mathbf{s}$ & \\
\hline CRAn 19 & 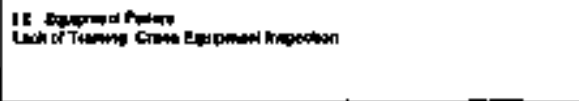 & $\$ 2$ & & 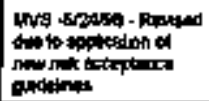 & F1 & $\mathbf{F a}$ & sı & \\
\hline CRE-19- & 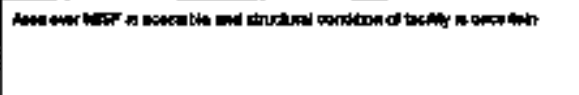 & 62 & & 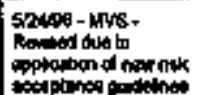 & F1 & & B1 & \\
\hline CRAs 20 & 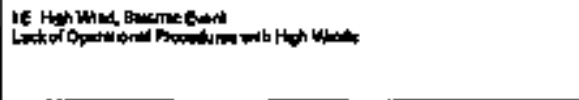 & $2 \mathbf{2}$ & & 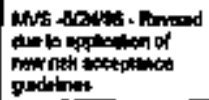 & F1 & $\mathbf{r s}$ & s1 & \\
\hline CRN-ZMH & 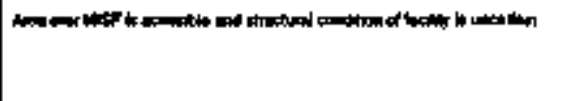 & 的 & & 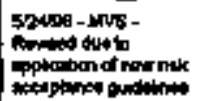 & Fo & & 自1 & \\
\hline 대사저 & 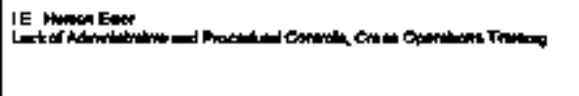 & 92 & & 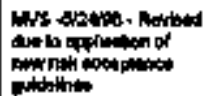 & $\mathbf{r}$ & P3 & $\$ 1$ & \\
\hline
\end{tabular}




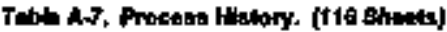

\begin{tabular}{|c|c|c|c|c|c|c|c|c|}
\hline 10 & MU⿴囗十⺝木 & 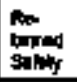 & tond & 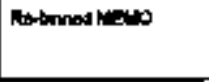 & $\operatorname{mop}_{0}$ & on & sing & himats \\
\hline CARktath & 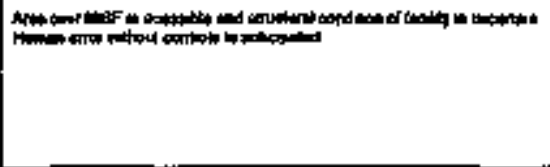 & $\$ 2$ & & 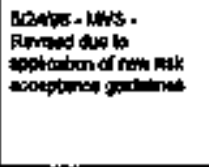 & $\mathbf{m}$ & & a1 & 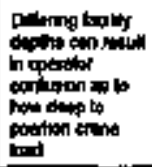 \\
\hline CRN-Z1- & 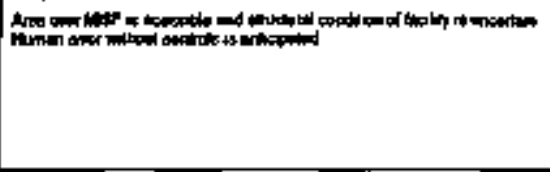 & sto & & 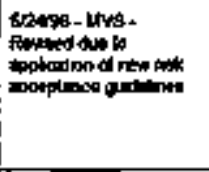 & F2 & & $\mathbf{s}$ & 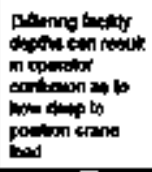 \\
\hline conat22 & 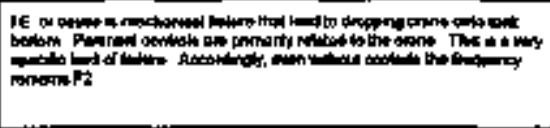 & क्ष & & 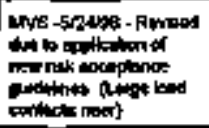 & $\mathbf{7 2}$ & $\mathbf{m}$ & B & \\
\hline CAnzerin & 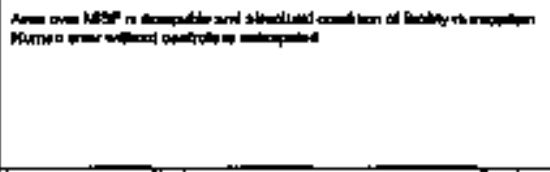 & at & & 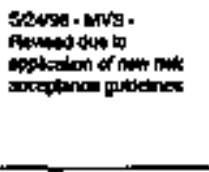 & Fi & & \$1 & 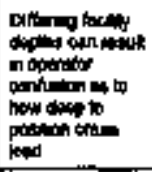 \\
\hline CRH-ZWF & 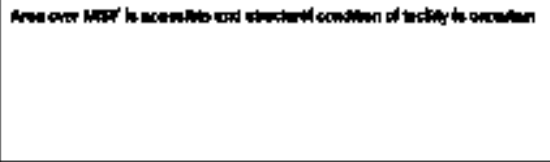 & 62 & & 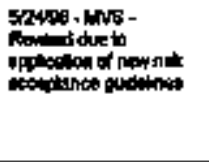 & F1 & & 81 & 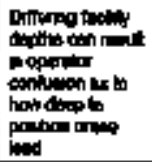 \\
\hline
\end{tabular}


Tuble A-7. Proona Holory. (116 atental

\begin{tabular}{|c|c|c|c|c|c|c|c|c|}
\hline u & NEAO NH & $\begin{array}{l}\text { Re } \\
\text { binind } \\
\text { Exity }\end{array}$ & $\begin{array}{l}\text { fintd } \\
\text { find }\end{array}$ & Ratimas hewo & ind & ing & $\operatorname{sing}$ & Rentito \\
\hline $\cos w 29$ & 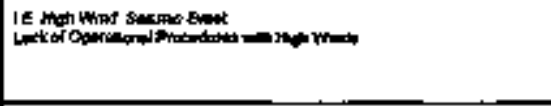 & sz & & 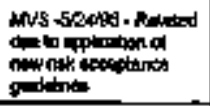 & 1 & $\mathbf{m}$ & $\mathbf{s 1}$ & \\
\hline contrath & 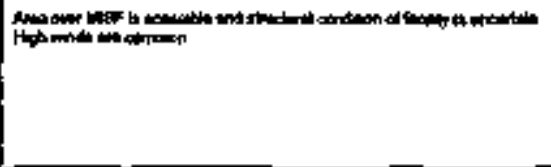 & $\leq 2$ & & 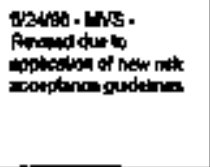 & 90 & & 91 & 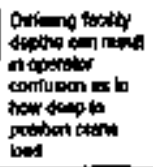 \\
\hline 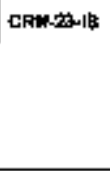 & 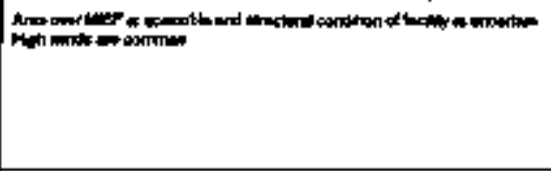 & $9 x$ & & 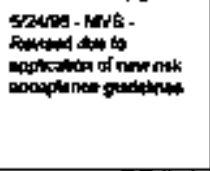 & Fo & & si & 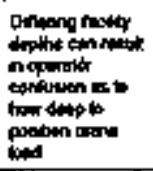 \\
\hline cents & & & & & $\mathbf{f 2}$ & & $\$ 1$ & \\
\hline costant & & & & & $\mathbf{m}$ & & $\mathbf{s 1}$ & 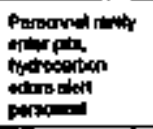 \\
\hline CRPar & & & & & F2 & & 81 & \\
\hline CPith251 A & & & & & $\mathbf{H 2}$ & & s1 & \\
\hline cotras & 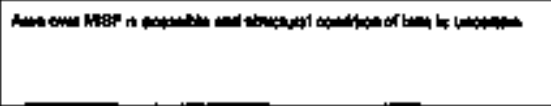 & $\$ 2$ & & 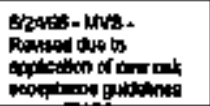 & $F \boldsymbol{Z}$ & & B1 & \\
\hline CAmax & & & & & $\mathbf{F 3}$ & & 31 & \\
\hline
\end{tabular}




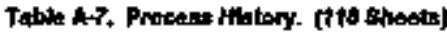

\begin{tabular}{|c|c|c|c|c|c|c|c|c|}
\hline 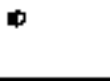 & watom & pantil & $\lim _{\lim }$ & Rentened thes & Fin & ord & $\mathrm{cos}^{0}$ & themaía \\
\hline 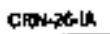 & & & & & F1 & & $\$ 1$ & \\
\hline consos & 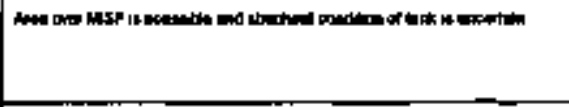 & ER & & 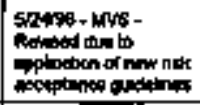 & $\mathbf{F I}$ & & \$1 & \\
\hline crint2r & & & & & $\mathbf{F 1}$ & & 91 & \\
\hline contasth & & & & & fo & & $\$ 1$ & \\
\hline CAREzt: & 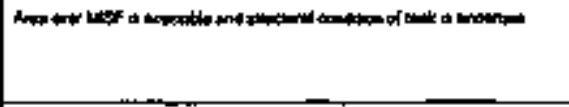 & ss & & 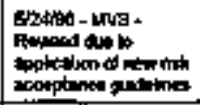 & mo & & s1 & \\
\hline CAREACI & 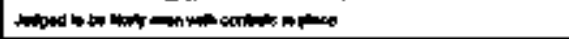 & & & & fo & & $\mathbf{s}$ & \\
\hline curas & 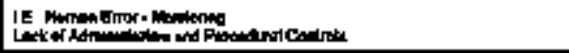 & & & & $\mathbf{F 9}$ & F & B1 & \\
\hline Cherend & 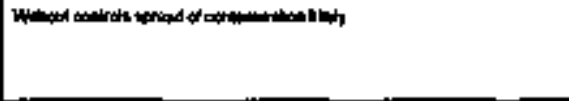 & & & & $\mathbf{m}$ & & st & 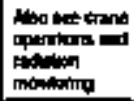 \\
\hline cuk-n & 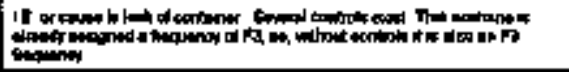 & & & & F & $\boldsymbol{F}$ & 61 & \\
\hline conteses & 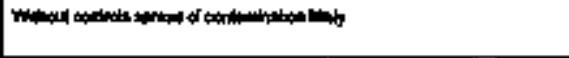 & & & & $\mathbf{m}$ & & st & (1) \\
\hline CMROS & 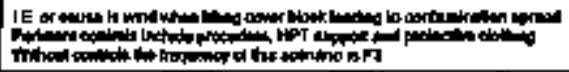 & & & & $m 2$ & $\mathrm{~F}$ & s1 & \\
\hline GVR-03-1 & 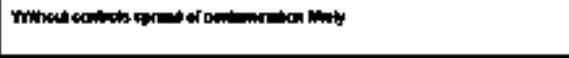 & & & & F2 & & 81 & 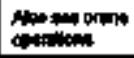 \\
\hline Evingut & IE & & & & 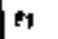 & F1 & s1 & \\
\hline
\end{tabular}




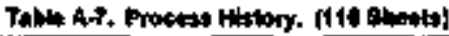

\begin{tabular}{|c|c|c|c|c|c|c|c|c|}
\hline ID & MENONG & dint & Finged & 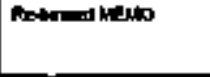 & Ong & 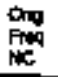 & Ond & 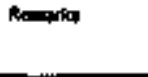 \\
\hline Chent & 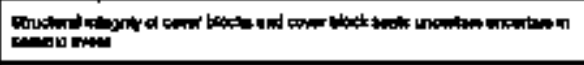 & & & & Fa & & $\$ 1$ & Nopos \\
\hline twhos & 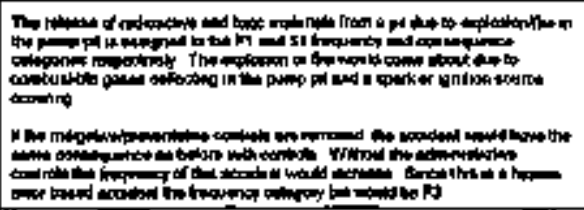 & sas & & 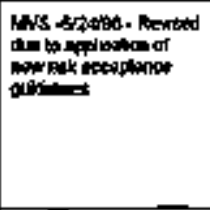 & $\mathbf{F}$ & $\mathbf{r y}$ & \$1 & \\
\hline Curios & 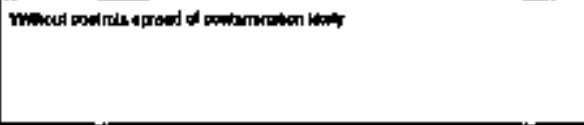 & & & & Fo & & $\mathbf{s 1}$ & 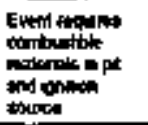 \\
\hline CVAR & & & & & $\mathrm{Fa}$ & & a1 & \\
\hline crincos & & & & & F2 & & s1 & 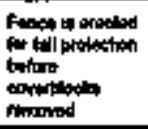 \\
\hline cimos & & & & & $\mathbf{k s}$ & & gis & \\
\hline CWAOT/4 & & & & & $\mathrm{Fz}$ & & $s_{1}$ & 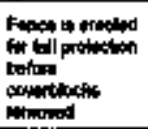 \\
\hline Crisod & & & & & $\mathbf{A s}$ & & s! & \\
\hline
\end{tabular}


Tha A-7. Pousts history. [116 antws]

\begin{tabular}{|c|c|c|c|c|c|c|c|c|}
\hline $\mathbf{0}$ & Manows & bintiod & Pin & Fe- & Onin & 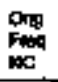 & $\begin{array}{l}009 \\
\text { switur }\end{array}$ & Pिm \\
\hline curasin & & & & & 72 & & $\$ 1$ & 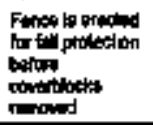 \\
\hline CUA- $\rightarrow$ & & & & & F1 & & 91 & \\
\hline CWR-0OA & & & & & $n$ & & 51 & 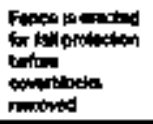 \\
\hline cute.10 & & & & & F1 & & B1 & \\
\hline Cuk-10-1 & & & & & $\boldsymbol{r}$ & & B1 & 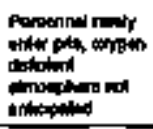 \\
\hline consi1 & & & & & 1 & & s & \\
\hline chast.1 & & & & & $\mathbf{F 1}$ & & 81 & 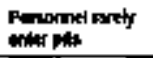 \\
\hline Gin-12-1 & 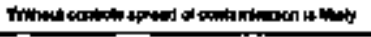 & & & & $\mathbf{F}$ & & \$1 & \\
\hline Exh-13:4 & . & & & & A & & 81 & \\
\hline CVR-14-1 & 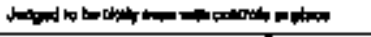 & & & & $\mathrm{F} \$$ & & 81 & \\
\hline CWR-15-4 & 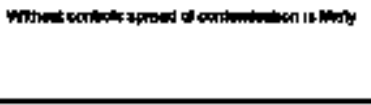 & 62 & & 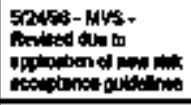 & $\mathbf{F 2}$ & & $\$ 1$ & \\
\hline DENLAI & & & & & P3 & & \$1 & \\
\hline DCAH-01-1 & & & & & $\mathbf{P z}$ & & ga & \\
\hline
\end{tabular}


Tuble A.T. Protews Hittory, (118 abutu)

\begin{tabular}{|c|c|c|c|c|c|c|c|c|}
\hline ID & WHiNO KC & Bot & $\lim _{\lim }$ & Sמשג & moq & 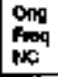 & क्og & Reminim \\
\hline DCN-OR & 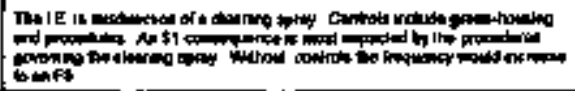 & & & & 2 & $\mathbf{F}$ & 61 & \\
\hline $\operatorname{OCN}-\infty \mathrm{a}-\mathrm{k}$ & 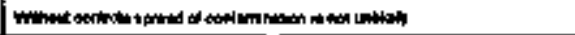 & & & & $\mathbf{F} 1$ & & $\$ 1$ & \\
\hline $\operatorname{Cos}-\cos -18$ & 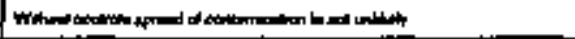 & & & & $\mathbf{r}$ & & 54 & \\
\hline DCN-DS & 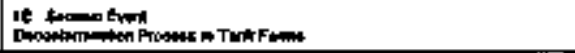 & & & & E1 & $\mathbf{F I}$ & si & \\
\hline OCN-DSA & 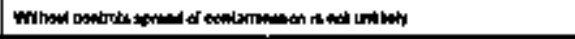 & & & & 60 & & $\$ 1$ & \\
\hline OCNA4 & IE & & & & 12 & 62 & $\$ 1$ & \\
\hline Desto44 & 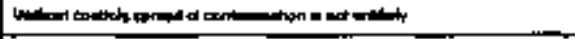 & & & & ro & & $\$ 1$ & \\
\hline Dopios. & & & & & $\mathrm{Fa}$ & & so & \\
\hline DCHADS- & & & & & $\mathbf{F}$ & & $\Delta 1$ & \\
\hline Dewos & & & & & $\mathbf{r z}$ & & 61 & \\
\hline Dewas-1 & & 81 & & 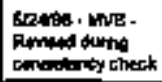 & $\mathrm{F2}$ & & $\$$ & \\
\hline Dentas & & & & & F & & so & \\
\hline DCWOTras & & & & & $\mathbf{F 2}$ & & a & \\
\hline EE-OI & IE Alut & & & & $\mathbf{F 1}$ & $F 1$ & 00 & \\
\hline
\end{tabular}




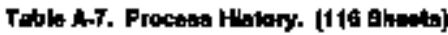

\begin{tabular}{|c|c|c|c|c|c|c|c|c|}
\hline 10 & Newawc & sofing & $\begin{array}{l}\text { Pos } \\
\text { Fined } \\
\text { Fond }\end{array}$ & Rabanda listo & ong & 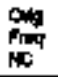 & ond & Reweriks \\
\hline exsiz & 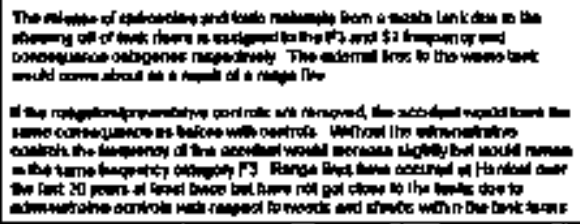 & & & & $\mathbf{f}$ & $\mathrm{Fq}$ & $\$ 1$ & \\
\hline EEE-10 & 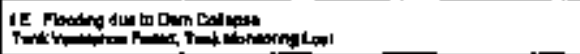 & & & & $\mathbf{F 2}$ & F2 & $\$ 1$ & \\
\hline EE-04: & 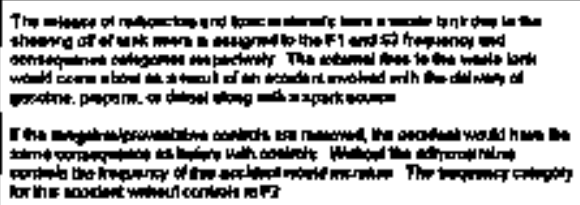 & & & & FI & $F$ & $\mathbf{s 3}$ & \\
\hline EE- $040 \mathrm{~b}$ & 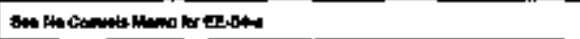 & & & & $\mathbf{F}$ & 82 & $\Leftrightarrow$ & \\
\hline ELAS- & 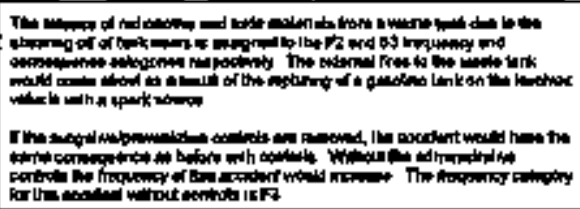 & & & & pz & $\mathbf{M u}$ & घా & \\
\hline gention & 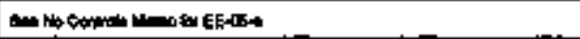 & & & & $\mathbf{z}$ & $\mathrm{fa}$ & st & \\
\hline
\end{tabular}


Tah't M-7. Proceses Hestory. (1tt aheats)

\begin{tabular}{|c|c|c|c|c|c|c|c|c|}
\hline ID & LEHOAC & Santid & Fined & Ro-bined MFinO & om & $\begin{array}{l}\lim _{\ln } \\
\ln \end{array}$ & $\sin$ & Requita \\
\hline Elets & 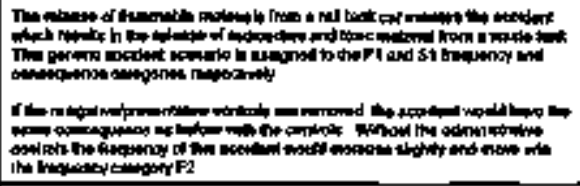 & & & & $\mathbf{P 1}$ & $\mathbf{F}$ & s1 & \\
\hline Ex-ot & & & & & Fo & & 60 & \\
\hline EE-eB & & & & & $n$ & & $\infty$ & \\
\hline EE $\rightarrow 0$ & & st & $\mathbf{6 s}$ & 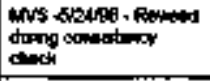 & Hs & & so & \\
\hline (T6-60 & 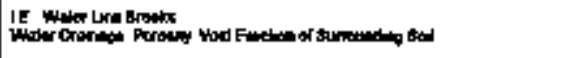 & & & & $\mathbf{r}$ & $\mathbf{E} 1$ & $\$ 1$ & \\
\hline EE-11 & 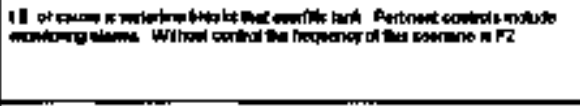 & 87 & & 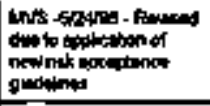 & $\mathbf{F 1}$ & $F_{2}$ & 91 & \\
\hline EE-12 & & s1 & & 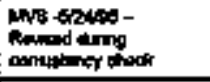 & $\mathbf{F u}$ & & so & \\
\hline EE-13 & & & & & Ps & & $\omega$ & \\
\hline EE.14 & & & & & 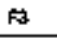 & & Af & \\
\hline EEE-16 & & $\$ 1$ & & 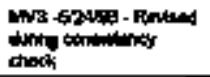 & $\mathrm{F} 3$ & & 90 & \\
\hline
\end{tabular}




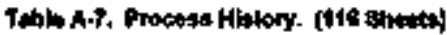

\begin{tabular}{|c|c|c|c|c|c|c|c|c|}
\hline ID & Memona & Din & $\lim _{\lim }$ & 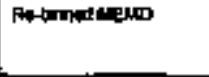 & Fig & $\begin{array}{l}\text { ing } \\
\text { Fin } \\
\text { iKC }\end{array}$ & orion & Anstaplex \\
\hline EE-16 & 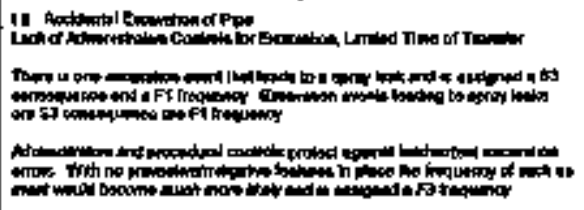 & & & & F1 & $\mathbf{r}$ & Bs & \\
\hline EE-IT & 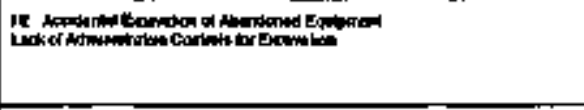 & 32 & & 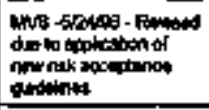 & F & 8 & $\$ 1$ & \\
\hline GRMOT & & & & & es & & $\$ 1$ & \\
\hline GNM-1.1 & & & & & Fz & & $\$ 1$ & 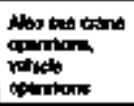 \\
\hline GAN@2 & & & & & f3 & & g: & \\
\hline GRN-WA & & & & & $m$ & & $\$ 1$ & 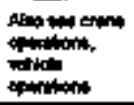 \\
\hline GaAto3H & & & & & $\mathbf{F}$ & & 51 & \\
\hline $\operatorname{loghat} 10$ & 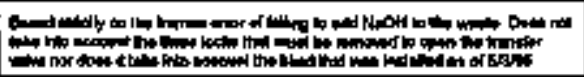 & & & & & & & \\
\hline 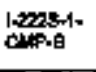 & 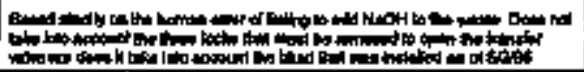 & & & & & & & \\
\hline
\end{tabular}




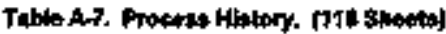

\begin{tabular}{|c|c|c|c|c|c|c|c|c|}
\hline $\mathbf{D}$ & MEMONE & Rimion & Finger & 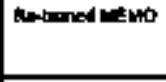 & ong & 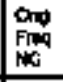 & ond & ineraks \\
\hline 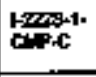 & Fars & & & & & & & \\
\hline 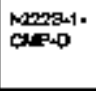 & 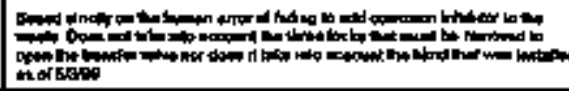 & & & & & & & \\
\hline censos. & 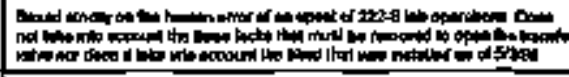 & & & & & & & \\
\hline $\operatorname{lin}^{\ln 2025-1-}$ & 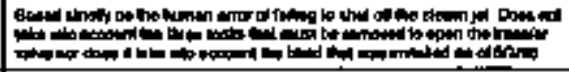 & & & & & & & \\
\hline 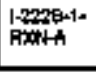 & 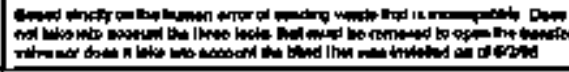 & & & & & & & \\
\hline Fint & 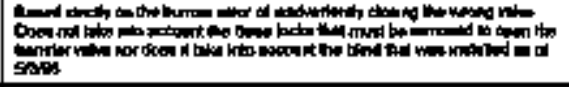 & & & & & & & \\
\hline $\log 5$ & 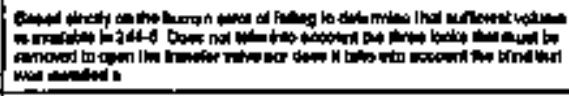 & & & & & & & \\
\hline FLWF & 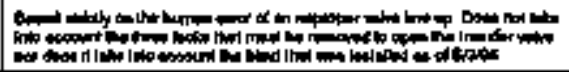 & & & & & & & \\
\hline FLWtit & 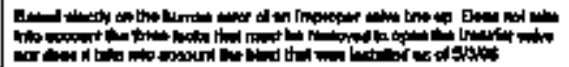 & & & & & & & \\
\hline $\operatorname{tog} 2$ & 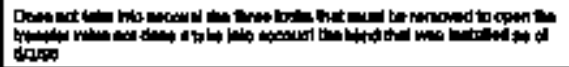 & & & & & & & \\
\hline
\end{tabular}




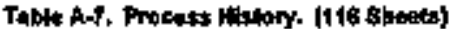

\begin{tabular}{|c|c|c|c|c|c|c|c|c|}
\hline$\Phi$ & بع & $\lim _{\text {simitad }}$ & 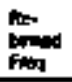 & the-buned Mtes & Frog & ing & Ding & Fenciles \\
\hline 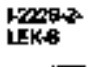 & 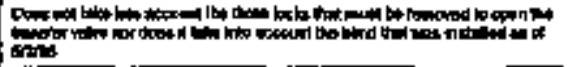 & & & & & & & \\
\hline 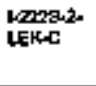 & 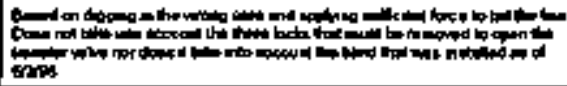 & & & & & & & \\
\hline $\log _{\log 5}$ & 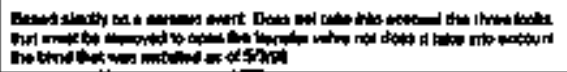 & & & & & & & \\
\hline $\begin{array}{l}\text { l.2029-2 } \\
\text { Lexe }\end{array}$ & 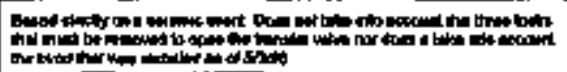 & & & & & & & \\
\hline $\operatorname{PRSA}_{1202}$ & 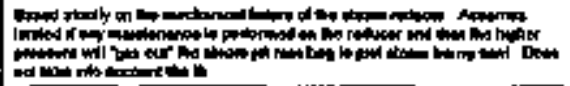 & & & & & & & \\
\hline $\begin{array}{l}\text { L2A2A4A } \\
\text { PLWA }\end{array}$ & 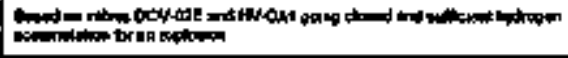 & & & & & & & \\
\hline 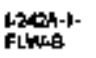 & 的 & & & & & & & \\
\hline $\begin{array}{ll}\text { roch-1- } \\
\text { rowe }\end{array}$ & 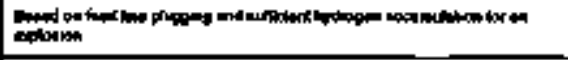 & & & & & & & \\
\hline anted & 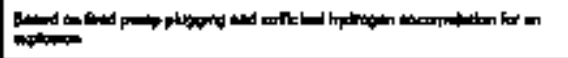 & & & & & & & \\
\hline Futwet & 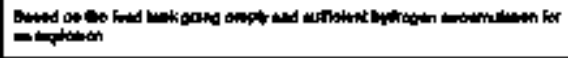 & & & & & & & \\
\hline \multicolumn{9}{|l|}{$\begin{array}{l}1-242+1+1+ \\
\text { Flwf }\end{array}$} \\
\hline $\begin{array}{l}\text { lowate-10 } \\
\text { Lexan }\end{array}$ & & & & & & & & \\
\hline
\end{tabular}




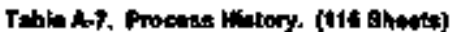

\begin{tabular}{|c|c|c|c|c|c|c|c|c|}
\hline $\mathbf{0}$ & HeNo & 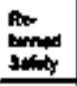 & $\begin{array}{l}\text { Ror } \\
\text { tonined } \\
\text { Prid }\end{array}$ & Dobanod Maseb & Ond & $\begin{array}{l}\text { Ody } \\
\text { Friec } \\
\text { its }\end{array}$ & Dentrir & Rametr \\
\hline \multicolumn{9}{|l|}{ 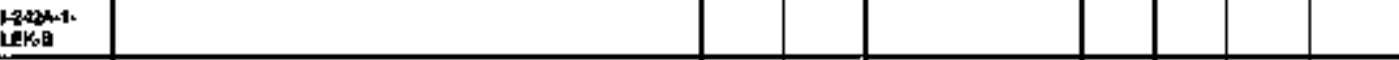 } \\
\hline $\begin{array}{l}\text { LZCEA-1r } \\
\text { LEKC }\end{array}$ & 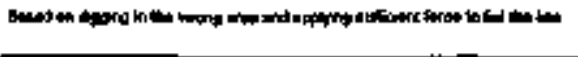 & & & & & & & \\
\hline $\begin{array}{l}1-2 \times 2011 \\
1 \times 160\end{array}$ & |ll & & & & & & & \\
\hline $\begin{array}{l}\text { logethit } \\
\operatorname{Lax} E\end{array}$ & Bund ontengen ment & & & & & & & \\
\hline \multicolumn{9}{|l|}{$\begin{array}{l}1.200+2-2 \\
\text { ctophe }\end{array}$} \\
\hline 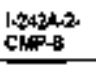 & 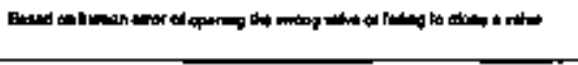 & & & & & & & \\
\hline 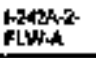 & 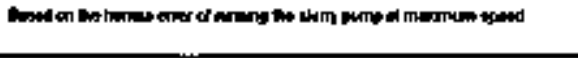 & & & & & & & \\
\hline 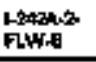 & 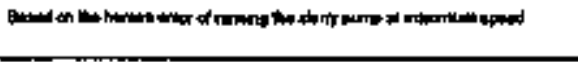 & & & & & & & \\
\hline \multicolumn{9}{|l|}{ 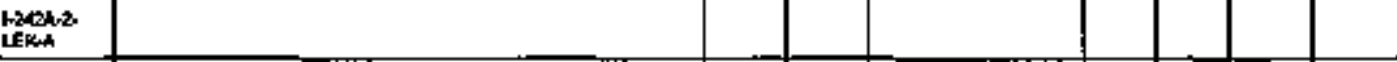 } \\
\hline \multicolumn{9}{|l|}{ Lever2 } \\
\hline $\begin{array}{l}\text { Lashin. } \\
\text { LEKC }\end{array}$ & 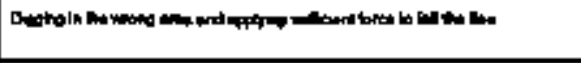 & & & & & & & \\
\hline $\begin{array}{l}10 \operatorname{sen} 2 \\
1 \exp 0\end{array}$ & 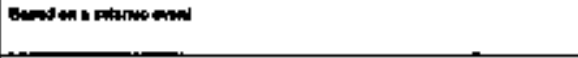 & & & & & & & \\
\hline loperte & 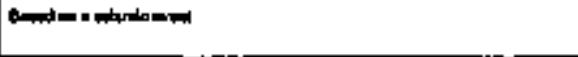 & & & & & & & \\
\hline $\begin{array}{l}\text { ladela } \\
\text { ThPA }\end{array}$ & & & & & & & & \\
\hline
\end{tabular}


Tablo A-T. Procede Hitry. (116 Btwats)

\begin{tabular}{|c|c|c|c|c|c|c|c|c|}
\hline I8 & Mano nc & $\begin{array}{l}\text { Mat } \\
\text { banned } \\
\text { Banit }\end{array}$ & Fined & Mosthned hath & ind & 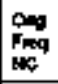 & ong & Him \\
\hline 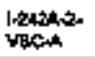 & 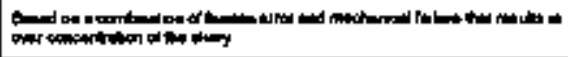 & & & & & & & \\
\hline 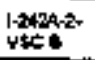 & & & & & & & & \\
\hline loglowhes & 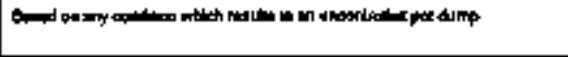 & & & & & & & \\
\hline Hates & 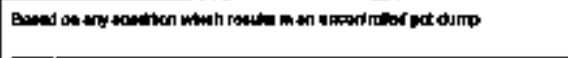 & & & & & & & \\
\hline FLenth & 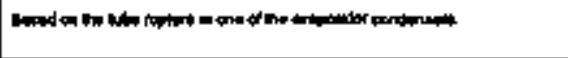 & & & & & & & \\
\hline Laph-4 & 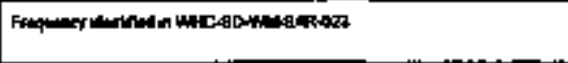 & & & & & & & \\
\hline Finte- & 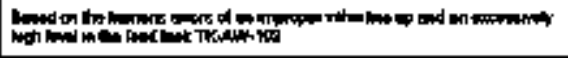 & & & & & & & \\
\hline Lapento & 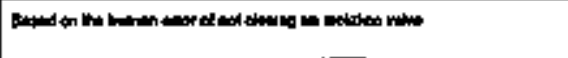 & & & & & & & \\
\hline rowat & 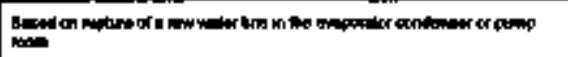 & & & & & & & \\
\hline Fintion & 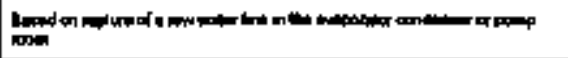 & & & & & & & \\
\hline rate & 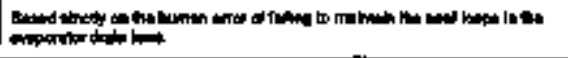 & & & & & & & \\
\hline $\lim ^{2} \operatorname{sen} x-1$ & 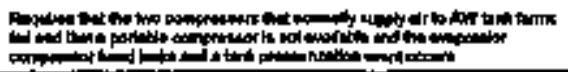 & & & & & & & \\
\hline Latert- & 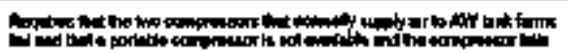 & & & & & & & \\
\hline
\end{tabular}


Table A.7. Proceas Hitory. [116 Brebts]

\begin{tabular}{|c|c|c|c|c|c|c|c|c|}
\hline $\mathbf{0}$ & 수구웅 & borm & Fined & 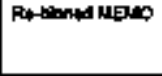 & Pind & $\lim _{\min }$ & Ont & Rention \\
\hline 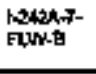 & 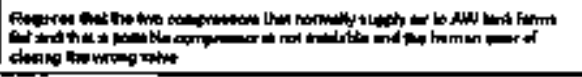 & & & & & & & \\
\hline 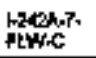 & 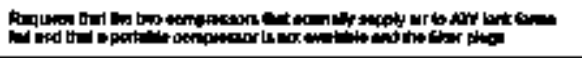 & & & & & & & \\
\hline $\operatorname{lomsin}^{2}$ & 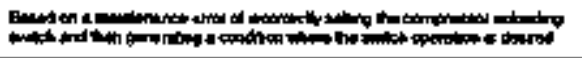 & & & & & & & \\
\hline peste-1- & 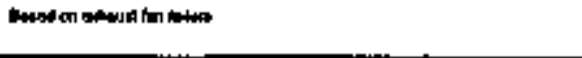 & & & & & & & \\
\hline 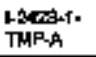 & 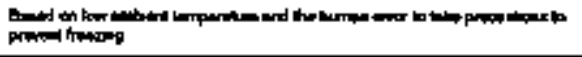 & & & & & & & \\
\hline 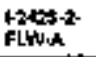 & 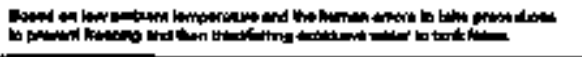 & & & & & - & & \\
\hline $\begin{array}{l}\operatorname{loces} 2 \\
\text { flint }\end{array}$ & 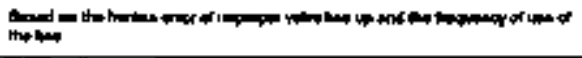 & & & & & & & \\
\hline 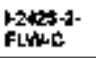 & 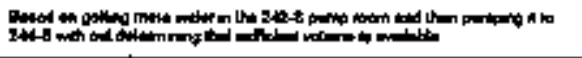 & & & & & & & \\
\hline Flogen & 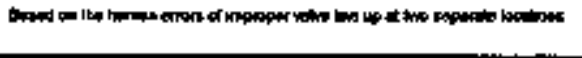 & & & & & & & \\
\hline Funte & 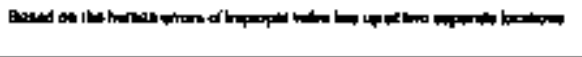 & & & & & & & \\
\hline $\log _{\log 20}$ & & & & & & & & \\
\hline $\begin{array}{l}1-2428-2 \\
1.248\end{array}$ & & & & & & & & \\
\hline $\begin{array}{l}1.24052 \\
\text { Lex-c }\end{array}$ & 管 & & & & & & & \\
\hline
\end{tabular}




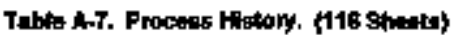

\begin{tabular}{|c|c|c|c|c|c|c|c|c|}
\hline 10 & 虾直 & for & $\begin{array}{l}\text { Rit } \\
\text { tomined } \\
\text { Find }\end{array}$ & Aethrued thesto & Ond & $\begin{array}{l}\text { Ong } \\
\text { Fing }\end{array}$ & ong & $R=1$ \\
\hline $\begin{array}{l}\text { lowisst- } \\
\text { Lexp }\end{array}$ & 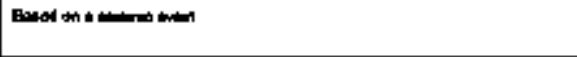 & & & & & & & \\
\hline 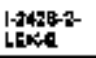 & 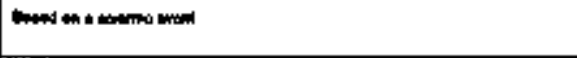 & & & & & & & \\
\hline $\begin{array}{l}\text { lostitset } \\
\text { Fuwat }\end{array}$ & 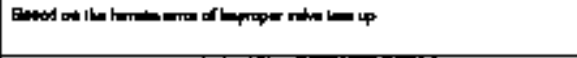 & & & & & & & \\
\hline -2428-S & 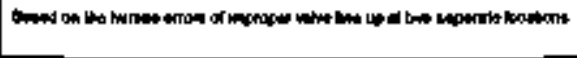 & & & & & & & \\
\hline $\begin{array}{l}1,2425-4 \\
F(W-C)\end{array}$ & 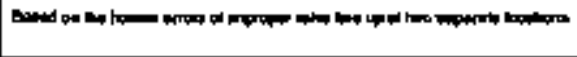 & & & & & & & \\
\hline fletes & 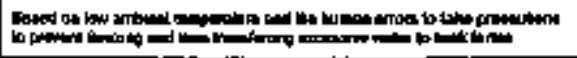 & & & & & & & \\
\hline 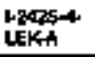 & & & & & & & & \\
\hline $\begin{array}{l}\text { hess-A } \\
\text { Leks }\end{array}$ & & & & & & & & \\
\hline 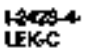 & S & & & & & & & \\
\hline $\begin{array}{l}\text { latst } \\
\text { LEx-b }\end{array}$ & Boged & & & & & & & \\
\hline 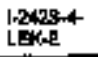 & 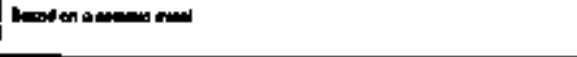 & & & & & & & \\
\hline L-2428-s- & 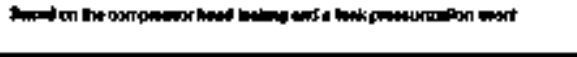 & & & & & & & \\
\hline 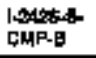 & 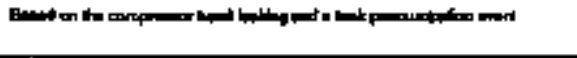 & & & & & & & \\
\hline 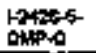 & 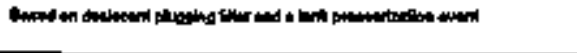 & & & & & & & \\
\hline
\end{tabular}




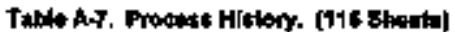

\begin{tabular}{|c|c|c|c|c|c|c|c|c|}
\hline 10 & 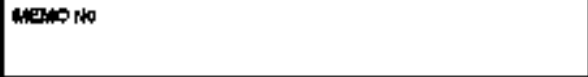 & tinnod & Fon- & 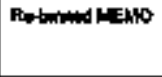 & Ong & Png & Ong & Ranmils \\
\hline 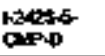 & 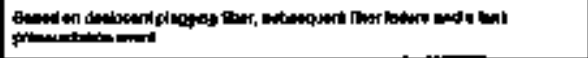 & & & & & & & \\
\hline kass-s- & 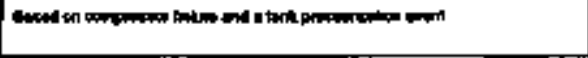 & & & & & & & \\
\hline $\begin{array}{l}12428.5 \\
74 m-3\end{array}$ & 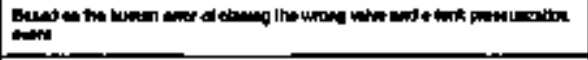 & & & & & & & \\
\hline $\begin{array}{l}\text { 1-27855- } \\
\text { nwas }\end{array}$ & 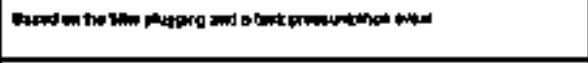 & & & & & & & \\
\hline $\begin{array}{l}\text { lo2t25s } \\
\text { PRREA }\end{array}$ & 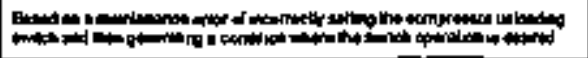 & & & & & & & \\
\hline 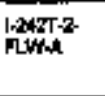 & 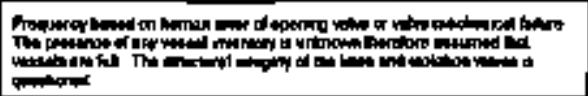 & & & & & & & \\
\hline $\begin{array}{l}-2+42 T-3- \\
\mid L W-4\end{array}$ & A & & & & & & & \\
\hline $\begin{array}{l}\text { Last-4 } \\
\text { FLWh- }\end{array}$ & 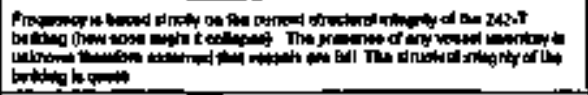 & & & & & & & \\
\hline Hapunt- & 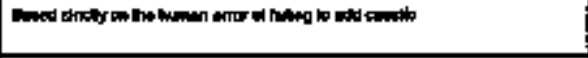 & & & & & & & \\
\hline 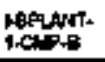 & 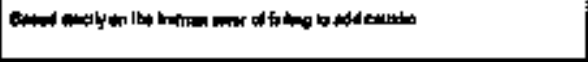 & & & & & & & \\
\hline 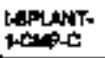 & 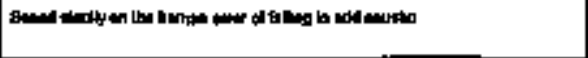 & & & & & & & \\
\hline 1.APLANT- & 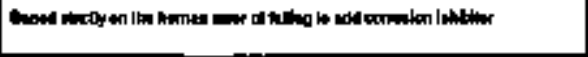 & & & & & & & \\
\hline
\end{tabular}




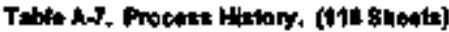

\begin{tabular}{|c|c|c|c|c|c|c|c|c|}
\hline 10 & Nater & Pos & $\begin{array}{l}\text { Pre } \\
\text { bond } \\
\text { from }\end{array}$ & Pabrend Mate & Ong & $\begin{array}{l}\text { Ond } \\
\text { Fins } \\
\text { its }\end{array}$ & Deng & Finmatin \\
\hline 1.9PUNT. & 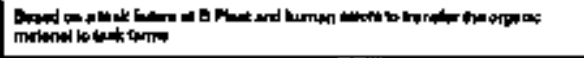 & & & & & & & \\
\hline 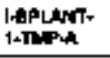 & 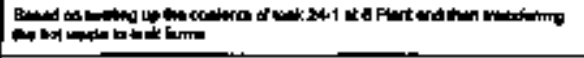 & & & & & & & \\
\hline \multicolumn{9}{|l|}{ 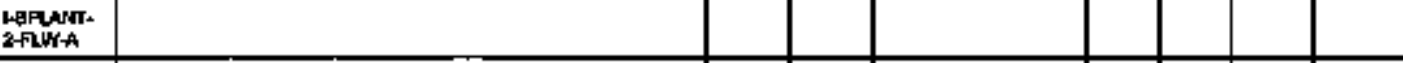 } \\
\hline $\begin{array}{l}\text { PE:LANT. } \\
\text { ZFLWWAB }\end{array}$ & 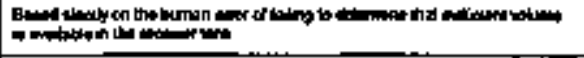 & & & & & & & \\
\hline LPFLANT- & 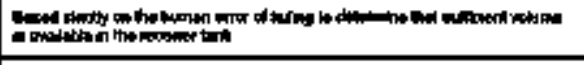 & & & & & & & \\
\hline $\begin{array}{l}\text { HEPLANT. } \\
\text { ZFLWE- }\end{array}$ & 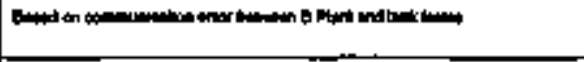 & & & & & & & \\
\hline \multicolumn{9}{|l|}{$\begin{array}{l}\text { 1-6PLANT- } \\
\text { 2CUKA- }\end{array}$} \\
\hline \multicolumn{9}{|l|}{$\begin{array}{l}\text { IAPLANT. } \\
\text { 2HEK }\end{array}$} \\
\hline 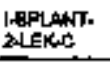 & 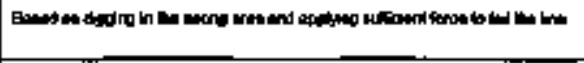 & & & & & & & \\
\hline $\begin{array}{l}\text { LEPLANT- } \\
\text { 2HEK-D }\end{array}$ & 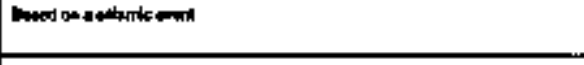 & & & & & & & \\
\hline $\begin{array}{l}\text { LOLWWT. } \\
\text { ZLEXE }\end{array}$ & 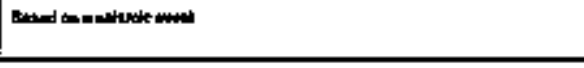 & & & & & & & \\
\hline Hatp-i- & 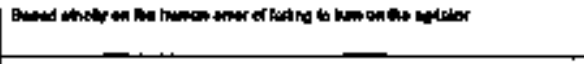 & & & & & & & \\
\hline $\begin{array}{lll}\text { LFFP-1 } \\
\text { Sam }\end{array}$ & 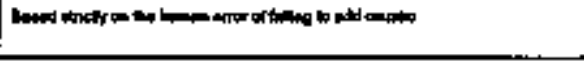 & & & & & & & \\
\hline $\begin{array}{l}\text { lefp.11 } \\
\text { ctepse }\end{array}$ & 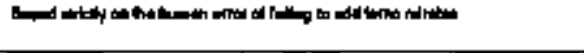 & & & & & & & \\
\hline
\end{tabular}


Tabla A7. Procese Hiatory. (116 shents)

\begin{tabular}{|c|c|c|c|c|c|c|c|c|}
\hline $\mathbf{m}$ & helog nat & $\begin{array}{l}\text { Ro- } \\
\text { hirned } \\
\text { Soling }\end{array}$ & $\lim _{\lim }$ & 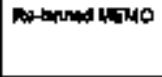 & Fran & $\begin{array}{l}\text { Ordo } \\
\text { Nem } \\
\text { Neq }\end{array}$ & Ong & Hertriald \\
\hline GAFP-1- & 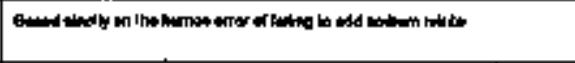 & & & & & & & \\
\hline$\underset{\operatorname{compl}-1-}{\operatorname{tmot}}$ & 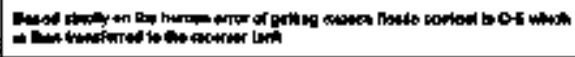 & & & & & & & \\
\hline $\begin{array}{l}\text { MPFP.1T } \\
\text { CAPPE }\end{array}$ & 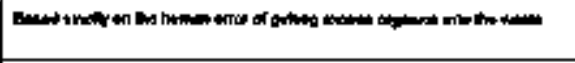 & & & & & & & \\
\hline $\begin{array}{l}\text { LPFP-1- } \\
\text { LWHA }\end{array}$ & 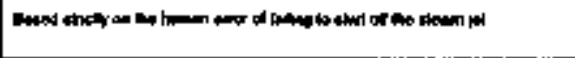 & & & & & & & \\
\hline |PFP.1. & 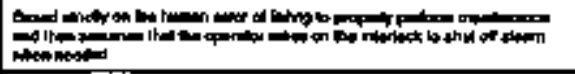 & & & & & & & \\
\hline loppa-s- & 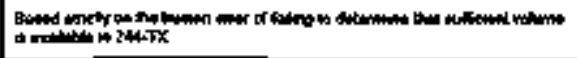 & & & & & & & \\
\hline LOPAP-2. & 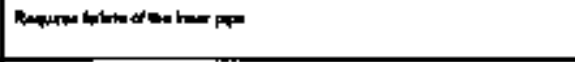 & & & & & & & \\
\hline \multicolumn{9}{|l|}{ 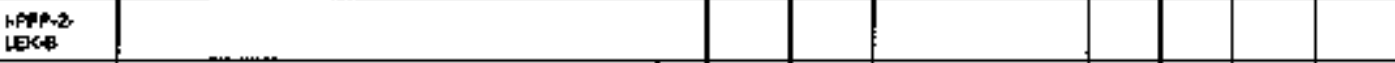 } \\
\hline $\begin{array}{l}\text { LFPR-2 } \\
\text { were }\end{array}$ & 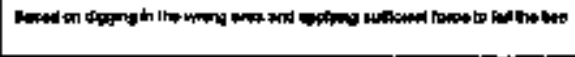 & & & & & & & \\
\hline 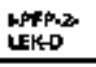 & 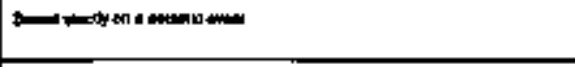 & & & & & & & \\
\hline Lepest.2. & 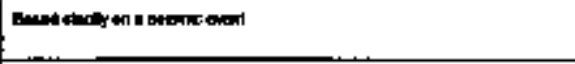 & & & & & & & \\
\hline LFPr-7 & 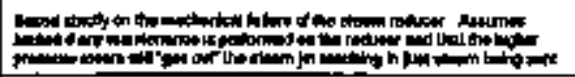 & & & & & & & \\
\hline IPFe & 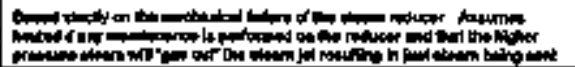 & & & & & & & \\
\hline
\end{tabular}


Tabte M-T. Procass Hutory, h16 9kents]

\begin{tabular}{|c|c|c|c|c|c|c|c|c|}
\hline $\mathbf{m}$ & MENo Ne & portad & $\lim _{\text {innod }}$ & 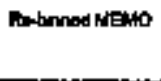 & Fan & $\begin{array}{l}\text { Ong } \\
\text { Fog } \\
\text { NO }\end{array}$ & Ong & Famiti \\
\hline $\begin{array}{l}\text { LFPs- } \\
\text { chinth }\end{array}$ & 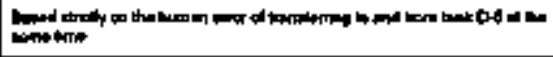 & & & & & & & \\
\hline $\begin{array}{l}\text { RPFPat } \\
\text { FLine }\end{array}$ & 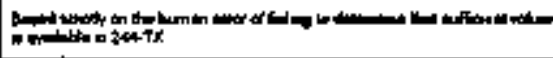 & & & & & & & \\
\hline $\begin{array}{ll}\text { Lepez- } \\
\text { Flinte }\end{array}$ & | & & & & & & & \\
\hline Letpis & 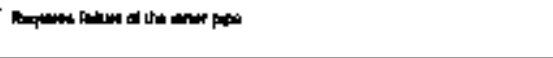 & & & & & & & \\
\hline lepp-3 & & & & & & & & \\
\hline $\begin{array}{l}\text { I.PPPA } \\
\text { LEX-C }\end{array}$ & 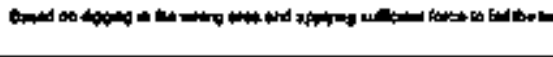 & & & & & & & \\
\hline $\begin{array}{l}\text { IfPp3 } \\
\text { WNo }\end{array}$ & 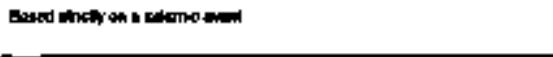 & & & & & & & \\
\hline $\begin{array}{l}\text { LAfPit } \\
\text { LakE }\end{array}$ & 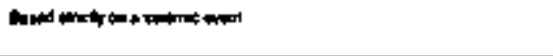 & & & & & & & \\
\hline 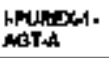 & 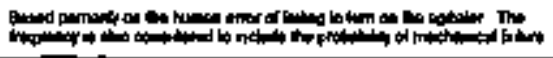 & & & & & & & \\
\hline HURTX-1- & 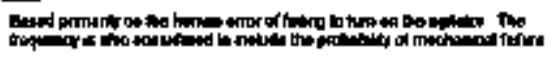 & & & & & & & \\
\hline $\begin{array}{l}\text { LFUh:-1. } \\
\text { AGT-C }\end{array}$ & 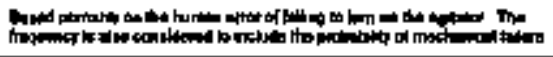 & & & & & & & \\
\hline 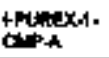 & 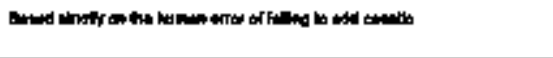 & & & & & & & \\
\hline 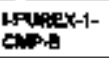 & 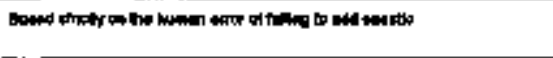 & & & & & & & \\
\hline 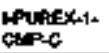 & & & & & & & & \\
\hline
\end{tabular}


Tabu A-7. Process Hertory. (116 thutt)

\begin{tabular}{|c|c|c|c|c|c|c|c|c|}
\hline ID & $M=M O M$ & Bintir & fined & 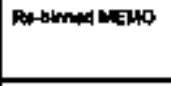 & on & 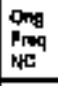 & ority & 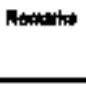 \\
\hline \multicolumn{9}{|l|}{ 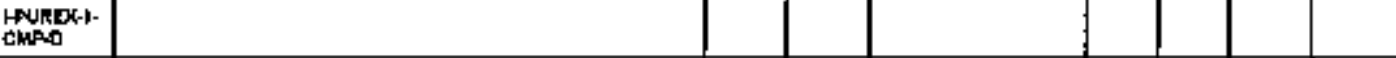 } \\
\hline 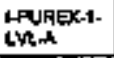 & 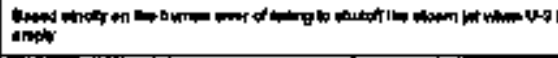 & & & & & & & \\
\hline $\begin{array}{l}\text { tPURDX-1 } \\
\text { THAMA }\end{array}$ & 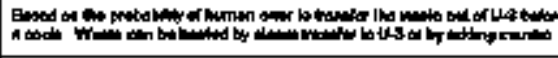 & & & & & & & \\
\hline \multicolumn{9}{|l|}{$\begin{array}{l}\text { PRARER } \\
\text { FLWA }\end{array}$} \\
\hline \multicolumn{9}{|l|}{ 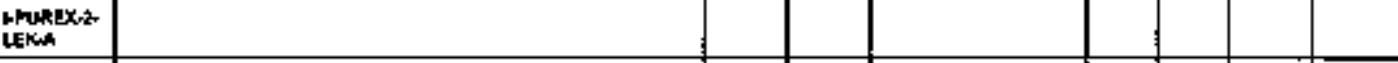 } \\
\hline \multicolumn{9}{|l|}{$\begin{array}{l}\text { LPLWEEX-2- } \\
\text { LEKA }\end{array}$} \\
\hline $\log _{0}$ & 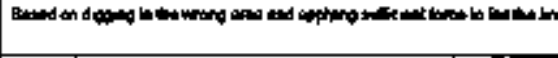 & & & & & & & \\
\hline 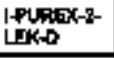 & 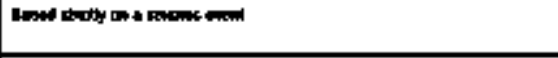 & & & & & & & \\
\hline $\begin{array}{l}\text { lofunex-2 } \\
\text { LEXEE }\end{array}$ & 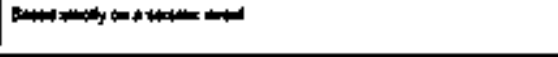 & & & & & & & \\
\hline 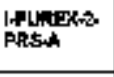 & 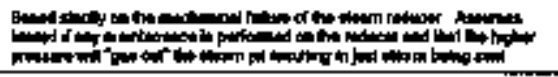 & & & & & & & \\
\hline Pangex-2 & 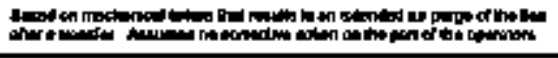 & & & & & & & \\
\hline \multicolumn{9}{|l|}{$\begin{array}{l}\text { LFUREX6. } \\
\text { Pres-C }\end{array}$} \\
\hline $\operatorname{linR}_{\text {int }}$ & 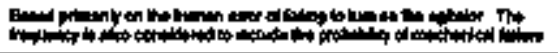 & & & & & & & \\
\hline
\end{tabular}




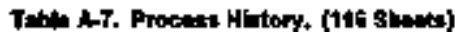

\begin{tabular}{|c|c|c|c|c|c|c|c|c|}
\hline 10 & Merm m & $\begin{array}{l}\text { hit } \\
\text { bhined } \\
\text { tatit }\end{array}$ & 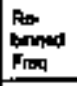 & Ptormead MES & Freq & Orig & ontion & Pentratsos \\
\hline $\begin{array}{l}\text { RFUREKa } \\
\text { AGT-8 }\end{array}$ & 然 & & & & & & & \\
\hline 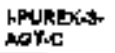 & 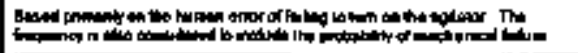 & & & & & & & \\
\hline $\begin{array}{l}\text { LPUREK3 } \\
\text { CAPA }\end{array}$ & 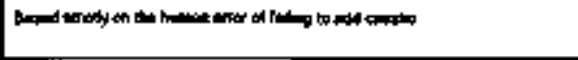 & & & & & & & \\
\hline 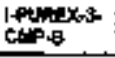 & 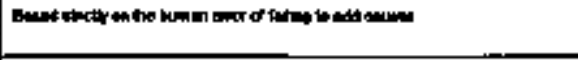 & & & & & & & \\
\hline \multicolumn{9}{|l|}{$\begin{array}{l}\text { letphex-3 } \\
\text { ctimic }\end{array}$} \\
\hline \multicolumn{9}{|l|}{ lepnats } \\
\hline 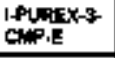 & 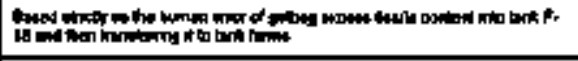 & & & & & & & \\
\hline $\begin{array}{l}\text { lempext- } \\
\text { Linn }\end{array}$ & 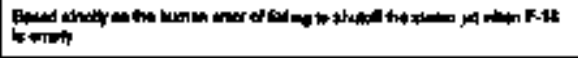 & & & & & & & \\
\hline 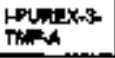 & 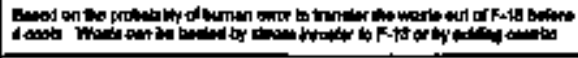 & & & & & & & \\
\hline $\begin{array}{l}\text { LPUREX-A } \\
\text { FUWF }\end{array}$ & 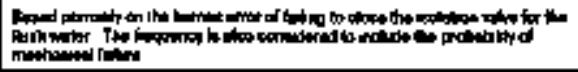 & & & & & & & \\
\hline \multicolumn{9}{|l|}{ LPUREX-4. } \\
\hline \multicolumn{9}{|l|}{$\begin{array}{l}\text { IPURET-4 } \\
\text { IUKe }\end{array}$} \\
\hline 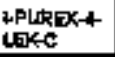 & 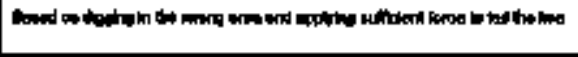 & & & & & & & \\
\hline
\end{tabular}




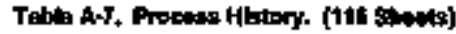

\begin{tabular}{|c|c|c|c|c|c|c|c|c|}
\hline $\mathbf{0}$ & weno wx & Ber. & fined & 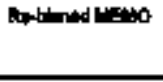 & ind & $\operatorname{mov}_{\operatorname{mog}}$ & sitit & fombits \\
\hline $\begin{array}{l}\text { l.PUREX-4 } \\
\text { LEK-0 }\end{array}$ & 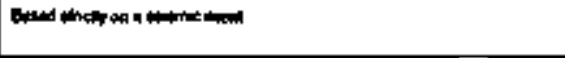 & & & & & & & \\
\hline $\begin{array}{l}\text { lefurgit } \\
\text { Leter }\end{array}$ & | & & & & & & & \\
\hline $\begin{array}{l}\text { 2PUREX-4 } \\
\text { PRAP }\end{array}$ & 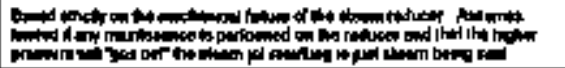 & & & & & & & \\
\hline 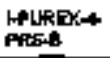 & 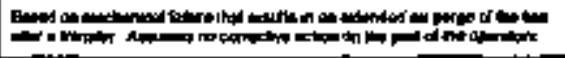 & & & & & & & \\
\hline $\begin{array}{l}\text { HPURES-4 } \\
\text { PRSC }\end{array}$ & & & & & & & & \\
\hline $\begin{array}{l}\text { HFURext- } \\
\text { FLWhin }\end{array}$ & 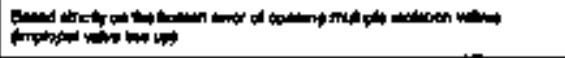 & & & & & & & \\
\hline 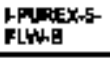 & 管 & & & & & & & \\
\hline 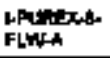 & 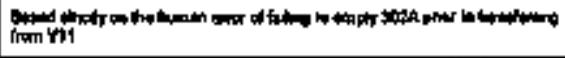 & & & & & & & \\
\hline 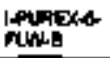 & 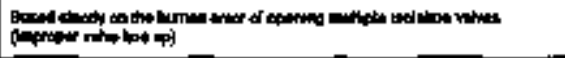 & & & & & & & \\
\hline $\begin{array}{l}\text { coutex-8- } \\
\text { Funte }\end{array}$ & 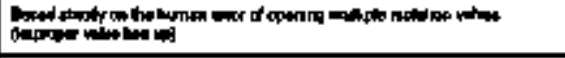 & & & & & & & \\
\hline 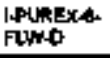 & & & & & & & & \\
\hline 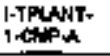 & 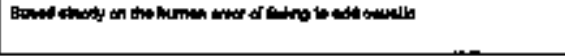 & & & & & & & \\
\hline 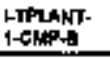 & 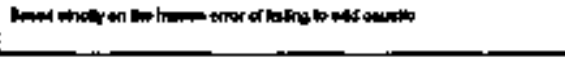 & & & & & & & \\
\hline
\end{tabular}




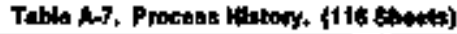

\begin{tabular}{|c|c|c|c|c|c|c|c|c|}
\hline 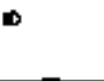 & HEDit & $\begin{array}{l}\text { Po. } \\
\text { solingy }\end{array}$ & 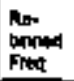 & Relinned ME & Fing & $\begin{array}{l}\text { hrd } \\
\text { Fra } \\
\text { lic }\end{array}$ & ton & fumerlo \\
\hline D-TPLinit- & 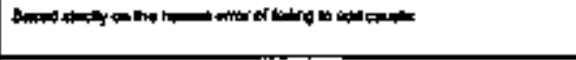 & & & & & & & \\
\hline 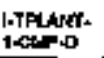 & 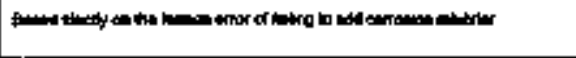 & & & & & & & \\
\hline 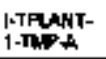 & 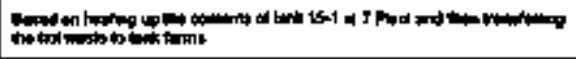 & & & & & & & \\
\hline 1.TPUANT. & 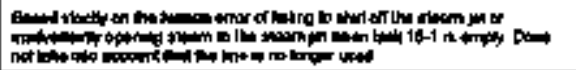 & & & & & & & \\
\hline $\begin{array}{l}\text { HIPLAT- } \\
\text { 2.PLWWh }\end{array}$ & 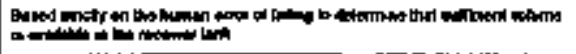 & & & & & & & \\
\hline 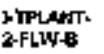 & 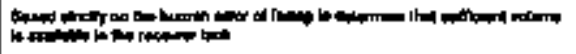 & & & & & & & \\
\hline 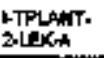 & & & & & & & & \\
\hline $\begin{array}{l}\text { PTPUNT- } \\
\text { 2-4EK:E }\end{array}$ & & & & & & & & \\
\hline $\begin{array}{l}\text { RTPLiT. } \\
\text { 24EKG: }\end{array}$ & 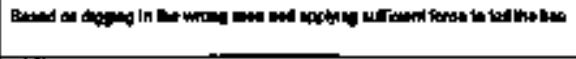 & & & & & & & \\
\hline $\begin{array}{l}\text { PTPLAMT. } \\
\text { 2HEKA: }\end{array}$ & 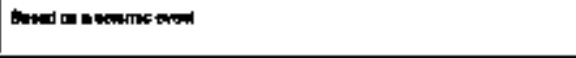 & & & & & & & \\
\hline $\begin{array}{l}\text { LTPLANY- } \\
\text { 2-LER-E }\end{array}$ & 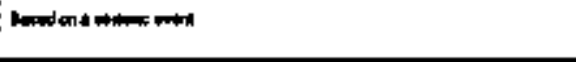 & & & & & & & \\
\hline 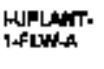 & 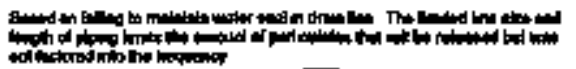 & & & & & & & \\
\hline $\begin{array}{l}\text { HuPLATT. } \\
\text { 1.LEKN }\end{array}$ & 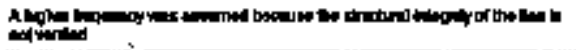 & & & & & & & \\
\hline
\end{tabular}




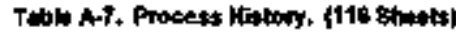

\begin{tabular}{|c|c|c|c|c|c|c|c|c|}
\hline ID & HENO NOC & $\operatorname{lom}_{\log }$ & $\lim _{\lim }$ & Folvent MEN & Pren & $\begin{array}{l}\text { Ong } \\
\text { Nin } \\
\text { NC }\end{array}$ & Oridis & Fesmerie \\
\hline $\begin{array}{l}\text { HPLANT. } \\
\text { 1.2EK.B }\end{array}$ & 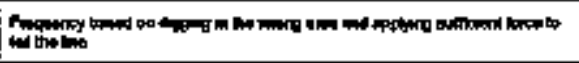 & & & & & & & \\
\hline 1-LPENAT: & 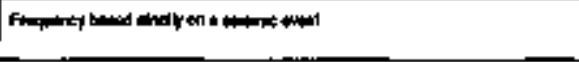 & & & & & & & \\
\hline H.RPLART- & 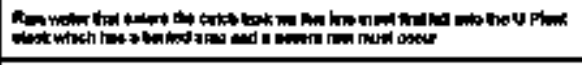 & & & & & & & \\
\hline rtroth & 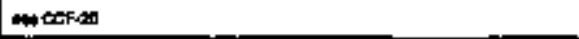 & & & & F 2 & $\mathbf{F t}$ & $\mathbf{s i}$ & \\
\hline mस्पा & 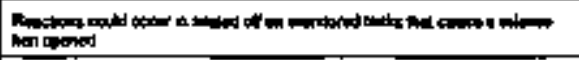 & & & & $\mathbf{F 1}$ & & 81 & \\
\hline ITKNOE & 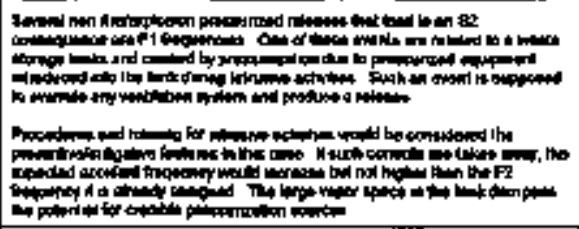 & & & & $\mathbf{n}$ & $\mathbf{n}$ & $\mathbf{s 1}$ & \\
\hline TKG & 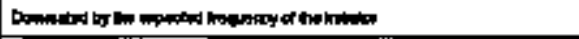 & & & & $\mathbf{F 1}$ & & 61 & \\
\hline ITK- & 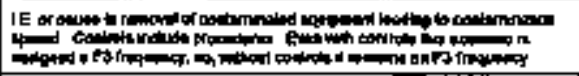 & & & & $\mathbf{P}$ & $\mathbf{r}$ & $\$ 1$ & \\
\hline$m k-\infty$ & 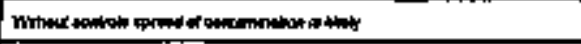 & & & & $n$ & & $\$ 1$ & \\
\hline inkous & 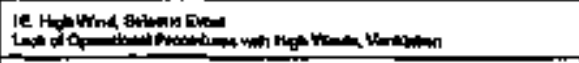 & & & & $\mathbf{F 1}$ & $\mathbf{F s}$ & si & \\
\hline ink-04 & 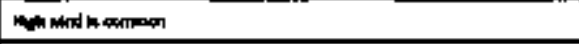 & & & & ro & & 81 & \\
\hline mKos & 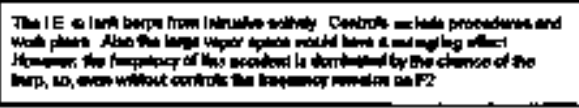 & 62 & & 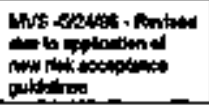 & $\mathbf{P} 2$ & $P 2$ & 81 & \\
\hline
\end{tabular}


Tubth M.3. Prockas Hiatory, (11t ahestr)

\begin{tabular}{|c|c|c|c|c|c|c|c|c|}
\hline 中 & MFino No & $\begin{array}{l}\text { Por } \\
\text { binned } \\
\text { Exhot }\end{array}$ & Por & 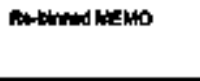 & ind & $\begin{array}{l}\text { ofid } \\
\lim _{1 \rightarrow 0}\end{array}$ & gras & Pemerit: \\
\hline mKats & 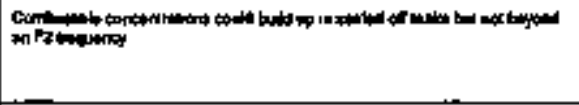 & $\mathbf{a z}$ & & 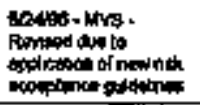 & $\mathbf{F 2}$ & & s1 & \\
\hline mK-at & 1E & & & & Fo & $\mathbf{F i}$ & \$2 & \\
\hline TKK-0Q & 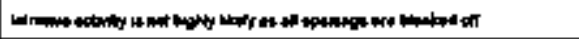 & & & & $\mathbf{F r}$ & & 82 & \\
\hline$\pi k-\phi$ & 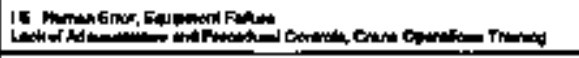 & & & & $\mathbf{F}$ & Fs & s) & \\
\hline ithentith & 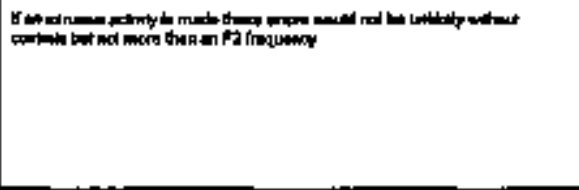 & & & & m & & st & 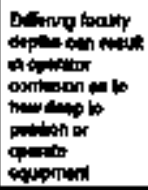 \\
\hline
\end{tabular}


Tebls A-7. Process thistory. (116 ghenta)

\begin{tabular}{|c|c|c|c|c|c|c|c|c|}
\hline 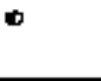 & (1) & inom & Frimed & fotolmad & $\lim _{\lim }$ & 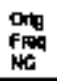 & $\sin$ & Nemplats \\
\hline ITKSONA & 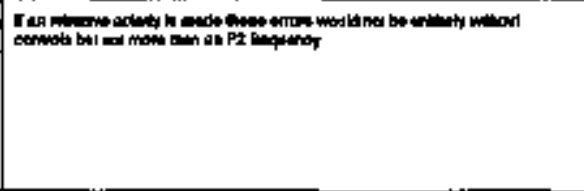 & & & & P2 & & $\mathbf{9 1}$ & 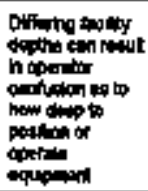 \\
\hline TKKOE & 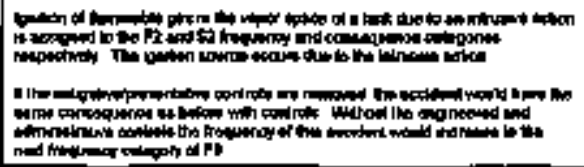 & $\$$ & & 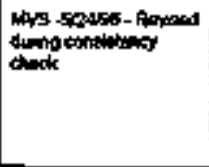 & F2 & $\mathbf{m}$ & 62 & \\
\hline mo-0e-1 & 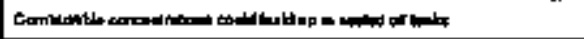 & & & & F1 & & 87 & \\
\hline mke0 & 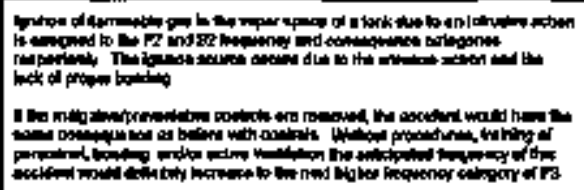 & 89 & & 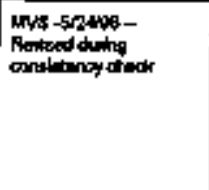 & $\mathbf{F}$ & $\mathbf{m}$ & $\mathbf{n}$ & \\
\hline ITKAs & 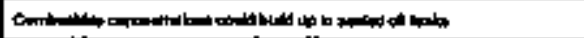 & & & & F & & $s$ & \\
\hline mKIOH & 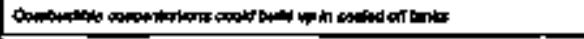 & & & & $\mathbf{F z}$ & & s2 & \\
\hline mkilas & 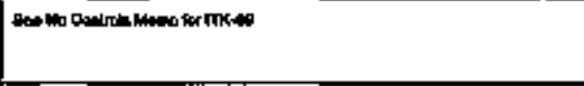 & ss & & 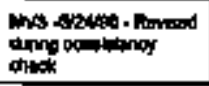 & $\mathbf{F} \mathbf{2}$ & H & $\$$ & \\
\hline mkitab & 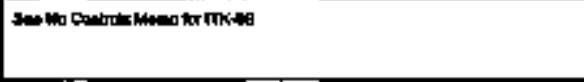 & 53 & & 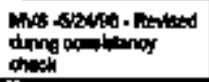 & $\mathbf{m}$ & Fa & 6 & \\
\hline
\end{tabular}




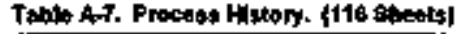

\begin{tabular}{|c|c|c|c|c|c|c|c|c|}
\hline $\mathbf{0}$ & Mandoc & $\lim _{\text {bimid }}$ & $\lim _{\substack{\operatorname{lom} \\
\lim }}$ & Rotannd Me & Ond & $\begin{array}{l}\text { Oro } \\
\text { friad } \\
\text { inc }\end{array}$ & ong & Rowestos \\
\hline BK-11 & 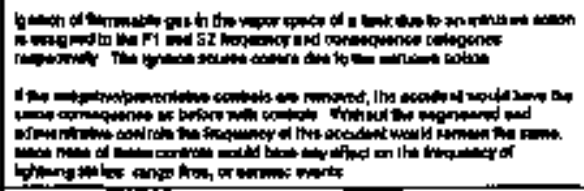 & $\$$ & & 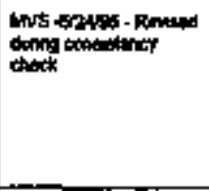 & F1 & $\mathbf{F}$ & 井 & \\
\hline mK-11- & 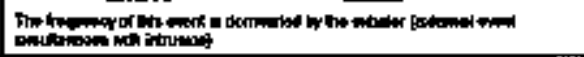 & & & & Fo & & 92 & \\
\hline ITK-PROT & 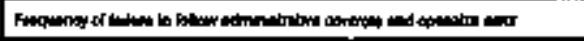 & & & & & & & \\
\hline ETK.FA. & 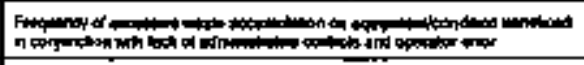 & & & & & & & \\
\hline ITKFFA-OS & lom & & & & & & & \\
\hline mK-Frou & 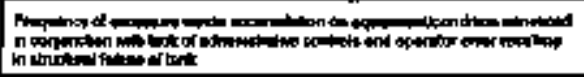 & & & & & & & \\
\hline 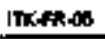 & Fapopay of of & & & & & & & \\
\hline InKfadet & 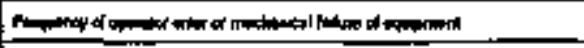 & & & & & & & \\
\hline TKKFR-OT & 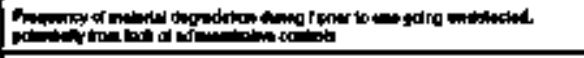 & & & & & & & \\
\hline ITKER-Ad & 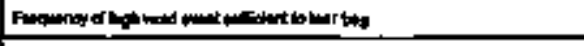 & & & & & & & \\
\hline ItKAFR-0 & 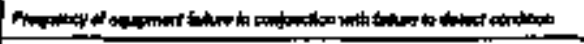 & & & & & & & \\
\hline TKKR:10 & 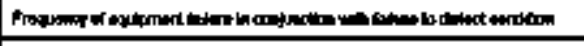 & & & & & & & \\
\hline MKKFE11 & 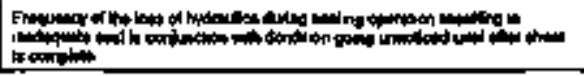 & & & & & & & \\
\hline
\end{tabular}


Tabl A-7+ Prockas Hefory. [116 shasts]

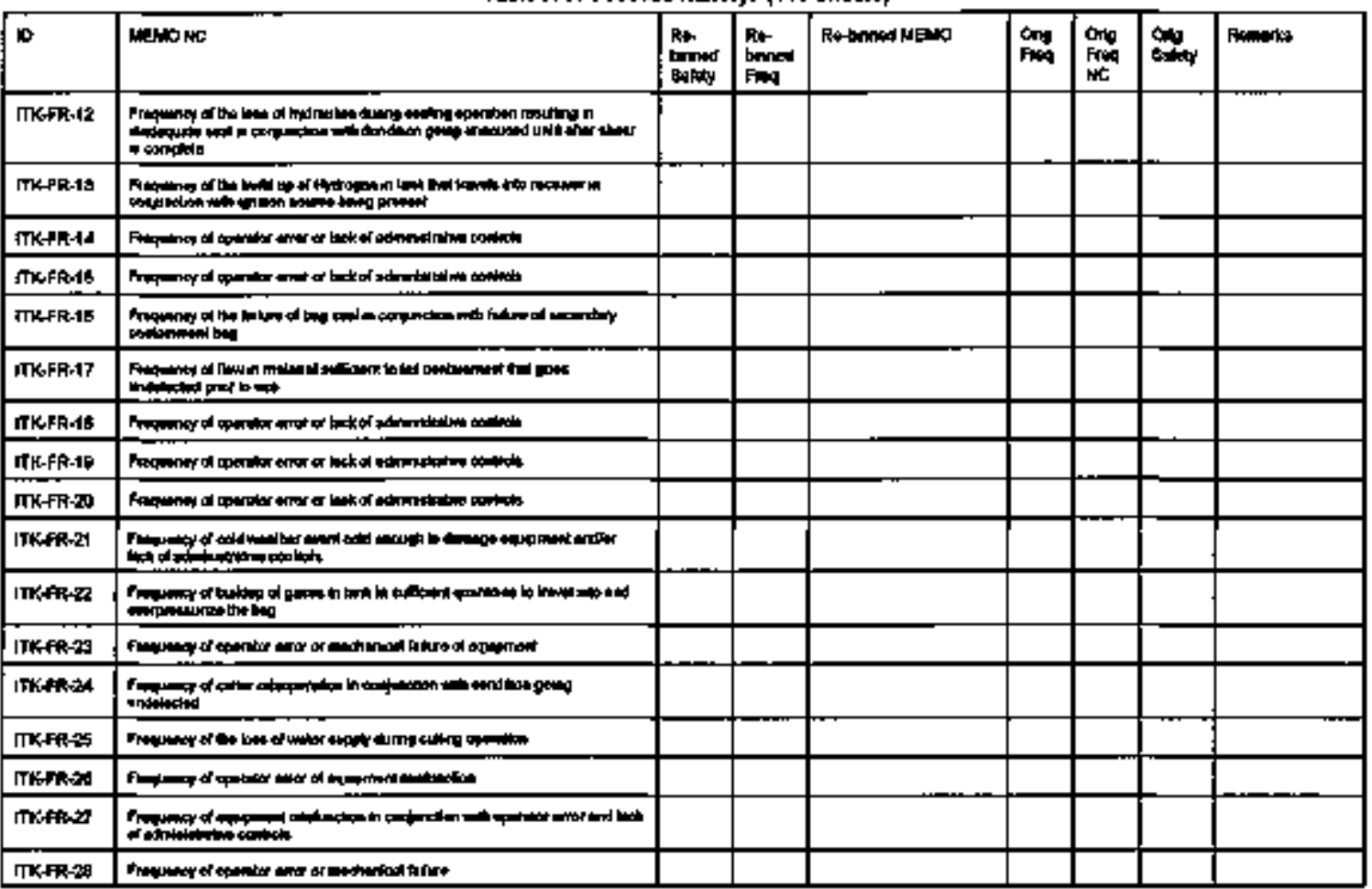


Tabfo A-7. Process Histery. (115 Shentsy

\begin{tabular}{|c|c|c|c|c|c|c|c|c|}
\hline 0 & אי סמשו & $\begin{array}{l}\text { Por } \\
\text { birned } \\
\text { selisy }\end{array}$ & $\begin{array}{l}\text { Rit } \\
\text { benned } \\
\text { Find }\end{array}$ & Robinnod AnENO & $\operatorname{ling}$ & $\lim _{i \rightarrow c}$ & Ond & Pemants \\
\hline NPAI & 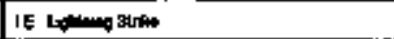 & & & & Fu & $\mathbf{s}$ & $\mathbf{s 1}$ & \\
\hline NPGR & 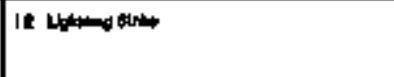 & 8 & & 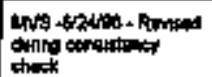 & F & $\mathbf{f}$ & 61 & \\
\hline D. & 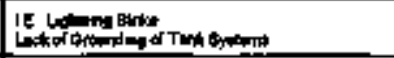 & & & & F1 & $\mathbf{m}$ & tha & \\
\hline 6P-65 & | & & & & f1 & F1 & 52 & \\
\hline $\operatorname{mes}$ & IE not wint & & & & F & $\mathbf{F 3}$ & st & \\
\hline$M P \cdot A$ & If B Bum Exht & & & & $\mathbf{F I}$ & $\mathbf{F i}_{1}$ & \$3 & \\
\hline 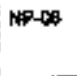 & 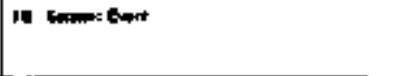 & 83 & & 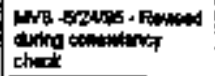 & FI & $\mathrm{Fz}$ & s1 & \\
\hline NPG & 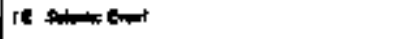 & & & & F1 & Fr & si & \\
\hline NP-10 & 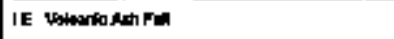 & & & & $\mathbf{r 2}$ & P2 & 81 & \\
\hline NNP-11 & IN IN & & & & Fo & Fo & 93 & \\
\hline$N P-12$ & 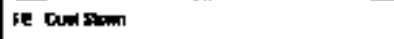 & & & & $\mathrm{Fs}$ & 73 & 31 & \\
\hline resala & 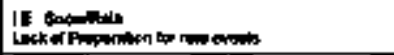 & & & & $\mathbf{F 1}$ & $\mathbf{F}$ & es & \\
\hline 10.14 & 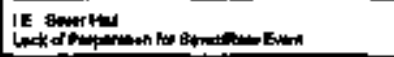 & & & & $\mathbf{r s}$ & P & 51 & \\
\hline Nop.15 & 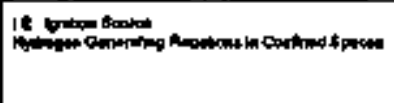 & 82 & & 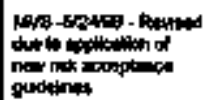 & $\mathbf{F}$ & $F$ & 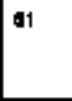 & \\
\hline
\end{tabular}


Tabto A-7. Prodeses Fifkory. 1116 theats

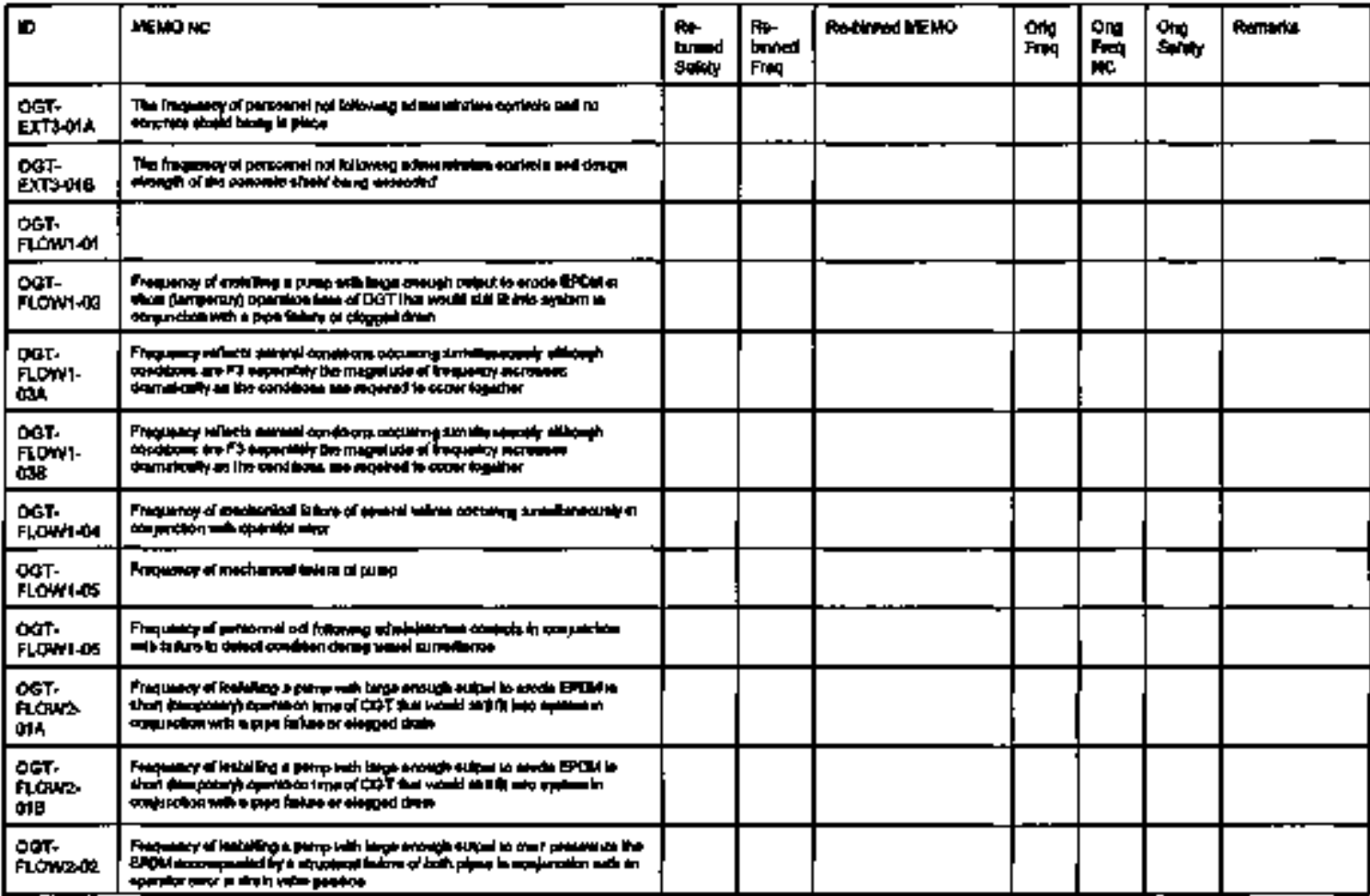


Talbe A-T. Process Hiskry. (119 9heats)

\begin{tabular}{|c|c|c|c|c|c|c|c|c|}
\hline 中 & udner & Row & Fingen & NWhinhad MENO & ore & 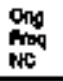 & and & 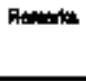 \\
\hline OogT: & 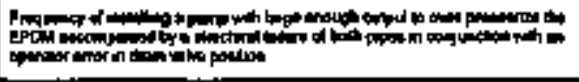 & & & & & & & \\
\hline 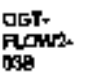 & | & & & & & & & \\
\hline OGT. & The & & & & & & & \\
\hline OCTI-NP3 & 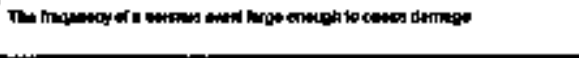 & & & & & & & \\
\hline $\begin{array}{l}\text { phr.Nopt } \\
\text { Da }\end{array}$ & 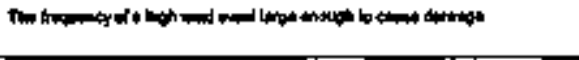 & & & & & & & \\
\hline OGI. & 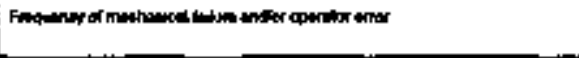 & & & & & & & \\
\hline $\begin{array}{l}\text { DoT. } \\
\text { PRetS }\end{array}$ & 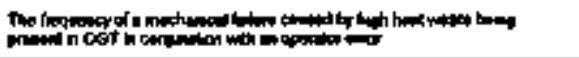 & & & & & & & \\
\hline Oat: & 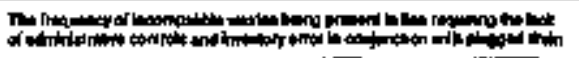 & & & & & & & \\
\hline DPT- & 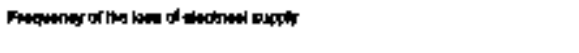 & & & & & & & \\
\hline Oxtr. & & & & & & & & \\
\hline OOT- & 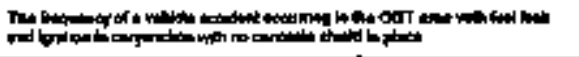 & & & & & & & \\
\hline oor- & 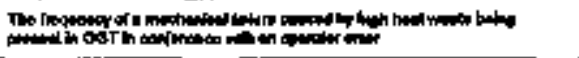 & & & & & & & \\
\hline Putoul & & & & & $\mathbf{p}$ & & s1 & \\
\hline
\end{tabular}


Table MA+ Process Hatory. (116 sheets)

\begin{tabular}{|c|c|c|c|c|c|c|c|c|}
\hline 10 & Utank & ber. & $\begin{array}{l}\text { Ror } \\
\text { bonnest } \\
\text { Prod }\end{array}$ & Restinned Hitio & $\operatorname{mon}$ & 员 & Dnt & Pennman \\
\hline AAOOHA & & & & & $m$ & & \$1 & 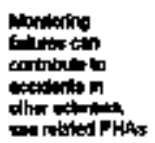 \\
\hline Redout & & & & & $\boldsymbol{F s}$ & & 81 & \\
\hline Rapdoe-1 & & & & & F1 & & si & 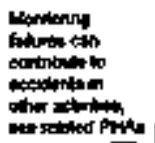 \\
\hline reaters & & & & & Fo & & 81 & \\
\hline RAD-03] & & & & & $F 2$ & & $\$ 1$ & 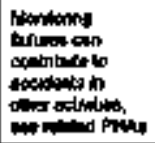 \\
\hline FESA-AI & 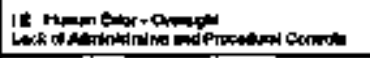 & & & & $F$ & $\mathrm{Fg}$ & s1 & \\
\hline estert & 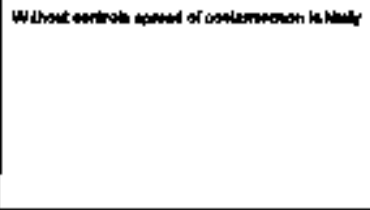 & & & & $\mathbf{F z}$ & & s1 & 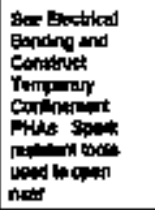 \\
\hline RER-0R & & & & & $\mathbf{P}$ & & $\$ 1$ & \\
\hline
\end{tabular}




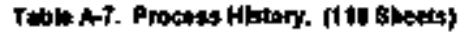

\begin{tabular}{|c|c|c|c|c|c|c|c|c|}
\hline סו & Whonc & 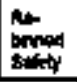 & 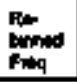 & 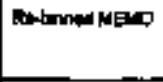 & $\ln _{n \rightarrow \infty}$ & $\begin{array}{l}\text { ONo } \\
\text { Fmo } \\
\text { NC }\end{array}$ & Solitir & Farpontion \\
\hline RBPR-1 & & & & & $\mathbf{F} 2$ & & s1 & 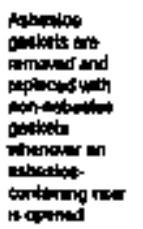 \\
\hline RPR-0S & & & & & $M 1$ & & $\$ 1$ & \\
\hline RSR-0:-1 & & & & & 71 & & s1 & 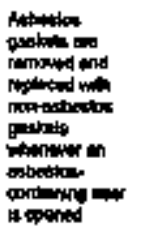 \\
\hline RsRont & & & & & 为 & & $\$ 1$ & \\
\hline Ex:Rotal & & & & & $\mathbf{F}$ & & E1 & \\
\hline RARE- & 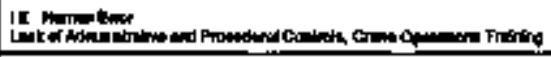 & & & & $\boldsymbol{f}$ & bo & 60 & \\
\hline insingsin & 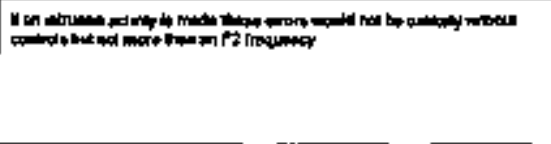 & & & & $\mathbf{F}$ & & $\$ 1$ & 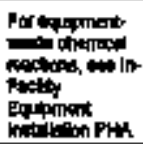 \\
\hline
\end{tabular}


Tubb A-7. Procuas Hisiory. (116 Dheets)

\begin{tabular}{|c|c|c|c|c|c|c|c|c|}
\hline ID & menow & 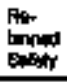 & $\begin{array}{l}\text { Pot } \\
\text { bnined } \\
\text { Find }\end{array}$ & 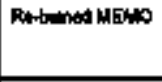 & On: & Pra & song & Rermentes \\
\hline NGRA5NG & 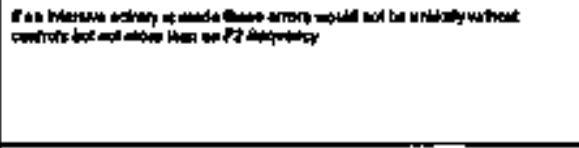 & & & & $\mathbf{n}$ & & st & 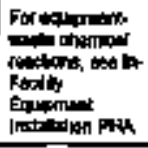 \\
\hline RSAMAB. I & 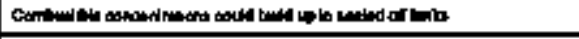 & & & & f2 & & s2 & \\
\hline RERAT:-1 & 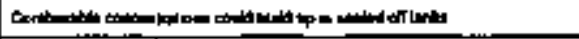 & & & & $\mathbf{r 2}$ & & 62 & \\
\hline Rsh-0Bul & & & & & F2 & & si & \\
\hline$\$ 5801$ & 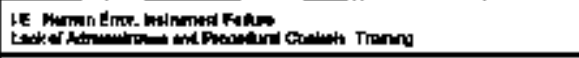 & & & & F3 & $\mathbf{F}$ & $\$ 1$ & \\
\hline sFFad & & & & & $\mathbf{r a}$ & & $s 1$ & 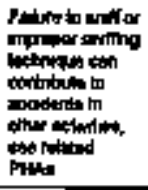 \\
\hline \multicolumn{9}{|l|}{ TF\&-1 } \\
\hline Ynot & 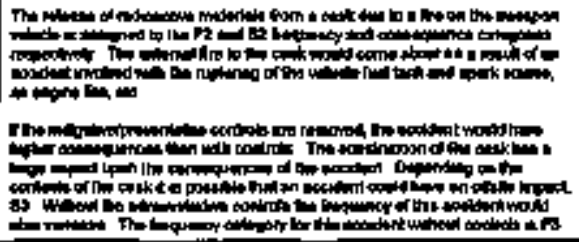 & & & & $\mathbf{m}$ & 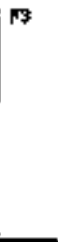 & $s$ & \\
\hline VEHAOPA & Th & & & & $\mathbf{F z}$ & & 刍 & \\
\hline
\end{tabular}




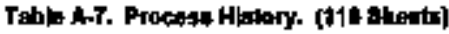

\begin{tabular}{|c|c|c|c|c|c|c|c|c|}
\hline 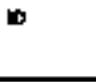 & Manow & nempint & Fonition & Astinind tato & $\infty_{\substack{\infty \\
0}}$ & $\begin{array}{l}\infty \\
\min \\
\min \end{array}$ & torly & $R=$ \\
\hline veters & 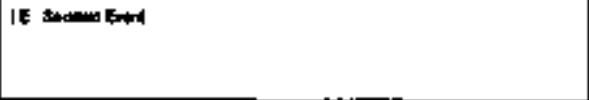 & $\$$ & & 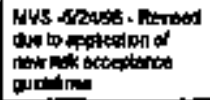 & $\mathbf{F}$ & $\mathbf{F}$ & 31 & \\
\hline valoed & 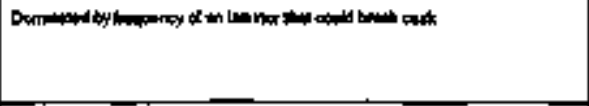 & E2 & & 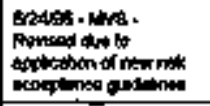 & Fu & & 81 & \\
\hline simen & 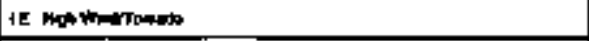 & & & & P2 & $n$ & $\$ 2$ & \\
\hline veros & 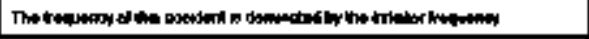 & & & & $\mathbf{m o}$ & & 32 & \\
\hline WEHOA & & का & & 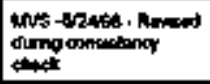 & Fs & & $\$ 0$ & \\
\hline WEOH & & & & & Fi & & ง1 & \\
\hline VEHAS & & & & & $\mathbf{k 2}$ & & 21 & \\
\hline VEH-DG- & & & & & $\mathrm{Fz}$ & & $\mathbf{3 1}$ & \\
\hline VEHEOH & & & & & $\mathbf{r}$ & & $\$ 1$ & \\
\hline VEHASH & & & & & $\mathbf{z}$ & & 由g & \\
\hline VEHAN & 管 & & & & $\mathbf{F} 2$ & $\mathrm{FB}$ & ap & \\
\hline
\end{tabular}


Table A-7. Process Hisiory. (116 thestrot

\begin{tabular}{|c|c|c|c|c|c|c|c|c|}
\hline 10 & MEHO ND & $\begin{array}{l}\text { Ror } \\
\text { inimad } \\
\text { Seltit }\end{array}$ & inim & 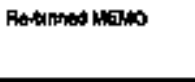 & ing & $\begin{array}{l}\text { Ong } \\
\operatorname{Fin} \\
\operatorname{Nin}\end{array}$ & $\sin _{\sin y}$ & Rentits \\
\hline Vemeral & 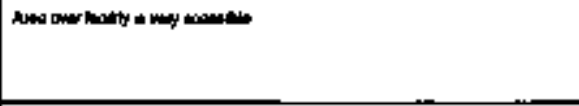 & $\$$ & & 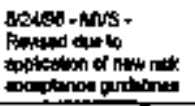 & $\mathbf{r 2}$ & & st & \\
\hline HEH WESA & 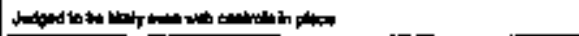 & & & & Fs & & 90 & \\
\hline 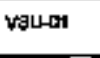 & 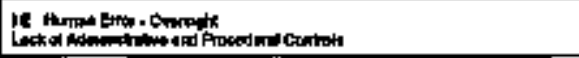 & & & & F3 & 9 & 81 & \\
\hline wastor & 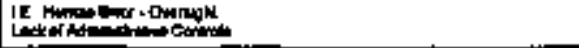 & & & & $* 3$ & $\mathbf{k}$ & $\$ 1$ & \\
\hline VFulas & 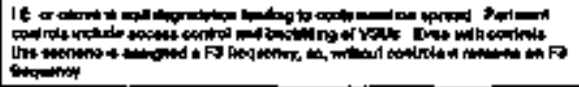 & & & & F3 & f9 & $\mathbf{s 1}$ & \\
\hline พвนด्A & 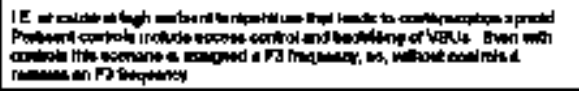 & & & & $\mathbf{F}$ & $\mathbf{F R}$ & 81 & \\
\hline vitumats & & & & & $\mathbf{F}$ & & si & \\
\hline visuted & & & & & $\mathbf{F g}$ & & B1 & \\
\hline Woss- & 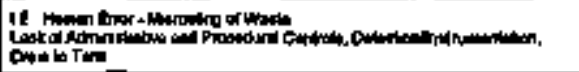 & & & & fi & $\mathbf{r s}$ & $\mathbf{s e}$ & \\
\hline nownos & 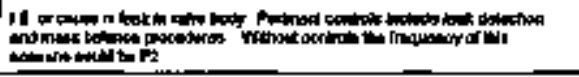 & & & & fa & $\mathbf{F}$ & $\$ 2$ & \\
\hline nimis. & 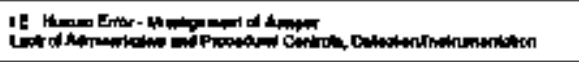 & & & & $\mathbf{F 1}$ & $\mathbf{H}$ & 8 & \\
\hline $\begin{array}{l}\text { nesto } \\
\text { Fiendoto }\end{array}$ & IE & & & & E1 & $\mathbf{F}$ & $s z$ & \\
\hline
\end{tabular}




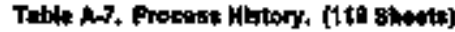

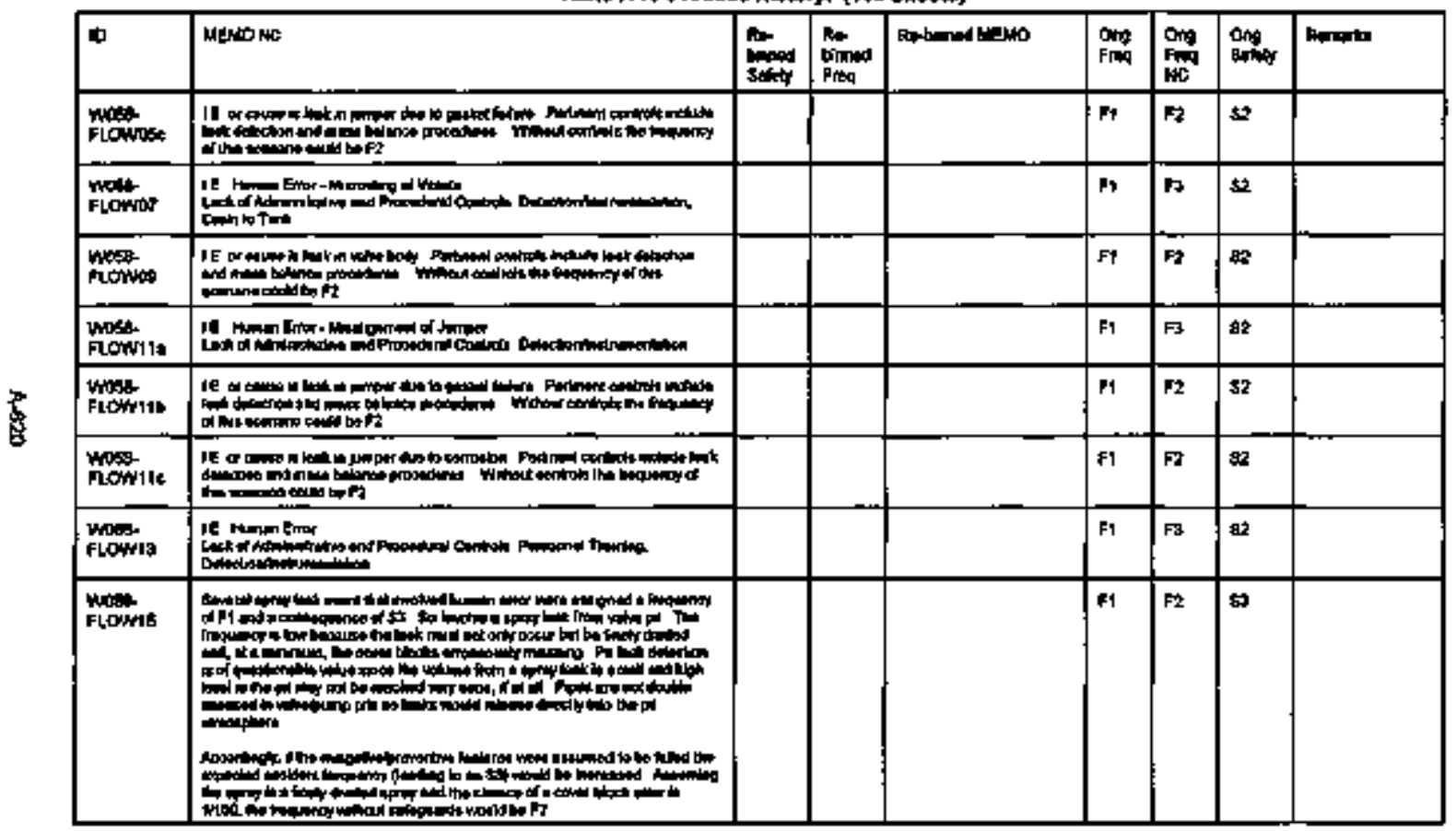


Tabe A-7. Processs Holory. (11t Mratts)

\begin{tabular}{|c|c|c|c|c|c|c|c|c|}
\hline 1 & Nate uc & 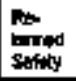 & intion & Rabinnos hemo & ond & $\lim _{\boldsymbol{m} \rightarrow \infty}$ & ont & Nombits \\
\hline $\begin{array}{l}\text { Wuss. } \\
\text { FLownt }\end{array}$ & 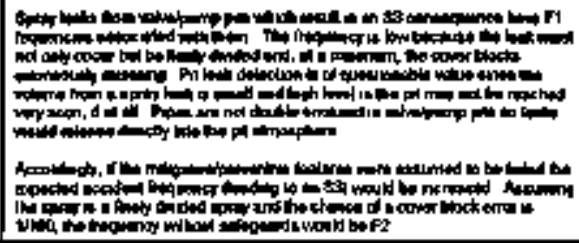 & & & & F1 & $\mathbf{F 2}$ & sa & \\
\hline $\begin{array}{l}\text { unote } \\
\text { Funtwith }\end{array}$ & 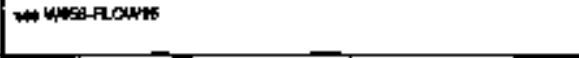 & & & & fi & $N_{2}$ & ss & \\
\hline $\begin{array}{l}\text { Moss } \\
\text { PLONWIT }\end{array}$ & 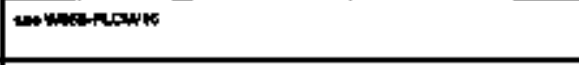 & & & & F1 & $\mathrm{Fz}$ & 5 & \\
\hline frowite & 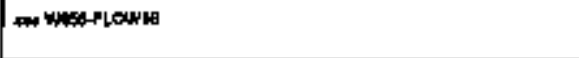 & & & & F1 & $\mathrm{F} 2$ & sa & \\
\hline $\begin{array}{l}\text { WOSB- } \\
\text { PLON1E }\end{array}$ & 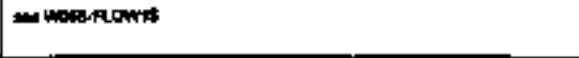 & & & & $\mathbf{F I}$ & $\mathbf{N 2}$ & 93 & \\
\hline $\begin{array}{l}\text { wosk } \\
\text { powis }\end{array}$ & 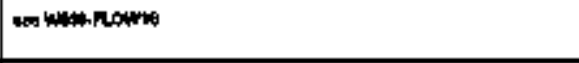 & & & & fs & F & 的 & \\
\hline Flow & 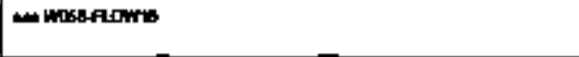 & & & & $\mathbf{m}$ & 72 & ss & \\
\hline FLowh & 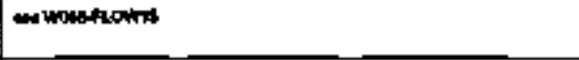 & & & & $\mathbf{F 1}$ & $\mathrm{Pr}$ & 93 & \\
\hline $\begin{array}{l}\text { wose } \\
\text { flowax }\end{array}$ & 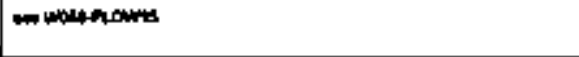 & & & & F1 & $F 2$ & 93 & \\
\hline west & 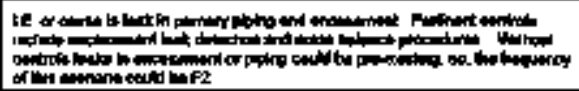 & & & & $\mathbf{m}$ & $\mathbf{F} 2$ & 故 & \\
\hline
\end{tabular}


Table A-7. Procesa Hatory. \{116 abeats]

\begin{tabular}{|c|c|c|c|c|c|c|c|c|}
\hline$D$ & Mesto & 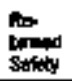 & $\begin{array}{l}\text { Ror } \\
\text { tonined } \\
\text { Fines }\end{array}$ & RAtrind MESO & $\lim _{\ln }$ & $\min _{\mathrm{Ne}}$ & ong & fitritatut \\
\hline Winsot & 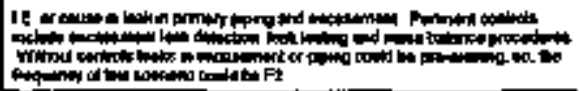 & & & & H & 2 & 32 & \\
\hline $\begin{array}{l}\text { WFoto- } \\
\text { INTEGOIG }\end{array}$ & 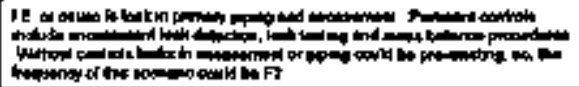 & & & & F1 & $\mathbf{p 2}$ & 32 & \\
\hline $\begin{array}{l}\text { Whol } \\
\text { INtEonoid }\end{array}$ & 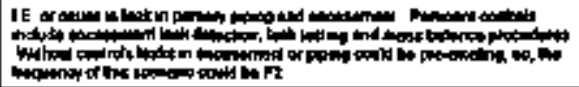 & & & & $\mathbf{F 1}$ & $\mathbf{F 2}$ & 32 & \\
\hline Wo6t. & 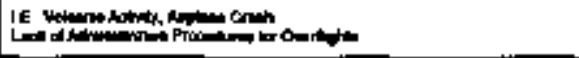 & & & & $k_{1}$ & $\mathbf{F}$ & $\leqslant 2$ & \\
\hline Wosecost & 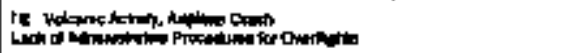 & & & & $\mathbf{F}$ & $\mathbf{F 2}$ & $\$ 2$ & \\
\hline nots & 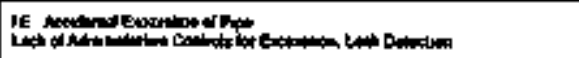 & & & & $\mathbf{F 1}$ & $\mathbf{F}$ & 32 & \\
\hline nefoross & 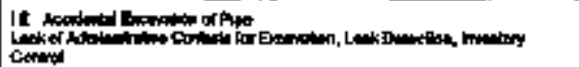 & & & & $\mathbf{P 1}$ & $\boldsymbol{r}$ & 8 & \\
\hline Иस: & 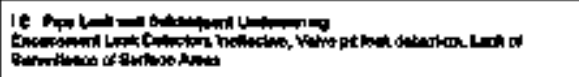 & & & & F1 & $\mathbf{F}$ & $\$ 2$ & \\
\hline 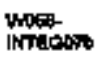 & 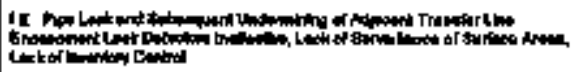 & & & & 1 & $\mathbf{r z}$ & 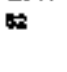 & \\
\hline whose & 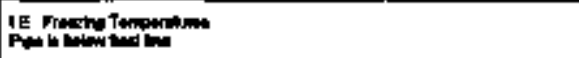 & & & & 81 & $\mathbf{F i}$ & 2 & \\
\hline Mots- & 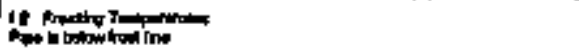 & & & & 81 & FI & 62 & \\
\hline
\end{tabular}


Table A-7. Process Histry. (116 Ohalk)

\begin{tabular}{|c|c|c|c|c|c|c|c|c|}
\hline $\mathbf{0}$ & 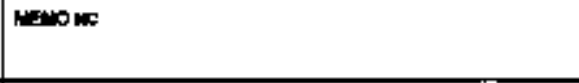 & $\begin{array}{l}\text { Rit } \\
\text { bented } \\
\text { Path }\end{array}$ & finged & Rabmed ing & $\lim _{\min }$ & ind & 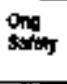 & Pentarists \\
\hline $\begin{array}{l}\text { West } \\
\text { WTES119 }\end{array}$ & 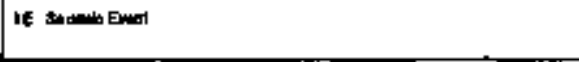 & & & & F1 & $\mathbf{F} 1$ & 82 & \\
\hline moss- & 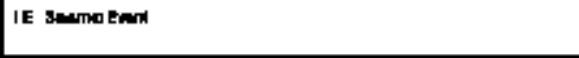 & & & & F1 & $\mathbf{F 1}$ & 82 & \\
\hline What & 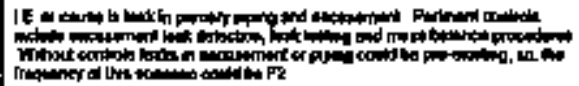 & & & & N & $\boldsymbol{r 2}$ & $\cong 2$ & \\
\hline $\begin{array}{l}\text { Wotas } \\
\text { INTESTIab }\end{array}$ & 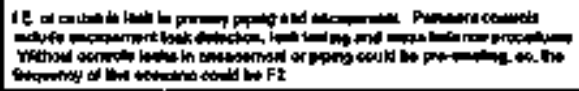 & & & & $\boldsymbol{M}$ & $\mathbf{P z}$ & $\$ 2$ & \\
\hline $\begin{array}{l}\text { Whith } \\
\text { INTEGIS0 }\end{array}$ & 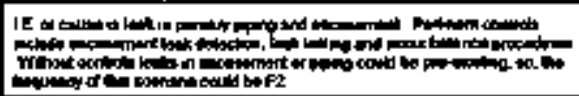 & & & & Fi & $\mathbf{r} 2$ & 52 & \\
\hline $\begin{array}{l}\text { wort: } \\
\text { wTtes id }\end{array}$ & 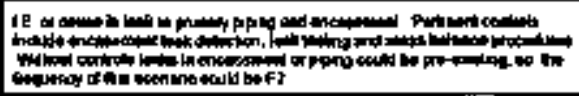 & & & & F1 & $\mathbf{F 2}$ & $\$ 7$ & \\
\hline Wist. & IE Vot & & & & $* 1$ & $\mathbf{F z}$ & $\mathbf{s}$ & \\
\hline $\operatorname{mox}$ & 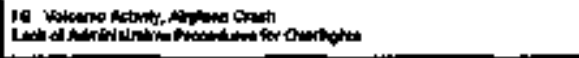 & & & & $\mathbf{F} 1$ & $F 2$ & $\$ 2$ & \\
\hline WTE: & It & & & & $F_{1}$ & 13 & 52 & \\
\hline $\begin{array}{l}\text { What } \\
\text { INTEQTIE. }\end{array}$ & Ie & & & & $\mathbf{F 1}$ & $\mathbf{f s}$ & $\$$ & \\
\hline nows. & 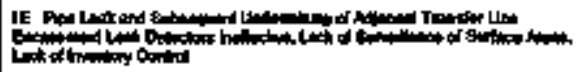 & & & & $\mathbf{F}$ & 2 & $\$ 2$ & \\
\hline
\end{tabular}


Tuble ArT. Provers Hetory. (11) ahents;

\begin{tabular}{|c|c|c|c|c|c|c|c|c|}
\hline סו & trence & Rar & Primed & Robtined MEWO & Orta & $\lim _{\substack{\infty \\
0}}$ & ong & Rententith \\
\hline $\begin{array}{l}\text { phos: } \\
\text { inteolis }\end{array}$ & 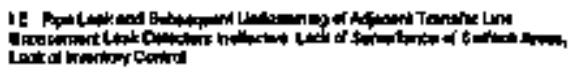 & & & & $F 1$ & $\mathbf{r} 2$ & 52 & \\
\hline wots- & Ie & & & & fa & $\boldsymbol{M}$ & $\$ 2$ & \\
\hline WTEFat & 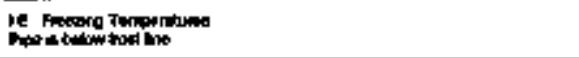 & & & & $\mathbf{F}$ & F1 & 92 & \\
\hline Nesis & re & & & & $\mathbf{f l}$ & $\neq 1$ & sz & \\
\hline noges & 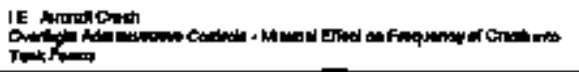 & & & & $\mathbf{F}$ & $\mathbf{F 1}$ & sy & \\
\hline $\operatorname{mos}$ & IE & & & & FI & $\mathbf{f 1}$ & a2 & \\
\hline 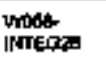 & 1e & & & & $\mathbf{r}$ & $\mathbf{F}$ & 安 & \\
\hline 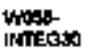 & IE SEmo Em & & & & $\mathbf{F}$ & $\mathbf{F 1}$ & 52 & \\
\hline wops. & 的 & & & & F1 & $\mathbf{F}$ & sq & \\
\hline
\end{tabular}




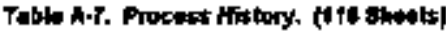

\begin{tabular}{|c|c|c|c|c|c|c|c|c|}
\hline 10 & HENONG & Bimed & $\lim _{\substack{\operatorname{lom} \\
\text { Finged }}}$ & 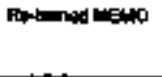 & Fin & ing & song & Rommits \\
\hline $\begin{array}{l}\text { WHo58- } \\
\text { 3NTESSM }\end{array}$ & All & & & & FI & P1 & 99 & \\
\hline Woep & 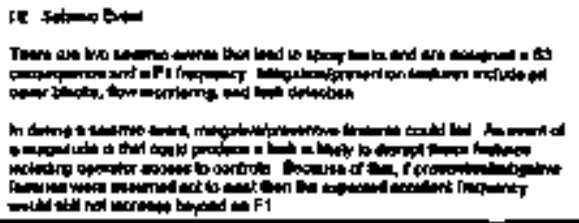 & & & & F1 & $F$ & 83 & \\
\hline $\begin{array}{l}\text { popto } \\
\text { INTESS }\end{array}$ & 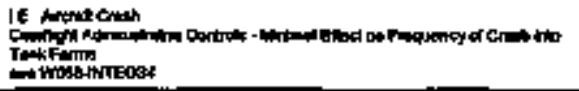 & & & & F1 & $\mathbf{F 1}$ & $\$ 3$ & \\
\hline $\begin{array}{l}\text { wots } \\
\text { WIteos? }\end{array}$ & 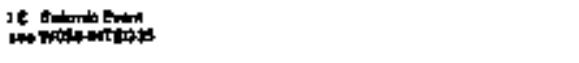 & & & & $\mathbf{F} 1$ & $\mathbf{F t}$ & $\mathbf{8}$ & \\
\hline $\begin{array}{l}\text { wota } \\
\text { phespor }\end{array}$ & IF & & & & F & $\mathrm{F}^{3}$ & $g z$ & \\
\hline nowe. & 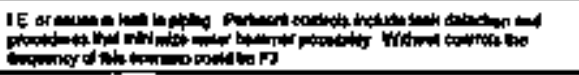 & & & & $\mathbf{m}$ & $\mathbf{F}$ & 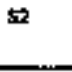 & \\
\hline
\end{tabular}




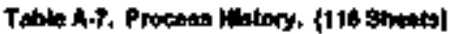

\begin{tabular}{|c|c|c|c|c|c|c|c|c|}
\hline$\omega$ & 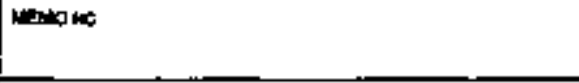 & $\lim _{\text {sined }}$ & 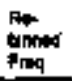 & 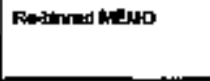 & ing & ind & ong & Rematis \\
\hline Xgoi- & 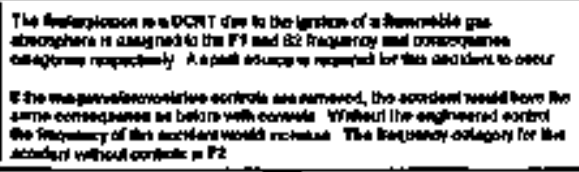 & & & & F1 & $\mathbf{2}$ & $\$ 2$ & \\
\hline Lonis & 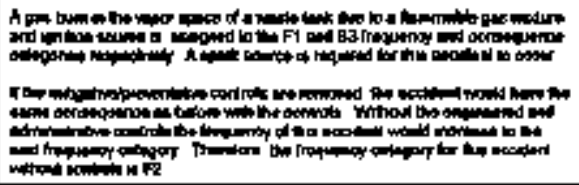 & & & & $\mathbf{F 1}$ & $F \mathbf{2}$ & $\mathbf{s}$ & \\
\hline $\begin{array}{l}\text { ye-or- } \\
\text { flowios }\end{array}$ & 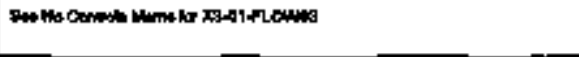 & & & & $\mathbf{r 1}$ & F & $\Leftrightarrow$ & \\
\hline 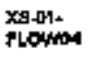 & 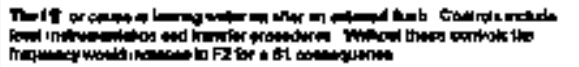 & & & & $\mathbf{F 1}$ & P文 & B1 & \\
\hline 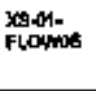 & 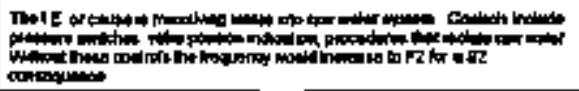 & & & & $\boldsymbol{F 1}$ & $\mathbf{r 2}$ & ss & \\
\hline $\begin{array}{l}\text { xtsol. } \\
\text { FLowids }\end{array}$ & 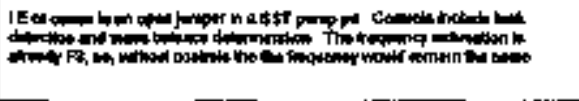 & $s$ & & 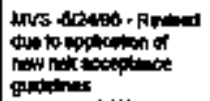 & As & es & s1 & \\
\hline XF-OA- & 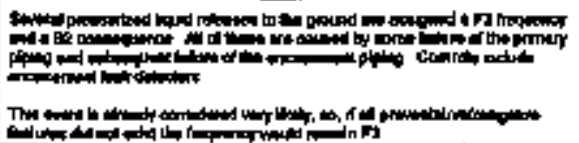 & 82 & & 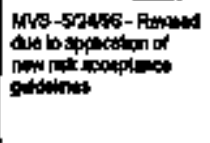 & $\mathbf{r a}$ & $F 3$ & B1 & \\
\hline
\end{tabular}




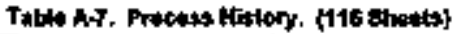

\begin{tabular}{|c|c|c|c|c|c|c|c|c|}
\hline 10 & MEMOAL & Bind & $\begin{array}{l}\lim _{\lim } \\
\text { Fread }\end{array}$ & Pि-tinned & Ong & $\lim _{\ln }^{\infty}$ & 00 & Rawerlat \\
\hline $\begin{array}{l}\text { Xs-01- } \\
\text { prestin }\end{array}$ & 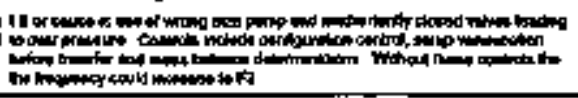 & & & & F1 & $\mathbf{F} \mathbf{2}$ & $\Leftrightarrow$ & \\
\hline $\begin{array}{l}\text { XSpos. } \\
\text { PREstog }\end{array}$ & 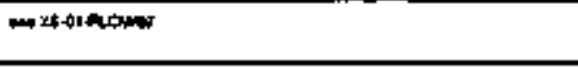 & & & & $\mathbf{F}$ & $\mathrm{FB}$ & B1 & \\
\hline $\begin{array}{l}\text { xger. } \\
\text { IENPM }\end{array}$ & 4.0se-da & & & & F2 & $\mathrm{F3}$ & 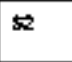 & \\
\hline 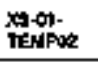 & 0000000 & & & & $\mathbf{F}$ & $F^{7}$ & Bi & \\
\hline $\begin{array}{l}x \cos x \\
\text { flosinon }\end{array}$ & 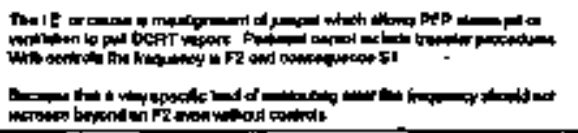 & & & & F2 & F & 51 & \\
\hline Ksing: & 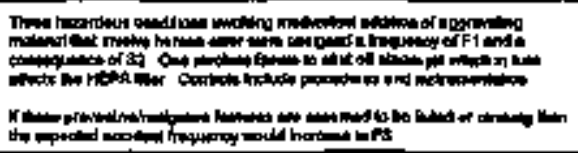 & 92 & & 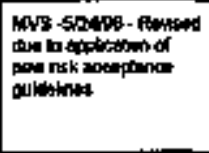 & $\mathbf{F 2}$ & $F$ & s1 & \\
\hline Poos & 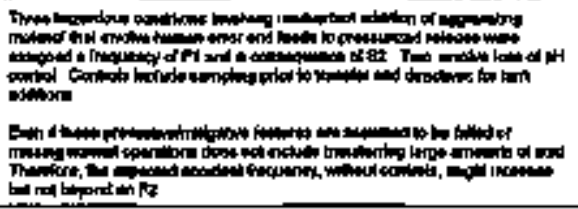 & 82 & & 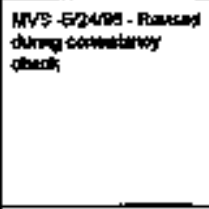 & $\mathbf{F 2}$ & $\mathbf{F}$ & 81 & \\
\hline rop- & 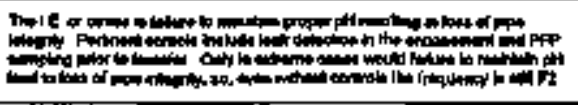 & 82 & & 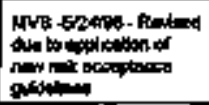 & $\mathbf{F 2}$ & 82 & 81 & \\
\hline
\end{tabular}




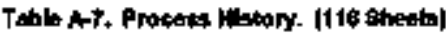

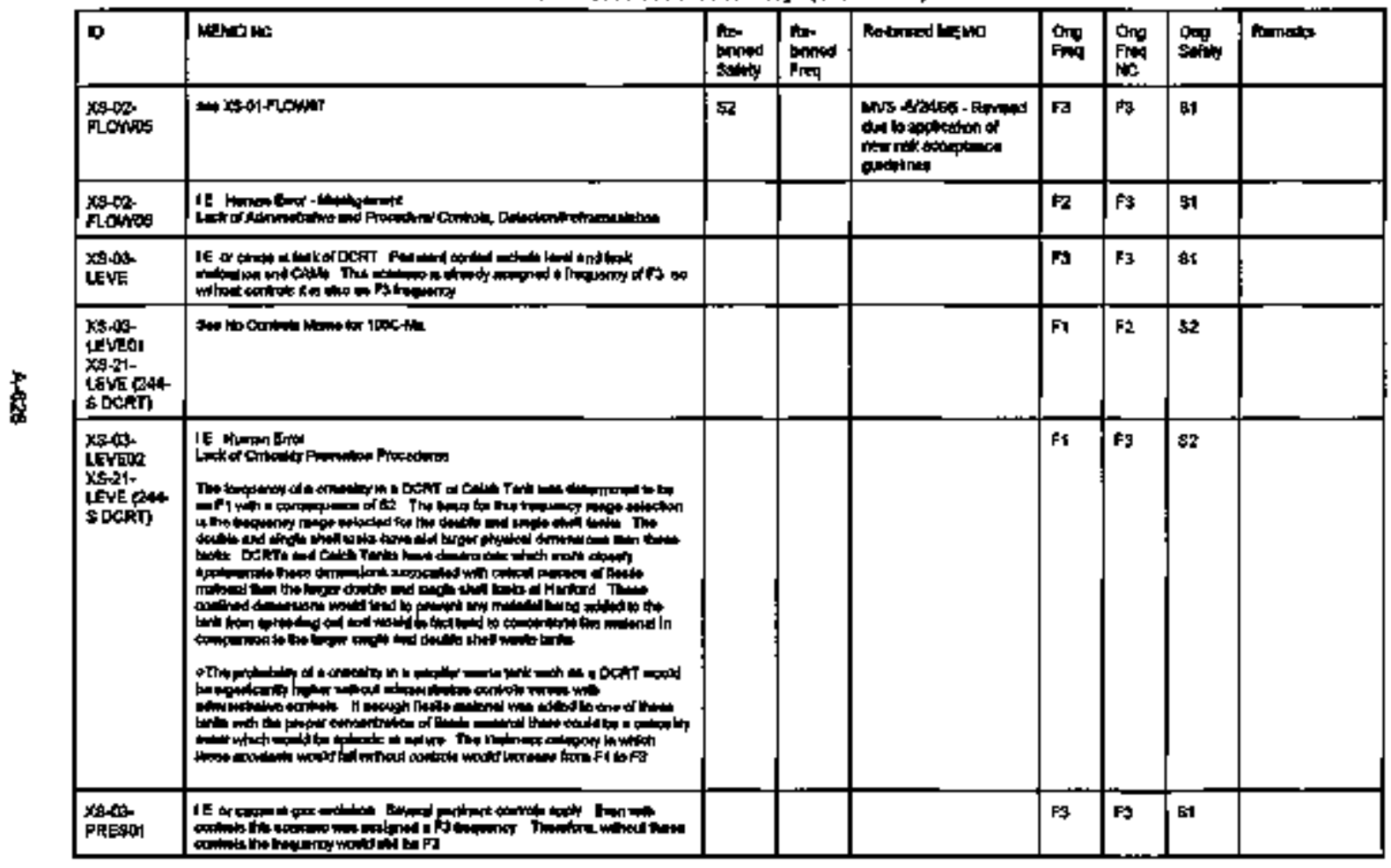




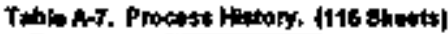

\begin{tabular}{|c|c|c|c|c|c|c|c|c|}
\hline $\mathbf{H O}$ & LENO W & 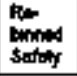 & At- & 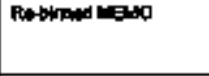 & Pon & $\begin{array}{l}\text { Ong } \\
\text { find } \\
\text { NC }\end{array}$ & ang & Amitan \\
\hline 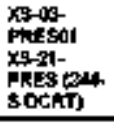 & 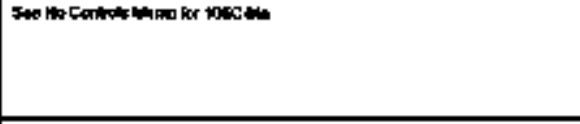 & & & & Fi & F? & 52 & \\
\hline 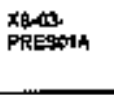 & 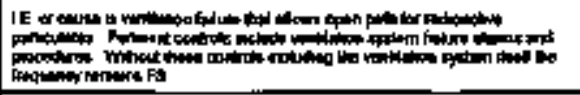 & & & & $\mathbf{F}$ & $\mathbf{k}$ & s1 & \\
\hline 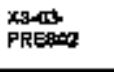 & 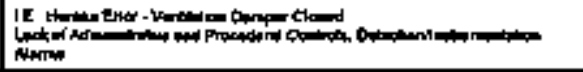 & & & & $\mathbf{k}$ & $\mathbf{f a}$ & $\$ 1$ & \\
\hline 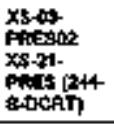 & 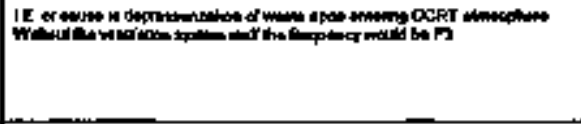 & $\$ 7$ & & 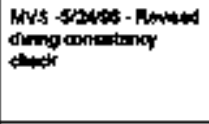 & $8 z$ & $\mathbf{F}$ & $\$ 1$ & \\
\hline$x$ & 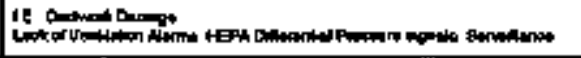 & & & & $\mathbf{t a}$ & $\mathbf{F} \mathbf{3}$ & 91 & \\
\hline 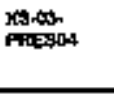 & 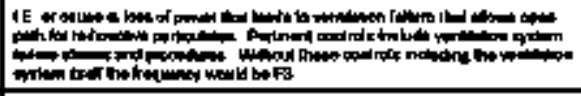 & & & & es & $\boldsymbol{n}$ & $\$ 1$ & \\
\hline $\begin{array}{l}\text { Xe-Go } \\
\text { PREEAt: }\end{array}$ & IE arar & 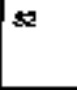 & & 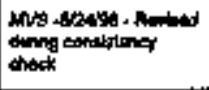 & 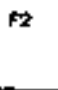 & $\boldsymbol{F} \boldsymbol{z}$ & s1 & \\
\hline
\end{tabular}


Tably A7, Prockas Hailory. (t16 Jhots)

\begin{tabular}{|c|c|c|c|c|c|c|c|c|}
\hline סו & MEMOKC & 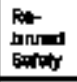 & $\lim _{\lim }$ & 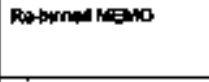 & Pon & $\underset{\substack{\infty \\
\infty}}{\infty}$ & and & Fenowion \\
\hline $\begin{array}{l}x S_{-103} \\
\text { TEMPOM }\end{array}$ & 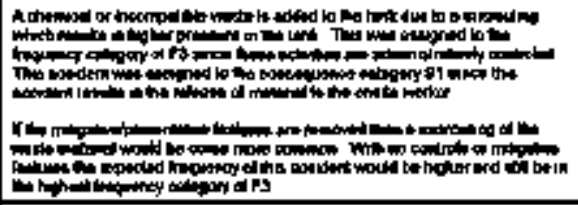 & $\$ 2$ & f2 & 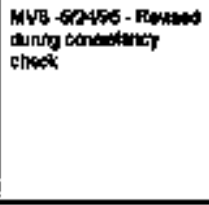 & $\mathbf{F 3}$ & $\mathbf{F}$ & 81 & \\
\hline 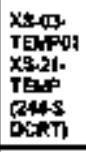 & IE & & & & F1 & $\mathrm{Ms}$ & \$1 & \\
\hline 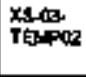 & 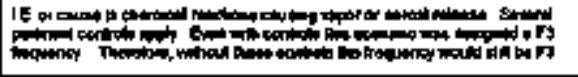 & as & Ez & 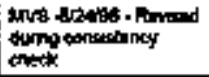 & Fs & $\mathrm{Ps}$ & \$1 & \\
\hline 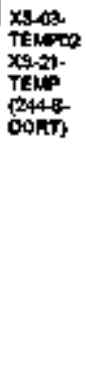 & 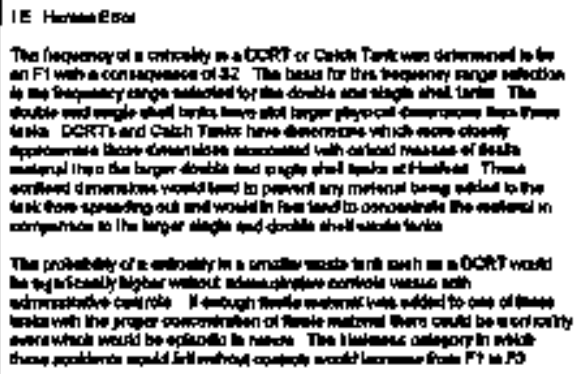 & & & & $\mathbf{F 1}$ & $\mathbf{F s}$ & s & \\
\hline
\end{tabular}


Tabla A.7. Proctate H|atory. [195 sumita]

\begin{tabular}{|c|c|c|c|c|c|c|c|c|}
\hline סו & MAONO & $\lim _{\sin }$ & Pon & 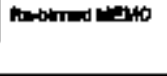 & Pig & Ond & sitis & Fanowh \\
\hline 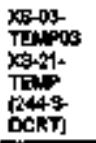 & 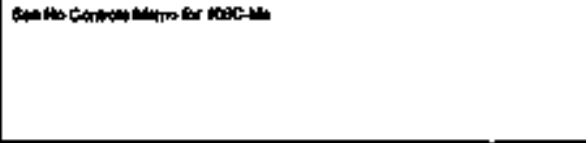 & & & & $\mathbf{F 1}$ & $\mathbf{F} 2$ & $8 \mathbf{2}$ & \\
\hline Tras. & 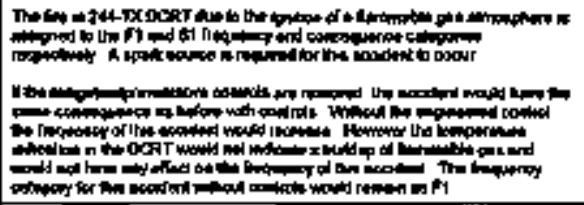 & & & & F & F & 31 & \\
\hline 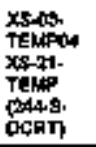 & 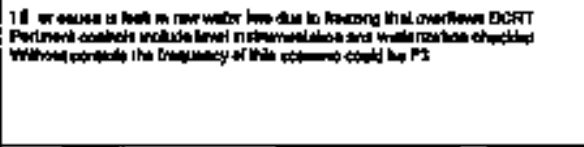 & & & & $\mathbf{P 2}$ & $\boldsymbol{F s}$ & 32 & \\
\hline TE-Ge: & 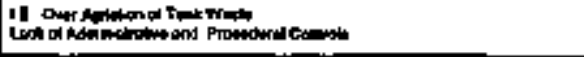 & & & & $\mathbf{r}$ & $P$ & 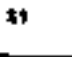 & \\
\hline 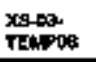 & 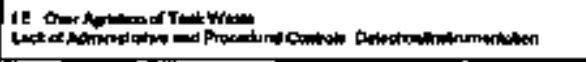 & & & & $F$ & $F$ & 81 & \\
\hline 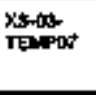 & 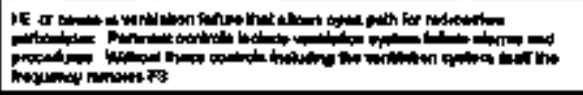 & & & & $\mathbf{A}$ & $\mathbf{P}$ & 11 & \\
\hline 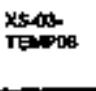 & 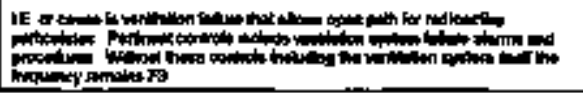 & & & & $\mathbf{F}$ & $\mathbf{F}$ & A1 & \\
\hline
\end{tabular}




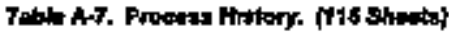

\begin{tabular}{|c|c|c|c|c|c|c|c|c|}
\hline $\mathbf{L}$ & 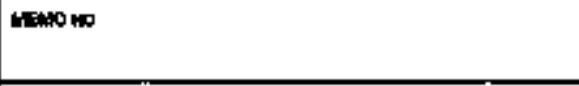 & 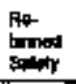 & $\begin{array}{l}\text { Ro- } \\
\text { bired } \\
\text { Fifi }\end{array}$ & Rolinnodhanas & $\operatorname{cop}_{i \rightarrow 0}$ & $\begin{array}{l}\text { ong } \\
\text { freq } \\
\text { inc }\end{array}$ & ond & Romwills \\
\hline $\operatorname{Tim}_{\sin }$ & IE of & & & & FI & $\$ 1$ & 58 & \\
\hline 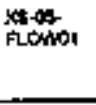 & 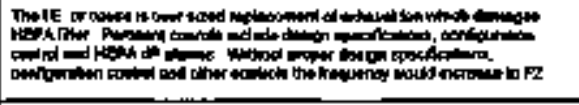 & 82 & & 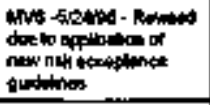 & F1 & rz & $\$ 1$ & \\
\hline $\begin{array}{l}\text { Xedos. } \\
\text { flownoz }\end{array}$ & 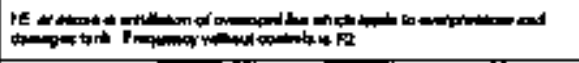 & & & & rq & FZ & s2 & \\
\hline$x \leqslant-4-\infty$ & 管 & & & & Ps & Fo & $\$ 2$ & \\
\hline $\operatorname{lng} x$ & 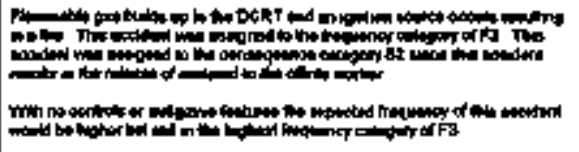 & & & & $F$ & $\mathbf{F a}$ & 92 & \\
\hline Lown & 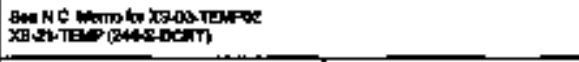 & & & & F1 & $\mathbf{m}$ & 52 & \\
\hline 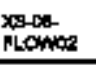 & 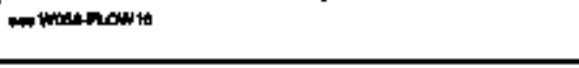 & & & & $\mathbf{P 1}$ & $F 2$ & 89 & \\
\hline 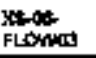 & 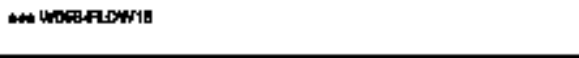 & & & & F1 & FZ & $s$ & \\
\hline
\end{tabular}


Tabb 47. Process HElary. (116 sheets)

\begin{tabular}{|c|c|c|c|c|c|c|c|c|}
\hline ID & MENOM & $\begin{array}{l}\text { Mor } \\
\text { troned } \\
\text { gentivity }\end{array}$ & $\lim$ & Norbimed ME:W & ontun & 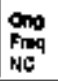 & org & Rention \\
\hline xistion & M mosbalow18 & & & & $\$ 1$ & $\mathbf{H}$ & $\Leftrightarrow$ & \\
\hline $\begin{array}{l}\text { Xsiog- } \\
\text { ponives }\end{array}$ & 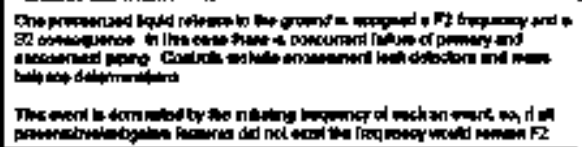 & & & & f2 & $F 2$ & $\$ 2$ & \\
\hline 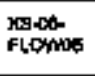 & 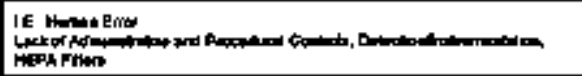 & & & & $\mathbf{F 1}$ & a & 51 & \\
\hline $\begin{array}{l}\text { xoton- } \\
\text { flowh }\end{array}$ & 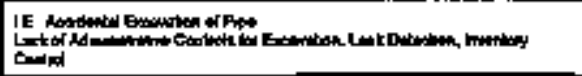 & & & & $\boldsymbol{m}$ & P & $\Leftrightarrow$ & \\
\hline $\begin{array}{l}\text { XE-ON- } \\
\text { PLONIOR }\end{array}$ & 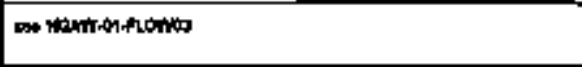 & & & & $\mathbf{F}$ & F & 8 & \\
\hline Ftown. & 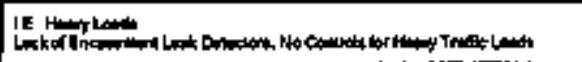 & & & & $\mathbf{m}$ & a & $\Leftrightarrow$ & \\
\hline ror. & It Betam Ever & & & & $\bar{m}$ & $n$ & 52 & \\
\hline 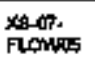 & 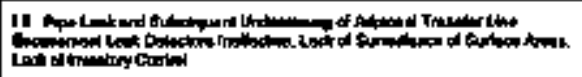 & & & & $\mathrm{Fa}$ & F & 62 & \\
\hline $\begin{array}{l}\text { xis of } \\
\text { Plointe }\end{array}$ & 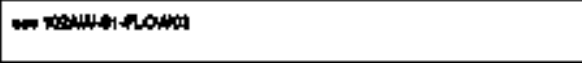 & & & & $\mathrm{FP}$ & f & 89 & \\
\hline 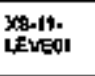 & 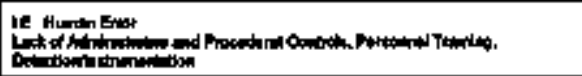 & & & & Fi & $m$ & bi & \\
\hline xSili. & 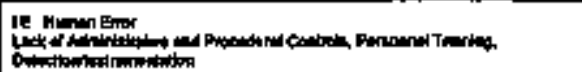 & & & & $\mathbf{P Z}$ & rs & $\mathbf{3 2}$ & \\
\hline
\end{tabular}


Thble h.7. Proceas Heslory. (116 Ghedts)

\begin{tabular}{|c|c|c|c|c|c|c|c|c|}
\hline 10 & (1) & ino & Fin & 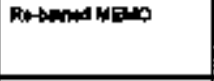 & fivis & $\begin{array}{l}\text { ond } \\
\text { fich } \\
\end{array}$ & कos & Remation \\
\hline $\begin{array}{l}x \in 11- \\
\text { LF }\end{array}$ & 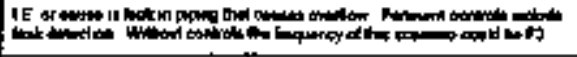 & & & & $\mathbf{M}$ & $\boldsymbol{m}$ & 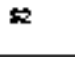 & \\
\hline KB-12. & 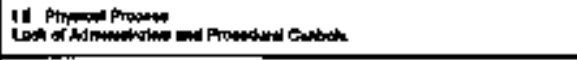 & & & & $\mathrm{F} 1$ & $\mathbf{F}$ & $\$ 1$ & \\
\hline $\begin{array}{l}\text { Xs.12, } \\
\text { FIOWhan }\end{array}$ & 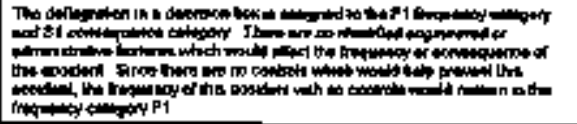 & $\$ 2$ & & 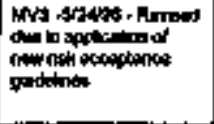 & $\mathbf{P 1}$ & $n$ & s1 & \\
\hline $\begin{array}{l}\text { xog-12- } \\
\text { FLoniog }\end{array}$ & 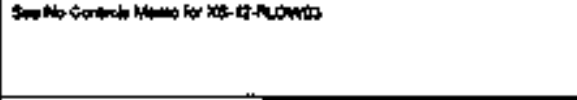 & 62 & & 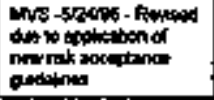 & F: & F1 & $\mathbf{9 1}$ & \\
\hline XSS-12 & 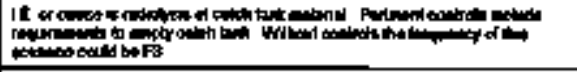 & & & & FI & Fu & $\mathbf{s 1}$ & \\
\hline Plowes & 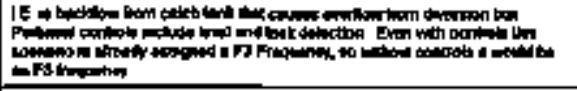 & & & & $\mathbf{F}$ & $F$ & 98 & \\
\hline $\begin{array}{l}\text { XE-t2- } \\
\text { PLowns: }\end{array}$ & 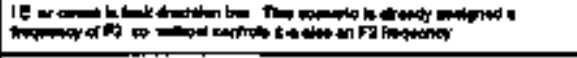 & & & & $\mathbf{F a}$ & $\mathbf{F}$ & $\$ 1$ & \\
\hline WEVE & 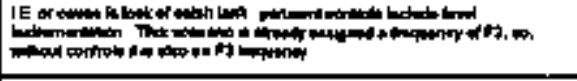 & 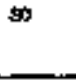 & & 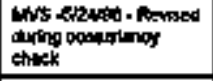 & $\mathbf{n}$ & $\mathbf{F}$ & ง1 & \\
\hline 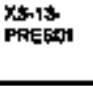 & 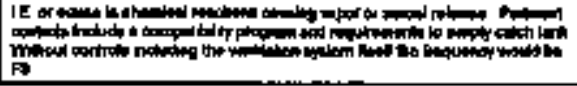 & & & & $\mathbf{m}$ & $\mathbf{F a}$ & $\ddot{2}$ & \\
\hline
\end{tabular}


Tubla A-7. Procoss Hiotery. (116 sheots)

\begin{tabular}{|c|c|c|c|c|c|c|c|c|}
\hline ID & MEMO W & intivit & $\begin{array}{l}\text { Pror } \\
\text { Froud }\end{array}$ & frothmed kewo & Orto & $\begin{array}{l}\lim _{\min } \\
\operatorname{lin}^{\mathrm{n}}\end{array}$ & Orto & fintitis \\
\hline $\begin{array}{l}\text { XSAl木 } \\
\text { PFEECQ }\end{array}$ & 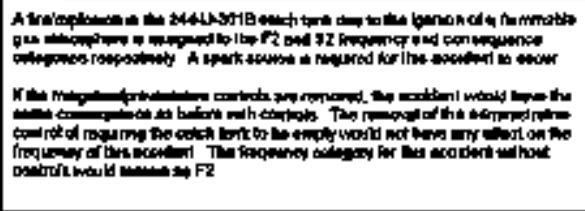 & $\mathbf{s}$ & & 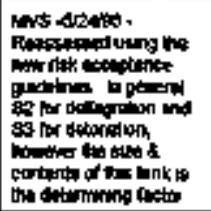 & $\mathbf{F}$ & $\boldsymbol{r}$ & az & \\
\hline $\begin{array}{l}\text { Pas-13k } \\
\text { PAEStos }\end{array}$ & 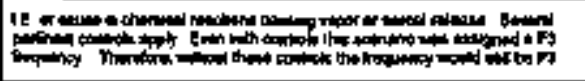 & 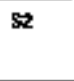 & 72 & 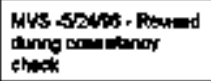 & $\mathbf{A}$ & $\mathbf{F}$ & 51 & \\
\hline 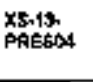 & 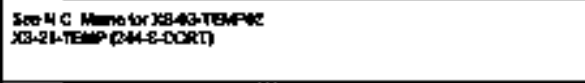 & & F1 & 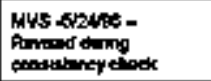 & 11 & $\boldsymbol{F}$ & th & \\
\hline Plotios & 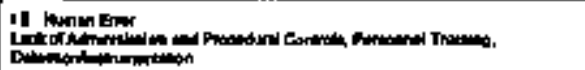 & & & & $\mathbf{F y}$ & $\mathbf{F}$ & 52 & \\
\hline $\begin{array}{l}x \cos \\
\text { Fontar }\end{array}$ & 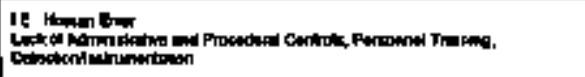 & $\$ 1$ & $\mathbf{F 2}$ & 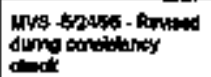 & $\mathbf{r g}$ & $\mathrm{F}$ & 32 & \\
\hline 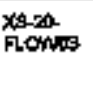 & 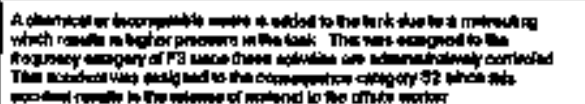 & & $\mathbf{F}$ & 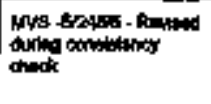 & $\mathbf{F y}$ & $\mathrm{FB}$ & 50 & \\
\hline $\begin{array}{l}\text { X:-20- } \\
\text { roting4 }\end{array}$ & 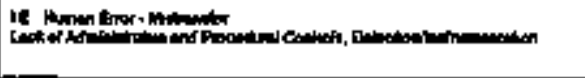 & & $\boldsymbol{F}$ & 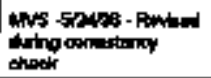 & $\mathbf{r y}$ & $\mathbf{F}$ & $\$ 2$ & \\
\hline
\end{tabular}




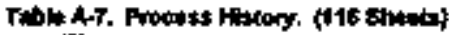

\begin{tabular}{|c|c|c|c|c|c|c|c|c|}
\hline 10 & 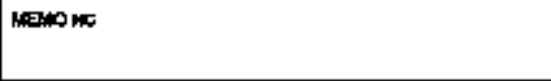 & Finting & $\begin{array}{l}\lim _{\lim } \\
\text { Fined }\end{array}$ & 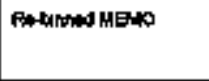 & oro & $\begin{array}{l}\text { Ong } \\
\text { fith } \\
\text { NC }\end{array}$ & ond & Pompras \\
\hline 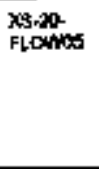 & 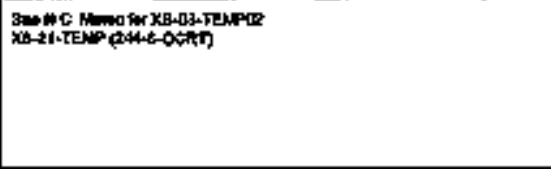 & & $F 1$ & 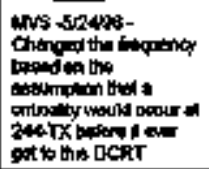 & $\mathbf{F 1}$ & F3 & sp & \\
\hline 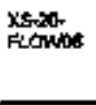 & 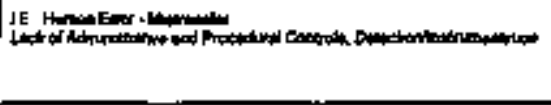 & st & $\mathbf{m}$ & 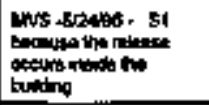 & $\mathbf{F s}$ & Pg & 92 & \\
\hline$x_{\text {atom }}^{x}$ & 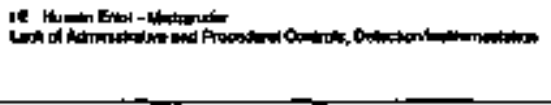 & 81 & +2 & 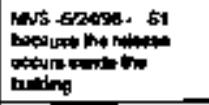 & $\mathrm{FB}$ & $\mathbf{F}$ & s & \\
\hline $\begin{array}{l}\text { xe-20. } \\
\text { PLoming }\end{array}$ & 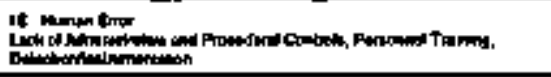 & & & & $\mathbf{F 2}$ & $\mathbf{F a}$ & 82 & \\
\hline 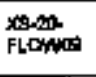 & 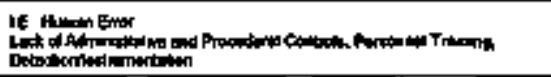 & & & & $\mathbf{F s}$ & $\mathbf{k}$ & ta & \\
\hline 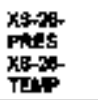 & 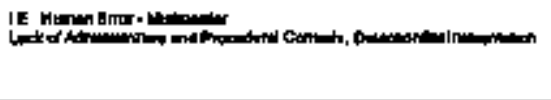 & & & & $\mathbf{n}$ & $\mathbf{F 3}$ & $\mathbf{b 2}$ & \\
\hline 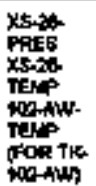 & (1) & & & & Fo & $\mathbf{F 1}$ & 32 & \\
\hline
\end{tabular}


Tabla M-7. Process Hiatony. (116 aments)

\begin{tabular}{|c|c|c|c|c|c|c|c|c|}
\hline ID & סוטים & sting & find & Ro-binod Malo & Onit & $\operatorname{ling}^{2}$ & Dow & Maristo \\
\hline 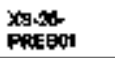 & 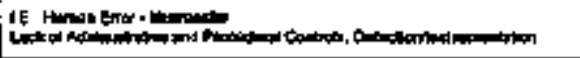 & & & & $\mathbf{r 2}$ & $\mathbf{r s}$ & 82 & \\
\hline 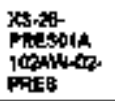 & 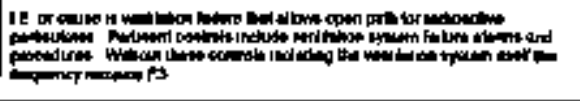 & & & & $\mathbf{m}$ & $\mathbf{F}$ & s1 & \\
\hline 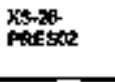 & 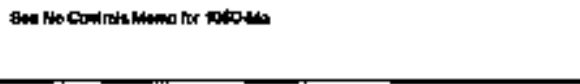 & $\mathbf{s s}$ & & 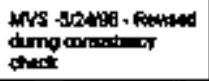 & $\mathbf{F 1}$ & $\mathbf{F 2}$ & st & \\
\hline 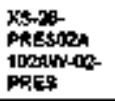 & 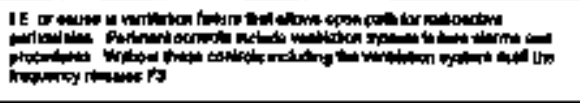 & & & & $\mathbf{r s}$ & $\mathbf{P 3}$ & s1 & \\
\hline 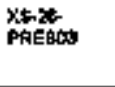 & 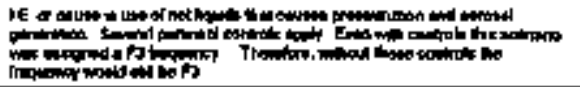 & & & & $\boldsymbol{m}$ & $\mathbf{F}$ & B1 & \\
\hline 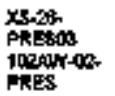 & 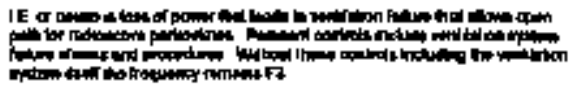 & & & & Ps & es & \$1 & \\
\hline 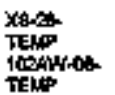 & IE of & & & & F1 & $\mathbf{F} 1$ & $\$ 1$ & \\
\hline
\end{tabular}


Table Ar. Proceds Findary. (116 shod:s)

\begin{tabular}{|c|c|c|c|c|c|c|c|c|}
\hline ID & MENONE & 最 & fing & 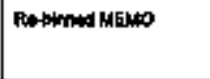 & ing & $\cos _{\operatorname{lin}}$ & gover & Reannilis \\
\hline 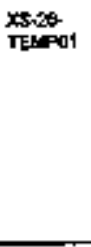 & 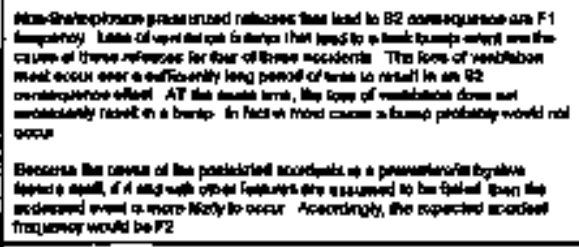 & & & & P1 & F2 & 62 & \\
\hline 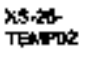 & 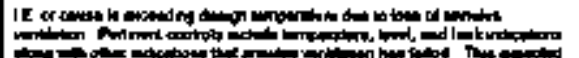 & & & & $\boldsymbol{F}_{1}$ & $\boldsymbol{P}_{1}$ & $\mathbf{s}$ & \\
\hline $\begin{array}{l}\text { Xfous } \\
\text { Fuover }\end{array}$ & 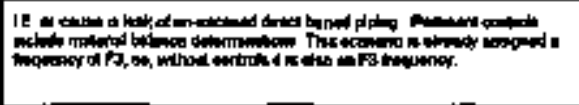 & $\$ 2$ & & 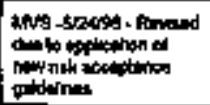 & F3 & f & 81 & \\
\hline 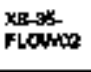 & 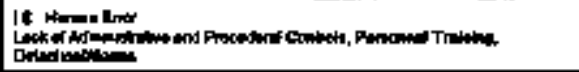 & & & & f2 & fy & $\approx$ & \\
\hline $\begin{array}{l}\text { xtsan. } \\
\text { Fontor }\end{array}$ & 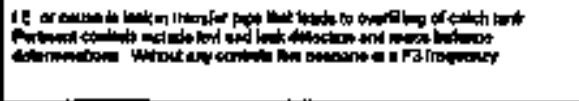 & $\$ 2$ & & 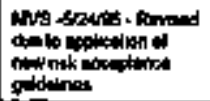 & $\boldsymbol{r z}$ & fa & $\$ 1$ & \\
\hline $\begin{array}{l}\text { xhat- } \\
\text { rowno }\end{array}$ & 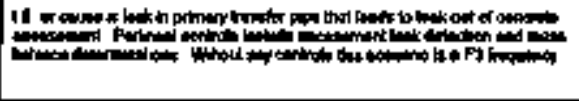 & 82 & & 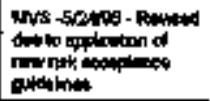 & $n$ & os & sit & \\
\hline 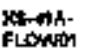 & 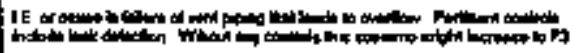 & & & & $\mathbf{F}$ & ค & 如 & \\
\hline
\end{tabular}


Table ANT. Proseas Halory. (116 sheats)

\begin{tabular}{|c|c|c|c|c|c|c|c|c|}
\hline $\mathbf{0}$ & $=10 \mathrm{n}$ & 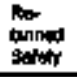 & $\lim _{\text {ind }}$ & 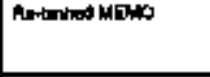 & ond & Fin & ond & Rowate \\
\hline $\begin{array}{l}\text { xsallit. } \\
\text { Flonwor }\end{array}$ & 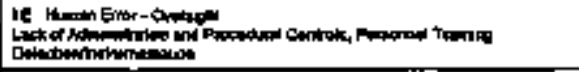 & & & & $\mathbf{B 3}$ & $\mathbf{F a}$ & $\$ 2$ & \\
\hline Lyetrot & 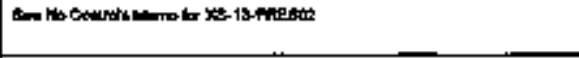 & & & & $\mathbf{r 2}$ & $\mathbf{r z}$ & 22 & \\
\hline $\begin{array}{l}\text { xy-nit. } \\
\text { LEVE-02 }\end{array}$ & 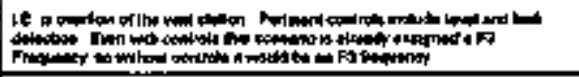 & & & & $\boldsymbol{F}$ & F3 & 31 & \\
\hline $\begin{array}{l}\text { xheass: } \\
\text { LEVEDS }\end{array}$ & 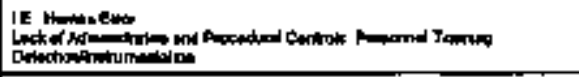 & & & & $\boldsymbol{m}$ & 舟 & s2 & \\
\hline 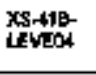 & 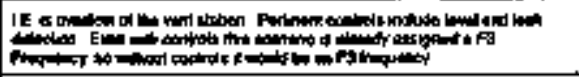 & & & & $\boldsymbol{F}$ & is & s1 & \\
\hline 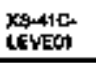 & 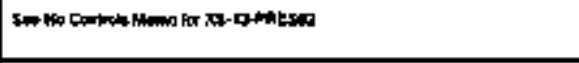 & & & & $\mathbf{F}$ & $\mathrm{F} z$ & az & \\
\hline 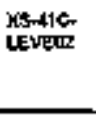 & 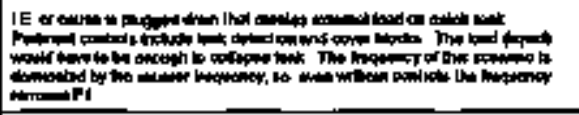 & & & & m & $\mathbf{F 1}$ & s1 & \\
\hline $\begin{array}{l}\text { XEAlc- } \\
\text { UEVIES }\end{array}$ & 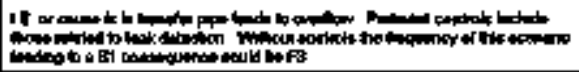 & & & & F1 & $\mathrm{FB}$ & g1 & \\
\hline 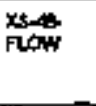 & 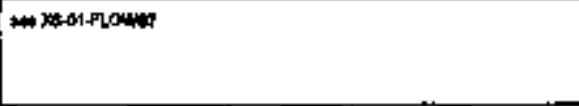 & sas & & 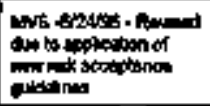 & $\mathbf{r a}$ & $\mathrm{Fs}$ & \$1 & \\
\hline rator- & 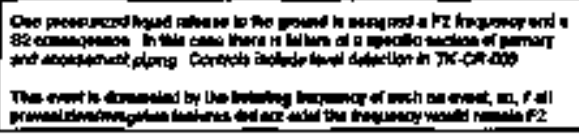 & & & & $\mathbf{m}$ & $\mathbf{F 2}$ & $\$ 2$ & \\
\hline
\end{tabular}


Table A-T. Process History. 1116 strot

\begin{tabular}{|c|c|c|c|c|c|c|c|c|}
\hline D & Heino ins & Rot & $\begin{array}{l}\text { Rit } \\
\text { tinner } \\
\text { Frop }\end{array}$ & Ro-dinned 15 SWO & 贺保 & $\begin{array}{l}\text { ond } \\
\text { incin }\end{array}$ & ond & Raratis \\
\hline How & 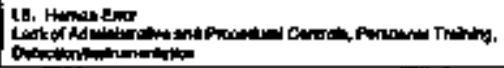 & & & & F2 & $\mathbf{F}$ & 32 & \\
\hline
\end{tabular}


HWF-SD-WH-TL-764, Rer. 1

\section{APFendox 8}

HAZARO TOPOGRAPHY DATABASE TAGLES 
HNF_SD-UMA-TR764, REY. 1

Thes page intenknally left byank. 
HNF-AD-INM-TI-764, REN I

UST OF THBLES

B.1 Pand Cod Oonth

B-1

B-2 Topogmplny Dati

B-23

B-3 Matenal at Rak Data

B-47

e-d Unque Phavomang Data - Pert 1

B.5 Unlaue Phenomena Data - Part 2

6-179

B-6 Facity Conluraton Cata

B-209

B-27? 
HNF-\$SD-WAL-TI.JEG, REN. 1

Thla perge Intenthonally left blenk. 


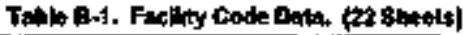

\begin{tabular}{|c|c|c|c|c|}
\hline Footry code & $\mathrm{Fm}$ & Fealtr $\mathrm{N}$ & Fald vame & Type \\
\hline ER & AFNRM & 216 Aी & 2104005 & Chas \\
\hline wit & A CHABA & $210-4005$ & 2to-inous & satmpte on \\
\hline ER & A FAred & 2104A2 & 210 Sast & Crits \\
\hline ER & A FARI & He-pos & $210 \cos 3$ & Cnts \\
\hline ER & AFHFM & gotrssh & 218+1-344 & ant \\
\hline ER & AFARA & 210 - itso: & 216 Arsis & Qnb \\
\hline in & A Fantar & 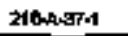 & 216-4nis & Cin \\
\hline$E R$ & AFARM & सबस-ख़-2 & $210-1372$ & En \\
\hline $\boldsymbol{E R}$ & A. FAFu & 215+39 & $215+49$ & Goto \\
\hline ER & A. FAF' & $218-4-45$ & 2104-45 & $\cos$ \\
\hline Tssmo & A Finty & 2Al-thetor & 2A1-thowit & 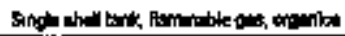 \\
\hline $\mathbf{T} \$ \mathbf{s}$ & A Fintrid & $241-1402$ & 2HAv16 & 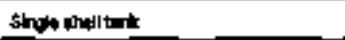 \\
\hline$T \mathrm{~B}=\mathrm{9}$ & A Fuford & $241+1409$ & $241+100$ & 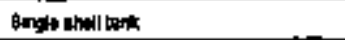 \\
\hline 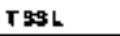 & A. FARA & 241-4004 & $2+1+4-104$ & 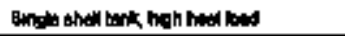 \\
\hline$T S S L$ & iristy & 2t+1-4utos & $241+40 x$ & 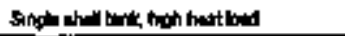 \\
\hline T As L & A Flotir & $2414-100$ & 2HAndat & 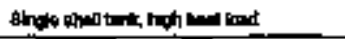 \\
\hline ng & A Fapde & 241-4-151 & $241+151$ & Dawation bex \\
\hline WD & A Finch & $2+1-4.56$ & $241+4-192$ & Dwhengen bow \\
\hline 2 & A Fivath & $241+4211$ & 24140271 & 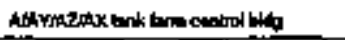 \\
\hline T CN & A FAbad & $246-1302-4$ & 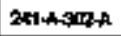 & 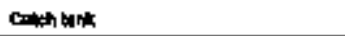 \\
\hline$T C D$ & A Phopa & $241+4000$ & $241+1000$ & DCRT tatth ban \\
\hline TCN & A FANAN & $24+-4+50$ & 2findis & Di \\
\hline
\end{tabular}




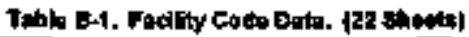

\begin{tabular}{|c|c|c|c|c|}
\hline Futhily cada & Farm & Fonity Mere & Fuld BDen & Type \\
\hline WD & $h F A$ Fin & $241+4+401$ & $241 \sqrt{10401}$ & 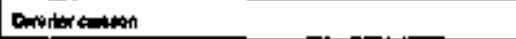 \\
\hline$z$ & A FARM & 24140101 & 741 & Mrithz corfonoer bod \\
\hline TCH & A FARA & $241-A-417$ & $241+417$ & conhmin \\
\hline $\mathbf{z}$ & NAMkH & 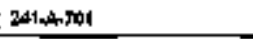 & $241-4=101$ & Sempnaroce buland \\
\hline$z$ & A Panter & $241+\operatorname{trnt}$ & $2414 \pi$ & 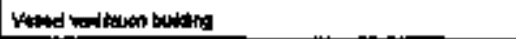 \\
\hline mo & A FAROA & 2ulaning & 2414 & Watep \\
\hline WF & A FAFA & 241-中-A & 241-AA & ruth Bt \\
\hline no & A FAR⿴囗十 & $2+1+A-6$ & $2+1-A+B$ & Wimed \\
\hline wr & A phatey & $2411+8$ & $241 \times 3$ & Dush po \\
\hline WB & A Frath & 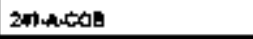 & 2Alliticos & cleanar bax \\
\hline wo & AFARM & $241-1$ & 241 & 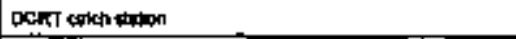 \\
\hline ShT & AFARH & A. Fentis Aectimution Ares & 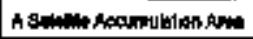 & Sontite Acetsmition Aram \\
\hline Wh & 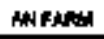 & 201-Nk-010 & 2At-ALHO & 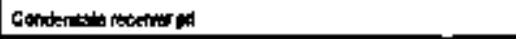 \\
\hline Tos in & Now find & 2414thosor & $241+4 m-104$ & 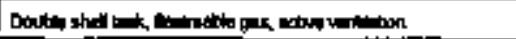 \\
\hline TD舟Y & NAFAR & $24+1-A k 108$ & $241-A N+10 B$ & 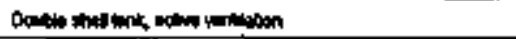 \\
\hline Tos HV & AMFAPIN & 261-AN-109 & $241+$ W-109 & 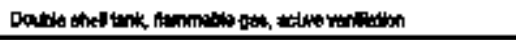 \\
\hline TDSHV & ANFArith & $2+1-1 \mathrm{~N}-10 \mathrm{M}$ & $241+4 k \cdot 10 \mathrm{~d}$ & 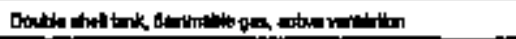 \\
\hline Tos $\mathrm{HW}$ & 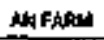 & 2Athin.tios & 24A4toto6 & 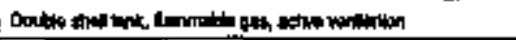 \\
\hline $\mathrm{T} 06 \mathrm{~V}$ & ANFARM & $241 \sqrt{14}+100$ & $201+N+200$ & 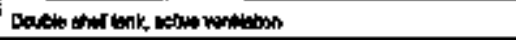 \\
\hline TDSHCP & AJ FAnM & 2414w-107 & $24+4 k-40)$ & 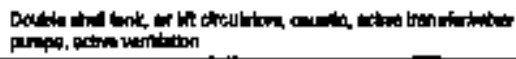 \\
\hline $\mathbf{z}$ & AHFAREM & 241-An-RT & 3⿻1-A4-2I1 & 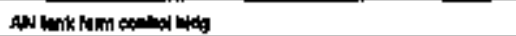 \\
\hline
\end{tabular}


Tuble 0-1. Paelity Codo Dala. 122 sinnotis

\begin{tabular}{|c|c|c|c|c|}
\hline Pbeteft code & Famp & Feciong Nomo & Fito Nom & Thes \\
\hline$z$ & Ak Futud & 2011 ankinas & $241444-271$ & 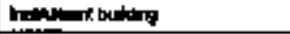 \\
\hline WF & NM RAPA & 241-AN4253 & $241-\sqrt{N-21277}$ & Sonxopd \\
\hline$z$ & ANFAMA & $2+1+\mathrm{A} *-701$ & $241 \times N-301$ & Campersost buting \\
\hline no & WN FAMAN & 241 AN A & 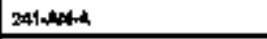 & Wtow on \\
\hline mo & NW FANA & 241-atkde & 2414440 & vation in \\
\hline ms & MAX Farul & $241+4 N 20 B_{1} 1$ & copons 1 & Cancept bas \\
\hline ws & AN FARM & $291-A+c 06-2$ & $\cos +4 i t a$ & clearoul bex \\
\hline Me & AN FAPA & $241 \omega N \cdot \cos -3$ & coshks & Comoudtax \\
\hline Hets & WN FANA & 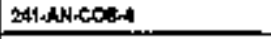 & 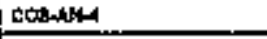 & clectinut the \\
\hline W & WN FAAM & 211 arkctitet & cotsining & clemenow bor \\
\hline w日 & MN FARM & 241AN-CDC6 & $\operatorname{COSAAN-4}$ & clamour bex \\
\hline we & AN FARM & $241 / n+\cos -7$ & $\cos \sin -7$ & Clamold be \\
\hline We & AN FAREN & 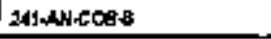 & cotastiked & Eletoul tor \\
\hline wi & NWAPSH & 241 and.FP & 241 Alep & Fling \\
\hline $\operatorname{sen}$ & AN FAPA & 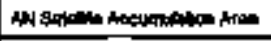 & 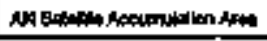 & 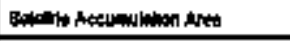 \\
\hline WF & APFAOA & 24140 & 241 - $4 p$ & Fueh in \\
\hline $\operatorname{Tos} V$ & AP FANM & 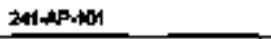 & $2+1+4 P-10$ & 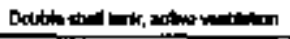 \\
\hline t De $y$ & AP FARAX & $2+1-A 0-106$ & 2A1-AB-104 & 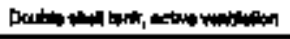 \\
\hline TDE & Ap Fapin & 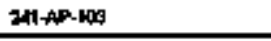 & $2+1+\operatorname{Np}_{100}$ & Doshom \\
\hline T DSV & NP FARH & 2H-AP-10 & $2414 \mathrm{~A}-106$ & 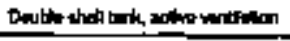 \\
\hline T 㕷V & NP FARM & $241-A P_{1} 10 t$ & $261-A P-165$ & 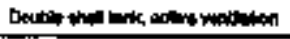 \\
\hline $\mathrm{T}$ deV & APFARA & 2*1AP-100 & SHAP-10: & 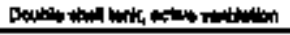 \\
\hline
\end{tabular}


Table B-1. Facialy Coda Dete (22 ahents)

\begin{tabular}{|c|c|c|c|c|}
\hline Fobitctes & ferm & Fralop lithe & fon Hand & Typu \\
\hline T DEs Y & AP FARA & 241-APमाबत & zaridention & 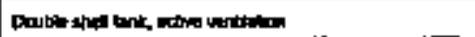 \\
\hline T DO Y & APFARA & $241+A P-105$ & 241APMOQ & 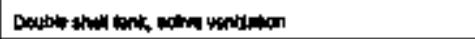 \\
\hline$z$ & AP FAFU & 241 AP.27I & $241 / A P-271$ & 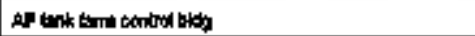 \\
\hline WF & AP FARA & 241 APXCB & 24140,an & gencept \\
\hline 2 & AP FARA & 2414004R3 & $2414+420$ & 5encebing \\
\hline$z$ & AP FARA & 241-AP-TO1 & 241APיतM & complaser bulding \\
\hline Mo & APFARM & 24liteith & 2A1-AP-A & What \\
\hline Ho & NP FAnIt & 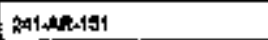 & 2.14.4R.151 & Dotitros bar \\
\hline SAT & NP FARA & 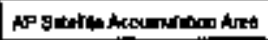 & 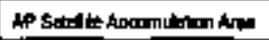 & 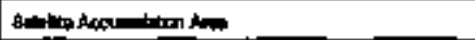 \\
\hline Fap4 & AP FARA & $A R-204$ & Nh-804 & 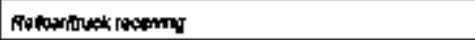 \\
\hline Fard & AP FARM & IN>244 & AR-24K & Vull \\
\hline MD & AN FHis & 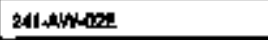 & Z11-AW-cez: & fadpumpal \\
\hline Tas $H V$ & AH RAFin & 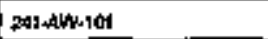 & $2011+4=10$ r & 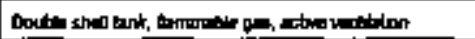 \\
\hline TOESEP & AWr FNAM & 241AW-1m & $241-1 \% 102$ & 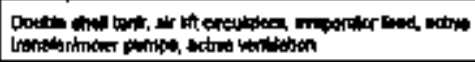 \\
\hline $\operatorname{Tos} v$ & 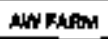 & 241+nutas & $241+4 W-100$ & 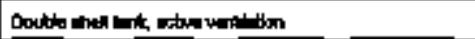 \\
\hline$T 0 Q v$ & AW FARM & 2*1-AW-1E4 & 241-AWH.HOH & 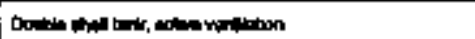 \\
\hline $\mathrm{T} D \boldsymbol{Y} \mathrm{Y}$ & AWN Fhrod & $24+h H-10$ & $201+104-105$ & 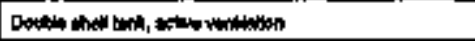 \\
\hline TDEY & Aw That & $241414-105$ & $241+4 N-100$ & 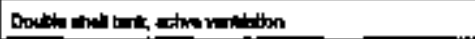 \\
\hline $\mathbf{z}$ & MW PARU & 241-AW271 & 2414niven & Aw bink how owdid bidg \\
\hline $\mathbf{z}$ & MW FARU & $2 n+4 w-272$ & 201-AW-207 & 管 \\
\hline$z$ & AW FANH & 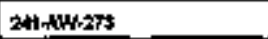 & 24h+4h-27d & 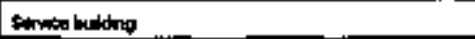 \\
\hline
\end{tabular}


Tubls E-1. Fucitity Cols Dale (22 Jheris)

\begin{tabular}{|c|c|c|c|c|}
\hline facing coll & Ferm & Fodtur Nom & Fand Ringer & Tyos \\
\hline $\mathbf{z}$ & antr Fandu & 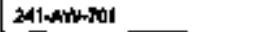 & 2414arvatiot & Compeor busing \\
\hline mo & AW FARU & ZIIAWA & 졔1-AMA-A & Yhing pt \\
\hline WO & AH FARIM & 2414AWH & 2414hing & Yatum ph \\
\hline Wo & AW FAmin & 2114intecos-1 & Cotominten & Ghoinold bot \\
\hline wit: & 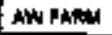 & 2m1-4hMoos-10 & Cotondur-10 & Dathon \\
\hline mo & AW FAFA & 241-ANMCOAN1 & tOB-AMit1 & Oteroul bix \\
\hline MB & AW FAPA & 241-AN-CDO-12 & CDB-NH-12 & Elomput tor \\
\hline WB & AUW FARU & 21jهin-c0s-2 & costhtar & tandroul bex \\
\hline Wh & Aw Pind & $2+1+4 \cos 3$ & cotatwas & cthoth beth \\
\hline $\operatorname{mi}$ & Aw Fistity & $2 \pi+1+\cos -4$ & CCOA-AW44 & trian bax \\
\hline MB & AW FAPA & $2+11$ AM-CDO-\$ & CDDBNW-65 & Clingoul ber \\
\hline we & AN FAPA & 2A1-AW-COB 6 & cododityot & Exameit ber \\
\hline M. & 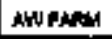 & $2+1+4600 \pi$ & CCadithar & Conthe bex \\
\hline nts & An Fatser & 2Hinumoses & cosenwets & t.coul tax \\
\hline พ⿻ & AW FAfred & 241-AW-COBB & coporityog & Ctingat bat \\
\hline$W F$ & AN FARM & 241-NWWFP & 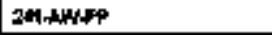 & 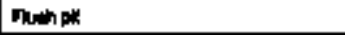 \\
\hline skT & AW Fintor & 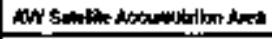 & 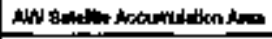 & 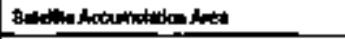 \\
\hline T.8gh & AX FARIU & 241-Ax-3vi & 241-AX-191 & 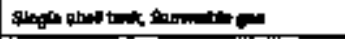 \\
\hline$T, 3940$ & AXFADid & $245-4 \times-100$ & $24+4 x-102$ & 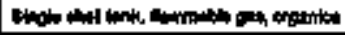 \\
\hline T.\$FH & 此 Fimind & 241460100 & 2thlutat & 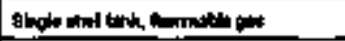 \\
\hline T와옹 & Ax Finfin & $24 \ln x-104$ & $2+1-4 x-104$ & gingle thelrongh \\
\hline ont & AxFAnd & $341-4 x-151$ & $241-4 x-151$ & 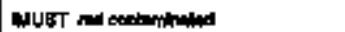 \\
\hline
\end{tabular}




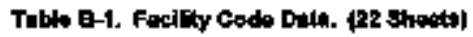

\begin{tabular}{|c|c|c|c|c|}
\hline fullo Cods & Fum & Fucing rienter & Fads Nant & The \\
\hline mo & WrFNA & $24+156151$ & $241 \cdot 6 \times 181$ & Drention tex \\
\hline $\mathbf{T} \mathbf{C N}$ & NKPAFM & $241+4 \times-153$ & 241-106018? & choth inat: \\
\hline mo & AXFARH & $24-4 x-15 x$ & $241+4 x-152$ & Draine bethen \\
\hline wo & NYSANGA & $141-1 \times-199$ & $2414 \times-153$ & Deweroten tox \\
\hline mo & MXFNA & $241+1 \times K=1$ 品 & $2414 \times-158$ & Drimeraben tox \\
\hline$z$ & MXPAN & 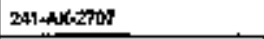 & 24140x-2017 & chaty hores \\
\hline wD & MX FAFO & $241-A \times-901$ & 241 Ax+501 & 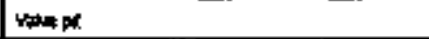 \\
\hline$W$ & AXFNAM & $241-4 \times-80$ & 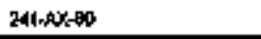 & 5aryos it \\
\hline Wr & MXFNMU & $241 \cdot d x=0$ & $2414 \times 4 \times 0$ & senctapt \\
\hline 16 & MX FARox & 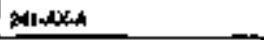 & Ald & vaturept \\
\hline$w F$ & Ax RnRad & $201 \mathrm{Ax} \times$ & $241+Q x-A$ & Fhan \\
\hline WD & AX FNOA & $241-A X-8$ & 241-Ax-g & Yeth pt \\
\hline WF & AXFANGA & $2414 x=9$ & 241 ateo & Puch pe \\
\hline 내요 & AX FARAI & $241-4 x+50$ & $241+4 \times-C O B$ & chent bas \\
\hline Ant & AXFARN & 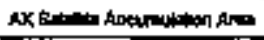 & 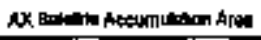 & 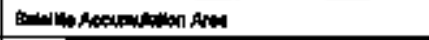 \\
\hline Tosiv & AY EARM & $2 \Delta 1$ AYr-101 & 21I-AY-101 & 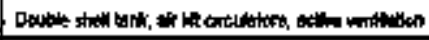 \\
\hline Tosint & HYPANM & $2+1+4 y-102$ & $2+1+h y=102$ & 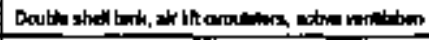 \\
\hline mo & AYFAAU & 241 AY 181 & 241-AY-161 & Pumporin \\
\hline no & AY FARH & 2HIAY-15\% & $21+1 / 152$ & Yandon \\
\hline wo & AYFAMH & 2h-hy-eol & 2hthen & 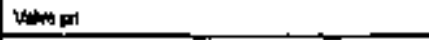 \\
\hline $\mathbf{z}$ & AYTARM & 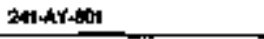 & 24thy+atir & 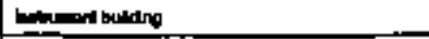 \\
\hline us: & AY FARU & ZA1-AY-CDA-1 & $241-k<-1$ & 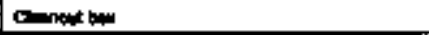 \\
\hline
\end{tabular}




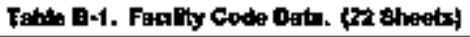

\begin{tabular}{|c|c|c|c|c|}
\hline Fistrycost & Fath & Fatho hine & fatiding & Tyos \\
\hline ght & ArFerigen & 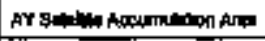 & 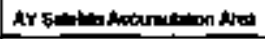 & 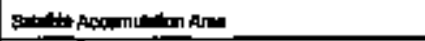 \\
\hline TDA A & AZ FAPA & 261-N2-101 & 201+NE-101 & 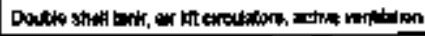 \\
\hline $\operatorname{soth} v$ & R Findu & $2414+17-102$ & $34+2 z-10 R$ & 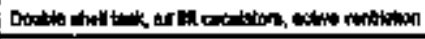 \\
\hline TEN & Nzt thate & 241 4.2151 & 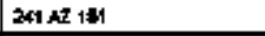 & CEtch tank \\
\hline Tcki & 12 Fafu & $24 t+2 \cdot 15$ & $2+1+2+407$ & Cfachton \\
\hline $\mathbf{m B}$ & AZFARM & $241-A 2-158$ & $241+22-1 \%$ & Baca trennior box \\
\hline WO & NZFARA & $241+27-101$ & 24hA2-152 & Dineration bor \\
\hline Wo & me ranul & 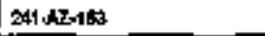 & 241ulf-130 & What pot \\
\hline Ton & AZFARM & $241+2154$ & 241 A2154 & Ctinh bux \\
\hline WF & AZ FARM & 241-A2-155 & 241-Az-1보 & 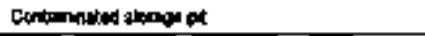 \\
\hline AD & AZ FARM & $24 a-12,+64$ & 24142-19: & 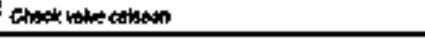 \\
\hline Wอ & AZ FARH & 2414tz-10 & $241+1 z-162$ & Check rimetomitch \\
\hline nto & AZ FARM & $241+12+50$ & 2414tzon & tange \\
\hline 7 & AX Farid & 2HAz-901A & 2s1-Az-610 & 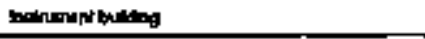 \\
\hline Wo & A2P的州 & $241+\cos -\cos -1$ & 2d1-中?-1 & Clingive bex \\
\hline We & AZ PARH & 2A14tz-c口a-10 & $241+2-10$ & chmoul be \\
\hline Wre & AZFAfied & $2 A 1-2-c 0,0-2$ & $211+2-2$ & chonout box \\
\hline $\mathrm{WB}$ & AzPARim & $2+1+2-0000$ & 2:11-A2-3 & chmouk the \\
\hline WD & A2 PAmin & $241-N$-ctat & $241+2-4$ & Chomot the \\
\hline wi & 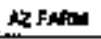 & $241-2 z-c 098-6$ & $241+120$ & Crenont bep \\
\hline Wh & 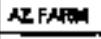 & $201-\cos -\cos$ & $211+x-6$ & ckinesk top \\
\hline M & Ar YAM & $11+22-000-7$ & 201AF 7 & chonit bo \\
\hline
\end{tabular}




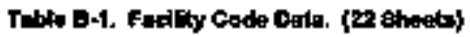

\begin{tabular}{|c|c|c|c|c|}
\hline Poctifitcode & Pam & 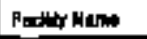 & fuld Binet & Tripa \\
\hline WB & Nz rakin & 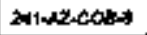 & 2tratere & Chementbor \\
\hline WO & AZFARA & $241-A Z-C C A D$ & $241-2-9$ & Ctempat bew \\
\hline $\mathbf{z}$ & NZF的和 & 200 bots & soow & 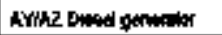 \\
\hline$z$ & Nzrian & star & 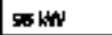 & 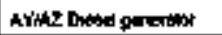 \\
\hline ER & B HAnth & 216403 & 다에 & Fand(at pend) \\
\hline ER & BFARY & 210-8 & $216-8.56$ & $\mathrm{Cnb}$ \\
\hline ER & B FAR & $216-802$ & $216-92$ & $c n b$ \\
\hline $\mathbf{E R}$ & D FHNM & $21 t-60$ & 21060 & Dath \\
\hline $\mathbf{T} \leqslant 5$ & A PARA & 24160101 & 241.8-101 & Engtathed bonk \\
\hline $\mathbf{T}$ & B FAFin & $2410-102$ & $241-8-19$ & Shoin theid lonk \\
\hline$T S 80$ & 口 FAPH & $2+1+108$ & $241-B-103$ & 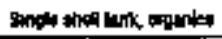 \\
\hline T35 & D Faㅐ & $24-104$ & $241 \cdot+104$ & 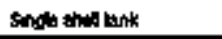 \\
\hline $\mathbf{T}$ 55 & 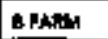 & $2+140-106$ & $2 A 1+B 16$ & Inglenthel bonk \\
\hline T g白 & DFARin & 2410-100 & $2+1-16$ & Eng and tort \\
\hline T $\$ \$$ & D FADH & $2410-167$ & 2ब1-श-10 & 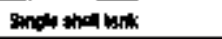 \\
\hline T\$s & D Pdenth & 240108 & $241+3.165$ & Singht thed bnk \\
\hline rab & BFHAM & 241-810 & $2018-100$ & 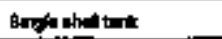 \\
\hline T 68 & DFABMA & $241-0-110$ & $241-110$ & 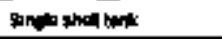 \\
\hline$T \$ \$$ & Danin & 244-111 & $241+6-141$ & 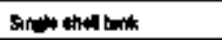 \\
\hline T 35 & G FHANA & $3446+12$ & 2418112 & Ginglentin tart \\
\hline$w t$ & B FARM & $741-8-151$ & $2+18-16$ & Danton tap \\
\hline דוה & D PNAM & $241-1-152$ & $2116-15$ & 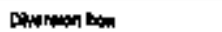 \\
\hline
\end{tabular}




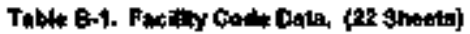

\begin{tabular}{|c|c|c|c|c|}
\hline Fundofy Cood & $\operatorname{sen}$ & Macting Nme & 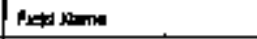 & ryper \\
\hline WD & BFARM & 241.0 .183 & $241-6-153$ & Dementan bax \\
\hline WD & B FARM & $2+1-6-13$ & $241-8 \cdot 154$ & Dowaven tax \\
\hline 553 & B FAFA & $241-4-211$ & $2418-201$ & 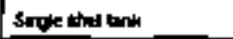 \\
\hline 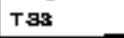 & B FARM & 241 B 202 & $241-\tan$ & Buncte bellons \\
\hline T昰 & BEARH & $24160 \mathrm{~ms}$ & $241-8-203$ & Bungle stewimn \\
\hline Ist & BFAReA & $241-8-204$ & $241-2014$ & 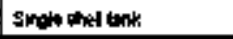 \\
\hline mo & E PAा: & $241+292$ & $241+42012$ & Dentrition Wir \\
\hline on & B FARA & 241 bato B & 21168019 & 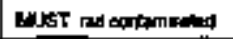 \\
\hline 19 & B Farad & $241+8+3028$ & $241-8-3028$ & 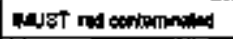 \\
\hline no & B FAPRA & 2-42-8-151 & $2428-151$ & Comman bex \\
\hline saT & D FANG & 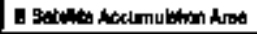 & 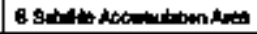 & 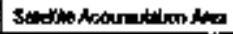 \\
\hline Tse & DKFARH & 241.EK. En & 241. $4 \times 1001$ & 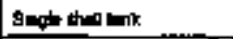 \\
\hline T移 & ExFAFH & 241. Bux.sng & $2418 x-10 \%$ & 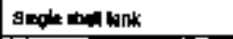 \\
\hline T B & EXFARH & $241-E x-103$ & $24+2 \times 5 \times 103$ & 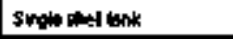 \\
\hline T35 & BX Find & $241.906,004$ & $241.0 \times \cdot 104$ & Single then then \\
\hline 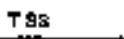 & DXFAFG & $241-D_{0}-106$ & $241-e x-106$ & Sugh hef trit \\
\hline TE\& & EXFNA & $241.8<400$ & 291-DK-106 & 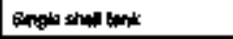 \\
\hline T路 & Daxpun & $2+1-p^{2} x-16$ & $201+0 \times 0,404$ & 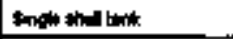 \\
\hline$T$ ts & BxFufy & $2410 \times 0 \times 00$ & 241-Bx.100 & Sngle that trit \\
\hline$r \$$ & AX FARAd & $241-4 x-10$ & $241-60-100$ & 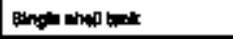 \\
\hline T 9t & Exphan & $201-8 \times-110$ & $2 \times 1-10 x-10$ & thath that \\
\hline$T 58$ & expary & $2+16-111$ & 241-Exh-11 & thest thel that \\
\hline
\end{tabular}


Tabl E-1. Facing codo Deth 122 shocts

\begin{tabular}{|c|c|c|c|c|}
\hline 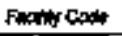 & Pwht & Fantr Mans & Foid Mtum & Tho \\
\hline T\&s & BU FARM & $241+\mathrm{BK}_{6} 112$ & $241 \cdot B \times-112$ & steph thin tonk \\
\hline out & ENCFARH & $241+8 \times(x)=29$ & 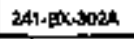 & 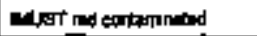 \\
\hline call & BXFAFA & $241-\mathrm{Ex}-3028$ & $241-6 x-303$ & 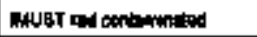 \\
\hline Ont & BCPrfind & $241-0 x-1000$ & $241=0 \times-300$ & WUST and conthinted \\
\hline om & BXFAP4 & 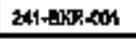 & $241.8 \times R=001$ & 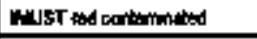 \\
\hline 해 & BX flpord & 241.000000 & 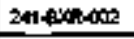 & 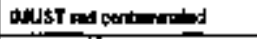 \\
\hline 6 & BXFAfiond & $241-80 \times 2-003$ & $241-8 \times x-0 \times 1$ & 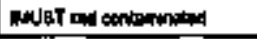 \\
\hline c애 & BXFNAFA & 241-DXR-M11 & 241-exp:01: & 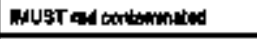 \\
\hline wo & IXFANA & $241-8 x R-151$ & 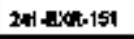 & Dreatosin ton \\
\hline Wo & Dxphot & $241-2 x p-103$ & $2+1.4 \times a t \cdot 152$ & Dowasn bey \\
\hline unt & BXFAford & $241-B \times p-159$ & 241-Bxtats & Denan bex \\
\hline$T C O$ & EY ENPA & $24-6 x$ & $24-1 x$ & 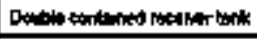 \\
\hline TCN & exNAPM & $2446 x$ & $24+2 x$ & 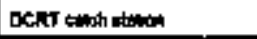 \\
\hline 积 & BrFant & $216-2 \times r a n$ & 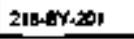 & 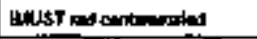 \\
\hline Tas & Br FARAM & 241-EY101 & $241-84+901$ & Engte ghall bon \\
\hline T 65 & EY EAPOA & $2+16 Y-102$ & $2+1-\nabla Y-100$ & 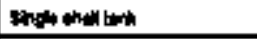 \\
\hline $\mathbf{T}$ \$6 $\mathrm{F}$ & OY FARK & $2414-44-105$ & $241.0 \% \cdot 1003$ & 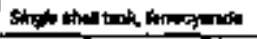 \\
\hline T的F & DrFARM & $2+1-6 Y-104$ & $241-87-104$ & 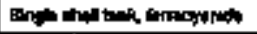 \\
\hline TEEF & EYFARAM & $2+1-6 y-160$ & $2 * 1-84-106$ & 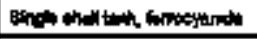 \\
\hline T $1 \mathrm{~F}$ & DrFiney & $24+1 / Y-100$ & $241-0 \times+104$ & 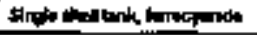 \\
\hline$T 58.6$ & WF FAAM & 2) - & $241-6 r+401$ & 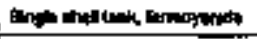 \\
\hline $\mathbf{T} \notin \mathbf{F}$ & GYFARH & $2 * 1-6 Y-10 \%$ & $261-8 Y-106$ & 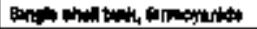 \\
\hline
\end{tabular}


Table B-1. Facindy Code Deta, (at Bhats)

\begin{tabular}{|c|c|c|c|c|}
\hline Handy tor & $\operatorname{Ranh}$ & Pathen & Fowd Hentre & $T_{p e}$ \\
\hline$T$ \$s & BY FAPA & $2418 Y+10$ & $241+4=1100$ & 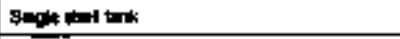 \\
\hline ISS F & er Fond & $241.0 \%-110$ & $241-8 \%-110$ & 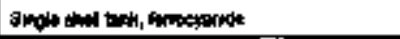 \\
\hline T ss F F & 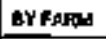 & 241-Er-111 & $241-8 y+111$ & 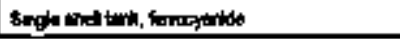 \\
\hline$T \mathbf{s g}$ & BY FAPAd & 241 BY 112 & $241+84 \cdot 112$ & 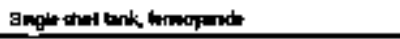 \\
\hline$z$ & BY FAPM & $2018+401$ & $241+4 y=01$ & 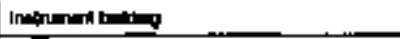 \\
\hline $\mathbf{z}$ & EYFAPA & $241-8 \%-302$ & $2+1+6 y-\sin$ & Componter watino \\
\hline Wa & EY FAmUt & 2A1-64R.152 & 241-1YR-152 & Dhardon bex \\
\hline ND & DY FARA & 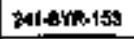 & 2H.SYR.1S3 & Drman ben \\
\hline wo & GY FARA & 20164kis & $2+1.4$ Yta-154 & Doopan wa \\
\hline Wo & BY FARH & 241-日T-1结 & 241-EYin-15S & Dresum ber \\
\hline TS3 & CFARM & $2+1-6.101$ & $24-6-104$ & Shale thellont \\
\hline Ts50 & CFAAN & $241-6-102$ & 241-6102 & sogit had thik \\
\hline $\mathbf{T} \mathrm{st}$ & CERpen & 2610test & 241000 & sngatill buk \\
\hline $\mathrm{I}$ 鵤 & C FARM & 241-6-104 & 241-c-104 & 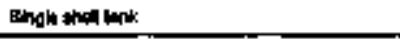 \\
\hline$T 596$ & C FARM & $2 n-6.105$ & $241-c-10$ & 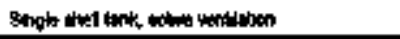 \\
\hline TsS LV & C Pafoul & $24400-106$ & 2410600 & 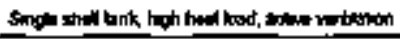 \\
\hline $\mathbf{T}$ \& & c Fapan & 2*1-c-10t & $241 \cdot \mathrm{C} \mathbf{k N}$ & 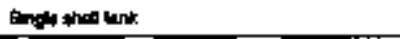 \\
\hline $\mathrm{I}+9 \mathrm{~F}$ & C FAR:A & $2 n-c-108$ & $241-6-190$ & 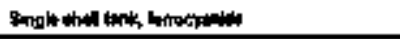 \\
\hline T\&\& F & crufar & $241 \cdot 0-100$ & MISAla & 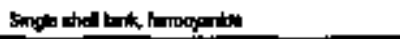 \\
\hline T 的 & C FAPGM & $24 t-c-100$ & 341 它110 & 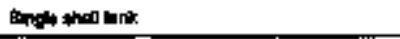 \\
\hline$T \Leftrightarrow \mathrm{r}$ & C Finis & $24-6-111$ & $241-4-111$ & 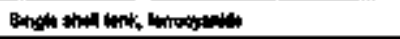 \\
\hline T $65 \%$ & conpis & $241-6-112$ & $201-6-112$ & 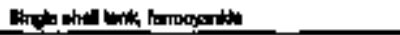 \\
\hline
\end{tabular}




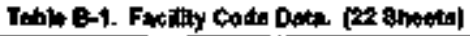

\begin{tabular}{|c|c|c|c|c|}
\hline Fmarcat & fore & Poctisy Hame & 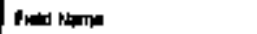 & Typex \\
\hline mo & C FNRH & $24 t-(-15)$ & $241-c-151$ & 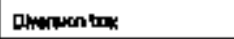 \\
\hline mo & CEARNA & $241+c-152$ & 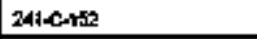 & Daweran bes: \\
\hline mo & C FARAd & 24156 & $249-6-107$ & Dmanten bex \\
\hline$m$ & C FARM & $3 \times 1<154$ & 241 C 154 & Daversenton \\
\hline $\mathrm{I} \$ 8$ & C ENRH & $2+1-\cos$ & 341-c-201 & 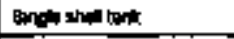 \\
\hline Iss & C FARPA & $2+1-c-202$ & $201<-202$ & sinet whalum \\
\hline Tst & C FAlsid & $241+c 200$ & $2+1+\cos$ & 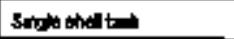 \\
\hline$T \lesssim s$ & CFAAIM & 2011004 & $2+1-c-201$ & Angle shal tuld \\
\hline WD & cFAFin & $241-1-20$ & $241-c-52$ & Dax \\
\hline Can & C PARA & 241-c-901c & astensic & 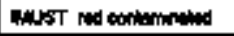 \\
\hline wo & G RAlEA & $24+C R-1 M$ & $2416 R_{1} 151$ & Dowerbin be \\
\hline WD & CFAFA & $241-C R-163$ & 241-CR-1\% & Domerar tor \\
\hline WD & CFAF:A & 241-CA-159 & $248-C R-153$ & Dexing \\
\hline $\mathbf{z}$ & C PARPA & 241-CR-2R1 & $241-\cos 27$ & Controt recen \\
\hline no & CFrina & $241.9 R .15$ & 241.ER-1) & Drmetin bax \\
\hline WD & CFAPA & 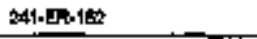 & $241-E R-15$ & Onden ber \\
\hline wP & CFAPaA & 241-ER-158 & 24LER-159 & 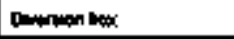 \\
\hline$T \mathrm{CN}$ & C. FNor & $211-6 \mathrm{R}-3+1$ & 2011-Ekd11 & Condtin \\
\hline no & C FAAEA & 241-dini1 & 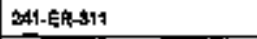 & Onnm beer \\
\hline out & C FARA & 2A1-ER-a11A & $241-E R-311 x$ & 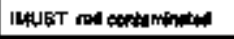 \\
\hline red & C एAMM & $244 C R$ & $244 \mathrm{Ck}$ & 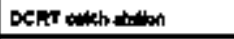 \\
\hline SAT & C Fank & 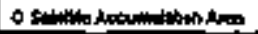 & 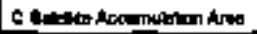 & 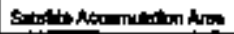 \\
\hline
\end{tabular}




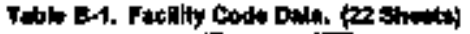

\begin{tabular}{|c|c|c|c|c|}
\hline 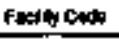 & Fave & Fuedtr Nom & 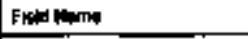 & Ime \\
\hline ser & NuScgШANEOUS & Xue & COE & 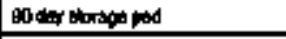 \\
\hline so & 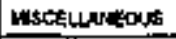 & 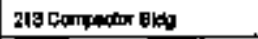 & 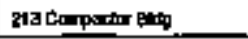 & 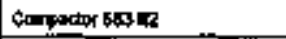 \\
\hline $\mathbf{E R}$ & MBBCELHEDOU & $216-2-20$ & $216-2-20$ & 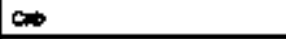 \\
\hline EF & WMTEELANEOUE & 216z-21 & $21+221$ & Pans \\
\hline wo & MACEEUANEOUS & $2+12 W-15$ & 2H-2H-151 & 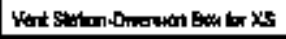 \\
\hline $\operatorname{mo}$ & MSCELLWVEOU & $8+1+460$ & 2011fans & 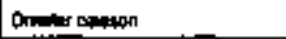 \\
\hline no & 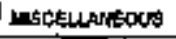 & $241+562$ & $2+1+1+500$ & wated \\
\hline wa & MgĆlaneOUE & $24+40 x-154$ & $241-4 x-151$ & 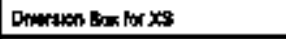 \\
\hline $\mathbf{s s}$ & WSCEULNEOUS & 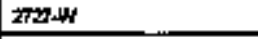 & $272 \pi+4$ & 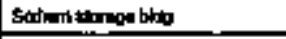 \\
\hline PQ & HECEUWNEOU & Dable Eraned Pipmp & Dable Enchad Ppap & Tronetr Ln Pperp \\
\hline W白 & 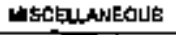 & 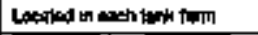 & 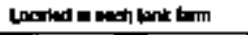 & cingoul bov \\
\hline WB & ISCERLANEOUE & 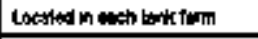 & 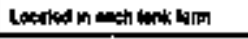 & 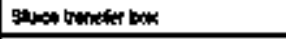 \\
\hline$w 0$ & MSCEUANEOUS & 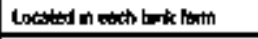 & Locosted in ench tow fin & Derowen bow \\
\hline WD & rascedunidous & 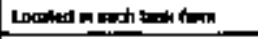 & 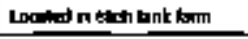 & Daverturan \\
\hline wD & 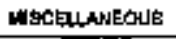 & 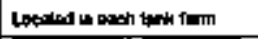 & 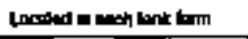 & Mmpp \\
\hline WD & MBCELANFOUS & 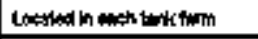 & 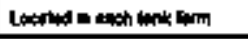 & Yman pox \\
\hline win & MIscerlwhous & loxind hit thth tonk fin & 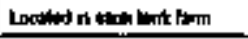 & ctin \\
\hline if & 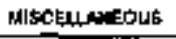 & 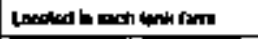 & Locoded n man mix thm & ctant \\
\hline MF & 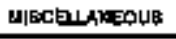 & 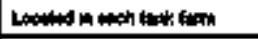 & & Finm pat \\
\hline wp & Mreserwhinous & Locind n then lank ton & 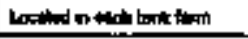 & Indtheston pt \\
\hline WF & Hitser Undectus & laceled nesch lank tore & Loceded n mint turt tain & Anncept \\
\hline sor & 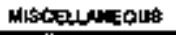 & 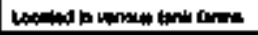 & 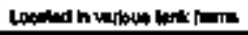 & 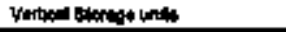 \\
\hline
\end{tabular}


Table B-1. Facelity Code Datar 122 Ehowta)

\begin{tabular}{|c|c|c|c|c|}
\hline Fartiy Pedn & $\mathrm{Fam}$ & far:eq nome & Dota Mare & Type \\
\hline PA & MIBCELLANEOULA & 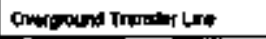 & 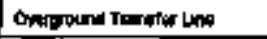 & 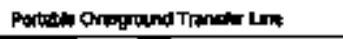 \\
\hline et & nuscerdunicons & DFP mond & FFP Pand & Motd \\
\hline ShT & MASCELUNEOUR & 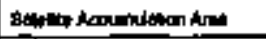 & 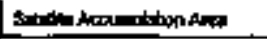 & 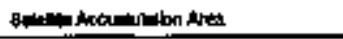 \\
\hline PC & MLBCELLANEDUKB & 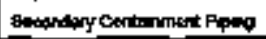 & Eucontery Centinnem Appig & Thendtor Lne Pyeng \\
\hline PB & HacelanEOU日 & 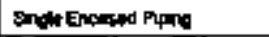 & 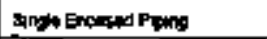 & Trwator Lon Pang \\
\hline$s 4$ & MESCELLANEOUS & 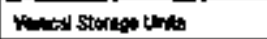 & 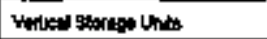 & I2:20 th dow \\
\hline Ek & $\operatorname{sindu}$ & $210-5 i x$ & $210-5-28$ & An \\
\hline $\mathrm{ER}$ & Q EARAM & 216-1200 & 210-80 & cos \\
\hline ChN & BFARM & $240-9002$ & $200-302$ & 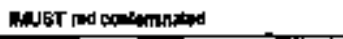 \\
\hline Ts\$ & SEARM & 2415.5101 & 2At-sictot & 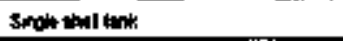 \\
\hline TSSHO & SFARM & $241-50102$ & $24+100$ & 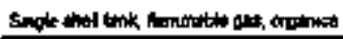 \\
\hline Tos & BPANEA & $2 / 1-8-103$ & 24150100 & 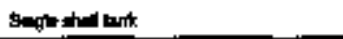 \\
\hline T EB & 8fariul & $24 \%-3-104$ & 241-8-104 & Sngik thell bonk \\
\hline T 93 & SFARM & $2414-105$ & 2015-105 & 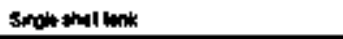 \\
\hline Tst & S PARAN & $2+1-8-106$ & $241+5100$ & Shoth othed task \\
\hline $\mathbf{T}$ 的 & A Fapes & $2+1-4-100$ & 241040 & 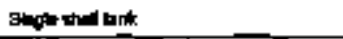 \\
\hline$T \Leftrightarrow 9$ & S FAPQM & $241-\$-106$ & 2415-1489 & Sheng that lonk \\
\hline Ts5 & soneses & $2+1840$ & $3 \times 1-190$ & Singlenthed bork \\
\hline T कם & a fips & $2+1-3-110$ & 24148410 & angth thed lit \\
\hline$T \$$ & SFafien & 2*1-6-111 & 265-8-111 & 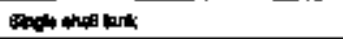 \\
\hline$T 68$ & 5 FAQM & $251-4.112$ & $241-6-112$ & 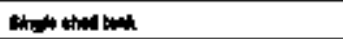 \\
\hline No & stangan & 2*14.นด1 & 241-6.4B & Otheroten then \\
\hline
\end{tabular}




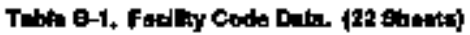

\begin{tabular}{|c|c|c|c|c|}
\hline 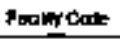 & ram & Pation Nom & Find Hont & Typo \\
\hline no & EtAKN & 241-5ild & $24 t-3+42$ & pmercen bes \\
\hline Dut & SFARM & $241-8-30201$ & $241-6-302 \mathrm{~A}$ & 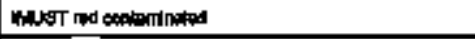 \\
\hline out & S FAPN & $241-5+3009$ & 241.5306 & 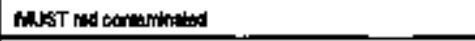 \\
\hline TсR & 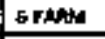 & 2415304 & 241 3-304 & C-tich toin \\
\hline $\operatorname{mo}$ & B PAkM & $201-6-k$ & $241-94$ & $\operatorname{tanp}$ \\
\hline mo & SFARM & $241-86$ & 2*1-8 & tatepd \\
\hline no & S FARM & $2 \mathrm{a} 1-5-\mathrm{C}$ & $24+5, C$ & vanat \\
\hline Who & \& FAREA & 241 \$D & 2011.50 & What in \\
\hline gr & G PAPAS & 241-5ivts & 2A1-8-র) & Letretstrequ Un: \\
\hline$T C D$ & SFARA & 246.8 & 2445 & 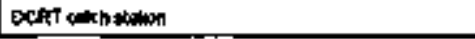 \\
\hline sik $T$ & 5fArew & 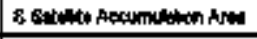 & 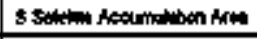 & 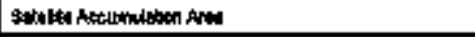 \\
\hline $\mathbf{z}$ & SXKAANA & & & Latera Wh" (t) \\
\hline$\tau \operatorname{sen} \mathrm{H}$ & SxTARAd & $2+1.5 \times 10.109$ & $291-6 x-101$ & 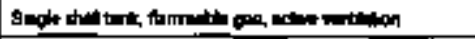 \\
\hline Tав н $\mathbf{Y}$ & EXXFARH & 245-8x-10h & $201-9 x-102$ & 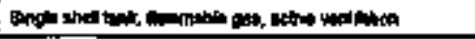 \\
\hline T53 ноУ & SXFARM & $241-5 \times-109$ & $241-5 x-100$ & 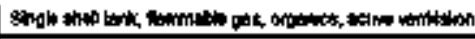 \\
\hline TSsiv & STCFAAL & $2 n-5 x-104$ & $241-5 x+104$ & 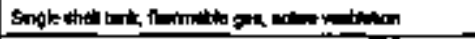 \\
\hline TESH H & 6x Fum & 241- Baktos & $2+1-8 x-16$ & 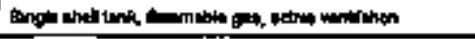 \\
\hline $\mathrm{T} \$ \mathrm{HOOY}$ & $\$ X F A N H$ & $2+1-93 x-100$ & $241-5 x-108$ & 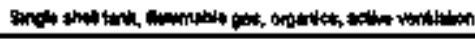 \\
\hline TsS L & sx Fanm & $34+5 \times 1000$ & 24160,107 & 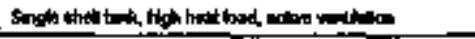 \\
\hline $\mathbf{T}$ en L V & EXFAR & 3a1-30, 100 & $241-\$ x-100$ & 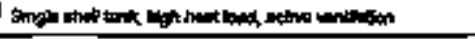 \\
\hline$T \$ 81 \mathrm{~V}$ & \$X Fenew & $241-8 x-100$ & $241-6 x-100$ & 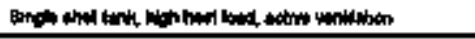 \\
\hline$T \notin S$ LV & EXPANin & $241-8 x-10$ & $3+1-4 x-110$ & 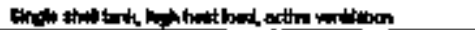 \\
\hline
\end{tabular}




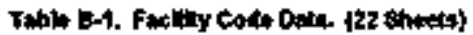

\begin{tabular}{|c|c|c|c|c|}
\hline Faneycont & Pra & 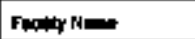 & Fitam: & Tpe \\
\hline T\$ & SXFARH & $241-93 x-111$ & $211-3 x-111$ & 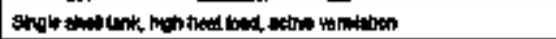 \\
\hline$T$ ss $4 \mathbf{Y}$ & sXFANH & $241-5 x-112$ & $241-5 x-112$ & 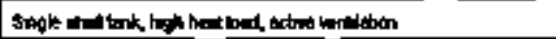 \\
\hline T & gXFARPA & $241-8 \times 2-113$ & $249-65015$ & 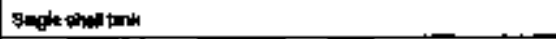 \\
\hline$T \& D L Y$ & פXFARM & $241-8 x-114$ & $2+15 \times 114$ & 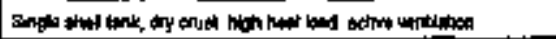 \\
\hline $\mathrm{T} \Leftrightarrow$ & EXFARM & $241-9 x-115$ & $241-3 x-115$ & Enoto thel tent \\
\hline mo & SXFARM & $241-5 \times-151$ & $241-3 x-151$ & Dherton ter \\
\hline wo & skrifos & $2414 x-182$ & $241-5 \times 152$ & Onersan pas \\
\hline cen & 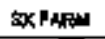 & $241-3 x-3 \times 2$ & $2+1-\$ x+3 a_{2}$ & 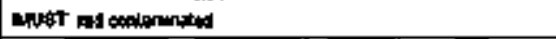 \\
\hline mo & BXFARR & $211-3 x-A$ & $241-3 \times-A$ & mand \\
\hline Ho & EXFNA & 241 कर: & $241+56013$ & $\sin p$ \\
\hline su & sxistifad & 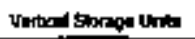 & 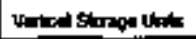 & 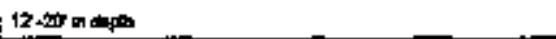 \\
\hline wF & SYPAFiqu & $241-8 Y$ & 241-sy & 3 ingan pal \\
\hline WT & BY Fugaw & $241-97-016$ & 2h-sY-Q1C & Lentedon PA \\
\hline WF & STFNAM & 241.8 Y0102 & $24+\$ 4002$ & 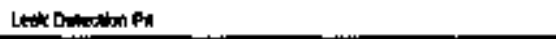 \\
\hline Wo & stropid & $2+1-5 y-128$ & 2an-SYate & Fond perpt pt \\
\hline$w r$ & BYFNF & 211-\$r-att & atsoratis & Fiph \\
\hline me & SYFAPM & $245-84-030$ & $241-87-09$ & L L \\
\hline TDSHPV & Srompan & $241.84-104$ & 241.96 .100 & 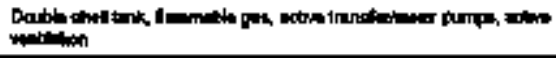 \\
\hline T DBP P & GY FAFA & $241-34-402$ & 241-4\%-100 & 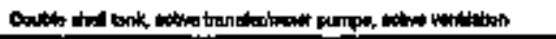 \\
\hline $\operatorname{Tos}$ & SY EMAM & $24,9 y+400$ & 241.694 .403 & 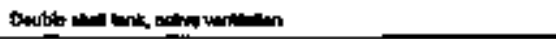 \\
\hline $\mathbf{z}$ & Sr Finkat & $245-3 \times 201$ & 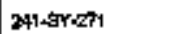 & 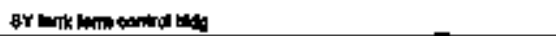 \\
\hline
\end{tabular}


Table O-1, Faclly Code Date (22 Sh+ota)

\begin{tabular}{|c|c|c|c|c|}
\hline Pocterircat & Fam & fargh & Ped Hand & tyo \\
\hline$z$ & GYFARAM & $241-8 Y-Z T 1+51$ & $241-56 / 2 t>1+P 1$ & 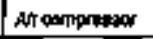 \\
\hline WD & SY FANA & 241 -syath & $241-8 \pi+A$ & valept. \\
\hline wF & Er falou & $261-94 \times 4$ & $241-5 S^{\prime}-A$ & Flowh pl \\
\hline wo & BY FArian & 2*1-5Y-B & $241+8 Y A B$ & How pt \\
\hline$w F$ & SY FARA & 241-EY-B & $241<8 Y 48$ & Patipla \\
\hline WB & ST FAnth & $241+5 y-000-1$ & 241-3Y-1 & Damod bex \\
\hline WB & sY Finga & $241-5 y-\cos 2$ & $241.83 \times 2$ & Ctabinoud box \\
\hline is & SYFARU & $241-94 \cos 4$ & $241-8 \times 4$ & Gonout bax \\
\hline$m$ & SY FARAN & $241-5 \%-c 004$ & $241-\$ Y 44$ & chanout bex \\
\hline$m 8$ & SY FARAI & $241+5 Y-200-5$ & 241-3Y-5 & chemat bes: \\
\hline ME & SYPARH & $244-59+60 \theta_{8}$ & 241-SY-6 & anthex bex \\
\hline ER & Tratin & 216-T.1 & 21E-T-1 & Inth \\
\hline EA. & Trapes & 216-T-32 & 216-T.ts & Cab \\
\hline ER & TENAM & 216-T-42 & $218-1-42$ & Onen \\
\hline ER & TVARM & 210-T. 7 & $21 \%-7$ & $a b$ \\
\hline wo & TFANM & $240-T 481$ & $240 \times 141$ & Anmedon tax \\
\hline wo & TFAAM & $200-15$ & 200-1-193 & Dim \\
\hline WD & T FARM & $2407 \cdot 163$ & 20-1-199 & Dintition ber \\
\hline Wo & TFAKM & 200Ttzes & 2ATSA2: & Alofdor bo \\
\hline wo & T FARA & 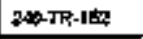 & 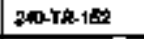 & Donerion ber \\
\hline WD & TFAR⿴囗十 & 20-Th-1ES & 20-TR-15 & Dtwailon bex \\
\hline T.6B & T FARM & 201-Tr19t & $24 k-T .101$ & atholh thithonk \\
\hline
\end{tabular}




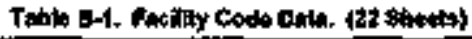

\begin{tabular}{|c|c|c|c|c|}
\hline Focerp Gods & Fware & 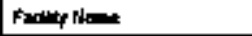 & 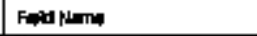 & Tpen \\
\hline$T$ st & TPAPAS & $2+1-T-102$ & 2+1-T.102 & Sholetiolank \\
\hline $\mathbf{I} 6 \mathrm{~s}$ & Trentus & $241-T \cdot 103$ & $2+1 \cdot T \cdot 108$ & shplationink \\
\hline T $\$ 6$ & TFAFN & 2-41.T-104 & $241+\mathrm{T}-104$ & Shigle bur bur \\
\hline 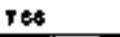 & TEARN & 241-T-105 & $201+T-105$ & Shipgent \\
\hline Ts\$ & TFAPM & $241-T-106$ & $261-T-108$ & Sincla \\
\hline $\mathbf{r}$ bs $\mathbf{P}$ & Tratas & $241 . T-10 \%$ & $2 \pi+T+10 \%$ & 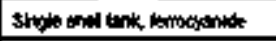 \\
\hline $\mathrm{T} \$ \mathrm{ga}$ & T FAFM & 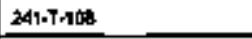 & $241, \tau-106$ & Inglt the bift \\
\hline TEB & TFAAM & $241 \cdot T \cdot 109$ & $241-T-100$ & Ingle pend brt \\
\hline$T \$ \$ H$ & TEARH & 241-T-110 & $241-T-110$ & 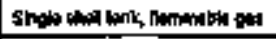 \\
\hline$T \leq s 0$ & TEANM & 241-T-111 & $241-T \cdot 111$ & stholow \\
\hline Tas & TFAPEA & $241 . T+112$ & $241 \cdot T \cdot 112$ & shgint thell thin \\
\hline WD & TFA艚 & $241.7-151$ & $241-T-161$ & Onnowan ben \\
\hline T\$3 & TFARM & 24I-T-201 & $241-T-201$ & Singloshom bor \\
\hline Tss & T FAREA & $24 t-T-202$ & all.T.ane & shote end tort \\
\hline $\mathbf{T}$ 的 & T FAFA & $241.7-270$ & $241 . T$ and & 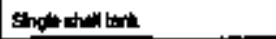 \\
\hline$T$ 189 & TFAPQM & $2+1.7-304$ & 241-†-264 & Engteshell bit \\
\hline on & TPAREM & 24l-T-301 & $241 \cdot T .301$ & 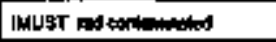 \\
\hline$z$ & TRAnis & $2+1+7=01$ & 241.7000 & Conprocior buting \\
\hline$z$ & TFAley & 241-TIOT & 2:1-T-Jo1 & 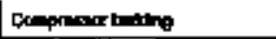 \\
\hline con & TPAPe⿻ & 30-1-15 & 24:T-15 & most \\
\hline ON & I FANW & $24-7 h-R 1$ & 20.ThNR1 & 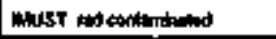 \\
\hline sate & TFAFA & 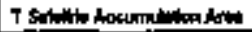 & 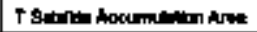 & 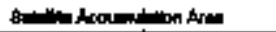 \\
\hline
\end{tabular}




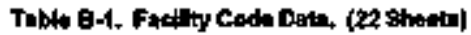

\begin{tabular}{|c|c|c|c|c|}
\hline Funery coode & Ram & Fanderime & Fati Mors & $T_{y p}$ \\
\hline sv & T FARM & 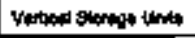 & 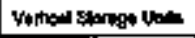 & 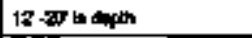 \\
\hline out & TXPNDAH & $2+1 . T \times 200$ & 2 ti-Tken & HUST Bdmpthmeded \\
\hline 에 & TXFARAM & $241-5 \times=002$ & $241+100002$ & Dust rad comamnaled \\
\hline oth & TXFARA & 241-TX-003 & $3+1-T<000$ & 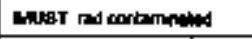 \\
\hline 159 & TXFARM & 241-ГХर-ю1 & 24t-Tx-101 & 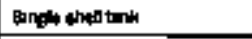 \\
\hline Tsts & TXFARA & $241+\pi, 102$ & $241+1 \times-102$ & Fing \\
\hline $\mathrm{I} \mathbf{5 s}$ & TXFARM & $241-7 K=100$ & $241.7 x<100$ & 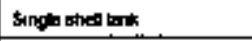 \\
\hline 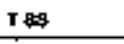 & Tx FAlun & 741-17K-104 & $241+10-104$ & Singla shed trink \\
\hline 1990 & TX FARM & 241-TXNos & $241-7 x-105$ & 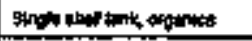 \\
\hline Tst & TXFAי人 & $241-T x-100$ & $241 . T x-100$ & single and ink \\
\hline Ess & IXFAxil & $241, T x_{-107}$ & $241-T x-107$ & stipe that thin \\
\hline T\$ & TXFAntwin & $2012 \pi x+100$ & 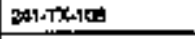 & 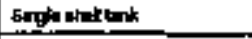 \\
\hline$T \$ 8$ & IX, PARAM & 2A1-TX-109 & 2A1-TR-109 & 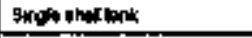 \\
\hline TSS & TXFAPris & $241 . T X-110$ & $201 \cdot 7 x-110$ & sino aratank \\
\hline Tst & TXFARA & 2A1-TX-111 & $241-T x-111$ & 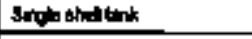 \\
\hline 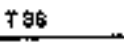 & 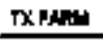 & $2+1 \cdot 7 x \cdot 112$ & $2+1-7 x-112$ & 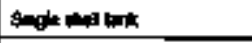 \\
\hline$\pi 58$ & TXFend & $341-T x-119$ & $2+1-T x-113$ & Shopond bit \\
\hline Tss & TX FNFM & $2+1 \cdot T \times 114$ & $241 \cdot T x \cdot 114$ & 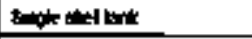 \\
\hline $\mathrm{T}$ 的 & TXPAn & 261-Tx-116 & $241.7 x-118$ & Angle the thet \\
\hline$T B S$ & TXFend & $2 \pi 1-T x-110$ & 2A1-TX-118 & 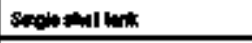 \\
\hline$T 35$ & TXFAR:A & $241 . T x-117$ & $2+1-T x-117$ & 㑭 \\
\hline T tas $\mathbf{F}$ & TXVAnim & $341-5 x-428$ & $2+1+5 x-148$ & 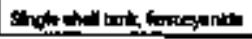 \\
\hline
\end{tabular}


Tabte B-1. Fadily Coda Oats. (202 shoela)

\begin{tabular}{|c|c|c|c|c|}
\hline Ferily Coda & Fam & Roctith Nalle & Mats Horme & Typ \\
\hline no & TXFNPA4 & $241-T x-169$ & 241.TX.1E2 & Druptwan bac \\
\hline mo & TXFARM & $2 n \cdot 5 x-15 s$ & $241-T x-154$ & Dawnam : \\
\hline mp & $T X \cap A R H$ & $241-7 x-194$ & $2+1-150-104$ & Denerabon bas \\
\hline $\operatorname{mp}$ & TXFAFU & 241 TX-1ES & 2A+7) & Dueraben bac \\
\hline can & TX FMARH & $241 \cdot T x-3012 x$ & $241-7 \times 30029$ & Must apdorimmind \\
\hline on & TXFARAM & $2+1-T x-x x=0 ; 0]$ & 241-TX-30090/R & 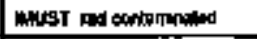 \\
\hline Ton & TKFAR: & $24 t-1 \times-4002$ & $241-T$ xatanc & Calten thik \\
\hline DNA & TKPARH & $242-1 \times \sec x$ & $241.7 x-300 x$ & 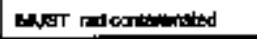 \\
\hline WD & TXFARA & 245-TXR:151 & $241 \cdot Y \times 2 \times 159$ & Dewrom beas \\
\hline WD & TXFARM & $24-1 \times 2 \times-152$ & $241-T 0 R-157$ & 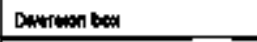 \\
\hline wo & TXFARU & $24 \%-1 \mathrm{KR} \cdot 199$ & 241-TXR-15s & Dueriontets \\
\hline T & TK FARU & $244 \mathrm{TX}$ & $2447 x$ & DCCAT Dalch withon \\
\hline SAt & TXFAla & 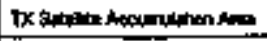 & 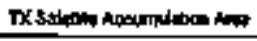 & 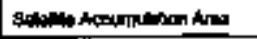 \\
\hline $\mathbf{s v}$ & DXFAfin & 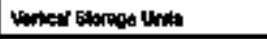 & 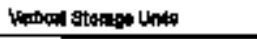 & 12,20 intaph \\
\hline on & TY FAMU & 218TYAOH & 216-TYzaM & 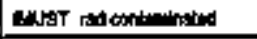 \\
\hline $\mathrm{T}$ 的 $\mathrm{F}$ & 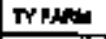 & 241-Tran & $241 . T Y+101$ & 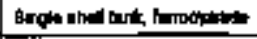 \\
\hline$T$ T & TY FAf: & 241-TY-No2 & $241 . T Y-102$ & 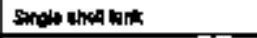 \\
\hline T s. & TYSAPM & $241 \cdot T Y+100$ & 2All-TY-10 & 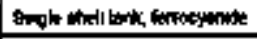 \\
\hline 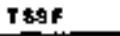 & Trzarat & 3*1.TY-104 & 2N1.TY.104 & 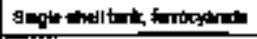 \\
\hline $\mathrm{T}$ ss & TrFitar & 2at-Tr-10s & 24a-TY-14 & 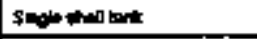 \\
\hline $\mathbf{1} 98$ & TY FNAR & 2ป1-TY-109 & 2N1-TY-100 & 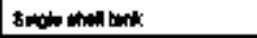 \\
\hline ar & Tr rNen & $2 / 1 . T+3000$ & 24:-Traxin & 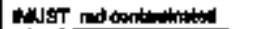 \\
\hline
\end{tabular}




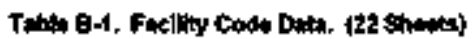

\begin{tabular}{|c|c|c|c|c|}
\hline Fopelep code & fam & Fatiey Wome & Fotd Nome & Trpa \\
\hline on & NFARAM & 241-ГY-\$200 & $2 n-T Y-300$ & 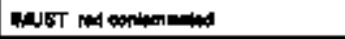 \\
\hline ar & Tr RARA & Whiteil sarepe Unts & Vatred Sarapa Unt: & 12,20 h dheth \\
\hline EF & u penpu & 215.4. 12 & 21:-12 & cob \\
\hline En & U FAfA & 218-U1t & 250-14-14 & Odeb \\
\hline $\mathbf{E R}$ & U FAAS & $20-4-17$ & 2 2N-4T & 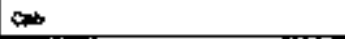 \\
\hline$T s s$ & U RAMUN & $211-4-104$ & 2ख1.1-104 & tingh atil lonk \\
\hline tos & UFAAM & $2011 \cdot 1 \cdot 102$ & $2414 \cdot 102$ & snot athel ton \\
\hline Т авн & UFARM & 2月1.ப.103 & $201-2-103$ & 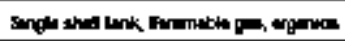 \\
\hline 589 & UFARH & $244+4 \cdot 504$ & $2 \mathrm{a} 1-\mathrm{U}-10 \mathrm{~A}$ & 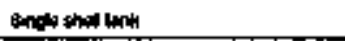 \\
\hline Tss HO & U FARM & $211-2-109$ & $2041+2+100$ & 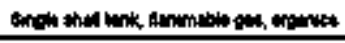 \\
\hline Tsto & u fasen & $2-211+\sqrt{1}+006$ & 20114405 & 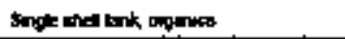 \\
\hline $\mathrm{T} 95 \mathrm{HO}$ & U Fareu & 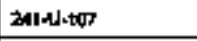 & $2 n-1-10 T$ & 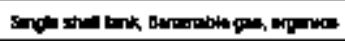 \\
\hline I 18 - H & UFAPMA & $2414 \cdot 109$ & $2: 11-1-100$ & 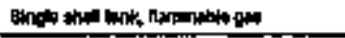 \\
\hline $\mathrm{ISSH}$ & UEARA & $241-100$ & $211+4-100$ & 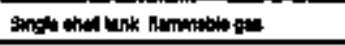 \\
\hline T ss & If FAl:A & $241+4110$ & 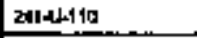 & stapte thel thin \\
\hline $1 \notin 0$ & UFAFA & $2414 \alpha_{111}$ & $2+12-111$ & 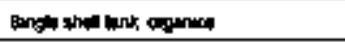 \\
\hline 159 & UFARA & $2+1-112$ & $2 a 1-4-112$ & Bangh then lenk \\
\hline Wo & UFANA & $301-2+181$ & 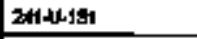 & Dantion ba \\
\hline 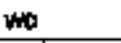 & UI PAFM & $241+4-152$ & $2+1-4-152$ & Dantant has \\
\hline mo & U FARA & $2+1-4159$ & 2utuhiss & Damach bou \\
\hline we & U FArey & $2+1-16$ & 2ndalst & 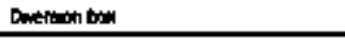 \\
\hline Tos & U F rawer & 2Hdu值 & 21140011 & slng and \\
\hline
\end{tabular}




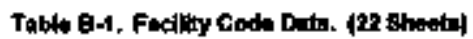

\begin{tabular}{|c|c|c|c|c|}
\hline Fachingods & Fmm & focentrome & Fats Nan & ris \\
\hline$T \leqslant s$ & U FAPQP & $2+1-2-202$ & $241-4-202$ & 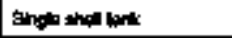 \\
\hline tsso & U FARPA & 2A1-4hatos & $241.1 \mathrm{~m} 2 \mathrm{~s}$ & 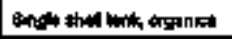 \\
\hline TEB & UFAFA & 311-20x & $241-4-204$ & 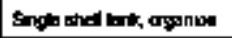 \\
\hline WD & UFAPA & $2 n-4=0$ & $x-y-z=2$ & Demergen text \\
\hline T CH & Ufarad & 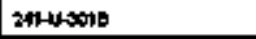 & 241-U-301B & cthol lenk \\
\hline 2 & vexpes & 2ntwites & 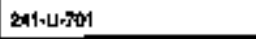 & 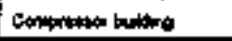 \\
\hline nop & Ufiated & 2410um & Fiduk & Notenge \\
\hline no & UFAPA & 24548 & $211-2-8$ & vatept \\
\hline no & UFAPA & $241-\sqrt{c}$ & $241-1)$ & yane ot \\
\hline no & UEAAH & $241+110$ & 2alatso & Walopet \\
\hline mo & U fantur & $241.17+181$ & 2014 & Dembion tex \\
\hline mo & UFABM & 241-4N-152 & $2+1-U R-16$ & Danten the \\
\hline no & UFARA & 26-UR-168 & 241-UR-163 & Demengen bes \\
\hline no & UFAPA & $240+460.154$ & 241.UR-15.54 & Draram tox \\
\hline TaN & u fintid & $24124 x-305 n$ & $20144 x \operatorname{sos}$ & Catth taik \\
\hline ס & U FARAK & 2440 & 2NAU & DCRT' \\
\hline ShT & UFABM & 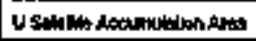 & 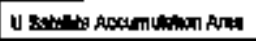 & 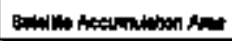 \\
\hline sy & UFATN & thet Storego Linte & 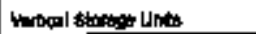 & 127ar houth \\
\hline
\end{tabular}




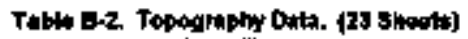

\begin{tabular}{|c|c|c|}
\hline 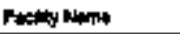 & The & 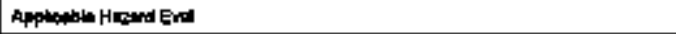 \\
\hline 2006 & 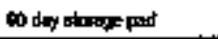 & Pun xas \\
\hline 213 Compoction Bofog & Compader ER 172 & Prin zatior \\
\hline $216-06$ & ch & PHA CDP \\
\hline $214-404$ & tompol pos & 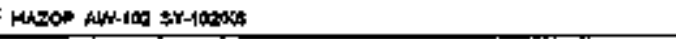 \\
\hline $210-4,26$ & cob & PWh CDP \\
\hline 216-4 30 & col & PH CDP \\
\hline 216-4ash & cnb & Pth cap \\
\hline $216+398$ & Gno & PIth CDP \\
\hline $210-4-47=1$ & tht & PHA CDP \\
\hline $216 \operatorname{sinta2}$ & Grb & PHA COP \\
\hline $216 \sin$ & Gin & PHA CpP \\
\hline $210 A-4$ & $\mathrm{Cnt}$ & PHA CDP \\
\hline $216 \times B=9$ & Find $\{$ and & PHA CDP \\
\hline 218.069 & Ent & PHA CDP \\
\hline $218-8-6$ & Cnth & PHA CDP \\
\hline $210-8-69$ & Eakn & PHA COP \\
\hline $210-5 \times 1+201$ & 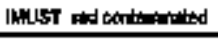 & 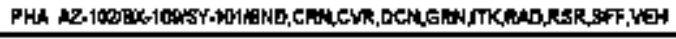 \\
\hline 210-60/5 & †nt & PHA CPP \\
\hline $218-9-29$ & $C_{n b}$ & PHA CDP \\
\hline 2lo-T-t & Dith & PHin cDP \\
\hline 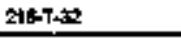 & the & PHA CAP \\
\hline $21<-1 \cdot 4-2$ & and & FHA 然 \\
\hline
\end{tabular}




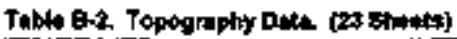

\begin{tabular}{|c|c|c|}
\hline Fieang & TyF & Appopictir Hand Ead \\
\hline 2115.7.7 & enb & PHA COP \\
\hline 215-TYROA & Imust ad contimmeted & 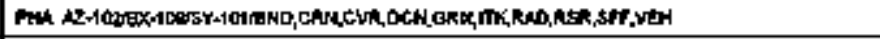 \\
\hline $210-12$ & Çnफ & FHA COP \\
\hline $216-4-14$ & Dakh & PHe cop \\
\hline 2Itskn-17 & sp & PHA COP \\
\hline 21502 & ent & PHA CDP \\
\hline $218-2-21$ & Pond & PHA CRP \\
\hline $240-5-302$ & 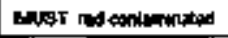 & 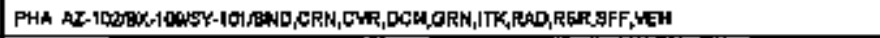 \\
\hline $240-T+151$ & Datewen be & 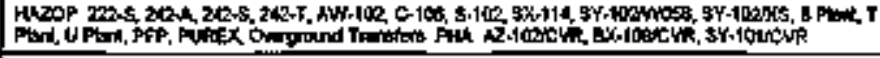 \\
\hline 240-T-158 & Dawnon bas & 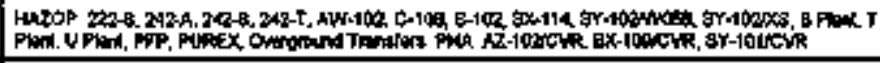 \\
\hline $240 \cdot 7 \cdot 165$ & Dntraton bax & 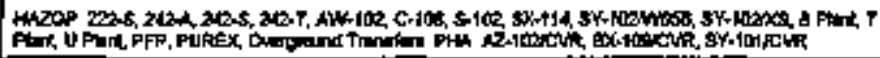 \\
\hline $240-T-200$ & Dontan ber & 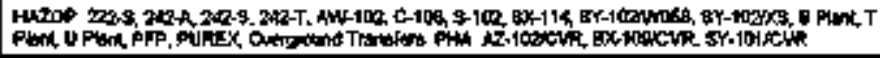 \\
\hline $240-T A-182$ & Ontrimin be & 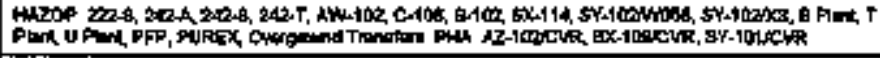 \\
\hline 2:10-Th.15s & 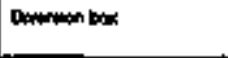 & 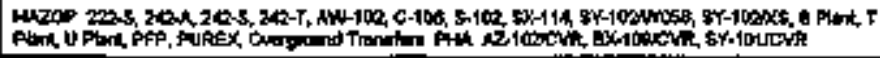 \\
\hline $291+A 101$ & 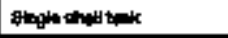 & 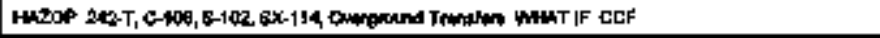 \\
\hline 2A14n+16s & 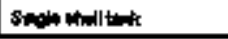 & 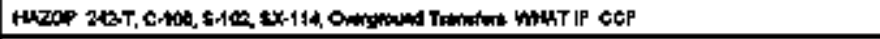 \\
\hline 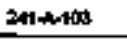 & Ahphedent tonk & 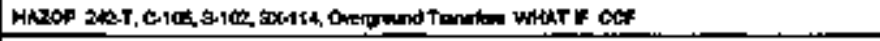 \\
\hline 261-A-104 & 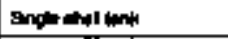 & 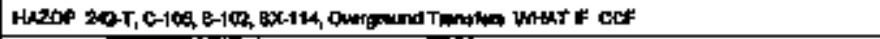 \\
\hline 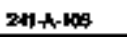 & 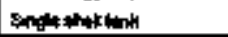 & 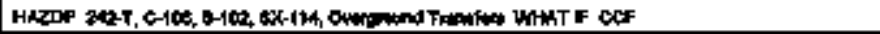 \\
\hline
\end{tabular}


Tahta 1-2, Topograpty Oake, (2s othets)

\begin{tabular}{|c|c|c|}
\hline 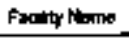 & Tpe & 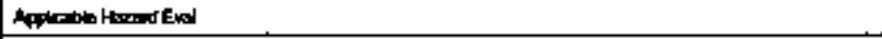 \\
\hline $241+1 \cdot 100$ & 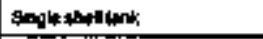 & 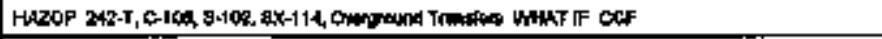 \\
\hline $241-4-151$ & Othosin ber & 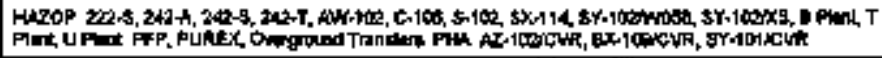 \\
\hline $26+1+152$ & Dewesen boer & 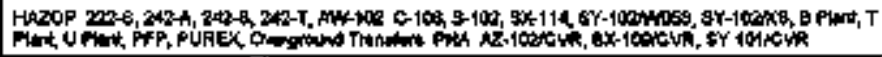 \\
\hline $241+\tan \sin A$ & Cuthtak & HeZOP sY.100Xs, PURFX \\
\hline $241+360$ & Gcket calah tom & 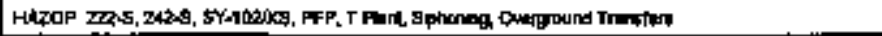 \\
\hline $241-4,360$ & 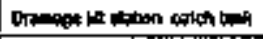 & 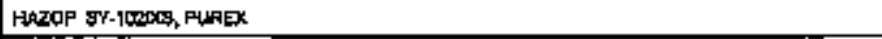 \\
\hline $2 \times 1+401$ & Drwint ctation & 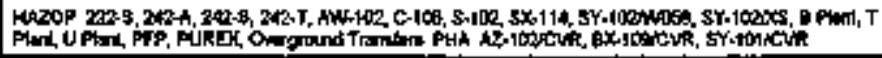 \\
\hline $241+4,4 t ?$ & istehponk & 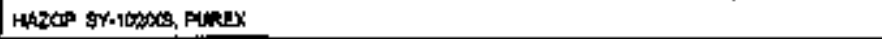 \\
\hline $2 * 1+A$ & Fualing & 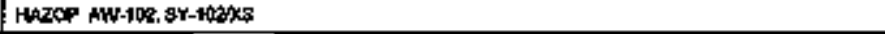 \\
\hline $241+4$ & beriation & 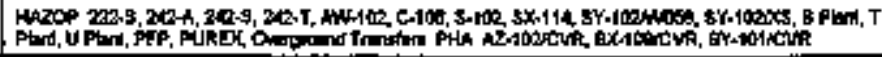 \\
\hline 241140 & Fhang in & 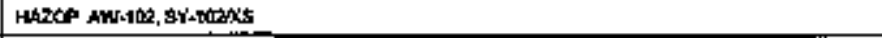 \\
\hline $24 ⿻$ A- & wat ph & 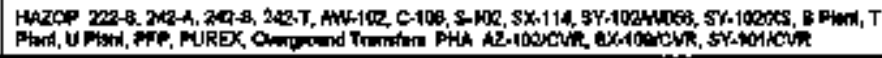 \\
\hline 2414000 & Chimout the & PHA col \\
\hline 241-AnONO & candenato meauer $\mathbf{m}$ & 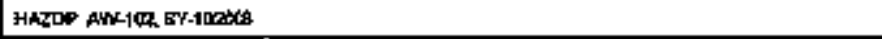 \\
\hline $2+1+4-101$ & Conoth then inter & 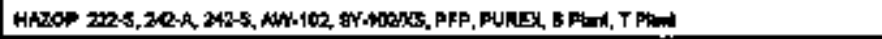 \\
\hline $201+44102$ & Oouth ind lath & 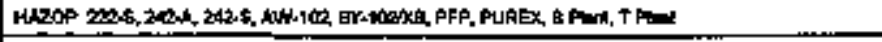 \\
\hline 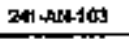 & Doutit and bot & 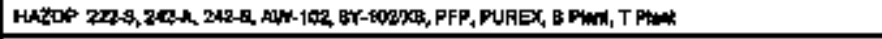 \\
\hline $241+4 \mathbf{4}-104$ & Donth thin wim & 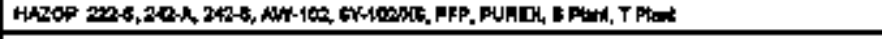 \\
\hline $24-4 k+165$ & 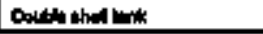 & 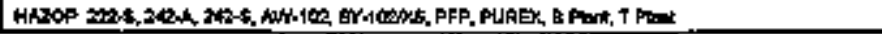 \\
\hline
\end{tabular}


Table B.2 Topogniphy Data. (23 shatsil

\begin{tabular}{|c|c|c|}
\hline Pocilit Now & Type & 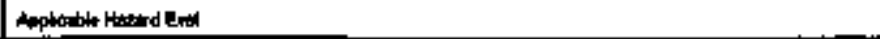 \\
\hline 21-AM-1E: & 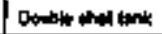 & 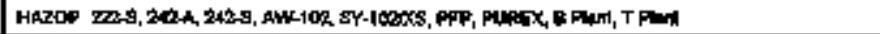 \\
\hline $26+1 \mathrm{~N}-10 \mathrm{~T}$ & Datte phaf bik: & 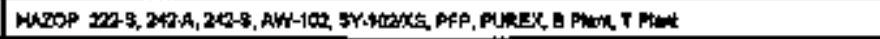 \\
\hline 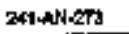 & Bamed pd & HuzOP AW-10z, EY-10000) \\
\hline $241-\mathrm{NHA}$ & Whin & 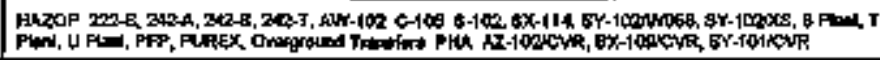 \\
\hline $241+1+6$ & thang ont & 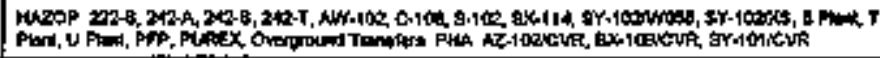 \\
\hline $241+N-f P$ & faum pa & HAZOP AW-102, BY-1000XB \\
\hline 241-AP & 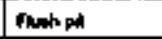 & 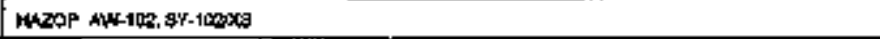 \\
\hline $241-A+101$ & Oovitu bhen tait & 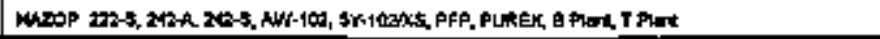 \\
\hline 2A1 AP.102 & Douth shed batt & 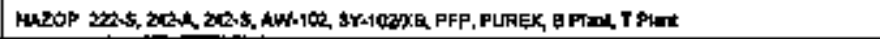 \\
\hline 2414ap.100 & oovith shed bert & 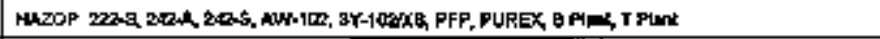 \\
\hline $241-40.104$ & 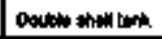 & 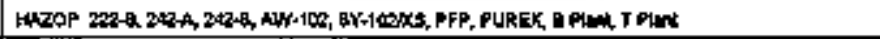 \\
\hline $241+4-165$ & 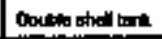 & 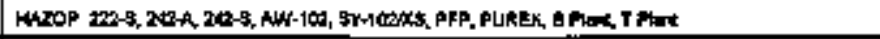 \\
\hline 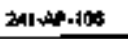 & Douth and tint & 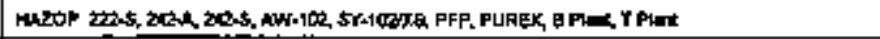 \\
\hline 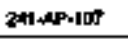 & 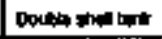 & 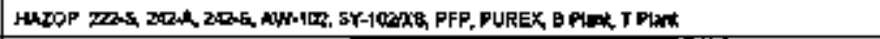 \\
\hline $2+1-10-100$ & 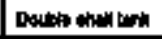 & 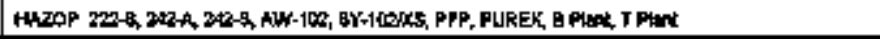 \\
\hline 2HAPrZT3 & seren pa & 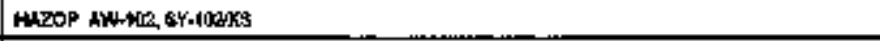 \\
\hline $241 \rightarrow P 4$ & $\min$ in & 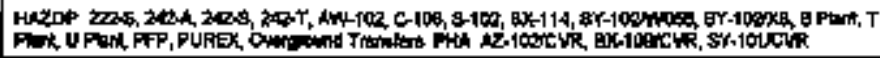 \\
\hline $241-A R-151$ & Dheden bax & 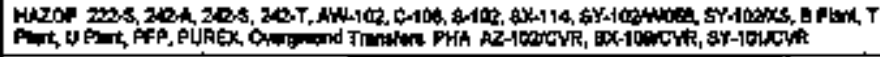 \\
\hline 2A1-Aw of: & Fad anpat & 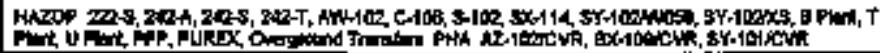 \\
\hline
\end{tabular}


Tahls E-2. Topograpty Dith (27 sheota)

\begin{tabular}{|c|c|c|}
\hline 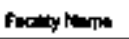 & Tye & Apphalin Hard Lad \\
\hline 241-AW-NO1 & Doutin inth bant & 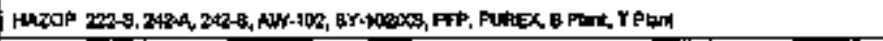 \\
\hline 2al1AWh-102 & Dorbin sinatian & 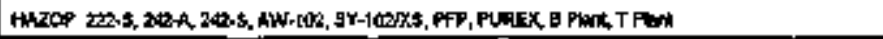 \\
\hline 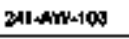 & 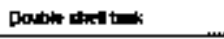 & 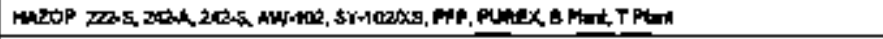 \\
\hline 2A1 AW-tod & Darbie theit tran & 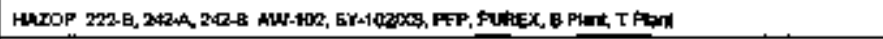 \\
\hline 2n-AM-105 & Dopuble thel tank & 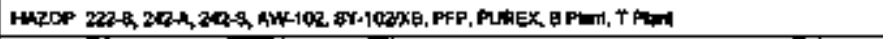 \\
\hline $2 \pi+A n-100$ & 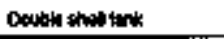 & 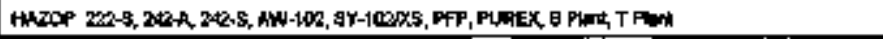 \\
\hline 2AI-AWh & Wate pat & 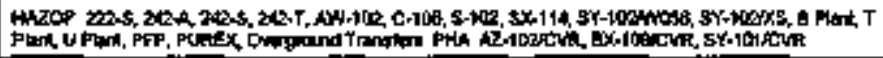 \\
\hline $341+\mathrm{m} m-\mathrm{B}$ & Whope & 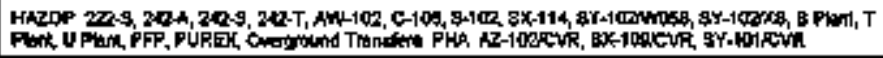 \\
\hline 2atanthese & fangat & 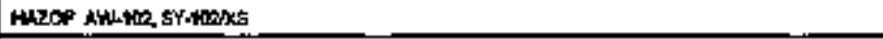 \\
\hline $24+40101$ & Angent whall tart & 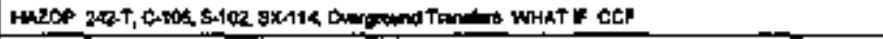 \\
\hline $241+N-102$ & 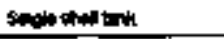 & 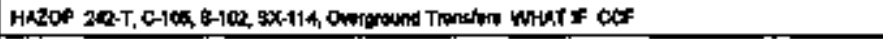 \\
\hline $241+N-100$ & 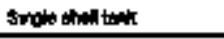 & 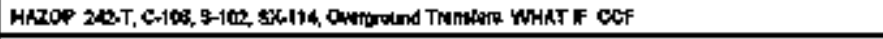 \\
\hline $241+4 x-104$ & Ampla thet th & 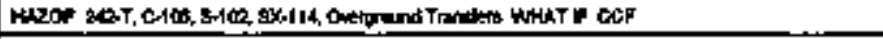 \\
\hline $741-4 \times-151$ & Denemos bax & 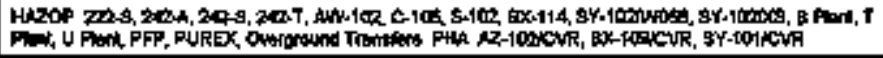 \\
\hline $241-4 x-151$ & 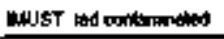 & 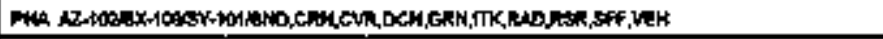 \\
\hline 24140.157 & atchtrat & 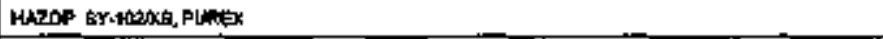 \\
\hline $291-A x-158$ & 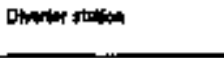 & 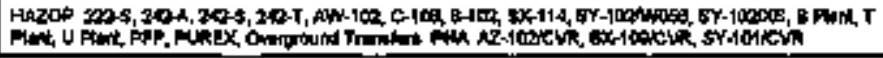 \\
\hline 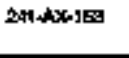 & phardo bar & 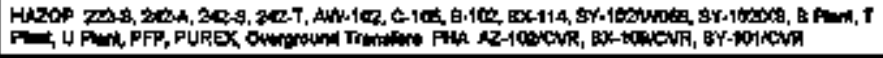 \\
\hline 2A1-PXe-16s & Dheraton back & 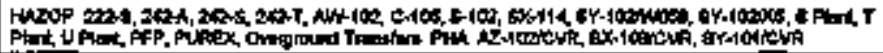 \\
\hline
\end{tabular}


Tabh 日-2. Topoglaphy Dah. (23 ahodt)

\begin{tabular}{|c|c|c|}
\hline 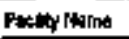 & 100 & 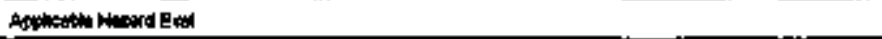 \\
\hline 24144Ka) & ent pat & 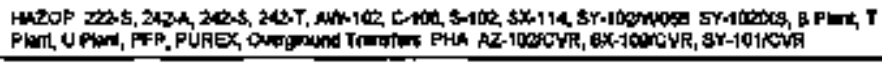 \\
\hline $2+14060$ & senwespt pat & 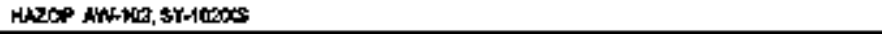 \\
\hline $241+4 \times d x$ & Nhention & 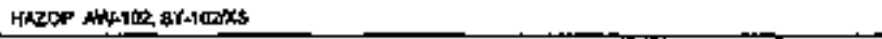 \\
\hline 241404 & on put & 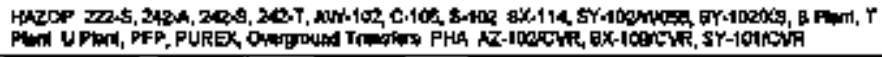 \\
\hline $2414 \mathrm{Ax}-\mathrm{I}$ & Fun pan & 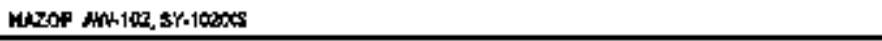 \\
\hline $2 \pi 146 \times$ & rater & 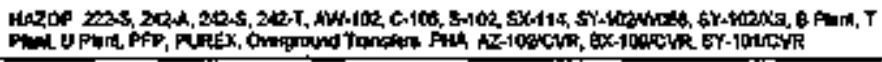 \\
\hline 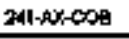 & Camand bow & PWh COB \\
\hline $241+4 r-1$ & chemarbes & Puh cod \\
\hline 24144Y-101 & Douth thel tain & 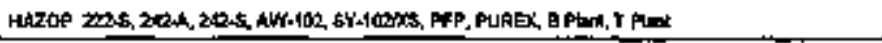 \\
\hline 2A1-AYY108 & 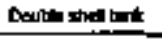 & 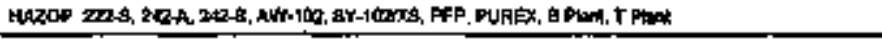 \\
\hline $201+\mathbf{Y Y - 1 5 1}$ & Pumpour m & 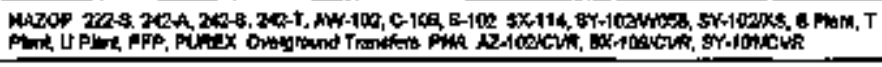 \\
\hline $241+4 y-482$ & then & 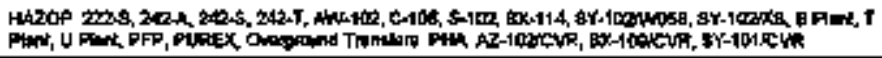 \\
\hline $241-\operatorname{liY}-501$ & tentor on & 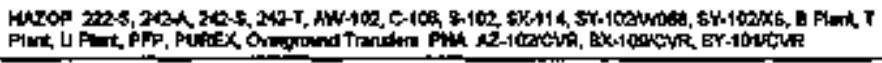 \\
\hline $241-k 2-1$ & Clementitie & PHA COA \\
\hline $2 \times 11-N 2-40$ & Cleninutban & PHh ool \\
\hline $241 \sqrt{2}-104$ & Doult Bhel thint & 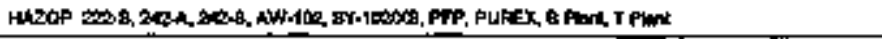 \\
\hline $241+2 \geq-109$ & 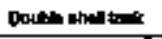 & 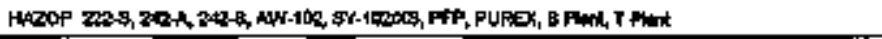 \\
\hline $211-12-151$ & Eatehinger & 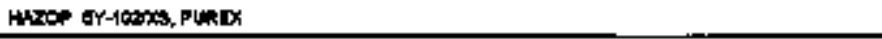 \\
\hline $241-12-1=3$ & Colathintik & HEOP MY-1002XS, PURDX \\
\hline
\end{tabular}




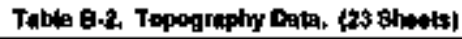

\begin{tabular}{|c|c|c|}
\hline Fagitr kum & The & Apolatite Hard Ext \\
\hline $2+11 A 2-15$ & Buce Trinder Bas & FFA, 000 \\
\hline 34112-150 & studotinstar bots & Prin cos \\
\hline $241+2+43$ & Whept & 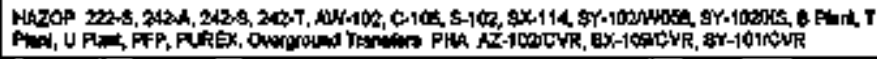 \\
\hline 201-A2-154 & catentont & 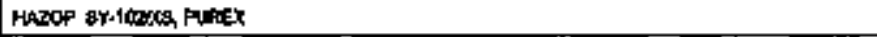 \\
\hline 24t-15.155 & 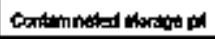 & Herop nW-10R, SW-100, \\
\hline $2+1+12+161$ & Ghed whe anan & 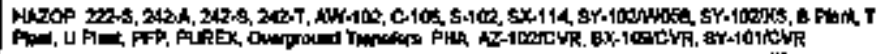 \\
\hline $24, k Z 16 z$ & 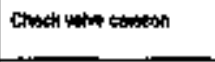 & 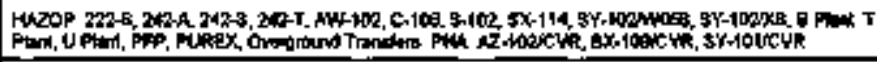 \\
\hline $241 A 2 A$ & Ctencut ber & PHa cod \\
\hline $241+2-3$ & Chanout bex & PHA COA \\
\hline $2 \times 1+2-1$ & CHonay bow & Din 000 \\
\hline 2211425 & Aletiout bex & PHA tos \\
\hline $241-42852$ & valupa & 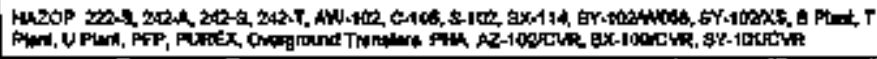 \\
\hline $311+20$ & 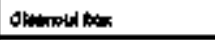 & pith \\
\hline $2414+27$ & claconoul box & PHA COS \\
\hline $241-428$ & Cheneutber & PHA COA \\
\hline 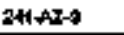 & 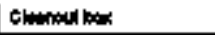 & PHA $60 \mathrm{~B}$ \\
\hline 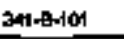 & 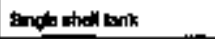 & 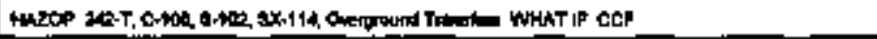 \\
\hline $24+8-102$ & 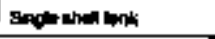 & 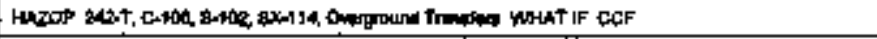 \\
\hline $241-8-109$ & 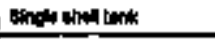 & 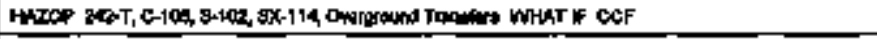 \\
\hline $2+19-104$ & thow whill bik & 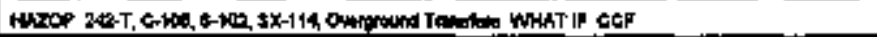 \\
\hline
\end{tabular}




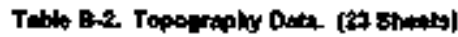

\begin{tabular}{|c|c|c|}
\hline Fanty lam & Trpa & 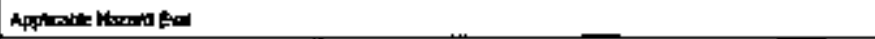 \\
\hline 241-6-106. & 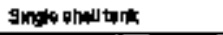 & 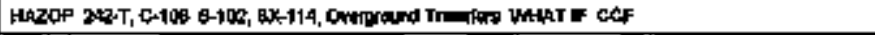 \\
\hline $2 n+8-108$ & stopla then torit. & 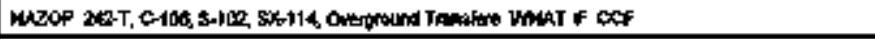 \\
\hline $241+8-10 \%$ & 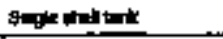 & 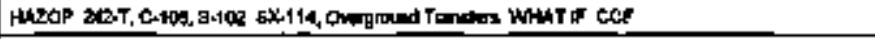 \\
\hline 241 B-100 & 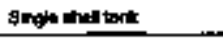 & 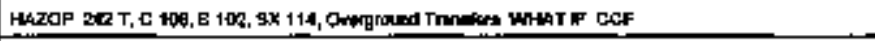 \\
\hline $241-8-109$ & 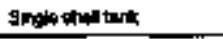 & 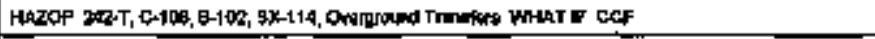 \\
\hline $241+8-110$ & stabandink & 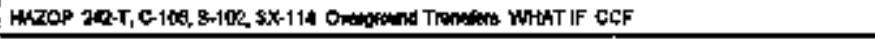 \\
\hline $241+111$ & Engla dethtitic & 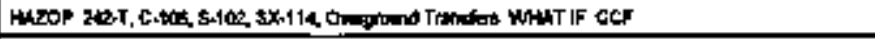 \\
\hline $241+8-112$ & gogle phitutir & 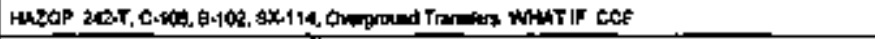 \\
\hline $241-8-151$ & Dinimangan & 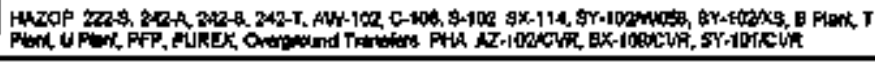 \\
\hline $2 A 1-5-152$ & Ornason ber & 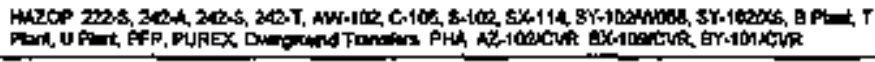 \\
\hline $241-8-15$ & Drwatian bex & 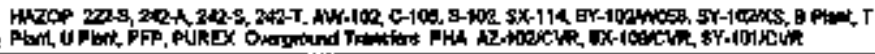 \\
\hline 241-404 & Daneon bow & 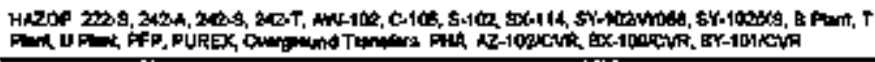 \\
\hline 241-8-101 & Enduthinimir & 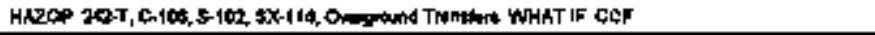 \\
\hline $241 \cdot 0-202$ & Angtediatinte & 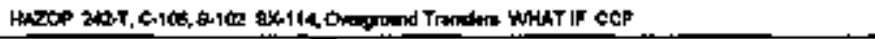 \\
\hline $241-\theta+203$ & Bang; and ank & 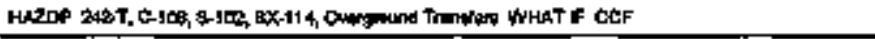 \\
\hline $2+1-6-204$ & 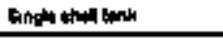 & 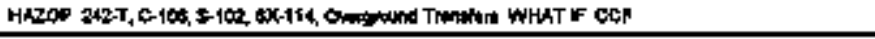 \\
\hline $24+4 a t$ & Deroonbom & 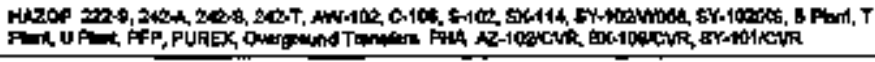 \\
\hline $241-8-3010$ & 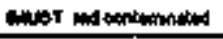 & 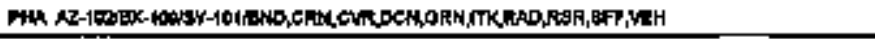 \\
\hline $2+1-400$ & 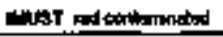 & 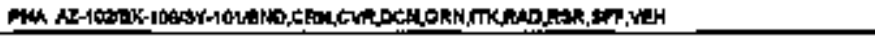 \\
\hline
\end{tabular}


Table E-2. Topograpty Dote. (23 Btoust)

\begin{tabular}{|c|c|c|}
\hline Pothy kam & Fon & 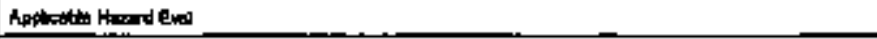 \\
\hline $291+90 \times 101$ & Angle mell bain & 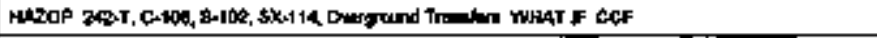 \\
\hline $241-B \times-102$ & 8nglo inall then & 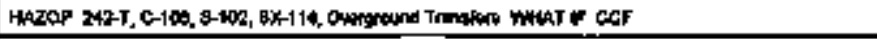 \\
\hline $24140-100$ & Stpolatilin: & 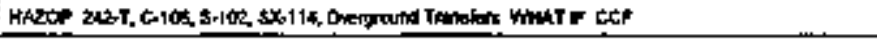 \\
\hline $241+8 \times-104$ & 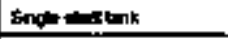 & 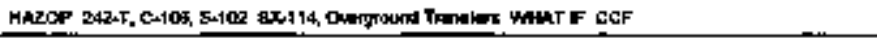 \\
\hline $2+1 \cdot 8 x+105$ & 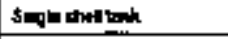 & 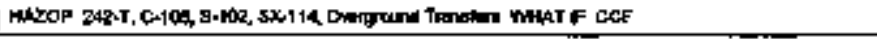 \\
\hline 2क-6x-104 & Single tholtunk & 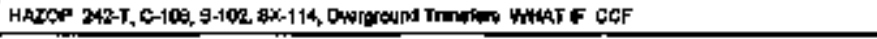 \\
\hline $2+1+6 \times-107$ & Endin than ink & 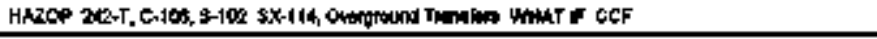 \\
\hline 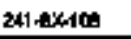 & sthopathal mint & 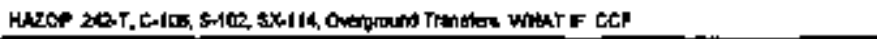 \\
\hline $241+8 x-169$ & 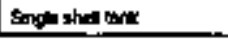 & 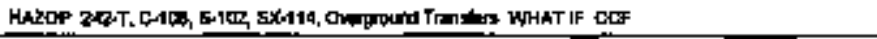 \\
\hline $241-8 \times-110$ & Sngth ahid bink. & 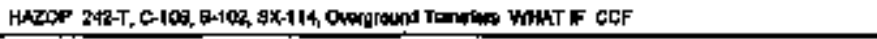 \\
\hline $241-5 x-111$ & 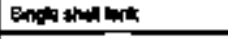 & 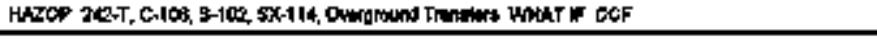 \\
\hline 241-EKA112 & Single thell terk & 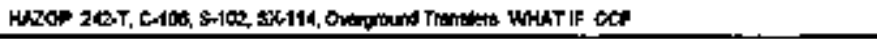 \\
\hline 241. Dexatis. & Wust redtombed & 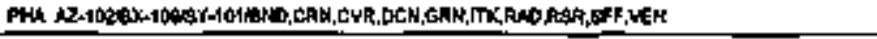 \\
\hline 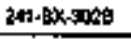 & 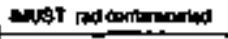 & 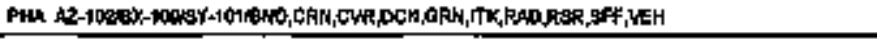 \\
\hline $241-8 x-300 x$ & 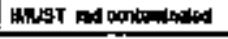 & 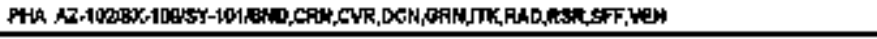 \\
\hline 341.BVR-COA & 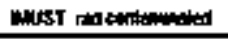 & 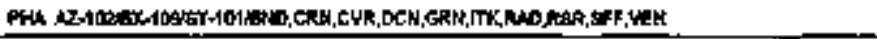 \\
\hline $241 \cdot-B \times 2 \times 2-002$ & 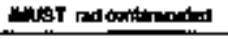 & 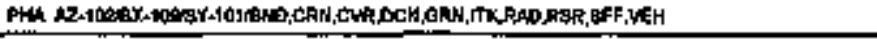 \\
\hline ZALEXR-003 & 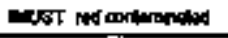 & 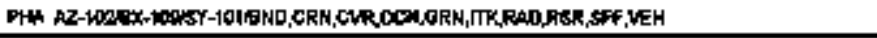 \\
\hline $24 k=B \times k=011$ & 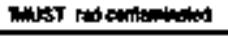 & 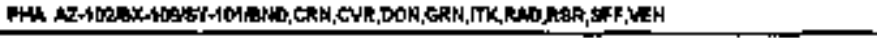 \\
\hline 241 \&XR-161 & Ond the & 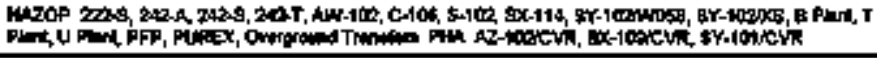 \\
\hline 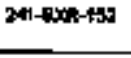 & Dawim & 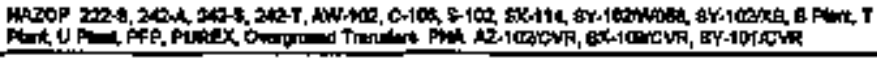 \\
\hline
\end{tabular}




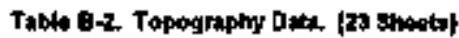

\begin{tabular}{|c|c|c|}
\hline 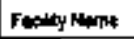 & Tov & 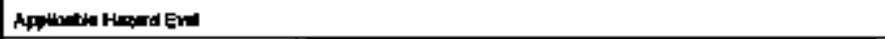 \\
\hline 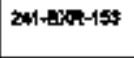 & Derodtion bor & 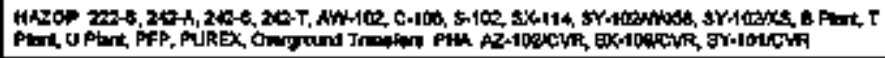 \\
\hline $241-8 Y-101$ & Sngothothe & 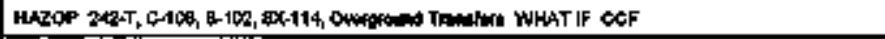 \\
\hline 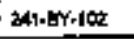 & Singthentink & 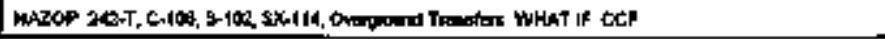 \\
\hline 2al-BY.10s & songt whal tonk & 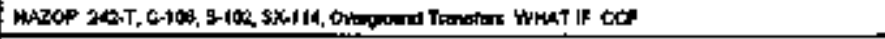 \\
\hline 241.8Y.104 & Sngte miltuk & 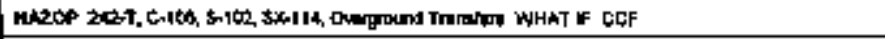 \\
\hline $241-8 Y-146$ & 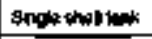 & 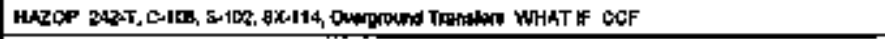 \\
\hline $241 \cdot B^{\prime} Y-108$ & 6noll then & 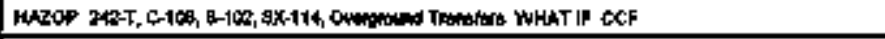 \\
\hline $241 \cdot 54 \cdot 10 ?$ & Sthon and tank & 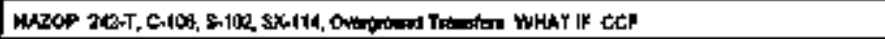 \\
\hline Z91.BY-1DS & 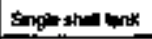 & 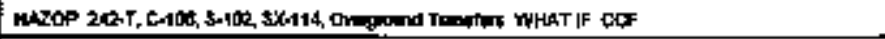 \\
\hline 241-8r-169 & 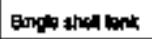 & 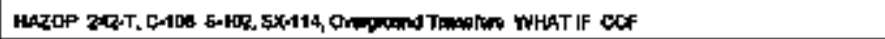 \\
\hline $241-6 \times-110$ & Bingth thel tenk & 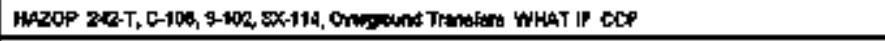 \\
\hline $2118 Y-111$ & Singte thall tant & 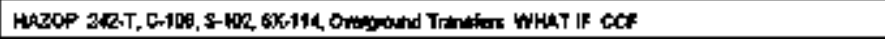 \\
\hline zhtserstes & shole shed bank & 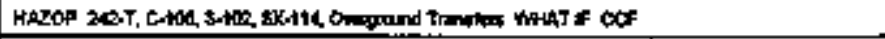 \\
\hline 2+1-6M:152 & Ouwaphow & 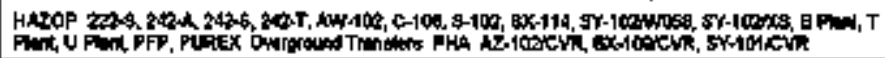 \\
\hline 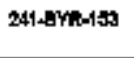 & Onmbintex & 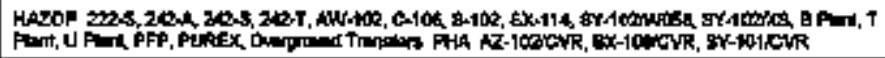 \\
\hline 241-8YR-15d & 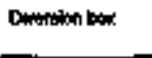 & 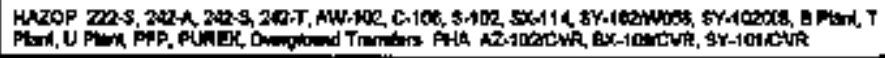 \\
\hline 241-日YR-14: & 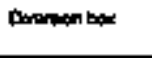 & 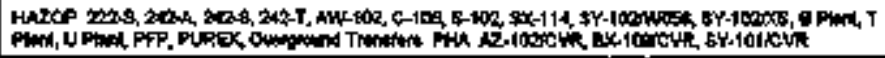 \\
\hline 241-C.101 & Shqu thelltu & 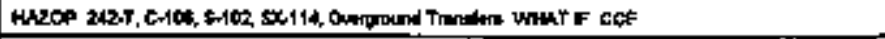 \\
\hline 24)c-10 & Sholosilitiok & 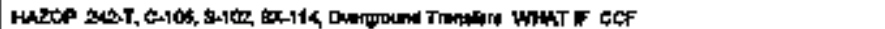 \\
\hline
\end{tabular}


Takto B-t. Topography bate (2A shats)

\begin{tabular}{|c|c|c|}
\hline Fathrom & Thw & 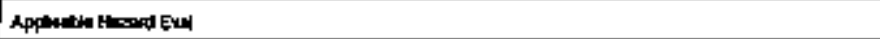 \\
\hline $201-100$ & 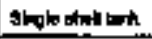 & 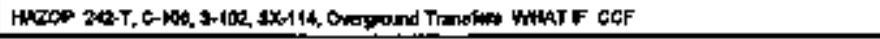 \\
\hline $241+40404$ & snglemelterk & 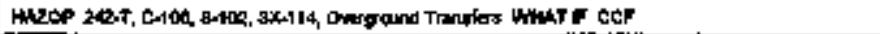 \\
\hline $241-6-\infty 0_{5}$ & Ondownotiok & 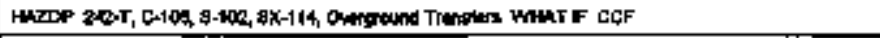 \\
\hline $241+c-1000$ & 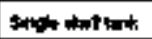 & 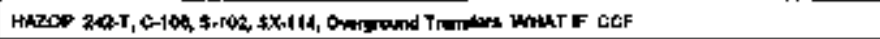 \\
\hline $241-t-100$ & 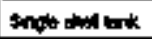 & 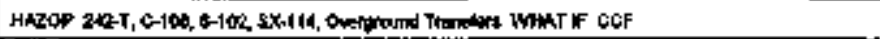 \\
\hline $241<0-100$ & topge the tank & 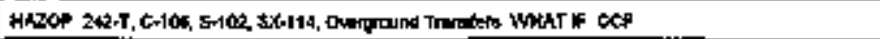 \\
\hline 341-C-109 & 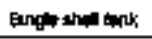 & 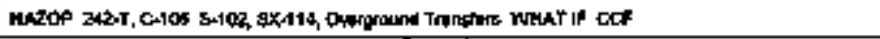 \\
\hline $241-c-110$ & 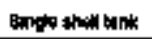 & 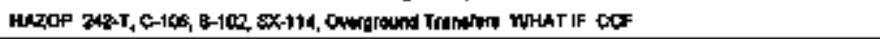 \\
\hline $241-6-111$ & Singte thell whin & 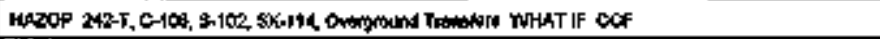 \\
\hline $241-C=112$ & songe trat tank & 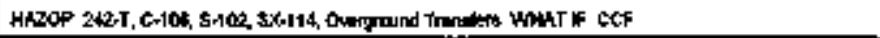 \\
\hline 241-C-15: & Dentem bas & 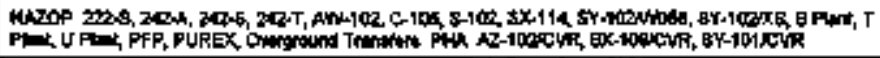 \\
\hline $24+c-152$ & Dwatom bet & 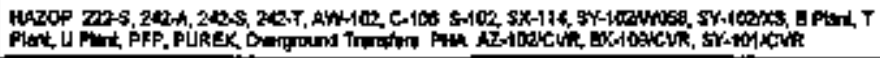 \\
\hline $2+1-6-150$ & Onerion tom & 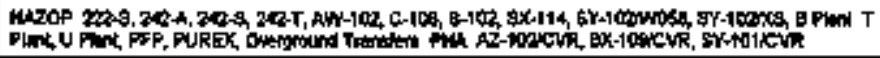 \\
\hline $241+c \mid-184$ & Dmentabn bax & 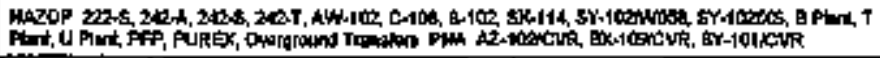 \\
\hline $241+\cos ^{2}$ & 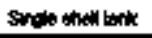 & 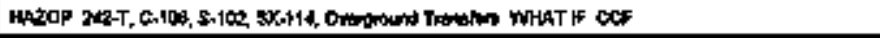 \\
\hline $241-0-2012$ & stople thell th & 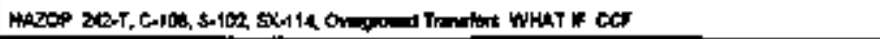 \\
\hline $241-C_{-203}$ & shoge ond box & 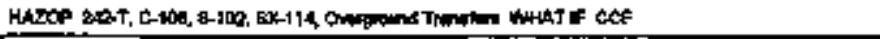 \\
\hline $211-c-204$ & 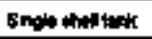 & Harces 2Q-T, \\
\hline $241 \cdot c 42$ & Onertidenter & 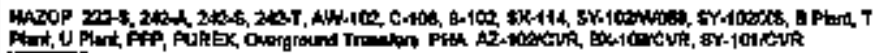 \\
\hline
\end{tabular}




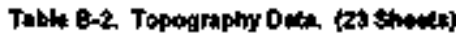

\begin{tabular}{|c|c|c|}
\hline 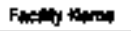 & TYpe & 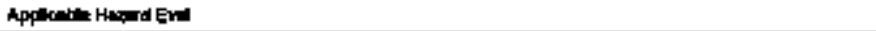 \\
\hline $2 \omega-63010$ & 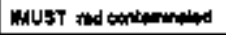 & 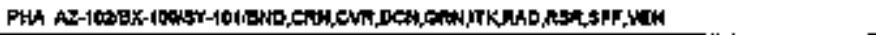 \\
\hline 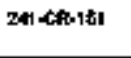 & Driaran bar & 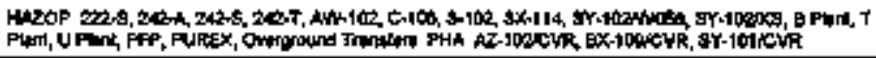 \\
\hline $241 \ldots 9,152$ & Dintrosin beth & 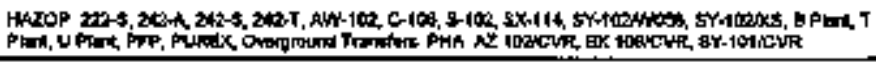 \\
\hline 2414RPAID & Dmarean bou & 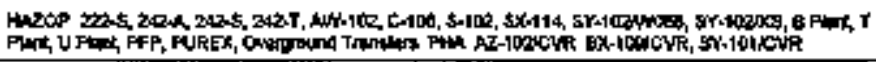 \\
\hline $2+1$-ER-151 & Druston bex & 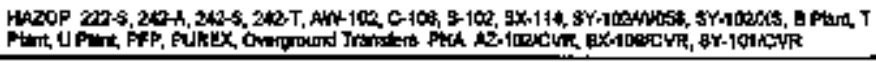 \\
\hline 241-ER-16\% & Dinspre pos & 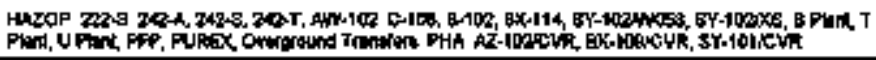 \\
\hline 241-ER-10s & Dintomen bow & 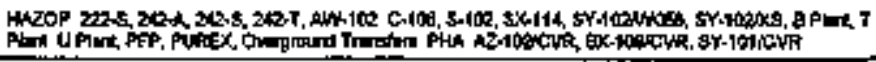 \\
\hline 241-ER-311 & Cistolinik & 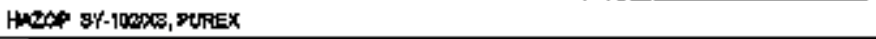 \\
\hline Zd1.ER311 & Dunotantow & 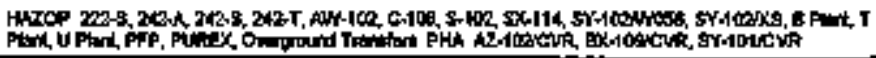 \\
\hline 241-ER-311A & 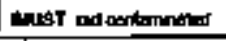 & 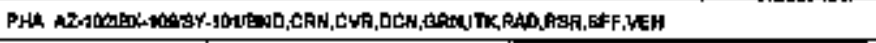 \\
\hline 241-F.955 & Duwter cainon & 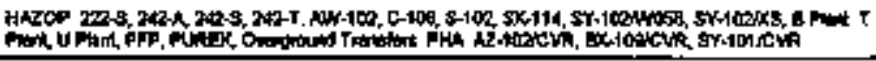 \\
\hline $2+14.6 \%$ & velept & 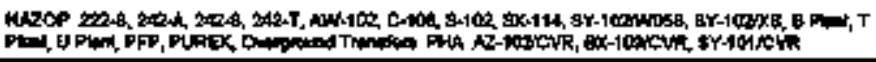 \\
\hline $2+4-3-10 t$ & 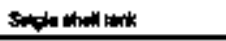 & 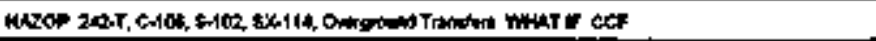 \\
\hline 241 4-102 & 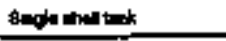 & 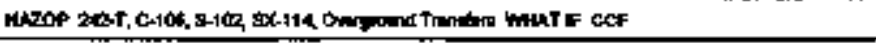 \\
\hline 241-\$-103 & 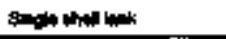 & 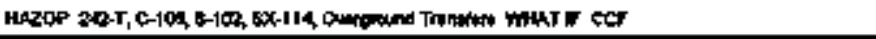 \\
\hline $241-4-104$ & 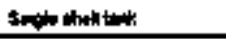 & 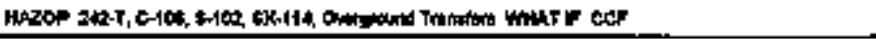 \\
\hline $241-9-106$ & Angtantheth & 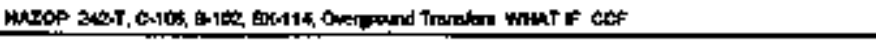 \\
\hline
\end{tabular}




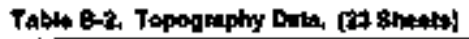

\begin{tabular}{|c|c|c|}
\hline Facitringe & Type & Applanit Hoond End \\
\hline $2+1+8-10 \%$ & 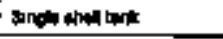 & 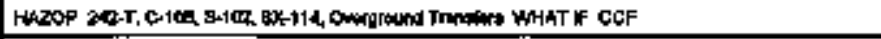 \\
\hline $241-3-101$ & ongen thim tand. & 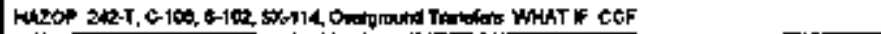 \\
\hline 241-3.16s & tongh ahe but & 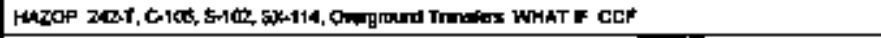 \\
\hline 241.8100 & ange bhel tart & 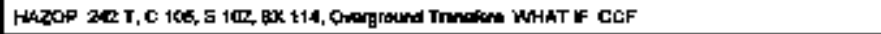 \\
\hline $241+8-110$ & sangh whin bent & 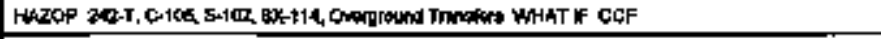 \\
\hline $2 n-5 n 111$ & Genow than teren & 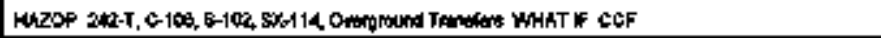 \\
\hline 241.4112 & 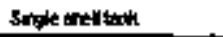 & 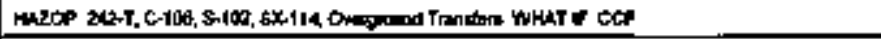 \\
\hline 241 \&+151 & Andenter & 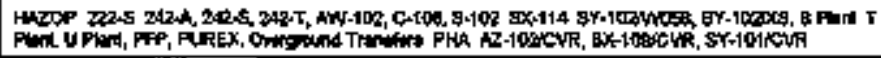 \\
\hline 2/1-3-132 & Conumation & 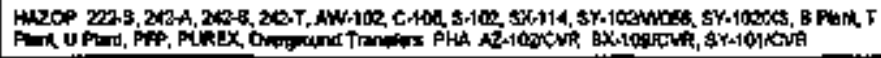 \\
\hline $241.8-3002$ & 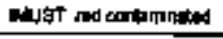 & 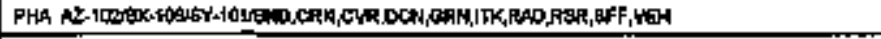 \\
\hline $241-3,3028$ & 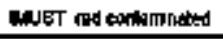 & 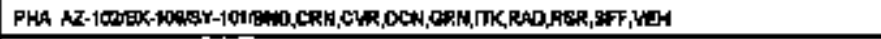 \\
\hline $241+8,304$ & Daten toin & 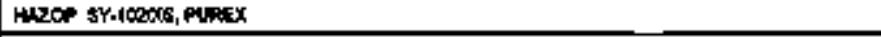 \\
\hline $2-1+3-4$ & 的 & 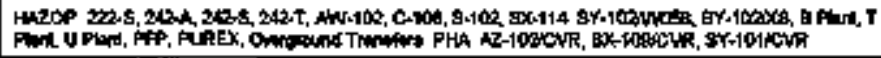 \\
\hline 24-5s & 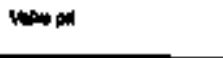 & 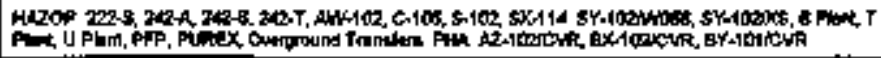 \\
\hline 2011-6-C & A & 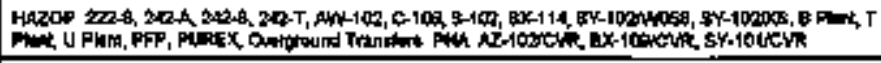 \\
\hline $2 n+30$ & kim m & 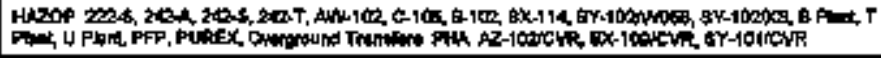 \\
\hline 201-8.4y & 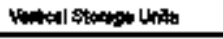 & mak vay \\
\hline 24t-2001 & 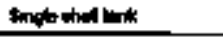 & 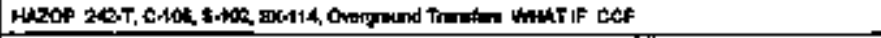 \\
\hline $24 t-6 x-k \mid+2$ & Anquabel bovt & 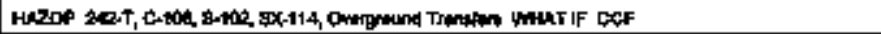 \\
\hline
\end{tabular}




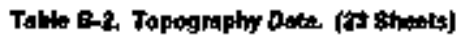

\begin{tabular}{|c|c|c|}
\hline Faxily Ntman & Typ* & 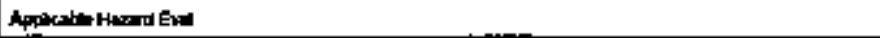 \\
\hline $241-9 x-100$ & 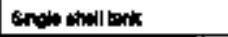 & 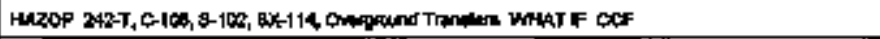 \\
\hline $241-5 \times-104$ & Engla binel ball & 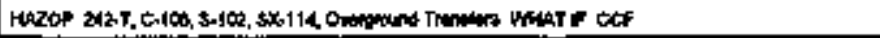 \\
\hline $241-8 x \cdot 905$ & Singin whell bur & 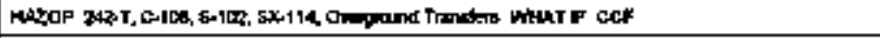 \\
\hline $241-4 \times 640$ & 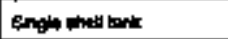 & 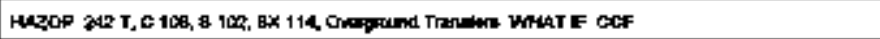 \\
\hline $241-9 x-107$ & Enele chat bork & 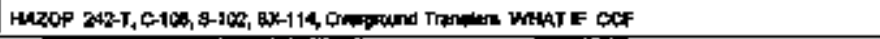 \\
\hline $241-\$ x-160$ & solve bhell bith & 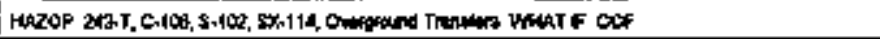 \\
\hline $241-\$ 10.100$ & Engla thell bak & 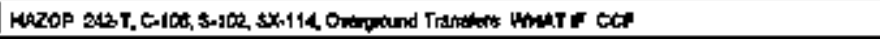 \\
\hline $741.9 \times-110$ & gngin thelltark & 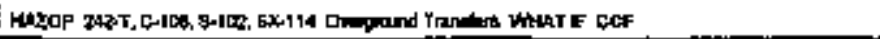 \\
\hline $241-9 x-111$ & 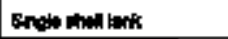 & 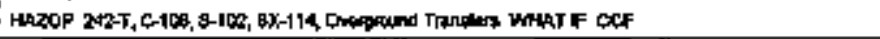 \\
\hline $261-5 \times-112$ & stoule bitall bart & 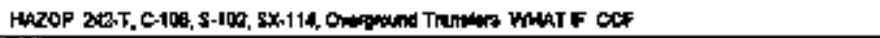 \\
\hline $2,1+5 x+13$ & Angte thiluk & 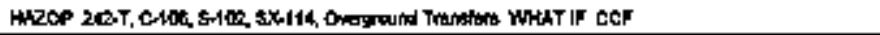 \\
\hline 241.50 .114 & 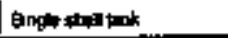 & 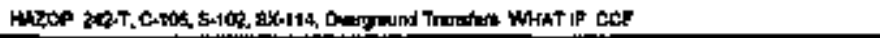 \\
\hline $24-6 \times-116$ & 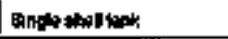 & 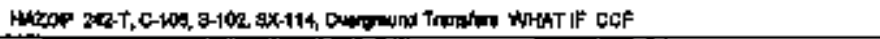 \\
\hline $281-80-151$ & Drwesin bex & 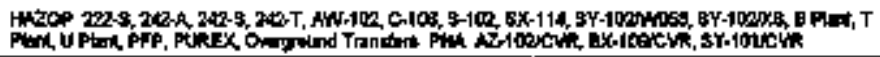 \\
\hline $244-3 x+18$ & Damerea por & 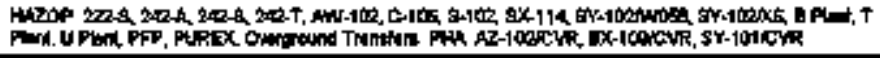 \\
\hline $241-5 \times 392$ & 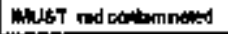 & 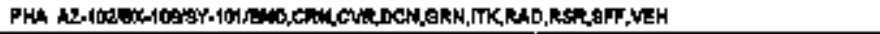 \\
\hline $2+1-8 \times 14$ & 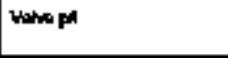 & 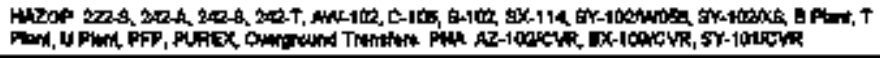 \\
\hline $24-5-5 x-1$ & 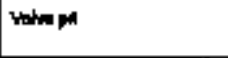 & 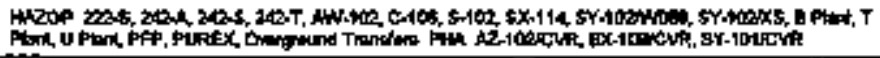 \\
\hline 241-8r & Parmat & 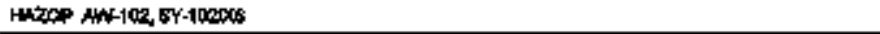 \\
\hline 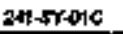 & Look Dowation Pk & 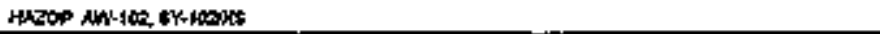 \\
\hline
\end{tabular}


Tebte E-2 Topogrephy Dect [23 aboto)

\begin{tabular}{|c|c|c|}
\hline Pugan Nome & Type & Apperbe Head end \\
\hline $2: 4-8 \div-006$ & Intr Detwoton PA & 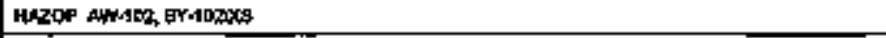 \\
\hline $241-5 Y-000$ & Fes anmpot & 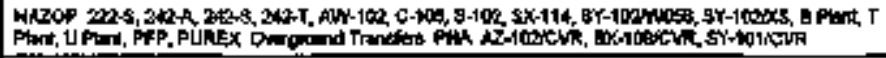 \\
\hline 2A1-SY-02E & Fush pa & HAZOP AW-102, \$Y-102XXB \\
\hline $241-\$ Y-03 C$ & 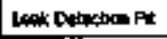 & HAZOP AW-102, 8YY102XX5 \\
\hline 2A1-5Y.1 & Clemaktber & PHA COA \\
\hline 201-6Y.101 & Dould bhed batk & 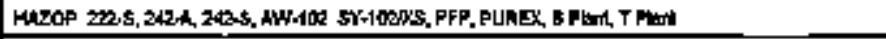 \\
\hline 2+1-9Y-140 & Ooutit whell trat & 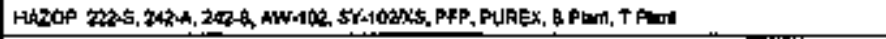 \\
\hline 261-5Y-103 & 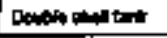 & 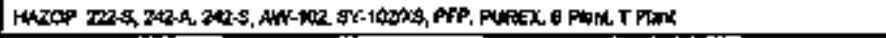 \\
\hline $241+\$ Y 2$ & Chondingen & PHA COP \\
\hline $241-5 Y A$ & thoulbex & PHA COA \\
\hline $241.85 \% 4$ & Conent tix & PHA CXe \\
\hline 2*1-8Y-5 & Gopent has & PHe Cod \\
\hline $241+9 Y-\theta$ & Clemant bex & PHA COS \\
\hline 2419-SYMA & Mothin & HKZOP AWiNe, st.10020S \\
\hline 24148YM & vent & 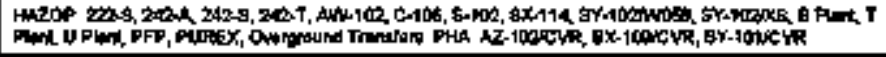 \\
\hline 241-5rig & Anth in & 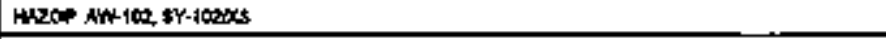 \\
\hline 2414Y-B & vent & 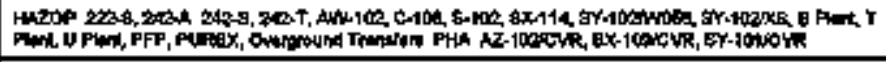 \\
\hline 241.T-109 & BNoth abell bitit & 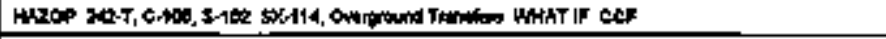 \\
\hline $241-T-102$ & Angle shell tart & 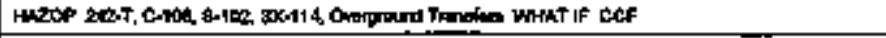 \\
\hline 2*1-T-1094 & gngin iniling & 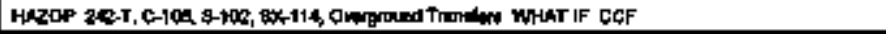 \\
\hline
\end{tabular}


Tabte B-2. Topograpmy Dath. 123 Stretsy

\begin{tabular}{|c|c|c|}
\hline Fatedy & $\mathbf{T}_{\mathbf{x}} \mathbf{*}$ & 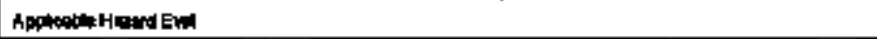 \\
\hline $241 \cdot T+104$ & 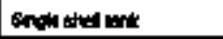 & 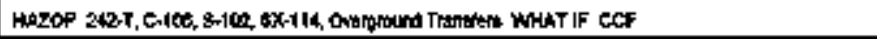 \\
\hline $245-T+10 d$ & 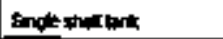 & 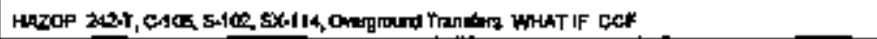 \\
\hline $241 . T-100$ & 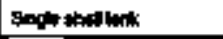 & 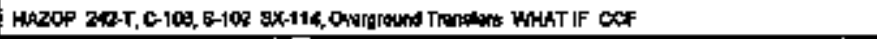 \\
\hline $2+1+7-107$ & End thenth the & 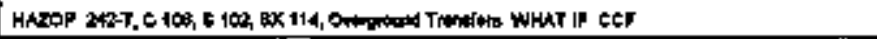 \\
\hline $241-T-109$ & sinch bhe batk & 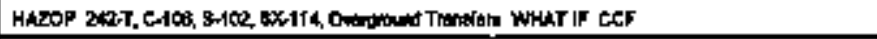 \\
\hline $241-\mathrm{T}-10 \mathrm{~A}$ & 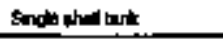 & 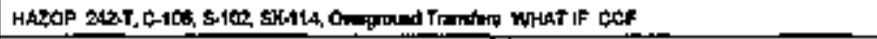 \\
\hline 241-T-110 & 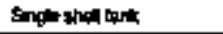 & 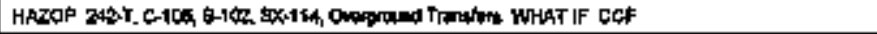 \\
\hline 241ㄴ.T-1:A & 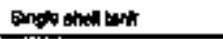 & 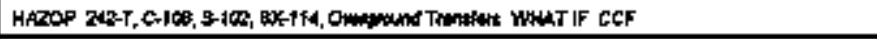 \\
\hline $241 \cdot T \cdot 112$ & Single thel t then & 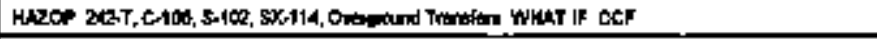 \\
\hline $241 \cdot T \cdot 151$ & Duremon bax & 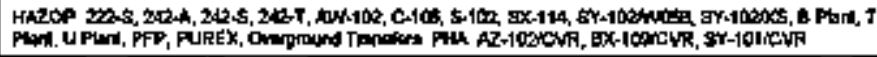 \\
\hline $241 \cdot T-209$ & 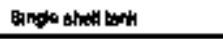 & 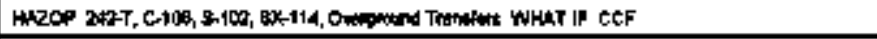 \\
\hline 2H.T202 & 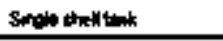 & 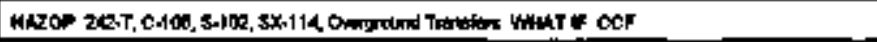 \\
\hline 241.T.2015 & 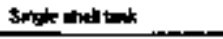 & 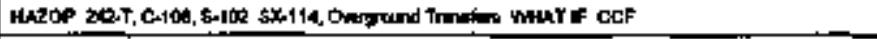 \\
\hline $2 * t-T-204$ & 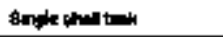 & 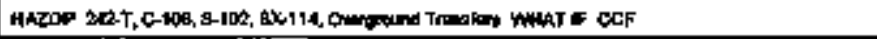 \\
\hline 24t-T-3001 & 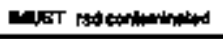 & 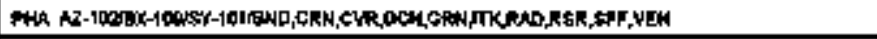 \\
\hline $2+1 \times 5 \times 004$ & 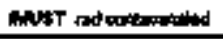 & 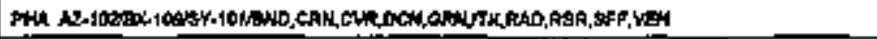 \\
\hline 241-Tx-002 & 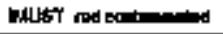 & 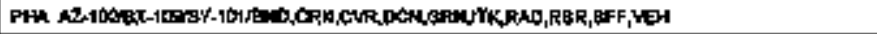 \\
\hline $241-\Gamma \times-000$ & 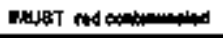 & 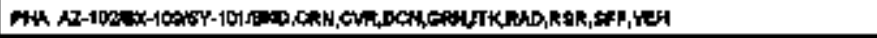 \\
\hline $2011-7 x-101$ & 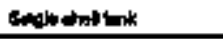 & 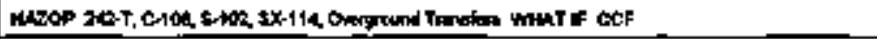 \\
\hline 241-Thatez & 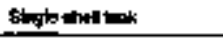 & 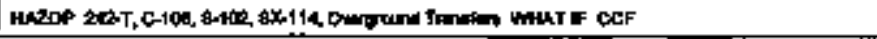 \\
\hline 241-TX-1K3 & 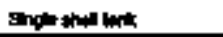 & 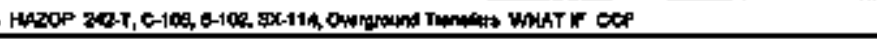 \\
\hline
\end{tabular}


Talo B-2. Topography DHth. (24 thents)

\begin{tabular}{|c|c|c|}
\hline Fonitir Mand & Typat & 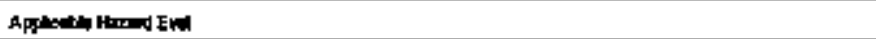 \\
\hline $2011.7 x_{0}+004$ & sthote stol tort & 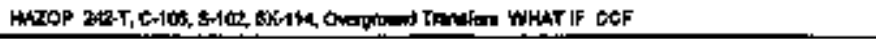 \\
\hline 291-Tx & 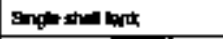 & 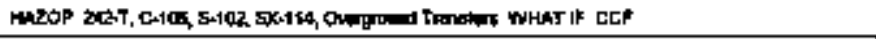 \\
\hline $241 \cdot T x-100$ & 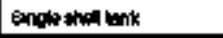 & 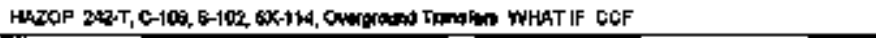 \\
\hline $241 . T X-107$ & enclusthin berk & 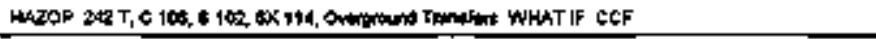 \\
\hline $24 \cdot T X-10 s$ & Singof thell thent & 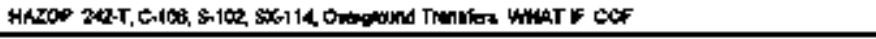 \\
\hline $2+1-T x-10$ & sngla shd lant & 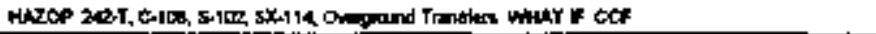 \\
\hline $241-T \times-110$ & Binge shopl turk & 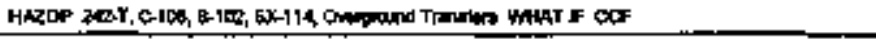 \\
\hline $241 \cdot T X-411$ & 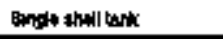 & 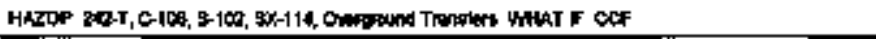 \\
\hline $2+1 \cdot T \times-112$ & single thetl but & 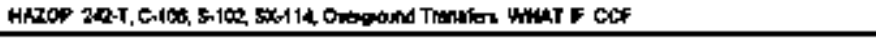 \\
\hline $241-T x, 193$ & sngle ahedl bert & 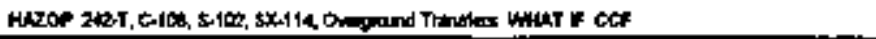 \\
\hline $241-\Gamma x-114$ & Binge shet tonk & 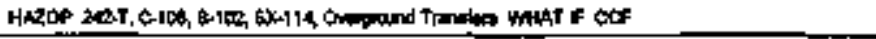 \\
\hline $2+41+5 x-215$ & Bangto ohe bark & 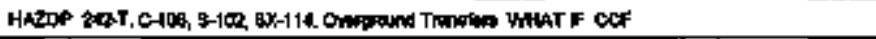 \\
\hline $241.7 \times-118$ & 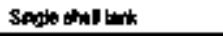 & 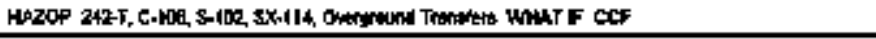 \\
\hline $241 .+7 x-117$ & 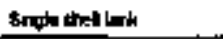 & 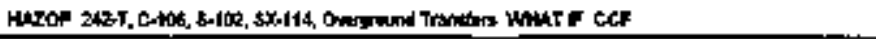 \\
\hline $241-T X-118$ & savit dhellimir & 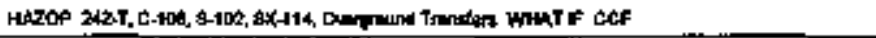 \\
\hline 241.TX15 & 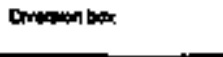 & 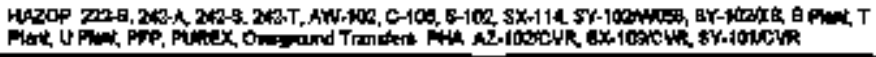 \\
\hline $2+1-T \times-163$ & Anm in bax & 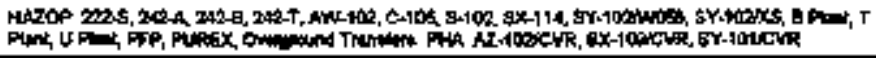 \\
\hline $201.7 x \cdot 1.64$ & 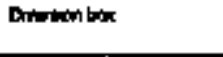 & 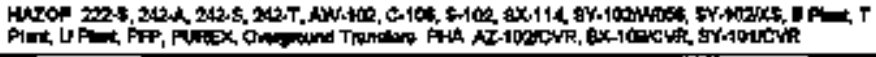 \\
\hline 24-7x-4 B & Drumaper & 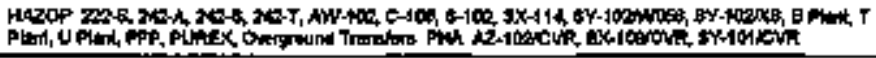 \\
\hline $24+1-1 \times-302 x$ & 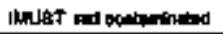 & 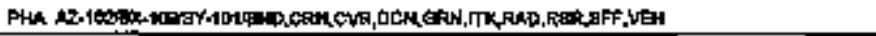 \\
\hline
\end{tabular}


Tobta B-2. Topogrephy Deth. 120 streats]

\begin{tabular}{|c|c|c|}
\hline Pocalentions & Typos & Apoleatu keard Bat \\
\hline 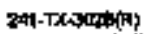 & 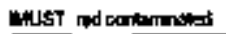 & 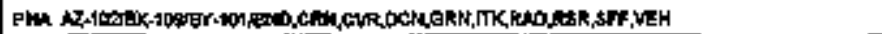 \\
\hline $241 . T X-90 x$ & Coulch tinit & 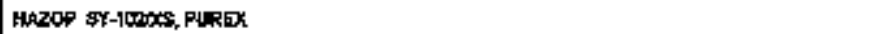 \\
\hline $2011 . T x-502 x$ & 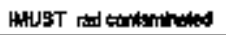 & 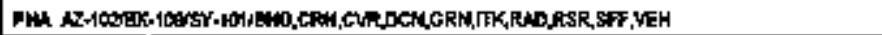 \\
\hline $241 \cdot 730 t \cdot 13 r$ & Onerain ber & 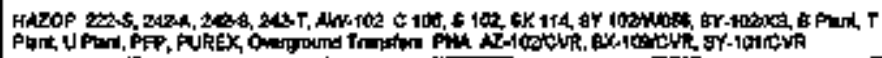 \\
\hline $241 \cdot T \times R-15 R$ & Onersom ber & 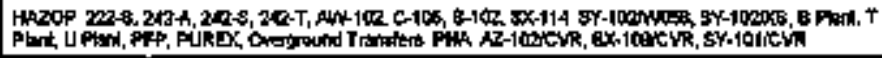 \\
\hline 241-TxR:-163 & Deraen bar & 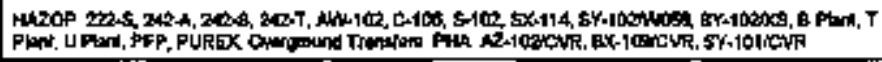 \\
\hline $241-T Y+101$ & 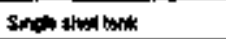 & 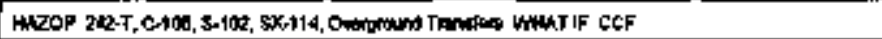 \\
\hline $241+\pi+102$ & singitu thent bork & 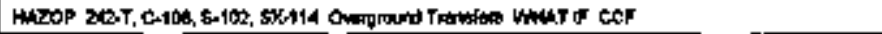 \\
\hline 241.TY-100 & 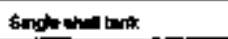 & 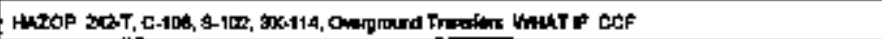 \\
\hline 241-TY-104 & 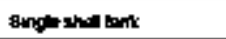 & 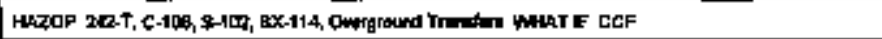 \\
\hline 24h-TY-105 & Songtion tank & 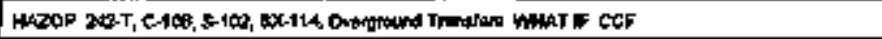 \\
\hline $241 . T+N \cdot 00$ & 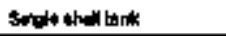 & 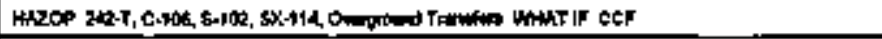 \\
\hline $241+\pi \operatorname{sic} 20$ & 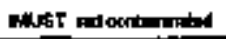 & 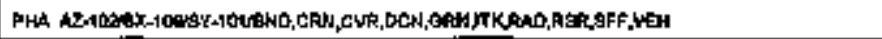 \\
\hline $241-T Y-3028$ & 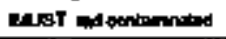 & 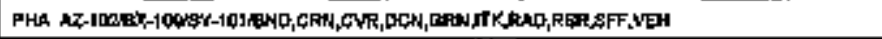 \\
\hline $201-4-601$ & 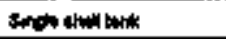 & 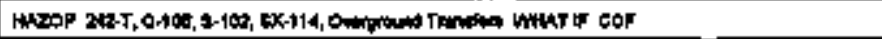 \\
\hline 2*A-10 & Encle the that & 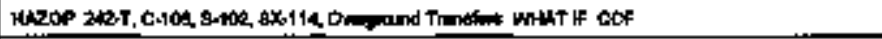 \\
\hline $291-4-100$ & Spala anall tert & 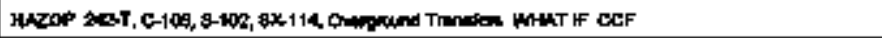 \\
\hline $2011-4-104$ & 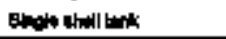 & 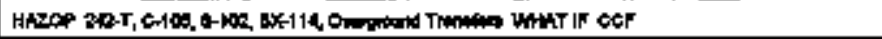 \\
\hline $241-4+160$ & surin thell bart & 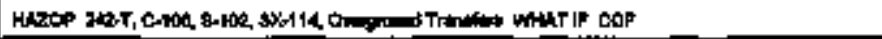 \\
\hline $2+14100$ & Angle thell the & 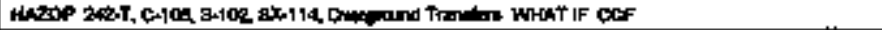 \\
\hline
\end{tabular}




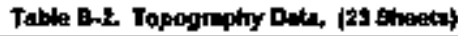

\begin{tabular}{|c|c|c|}
\hline Facty nam & Nom & 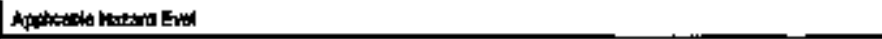 \\
\hline 241-4-1बग & 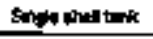 & 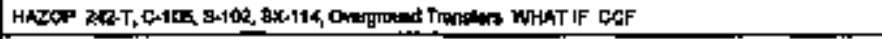 \\
\hline $241+108$ & Bnola aheil tork & 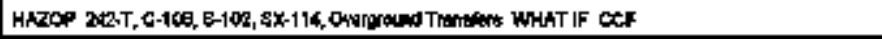 \\
\hline $241+160$ & Fingle thell thits & 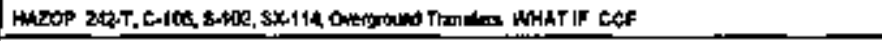 \\
\hline $241 / 110$ & 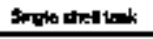 & 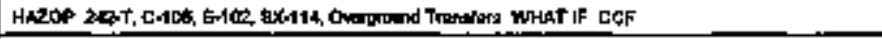 \\
\hline $244-4-111$ & 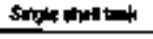 & 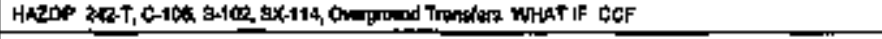 \\
\hline $241+4-912$ & 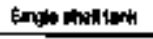 & HAZOP 202,T, C-106, E-102 SX-114, Oroporaned Traterts WHAT IF CCF \\
\hline 244.45 .151 & Devition ber & 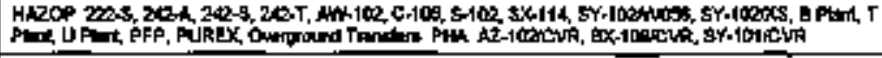 \\
\hline $2 * 1+15$ & Damore ber & 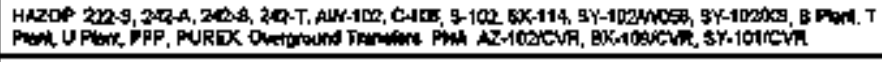 \\
\hline 241.U.t.6s & Ontersid bax & 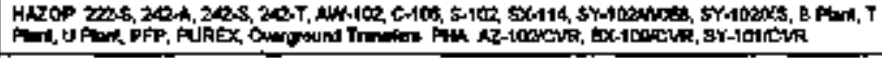 \\
\hline $241-4-154$ & Mnatumber & 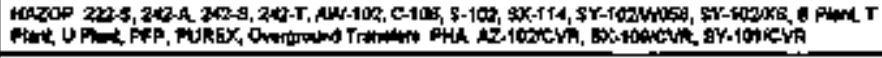 \\
\hline $241 \cdot 10201$ & Sngtedol bith & 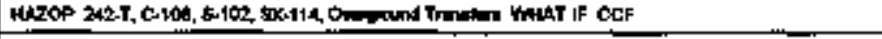 \\
\hline 241-u-202 & Enolent mik & 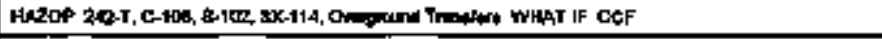 \\
\hline $241-4-203$ & Beglestinaterik & 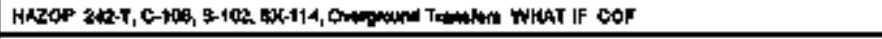 \\
\hline $24+14-204$ & 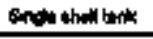 & 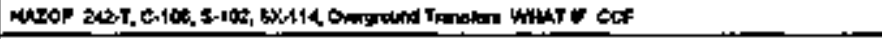 \\
\hline 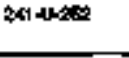 & Drenemban & 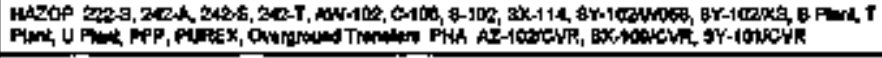 \\
\hline $2+103010$ & Cutwhtat & 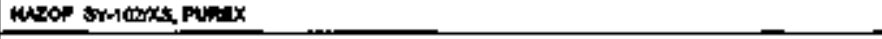 \\
\hline $20+1 \mathrm{dma}$ & Winpe & 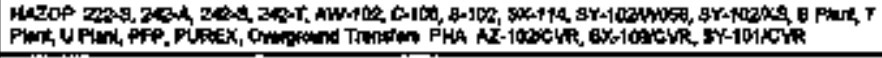 \\
\hline $241+46$ & wan & 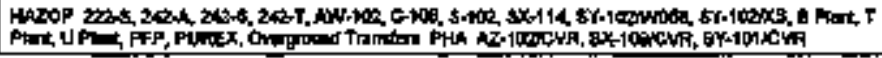 \\
\hline
\end{tabular}


Table B-8. Topography Data (25 ahents)

\begin{tabular}{|c|c|c|}
\hline Patay Nom & The & 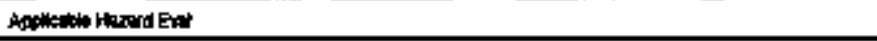 \\
\hline $201-20$ & Vatpr & 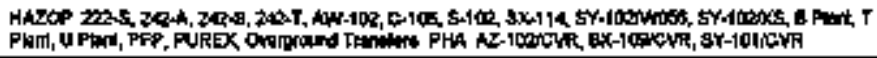 \\
\hline $2+1.00$ & whet pt & 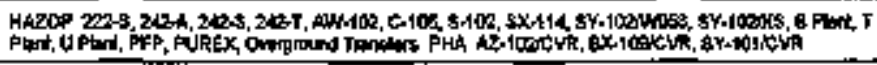 \\
\hline $241-46-151$ & Dimatom bou & 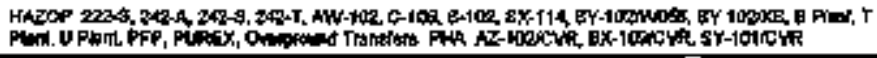 \\
\hline $201+16.152$ & Dentitin bax & 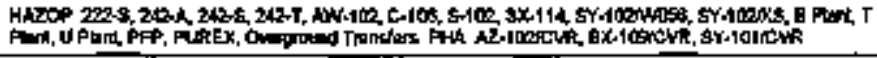 \\
\hline 24-14R-190 & Dnambing & 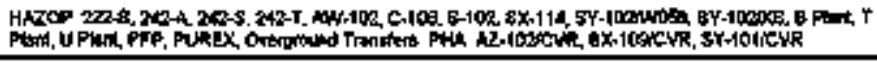 \\
\hline 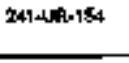 & Dimeronber & 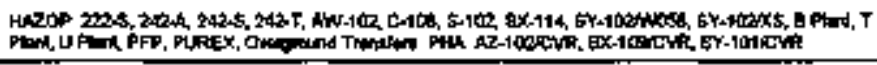 \\
\hline $241-4 x-3024$ & chath & HAZOO BY-1002XS, PUREX \\
\hline 240494 & Dimberan ber & 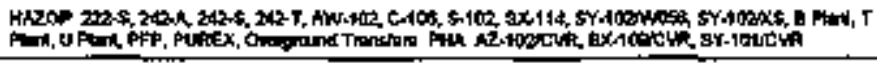 \\
\hline $2 \times 8-1-135$ & 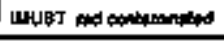 & 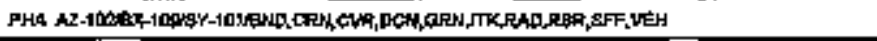 \\
\hline 2Na-TAR1 & 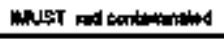 & 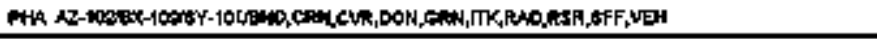 \\
\hline 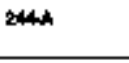 & 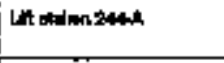 & 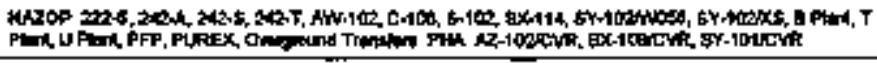 \\
\hline $2448 x$ & 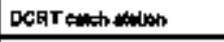 & 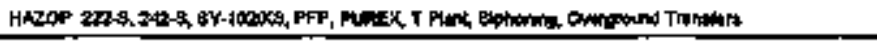 \\
\hline 1440R & DORT cothen math & 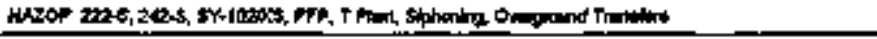 \\
\hline 2446 & GCRT ontan & 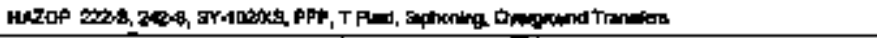 \\
\hline 24-TK & OCFT ceteh twan & 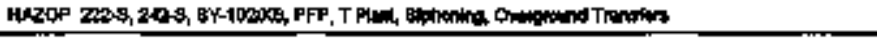 \\
\hline $24+14$ & OCRT cath mosth & 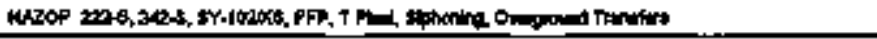 \\
\hline ITLF & sodem mong bitp & min 2rentw. \\
\hline
\end{tabular}


Table B-2. Topography Dete (25 aluols)

\begin{tabular}{|c|c|c|}
\hline Falty keme & Tyon & 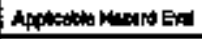 \\
\hline 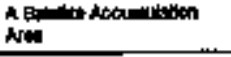 & 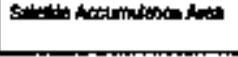 & PHA Dee \\
\hline 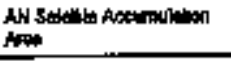 & 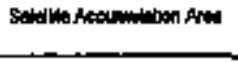 & PHA wee \\
\hline 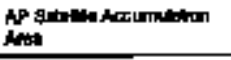 & 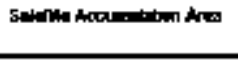 & PHA 200E \\
\hline MR-aAd & 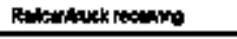 & HEOP XUAR \\
\hline AR $2 ? 4$ & that & PHA 244kt \\
\hline $\ln _{\text {sid }}$ & 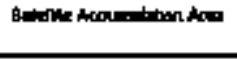 & DHR Zâe \\
\hline 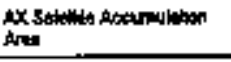 & 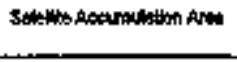 & Puth ace \\
\hline 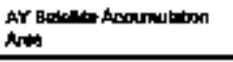 & 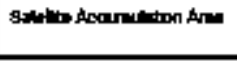 & DH. 205 \\
\hline 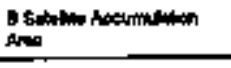 & 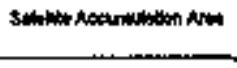 & puld ace \\
\hline 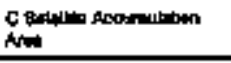 & 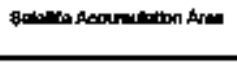 & FHA 200. \\
\hline cosentw & Cteonoul ber. & Prth col \\
\hline coteng & chemoul bax & $\operatorname{Pan} \cos$ \\
\hline $\cos x \times 40$ & 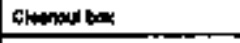 & Pthe $\cos$ \\
\hline $\cos a w n$ & Sletmoll thes & Prth of: \\
\hline cotoments & cleamul bax & Prin con \\
\hline $\cos \theta \sin \theta$ & ammont max & PHA 009 \\
\hline $\cos A \mathrm{M}$ & X & PHA coll \\
\hline conomentes & 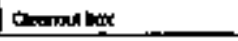 & PHa cot \\
\hline
\end{tabular}




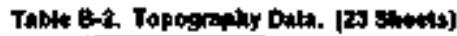

\begin{tabular}{|c|c|c|}
\hline 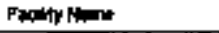 & Trpe & 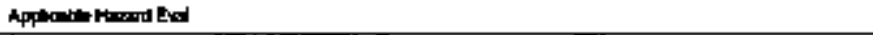 \\
\hline Copaintor & Alumat bed & Pancos \\
\hline cosditular & Cment bou & Put col \\
\hline COPBAn-03 & Cronout bor & PHA CDe \\
\hline COBAANLOA & conoriant bou & PAH $c 00$ \\
\hline CDBAHFOS & cluntet beil & DHA $c 00$ \\
\hline cosinktust & Clesthart bon & PHA COB \\
\hline COA-AW-LT & cheneut hax & PHA COA \\
\hline Cosintos & Cleonout bas & PHe chos \\
\hline cos-nhtos & cumeut bex & PHA 900 \\
\hline CogentMo & Clesioutbox & PHA COS \\
\hline Colinwit & Clenour bax & PHon COA \\
\hline $\cos -1 \mathrm{x}-1 \mathrm{x}$ & Cleoneut hex & PHA COA \\
\hline 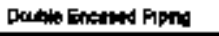 & 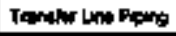 & 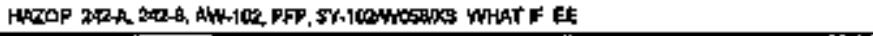 \\
\hline 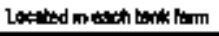 & Catabion & HAEOP AW-1CR, BY-102XS: \\
\hline 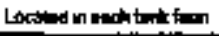 & Cokh but pa & 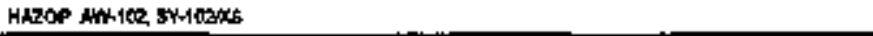 \\
\hline 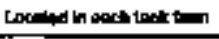 & Choul bor & BHA \\
\hline 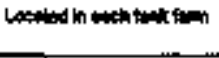 & 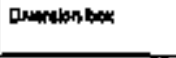 & 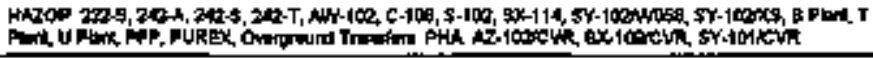 \\
\hline | & Danderation & 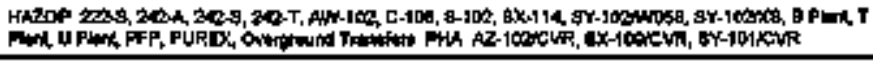 \\
\hline 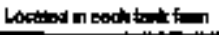 & Phoh pal & 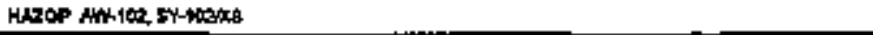 \\
\hline | & 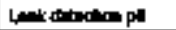 & 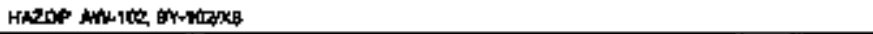 \\
\hline
\end{tabular}




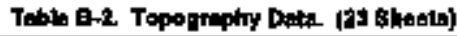

\begin{tabular}{|c|c|c|}
\hline Party kn & Type & 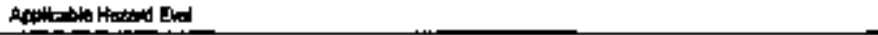 \\
\hline 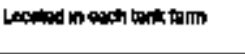 & Pumpp & 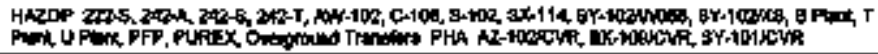 \\
\hline 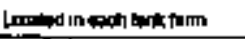 & sermpan & 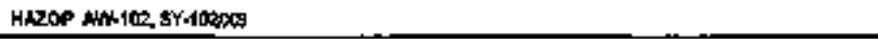 \\
\hline 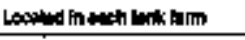 & 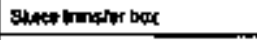 & PHA COS \\
\hline 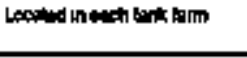 & whet pt & 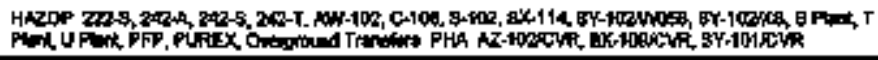 \\
\hline 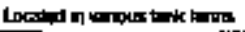 & Hertad shanges unte & Puth vat \\
\hline Ohropond Triafor Lho & $\begin{array}{l}\text { Portat Oungond Tramplar } \\
\text { Ind }\end{array}$ & HezoP Onground Tonafors \\
\hline FPP Pond & Pond & Puh CDP \\
\hline 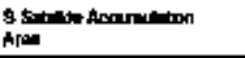 & 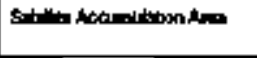 & Pwh Xac \\
\hline 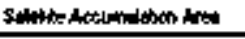 & 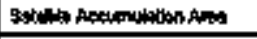 & DWh 2005 \\
\hline Pecombing Centhimert & Trumbitu Und Prong & 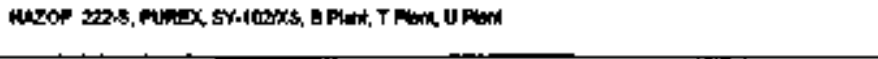 \\
\hline 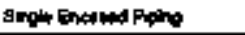 & Tronefer Une Ppang & Hazop AK-IDC, B-102, Br-1000\%s \\
\hline 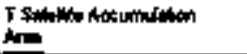 & 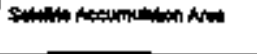 & PHS NOE \\
\hline 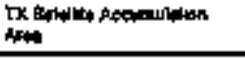 & 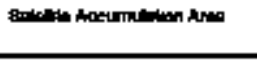 & PHA zote \\
\hline 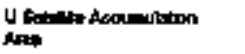 & 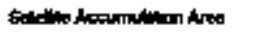 & PHA zad \\
\hline Varthod storag then & $12-x$ intuph & FHA wou \\
\hline 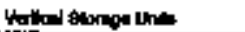 & It-all hd dith & PHA wast \\
\hline
\end{tabular}


HWNF-9D-WMH-TR-784, RoY. 1

Thls poge intentlonely let blank. 


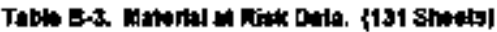

\begin{tabular}{|c|c|c|c|c|c|c|c|c|c|c|}
\hline Find & 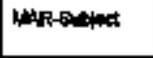 & MARR-Daporipton & Mlar. & cravely & Thenes & Proped & (19) & Dringatid & oumly & Conmento \\
\hline 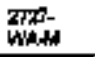 & Tand Cordets & Hore & $\mathbf{~} \mathbf{4 h}$ & $m$ & $\boldsymbol{H i n}$ & rath & Nh & Nat & $\mathbf{m h}$ & 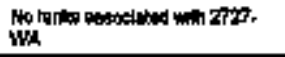 \\
\hline Whati. & Tank & Nons & Nh & & $\mathbf{m}$ & NA & Nh. & Na & in & 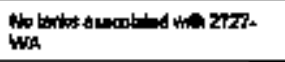 \\
\hline Trzing & 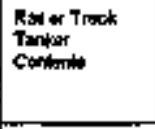 & Torle thute nat & Not & 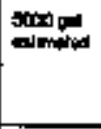 & 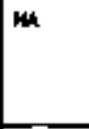 & 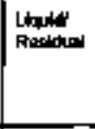 & Hed of thand & Ym & $\mathbf{m}$ & 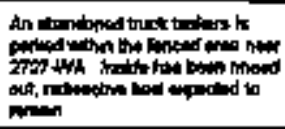 \\
\hline 2727-WA & conimination & 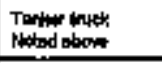 & $\mathrm{NAS}$ & NAx & UA & Ruratist & 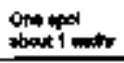 & $Y *$ & HA & 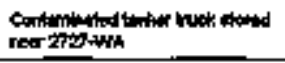 \\
\hline WTH. & 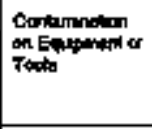 & None & ine & Nh & $\mathrm{mh}$ & NA & MA & Nik & $\mathbf{m e n}$ & 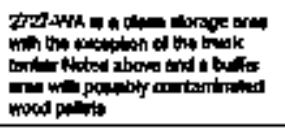 \\
\hline Trar- & 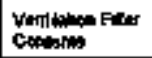 & Hons & whe & NAh & NAn & Wh & and & Nh & Nah & 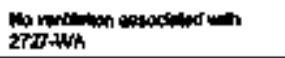 \\
\hline trd: & 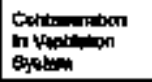 & Dratrantios & sent & $\mathrm{m}$ & NA & ath & $\mathbf{m}$ & $\mathbf{m}$ & $m$ & No teothinenes \\
\hline aty. & 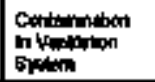 & Stat & Wh & $\mathrm{Mh}$ & in & $\mathrm{Hah}$ & $m$ & $\mathbf{m h}$ & Mu & m \\
\hline 202. & 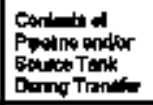 & an & Mh & $M$ & Non & in & $\mathrm{mh}$ & $m$ & $\mathrm{Nin}$ & 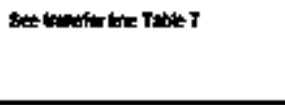 \\
\hline Whin & Oontits of & Ninn & nat & Im & in & and & $\mathbf{m}$ & $m$ & sh & 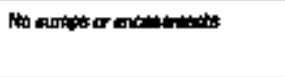 \\
\hline
\end{tabular}


Tuble B-3, Nateral al Rbak Dake (131 \$heqtey

\begin{tabular}{|c|c|c|c|c|c|c|c|c|c|c|}
\hline הid & MARstiptet & Whathompon & lets & Canpectit & 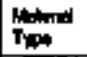 & $\begin{array}{l}\text { Phyoked } \\
\text { Ron }\end{array}$ & Folumaty & Trandent & Qundey & ctame de \\
\hline $\begin{array}{l}272 \\
\text { Whis }\end{array}$ & $\begin{array}{l}\text { Contionation } \\
\text { in Pit or Bon }\end{array}$ & Nom & in & $\boldsymbol{m}$ & $\mathbf{M h}$ & NA & in & th & Nin & 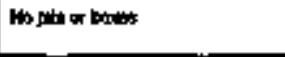 \\
\hline $\begin{array}{l}\text { rrat- } \\
\text { Whith }\end{array}$ & Sinpid at 3 & Neen & $\mathbf{m}$ & HA & in & Nin & nith & NA & $\mathbf{m a k}$ & $\mathbf{m a n}$ \\
\hline $\begin{array}{l}2 \pi 27 . \\
\text { Whis }\end{array}$ & $\operatorname{con} x$ & Urimbind & Unionitin & Sh & res & 1ntim & Longow, up & $y=$ & 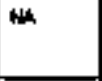 & 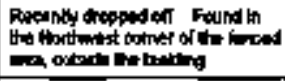 \\
\hline Whis & Thent & Cotation & 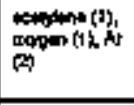 & 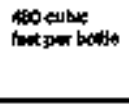 & NA & Vepoer & 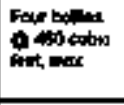 & $Y \omega$ & 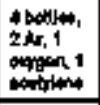 & 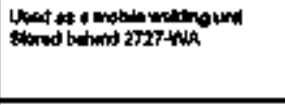 \\
\hline $\begin{array}{l}\text { 2757- } \\
\text { WA. }\end{array}$ & Dant & Calkemm & 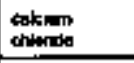 & Sotation & an & 64 & 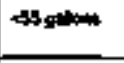 & $Y=$ & Dentene & 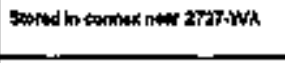 \\
\hline Whi: & Ober & Streted in & setatan & tot pakars & wer & Lim & togitom & $\mathrm{Yen}$ & 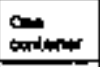 & 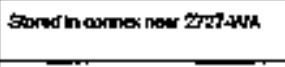 \\
\hline $\begin{array}{l}2727 \\
\text { What }\end{array}$ & Danf & 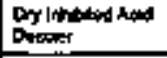 & 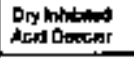 & 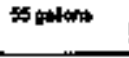 & NA & sold & क्स & Yos & on & 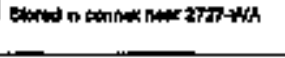 \\
\hline trat: & Dther & Noutronat & Neminest & sinte & Na & Lnid & $30 g^{0}$ & Yeq & intan & 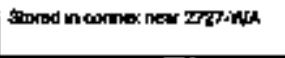 \\
\hline what: & Other & Kand DE, caith & 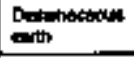 & os poikat & wath & Solid & 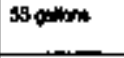 & Yes & in & 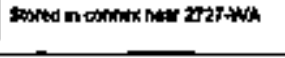 \\
\hline $\begin{array}{l}\text { max. } \\
\text { Whos }\end{array}$ & othe & sedon artenta & codum & 110 gubon & $m a$ & Lqd & So oflun & Y⿻ & One & 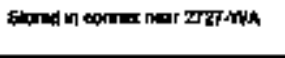 \\
\hline WTA. & Other & nowerit 2 & rontion & 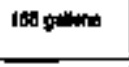 & Nin & sollo & 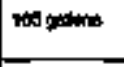 & $r=$ & $\min _{\text {and }}$ & 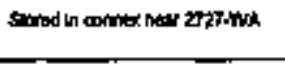 \\
\hline This & $60 \mathrm{mox}$ & Aneoviont & sold & d & $\mathbf{M h}$ & Bold & 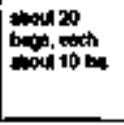 & Yes & 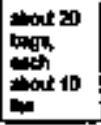 & 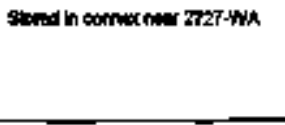 \\
\hline
\end{tabular}




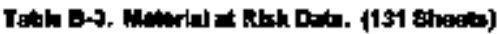

\begin{tabular}{|c|c|c|c|c|c|c|c|c|c|c|}
\hline 部 & MHR-S的望 & 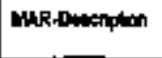 & Mhr- & Capecty & Hetser & $\begin{array}{l}\text { Finawed } \\
\text { Form }\end{array}$ & thilume or & Intmanint & Duncisy & Commention \\
\hline $272 \pi+w$ & Tom Contanta & Tonk I & sodum & 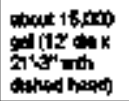 & NA & sond & 300 How & $\mathbf{m}$ & Nh & 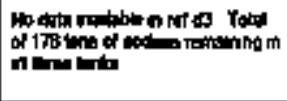 \\
\hline $2 \pi m+w$ & Tancomitenth & Tsoin 2 & Soturn & 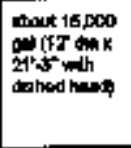 & NAS & Sald & 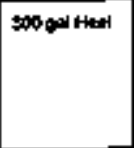 & $\mathbf{N o}$ & Nin & 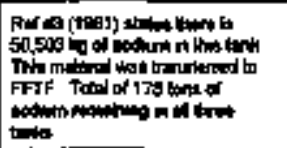 \\
\hline $2 \pi 7-W$ & Tant Consunth & Tank 3 & sontur. & 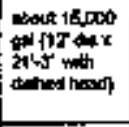 & and & soles & 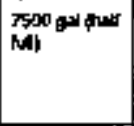 & Ho & $\mathrm{MA}$ & 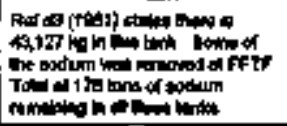 \\
\hline Etr.w & Tuk Eontertix & Tnak 4 & thenth & 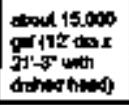 & $m$ & sid & 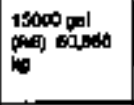 & No & $\mathrm{Nh}$ & 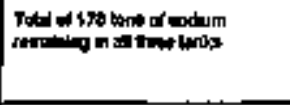 \\
\hline Ixtron & Tart conterte & Terks & toding & 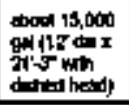 & $\operatorname{men}$ & sond & 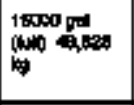 & No & $\boldsymbol{m h}$ & 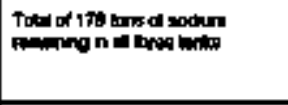 \\
\hline arerin & Turt & Trank : & Mator & $\mathrm{NH}$ & an & Vupar & 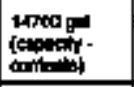 & $N$ & Wh & 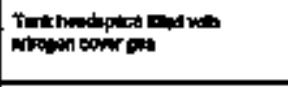 \\
\hline $272+4$ & Twat: & Thint & Heregen & Nh & $\boldsymbol{m}$ & Wher & imponit. & No & 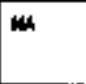 & 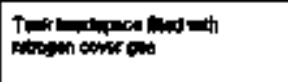 \\
\hline 2725 & Tum & Teth & 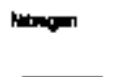 & Nh & un & mper & rasidit. & Ho & $\mathrm{m}$ & Therim \\
\hline
\end{tabular}




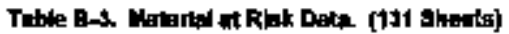

\begin{tabular}{|c|c|c|c|c|c|c|c|c|c|c|}
\hline imat & MAkstitad & Whit:Detorikn & MrR. & Coptidy & Typom & 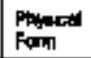 & 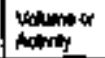 & Tetmetrl & 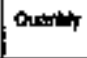 & Conments \\
\hline $2 \pi n+4$ & Tent & Tank 4 & Arragm & Wh & Nh & Veax & mmonal & No & $\mathrm{Nh}$ & 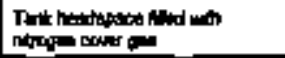 \\
\hline 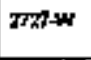 & Tratripes & Taxt: & mropen & Nh & Nhe & Vitper & mant & $\mathrm{mb}$ & Wh & 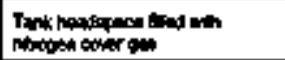 \\
\hline $2727 w$ & $\begin{array}{l}\text { Ron or Thuek } \\
\text { Tanker } \\
\text { Corterits }\end{array}$ & Wan & MA & NA & Nh & WA & $\mathbf{m}$ & Nh & NA & 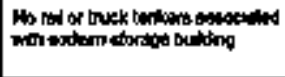 \\
\hline m2r-w- & 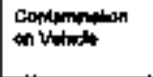 & nenes & $\mathbf{m}$ & wa & $\mathrm{Mh}$ & Nh & NA & NA & Nath & 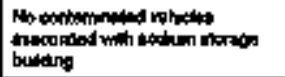 \\
\hline $3^{3-n k}$ & 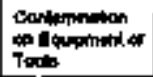 & Nan & MA & NA & Mu & $m$ & NA & wa & un & 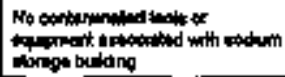 \\
\hline 2tzatis. & Contistin & Mene & ma & MA & $\mathbf{m e}$ & $m$ & NA & Wh & $\mathrm{en}$ & 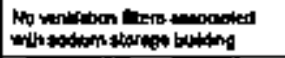 \\
\hline $2720-4$ & 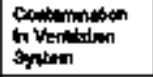 & $\operatorname{Ninh}$ & NA & NA & $\mathrm{mon}$ & WA & Wh & WA & Mh & 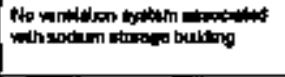 \\
\hline $2727+4+$ & 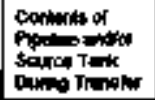 & ans & NA & NA & 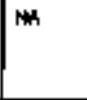 & $\mathbf{m}$ & NA & Wh & m & 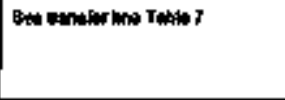 \\
\hline num & 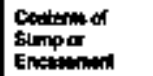 & Nitune & Na & mo & $\mathrm{min}$ & NA & Nh & Wh & u & 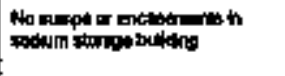 \\
\hline$M^{272 J+M}$ & Contmithosen & Ninis & NA & NA & $\mathrm{man}$ & wh & WA & HA & $\mathbf{m}$ & 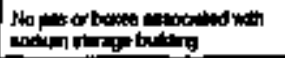 \\
\hline$m^{2}$ & S4FA Fia & Nont & Wh & $m$ & Ma & Ma & pith & m & M & Nh \\
\hline
\end{tabular}


Tuble D-3, Mrterial at Rlah Dofe (131 6hetiol

\begin{tabular}{|c|c|c|c|c|c|c|c|c|c|c|}
\hline inding & MARSthed & 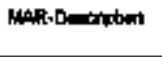 & Chas. & Cappoctr & Tipend & $\begin{array}{l}\text { Plyoted } \\
\text { Pond }\end{array}$ & untoridy & Trthent & 要 & Conminents \\
\hline 27r2an. & Other & Iimo & Nh & $\mathbf{m}$ & Hh & MA & Mh & $M$ & NAS & Nhe \\
\hline $200-6$ & Tenth Corlemts & Matie & $\mathbf{m}$ & NA & $\mathbf{r e n}$ & MA & $\mathrm{Na}$ & Mh & NA & an \\
\hline $200 E$ & 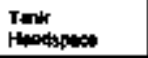 & $\mathrm{ran}$ & $\mathbf{m a}$ & NA & un & Na & NA & in & HA & 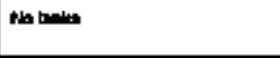 \\
\hline 20064 & 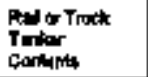 & What & wh & $\boldsymbol{H}$ & Mn & $\mathrm{MH}$ & $\mathrm{NA}$ & H & Nan & 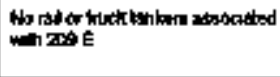 \\
\hline 2000.5 & 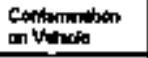 & Nom & Mh & NA & $M$ & Nh & Nha & NA & wh & 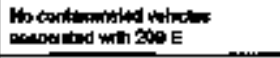 \\
\hline 300EE-11 & 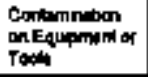 & $\mathrm{Nem}$ & $\mathbf{w h}$ & Na & $\mathbf{r m}$ & Nh & $\mathrm{Na}$ & M & NA & 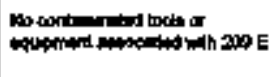 \\
\hline XIREAN & 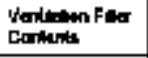 & Henen & $m$ & $\mathrm{NH}$ & Nh & $\boldsymbol{m}$ & Nh & Na & $\mathbf{m}$ & El \\
\hline $200-E+4$ & 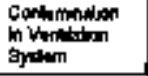 & 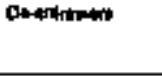 & MA & NA & NA & NA & $\mathrm{Nh}$ & NA & $\mathrm{ma}$ & 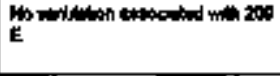 \\
\hline $206-1+1$ & 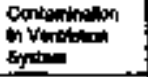 & إن & NA & NA & nith & M & Nak & NA & Bh & 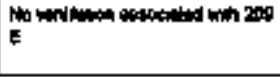 \\
\hline 209E-W & 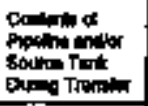 & $\mathrm{mn}$ & Ban & wa & Ma & $\mathbf{m}$ & $\mathrm{Wh}$ & nes & $\mathbf{m}$ & 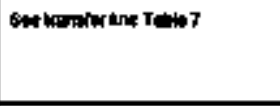 \\
\hline $\operatorname{mos}=$ & $\begin{array}{l}\text { Continite of } \\
\text { sump of } \\
\text { Encintentax }\end{array}$ & Hime & NA & $\mathbf{m}$ & Wh & Hh & $\mathbf{H h}$ & Wh & wat & No م \\
\hline $2005+4$ & $\begin{array}{l}\text { Contioningling } \\
\text { in Ptor Bon. }\end{array}$ & $\min$ & MA & NA & Nh & $\mathbf{M}$ & $\mathbf{M H}$ & 钫 & m & 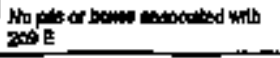 \\
\hline
\end{tabular}




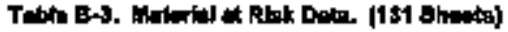

\begin{tabular}{|c|c|c|c|c|c|c|c|c|c|c|}
\hline Ditid & MHR-Sulent & 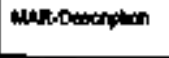 & MAR- & Capuetry & Thiontal & Pander & Hoing of & Thenownt & क्षority & Collnm:ाt: \\
\hline 200-EIA & BARA \$3 & Prowam & Wh & 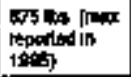 & an & Gowd & 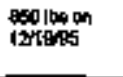 & Yeڤ & $\mathbf{m}$ & 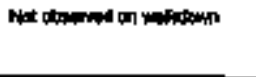 \\
\hline $200-5-4$ & SARA Gr3 & chenuler uran & MAH & $\begin{array}{l}300 \text { bi finer } \\
\text { nperlad in } \\
\text { 19gal }\end{array}$ & NRA & sond & 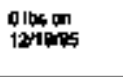 & Tos & $\boldsymbol{N h}$ & 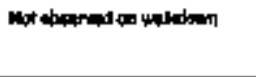 \\
\hline aterter & GARd ats & 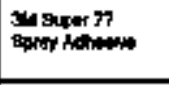 & Hin & 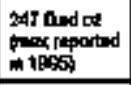 & $\mathrm{man}$ & Land & $\begin{array}{l}192 \text { hudex } \\
\text { on } 1 \text { 2919xis }\end{array}$ & $\mathrm{Ya}$ & Mh & 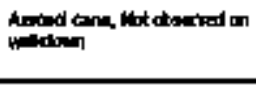 \\
\hline tated & gARd b3 & 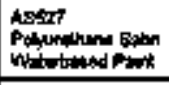 & 滥 & $\begin{array}{l}\text { rot ind os } \\
\text { (mac mpated } \\
\text { n 1009) }\end{array}$ & Nh & Inpd & 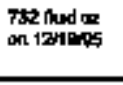 & Ye & NA & 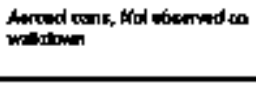 \\
\hline Shen & ANRH 313 & $\operatorname{tan=n} \mid v^{\prime}$ & wh & 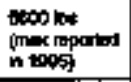 & NA & sotid & $\begin{array}{l}\text { 1600tit on } \\
\text { 12yition }\end{array}$ & $Y=$ & Wh & 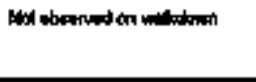 \\
\hline $200-4$ & StARA 919 & $\begin{array}{l}\text { chete-zo } \\
\text { Werowo }\end{array}$ & HA & 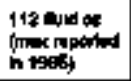 & wh & |A & 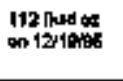 & Yw & NA & 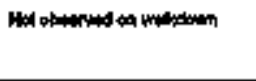 \\
\hline $200-5-1$ & $\begin{array}{l}\text { SAFA } 39 \\
\text { Motarein }\end{array}$ & 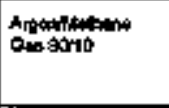 & $\mathbf{m}$ & 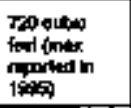 & me & on & 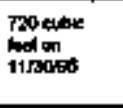 & $n *$ & Hh & 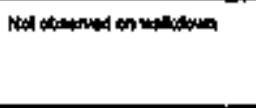 \\
\hline $200-4$ & $\begin{array}{l}\text { sitedsis } \\
\text { Mremix }\end{array}$ & 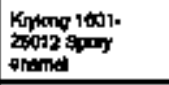 & wh & 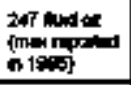 & Nh & ind & 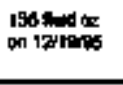 & res & Nh & 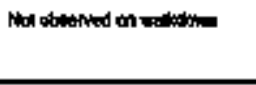 \\
\hline $\operatorname{lng} x$ & sithias & Prupan & $\boldsymbol{m}$ & 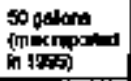 & 缋 & | & 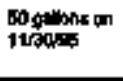 & $y=$ & Nh & 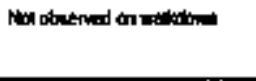 \\
\hline 2005 EA & spin 313 & 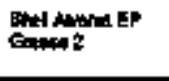 & HA & 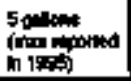 & 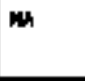 & Inிل & 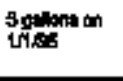 & Yat & $\mathbf{H}$ & 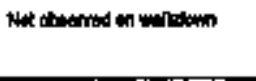 \\
\hline
\end{tabular}




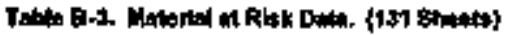

\begin{tabular}{|c|c|c|c|c|c|c|c|c|c|c|}
\hline Find & uturisuryead & 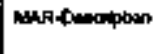 & Makr & crapecty & Then & $\begin{array}{l}\text { Ptpayod } \\
\text { Form }\end{array}$ & Vome or & Trofend & Quntity & Cemmete \\
\hline aba-tu & 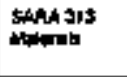 & \$prapar Antets & Nh & 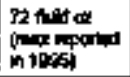 & $\mathrm{Buh}$ & upd & 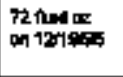 & $Y$ rod & an & Wht atoorrod on ardan \\
\hline ate-4 & 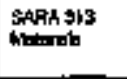 & THD & NA & 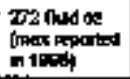 & $\mathrm{Nat}$ & ing & 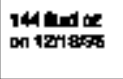 & Yot & H & lat oboditidd on aldonn \\
\hline $200-E-0$ & Othat & Drana & 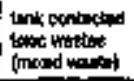 & Fon & IH & Solw & latherer F & Yes & 41 dxका & $\mathbf{H A}$ \\
\hline $20 \%-E-0$ & pthen & DOT boms (TA] & tank contestid & 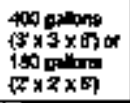 & H & Esing & 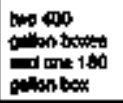 & Yoq & thent & MH \\
\hline $\cos [10$ & Othw & DOT $\operatorname{Bos}(\nabla A)$ & 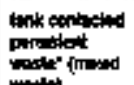 & 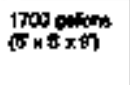 & m & sond & 象 1700 & $Y a$ & an bon & 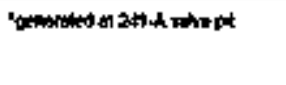 \\
\hline $\sin 60$ & Orlare & Drumt & 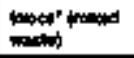 & 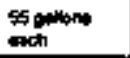 & $M$ & لبهي & 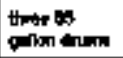 & $Y$ (H) & 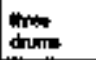 & d \\
\hline ane-O & Othr & Drma & 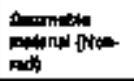 & tions & H & 5old & tou 55 gintad & Yes & $\lim _{1 \rightarrow \infty}$ & $\mathrm{MA}$ \\
\hline 206_ED & cerer & Drum & 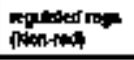 & Afgan & wa & 80ived & and Expen & $Y * 4$ & and and & Ma \\
\hline 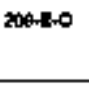 & Otwr & Drin & 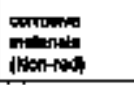 & spalm & Nat & sat & $\begin{array}{l}\text { mestigan } \\
\text { mint }\end{array}$ & res & one them & 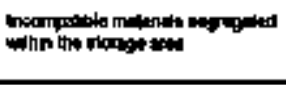 \\
\hline $200-E-0$ & ond & Dnim & pitimate & 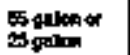 & Nh & Lend & 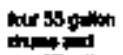 & TEs & the & Nh \\
\hline
\end{tabular}


Tabte B-3. Malerdal at Rish Drte (131 Ghats)

\begin{tabular}{|c|c|c|c|c|c|c|c|c|c|c|}
\hline Faids & Har-subloct & MAR-D Deqposh & MaR- & Cenestr & Mond & Pataled & 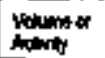 & Tranaest & averints & Canilats \\
\hline AR-204 & 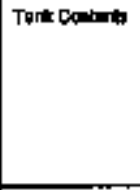 & $\operatorname{lank} 1$ - CAkh & 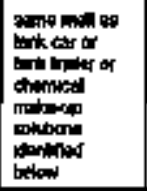 & 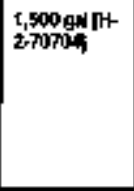 & MH & 10010 & $0000 \mathrm{At}$ & No & MA & 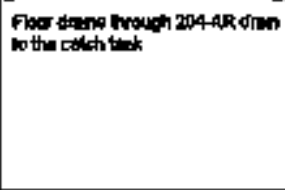 \\
\hline AR 200 & Totat Dententh & Tolk2-Chemed & 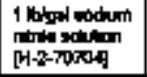 & 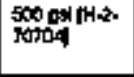 & $\mathrm{N} / \mathrm{h}$ & Utand & الهم 120 & $\infty$ & No & Mh \\
\hline AR-ZM & 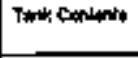 & 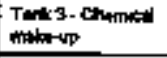 & 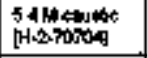 & 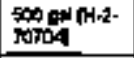 & $\mathrm{NA}$ & Noqus & 19: & $\infty$ & Nh & $m$ \\
\hline ARABOA & 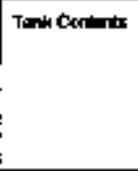 & 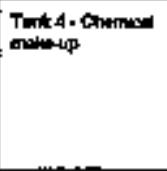 & 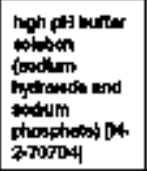 & $2000001+1+2-$ & NA & uqued & 00 & $\mathbf{H o}$ & ing & 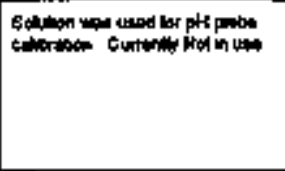 \\
\hline$A R-204$ & Tentik Gorlemts & Tad s. chancoll & 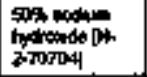 & 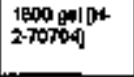 & Wh & Land & apen & No & M & Cunnoly Nax in wis \\
\hline Aktatat & Twnk cartents & 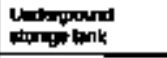 & Dand & $\sin ^{2003}$ & wh & الفها & sapow & No & $\mathbf{m}$ & 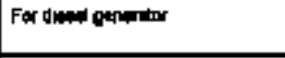 \\
\hline $\operatorname{lR} \rightarrow 204$ & Ttank & Cald tork & Nh & Na & Wh & $\mathbf{m k}$ & Nin & NA & in & 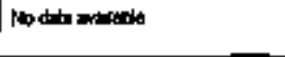 \\
\hline AR-20A & Tat & 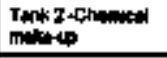 & $\mathbf{m}$ & $m$ & $\mathbf{M r}$ & NA & $\mathbf{m}$ & Nhe & M & 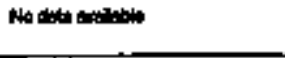 \\
\hline $\sin x$ & Turt & 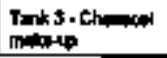 & $\mathbf{m}$ & $\mathbf{m}$ & $m$ & Nh & in & NA & $\boldsymbol{m}$ & 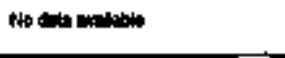 \\
\hline
\end{tabular}




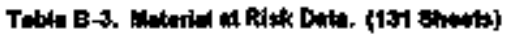

\begin{tabular}{|c|c|c|c|c|c|c|c|c|c|c|}
\hline Andd & MHR-sabinal & 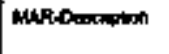 & MAR: & Capucty & Thisina & $\begin{array}{l}\text { Fripuced } \\
\text { Fonil }\end{array}$ & Wolung or & 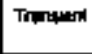 & Cynerif & Cormments \\
\hline NR-204 & 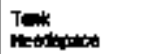 & 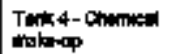 & NA & Ah & Na & Don & Nar & $\mathbf{m}$ & Nk & W \\
\hline ARPOA & Haik & 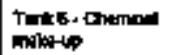 & $\mathrm{NH}$ & $\mathbf{m}$ & Nh & $\mathbf{M A}$ & 执 & then & Wh & 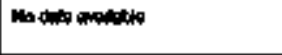 \\
\hline AR-204 & Imikn & 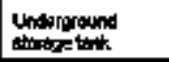 & $M_{H}$ & 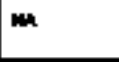 & in & man & Nin & but & Wh & 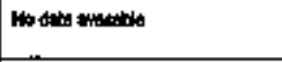 \\
\hline ARLOC & 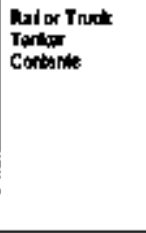 & Prened bitter & 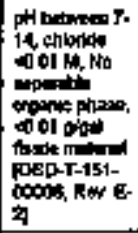 & 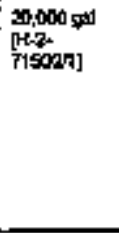 & $\mathbf{H a t}$ & LFed & 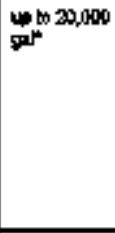 & Ho & NR & 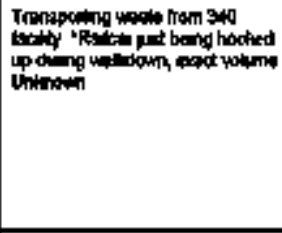 \\
\hline AR-aP4 & 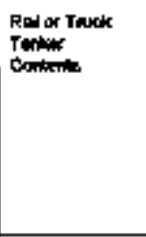 & Tonil truler & 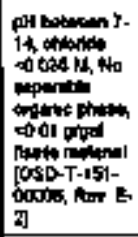 & 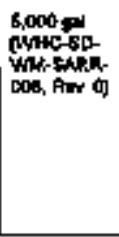 & MA & Lnutió & بr & No & Wh & 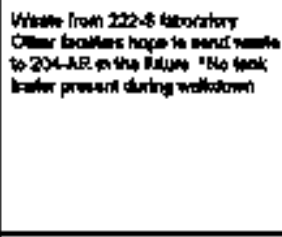 \\
\hline AR & 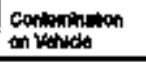 & 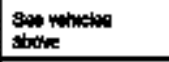 & Nh & $r$ & and & $\mathbf{M n}$ & wes & $\mathbf{~ H h}$ & Nin & $\mathbf{m}$ \\
\hline Ansart & 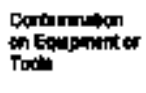 & 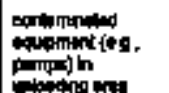 & 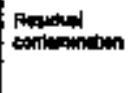 & math & wh & 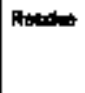 & Nh & Tel & Nh & $\mathbf{m}$ \\
\hline
\end{tabular}




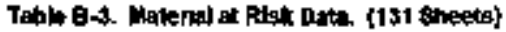

\begin{tabular}{|c|c|c|c|c|c|c|c|c|c|c|}
\hline Fish & 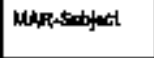 & HAR-piacorpon & Mink & Crencty & The & $\begin{array}{l}\text { Pigucen } \\
\text { Form }\end{array}$ & 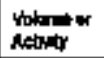 & Thandet & Croth & Conroming \\
\hline 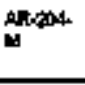 & 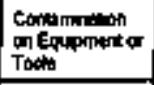 & mod & wind lavily? & in & hh & soly & Mh & $Y=t$ & Mh & 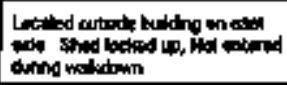 \\
\hline NRPM4 & 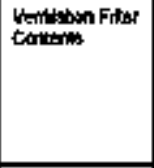 & 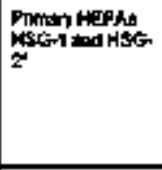 & HA & 1 fift all tar & man & $\begin{array}{l}\text { Partimatat } \\
\text { - }\end{array}$ & 塩 & 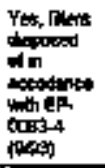 & Na & 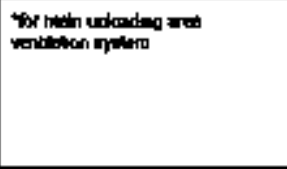 \\
\hline$\underset{\text { it }}{A R-204}$ & 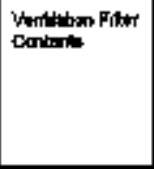 & MEPA MSG & NA & $\begin{array}{l}\text { I fint } \text { : then } \\
\text { Inchen }\end{array}$ & NA & Partualt & An & 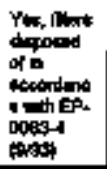 & NA & for cton tank wontibon \\
\hline M & 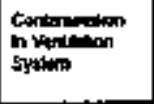 & $\begin{array}{l}\text { Mortive } \\
\text { exporitars }\end{array}$ & Protat & Mr & Non & Uqud & $\mathbf{m}$ & mo & zotion & 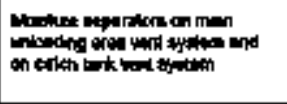 \\
\hline Ningent & 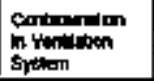 & sal lopp & Nh & $\operatorname{man}$ & NR & NA & $\mathbf{m}$ & HA & WA & 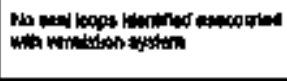 \\
\hline Mintar & & Capterta of & $\mathrm{Mh}$ & $\mathrm{NH}$ & Hh & m & Nh & ven & $\mathbf{w h}$ & 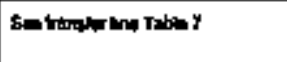 \\
\hline MRTh4 & $\begin{array}{l}\text { Conlwite of } \\
\text { sumpar } \\
\text { Encement }\end{array}$ & Cochtant in & thaner & 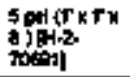 & Nh & wh & Hon: & rh & MH & 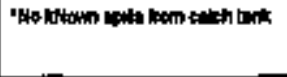 \\
\hline MN204 & 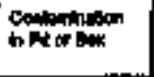 & 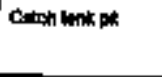 & Ihne: & 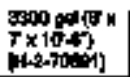 & m & MA & Rines: & NA & rin & 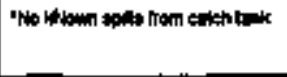 \\
\hline
\end{tabular}




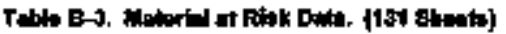

\begin{tabular}{|c|c|c|c|c|c|c|c|c|c|c|}
\hline $\begin{array}{l}\text { Fidd } \\
\text { Meine }\end{array}$ & $M A R-e_{0}$ & MuR-Denappen & Whe & Capuctir & Thined & $\begin{array}{l}\text { Pboingl } \\
\text { Poim }\end{array}$ & vinty & Trewand & Axinty & Danfmim \\
\hline A 1 & SARM \$ạ & 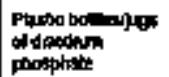 & popdum & 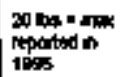 & M & Eatu & 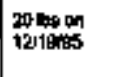 & $Y=$ & Mat & 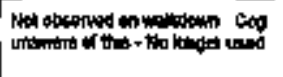 \\
\hline ARs:304 & 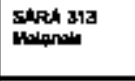 & $\begin{array}{l}\text { Bey of mothm } \\
\text { bythous }\end{array}$ & 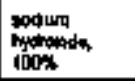 & 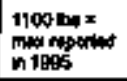 & $\mathrm{ru}$ & Eatd & 要010404 & $\mathbf{Y}_{4}$ & H & 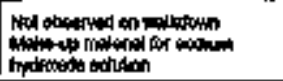 \\
\hline in & 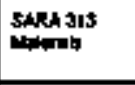 & 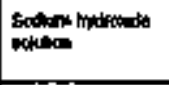 & 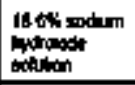 & 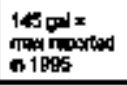 & ith & |d & 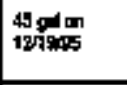 & Ye & 처 & 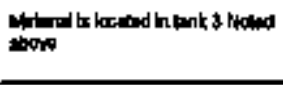 \\
\hline HA & torR & $\begin{array}{l}\text { Sadim fillte } \\
\text { nolution }\end{array}$ & 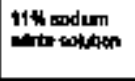 & 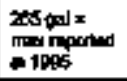 & Wh & همان & 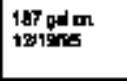 & Tex & NA & 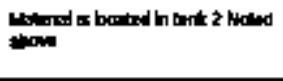 \\
\hline in & SARA J|3 & Denal fal & Dnosed lud & 50 arb & NA & Lys & 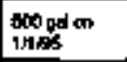 & $m$ & Nh & 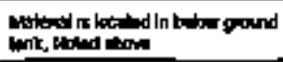 \\
\hline A & Other & $204 \times 14$ and & 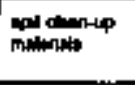 & Gne- thed" & Ha & sold & NA & Yet & NA & 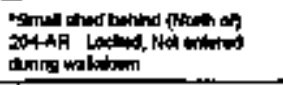 \\
\hline 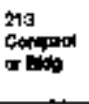 & Tank torinerta & $213+N-T h+1$ & 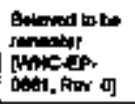 & 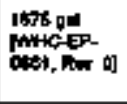 & ta & Lnued & 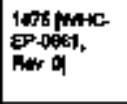 & No & an & 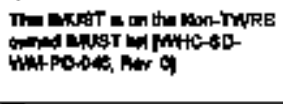 \\
\hline 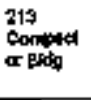 & 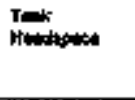 & 219-WTK-1 & NA & in & $\mathrm{NH}$ & Na & 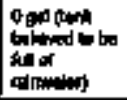 & NA & Nh & 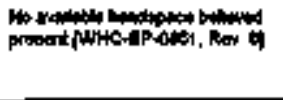 \\
\hline $\begin{array}{l}213 \\
\text { Conpoct } \\
\text { of alder }\end{array}$ & $\begin{array}{l}\text { Ras of Toukt } \\
\text { Tanti } \\
\text { Contonto }\end{array}$ & Notion & $\mathrm{NA}$ & Mh & ut & Wh & H & $\mathrm{NH}$ & $\operatorname{an}$ & 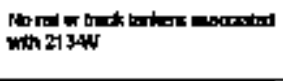 \\
\hline 219 & 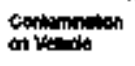 & Nitone & $\mathrm{MH}$ & HA & $\mathbf{M h}$ & NA & Nu & Nh & $\mathbf{M h}$ & 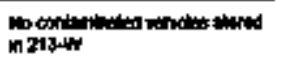 \\
\hline
\end{tabular}




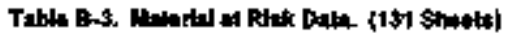

\begin{tabular}{|c|c|c|c|c|c|c|c|c|c|c|}
\hline Find & Abur:subjed & MR-Datinglian & 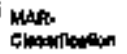 & copoctyy & Tyend & Finglred & $\begin{array}{l}\text { Vodumegr } \\
\text { Axtmill }\end{array}$ & Traneivat & Murty & 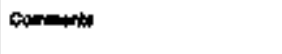 \\
\hline $\begin{array}{l}\text { 21s } \\
\text { compons } \\
\text { or tion }\end{array}$ & 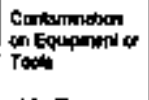 & 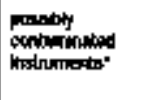 & NA & 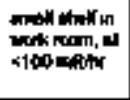 & $B M$ & Roulder & sitconenthr & $\operatorname{mos}$ & Hin & 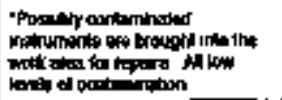 \\
\hline $\begin{array}{l}213 \\
\text { compan } \\
\text { or Bist }\end{array}$ & 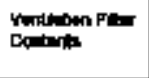 & 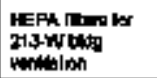 & wh & 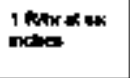 & m & Reuter & Nh & Yes & ArePh & 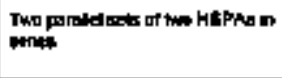 \\
\hline 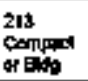 & 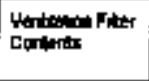 & 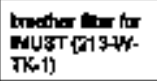 & NA & $\begin{array}{l}1 \text { Rhe of ex } \\
\text { nchex }\end{array}$ & 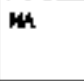 & Rtobdue & Nin & Yex & Nh & 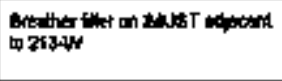 \\
\hline 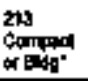 & 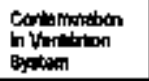 & Detutingere & MA & WA & Nh & BH & Nh & Nik & Nh & 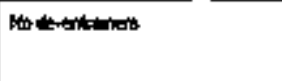 \\
\hline $\begin{array}{l}\text { 217 } \\
\text { Conged }\end{array}$ & 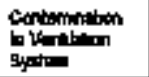 & sect lat & NA & NA & Nh & MA & 塩 & Nh & $\mathbf{H}$ & 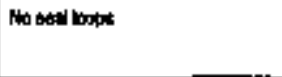 \\
\hline $\begin{array}{l}219 \\
\text { Candpoct } \\
\text { a Bldg }\end{array}$ & 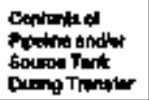 & M & JaA & aA & in & Nah & MA & Nh & $\mathbf{M n}$ & 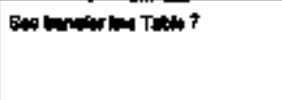 \\
\hline $\begin{array}{l}215 \\
\text { Canpeot } \\
\text { at 64tit }\end{array}$ & 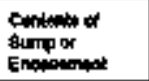 & Nhin & MA & 1h & whe & NA & in & wh. & MH & No tom \\
\hline $\begin{array}{l}\text { th } \\
\text { Campit } \\
\text { of Bitg }\end{array}$ & 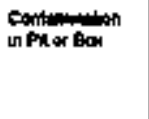 & muts p p & lopind twok & 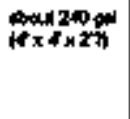 & NA & Nh & 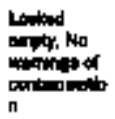 & $\mathbf{W}$ & $\mathrm{kH}$ & 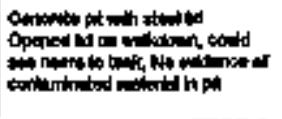 \\
\hline 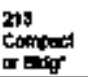 & Miph & Nann & $\mathbf{m}$ & un & Nh & NA & MA & $\mathbf{M H}$ & MA & Nh \\
\hline
\end{tabular}




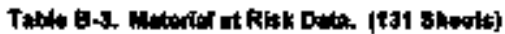

\begin{tabular}{|c|c|c|c|c|c|c|c|c|c|c|}
\hline Name & NAR-ER:mat & WAP-Dianglaten & INA- & copeaty & Typa & Phyming & Folumity & Trunewat & danetry & Commints \\
\hline $\begin{array}{l}213 \\
\text { comprat } \\
\text { or } E \text { ity }\end{array}$ & other & caphen trifon & orompoing & es gard drum & NA & $\mathbf{m}$ & 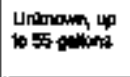 & $r=$ & wan & 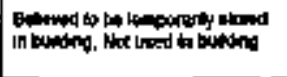 \\
\hline of :-ing & berer & 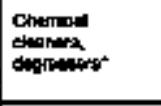 & ootrit & $\begin{array}{l}\text { wad } \\
\text { qunitom at }\end{array}$ & $\mathrm{wh}$ & iqura & 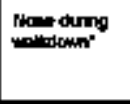 & $\mathrm{re}$ & man & 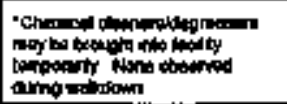 \\
\hline AR-244 & Tart Curtent & 244AR-OB1 & Untam the & 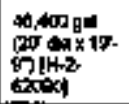 & wh & Iqed & ZMO ga & No & Wh & Th⿻ \\
\hline AR-244 & Turt Conimb & 244AR-C02 & 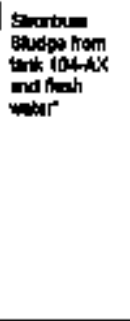 & 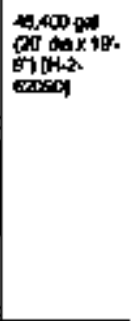 & $\begin{array}{l}\text { lyid and } \\
\text { shinge }\end{array}$ & lowd & 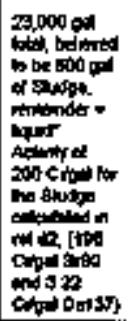 & H. & Wh & 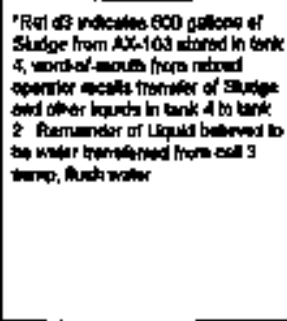 \\
\hline MAR44 & Tanh Contents & 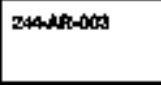 & Urtinown: & 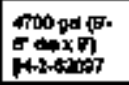 & ma & Iquad & 2000 & Non & in & 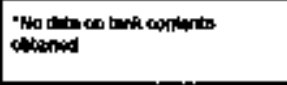 \\
\hline $\min 244$ & 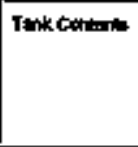 & 244ith-ob4 & Unichewn" & 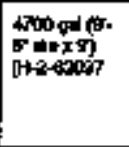 & ren & ing & $400 \%$ & $\mathrm{No}$ & $\mathrm{m}$ & 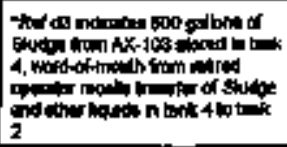 \\
\hline
\end{tabular}




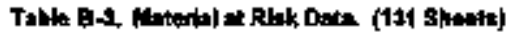

\begin{tabular}{|c|c|c|c|c|c|c|c|c|c|c|}
\hline $\begin{array}{l}\text { Finid } \\
\text { Ninnim }\end{array}$ & Minstubjod & MhrDenchiton & 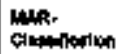 & Capect & $\frac{1}{T+1}$ & $\begin{array}{l}\text { Phpord } \\
\text { Fopen }\end{array}$ & 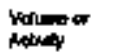 & Trenewt & Dryanity & Cammanity \\
\hline Ifa-244 & 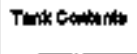 & 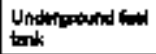 & Ghal lud & 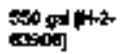 & $\operatorname{man}$ & Lrined & 100 & $\mathbf{k}$ & Nin & 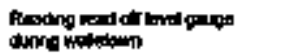 \\
\hline AR-241 & $\begin{array}{l}\text { Tert } \\
\text { Howionest }\end{array}$ & 264th-601 & Nohylogen & $\mathrm{NH}$ & NAM & vimpor & tout 44, 200 & $\mathbf{m}$ & HA & Mat \\
\hline ARr244 & Triation & $24+4 R-600$ & 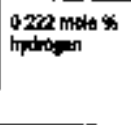 & 蚔 & Nh & Wapr & 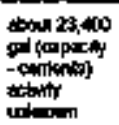 & $m$ & Nh & beth \\
\hline$A R-244$ & $\begin{array}{l}\text { Toric } \\
\text { Heodapuse }\end{array}$ & ZCAnR & $\begin{array}{l}\text { ilo bythege } \\
\text { of ergulist }\end{array}$ & MA & NIh & Whor & 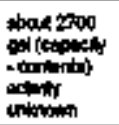 & No & Wh & NA \\
\hline $14 \times 244$ & Thention & $2440 R=004$ & $\begin{array}{l}\text { Ho hadioges } \\
\text { or orptox }\end{array}$ & $\boldsymbol{M}$ & $\mathbf{M h}$ & Vtoor & 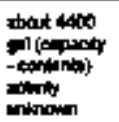 & $\mathrm{Na}$ & wh & Na \\
\hline AR-2014 & $\begin{array}{l}\text { Res or Trut } \\
\text { Tanker } \\
\text { etolunte }\end{array}$ & $\mathrm{Hm}$ & wh & $\operatorname{man}$ & $\mathbf{u h}$ & NA & $\mathbf{m}$ & $\mathbf{M A}$ & MA & 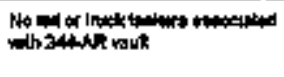 \\
\hline Mr-244 & Contamubas & Dhe & wh & HA & un & HA & $\mathbf{m}$ & wa & MH & 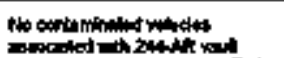 \\
\hline
\end{tabular}




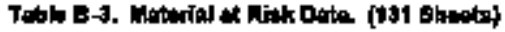

\begin{tabular}{|c|c|c|c|c|c|c|c|c|c|c|}
\hline $\begin{array}{l}\text { fud } \\
\text { Hัगय }\end{array}$ & Mukststyed & Whathingin & Whit: & Copoitis & Tripend & $\begin{array}{l}\text { Promitis } \\
\text { Pam }\end{array}$ & Aching & Tftitanot & aninity & Cenmanis \\
\hline Mhrz) & 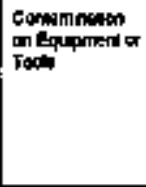 & 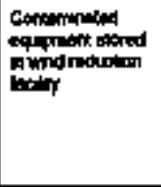 & 幅 & 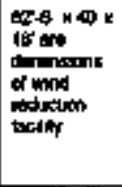 & NA & 3014 & Unknowrt" & $y+\boldsymbol{p}^{2}$ & Nah & 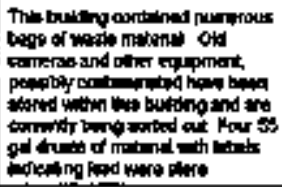 \\
\hline MAOQ⿻4 & 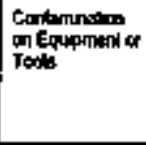 & 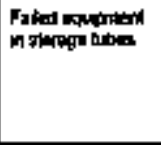 & $\mathrm{NA}$ & 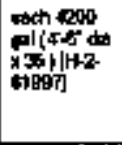 & Mat & Soind & throbuth & Yos & m & 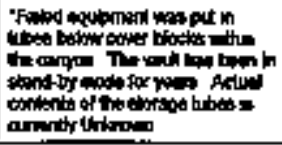 \\
\hline 4 & 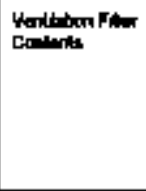 & 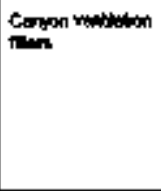 & Wh & Wh & $\mathrm{NA}$ & RAmato & Unkhown" & Yes & $\mathbf{N A}$ & 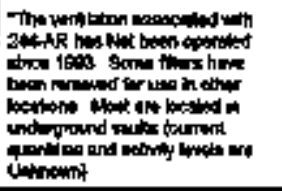 \\
\hline A & 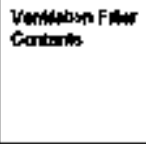 & 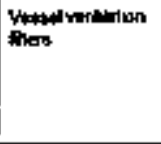 & $\mathbf{m h}$ & $\mathbf{m}$ & Nh & Fentitue & Lntvemir & T\& & man & 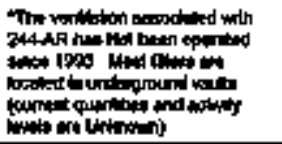 \\
\hline AR2014 & 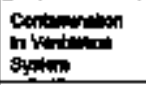 & Ox-4ingunts & $\mathbf{m}$ & NA & Nha & NA & NA & H & Na & 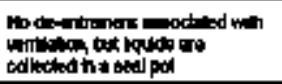 \\
\hline AR-24S & 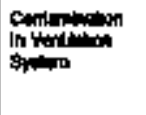 & gndpal & Propos & 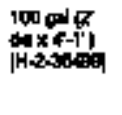 & $w h$ & الهinkل & "أستصا" & No & wh & 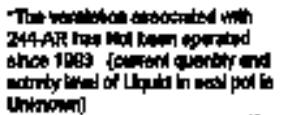 \\
\hline
\end{tabular}




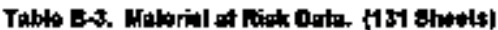

\begin{tabular}{|c|c|c|c|c|c|c|c|c|c|c|}
\hline Find & 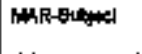 & 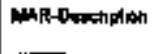 & Mha- & Gound & 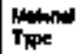 & 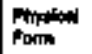 & Powmon कि & Tamient & Bunits & 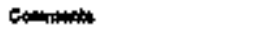 \\
\hline in & 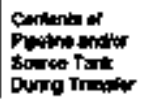 & min & wh & Nh & an & NA & an & $\mathbf{m a n}$ & $\mathbf{m a n}$ & 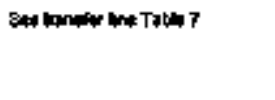 \\
\hline $10: 244$ & 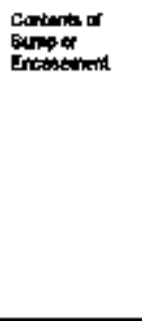 & Vent oen whin & $\mathrm{MH}$ & 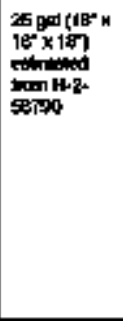 & NA & Lquat & 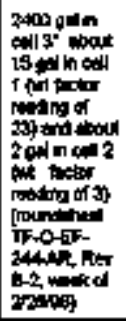 & No & 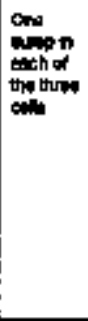 & 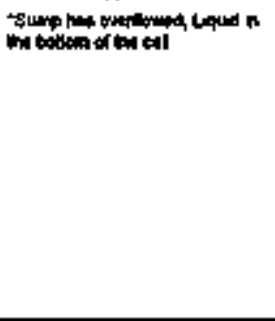 \\
\hline Nats & $\begin{array}{l}\text { Condinintbon } \\
\text { in Atar Bar }\end{array}$ & Whan of & Rended & 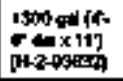 & Nh & Geostat & BA & مa & NA & 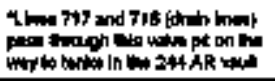 \\
\hline Nin-st & 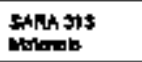 & Rlow" & Nh & MA & Nh & Wh & HA & Wh & Wh & Oned hed Rtabdedn \\
\hline Ma-244 & Othes & Niceng & WA & un & NHA & MA & MA & in & MA & Wh \\
\hline $\begin{array}{l}2+1+9 k- \\
161^{*}\end{array}$ & Touchotute & WA & Wh & mh & Nh & wh & BA & Wh & Nh & 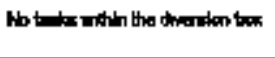 \\
\hline $\begin{array}{l}241+4 R \\
+31^{*}\end{array}$ & This & NA & NA & ma & NA & 的 & Wh & ant & NA & 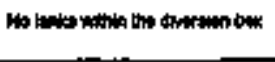 \\
\hline $\begin{array}{l}241+46 . \\
151^{\circ}\end{array}$ & tolar Truck & Here & wh & sh & Wh & uh & wh & $\mathbf{m}$ & HA & 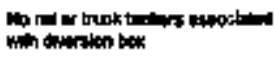 \\
\hline
\end{tabular}




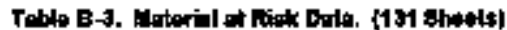

\begin{tabular}{|c|c|c|c|c|c|c|c|c|c|c|}
\hline rivine & MhR-gabjagl & 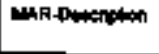 & $\begin{array}{l}\text { WAFt- } \\
\text { CBr. }\end{array}$ & 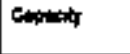 & 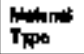 & $\begin{array}{l}\text { Phutilow } \\
\text { Fomm }\end{array}$ & volum of & Temanatex & curailty & Comats \\
\hline $\begin{array}{l}241+4 R-2 \\
131^{*}\end{array}$ & $\begin{array}{l}\text { Cont ringan } \\
\text { en trinuelo }\end{array}$ & None & NAR & Nh & $\mathbf{m}$ & Nh & nid & MA & Wh & No cortiminimow wistod \\
\hline 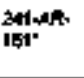 & 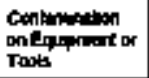 & $\operatorname{Non}$ & N & $\mathrm{Man}$ & NA & Noh & $m$ & $m$ & $\mathbf{N o n}$ & 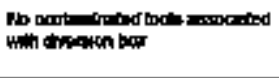 \\
\hline $\begin{array}{l}\text { X4h-AR- } \\
\text { 15I" }\end{array}$ & Vintion $F$ & PAme & WA. & M & NA & NA & $m$ & $\mathbf{m h}$ & NAS & 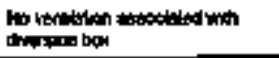 \\
\hline $\begin{array}{l}74+4 R \text {. } \\
151^{4}\end{array}$ & 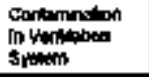 & Detstram & $\mathbf{u n}$ & Ma & MA & wa & bh & $\mathbf{m}$ & Wh & 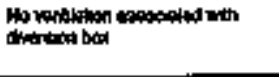 \\
\hline $\begin{array}{l}241 \text { HA. } \\
151^{*}\end{array}$ & 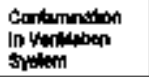 & stal hop & $\mathbf{H}$ & xas & Mar & hil & Mh & an & Nh & 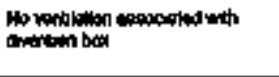 \\
\hline 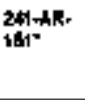 & 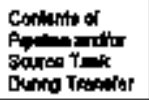 & Wh & Hh & $\mathbf{M r}$ & Nh & 篮 & HA & Mat & Nh & sentreter the Tulto 7 \\
\hline $\begin{array}{l}\text { 2414AR. } \\
151^{*}\end{array}$ & 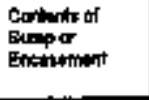 & Norte & Mm & MA & $\mathbf{m}$ & in & NA & $\mathbf{m a n}$ & un & 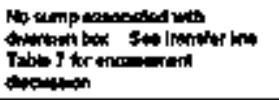 \\
\hline $\begin{array}{l}341-A R-2 \\
1 \leqslant 1\end{array}$ & 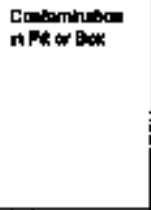 & Dovempan & Raratill & 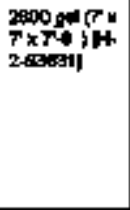 & Nh & Papidue" & nen & $N_{\phi}$ & Nh & 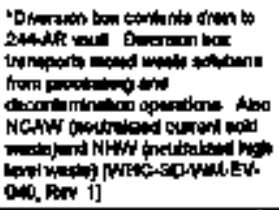 \\
\hline $\begin{array}{l}241-A R- \\
15 y^{*}\end{array}$ & gARR 919 & Nann & MA & Mh & H & $\mathbf{m}$ & MA & Ma & $\mathbf{m e t}$ & Na \\
\hline
\end{tabular}




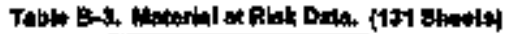

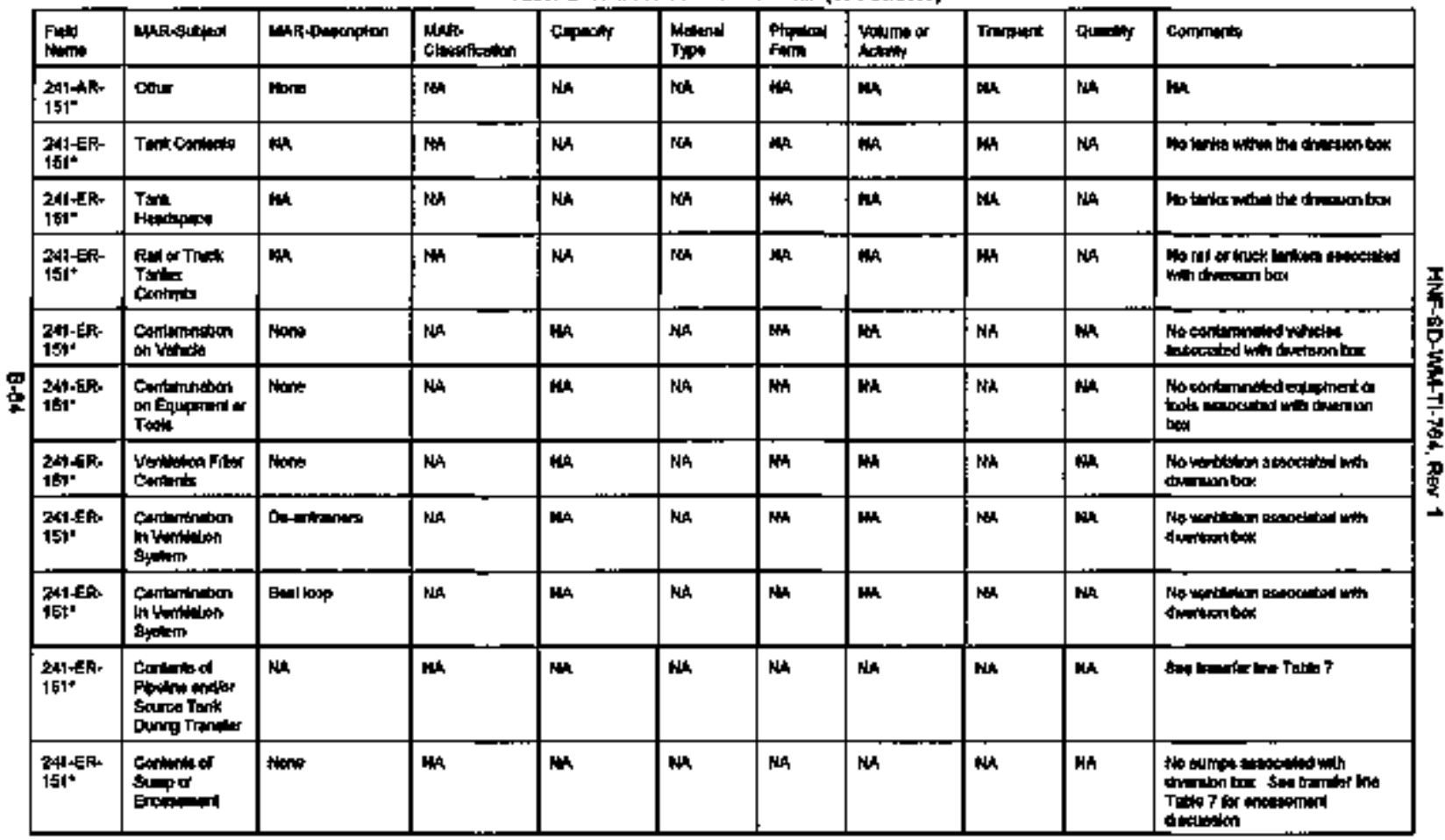




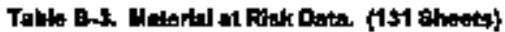

\begin{tabular}{|c|c|c|c|c|c|c|c|c|c|c|}
\hline Pand & Mafisubyed & MAR-0Ecripton & chisir & Copery & $\begin{array}{l}\text { rybal } \\
\text { Then }\end{array}$ & $\begin{array}{l}\text { Pryoural } \\
\text { Fomm }\end{array}$ & $\begin{array}{l}\text { Wothme or } \\
\text { inglenty }\end{array}$ & Trument & Olvatby & Cammats \\
\hline 161-5i- & $\begin{array}{l}\text { Continhatan } \\
\text { n RA कo Box }\end{array}$ & Whets Py & Rosmon & 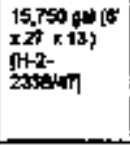 & $\mathbf{M h}$ & Rotuder & Nh & 16 & NA & 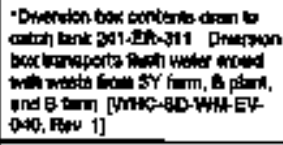 \\
\hline $\begin{array}{l}\text { 74-ER- } \\
151\end{array}$ & 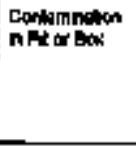 & $P \& F_{1}$ & Rectur & 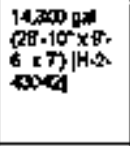 & wh & Rिका中国" & MA & a & NA & 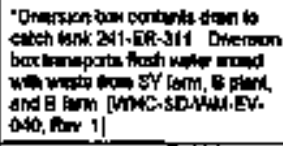 \\
\hline $\begin{array}{l}\text { 241-ER } \\
151\end{array}$ & 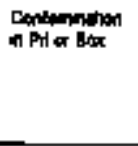 & 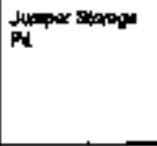 & Rescon & 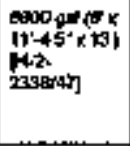 & NA & "مtace" & MA & 16.6 & $\mathrm{Hh}$ & 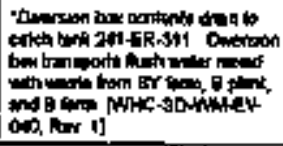 \\
\hline $\begin{array}{l}\text { श्याही } \\
1510\end{array}$ & GARA3 & Nome & Wh & ah & NRA & Nh & in & $\mathbf{m}$ & $\mathrm{m}$ & MA \\
\hline $\begin{array}{l}241-6 h \\
161^{*}\end{array}$ & Othe & None & MH & wh & $\mathrm{NA}$ & 粗 & Hh & nes & Wh & Nh \\
\hline $\begin{array}{l}\text { 2H1EA } \\
159\end{array}$ & TaukCGontiola & $\mathbf{m}$ & Wh & $\mathbf{m x}$ & NA & $\mathbf{N h}$ & $\operatorname{an}$ & $\mathbf{m}$ & $\mathbf{m}$ & 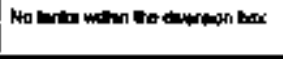 \\
\hline 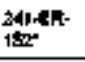 & Thex & ind & Nh & $\mathbf{m}$ & non & H & Wh & in & $\mathbf{H}$ & 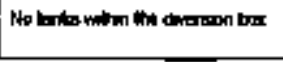 \\
\hline $\begin{array}{l}\text { P4LER } \\
152^{\circ}\end{array}$ & Pred Trukk & Nonte & Nh & Wh & MN & NA & Wh & wh & 倍 & 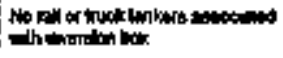 \\
\hline $\begin{array}{l}241-E R- \\
150^{+}\end{array}$ & conimination & $\ln$ & Wh & MA & Wh & mh & wh & NA & Mh & 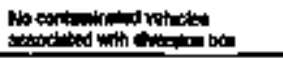 \\
\hline
\end{tabular}




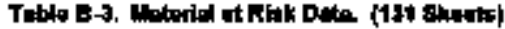

\begin{tabular}{|c|c|c|c|c|c|c|c|c|c|c|}
\hline Pont & HAR-\$ybyed & MAR-D therem & Cuti- & 16 & Typer & Phingal & tollining on & Trumintint & oustor & Comintinte \\
\hline $\begin{array}{l}\text { 2N1-EF- } \\
\text { ISt2 }\end{array}$ & 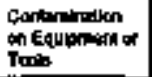 & thane & NA & NA & $\mathbf{m a n}$ & $\mathbf{m}$ & NA & NA & $\mathbf{m}$ & 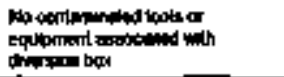 \\
\hline $\begin{array}{l}201+\mathrm{EA} \\
152^{\circ}\end{array}$ & 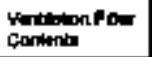 & Nome & NA & NaA & $\mathbf{m}$ & $\mathbf{m}$ & $\mathrm{Nan}$ & NA & $\mathbf{m}$ & 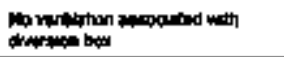 \\
\hline 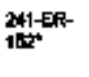 & 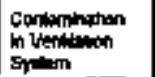 & 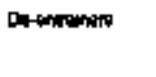 & NA & NA & $\mathbf{m}$ & $m$ & MA & NA & kh & 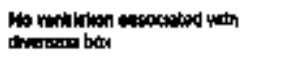 \\
\hline 241-日R- & 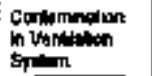 & Ent loos & MA & Na & $\mathbf{m}$ & $\mathbf{m}$ & MA & NA & HA & 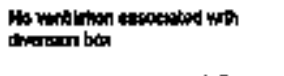 \\
\hline $\begin{array}{l}2+1-E R-1 \\
132\end{array}$ & 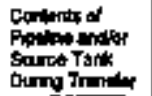 & M & NA & NA & MH & $\mathbf{m}$ & NA & ma & MA & 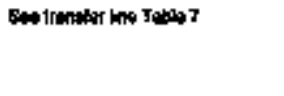 \\
\hline 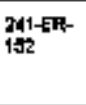 & 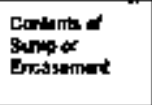 & 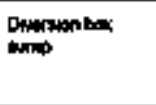 & $\begin{array}{l}\text { Redital } \\
\text { Continimition }\end{array}$ & 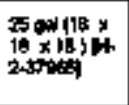 & 幽 & คrosuch & Na & $\mathrm{No}$ & NA & 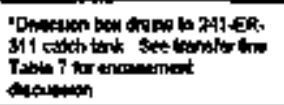 \\
\hline $\begin{array}{l}241-e R \\
100\end{array}$ & hPt on Bom & Dimatist & 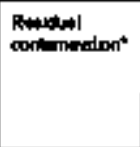 & 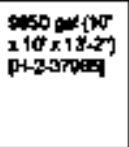 & 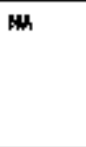 & Rualdor & Ma & No & NA & 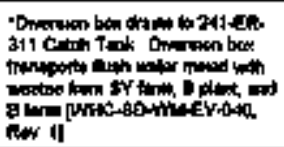 \\
\hline 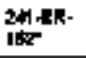 & AARs 10 & None & $\mathbf{m}$ & $m$ & NA & NA & mak & $m$ & Nat & NH \\
\hline $\begin{array}{l}2+1+6 A^{2} \\
150^{\circ}\end{array}$ & other & Fon & $\mathbf{m}$ & $\mathbf{m}$ & NA & Na & m & $\mathbf{m}$ & Wh & NA \\
\hline 2W15. & Tankcosints & m & $\boldsymbol{\omega}$ & $\mathbf{m k}$ & Nath & Non & $\mathbf{m}$ & ath & Nh & 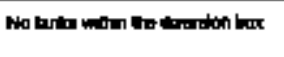 \\
\hline
\end{tabular}




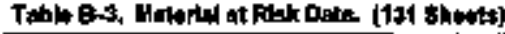

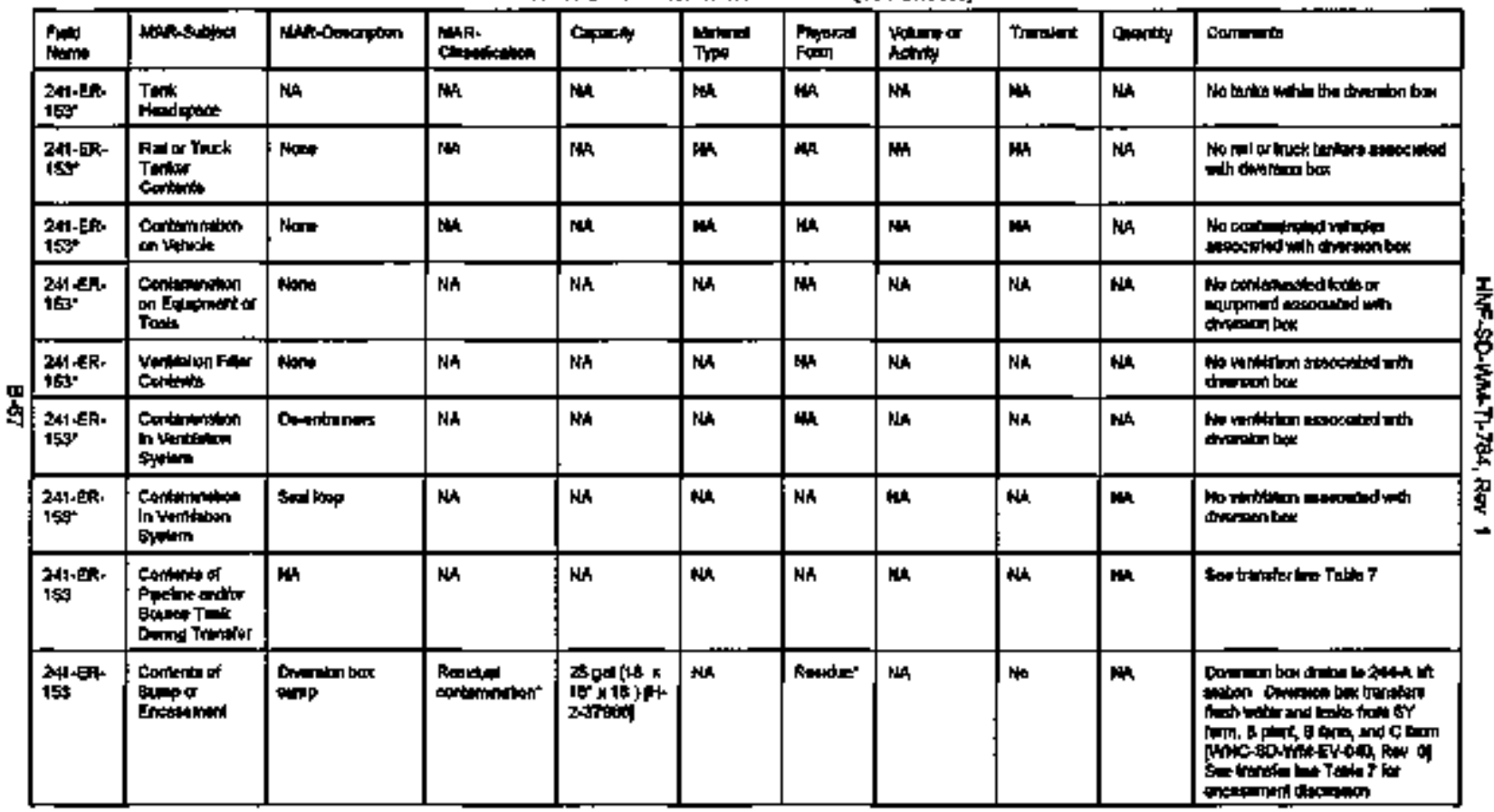




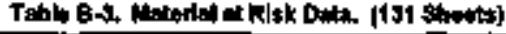

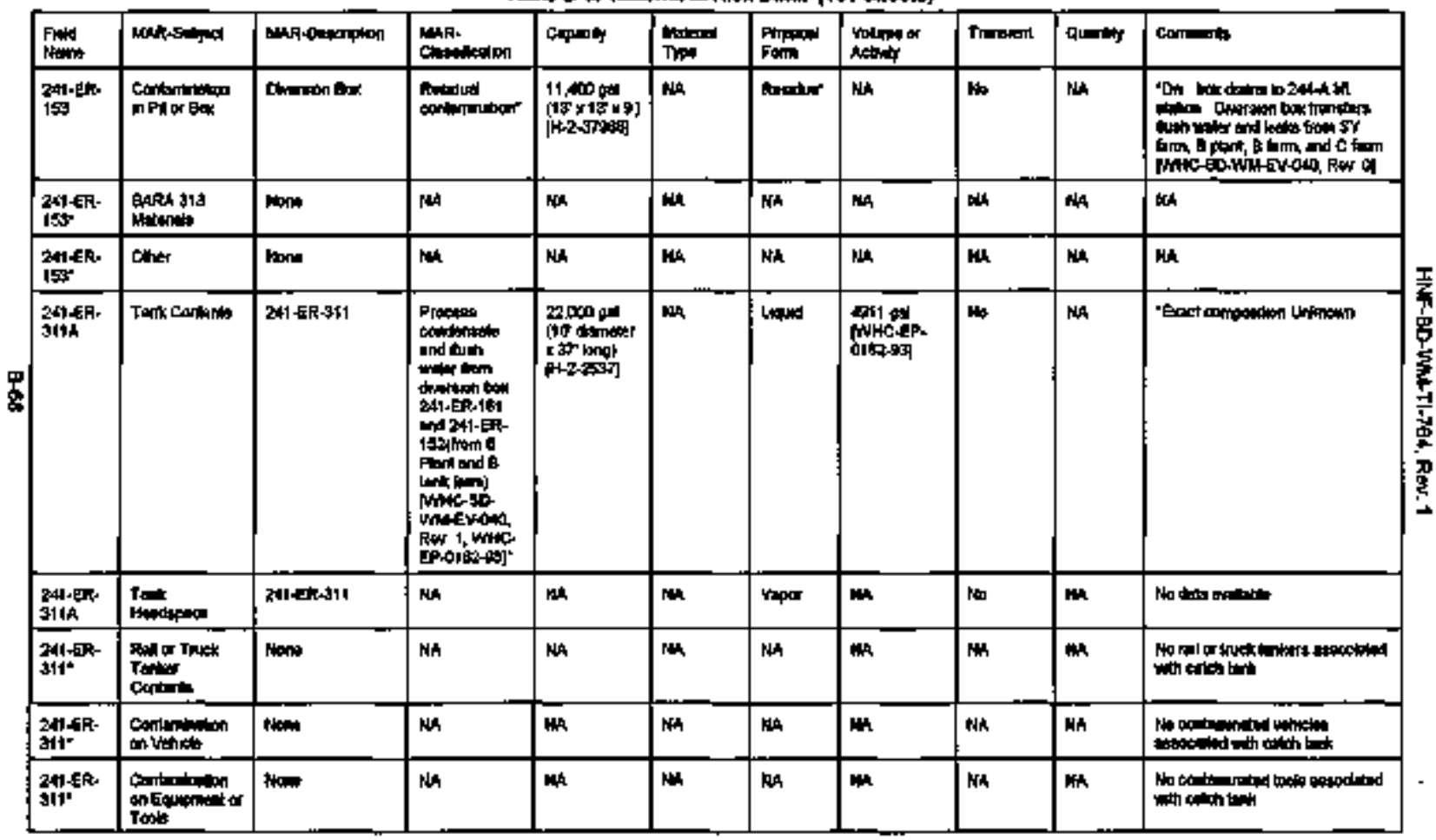




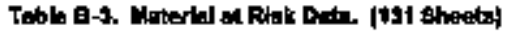

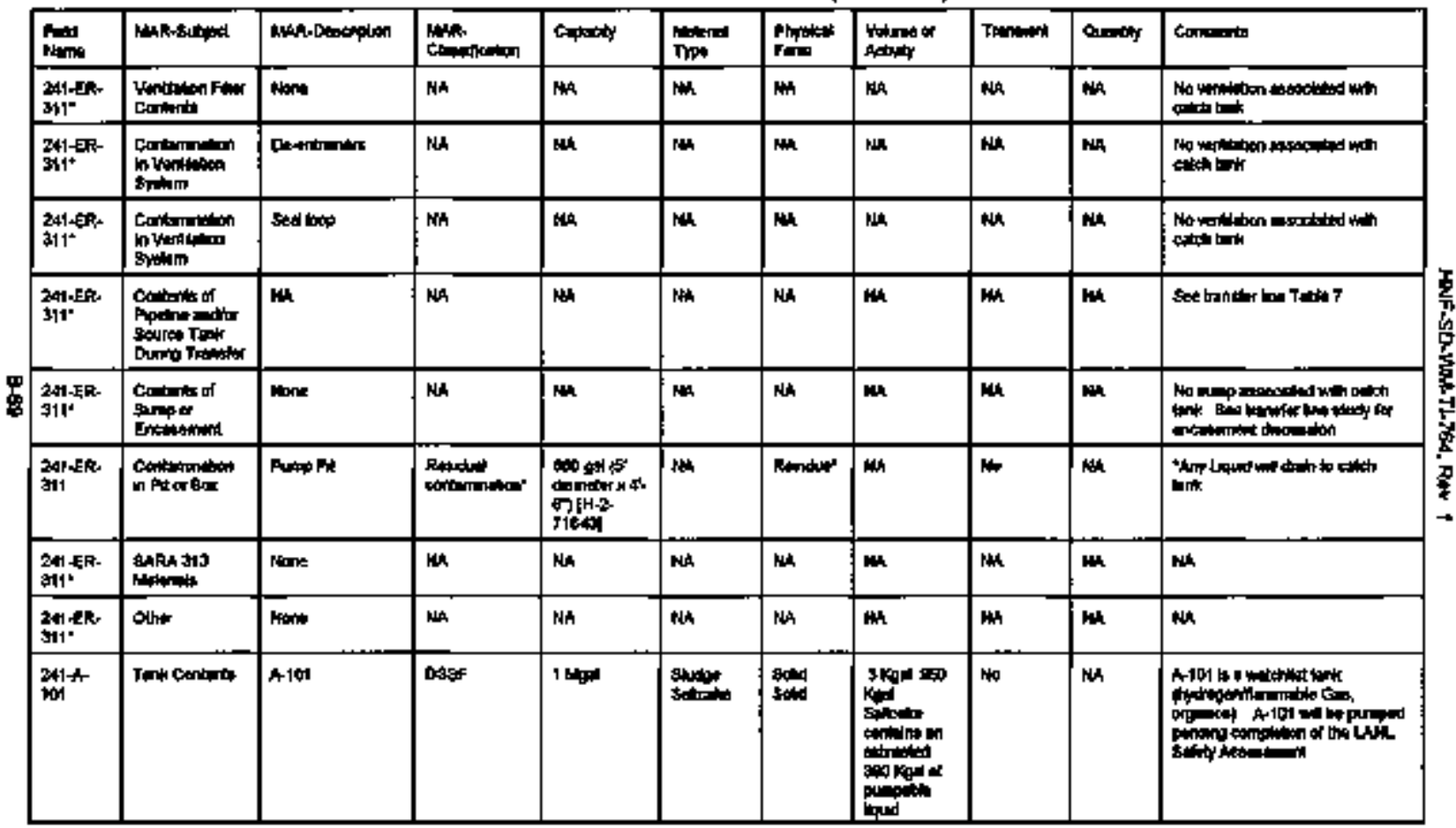




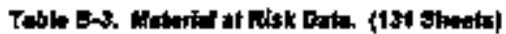

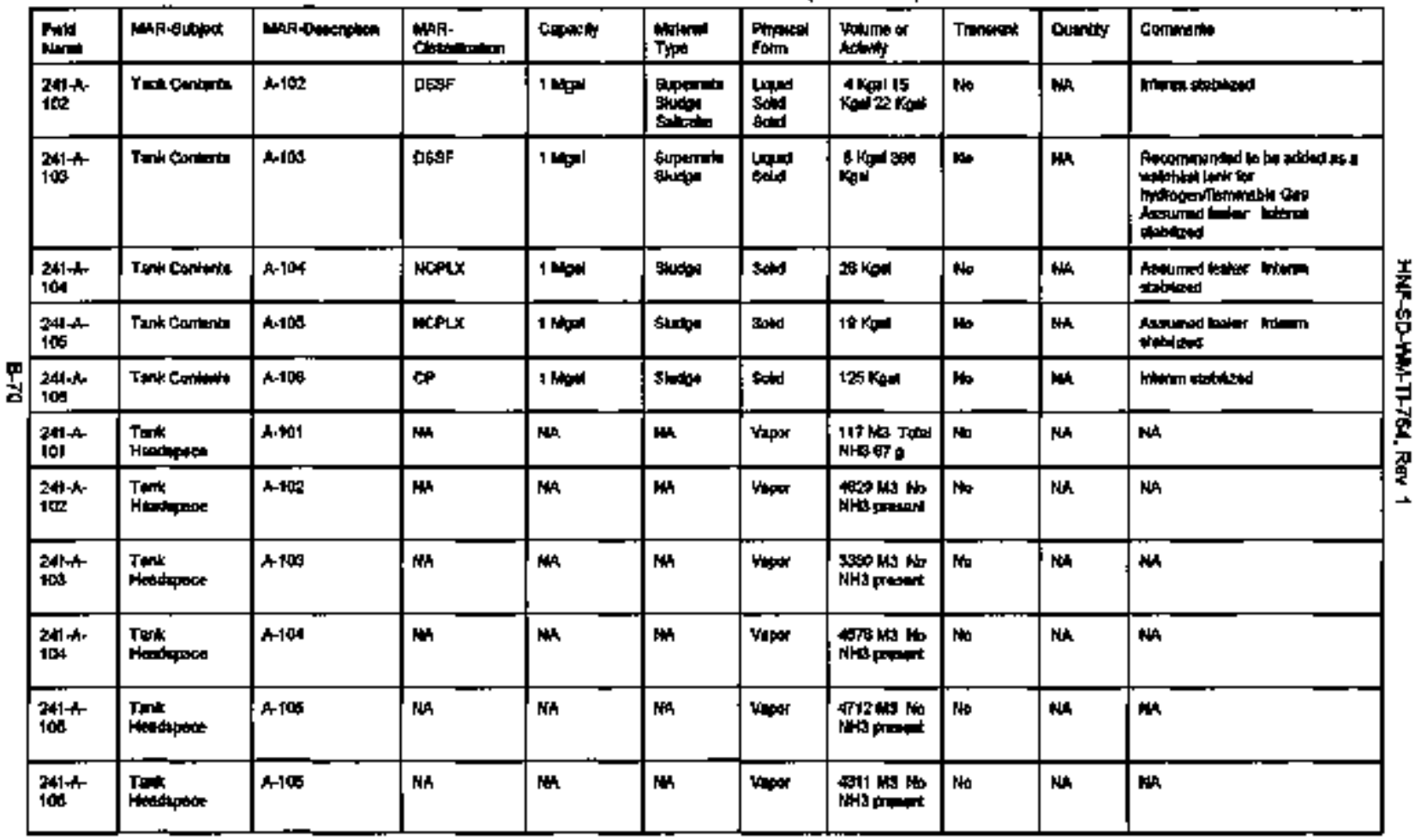




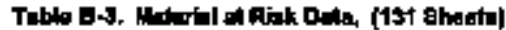

\begin{tabular}{|c|c|c|c|c|c|c|c|c|c|c|}
\hline Thend & 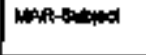 & MHR-Dutenguth & 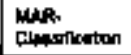 & cespertr & Type & $\begin{array}{l}\text { Phytors } \\
\text { Ramp }\end{array}$ & $\begin{array}{l}\text { Wotiride of } \\
\text { Antioty }\end{array}$ & Thanthe & Ouands & connutits \\
\hline $2 \cdot 1 A^{2}$ & 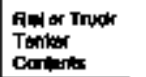 & mh & M & NA & NA & MA & Hah & Nh & $\mathbf{m a t}$ & 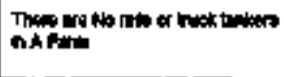 \\
\hline 2র1A & $\begin{array}{l}\text { Combinmenn } \\
\text { an Vingle }\end{array}$ & MA & M & NA & NA & WA & EA & Nh & MA & There ard ho Yotioles \\
\hline $2 \cdot 1 \phi^{+}$ & 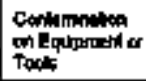 & $\mathbf{W}$ & Nd & MA & MA & NA & $\mathbf{m}$ & Non & NA & 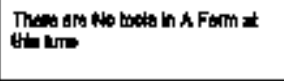 \\
\hline 2A1 & 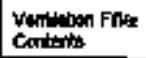 & Pritar & Mn & $M$ & NA & HA & $\mathbf{k H}$ & Fin & m & Nh \\
\hline $201 A^{*}$ & $\begin{array}{l}\text { Whition Fan } \\
\text { Contions }\end{array}$ & Mritus & xA & Nh & NA & un & NA & hy & $\mathbf{m}$ & 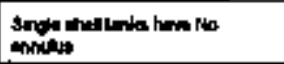 \\
\hline $2011 \hat{A}^{\prime}$ & 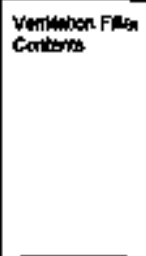 & Busslinar & $\mathrm{KAs}$ & $\begin{array}{l}\text { I Ruthr ad ax } \\
\text { nethes. }\end{array}$ & MA & Pantintatst & 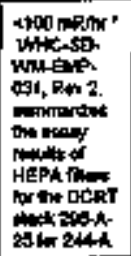 & 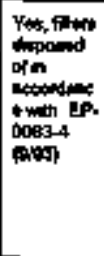 & wh & 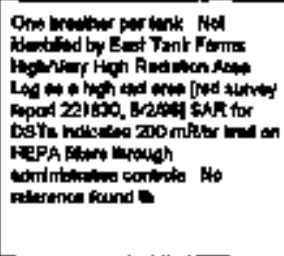 \\
\hline $241+4$ & 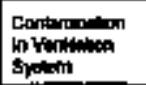 & De-entunen & Procias & ant & Nh & لهiqu & Requin & an & Nan & m \\
\hline $241-4$ & 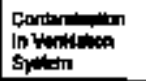 & קוp & and tant & ה & NA & |diplat & Rapulat & an & Nh & 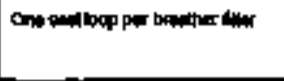 \\
\hline $241+4$ & Çontipin of & NA & MA & wh & NA & $\sin$ & Nh & m & MH & 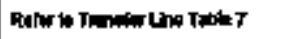 \\
\hline
\end{tabular}




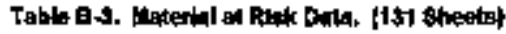

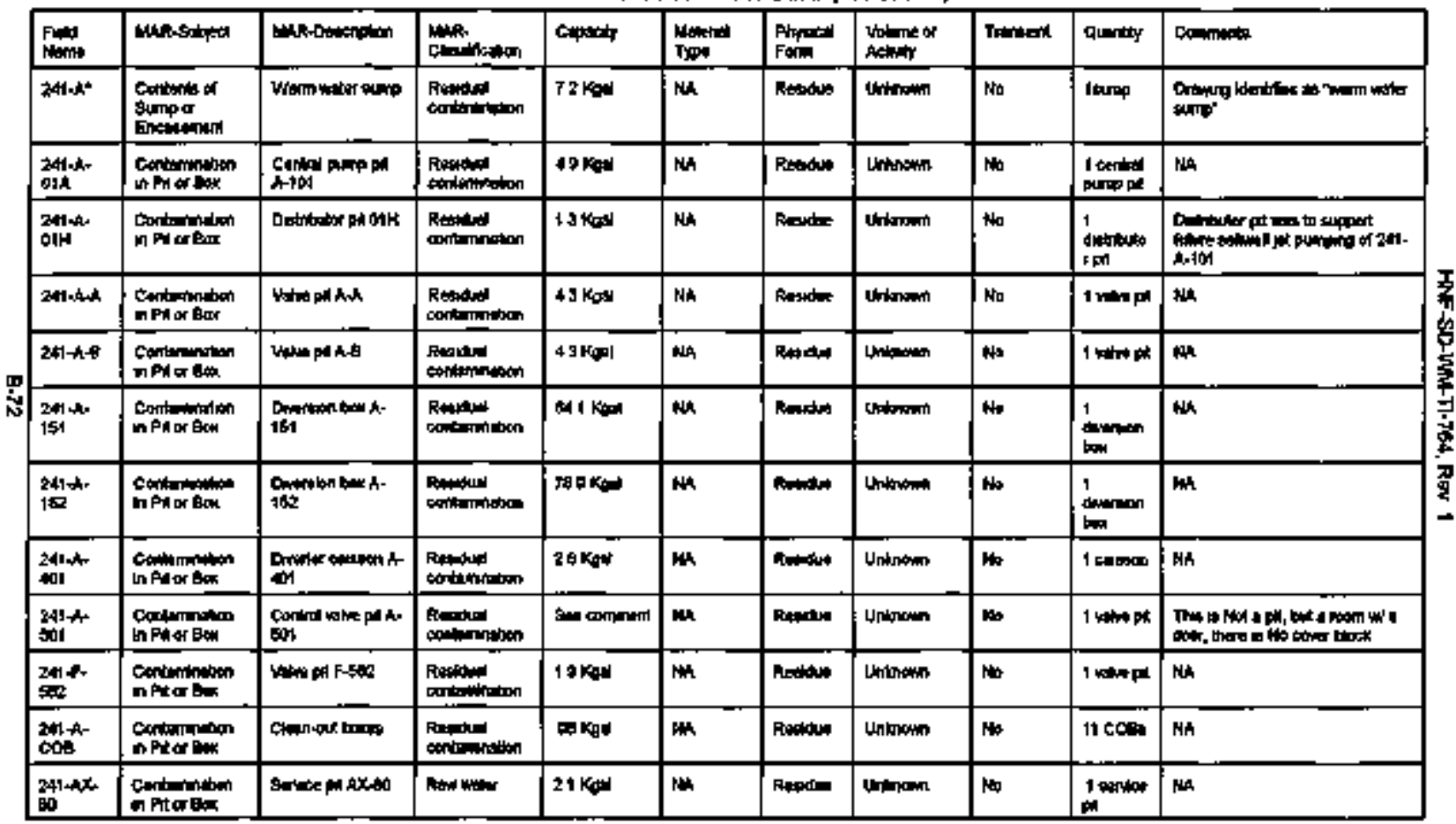




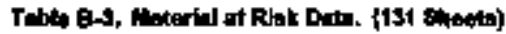

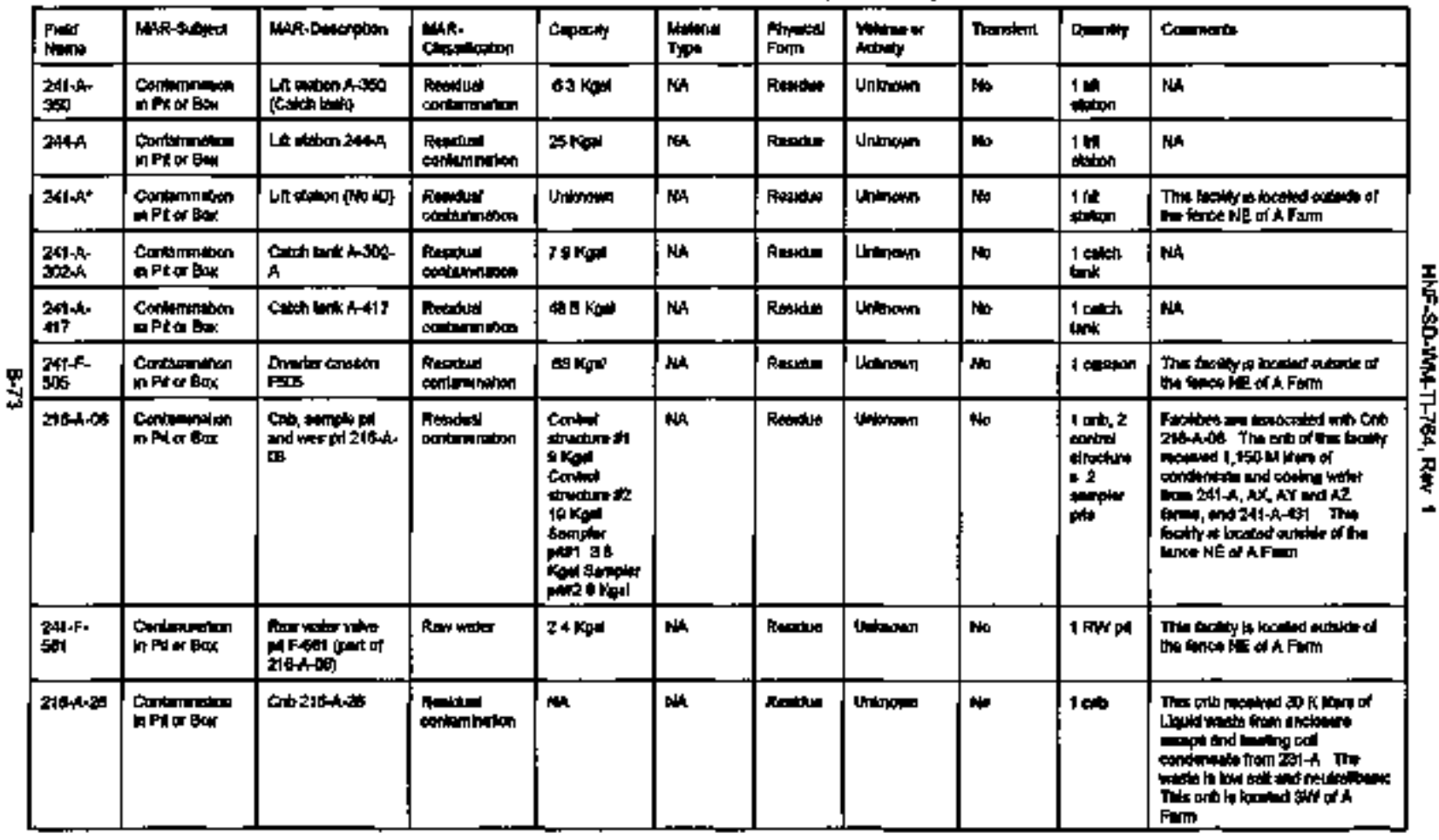


Table B-s. Material at Riak Oath, \{131 shoets\}

\begin{tabular}{|c|c|c|c|c|c|c|c|c|c|c|}
\hline$\underset{M=10}{\text { Pand }}$ & Whestopact & Muts.0. & $\begin{array}{l}\text { ann. } \\
\text { claninoston }\end{array}$ & capory & $\begin{array}{l}\text { Minita } \\
\text { Typ }\end{array}$ & $\begin{array}{l}\text { Phare } \\
\text { form }\end{array}$ & votume of & Tramed & cuanty & Earintita \\
\hline 216 的 & 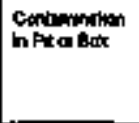 & ab 216 An & 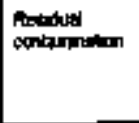 & NA & NA & fituda & 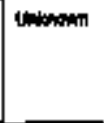 & $\omega$ & $1 \mathrm{enb}$ & 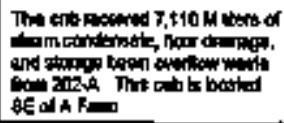 \\
\hline 2104+4 & $\begin{array}{l}\text { Cantatridon } \\
\text { in Rtar Bar }\end{array}$ & tht 21 thentath & frepiud & $\mathrm{BH}$ & MH & ficturatu & Uhtisten & bo & 1 chb & 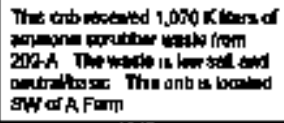 \\
\hline$\underset{306}{210}$ & 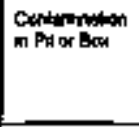 & ab 216-4nas & Peondust & $\mathbf{M H}$ & NA & fiendet & 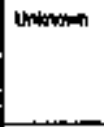 & mo & 1 eto & 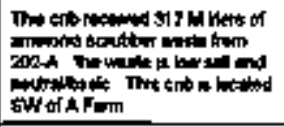 \\
\hline $218-1$ & 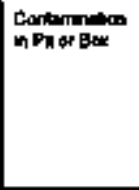 & Cribstan-34 & Ropulat & Nh & m. & Ravale & Uninoun & tho & 1 arth & 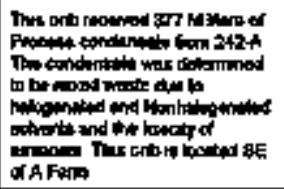 \\
\hline $\begin{array}{l}210-2 \\
37-2\end{array}$ & $\begin{array}{l}\text { Contuminaban } \\
\text { n Pla be }\end{array}$ & cht210-5?-2 & Rosing & NA & $\mathbf{M h}$ & Routur & Unifonom & $\mathbf{H o}$ & $1 \leqslant 6$ & 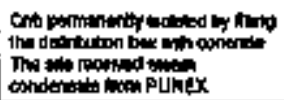 \\
\hline 210 & $\begin{array}{l}\text { Coitsmiban } \\
\text { hPAor Bas }\end{array}$ & chb 210-4-10 & Faind & $\mathrm{Nh}$ & $\mathbf{k}$ & Feanter & Unthom & $\mathbf{N o}$ & 1 thb & 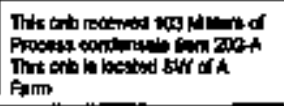 \\
\hline $244 k^{*}$ & saph 313 & Chin low for & $10 \times$ inminal & $\Leftrightarrow$ at betil & Wh & aes & NA & ve & 2 botion & 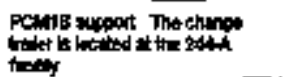 \\
\hline
\end{tabular}




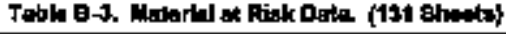

\begin{tabular}{|c|c|c|c|c|c|c|c|c|c|c|}
\hline Find & WhRspling & Hiktouspoin & Ming & capusty & 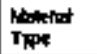 & $\begin{array}{l}\text { Prumica } \\
\text { Kam }\end{array}$ & Volnum of & Tromand & coninity & Cemantios \\
\hline $241+t^{*}$ & $\begin{array}{l}\text { FARA 13 } \\
\text { Htats }\end{array}$ & 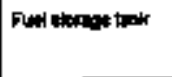 & Lhinawn & vmenn & ren & Mingen & | & 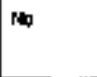 & Unmonnt & 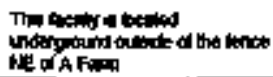 \\
\hline 241भ" & Othe & Fandoup & S4 coprmint & MA & Eximitith & Bowd & 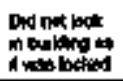 & Yel & Uitoman & 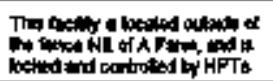 \\
\hline $241+4$ & Dthe & conderen & low & Wh & $\mathbf{r a n}$ & sold & tritst & bo & $\begin{array}{l}\text { lonkind } \\
\text { oid } \\
\text { onduntes } \\
\text { n }\end{array}$ & 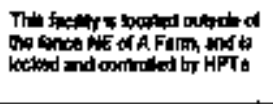 \\
\hline $241 \mathbf{N}^{*}$ & olne & bural bormed & 的 & $\mathbf{~ H h}$ & NA & sold & Low tard & Yex & 2 howes & an \\
\hline 2402 & Olhe & 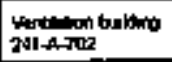 & Low low & Anh & Nin & frestue & Low hind & No & 1 buldng & $\mathbf{m h}$ \\
\hline $2+1-k^{*}$ & arr & $\begin{array}{l}\text { len mabonga } \\
\text { fitivy }\end{array}$ & 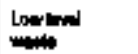 & MA & Nh & Baratu & لمew & No & I traing & Inachan \\
\hline 241. & onr & 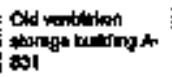 & 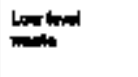 & NA & Nh & hrodve & Low lond & $* \$$ & 1 buiding & BAS \\
\hline 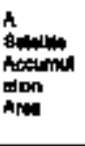 & Char & 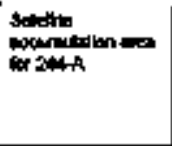 & $\begin{array}{l}\text { waterntod } \\
\text { mente }\end{array}$ & 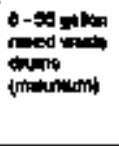 & 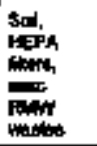 & toda & 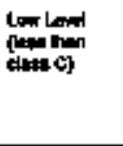 & $Y *$ & 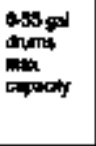 & $\mathrm{MH}$ \\
\hline $2 \boldsymbol{H}+\boldsymbol{A}^{*}$ & Cotar & on & Comprobos of & 2 ants & Hoth & Itapin & 2 pasts & Yas & $\begin{array}{l}1 \\
\text { eqnangmang }\end{array}$ & \\
\hline
\end{tabular}




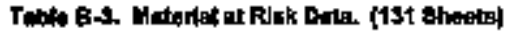

\begin{tabular}{|c|c|c|c|c|c|c|c|c|c|c|}
\hline Fimed & 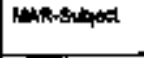 & 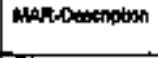 & Whis. & ceptity & 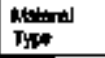 & $\begin{array}{l}\text { Phytin } \\
\text { Form }\end{array}$ & $\begin{array}{l}\text { Wolume or } \\
\text { tultisty }\end{array}$ & Trantert & onting & corimetime \\
\hline 2N14 & ons & 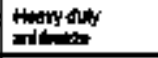 & 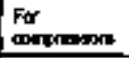 & 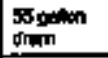 & Na & How & Sortdoun & Yes & Tdum & 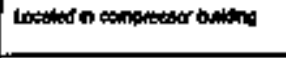 \\
\hline $241-4012$ & Tank Domberth & Abastó & DN & 1,164 LF & apariat & Lqund & $1000 \mathrm{Kg}$ & Mo & UA & 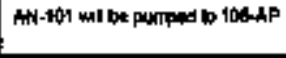 \\
\hline 2418tat & Tenk Conlinoto & AH 162 & $\mathrm{cc}$ & 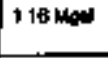 & saperids & Lapd & $\begin{array}{l}503 \mathrm{Kad} \\
\mathrm{KOA}\end{array}$ & $\mathrm{Mo}$ & MA & $\mathbf{m}$ \\
\hline 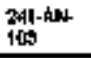 & Tamt Canterts & ANtics & 口的 & 118 4p & Cos & tont & ost kged & in & NA & NA \\
\hline $\begin{array}{l}241 \cdot k 6 t \\
104\end{array}$ & Tank Contarto & $10+104$ & Dssp & 116 log & shomy & Lon & now kpot & $* 0$ & Nh & HA \\
\hline $\begin{array}{l}2+1 \text { Ave } \\
105\end{array}$ & Tortcortents & AN-10S & toga & 1 18450 & $\sin \gamma$ & L्qud & 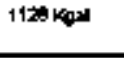 & mb & MA & NA \\
\hline $\begin{array}{l}241 \text { thy } \\
105\end{array}$ & 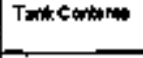 & Ak-108 & DS\$P & 116 wbol & $\begin{array}{l}\text { supertht } \\
\text { soled }\end{array}$ & howd & 1700 & No & $\mathrm{NA}$ & Nh \\
\hline $\begin{array}{l}241+\mathrm{AN} \\
10 \mathrm{H}\end{array}$ & Tatcituth & NN-107 & sc & 1 1日明和 & $\begin{array}{l}\text { Exporit. } \\
\text { stid }\end{array}$ & $\begin{array}{l}\text { Loped } \\
\text { Cold }\end{array}$ & 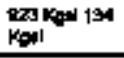 & Wb & NA & NA \\
\hline $24+44 t$ & 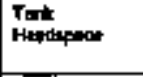 & $A N-1 O M$ & in & Nh & wh & Maper & 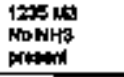 & wo & Nh & Na \\
\hline $\begin{array}{l}241+81 t \\
102\end{array}$ & Tah & $A N+102$ & $N$ & Wh & roh & V) & 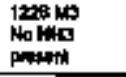 & $\omega$ & Wh & $\mathrm{m}$ \\
\hline $\begin{array}{l}241+1 \mathrm{~N}- \\
103\end{array}$ & Iothe & 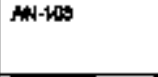 & $\mathrm{W}$ & $\mathrm{HA}$ & $\mathbf{m h}$ & Vhaps & 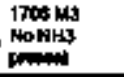 & 16 & in & $\mathrm{NH}$ \\
\hline 2019404 & Tank & $4+104$ & $\omega$ & ne & Nan & 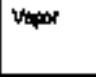 & 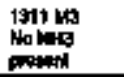 & in & $\mathbf{n k}$ & man \\
\hline
\end{tabular}




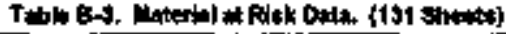

\begin{tabular}{|c|c|c|c|c|c|c|c|c|c|c|}
\hline Find & Muthothet & $M A F \cdot 0$ matim & $\begin{array}{l}\text { MuRp } \\
\text { Clatalikerion }\end{array}$ & chengiा & $T_{15}=1$ & $\begin{array}{l}\text { Finatcal } \\
\text { Foim }\end{array}$ & Ylotwn or & Thent & qumbtr & Commintin \\
\hline 100 & Tolil & instos & $\mathrm{ran}$ & Hat & NA & vaptar & 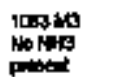 & ath & N & w \\
\hline $\tan _{104}^{204 k}$ & Turl & intos & ind & net & NA & Whor & 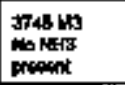 & $\mathbf{m}$ & HA & wh \\
\hline 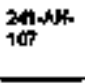 & Treir & AN-10 & Wh & NA & Nh & traper & 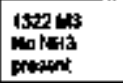 & $\mathrm{No}_{0}$ & Hh & in \\
\hline 24 are & 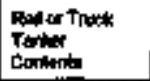 & 새 & NA & NA & NA & Hon & ف & m & NA & 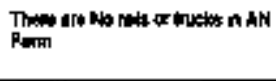 \\
\hline $241+10$ & 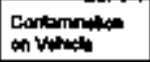 & MA & NA & Nar & wh & WA & mh & Nik & MA & 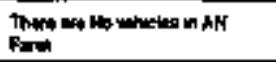 \\
\hline 241 hit* & 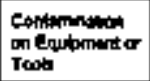 & M & $N A$ & NA & H & NA & $\mathbf{m}$ & $N k$ & in & 缋 \\
\hline $241-H_{N}$ & 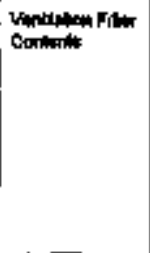 & Pintion & wh & 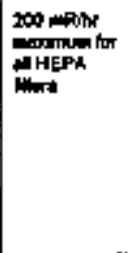 & $\mathbf{m}$ & Periknin & 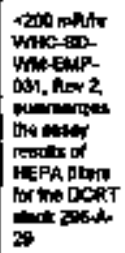 & 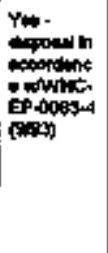 & HA. & 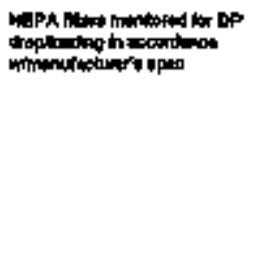 \\
\hline 2Alvkt & 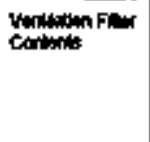 & Anwing & HA & 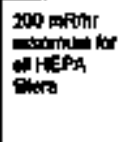 & an & $\begin{array}{l}\text { Patientat } \\
\text { a }\end{array}$ & 200 mithr & 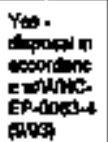 & Nh & 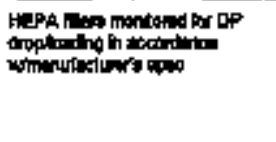 \\
\hline
\end{tabular}




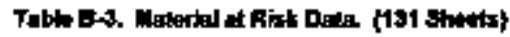

\begin{tabular}{|c|c|c|c|c|c|c|c|c|c|c|}
\hline Pretd & MAR-subjett & 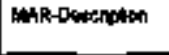 & 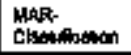 & Cosentr & thend & $\begin{array}{l}\text { Propted } \\
\text { Rom }\end{array}$ & Voinger or & Troneiving & Qminty & Commints \\
\hline $2414 \mathbf{H}^{*}$ & 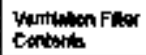 & Brenter & MR & an & NA & NA & NA & NA & KA & 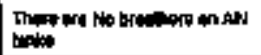 \\
\hline $201+$ Wh & 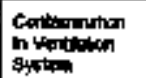 & Datrotis: & Proctst & inth & 새 & 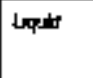 & nemotut & ה & Hh & $\mathrm{MA}$ \\
\hline $241+4 k$ & 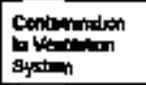 & 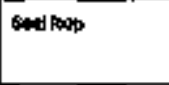 & $\begin{array}{l}\text { Proces } \\
\text { otidehet }\end{array}$ & MA & NA & Unqud & Rectur & Nh & MA & MA \\
\hline 2At-Ak & 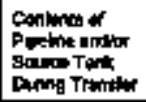 & $\mathbf{M M}_{\mathbf{H}}$ & ANA & MA & AH & nith. & Wh & Nad & MA & 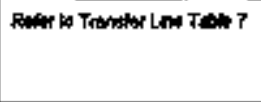 \\
\hline 241 and & 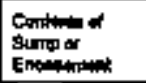 & NA & Nh & Hoh & Wh & Mh. & wh & Wh & Wh & 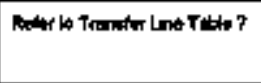 \\
\hline 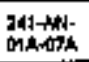 & 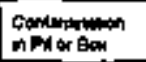 & 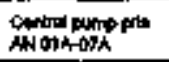 & Findus & 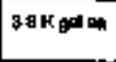 & $\mathbf{m e}$ & Fitmonto & 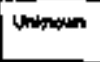 & Ws. & $70 * 4$ & NA \\
\hline $\begin{array}{l}241-4 N- \\
01 B-07 B\end{array}$ & 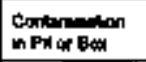 & 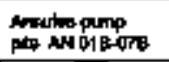 & Potuhd & $17 \mathrm{~K}$ pal $=$ & $\mathbf{m k}$ & Renenden & Uabornen & $\mathbf{H o}$ & $\mathbf{r}_{\mathbf{p}}$ & Wh \\
\hline $\begin{array}{l}24104 k \\
010-0 T c\end{array}$ & 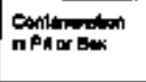 & 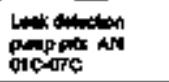 & Ratidun & 17 Ko & in & 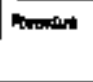 & A. & $\mathbf{W}$ & 7 in & NA \\
\hline 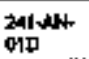 & 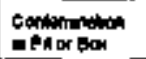 & 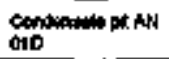 & Ren-dund & gtkous & m & Roduta & iniminim & $\boldsymbol{H}$ & 2 pte & mh \\
\hline 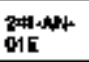 & 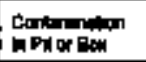 & 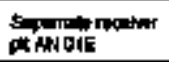 & Alcodus & $14 \mathrm{Kg}$ & $\mathrm{m}$ & Reqpivat & Whonen & $\mathbf{W b}$ & $1 \mathrm{pt}$ & $\mathrm{min}$ \\
\hline 241 aty & 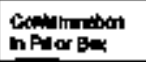 & AM tonk p & Rinutes & $19 \mathrm{~kg}$ & ath & Rasodva & Unthewn & Now & Ipto & 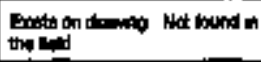 \\
\hline 2il- & $\begin{array}{l}\text { Coghimning } \\
\text { in pilor Bex }\end{array}$ & Yhin PEANA & 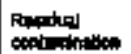 & $54 \mathrm{Kg}$ & mh & 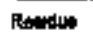 & Uninown & $\boldsymbol{N}$ & Intum pol & ma \\
\hline
\end{tabular}




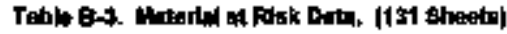

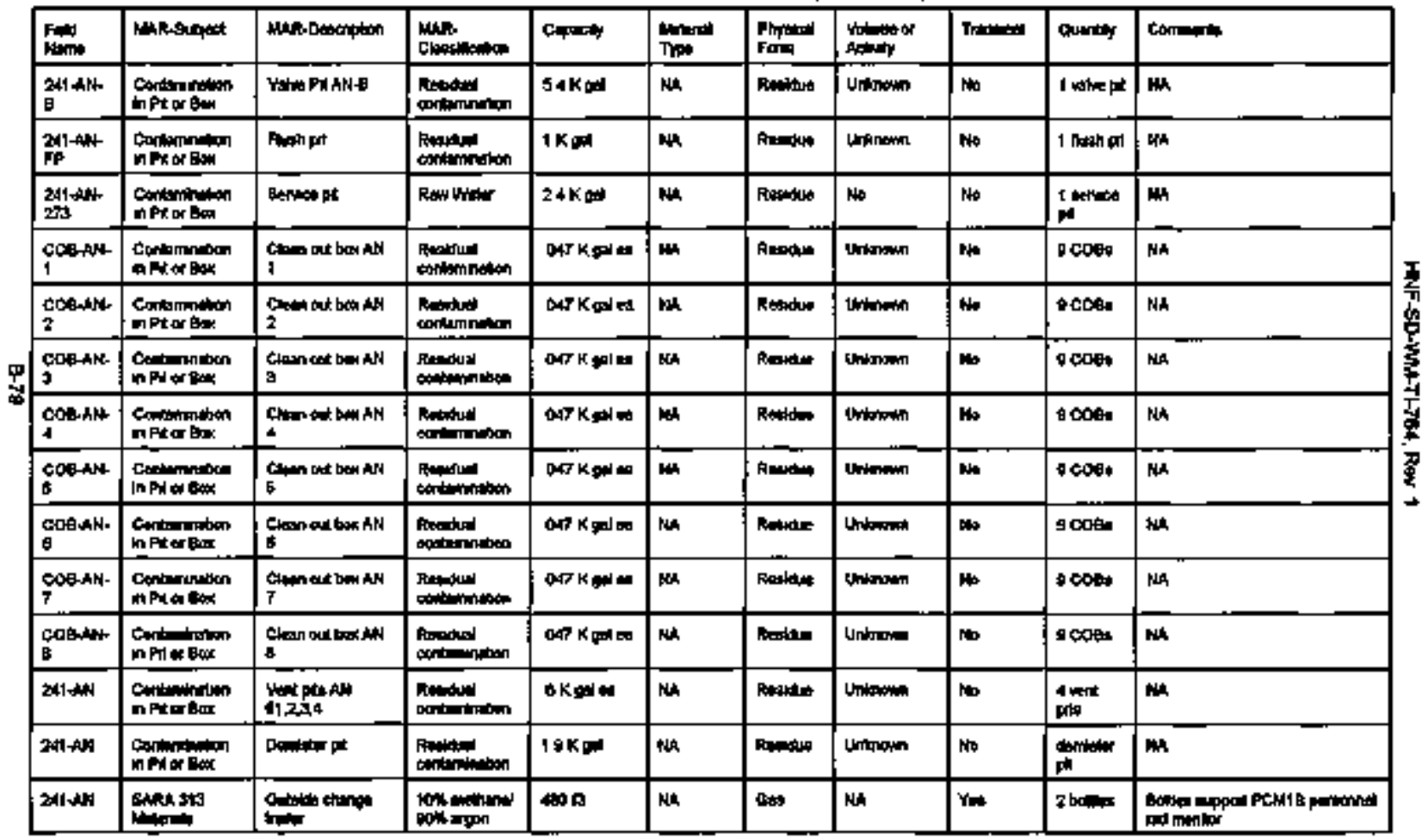




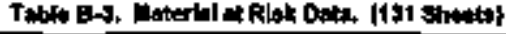

\begin{tabular}{|c|c|c|c|c|c|c|c|c|c|c|}
\hline Fund & MAstiting & 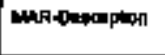 & $\begin{array}{l}\text { Mar. } \\
\text { Chaniletan }\end{array}$ & Cepecty & Wy:man & $\begin{array}{l}\text { Prymin } \\
\text { form }\end{array}$ & 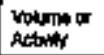 & Traned & Duraty & tomlatiolat \\
\hline 2414k & Cther & 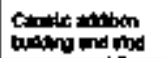 & secommint & 1 une & Nh & in & Nh & $\mathrm{NH}$ & Wh & 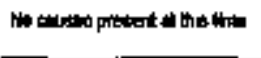 \\
\hline 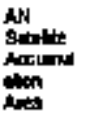 & Othe & Brimb & $\begin{array}{l}\text { RicRih Howd } \\
\text { Whate }\end{array}$ & 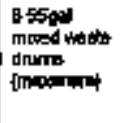 & 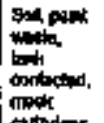 & \$od & 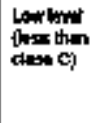 & Yes & 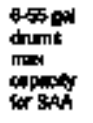 & 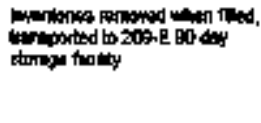 \\
\hline $241+4 \mathrm{~N}$ & otin & Dutdange & Lomind & 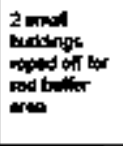 & 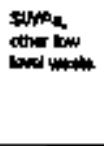 & fold & Low lowel & $Y=$ & Cod Nod & 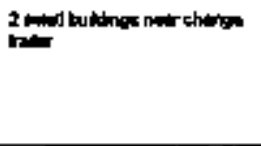 \\
\hline 201 AP, & T:thil Canterina & 241 AP-101 & Destr & 1 10 40:4 & Bapurith & 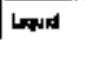 & Fes rod & $\mathbf{N o}$ & 蝁 & N4 \\
\hline tin & Trat:Donimis & 2414AP-102 & EP & 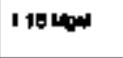 & Suptents & Lopin & $1000 \mathrm{kod}$ & $\mathbf{m}$ & MA & in \\
\hline $\begin{array}{l}241019 \\
103\end{array}$ & Tent Conterime & 241 AP 103 & DN & I to ingen & 9upantw & 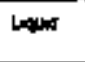 & 2a rpad & $\mathbf{H o}$ & in & mh \\
\hline $\begin{array}{l}241+49 \\
104\end{array}$ & Trank Cuntorits & $241-4 P-104$ & DN & 1 18 knin & Fuperain & Lond & $1129 \mathrm{Kod}$ & No & Wh & $m$ \\
\hline $\begin{array}{l}241+10 \\
105\end{array}$ & Thecorthoth & 2414pido. & c189F & 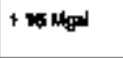 & stitge & fold & $15 \mathrm{~K}$ & bo & Mn & Wh \\
\hline 24104 & TarkConthts & 21149.100 & DN & 1 to lows & Syperiate & Lafus & $10 \%$ & $\mathbf{b}$ & wh & Wh \\
\hline
\end{tabular}




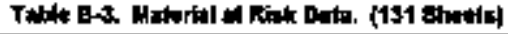

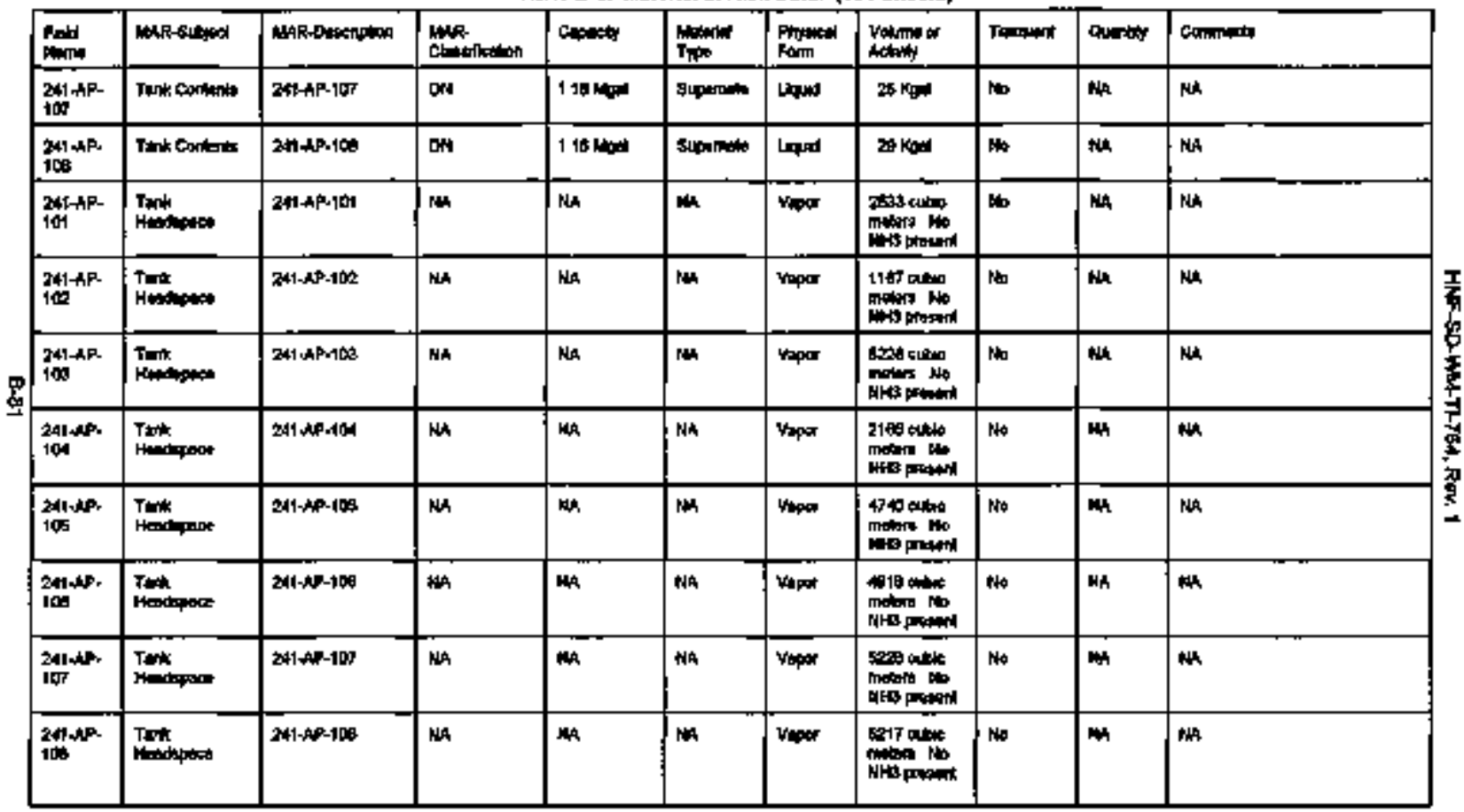




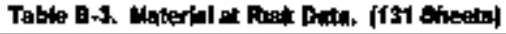

\begin{tabular}{|c|c|c|c|c|c|c|c|c|c|c|}
\hline Fid & MHR-orfictit & 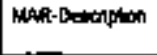 & 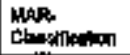 & Coparty & 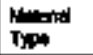 & $\begin{array}{l}\text { Plptitad } \\
\text { Fond }\end{array}$ & Hectumat or & Thandil & Guardity & topmantin \\
\hline 241040 & 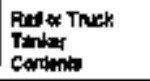 & Kans & NA & NA & $\mathbf{H}$ & Hh & MA & MH & Hh & 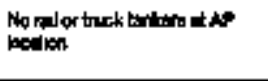 \\
\hline مولها|2ح & 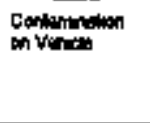 & Conbentind & 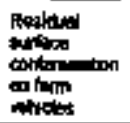 & Rertostal & Rotactual & Mh & Untonewn & $N A$ & Mh & 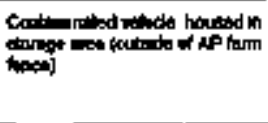 \\
\hline 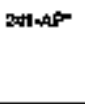 & 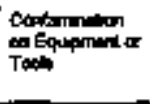 & Norte & Ha & then & Nin & NA & HA & Wh & NA & 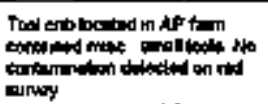 \\
\hline 2A1-AP & 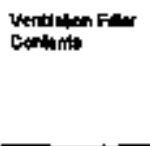 & Prmery & $\mathbf{m}$ & 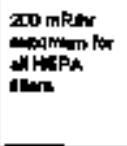 & Nor & Printed & Lothramn & 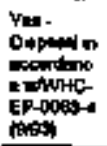 & MA & 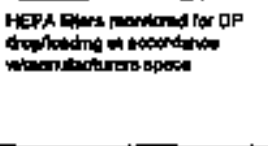 \\
\hline Z414PP & $\begin{array}{l}\text { Veringer fiter } \\
\text { Cootints }\end{array}$ & Annte & vin & 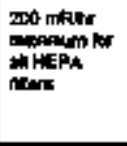 & Nin & Pertalat & Ih & 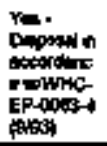 & NA & 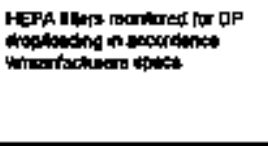 \\
\hline 7서 & 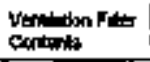 & 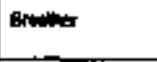 & Mh & wh & Nh & $\mathrm{Hh}$ & 散 & $\mathbf{M h}$ & Nh & $\mathrm{m}$ \\
\hline 2H-APr & 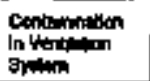 & De-4itbatins & PTectods & Wh & NA & Linua & Reminas & Wh & NA & hin \\
\hline 241.04 & 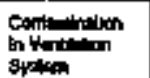 & sallos & Proctit & $\mathbf{~ H h}$ & Ha & Lquil & Rivatus & $\mathbf{H h}$ & Nin & ba \\
\hline
\end{tabular}




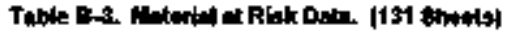

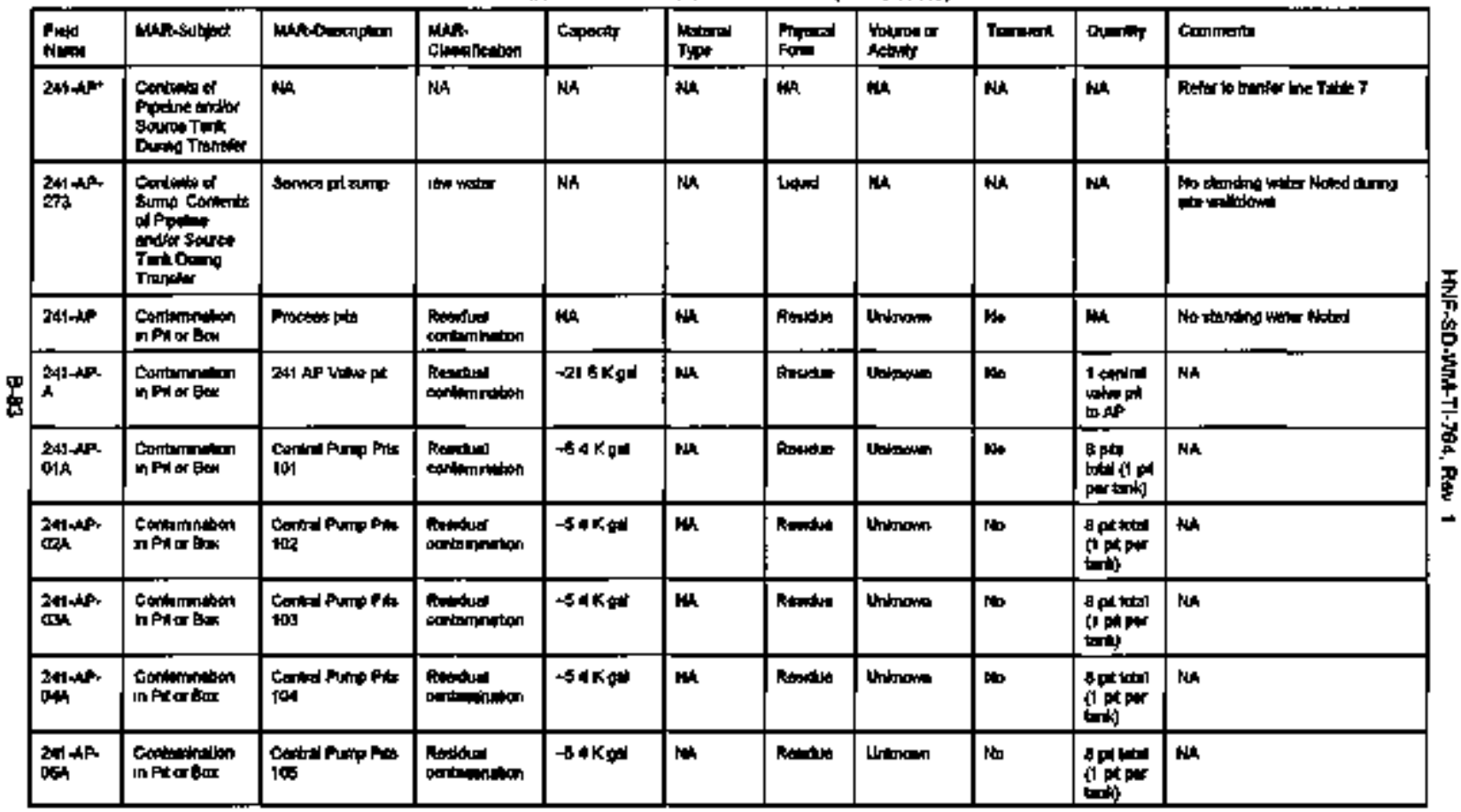




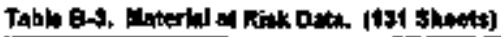

\begin{tabular}{|c|c|c|c|c|c|c|c|c|c|c|}
\hline Fald & 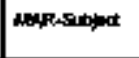 & 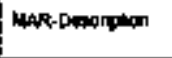 & 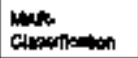 & Capait & $\begin{array}{l}\text { Molene } \\
\text { TYpa }\end{array}$ & $\begin{array}{l}\text { Fing } \\
\text { Fontil }\end{array}$ & tomengen & Trement & dumby & Comport \\
\hline 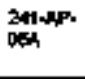 & $\begin{array}{l}\text { Eartammibon } \\
\text { n Ptar Bur }\end{array}$ & Central Purpop Pit & $\begin{array}{l}\text { Ritadual } \\
\text { ontemanaion }\end{array}$ & -54 Ko에 & $\mathbf{H}$ & Areath & | & $m$ & 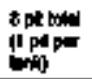 & Non \\
\hline $\begin{array}{l}241 \\
\text { Dithen }\end{array}$ & 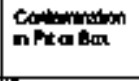 & $\begin{array}{l}\text { Condrat Pump Pitt } \\
\text { 10r }\end{array}$ & Restitid & $-34 \mathrm{~K}+\mathrm{ar}$ & MA & Rethe & Uh'ongun & $\mathrm{Na}$ & $\begin{array}{l}6 \text { pt thes } \\
\text { if pil not } \\
\text { int) }\end{array}$ & $\mathrm{Nh}$ \\
\hline $2 \times 1+45$. & 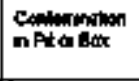 & $\begin{array}{l}\text { Dentre Pump Pls } \\
\text { 100 }\end{array}$ & 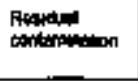 & $-54 \mathrm{~K}$. & MA & Rotive & Uningun & No & 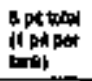 & NA \\
\hline $241+4 P^{\prime}$ & 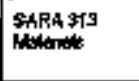 & 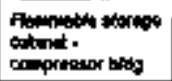 & NA & kh & NA & vanit & Wnimean & $Y \leftrightarrow$ & NA & 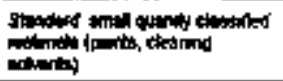 \\
\hline $341-A p^{2}$ & BARA 313 & funder & Na & NA & Wh & |4⿴囗十) & Untenown & Yodo & UK & 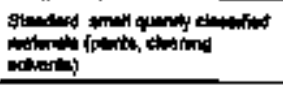 \\
\hline 2A1-AF & other & 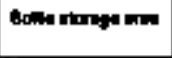 & 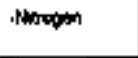 & $\begin{array}{l}\text { 2at bottien } \\
\text { (nomed }\end{array}$ & M & ib & क th & $Y=$ & Ldotime & 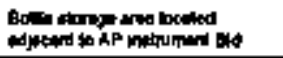 \\
\hline 24 मP+ & Drat & 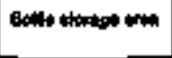 & Hutrogen & 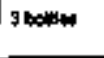 & MA & G* & 4908 & $Y \omega$ & 1 botin & 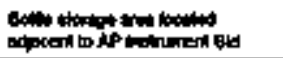 \\
\hline 241-4P & other & 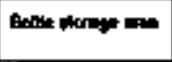 & 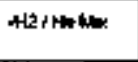 & 14 botical & Nh & 8an & aond & ret & dondes & 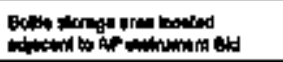 \\
\hline 24114ap & फिब & 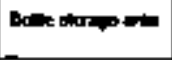 & 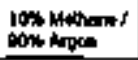 & 14 bountat & HA & 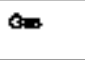 & ה & Yas & t binkt & 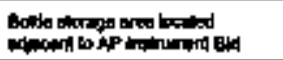 \\
\hline $24 \boldsymbol{A f}$ & Dtatr & Outiole obengen & 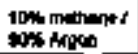 & 2tottin & Nh & On: & Dn n & $\gamma *$ & 2tonal & 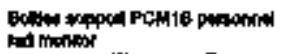 \\
\hline
\end{tabular}




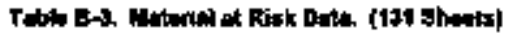

\begin{tabular}{|c|c|c|c|c|c|c|c|c|c|c|}
\hline Fing & MAR-pubuncl & 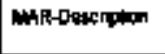 & $\begin{array}{l}\text { MAR. } \\
\text { Chabetikthal }\end{array}$ & Gepertir & $\begin{array}{l}\text { Motan: } \\
\text { Type }\end{array}$ & $\begin{array}{l}\text { Pholicind } \\
\text { Fomm }\end{array}$ & hating & Trnimeth & tounity & Comnnemin \\
\hline 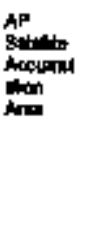 & othe & tom & moten ling & 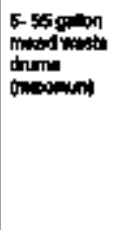 & 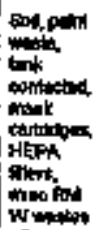 & Boid & 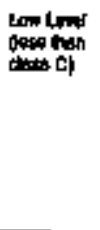 & $r=$ & 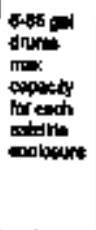 & 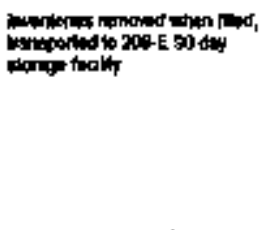 \\
\hline $\begin{array}{l}241-A W- \\
101\end{array}$ & Tunir Centandi & 211-AN-1aI & $\cos \theta f$ & $1 \mathrm{ls} \mathrm{mgr}$ & Supunata & Laqued & 1400 kged & w & ma. & wh \\
\hline $\begin{array}{l}2+1+\text { syrs } \\
10 t\end{array}$ & Tark Centurite & 2011-4h-101 & ostr & Nin & stimbotat & sold & M lad & $\boldsymbol{b}$ & Nh & $\mathbf{m}$ \\
\hline 204-4h- & 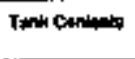 & 211-AW-4C? & Dw & 118 bugn & \$upwink & Lqqud & as how & w. & WA & $\mathbf{M H}$ \\
\hline $\begin{array}{l}245-14 y- \\
\operatorname{lat}\end{array}$ & Tanil Centants & 2HAwh-10 & ON & Nh & botwe & soid & 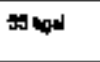 & $\mathbf{H *}$ & Nin & $\mathbf{m}$ \\
\hline $241-\operatorname{lit}$ & Tank Carkend & $211-44-103$ & ㅁN맘 & 118 Nata & 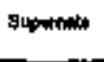 & 10. & 18 19:4 & Hs & wh & NA \\
\hline $\begin{array}{l}24140 \% . \\
108\end{array}$ & Tank Conterta & $241-10 \times-100$ & DrepD & $\mathbf{m}$ & 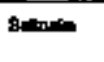 & sond & 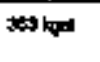 & $\mathbf{m o}$ & wh & NA \\
\hline $\begin{array}{l}24104 \mathrm{t} . \\
104\end{array}$ & Tank Canimis & $241+44-104$ & ow & $10 \mathrm{Kard}$ & ouparitat & und & क्ष & w & NA & man \\
\hline $\begin{array}{l}241-4,4- \\
104\end{array}$ & 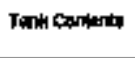 & 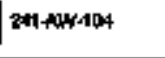 & DeA & MA & gntelns & Bons & realend & is & HA & MA \\
\hline 2010 & Tark camanta & 24t-AlWAdo5 & DNPD & 1 164bald & sxpmath & inala & 1384tipa & Not & $\mathbf{H h}$ & Nh \\
\hline $\begin{array}{l}341 \text { 샌 } \\
105\end{array}$ & Tenk Comimits & 201-AN-105 & DיW/P & 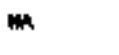 & 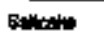 & sold & Folus & Ha & $\mathbf{m}$ & $\mathrm{kn}$ \\
\hline
\end{tabular}




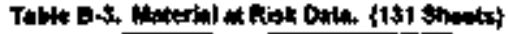

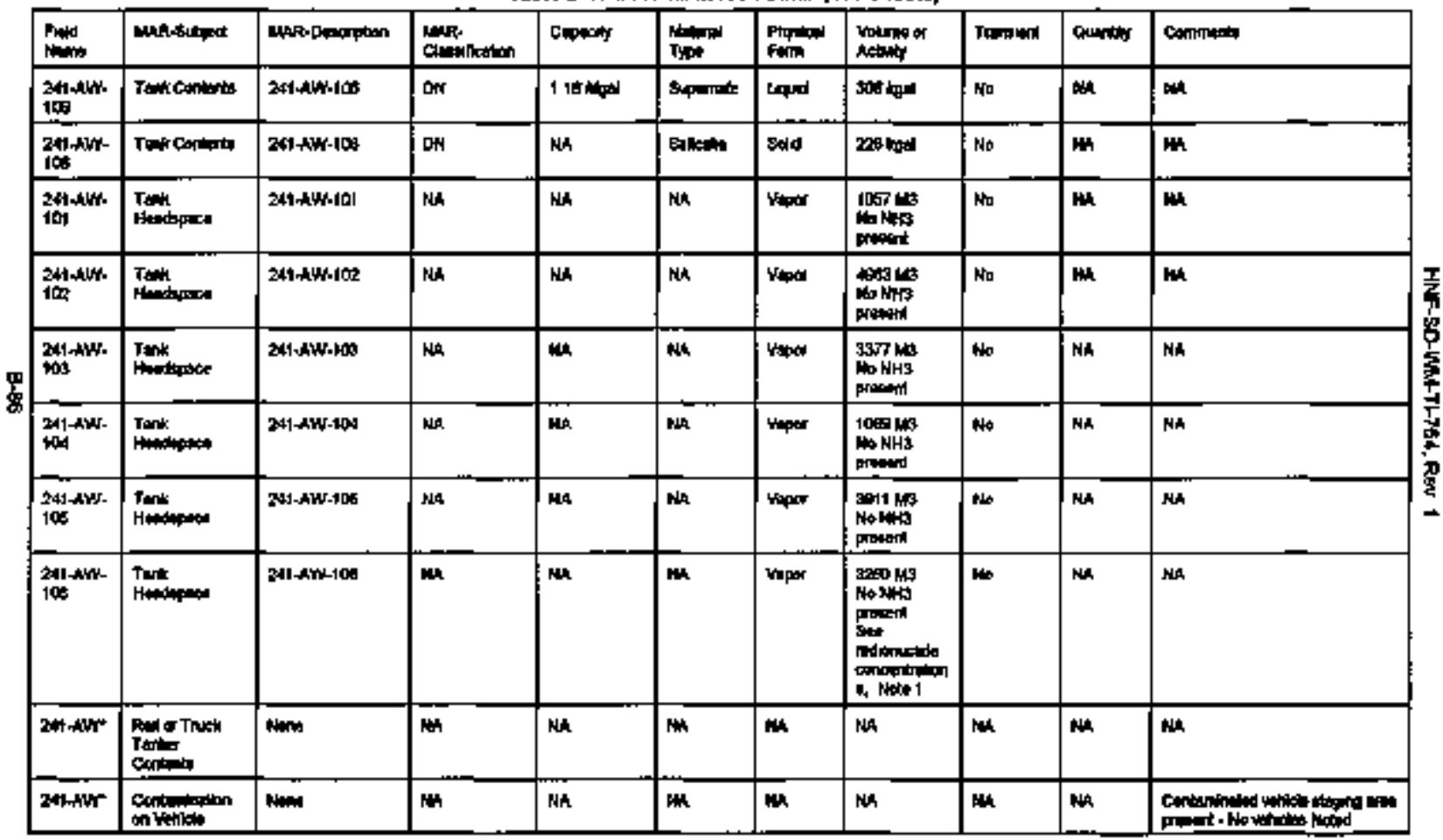




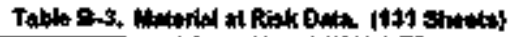

\begin{tabular}{|c|c|c|c|c|c|c|c|c|c|c|}
\hline $\begin{array}{l}\text { Proid } \\
\text { Nend }\end{array}$ & 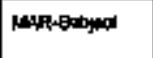 & 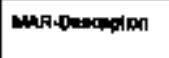 & $\begin{array}{l}\text { MARr } \\
\text { Clatankatim }\end{array}$ & Coppoty & Typing & Fonten & tophin or & Trmatum & curatis & 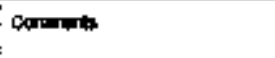 \\
\hline 2andthr & 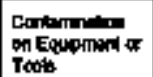 & then & $\mathbf{r a t}$ & Nh & Nin & Nh & in & Mr & man & Nh \\
\hline 34t-nut & 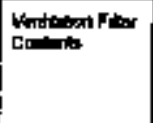 & Pintry & $\mathrm{ma}$ & 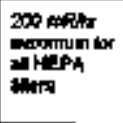 & Nh & Partivily & 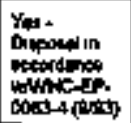 & ret & WA & 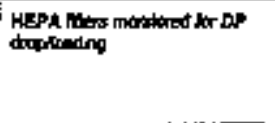 \\
\hline 241 Aly & Yaidion Filar & Anntuse & NA & 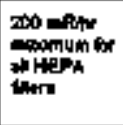 & IA & $\begin{array}{l}\text { Porturita } \\
\text { : }\end{array}$ & 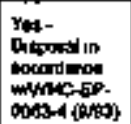 & Yed & MA & 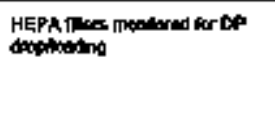 \\
\hline $241+N 4$ & 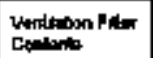 & Bry & Nin & HA & Wh & mo & Mh & $\ln$ & HA & $\mathrm{MH}$ \\
\hline 241-Ain & 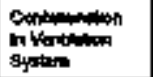 & De-miniminte & 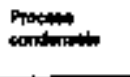 & $\mathrm{kn}$ & $\mathbf{~ H h}$ & 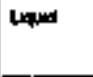 & R/Ach & $N / N$ & NA & HA \\
\hline 2Alatir & 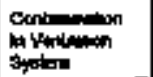 & seap loop & PTeoten: & ent & $\mathbf{m h}$ & Lapd & Acquon & NAS & Nh & Wh \\
\hline 2A14hy & 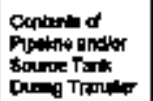 & Mans & wa & ats. & $\mathbf{m}$ & $\mathbf{m}$ & MA & MA & Nan & wh \\
\hline $2+1-44$ & caninge of & MA & wh. & WA & im & m & NA & Nh & MA & 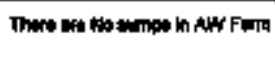 \\
\hline $\begin{array}{l}\text { zat - } 14 y- \\
\text { ois-068 }\end{array}$ & Continginion & Annoppong & Peopopiat & 1 akm & $\mathbf{m}$ & Penenger & Lniknow & 10 & apts & $\mathbf{m}$ \\
\hline $\begin{array}{l}\text { 20f-NH. } \\
\text { olv. }\end{array}$ & $\begin{array}{l}\text { continimation } \\
\text { op PA or Bos }\end{array}$ & Anvingetpt & Rtocitud & $15 \mathrm{KO}$ & $\mathbf{H}$ & Ruldut & Unthove & Ho & 1 i & $\mathbf{~ h}$ \\
\hline
\end{tabular}




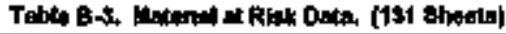

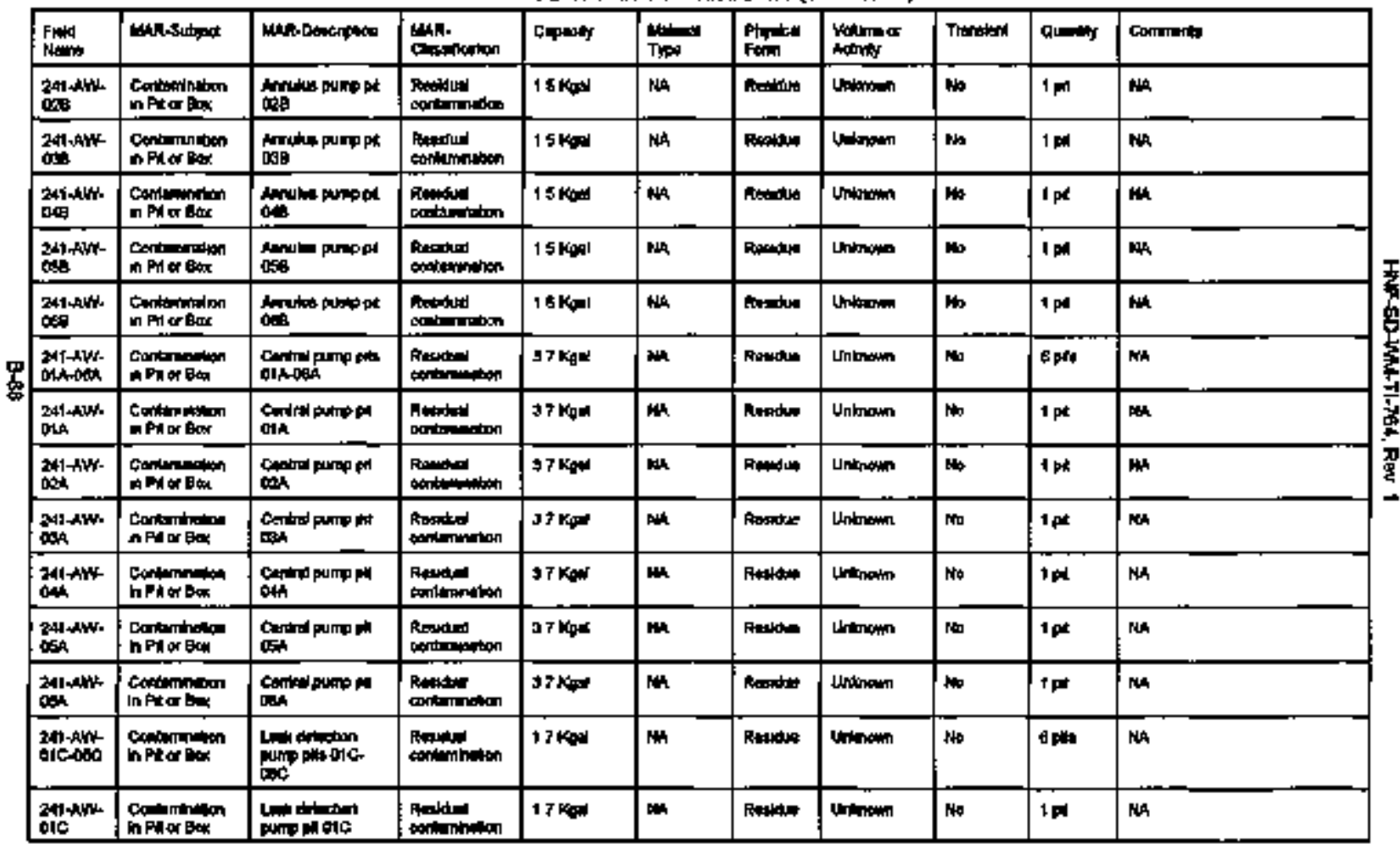




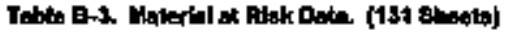

\begin{tabular}{|c|c|c|c|c|c|c|c|c|c|c|}
\hline Pats & MuRs:- & MAR-D:tenober & Chis: & Aparay & Tyok & $\begin{array}{l}\text { Plinuled } \\
\text { Forn }\end{array}$ & $\begin{array}{l}\text { Woterier a } \\
\text { notivity }\end{array}$ & Trankert & tongy & Camerists \\
\hline $\begin{array}{l}2+1-A) N . \\
0 \times 2\end{array}$ & 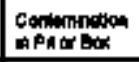 & pump pit tot & $\begin{array}{l}\text { Potedjod } \\
\text { rortemiterion }\end{array}$ & $17 \mathrm{Kat}$ & an & Ritatus: & Uniming & $\mathbf{W 0}$ & tot & MA \\
\hline 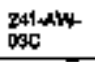 & $\begin{array}{l}\text { Combammati } \\
\text { A PA or Bas }\end{array}$ & 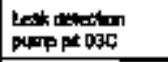 & Fotian & $14 K_{4}$ & m & Rante & (kat: & No & 1 pt & Man \\
\hline $\begin{array}{l}241-A h t-2 \\
045\end{array}$ & 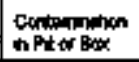 & 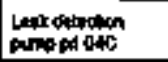 & Rowor:al & $17 \mathrm{kgN}$ & NA & Fonatup & Unknowim & No & 1 et & Nh \\
\hline 24lathth & $\begin{array}{l}\text { Cant wivan } \\
\text { oft of Bax }\end{array}$ & 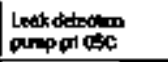 & Apexilu & $17 \mathrm{KW}$ & MA & frodu & Unkonom & $\mathbf{H o}$ & $1 p t$ & NA \\
\hline $\begin{array}{l}241 .+A \omega . \\
00 \%\end{array}$ & $\begin{array}{l}\text { Contominalion } \\
\text { n Pta ber }\end{array}$ & 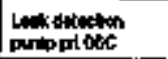 & Rtudtua & 17 Has & wh & Potrita & Unionothe & $\mathrm{kb}$ & 1 t & Nh \\
\hline $\begin{array}{l}241 \text { anthe } \\
\text { tres }\end{array}$ & 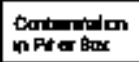 & 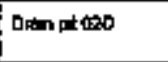 & 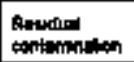 & $31 \times 0$ & NA & Findulu & Unkoum & $\mathbf{H o}$ & 1 pt & NAs \\
\hline $\begin{array}{l}2 \|+A W . \\
n\end{array}$ & 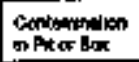 & Wal at AW-h & Randuad & 14ked & $\mathrm{Nh}$ & 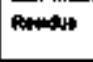 & Unkn:4nm & No & 1 that & then \\
\hline $\begin{array}{l}\text { 241-4⿴囗十. } \\
\text { 日 }\end{array}$ & $\begin{array}{l}\text { Conturimen } \\
\text { th PN of Bis }\end{array}$ & 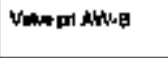 & 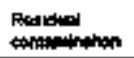 & $44 \mathrm{Kq}$ & Wh & Prewdue & |rtenam & $\mathrm{Nog}$ & I pats & an \\
\hline 24-Aw: & 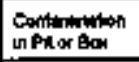 & 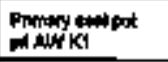 & Randed & اamb & Wh & 月hatht & Untartom & No & I pd & wh \\
\hline $\begin{array}{l}241+4 k x- \\
10\end{array}$ & $\begin{array}{l}\text { conturnaton } \\
\text { In PA of Bes }\end{array}$ & 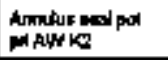 & Rerber & Uniknman & NA & Presidue & Intann & $\mathrm{Na}$ & I plat & wh \\
\hline 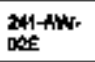 & $\begin{array}{l}\text { Conttimition } \\
\text { n PA or Bas }\end{array}$ & 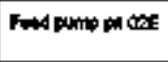 & Rosdiditidin & $3 k d$ & Nh & Dondur & Untortion & No & 1 para & Wh \\
\hline $241-4 w$ & 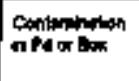 & 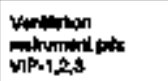 & 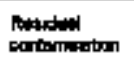 & 52 K녕 & Nh & Riondus & Unimoint & $\mathrm{Na}$ & I phlo & Nk \\
\hline PIAAnt- & 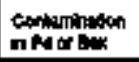 & Fuangt & poustual & $5 \mathrm{kOA}$ & $\mathbf{M H}$ & Rosintos & Unmintin & Wo & $1 \mathrm{ph}$ & $\mathrm{man}$ \\
\hline $\begin{array}{l}\text { Cot: } \\
\text { AN1/12 }\end{array}$ & 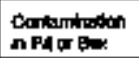 & 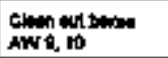 & 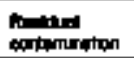 & at ropd & $\mathbf{m}$ & 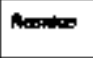 & 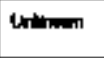 & Ha & $2 \cos$ & an \\
\hline
\end{tabular}




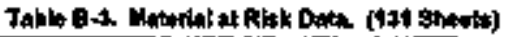

\begin{tabular}{|c|c|c|c|c|c|c|c|c|c|c|}
\hline Fom & 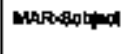 & 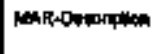 & Hapi. & Capingy & Fyom & $\begin{array}{l}\text { Phaved } \\
\text { Fant }\end{array}$ & Wotumpor & Thopumt & Dusitiy & Complapt \\
\hline cos & 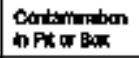 & $\begin{array}{l}\text { Chen axt benat } \\
\text { AW } D_{1} 19\end{array}$ & Rencinarion & arr kal & Nh & stadt & שמדיטג & Ho & 2608 & Nan \\
\hline cop & $\begin{array}{l}\text { Conbenmiben } \\
\text { o Pit ti Bon }\end{array}$ & 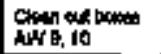 & Rouktud & का kent & NA & 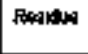 & Whivin & No & 2000 & NA \\
\hline 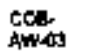 & 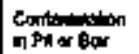 & 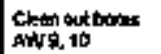 & 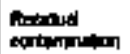 & 047 KaAl & Nh & Tratud & Uhisloun & Wb & 2000 & Nin \\
\hline cos- & 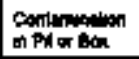 & $\begin{array}{l}\text { Clagh out basto } \\
\text { NWS, } 10\end{array}$ & 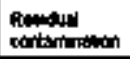 & Dar Real & NA & Reradus & Uh⿱一𫝀口⿰丨丨 & $\mathbf{H o}$ & 2004 & Wh \\
\hline cch. & 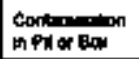 & $\begin{array}{l}\text { Ch:m oulbors } \\
\text { ANS 1D }\end{array}$ & 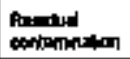 & 0* Kord & Nh & farlua & Unlonou & wo & $2 \cos t$ & N/4 \\
\hline coce- & condromimon & $\begin{array}{l}\text { Clima outbars } \\
\text { Ait o, iD }\end{array}$ & 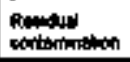 & Day Rorl & NA & Rominas & Lhongma & $\mathbf{H}$ & 2 Cota & NAB \\
\hline cos. & $\begin{array}{l}\text { cont- locion } \\
\text { in PA ar Bar }\end{array}$ & $\begin{array}{l}\text { Chratbons out } \\
\text { AWg 10 }\end{array}$ & Anidud & | & Hh & Anedua & Untoroun & $\omega$ & 2cost & Nh \\
\hline cop日- & 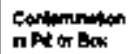 & 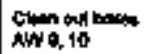 & 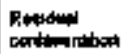 & W & $M$ & Rindur & Univim & Not & 290 & man \\
\hline $\begin{array}{l}\text { COB. } \\
\text { AWE }\end{array}$ & $\begin{array}{l}\text { Contumniman } \\
\text { n Fis of Bex }\end{array}$ & $\begin{array}{l}\text { Com oul bows } \\
\text { AW Q } 10\end{array}$ & 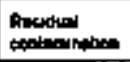 & $04 \mathrm{~kg}$ & uh & Rreudue & Umbown & Na & $2 \cos$ & in \\
\hline $\begin{array}{l}\text { cos- } \\
\text { AlW-10 }\end{array}$ & 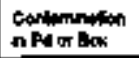 & 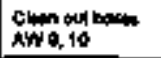 & Dowdon & OP & $\mathbf{M W}$ & 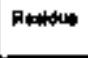 & Unb/nin & $N$ & 3000 & ath \\
\hline COH & 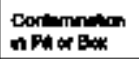 & 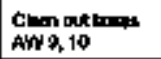 & 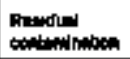 & الAp & $\mathbf{m}$ & Prestor. & Unknoyn & $\mathrm{No}$ & $2 \cos \theta$ & $m$ \\
\hline هol- & $\begin{array}{l}\text { Contembinton } \\
\text { n Prea Bax }\end{array}$ & $\begin{array}{l}\text { Obad oul bona } \\
\text { MW } 0,10\end{array}$ & Aptding & OAl & $\mathbf{w}$ & heddua & Unisount: & ite & 2 colla & Nh \\
\hline $341-44 n^{2}$ & GARA 343 & Gominer & 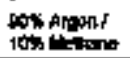 & 404 ant & nes & Qus & 4004t & Yos & 2 bonist & For PCAN1-6 \\
\hline 241-AWr & Other & samor butkly & تعلها ستر & Mh & $\ln$ & Neode & Nh & $\mathbf{N o}$ & Nk & m \\
\hline
\end{tabular}




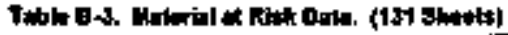

\begin{tabular}{|c|c|c|c|c|c|c|c|c|c|c|}
\hline Find & MAR-suman & MNR-Ovaciplon & MAR: & casusty & The & $\begin{array}{l}\text { Poumeal } \\
\text { tomm }\end{array}$ & watim on & Trandimat & curitg & Cerminumbats \\
\hline 241-ANN & Dthr & Crange lraper & إlo & NA & 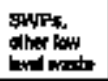 & Sowtd & 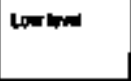 & Yas & NA & Cantoles HPT \\
\hline ZAF+AIT & Othe & 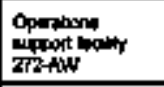 & sted cominnt & Sow 9am-it & 9ut & Bing & tet owmmat & $\operatorname{sen}$ & get & 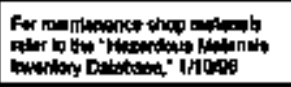 \\
\hline 241 & Olhe & 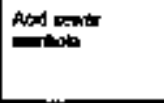 & Sod ctirmat: & 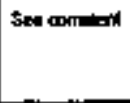 & $\begin{array}{l}\text { Set } \\
\text { ecrment }\end{array}$ & डex & $\begin{array}{l}\text { ser } \\
\text { crement }\end{array}$ & sec & soc & 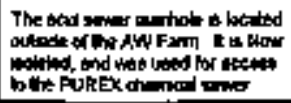 \\
\hline 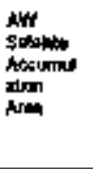 & One & 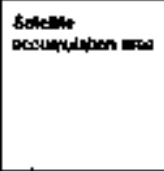 & $\begin{array}{l}\text { heled mond } \\
\text { wotio }\end{array}$ & 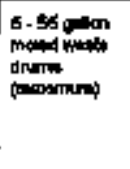 & 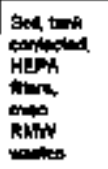 & Bold & 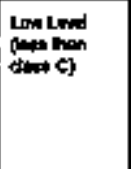 & res & 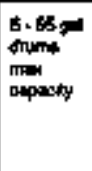 & Wh. \\
\hline $241+4 x$ & Tonu centaris & Axas & begaf & 10 onder & gandick & sold & SKand & Ho & MA & 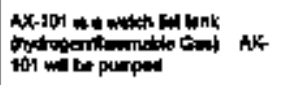 \\
\hline 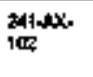 & Tart Conthith & Axinte & $\operatorname{cc}$ & 10 10 & 3upankit & $\underset{\text { ind }}{\text { sald }}$ & 1Kat? & Nho & nth & 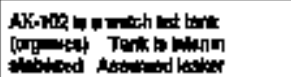 \\
\hline $\operatorname{lig}_{10}^{240}$ & Terth Cathate: & AKowtot & ce & $10 \mathrm{Mr}$ & shitat & sold & $2 \mathrm{Ked}+\mathrm{N}$ & Nop & $\mathrm{Nh}$ & 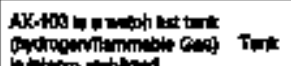 \\
\hline $\begin{array}{l}2+14 k \\
104\end{array}$ & Tat Cartanth & Ax-10d & HeFu & $1 \mathrm{dH} / \mathrm{CH}$ & Stridpa & Anind & $7 \mathrm{kot}$ & Ho & wh & 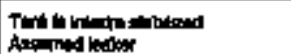 \\
\hline
\end{tabular}


Tabh E-3. Hobrin at Kisk Datu, \{131 shots|

\begin{tabular}{|c|c|c|c|c|c|c|c|c|c|c|}
\hline Fidu & Mars-pabjest & MHR-DEm & maki- & conopy & Thenol & $\begin{array}{l}\text { Phoikel } \\
\text { Ponin }\end{array}$ & vingex & Tronuiant & Dathy & Cotemtits \\
\hline${ }_{101}^{241+4 x}$ & 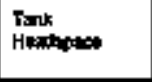 & $M X-101$ & Ma & na & $M A$ & VIm & 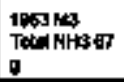 & NA & m & Nh \\
\hline 241004 & $\begin{array}{l}\text { Thik } \\
\text { Hendipenpe }\end{array}$ & $M x \cdot 102$ & Nh & Na & ma & Vwar & 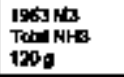 & wh & m & Nh \\
\hline $\begin{array}{l}241 \\
100\end{array}$ & Tetk & $A x \cdot 103$ & Me & Wh & $\mathrm{kh}$ & Whor & 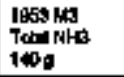 & $\mathrm{NA}$ & $\mathbf{m}$ & Wh \\
\hline 2014tix. & $\begin{array}{l}\text { Twik } \\
\text { Hent/pot }\end{array}$ & $M x \cdot 104$ & Nh & 情 & $\mathrm{m}$ & 1 & 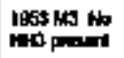 & Nh & $\mathbf{r a h}$ & Wh \\
\hline 2ब114X" & 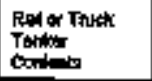 & Wine & Nh & NA & M & $\mathbf{m}$ & MA & Ne & $\mathrm{rm}$ & Wh \\
\hline $2 \tan -\cos$ & 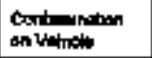 & Nome & Wh & HA & Na & Na & NA & un & Nh & $\mathbf{M}$ \\
\hline $2+1+h x^{2}$ & 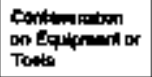 & Nhan & $M$ & NA & Nik & MA & $\mathrm{man}$ & wh. & NA & in \\
\hline $241+4 x^{2}$ & 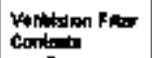 & Pimm & Wh & NA & Nin & M & $m \alpha$ & wh. & Nh & $\mathbf{m}$ \\
\hline $241-A x^{+}$ & 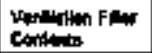 & 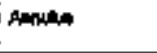 & HA & NA & NA & WA & NA & He & Nin & MH \\
\hline
\end{tabular}




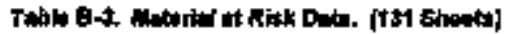

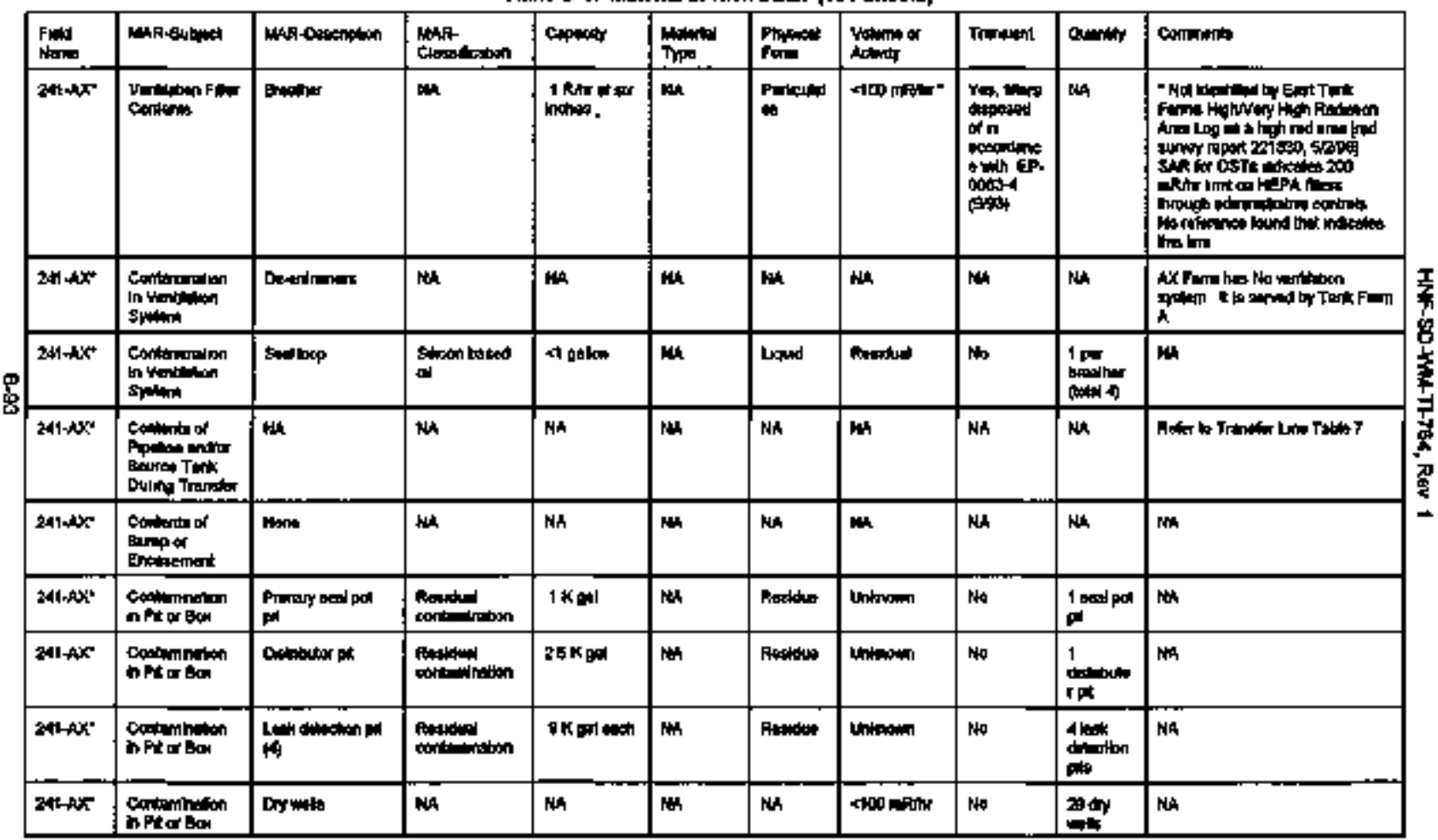


Tabla B-3. Antarid at Rlak Date. [131 Shuote]

\begin{tabular}{|c|c|c|c|c|c|c|c|c|c|c|}
\hline $\begin{array}{l}\text { Pudd } \\
\mathrm{Nan}\end{array}$ & Mnsthtod & 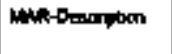 & Hak. & capescing & $\begin{array}{l}\text { theded } \\
\text { Thpe }\end{array}$ & $\begin{array}{l}\text { Phyoded } \\
\text { Foen }\end{array}$ & 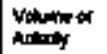 & Trindinth & 中故 & Comenten \\
\hline 241 -A口" & 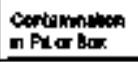 & Drin pt & Uningerm & Untantin & NA & Undown & 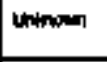 & Mo & 1 tath & $N$ \\
\hline $\begin{array}{l}2414 x . \\
151\end{array}$ & $\begin{array}{l}\text { conterarion } \\
\text { in Pin er 60\% }\end{array}$ & $\begin{array}{l}\text { Ometer whan } \\
\text { AX-151 }\end{array}$ & foreding & $310 \mathrm{kgr}$ & m & Finglue & Chtonom & No & 1 gtrpiner & Ha \\
\hline $\begin{array}{l}24 t-h x- \\
10 x\end{array}$ & $\begin{array}{l}\text { Contininopon } \\
\text { in Pin of for }\end{array}$ & 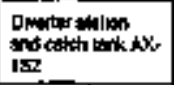 & Pandun & $212 \mathrm{Kan}$ & Wh & Reopedus & Uhtmoun & טلر & 1 inniar & $M h$ \\
\hline $241+4 x$ & $\begin{array}{l}\text { Contimmeginn } \\
\text { in Fin or Ben }\end{array}$ & ven pt it & 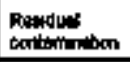 & GKod & sh. & Respotus & Untencun & מה & I yerion at & $\mathrm{Mh}$ \\
\hline $\begin{array}{l}241.4 x . \\
150\end{array}$ & 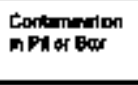 & 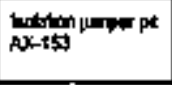 & $\begin{array}{l}\text { Ansdur } \\
\text { centeminutor }\end{array}$ & $1 \mathbf{k} \mathbf{k t}$ & $\mathbf{~ N h}$ & Anidue & Lntenown & $\omega$ & $\begin{array}{l}1 \\
\text { incluber } \\
\text { imper pit }\end{array}$ & HA \\
\hline 2011-4k. & 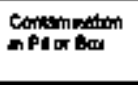 & 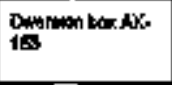 & Deadus & $35 K$ 证 & Hh & Motadub & Uridnewn & $\mathbf{m}$ & $\begin{array}{l}1 \\
\text { tam }\end{array}$ & be \\
\hline E11 & 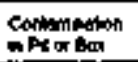 & Wetuen Axsor & Metudusl & $11 \mathrm{~K}$ as & $\mathbf{A n h}$ & Riscisus & Unboun & $\omega$ & 1 xater pat & Buh \\
\hline $\operatorname{lp}^{71-A x-}$ & 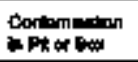 & Vhevep & Rpodus & $32 \mathrm{kgd}$ & Nh & Rosentur & Urtenoun & $\mathbf{m}$ & I man pt & Nh \\
\hline $241+4 \times 14$ & $\begin{array}{l}\text { Contminminim } \\
\text { A Pt or Bces }\end{array}$ & Wan id Axid & Andoded & $\underset{\cot }{4 J K}$ & He & Fistan & Utinnom & 16 & 1 mant & $\mathbf{m e}$ \\
\hline $241-A x-\theta$ & Dentom & Wasp $p \times x$ & 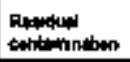 & A aka & Mn & Requatus & Unteun & $\mathbf{m}$ & I nowe in & BH \\
\hline $\begin{array}{l}241+4 x \\
\text { spa }\end{array}$ & 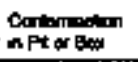 & Bamcr pt & Rere woter & 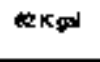 & Nh & ipd & Nh & ib & 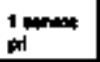 & $\mathrm{NH}$ \\
\hline $2+1+k x$ & Contmint & Push paty & Prow nats & e K & Nah & L & NA & $\boldsymbol{H}$ & 1 hushp: & ren \\
\hline $241+4 x-0$ & 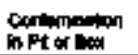 & Fuphpt & Raw h-in & $4 \mathrm{~K}$ Ko & Nh & Llowed & NA & $\mathbf{m}$ & 1 hushed & Nah \\
\hline
\end{tabular}




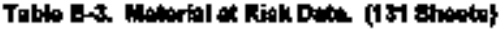

\begin{tabular}{|c|c|c|c|c|c|c|c|c|c|c|}
\hline Fild & MAR_Babjeot & Muth-Danplim & Mar- & 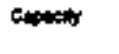 & Mrinat & $\begin{array}{l}\text { Ptyuticen } \\
\text { Rown }\end{array}$ & vinitio or & Thindih & Ondady & Commitat \\
\hline $\begin{array}{l}\text { 2414 } \\
\text { cos }\end{array}$ & 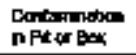 & Ckemout boreal & pountum & or $\mathrm{K}_{\text {gen }}$ & Min & Fandun & yomen & $\mathbf{H o}$ & 100009 & NA \\
\hline $241-A K=$ & EARA 310 & Not & Wh & $\mathrm{MH}$ & $N$ & HA & Min & $\mathbf{m}$ & me & $\mathbf{r a n}$ \\
\hline 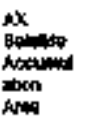 & Dther & 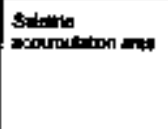 & $\begin{array}{l}\text { nctich moved } \\
\text { wath }\end{array}$ & 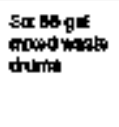 & 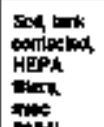 & soted & 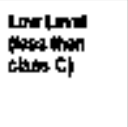 & Yat & 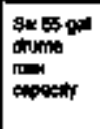 & 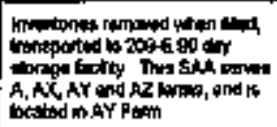 \\
\hline $\begin{array}{l}\text { 241-MY- } \\
\text { CO1 }\end{array}$ & 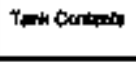 & $A Y>1 d \mid$ & DC & I 18 Har & gaponita & Leand & $\operatorname{Kag}_{0=1}$ & $\mathrm{Na}$ & H & Nid \\
\hline $\begin{array}{l}241-N y= \\
100\end{array}$ & 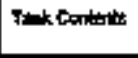 & AYT10Z & DW & 1 vtitgd & $\begin{array}{l}\text { bupenth } \\
\text { \$oind }\end{array}$ & Ind & 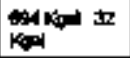 & Ma & M & WHA \\
\hline $\begin{array}{l}241-A Y- \\
101\end{array}$ & Tan & Arstid & NA & b4 & MA & Wopar & 1 reska ks & No & NA & Nin \\
\hline $\begin{array}{l}241-A Y, \\
100\end{array}$ & Trak & $M Y-102$ & NA & m & MA & Mnper & 15t2 un kl & שמ & $N A$ & Wh \\
\hline 24) & $\begin{array}{l}\text { Rer er Trut } \\
\text { Tanlier } \\
\text { Conteite }\end{array}$ & Btene & NA & eh & wh & NA & NA & WA & wh & 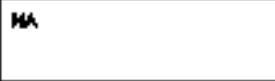 \\
\hline 241-AY" & 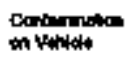 & 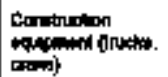 & Pesilynd & MA & Na & Raying & Uritioun & Y* & 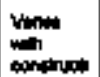 & 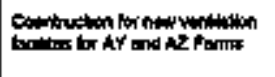 \\
\hline
\end{tabular}




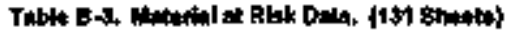

\begin{tabular}{|c|c|c|c|c|c|c|c|c|c|c|}
\hline Fion & MAR-\$Himt & MAR-Doperpman & Mart- & cenocts & Ther & 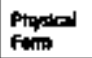 & Whime or & trenemt & Arditit & Comments \\
\hline 241+Apr & 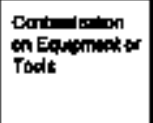 & 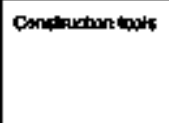 & Paintid & Nan & res & Poodue & |lom & $\mathrm{res}$ & 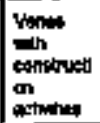 & 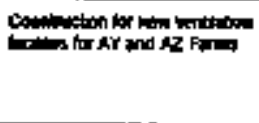 \\
\hline 2441-사" & 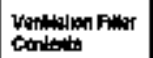 & Pommy & WA & MA & NH & $\mathbf{m}$ & $\mathrm{m}$ & in & Nan & wh \\
\hline 3414Ar & $\begin{array}{l}\text { Wertingen fiter } \\
\text { Centento }\end{array}$ & Annitus & wA & 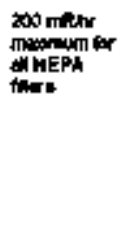 & mo & Paticalax & 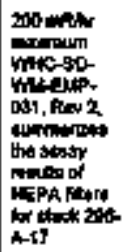 & 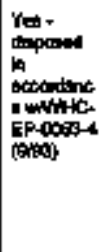 & Nh & 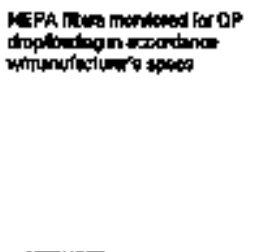 \\
\hline 2*⿻川⿲丶丶丶 & Hatim & Erithe & HA & Nh & Wh & MA & en & MA & Wh & uh \\
\hline 2UIAr & 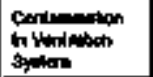 & De-dinapas & Prapalat. & NA & $\boldsymbol{\mu}$ & Uapd & Posaturd & $\mathbf{w h}$ & $\mathrm{NH}$ & Wh \\
\hline 3atilur & 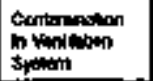 & gav loep & Proons & Nan & ran & Lend & 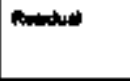 & Wh & Wh & Wh \\
\hline 2011 wer & conturit of & Ma & $\mathbf{m}$ & Nat & man & $\mathbf{w h}$ & $\mathbf{m}$ & $\operatorname{sen}$ & Nan & 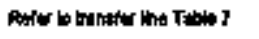 \\
\hline $2,41+4 y^{*}$ & 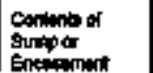 & 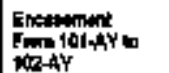 & 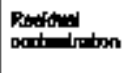 & $12 \mathrm{Kad}$ & $M$ & Paspqua & 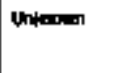 & $\mathrm{rat}$ & NA & 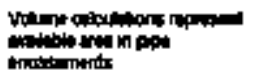 \\
\hline
\end{tabular}




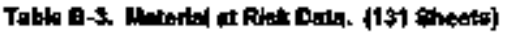

\begin{tabular}{|c|c|c|c|c|c|c|c|c|c|c|}
\hline Fied & Mustotact & MHRTDaropton & Rakr & chipoly & 19. & $\begin{array}{l}\text { Ptyond } \\
\text { Fom }\end{array}$ & Nowing & Tranewnt & Onumity & Comate \\
\hline ZAt-Âr & $\begin{array}{l}\text { Coumite of } \\
\text { Surp or } \\
\text { Encominent }\end{array}$ & 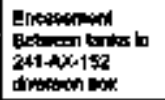 & Reidiv & $99 \mathrm{Kar}$ & $\mathbf{m}$ & Ratades & Unthom & wo & Hh & 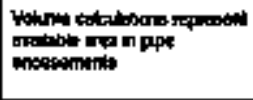 \\
\hline $\begin{array}{l}\text { Z4|rktr. } \\
\text { ald }\end{array}$ & 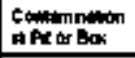 & Pumb bald & 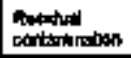 & $36 \mathrm{KH}$ & wh & hrodus & 17. & ap & 1 pros & $\mathrm{Nan}$ \\
\hline 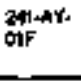 & 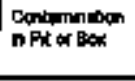 & $\begin{array}{l}\text { Anmofus pumpect } \\
\text { D\& ㅂF }\end{array}$ & 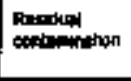 & I БК⿴囗十 & WA & R日ingr & | & b & $\begin{array}{l}1 \\
\text { pumpat }\end{array}$ & $\mathrm{KOA}$ \\
\hline $\begin{array}{l}\text { ZS1 Wh. } \\
\text { OIBEE }\end{array}$ & 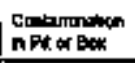 & 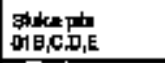 & 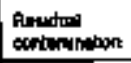 & $28 K_{\text {gal }}$ & 14h & Fanots & 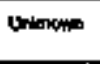 & 16 & $\begin{array}{l}4 \text { fonct } \\
\text { Dilo }\end{array}$ & Nh \\
\hline 2해부 & 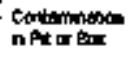 & 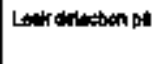 & 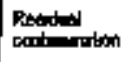 & 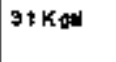 & bu & Recotut & Lthotoun & Ho & I tat pt & NA \\
\hline Z41-AY" & $\begin{array}{l}\text { Deninumpace } \\
\text { on Pit ow Box }\end{array}$ & 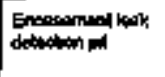 & Rymber| & 31 K & m & 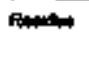 & 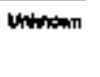 & $\mathbf{H b}$ & 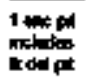 & 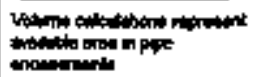 \\
\hline 2Al|ATr & $\begin{array}{l}\text { Coniminaten } \\
\text { In PA or Ban }\end{array}$ & Dopicionk on $h$ & focaktur & atron & $\mathrm{MA}$ & Rearotus & 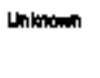 & No & $\lim _{\text {at }}^{1}$ & 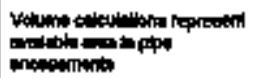 \\
\hline $\begin{array}{l}24 \mid A \text { AY- } \\
\text { itah }\end{array}$ & 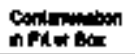 & Phip of 02 h & 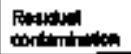 & $30 \times \mathrm{Ka}$ & NA & Ranchat & 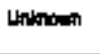 & ing & pt & 积 \\
\hline
\end{tabular}




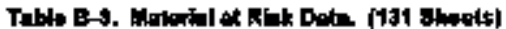

\begin{tabular}{|c|c|c|c|c|c|c|c|c|c|c|}
\hline rimd & HAR-Sybingl & 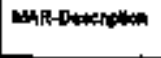 & ther- & Gtonctror & $\underset{\text { The }}{\text { Thingnd }}$ & Plptid & Folume of & Trtnnemt & atdily & Comomertit \\
\hline $2 \times 1+1$ & 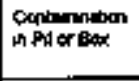 & $\begin{array}{l}\text { minutis purpout } \\
\text { pet }\end{array}$ & Rasing & 90 Ka & ran & 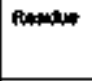 & Vodnown & to & $\begin{array}{l}1 \\
\text { pumpodil } \\
\text { Fil }\end{array}$ & NA \\
\hline $\begin{array}{l}\text { 241-AY- } \\
028-1\end{array}$ & $\begin{array}{l}\text { Contarixion } \\
\text { in Pu of Bur }\end{array}$ & $\begin{array}{l}\text { stweptr } \\
\text { DQRCDE }\end{array}$ & $\begin{array}{l}\text { Aroded } \\
\text { ochlominter }\end{array}$ & $25 \mathrm{Kg}=$ & Nh & Pappatup & Unmonan & Mo & 4 ingen & ah \\
\hline $2414 Y^{*}$ & $\begin{array}{l}\text { Conimingipn } \\
\text { in Pin of Bex }\end{array}$ & 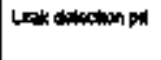 & 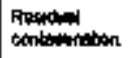 & $9 \mathrm{Kad}$ & NA & Re:acus & Unknom & 14 & I land & wh \\
\hline 2tithir & $\begin{array}{l}\text { Conidimnetan } \\
\text { on Pit on Bat }\end{array}$ & 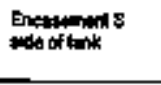 & 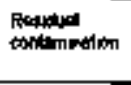 & اعث K & NR & Rotudis & עח & 的 & $\begin{array}{l}1 \\
\text { and } \\
\text { ard }\end{array}$ & MA \\
\hline $\begin{array}{l}2011+Y Y- \\
50 ;\end{array}$ & 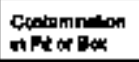 & 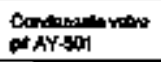 & 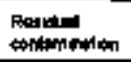 & $4 \mathrm{Kar}$ & Wh & Rषudu & Lntmove & $\mathbf{H}$ & 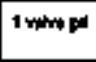 & MA \\
\hline 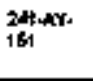 & 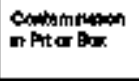 & Plinpout ot:AY- & Rotitud & $13 \mathrm{kpH}$ & Wh & Rentus & Unthomen & Ath & pupat & nes \\
\hline 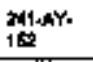 & $\begin{array}{l}\text { Contiminton } \\
\text { in Pite Bax }\end{array}$ & 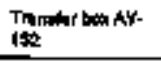 & Romity & 4t Ka & $\operatorname{ban}$ & Roudn & 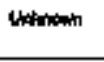 & $\mathrm{Nog}$ & $\begin{array}{l}1 \text { indere } \\
\text { bein }\end{array}$ & Nar \\
\hline 241-AYt & Cantrinoch & Reymierpte & 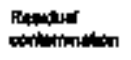 & $\begin{array}{l}2812 k 9 \\
0.42 x\end{array}$ & $\mathbf{m}$ & Pitalute & 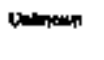 & Not & 11 the & $\mathrm{MH}$ \\
\hline $24144 \gamma$ & 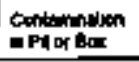 & 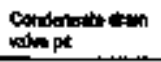 & 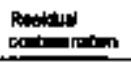 & $1 \mathrm{~K}$ 国 & Mh & Rostrg & thintom & ita & $\begin{array}{l}1 \text { condt } \\
\text { in }\end{array}$ & Na \\
\hline ZAIAY-1 & Contine & 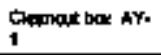 & contron & W & Hen & 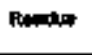 & Whk & 10 & 1000 & Nh \\
\hline
\end{tabular}




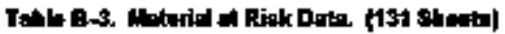

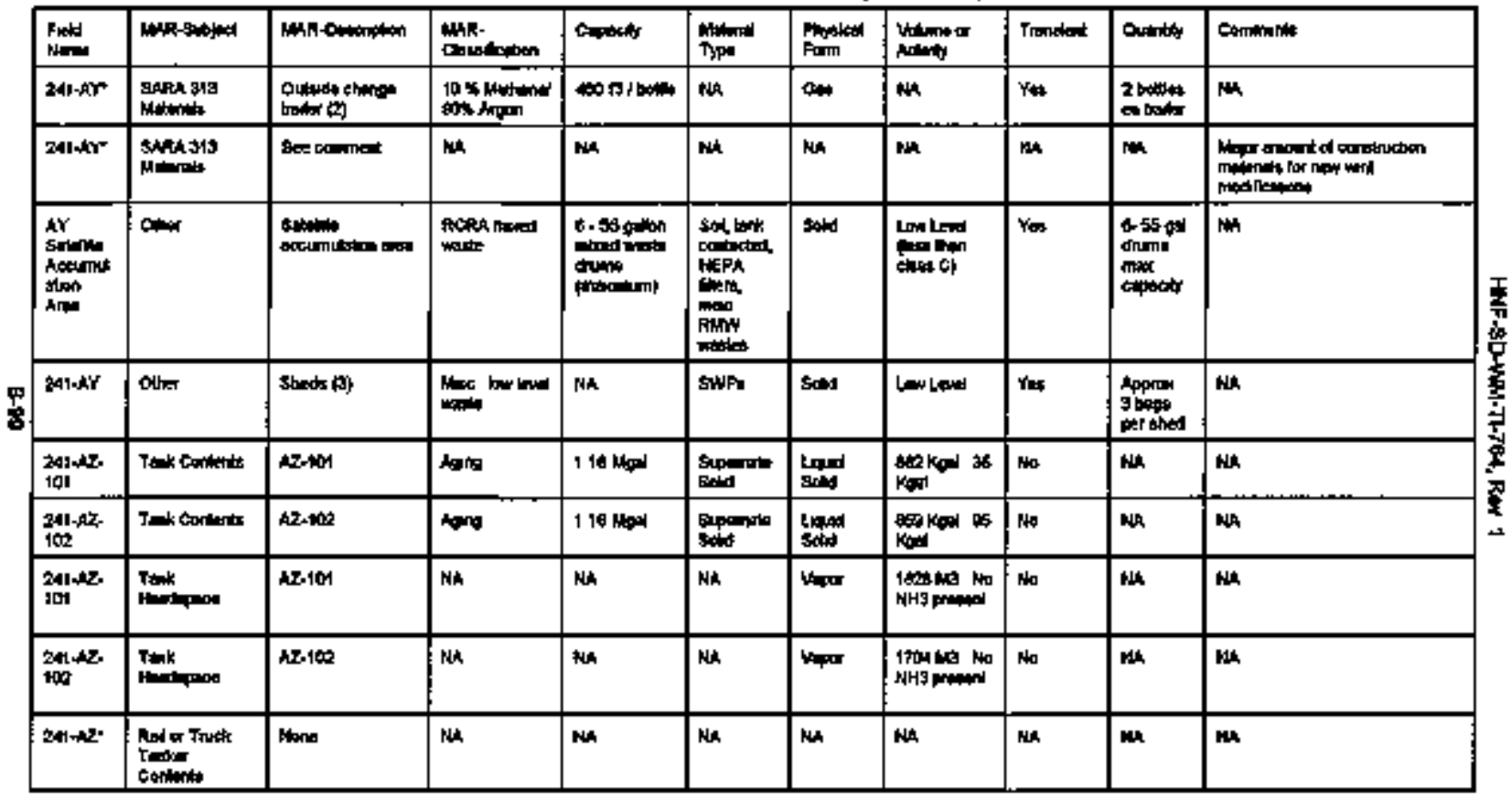




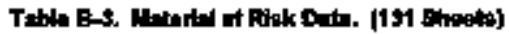

\begin{tabular}{|c|c|c|c|c|c|c|c|c|c|c|}
\hline (1) & BAR-Srejert & WhAthescopton & 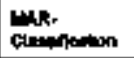 & Eaprocts & Typer & $\begin{array}{l}\text { Mhine } \\
\text { forle }\end{array}$ & Notrity of & Teminat & ansions & Colmpintiv \\
\hline $2+1+z^{*}$ & 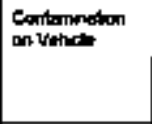 & 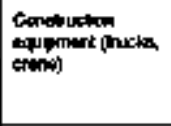 & 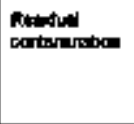 & NA & $\mathrm{Nh}$ & ReAldis & UThorown & Yט & 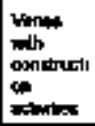 & 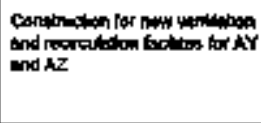 \\
\hline 2414Ar & 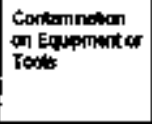 & Constructimbots & $\begin{array}{l}\text { Aleodud } \\
\text { continimben }\end{array}$ & Man & $\mathrm{md}$ & Artoldos & Undmown & res & 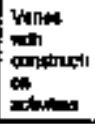 & 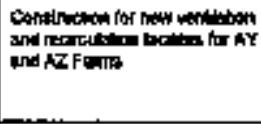 \\
\hline $2412 z^{*}$ & $\begin{array}{l}\text { Yodivion Fes } \\
\text { Conterts }\end{array}$ & Pontsly & MA & NA & $\boldsymbol{H A}$ & NA & NA & NA & Wa & uh \\
\hline 241-4t & 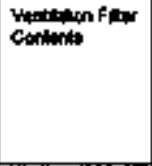 & Anpilus: & uh & 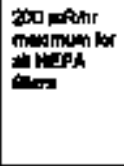 & un & Partadid & mo mkth & 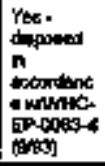 & Nin & 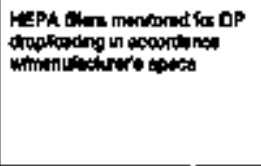 \\
\hline $241-\rightarrow 7^{\prime}$ & Coritum & Panter & in & Na & Wh & NA & Ma & $\boldsymbol{H}$ & Noh & $\mathbf{m a n}$ \\
\hline $241-\mathbf{N}^{*}$ & 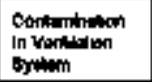 & Deterimenters: & Proctiot & M & $m$ & Land & Rumitur & $m$ & Nh & Mh \\
\hline $24104 z^{\circ}$ & 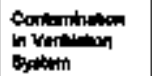 & for losp & Pondet. & Nh & $m$ & Land & 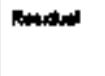 & Mh & Nh & $\mathbf{m}$ \\
\hline 241.45 & 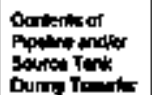 & $\operatorname{man}$ & Nah & $\mathrm{NAm}$ & ath & MA & Nh & NA & Nh & 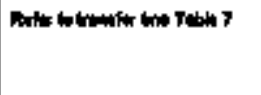 \\
\hline
\end{tabular}


Table B-t. Malerted at Rilat Date (191 Bheotey

\begin{tabular}{|c|c|c|c|c|c|c|c|c|c|c|}
\hline Fiedd & MArrstupex & Whe-Dowatpthon & 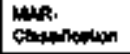 & Eopanty & Trend & $\begin{array}{l}\text { Phethend } \\
\text { Fand }\end{array}$ & 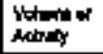 & 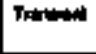 & ouritity & Connespts \\
\hline $241 \sqrt{2} z^{+}$ & 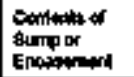 & Nopth fortummint & 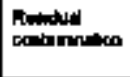 & $12 \mathrm{Kpd}$ & Whe & 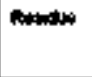 & Unbnatr & No & in & 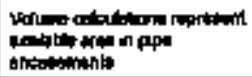 \\
\hline $2+1+x$ & 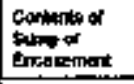 & Bouth ahstammerl & 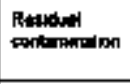 & $51 \mathrm{~kg}$ & M & Reondus & Urmangan & $\mathrm{No}$ & $\operatorname{lok}$ & 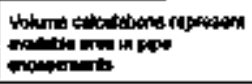 \\
\hline $2 \times 1-\alpha$ & 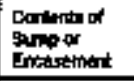 & 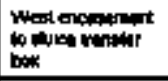 & 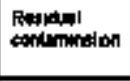 & $6 \mathrm{BK}$ \% & $\mathbf{r e n}$ & Be:mon & Untmon & ra & KA & 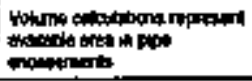 \\
\hline $241+12$ & 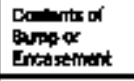 & 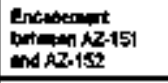 & R/atyed & $7 \mathrm{kgd}$ & HAR & Rerodue & untan & tho & MA & 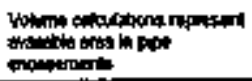 \\
\hline 241427 & 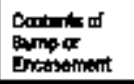 & 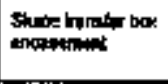 & Ravid & $4 \mathrm{~K}$ & NA & Reodu & U-10=1 & ito & NA & 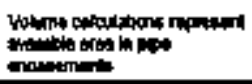 \\
\hline 24142 & 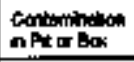 & AZ-10r & & & & & & & & \\
\hline $\begin{array}{l}241-A 2 \\
014\end{array}$ & 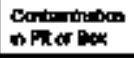 & Pump pan Dik & 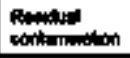 & $34 \mathrm{Kal}$ & NA & Rpospun & 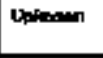 & Ma & 1 pho & Nhen \\
\hline $\begin{array}{l}2410 \sqrt{2} . \\
\text { Dif }\end{array}$ & 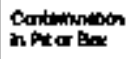 & 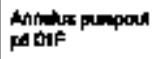 & 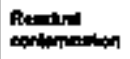 & 1*KG| & Na & Rendos & 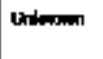 & ito & $\begin{array}{l}1 \\
\text { intwinth }\end{array}$ & Mh \\
\hline 24l-kz- & 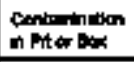 & 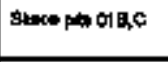 & Dontemingen & tok日l & $\mathrm{NAN}$ & 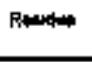 & 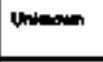 & Wo & $\begin{array}{l}\text { zoluse } \\
\text { phe }\end{array}$ & Nh \\
\hline $\begin{array}{l}241+42, \\
\text { alp }\end{array}$ & 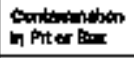 & aknototos & fiendud & 2ثKFl & NH & Ronldes & Intrimen & $\mathbf{W o}$ & 1 arkot & $\mathbf{m}$ \\
\hline $\begin{array}{l}\text { OIL } \\
\text { old }\end{array}$ & 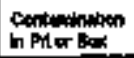 & Shest pulde & 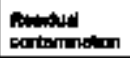 & 15Kक현 & w & Ratula & Unimin & $\boldsymbol{m}$ & $\begin{array}{l}1 \text { ahere } \\
\text { od }\end{array}$ & $\mathbf{k}$ \\
\hline
\end{tabular}




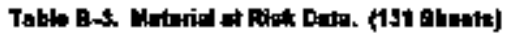

\begin{tabular}{|c|c|c|c|c|c|c|c|c|c|c|}
\hline Find & MAR-suldict & 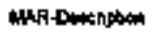 & MWh- & copocty & Typand & $\begin{array}{l}\text { Finnald } \\
\text { fom }\end{array}$ & Wation & Trendart & anda & Comintim \\
\hline $241-12$ & 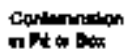 & 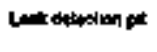 & Fin:mint & $3 k=d$ & M & Resioun & Whithom & No & $1 \ln$ & $\operatorname{mos}$ \\
\hline $2414 x$ & Cantar robon & For when & Rerer & $13 K_{\text {gar }}$ & $\mathbf{m}$ & Enapdre & 10 & 20 & 15 & NA \\
\hline 158 & 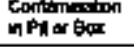 & Yert role pi & 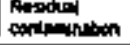 & F $\mathrm{kg}$ & $\mathbf{r a r}$ & Res:mety & Untenown & $\mathbf{N o}$ & trit & Wh \\
\hline $241+h$ & $\begin{array}{l}\text { Conkenninen } \\
\text { in Pil or Bar }\end{array}$ & 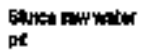 & Raw res & $2 \mathrm{Kag}$ & Nat & Putidut & $\mathrm{Nata}$ & $\mathrm{No}$ & of thuct & Nh \\
\hline $241+12$ & 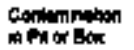 & $12-102$ & & & & & & & & \\
\hline $\begin{array}{l}241+42 . \\
020\end{array}$ & $\begin{array}{l}\text { Conktimition } \\
\text { a Pin or Bax }\end{array}$ & Pumpolosh & 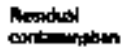 & $34 \mathrm{~K} F$ & Nh & Puentua & Untrown & No & 1 parip & $\mathbf{M h}$ \\
\hline $\begin{array}{l}241+12- \\
0 \neq 1\end{array}$ & 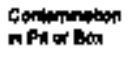 & Amutim anmpart & Ropiptuin & $14 \mathrm{~K}$ 新 & NN & 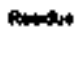 & 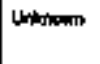 & No & 1 & $\boldsymbol{M}$ \\
\hline 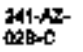 & 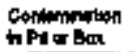 & 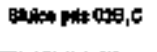 & Rtainivin & $28 \mathrm{~K}$ 헤네 & $\mathrm{NA}$ & 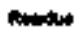 & mbdinem & Not & $\begin{array}{l}\text { 7othen } \\
\text { ptis }\end{array}$ & $\boldsymbol{M}$ \\
\hline $241-12$ & $\begin{array}{l}\text { Contuminitan } \\
\text { of Ri or Bum }\end{array}$ & 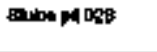 & Fatintion & 28 К리 & Wh & Patentury & 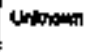 & No & 1 ats & $\mathbf{M A}$ \\
\hline $\begin{array}{l}241 \sqrt{2} 2- \\
0 \times C\end{array}$ & 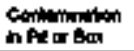 & 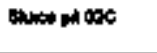 & 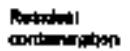 & 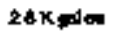 & Nin & Finditura & 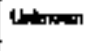 & $\mathrm{No}$ & 1 ature & $\mathbf{m}$ \\
\hline
\end{tabular}


Tobos B-3, Wotertal at Ritk Dote. (131 Bhots)






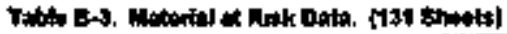

\begin{tabular}{|c|c|c|c|c|c|c|c|c|c|c|}
\hline Finge & 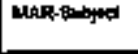 & Mr-Desorplas & $\begin{array}{l}\text { HAR- } \\
\text { Olintimithan }\end{array}$ & Contity & thing: & $\begin{array}{l}\text { Pluminis } \\
\text { Form }\end{array}$ & $\begin{array}{l}\text { Wolume or } \\
\text { nothink }\end{array}$ & Tannamk & coning & Cotiminitata \\
\hline $241+2-4$ & 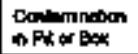 & Genout bow $\mathbf{N Z}_{4}$ & Resuted & $\mathrm{DeKg}$ & NA & Raskde & Un:mon & $\mathrm{No}$ & to cops & nh \\
\hline $241+425$ & 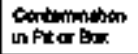 & 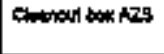 & |antitum & oskgiol at & $N A$ & Roudht & Lthomint & bo & $10 \cos$ & int \\
\hline 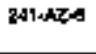 & $\begin{array}{l}\text { Cand mabon } \\
\text { in Pitor Bar }\end{array}$ & Cheoroul bo 128 & 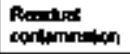 & Dok Ka: & Nh & Reandr & Unkrower & $\mathbf{H o}$ & 10 cons & $\mathbf{m e}$ \\
\hline $241+427$ & 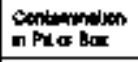 & cloctoul bous $A$ & formitur & 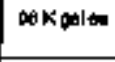 & Nh & Rostent & Unknimin & $\mathbf{W o}$ & 10 c内bo & $\mathbf{m}$ \\
\hline $241-A 28$ & $\begin{array}{l}\text { font and } \\
\text { in fil or } \mathrm{Bax}\end{array}$ & Chaout bo & Fas.ilud & $05 \mathrm{~K} p=0$ & Wh & Ricacter & Uninovin & ito & $10 \mathrm{COB}$ & HA \\
\hline $2 n+2+8$ & 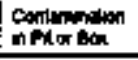 & 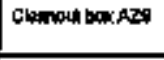 & Ropitud & $\omega \mathrm{K}$ & Wh & Romintat & Unknown & Ho & $10 \cos 8$ & an \\
\hline $\begin{array}{l}241+22 \\
10\end{array}$ & 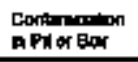 & $\begin{array}{l}\text { Clenou bou } \\
\text { Al10 }\end{array}$ & Prifie & 6 & $\mathbf{M A}$ & Fatiplu & بnkngme & 10 & 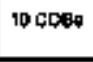 & $\mathbf{H A}$ \\
\hline $\begin{array}{l}241-z+2 \\
181\end{array}$ & 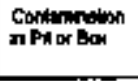 & 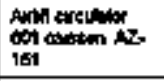 & $\mathbf{M h}$ & 8目 & $\mathbf{H h}$ & Hond & MA & in & I toristan & m \\
\hline $\begin{array}{l}241+k z \\
102\end{array}$ & $\begin{array}{l}\text { Contamnitist } \\
\text { IPA or Bow }\end{array}$ & 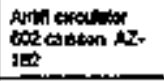 & $\mathbf{M}$ & $73 \mathrm{~K}$ 명 & $\mathbf{H}$ & What & Nan & rah & 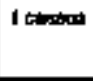 & $\mathrm{man}$ \\
\hline 24114tz- & 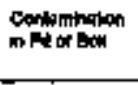 & $\begin{array}{l}\text { ginecostanalta bat } \\
12.152\end{array}$ & Rasidion & $75 \mathrm{Kpa}$ & $\mathbf{H}$ & Prewitys & 17lown & 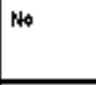 & 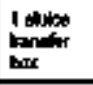 & Mth \\
\hline 241- & Cocingen & 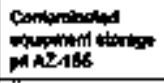 & Rpaptil| & 587 K 0.1 & H & Prowdus & Uninewn & No & 1 on & Nh \\
\hline 241-A2- & 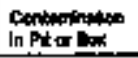 & 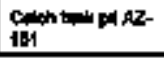 & Rayplyal & $20 \mathrm{~K}=\mathrm{w}$ & $\mathrm{res}$ & Thath & Uthom & $\mathrm{No}$ & thent & Nh \\
\hline
\end{tabular}




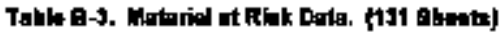

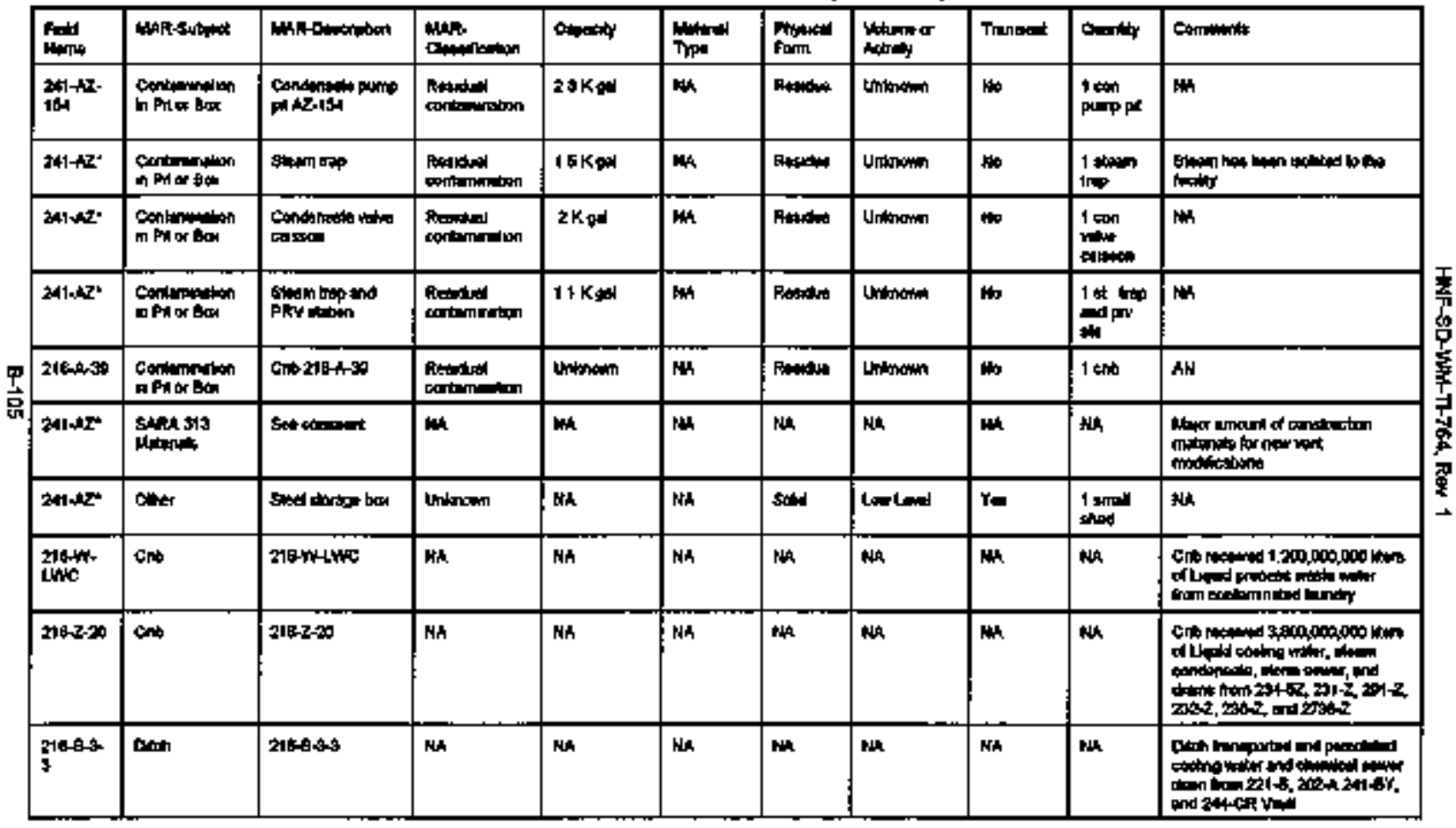


Tahle 8-1. Matedel at Rinh Date (1s1 sheata)

\begin{tabular}{|c|c|c|c|c|c|c|c|c|c|c|}
\hline Find & Whatimpod & 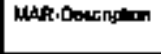 & 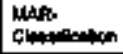 & Aneatir & Then & $\begin{array}{l}\text { Phyotril } \\
\text { Fon }\end{array}$ & Whom or & Trantitin & andition & Osmmerta \\
\hline Prond & Fond & PrP Pond & NA & WA & $\mathrm{mon}$ & NA & M & NA & MA & 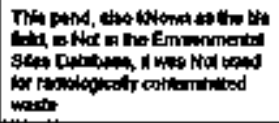 \\
\hline $210-2.21$ & Cnb & 2152026 & Nh & nes & res & m & MA & M & Nh & 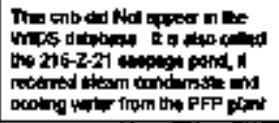 \\
\hline 2941-E & 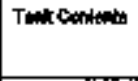 & 8101 & Meptx & 590 ind & giter & sold & | & $\mathbf{H}$ & H4 & 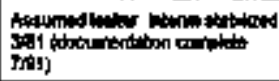 \\
\hline 24le- & Twin Centwo & -100 & WCPLX & 590 내헤 & 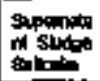 & $\begin{array}{l}\operatorname{low} \\
\sin \\
\operatorname{son}\end{array}$ & 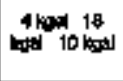 & $\mathbf{H a}$ & Wh & 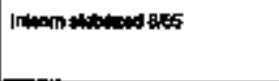 \\
\hline $\begin{array}{l}2418- \\
105\end{array}$ & Tenil Coriming & B-108 & moptx & $590 \mathrm{~lm}$ & stude & sat & $\operatorname{sen}$ & No & un & 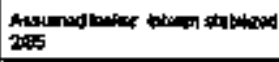 \\
\hline $\begin{array}{l}241-5 \\
104\end{array}$ & Tall cantis & B.104 & HCPLX & $5 \mathbf{~}$ & 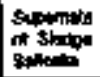 & $\begin{array}{l}\text { Uoluid } \\
\text { solid } \\
\text { sodid }\end{array}$ & I lim & W & NA & hinm atsolated tatos \\
\hline $\begin{array}{l}24164 \\
105\end{array}$ & 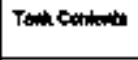 & $6-105$ & HCPLX & 15y & strost & sold & $\ln 20 \%$ & wo & Wh & 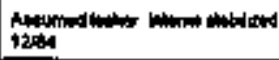 \\
\hline $2+1+8$ & Twen Cerinang & E-108 & wePLx & 580 knd & oppontion & Inid & 1 hist 118 & Ho & NA & 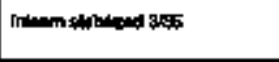 \\
\hline $\begin{array}{l}2+1 \\
1007\end{array}$ & 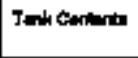 & E-10t & $\operatorname{mct} 2 x$ & exd kpal & in sutes & Leind & $1 \mathrm{~kg}=1 \mathrm{t}$ & ib & $\mathrm{Nh}$ & 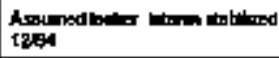 \\
\hline 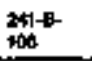 & Tenth Ganinntith & E-165 & wertx & 580 kad & aturaten & sold & othen & $\mathbf{H o}$ & en & 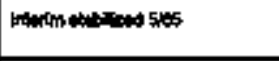 \\
\hline $241-4$ & Yak Carlenth & $0 \times 109$ & mcpex & lad ipa & 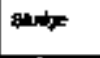 & batd & 12 أl & No & $\operatorname{lin}$ & binm ofited exted \\
\hline
\end{tabular}




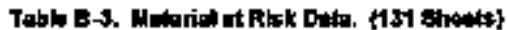

\begin{tabular}{|c|c|c|c|c|c|c|c|c|c|c|}
\hline itima & MMR-Bubuad & MAR-Dengan & IFis- & Cripindind & Type & $\begin{array}{l}\text { Pliticen } \\
\text { form }\end{array}$ & $\begin{array}{l}\text { Vohnt or } \\
\text { inclint }\end{array}$ & 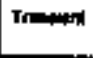 & Cuinity & Cetarints \\
\hline $\begin{array}{l}2+10 \\
110\end{array}$ & Tank Corlemt: & E-110 & metx & Exp kqu & 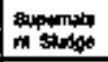 & lapid & 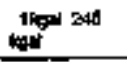 & N & nh & 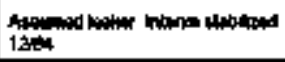 \\
\hline $\begin{array}{l}2+1 \\
111\end{array}$ & Tent Conints & e-111 & nicte & Stat mas & 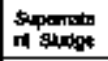 & IFatd & 1 int 290 & $\boldsymbol{H}$ & Wh & 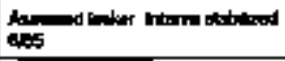 \\
\hline $\begin{array}{l}341+8 \\
112\end{array}$ & Tart Cantate & $\theta \cdot 112$ & NCFIX & استها & 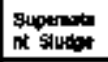 & Eipd & ind & No & Ma & 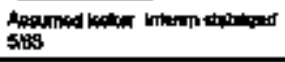 \\
\hline 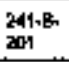 & Tente Contion & $B-201$ & NCPex & 被, 0000 & 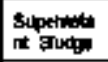 & $\begin{array}{l}\text { Lidud } \\
\text { sold }\end{array}$ & $1 \mathrm{mos} 29$ & No & NA & 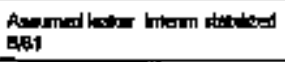 \\
\hline $\begin{array}{l}\text { 3a1-B } \\
\text { and }\end{array}$ & Turt Contantu & B.9\% & FCPXX & 표,0000 & Elatho & Bond & Z7 tyd & No & MH & 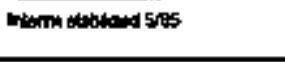 \\
\hline 2ud & Tartik Contenth & B-200 & NCPLX & 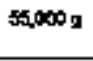 & $\begin{array}{l}\text { supernata } \\
\text { it budi }\end{array}$ & $\begin{array}{l}\text { Litid } \\
\text { soid }\end{array}$ & $1 \mathrm{kgd} 50$ & No & MA & 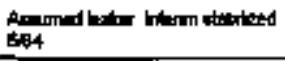 \\
\hline $201-1$ & 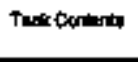 & Butat & MCPXX & $\$ 0000$ & $\begin{array}{l}\text { Euowima } \\
\text { it studed }\end{array}$ & Loind & 1 logd & Mo & Ma & 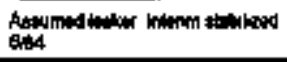 \\
\hline $\begin{array}{l}\text { 241.t. } \\
\text { tols }\end{array}$ & Tont cathoth & 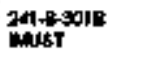 & 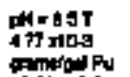 & $3 \operatorname{son}$ & $\begin{array}{l}\text { Supernolit } \\
\text { mat Sold }\end{array}$ & $\begin{array}{l}\text { Lond } \\
\text { and } \\
\text { Soltols }\end{array}$ & 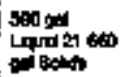 & in & Ma & latind 150 . \\
\hline 2017-7 & Thak Conturita & 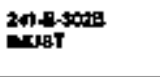 & 4 part woster & 17504 प & $\begin{array}{l}\text { supalen } \\
\text { ndind } \\
\text { studse }\end{array}$ & $\begin{array}{l}\text { Lqued } \\
\text { ind } \\
\text { Sotid }\end{array}$ & ف0 & Ho & Nith & 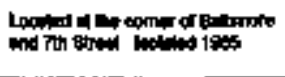 \\
\hline
\end{tabular}




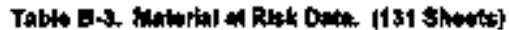

\begin{tabular}{|c|c|c|c|c|c|c|c|c|c|c|}
\hline Find & 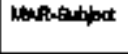 & 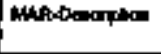 & MAR- & coprosins & Timed & 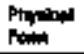 & Walen ax & Tranumptr & trantis & Camminta \\
\hline $\begin{array}{l}241-6 \\
101\end{array}$ & 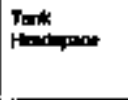 & $=101$ & 㥏 & NA & wh & Wopr & 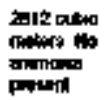 & $\mathbf{m e}$ & man & whe \\
\hline $\begin{array}{l}241 \cdot \mathrm{B} . \\
102\end{array}$ & Then & $1-102$ & ex & NA & $\mathbf{m}$ & Whor & 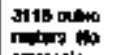 & ho & NA & $\mathrm{m}$ \\
\hline 103 & $\begin{array}{l}\text { Turt } \\
\text { Headenenes }\end{array}$ & 100 & wh & Nh & ran & Wyor & 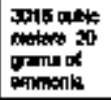 & 14 & NA & $\mathbf{m}$ \\
\hline $\begin{array}{l}2+1-8- \\
104\end{array}$ & 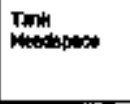 & 8.104 & HA & in & NA & Hear & 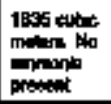 & $\mathrm{NHO}$ & HH & MH \\
\hline 2014 & Tank & D-105 & MA & $\mathbf{m}$ & Nht & Haper & 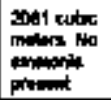 & $\mathrm{No}$ & loh & Na \\
\hline $\begin{array}{l}241-6 \\
106\end{array}$ & Tink & $D-109$ & NA & $\mathbf{m k}$ & hith & Yrapg & 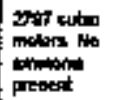 & No & BCA & Nid \\
\hline $\begin{array}{l}241-8-2 \\
109\end{array}$ & Tank & Braf & HA & $\mathbf{m}$ & $\mathbf{m k}$ & vaper & 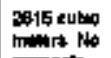 & $\mathrm{No}$ & BCh & Nh \\
\hline $241-8$ & Torth & $\mathrm{B}-100$ & $\mathbf{H A}$ & mh & wh & Maper & atod outctic & $\$ 0$ & Nh & Nh \\
\hline
\end{tabular}




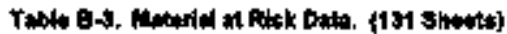

\begin{tabular}{|c|c|c|c|c|c|c|c|c|c|c|}
\hline Prow & MHF-F(t) & WAR-DAamplon & Mar- & cenpeotr & Tyind & $\begin{array}{l}\text { Fryand } \\
\text { Fomm }\end{array}$ & Holking of & Tranawh & Qun & Commita \\
\hline 2Al-B & Tric & B-16 & NA & $\operatorname{lat}$ & Nin & reforar & 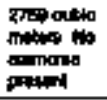 & $\mathbf{m}$ & $\mathbf{N i n}$ & $\mathbf{N u}$ \\
\hline 110 & Tonstapas & B-110 & NA & NA & Nin & Vepor & 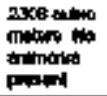 & $\mathbf{r a t}$ & $\mathbf{N}$ & $\mathbf{N}$ \\
\hline $\begin{array}{l}\text { 24tb- } \\
\text { I1t }\end{array}$ & Tent: & $8-111$ & NA & NA & NW & hipor & 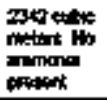 & $\mathbf{H o}$ & NA & NA4 \\
\hline $\begin{array}{l}241+4 \\
113\end{array}$ & Tank & 6.112 & Nh & NA & Wh & Ther & 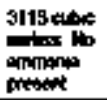 & No & Nh & Nh \\
\hline $\begin{array}{l}241-8 \\
701\end{array}$ & $\begin{array}{l}\text { Tart } \\
\text { Hendterea }\end{array}$ & 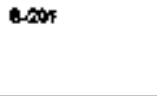 & Nh & N4 & Wh & Hepor & 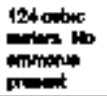 & Noto & Wh & Nh \\
\hline $\begin{array}{l}241 \cdot 8 \\
200\end{array}$ & $\begin{array}{l}\text { Tart } \\
\text { Hoontepect }\end{array}$ & B-202 & mat & Wh & $\mathbf{m e}$ & Trapg & 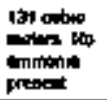 & No & wh & $m$ \\
\hline$\frac{241-k-}{200}$ & 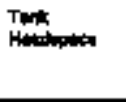 & B-2:03 & 帅 & ma & uh & Yipper & $\begin{array}{l}\text { Donis } \\
\text { Dimiona } \\
\text { Drami }\end{array}$ & No & $\mathbf{m a n}$ & $m$ \\
\hline$\frac{2414}{204}$ & $\begin{array}{l}\text { Taht } \\
\text { Medipen. }\end{array}$ & 1-201 & $M h$ & WA & Mh & Yaper & 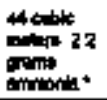 & $\mathrm{No}$ & Bh & 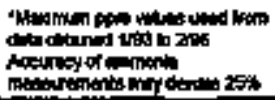 \\
\hline
\end{tabular}




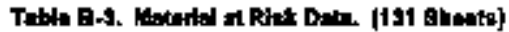

\begin{tabular}{|c|c|c|c|c|c|c|c|c|c|c|}
\hline Fad & MHRSLipat & 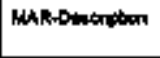 & KAls- & Copen' & $\begin{array}{l}\text { Mrictad } \\
\text { Typu }\end{array}$ & $\begin{array}{l}\text { Pupmid } \\
\text { Form }\end{array}$ & 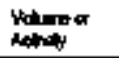 & Totminnt & Oratity & Con \\
\hline 2010 & Thed & $241-B-3018$ & exh & Hent & Wh & Whr & $\begin{array}{l}\text { 13rop ot } \\
\text { Uninown } \\
\text { enperthen }\end{array}$ & $\boldsymbol{W}$ & Hin & Nh \\
\hline 2410 & Tonin & $211-8-5 x$ & $\mathbf{H A}$ & NA & 性 & What & 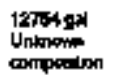 & $\mathbf{H O}$ & 鄅 & Nos \\
\hline $241-8$ & $\begin{array}{l}\text { Red a Trut } \\
\text { Tentid } \\
\text { Ctimlents }\end{array}$ & Nom & $\mathbf{H A}$ & MA & uh & HA & NA & ma & $\mathbf{m}$ & 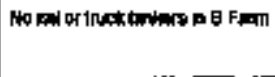 \\
\hline $241+8$ & 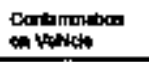 & $\mathrm{NaO}$ & Nh & Wh & $\mathbf{m}$ & NA & wa & N4 & wh & 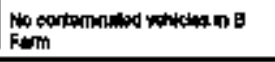 \\
\hline $241 \cdot B$ & 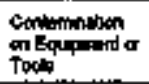 & 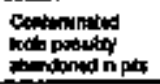 & 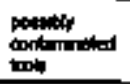 & $\mathrm{NA}$ & $\mathbf{m}$ & sodd & Latidiontr & $N+\sigma^{2}$ & Hh & 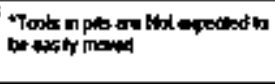 \\
\hline $2+6$ & 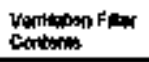 & Phrnar & Nh & NA & H & MA & NA & Wh & MH & H \\
\hline $2+14$ & 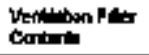 & Annilet & $\mathbf{N}$ & thin & in & Wh & Nin & Nh & $N$ & hus \\
\hline $241+8$ & 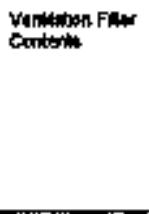 & Brentwit & NA & 1 Rinr ax ax & Non & $\begin{array}{l}\text { Pationila } \\
\text { a }\end{array}$ & 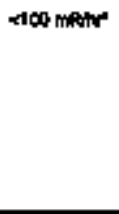 & 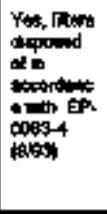 & NA & 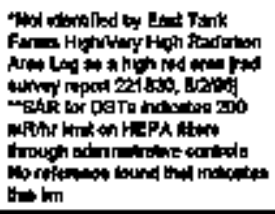 \\
\hline $241+$ & 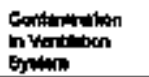 & 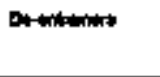 & in & mh & $\mathrm{Nh}$ & Hh & $\mathbf{h k}$ & wh & $\mathrm{Nh}$ & $\mathbf{m}$ \\
\hline
\end{tabular}




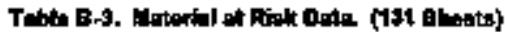

\begin{tabular}{|c|c|c|c|c|c|c|c|c|c|c|}
\hline Find & HNR-8ubing & Wha-Dactiphat & 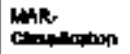 & teapeart & Typentan & $\begin{array}{l}\text { Prifked } \\
\text { Rom }\end{array}$ & $\begin{array}{l}\text { Katmes } \\
\text { Aothing }\end{array}$ & Tramed & Onntry & Amment \\
\hline $2 *-6$ & 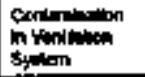 & Eardaces & 해 & × 1 요 & Wh & upind & Rt:Adtat & 禹 & Onepir & 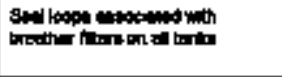 \\
\hline $2+1-6$ & 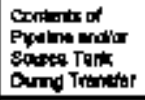 & NA & HA & Mh & Wh & MA & Hah & m & in & 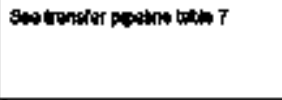 \\
\hline $251-9$ & 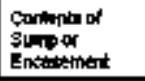 & Nhen & NA & MA & NA & MA & NA & m & Wh & 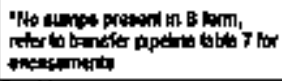 \\
\hline $241-8$ & $\begin{array}{l}\text { Contominito } \\
\text { nPA or Bow }\end{array}$ & P:s & Reantas & NA & an & Bastin & Wh & No & $\mathbf{M h}$ & 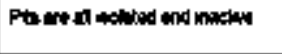 \\
\hline $244 \cdot 0$ & 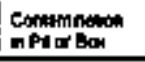 & Onadin bin & Ropider & Hal & $\mathbf{H h}$ & fintule & Nh & Na & $\mathbf{m}$ & 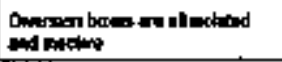 \\
\hline $\begin{array}{l}241-18 \\
151\end{array}$ & 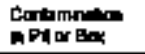 & Crand thax & 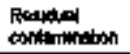 & HA & $\mathbf{m}$ & Geonit & Nh & No & Wh & 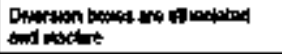 \\
\hline $\begin{array}{l}241 \cdot B \\
150\end{array}$ & $\begin{array}{l}\text { Conlaminion } \\
\text { nfe or Bax }\end{array}$ & Owboth box & Ranktal & 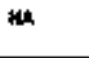 & $\boldsymbol{W h}$ & Abthore & Nin & No & $\mathbf{m}$ & 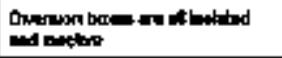 \\
\hline 244-B- & 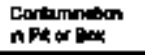 & 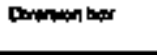 & 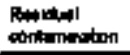 & Wh & mat & Paren's & Noh & No & MH & 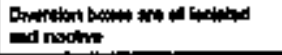 \\
\hline 2414 & 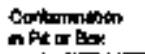 & Dorem in bax & periol & $\mathbf{m h}$ & $m$ & Bacdue & Wh & Noto & m & 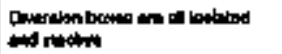 \\
\hline tile & 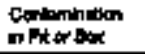 & 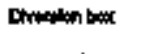 & Rointivid & wh & Mh & Ronsfun & Nh & No & ak & 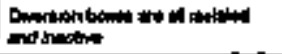 \\
\hline $\begin{array}{l}242-8- \\
+191\end{array}$ & $\begin{array}{l}\text { Gata don } \\
\text { in Piy ar Bax }\end{array}$ & Danowen bew & Repingur & Mn & MR & Fiestater & Mh & W & NA & 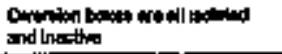 \\
\hline $241+8$ & MARA जt3 & Nhen & $\mathbf{m}$ & Wh & $\mathrm{NH}$ & $M$ & ant & MA & wh & $\mathrm{min}$ \\
\hline
\end{tabular}




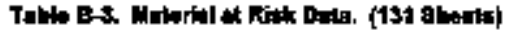

\begin{tabular}{|c|c|c|c|c|c|c|c|c|c|c|}
\hline Find & MAR-gatidal & BAR-Dtheripten & 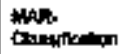 & ctopsing & Thiond & $\begin{array}{l}\text { Pinend } \\
\text { Fom }\end{array}$ & Wotumes & Tameinat & Dientit & Camentis \\
\hline 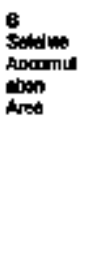 & C)क & 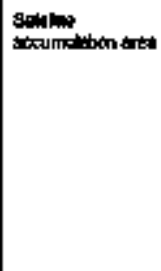 & RCFRA masd & 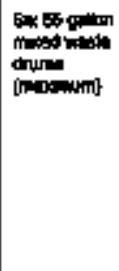 & 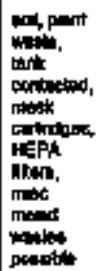 & Sold & low low어에 & $Y \oplus 9$ & |ck & 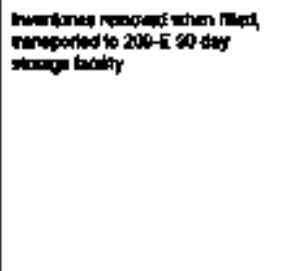 \\
\hline 216B-s5 & Othe & Cnt 265-日.8S & wh & nid & $\mathrm{Nh}$ & NA & WA & Wh & Wh & 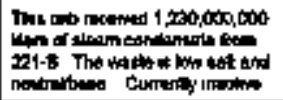 \\
\hline 218-日E & Qther & 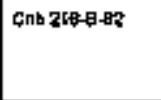 & MA. & N14 & NA & MA & HA & NA & Nh & 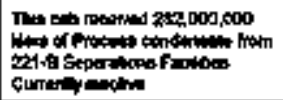 \\
\hline $218-8-68$ & Ollw & $D$ Dhh $216-8-63$ & MA & NA & NA & $N \mathrm{H}_{1}$ & Wh & NA & NA & 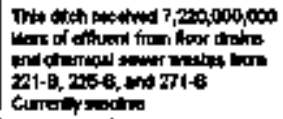 \\
\hline साक्ष.4 & ירים & Ppond zaphas & $\mathbf{m i n}$ & $\operatorname{man}$ & Nh & Laqud & NA & Y⿻肀 & m & 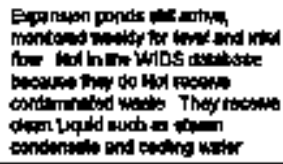 \\
\hline toil- & Tank Carlonth & Cx-101 & kevix & 500 kad & sopening & Uand & $1 \mathrm{ken}$ की & Ho & $\mathbf{m}$ & 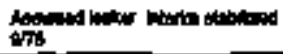 \\
\hline
\end{tabular}




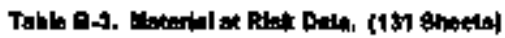

\begin{tabular}{|c|c|c|c|c|c|c|c|c|c|c|}
\hline $\lim$ & MUR-steyart & IHRAacnplan & 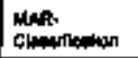 & Aporty & Tymal & $\begin{array}{l}\text { Pinfingel } \\
\text { Fomp }\end{array}$ & $\begin{array}{l}\text { voumea } \\
\text { Adinty }\end{array}$ & Tanchat & Ounty & Cosmata \\
\hline $\begin{array}{l}241+0 K \\
108\end{array}$ & Tartic cominita & $\Delta x-1 x^{2}$ & HePX & $5 \mathrm{sol}$ ket & studip & soid & bolkps & Wh & MH & 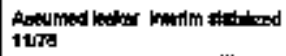 \\
\hline $\begin{array}{l}241-8 x \\
100\end{array}$ & Turt Colestit & ex-103 & Hafix & $500 \mathrm{~kg}$ & 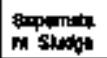 & soind & 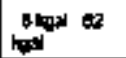 & Mo & Na & 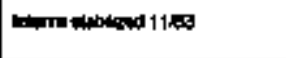 \\
\hline $\begin{array}{l}241-B: 5 \\
104\end{array}$ & Tente Contento & Ex.104 & MCPUX & $500 \mathrm{~kat}$ & in stritod & sold & 3 kgin 89 & 16 & Mh & 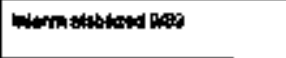 \\
\hline $\begin{array}{l}341-8 x- \\
105\end{array}$ & Tant Dontemis & Ex-105 & $\operatorname{mefux}$ & $5300 \mathrm{yg}$ & 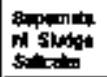 & $\begin{array}{l}\text { Lopd } \\
\text { sold }\end{array}$ & 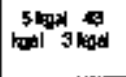 & wa & Nah & 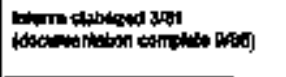 \\
\hline $200-3 x$ & Tarteontets & BX-109 & $\operatorname{met} x$ & $630 \mathrm{bg}$ & sluder & sold & 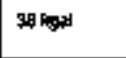 & $\mathbf{m}$ & Na & 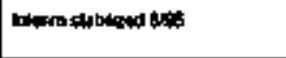 \\
\hline 241-8x & Touk Contints & Ex.to? & wix & 590 ind & 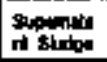 & $\begin{array}{l}\text { Lowd } \\
\text { sold }\end{array}$ & 1 ling $\$ 4$ & $\mathbf{H o}$ & MA & 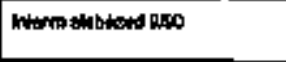 \\
\hline $\begin{array}{l}241-10 \\
108\end{array}$ & JakContete & Ex-108 & $\operatorname{sen} x$ & $\cot 10$ & suref & boly & 20.나에 & $\mathbf{m o}$ & Na & 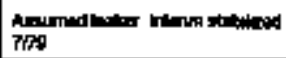 \\
\hline $\begin{array}{l}2+10 x \\
15:\end{array}$ & Toth Contionts & DX.106 & WCPLX & 530 intol & औidts & Sold & $\operatorname{tad} \operatorname{lng}$ & Wo & $\mathrm{MH}$ & 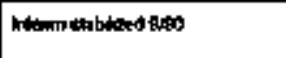 \\
\hline $241-8 x-$ & Tarir Centerts & Dx-110 & $\omega c \ln x$ & sero legd & 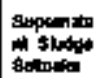 & $\begin{array}{l}\text { uquad } \\
\text { sold } \\
\text { sold }\end{array}$ & 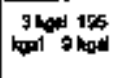 & ma & MA & 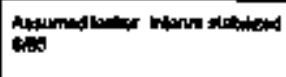 \\
\hline $\begin{array}{l}241-E x \\
111\end{array}$ & Tank conlentin & $E x-111$ & سㅜㄴㅈㅓ & trot & 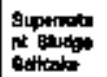 & $\begin{array}{l}\text { uqad } \\
\text { \$old } \\
\text { fold }\end{array}$ & 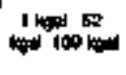 & Nom & an & 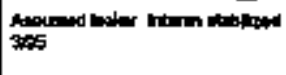 \\
\hline 341-ex & Tark corkents & $B x-117$ & meplx & $\operatorname{leg}$ & 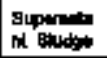 & Sendidid & 1 kad 184 & No & wh & 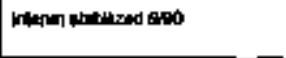 \\
\hline $24400 \mathrm{C}$ & Tart Conturits & 244:x DFAT & 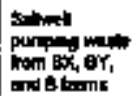 & it kp & ine & Ind gind & 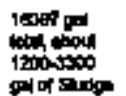 & Nfa & $\mathbf{H}$ & 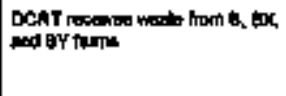 \\
\hline
\end{tabular}




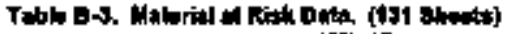

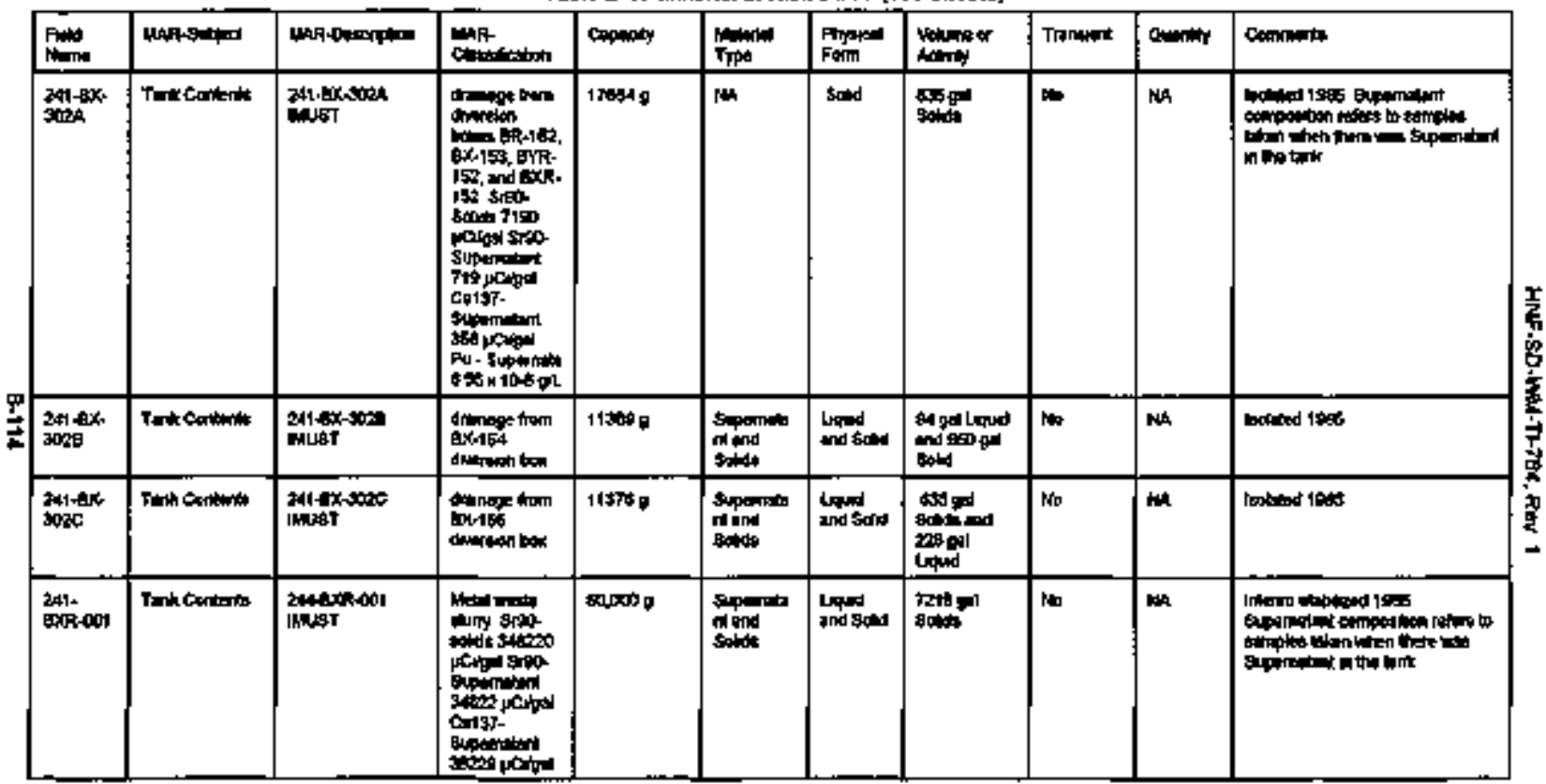




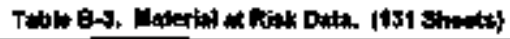

\begin{tabular}{|c|c|c|c|c|c|c|c|c|c|c|}
\hline Find & Awntokibied & HAR-Omaciptopn & Mhr. & Cppant & 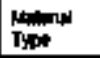 & $\begin{array}{l}\text { Ptyonat } \\
\text { Fon }\end{array}$ & Yow & Thenpapt & angity & tomlarim \\
\hline $\begin{array}{l}\text { 2A1. } \\
\text { ExR-Don }\end{array}$ & 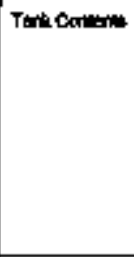 & 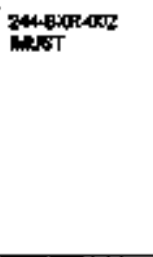 & 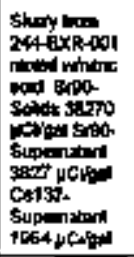 & $18,000 \mathrm{~g}$ & sapmital & Lapld & 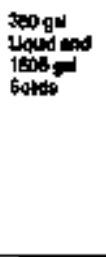 & 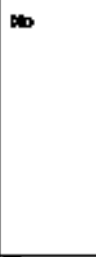 & NA & 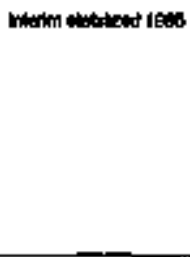 \\
\hline 241- & Tun contiants & $\begin{array}{l}3440 \times 18-603 \\
\text { WUST }\end{array}$ & 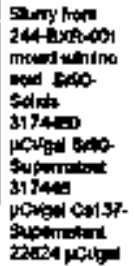 & 15,000 & 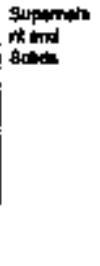 & 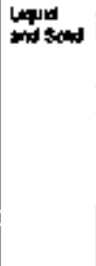 & 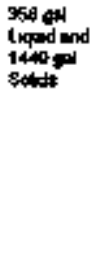 & $\mathbf{N o}$ & NA & 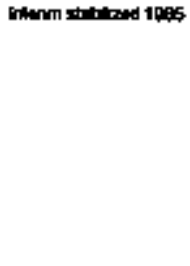 \\
\hline
\end{tabular}


Tubto B-3. Meterial at Rak Dade (131 thotwa)

\begin{tabular}{|c|c|c|c|c|c|c|c|c|c|c|}
\hline Predd & Whatitipact & What Datenpoan & Mtre. & Capent & $\begin{array}{l}\text { Neternt } \\
\text { Typa }\end{array}$ & $\begin{array}{l}\text { Miponad } \\
\text { Form }\end{array}$ & 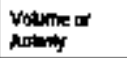 & Tathent & ounty & tomats \\
\hline 241. & Tonscontants & $\begin{array}{l}\text { 206xf-91 } 1 \\
\text { must }\end{array}$ & 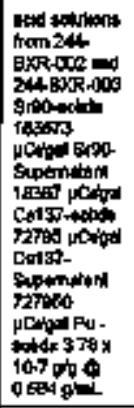 & 50,000 & $\begin{array}{l}\text { Serpandala } \\
\text { ro and } \\
\text { Eolite }\end{array}$ & Land sud & 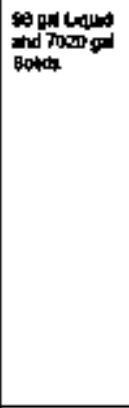 & Ho & HA & 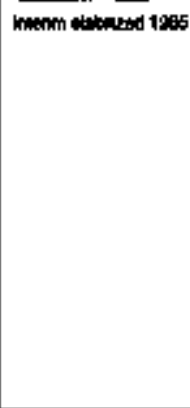 \\
\hline $\begin{array}{l}341-\operatorname{tax} \text { - } \\
101\end{array}$ & 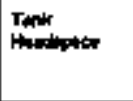 & Ex-1ar & Ma & Ma & Nh & Vuper & 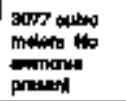 & No & NA & NA \\
\hline $\begin{array}{l}2+1-\mathrm{BK}^{2} \\
\text { I由 }\end{array}$ & Tent: & ax-100 & 채 & 站 & wa & Vomer & 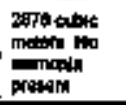 & No & NA & $m a$ \\
\hline $\begin{array}{l}241 \cdot 5 x . \\
1901\end{array}$ & Thik & Ma.105 & Nh & Wh & wh & Wtopr & ation & wh & $m$ & in \\
\hline $201-6 x$ & Thet & ex-104 & 16 & Neth & M & Viper & 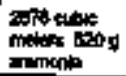 & W & NA & घh \\
\hline
\end{tabular}




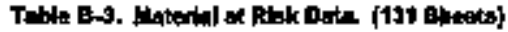

\begin{tabular}{|c|c|c|c|c|c|c|c|c|c|c|}
\hline $\begin{array}{l}\text { Prodid } \\
\text { Nmink }\end{array}$ & Mtarstived & MaA-Ditery & Whe & caspestrt & Thent & $\begin{array}{l}\text { Pryoked } \\
\text { Fomm }\end{array}$ & Wother & Thomitat & Ousonty & Conmets \\
\hline $\begin{array}{l}241-896.0 \\
105\end{array}$ & Thin & Ex-106 & Nh & Nh & m & Hepor & 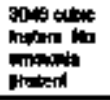 & 10 & MH & Nh \\
\hline $24 \|-4 x$ & $T_{\text {Thenditace }}$ & $\operatorname{ex}-109$ & ath & $\mathrm{NA}$ & ton & $\operatorname{vap}$ & 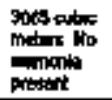 & 饰 & Wh & $\mathbf{N}$ \\
\hline 24letex. & $\begin{array}{l}\text { Turt } \\
\text { Hentepoor }\end{array}$ & $\mathrm{ax}-10 \mathrm{~s}$ & $\boldsymbol{M n}$ & $\mathrm{MH}$ & Nid & Wapr & 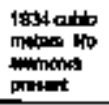 & No & wh & Wh \\
\hline $\begin{array}{l}241 \cdot 4 x- \\
108\end{array}$ & Tart & $\mathbf{a s}_{-100}$ & en & Na & Na & Vhps & 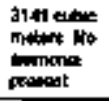 & $\mathbf{H o}$ & WA & NAS \\
\hline $\begin{array}{l}241-8 x \\
100\end{array}$ & Timk & $\theta x-16$ & Na & un & Nh & vipor & 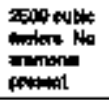 & Noto & $m$ & $\mathrm{Mh}$ \\
\hline $\begin{array}{l}241-6 \times- \\
110\end{array}$ & Tronk & $E x-110$ & NA & wh & Nh & vapor & 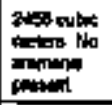 & No & m & $\mathrm{m}$ \\
\hline $\begin{array}{l}241+4 x . \\
111\end{array}$ & Trah & $0 x-111$ & Nh & $\mathrm{WA}$ & Nh. & vepor & 隹 & No & $\mathbf{m}$ & $\mathrm{men}$ \\
\hline 2011-8X & 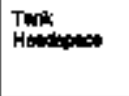 & $0 \times-112$ & MA & $m$ & Mh & Vipor & 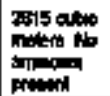 & No & Nh & Wh \\
\hline
\end{tabular}




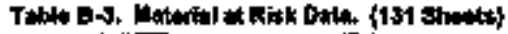

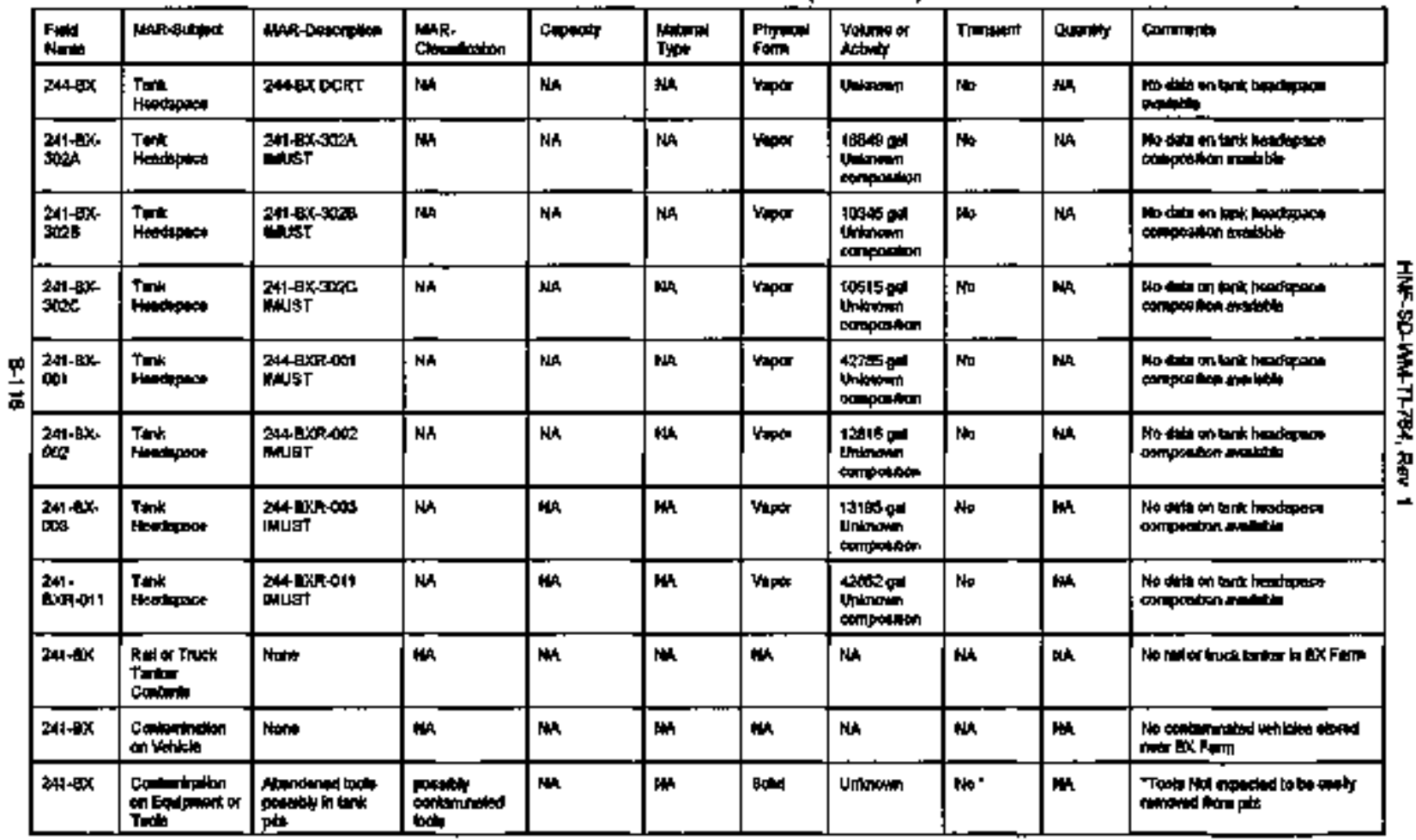




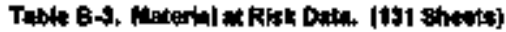

\begin{tabular}{|c|c|c|c|c|c|c|c|c|c|c|}
\hline 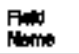 & 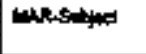 & MuRobartipo & 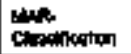 & cepreility & $\frac{1}{T h}$ & $\begin{array}{l}\text { Elpored } \\
\text { Foim }\end{array}$ & Yodines or & Trthowd & קולמשום & Cartinas: \\
\hline $241+\operatorname{tax}$ & 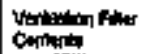 & Pronary & $\mathbf{H i t}$ & Nh & NA & an & Ha & Mh & MA & Nh \\
\hline $2+1+8 x$ & Wontulim Fum & Anrulas & 中h & NA & MA & Wh & M & Wh & Na & 的 \\
\hline 241 & 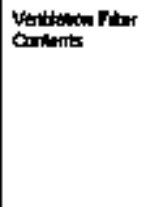 & Dextiter & $\mathbf{m t}$ & 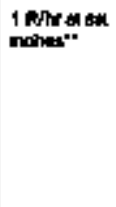 & Nh & Patborlat & K100 What & 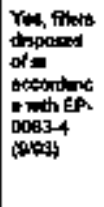 & Wh & 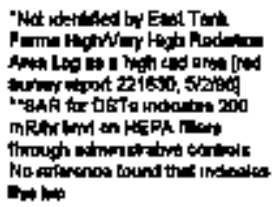 \\
\hline $2410 x$ & 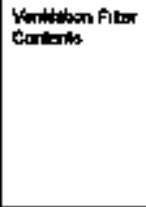 & 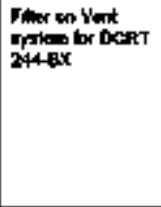 & in & 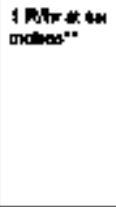 & Wh & Partialt & tobowntr & $v_{m}$ & NH & 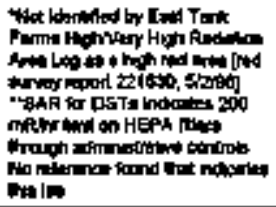 \\
\hline $24+x$ & 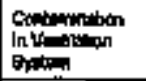 & Dodiftuman & inh & Wh & H & Non & m & Noh & $m$ & 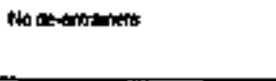 \\
\hline $241+x$ & 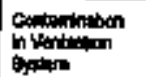 & 些 & 영 & C I o & Nin & هيما & Ridudud & No & Ont pus & 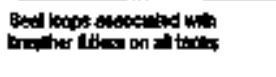 \\
\hline $211+8 x$ & Conimat d & wh & Wh & Nh & $\mathbf{H h}$ & 哺 & $\mathbf{H h}$ & Wh & $m$ & 7obs \\
\hline
\end{tabular}




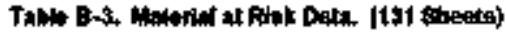

\begin{tabular}{|c|c|c|c|c|c|c|c|c|c|c|}
\hline Fatd & Mukstyen & 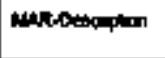 & Wher & CFpond & Tripe & $\begin{array}{l}\text { Phipil } \\
\text { Fam }\end{array}$ & $\begin{array}{l}\text { vohmet tr } \\
\text { netrint }\end{array}$ & Thanote & ث口माम & Dom ats \\
\hline $2416 x$ & 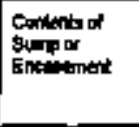 & " & $\mathrm{km}$ & $15 \times 00^{\circ}$ & Hit & wh & NA & No & $\mathrm{Hh}$ & 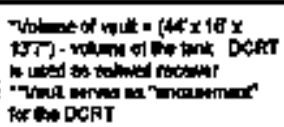 \\
\hline $241-8 x$ & $\begin{array}{l}\text { Contumnthar } \\
\text { AP/ or Bot }\end{array}$ & 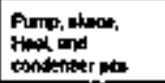 & Revated & Wh & $\mathbf{m}$ & Anudun & Hin & ino & Wh & 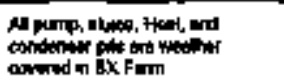 \\
\hline $241-\operatorname{Ex}$ & $\begin{array}{l}\text { Conlummation } \\
\text { DPA ar Box }\end{array}$ & $\begin{array}{l}\text { Ran mone sernce } \\
\text { pit }\end{array}$ & minter & 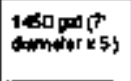 & $\mathbf{m i n}$ & Lnad & MA & No & wh & 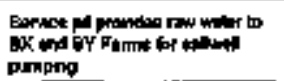 \\
\hline 24I-BX & $\begin{array}{l}\text { Contaminam } \\
\text { n Fitareas }\end{array}$ & 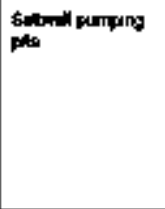 & Reriannation & 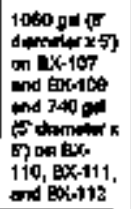 & $\mathbf{m}$ & Routh & mh & $\mathbf{n}$ & NA & 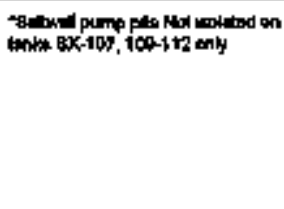 \\
\hline $2449 x$ & $\begin{array}{l}\text { contentrian } \\
\text { in Ptarexp }\end{array}$ & 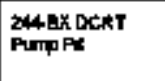 & fingud & $\begin{array}{l}2,000 \% 9 \\
17 \times 17 \times 7 \\
6 j\end{array}$ & $\mathrm{ran}$ & Roukt & $\mathbf{m}$ & mo & man & $\mathbf{w}$ \\
\hline $2+4: x$ & 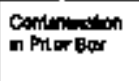 & $\begin{array}{l}\text { 2448x DCRT } \\
\text { Pith Put }\end{array}$ & Ritudid & 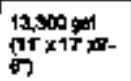 & Nh & Pontand & wh & th & in & fats \\
\hline $244+2 x$ & $\begin{array}{l}\text { Contindrion } \\
\text { on Pite Box }\end{array}$ & 244BX DCRT & fibtad & 60 gitur & Nh & Ponth & Wh & Wh & $\mathrm{Nh}$ & Wh \\
\hline $\begin{array}{l}\text { 241. } \\
\text { EXR-151 }\end{array}$ & $\begin{array}{l}\text { Contunnitan } \\
\text { In Pid of Bew }\end{array}$ & Dintrationbax & ritadian & m & Nh & findun & wh & No & ist & 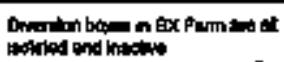 \\
\hline 24indes & 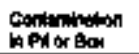 & Danrwontow & Rinton & NA & NH & Panctuta & HA & 的 & Hh & 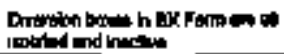 \\
\hline
\end{tabular}




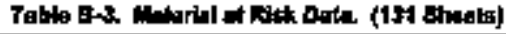

\begin{tabular}{|c|c|c|c|c|c|c|c|c|c|c|}
\hline Fadd & 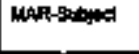 & 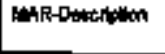 & $\begin{array}{l}\text { MHR- } \\
\text { C.r.pingan }\end{array}$ & Crepety & Thind & $\begin{array}{l}\text { Powmed } \\
\text { Romm }\end{array}$ & $\begin{array}{l}\text { Vaumet } \\
\text { intomfy }\end{array}$ & Tranument & Duanity & Comnerika \\
\hline 241- & 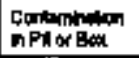 & Conden bux & Reapidid & NA & Wh & Rasko: & NA & No & An & 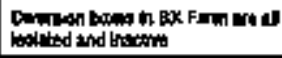 \\
\hline $241+B X$ & Serrats ais & 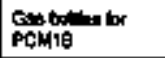 & $\begin{array}{l}\text { 60ik in } 10 \% \\
\text { lation }\end{array}$ & atraba & $\mathbf{H}$ & Bax & 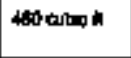 & $Y=$ & 2bottle: & When \\
\hline 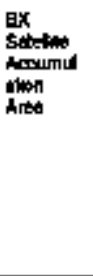 & Coth & 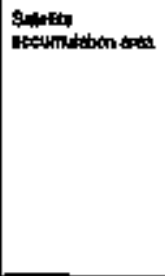 & 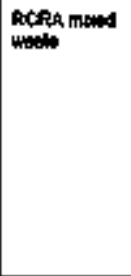 & 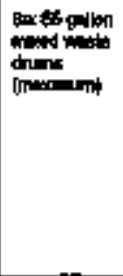 & 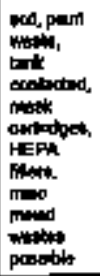 & Baind: & low lewil outiol & Yes & 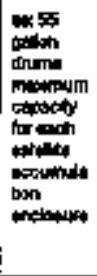 & 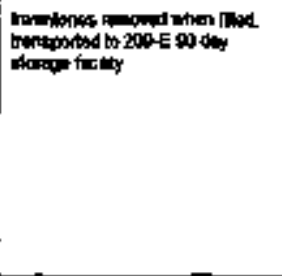 \\
\hline $\mathbf{2 + 1 - 9 x}$ & other & 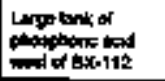 & phespitions & $\begin{array}{l}\text { hooo } \\
\text { chlorits? }\end{array}$ & Non & Loped & 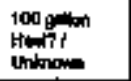 & Y\$: & $1 \mathrm{kmm}$ & 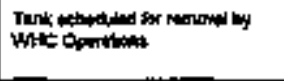 \\
\hline $24 \mid \cdot B r:$ & Taitcocterts & Err.HOH & NEPLX & tado,ood & Eudie & gotd & 100 low 208 & $\mathbf{m}$ & KA & 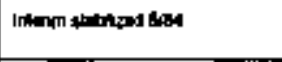 \\
\hline $\begin{array}{l}2402 \\
102\end{array}$ & Tint Contands & ENROZ & MEPUX & $1=000$ & onilensing & fond & $2 m$ d & H. & NA & 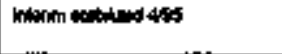 \\
\hline $\begin{array}{l}241+84 . \\
103\end{array}$ & Tank certan & trr-xos & HсPபx & TBa,ood & Budie & $\operatorname{sind}$ & 6lag & Ap & NA & 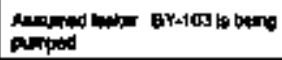 \\
\hline $\begin{array}{l}\text { 30헌 } \\
104\end{array}$ & Tonitcothins & Groth & NEFபx & Trad,000 & Bindos & sand & phom & $\mathbf{m}$ & Nh & 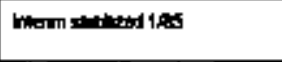 \\
\hline $\begin{array}{l}241-1 y- \\
104\end{array}$ & Tonk Corimb & BY-10S & HCFX & $T 58,040$ & gtaptor & soind & 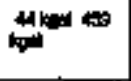 & Noth & Nh & 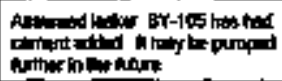 \\
\hline $\begin{array}{l}241-t y \\
100\end{array}$ & Tank Corimin & $\theta r+10 d$ & KCPUx & 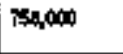 & otidis & solid & Fin 5if & 14.4 & Nh & 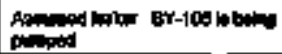 \\
\hline
\end{tabular}




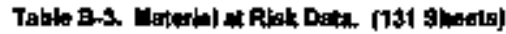

\begin{tabular}{|c|c|c|c|c|c|c|c|c|c|c|}
\hline $\begin{array}{l}\text { Piatd } \\
N \in \operatorname{mon}\end{array}$ & MuR-sut|ct & Mterdagpton & Cint. & Cipaty & The & 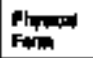 & 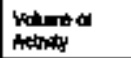 & Trartats & ontity & conmonta \\
\hline 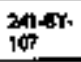 & Taincoilth & EY.IOF & HCPUX & 狩, & sold: & $\begin{array}{l}\text { Sold } \\
\text { bold }\end{array}$ & bollat 203 & No & $\mathbf{m}$ & 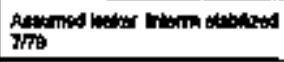 \\
\hline $\begin{array}{l}\text { 241-4r- } \\
\text { lot. }\end{array}$ & TـhConists & Ertan & NEPLX & rt:-0.000 & Eudqu & sold & 154 low 74 & No & $\mathbf{m k}$ & 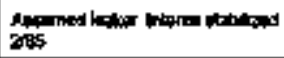 \\
\hline $\begin{array}{l}241-E Y, \\
100\end{array}$ & Tenil Conturts. & Errog & mefr & एम्भ,000 & studed & Sand & ofinged 340 & m & $M A$ & 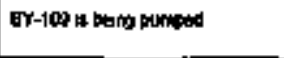 \\
\hline $\begin{array}{l}261-8 \mathrm{r}- \\
110\end{array}$ & Talk Comineng & 白Y-110 & $\operatorname{mat} x$ & tots, toon & Buction & sand & 10 lag & m & Ban & 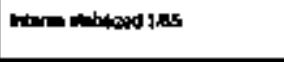 \\
\hline $\begin{array}{l}241.5 \% . \\
111\end{array}$ & Tatik Cormits & $6 \%+111$ & $\operatorname{matx}$ & 850,00 & $\begin{array}{l}\text { shrotpe } \\
\text { stiplete }\end{array}$ & sold & 21 log 406 & W & NA & 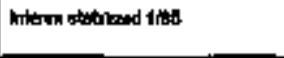 \\
\hline $\begin{array}{l}241-E Y- \\
1+12\end{array}$ & Tarth Conkits & Er:112 & repex & rostow & 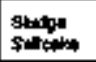 & satid & $\underset{2000}{5}$ & $\mathbf{m}$ & mon & 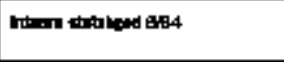 \\
\hline $\begin{array}{l}2010-5- \\
201\end{array}$ & Tonk Conlents & $\begin{array}{l}216-84-201 \\
\text { DIST }\end{array}$ & 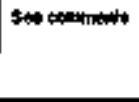 & 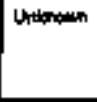 & thintion & Uhworan & Untornotn & $\mathbf{W}$ & MA, & 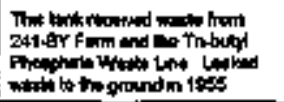 \\
\hline $\begin{array}{l}\text { 2Al-Err } \\
101\end{array}$ & 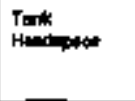 & $6 r+10$ & NA & NA & m & Yapar & 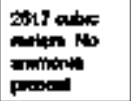 & Ab & Mh & wh \\
\hline$\underset{102}{241-6 Y}$ & Trestepict & Eritaz & wh & nos & ra & waper & 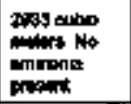 & No & end & NG \\
\hline $\operatorname{lnt}_{105}$ & Tint & EY-103 & $\mathrm{kH}$ & Wh & wh & thor & 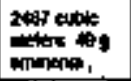 & $H$ & NA & 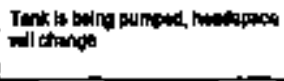 \\
\hline 104- & 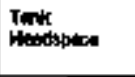 & घY-104 & An & WA & $\mathrm{man}$ & प̆ap: & 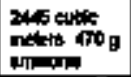 & $\mathrm{NH}$ & mh & NA \\
\hline
\end{tabular}




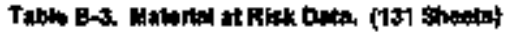

\begin{tabular}{|c|c|c|c|c|c|c|c|c|c|c|}
\hline $\begin{array}{l}\text { Finid } \\
\text { Patits }\end{array}$ & NAR-Subuct & 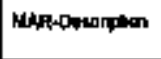 & 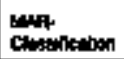 & Cropesty & Typtom & $\begin{array}{l}\text { Phyladid } \\
\text { Form }\end{array}$ & Notint & Tt: & Qupinter & cormments \\
\hline $\begin{array}{l}2410 \%+ \\
105\end{array}$ & $\begin{array}{l}\text { That } \\
\text { Hinedupused }\end{array}$ & DY.10s & Wh & NA & Nh & Vtpor & 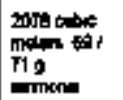 & Not & $\mathrm{WH}$ & Tak Grbe pumped n lhe \\
\hline $\begin{array}{ll}2+16 Y \\
169\end{array}$ & Tand & EYमक & $\mathbf{m}$ & NA & wh & trapd & 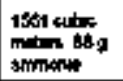 & Na & Nh & 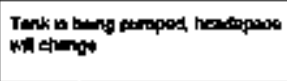 \\
\hline $\begin{array}{l}241 \text { Gr. } \\
100\end{array}$ & Thank & Br.tol & $\mathrm{man}$ & Had & $\mathbf{m}$ & Wapor & 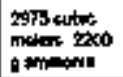 & Ho & Wh & MA \\
\hline $\begin{array}{l}241+4 r . \\
168\end{array}$ & Tink & Drustid & $\mathrm{mum}$ & has & $\mathbf{M t}$ & Whap & 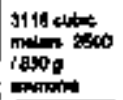 & No & Wh & Nh \\
\hline $\begin{array}{l}241 \cdot \mathrm{BY} . \\
110\end{array}$ & Tum & tYr110 & wa & $\mathbf{m}$ & $\mathrm{kn}$ & Wapor & 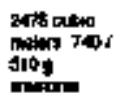 & $\mathbf{m}$ & MA & wa \\
\hline $\begin{array}{l}\text { 2A1-BY- } \\
\text { dII }\end{array}$ & Think & GY-111 & NA & Wh & $\mathbf{H A}$ & Wener & 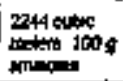 & Nin & wh & WA \\
\hline $\begin{array}{ll}2114 \\
112\end{array}$ & Torle & EY.112 & NA & $\mathbf{H h}$ & $M$ & riper & Pad oflo & $\mathbf{H b}$ & HA & Nh \\
\hline 2lantr. & Trok & रस्सासा & Latam & $\mathrm{kH}$ & Undentent & 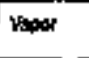 & Latolomenten & Nh & HA & 㔣 \\
\hline
\end{tabular}




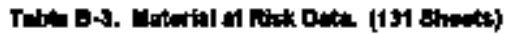

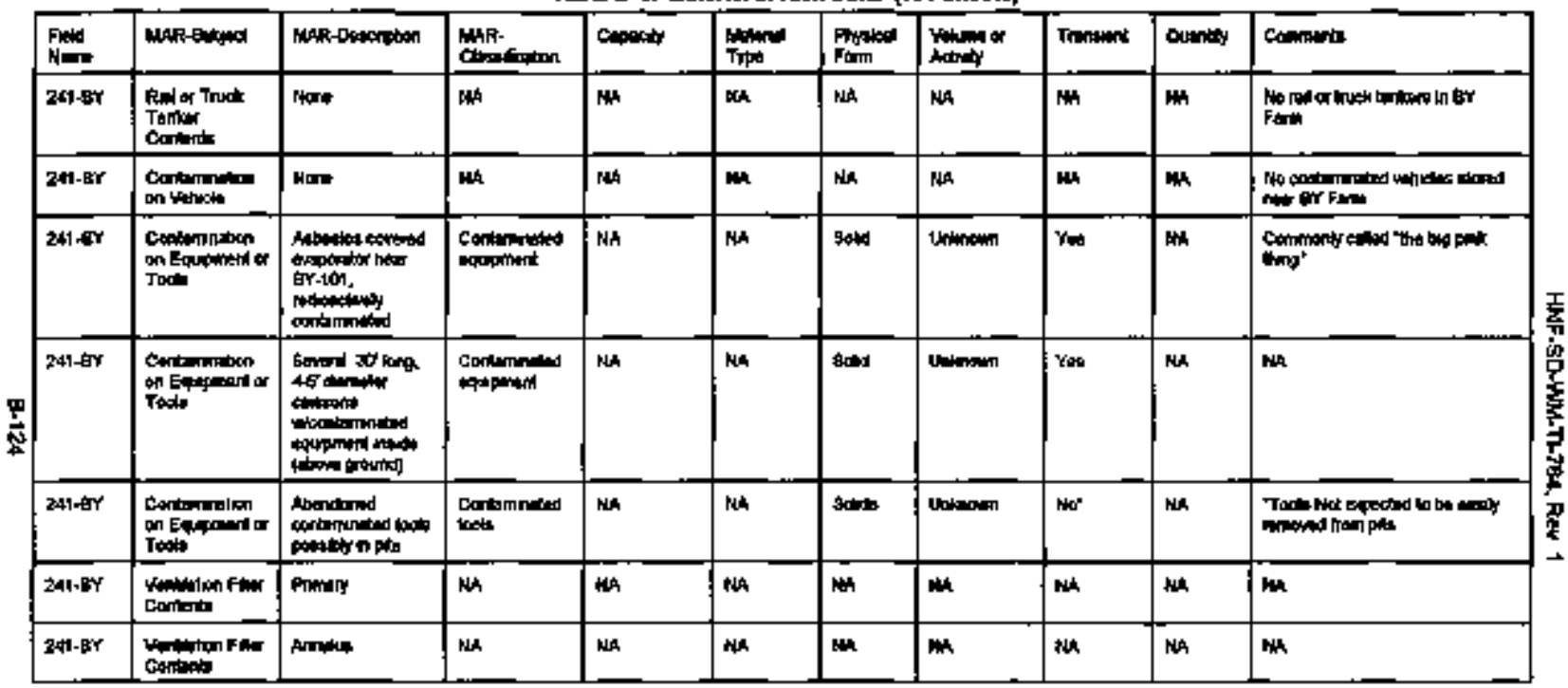




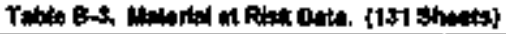

\begin{tabular}{|c|c|c|c|c|c|c|c|c|c|c|}
\hline Find & Nak-guting & 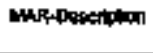 & nation- & Cenpactity & Typum & $\begin{array}{l}\text { Fowow } \\
\text { Fom }\end{array}$ & volum er & Trtment & Ounty & 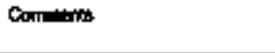 \\
\hline 241.sr & Volithonfers & Eroster & $\mathbf{m}$ & I Aln t. & Nh & 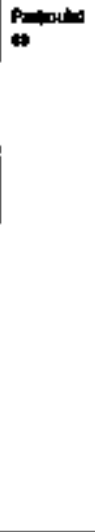 & 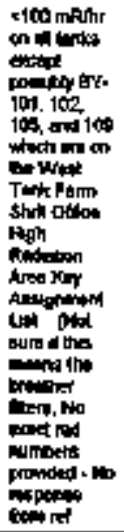 & 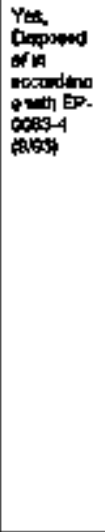 & Nin & 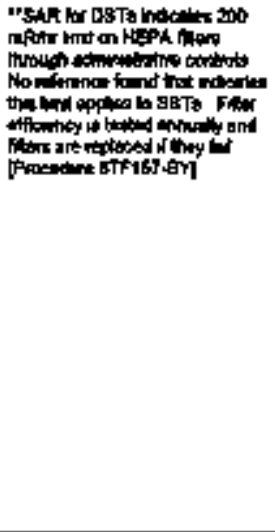 \\
\hline $2414.8 Y$ & 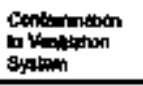 & Detentianerts & Nh & NA & Ma & in & Wh & Ha & Kh & 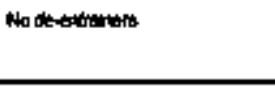 \\
\hline $241.5 Y$ & 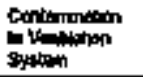 & 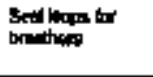 & an & لالبو 1ج & M & Laqued & Fitoder & $\omega$ & Ones & 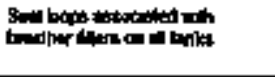 \\
\hline $24+48$ & 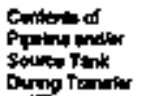 & Nh & Ha & $\mathrm{m}$ & NH & 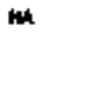 & NA & MN & MA & 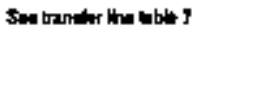 \\
\hline
\end{tabular}




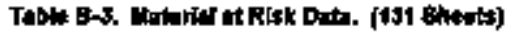

\begin{tabular}{|c|c|c|c|c|c|c|c|c|c|c|}
\hline Fand & MAREGblingt & 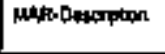 & 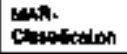 & Cngonts & wirtod & $\begin{array}{l}\text { Phirivis } \\
\text { Fand }\end{array}$ & 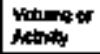 & Tnलment & Dunchy & Comatoris \\
\hline $245-6 r$ & 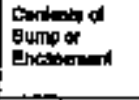 & Nh & $\mathbf{w h}$ & NA & Wh & H & Noh & Wh & NA & 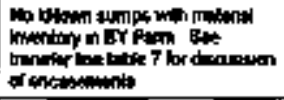 \\
\hline $741-E Y$ & 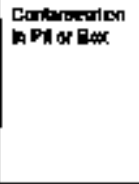 & sulwel pum git & 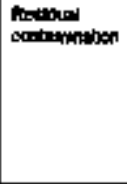 & 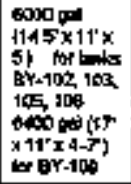 & NH & Pretmitus & NA & $\mathbf{H o}$ & NA & 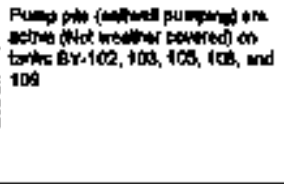 \\
\hline $241-B r$ & $\begin{array}{l}\text { Contamansont } \\
\text { in Adar Bor }\end{array}$ & 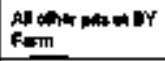 & Rindus & Wh & $\mathbf{m}$ & Areadue & NA & $\mathrm{kho}$ & $\mathbf{m h}$ & N win roept pula pat Hoted \\
\hline OMk-15: & 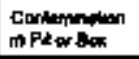 & Dwerant tax & Randur & wh & wh & 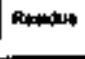 & wa & $\mathbf{N o}$ & $\cos$ & 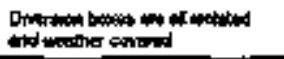 \\
\hline $\begin{array}{l}\text { 241. } \\
\text { BYR-15: }\end{array}$ & $\begin{array}{l}\text { Coptmondan } \\
\text { In Ptor Bew }\end{array}$ & Orthten bex & 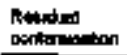 & inth & ren & Reuder & wh & No & bch & 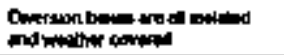 \\
\hline $\begin{array}{l}\text { 2H: } \\
\text { BYrR-154 }\end{array}$ & $\begin{array}{l}\text { Cotaminan } \\
\text { nP Ptor Den }\end{array}$ & Cneron the & 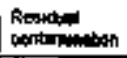 & ux & an & Relevolus & wh & No & wh & 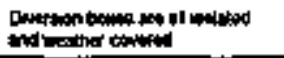 \\
\hline 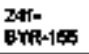 & $\begin{array}{l}\text { Cutitimatinan } \\
\text { in Pitereas }\end{array}$ & Ontex & Rukt. & int & $\mathbf{r a n}$ & Amodr & Wh & o & Noh & 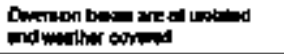 \\
\hline 2†-EY & SArk 319 & 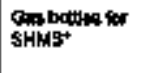 & Hotrogens' & 490 ants & Non & Qen & topantas & Yo* & $\begin{array}{l}\text { 3bedilat } \\
\text { pertarte }\end{array}$ & 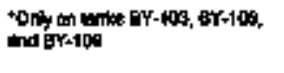 \\
\hline 24t=y & Othex & Hond & Nh & Wh & How & kb & HA & NA & wh & \\
\hline 2 & Taitconturits & $c-101$ & $\operatorname{Mc} i x$ & 500 ked & studo & Sold & B8lasin & $\mathbf{k}$ & Nh & 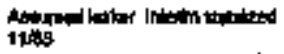 \\
\hline $\begin{array}{l}241-c- \\
\text { tod }\end{array}$ & Tolecenturits & ton & $\mathbf{D E}$ & 601010 & Endes & gend & 3*10 & Ho & Na & 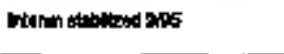 \\
\hline
\end{tabular}




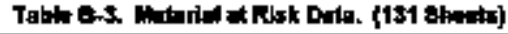

\begin{tabular}{|c|c|c|c|c|c|c|c|c|c|c|}
\hline Prdd & WAft-Gatinct & 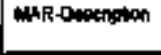 & Mols & Cupary & Therend & $\begin{array}{l}\text { Pholiev } \\
\text { Fom }\end{array}$ & $\begin{array}{l}\text { Votume ar } \\
\text { Mothep }\end{array}$ & Tranaser & Ounery & Comentite \\
\hline 2ha- & 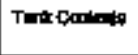 & c-100 & $\operatorname{NcF} x$ & 5980 ha에 & it steing & Lind & $\lim _{\log }$ & M & m & 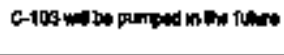 \\
\hline 2416 & Tont Canitalt & Coston & cc & 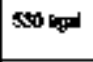 & shudet & odd & 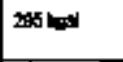 & lb & wh & 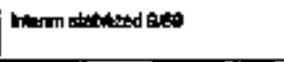 \\
\hline 205 & Tritcoing & c-16s & NCFux & 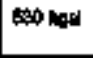 & anpintim & Lown & 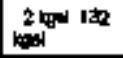 & w & IA & Nimen thinged 1075 \\
\hline 2410 & Torilentions & 106 & mats & Bat kagl & $\begin{array}{l}\text { supeitath } \\
\text { nt Eindor }\end{array}$ & ingerd & la & Wh & wh & 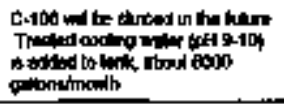 \\
\hline $241-c-$ & Tret Corknita & $\cos \theta$ & $\mathrm{pC}$ & 530t nagl & surap & Bend & لإبا & us & MA & Inknm ditend ans. \\
\hline $241-C-$ & Tand Conkrm & $6-108$ & wePx & 590 相标 & Exwot & 5064 & 9 & $m$ & NAS & 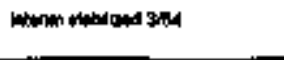 \\
\hline 100 & Tara Corminte & c-1bo & mcPLx & Stat in & 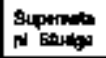 & Upat & 1109 की & $\mathbf{N e}$ & MA & 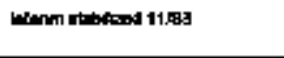 \\
\hline $\begin{array}{l}2+19-5- \\
110\end{array}$ & Tran Cordinge & $0-110$ & oc & $500 \mathrm{~kg}$ & 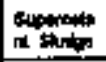 & boid & $1 \mathrm{~h}=\mathrm{in}$ & mon & NA & 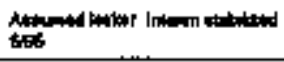 \\
\hline $\begin{array}{ll}241-C-C \\
111\end{array}$ & fand cankertis & c-t11 & 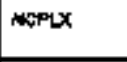 & 530 wat & stritgen & Baid & artyed & No & HA & اله \\
\hline $241-0$ & Tonk Canatos. & $6-112$ & ax & d & Alindot & sotid & $10+101$ & $\mathrm{Ma}$ & Wh & htem \\
\hline 201 & Truk Gophesks & $c-201$ & M⿻上丨 & $56 \mathrm{kenl}$ & Enofot & Bolat & 2 wed & No & NA & 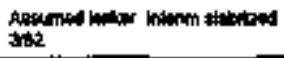 \\
\hline 202 & Tank coukente & cale & ENPTY & 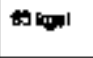 & tontipa & Bold & $1 \mathrm{~kg}$ & No & MA & 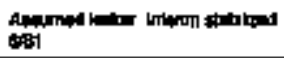 \\
\hline $241-t$ & Tenk Coninots & $\cos \theta$ & $\operatorname{mex}$ & 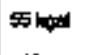 & squases & sand & $5 \mathrm{kpl}$ & Ha & H & 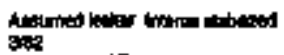 \\
\hline
\end{tabular}




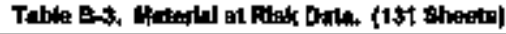

\begin{tabular}{|c|c|c|c|c|c|c|c|c|c|c|}
\hline $\begin{array}{l}\text { Fipld } \\
\text { Henth }\end{array}$ & MRsibea & 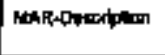 & 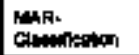 & Expenty & Ty & $\begin{array}{l}\text { Phym } \\
\text { Foim }\end{array}$ & $\begin{array}{l}\text { Undind of } \\
\text { intinty }\end{array}$ & Tomom & ontronst & Conterime \\
\hline 2412 & Tank Contwess & $\cos 4$ & Replx & 夏战 & slutst & sod & $3 \mathrm{~mol}$ & Nta & Nh & 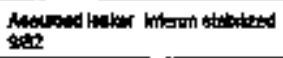 \\
\hline $244-C R$ & Tenk Coments & CR Hall lant DO9 & הinon & SAlon & Whangen & Unionom & Vuangen & No & Nith & 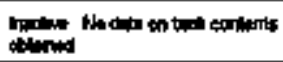 \\
\hline 244er & Tank Conions & 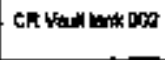 & Unthem & $15 \mathrm{~kat}$ & Intim & Uhberan & Intakn & No & NA & 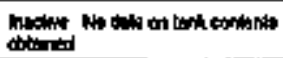 \\
\hline THA-CR & Tarik Cortente & 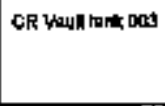 & 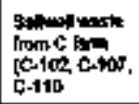 & IE & Nan & ind sond & |0se & Noto & wh & 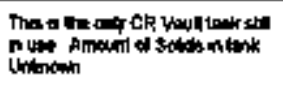 \\
\hline $244-69$ & Tank Content & CR Yand hand Di1 & Uhiphye & الهيh & Unkapan & Untomph & 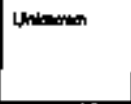 & $\mathrm{Not}$ & An & 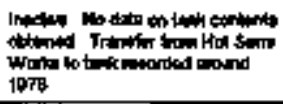 \\
\hline $\begin{array}{l}\text { zall-c- } \\
\text { 3otc }\end{array}$ & 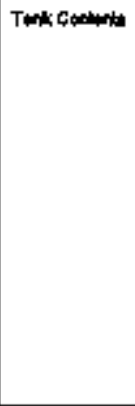 & $\begin{array}{l}\text { 2h-c-3otc } \\
\text { EAt }\end{array}$ & 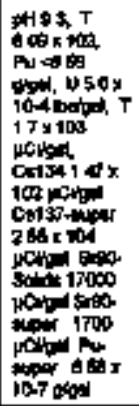 & 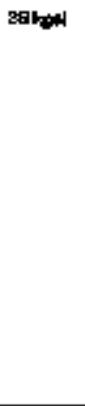 & $\begin{array}{l}\text { Bafinim } \\
\text { indand } \\
\text { andot }\end{array}$ & and sold & 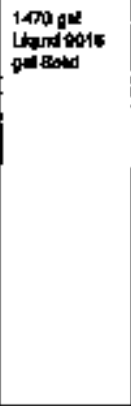 & 幅 & $\mathbf{m}$ & لبهاسابط| \\
\hline $\begin{array}{l}241 / 45 \\
101\end{array}$ & Toll & tin & $\mathbf{w n}$ & the & Nh & varor & 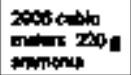 & $\boldsymbol{m}$ & Bh & Hik \\
\hline
\end{tabular}




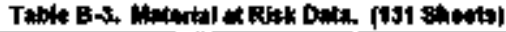

\begin{tabular}{|c|c|c|c|c|c|c|c|c|c|c|}
\hline Find & 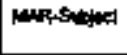 & with-Dentrphes & chater & C7ond & Tytom & $\begin{array}{l}\text { Pinford } \\
\text { Fomm }\end{array}$ & voluma & Thempat & quartity & 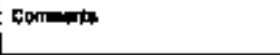 \\
\hline 1002 & $\begin{array}{l}\text { Tank } \\
\text { Honispose }\end{array}$ & c.ton & the & HA & Nh & Whor & 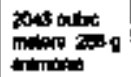 & blo & Nin & Int \\
\hline 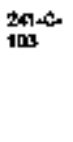 & Thak & coris & H & Ha & hik & Vher & 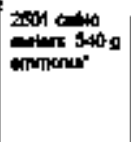 & ib & in & 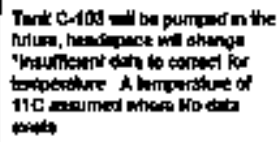 \\
\hline 2412 & Trank & $c-104$ & $\mathbf{m}$ & NA & Wh & riphor & 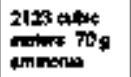 & $\mathrm{kb}$ & Nh & HA \\
\hline $\begin{array}{l}\text { 2a1-c- } \\
105\end{array}$ & Twindipaes & c-1es & $\mathrm{MAN}$ & MA & Wh & 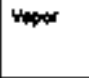 & 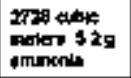 & 10 & Wh & MH \\
\hline 100 & Timbingere & C-10d & $\mathbf{H}$ & MA & ith & Hopor & 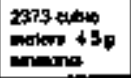 & wo & wh & 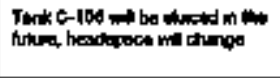 \\
\hline $\begin{array}{l}211-0 \\
\text { 10? }\end{array}$ & 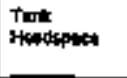 & \& & NA & $\operatorname{ch}$ & w & Yapor & 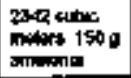 & ra & Wh & NA \\
\hline $\begin{array}{l}211-0 . \\
103\end{array}$ & Twatesea & تهم & NA & $m$ & $\mathbf{m}$ & Yepor & 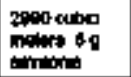 & $\mathbf{r a t}$ & wh & wh \\
\hline $\begin{array}{l}\text { 291-6- } \\
\text { IDe }\end{array}$ & 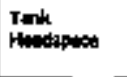 & 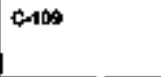 & H & Nh & $\mathbf{H}$ & Vippr & 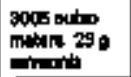 & 10 & $N$ & NA \\
\hline 2110 & Tak & 中110 & Wh & $\mathbf{m}$ & Hoh & Vhop & 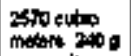 & No & $N$ & NA \\
\hline
\end{tabular}


Talke B-3, Material at Risk Dati. (191 theets)






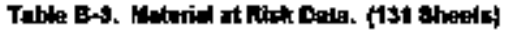

\begin{tabular}{|c|c|c|c|c|c|c|c|c|c|c|}
\hline Find & MHR-SIt:ct & 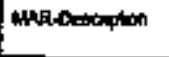 & MAR: & Copatily & Typt & $\begin{array}{l}\text { Fiptital } \\
\text { Fome }\end{array}$ & $\begin{array}{l}\text { Wolume of } \\
\text { Antiod }\end{array}$ & Tr:mith & arontry & Commant: \\
\hline $\begin{array}{l}\text { 24i.c. } \\
\text { अâc }\end{array}$ & 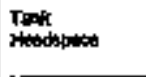 & 211-conct & Nh & Mh & NA & war & 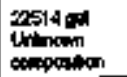 & $\mathbf{N o}$ & MA & 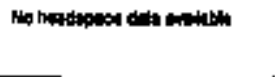 \\
\hline $2+1-c$ & 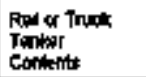 & none & NA & Mh & nh & Nh & $\mathbf{m}$ & NA & NAh & 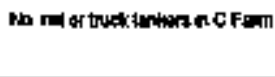 \\
\hline 24t-c & 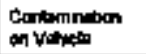 & Hban & Wh & Nh & H & Nh & wh & NA & Wh & 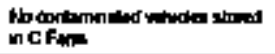 \\
\hline $2 *-6$ & 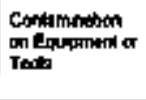 & 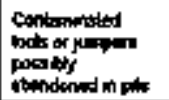 & contiminilid & Nh & Nh & scdd & Untabuth & Notot & Nh & 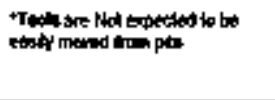 \\
\hline 2414 & 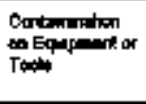 & 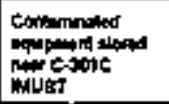 & $\begin{array}{l}\text { vortomintual } \\
\text { equpment }\end{array}$ & 乵 & Bh & boid & Unknom & Yan & wh & Bemo and ofnminum \\
\hline 241: & $\begin{array}{l}\text { Verriliben Fino } \\
\text { Conterls }\end{array}$ & 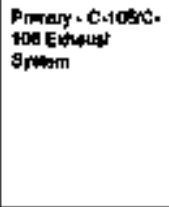 & 䖵 & 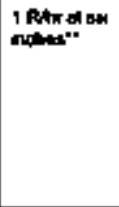 & Bh & Perbata & 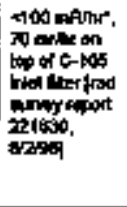 & 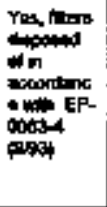 & $\mathbf{m}$ & 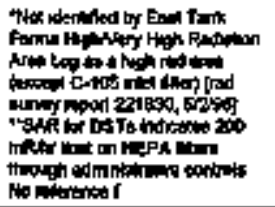 \\
\hline $241-C$ & 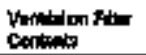 & |aviq Entart & wh & $\mathbf{m}$ & w & NA & Nih & $\mathbf{m}$ & wh & M \\
\hline
\end{tabular}


Table Bas. Malarnlat Rask Dirla, (131 theats)

\begin{tabular}{|c|c|c|c|c|c|c|c|c|c|c|}
\hline Patd & Matk-sich, & 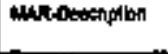 & MAR- & caposty & Tripe & $\begin{array}{l}\text { Fintitid } \\
\text { Fomm }\end{array}$ & Holimin of & Troblat & Ouintity & 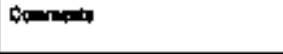 \\
\hline 2016 & Vowingen Fim & Bxather & MA & 1 Rnx $10 x$ & $\mathbf{m}$ & Parietal & $\therefore 104$ nifner & 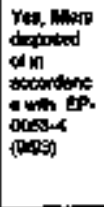 & Wh & 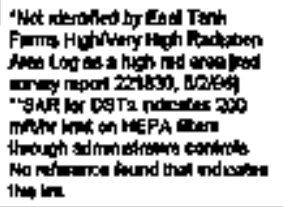 \\
\hline $244 C B$ & Wonition Fibr & 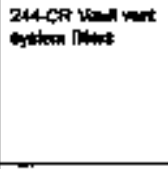 & $\operatorname{man}$ & 1 fronr 2 an & $\mathbf{r a s}$ & Pation & Lrtenoun & 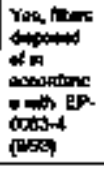 & m & 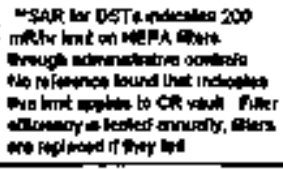 \\
\hline $241-6$ & 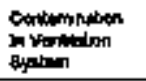 & Do-4nthet & 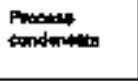 & NA & MA & LAud & Pophiped & *ta & MA & 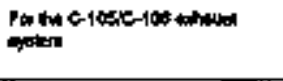 \\
\hline $24-6$ & 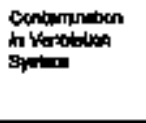 & 5ev1 10009 & condenento & 4100 & NA & 1001 & Prasulat & Nto & 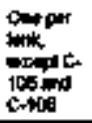 & 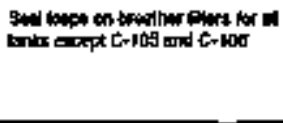 \\
\hline $2+1-C$ & 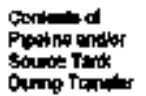 & 14a & $\mathrm{Kan}$ & uh & Wh & MA & $\mathbf{i n t}$ & $\mathbf{m h}$ & N & 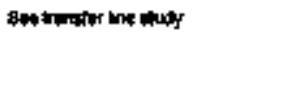 \\
\hline 244-CR & $\begin{array}{l}\text { Contimind } \\
\text { bing of } \\
\text { Encentint }\end{array}$ & CR Woll Bpon & 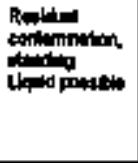 & 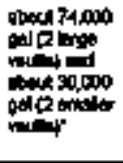 & wh & 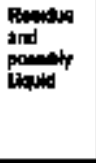 & 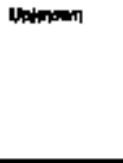 & 40 & 4thin & 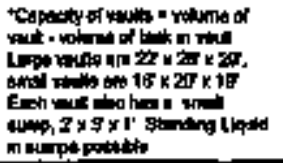 \\
\hline
\end{tabular}


Table B-3, Wataftal at Riak Dam (131 SMabs)

\begin{tabular}{|c|c|c|c|c|c|c|c|c|c|c|}
\hline Find & Manstingt & MH:Deciphon & 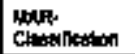 & cranent & $T_{y=4}$ & Foniln & intinger & It & Guanitis & 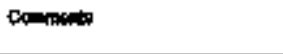 \\
\hline 2440R & 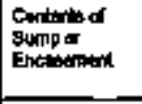 & 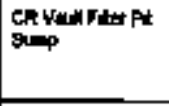 & Rosded & 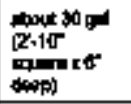 & $\mathrm{NH}$ & Radt & $\mathbf{M m}$ & to & 1 & $\mathrm{NH}$ \\
\hline 244 & 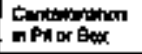 & nhtre & Nesider & NA & Nan & Rante & Nh & $\mathbf{m o}$ & WA & 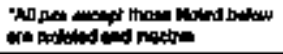 \\
\hline $241+c$ & $\begin{array}{l}\text { Coninimian } \\
\text { of PAl of Bac }\end{array}$ & Proppots" & Rosules & 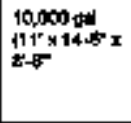 & Nh & Powatur & NA & Who & $\mathbf{H h}$ & 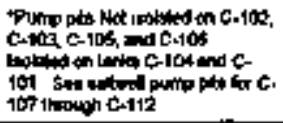 \\
\hline $3 \mathbf{4 I - C}$ & $\begin{array}{l}\text { Contaminan } \\
\text { in PA or Btw }\end{array}$ & Hen por & Ranital & $\begin{array}{l}2000 \text { at }\{[x x \\
5 \rightarrow-10\}\end{array}$ & Wh & Reodue & HA & $M$ & $\mathbf{N A}$ & 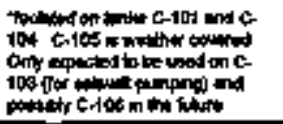 \\
\hline $241-t$ & 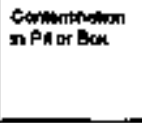 & 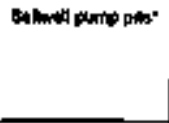 & Roskint & 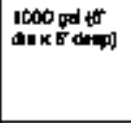 & in & prither & Nh & Ho & ah & 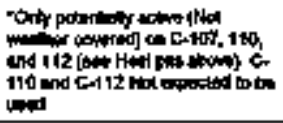 \\
\hline $24+6$ & $\begin{array}{l}\text { Cantiming } \\
\text { In Ptarexk }\end{array}$ & shrept & Plectud & 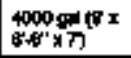 & $\mathbf{m h}$ & Rarode & MA & No & NA & 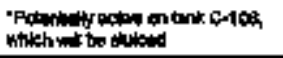 \\
\hline $2+16$ & 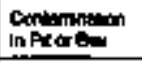 & Whw at 24i-c & | & 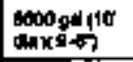 & $\mathbf{m}$ & filtode & 敌 & Ho & ma & 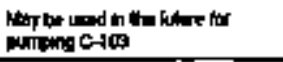 \\
\hline $241-c$ & $\begin{array}{l}\text { contioniben } \\
\text { in Piter Don }\end{array}$ & 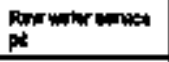 & ren neimi & Nh & 笛 & (n) & inh & $\mathbf{H}$ & Nh & 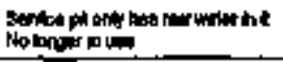 \\
\hline 244CK & $\begin{array}{l}\text { Cont lopan } \\
\text { in Piter } \operatorname{tax}\end{array}$ & 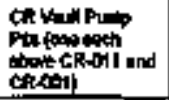 & cencinin & $\begin{array}{l}3000001 \\
{[20 \times 8 \times 18 .} \\
47\end{array}$ & $\mathbf{~ H a n}$ & Amatale & m & $\boldsymbol{n}$ & MA & HA \\
\hline
\end{tabular}


Talth B-3. Molaflal at Rtak Orgta (131 shets)

\begin{tabular}{|c|c|c|c|c|c|c|c|c|c|c|}
\hline Fand & MAR-Shthet & Whatedoritbn & cher. & coparty & What & $\begin{array}{l}\text { Fingtid } \\
\text { Fond }\end{array}$ & 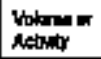 & Trument & oxamly & Comitionila \\
\hline 244Cח & $\begin{array}{l}\text { Caningallon } \\
\text { mPtarim }\end{array}$ & 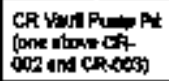 & |litud & 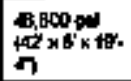 & 㮃 & Rantert & MA & h & m & Nh \\
\hline 244GR & in Pin or Bex & CR Veut Filor PN & Rotadid & 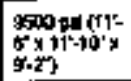 & NA & Ponmothe & Non & m & HA & Wh \\
\hline 2A4CR & $\begin{array}{l}\text { Contancion } \\
\text { in Ptextom }\end{array}$ & 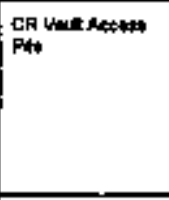 & Rocitur & 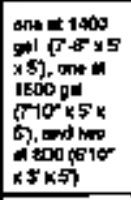 & MA & Pearity & NA & Wa & NA & 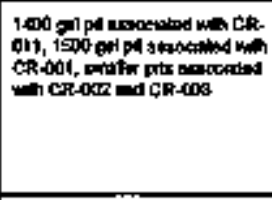 \\
\hline $24-t$ & Conkinator & Onorom tosts & Raskdod & ant & $\mathrm{nth}$ & firmatue & wh & $\mathbf{m}$ & $N A$ & 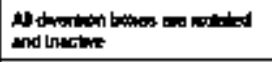 \\
\hline 2h16- & 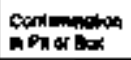 & Duprapn bow & Ropicin & WA & NA & Pipandus: & Wh & Nos & MA & 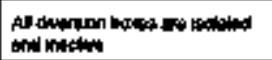 \\
\hline 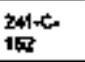 & $\begin{array}{l}\text { Coninnowose } \\
\text { a Pit or Bax }\end{array}$ & Dentambon bon & Randill & NA & Nh & Bume & $\mathbf{m}$ & $N o$ & NA & 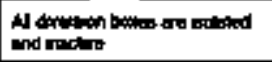 \\
\hline $\begin{array}{l}241-c-6 \\
103\end{array}$ & $\begin{array}{l}\text { Contaringingm } \\
\text { n Pil or bax }\end{array}$ & 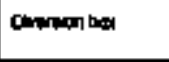 & 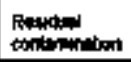 & NA & Nuh & Penoflus & An & No & MA & 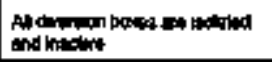 \\
\hline 154 & 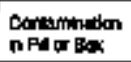 & Dntrint bow & hentril & $\mathrm{NH}$ & ih & Ratade & an & No & Nh & 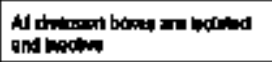 \\
\hline $\log _{200}^{241 x}$ & 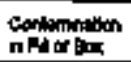 & Drmacin bay & Rosidjal & NH & Fh & Rauter & Wh & $\mathrm{No}$ & NA & 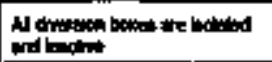 \\
\hline $\begin{array}{l}241 \cdot c R= \\
151\end{array}$ & 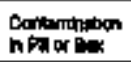 & 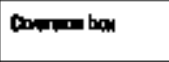 & Rachl & Nh & wh & An:ddin: & min & No & Nin & 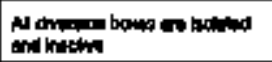 \\
\hline $\begin{array}{l}\text { 241-chr } \\
\text { 1요 }\end{array}$ & 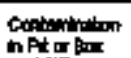 & Dintitan bax & 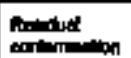 & $\mathbf{H}$ & $\mathbf{M h}$ & Rould & wh & ka & Wh & 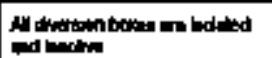 \\
\hline
\end{tabular}




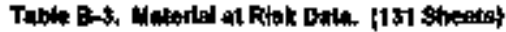

\begin{tabular}{|c|c|c|c|c|c|c|c|c|c|c|}
\hline Priditat & 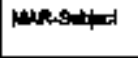 & 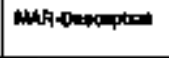 & Cintionition & Copery & Ther & 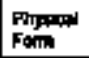 & uphen & Truabet & ytaty & Cammitis \\
\hline 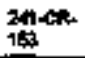 & 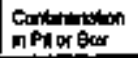 & Damiton be & $\begin{array}{l}\text { Reablud } \\
\text { contmintion }\end{array}$ & Na & 塩 & 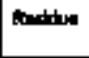 & Nh & b b & N动 & 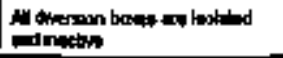 \\
\hline $241+c$ & $\begin{array}{l}\text { BARA } \$ 13 \\
\text { Ititasts }\end{array}$ & $\begin{array}{l}\text { Gepoptian for } \\
\text { PCHA B }\end{array}$ & 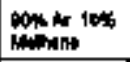 & intor & $\mathbf{m}$ & an & folabwt & $Y \in 9$ & 2 botilat & MA \\
\hline 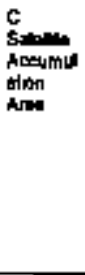 & ont & 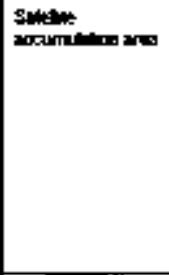 & $\begin{array}{l}\text { RCP hand } \\
\text { note }\end{array}$ & 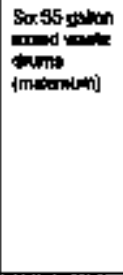 & 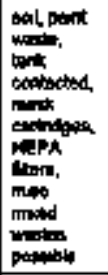 & stad & $\begin{array}{l}\text { bay latid imos } \\
\text { han cbes ch }\end{array}$ & Yed & 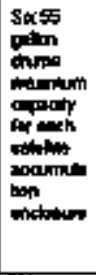 & 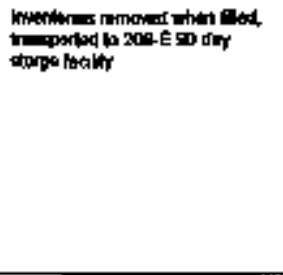 \\
\hline 24110 & Othe & cen b-tilest & 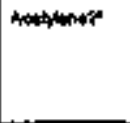 & ind & Nom & ter & 100 anbt & You & 2 todlat & 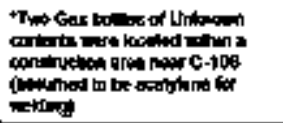 \\
\hline iक्या-8 & 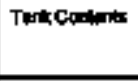 & 241-8-101 & NEPLX & Tाब kqu & 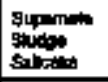 & $\begin{array}{l}\text { Longt } \\
\text { solid } \\
\text { polit }\end{array}$ & 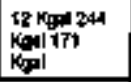 & 140 & $m$ & 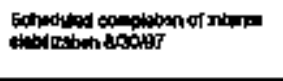 \\
\hline $\begin{array}{l}241 \% \\
102\end{array}$ & tenkctentrets & $2 \times 140112$ & Dost & $\mathrm{Hat}$ & Budpe & sold & 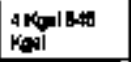 & we & Wh & 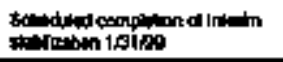 \\
\hline $\begin{array}{l}241-5.5 \\
\text { ins }\end{array}$ & Taticodtath & $241 \leqslant-105$ & Dos & $T 3 k^{\prime}$ & sopamint & $\begin{array}{l}\text { Lquid } \\
\text { sold } \\
\text { soid }\end{array}$ & 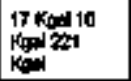 & be & BA & 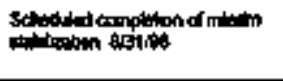 \\
\hline 204-5. & TalcCotititis & $211+504$ & NEPU & Tod & Bopank & Lquid & 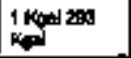 & he & Wh & intild \\
\hline
\end{tabular}




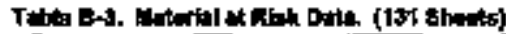

\begin{tabular}{|c|c|c|c|c|c|c|c|c|c|c|}
\hline Prad & MWR-gapjock & MAR-D Finquan & 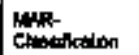 & capostil & dytion & Prowes & valum or & Tomant & 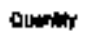 & Comitiets \\
\hline $\begin{array}{l}261-9 \\
106\end{array}$ & Tort comtorits & $241-5+10$ & ntplx & IJs $\mathrm{kgd}$ & 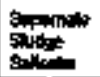 & $\begin{array}{l}\operatorname{lom}_{\text {sedd }} \\
\text { sidd }\end{array}$ & 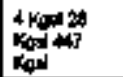 & in & NA & 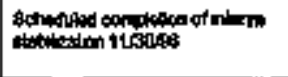 \\
\hline $\begin{array}{l}\text { 261-s } \\
10\end{array}$ & Trik Cuments & $241-5107$ & ntPlx & II & 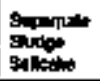 & $\begin{array}{l}\text { Iring } \\
\text { govd } \\
\text { sold }\end{array}$ & 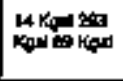 & mo & NA & 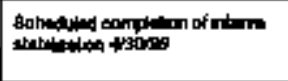 \\
\hline $\begin{array}{l}241-5 \\
106\end{array}$ & Tonctintats & $241-5.108$ & MEPLX & $T_{s}=\mathrm{kp}$ & 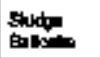 & $\begin{array}{l}\text { sold } \\
\text { Bowd }\end{array}$ & 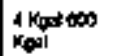 & $m$ & wh. & 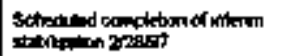 \\
\hline $241-5$ & Tontccosinitis & $791-8-100$ & KстPU & Ist & Brude: & gold & $\begin{array}{l}13 \text { Kont伍 } \\
\text { Kal }\end{array}$ & $\mathbf{N e}$ & NA & 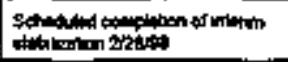 \\
\hline $\begin{array}{l}241-3 \\
110\end{array}$ & Trat Contart & $201-5+110$ & NCPLX & $\pi$ & sudpe & $\begin{array}{l}\text { sovd } \\
\text { goted }\end{array}$ & 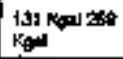 & $N 0$ & NA & 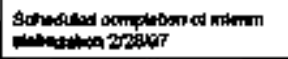 \\
\hline $\begin{array}{l}241-8-2 \\
111\end{array}$ & TanicCerisuman & 2*1-3-111 & Nefex & $758 K_{04}$ & 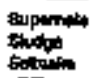 & $\begin{array}{l}\text { Loped } \\
\text { sold } \\
\text { bold }\end{array}$ & 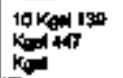 & No & Mh & 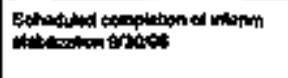 \\
\hline 112 & Tercentarb & $2+1<6-112$ & NEFIX & TES kad & gividit & fowd & $\log _{0 \rightarrow 16}$ & No & wh & 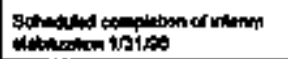 \\
\hline 2445 & Tank Coiluents & OCKT 244-5 & 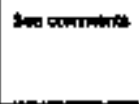 & st, $720 \mathrm{~kg}$ & tom & tor & Uhtomenn & No & MA & 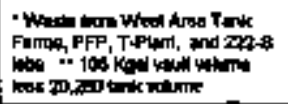 \\
\hline 2*1: & Tank Cortipinta & $\operatorname{coth}_{0 \rightarrow 4}$ & 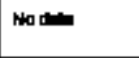 & 6.00 & bodal & Wodta & Zुत्ञ & Ha & $m$ & ren \\
\hline $200 \mathrm{k}$ & Tarik Contunitu & warsт $20-300$ & 10 & 17084 195 & Watat & And & 2070 & Ho & nat & 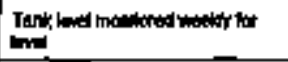 \\
\hline $2000-$ & 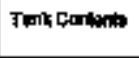 & 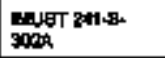 & 1904 & $17 \times 8940$ & 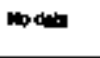 & Hoth & 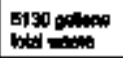 & Nin & MA & 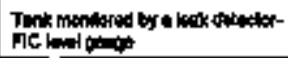 \\
\hline $\log _{0}$ & Turk Coriteiti & 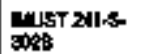 & $\mathbf{H A n}$ & $14 \div 49$ & 000 & ith & 0 & Ha & MA & MA \\
\hline
\end{tabular}




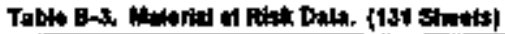

\begin{tabular}{|c|c|c|c|c|c|c|c|c|c|c|}
\hline Fuld & MAN-gutint & MHR-Deactiven & thes- & Conpaly & Thpor & 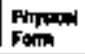 & 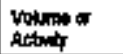 & To. & Qunty & Commonth \\
\hline $210-8-25$ & Tapk Cortusth & काpर्टाt-3-26 & 14 & Wh & wh & Nat & $\mathbf{m}$ & Wh & $\mathbf{w h}$ & 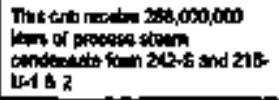 \\
\hline $2+8-2-20$ & Trak Comitent & Crb215-sexs & NAS & Wh & $\mathbf{m}$ & $m$ & NA & wh & $\mathrm{ma}$ & 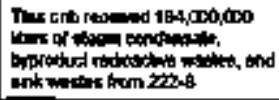 \\
\hline $\begin{array}{l}241-5 \\
101\end{array}$ & Twather & $2 \times 1-5=104$ & mak & wh & Na & Hapor & $\begin{array}{l}220503 \\
v 2000143\end{array}$ & מ & KCA & NA \\
\hline $\begin{array}{l}241-8-2 \\
160\end{array}$ & TH: & $241-7+102$ & $\mathbf{m k}$ & an. & en & Hepor & 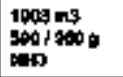 & No & wh & NA \\
\hline 105 & 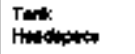 & 2A1-8-103 & MA & an & HA & Yrapor & 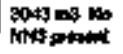 & No & in & Nan \\
\hline $\begin{array}{l}245=3 . \\
104\end{array}$ & $\begin{array}{l}\text { Tart } \\
\text { Hapilypop }\end{array}$ & 241<-104 & $\mathbf{m a n}$ & wh & Wh & Vops & 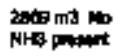 & Ho & Ma & Wh \\
\hline $\begin{array}{l}2 \times 1-5 . \\
105\end{array}$ & Tut & $2+1-5-104$ & $\mathbf{m}$ & wh & ran & vepor & 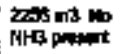 & to & $\cos$ & MA \\
\hline $\begin{array}{l}241-5 . \\
108\end{array}$ & Thatp & $241-5-106$ & an & wh & Nin & Wopt & 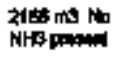 & ito & $N$ & Nh \\
\hline $\begin{array}{l}2 \times 1-9 . \\
\text { vण }\end{array}$ & Thatipen & $2+14000$ & ben & $\mathbf{m h}$ & Nh & vaph & अon ma & ib & $\mathrm{NA}$ & 缋 \\
\hline 261-s. & Thint & 241860 & $\mathbf{m}$ & $\mathbf{H}$ & NA & Shper & 1605 mo h & $\mathbf{H o}$ & NA & Nh \\
\hline
\end{tabular}




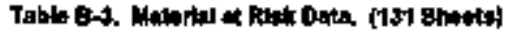

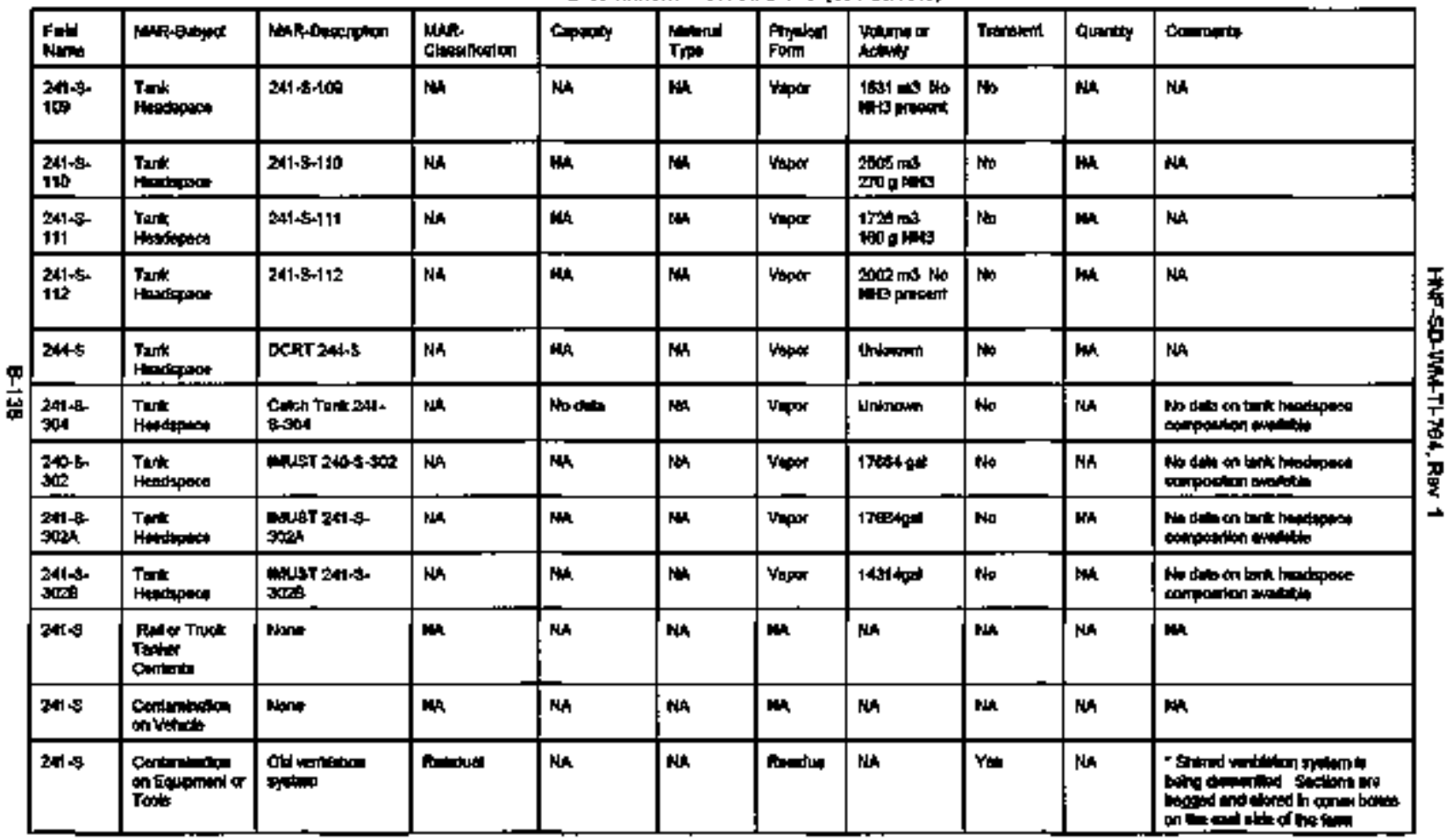




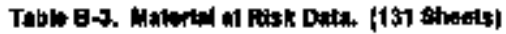

\begin{tabular}{|c|c|c|c|c|c|c|c|c|c|c|}
\hline Find & MARSutid & intritheripton & Whes & cepanty & Trim & Pithill & 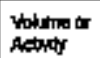 & 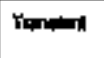 & Guintity & Comperte \\
\hline $241-6$ & 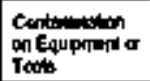 & Conet Euctat & Roctutes & wh & Nh & fintodve & Nat & $y=$ & $\mathbf{m k}$ & 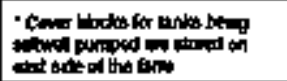 \\
\hline 24 舟 & 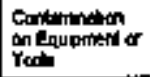 & Eate rad & man & ent & $\boldsymbol{M h}$ & Ah & 妕 & $Y=$ & mo & 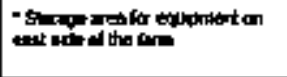 \\
\hline $2 \times 1+5$ & 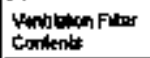 & Promay & NH & Wh. & Mh & MH & NA & Nh & HA & $N$ H \\
\hline $241-8$ & 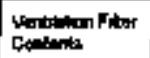 & Annater & ran & MA & $\mathbf{m}$ & $\mathbf{m}$ & Hin & NA & UA & in \\
\hline 241,5 & 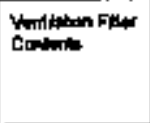 & ה & NA & $\begin{array}{l}1 \text { phat } 6 \\
\text { neher }\end{array}$ & $\mathrm{man}$ & Pubtat & $\mathrm{me}$ & 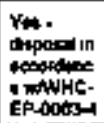 & Nh & 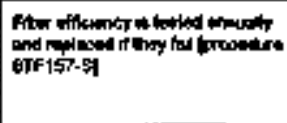 \\
\hline $241-3$ & 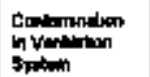 & Dequitraners & Nh & NA & $\operatorname{lat}$ & NA & $\mathbf{m}$ & $\mathbf{m}$ & Na & $\mathbf{m}$ \\
\hline $2 / 1-3$ & 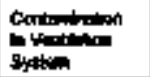 & Dodkap & on andinter & 1 сир & Nh & Hqud & Rontad & Ho & l per & 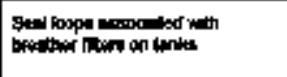 \\
\hline $241-5$ & Conterts of & $\mathrm{man}$ & Wh & Ma & NA & Mat & int & men & $\boldsymbol{m}$ & gentroter the thite \\
\hline $24-4$ & $\begin{array}{l}\text { Continin of } \\
\text { Simp of } \\
\text { Enomet }\end{array}$ & 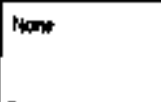 & ma & NA & WA & NA & wh & Wh & RA & 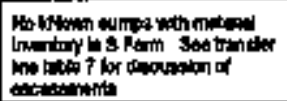 \\
\hline
\end{tabular}




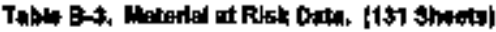

\begin{tabular}{|c|c|c|c|c|c|c|c|c|c|c|}
\hline Fisd & 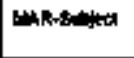 & 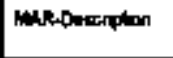 & 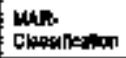 & cepricty & $\begin{array}{l}\text { The } \\
\text { Ther }\end{array}$ & $\begin{array}{l}\text { Ptopy- } \\
\text { Fonn }\end{array}$ & 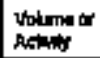 & trumant & Auraty & corrematis \\
\hline $241 \leqslant$ & $\begin{array}{l}\text { Conkerintion } \\
\text { In PK or Ben }\end{array}$ & 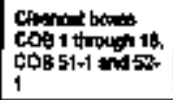 & 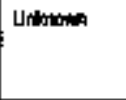 & M & NA & Uhistom & Lritatm & $\mathrm{rab}$ & $211 \mathrm{bath}$ & $\mathbf{m h}$ \\
\hline 241-t & $\begin{array}{l}\text { Coniminiben } \\
\text { oftaria }\end{array}$ & H, L, C, D & Patrias & 51420 & NA & kreider & Umonem & No & 4 phs & NA \\
\hline $291-3-4$ & 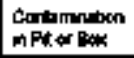 & 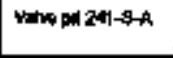 & Reatiol & M420d & Wh & Reantwa & Varmon & $\mathrm{No}$ & t pt & $\mu \mathrm{H}$ \\
\hline $241<5<\theta$ & 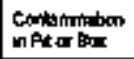 & 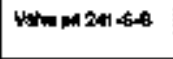 & Resiobl & औ42 & Nh. & Ansuder & Utomen & No & 1 pt & NA \\
\hline $241-8-6$ & 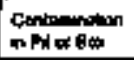 & $w_{k} \Rightarrow \operatorname{DA} 241-5-c$ & Presqudud & 3147 에세 & $\mathbf{m}$ & Rumply & 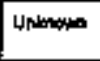 & 16 & 10 & Na \\
\hline $24.5-0$ & 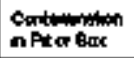 & Vtria pe 241.65 & fiec-dual & 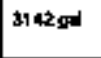 & bur & 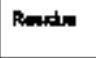 & Introm & ins & 1 pal & $N A$ \\
\hline 2*1-5- & Deniming & $\begin{array}{l}\text { Dawnen Bas 241- } \\
\text { B-151 }\end{array}$ & Rourdual & 33510 ow & $\mathbf{m}$ & Rututy & 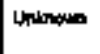 & $\mathbf{W o}$ & 10 & MA \\
\hline 204k- & 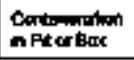 & $\begin{array}{l}\text { Denerom Bex 241. } \\
\text { S-15: }\end{array}$ & Asedid: & ret & ar & Resule & Uhinane & ine & $1 p$ & Nin \\
\hline tकis & in Pin or Bom & 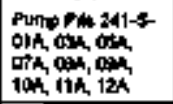 & Hinduil & $2 \pi 350$ & $\mathrm{~kg}$ & Pintat & Utheratn & in & 中 pow & NA \\
\hline 241-5. & 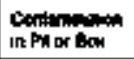 & 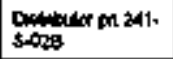 & Runded & tod & MA & Rocitud & Uhtunoth & mo & 1 F & Wh \\
\hline $241-5$ & 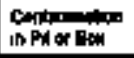 & Ornw bils & Fpeldun & NA & MA & Renching & Unthown & m & NA & 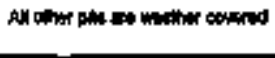 \\
\hline 2414 & SARR 513 & Hont & $\mathbf{r a h}$ & MA & $\mathrm{m}$ & an & Nh & $\mathbf{m}$ & Mh & wh \\
\hline
\end{tabular}




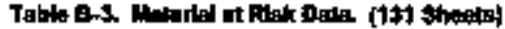

\begin{tabular}{|c|c|c|c|c|c|c|c|c|c|c|}
\hline Fand & MArstopa & 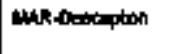 & 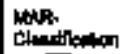 & Aaptity & Thentaly & $\begin{array}{l}\text { Pryotsta } \\
\text { Fom }\end{array}$ & $\begin{array}{l}\text { Wohme a } \\
\text { Actupt }\end{array}$ & Transient & Oumity & Cominterts \\
\hline $2+1-5$ & Othe & 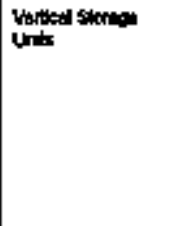 & M & NA & Satd & Uniniman & Yos " & MH & 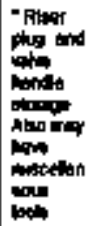 & Mh \\
\hline $241-\$$ & Other & Fiamition & " & 虫 & Wh & $\mathrm{MA}$ & Wh & $r$ & NA & 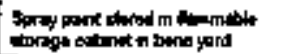 \\
\hline $2+1-8$ & קוtro & Propanc botas & Prepert* & 2 botsog & en & Bes & me & Y*Q & 2bottien & 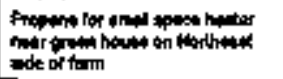 \\
\hline $241-8$ & oftop & Traner & iped nimogen & 2 the & $\mathbf{m}$ & Hax & $\mathbf{m}$ & Y*A & $2 \tan k$ & 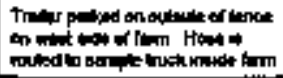 \\
\hline 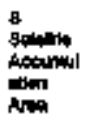 & Othes: & 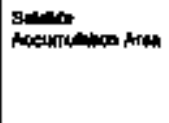 & $\begin{array}{l}\text { RCRd Mred } \\
\text { Woine }\end{array}$ & 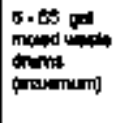 & $\operatorname{Ban}$ & soid & $\begin{array}{l}\text { lon kand } \\
\text { cosition tip }\end{array}$ & Yex & 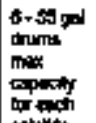 & 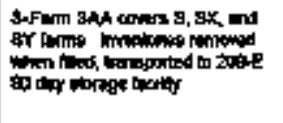 \\
\hline $\begin{array}{l}241-5 x- \\
101\end{array}$ & Tank oontinte & 2al-sx-101 & bc & 1 itten & songenting & $\begin{array}{l}\text { Land } \\
\text { sald } \\
\text { sold }\end{array}$ & $\begin{array}{l}1 \log 112 \\
\operatorname{Kog} 340 \\
\operatorname{Key}\end{array}$ & No & NA & 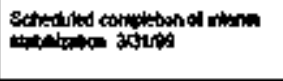 \\
\hline $\begin{array}{l}241+5 x \\
100\end{array}$ & Tark Caindit & 2 기연-102 & ossf & 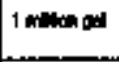 & sindist & Sold & 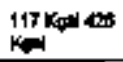 & Ho & NA & 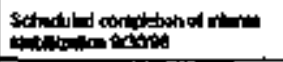 \\
\hline $\begin{array}{l}241-2 x x \\
100\end{array}$ & Terik Coninith & $2,11-3 x-100$ & $\operatorname{meptx}$ & 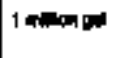 & 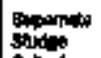 & Stoid & 1160115 & No & NA & sechind conginbent Fimne \\
\hline
\end{tabular}




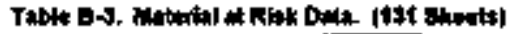

\begin{tabular}{|c|c|c|c|c|c|c|c|c|c|c|}
\hline $\begin{array}{l}\text { Findad } \\
\text { Niturd }\end{array}$ & MHBS\$ubigot & 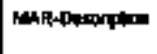 & Mark & Geapol & 1) & 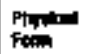 & Aching & Tranapat & axintr & Commonts \\
\hline 204 & Tat Cohtwes & 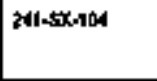 & Dost $\mathrm{F}$ & 1 angh & 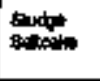 & sold & 135 Feg बrरs & No & wh & 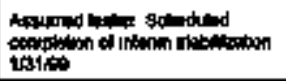 \\
\hline $\begin{array}{l}\text { 2서 } 4 \text { Ax } \\
\text { 100 }\end{array}$ & Thin Conthe & 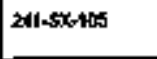 & DSs & 1 mbngal & shodet & $\begin{array}{l}\text { sold } \\
\text { sodd }\end{array}$ & ratoloto & Mb & Wh & 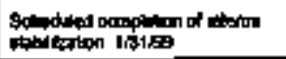 \\
\hline $241-9 x$ & Tank forterta & $2+1-8 x-105$ & NCFEX & 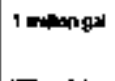 & 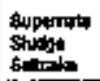 & $\begin{array}{l}\text { latid } \\
\text { sold } \\
\text { sdit }\end{array}$ & 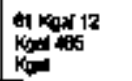 & $\mathbf{N o}$ & Nh & 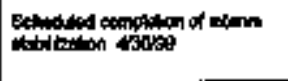 \\
\hline $\begin{array}{l}241+9 x \text {. } \\
100\end{array}$ & Tank Combta & $2 * 1-8)<107$ & NLEF & 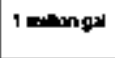 & stope & Inengr & Ias $\mathrm{kod}$ & $\mathbf{N o}$ & ah & 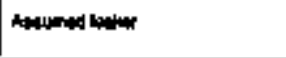 \\
\hline $\begin{array}{l}241-5 \times x \\
10 d\end{array}$ & Tonk Conimento & $241-3 x-100$ & ACHX & 1 neimengral & shodpd & todd & $87 \mathrm{Kod}$ & Noto & in & 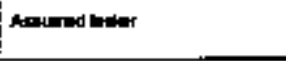 \\
\hline $\begin{array}{l}24145 x \\
100\end{array}$ & Tank Conturts & $2+1-8 x-16$ & $\operatorname{lnc} 2 \mathrm{x}$ & 1 mitongr & stow & boind & $244 k 00$ & Not & m & שvid \\
\hline $\begin{array}{l}241-5 \mathrm{~K}- \\
110\end{array}$ & 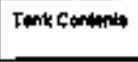 & $2+1-5 x-110$ & NCMX & 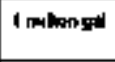 & sketat & stat & as Kar & No & hat & Mathed herer \\
\hline $\begin{array}{l}241-6 x- \\
111\end{array}$ & Tart Cumerts & $241-8 x-111$ & HCFIX & I nertonged & shopo & sold & 175 ked & No & $\mathbf{m}$ & 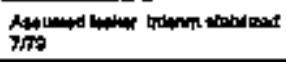 \\
\hline $\begin{array}{l}2+11 \cdot+5 r \\
112\end{array}$ & Tart Combend & $2+15 x+112$ & MCPUX & 1 matongl & strito & Cold & 此 & No & an & 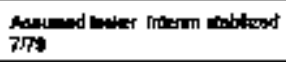 \\
\hline $\begin{array}{l}34-8 x- \\
11 \geqslant\end{array}$ & Tert Conkene & $2+1-\$ x-113$ & $\operatorname{men} x$ & 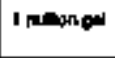 & Matge & sold & a kan & No & m & 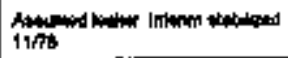 \\
\hline $\begin{array}{l}24 \operatorname{tin} 1 x \\
114\end{array}$ & Tanteritents & $24 t-a x-114$ & Marex & 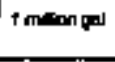 & she & sod & lat & inde & nes & 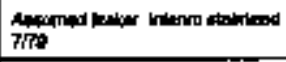 \\
\hline $\begin{array}{l}2+1-9 x- \\
115\end{array}$ & Treik Contonta & $241+5 x-1+5$ & mexx & סק רחמוm 1 & studs & satd & $\mathbf{r} \mathbf{k}$ & m & $\mathrm{NH}$ & 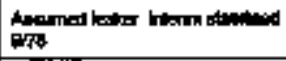 \\
\hline 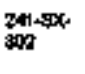 & Tackertats & 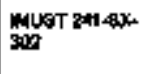 & $\underset{m=1 x}{\operatorname{cP}}$ & 17004 & inh & 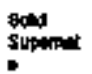 & 1050 ars & No & NA & 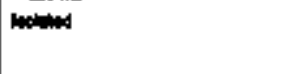 \\
\hline
\end{tabular}




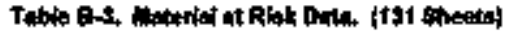

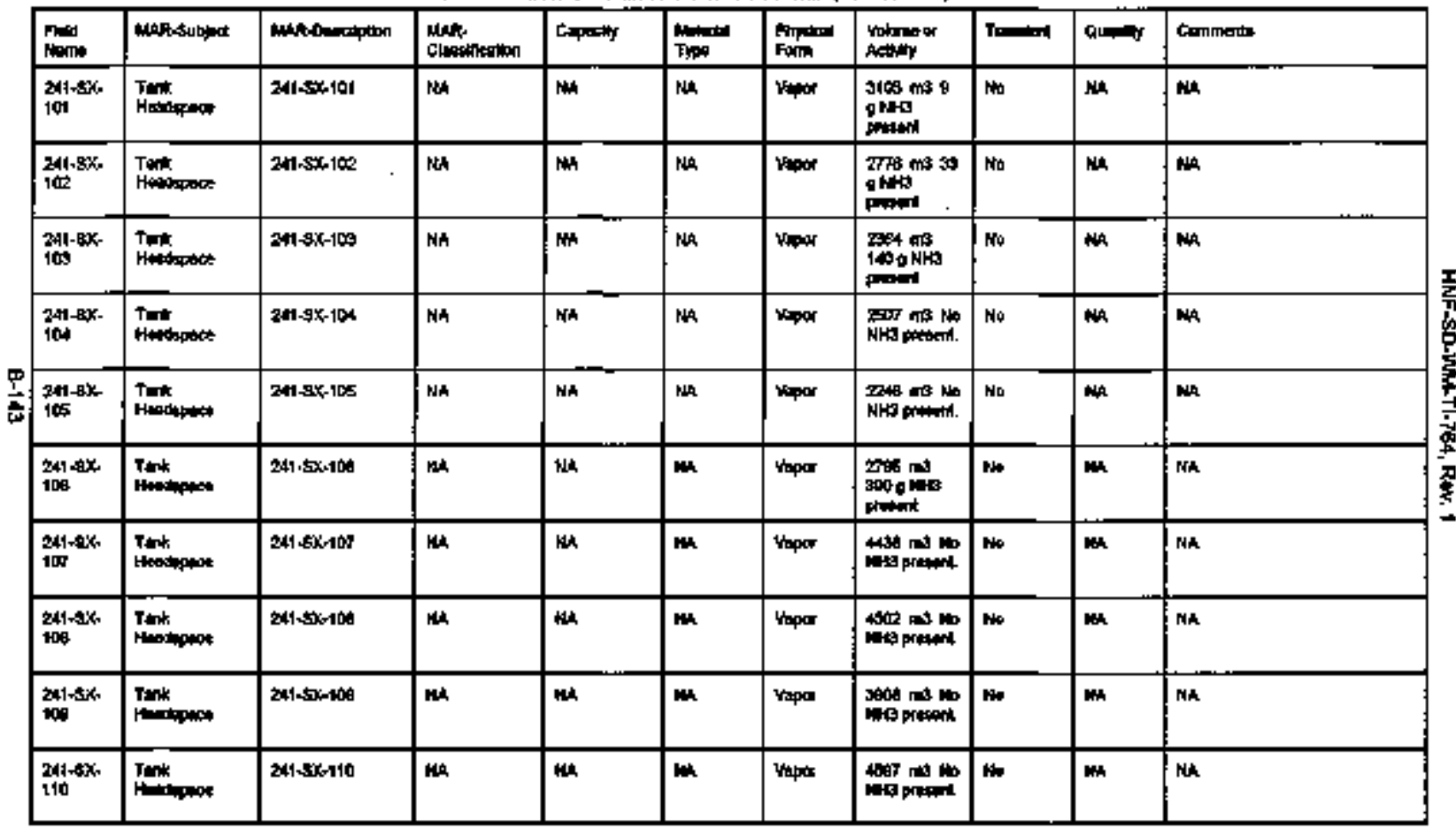




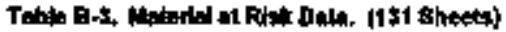

\begin{tabular}{|c|c|c|c|c|c|c|c|c|c|c|}
\hline Fieted & Meksutitgit & 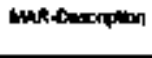 & Makr. & capoctis & Thend & $\begin{array}{l}\text { Fingan } \\
\text { Form }\end{array}$ & rencin & Tran & (1) & Com ate \\
\hline $\begin{array}{l}211-5 \% \\
111\end{array}$ & Tank & $211-3 x-111$ & and & $\mathrm{m}$ & Nh & vapor & 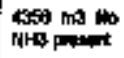 & W & NA & Wh \\
\hline $24125 x$ & Trink & $24-5 \times 112$ & $\mathrm{Mh}$ & Wh & wh & tratar & Nith ms Not & Noto & Nh & Nh \\
\hline 201-9x- & Tom & $241-5 \times-113$ & NA & $\min$ & $\mathbf{m}$ & Yeper & 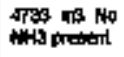 & $\mathrm{NoO}$ & $\mathbf{w h}$ & 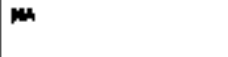 \\
\hline $2+1-9 x-$ & $\begin{array}{l}\text { Tonk } \\
\text { Howlositer }\end{array}$ & $241-E X-114$ & NA & $\mathrm{ma}$ & $\mathbf{m}$ & teros & 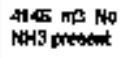 & No & Wh & and \\
\hline $241-3 x$ & Trut & $241-6 x-115$ & HA & NA & m & voper & Fes & No & $m$ & un \\
\hline $241 . \$ 3 x$ & Turt & 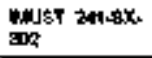 & Na & $\mathrm{NH}$ & $m$ & Vupser & $10 \times 285$ & toto & Wh & 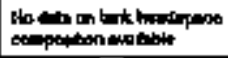 \\
\hline $241-8 x$ & 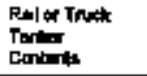 & $\operatorname{lon}$ & M & HA & Nuh & NA & NA & $\mathrm{NAS}$ & en & Wh \\
\hline $24-8 x$ & 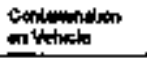 & Nom & Nh & NAS & $\mathbf{m}$ & Nh & NA & NA & NAS & MA \\
\hline $2+1-3 x$ & 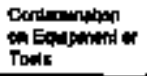 & $\operatorname{Nan}$ & MA & mal & NA & $\min$ & $\mathbf{m}$ & $\mathbf{m a}$ & ina & Na \\
\hline $241-5 x$ & 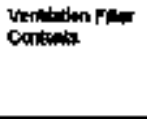 & Arm & $\mathrm{man}$ & 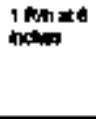 & in & Pariaula & $\mathbf{m k}$ & 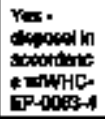 & roh & Nan \\
\hline
\end{tabular}




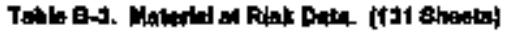

\begin{tabular}{|c|c|c|c|c|c|c|c|c|c|c|}
\hline $\begin{array}{l}\text { Hidd } \\
\text { Nitat }\end{array}$ & Hithestyost & AHP-Owenewhin & 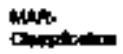 & trpacty & 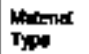 & $\begin{array}{l}\text { fiptral } \\
\text { Folm }\end{array}$ & $\begin{array}{l}\text { Aphite of } \\
\text { Aptoxt }\end{array}$ & Tranent & 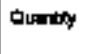 & Com mpan \\
\hline $241-5 x$ & 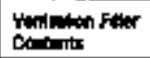 & mantes & MA & NA & $\mathbf{M h}$ & $\mathbf{m}$ & mh & NA & 塩 & $\mathrm{man}$ \\
\hline 2AI-EX & $\begin{array}{l}\text { Vertion Find } \\
\text { Contents }\end{array}$ & Eresther & Wh & $\begin{array}{l}1 \text { Fon ज्ञा } \\
\text { novido }\end{array}$ & $\mathbf{m}$ & 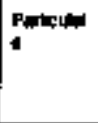 & NH & 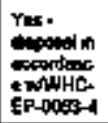 & wh & 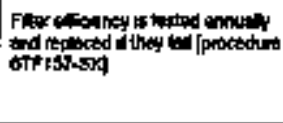 \\
\hline $241-5 x$ & 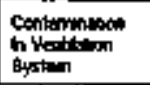 & De-wineners & Nh & Wh & $\mathrm{NA}$ & MA & Nh & Wh & im. & Nh \\
\hline $2+1-9 x$ & 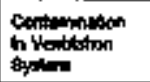 & sarbep & 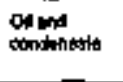 & 1 cap & NA & Land & Pansum & 16 & ond per & 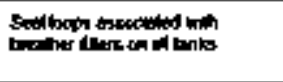 \\
\hline $241-E x$ & 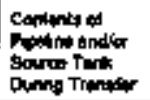 & Mu & $\mathbf{M A}$ & $\mathbf{M A}$ & Na & NAB & Nh & MA & in & 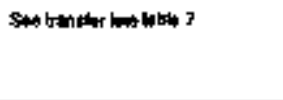 \\
\hline $2 *-5 x$ & $\begin{array}{l}\text { Caminit of } \\
\text { surtip or } \\
\text { Encenement }\end{array}$ & an & NA & wh & M & NA & Nh & MA & M & 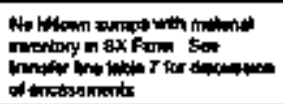 \\
\hline $\begin{array}{l}241-96- \\
011\end{array}$ & 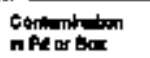 & $\underset{\text { Ond }}{\text { Pund }}$ & Artadur & 20000 & $N$ H & finctits & $\mathrm{m}$ & No & 1 म्ल & $\mathbf{~ w h}$ \\
\hline $\begin{array}{l}241-60 k . \\
04 A\end{array}$ & 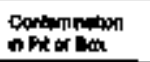 & 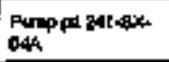 & Rasader & $22 \pi 5$ 여 & MA & Ronatists & $\operatorname{man}$ & No & I pal & $M$ \\
\hline $\begin{array}{l}\text { 241-sx. } \\
\text { 06h }\end{array}$ & $\begin{array}{l}\text { contmintion } \\
\text { in Re or Box }\end{array}$ & $\operatorname{Pu}_{0 \rightarrow 4} p p t 241-s x$. & Andefter & 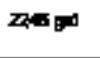 & ind & R4: & $\mathrm{m}$ & $\mathrm{No}$ & 1 p & Nh \\
\hline $\cos _{\text {and }}^{241-8 x}$ & $\begin{array}{l}\text { Conk:mnion } \\
\text { in } R: \text { tr Ban }\end{array}$ & $\begin{array}{l}\text { Punp pt 24-9x } \\
\text { OSi }\end{array}$ & Rembial & sopen & $\mathbf{M A}$ & 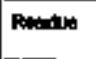 & $\mathrm{ren}$ & Ho & 1 pal & $M$ \\
\hline $\begin{array}{l}24154 \\
1009\end{array}$ & 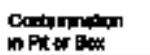 & 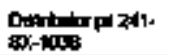 & 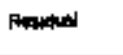 & 매 & Pan & Residup & MA & $\mathrm{No}$ & 1 pll & Mh \\
\hline
\end{tabular}




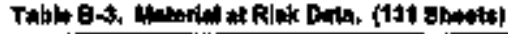

\begin{tabular}{|c|c|c|c|c|c|c|c|c|c|c|}
\hline Finid & MAs-sutp=r & $M / k_{0}$ & Mth: & Cypaty & Ty: & $\begin{array}{l}\text { Pincral } \\
\text { Fơाin }\end{array}$ & Hotumea & Tondul & Quthot & Com \\
\hline $\begin{array}{l}241.5 \times 2 \\
150=\end{array}$ & $\begin{array}{l}\text { Conimimation } \\
\text { mRter Ben }\end{array}$ & 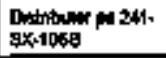 & 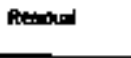 & 400t pat & $\mathbf{W h}$ & Ratadat & HA & Ho & $1 \mathrm{pt}$ & Nh \\
\hline $2+15 x+1$ & 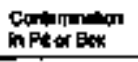 & Wha box $241-8 x$ & Rim & 3142 gd & NA & Rayaden & धh & Noto & 1 beth & MA \\
\hline 2허 -5저용 & 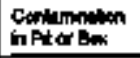 & 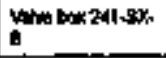 & Paterthad & sic a d & Wh & Ruchath & ath & No & I bac & Mu \\
\hline $\begin{array}{l}2+15 x- \\
151\end{array}$ & 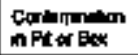 & $\begin{array}{l}\text { Dicrige pou 201- } \\
\text { SK.151 }\end{array}$ & Fiandur & 4h & NA & Rayath & uh & Nob & $1 \mathrm{bex}$ & 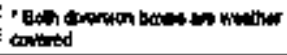 \\
\hline $\begin{array}{l}241+9 x \\
1 \leq 2\end{array}$ & 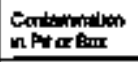 & 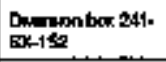 & Mrocher & Nh & an & Reche & Na & wo & Ibar & 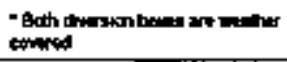 \\
\hline $241+5 x$ & $\begin{array}{l}\text { Cont wimon } \\
\text { in Pil or Box }\end{array}$ & $\begin{array}{l}\text { Cotue pits and } \\
\text { bowos }\end{array}$ & Rasudhw & HA & m & Reratua & MA & 14 & HA & 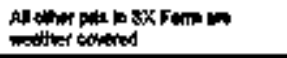 \\
\hline $241-5 x$ & 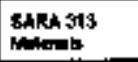 & 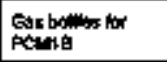 & $\begin{array}{l}\text { Gow ingons } \\
\text { 10w tringes }\end{array}$ & $\operatorname{sid}_{\text {tod }}$ & Mut & Gar & $\begin{array}{l}\text { Woutowo } \\
\text { thet }\end{array}$ & $r=$ & 2bettlea & Whe \\
\hline $24 y-4 x$ & other & 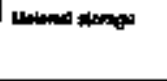 & 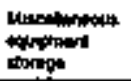 & Wh & $\boldsymbol{H h}$ & Band & мᄉ & $x *$ & M & 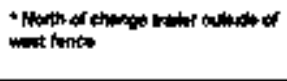 \\
\hline $\begin{array}{l}241-5 y- \\
101\end{array}$ & Tand Cartan & 241 EV-19 & cc & 1 18 & M & puv & 1100 Kt & $\mathbf{H}$ & M & 14 \\
\hline $\begin{array}{l}2 \%-5 \gamma \\
102\end{array}$ & Tank Conkinat & $24-5 y-102$ & DNITT & 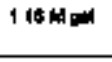 & $\mathrm{moh}$ & $\begin{array}{l}\text { lopid } \\
\text { Bdild }\end{array}$ & Mo & Ma & Nh & $m$ \\
\hline $\begin{array}{l}2 *-6 \gamma- \\
\text { 100 }\end{array}$ & Tonk Centand & $245-8 Y-143$ & cᄃ & 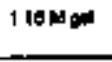 & Nin & Lquad & 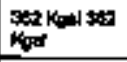 & $\mathbf{H}$ & $\mathbf{M A}$ & wh \\
\hline $\begin{array}{l}241-3 Y 2 \\
101\end{array}$ & $\begin{array}{l}\text { Tark } \\
\text { Hentapiot }\end{array}$ & 241-3ry-10n & in & wh & wh & inear & 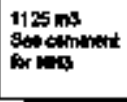 & No & HA & 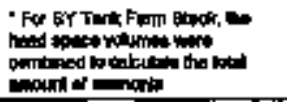 \\
\hline
\end{tabular}




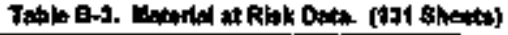

\begin{tabular}{|c|c|c|c|c|c|c|c|c|c|c|}
\hline Pleld & MApsthot & Mundpechoon & $\begin{array}{l}\text { MAR. } \\
\text { CHatinising }\end{array}$ & Caporty & 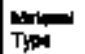 & $\begin{array}{l}\text { Ptared } \\
\text { fofin }\end{array}$ & rofing or & Trandat & Ouath & Cormm \\
\hline $\begin{array}{l}241-5 r^{2} \\
108\end{array}$ & 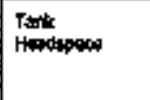 & $2 n-4 y-1$ th & wh & $\mathrm{NA}$ & Wh & Vtapr & 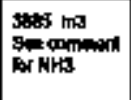 & Ho & Nh & 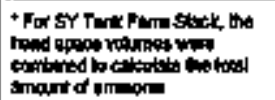 \\
\hline$\underbrace{2016 r}_{100}$ & Fank & $24.5 Y-1 \$ 5$ & $\boldsymbol{m}$ & $m$ & $\mathrm{HW}$ & Hepor & 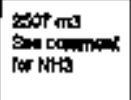 & Wo & $w$ & 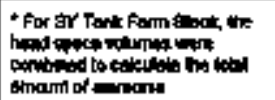 \\
\hline 24|-6y & Jom & sr-smet & Wh & $\mathbf{w h}$ & $\mathrm{ma}$ & veror & 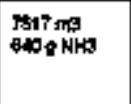 & Ha & ah & 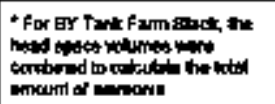 \\
\hline PH-SY & 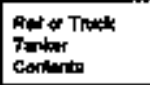 & Nom & Wh & uh & $\mathbf{k h}$ & un & MA & MA & m & wh \\
\hline 241-94 & 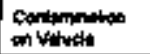 & Nate & Wh & ah & $M$ & Hh & HA & MA & H & an \\
\hline $141-6$ & $\begin{array}{l}\text { Contmonico } \\
\text { on Equpmenter } \\
\text { Toph }\end{array}$ & 101-SY HFm & Thot whom & HA & ins & NA & Nh & Nat & $\mathrm{NH}$ & 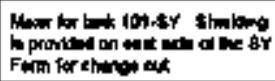 \\
\hline $141-6 \gamma$ & rertids & Pnomiry & NA & 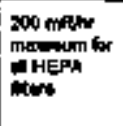 & BM & Pratuin & 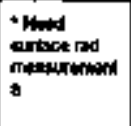 & 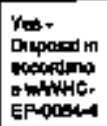 & MA & 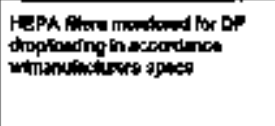 \\
\hline $241+8 r$ & 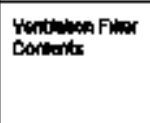 & Arotus: & HA & 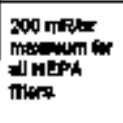 & $m$ & Rertertal & Mod & 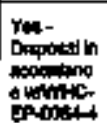 & Mh & 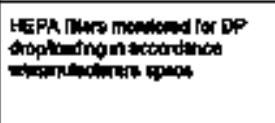 \\
\hline
\end{tabular}




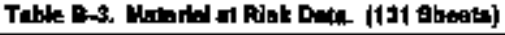

\begin{tabular}{|c|c|c|c|c|c|c|c|c|c|c|}
\hline $\begin{array}{l}\text { Fond } \\
\text { Nempe }\end{array}$ & Malkstitpod & Mentoditum & $\begin{array}{l}\text { WHP. } \\
\text { Craphophen: }\end{array}$ & Eapocity & Ipes & Ply & wother of & Tewnintit & 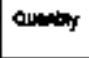 & com \\
\hline $241+5 Y$ & 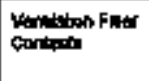 & Intul Bantion & WA & 1 Ran 18 & Wh & Pertaly & wh & 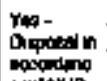 & HA & 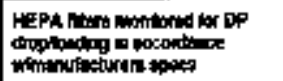 \\
\hline $241-54$ & 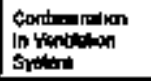 & 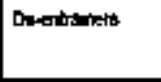 & Cendera- t: & ma & hH & Uqued & wh & Hh & 帆 & wh \\
\hline $241-5 Y$ & 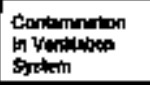 & searbop & contengera & ה N & M & Ind & wh & Nin & $\mathbf{H h}$ & Orm b state dranpt \\
\hline 241.54 & 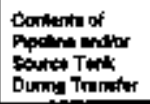 & Wh & Mn & 㠹 & man & H & Nat & NA & Bh. & 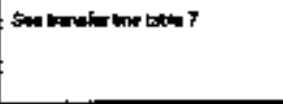 \\
\hline $241-6 \mathbf{r}$ & 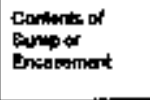 & hin & Mh & wh & $\mathbf{r a h}$ & MA & NA & Nan & ben & 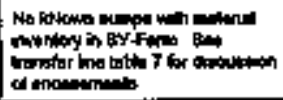 \\
\hline $\begin{array}{l}\text { 241-6r. } \\
\text { old }\end{array}$ & 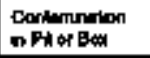 & 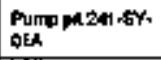 & heoder & xridgal & Mh & Aradur & Untonom & & 1 pd & Nen \\
\hline tad- & 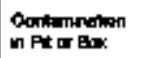 & Purto pal 2+1-SY- & Ruploted & HTH & NH & Rlodes & Untoremt & Nto & 1 gin & wh \\
\hline $\begin{array}{l}\text { 241-sy. } \\
\text { ops }\end{array}$ & 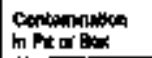 & Pump pa 241-gY. & Rexted & 25130 היה & NA & 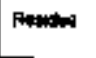 & 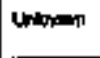 & 100 & $1 \mathrm{pt}$ & 蚶 \\
\hline $\begin{array}{l}241-8 Y . \\
\text { O1B }\end{array}$ & 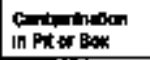 & 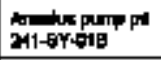 & Rowhed & ||$_{\alpha} \mid$ & NA & 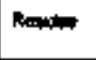 & 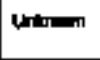 & Ho & $1 \mathbf{p t}$ & NA \\
\hline $\begin{array}{l}241-8 Y . \\
020\end{array}$ & 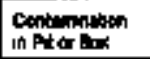 & 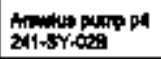 & Frouster & 1237 & NA & Proultat & 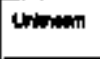 & No & $1 \mathrm{pt}$ & H. \\
\hline $\begin{array}{l}\text { DH-\$YY. } \\
00 B\end{array}$ & 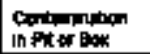 & 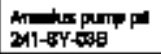 & Ratale & $181 \mathrm{~g}$ & NA & Reasivo & 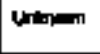 & Nla & $1 \mathbf{p t}$ & NA \\
\hline
\end{tabular}




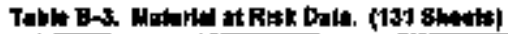

\begin{tabular}{|c|c|c|c|c|c|c|c|c|c|c|}
\hline Fid & MMR-\$tinct & 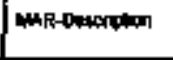 & MAR. & Copacts & 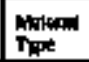 & $\begin{array}{l}\text { Plinius:a } \\
\text { Ftim }\end{array}$ & 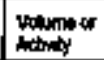 & Trenichat & ounth & Comminto \\
\hline $\begin{array}{l}\text { 241-EY. } \\
\text { MCE }\end{array}$ & 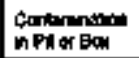 & 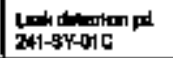 & forides & $13 \%$ gat & $\ln$ & Raytan & Whangen & No & $1 \mathbf{m ~}$ & NA \\
\hline $\begin{array}{l}241.5 Y . \\
020\end{array}$ & 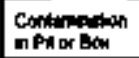 & 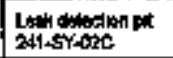 & Reotrd & 1358 130 & in & Pbade & Inithenth & klo & 1 t & $\mathrm{NH}$ \\
\hline $\begin{array}{l}\text { P41-Er. } \\
030\end{array}$ & 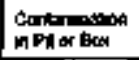 & 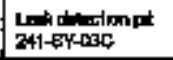 & Ractel & jast pat & $\mathbf{m u}$ & Rnude & Undongen & $\mathrm{kto}$ & $\mathbf{1 p}$ & NA \\
\hline $\begin{array}{l}241-54 . \\
\text { 620 }\end{array}$ & $\begin{array}{l}\text { Corimnimon } \\
\text { n Ad or Box }\end{array}$ & $\begin{array}{l}\text { Dran pt Z41.94. } \\
000\end{array}$ & freodus & 1459 ad & run & Restiva & Untorount & $\omega$ & $1 \mathrm{pd}$ & Wh \\
\hline $\begin{array}{l}241-94 . \\
\text { Qtel }\end{array}$ & $\begin{array}{l}\text { Corimmnoon } \\
\text { n Pid or Bex }\end{array}$ & 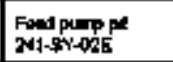 & Punder & $2474 \mathrm{gd}$ & $\mathbf{M H}$ & Rqabut & Unnowa & Ma & $1 \mathrm{pm}$ & NAR \\
\hline $249-\$ Y h$ & 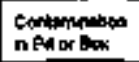 & 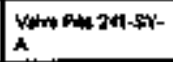 & fromets & 413 ad & Hh & Rentat & tInitalotim & is & $1 \mathrm{~m}$ & Nh \\
\hline 2*1-9Y-8 & 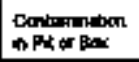 & $\begin{array}{l}\text { Yakn At 241-\$r- } \\
\text { P }\end{array}$ & Rendup & 44130 & NA & 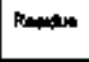 & חוmentur & $N$ & I pd & $\mathrm{kh}$ \\
\hline $\begin{array}{l}\text { sotesy. } \\
1-9\end{array}$ & 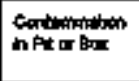 & 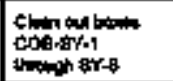 & Averdif & 181 \% & Nkh & Resciva & 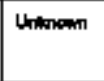 & $\mathbf{W b}$ & dbouss & $\mathbf{m}$ \\
\hline 24hn\$Y1 & 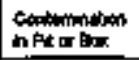 & 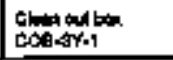 & Anerivd & 181 芇 & ran & Prositua & | & $\mathbf{m}$ & $1 \mathrm{bar}$ & Nh \\
\hline $241-9 \times a$ & $\begin{array}{l}\text { Conts miston } \\
\text { in Prt \& Bar }\end{array}$ & $\begin{array}{l}\text { Lemat bon } \\
\cos -6 \gamma^{2}\end{array}$ & 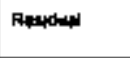 & 10: & Nh & Aypofun & In:men & $\mathrm{No}$ & 1 bqu & Wh \\
\hline $241-8 y-3$ & 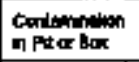 & 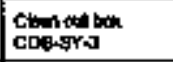 & |cudact & 141 t) & Nhe & ع. & Lrلm & Non & $1 \mathrm{bon}$ & an \\
\hline $241-6 Y Y-4$ & 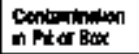 & 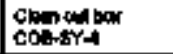 & 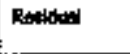 & لهa 191 & NA & 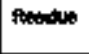 & 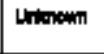 & No & 1 & $\mathbf{m}$ \\
\hline $241-875$ & 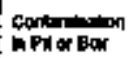 & $\begin{array}{l}\text { Can at bos } \\
\text { cop-8it-5 }\end{array}$ & RAaktal & 1무 ga & $\mathbf{m A}$ & frosinge & 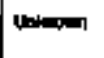 & $\mathrm{kos}$ & $1 \mathrm{bat}$ & $\mathrm{m}$ \\
\hline $211.8 Y 4$ & 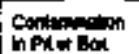 & $\begin{array}{l}\text { cimout bour } \\
\text { cosests }\end{array}$ & Rabidid & 191 a & Nh & Pratalus & Un:mim & $\mathrm{Na}$ & 1 bet & $\mathrm{mu}$ \\
\hline
\end{tabular}


Tabls D-3. Watarle it Rtst Data. (131 Shests|

\begin{tabular}{|c|c|c|c|c|c|c|c|c|c|c|}
\hline Find & 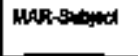 & MotR-Datentosh & 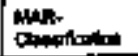 & Capar & Thind & $\begin{array}{l}\text { Plysucal } \\
\text { Fonm }\end{array}$ & Woingut af & Dramoin & Quntio: & Contmin \\
\hline $241-5 Y$ & $\begin{array}{l}\text { EARA } \$ 13 \\
\text { Whatsit }\end{array}$ & 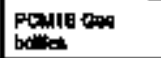 & 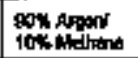 & topention & $\mathbf{m A}$ & an & 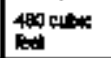 & Yen & 2 bonition & Nh \\
\hline Z41 wy & colver & Iqpd aphod & Ntogen & البه 18c0 & $\mathbf{w n}$ & ban & Un'som & $\mathrm{rb}$ & $\begin{array}{l}1 \text { lage } \\
\text { lanks }\end{array}$ & 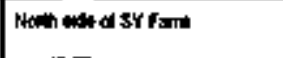 \\
\hline $241-6 Y$ & Dthe & Dwail ponerifor & שي & WA & un & Lquad & Untruman & Yan & 1 consolar & 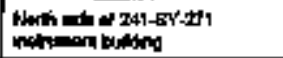 \\
\hline 2414 ar & odher & Hadroper bottles & 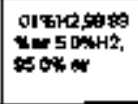 & thectibo & Ban & ter & 100 but & Yex & 2 battect & 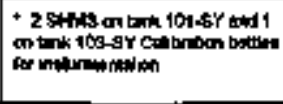 \\
\hline $\begin{array}{l}241-T- \\
101\end{array}$ & Tart Cuntents & 24I-T-101 & КгPLX & SeO Kps & $\begin{array}{l}\text { Superrorts } \\
\text { Eivege }\end{array}$ & toud & $\sum_{k \rightarrow 1}^{5} \mathrm{~K}_{0 \rightarrow 1}$ & Non & Wh. & 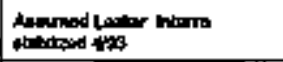 \\
\hline $\begin{array}{l}241-T- \\
102\end{array}$ & 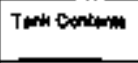 & $241-T-102$ & NCHX & \$OK K & Exparam & tod & 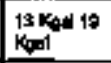 & $\mathrm{No}$ & $\mathbf{m}$ & 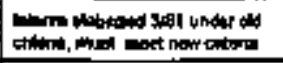 \\
\hline $341-T-$ & TaristCanieria & 2011-T-100 & MCFLX & $80 \mathrm{~kg}$ & $\begin{array}{l}\text { Bupermit } \\
\text { Ginge }\end{array}$ & uquad & A 14palizg & No & $\mathbf{m}$ & 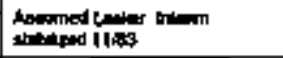 \\
\hline $\begin{array}{l}241 . T . \\
104\end{array}$ & Terh corthols & 241.7 .104 & NCPDr & 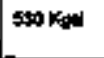 & $\begin{array}{l}\text { supamina } \\
\text { sudip }\end{array}$ & Sold & 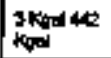 & $\mathrm{Nag}$ & wa & 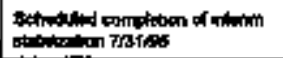 \\
\hline $\begin{array}{l}2+1-T- \\
10 \$\end{array}$ & 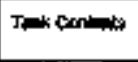 & 241.T:106 & mopux & 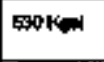 & sudge & 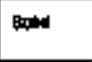 & sak kod & 10 & $\mathbf{m}$ & 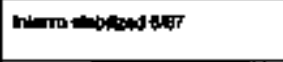 \\
\hline $\begin{array}{l}2 \times 1 \cdot T \cdot \\
106\end{array}$ & 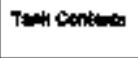 & $241 \cdot T+10 \theta$ & nem & Eot Kal & Bromine & Loquts & $2 \mathrm{Kad} 19$ & No & $\mathbf{m}$ & 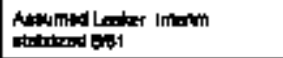 \\
\hline $\begin{array}{l}241-T- \\
\text { IOT }\end{array}$ & Tentr Coxtinit. & 2A1-T-tor & NCPU: & 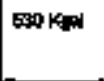 & Expotg & Baid & 1 rakea & $\mathrm{No}$ & $\mathbf{m}$ & 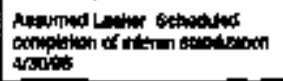 \\
\hline 201-T. & 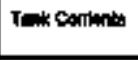 & $245-T-108$ & $\operatorname{MeP} x$ & Exa keal & Bados & sov & 4 Keal & is & H & 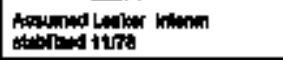 \\
\hline 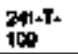 & $T_{\text {ma }}$ & $2 \times 1 \cdot T=100$ & $\operatorname{mep} x$ & stokn & stude & 90ind & 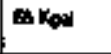 & $\$ 0$ & $\mathbf{m}$ & ith \\
\hline
\end{tabular}




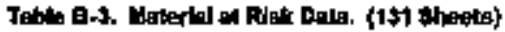

\begin{tabular}{|c|c|c|c|c|c|c|c|c|c|c|}
\hline Find & Mhrsthed & Mink-DLdenplath & MAR- & Captity & Typent & $\begin{array}{l}\text { Findted } \\
\text { Form }\end{array}$ & $\begin{array}{l}\text { Whind or } \\
\text { Axtmily }\end{array}$ & Teustarin & Duanity & Comenth \\
\hline $\begin{array}{l}241-T, \\
1+4\end{array}$ & Tant cortusts & $241+T-110$ & M⿻P口X & $5 \mathbf{s}\} \mathrm{Kod}$ & $\begin{array}{l}\text { Gupeminti } \\
\text { Striga }\end{array}$ & Eavd & $3 K_{0}$ & 相 & in & 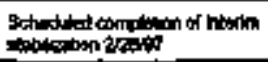 \\
\hline $\begin{array}{l}241+E \\
111\end{array}$ & Tort Contum & $241+T+111$ & NEPLX & Bath & \$tron & Satd & $48 K_{t}$ & b b & hit & 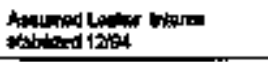 \\
\hline $\begin{array}{l}241-T- \\
112\end{array}$ & Tond Continita & 241-T-113 & MCPिए & $5 \mathbf{5}: \mathbf{K} \times \mathbf{m}$ & $\begin{array}{l}\text { Eupumint } \\
\text { stodge }\end{array}$ & Inowd & 7 Kand 6 & 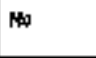 & in & 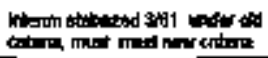 \\
\hline $\begin{array}{l}\text { P41-7. } \\
201\end{array}$ & Tort Conim & $241+72001$ & N亡PLX & $56 \mathrm{Kq}$ & 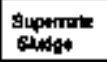 & Soid & $1 \mathrm{kgn}$ & Noto & $\mathrm{m}$ & NA \\
\hline 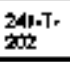 & Totk Conlerith & $241 . T-202$ & кся & $53 \mathrm{kor}$ & strope & sond & 2) & No & Hh & Nin \\
\hline 241.T. & Twi Carkenta & 241.T.庇 & MCPLX & BS Kol & Eudpt & sod & as $\mathrm{kpd}$ & Ho & Nh & MA \\
\hline $\begin{array}{l}2 \text { itt-T- } \\
204\end{array}$ & Tht conturith & 2n-T-204 & NCIX & 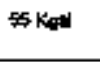 & stor & Tolom & 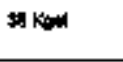 & No & HA & Nh \\
\hline $\begin{array}{l}\text { 2at- } T \text {. } \\
\text { क्रr }\end{array}$ & 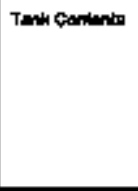 & 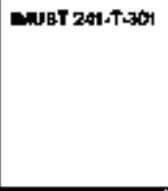 & 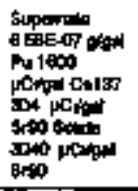 & 30000 마 & NA & $\begin{array}{l}\text { Boud } \\
\text { Bupinan } \\
-\end{array}$ & Fis & Ho & Nh & MA \\
\hline $\begin{array}{l}242-T . \\
1 \$ 6\end{array}$ & Tat do tents & ELst 242.T-135 & wodala & Lhtonm & Not & $\mathbf{m}$ & MA & wh & Nh & But \\
\hline $\begin{array}{l}241-T . \\
101\end{array}$ & 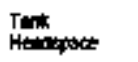 & 241-T-101 & $\mathbf{m}$ & WA & Nh & Whor & 2053 ma na & $\omega$ & Wh & $\boldsymbol{L}$ \\
\hline $\begin{array}{l}241-\mathrm{T} . \\
102\end{array}$ & Tritk & 241-T-102 & $m$ & NA & Nh & weror & 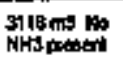 & $\omega$ & NA & Mh \\
\hline
\end{tabular}




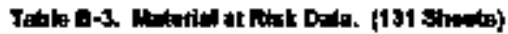

\begin{tabular}{|c|c|c|c|c|c|c|c|c|c|c|}
\hline Flatd & MAR-sublect & MAR-Ocasortibn & MAR- & Daty & Noted & $\begin{array}{l}\text { Plipinde } \\
\text { form }\end{array}$ & Yaingen & Tentandal & Ouanity & Commitath \\
\hline $\begin{array}{l}341-7= \\
103\end{array}$ & Hotionact & $201-T-104$ & M & WA & MA & Wepr & 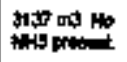 & W & $\mathbf{H}$ & $\mathbf{k h}$ \\
\hline $\begin{array}{l}\text { 241.T. } \\
\text { IDS }\end{array}$ & $\begin{array}{l}\text { Tank } \\
\text { Henctipex }\end{array}$ & $241 \times 1+104$ & NA & MA & Wh & Vepor & 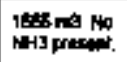 & No & $\mu \omega$ & M \\
\hline $\begin{array}{l}\text { Z4I-T. } \\
\text { ILE }\end{array}$ & $\begin{array}{l}\text { Tank } \\
\text { Hencenpes }\end{array}$ & $24,-4-405$ & $\mathrm{NH}$ & wh & Wh & Ver & 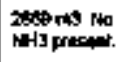 & No & Na & NA \\
\hline $\begin{array}{l}241-T . \\
100\end{array}$ & Thin & 2्41,T-105 & wh & NA & m & Yrepar & $\begin{array}{l}5100 \text { mis No } \\
\text { lars proctit. }\end{array}$ & no & NA & Na \\
\hline $241 . T$. & Thandiper & $241-T-107$ & Wh & $\mathrm{MA}$ & BH & Hops & 2004 ms & No & Man & Na \\
\hline $\begin{array}{l}241-T- \\
100\end{array}$ & $\begin{array}{l}\text { Jort } \\
\text { Henteptot }\end{array}$ & $241 . T=168$ & wh & NA & $\mathrm{mon}$ & Veper & 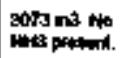 & ite & NA & NA \\
\hline $\begin{array}{l}241<\mathrm{T} . \\
109\end{array}$ & $\begin{array}{l}\text { Tant } \\
\text { Hatedeseon }\end{array}$ & $241-F-103$ & $\mathbf{m i n}$ & Ian & $\mathrm{ngh}$ & vaper & 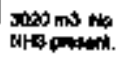 & $a b$ & we & NA \\
\hline $\begin{array}{l}241 . T . \\
110\end{array}$ & 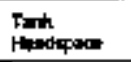 & 2a1-T.116 & $\mathbf{m h}$ & na & ran & vepor & 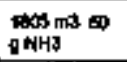 & $\mathbf{m o}$ & Wh & NA \\
\hline $\begin{array}{l}2+1 . T . \\
111\end{array}$ & Trenk & 241-T-111 & wh & WA & $\mathrm{mon}$ & Woper & $\operatorname{mon}_{0}$ & $\mathbf{W}$ & Nh & $\mathbf{N}$ \\
\hline $\begin{array}{l}\text { 741-T. } \\
112\end{array}$ & $\begin{array}{l}\text { Trulc } \\
\text { Howdaceor }\end{array}$ & 2.11-T.112 & wh & MA & $\mathrm{rat}$ & verpr & $\operatorname{mon}_{\mathrm{NOH}}$ & 40 & Nh & Nh \\
\hline 241-T- & Tank & $24 . \mathrm{T}-2 \mathrm{M}$ & Nh & Nin & $N$ & 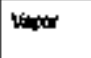 & 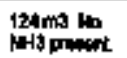 & $N a$ & $\mathrm{~m}$ & $m$ \\
\hline
\end{tabular}




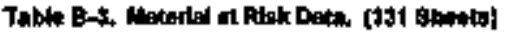

\begin{tabular}{|c|c|c|c|c|c|c|c|c|c|c|}
\hline Find & Muktsublect & 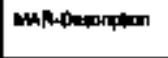 & Clapt- & capoch & Thyos & $\begin{array}{l}\text { Pligred } \\
\text { Fơm }\end{array}$ & $\begin{array}{l}\text { Hotume or } \\
\text { Helentil }\end{array}$ & Tr.t & Gutity & Corntert: \\
\hline $\begin{array}{l}241-T+ \\
202\end{array}$ & Trik & 34t-T-xit2 & $\mathrm{man}$ & Mh & Nh & vapux & 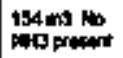 & $\mathrm{Na}$ & $\mathbf{H}$ & Wh \\
\hline $241-T$ & $\begin{array}{l}\text { Think } \\
\text { Hethotpace }\end{array}$ & $241 \cdot \mathrm{T}=45$ & $\mathrm{MA}$ & Mh & Nh & Venx & tol mo Nh & $\mathrm{Na}$ & $\mathbf{M H}$ & Wh. \\
\hline 2041-T- & Trith & 241-T-203 & NAS & NA & Wh & Hest & poms las & $\mathrm{NOO}$ & uh & NA \\
\hline $\begin{array}{l}241-T- \\
\Delta 1\end{array}$ & $\begin{array}{l}\text { Tart } \\
\text { Heod pipate }\end{array}$ & INपST 261-T-301 & ms. & WA & wh & Inepr & 19754 a d & on & NA & 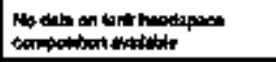 \\
\hline $\begin{array}{l}241.7 . \\
135\end{array}$ & $\begin{array}{l}\text { Tork } \\
\text { Bestapoest }\end{array}$ & 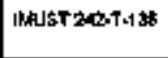 & wh & Natem & m & Wh & Na & Nh & $\mathrm{man}$ & ru \\
\hline 241-T & 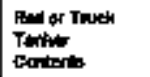 & Kant & as. & Wa & un & an & MA & NA & $\mathrm{km}$ & $\mathbf{H}$ \\
\hline $241-T$ & $\begin{array}{l}\text { Ceningumace } \\
\text { on thithe }\end{array}$ & rent & Wh & wa & an & $\mathbf{m}$ & Na & wa & kn & $\mathbf{H A}$ \\
\hline 241-T & 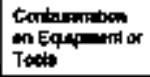 & Hond Trobs & mA & MA & MA & $\mathbf{k}$ & man & $Y=s$ & M & 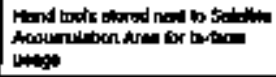 \\
\hline $241-7$ & 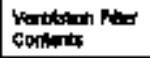 & Pmbl & NA & inth & NA & MA & Nh & $\mathbf{w h}$ & Nh & wh \\
\hline $241-\mathbf{T}$ & Contention & Aminan & NA & wh & NA & nch & Nh & wh & Nh & NA \\
\hline $241-T$ & Conlinition & sther & Nh & 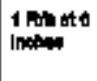 & Non & Fintorid & UA & Ye. & Nh & 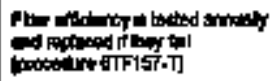 \\
\hline
\end{tabular}




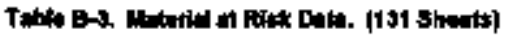

\begin{tabular}{|c|c|c|c|c|c|c|c|c|c|c|}
\hline Fund & MHR-Eustet & 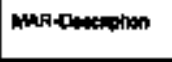 & Mup. & Govertir & Type & $\begin{array}{l}\text { Piotacy } \\
\text { Fom }\end{array}$ & inction of & Trmengit & Quindty & Camonents \\
\hline $2 \times 1.7$ & 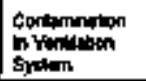 & De-edranerte & Hit & $w_{k}$ & Nh & $\mathbf{m}$ & Ин & wh & NA & rw \\
\hline $241+T$ & 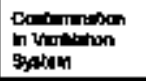 & S.d bop & Od and & 1 pap & $\mathbf{m}$ & Lipd & NA & m & $\mathbf{m}$ & 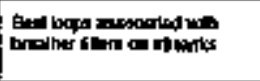 \\
\hline $241-T$ & 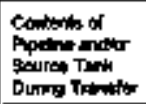 & Wh & Wh. & NA & $\mathbf{M n}$ & NA & Ma & Mat & $\boldsymbol{k n}$ & 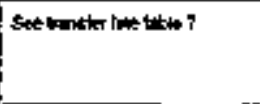 \\
\hline 241.7 & 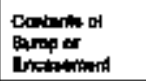 & $\mathbf{M h}$ & $m$ & HA & $\mathbf{m h}$ & $N$ & Ma & Nath & $\mathbf{m}$ & 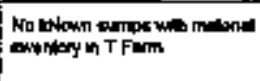 \\
\hline $241-T$. & $\begin{array}{l}\text { Contaniston } \\
\text { ज Fit \& Bax }\end{array}$ & Pupp 241.T. & Rentall & 1058 pal & Mas & Freode & Loincen & $\mathrm{No}$ & 12 & $\mathbf{N h}$ \\
\hline 241.T- & 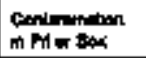 & Pus $10 x$ 241-T- & Resudel & 10 묘 범 & Na & Randth & |n:m & No & 1gt & Nh \\
\hline $\begin{array}{l}241-T . \\
\text { ath }\end{array}$ & 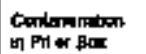 & $\ln _{107}=241-T$. & Rtondal & 1057 pal & in & Ponere & Lximom & Nog & Ipt & $\mathbf{N}$ \\
\hline 24I.T- & $\begin{array}{l}\text { Conirninimin } \\
\text { m Pit of Bain }\end{array}$ & $\operatorname{Pump}_{104}$ & Ropudeal & 1657 and & $\mathrm{Han}$ & 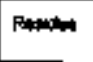 & Unimown & $\mathrm{No}$ & $1 \mathrm{M}$ & Nu \\
\hline 201-7. & $\begin{array}{l}\text { Contemrinton } \\
\text { nPM } \mathrm{Bar}\end{array}$ & $\underset{110}{P \text { PIP }}$ & Resited & 1069 gid & Na & Arody & Whisom & Ho & $1 \mathbf{F}$ & $\mathbf{m}$ \\
\hline $\begin{array}{l}\text { 2+1-T. } \\
\text { 1บ4 }\end{array}$ & $\begin{array}{l}\text { Conimminiben } \\
\text { h PAl or Ban }\end{array}$ & Pitap ot 241-T. & Reanten & 105 & M & Poonduta & 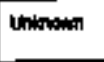 & No & $1 \mathrm{man}$ & Mh \\
\hline $\begin{array}{l}\text { 2n-t- } \\
\text { o18 }\end{array}$ & $\begin{array}{l}\text { Contimnimiton } \\
\text { h Pil of Pox }\end{array}$ & Hei pH-2*1-T×018 & Repiter & tido ord & wa & Fing & Uningen & ilo & $1 \mathrm{man}$ & $\mathbf{m}$ \\
\hline
\end{tabular}




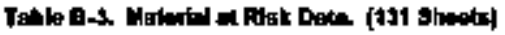

\begin{tabular}{|c|c|c|c|c|c|c|c|c|c|c|}
\hline fath & Wrstojet & 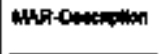 & Mition: & Gtaty & 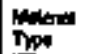 & Pmpled & Hoinger or & Thtmet & Qubby & combatits \\
\hline $\begin{array}{l}\text { 241-T- } \\
\text { ont }\end{array}$ & $\begin{array}{l}\text { Conimminion } \\
\text { In PA or Bas }\end{array}$ & 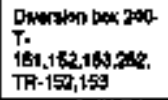 & Rtudud & NA & Mh & Ponulat & Uniknowrt & wo & למידמ 6 & 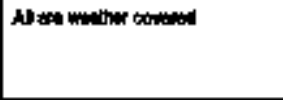 \\
\hline $\begin{array}{l}240-T- \\
101\end{array}$ & Loniminion & $\begin{array}{l}\text { Dawson bex 2ath } \\
\text { T.151 }\end{array}$ & Rulduat & $\mathrm{MH}$ & Ma & Promitut & Unknown & wo & 1 bod & 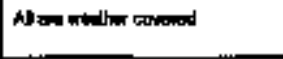 \\
\hline $\begin{array}{l}240 . T . \\
152\end{array}$ & 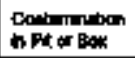 & 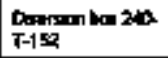 & Rertus & Nh & NH & Peodue & 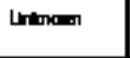 & 40 & 1 wa & 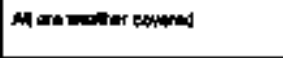 \\
\hline 200.7. & 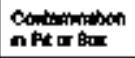 & 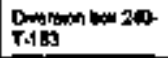 & Reorstrat & in & now & netedub & Unidingm & $\omega$ & $1 \mathrm{bow}$ & Mre mather poverd \\
\hline 200 & 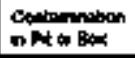 & 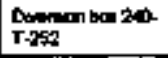 & 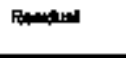 & NA & KH & Respdun & 1" & ats & 1 w & 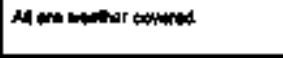 \\
\hline $\begin{array}{l}\text { 20-TR- } \\
162\end{array}$ & 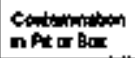 & 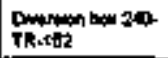 & Reminat & NA & m & Mtudut & Untom & tor & $1 \mathrm{tom}$ & 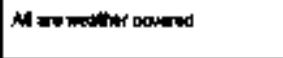 \\
\hline $\begin{array}{l}\text { 200-TR. } \\
15 \%\end{array}$ & 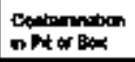 & 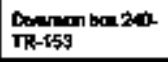 & Randind & NA & $\mathbf{~ H 4}$ & Feondue & 1" & $\omega$ & 1 | & 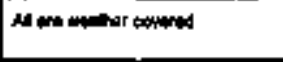 \\
\hline $241-T$ & gaks oft & 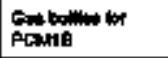 & $\begin{array}{l}\text { 60togon } \\
\text { 104 methen }\end{array}$ & ind cable & $N \lambda$ & Whor & Weltit & $Y=x$ & 2 botse & Leoted oumkh dhange trefer \\
\hline 241-T & 엪 & Comex sionge & wh & HA. & Wh & Wh & uh & W & 10 & 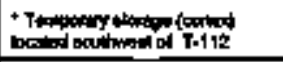 \\
\hline 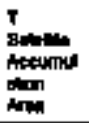 & other & Stimith & $\begin{array}{l}\text { Achthmod } \\
\text { math }\end{array}$ & din & Nin & man & wh & Ma & Nane & - Locited with of ocnac ibage \\
\hline 241-T & catho & 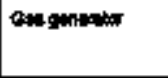 & in & Hh & NA & $\mathbf{m}$ & Hin & $\mathbf{H}$ & $\begin{array}{l}1 \\
\text { andinger }\end{array}$ & 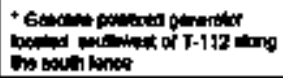 \\
\hline
\end{tabular}




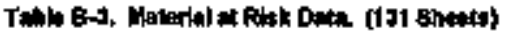

\begin{tabular}{|c|c|c|c|c|c|c|c|c|c|c|}
\hline Find & 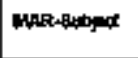 & 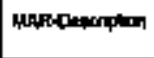 & Kolp- & Capedily & Tying & $\begin{array}{l}\text { Preycel } \\
\text { Fanm }\end{array}$ & valune or & Trminat & Quाby & 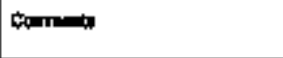 \\
\hline $241-T$ & Othe & Tod binep & $\mathbf{m}$ & Nin & NA & N & Mu & ath & 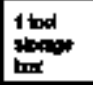 & 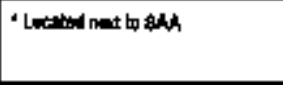 \\
\hline $241-T$ & Other & 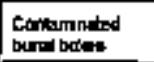 & $\mathbf{m}$ & Nh & min & Nh & ma & uh & 2 bows. & 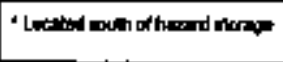 \\
\hline 241-T & Oiner & Aoting & NA & $\mathbf{m}$ & WA. & $\mathbf{m}$ & Wh & NA & $\mathbf{r a n}$ & 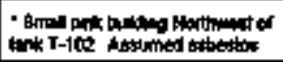 \\
\hline $215-14$ & odhe & 0.th 210.T.1 & MA & man & Nh & $\mathbf{k t}$ & $\mathbf{N h}$ & NA & han & 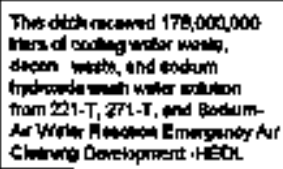 \\
\hline $\begin{array}{l}215 T+4 \\
2\end{array}$ & Oiner & ant $210 T+2$ & NA & $\mathrm{me}$ & Na & $\mathrm{min}$ & Na & NA & rah & 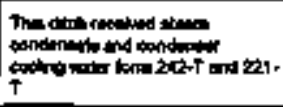 \\
\hline 24:Thy & cather & Gab 215-Tat & NA & Mu & $\mathbf{m}$ & NA & in & NA & Nh & 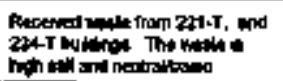 \\
\hline 216.1 42 & cater & Crb 215-7.72 & NA & Ma & m & wh & $\mathrm{Nh}$ & NHA & NA & 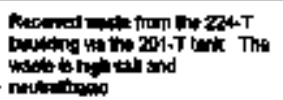 \\
\hline $\begin{array}{l}241-T / 2 \\
104\end{array}$ & Tank Cenlmo & 241-De-109 & $\operatorname{mep} x$ & ToK & 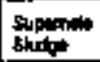 & Lifid & 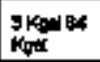 & $\mathrm{Ha}$ & NA & 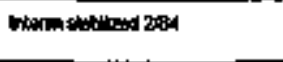 \\
\hline $\begin{array}{l}241+7 x . \\
100\end{array}$ & 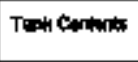 & $241+7 x-10$ & NCFU & $7+4 \mathrm{~K}$ & S4: & siged & $217 \mathrm{kgm}$ & $\mathrm{No}$ & NA & 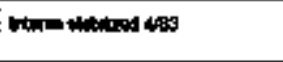 \\
\hline$x_{101}^{241 . T x-}$ & Tente Conimails & $241-T \times 103$ & hast & Fon & t8lad & sond & 15 Kas & m & Nh & 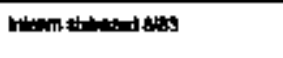 \\
\hline
\end{tabular}




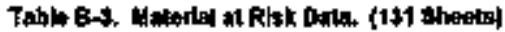

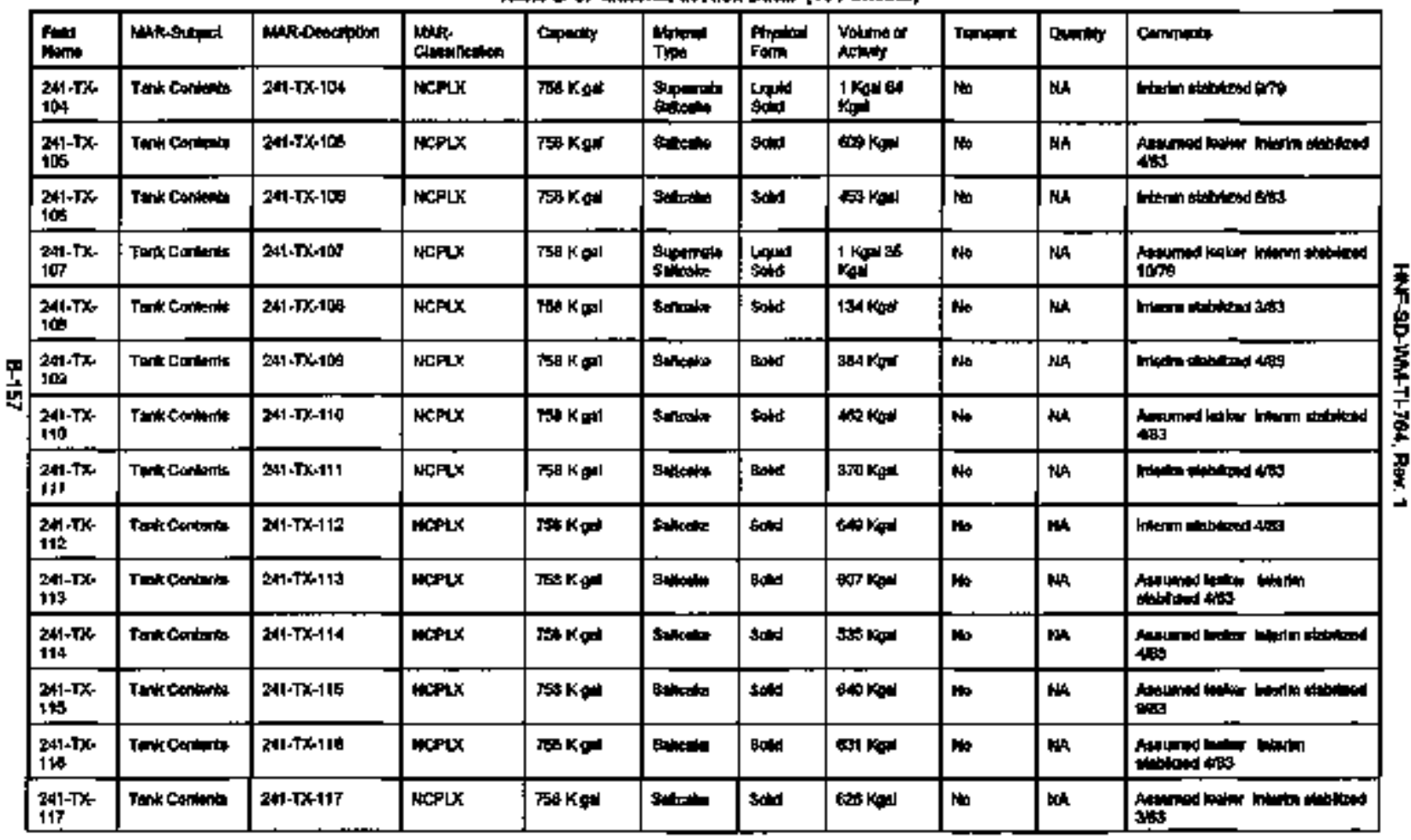




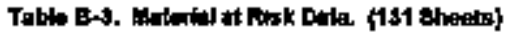

\begin{tabular}{|c|c|c|c|c|c|c|c|c|c|c|}
\hline Find & MAR-Bubjot & MHRLotedilon & MtR. & capacty & That & $\begin{array}{l}\text { Fifical } \\
\text { Fomit }\end{array}$ & Whima of & Tranteol & Ounby & Colments \\
\hline $\begin{array}{l}241-1 x- \\
110\end{array}$ & Yalk Co.mtr & 241-TX-118 & NGPAX & $\pi \times \mathbb{K}$ & Batoner & 5메 & 3\$ Kad & No & $\mathbf{H}$ & 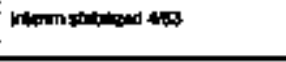 \\
\hline $\begin{array}{l}241-7 x \text {. } \\
302 A\end{array}$ & Tant Canimito & $\begin{array}{l}\text { 241-TX-30t2k } \\
\text { WUण }\end{array}$ & 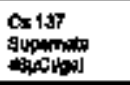 & $17 t 64$ & Nh & 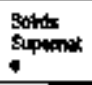 & 248010 & Ha & man & in \\
\hline $\begin{array}{l}2+1-T x- \\
\operatorname{son} \cdot-4 x \mid\end{array}$ & Tomt Contimlis & 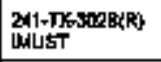 & Hh & 12000 & Nh & Nito fat & 0gal & Ho & H & m \\
\hline $\begin{array}{l}241.7 x \\
3028\end{array}$ & Tart Cantens & $\begin{array}{l}\text { 741-[x-3028 } \\
\text { ImvsT }\end{array}$ & MA & 17: & Nh & Nat doth & $1930=$ & No & M & NA \\
\hline $\begin{array}{l}241 . T x_{2} \\
\text { atore }\end{array}$ & Tonk Conitints & 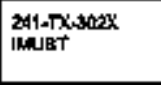 & int & |4\$d & Wh & $\begin{array}{l}\text { Soth } \\
\text { supernt } \\
\text { t }\end{array}$ & 10d 348 & Ha & $\ln$ & $\mathrm{NA}$ \\
\hline $\begin{array}{l}249-T x- \\
61\end{array}$ & Tort Cante is & $\begin{array}{l}\text { 244-Tस001 } \\
\text { I|w }\end{array}$ & 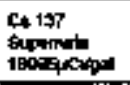 & spepos & MA & $\begin{array}{l}\text { Bntit } \\
\text { Sylomint } \\
0\end{array}$ & 2001 & No & ins & NA \\
\hline $\cos ^{241-T x-}$ & Tertentain & 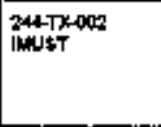 & 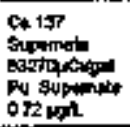 & 1 15xक & MA & Ecots & 14040 & ks & Nan & nat \\
\hline $\begin{array}{l}241 \cdot T K \\
0 \times B\end{array}$ & Tarit Gonterin & $\begin{array}{l}244 T x-000 \\
\text { MUST }\end{array}$ & m & 16000 & Mh & solde & E*03 ga & Bo & $\mathrm{kn}$ & $\mathbf{N}$ \\
\hline $\begin{array}{l}242-T A- \\
\text { h1 }\end{array}$ & 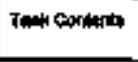 & 2at.TAR1 & No data + & Not th & Hen & Whatata & No dota & $\mathrm{Wb}$ & Nh & 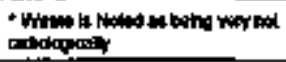 \\
\hline $\begin{array}{l}\text { 241-7x. } \\
302 C\end{array}$ & Tas conterts & 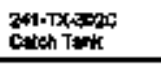 & 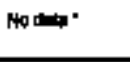 & 14006 & ren & Whth & 40000 & No & wh & 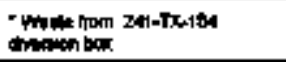 \\
\hline $\begin{array}{l}241-T K \\
101\end{array}$ & 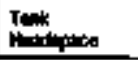 & 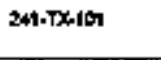 & Mn & Nhe & ned & Inotrin & ats ms & No & H. & $\mathrm{kH}$ \\
\hline $\begin{array}{l}241+\mathbf{T X} . \\
102\end{array}$ & Thes & $241-7 x-162$ & NA & NA & H & Woor & II:0) & $\mathbf{N o}$ & Wh & $\mathbf{m}$ \\
\hline
\end{tabular}




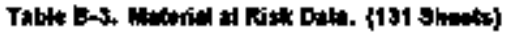

\begin{tabular}{|c|c|c|c|c|c|c|c|c|c|c|}
\hline $\begin{array}{l}\text { Find } \\
\text { Not: }\end{array}$ & Hatr-ganojed & MHROWeTtion & 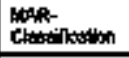 & apincity & 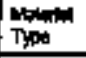 & pont & volum & Tringentit & Qu:aty & Conlmits \\
\hline${ }_{100}^{241-\pi R .}$ & $\begin{array}{l}\text { Tort } \\
\text { Hexpouse }\end{array}$ & 241-TX-103 & NA & int & $\mathbf{m a t}$ & Yapar & 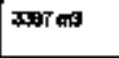 & No & $\mathbf{m}$ & Wh \\
\hline $\begin{array}{l}241-7 x . \\
104\end{array}$ & $\begin{array}{l}\text { Tark } \\
\text { Hondipace }\end{array}$ & 241-TK-194 & NA & Nh & $M$ & Yaper & J758 ma & Not & $\mathrm{m}$ & Nor \\
\hline $\begin{array}{l}241-\mathrm{Tx} . \\
106\end{array}$ & $\begin{array}{l}\text { Turt } \\
\text { Houspapes }\end{array}$ & $241-t x-116$ & Nur & Nin & $\omega$ & Hepor & 10 & No & wh & Wh \\
\hline $\begin{array}{l}241+T x . \\
100\end{array}$ & $\begin{array}{l}\text { Thin } \\
\text { Hed }\end{array}$ & $241+T \times 106$ & MA & Nh & Mh & topor & $2 x+3=5$ & $\mathrm{~N}_{\mathrm{t}}$ & $\mathbf{M n}$ & Nin \\
\hline $\begin{array}{l}241-T R- \\
108\end{array}$ & Tut & $241+\Gamma x-10 s$ & MA & Wh & ruh & Wepe & 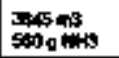 & No & $\mu$ & Nhth \\
\hline $\begin{array}{l}241 . T \times-7 \\
100\end{array}$ & Toin & $241-7 x+106$ & Nh & NAS & in & Veper & $\sin 4 \sin$ & $\mathrm{Not}$ & An & Nut \\
\hline $\begin{array}{l}241-T x- \\
109\end{array}$ & Turk & $241-T x-109$ & Na & ah & $\mathbf{H A}$ & Waper & 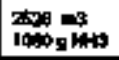 & $\mathrm{No}$ & $\boldsymbol{\mu}$ & Nh \\
\hline $\begin{array}{l}241 \cdot T X- \\
110\end{array}$ & Thith & $24,-7 x, 710$ & $\mathrm{Nh}$ & $m$ & $\operatorname{mon}$ & Hopr & 200 & No & M & wh \\
\hline $\begin{array}{l}\text { 311-TX- } \\
111\end{array}$ & Trok & $241-T x-111$ & NA & $\mathbf{m}$ & $\mathbf{H A}$ & vaper & 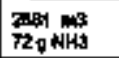 & $\mathrm{Not}$ & Nu & Nh \\
\hline $\begin{array}{l}211.7 X- \\
112\end{array}$ & $\begin{array}{l}\text { Thike } \\
\text { Hesodapose }\end{array}$ & $241 \cdot T x, 112$ & $\mathrm{NH}$ & ma & an & them & $1 \cos x=3$ & No & MH & wh \\
\hline $\begin{array}{l}241-T x- \\
119\end{array}$ & Tintr & $211-T X-113$ & NA & m & NH & vaper & 1604 mats & $\mathrm{Hot}$ & M & Nh \\
\hline $\begin{array}{l}\text { 341-Tx. } \\
1 / 4\end{array}$ & Trit & $241-T \times-914$ & $\mathbf{m}$ & Na & Na & vipor & tiscents & $\mathbf{m}$ & mk & $\operatorname{mon}$ \\
\hline $\begin{array}{l}241-7 x . \\
1+8\end{array}$ & Tint & $241-T x_{0}+16$ & $\mathbf{m}$ & Nat & $N$ & vepor & ats mat & $\mathbf{m}$ & Wh & $\mathrm{mon}$ \\
\hline${ }_{119}^{241-\pi x .}$ & Tris & $2+1-T X-410$ & $M$ & M & Na & $\sqrt{n}$ & $160 \mathrm{ma}$ & $\mathbf{m}$ & Nh & $M$ \\
\hline
\end{tabular}




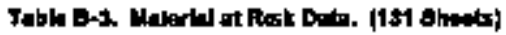

\begin{tabular}{|c|c|c|c|c|c|c|c|c|c|c|}
\hline Dive & Muspubtal & MHR-Docapinn & 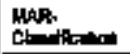 & capisty & $\begin{array}{l}\text { Mtutat: } \\
\text { TTPa }\end{array}$ & Plipuct & vonity & Tranenth & Outhy & Camintin \\
\hline $\begin{array}{l}241-T \times 6 \\
1+1 ?\end{array}$ & 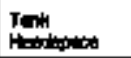 & 261.TX(-117 & NA & WA & Wh & Waper & 1012 ms & No & Nh & BCh \\
\hline $\begin{array}{l}241+T x \\
119\end{array}$ & Tank & $2 \hbar \cdot-T X-110$ & Nh & 螒 & wh & 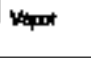 & $\begin{array}{l}\text { xtot int at } \\
\text { cking }\end{array}$ & Not & Nin & RCA \\
\hline $\begin{array}{l}\text { 3a1-Tx. } \\
\text { 3ank }\end{array}$ & Trot & $\begin{array}{l}\text { 241.T2402h } \\
\text { WU5T }\end{array}$ & Wh & wh & Mh & 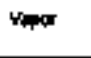 & 16004 D. - & 100 & $m_{2}$ & 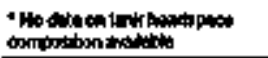 \\
\hline 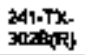 & $\begin{array}{l}\text { Tant } \\
\text { Hand-poct }\end{array}$ & 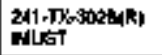 & Wh & wh & $\mathrm{ma}$ & vepor & 12000 四 & Ha & $\cos$ & 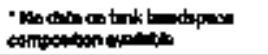 \\
\hline $\begin{array}{l}\text { 24a-TX } \\
\text { ates }\end{array}$ & Tonth & $\begin{array}{l}\text { X41-Tx-3028 } \\
\text { WเหsT }\end{array}$ & Nh & Hh & $\mathrm{mh}$ & Wapor & + 178940 & No & $\mathbf{K A}$ & 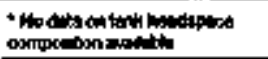 \\
\hline $\begin{array}{l}\text { 241-Tx } \\
\text { xirtix }\end{array}$ & $\begin{array}{l}\text { Terk } \\
\text { Hand-poot }\end{array}$ & 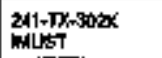 & H & $\boldsymbol{M h}$ & $\mathrm{man}$ & veor & 13너 & Ho & bon & 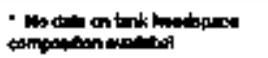 \\
\hline 201-TX & Tinder & $\begin{array}{l}\text { 244tx-001 } \\
\text { Wy:T }\end{array}$ & wh & NA & Nh & Wheper & (4) + & ma & Nh & 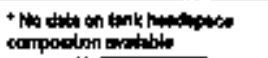 \\
\hline $\begin{array}{l}2+1 \cdot T x \\
002\end{array}$ & Tinctipace & 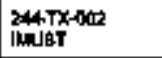 & wh & Nh & Wh & Voper & 1203 squ = & $\mathbf{m}$ & MA & 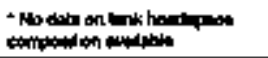 \\
\hline $\cot ^{2+1-T X-}$ & THedspers & $\begin{array}{l}\text { 244Tx-040 } \\
\text { Invot }\end{array}$ & uh & NA & Nh & Yuper & $040=10$ & w & Wh & 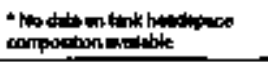 \\
\hline 2At & Tofit & 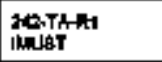 & int & wh & Wh & Vtepo & beinow & Ho & MA & 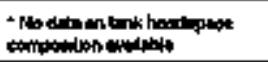 \\
\hline $\begin{array}{l}2+1-7 x \\
3000\end{array}$ & Then & 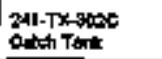 & wh & MA & $\omega \wedge$ & vars & 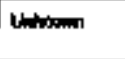 & w & nin & 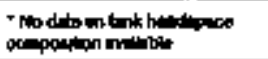 \\
\hline $241=7 x$ & $\begin{array}{l}\text { Rad of Thust } \\
\text { Tankif } \\
\text { Corkmt }\end{array}$ & Naon & Nin & Ner & WA & NA & Hh & Ha & m & $\mathbf{m}$ \\
\hline 741-1XX & 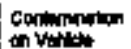 & 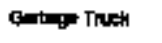 & $N A$ & MA & $\omega_{h}$ & NA & Bh & Yop & ith & 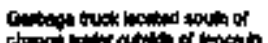 \\
\hline
\end{tabular}


Table B-3, Fintorlal at Risk Dith. (691 Shwts)

\begin{tabular}{|c|c|c|c|c|c|c|c|c|c|c|}
\hline Find & MAR-sybiacd & 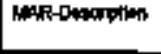 & $\begin{array}{l}\text { MARr } \\
\text { Chrindechoh }\end{array}$ & Cepouctir & $\begin{array}{l}\text { Mripla } \\
\text { Tytit }\end{array}$ & Pthinstill & 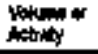 & Thotaw & anditis & Comante \\
\hline $241+1 x$ & 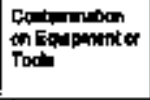 & than & m & man & M & Hit & Wh & NA & Heh & 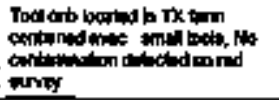 \\
\hline $241+1 \times$ & 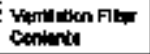 & Prtary & Mh & man & m & 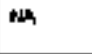 & WA & MA & Ha & NA \\
\hline $241 \cdot 7 x$ & 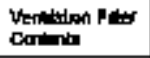 & Arruth & NA & NA & Nin & eh & Nh & NA & NA & Nh \\
\hline $24 y-T x$ & Yaniwiman Fifre & Broper & NA & 1 Prans 6 & Nh & Petcolfo: & NA & 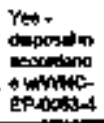 & NA & 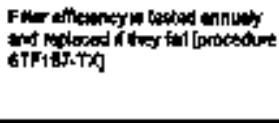 \\
\hline 241.TX & 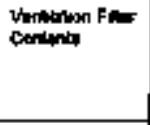 & 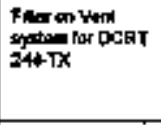 & Nh & ifhes & Na & Penorinal & in & 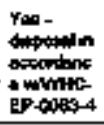 & MH & 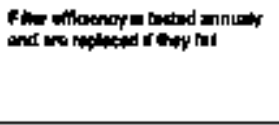 \\
\hline 2*1-TX & 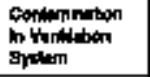 & De-tidentan & WA & NA & $\mathbf{M A}$ & NA & $\mathrm{Wh}$ & Mh & Wh & uh \\
\hline $241+1 X$ & 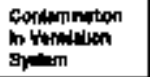 & 8act bcp & $\begin{array}{l}\text { on and } \\
\text { onderents }\end{array}$ & 1 Gap & MA & Lond & 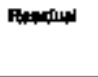 & MA & Nh & 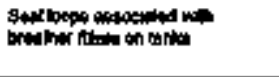 \\
\hline $241+10 x$ & Conlmitg of & Nh & NA & MA & 许 & MH & th & Wh & Wh & 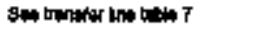 \\
\hline $2447 x$ & $\begin{array}{l}\text { Corteritzor } \\
\text { Expas }\end{array}$ & 244TX DCRT & H & E4,000 해 · & wh & $\mathbf{N H}$ & $\mathbf{m h}$ & uh & wh & 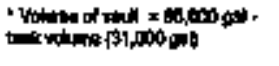 \\
\hline
\end{tabular}




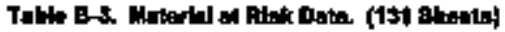

\begin{tabular}{|c|c|c|c|c|c|c|c|c|c|c|}
\hline Fid & MARrGitipd & Mritboxpom & 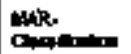 & Ceping & Mtan & Popel & Nodutity of & Tranomin: & |rint & Cotratheth \\
\hline $241-T x$ & 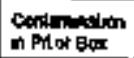 & Fump phas & Resulted & WA & m & Rascitua & Wh & 100 & MH & 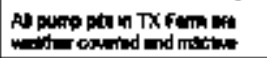 \\
\hline $341 \cdot \pi x$ & 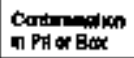 & gluce pit & 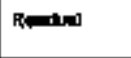 & Mn & ra & Ra:nety & on & Na & mon & 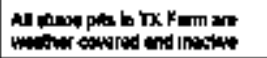 \\
\hline $241-T x$ & 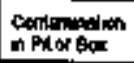 & Wat pt & Peratud & ma & MH & Ration & Wh & 100 & un & 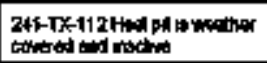 \\
\hline $\begin{array}{l}341 . \\
\text { TXR:- } \\
14143\end{array}$ & $\begin{array}{l}\text { Canturingion } \\
\text { n PAl or Bar }\end{array}$ & 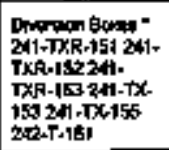 & Reater & in & $\mathrm{m}$ & 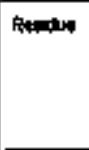 & WA & kla & MA & 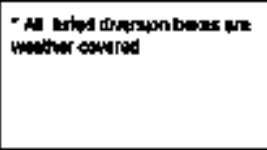 \\
\hline $\begin{array}{l}\text { 241. } \\
\text { TXRR-151 }\end{array}$ & 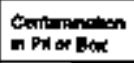 & $\begin{array}{l}\text { Durrace Bax } 241 . \\
\text { DXR-15. }\end{array}$ & Restud & wh & MA & Recitua & HA & klo & Na & 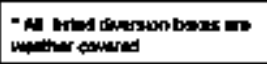 \\
\hline Txi-152 & 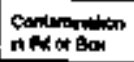 & 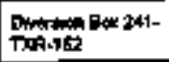 & Riminded & WA & NAM & R丨m & NA & No & Nan & 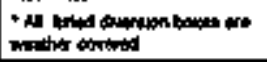 \\
\hline $\begin{array}{l}\text { 2A1: } \\
\text { TXR-1EB }\end{array}$ & 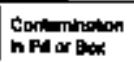 & 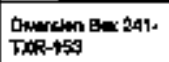 & Proodur & $M$ & MA & Proder & NAd & 10 & Nh & 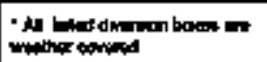 \\
\hline $\begin{array}{l}2+1-T X \\
160\end{array}$ & 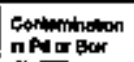 & 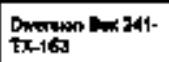 & Mindwal & $\mathbf{m e}$ & Nh & Punder & Na & $\mathbf{m}$ & NAS & "All \\
\hline $\begin{array}{l}241-T X \\
15\end{array}$ & 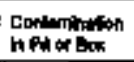 & Dixim Ben 241- & Rendur & $\mathbf{m}$ & Wh & Prapolut & NA & us & Nith & " \\
\hline 215-T- & $\begin{array}{l}\text { Cophumadon } \\
\text { n PA G Baw }\end{array}$ & $\begin{array}{l}\text { Derawan Dex 241. } \\
\text { T-151 }\end{array}$ & 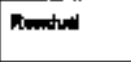 & Mh & Nh & fordutus & Nh & $\boldsymbol{w}$ & NA & - \\
\hline 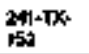 & $\begin{array}{l}\text { Conilmation } \\
\text { n Fid of tan }\end{array}$ & $2 \pi+7 x-152$ & Rooldow & 20020 & Nh & Pesidus & NA & b & Nh & H \\
\hline $\begin{array}{l}\text { 2*1-Tx. } \\
154\end{array}$ & 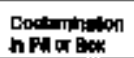 & 2小1 & Wh:ther & 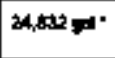 & Wh & Fanopole & Na & ito & math & 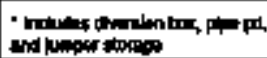 \\
\hline
\end{tabular}




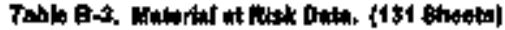

\begin{tabular}{|c|c|c|c|c|c|c|c|c|c|c|}
\hline $\begin{array}{l}\text { Field } \\
\text { Nown }\end{array}$ & 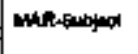 & MuR-baccapton & Whis: & cospary & Trind & $\begin{array}{l}\text { Proncer } \\
\text { Form }\end{array}$ & Yotuma or & Tonden & Qxumity & Commenting \\
\hline $241.7 x$ & SARA dist & $\begin{array}{l}\text { Gor bonter lor } \\
\text { PCAN1 日: }\end{array}$ & 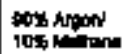 & 4tot autar & $\mathbf{m}$ & 的 & atrabe & $Y \mathbf{H}$ & 2 bottie: & 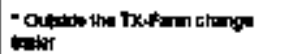 \\
\hline $241-T x$ & Qthe & 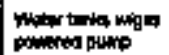 & wiles & - $\sec$ & Ah & LNWat & Unformen & Yes & 2 tancs & 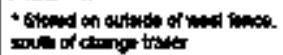 \\
\hline $241 \cdot T x$ & Other & Hetracts & Hertedu & -25 bath & $\mathbf{k}$ & Sand & Untantin & Yes & +2 & 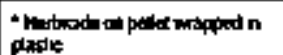 \\
\hline 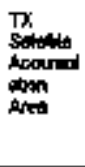 & Qthe & gatainie & $\begin{array}{l}\text { FCFA nind } \\
\text { Whethat }\end{array}$ & 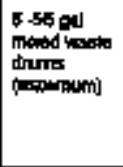 & $\mathbf{m}$ & Beind & 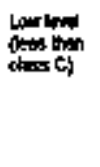 & Yas & 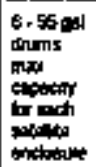 & 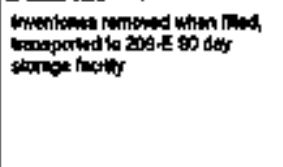 \\
\hline $241 \cdot \mathrm{TX}$ & othet & 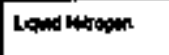 & & 2 beinlet & M & Haper & 的 & Y* & 26 attien & 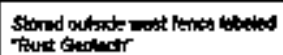 \\
\hline $\begin{array}{l}2+41+T Y . \\
101\end{array}$ & Tank Cendatm & $241-T=101$ & WePtK & ros kqu & Enudget & Boid & 114 Mra & $\mathrm{Ho}$ & NA & 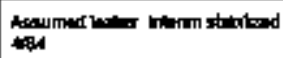 \\
\hline $\begin{array}{l}\text { T41-TY- } \\
102\end{array}$ & Tank Sonkwak & 2ul-TY-10? & wertx & Jss kif & Salichtos & 5044 & $04 k+1$ & w & NA & 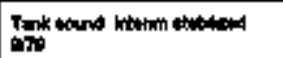 \\
\hline $\begin{array}{l}241-T Y . \\
109\end{array}$ & Tank Coritant & 2*1.Tr+16s & 1xPLX & 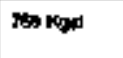 & Enogen & 8ded & $t: 2 \mathrm{Kp}$ & ws & NA & 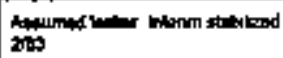 \\
\hline $\begin{array}{l}\text { 2d1.TY. } \\
104\end{array}$ & Tenk Conter & $241-T Y-104$ & 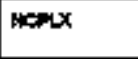 & JE: & 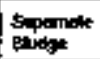 & $\begin{array}{l}\text { Uquid } \\
\text { spoed }\end{array}$ & $4 \mathbf{k}$ & $\boldsymbol{H}$ & Nik & 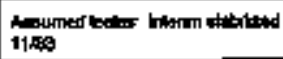 \\
\hline $\begin{array}{l}\text { 241-TY- } \\
105\end{array}$ & Tank Comilinin & 2H-TY-14 & แผPบx & $\mathrm{H}=\mathrm{MPN}$ & Barates & fold & $231 \mathrm{~kg}$ & Ho & NA & ה \\
\hline $\begin{array}{l}241-T r \\
109\end{array}$ & Tank Corthen & 2A1-TY-100 & Ka) & T: Ko & studye & satd & 17 Npal & $m$ & Ma & 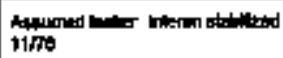 \\
\hline $\begin{array}{l}\text { 24I-TY- } \\
\text { stent }\end{array}$ & Twok Cankete & 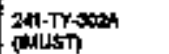 & en & لبم 17: & sindes & told & 100 & W & Nin & 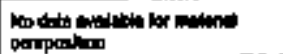 \\
\hline
\end{tabular}




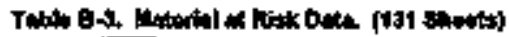

\begin{tabular}{|c|c|c|c|c|c|c|c|c|c|c|}
\hline Find & RAR-bunnot & 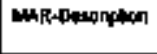 & MAR & Cepentity & Tyland & $\begin{array}{l}\text { Phylitid } \\
\text { Foniw }\end{array}$ & Volven ar & Thenand & Qunets & Comminto \\
\hline $\begin{array}{l}\text { P41-TY. } \\
\text { 302B }\end{array}$ & Tani Cotats & 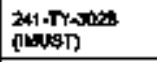 & MA & 14,214 pal & Nh & wh & ${ }^{0} \mathrm{~g}$ & $\mathbf{H o}$ & wh & 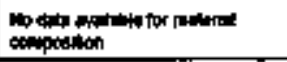 \\
\hline $\begin{array}{l}\text { 216-TY- } \\
\text { Zad }\end{array}$ & Tan Conting & $\begin{array}{l}\text { 2A-TY-201 } \\
\text { (It):T) }\end{array}$ & NA & $19200 \mathrm{pas}$ & Notata & Wodata & Not & Notata & NA & 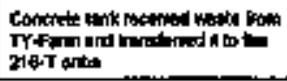 \\
\hline $\begin{array}{l}\text { 2AI +TY. } \\
10 \mathrm{~A}\end{array}$ & Tank & 341.TY<101 & Wh & NA & WA & Vhapr & 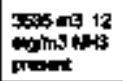 & $\mathbf{N o}$ & NA & 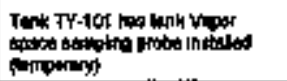 \\
\hline $\begin{array}{l}201 \cdot T Y \\
102\end{array}$ & Tank & $241+T Y+10 \mathbf{2}$ & Wh & Wh & WA & vepor & 9789 & $N$ & NA & 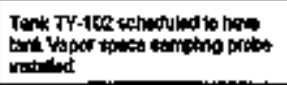 \\
\hline $\begin{array}{l}\text { 241.Tr. } \\
109\end{array}$ & Thak & 241.Trsta & wh & whe & Wh & Uner & 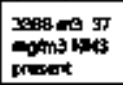 & $\mathrm{No}$ & Wh & 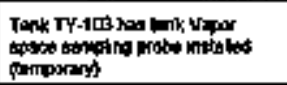 \\
\hline $\begin{array}{l}201 . \mathrm{TV} \\
104\end{array}$ & $\operatorname{Tink}_{\text {Kadtape }}$ & $241 . T Y-104$ & Nh & Nin & 14 & Ver & 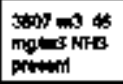 & $\mathrm{Na}$ & Wh. & 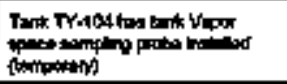 \\
\hline $\begin{array}{l}241-T Y- \\
106\end{array}$ & Tint & 211-TY-10s & Mh & sh & nom & Vyen & 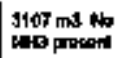 & No & NA & $\mathrm{NH}$ \\
\hline 10d-TY- & Tint & ZHA-TY-105 & Mh & th & $\mathrm{man}$ & Vyen & 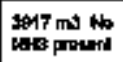 & 10 & NA & Nul \\
\hline $\begin{array}{l}241-T Y- \\
\text { stotin }\end{array}$ & Trat & 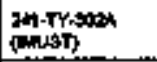 & MA & H. & mon & then & $17,24 \%$ & Wo & Nh & $\mathrm{man}$ \\
\hline $\begin{array}{l}241-T Y . \\
3008\end{array}$ & Tofi & $\begin{array}{l}\text { 2Hitrknes } \\
\text { inusT }\end{array}$ & en & $\mathrm{kn}$ & NA & Vepoce & 14:4140 & No & NA & NA \\
\hline $\begin{array}{l}216-T r \\
\text { 201 }\end{array}$ & Trak & $\begin{array}{l}\text { 216-TY241 } \\
\text { (14) }\end{array}$ & nth & $\mathrm{Nht}$ & $\mathrm{ran}$ & Yypa & $1 \mathrm{~A}, \mathrm{ax}$ & No & nen & Nh \\
\hline
\end{tabular}




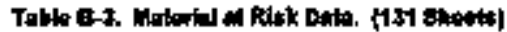

\begin{tabular}{|c|c|c|c|c|c|c|c|c|c|c|}
\hline $\begin{array}{l}\text { Fitd } \\
\text { Mande }\end{array}$ & 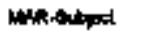 & MterDosctoban & Mhivithorim & Compistr & Mintex & $\begin{array}{l}\text { Fintifind } \\
\text { Tomm }\end{array}$ & Wound of & Tapratid & quming & क्राm-t: \\
\hline $241-\mathrm{TY}$ & $\begin{array}{l}\text { Ron or Thusk } \\
\text { Tanker } \\
\text { Conlent }\end{array}$ & Whot & NA & NA & $\mathbf{1 4}$ & WA & $\mathbf{~ w h}$ & Ne & Wh & 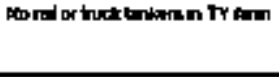 \\
\hline $241-T Y$ & 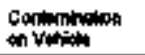 & Want & NA & MA & $\mathbf{m}$ & an & $\mathbf{~} \mathbf{m}$ & Na & wh & Nat \\
\hline 2A1.TY & 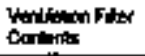 & Fith & NA & HA & m & NA & $\mathbf{m}$ & WA & Wh & 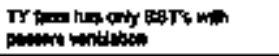 \\
\hline 2A1-TY & Yandition Fiter & Aaning & NA & Wh & NA & $\mathrm{m}$ & in & Nh & Wh & 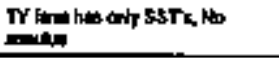 \\
\hline 24I.TY & 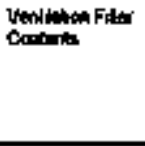 & Betwer & Rotudial & I Rin w日 & NA & Fartuoutat & in & 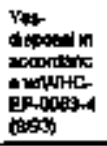 & 1 per & 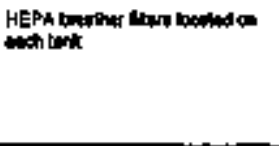 \\
\hline 241-TY & 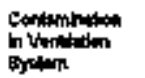 & 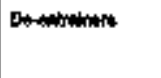 & NA & HA & MH & HA & $\mathbf{m a}$ & No & hid & 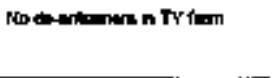 \\
\hline ZA1-TY & 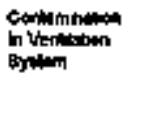 & 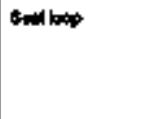 & Ruthindw & 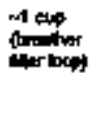 & wh & (4) & $\mathbf{m}$ & Ho & 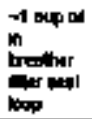 & 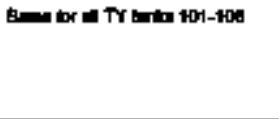 \\
\hline 24I-TY & 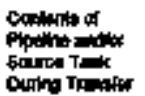 & None & WA & $\mathbf{m}$ & m & Nh & $\mathbf{m a}$ & No & mat & Reter blowetwe Talle? \\
\hline
\end{tabular}




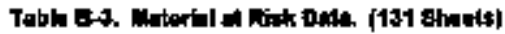

\begin{tabular}{|c|c|c|c|c|c|c|c|c|c|c|}
\hline Fidd & MAR-gubinst & 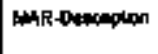 & Mour & capostr & The & Fon & volume or & Trenequid & surithy & Commenta \\
\hline $241+T Y$ & 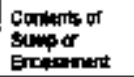 & Nond & ren & MA & $\mathbf{m}$ & NA & MA & No & $\mathrm{m}$ & 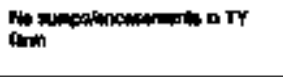 \\
\hline $241 \cdot T Y$ & 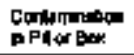 & Ninte & Non & an & $\mathrm{man}$ & NA & Fis: itu & No & $\mathrm{Nh}$ & in porn TY-Fwn ma yenter \\
\hline 241-TY & 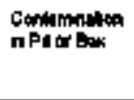 & What bot & ra & Wh & m & NA & focantus & $\mathrm{NO}$ & $\mathbf{m}$ & 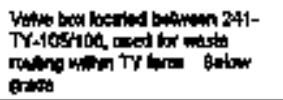 \\
\hline 24I.TY & sinkins & Notos & $\mathrm{NA}$ & kh & $\mathrm{min}$ & NA & $\mathbf{m h}$ & 恢 & NA & 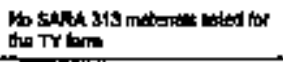 \\
\hline 2H-TY & Othe & Nank & Wh & $\mathrm{kM}$ & $M$ & Ha & $\boldsymbol{m}$ & Nat & No & int \\
\hline $\begin{array}{l}\text { 241-L } \\
\text { 101 }\end{array}$ & Teitcontintin & $2+1-101$ & HeF & $500 \mathrm{Kod}$ & atione & $\begin{array}{l}\text { Lopund } \\
\text { sinid }\end{array}$ & $\begin{array}{l}3 \mathrm{Kog} 72 \\
\operatorname{Kow}\end{array}$ & all & Na & 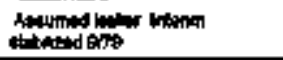 \\
\hline $\begin{array}{l}\text { 24tidtit } \\
\text { Im }\end{array}$ & Tatcontatis & $2 A 1-1)+10$ & MCPLX & Aso K K F & 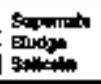 & $\begin{array}{l}\text { Lood } \\
\text { Gowd } \\
\text { Sold }\end{array}$ & $\begin{array}{l}18 \log _{0}+40 \\
\operatorname{lom}_{313}\end{array}$ & ib & nh & 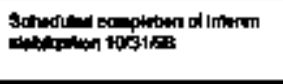 \\
\hline 106 & Tritcontate & $2,11-U-1 \infty$ & NCPUX & sto Km & Ention & $\begin{array}{l}\text { Linged } \\
\text { Boud } \\
\text { Bowd }\end{array}$ & 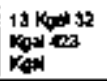 & No & NA & 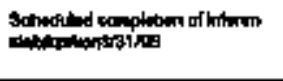 \\
\hline $\begin{array}{l}2+4-4 \\
154\end{array}$ & Tatchentats & $2,11-1-104$ & NCPX: & gso K $\mathrm{K}$ & sudpes & Sold & $12 \mathrm{kgd}$ & w & NA & 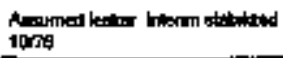 \\
\hline $\begin{array}{l}241-11 \\
105\end{array}$ & Tonin carlanta & 241-10.106 & ncpex & $\mathrm{KN} \mathrm{Kg}$ & 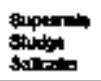 & Laved & 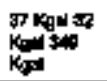 & $\mathbf{W}$ & NA & 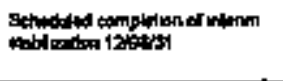 \\
\hline $\begin{array}{l}24144 . \\
100\end{array}$ & Tonll Carimint & 2414100 & ncpex & 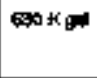 & 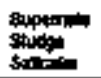 & $\begin{array}{l}\text { luguld } \\
\text { sidid } \\
\text { sodd }\end{array}$ & 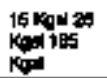 & Ho & wh & 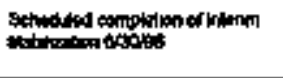 \\
\hline
\end{tabular}




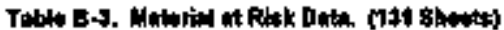

\begin{tabular}{|c|c|c|c|c|c|c|c|c|c|c|}
\hline Find & MUA_Butjeof & 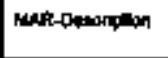 & MAR- & 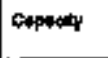 & Thint & Finaled & Poind ex & Trenayot & aximity & 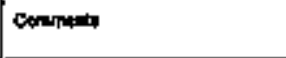 \\
\hline 2410 & Tent contertis & 2a1-ل/10 & DasF & La Ko & 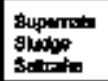 & inid & 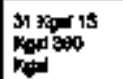 & mo & Wh & 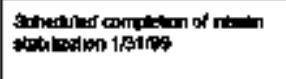 \\
\hline $\begin{array}{l}241416 \\
100\end{array}$ & Trak फ0nterts & 2.11-1ل-100. & $\operatorname{mat} x$ & $\mathrm{Bx} \mathrm{K}$ g & 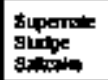 & $\begin{array}{l}\text { Lipid } \\
\text { Eotd } \\
\text { Entd }\end{array}$ & $\sum_{\substack{0 \rightarrow 16 \\
k_{0}}}$ & $\mathbf{m}$ & Na & 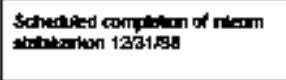 \\
\hline $\begin{array}{l}241+4 k \\
109\end{array}$ & Tert Canting & $2: 11-4-100$ & HCPLX & 550 Kalal & 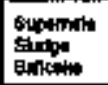 & $\begin{array}{l}\text { balug } \\
\text { sold } \\
\text { 5otal }\end{array}$ & 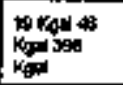 & $m$ & $\mathrm{NA}$ & 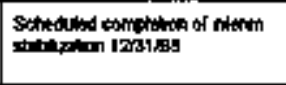 \\
\hline 2410. & Tente Contionla & $2+1-110$ & WPAX & 560 Kat & Standa & Fota & tag Kay & Wh & MA & 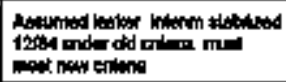 \\
\hline $\begin{array}{l}241.4- \\
111\end{array}$ & Tart Conbiols & $251-4-111$ & ossf & 501K01 & solve & $\begin{array}{l}\text { gotd } \\
\text { sotd }\end{array}$ & $\begin{array}{l}29 \text { Kat } 700 \\
\text { Kod }\end{array}$ & Non & Nh & 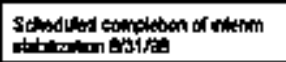 \\
\hline $\begin{array}{l}241 \cdot 4 \\
117\end{array}$ & Tartectitist & $24+24-112$ & MEPLX & $\operatorname{sen} \times$ & $\begin{array}{l}\text { Supmate } \\
\text { bivotpe }\end{array}$ & Uoned & $\mathrm{Kkp}_{\mathrm{p}}$ & ito & Wh & 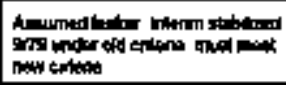 \\
\hline 2버산 & Toritcerilotit & $241+15201$ & سeקx & is $k$ ind & $\begin{array}{l}\text { Supormete } \\
\text { Evofot }\end{array}$ & Lond & $1 \mathrm{Kor}+\mathrm{Kqd}$ & No & Wh & HA \\
\hline $2+1-12$ & Therconimation & $241-1078$ & 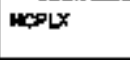 & 至K6 & $\begin{array}{l}\text { Baparnin } \\
\text { Gudgos }\end{array}$ & lond & 1 Kow 4 Kan & Noto & Nh & m \\
\hline $\begin{array}{l}241+25 \\
209\end{array}$ & Tencorlat & 241 ل & 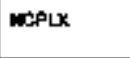 & es & 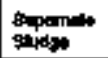 & Letid & 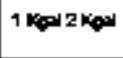 & $\mathrm{NO}$ & NA & un \\
\hline $241-4$ & Tonit Coriting & $2 * 1-4-204$ & Hepox & Sis & 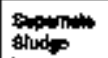 & latid & $1 \mathrm{ked} 2 \mathrm{Ktal}$ & $\mathrm{No}$ & H & nom \\
\hline 3444 & Trarcerterth & 244W DefT & BA & 29000 and & Nh & $\mathbf{m}$ & Mh & M & in & 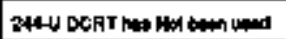 \\
\hline tat-v- & Tenil Conimith & 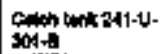 & 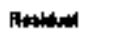 & 100 & intin & Motula & 7000 & No & $\mathbf{H h}$ & 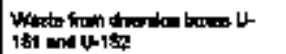 \\
\hline
\end{tabular}




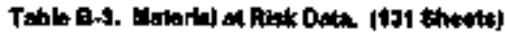

\begin{tabular}{|c|c|c|c|c|c|c|c|c|c|c|}
\hline Findd & Mukstolejent & 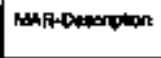 & Kuk. & caspoctip & Thind & $\begin{array}{l}\text { Ptquiqual } \\
\text { Fenth }\end{array}$ & 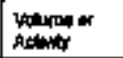 & Trunoat & Exald & Commentip \\
\hline $\begin{array}{l}241.106 \\
300 k \\
3\end{array}$ & Tunt Cotinntx & 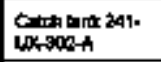 & heotud & 17roo as & Not the & Wodta & $2 t \geqslant p$ & $\mathbf{N o}$ & $\mathbf{m}$ & 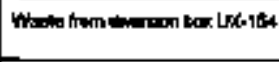 \\
\hline $\begin{array}{l}\text { 241-4 } \\
101\end{array}$ & Trnk & $241-4-101$ & WA & $\mathbf{~ H h}$ & ra & Whar & $245 m^{3}$ & Nato & an & nih \\
\hline $\begin{array}{l}24144 \\
102\end{array}$ & Trmik & 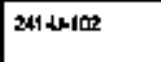 & and & Wh & Mat & Whor & 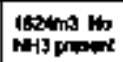 & $\mathbf{H o}$ & an & 咥 \\
\hline It|d & Trank & $241.14-103$ & int & ah & $M_{A}$ & Whor & 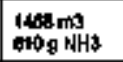 & No & wh & ph \\
\hline $\begin{array}{l}2+1-4 \\
106\end{array}$ & Tmk & $241-U 104$ & MA & Ne & NA & Vupor & 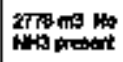 & No & un & nat \\
\hline $\begin{array}{l}2410 \\
105\end{array}$ & T=1k & $241-210 x$ & $\mathbf{~ H h}$ & NA & Na & $v z$ pr & $\begin{array}{l}105 \mathrm{mb} \\
1008900 \\
145\end{array}$ & wo & NA & in \\
\hline $\begin{array}{l}\text { 2410 } \\
105\end{array}$ & Talk & 2414nets & $\mathrm{kn}$ & $\mathrm{NH}$ & NA & veror & 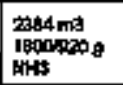 & mo & NA & $\mathrm{m}$ \\
\hline 244.4. & $\begin{array}{l}\text { Tait } \\
\text { Hedipest }\end{array}$ & 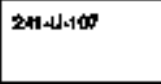 & $\mathrm{NH}$ & $M A$ & Nh & Vapor & 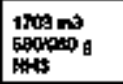 & $\mathrm{m}$ & $N M$ & Man \\
\hline $\begin{array}{l}241 \cdot 4 . \\
100\end{array}$ & 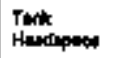 & 2/1-dedot & Na & NA & Nh & Vapor & 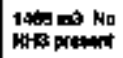 & No & NM & Man \\
\hline 106 & $\begin{array}{l}\text { Twh } \\
\text { Heotapsot }\end{array}$ & 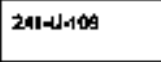 & $\mathrm{NH}$ & 的 & Nh & rapar & $\begin{array}{l}1467 \mathrm{~ms} \\
440, \mathrm{rats}\end{array}$ & No & Nh & $N$ \\
\hline علاء3410 & Tint & $211+1-110$ & W & PA & m & Vepor & 2545 ms No & N & NA & $\mathrm{ras}$ \\
\hline
\end{tabular}




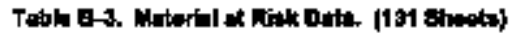

\begin{tabular}{|c|c|c|c|c|c|c|c|c|c|c|}
\hline Find & ItNR-Sybutst & MHA-0wangipn & 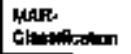 & cenpesty & Time & $\begin{array}{l}\text { Pifriond } \\
\text { Pofin }\end{array}$ & tolume ox & Tamanerd & tovintint & Canumatb \\
\hline $\begin{array}{l}341-4- \\
111\end{array}$ & 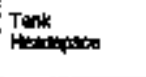 & $2 \times 14111$ & ren & ma & an & ver & $\begin{array}{l}1094 \mathrm{ma} \\
1000100 \\
N+9\end{array}$ & bat & $\operatorname{an}$ & wh \\
\hline $\begin{array}{l}349-4= \\
112\end{array}$ & Tenk & 2614117 & $\mathrm{mu}$ & an & un & veror & 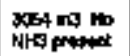 & ats & $\operatorname{an}$ & Wh \\
\hline $\begin{array}{l}241.4 . \\
201\end{array}$ & Tank & $241+4-201$ & Mas & in & $\mathbf{H}$ & vitodr & 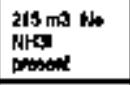 & w & wh & Nh \\
\hline$\underset{x \rightarrow 2}{241-4 h}$ & $\begin{array}{l}\text { Tut } \\
\text { Hendepeos }\end{array}$ & $241+4 k-202$ & Nh. & Non & $\mathrm{mum}$ & Whats & 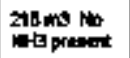 & 100 & NA & Nh \\
\hline 2allsh & Talt & 241.40203 & Nh & Mh & $\mathrm{mon}$ & Weatr & 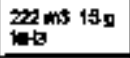 & No & NA & 蚶 \\
\hline $\begin{array}{l}2014 \\
201\end{array}$ & 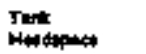 & $241.10 \times 44$ & Nh & $\mathbf{m a n}$ & $\mathbf{m}$ & Vheor & 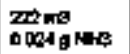 & $\mathrm{No}$ & Nh & his \\
\hline 2440 & Thtit & 2H4U DCकRT & Na & Nht & $\mathrm{m}$ & Na & 14: & $\mathrm{NH}$ & NA & 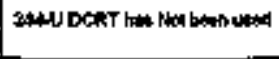 \\
\hline $\begin{array}{l}\text { 2011日 } \\
\text { 2018 }\end{array}$ & Twit & 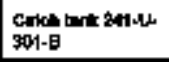 & kin & Kodta & He & Vepor & lation & No & NA & NA \\
\hline 2ums & $\begin{array}{l}\text { Tart } \\
\text { Hotapeced }\end{array}$ & cothing $241-$ & Nh & Nht & $\mathrm{mm}$ & Yopor & 168000 & No & Wh & 饾 \\
\hline $241-4$ & $\begin{array}{l}\text { Row of Then } \\
\text { Tanter } \\
\text { Canient }\end{array}$ & Nome & wh & HA & NA & un & $\mathbf{m}$ & wh & $\mathbf{m}$ & wh \\
\hline 2414 & $\begin{array}{l}\text { conderiminan } \\
\text { on thate }\end{array}$ & Nomin & $\mathbf{m}$ & Wh & Wh & MA & $\mathbf{m}$ & Wh & NA & wh \\
\hline 2414 & 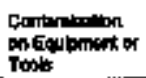 & 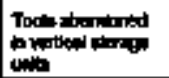 & Cohtemnitad & Wh & wh & Sods & Whipoen & $\mathbf{N e}$ & H & - Teque be dapond of \\
\hline
\end{tabular}




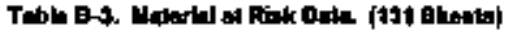

\begin{tabular}{|c|c|c|c|c|c|c|c|c|c|c|}
\hline Patd & 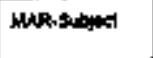 & 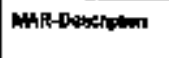 & HAR & captery & 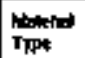 & $\begin{array}{l}\text { Plitucad } \\
\text { Pomm }\end{array}$ & Nation th & Thafidat & Durity & Dan ans \\
\hline $2 \boldsymbol{n}$ & 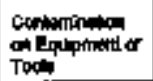 & Hand lood & Tols & WA & m & $\sin$ & Unimanting & Yat: & min & 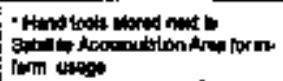 \\
\hline $241-4$ & Vontipen Ft: & Pि|rar & WA & $\ln$ & m & Na & NA & MH & MA & NA \\
\hline $241+0$ & 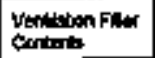 & Annte & wh & $\mathrm{mh}$ & $\mathrm{mu}$ & NA & HA & $\mathbf{M H}$ & Wh & Nin \\
\hline $241-4$ & 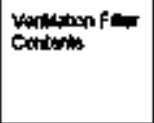 & En:the & Wh & 1 Rings & wh & Porticular & MA & 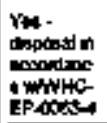 & MA & 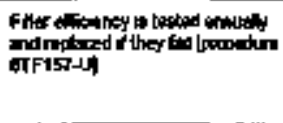 \\
\hline $2414 J$ & 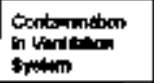 & Dathithenem & $\mathbf{m h}$ & $\mathrm{Mh}$ & 194 & Wh & Na & m & nen & Wh \\
\hline $241-4$ & 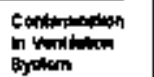 & poeslap & ONind & 1 anp & Nh & Ined & Fonted & $m$ & Nin & 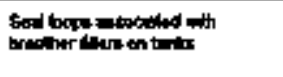 \\
\hline $241-4$ & 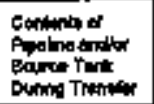 & NA & NA & MA & Nh & HA & Nh & $\mathrm{m}$ & Nin & 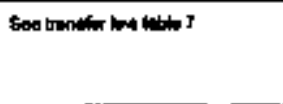 \\
\hline $2+1-4$ & 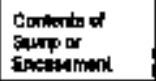 & NA & Nah & NA & $\mathbf{N h}$ & wh & Ma & $\mathbf{r a n}$ & NA & $\mathbf{m}$ \\
\hline $\begin{array}{l}241+0 \\
011004\end{array}$ & totamation & Puno pots L oth & ho tud & 1 & en & kedder & 10.0. & no & 口p & Mh \\
\hline $2+14$ & 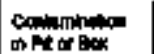 & Puाp pu प O1 & Purintur & $\cos \theta+1$ & $\mathbf{M n}$ & Randin & Minim & No & $1 \mathrm{pe}$ & Mh \\
\hline $241+4$ & Co:Amint & 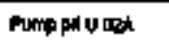 & Rether & 16ter & wh & An:dute & Vasingen & No & 1 献 & Mh \\
\hline
\end{tabular}




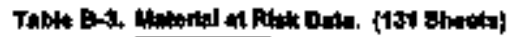

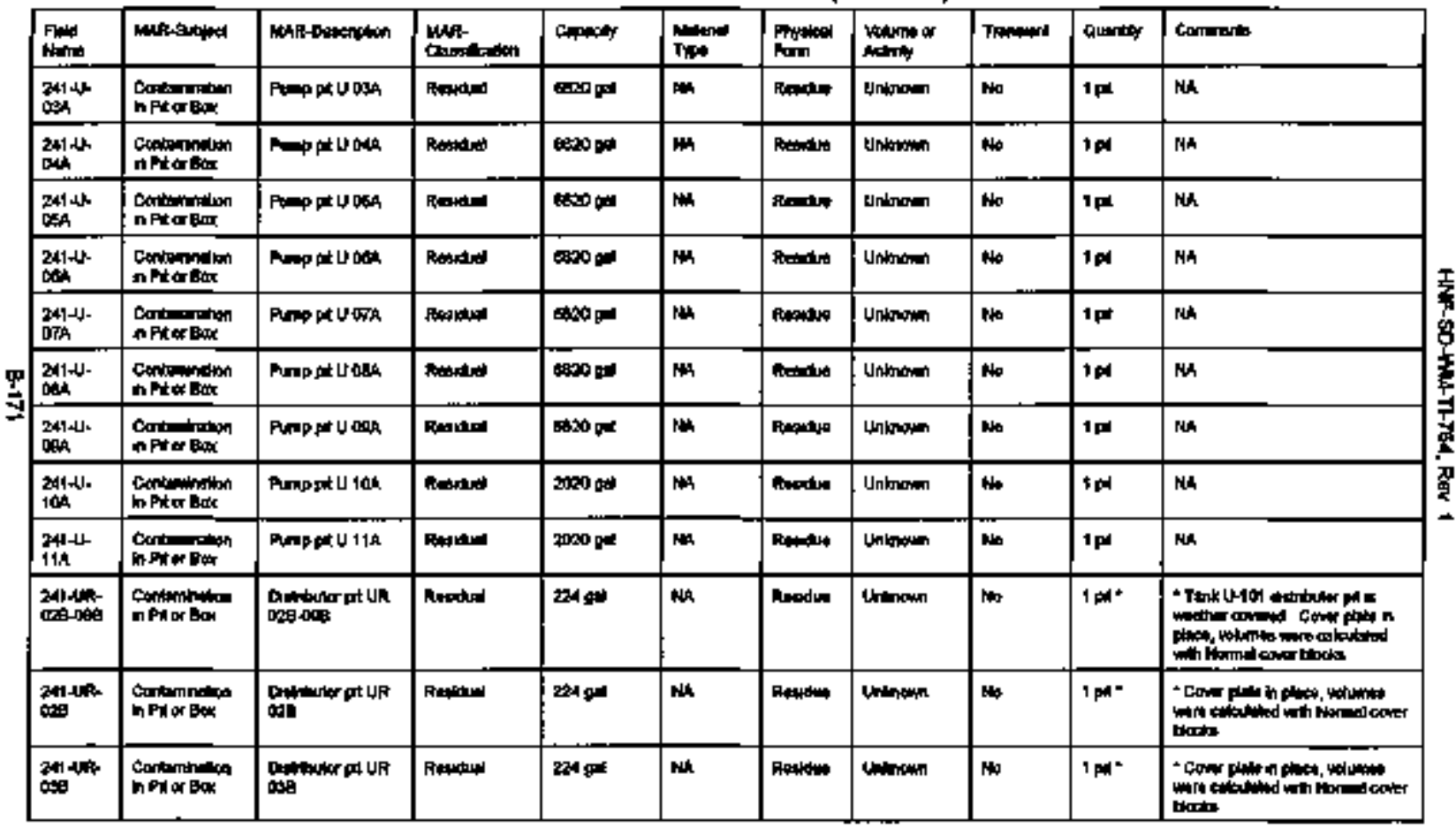




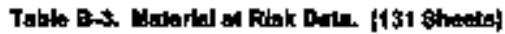

\begin{tabular}{|c|c|c|c|c|c|c|c|c|c|c|}
\hline $\begin{array}{l}\text { frad } \\
\text { nimen }\end{array}$ & Itolkstspyect & whllosecopion & Hint- & caparty & 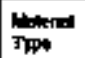 & $\begin{array}{l}\text { Alpara } \\
\text { Foln }\end{array}$ & rodume or & Thincintin & Ourtity & con th \\
\hline $\begin{array}{l}\text { 24tAR- } \\
\text { D4B }\end{array}$ & 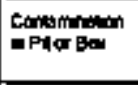 & Detroutor an UR & Poondual & 201 od & an & Riswotu & Lrithown & No & 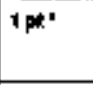 & 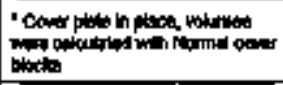 \\
\hline 241-4n- & 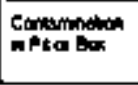 & 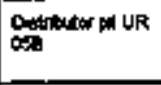 & Re:pinum & 224 sar & 배 & Rouncua & Unimathn & No & 1 pt" & 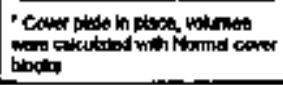 \\
\hline 241 & $\begin{array}{l}\text { Corlommetom } \\
\text { A Pta Bes }\end{array}$ & 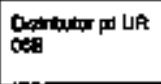 & finctur & वस का & wh & Resolut & Uninewn & No & 1 pt* & 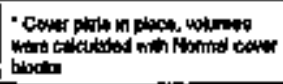 \\
\hline $\begin{array}{l}\text { OHALA } \\
\text { WTB }\end{array}$ & $\begin{array}{l}\text { Coniminan } \\
\text { mPint Ext }\end{array}$ & 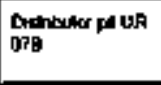 & Resuder & 200 & ren & Aasude & Volanom & No & 1p * & 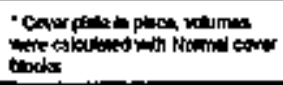 \\
\hline $\begin{array}{l}\text { Z4ILAR } \\
0 \% 8\end{array}$ & 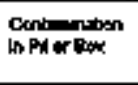 & $\begin{array}{l}\text { Cathbuler pa tok } \\
\text { pig }\end{array}$ & Reouda & 204 & $\mathrm{rat}$ & Prenden & Unkmon & tho & $1 \mathbf{p s}^{*}$ & 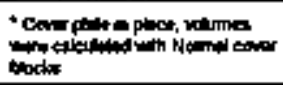 \\
\hline OAB & $\begin{array}{l}\text { Contiofibst } \\
\text { in Piler Bar }\end{array}$ & 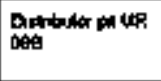 & Ruflatum & 224 & $\ln$ & Rinudent & Intormm & No & 100 & 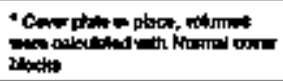 \\
\hline $\begin{array}{l}\text { 241-UR- } \\
\text { 1018 }\end{array}$ & 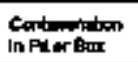 & 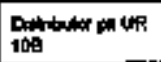 & Rondun & 1TH & Non & Rathat & Utrimn & $\omega$ & 10 & Nh \\
\hline $\begin{array}{l}241-16- \\
115\end{array}$ & 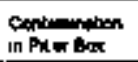 & 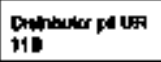 & Alesid- & ITB & $\mathbf{m}$ & Residum & 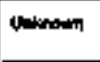 & NH & Ipt & NA \\
\hline $2+410$ & $\begin{array}{l}\text { contemnston } \\
\text { nPN Or Bos }\end{array}$ & sureptr & Retrater & NM & Nh & Powativer & Untoren & $\mathbf{M h}$ & 9 & 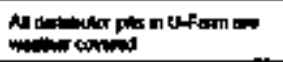 \\
\hline $2 * 140$ & 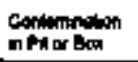 & candensor pta & Reanites: & NA & Wh & Risting & Unknom & $M$ & $14 \mathrm{Fit}$ & 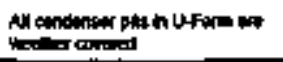 \\
\hline $2 \pi n$ & 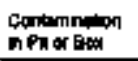 & solmed & Rowiver & NM & sin & horite & Untmonan & $\mathbf{m}$ & $1 \mathbf{p r}$ & 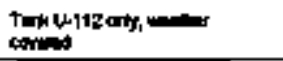 \\
\hline 2 & 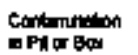 & 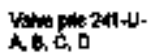 & Poraldual & $310 \%$ & Nh & Rentut & Ih'shth & $m$ & 4 alt: & Nh \\
\hline
\end{tabular}




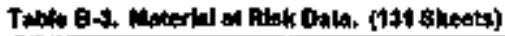

\begin{tabular}{|c|c|c|c|c|c|c|c|c|c|c|}
\hline Fid & 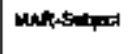 & 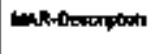 & Cari. & Gepent & $\begin{array}{l}\text { Nhatenal } \\
\text { Typa }\end{array}$ & $\begin{array}{l}\text { Phplanl } \\
\text { Form }\end{array}$ & roting & Tramant & andy & Com \\
\hline $241+14$ & 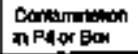 & 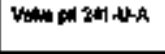 & Ptetidud & $\mathbf{s} \mid \mathbf{Z}$ gil & $\mathbf{m h}$ & Fbodtr & Unistith & Wh & 1 p & Mh \\
\hline $241-4-8$ & 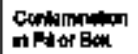 & 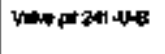 & Panmisud & 314200 & $\mathbf{m}$ & Rounow & Whangan & $\mathbf{m a}$ & $1 \mathrm{Bd}$ & MA \\
\hline 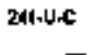 & 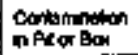 & 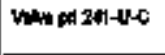 & Restutulu & $31 \mathbf{Z}_{\text {gr }}$ & Hh & Nater & Uhistim & $\mathbf{W h}$ & $1 \mathrm{pr}$ & Nh \\
\hline $2+1-0$ & 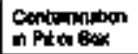 & 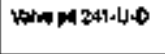 & Ratural & 9109 & NA & Roulde & Unanown & Hh & $1 \mathrm{pm}$ & Nh \\
\hline $\begin{array}{l}2414 k \\
151\end{array}$ & $\begin{array}{l}\text { Cont maton } \\
\text { m Pis ar Bax }\end{array}$ & $\begin{array}{l}\text { Dreneonbox 241. } \\
\text { U-161 }\end{array}$ & Anodatar & Iastapgan & NH & Rente & Lrtooun & m & $1 \operatorname{lom}$ & Wh \\
\hline $\begin{array}{l}24-11 \\
152\end{array}$ & 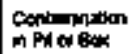 & $\begin{array}{l}\text { terremonbax } 241 . \\
\text { L-157 }\end{array}$ & Retwow & 1931 1931 & NA & Ratatur & 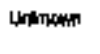 & M & $1 \mathrm{~b}$. & Hh \\
\hline $\begin{array}{l}\text { 201 d 자 } \\
164\end{array}$ & 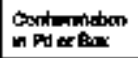 & 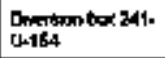 & Reuden & atset & NH & Renntu & 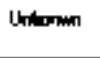 & in & 1 bese. & 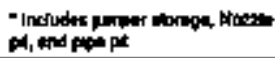 \\
\hline $2+1-u$ & 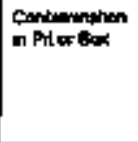 & 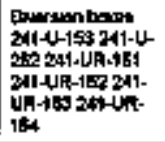 & 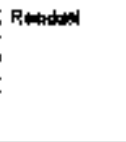 & Nh & NA & 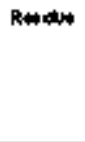 & 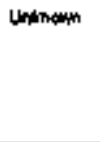 & Mes & 6 boum & 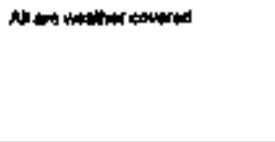 \\
\hline $\operatorname{lin}_{1 \rightarrow 0}^{241-\downarrow-}$ & o PA or Bor & 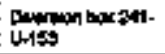 & Ritatut & Wh & Wh & 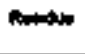 & |W & $N A$ & 1 bou & 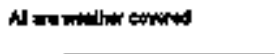 \\
\hline $\begin{array}{l}241 \cdot 1-1 \\
20\end{array}$ & $\begin{array}{l}\text { Contaringon } \\
\text { in PA or Bax }\end{array}$ & 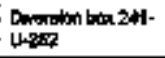 & Reving & $\mathbf{m}$ & wh & Preme & תחרen & Na & 1 bow & 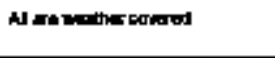 \\
\hline $\begin{array}{l}\text { 341-UR } \\
\text { 1รี }\end{array}$ & 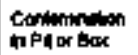 & Davown bac 201. & 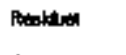 & MA & Wh & fiecolda & | & Nik & 1 bow & 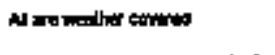 \\
\hline $\begin{array}{l}249-49 . \\
150\end{array}$ & 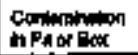 & UR-15: & repatual & Wh & wh & Propoty & Mnomin & Ma & 160 & 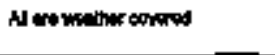 \\
\hline
\end{tabular}




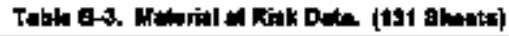

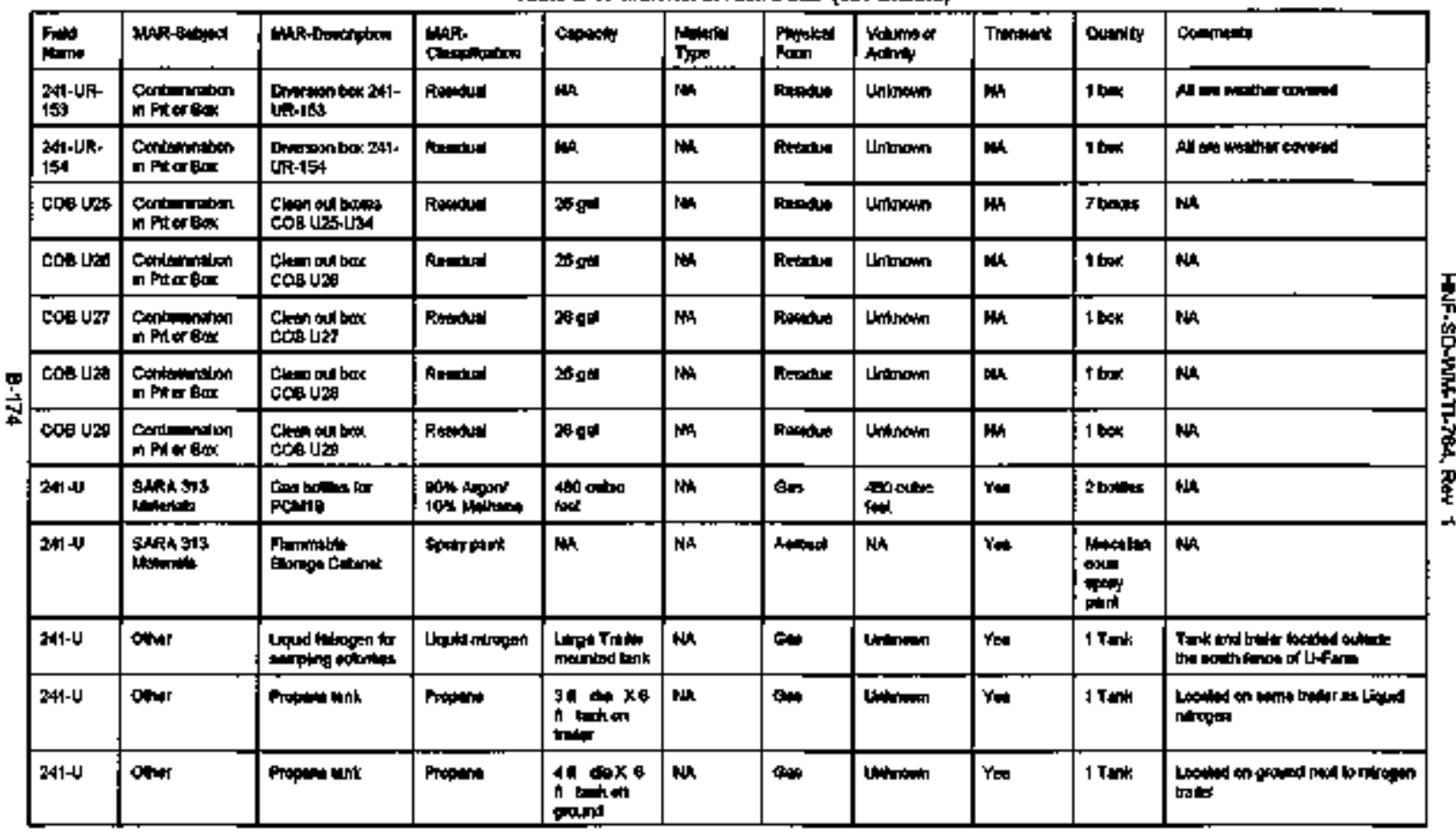


Tuble B-3. Mrierial ad Fiet Doll. (131 theots)

\begin{tabular}{|c|c|c|c|c|c|c|c|c|c|c|}
\hline Fand & 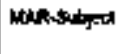 & 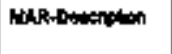 & latis- & ctoptests & homita & $\begin{array}{l}\text { Ptipkat } \\
\text { Fom }\end{array}$ & velume or & Tnotand & ounaty & Cornmets \\
\hline $2+8-4-12$ & प्मा1 & crarte-12 & $\mathbf{H A}$ & NA & $\mathbf{m}$ & Hon & NA & Hh. & NA & 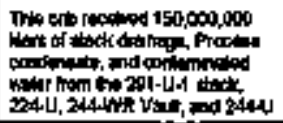 \\
\hline 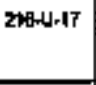 & Dher & Gmb216-1] & Mh & Nh & $\mathbf{M h}$ & Nin & 妕 & $\mathbf{M h}$ & Wh & 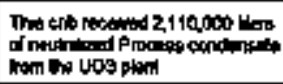 \\
\hline 2भA-1].14 & Other & Duth 21thl-14 & Mh & Nh & $\mathbf{m}$ & NA & 妕 & rath & Wh & 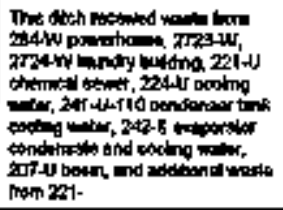 \\
\hline 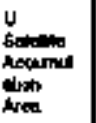 & Other & simition & $\begin{array}{l}\text { RCRA vind } \\
\text { Whatta }\end{array}$ & 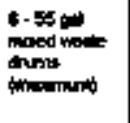 & $m$ & sotd & 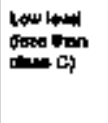 & $Y *$ & 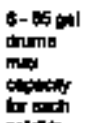 & 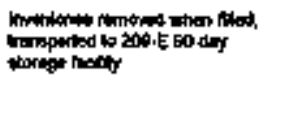 \\
\hline $\begin{array}{l}2+12 W \text {. } \\
151\end{array}$ & 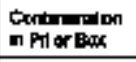 & movex pd & $\begin{array}{l}\text { Roshid } \\
\text { continitum }\end{array}$ & 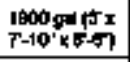 & man & Renter & $\mathbf{m h}$ & $\mathbf{N a}$ & $\mathbf{m}$ & $\mathrm{man}$ \\
\hline $\begin{array}{l}\text { 241-EW. } \\
151\end{array}$ & $\begin{array}{l}\text { Conkinam } \\
\text { in PMor Ber }\end{array}$ & 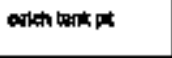 & 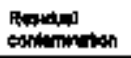 & 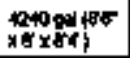 & MA & Pucalotua & Bh & $1+6$ & HA & NA \\
\hline $\begin{array}{l}2+1-2 W . \\
1 \$ 1\end{array}$ & $\begin{array}{l}\text { Centimang } \\
\text { ID PAl of Dow }\end{array}$ & 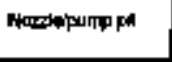 & 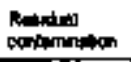 & $\begin{array}{l}13009+10 x \\
8 \times 507\end{array}$ & $\boldsymbol{M}$ & $R=0$ & But & No & MA & Nh \\
\hline $\begin{array}{l}241 \cdot 144 \\
181\end{array}$ & Gitemnim & Doditumbits & MA & Nhan & Nh & $\mathbf{k}$ & NA & $\mathbf{H k}$ & Nin & 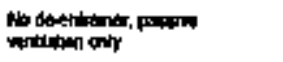 \\
\hline
\end{tabular}




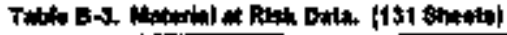

\begin{tabular}{|c|c|c|c|c|c|c|c|c|c|c|}
\hline Field & Mariforlyad & 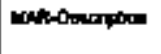 & $\begin{array}{l}\text { MAR- } \\
\text { Clablingunen }\end{array}$ & Gepart & الו & الج & votima & Troment & \&unty & 00 (a) \\
\hline $\begin{array}{l}24 \cdot d / 2 k \\
151\end{array}$ & 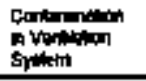 & $\operatorname{ses} \operatorname{losp}$ & Pandinento & Nht & rat & blaped & הativin & $\mathbf{k}$ & one & 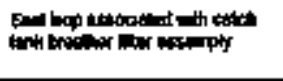 \\
\hline 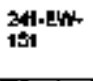 & 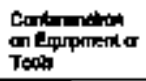 & Who & min & Hh & he & Nht & HA & Wh & wh & 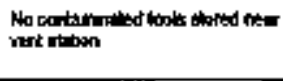 \\
\hline 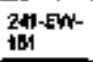 & $\begin{array}{l}\text { Conterinison } \\
\text { on thecte }\end{array}$ & $\operatorname{Man}$ & NA & $\mathbf{k H}$ & ren & Nht & NA & $\boldsymbol{M H}$ & wh & 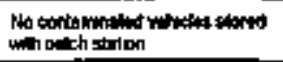 \\
\hline $\begin{array}{l}241 \text { EW. } \\
+51\end{array}$ & 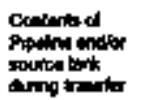 & ma & int & MA & Nh & NA & $\mathbf{w h}$ & reh & MA & 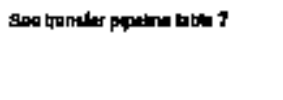 \\
\hline $\begin{array}{l}241-E N- \\
151\end{array}$ & $\begin{array}{l}\text { Contind el } \\
\text { Somper } \\
\text { Enowemal }\end{array}$ & Noen & MA & NA & NA & NA & MA & m & MA & 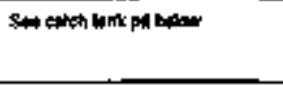 \\
\hline $\begin{array}{l}241 \text { EW. } \\
151\end{array}$ & othe & $\mathrm{Nan}$ & wh & Ma & NA & NA & $\mathbf{m}$ & M & NA & Wh \\
\hline $\begin{array}{l}241-E W- \\
161\end{array}$ & $\begin{array}{l}\text { fill or Taxk } \\
\text { Tantit } \\
\text { Conting }\end{array}$ & Nen: & wh & $M_{h}$ & 蚶 & NA & Wh & m & Na & 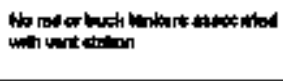 \\
\hline $\begin{array}{l}\text { 241-ent } \\
151\end{array}$ & 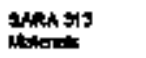 & ptom & $\mathrm{Ath}$ & im & Hh & WA & MA & $M$ & wh & Wh \\
\hline $\begin{array}{l}\text { 241-Enk } \\
151\end{array}$ & Thin Cerrenten & 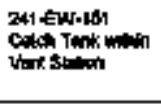 & 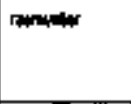 & $200 \mathrm{~g}$ & an & Lowid & 3610 & No & Hh & 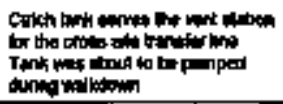 \\
\hline $\begin{array}{l}241-5 \times 2 \\
151\end{array}$ & Tenir & $\begin{array}{l}\text { 241+GN-15-1 } \\
\text { calth tant }\end{array}$ & $\mathrm{mh}$ & 1901 & in & WA & HA & N & Hin & 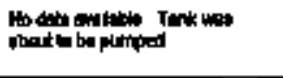 \\
\hline
\end{tabular}




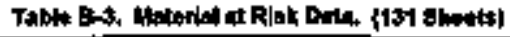

\begin{tabular}{|c|c|c|c|c|c|c|c|c|c|c|}
\hline Fifd & MnNSitida & MR & 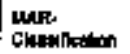 & 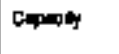 & Typol & Prim & 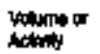 & Than & קטח & crovente \\
\hline $\begin{array}{l}2024.4 \\
151\end{array}$ & $\begin{array}{l}\text { W-ithton Pth } \\
\text { Certints }\end{array}$ & 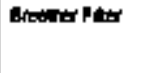 & 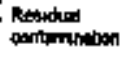 & 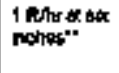 & NA & Ptouldua & 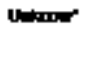 & $Y=$ & Nin & 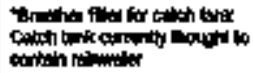 \\
\hline
\end{tabular}

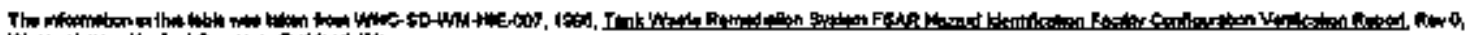

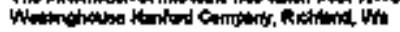

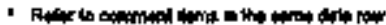

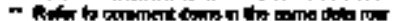

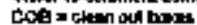

DOT $=4$ \$ Deparimon of Tnamportion

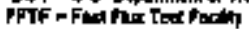

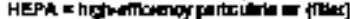

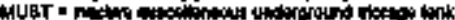

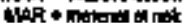

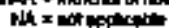

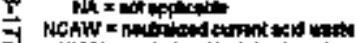

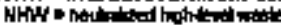

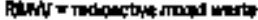

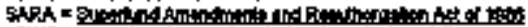

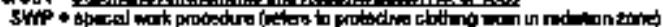

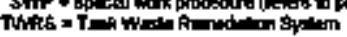


HNF-SO-MNATT-784,RW. 1

Thte pege intentlen:ty left btank. 


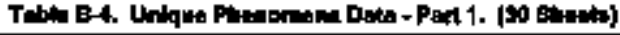

\begin{tabular}{|c|c|c|c|c|c|c|}
\hline 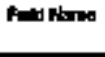 & Pbأाय & Tyon & Pentipatide & 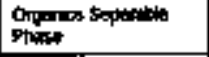 & roc interats & 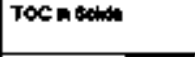 \\
\hline $241+101$ & 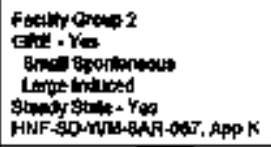 & Englestert innk & 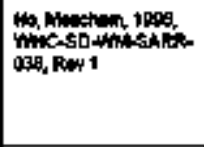 & 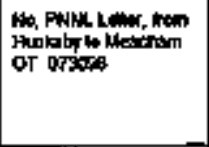 & 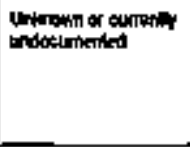 & 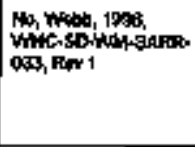 \\
\hline $2411+160$ & 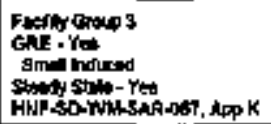 & 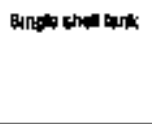 & 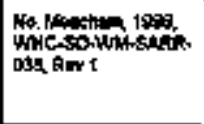 & 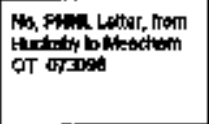 & 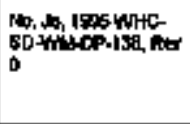 & 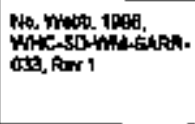 \\
\hline 2HA-1D3 & 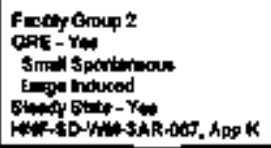 & single shell both & 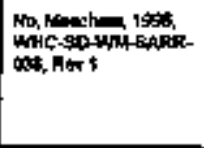 & 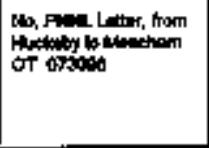 & 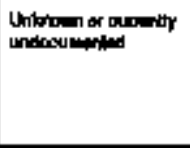 & 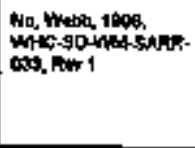 \\
\hline 341A-100 & 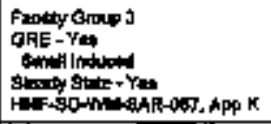 & 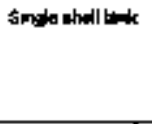 & 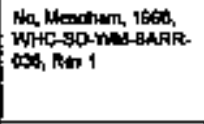 & 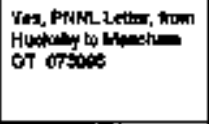 & 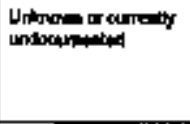 & Uhteowner curremity \\
\hline 241 thes & 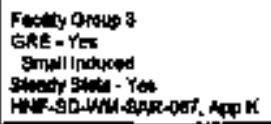 & Sloglo when the & 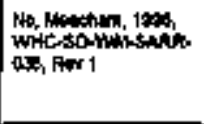 & 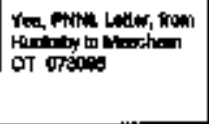 & 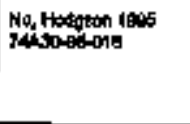 & 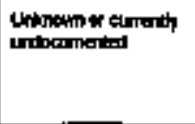 \\
\hline $2+1+400$ & 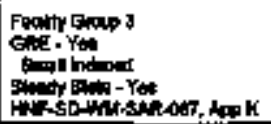 & 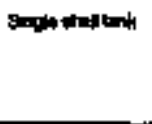 & 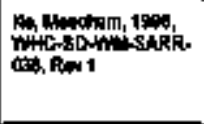 & 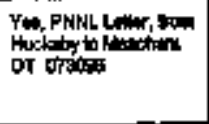 & Lafoum ax eurming & 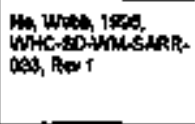 \\
\hline
\end{tabular}




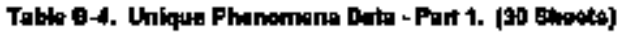

\begin{tabular}{|c|c|c|c|c|c|c|}
\hline Fad $\mathrm{N}$. & 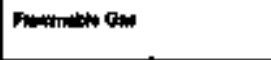 & $T m$ & Pastopthin & 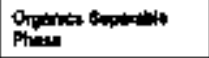 & Tex in Lلهith & TOC In 3olst \\
\hline $2+1-+1+2+21$ & 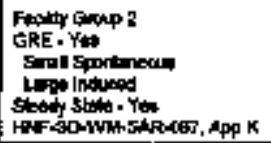 & Oowplespeil tonir & 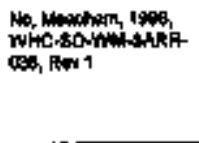 & 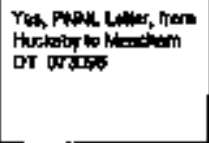 & 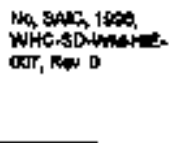 & 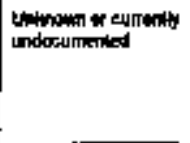 \\
\hline $241-\not W 4-162$ & 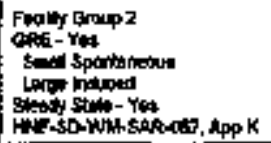 & 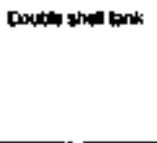 & 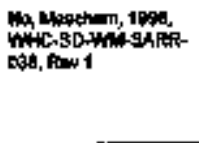 & 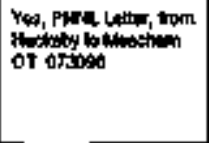 & 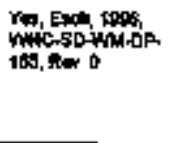 & 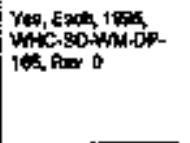 \\
\hline 201-AW-10 & 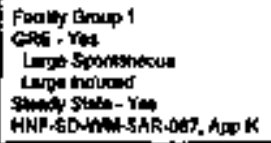 & Couth she lonk & 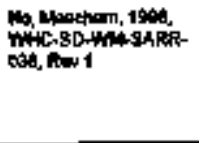 & 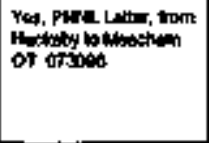 & 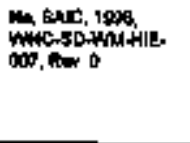 & 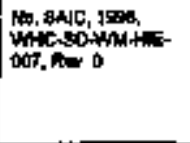 \\
\hline $241+1 / 104$ & 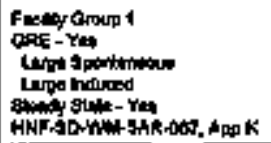 & Douth uhell trat & 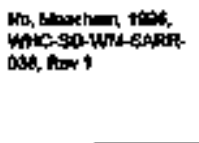 & 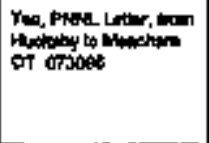 & 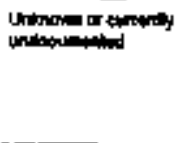 & 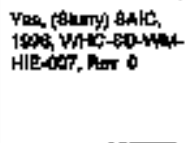 \\
\hline $2414 / 4-106$ & 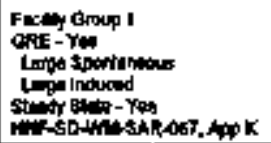 & Coulte end tait & 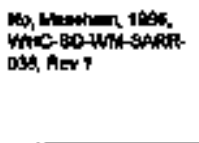 & 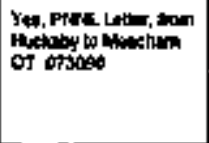 & 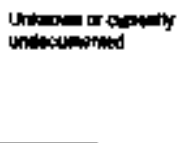 & 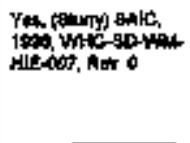 \\
\hline $241-4 k+108$ & 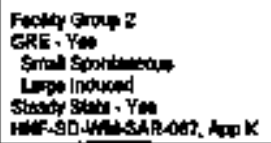 & Dorkin thall thet & 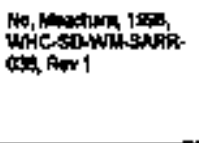 & 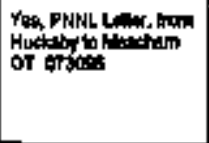 & 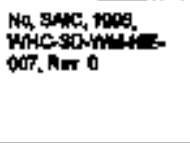 & 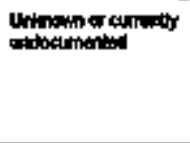 \\
\hline
\end{tabular}




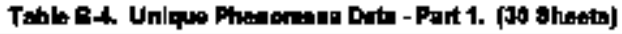

\begin{tabular}{|c|c|c|c|c|c|c|}
\hline Fred kbre & Plamolth ot & Type & 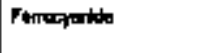 & Oriments & TOCh h Lutts & 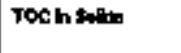 \\
\hline 2a1+4*-107 & 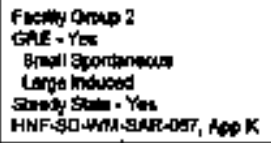 & Dodoth that then & 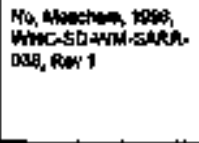 & 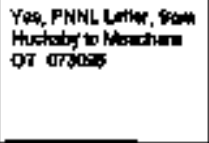 & 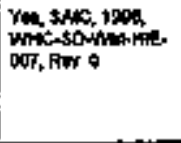 & 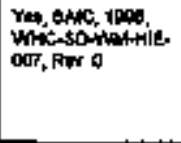 \\
\hline $2+1+9-101$ & 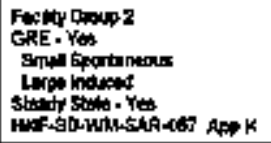 & Doublo alail tork & 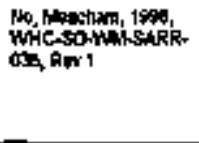 & 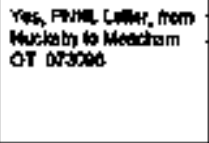 & 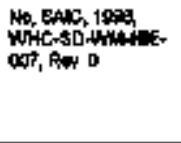 & 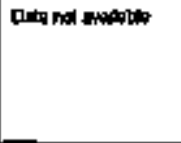 \\
\hline $2+1+2-102$ & 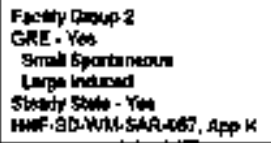 & 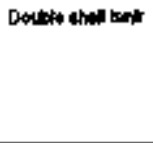 & 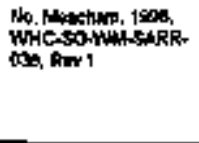 & 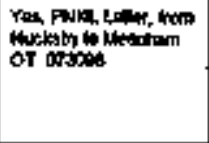 & 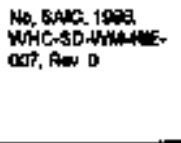 & 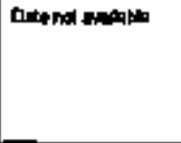 \\
\hline 241AP-10 & 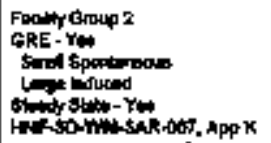 & |' & 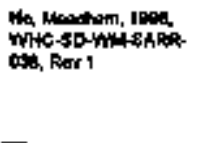 & 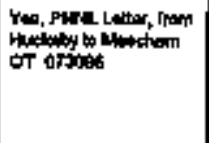 & 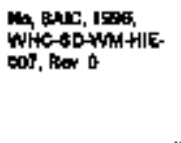 & 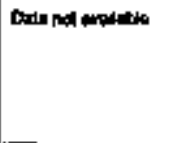 \\
\hline $241-A, P \cdot 104$ & 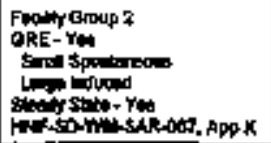 & 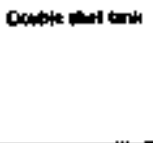 & 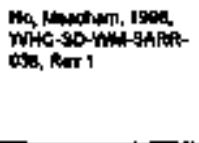 & 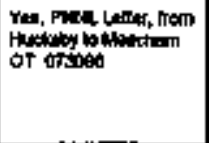 & 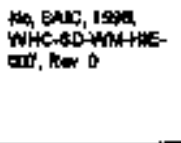 & 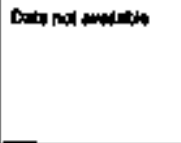 \\
\hline 241-AP-105 & 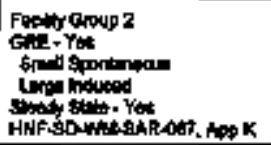 & 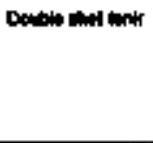 & 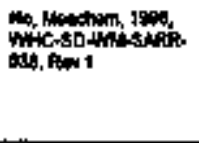 & 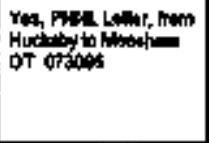 & 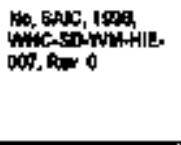 & 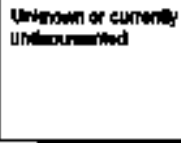 \\
\hline
\end{tabular}


Tabla B-4. Uniqua Phenomene Data - Par 1. [10 Pateata)

\begin{tabular}{|c|c|c|c|c|c|c|}
\hline 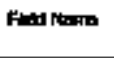 & f mabte con & Tpre & farospontio & 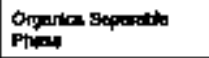 & 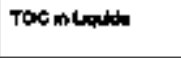 & Tot in bafit \\
\hline $247-4 \cdot 100$ & 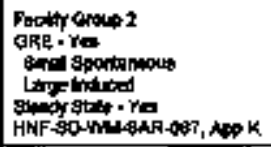 & 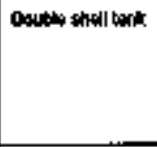 & 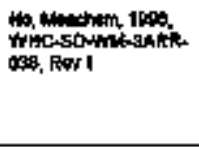 & 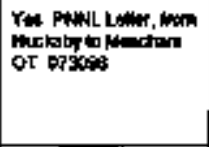 & 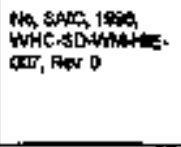 & 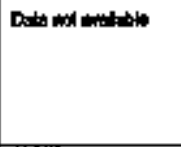 \\
\hline 241-48.107 & 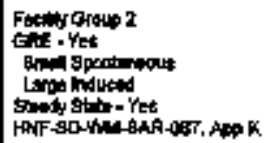 & Douls whallats & 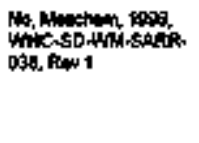 & 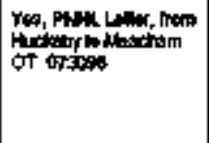 & 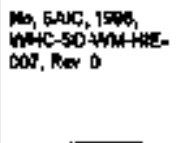 & 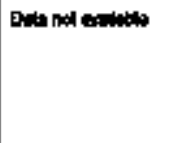 \\
\hline 241-Af: 108 & 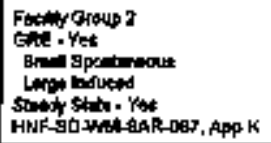 & 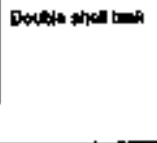 & 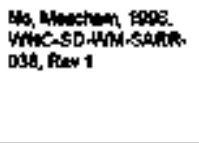 & 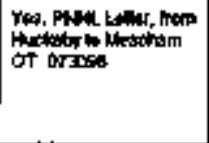 & 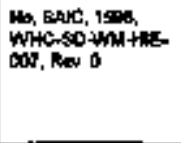 & Dalla \\
\hline 241-AM-101 & 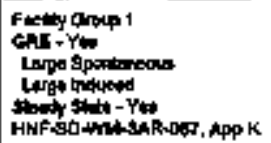 & 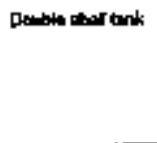 & 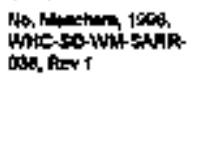 & 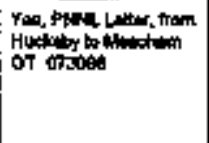 & 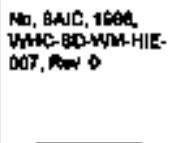 & 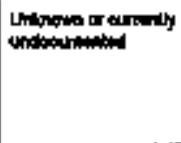 \\
\hline 2-1-AYH-108 & 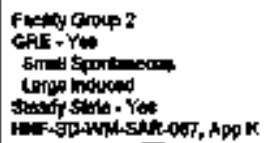 & Dohblt itelimk & 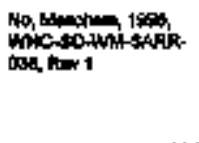 & 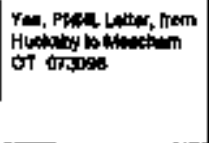 & 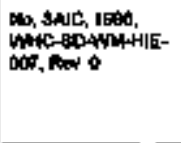 & 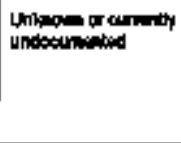 \\
\hline $241-46.103$ & 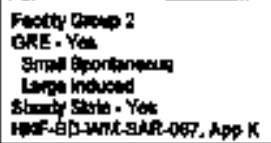 & Onublosteri lonit & 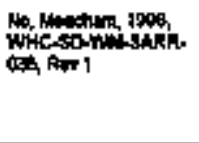 & 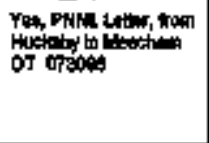 & 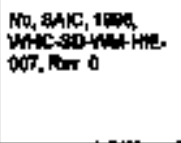 & 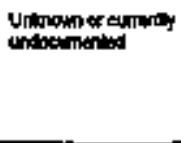 \\
\hline
\end{tabular}




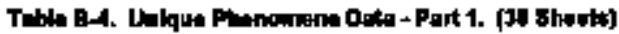

\begin{tabular}{|c|c|c|c|c|c|c|}
\hline Fit & Premetos & Typ & foocogatinte & $\begin{array}{l}\text { Oramiks onpulin } \\
\text { Phabe }\end{array}$ & 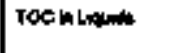 & 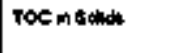 \\
\hline $241+4 W / 204$ & 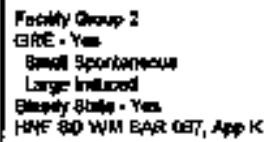 & 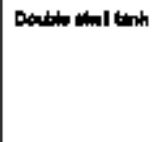 & 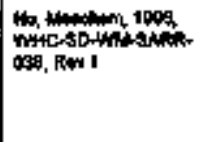 & 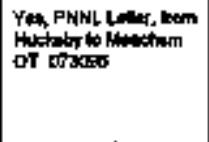 & 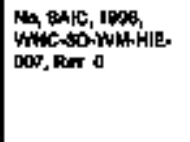 & 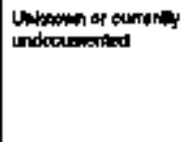 \\
\hline 241 औस-105 & 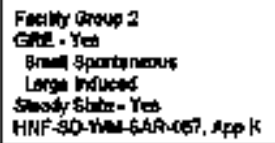 & 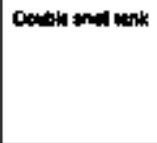 & 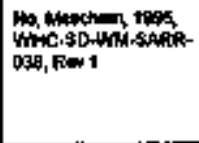 & 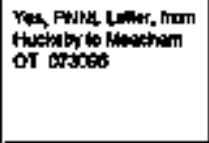 & 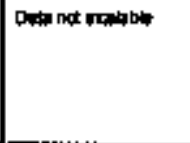 & 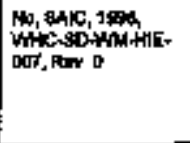 \\
\hline $241-a+w-106$ & 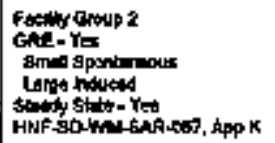 & 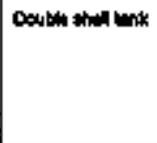 & 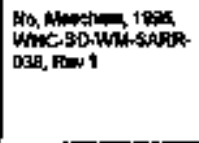 & 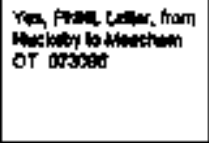 & 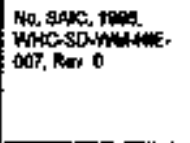 & 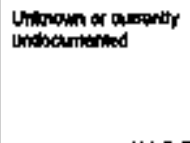 \\
\hline $2+1-\hat{A r}-101$ & 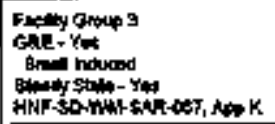 & 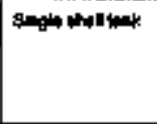 & 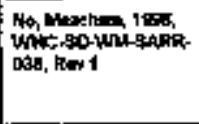 & 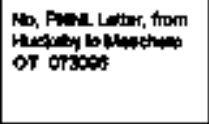 & 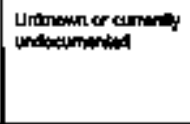 & 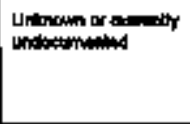 \\
\hline 201-AX-102 & 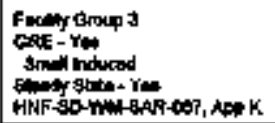 & Anqua whel tark & 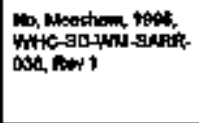 & 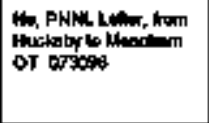 & 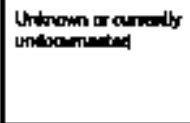 & 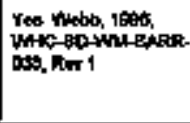 \\
\hline 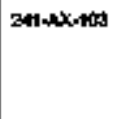 & 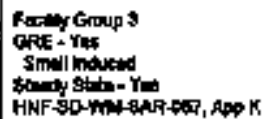 & 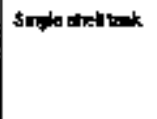 & 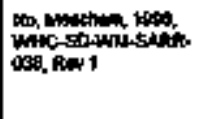 & 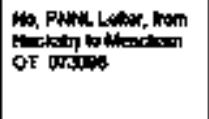 & Unknown of clinewly & 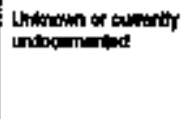 \\
\hline
\end{tabular}




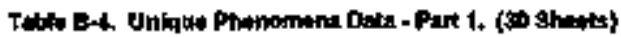

\begin{tabular}{|c|c|c|c|c|c|c|}
\hline Fapd Nene & fumptat Cos & The & Fomocyands & 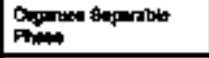 & 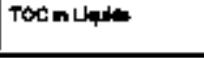 & Tos m fofit \\
\hline $245-2 x-104$ & 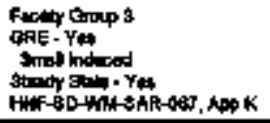 & 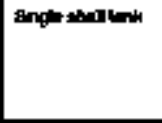 & 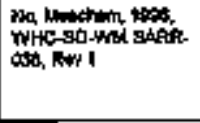 & 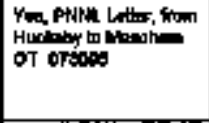 & 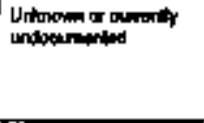 & 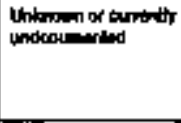 \\
\hline 241-AYY-101 & 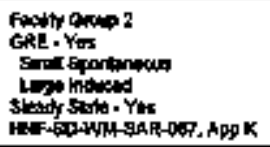 & 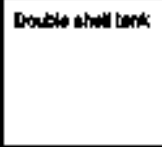 & 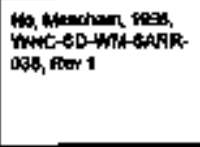 & 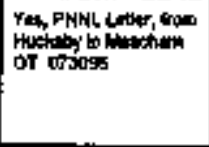 & 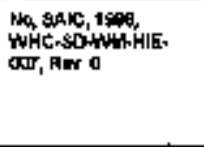 & 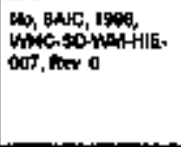 \\
\hline 241 AY -102 & 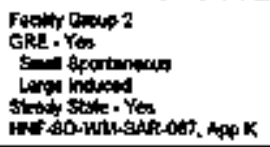 & Dabil thellogk & 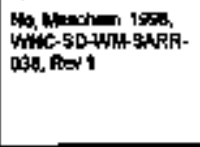 & 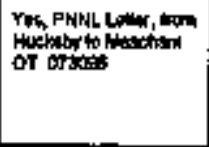 & 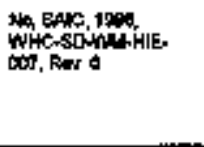 & 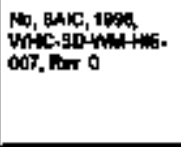 \\
\hline $211+A z-101$ & 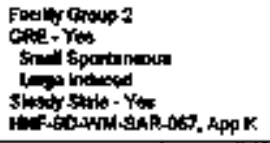 & 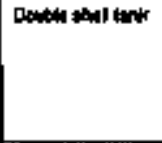 & 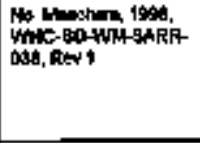 & 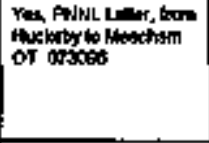 & 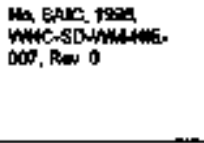 & 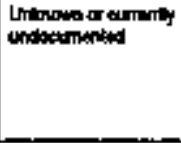 \\
\hline $241-42-602$ & 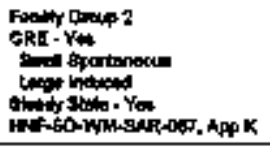 & 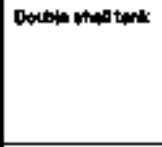 & 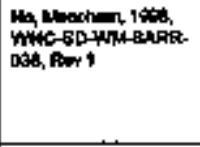 & 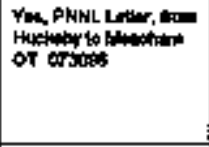 & 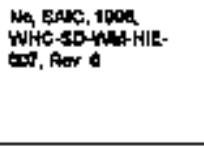 & 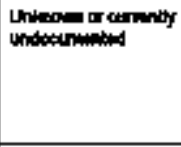 \\
\hline $2416-191$ & 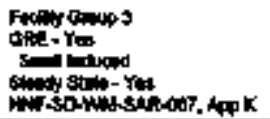 & 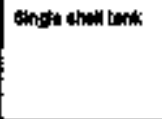 & 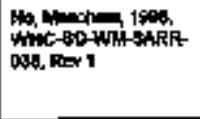 & 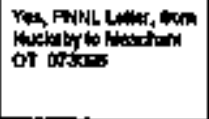 & 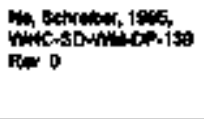 & 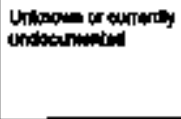 \\
\hline
\end{tabular}


Table 0-4. Uniqua Phonomena Data -Pant 1. (30 ghnats)

\begin{tabular}{|c|c|c|c|c|c|c|}
\hline 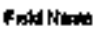 & Fbmall Gat & Th & 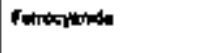 & 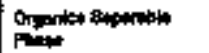 & 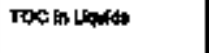 & TOCA Buth \\
\hline $2+1-102$ & 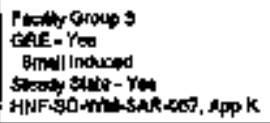 & Bungto ahell tark & 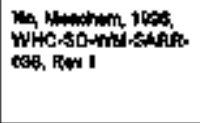 & 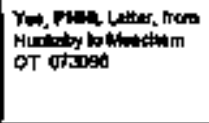 & 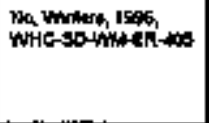 & 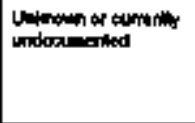 \\
\hline $245+100$ & 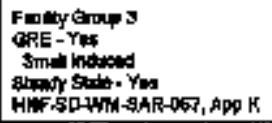 & 5raje bien tart & 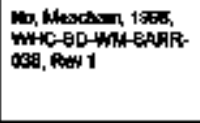 & 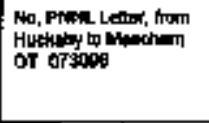 & Nin & 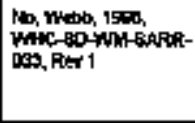 \\
\hline $241+104$ & 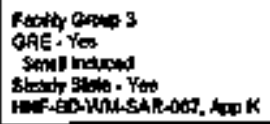 & Sade intillark & 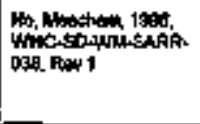 & 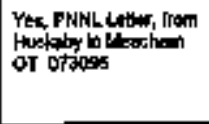 & 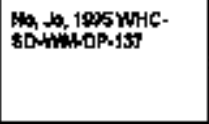 & 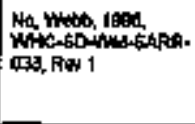 \\
\hline $249-8-105$ & 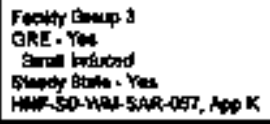 & 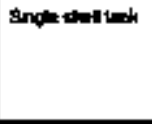 & 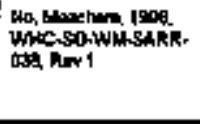 & 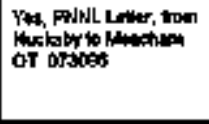 & 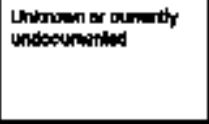 & 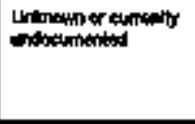 \\
\hline 241-B-100 & 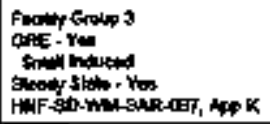 & inglethed the & 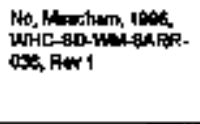 & 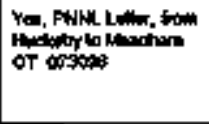 & 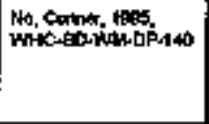 & 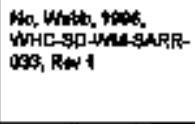 \\
\hline $24+10$ & 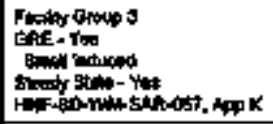 & Bande thind int & 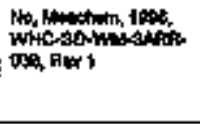 & 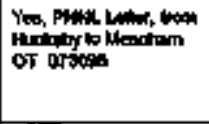 & 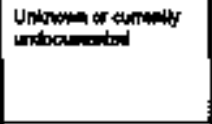 & 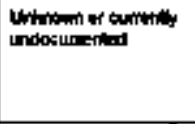 \\
\hline 241-6-609 & 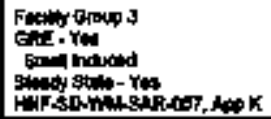 & 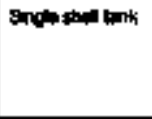 & 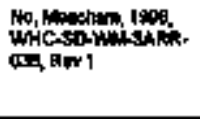 & 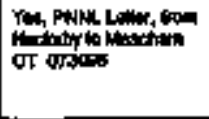 & 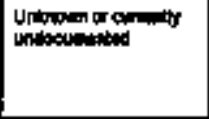 & 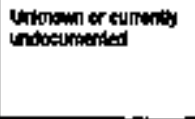 \\
\hline
\end{tabular}




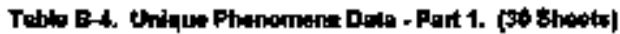

\begin{tabular}{|c|c|c|c|c|c|c|}
\hline Favd Katum & 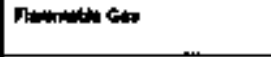 & Tyos & Phingand & 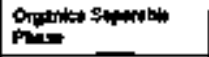 & Toc In & Toc in 5own \\
\hline 2418-409 & 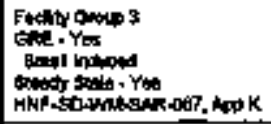 & 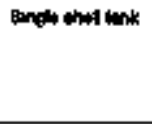 & 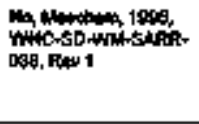 & 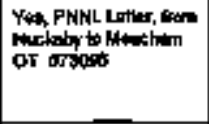 & 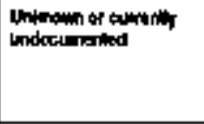 & 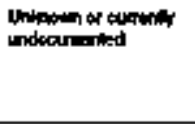 \\
\hline $2+18-10$ & 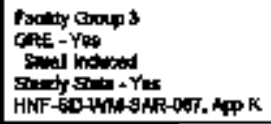 & Stang sfod tonk & 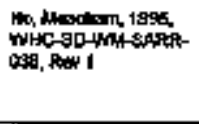 & 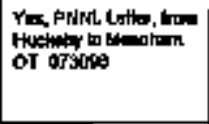 & 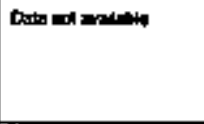 & 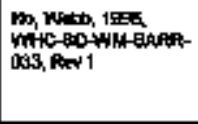 \\
\hline 241-8-111 & 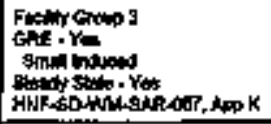 & 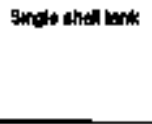 & 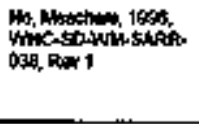 & 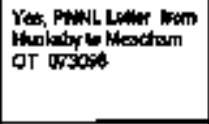 & 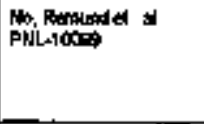 & 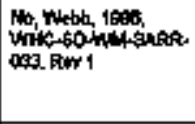 \\
\hline $2+18-112$ & 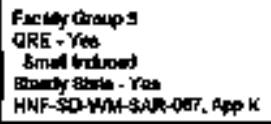 & 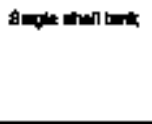 & 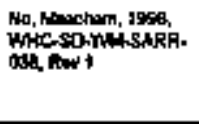 & 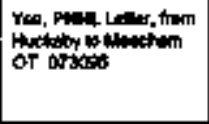 & 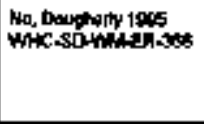 & 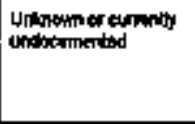 \\
\hline $24(18-20)$ & 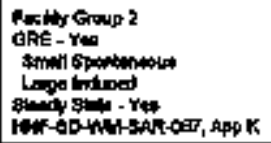 & 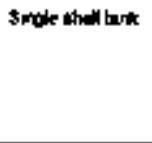 & 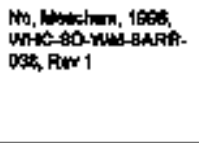 & 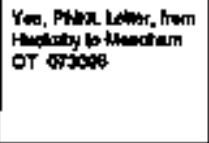 & 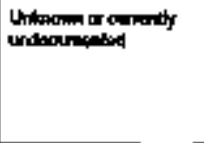 & 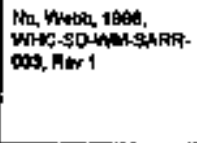 \\
\hline 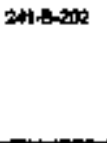 & 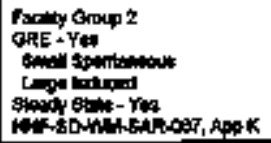 & Shot diclitom & 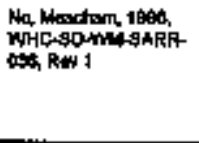 & 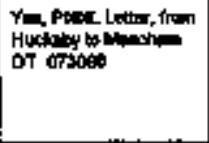 & Dtenct or & Litnomer cumpilly \\
\hline
\end{tabular}




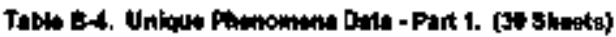

\begin{tabular}{|c|c|c|c|c|c|c|}
\hline Fald thom & Fbrmabla oal & Than & Forracyando & 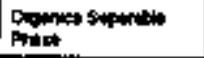 & TOC-h Laqu & TOC m in lath \\
\hline $24 t-6-2000$ & 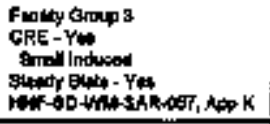 & 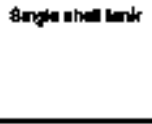 & 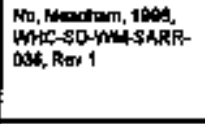 & 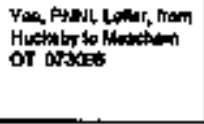 & 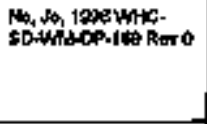 & 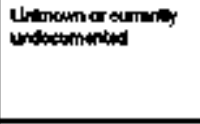 \\
\hline $241-0-204$ & 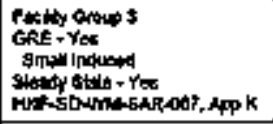 & Sinterth thes timh & 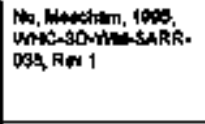 & 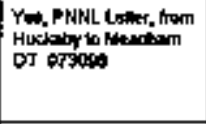 & 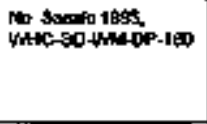 & 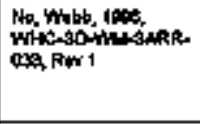 \\
\hline $241-8 x-101$ & 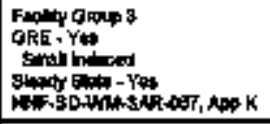 & 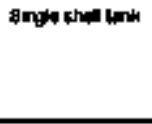 & 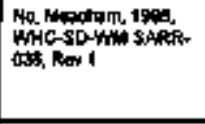 & 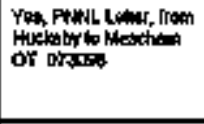 & 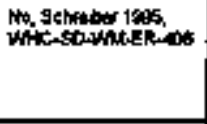 & 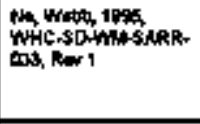 \\
\hline 241.ak.102 & 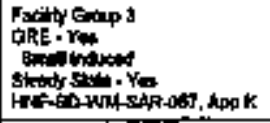 & 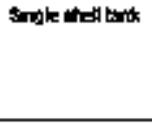 & 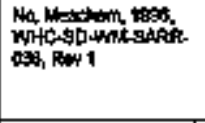 & 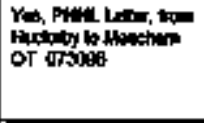 & 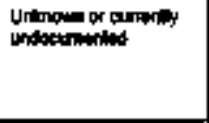 & 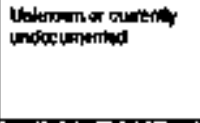 \\
\hline $24-8 \times-103$ & 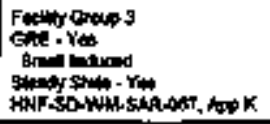 & 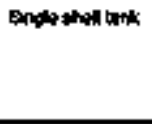 & 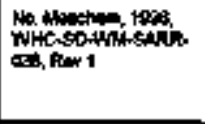 & 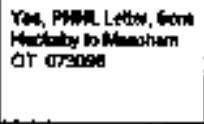 & 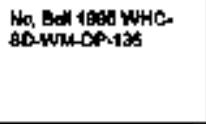 & $\begin{array}{l}\text { Untanown or curritit } \\
\text { underu dinted }\end{array}$ \\
\hline 241Ex-104 & 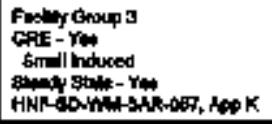 & Angtanelt at & 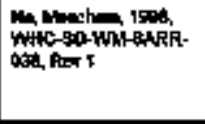 & 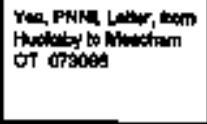 & 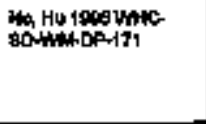 & 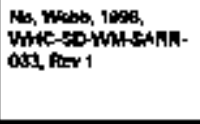 \\
\hline $241-4 x-105$ & 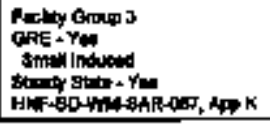 & shpla bhallonk & 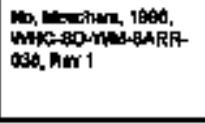 & 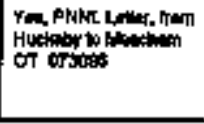 & Whe How 1996 wat & 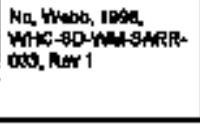 \\
\hline
\end{tabular}




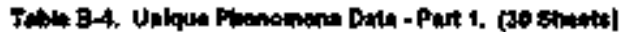

\begin{tabular}{|c|c|c|c|c|c|c|}
\hline Fad Hame & 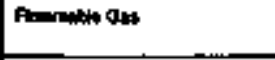 & The & Faroogent: & 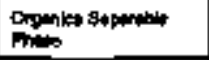 & Tosth thy & Tore in foulda \\
\hline $241-16400$ & 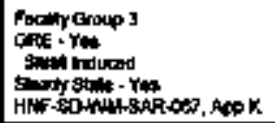 & thale fict tom & 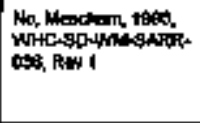 & 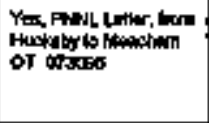 & 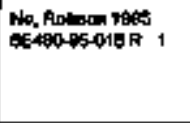 & 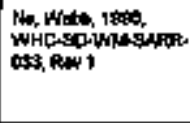 \\
\hline $241-8 x-107$ & 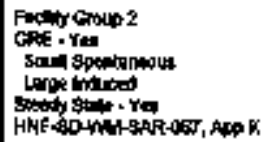 & onds shod tomir & 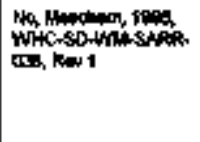 & 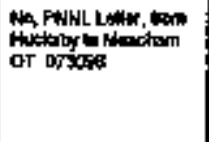 & 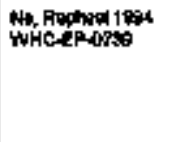 & 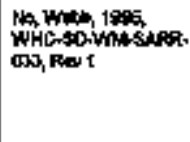 \\
\hline $241-8 x-106$ & 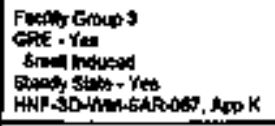 & Sondill thal ind & 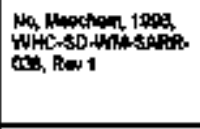 & 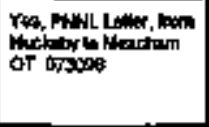 & NA & 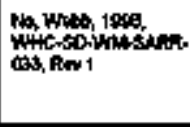 \\
\hline $291 .=x \cdot 100$ & 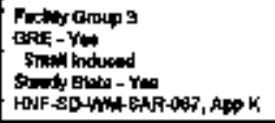 & Buncte that bak & 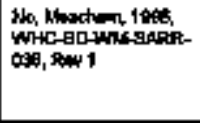 & 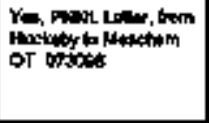 & 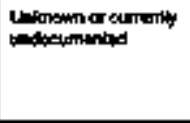 & 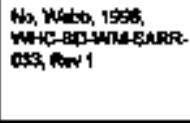 \\
\hline $241-9 x-110$ & 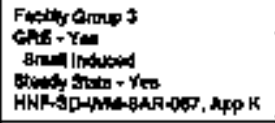 & Anoth then tank & 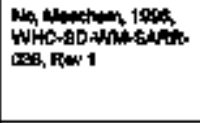 & 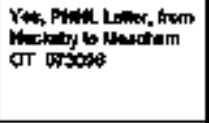 & 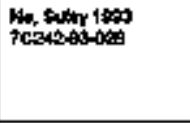 & 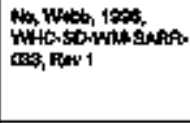 \\
\hline 2:19x-111 & 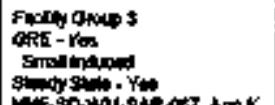 & Bnde the lank & 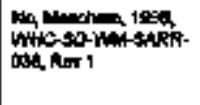 & 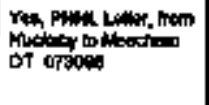 & 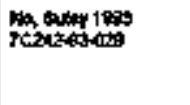 & 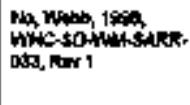 \\
\hline
\end{tabular}




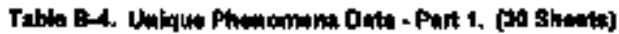

\begin{tabular}{|c|c|c|c|c|c|c|}
\hline Find wamp & 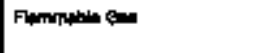 & Tye & Ferpeqpentaty & 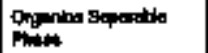 & Tocin Llapula & 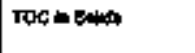 \\
\hline $241,0<6-117$ & 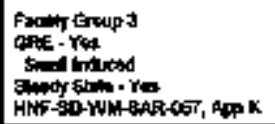 & 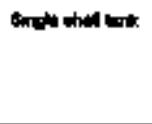 & 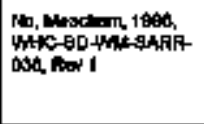 & 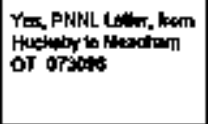 & 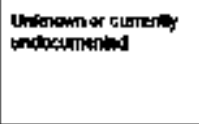 & 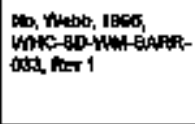 \\
\hline $241-B^{\prime} Y-101$ & 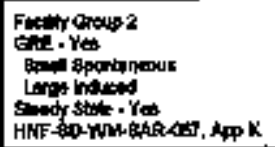 & 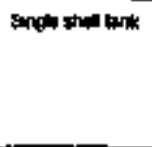 & 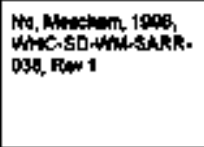 & 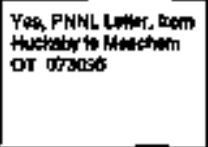 & 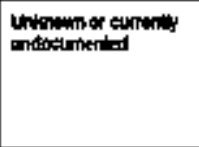 & 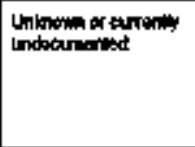 \\
\hline 2A1-BYY-102 & 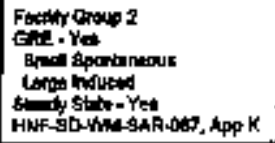 & 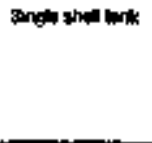 & 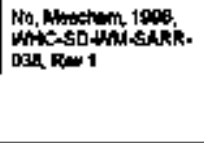 & 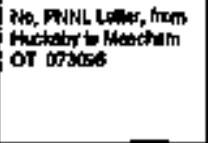 & 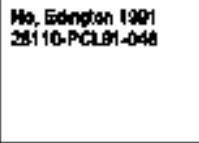 & 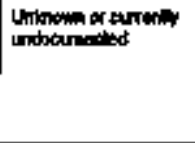 \\
\hline 241-8Y-109 & 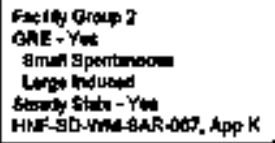 & Bngle uhegt tor & 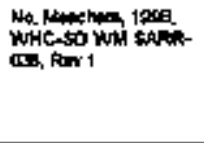 & 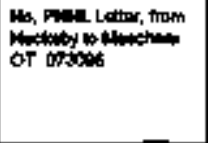 & 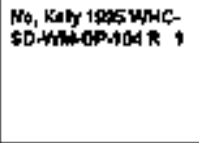 & 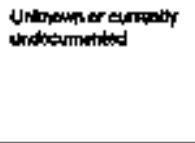 \\
\hline $2+6 Y-10$ & 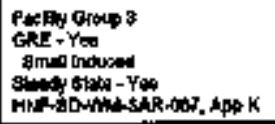 & Bngle unvith & 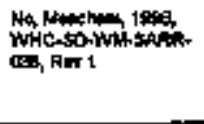 & 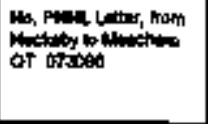 & 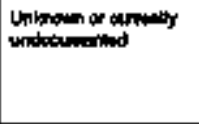 & 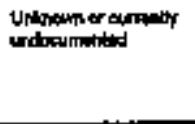 \\
\hline $241.5 y \cdot 100$ & 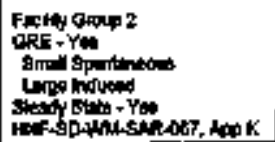 & stroje tondtok & 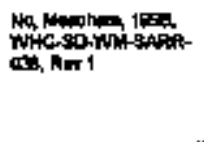 & 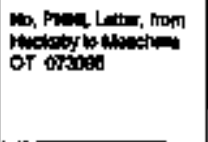 & 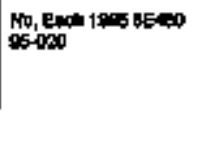 & 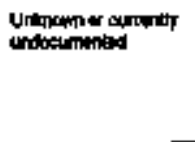 \\
\hline
\end{tabular}




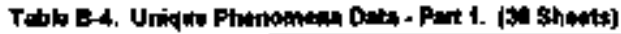

\begin{tabular}{|c|c|c|c|c|c|c|}
\hline Floid kamplo & 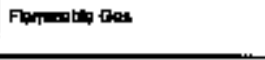 & Tyo & 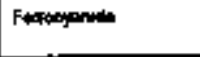 & 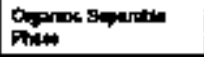 & TCC in Liquilin & TOC in Bellofig \\
\hline $2<1-6 y=100$ & 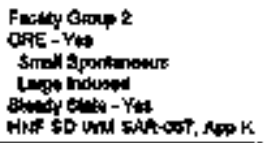 & 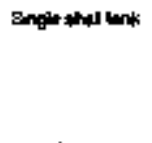 & 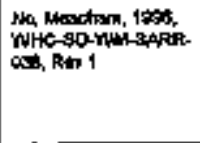 & 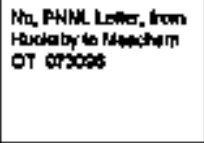 & 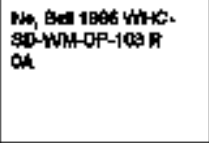 & 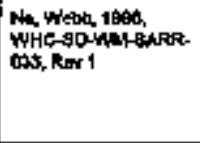 \\
\hline 241 en-107 & 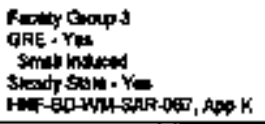 & sngm gheld bonk & 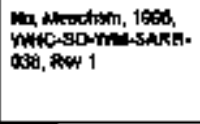 & 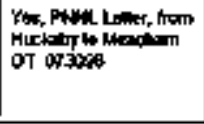 & 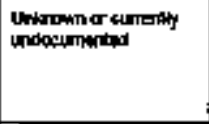 & 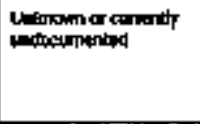 \\
\hline $241+9 Y-108$ & 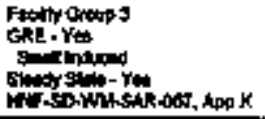 & Singth thell tast & 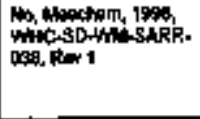 & 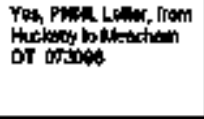 & 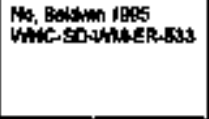 & 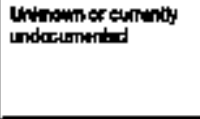 \\
\hline $2+1-8 \%+100$ & 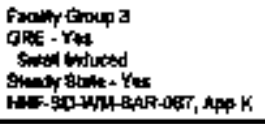 & 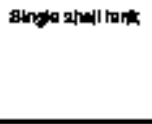 & 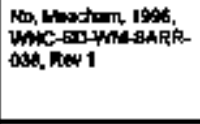 & 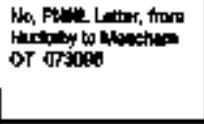 & 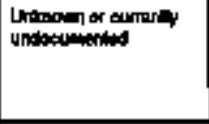 & 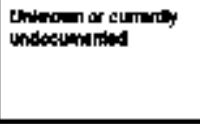 \\
\hline 241-0Y-110 & 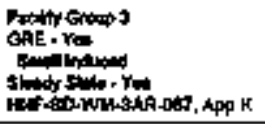 & Singta bhell bort & 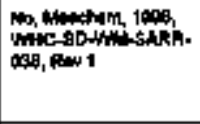 & 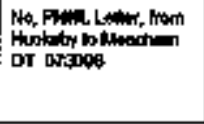 & 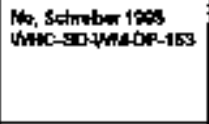 & 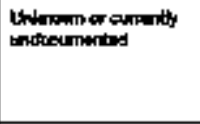 \\
\hline $241-B Y-111$ & 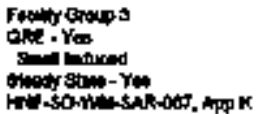 & 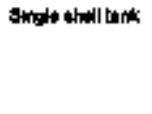 & 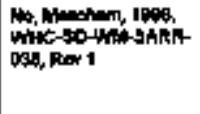 & 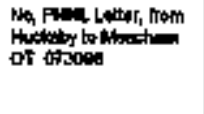 & 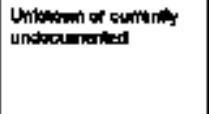 & 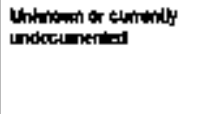 \\
\hline
\end{tabular}




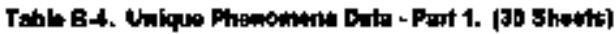

\begin{tabular}{|c|c|c|c|c|c|c|}
\hline Prid Whind & Flimmoted Cas & Typos & 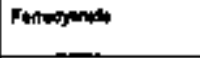 & 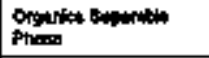 & TOC n & Toc in sists \\
\hline 2HEY-112 & 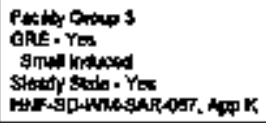 & 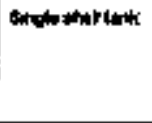 & 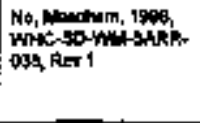 & 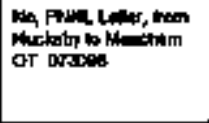 & 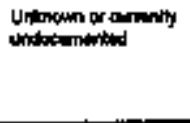 & 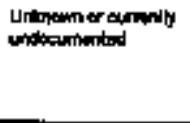 \\
\hline 241010104 & 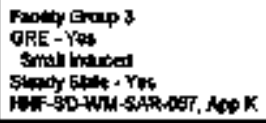 & Englatiol trok & 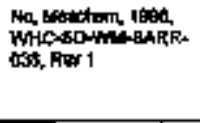 & 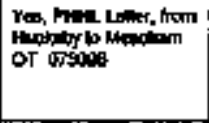 & Otand the & 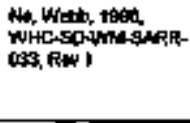 \\
\hline $241+C .1002$ & 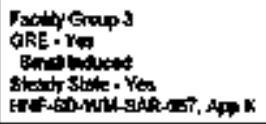 & Sinde thed think & 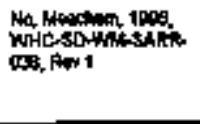 & 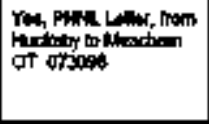 & 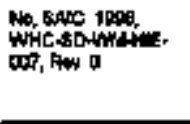 & 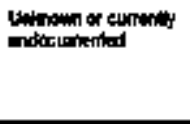 \\
\hline $241-c-103$ & 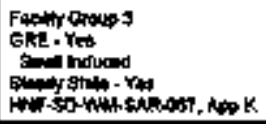 & 8min then bonk & 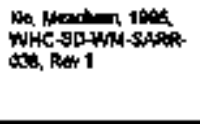 & 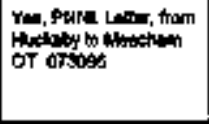 & 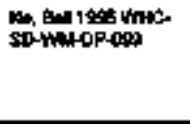 & 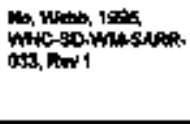 \\
\hline 241.c-104 & 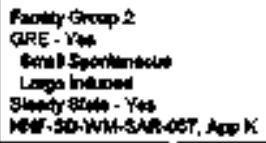 & Anquent tonk & 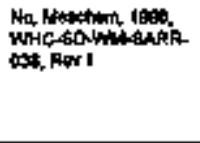 & 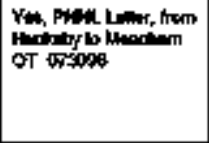 & 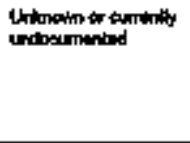 & 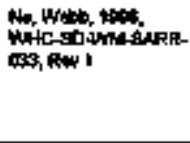 \\
\hline $241-1015$ & 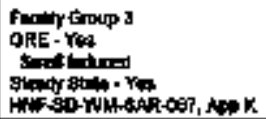 & 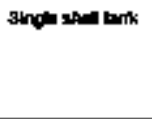 & 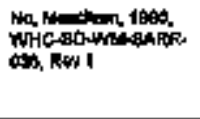 & 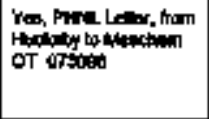 & 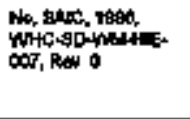 & 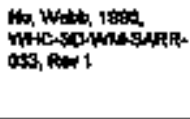 \\
\hline
\end{tabular}




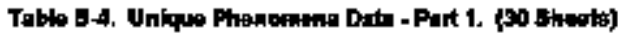

\begin{tabular}{|c|c|c|c|c|c|c|}
\hline 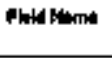 & 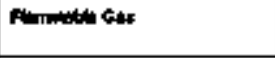 & Tim & 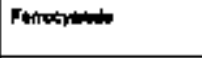 & 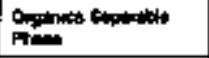 & TOC M L L W & Toc In ortat \\
\hline $2+16-100$ & 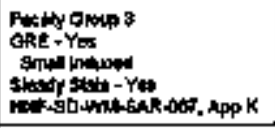 & 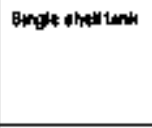 & 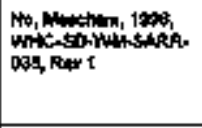 & 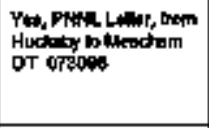 & 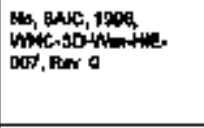 & 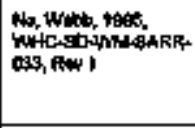 \\
\hline 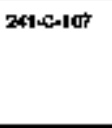 & 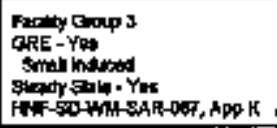 & stople ohed tank & 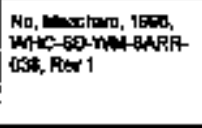 & 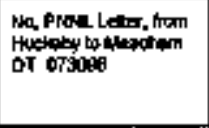 & 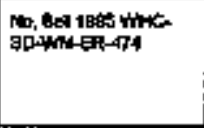 & 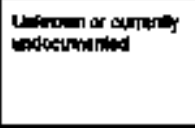 \\
\hline 24103 & 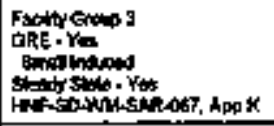 & 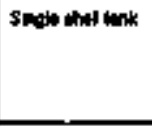 & 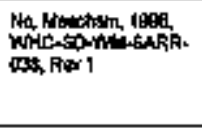 & 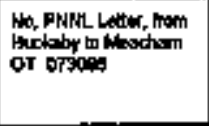 & 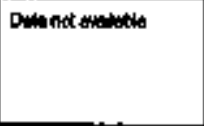 & 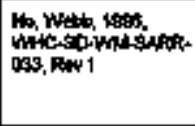 \\
\hline 241-C-1Cs & 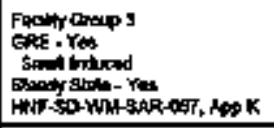 & Angledted bork & 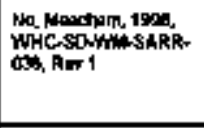 & 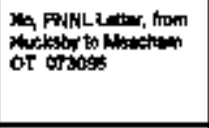 & 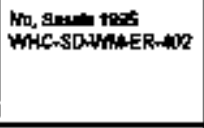 & 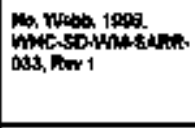 \\
\hline $241-0+10$ & 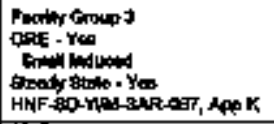 & 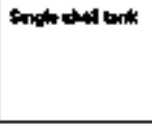 & 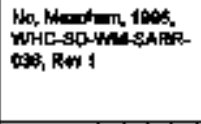 & 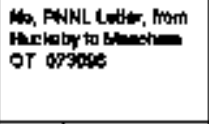 & 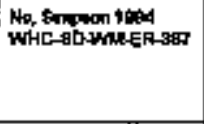 & 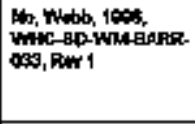 \\
\hline $2|1|-111$ & 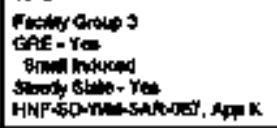 & onsing and & 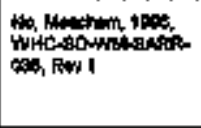 & 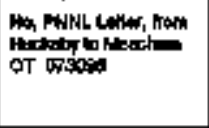 & WH & 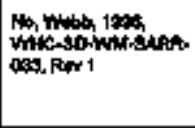 \\
\hline $201-6-112$ & 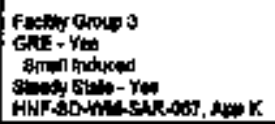 & 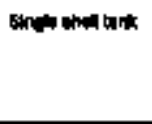 & 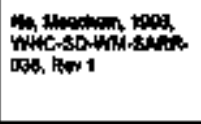 & 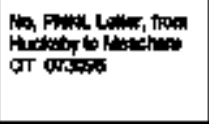 & 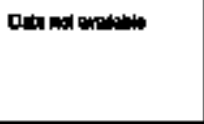 & 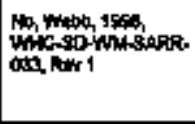 \\
\hline
\end{tabular}




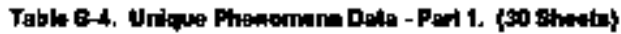

\begin{tabular}{|c|c|c|c|c|c|c|}
\hline 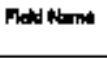 & Plammate Gat & The & Ftrmitimith & 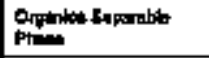 & Toc n iqunn & Fof = Sellit. \\
\hline $241-c-201$ & 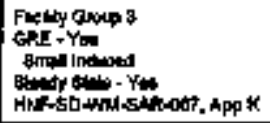 & 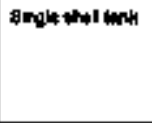 & 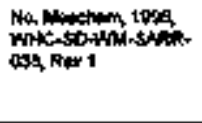 & 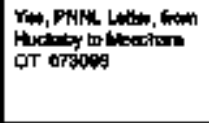 & 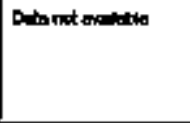 & 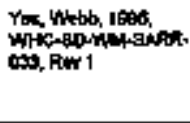 \\
\hline 241402012 & 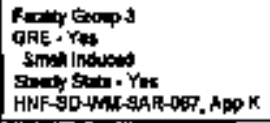 & thot aded tonk & 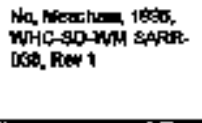 & 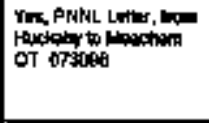 & atip not matitis & 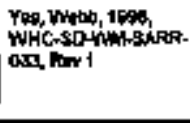 \\
\hline $211-6-2009$ & 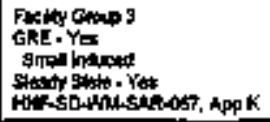 & SFalo stad ind & 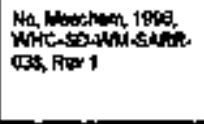 & 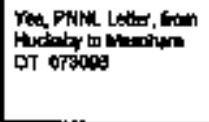 & Dhonct ractil & 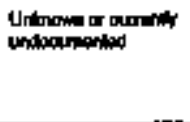 \\
\hline 261-c204 & 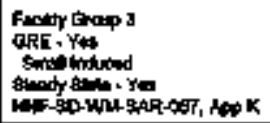 & Ingh stive tart & 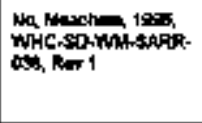 & 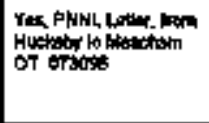 & 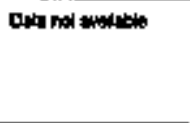 & 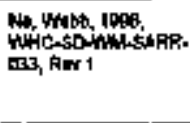 \\
\hline $241+6.701$ & 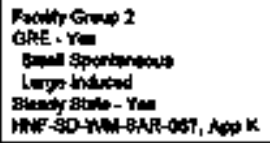 & sthend thest bont & 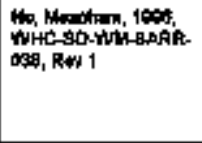 & 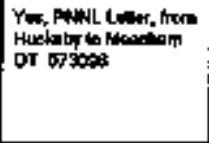 & 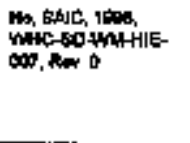 & 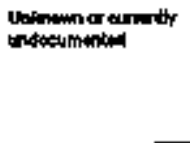 \\
\hline $2+1 \leqslant-102$ & 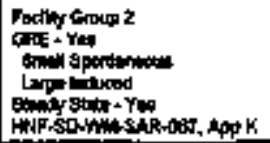 & 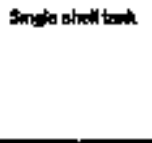 & 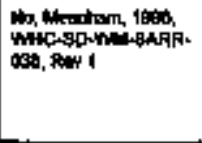 & 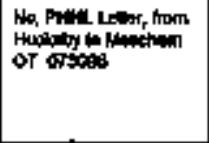 & 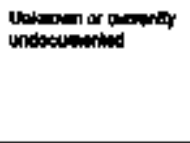 & 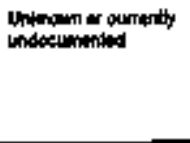 \\
\hline
\end{tabular}




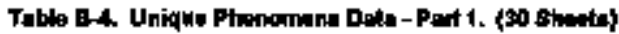

\begin{tabular}{|c|c|c|c|c|c|c|}
\hline Fata Bum & 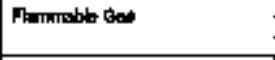 & Trna & Fwrosyntosto & 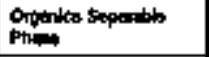 & Toc: in Uanidst & TOC in 9ows \\
\hline $241-8-103$ & 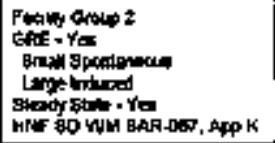 & 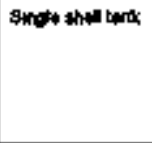 & 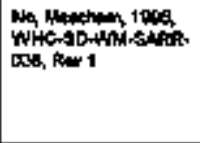 & 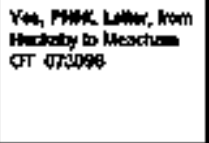 & 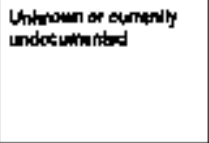 & 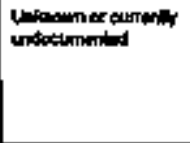 \\
\hline $241-9-104$ & 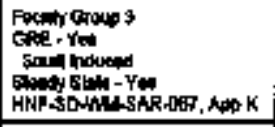 & 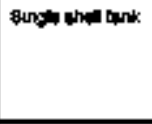 & 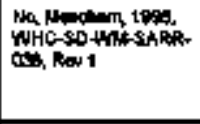 & 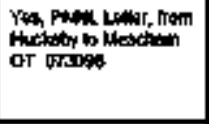 & 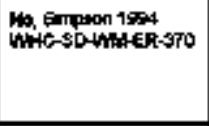 & 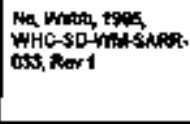 \\
\hline 241 S.405 & 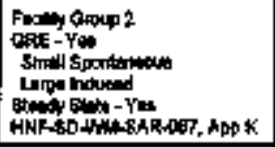 & 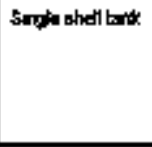 & 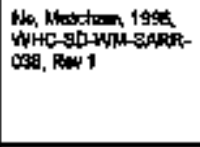 & 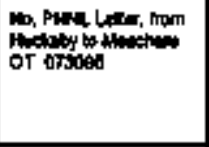 & 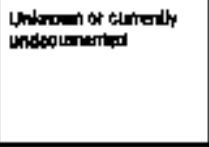 & 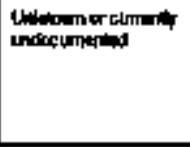 \\
\hline $241-5105$ & 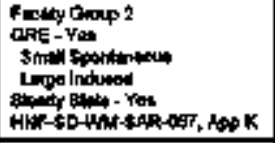 & EFole thes tait & 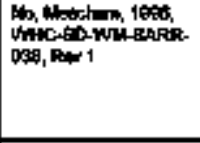 & 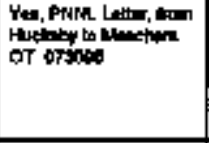 & 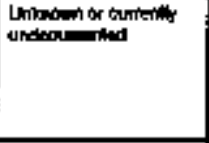 & 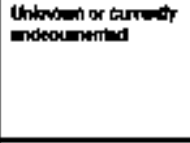 \\
\hline $2+1-6107$ & 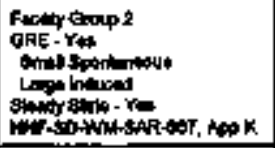 & Snglethl thit & 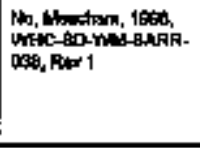 & 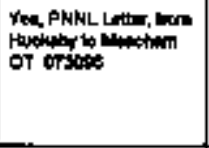 & 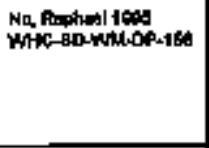 & 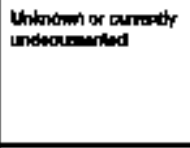 \\
\hline 3615-116 & 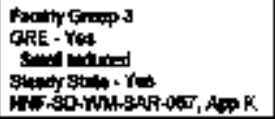 & 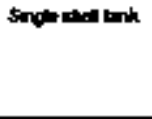 & 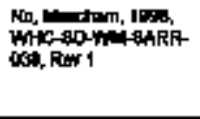 & 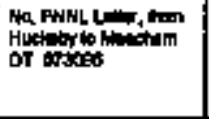 & 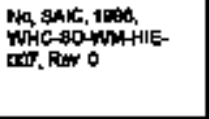 & 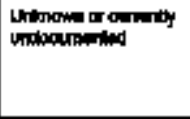 \\
\hline
\end{tabular}




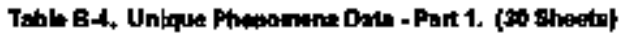

\begin{tabular}{|c|c|c|c|c|c|c|}
\hline Fuild nam & 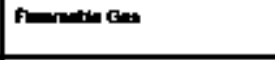 & Type & Furmocyantid & 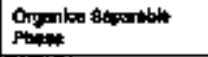 & 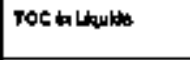 & $T O C$ in folds \\
\hline 211 -510 & 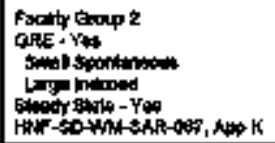 & Nopotal thenk & 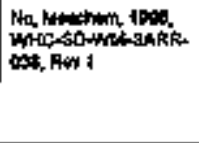 & 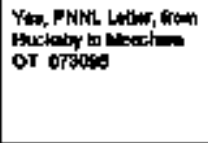 & 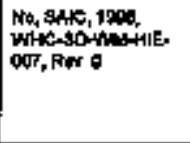 & 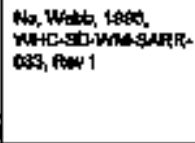 \\
\hline $241-5-110$ & 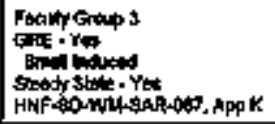 & Sndistat terik & 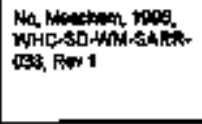 & 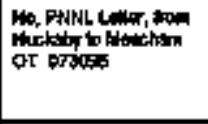 & 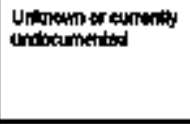 & 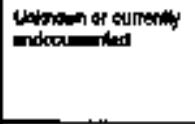 \\
\hline $201-5-111$ & 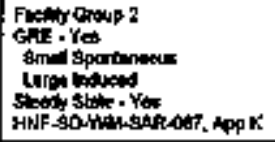 & Sngto shell torth & 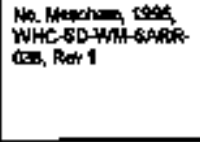 & 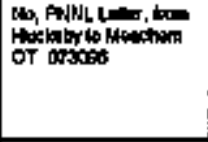 & 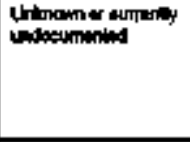 & 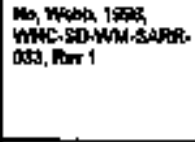 \\
\hline $241-8-11 ?$ & 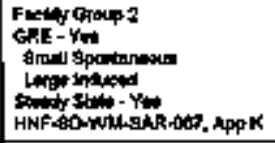 & 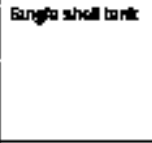 & 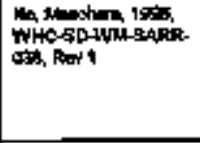 & 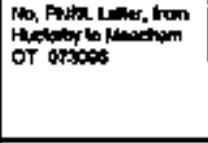 & 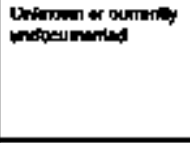 & 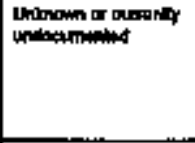 \\
\hline $241-3 x-101$ & 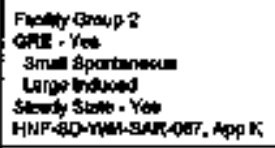 & 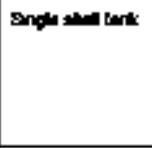 & 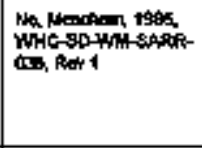 & 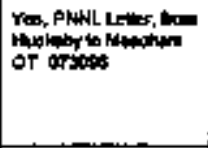 & $\begin{array}{l}\text { No. When 1800 } \\
\text { IFr-PCAS0-049 }\end{array}$ & Unbrom ar ourertily \\
\hline $241-5 x-102$ & 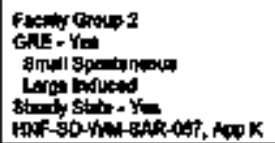 & Stinte shot tort & 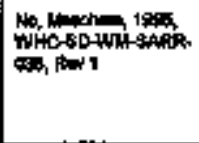 & 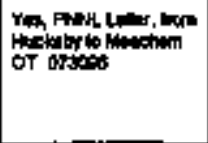 & 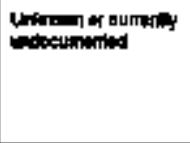 & 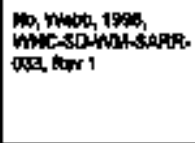 \\
\hline
\end{tabular}




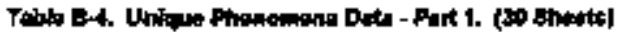

\begin{tabular}{|c|c|c|c|c|c|c|}
\hline P & 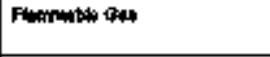 & Typos & 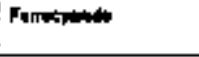 & 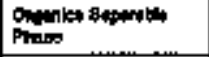 & TOC mLthats & Toc In Soldta \\
\hline $2+14 x-100$ & 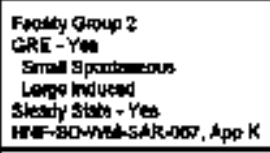 & 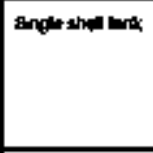 & 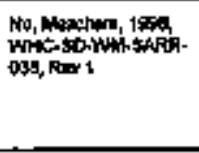 & 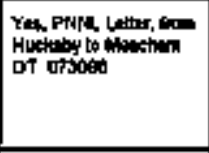 & 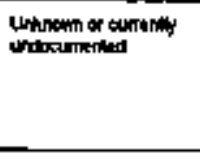 & 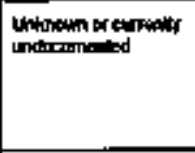 \\
\hline $241.5 \times \cdot 104$ & 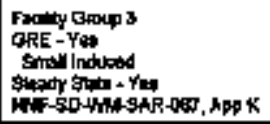 & Engla shel bots & 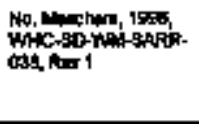 & 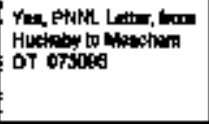 & 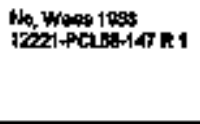 & 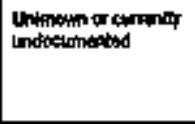 \\
\hline $241-5 x \cdot 105$ & 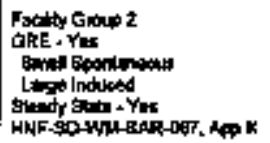 & single thell thent & 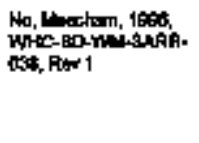 & 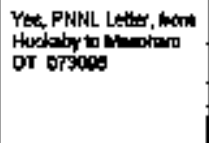 & Ihimewn of oumbity & 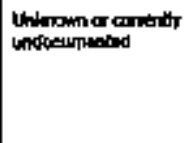 \\
\hline $241-8 \times-108$ & 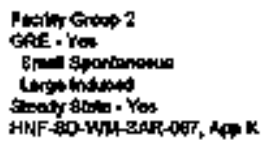 & 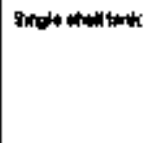 & 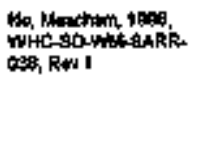 & 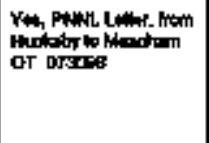 & 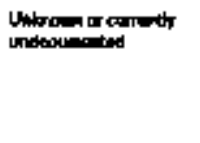 & 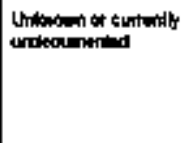 \\
\hline $241-6 x-10 \pi$ & 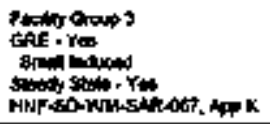 & 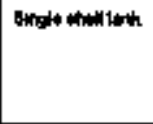 & 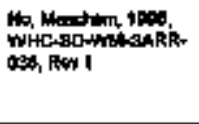 & 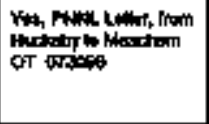 & 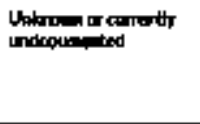 & 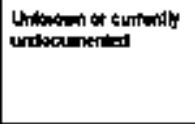 \\
\hline $2+1-8 x-106$ & 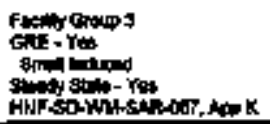 & 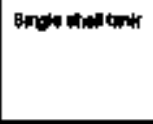 & 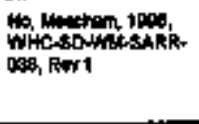 & 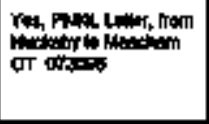 & ith & 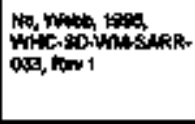 \\
\hline
\end{tabular}




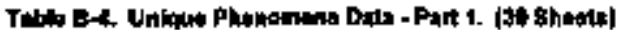

\begin{tabular}{|c|c|c|c|c|c|c|}
\hline Find Remo & 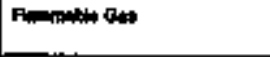 & Typo & 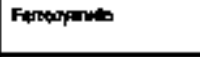 & 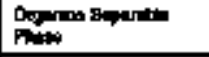 & Toć n أinj: & 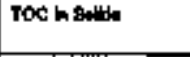 \\
\hline 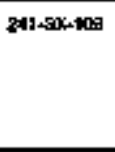 & 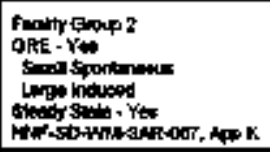 & Bangle thet ank & 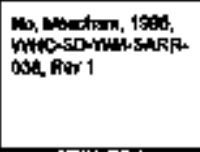 & 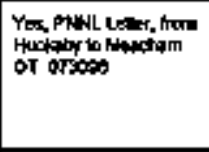 & 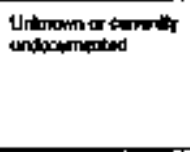 & 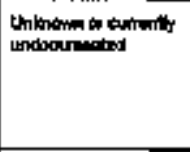 \\
\hline $241-6$ - & 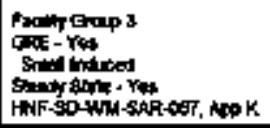 & 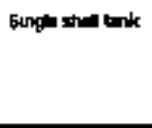 & 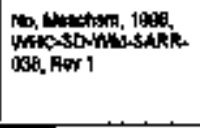 & 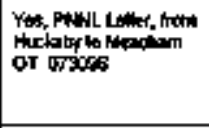 & Non & $\begin{array}{l}\text { Uninouner eurnwing } \\
\text { unboumetided }\end{array}$ \\
\hline $241-5 x-111$ & 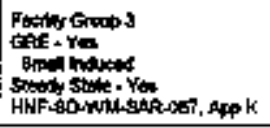 & Singte thed the & 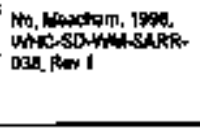 & 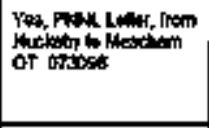 & Unimannes andunior & 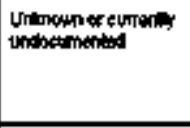 \\
\hline 245-Ex-112 & 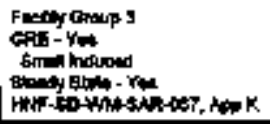 & Singlo angla bank & 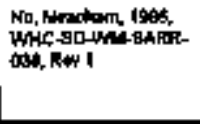 & 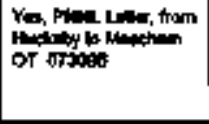 & 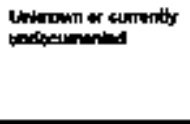 & 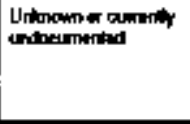 \\
\hline $241-5 x+113$ & 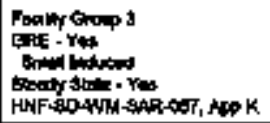 & fingte bred tonk & 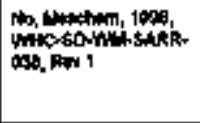 & 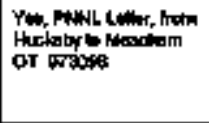 & Non & 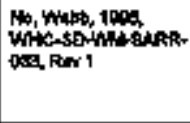 \\
\hline $24 t-8 x-114$ & 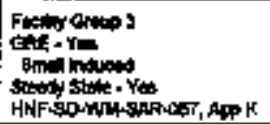 & 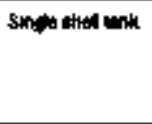 & 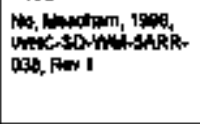 & 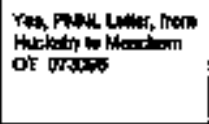 & 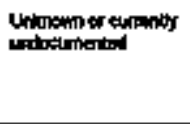 & 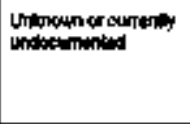 \\
\hline
\end{tabular}




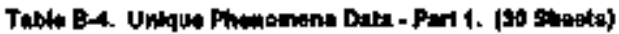

\begin{tabular}{|c|c|c|c|c|c|c|}
\hline Find & 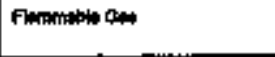 & Then & Feтtagn: & 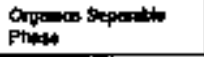 & TOC m Lalk & TOC m solts \\
\hline $241+60 \times-118$ & 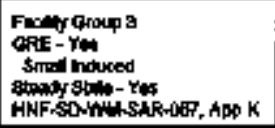 & ancle ahel brt & 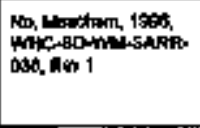 & 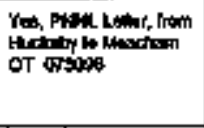 & NMIN & 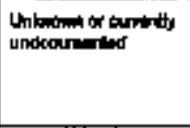 \\
\hline $241-3+2+01$ & 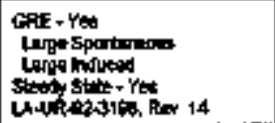 & Cowbt the int & 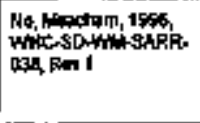 & 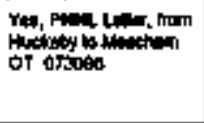 & 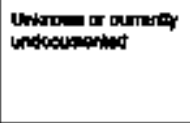 & 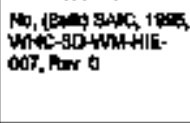 \\
\hline $2+1-34-102$ & 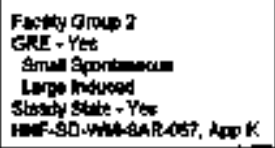 & Doulte thet lonk & 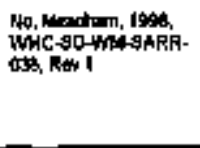 & 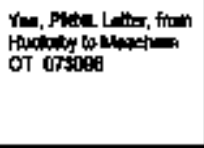 & 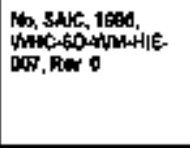 & 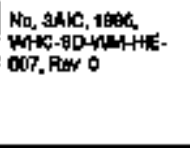 \\
\hline 241-\$Y-100 & 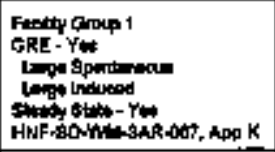 & Douth thel unk & 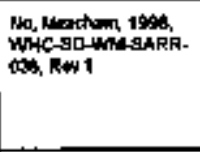 & 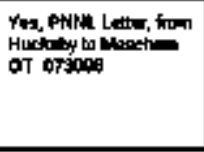 & 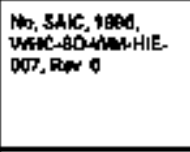 & 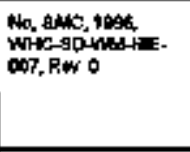 \\
\hline 241-T-k91 & 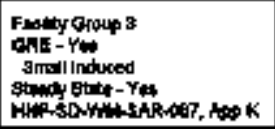 & Angen whel tart & 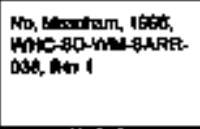 & 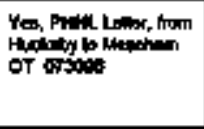 & 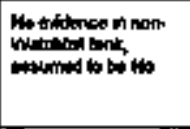 & 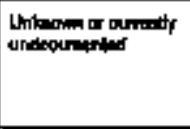 \\
\hline $24 t-T+102$ & 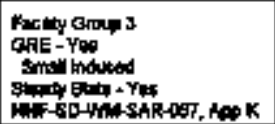 & 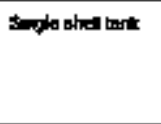 & 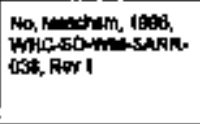 & 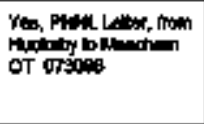 & Wh, PH/10101 & 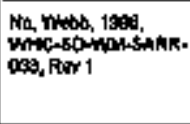 \\
\hline
\end{tabular}




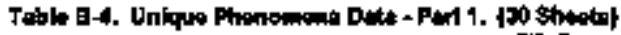

\begin{tabular}{|c|c|c|c|c|c|c|}
\hline 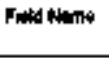 & 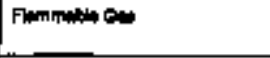 & Tho & 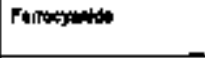 & 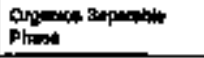 & TOCh h ا & $T O C=0$. \\
\hline $211-T-1103$ & 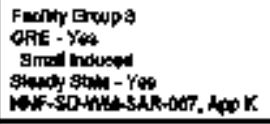 & 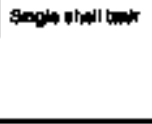 & 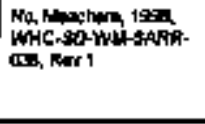 & 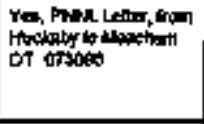 & 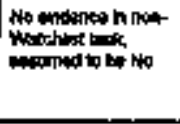 & Unknown or atementy \\
\hline $241-T-10 \mathrm{~d}$ & 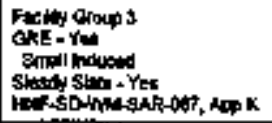 & Smpte tateit thk & 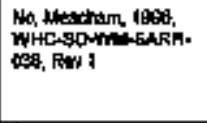 & 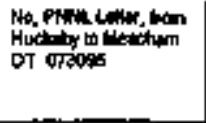 & 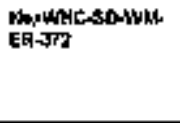 & 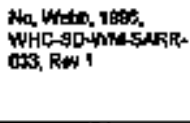 \\
\hline 241-T-106 & 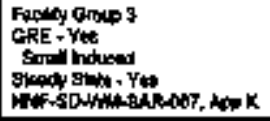 & Sunglis thit ink & 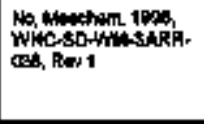 & 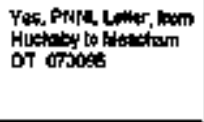 & ER, WHC-SOMAM & 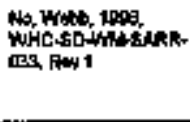 \\
\hline $241-\mathbf{T}-106$ & 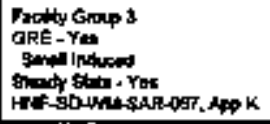 & 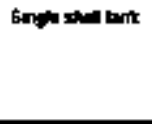 & 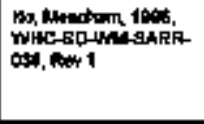 & 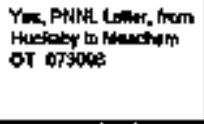 & 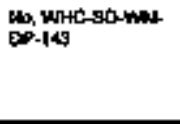 & 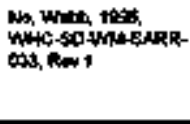 \\
\hline $241-7-107$ & 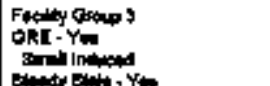 & 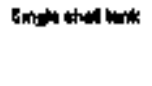 & 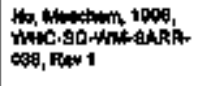 & 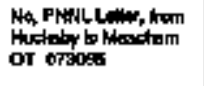 & 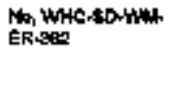 & 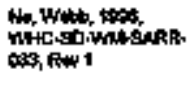 \\
\hline 241-T-196 & 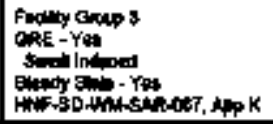 & 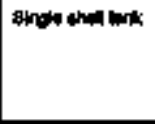 & 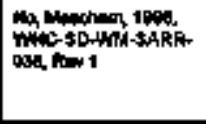 & 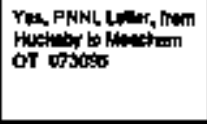 & 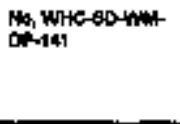 & 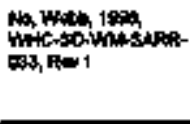 \\
\hline 2:1.T.100 & 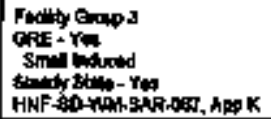 & shote what bert & 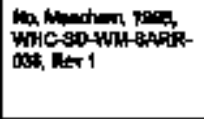 & 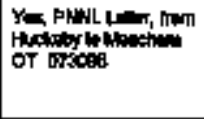 & 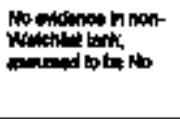 & Whing \\
\hline
\end{tabular}




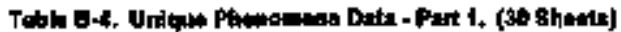

\begin{tabular}{|c|c|c|c|c|c|c|}
\hline Fratd timm & 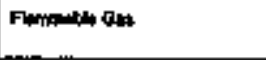 & mos & Fetpoganto & 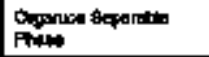 & TOC niquent & 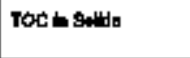 \\
\hline 241.T-110 & 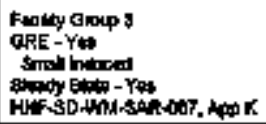 & Angle intipenk & 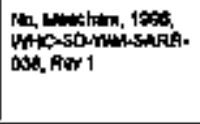 & 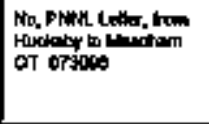 & 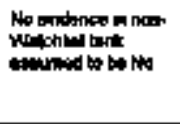 & 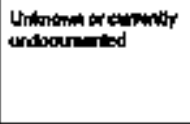 \\
\hline 241-T-111 & 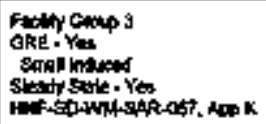 & stripk the thk & 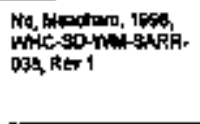 & 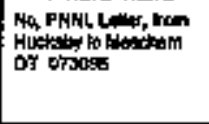 & 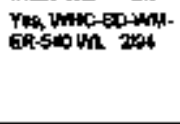 & 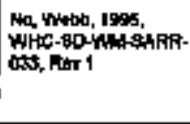 \\
\hline 2*1-T-11? & 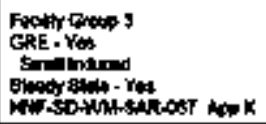 & Endinstiol lonk & 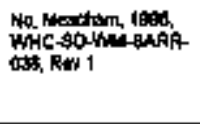 & 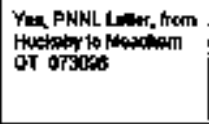 & 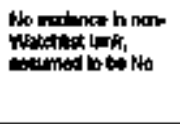 & 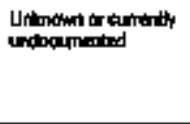 \\
\hline $241.7-201$ & 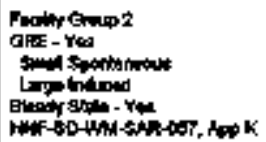 & singta grted bork & 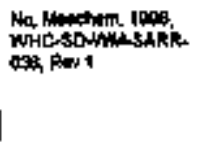 & 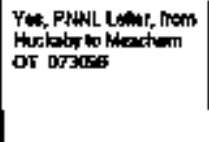 & 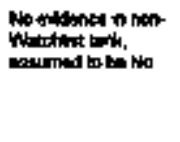 & 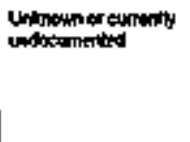 \\
\hline 241-T-20 & 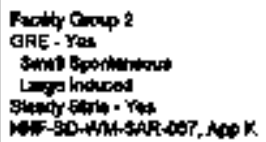 & tangle atel tonk & 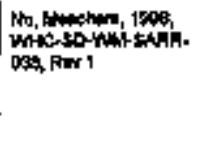 & 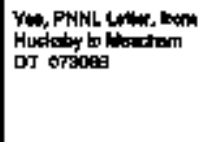 & 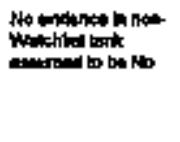 & 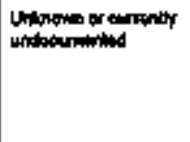 \\
\hline $241 \cdot T=200$ & 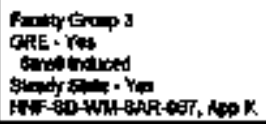 & Inqtented thik & 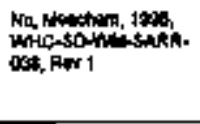 & 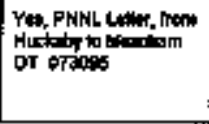 & 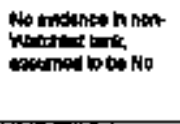 & 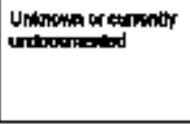 \\
\hline
\end{tabular}




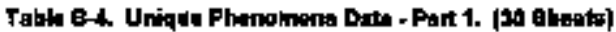

\begin{tabular}{|c|c|c|c|c|c|c|}
\hline Dats latem & 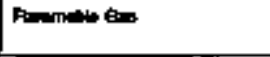 & Tyos & 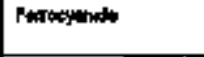 & 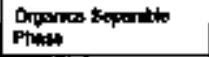 & TOC in & TOS in sconds \\
\hline 2:11-T-204 & 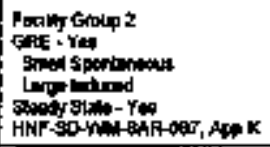 & 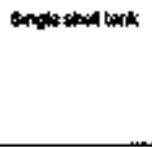 & 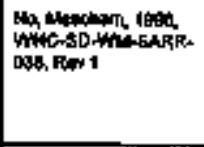 & 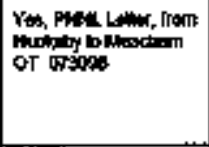 & 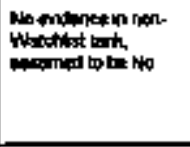 & Undingen of armitit \\
\hline 2AI-TR-101 & 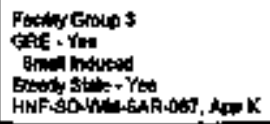 & 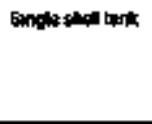 & 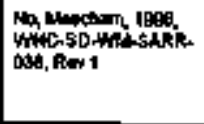 & 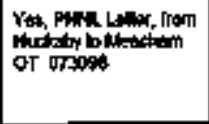 & 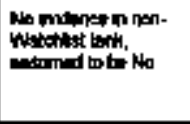 & 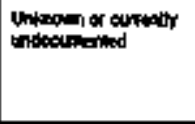 \\
\hline $241.7 x+102$ & 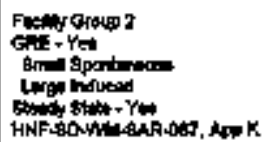 & 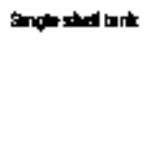 & 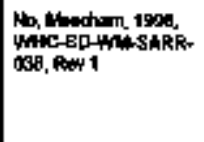 & 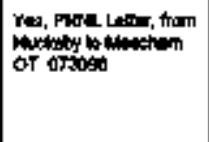 & 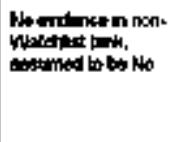 & 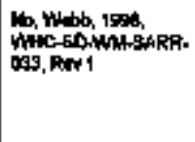 \\
\hline $2+1-7 x+100$ & 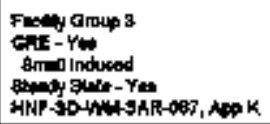 & 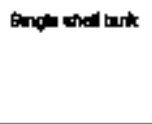 & 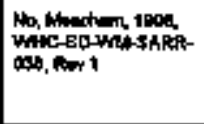 & 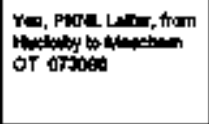 & 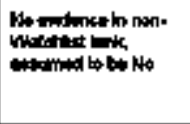 & Unkmomin or ours.tir \\
\hline $241-\pi x-104$ & 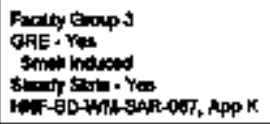 & 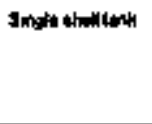 & 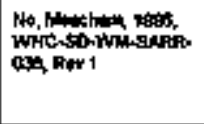 & 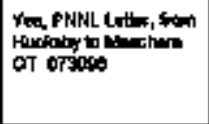 & 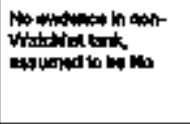 & 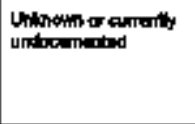 \\
\hline $241-\Gamma x-105$ & 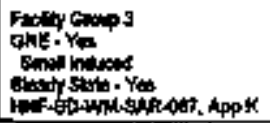 & 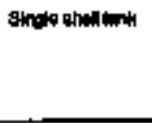 & 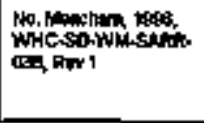 & 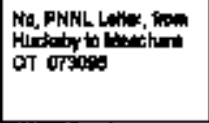 & 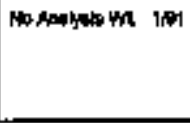 & 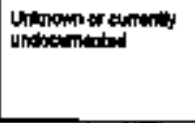 \\
\hline
\end{tabular}




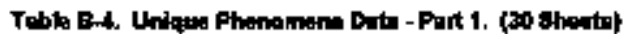

\begin{tabular}{|c|c|c|c|c|c|c|}
\hline 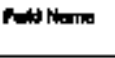 & Ftmidin OA & Typt & Fonosponde & 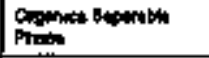 & TOC n nuguts & Toc in solds \\
\hline $241-\Gamma x, 100$ & 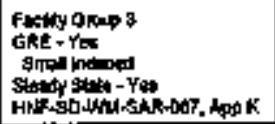 & sanglaten I lank & 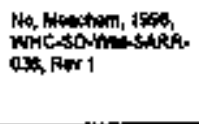 & 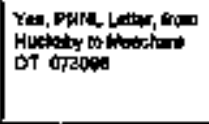 & 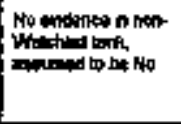 & 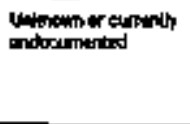 \\
\hline 241-Th-1ET & 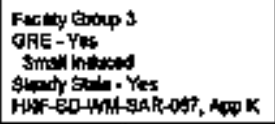 & sngt tent tonk & 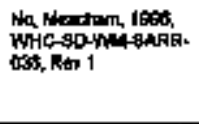 & 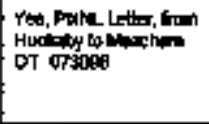 & 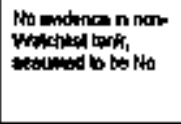 & 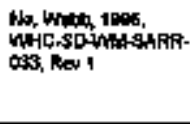 \\
\hline $241 \cdot \Gamma \times \cdot 106$ & 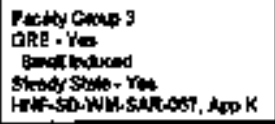 & Emple thel thk & 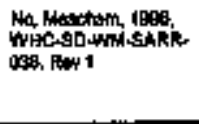 & 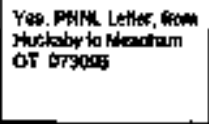 & 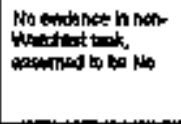 & 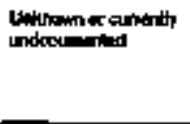 \\
\hline $241-T X-100$ & 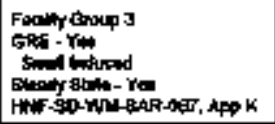 & fonghe shel tant & 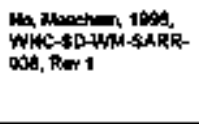 & 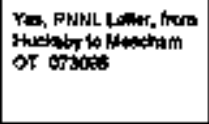 & 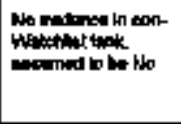 & 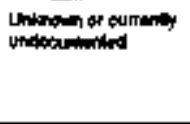 \\
\hline $2011-T \times 110$ & 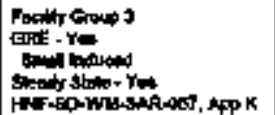 & Endw aheal tart & 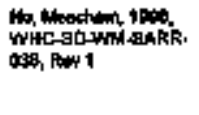 & 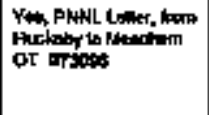 & 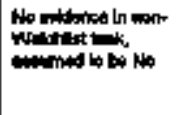 & 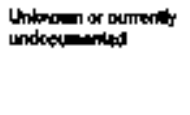 \\
\hline $241-T \times-111$ & 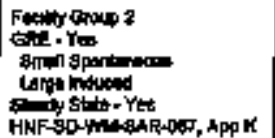 & 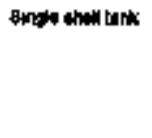 & 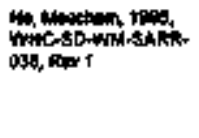 & 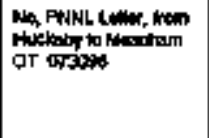 & 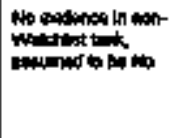 & 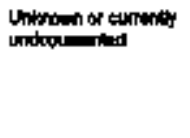 \\
\hline
\end{tabular}




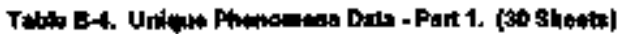

\begin{tabular}{|c|c|c|c|c|c|c|}
\hline 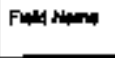 & Fumblat on & Typo & 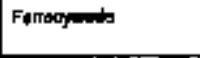 & 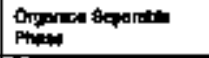 & TOC inlupula & TOS n sditos \\
\hline $241.7 x-112$ & 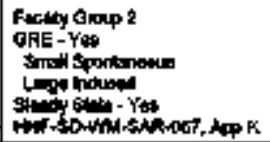 & sangen ther lank & 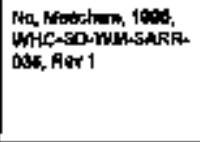 & 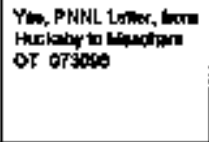 & 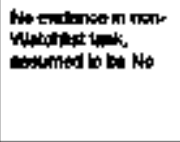 & 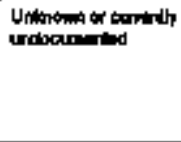 \\
\hline $241.7 x-113$ & 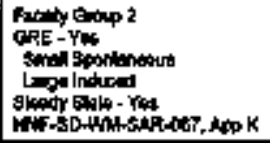 & 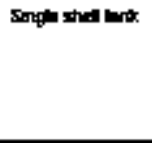 & 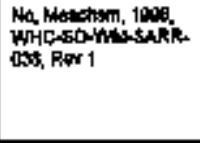 & 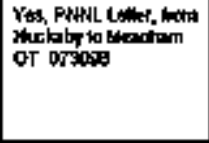 & 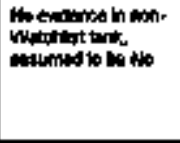 & 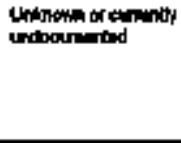 \\
\hline $2+1-T x-114$ & 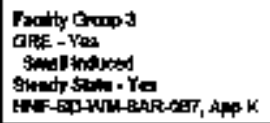 & Eunge shed bot & 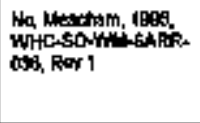 & 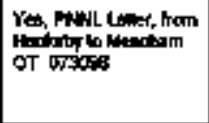 & 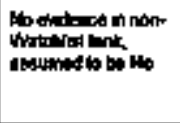 & 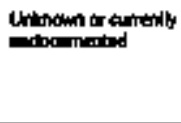 \\
\hline $241-1 \times-115$ & 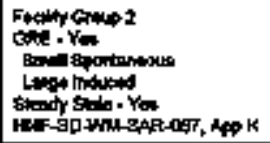 & Sind to thet tatit & 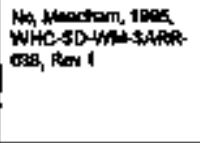 & 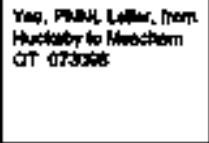 & 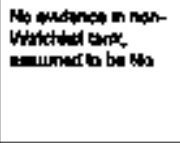 & 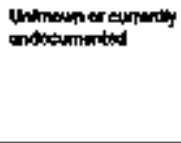 \\
\hline $24-T X-110$ & 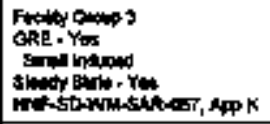 & Sing whell wh & 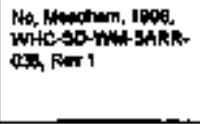 & 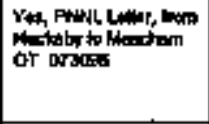 & 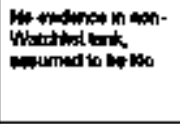 & 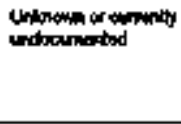 \\
\hline $241-5 \times 117$ & 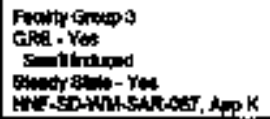 & 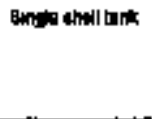 & 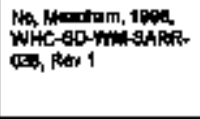 & 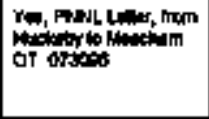 & 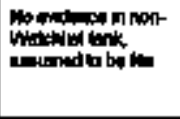 & 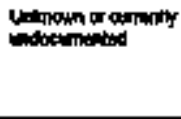 \\
\hline
\end{tabular}




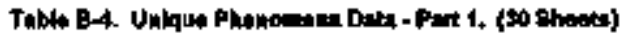

\begin{tabular}{|c|c|c|c|c|c|c|}
\hline Fend Nerest & Funnitis on: & Then & Farowede & 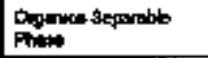 & 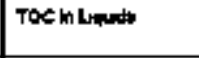 & ToS in Golkto \\
\hline 245-TX.1 1t & 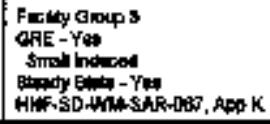 & Angle ahel tait & 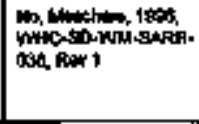 & 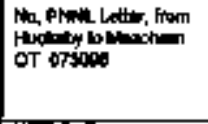 & 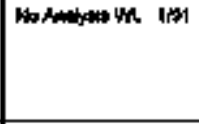 & 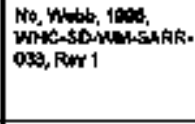 \\
\hline $241-T Y-101$ & 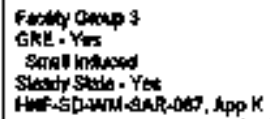 & Sinelo whell tor & 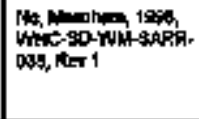 & 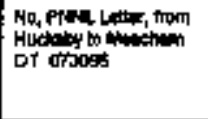 & 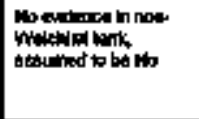 & 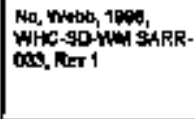 \\
\hline $241-t r-102$ & 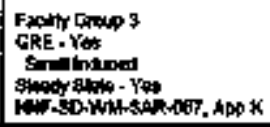 & Inde whell trit & 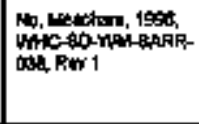 & 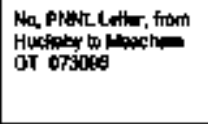 & 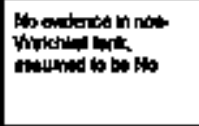 & 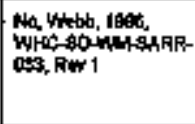 \\
\hline $241-T / 2+400$ & 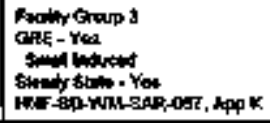 & solve ther that & 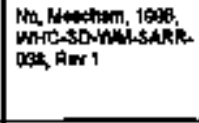 & 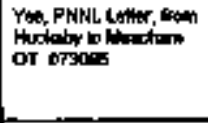 & 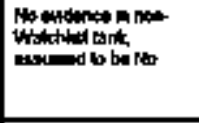 & 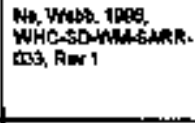 \\
\hline $241-T Y-104$ & 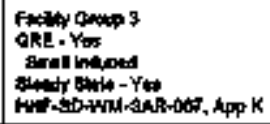 & 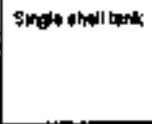 & 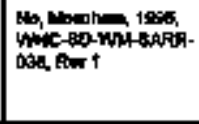 & 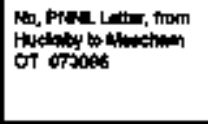 & 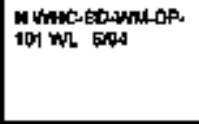 & 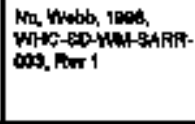 \\
\hline : & 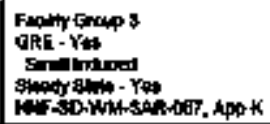 & 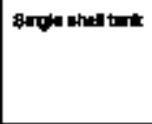 & 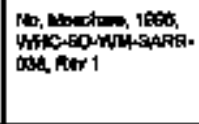 & 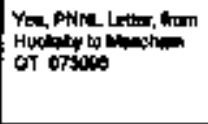 & 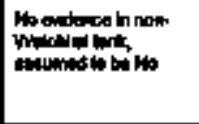 & 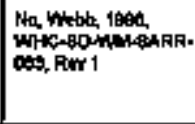 \\
\hline $241 \cdot T+265$ & 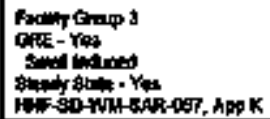 & 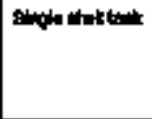 & 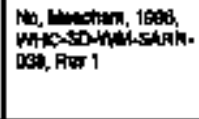 & 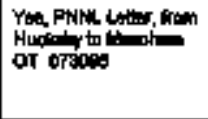 & 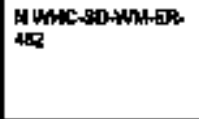 & 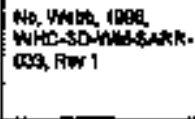 \\
\hline
\end{tabular}


Tabta B-4, Uniqua Phanomane Dada - Part 1. [30 Blwates)

\begin{tabular}{|c|c|c|c|c|c|c|}
\hline 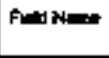 & 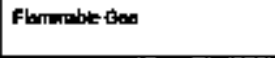 & Type & Fumbaporide & 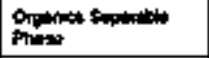 & 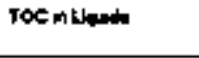 & Toc in fodsts \\
\hline 2ง1.ப.10 & 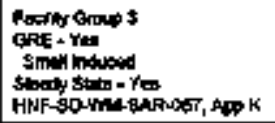 & Singth ahdil tarh & 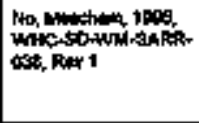 & 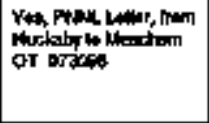 & 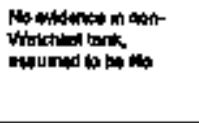 & 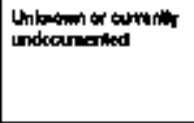 \\
\hline $211-4100$ & 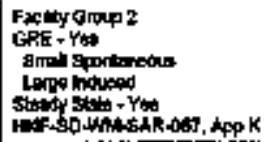 & Engien whallonis & 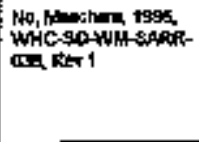 & 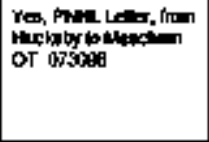 & 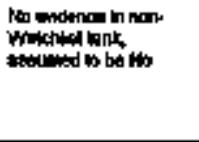 & 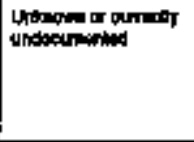 \\
\hline $2+1-4-103$ & 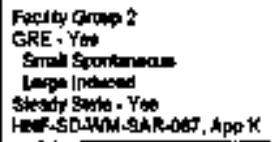 & Aingle ptollonk & 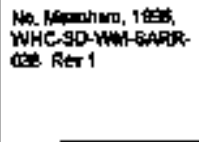 & 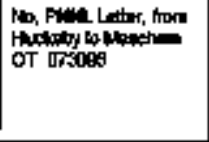 & No Antite wh sto & 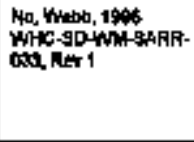 \\
\hline $24113-104$ & 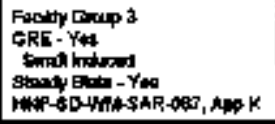 & Anoledter lork & 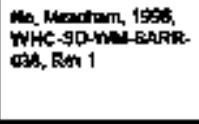 & 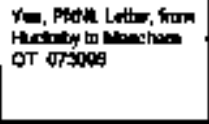 & 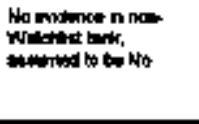 & 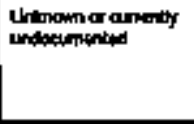 \\
\hline $241-10 s$ & 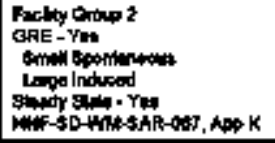 & 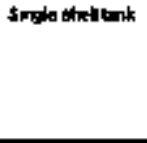 & 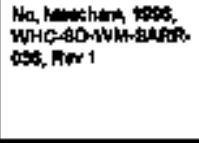 & 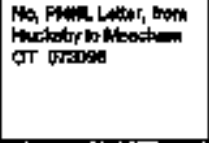 & 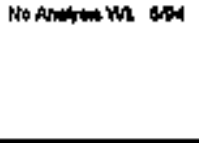 & 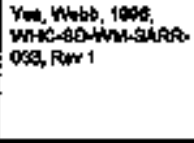 \\
\hline $241+4106$ & 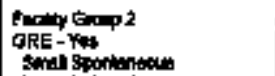 & 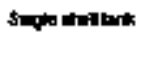 & 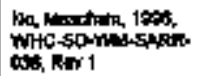 & 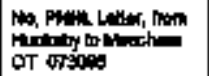 & 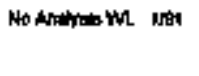 & 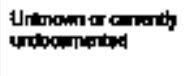 \\
\hline
\end{tabular}




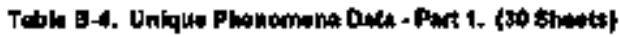

\begin{tabular}{|c|c|c|c|c|c|c|}
\hline Fund & Flammable Ben & Tho & 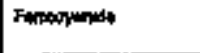 & 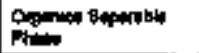 & TOC in Liquals & 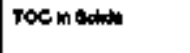 \\
\hline 241-10 & 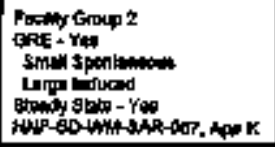 & Angle whellitis & 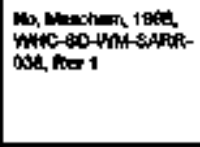 & 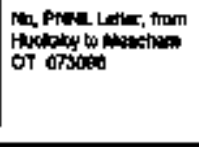 & No Anstane Wh, 1 Ar & 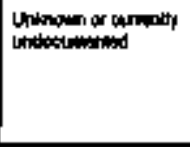 \\
\hline 2414h100 & 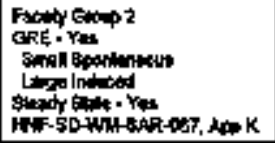 & singlip and bank & 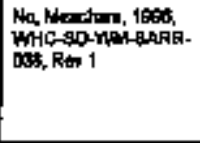 & 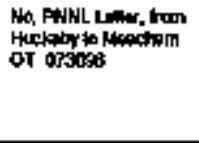 & 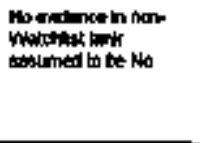 & 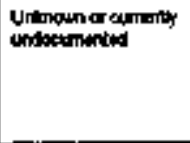 \\
\hline $2414 k 100$ & 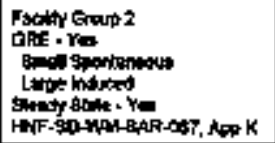 & 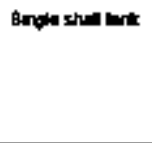 & 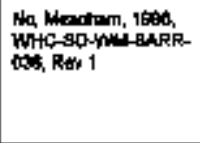 & 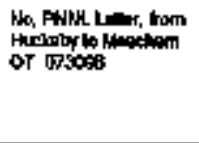 & 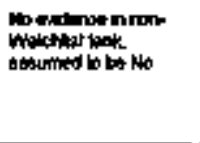 & 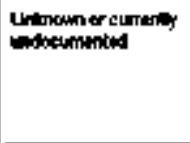 \\
\hline $241-24-110$ & 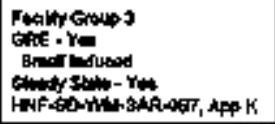 & lengla ahed bert & 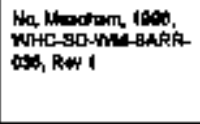 & 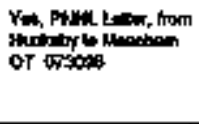 & 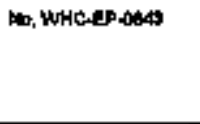 & 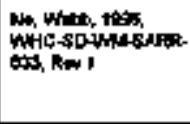 \\
\hline $241-4-11 t$ & 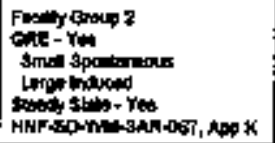 & 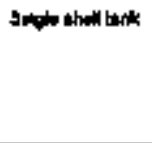 & 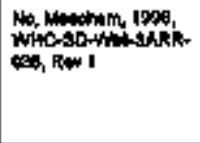 & 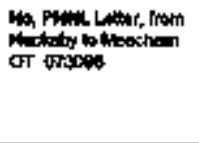 & $M, A=16 n .30$ & 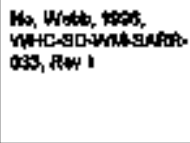 \\
\hline $241+1-112$ & 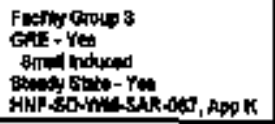 & 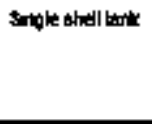 & 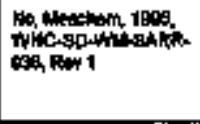 & 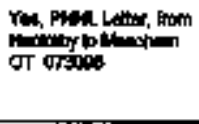 & 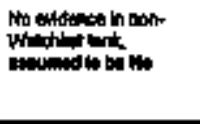 & 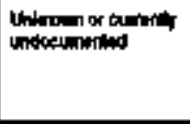 \\
\hline
\end{tabular}




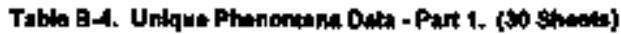

\begin{tabular}{|c|c|c|c|c|c|c|}
\hline 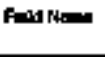 & 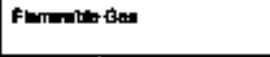 & The & Fanoplabe & 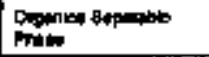 & 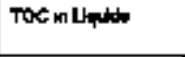 & The h h gats \\
\hline 241 - (1-201 & 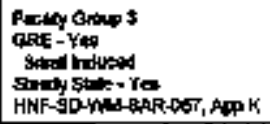 & 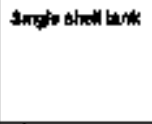 & 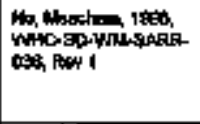 & 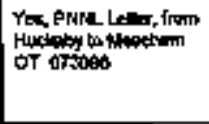 & 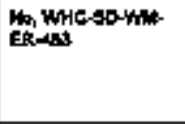 & 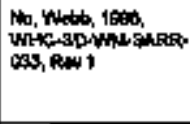 \\
\hline $241+4-2 x$ & 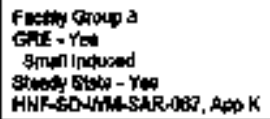 & 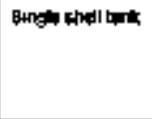 & 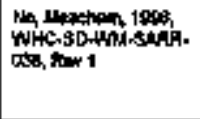 & 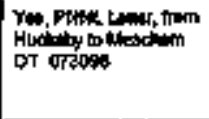 & $\mathrm{mb}_{\mathrm{ER}, \mathrm{WH}}$ & 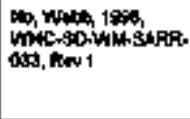 \\
\hline 241，山证了 & 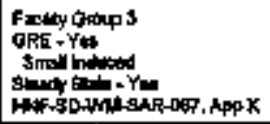 & 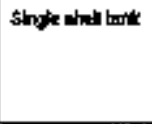 & 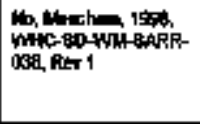 & 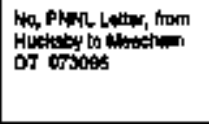 & 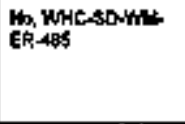 & 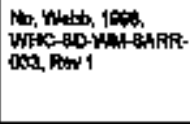 \\
\hline $241+40.20$ & 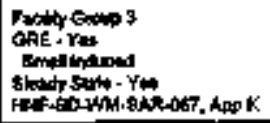 & 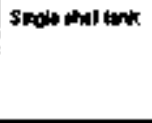 & 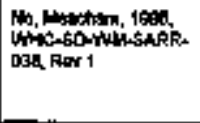 & 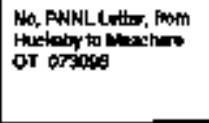 & Wh, WHC-SD-Wht & 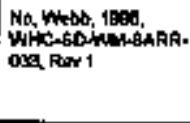 \\
\hline $2 \times 1 \mathrm{~A}$ & 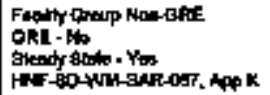 & tefft & 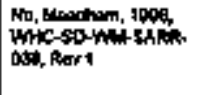 & 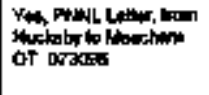 & 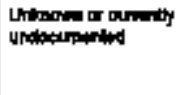 & Whoun or oprowity \\
\hline $240 x$ & 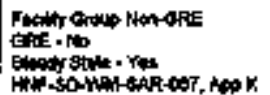 & DeRT & 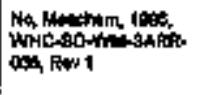 & 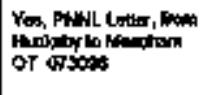 & 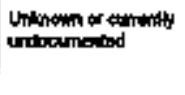 & 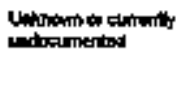 \\
\hline
\end{tabular}




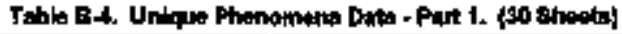

\begin{tabular}{|c|c|c|c|c|c|c|}
\hline Pledilame & fumbot Ges & Type & Paroganil: & 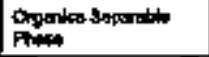 & Toc h uputidx & Tot $n$ solida \\
\hline 24akt & 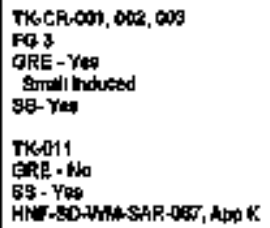 & vall & 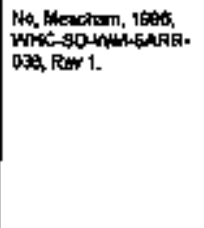 & 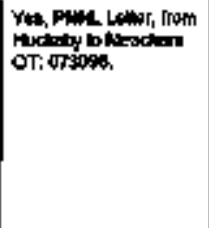 & 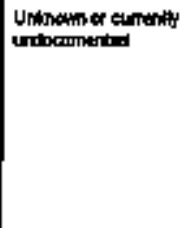 & 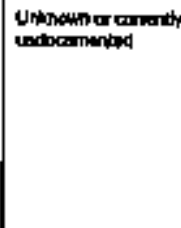 \\
\hline 2449 & 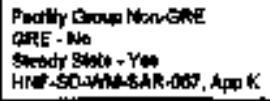 & DCRT & 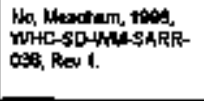 & 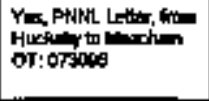 & 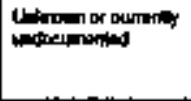 & 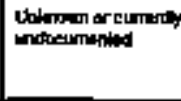 \\
\hline 244TX & 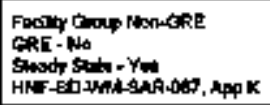 & GCAT & 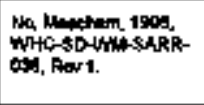 & 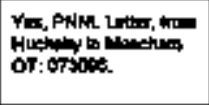 & 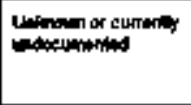 & 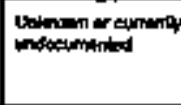 \\
\hline 2446 & 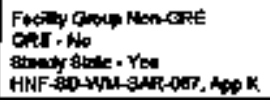 & DCRT & 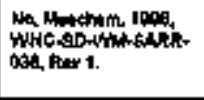 & 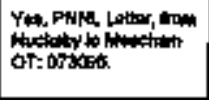 & Nh & wh \\
\hline
\end{tabular}




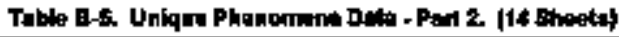

\begin{tabular}{|c|c|c|c|c|c|}
\hline Dוח & Hohn & Pyoptornes & towhing & coldesanty & Colosing \\
\hline $241-4+101$ & 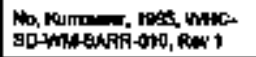 & 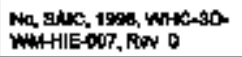 & 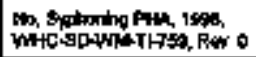 & 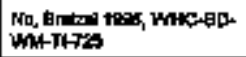 & 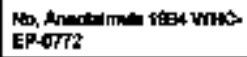 \\
\hline 2A1A-102 & 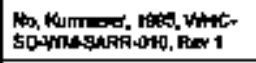 & 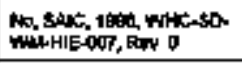 & 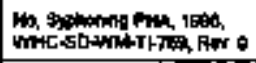 & 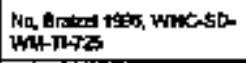 & 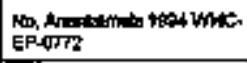 \\
\hline $241 / 4+109$ & 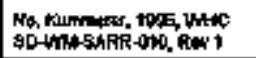 & 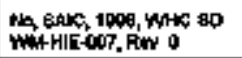 & 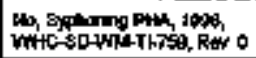 & 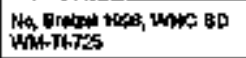 & 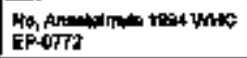 \\
\hline $241+$-104 & 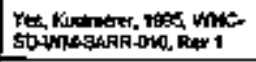 & 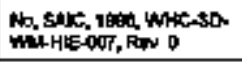 & 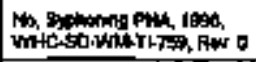 & 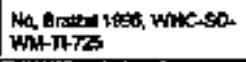 & 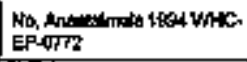 \\
\hline 24A-195 & 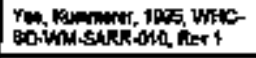 & 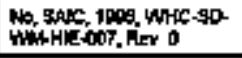 & 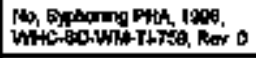 & 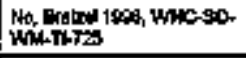 & 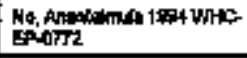 \\
\hline $2+1-k-100$ & 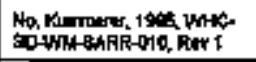 & 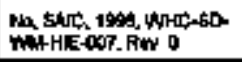 & 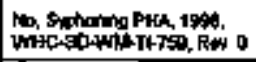 & 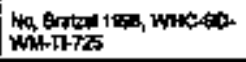 & 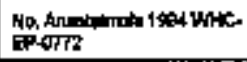 \\
\hline $261+2101$ & 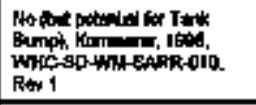 & 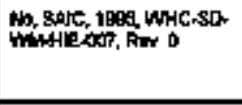 & 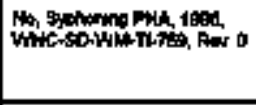 & 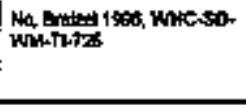 & 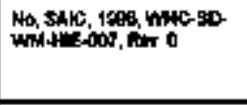 \\
\hline $241-404-102$ & 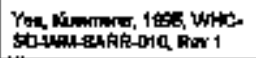 & 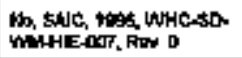 & 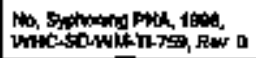 & 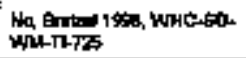 & 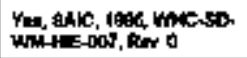 \\
\hline 241-AN-10 & 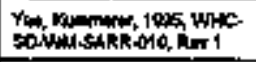 & 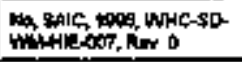 & 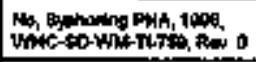 & 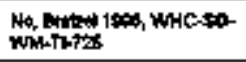 & 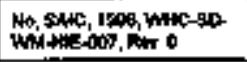 \\
\hline $241-4 N-104$ & 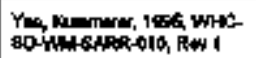 & 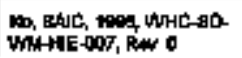 & 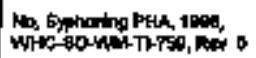 & 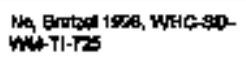 & 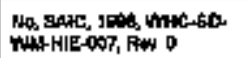 \\
\hline $211+4+106$ & 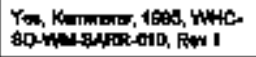 & 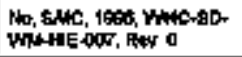 & 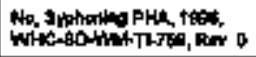 & 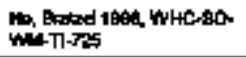 & 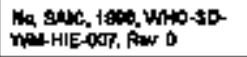 \\
\hline $241-4+109$ & 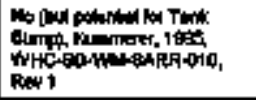 & 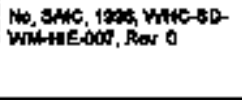 & 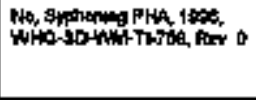 & 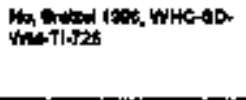 & 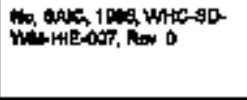 \\
\hline 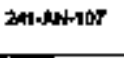 & 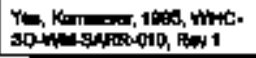 & 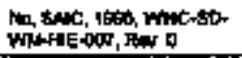 & 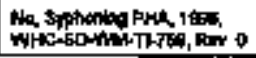 & 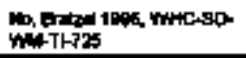 & 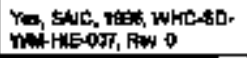 \\
\hline
\end{tabular}




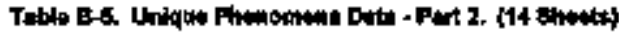

\begin{tabular}{|c|c|c|c|c|c|}
\hline 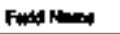 & Hygh Ham & 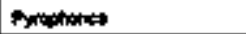 & Fropentes & 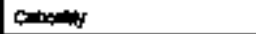 & Corrocentr \\
\hline $24 t+4 P-101$ & 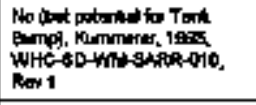 & 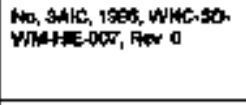 & 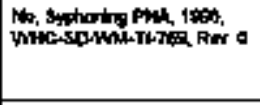 & 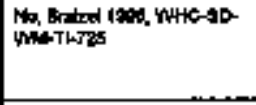 & 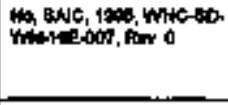 \\
\hline $241-1 P=102$ & 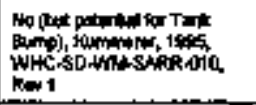 & 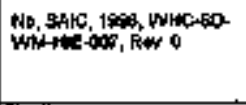 & 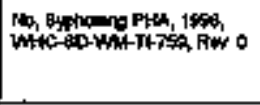 & 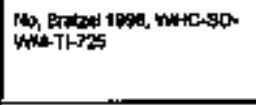 & 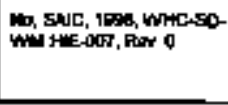 \\
\hline 2-11-AP-103 & 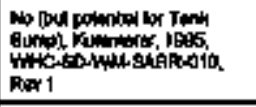 & 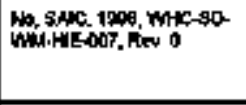 & What Shonwe PHA, 1909 , & 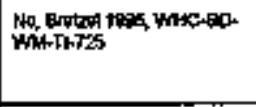 & 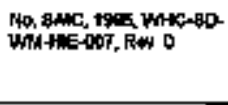 \\
\hline 241-40-104 & 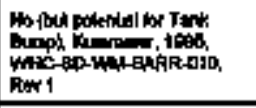 & 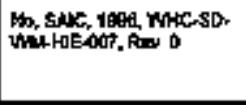 & 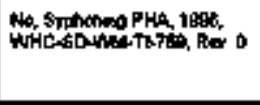 & 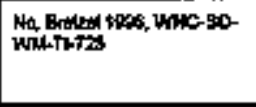 & 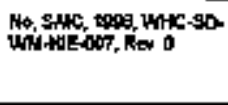 \\
\hline $241-4 \times-105$ & 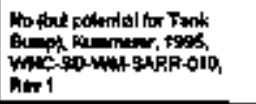 & 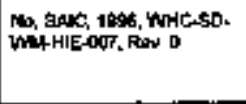 & 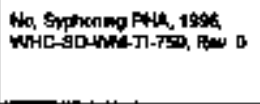 & 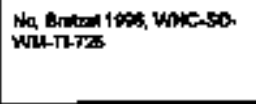 & 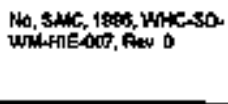 \\
\hline 2614P.100 & 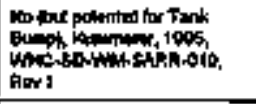 & 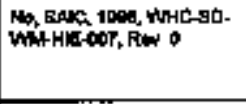 & 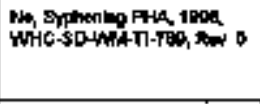 & 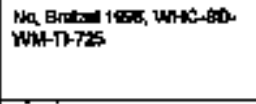 & 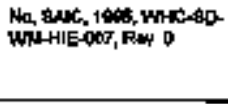 \\
\hline $2 \$ 1-A P-107$ & 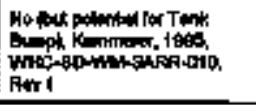 & 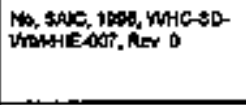 & 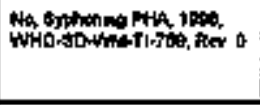 & 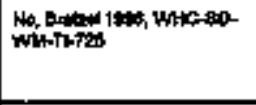 & 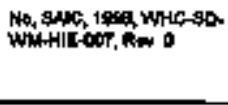 \\
\hline 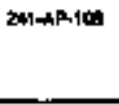 & 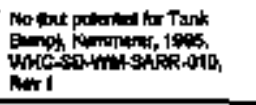 & 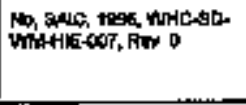 & 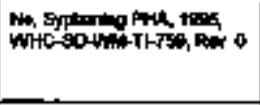 & 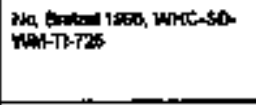 & 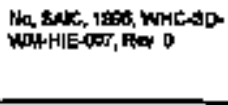 \\
\hline $241-4 w-101$ & 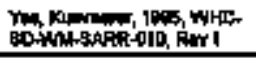 & 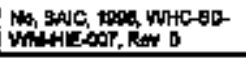 & 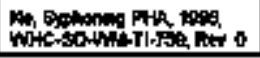 & 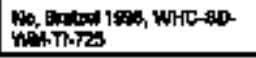 & 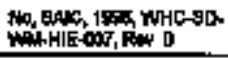 \\
\hline
\end{tabular}




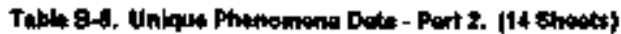

\begin{tabular}{|c|c|c|c|c|c|}
\hline Faly Pat & بهش| & Aproptanes & stionsto & Combing & costoming \\
\hline $2 \times 1-A M-102$ & 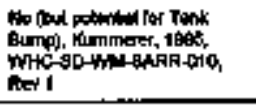 & 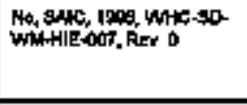 & 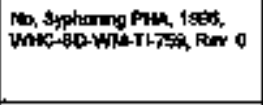 & 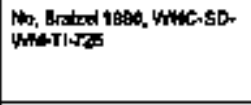 & 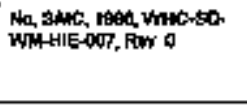 \\
\hline 241 anw-108 & 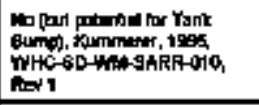 & 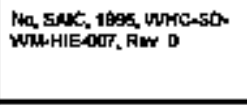 & 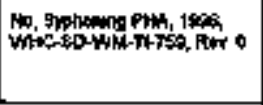 & 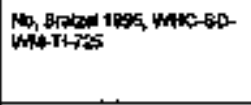 & 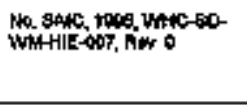 \\
\hline 201 4hW-154 & 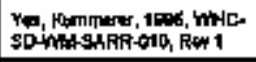 & 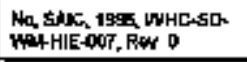 & 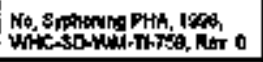 & 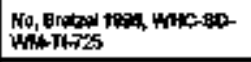 & 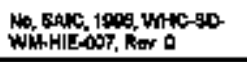 \\
\hline $2 \times 1 A W \cdot 108$ & 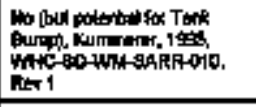 & 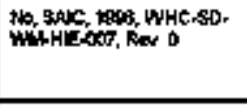 & 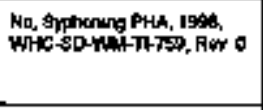 & 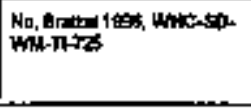 & 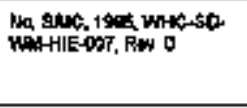 \\
\hline 241 AW. Het & 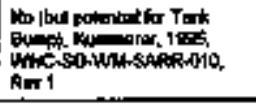 & 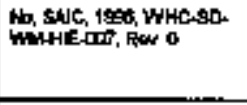 & 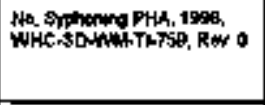 & 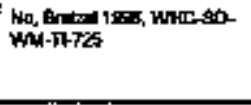 & 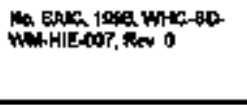 \\
\hline 241-AX-101 & 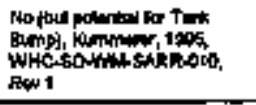 & 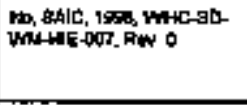 & 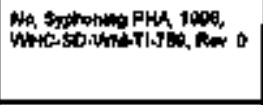 & 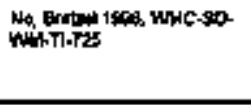 & 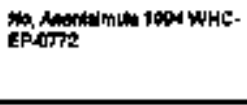 \\
\hline $241-k x-102$ & 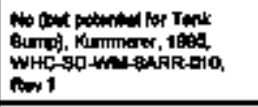 & 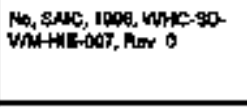 & 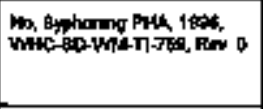 & 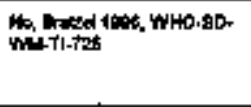 & 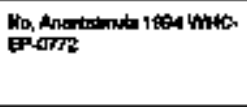 \\
\hline $2+14 \times 20109$ & 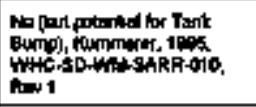 & 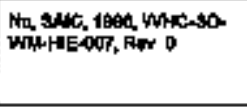 & 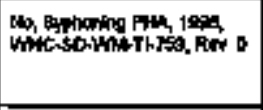 & 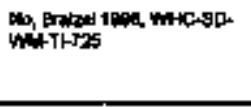 & 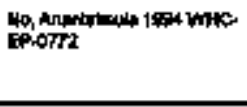 \\
\hline 26$)+(x-104$ & 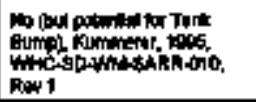 & $\begin{array}{l}\text { Na, Gak, } 100, \text { WHC-SO } \\
\text { Wh, }\end{array}$ & 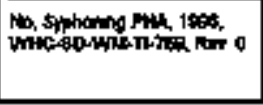 & 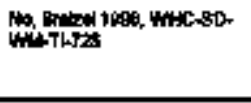 & 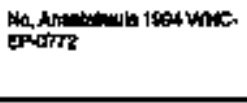 \\
\hline
\end{tabular}




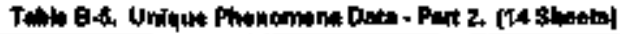

\begin{tabular}{|c|c|c|c|c|c|}
\hline Fand Nom & Hgh Hast & Ayphate & Eypony & Cintey & Comayoty \\
\hline $241+Y-101$ & 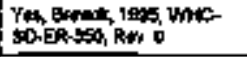 & 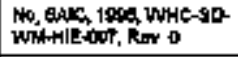 & 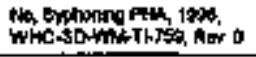 & 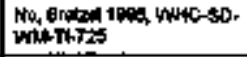 & 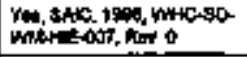 \\
\hline 241-AY-160 & 货 & 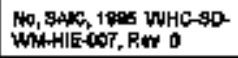 & 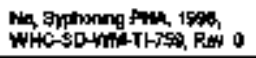 & 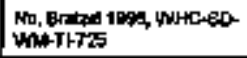 & 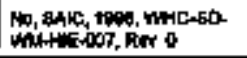 \\
\hline $2+1-2+2-21$ & 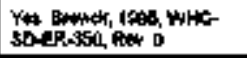 & 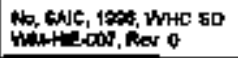 & 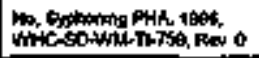 & 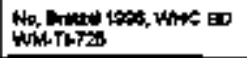 & 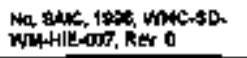 \\
\hline 211-A2-102 & 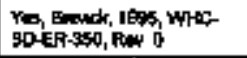 & 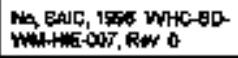 & 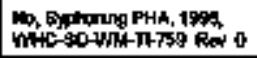 & 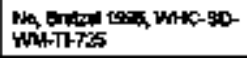 & 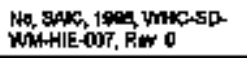 \\
\hline 241-8101 & 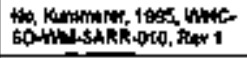 & 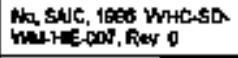 & 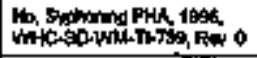 & 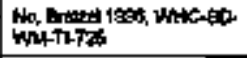 & 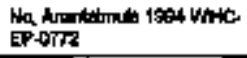 \\
\hline 24t-9-102 & 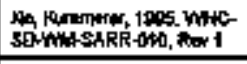 & 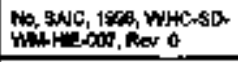 & 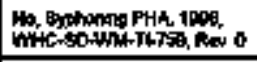 & 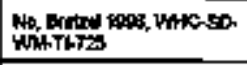 & 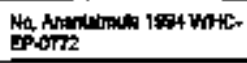 \\
\hline 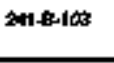 & 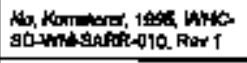 & 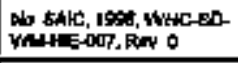 & 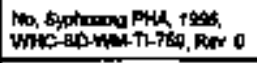 & 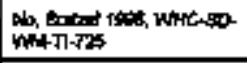 & 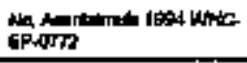 \\
\hline $2 n+504$ & 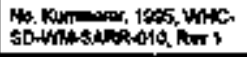 & 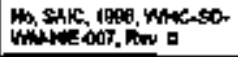 & 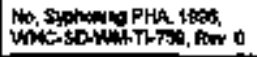 & 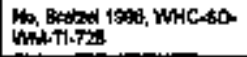 & 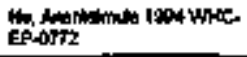 \\
\hline 241-8-145 & 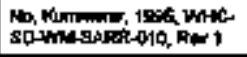 & 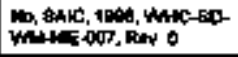 & 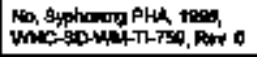 & 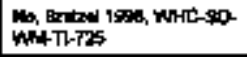 & 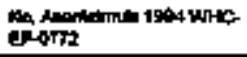 \\
\hline $241+40-405$ & 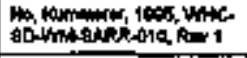 & 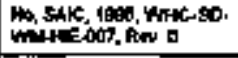 & 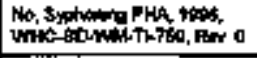 & 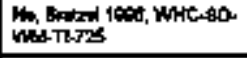 & 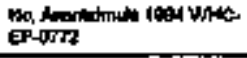 \\
\hline $2+18-107$ & 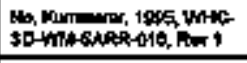 & 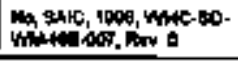 & 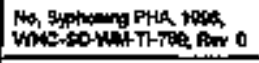 & 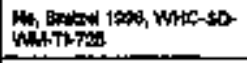 & 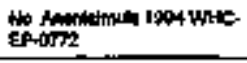 \\
\hline 241-8-106 & 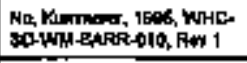 & 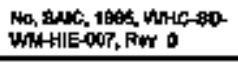 & 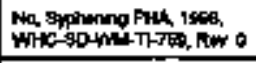 & 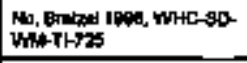 & 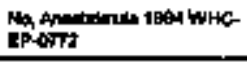 \\
\hline $241+806$ & 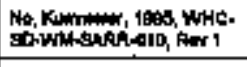 & 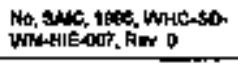 & 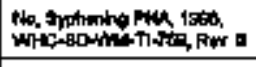 & 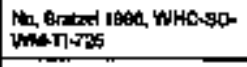 & 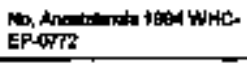 \\
\hline $241-8-16$ & 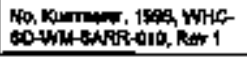 & $\begin{array}{l}\text { No, sAC, } 190, \text { who-so- } \\
\text { WhH }\end{array}$ & A & 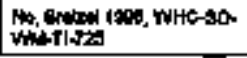 & 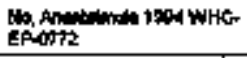 \\
\hline
\end{tabular}




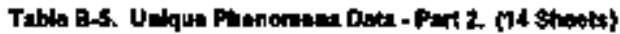

\begin{tabular}{|c|c|c|c|c|c|}
\hline 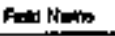 & Hyph Heal & Pypqtonce & typhaman & CInchy & Contoenty \\
\hline 241-8-111 & 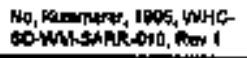 & 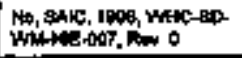 & 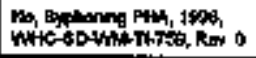 & 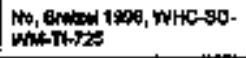 & 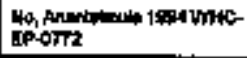 \\
\hline $241+8112$ & 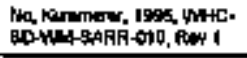 & 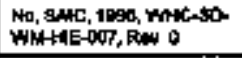 & 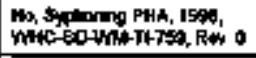 & 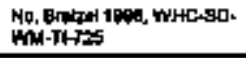 & 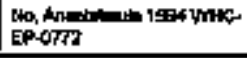 \\
\hline 2440201 & 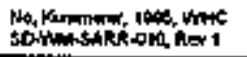 & 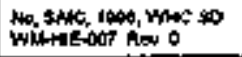 & 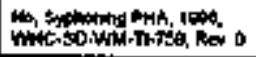 & 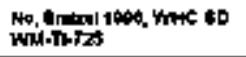 & 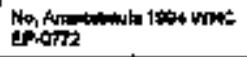 \\
\hline 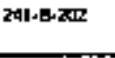 & 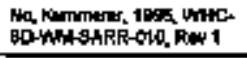 & 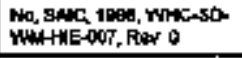 & 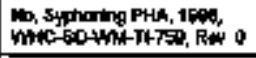 & 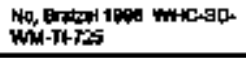 & 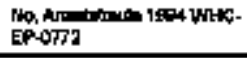 \\
\hline $241+B 205$ & 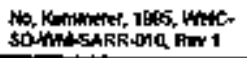 & 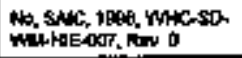 & 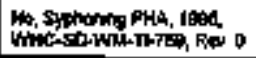 & 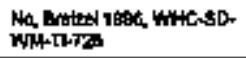 & 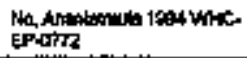 \\
\hline 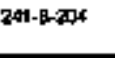 & 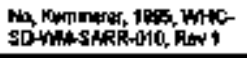 & 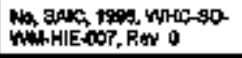 & 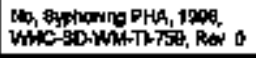 & 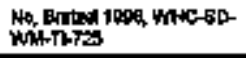 & 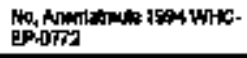 \\
\hline $24-4 x-41$ & 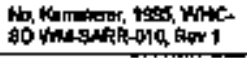 & 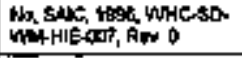 & 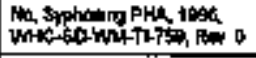 & 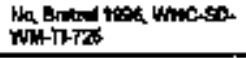 & 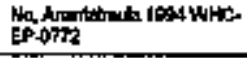 \\
\hline $261-6 x-102$ & 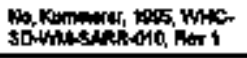 & 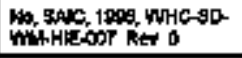 & 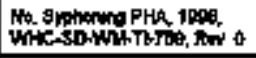 & $\begin{array}{l}\text { Ho, Brotal } 1928, \text { wacso } \\
\text { WH-Th }\end{array}$ & 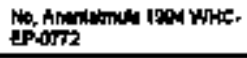 \\
\hline 2450 & 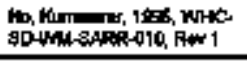 & 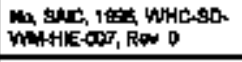 & 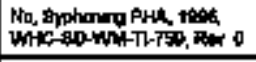 & 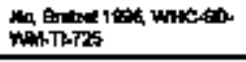 & 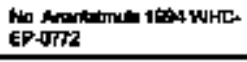 \\
\hline $241-10 \times 104$ & 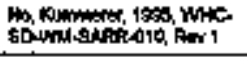 & 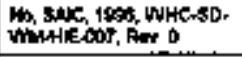 & 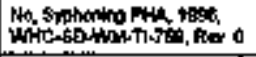 & 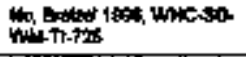 & 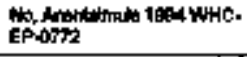 \\
\hline $2 \times 1-8 x-105$ & 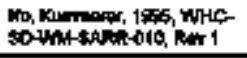 & 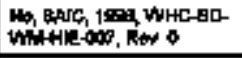 & 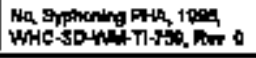 & 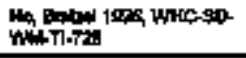 & 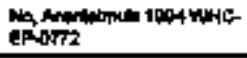 \\
\hline $241+0 x-100$ & 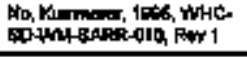 & 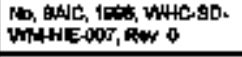 & 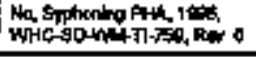 & 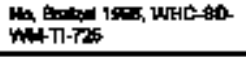 & 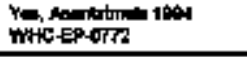 \\
\hline $241-0 k-107$ & 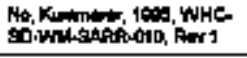 & 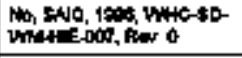 & 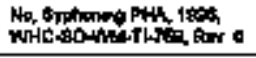 & 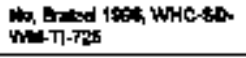 & 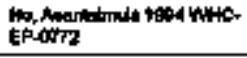 \\
\hline $241-8 x-169$ & 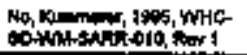 & 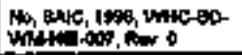 & 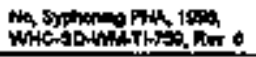 & 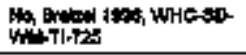 & 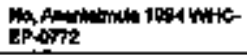 \\
\hline
\end{tabular}




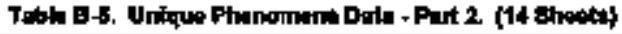

\begin{tabular}{|c|c|c|c|c|c|}
\hline Patd leme & Haph 1hat & 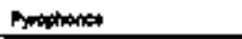 & Byomoris & Gotictiny & Aanoskaty \\
\hline $2+19 x-1010$ & 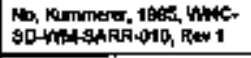 & 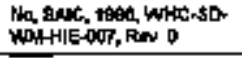 & 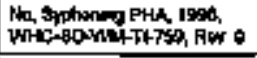 & 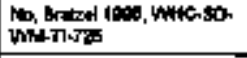 & 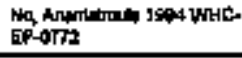 \\
\hline $241+4 x-110$ & 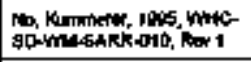 & 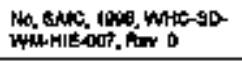 & 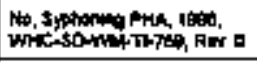 & 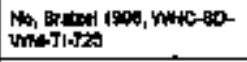 & 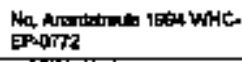 \\
\hline $2+1+9 x-111$ & 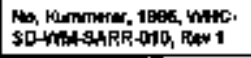 & 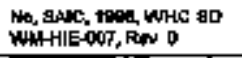 & 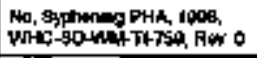 & 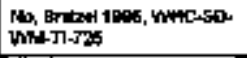 & 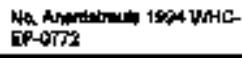 \\
\hline $2 n+4 x_{112}$ & 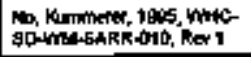 & 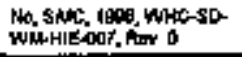 & 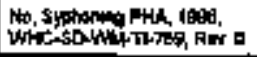 & 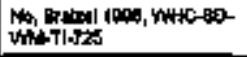 & 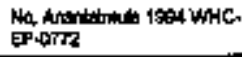 \\
\hline $241+8 Y-101$ & 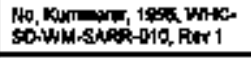 & 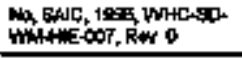 & 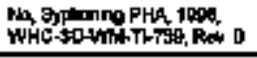 & 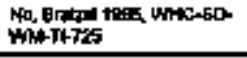 & 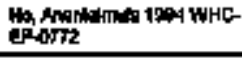 \\
\hline $241+4 Y=10 z$ & 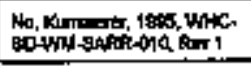 & 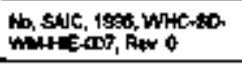 & 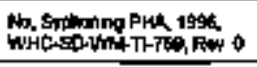 & 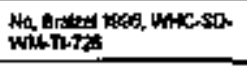 & 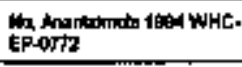 \\
\hline 2A1-BY.103 & 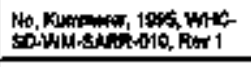 & 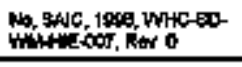 & 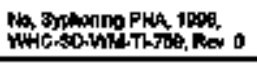 & 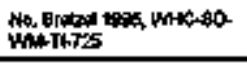 & 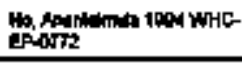 \\
\hline $241+4 r y-104$ & 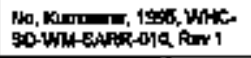 & 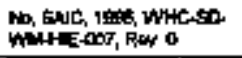 & Wh, Sming PHA 19.4 & 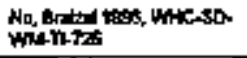 & 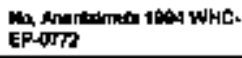 \\
\hline $2 \times 1+8 y-405$ & 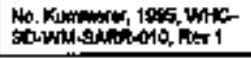 & 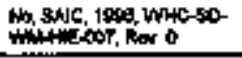 & 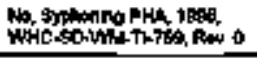 & 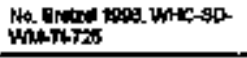 & 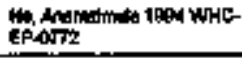 \\
\hline $241+47-109$ & 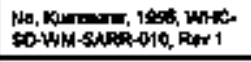 & 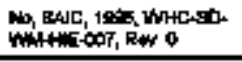 & 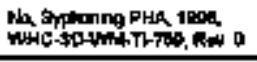 & 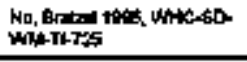 & 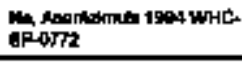 \\
\hline $2+1 \mathrm{Br}-\mathrm{W} n$ & Mo, Kaner, 1000, WHC- & 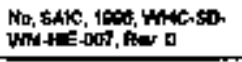 & 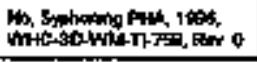 & 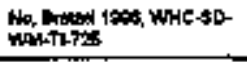 & 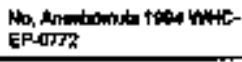 \\
\hline $2+1-5 x-20$ & 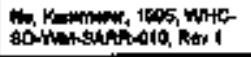 & 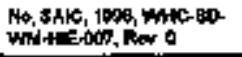 & 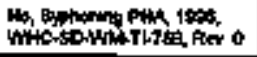 & 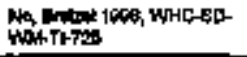 & 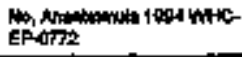 \\
\hline $2+164-100$ & 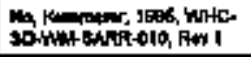 & 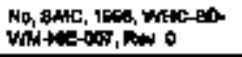 & 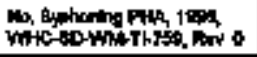 & 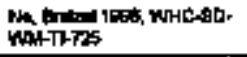 & 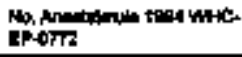 \\
\hline $2164-110$ & 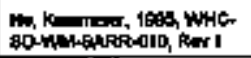 & 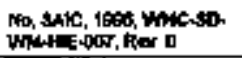 & 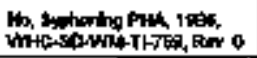 & 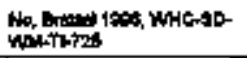 & 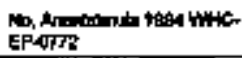 \\
\hline
\end{tabular}




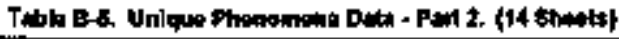

\begin{tabular}{|c|c|c|c|c|c|}
\hline 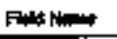 & Hhigh Hont & Propothent & stophing & Cominesty & cencouting \\
\hline 241 . WY.111 & 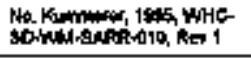 & 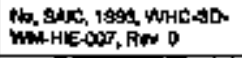 & 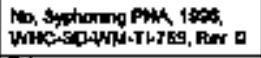 & 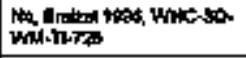 & 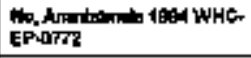 \\
\hline $241-B Y-112$ & 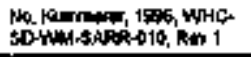 & 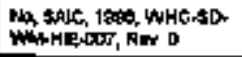 & 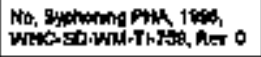 & 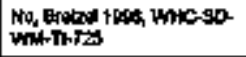 & 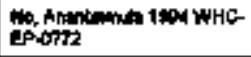 \\
\hline 241 C 10 어 & 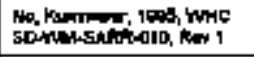 & 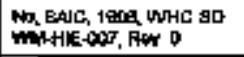 & 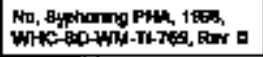 & $\begin{array}{l}\text { Nh, Brated 199, Who so } \\
\text { Wh-n-TS }\end{array}$ & 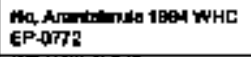 \\
\hline $2 d 1-C-\infty 02$ & 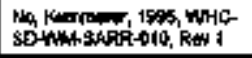 & 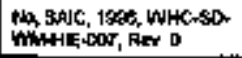 & 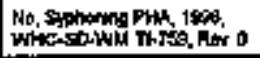 & 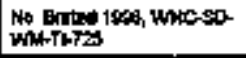 & 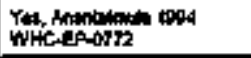 \\
\hline $249-103$ & 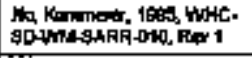 & 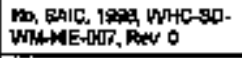 & 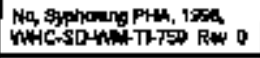 & 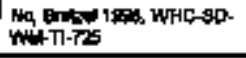 & 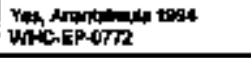 \\
\hline $2 \pi \mid-104$ & 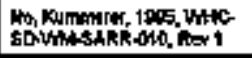 & 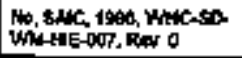 & 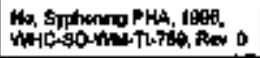 & 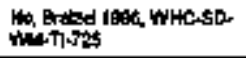 & 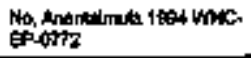 \\
\hline $241-c-145$ & 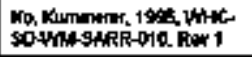 & 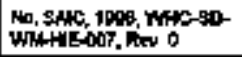 & 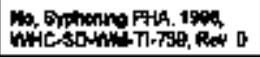 & 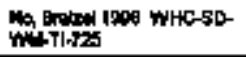 & 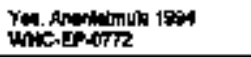 \\
\hline 241 10\% & 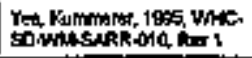 & 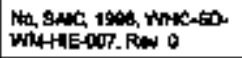 & 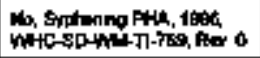 & 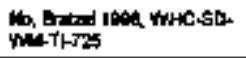 & 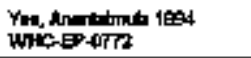 \\
\hline $241-c-107$ & 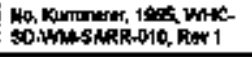 & 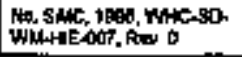 & 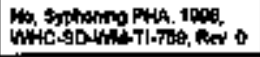 & 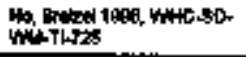 & 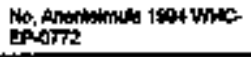 \\
\hline 2414 & 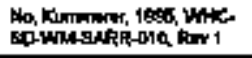 & 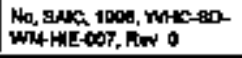 & 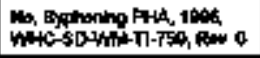 & 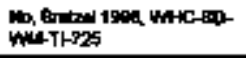 & 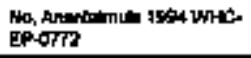 \\
\hline $241-c-100$ & 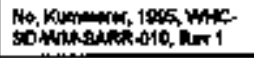 & 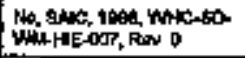 & 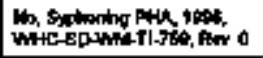 & 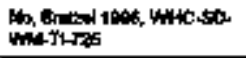 & 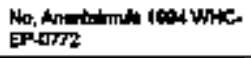 \\
\hline $241-C-110$ & 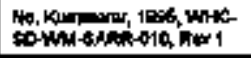 & Nac, nos, whe-so & 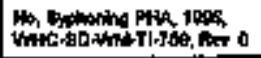 & 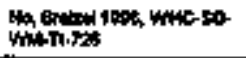 & 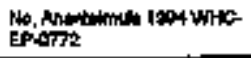 \\
\hline 241-C-111 & 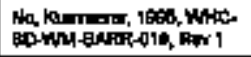 & 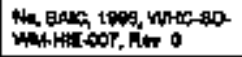 & 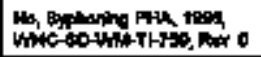 & 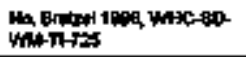 & 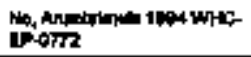 \\
\hline 3all-c-112 & 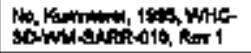 & 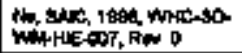 & 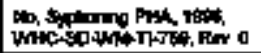 & 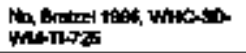 & 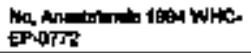 \\
\hline
\end{tabular}




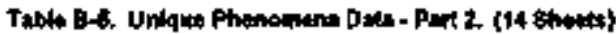

\begin{tabular}{|c|c|c|c|c|c|}
\hline Fivilen & Hoght & Proptonce & spptorng & 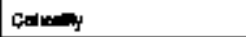 & Donouritr \\
\hline $2,11-6-301$ & 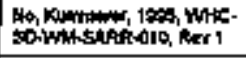 & 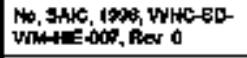 & 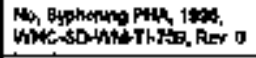 & 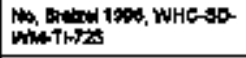 & 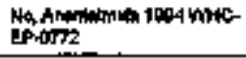 \\
\hline $241-c-208$ & 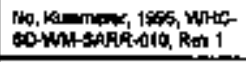 & 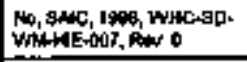 & 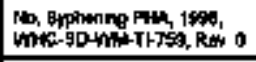 & 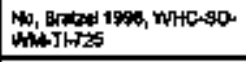 & 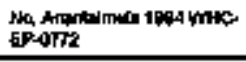 \\
\hline 2010300 & 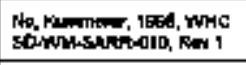 & 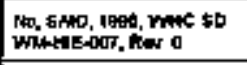 & 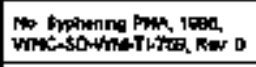 & 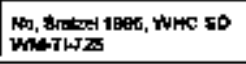 & 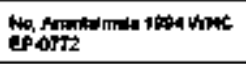 \\
\hline $2+1-6-304$ & 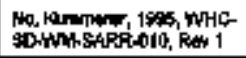 & 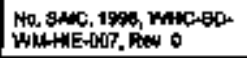 & 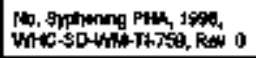 & 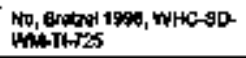 & 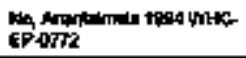 \\
\hline 261-86-30 & 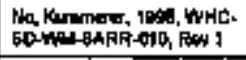 & 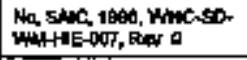 & 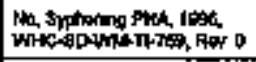 & 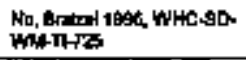 & 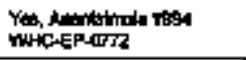 \\
\hline $261-100$ & 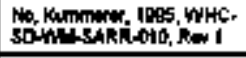 & 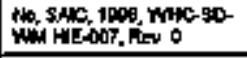 & 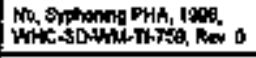 & 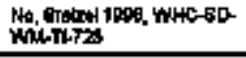 & 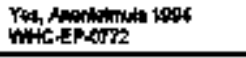 \\
\hline $241+100$ & 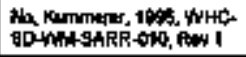 & 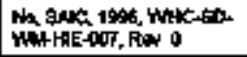 & 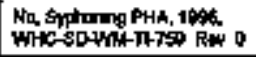 & 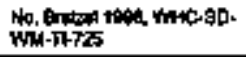 & 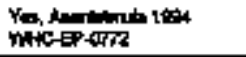 \\
\hline $241-5-104$ & 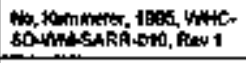 & 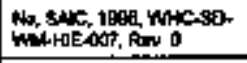 & 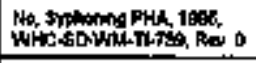 & 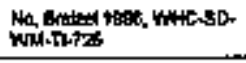 & 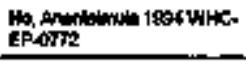 \\
\hline $241-8-105$ & 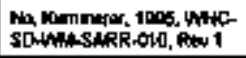 & 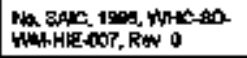 & 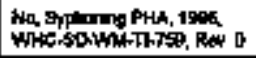 & 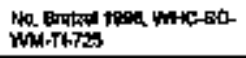 & 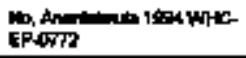 \\
\hline $241-5-100$ & 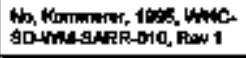 & 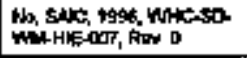 & 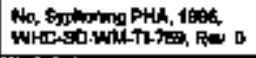 & 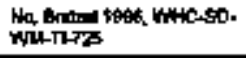 & 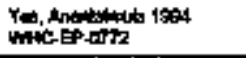 \\
\hline $241-8-107$ & 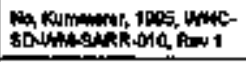 & 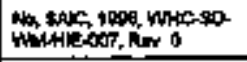 & 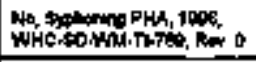 & 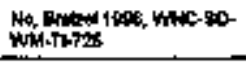 & 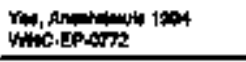 \\
\hline $341-8-106$ & 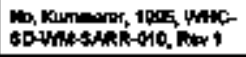 & 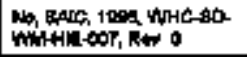 & 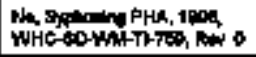 & 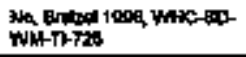 & 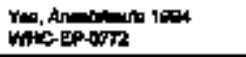 \\
\hline 2स1-क्षिक & 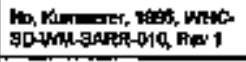 & 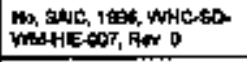 & 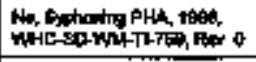 & 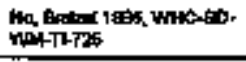 & 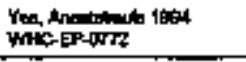 \\
\hline $2+1+110$ & 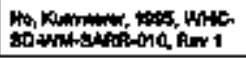 & 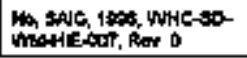 & 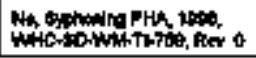 & 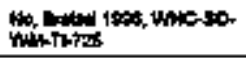 & 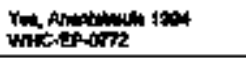 \\
\hline
\end{tabular}




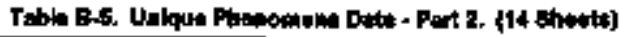

\begin{tabular}{|c|c|c|c|c|c|}
\hline Fed Nore & Hot & Prophenos & siptroinges & athonity & Compinting \\
\hline $241-s-114$ & 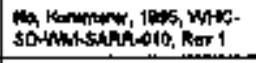 & 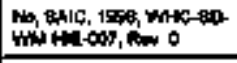 & 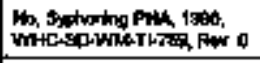 & 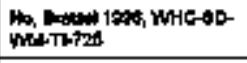 & 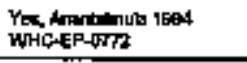 \\
\hline 241 \& 112 & 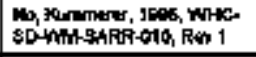 & 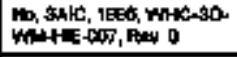 & 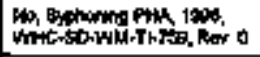 & 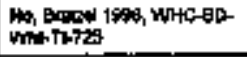 & 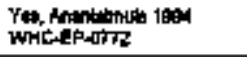 \\
\hline $2415 \times 101$ & 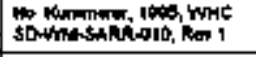 & mots & 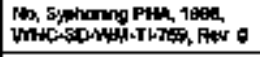 & 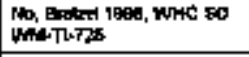 & 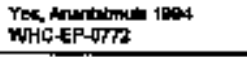 \\
\hline $241-5)-102$ & 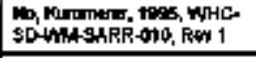 & 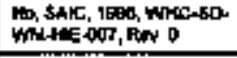 & 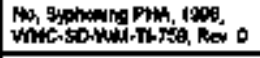 & 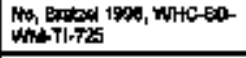 & 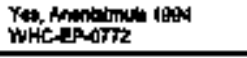 \\
\hline $241-516-100$ & 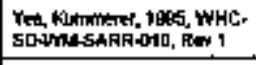 & 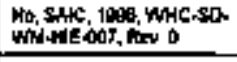 & 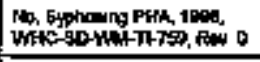 & 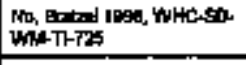 & 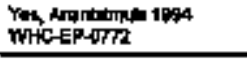 \\
\hline $211-8 x-104$ & 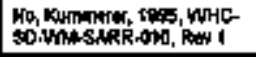 & 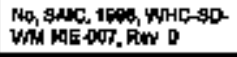 & 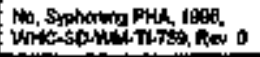 & 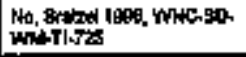 & 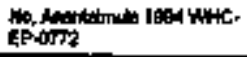 \\
\hline $241-5 x+100$ & 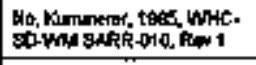 & 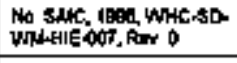 & 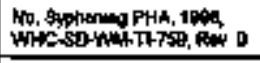 & 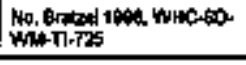 & 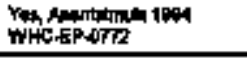 \\
\hline $241-35-100$ & 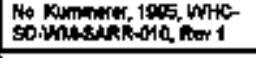 & 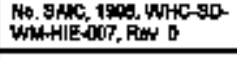 & 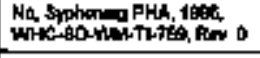 & 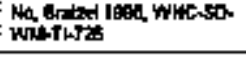 & 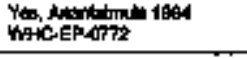 \\
\hline $2414 x-100$ & 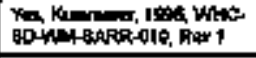 & 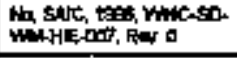 & 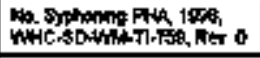 & 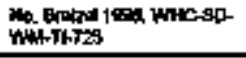 & 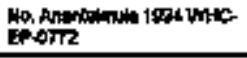 \\
\hline $241+5 x_{6} 108$ & 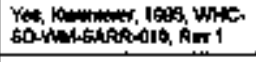 & 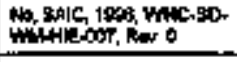 & 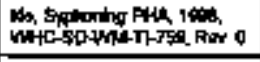 & 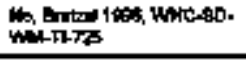 & 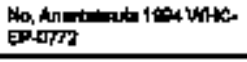 \\
\hline $2-11-E x-100$ & 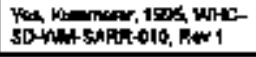 & 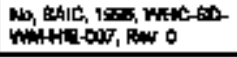 & 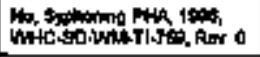 & 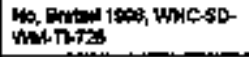 & 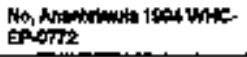 \\
\hline $291-60110$ & 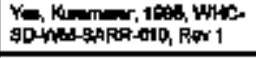 & 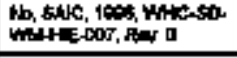 & 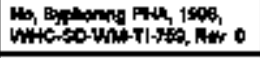 & 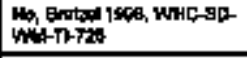 & 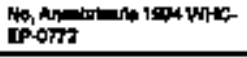 \\
\hline $241-86111$ & 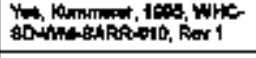 & 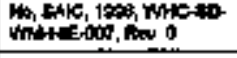 & 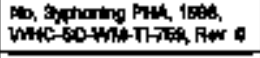 & 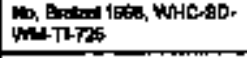 & 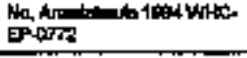 \\
\hline 241-8X-112 & 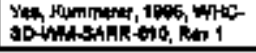 & 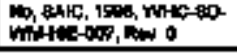 & 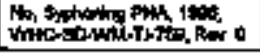 & 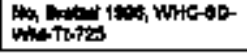 & 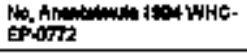 \\
\hline
\end{tabular}




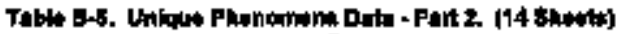

\begin{tabular}{|c|c|c|c|c|c|}
\hline Fald dim & Hothent & Proplanes & Esphoring & 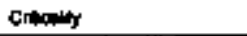 & correasity \\
\hline $211-516-113$ & 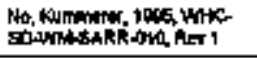 & 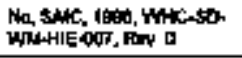 & 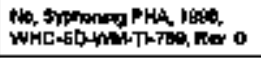 & 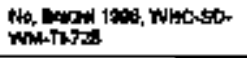 & 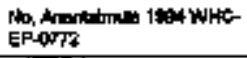 \\
\hline $24-3 \%-114$ & 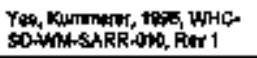 & 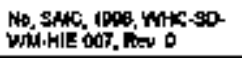 & 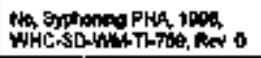 & 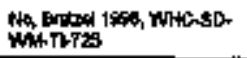 & 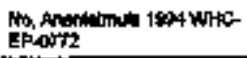 \\
\hline 241-8x-116 & 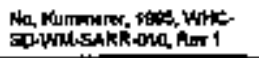 & 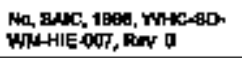 & 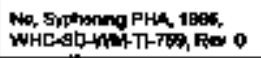 & 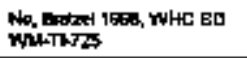 & 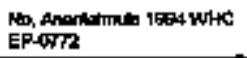 \\
\hline $2+1-5 \%+101$ & 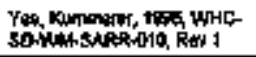 & 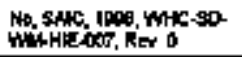 & 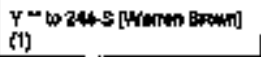 & 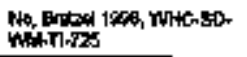 & 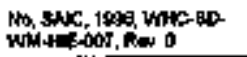 \\
\hline $241.9 Y-19$ & 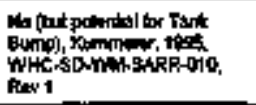 & 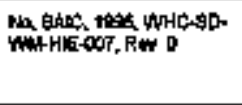 & 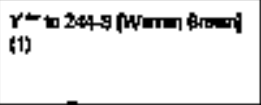 & 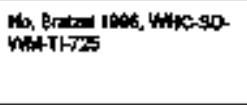 & 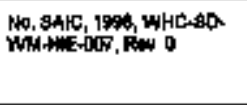 \\
\hline $241-5 \gamma-100$ & 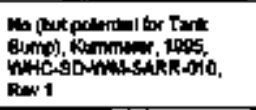 & 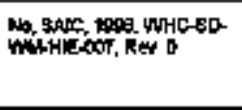 & 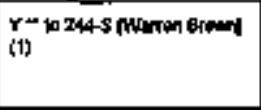 & 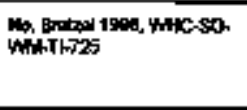 & 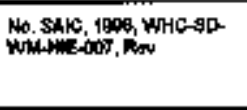 \\
\hline $241-T-101$ & 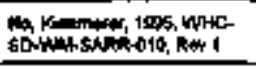 & 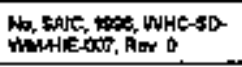 & 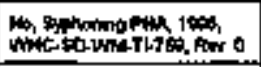 & 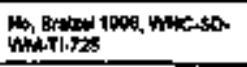 & 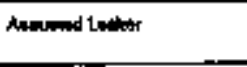 \\
\hline $241+T-102$ & 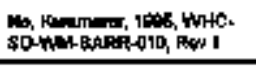 & 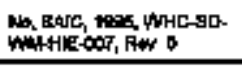 & 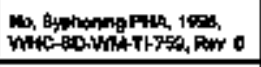 & 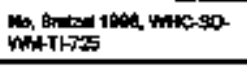 & 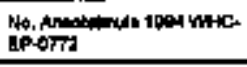 \\
\hline 2A1-T-10 & 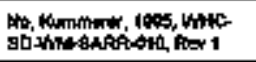 & 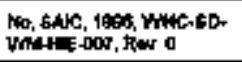 & 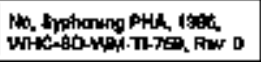 & 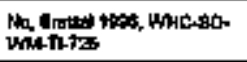 & 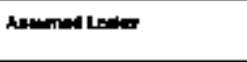 \\
\hline 241-T-104 & 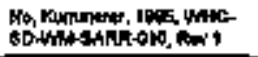 & 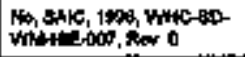 & 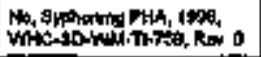 & 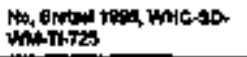 & 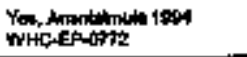 \\
\hline $241 \cdot T \cdot 9 \mid 53$ & 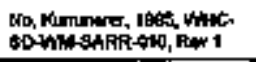 & 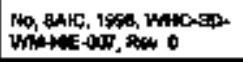 & 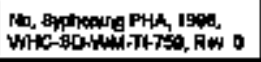 & 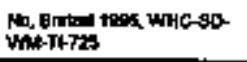 & 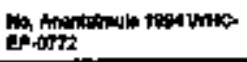 \\
\hline $341 .+\cdot 116$ & No, Nammare, ins, hick & 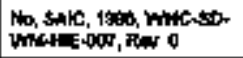 & 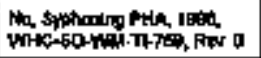 & 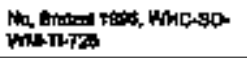 & 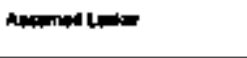 \\
\hline $241-T .197$ & 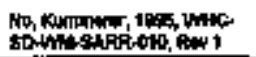 & 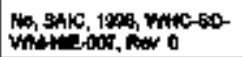 & 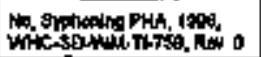 & 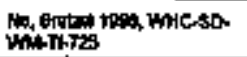 & 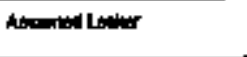 \\
\hline
\end{tabular}




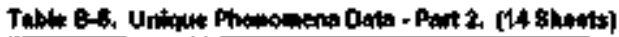

\begin{tabular}{|c|c|c|c|c|c|}
\hline Fued Kam & Hght He= & Proptorkax & appherny & Crententy & Cogroentif \\
\hline 241-T415: & 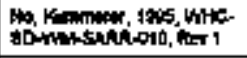 & 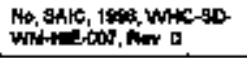 & 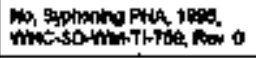 & 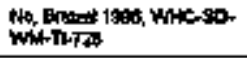 & Andurnow \\
\hline $241, T-108$ & 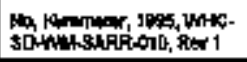 & 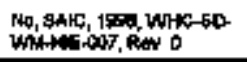 & 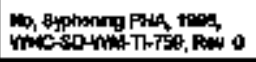 & 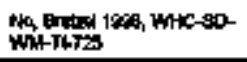 & 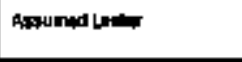 \\
\hline 2411-T-110 & 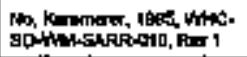 & 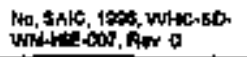 & 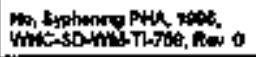 & 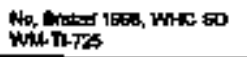 & 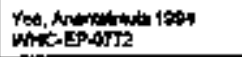 \\
\hline 201-T-111 & 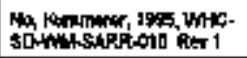 & 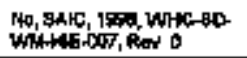 & 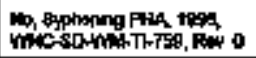 & 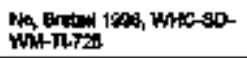 & 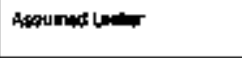 \\
\hline 241.T.112 & 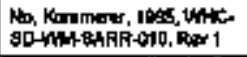 & 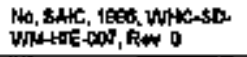 & 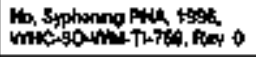 & 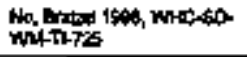 & 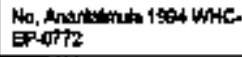 \\
\hline 241-T-2001 & 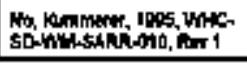 & 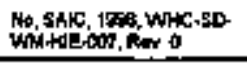 & 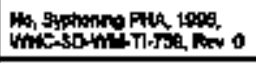 & 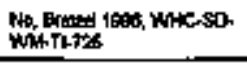 & 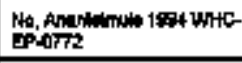 \\
\hline $241 . T-202$ & 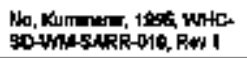 & 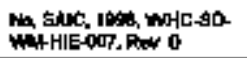 & 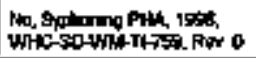 & 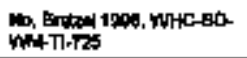 & 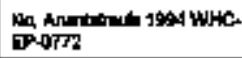 \\
\hline $241 \cdot T-200$ & 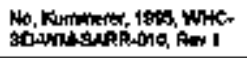 & 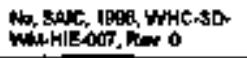 & 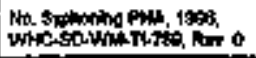 & 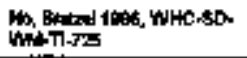 & 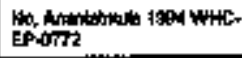 \\
\hline $241 \cdot T-804$ & 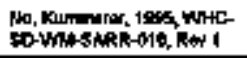 & 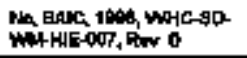 & 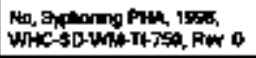 & 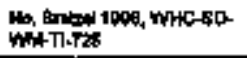 & 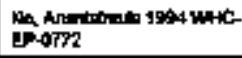 \\
\hline $241.75,-401$ & 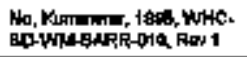 & 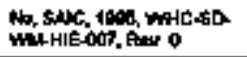 & 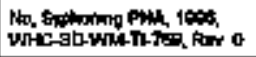 & 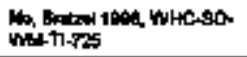 & 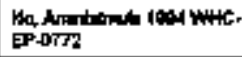 \\
\hline 241-TX-102 & 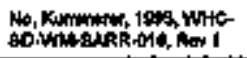 & 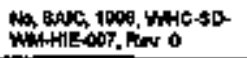 & 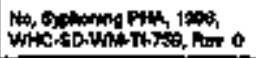 & 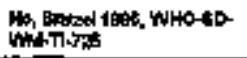 & 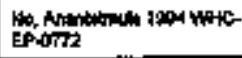 \\
\hline 241-T2-1035 & 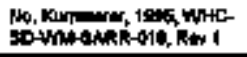 & 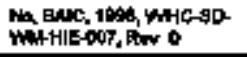 & 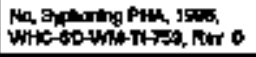 & 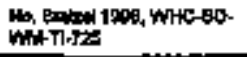 & 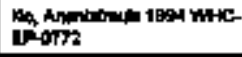 \\
\hline $241-76104$ & 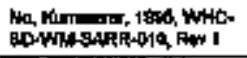 & 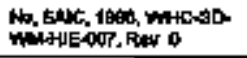 & 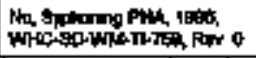 & 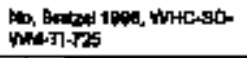 & 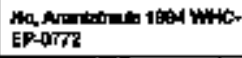 \\
\hline 241-TX-106 & 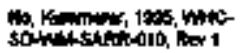 & $m_{1}$ silc, & 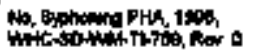 & 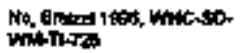 & 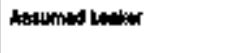 \\
\hline
\end{tabular}




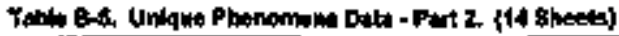

\begin{tabular}{|c|c|c|c|c|c|}
\hline Flatd VIento & Honhant & Pyosphonga & syppente & Crbcraly & Cormenty \\
\hline $241-T \times-100$ & 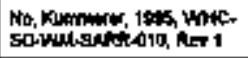 & 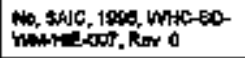 & 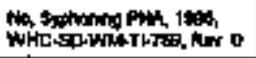 & 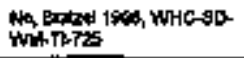 & 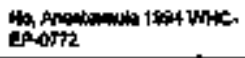 \\
\hline $241-\pi x-10 \%$ & 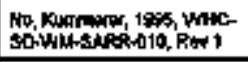 & 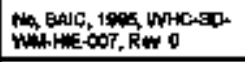 & 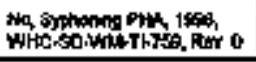 & 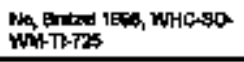 & 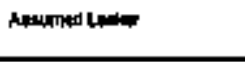 \\
\hline 24-TX-100 & 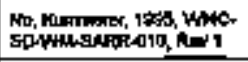 & 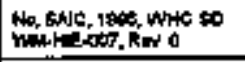 & 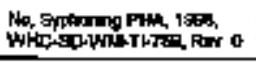 & 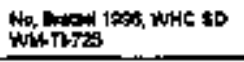 & 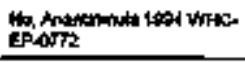 \\
\hline $241-1 \times-100$ & 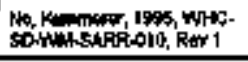 & 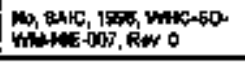 & 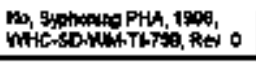 & 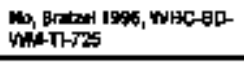 & $\begin{array}{l}\text { No, An } \\
\text { EPणTR }\end{array}$ \\
\hline $241+10-110$ & 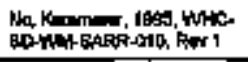 & 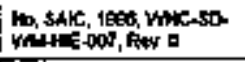 & 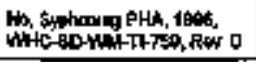 & 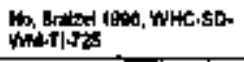 & 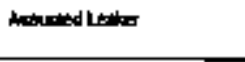 \\
\hline $241+\Gamma-111$ & 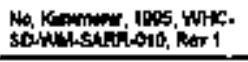 & 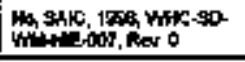 & 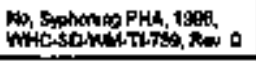 & 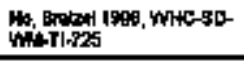 & 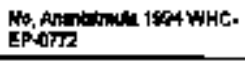 \\
\hline $241-T x-112$ & 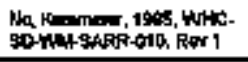 & 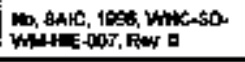 & 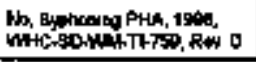 & 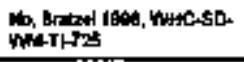 & 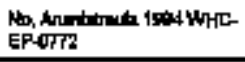 \\
\hline $241 . T<-113$ & 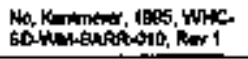 & 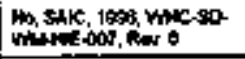 & 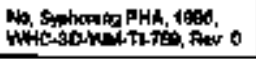 & 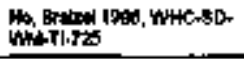 & 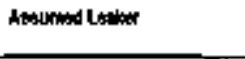 \\
\hline $211-T x-114$ & 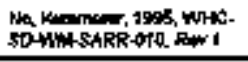 & 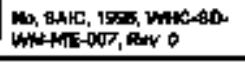 & 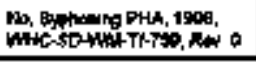 & 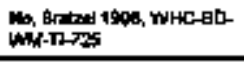 & Hourd Inster \\
\hline 201.TXم118 & 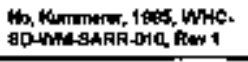 & 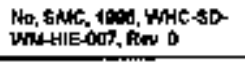 & 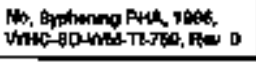 & 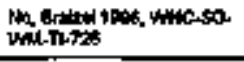 & Mnanted toker \\
\hline $24-7 x-110$ & 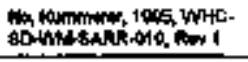 & 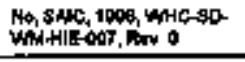 & 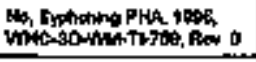 & 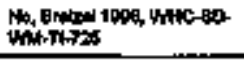 & 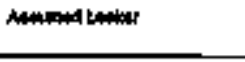 \\
\hline 241-TX-147 & 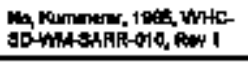 & 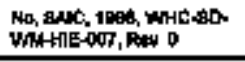 & 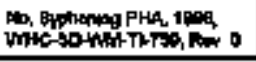 & 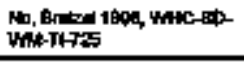 & 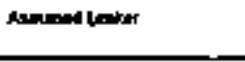 \\
\hline 201-txal110 & 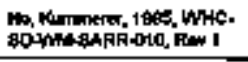 & 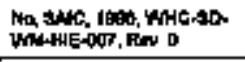 & 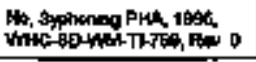 & 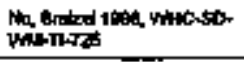 & loor laver \\
\hline 24).TY.101 & 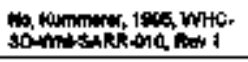 & 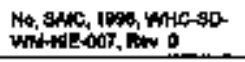 & 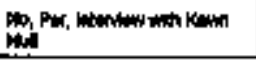 & 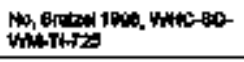 & 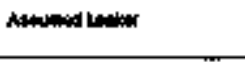 \\
\hline
\end{tabular}




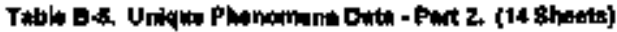

\begin{tabular}{|c|c|c|c|c|c|}
\hline 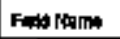 & Hon Heil & Pyropticeson & Apporing & Cinter & Dorpenty \\
\hline $241-T Y \cdot 102$ & 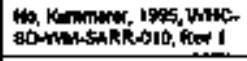 & 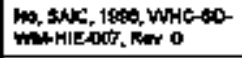 & 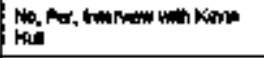 & 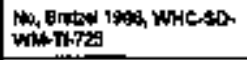 & 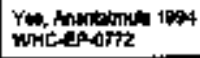 \\
\hline $241 \cdot \pi \gamma-100$ & 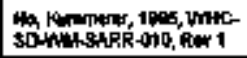 & 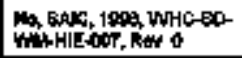 & 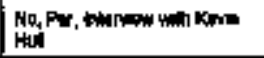 & 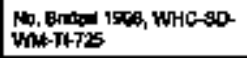 & 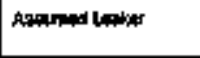 \\
\hline $241 \cdot \Gamma+104$ & 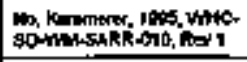 & 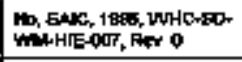 & 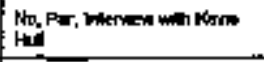 & 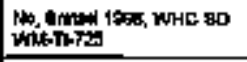 & 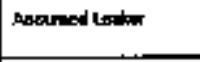 \\
\hline $241-T Y-105$ & 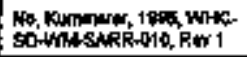 & $\begin{array}{l}\text { Ho, shic, } 196, \text { mec } \\
\text { What }\end{array}$ & 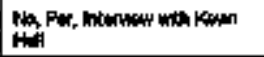 & 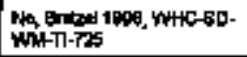 & AlumW \\
\hline $241-T Y-10 \%$ & 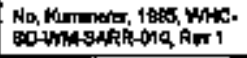 & 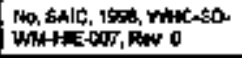 & 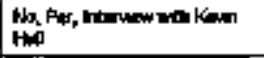 & 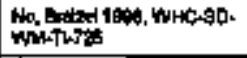 & Maymed Leter \\
\hline 241.4-10b & 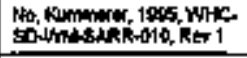 & 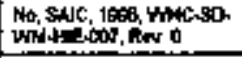 & Wh, Sthondo PHA 189 , & 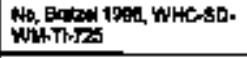 & 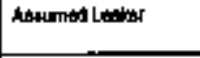 \\
\hline $2+1-4-160$ & 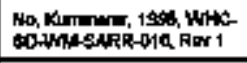 & 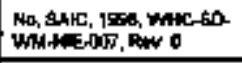 & 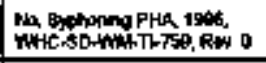 & 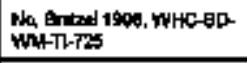 & 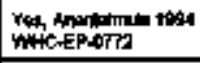 \\
\hline $2411-1 \cdot 100$ & 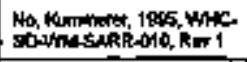 & 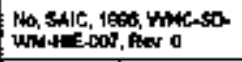 & 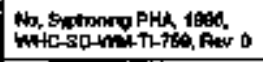 & 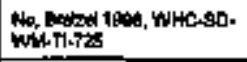 & 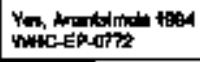 \\
\hline $211-4-104$ & 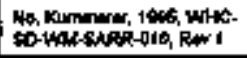 & 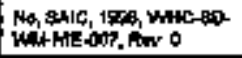 & Wh Papho PHA, 1009 , & 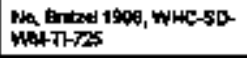 & 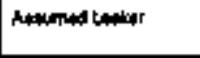 \\
\hline $291-2-106$ & 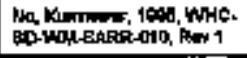 & 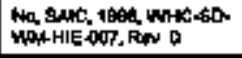 & 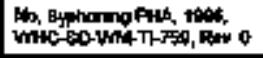 & 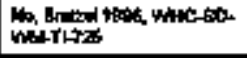 & 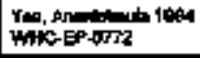 \\
\hline $2+1+404$ & 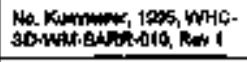 & 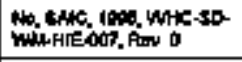 & 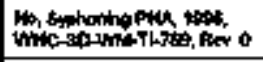 & 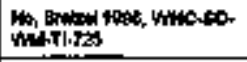 & 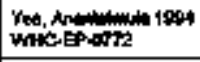 \\
\hline $201+2-108$ & 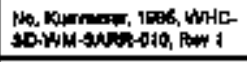 & 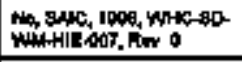 & 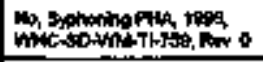 & 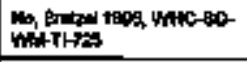 & 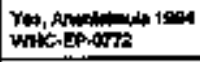 \\
\hline $241+106$ & 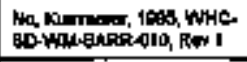 & 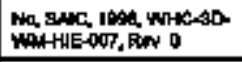 & 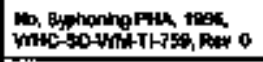 & 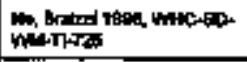 & 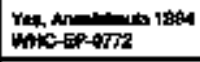 \\
\hline $241+4-106$ & 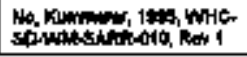 & 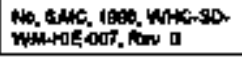 & 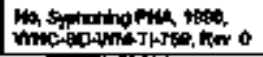 & 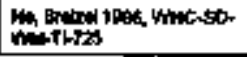 & 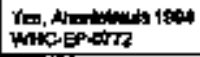 \\
\hline
\end{tabular}




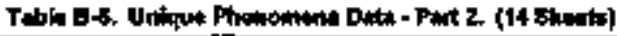

\begin{tabular}{|c|c|c|c|c|c|}
\hline Firid & Huth Hat & Propinones & spptentre & Cincienty & Comaneity \\
\hline 241-L-110 & 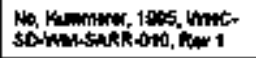 & 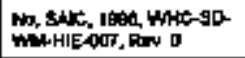 & 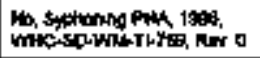 & 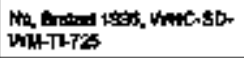 & 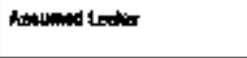 \\
\hline $241.4-111$ & 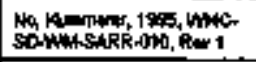 & 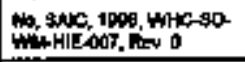 & 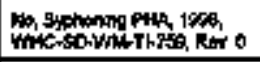 & 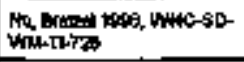 & 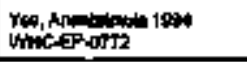 \\
\hline 241.1K112 & 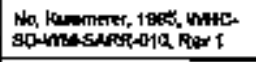 & 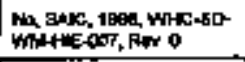 & 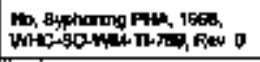 & 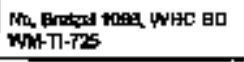 & Arourred LCب \\
\hline $2 A 1-1-201$ & 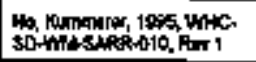 & 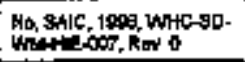 & 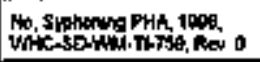 & 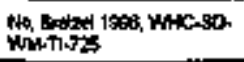 & 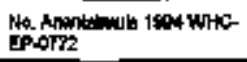 \\
\hline $2+1-x+2$ & 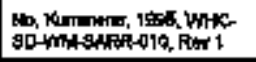 & 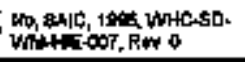 & 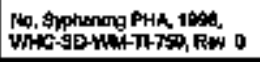 & 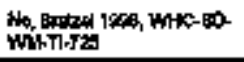 & 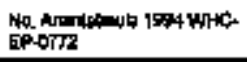 \\
\hline $2+1+$ thens & 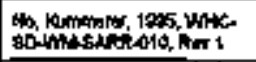 & 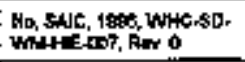 & 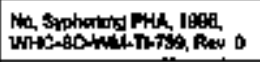 & 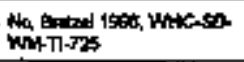 & 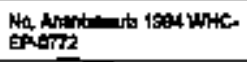 \\
\hline $2,1-204$ & 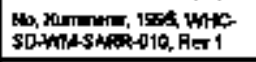 & 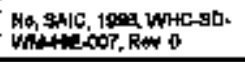 & Wo. Spploning PHA, 1904, & 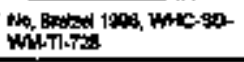 & 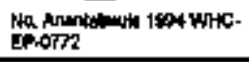 \\
\hline 344 & 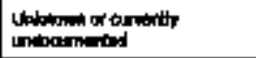 & 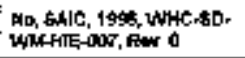 & 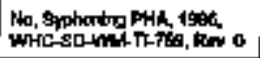 & 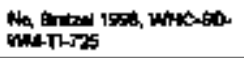 & 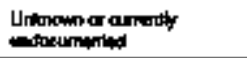 \\
\hline $2448 X$ & 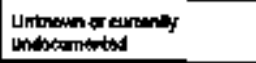 & 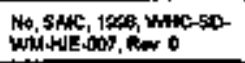 & Nh, Sphonng PHA, nas & 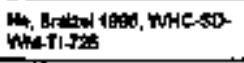 & 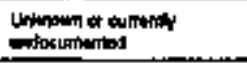 \\
\hline 244-CR & 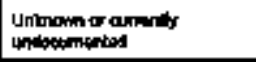 & 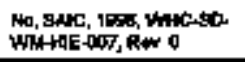 & 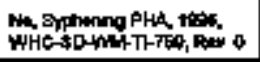 & 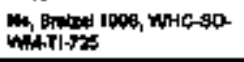 & 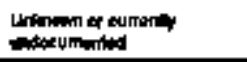 \\
\hline 2449 & 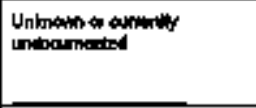 & 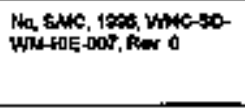 & 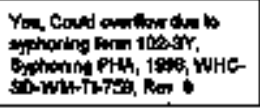 & 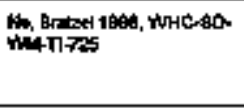 & Untorim or armerin \\
\hline $244 \pi x$ & 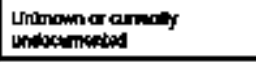 & 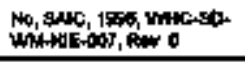 & 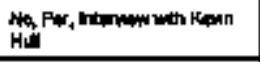 & Mathen Ism, MHC-so & 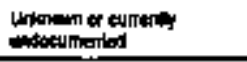 \\
\hline 244 & 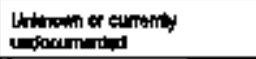 & 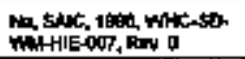 & 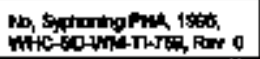 & 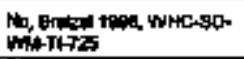 & 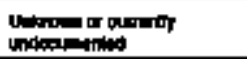 \\
\hline
\end{tabular}




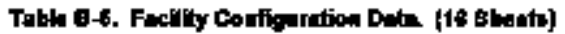

\begin{tabular}{|c|c|c|c|c|c|c|c|c|c|}
\hline find Rom & Thpe & 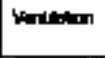 & Comintits & Alc & Gomber ALC & 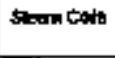 & cammind sc & 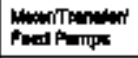 & Commenots in \\
\hline $2414-104$ & 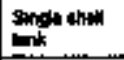 & 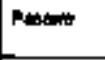 & 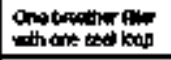 & $\omega$ & & No & & $\mathrm{No}$ & \\
\hline $241+102$ & gings: then & Prans & 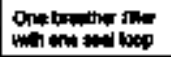 & $N$ & & No & & No & \\
\hline 2111.403 & sing & 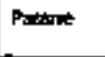 & Ont brember for & $\mathrm{NoO}$ & & h & & No & \\
\hline 2A1-A-106 & | & 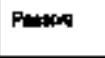 & 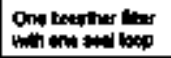 & No & & $\mathbf{N o}$ & & $\mathrm{No}$ & \\
\hline 2011) & $\sin _{\log }$ & Pasve & Onelartive cher & No & & $b$ & & No & \\
\hline 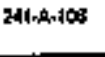 & Intion & Pansin & 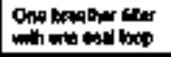 & No & & $\omega$ & & Ho & \\
\hline $\begin{array}{l}\text { 261-AN/. } \\
\text { I91 }\end{array}$ & Oovele whel & Ante & 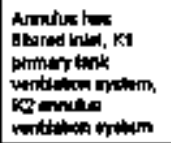 & No & & ito & & $\omega$ & \\
\hline $\begin{array}{l}241+1016 \\
102\end{array}$ & Oovite bhell & Nonise & 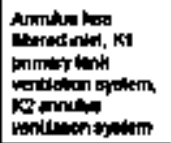 & No & & $\mathbf{m o}$ & & No & \\
\hline 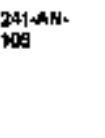 & 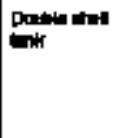 & Aater & 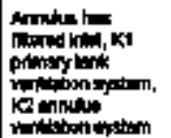 & 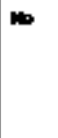 & & No & & $\mathbf{b b}$ & \\
\hline
\end{tabular}




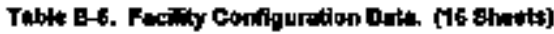

\begin{tabular}{|c|c|c|c|c|c|c|c|c|c|}
\hline 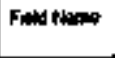 & $T_{\text {Yot }}$ & Vartaim & Comman & AxC & COAmmin ALCF & Sats Gots & 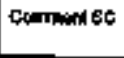 & 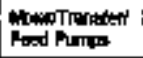 & 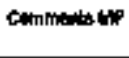 \\
\hline 241 AN & $\begin{array}{l}\text { Donbla find } \\
\text { fortk }\end{array}$ & whe & 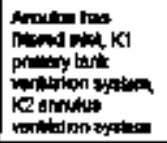 & No & & No & & Ho & \\
\hline $\begin{array}{l}2+1+A H \\
105\end{array}$ & Omitcoted & natur & 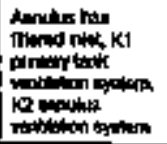 & $N$ & & $\mathrm{kto}$ & & $\mathbf{b}$ & \\
\hline 100 & 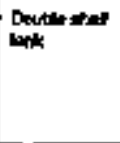 & Antitat & 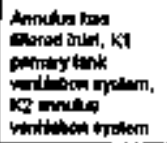 & No & & No & & bl & \\
\hline $\begin{array}{l}\text { 2011AM. } \\
\text { 167 }\end{array}$ & Comite & Netm & 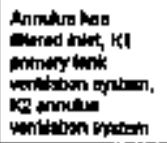 & Yex & & No & & $m$ & \\
\hline 241 A.101 & $\begin{array}{l}\text { Doutsite and } \\
\text { twit }\end{array}$ & indret & 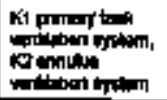 & mo & & mo & & wo & \\
\hline $241-A P-162$ & 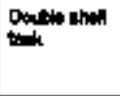 & Apter & גations & Ho & & W & & 禹 & \\
\hline
\end{tabular}




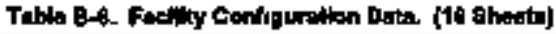

\begin{tabular}{|c|c|c|c|c|c|c|c|c|c|}
\hline Fit Pteme & Trpe & 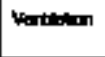 & Edoment & אעו & Commertivise & 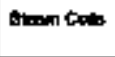 & Comeract & 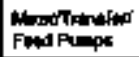 & 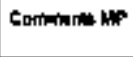 \\
\hline $24+4 \cdot-100$ & $\begin{array}{l}\text { Doutio that } \\
\text { tolt }\end{array}$ & whth & 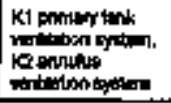 & 160 & & W & & No & \\
\hline 2414AP-104 & $\begin{array}{l}\text { Dorkida dinith } \\
\text { tink }\end{array}$ & Actre & 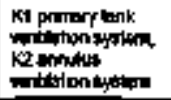 & $\omega$ & & Na & & bo & \\
\hline $241 \rightarrow P-105$ & $\begin{array}{l}\text { poptik dhel } \\
\text { init }\end{array}$ & Aciven & 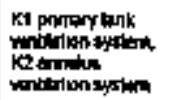 & 10 & & No & & No & \\
\hline $241+0.909$ & $\begin{array}{l}\text { Couphotat } \\
\text { think }\end{array}$ & Nebove & 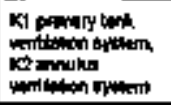 & Noto & & No & & $m$ & \\
\hline 241.4P.907 & 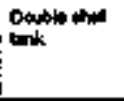 & Antmo & 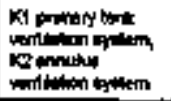 & No & & No & & is & \\
\hline 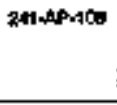 & 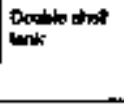 & Natas & 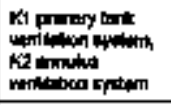 & No & & $\mathbf{W}$ & & $\omega$ & \\
\hline iton & Doutit and & Astit & 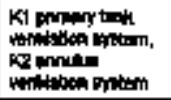 & 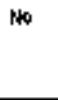 & & w & & $N$ & \\
\hline $\begin{array}{l}241+402 \\
102\end{array}$ & 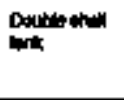 & interte & 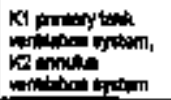 & Yos & & $\mathbf{w}$ & & 10 & \\
\hline
\end{tabular}




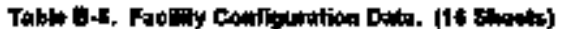

\begin{tabular}{|c|c|c|c|c|c|c|c|c|c|}
\hline Fatd Nume & $\operatorname{Trn}$ & Yuntibithont & 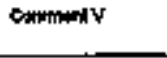 & MC & Commint ald & 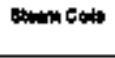 & Dempont at: & 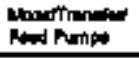 & Dommell, uP \\
\hline 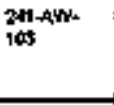 & 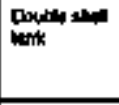 & 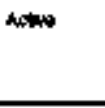 & 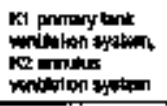 & $\mathrm{NoO}$ & & No & & No & \\
\hline 104 & $\begin{array}{l}\text { Doult thatl } \\
\text { barle }\end{array}$ & Netrit & 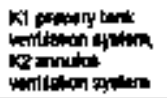 & No & & No & & bا & \\
\hline $\begin{array}{l}241+N W: \\
105\end{array}$ & Doubte thell & Ache & 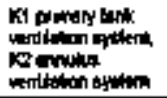 & No & & ib & & Ho & \\
\hline $\begin{array}{l}241 \text { ald } \\
109\end{array}$ & 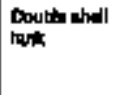 & Actur & 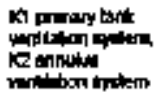 & Ho & & wh & & $b$ & \\
\hline 2N1AX-901 & $\begin{array}{l}\text { Bnyte she } \\
\text { intic }\end{array}$ & Drater & 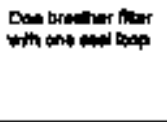 & la & list & ws & & no & \\
\hline 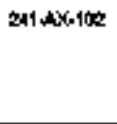 & $\begin{array}{l}\text { Singer atwal } \\
\text { ind }\end{array}$ & Powe & $\begin{array}{l}\text { Do broother hlar } \\
\text { wth ane wes bopp }\end{array}$ & the & 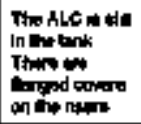 & $\mathbf{m o}$ & & $m$ & \\
\hline $241+0103$ & 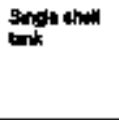 & Paverate & 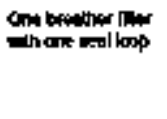 & $N$ & 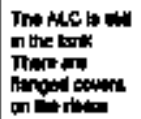 & $\omega$ & & $\omega$ & \\
\hline
\end{tabular}




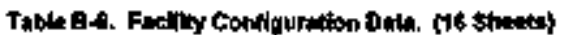

\begin{tabular}{|c|c|c|c|c|c|c|c|c|c|}
\hline Farda Neme. & $T_{y p}$ & Mrintan & Comed V & ALCC & 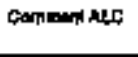 & 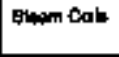 & Cormend EC & 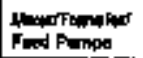 & 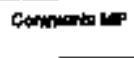 \\
\hline $241 \wedge x-104$ & $\begin{array}{l}\text { Engor ahed } \\
\text { indik }\end{array}$ & Parowe & 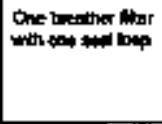 & No & 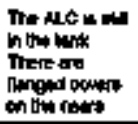 & No & & No & \\
\hline 2A1AYY.101 & Countostall & Antors & 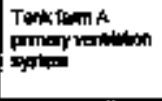 & $Y=9$ & & No & 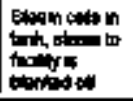 & ib & \\
\hline 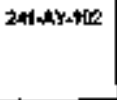 & $\begin{array}{l}\text { Doultivet thet } \\
\text { bit }\end{array}$ & Achitu & 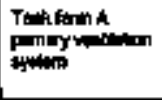 & $Y=$ & & No & 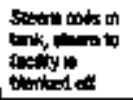 & $\mathrm{lb}$ & \\
\hline $24-A \geq-101$ & Doutt titel & Aoton & 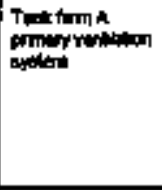 & Yex & & $N 0$ & 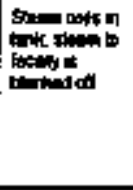 & $\mathbf{b}$ & 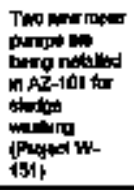 \\
\hline $261+42 \cdot 102$ & $\begin{array}{l}\text { Dontow that } \\
\text { tex }\end{array}$ & Notsotsots & 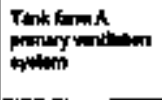 & $r$ & & No & 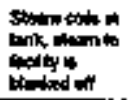 & Ho & \\
\hline $241-8-101$ & anpor thet & Paneren & 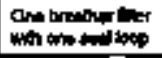 & ib & & $\omega$ & 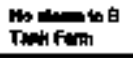 & $\mathbf{H}$ & \\
\hline $241+100$ & $\begin{array}{l}\text { singt ahed } \\
\text { lnk }\end{array}$ & Pandere & Che brithe char & No & & $\mathbf{m}$ & 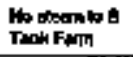 & $n$ & \\
\hline $241+4-103$ & $\begin{array}{l}\text { Englosing } \\
\tan \end{array}$ & Poran & 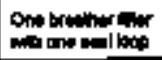 & 10 & & No & L & $w$ & \\
\hline $241-8-104$ & 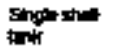 & מרוק & 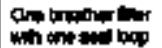 & wa & & Now & 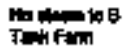 & $\boldsymbol{H}$ & \\
\hline
\end{tabular}


Tablo B-6. Fuelly Configuration Data (16 Ginets)

\begin{tabular}{|c|c|c|c|c|c|c|c|c|c|}
\hline FoddNon & $T_{x}$ & Herminkm & Comminent & Me & $C$ & sth & Conment SC & 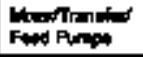 & م Comentis \\
\hline $246-105$ & $\begin{array}{l}\text { Bngte the } \\
\text { then }\end{array}$ & 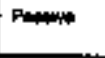 & 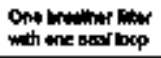 & No & & Ho & $\begin{array}{l}\text { Nothomol } \\
\text { Font Fom }\end{array}$ & $\mathrm{No}$ & \\
\hline $24+8-100$ & 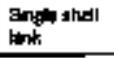 & Pand & 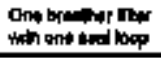 & Ho & & No & 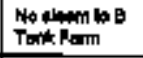 & it & \\
\hline $2+14-16$ & tong: the & Prosin & 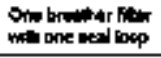 & $\mathrm{NoH}$ & & No & 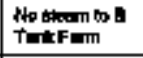 & $\mathbf{N o}$ & \\
\hline $241-8-100$ & $\lim _{\lim }$ & Pansin & 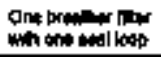 & 160 & & $\mathbf{m o}$ & 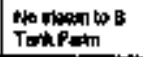 & $\omega$ & \\
\hline $2494 \div 109$ & singe the & Pustrum & 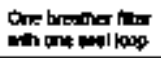 & Mo & & 10 & 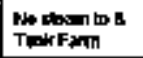 & $\mathbf{H b}$ & \\
\hline $341-8-110$ & ال: & Pressin & Ore bather ine & *6 & & $\boldsymbol{M}$ & 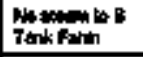 & W6 & \\
\hline $241 \cdot \beta-111$ & 6ogt hed & Ptate & 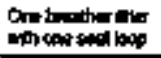 & wa & & No & 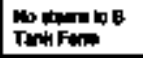 & 16 & \\
\hline $241 \cdot 8-112$ & $\begin{array}{l}\text { Singin thel } \\
\text { bati }\end{array}$ & Panow & 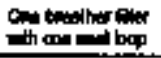 & $\omega$ & & Ho & 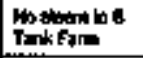 & in & \\
\hline $2416-201$ & $\begin{array}{l}\text { Singl that } \\
\text { torn }\end{array}$ & Pand & 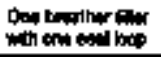 & $\mathbf{m}$ & & Non & 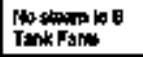 & $\omega$ & \\
\hline $241+402$ & 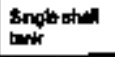 & 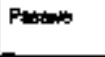 & 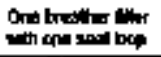 & bo & & No & 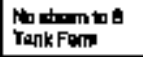 & No & \\
\hline $241+1-200$ & $\begin{array}{l}\text { Sholn ahen } \\
\text { the }\end{array}$ & Panew & 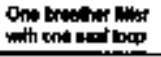 & $\omega$ & & $\mathrm{NO}$ & 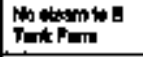 & $N 0$ & \\
\hline $24+6-204$ & $\begin{array}{l}\text { Sholorat } \\
\text { botit }\end{array}$ & Pan & 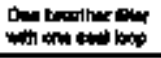 & $\mathbf{w n}$ & & $\mathbf{~} \mathbf{b}$ & The & $\omega$ & \\
\hline 241-4xact & $\begin{array}{l}\text { angle ahy } \\
\text { tanc }\end{array}$ & Nant & 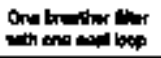 & $\mathbf{m}$ & & No & 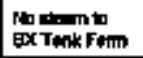 & Non & \\
\hline 2011-10k-102 & $\begin{array}{l}\text { 6nd the the } \\
\text { tank }\end{array}$ & Fomingen & 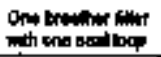 & $\omega$ & & ito & 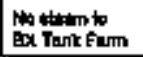 & $\mathrm{Ha}$ & \\
\hline
\end{tabular}




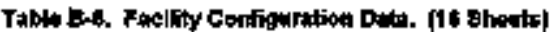

\begin{tabular}{|c|c|c|c|c|c|c|c|c|c|}
\hline Find & Typ & 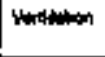 & ctoment & تصام & 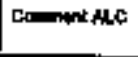 & Bath collo & Conmed & Food Ponper & Oanmatio in \\
\hline 241 exdos & sangen whed & Pн⿻上丨 & 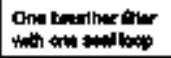 & $\mathbf{N o}$ & & 100 & $\begin{array}{l}\text { No mosm b } \\
\text { MK Tank Fum }\end{array}$ & No & \\
\hline 241-botod & $\begin{array}{l}\text { Injed anel } \\
\text { bon }\end{array}$ & Fanm & 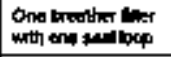 & No & & no & $\begin{array}{l}\text { No shom to } \\
\text { BX Turk Finn }\end{array}$ & No & \\
\hline $241-B \times 105$ & 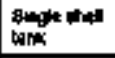 & Fospano & 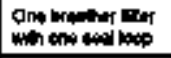 & No & & Mos & 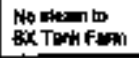 & No & \\
\hline $241+0$ - & $\begin{array}{l}\text { sige thed } \\
\text { biti }\end{array}$ & Fanene & 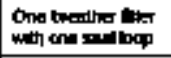 & no & & No & 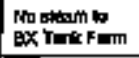 & No & \\
\hline $241-8 x-197$ & 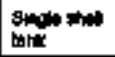 & Patstont & One thather linor & No & & w & 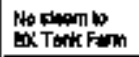 & ito & \\
\hline $241-B x_{10}$ & $\begin{array}{l}\text { Sngh the } \\
\text { toptin }\end{array}$ & Pasain & 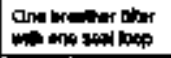 & Ho & & $\mathbf{m}$ & 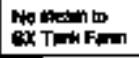 & No & \\
\hline $241-B \times-109$ & البn the & Patse & 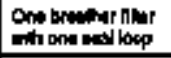 & $\mathbf{N o}$ & & W & 的 & 16 & \\
\hline $2410 \times 110$ & Singl* then & Poner & 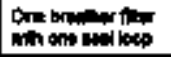 & $\mathrm{NOO}$ & & 10 & 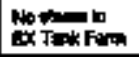 & mos & \\
\hline $241-46-111$ & $\begin{array}{l}\text { Sindththel } \\
\text { but }\end{array}$ & Pane & 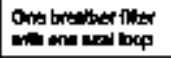 & No & & 10 & 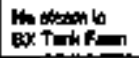 & 16 & \\
\hline $26-8 \times-112$ & $\begin{array}{l}\text { Bnolu and } \\
\text { bift }\end{array}$ & Patsots & 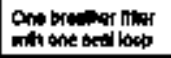 & 10 & & 6 & 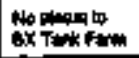 & $\omega b$ & \\
\hline $241-8 \times 101$ & Single the & Desond & 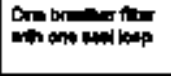 & $\mathrm{Ha}$ & 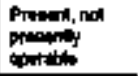 & ito & 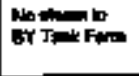 & $b$ & \\
\hline $241+\theta 4 \cdot 1+202$ & 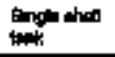 & Pwand & 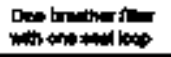 & a & & No & 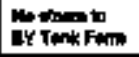 & Wo & \\
\hline 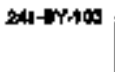 & thet athal & Pant & 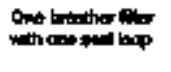 & 10 & 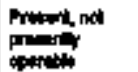 & $\mathrm{No}$ & 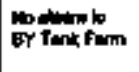 & No & \\
\hline
\end{tabular}




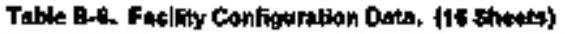

\begin{tabular}{|c|c|c|c|c|c|c|c|c|c|}
\hline 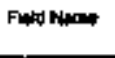 & Type & Verimben & comant & ANF & 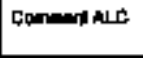 & Sate & Cormextst & 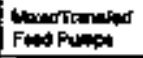 & Cominger \\
\hline $291 / 4 T-104$ & 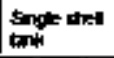 & Pander & 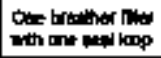 & No & & Ila & 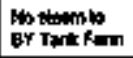 & $\mathrm{Na}$ & \\
\hline 2A1.EY-105 & $\begin{array}{l}\text { Sngh tho } \\
\text { and }\end{array}$ & 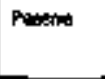 & Oan minther lnor & No & 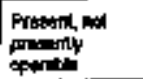 & *to & 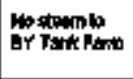 & No & \\
\hline $20-8$ r-104 & 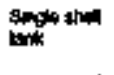 & Pasans & 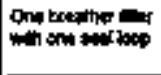 & $\mathbf{H o}$ & Phand nok & $\mathbf{m o}$ & 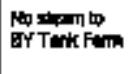 & $\mathbf{A b}$ & \\
\hline 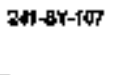 & 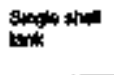 & Pт⿻上丨 & 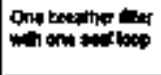 & Wo & Parand $n \alpha$ & $\mathbf{m}$ & 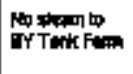 & $\mathbf{1 6}$ & \\
\hline $2 \Leftrightarrow-10 Y-109$ & 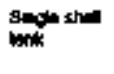 & Passana & Ond betither & us & Pandis nd & $\mathbf{1 6}$ & 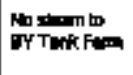 & ab & \\
\hline $241-8 \times-100$ & Ingent then & Panom. & 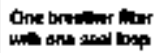 & No & & $\mathbf{N b}$ & 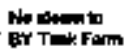 & Hs & \\
\hline $241.9 Y-110$ & 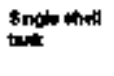 & Pantent & 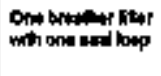 & No & 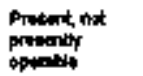 & m & ofto & m & \\
\hline $241-a Y-111$ & 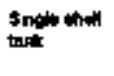 & Pantont & 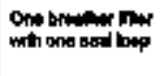 & Not & 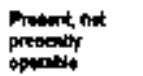 & $\omega$ & Not Tank Fam & ib & \\
\hline $241+a y-192$ & ln & Fmomt & 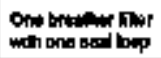 & Not & & No & 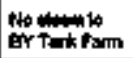 & $m$ & \\
\hline 2011-c-ky & 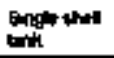 & Propenos & 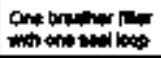 & No & & Not & C & No & \\
\hline 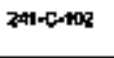 & 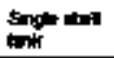 & Popran & 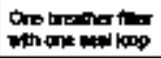 & 140 & & 100 & Thin Find & Not & \\
\hline 2A-6NOS & Singlent & 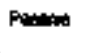 & 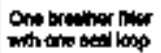 & No & & Nota & 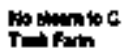 & No & \\
\hline
\end{tabular}




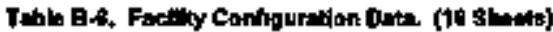

\begin{tabular}{|c|c|c|c|c|c|c|c|c|c|}
\hline Food hbment & 7 rax & Vathon & Awrmert $v$ & عله & 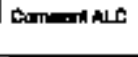 & 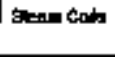 & Cevinent axc & 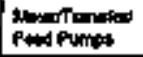 & 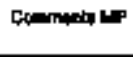 \\
\hline $201=-104$ & $\begin{array}{l}\text { sarde ahdil } \\
\text { brith }\end{array}$ & Pan & 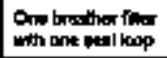 & Noto & & No & 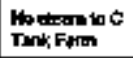 & h & \\
\hline $241-6-185$ & 8nots athil & Aeئing & Ediandier & $\mathrm{NHO}$ & & ito & 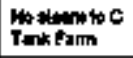 & $\omega$ & \\
\hline $245-100$ & Siloge ahel & Adero & EAromerer & $\mathrm{No}$ & & $\mathbf{N o}$ & 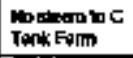 & $\mathrm{mb}$ & \\
\hline $241-c-10 r$ & singenthe & Panaw & 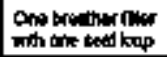 & NoO & & 10 & 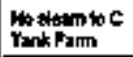 & Ho & \\
\hline 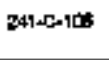 & $\begin{array}{l}\text { strige thed } \\
\text { tonte }\end{array}$ & Panen & 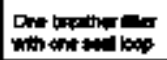 & No & & $\mathrm{Ha}$ & Trink Fam in & $\mathbf{m a}$ & \\
\hline $2+1+C-109$ & $\begin{array}{l}\text { Snowe thell } \\
\text { boit }\end{array}$ & Paterto & 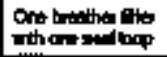 & No & & o & 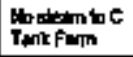 & its & \\
\hline 241-c-110 & $\begin{array}{l}\text { Sngle the } \\
\text { tont }\end{array}$ & Prisus & 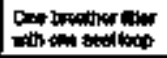 & No & & 10 & 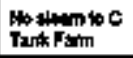 & $\omega$ & \\
\hline 2A1-t_111 & snjent thel & Ptitur & 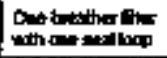 & Ho & & bo & 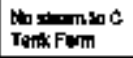 & bb & \\
\hline $241-c-112$ & land & Pantis & 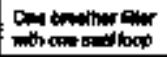 & No & & k0 & 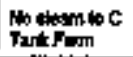 & m & \\
\hline $261<4001$ & singterind & Pand & 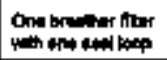 & Wb & & No & 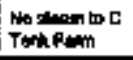 & Not & \\
\hline $241<-202$ & 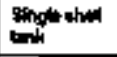 & Rumpt & 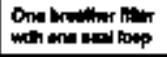 & No & & No & 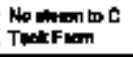 & $N$ & \\
\hline $251-6-304$ & gande thin & 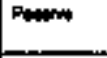 & 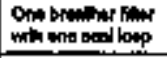 & $\mathbf{H o}$ & & N & The & Note & \\
\hline $2 \times 1-2004$ & $\begin{array}{l}\text { angle and } \\
\text { lank }\end{array}$ & Anen & 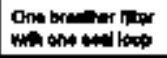 & $\mathrm{No}$ & & $\mathrm{Na}$ & 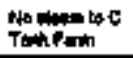 & No & \\
\hline $241-4-10 t$ & $\begin{array}{l}\text { singt ahed } \\
\text { inip }\end{array}$ & Panowe & 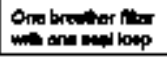 & No & & Non & 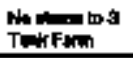 & $\mathrm{No}$ & \\
\hline
\end{tabular}




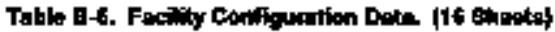

\begin{tabular}{|c|c|c|c|c|c|c|c|c|c|}
\hline Nuta bonte & Type & Vorilfhent & cermat $V$ & ALC & Comentithto & 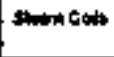 & Cknment & 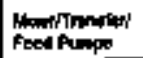 & Commonth kn \\
\hline $241-5-102$ & Fing the & Poorina & Ond boniline flum & up & & No & 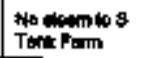 & 10 & \\
\hline $741-8-104$ & Eingh & Panpin & 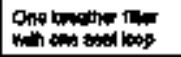 & ito & & Ho & 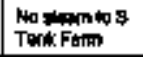 & mo & \\
\hline 24t-s-104 & 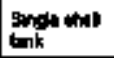 & Powors & 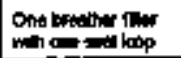 & Wo & & $\mathrm{NHO}$ & 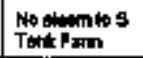 & 16 & \\
\hline 241-E-106 & șige par & Pappin: & 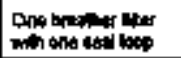 & Na & & ino & 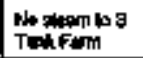 & No & \\
\hline $241-5-100$ & $\begin{array}{l}\text { sngt the } \\
\text { tont }\end{array}$ & Portim & 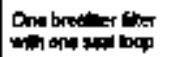 & Noto & & Ho & 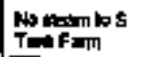 & Noto & \\
\hline $241-5-100$ & Shen & Prapon & 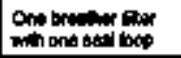 & $\mathrm{NoO}$ & & No & 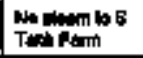 & $N$ & \\
\hline 241-2010s & Snctind & Prever & 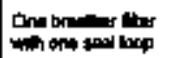 & Nó & & in & 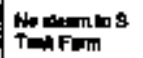 & Noto & \\
\hline $2,18-103$ & 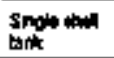 & Patown & 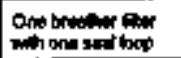 & No & & no & Wh betom 5 & No & \\
\hline 2*1-5-190 & $\begin{array}{l}\text { ingle thed } \\
\text { turir }\end{array}$ & 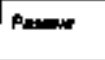 & 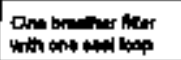 & Non & & $\mathbf{m b}$ & 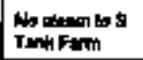 & Ma & \\
\hline $2 h-b-111$ & 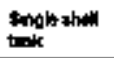 & Pantot & 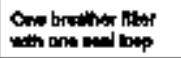 & No & & $\mathbf{1 0}$ & 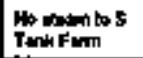 & No & \\
\hline 24-8-112 & 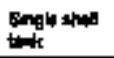 & 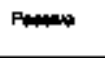 & 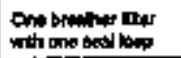 & No & & No & 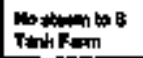 & 100 & \\
\hline 201-\$x-101 & tengletat & Antro & 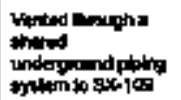 & No & & wo & 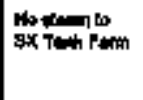 & No & \\
\hline $241-5 x-102$ & Englatsol & Antin & 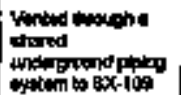 & No & & w & $\operatorname{lom}_{\mathrm{S}}$ & in & \\
\hline
\end{tabular}




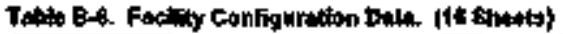

\begin{tabular}{|c|c|c|c|c|c|c|c|c|c|}
\hline Fow Kan & Typ & Verianimen & Carimertit & تصب & Comintim all? & shmoch & corment $3 c$ & 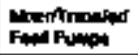 & 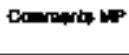 \\
\hline $2 * 1-\$ x-140$ & $\begin{array}{l}\text { Songle boll } \\
\text { tion }\end{array}$ & Natome & 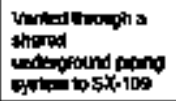 & $m$ & & $\mathbf{H o}$ & 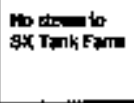 & $\mathbf{w o}$ & \\
\hline $2+1-6 x-104$ & 舟 & Nothen & 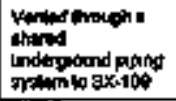 & $\mathrm{Mat}$ & & m & $\begin{array}{l}\text { No starmito } \\
\text { EX Torte Fomm }\end{array}$ & $\Delta$ & \\
\hline $241+5 \times 105$ & 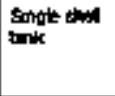 & hotat & 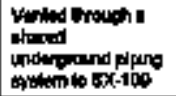 & Ha & & $\omega$ & 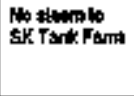 & Bat & \\
\hline 341 自x $x-100$ & tomple & natm & 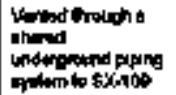 & Non & & $\omega$ & 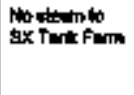 & $m$ & \\
\hline $2-19-8 x-10 x$ & 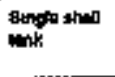 & مA & 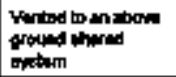 & No & & Na & $\begin{array}{l}\text { No weemb } b \\
\text { BXX Tart Fump }\end{array}$ & No & \\
\hline $241-82 x-108$ & 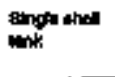 & indin & 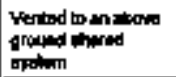 & No & & to & 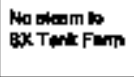 & No & \\
\hline $241+60<-109$ & 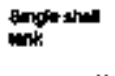 & Actron & 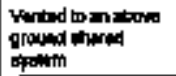 & $\mathbf{N a}$ & & Do & 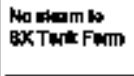 & to & \\
\hline $2+1-8 x-110$ & 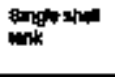 & Anthes & 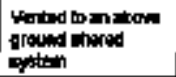 & $\mathrm{No}$ & & ab & 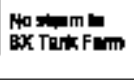 & 140 & \\
\hline $2+1-3 x-111$ & Shon & phas & 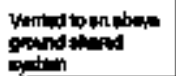 & he & & $N$ & 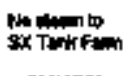 & $* 0$ & \\
\hline
\end{tabular}




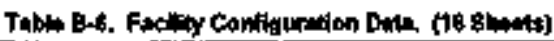

\begin{tabular}{|c|c|c|c|c|c|c|c|c|c|}
\hline 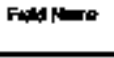 & Trpe & Voriluthen & $C(-m p d v$ & All C & Cemment the & 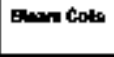 & Cen meal se & 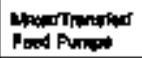 & CTamene MP \\
\hline $241-a_{x}-112$ & Sthot dhet & Ants & 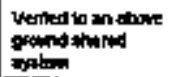 & No & & Not & 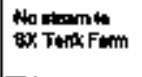 & No & \\
\hline $241-3 \times-1+3$ & Singer thel & Pawe & & $\mathrm{Ho}$ & & Nto & 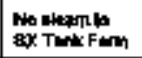 & No & \\
\hline $241-8 x-114$ & Englath & Antis & 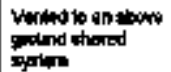 & Nop & & No & 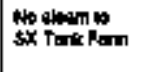 & Ho & \\
\hline $241-9 x-115$ & and & Pepentin & & No & & No & 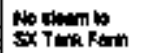 & Ho & \\
\hline $201-5 \%-101$ & $\begin{array}{l}\text { Dowela the } \\
\text { Initi }\end{array}$ & thatat & 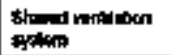 & No & & No & No mento & $Y=$ & \\
\hline $2+1-5 \times 102$ & $\begin{array}{l}\text { Ooxth bhell } \\
\text { link }\end{array}$ & Nente & 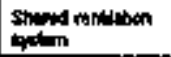 & $\mathrm{NHO}$ & & No & S4 & $Y_{t}$ & \\
\hline $2+1-8 Y-106$ & $\begin{array}{l}\text { Doupke phetl } \\
\text { ink }\end{array}$ & nothen & tophen & No & & Ho & ST Tok fow & $\mathbf{m}$ & \\
\hline 241-T.401 & 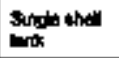 & מה:מק & 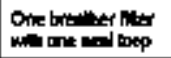 & Ho & & Ho & & $\mathbf{l b}$ & \\
\hline $241-T-202$ & singor and & Puasta & 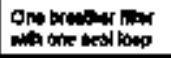 & Noto & & 190 & & W & \\
\hline $241+7=100$ & 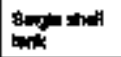 & Panter & 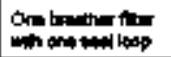 & $\mathrm{Ha}$ & & Ho & & $\mathbf{W b}$ & \\
\hline $241-T-104$ & tim & Pанған & 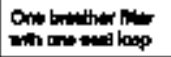 & to & & tw & & wo & \\
\hline $741-7-105$ & اله & Pronis & 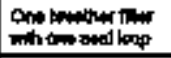 & No & & $\mathbf{m o}$ & & N & \\
\hline $34 \mid-T-10 d$ & $\begin{array}{l}\text { shose the } \\
\text { and }\end{array}$ & Penere & Drebother the & a. & & $\mathbf{m b}$ & & No & \\
\hline
\end{tabular}




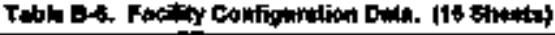

\begin{tabular}{|c|c|c|c|c|c|c|c|c|c|}
\hline 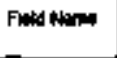 & Typ & Yantuliten & CommentY & ALC & Comment all & gatum Cody & Cominumat & 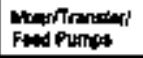 & Cammentap up \\
\hline 2स1-TAE & 6ngin biel & Paner & 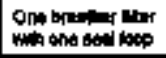 & Wh & & wo & & $\mathbf{N a}$ & \\
\hline 2andtaras & sidget thets & האונה & 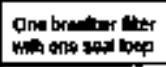 & $\omega$ & & $\mathrm{No}$ & & ino & \\
\hline 2n-T-109 & $\operatorname{sing}_{\text {the }}$ & Ратин: & 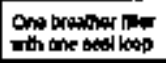 & $\mathrm{No}$ & & $m$ & & $\omega$ & \\
\hline $2+1 \cdot \mathbf{r}-1$ th & $\operatorname{sinq}$ & Patare & 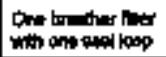 & $\mathrm{Na}_{\mathrm{b}}$ & & $\boldsymbol{b}$ & & ib & \\
\hline 2k1.T.1t1 & 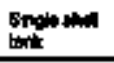 & Pa:me & $\begin{array}{l}\text { Ont bot:ther low } \\
\text { wh ont and losp }\end{array}$ & No & & $\mathbf{W}$ & & $\mathbf{m}$ & \\
\hline $2 \times 1-T-162$ & Sngte and & 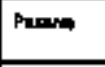 & Dre & $\mathrm{NHa}$ & & no & & b & \\
\hline $244.7-301$ & single & Pators & 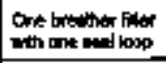 & No & & w & & $\omega$ & \\
\hline $241-T-200$ & andis and & Prean & 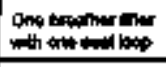 & $\mathrm{No}$ & & Non & & $\boldsymbol{n}$ & \\
\hline $241+7,200$ & 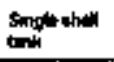 & Ptring & 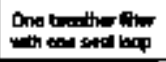 & No & & 禹 & & $\omega$ & \\
\hline 2)1-7-204 & $\begin{array}{l}\text { Bingle 4h } \\
\text { the }\end{array}$ & 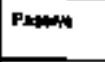 & 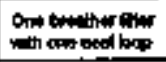 & $\mathrm{No}$ & & $\mathrm{Nu}$ & & 14 & \\
\hline $241-17 \mathrm{X}-101$ & andiatid & Pand & 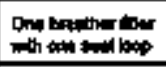 & No & & in & & $\mathbf{m}$ & \\
\hline 2a1-TK-10大 & $\begin{array}{l}\text { Singte ahe } \\
\text { int }\end{array}$ & Ponche & 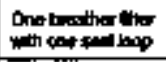 & No & & No & & $m$ & \\
\hline $241.5 x-100$ & Sill & Panom & 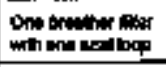 & $\omega$ & & $\mathrm{NO}$ & & No & \\
\hline 241-Tर-104 & 5nd & $\mathrm{R}$ & 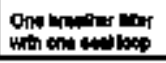 & na & & Na & & Non & \\
\hline
\end{tabular}


Table B-6. Factity Configuration Deth. (14 shocks)

\begin{tabular}{|c|c|c|c|c|c|c|c|c|c|}
\hline 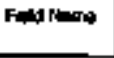 & Typo & Natim & corrmert V & ALC & 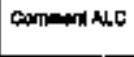 & gient Co:s & Conmert ac & 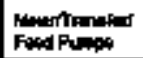 & 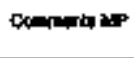 \\
\hline 345.Tx-10s & أingli & PMne & 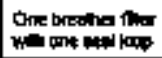 & $\mathbf{m b}$ & & $\mathbf{b}$ & & mo & \\
\hline $241-T \times \cdot 106$ & 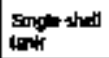 & Patom & 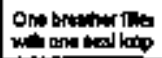 & m & & is & & 为 & \\
\hline $241 \cdot \mathrm{T}=10 \mathrm{~T}$ & $\begin{array}{l}\text { Bngtip thal } \\
\text { tank }\end{array}$ & Prape & 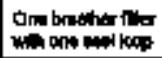 & $\mathbf{m o}$ & & no & & mo & \\
\hline $241-T K=104$ & $\begin{array}{l}\text { tonglevell } \\
\text { ind }\end{array}$ & Patste & 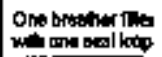 & Ho & & $\mathrm{m}$ & & 急 & \\
\hline $241-\Gamma x-100$ & $\begin{array}{l}\text { Singte and } \\
\text { tonk }\end{array}$ & Pration & 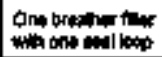 & $\mathbf{W 0}$ & & Ho & & 10 & \\
\hline $241-T K$ - tho & الهh & Prene & 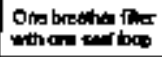 & wb & & ib & & ib & \\
\hline $241-T x-511$ & shotet thet & 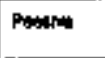 & 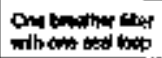 & No & & No & & No & \\
\hline $241+T x_{1}+12$ & أس & Panr & 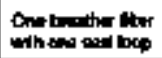 & No & & No & & Noto & \\
\hline 241-TX-113 & bإ & Punt & 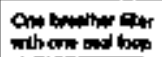 & No & & $\mathrm{Nos}$ & & $\mathrm{km}$ & \\
\hline श्या-Tx-11 & 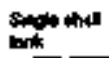 & Pansen & 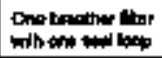 & Mo & & Nob & & Nto & \\
\hline $201-7 x-1+15$ & 8) & Panror & 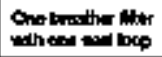 & No & & No & & No & \\
\hline $2+1-T X-110$ & thel thet & Pantant & 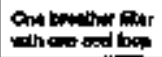 & No & & No & & no & \\
\hline $2+1 \cdot T \times-117$ & 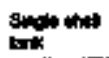 & Romisn & 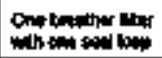 & No & & No & & No & \\
\hline $241 \cdot \mathrm{T}_{6} 119$ & Sping & Pante & 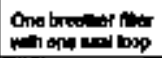 & No & & No & & $\omega$ & \\
\hline
\end{tabular}




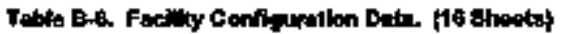

\begin{tabular}{|c|c|c|c|c|c|c|c|c|c|}
\hline Peid neme & Then & Verivinien & Commint $v$ & عله & Cominel the & Btamcals & 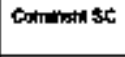 & 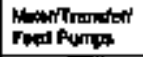 & Commenas MP \\
\hline $2 n-\Gamma Y-101$ & 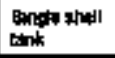 & Porain & 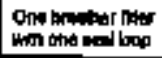 & $\mathbf{W o}$ & & ite & & Ho & \\
\hline $241-\Gamma Y-102$ & \begin{tabular}{|l} 
sagye thel \\
imin
\end{tabular} & Pater: & 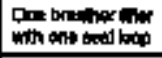 & No & & $\mathbf{m b}$ & & No & \\
\hline $241 \cdot T Y=103$ & 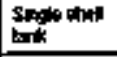 & Pa:me & 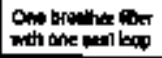 & into & & W & & No & \\
\hline 241-ГY-104 & Single thel & Prapu: & 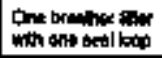 & Not & & $\mathbf{w}$ & & No & \\
\hline $241 \cdot T Y \cdot 100$ & stinge thel & Prester & Ore brtith a lines & Non & & $\mathbf{W}$ & & $\mathrm{Na}$ & \\
\hline $241-T \gamma+108$ & 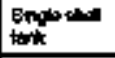 & Decomp & 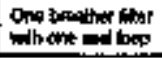 & No & & H. & & $\omega$ & \\
\hline अH-Utor & Ingt dis & Pane & 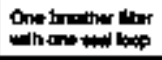 & No & & $N$ & & $\mathrm{bb}$ & \\
\hline 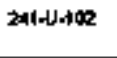 & Inter & 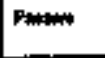 & 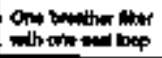 & No & & $\omega$ & & ob & \\
\hline $241-4-103$ & Angt & P=chan & 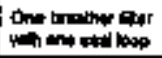 & $\mathrm{NoO}$ & & $N$ & & in & \\
\hline 241<1104 & $\begin{array}{l}\text { Singh ahdill } \\
\text { tontr }\end{array}$ & Panotwe & 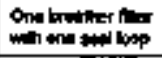 & no & & No & & $m$ & \\
\hline $2414-105$ & 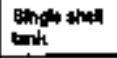 & Puitin & 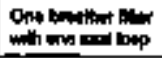 & 16 & & No & & w & \\
\hline $24-4-106$ & angtand & Pusern & 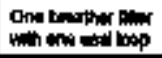 & 10 & & No & & 10 & \\
\hline $24 t+410$ & أling & Pand Pand & 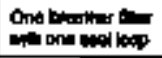 & no & & No & & ib & \\
\hline $2+1+4 \mid 100$ & 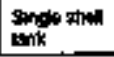 & Pawion & 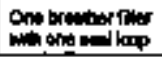 & $\omega$ & & in & & 的 & \\
\hline
\end{tabular}




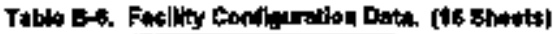

\begin{tabular}{|c|c|c|c|c|c|c|c|c|c|}
\hline Fied name & Y.pe & Vertiaten & Comanow $\forall$ & CLN & 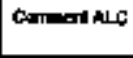 & Bnin con & comert gc & 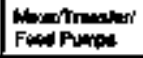 & cormenta \\
\hline 241 -1hates & 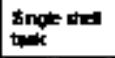 & Posine & Dre & tho & & No & & $\mathrm{Na}$ & \\
\hline $2+1-4-110$ & 6noll & Patain & 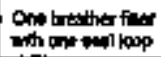 & tho & & Nor & & No & \\
\hline $241-2+111$ & $\begin{array}{l}\text { Ingle dhe } \\
\text { beik }\end{array}$ & Pr: & 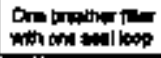 & tho & & No & & $\mathrm{Na}$ & \\
\hline $24-4-112$ & 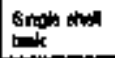 & Patsow & 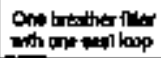 & No & & Not & & $\mathrm{No}$ & \\
\hline 241 山аख & Fingen shes & Paspan & 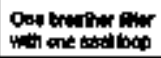 & $\mathrm{Na}$ & & No & & in & \\
\hline 241 小些 & $\begin{array}{l}\text { singoted ahel } \\
\text { ink }\end{array}$ & Pasone & 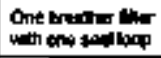 & No & & Ho & & $\mathrm{Ne}$ & \\
\hline $241-200$ & Find the & Pam:an & 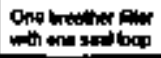 & Ho & & No & & ins & \\
\hline 241 dM:D4 & Enche shed & $\mathrm{P}=\mathrm{m}$ & Ond & $\mathrm{No}$ & & $\mathrm{HoO}$ & & ble & \\
\hline
\end{tabular}




\section{DLSTRIBUTION SHEET}

\begin{tabular}{|c|c|c|c|c|c|}
\hline \multirow{2}{*}{$\begin{array}{l}\text { To } \\
\text { Distribution }\end{array}$} & \multirow{2}{*}{\multicolumn{3}{|c|}{$\begin{array}{l}\text { From } \\
\text { B.d. Niems }\end{array}$}} & \multicolumn{2}{|l|}{ Pagt 1 of 1} \\
\hline & & & & \multicolumn{2}{|c|}{ Date $8 / 1 / 97$} \\
\hline \multirow{2}{*}{\multicolumn{4}{|c|}{$\begin{array}{l}\text { Frojact Tide/Nork Order } \\
\text { HWF-SD-in-TJ-764, Revision 1, Hazard Analysis Database Report }\end{array}$}} & \multirow{2}{*}{\multicolumn{2}{|c|}{$\begin{array}{l}\text { EOT No. } \mathrm{N} / \mathrm{A} \\
\text { ECN No. } 629254\end{array}$}} \\
\hline & & & & & \\
\hline Name & MSIN & $\begin{array}{l}\text { Toxt } \\
\text { With All } \\
\text { Attach. }\end{array}$ & Taxt Only & $\begin{array}{l}\text { Attuch } / \\
\text { Appendix } \\
\text { Only }\end{array}$ & $\begin{array}{c}\text { EDTJEON } \\
\text { Ondy }\end{array}$ \\
\hline 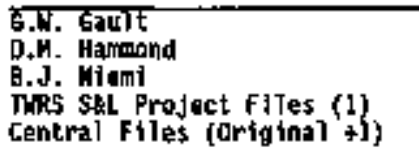 & $\begin{array}{l}k]-14 \\
R]-14 \\
k]-14 \\
k 2-26 \\
A 3-88\end{array}$ & $\begin{array}{l}x \\
x \\
x \\
x \\
x\end{array}$ & & & \\
\hline
\end{tabular}

